\title{
CHEMICAL COMPOSITION OF FERROMANGANESE CRUSTS IN THE WORLD OCEAN: A REVIEW AND COMPREHENSIVE DATABASE
}

Edited by

F.T. Manheim and C.M. Lane-Rostwick

Open-File Report 89-020

Version 1.1

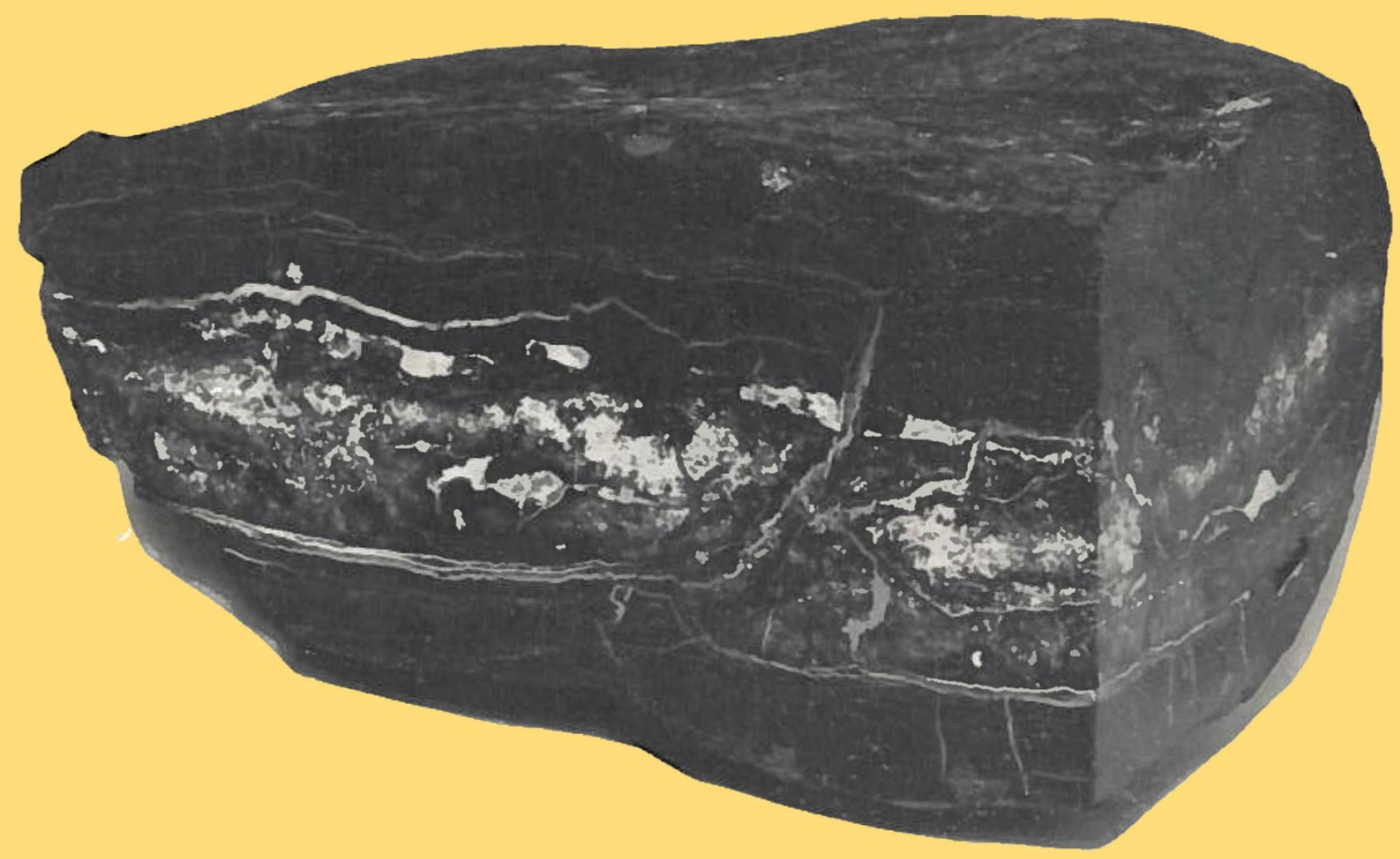

This report is preliminary and has not been reviewed for conformity with U.S. Geological Survey editorial standards and stratigraphic nomenclature. Any use of trade names is for descriptive purposes only and does not imply endorsement by the U.S.G.S. or M.M.S.

Woods Hole, MA 02543 



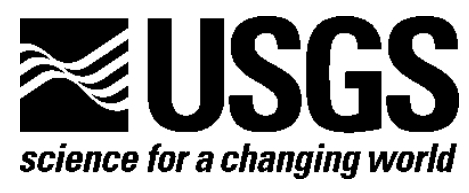

\section{Chemical Composition of Ferromanganese Crusts in the World Ocean: A Review and Comprehensive Database}

By Frank T. Manheim and Candice M. Lane-Bostwick

Open-File Report 89-020

Version 1.1, November 2014

U.S. Department of the Interior

U.S. Geological Survey 


\title{
U.S. Department of the Interior SALLY JEWELL, Secretary
}

\section{U.S. Geological Survey \\ Suzette M. Kimball, Acting Director}

\author{
U.S. Geological Survey, Reston, Virginia \\ First release: 1989 [in print] \\ Revised: October 2014 (ver. 1.1) [online]
}

For more information on the USGS-the Federal source for science about the Earth,

its natural and living resources, natural hazards, and the environment-visit

http://www. usgs.gov or call 1-888-ASK-USGS (1-888-275-8747)

For an overview of USGS information products, including maps, imagery, and publications, visit http://www.usgs.gov/pubprod

To order this and other USGS information products, visithttp://store.usgs.gov

Any use of trade, firm, or product names is for descriptive purposes only and does not imply endorsement by the U.S. Government.

Although this information product, for the most part, is in the public domain, it also may contain copyrighted materials as noted in the text. Permission to reproduce copyrighted items must be secured from the copyright owner.

Suggested citation:

Manheim, F.T., and Lane-Bostwick, C.M., eds., 2014, Chemical composition of ferromanganese crusts in the world ocean-A review and comprehensive database (ver. 1.1, November, 2014): U.S. Geological Survey Open-File Report 89-20, 476 p., http://dx.doi.10.3133/ofr8920V.1.1.

ISSN 2331-1258 (online) 


\section{U.S. Department of the Interior \\ Geological Survey}

\section{CHEMICAL COMPOSITION OF FERROMANGANESE CRUSTS IN THE WORLD \\ OCEAN: A REVIEW AND COMPREHENSIVE DATABASE}

\section{Edited by}

Frank T. Manheim and Candice M. Lane-Bostwick

Open File Report

$89-20$

Office of Energy and Marine Geology

Atlantic-Gulf Branch

Woods Hole, MA 02543

This report is preliminary and has not been reviewed for conformity with U.S. Geological Survey editorial standards and stratigraphic nomenclature. Any use of tradenames is for descriptive purposes only and does not imply endorsement by the USGS or MMS. 
CHEMICAL COMPOSITION OF FERROMANGANESE CRUSTS IN THE WORLD OCEAN

A REVIEW AND COMPREHENSIVE DATABASE

\section{Edited by}

Frank T. Manheim and Candice M. Lane-Bostwick

$$
\text { with contributions by }
$$

Vesna Marchig, German Geologic Survey, Hannover, FRG

Peter Halbach, Technical University Clausthal, Clausthal-Zellerfeld, FRG

Doris Puteanus, Technical Univerisity Clausthal, Clausthal-Zellerfeld, FRG

Benjamin W. Haynes, U.S. Bureau of Mines, Avondale, MD

Philip J. Aruscavage, U.S. Geological Survey, Reston, VA

Floyd M. Brown, U.S. Geological Survey, Reston, VA

Herbert K. Kirschenbaum, U.S. Geological Survey, Reston, VA

David M. Root, U.S. Geological Survey, Reston, VA

Judith A. Commeau, U.S. Geological Survey, Woods Hole, MA

Candice M. Lane-Bostwick, U.S. Geological Survey, Woods Hole, MA

Timothy H. Ling, U.S. Geological Survey, Woods Hole, MA

Frank T. Manheim, U.S. Geological Survey, Woods Hole, MA 
I INTRODUCTION by F.T. Manheim................... I

II HISTORY OF INVESTIGATION OF FERROMANGANESE OXIDE CRUST

by F.T. Manheim............................ 3

Shallow marine oxides...................... 10

Post-World War II advances; Mero's nodule mining

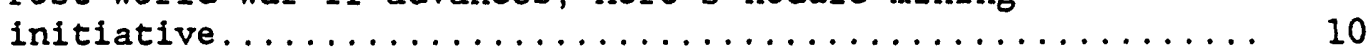

The 1970's and the Scripps Nodule Data Bank (SNDB)........ 11

National studies: Japanese, French, Russian............. 14

German studies and the "Midpac I" cruise.............. 16

U.S. national program and USGS Ferromanganese crust

database................................ 23

III ARCHIVE COLLECTIONS by T.H. Ling and F.T. Manheim........ 27

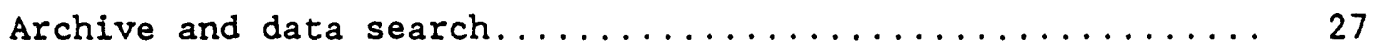

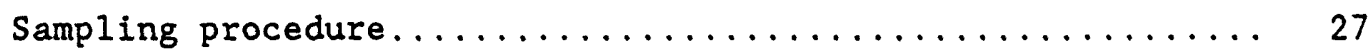

Institutional listings..................... 30

IV COMPUTER DATABASE DEVELOPMENT by C.M. Lane-Bostwick and

F.T. Manheim............................ 36

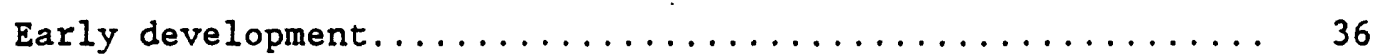

Scripps Institution Nodule Databank (SNDB) ............ 36

UNIX and IBM-PC - compatible hardware ............. 37

New data acquisitions...................... 37

$\checkmark$ ANALYTICAL METHODS: Sample preparation, water content and major element determination by $x$-ray fluorescence spectrometry

by J.A. Commeau and F.T. Manheim.................. 39

Sample selection and preparation................ 39

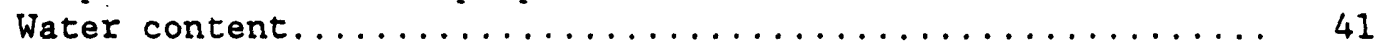

$\mathrm{X}$-ray fluorescence spectrometry................. 42

VI ANALYTICAL METHODS: The determination of 27 elements in

ferromanganese materials by P.J. Aruscavage and others........ 44

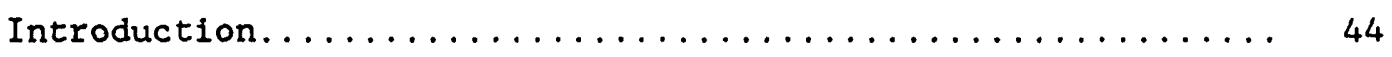

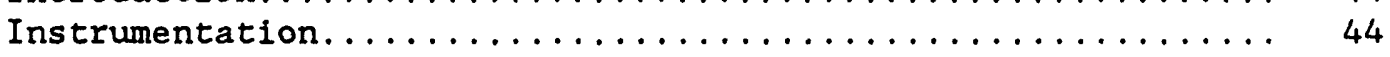

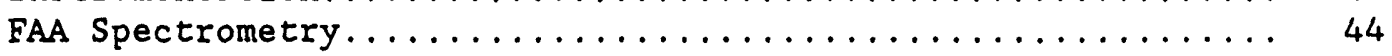

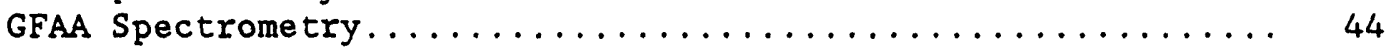

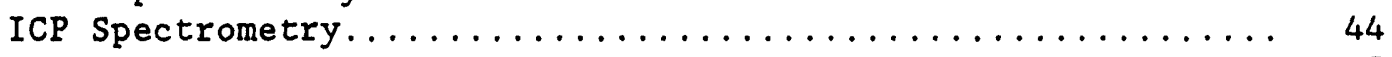

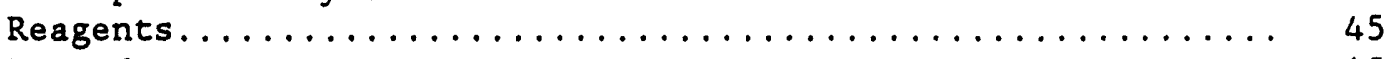

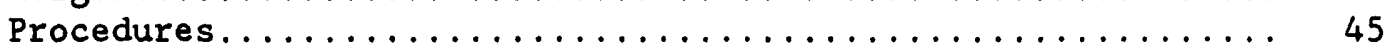

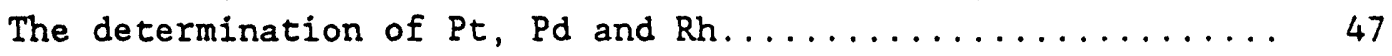

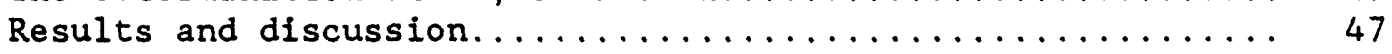


VII INTERIABORATORY COMPARISONS by F.T. Manheim, B.J. Haynes,

J.A. Commeau, and D. Root (with data of German Geological

Survey, V. Marchig; and Technical University, Clausthal-

Zellerfeld, P. Halbach and D. Puteanus)............... 54

Influence of grinding and sieving on analytical values..... 54

Interlaboratory calibration.................... 54

$\mathrm{H}_{2} \mathrm{O}^{+}$values............................... 56

VIII DATA by C.M. Lane-Bostwick and F.T. Manheim............. 59

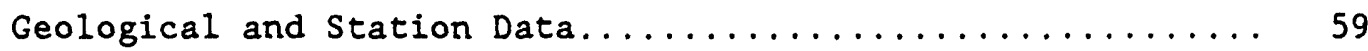

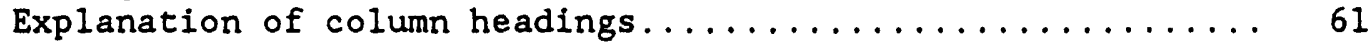

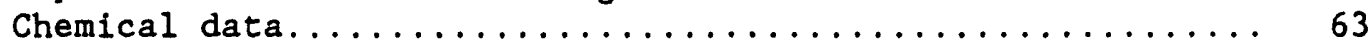

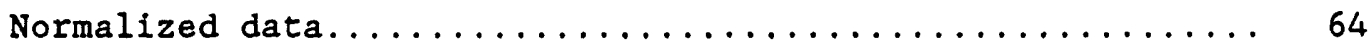

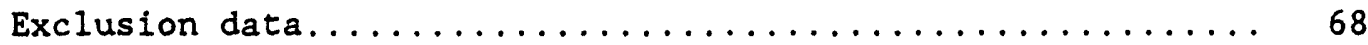

IX DATA INTERPRETATION AND MAPPING by F.T. Manheim and C.M. Lane-

Bostwick................................ 70

Frequency descriptions and regional means............ 70

Elemental concentration vs. water depth............. 77

Interelement plots........................ 77

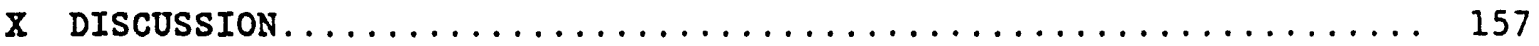

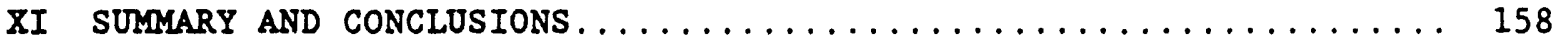

XII ACKNOWLEDGMENTS ............................ 159

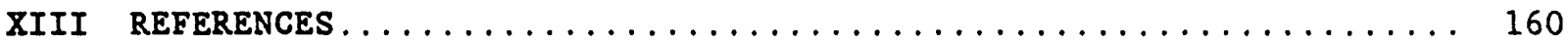

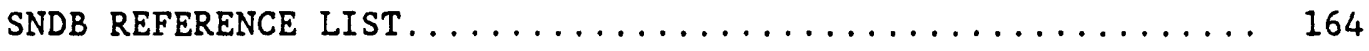

USGS SUPPLEMENTARY REFERENCE LIST. . . . . . . . . . . . 179

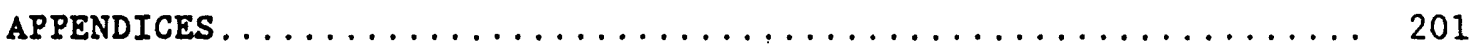

APPENDIX A: USGS STATION DATA
A1 Marshall Islands
A2 North Pacific
A3 South Pacific
A4 U.S. West Coast
A5 Blake Plateau
A6 Southeast Atlantic margin, North America
A7 New England Seamounts
A8 Other areas
A9 NGDC data
Al0 Reference data 
APPENDIX B: SNDB STATION DATA

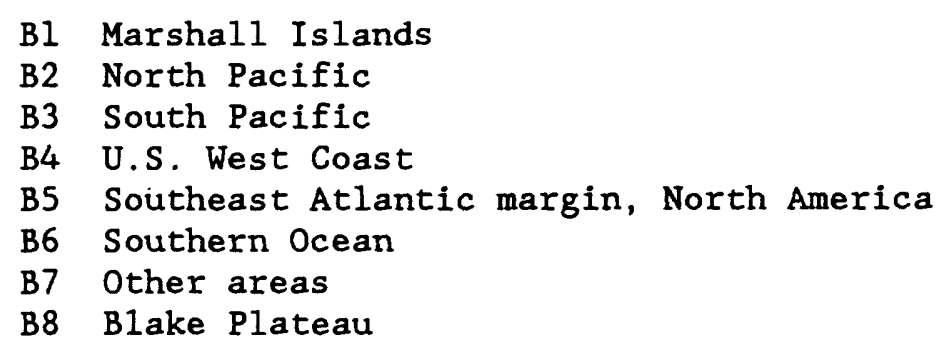

APPENDIX C: CHEMICAL DATA

Table 1 USGS Reston Laboratory data

Table 2 USGS Woods Hole Laboratory data

Table 3 Bureau of Mines Avondale data

Table 4 German Geological Survey data

Table 5 Portaspec shipboard data

Table 5a Midpac $2 a$ and $2 b$ data

Table 6 Japanese (A.Usui) data

Table 7 Analytical Services (cruise KK84) data

Table 8 Aplin thesis data -Major and Minor analyses

Table 8a Aplin thesis data - REE

Table 9 SNDB major and minor analyses

Table 10 SNDB trace analyses

Table 11 NGDC data - major and minor analyses

Table 12 Dillard and Crowther (1984), Exon (1982)

Table 13 Volkov (1976), Dymond and others (1984)

Table 14 Goddard and others (1987)

Table 15 Goddard and others (1987)

Table 16 DeCarlo and others (1987)

Table 17 DeCarlo and others (1987)

Table 18 Hein and others (1987) OF87-281 
Table II - 1. Chemical composition of marine ferromanganese oxides (from Buchanan, 1891)

Table II - 2. Distribution of Co concentration with depth in various environments (from Frazer and Fisk, 1980).

Table II - 3. Table of metal values in crusts and nodules (from Halbach and Manheim, 1984).

Table III - 1. Individual archive 1istings

Table IV - 1. Sources of crust samples in the SNDB

Table IV - 2. Sources of crust samples in the USGS collection

Table V-1. Moisture content and ignition loss for a Fe-Mn

Table V - 2 . phosphorite pavement sample from the Blake Plateau

fluorescence determinations, USGS laboratory, Woods Hole, MA.

Table VI - 1. Operating conditions for FAAS determinations

Table VI - 2. Operating conditions for GFAAS determinations

Table VI -3a. Operating conditions for ICP determinations

Table VI - 4. Values found for standard samples

Table VI - 5. ICP spectral interfaces

Table VI - 6. Determination limits

Table VII - 1. Comparison of moisture analyses for USGS

laboratories, Woods Hole and Reston.

Table VII - 2. Analysis of variance for Fe using five data sets

Table VII - 3. Analysis of variance and comparison of 'lab effect'

Table VIII - 1. Example of the USGS station data tables as found in Appendix $A$.

Table VIII - 2. Example of the Analytical lab's chemical data tables, in oxide form, as in Appendix $\mathrm{Cl}$.

Table VIII - 3. Example of the SNDB chemical data tables as in 
Table IX - 2. Histogram of Co values for SNDB data set: a) raw data b) exclusion data set, and c) normalized data set.

Table IX - 3. Descriptive statistics of elemental values for the Atlantic Ocean area.

Table IX - 4. Descriptive statistics of elemental values for the

Table IX - 5. Correlation matrix, Mid-Pacific area, based on USGS

Table IX - 6. Correlation matrix, Mid-Pacific area, based on SNDB data set: a) unmodified data, b) exclusion data set.

Table IX - 7. Correlation matrix, a) Southern Line Islands - French

Polynesia area, USGS + SNDB " exclusion" data,

b) Marshall Islands USGS exclusion data set.

Table IX - 8. Correlation matrix, a) Indian Ocean, and b) Atlantic

Table IX - 9. Correlation matrix of Total pacific Ocean, USGS exclusion data set.

Table IX - 10. Comparison of correlation coefficients for the Mid-Pacific area, unmodified vs. exclusion USGS data, plus Lee data, Mid-Pacific -Line Islands.

Table IX - 11. Pearson biserial correlation coefficients for Mid-Pacific area, $\mathrm{N}=259$.

Table IX - 12. Spearman Rank correlation coefficients for Mid-Pacific area, $\mathrm{N}=2.59$.

Table IX - 13. Principal features of chemical constituents as evident 
Figure II-1 Typical botryoidal surface of thick crust, Line Islands Mid-Pacific area (Midpac I expedition)

Figure II-2 Crusts on hyaloclastic breccia, Necker Ridge, Mid-Pacific Mountains area (S.P. Lee expedition)

Figure II-3 Closeup of crusts on altered basalt, Necker Ridge,

Figure II-4 Massive ferromanganese pavements and slabs on Blake

Plateau off South Carolina, approximately $31^{\circ} \mathrm{N} 78^{\circ} 56^{\prime} \mathrm{W}$, water depth $-760 \mathrm{~m}$.

Figure II-5 R/V Sonne, docking in Western Samoa at beginning of Midpac I expedition.

Figure II-6 Dr. Peter Halbach inspecting crusts on Midpac I

Figure II-7 Cavernous phosphatized carbonate from Line Islands area,

Figure II-8 Relationships between cobalt, nickel concentrations and water depth for crusts in the Line Islands, Mid-Pacific Mountains region (from Halbach and Manheim, 1984, and Halbach, 1986).

Figure II-9 R/V S.P. Lee at anchor in Pago Pago harbor, Tutuila, American Samoa, on completion of cruise to Line Islands, 1983.

Figure II-10 Boulder of volcanic mudstone-breccia, on shore at location in Figure II - 8. This material is similar to crust substrates found in altered state throughout Line Islands and Mid-Pacific Mountains.

Figure III-1 T. H. Ling driving forklift truck during sampling visit to Scripps Institution sample storage area in Camp Edwards Military Reservation, Claremont Mesa, California.

Figure IV-1 Analytical scheme for the determination of 27 elements in Ferromanganese materials.

Figure VIII-1 Location plot of ferromanganese crust sampling stations in world oceans.

Figure VIII-2 Histogram of $\mathrm{Mn}+\mathrm{Fe}$ for USGS data set.

Figure IX-1 Histogram of Co values for USGS data set: a) raw data b) exclusion data set, and c) normalized data set. 
Figure IX-2 Histogram of Co values for SNDB data set: a) raw data

b) exclusion data set, and c) normalized data set.

Figure IX-3 Plots of water depth vs. Co/Cu, Ni, Co, Mn, Mo, Pb for Mid-Pacific Ocean.

Figure IX-4 Plots of water depth vs. $\mathrm{Ca}, \mathrm{CO}_{2} \mathrm{Mg}, \mathrm{P}, \mathrm{Si} / \mathrm{Al}, \mathrm{Co} / \mathrm{Mn}$ for Mid-Pacific Ocean.

Figure IX-5 Plots of water depth vs. Fe, Cu, Si, Al, K, Ti for Mid-Pacific Ocean.

Figure IX-6 Plots of water depth vs. As, $\mathrm{Ba}, \mathrm{Ce}, \mathrm{Sr}, \mathrm{V}, \mathrm{Y}$ for

Figure IX-7 Plots of $\mathrm{Mn}$ vs., $\mathrm{Mn}+\mathrm{Fe}, \mathrm{Mn} / \mathrm{Fe}$ vs. $\mathrm{Mn}+\mathrm{Fe}, \mathrm{Mn}$ vs. Co, $\left(\mathrm{Al}_{2} \mathrm{O}_{3} \mathrm{Al}_{2} \mathrm{O}_{3} *\right) / \mathrm{H}_{2} \mathrm{O}^{+}$vs. $\mathrm{SiO}_{2}$ for complete USGS data set.

Figure IX-8 Plot of $A 1$ vs. $S i$ (USGS), $A I$ vs. Si (SNDB), Si vs. vs. Si.

Figure IX-9 Plot of Mn vs. Fe, Co, Ni, Zn, Pb, Cu for Mid-Pacific Ocean.

Figure IX-10 Plot of Mn vs. As, Ba, Cd, Ce, Mo, V, for Mid-Pacific Ocean.

Figure IX-11 Plot $A 1, \mathrm{Ni}, \mathrm{Si}, \mathrm{Fe}$ vs. Ti, Al vs. $\mathrm{Mg}, \mathrm{Al}$ vs. $\mathrm{k}$ for Mid-Pacific Ocean.

Figure IX-12 Plot of Mn vs. Mn+Fe, Co vs. Ni, Y vs. Ce, Fe vs. Si, $\mathrm{Ca}$ vs. Sr, P vs. Ca for Mid-Pacific Ocean.

Figure IX-13 Histograms of $\mathrm{Fe}, \mathrm{Mg}, \mathrm{Ti}, \mathrm{CO}_{2}, \mathrm{~K}, \mathrm{Na}$.

Figure IX-14 Histograms of $\mathrm{As}, \mathrm{Ba}, \mathrm{Cd}, \mathrm{Ce}, \mathrm{Cr}$, Mo.

Figure IX-15 Histograms of $\mathrm{Pb}, \mathrm{Sr}, \mathrm{V}, \mathrm{Y}, \mathrm{Zn}$.

Figure IX-16 Histograms of $\mathrm{H}_{2} \mathrm{O}^{-}, \mathrm{H}_{2} \mathrm{O}^{+}, \mathrm{Mn}+\mathrm{Fe}$.

Figure IX-17 Histograms of $\mathrm{Ni}, \log (\mathrm{Ni}), \mathrm{Mn}, \log (\mathrm{Mn}), \mathrm{Mn} / \mathrm{Fe}$, $\log (\mathrm{Mn} / \mathrm{Fe})$.

Figure IX-18 Histograms of Si, $\log (\mathrm{Si}), \mathrm{Co}, \log (\mathrm{Co}), \mathrm{Cu}, \log (\mathrm{Cu})$.

Figure IX-19 Histograms of $A 1, \log (A 1), C a, \log (C a), P, \log (P)$.

Figure IX-20 Elemental concentration of normalized Cobalt in the 
Figure IX-21 Elemental concentration of Cor in the Pacific Ocean, c.i. $=.18$

Figure IX-22 Gray scale map of Coz in the Pacific Ocean.

Figure IX-23 Elemental concentration of Cur in the Pacific Ocean;

Figure IX-24 Gray scale map of LOG3Cur in the Pacific Ocean.

Figure IX-25 Elemental concentration of Fes in the Pacific Ocean,

Figure IX-26 Gray scale map of Fez in the Pacific Ocean.

Figure IX-27 Elemental concentration of Mnz in the Pacific Ocean,

Figure IX-28 Gray scale map of Mnz in the Pacific Ocean.

Figure IX-29 Elemental concentration of Moz in the Pacific Ocean,

Figure IX-30 Gray scale map of Moz in the Pacific Ocean.

Figure IX-31 Elemental concentration of Niz in the Pacific Ocean,

Figure IX-32 Gray scale map of Niz in the Pacific Ocean.

Figure IX-33 Elemental concentration of Niz in the Pacific Ocean,

Figure IX-34 Gray scale map of LOG3Niz in the Pacific Ocean.

Figure IX-35 Elemental concentration of $\mathrm{Zn}$ in the Pacific

Figure IX-36 Gray scale map of $\mathrm{Zn}$ in the Pacific Ocean.

Figure IX-37 Accumulation rate Rmm/m.y. in the Pacific Ocean,

Figure IX-38 Gray scale map of Rmm/m.y. in the Pacific Ocean.

Figure IX-39 Elemental concentration of Co/Mng in the Pacific

Figure IX-40 Gray scale map of Co/Mns in the Pacific Ocean.

Figure IX-41 Elemental concentration of $\mathrm{Mn} / \mathrm{Fe}$ in the Pacific 
Figure IX-42 Gray scale map of $\mathrm{Mn} / \mathrm{Fe} z$ in the Pacific Ocean.

Figure IX-43 Elemental concentration of $\mathrm{Ti} /(\mathrm{Mn}+\mathrm{Fe})(z)$ in the

Pacific Ocean, c.i. $\mathrm{n}=.1$ for $\mathrm{Ti} /(\mathrm{Mn}+\mathrm{Fe})=10 \mathrm{n}$.

Figure IX-44 Gray scale map of $\mathrm{Ti} / \mathrm{Mn}+\mathrm{Fe}(8)$ in the Pacific Ocean.

Figure IX-45 Elemental concentration of $\mathrm{Al}_{2} \mathrm{O}_{3}$ in the Pacific

Ocean, c.i. $=18$

Figure IX-46 Gray scale map of $\mathrm{Al}_{2} \mathrm{O}_{3}$ in the Pacific Ocean.

Figure IX-47 Elemental concentration of $\mathrm{CaO}$ in the Pacific

Figure IX-48 Gray scale map of $\mathrm{CaO}$ in the Pacific Ocean.

Figure IX-49 Elemental concentration of $\mathrm{SiO}_{2}$ in the Pacific

Ocean, c.i. $=28$

Figure IX-50 Gray scale map of $\mathrm{SiO}_{2}{ }^{2}$ in the Pacific Ocean.

Figure IX-51 Elemental concentration of $\mathrm{TiO}_{2} 8$ in the Pacific

Ocean, c.i. $=.28$

Figure IX-52 Gray scale map of $\mathrm{TiO}_{2}{ }^{2}$ in the Pacific Ocean.

Figure IX-53 Gray scale map of Asz in the Pacific Ocean.

Figure IX-54 Gray scale map of Baz in the Pacific Ocean.

Figure IX-55 Gray scale map of Cdo in the Pacific Ocean.

Figure IX-56 Gray scale map of Cez in the Pacific Ocean.

Figure IX-57 Gray scale map of Cro in the Pacific Ocean.

Figure IX-58 Gray scale map of Cur in the Pacific Ocean.

Figure IX-59 Gray scale map of $\mathrm{Pb}$ in the Pacific Ocean.

Figure IX-60 Gray scale map of Srz in the Pacific Ocean.

Figure IX-61 Gray scale map of $V_{8}$ in the Pacific Ocean.

Figure IX-62 Gray scale map of Yo in the Pacific Ocean.

Figure IX-63 Gray scale map of Si/Als in the Pacific Ocean.

Figure IX-64 Gray scale map of MgOs in the Pacific Ocean. 


\section{INTRODUCTION by F.T. Manheim}

We define ferromanganese oxide crusts (hereafter referred to only as crusts) as encrustations forming largely on hard substrates that are at least periodically kept free from detrital sedimentation by strong currents, steep slopes, or both. In near-shore or hydrothermal environments where metal supply is much greater than it is in most of the open ocean, crusts may also form on soft sediments. Thus, the open ocean crusts, which are largely found in raised areas like seamounts and ridges, contrast with abyssal nodules, which are found lying on or in soft sediments, usually at depths greater than $4500 \mathrm{~m}$. The chemical composition, mineralogy, and source of metals for crusts are significantly different than for abyssal nodules (Figs. II-1 to II -4).

In the years before the increased interest in crusts (1981) most investigations categorized ferromanganese oxide phases in the oceans under the blanket term "nodules", which confuses terminology as well as understanding of habitats. In the historical summary we refer to the collective "nodules" as ferromanganese oxide deposits, whereas abyssal nodules, specified or presumed, are referred to simply as abyssal nodules. The abyssal nodules will not be discussed in this report except for comparison or background purposes, since an extensive literature exists for them (see Glasby, 1977; Meylan and others, 1981; Haynes and others, 1982; McKelvey and others, 1983; Murdmaa and Skornyakova, 1986, and Baturin, 1986 for background references).

The pioneering German "Midpac I" investigation of cobalt-rich ferromanganese oxide phases in the Central Pacific in 1981 set in motion several series of events. On the scientific front, interest in the processes causing cobalt enrichment in seamount "nodules" and crusts was heightened. Recognition of the possible resources in cobalt rich crusts within 200 miles of U.S. and territorial coasts stimulated environmental impact studies pursuant to possible lease sales by the Minerals Management Service and cooperative reconnaisance cruises by the U.S. Geological Survey. Interest in cobalt resources was among background factors cited in supporting documentation for the declaration of an Exclusive Economic Zone by President Reagan in March, 1983.

Crusts pose more serious problems than do abyssal nodules (see definition in Chapter II) in terms of a comprehensive chemical overview. Unlike nodules, crusts tend to occur in a highly localized manner on and around seamounts and ocean ridges, to some extent on certain current-swept terraces and plateaus, and on some steep island slopes. Their habitat is often rough and irregular. Systematic and effective sampling and definitive descriptions of these habitats have not yet been accomplished.

Second, unlike nodules, which form as discrete hard objects in a soft sediment matrix, crusts generally adhere to hard substrates in such a fashion that they cannot easily be separated from underlying substrate during recovery. Third, there is a complete spectrum of occurrence from oxide layers that are little more than stains to those that are as much as $25 \mathrm{~cm}$ oxide thick. Elemental composition of crusts varies from nearly pure manganese or iron oxides (both frequently hydrothermal in origin) to the fairly consistent admixtures that characterize cobalt-rich crusts. What do we choose as "dividing lines", and how does one compare chemical compositions from such diverse material? Finally, 
earlier literature data information is often highly variable in quality, and less consistent and reliable than are data from newer, targeted cruises. Earlier crust specimens were often by-products of sampling for igneous rocks, and sample preservation, contamination, labelling, and analytical efforts were less carefully controlled. Yet, the regional coverage provided by this material is valuable, and warrants effort to whenever possible retain the useful information in it.

Our primary effort has been to collect and evaluate all available samples and information, to analyze new samples by means of well-controlled and defined analytical procedures, and to present all information through the end of 1987 in a consistent and complete form. As a part of this effort, we have classified and normalized all data for mapping purposes so that information from diverse sources can be compared over larger geographic areas, as discussed below.

For resolving the difficult problem of contamination and water treatment, described later, we have chosen a basic procedure that normalizes data to a constant $\mathrm{Fe}+\mathrm{Mn}$ content. As the basis of the vernadite $\left(\delta \mathrm{MnO}_{2}\right)$ mineral phase that dominates most crusts, Fe+Mn oxide content will naturally tend to vary inversely with degree of substrate contamination and water content. When the effect of the latter two variables is minimized, $F e$ and Mn have been shown to have an inverse relationship. There are occasional exceptions to this but they do not invalidate its general utility; in fact, these cases may involve geochemical or mineralogical phenomena of special interest. We are aware that iron is involved in phases other than the oxide phase, like silicates and phosphates. However, we believe that complications and limitations such as on geographical distribution of samples introduced by making more refined corrections outweigh the benefits of more precise correction at this time. Investigators who wish to use other means of dealing with the comparability problem have the complete basic information in our database to create their own models.

The goal of this report is to present the data base in a form that can be used in computer-addressable and paper format, along with a basic set of descriptive output like regional means, frequency plots, interelement plots, and contoured maps based on gridded data. These enhance the description of the database as well as offer examples of the kinds of treatments that may yield useful information to investigators.

Magnetic tapes and floppy disks will be made available to major information repositories, primarily the National Geologic Data Center (NOAA), in Boulder Colorado, from which the data can be readily retrieved. Our office will not continue complete encoding and support of newly available information, as has been done to date. However, we will be glad to assist inquiries about the interpretation and retrieval of data from the database and may update selected portions of the data. 


\section{HISTORY OF INVESTIGATION By F.T. Manheim}

Ferromanganese oxide crusts are members of a family of manganese and iron oxide precipitates that are widely distributed in the lithosphere. Scientists as early as Alexander von Humboldt and Jens Jakob Berzelius in the late 18th and early 19 th century (Boussingault, 1882) recognized that manganese oxide precipitates are omnipresent wherever aqueous media are in contact with solid surfaces for a long period of time in the presence of oxygen. On land, oxide concretions and crusts are widespread in lakes and bogs of glaciated terranes, e.g. in Scandinavia, Britain, northern Germany, and European Russia, and were used as early as 800-500 B.C. for iron production (Arrhenius, 1959; Tylecote, 1962; R. Maddin, 1987, oral communication). The iron was reduced with charcoal at temperatures around $1100^{\circ} \mathrm{C}$ with the aid of a bellows, and the resulting sponge was hammered to remove slag and impurities. Further forging and hammering produced nails, swords, and iron implements of all kinds. Swedenborg (1734) in his great monograph on iron (De Ferro) gives a knowledgeable account of both the location of lake and bog ores, as well as a quite "modern" summary of their origin by precipitation from solution, with metal supply attributed to soil leaching and ground water. Bog and lake ore was used in North America in the Colonial era and into the 19th century; it continued to be used in Sweden until the early 1920's for local iron production (Naumann, 1922). Although historically the ores served only as a source of iron, they were temporarily used in Finland as a source of manganese during World War II, when that country was cut off from external supply (Vaasjoki, 1956).

The freshwater nodule deposits had been largely forgotten until the awakening of interest in abyssal manganese nodules in the 1960's. New studies showed extensive freshwater nodules in Green Bay, Wisconsin, as well as in other lakes (Callender and Bowser, 1976 and references cited). Though all natural freshwater ferromanganese oxides have much lower trace metal content than do marine oxides, the Green Bay as well as Blake Plateau crusts and deep ocean nodules were revealed to have potential use as catalyst material for petroleum refining, as demonstrated by American and foreign patents and reports in the middle 1970's (Van Heck and others, 1973, and authors cited in Hubred, 1981).

The most widely noted ferromanganese oxide phase, abyssal nodules, was discovered in the deep oceans by the Challenger Expedition of 1873-4 (Murray, 1876; Buchanan, 1876). The 532 page report on bottom materials from the Challenger expedition (Murray and Renard, 1891) long yielded the dominant body of deepsea information on manganese oxide concretions, and the archived collection of concretions in the British Museum has continued to serve scientific research up to the present time. The Challenger dredge hauls yielded not only the classical black manganese nodules from abyssal depths $(4500-6000 \mathrm{~m})$, often containing nuclei of sharks' teeth, whale earbones, pumice, volcanic rock or fragments of nodules, but also supplied a less noted variety of oxide coatings, layerings, and crusts (even slabs) of ferromanganese oxide material from lesser depths, some as shallow as $370 \mathrm{~m}$.

The chemist on board H.M.S. Challenger, J.Y. Buchanan, and his coworkers analyzed the newly-discovered deep-ocean concretions, demonstrating that they contained significant minor admixtures of copper, nickel and cobalt (Buchanan, 1876). These and other elements including thorium and thallium, which were not to be 
further investigated for another 60 years, were first detected qualitatively by the then quite new spectroscope. In a later paper, Buchanan (1891) showed that the abyssal manganese nodules contained much higher concentrations of minor metals than did shallow marine (estuarine) nodules from Loch Fyne in Scotland (TableII-1). He also demonstrated that the oxidation state of the deep ocean forms was considerably higher than that of the nearshore forms (Manheim, 1965). However, Buchanan apparently did not analyze any of the crusts from shallower oceanic depths for trace constituents. The differences in chemical composition and origin between the abyssal nodules and crusts remained as a task for much later investigators, and is the principal subject for this database effort. Typical crusts from the Mid Pacific area are shown in (Figs. II-I to II-4). 
Table II-1. Chemical composition of marine ferromanganese oxides from deep ocean and littoral manganese nodules (from Buchanan, 1891). Samples I-V are deep-ocean concretions, whereas $M-R$ represent littoral nodules from Loch Fyne, at water depths of $100-190 \mathrm{~m}$. Samples marked with $*$ refer to sum of $\mathrm{NiO}+\mathrm{CoO}$. Available oxygen ( 0 ) determined by iodometric titration according to Bunsen. $X$ refers to the state of oxidation of $O$ in $\mathrm{MnO}_{\mathbf{x}}$. All values are in weight percent.

Sample $\begin{array}{ccccc}0 & \mathrm{MnO} & \mathrm{NiO} & \mathrm{Co}_{3} \mathrm{O}_{4} & \mathrm{X}\end{array}$

$\begin{array}{rlllll}\text { I } & 5.47 & 25.76 & 1.11 & 0.19 & 1.945 \\ \text { II } & 5.80 & 26.94 & 1.32 & 0.19 & 1.95 \\ \text { III } & 5.27 & 23.91 & 0.90 & 0.21 & 1.97 \\ \text { IV } & 4.07 & 18.69 & 0.68 & 0.19 & 1.95 \\ \mathrm{~V} & 4.25 & 19.30 & 0.42 & 0.34 & 1.97 \\ \mathrm{M} & 3.96 & 31.35 & 0.02 * & & 1.56 \\ \mathrm{~N} & 2.48 & 27.90 & 0.02 * & & 1.39 \\ \mathrm{P} & 3.60 & 28.54 & 0.05 * & & 1.56 \\ \mathrm{Q} & 3.35 & 29.34 & 0.03 * & & 1.51 \\ \mathrm{~K} & 6.28 & 37.15 & & & 1.75 \\ \mathrm{R} & 3.38 & 29.55 & & & 1.51\end{array}$




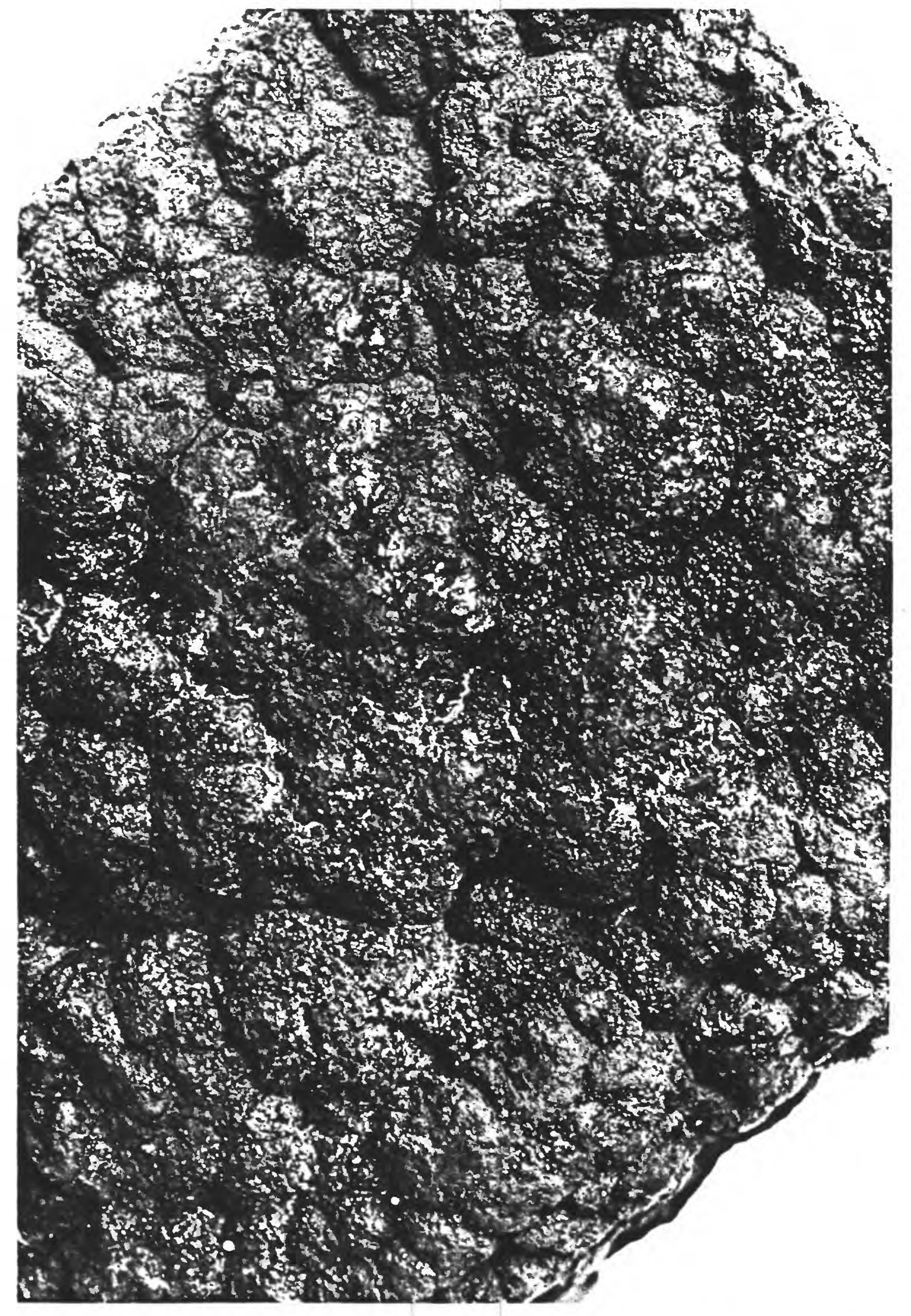

Figure II - 1 Typical botryoidal surface of thick crust, Line Islands Mid-Pacific area ( Midpac I expedition) 


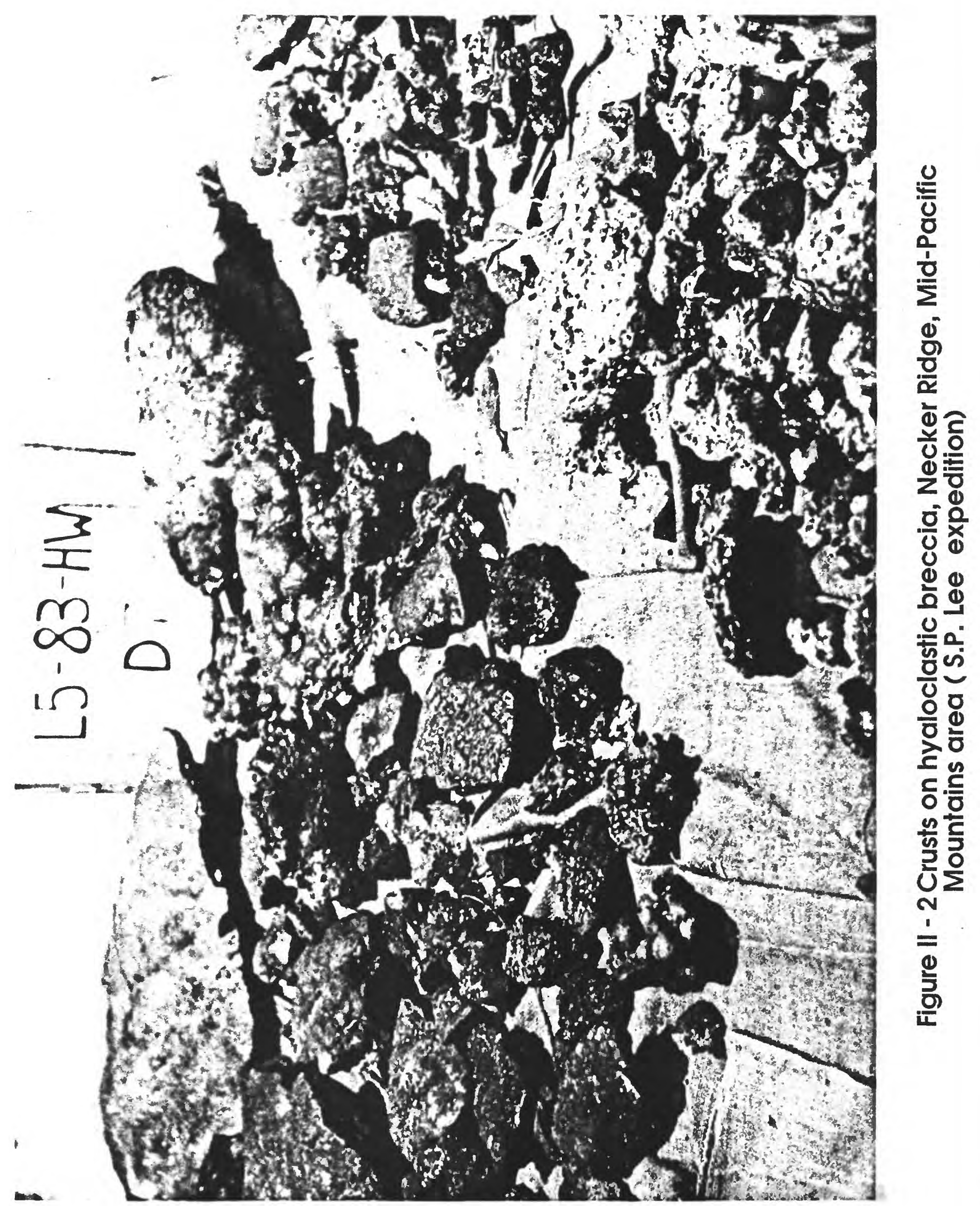




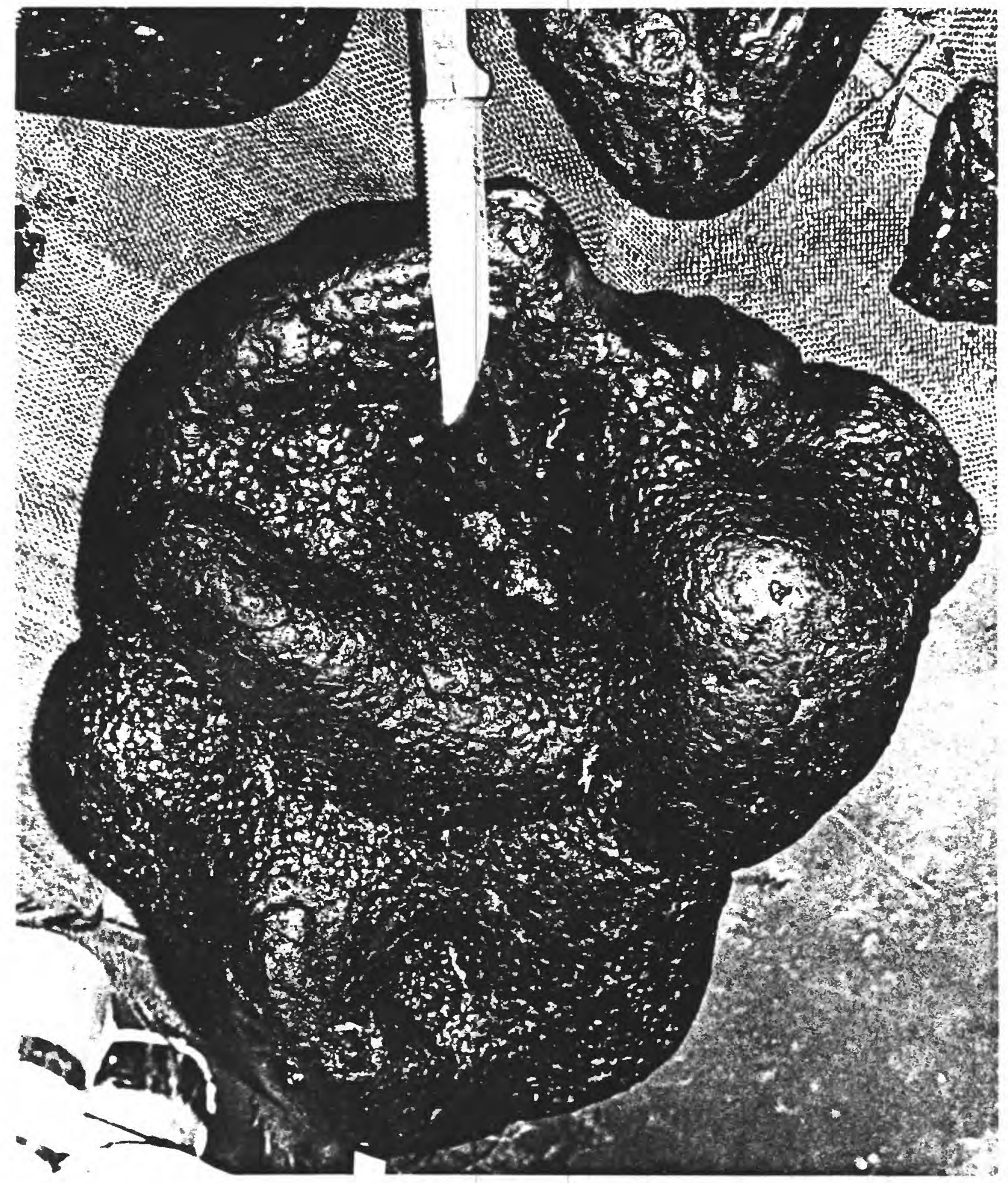

Figure II - 3 Closeup of crusts on altered basalt, Necker Ridge, Mid-Pacific Mountains area (S. P. Lee expedition) 

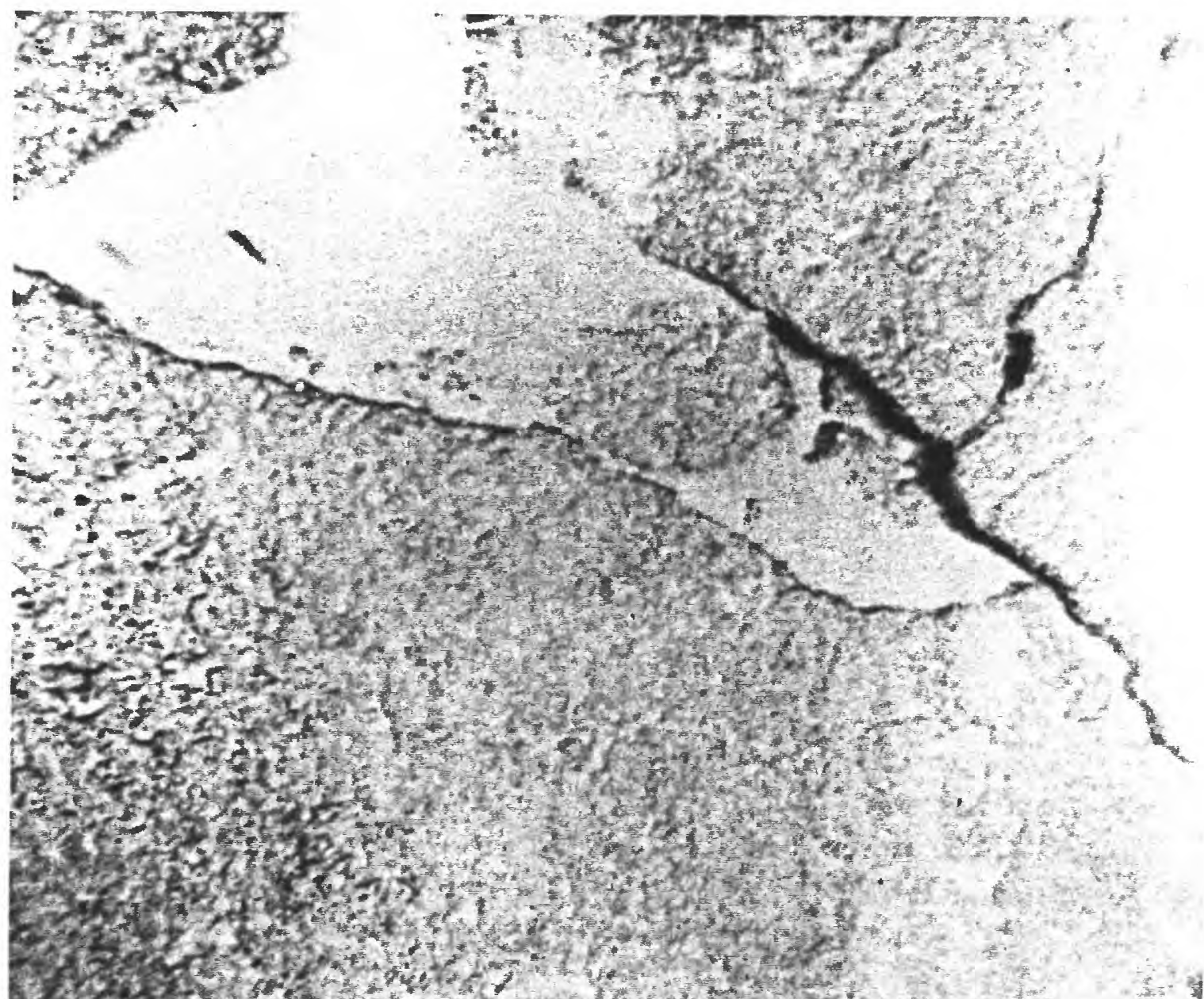

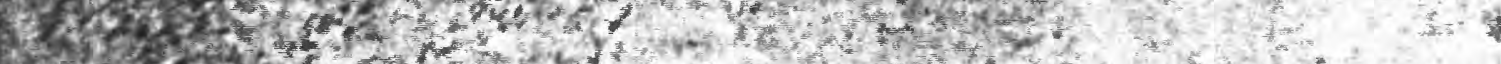
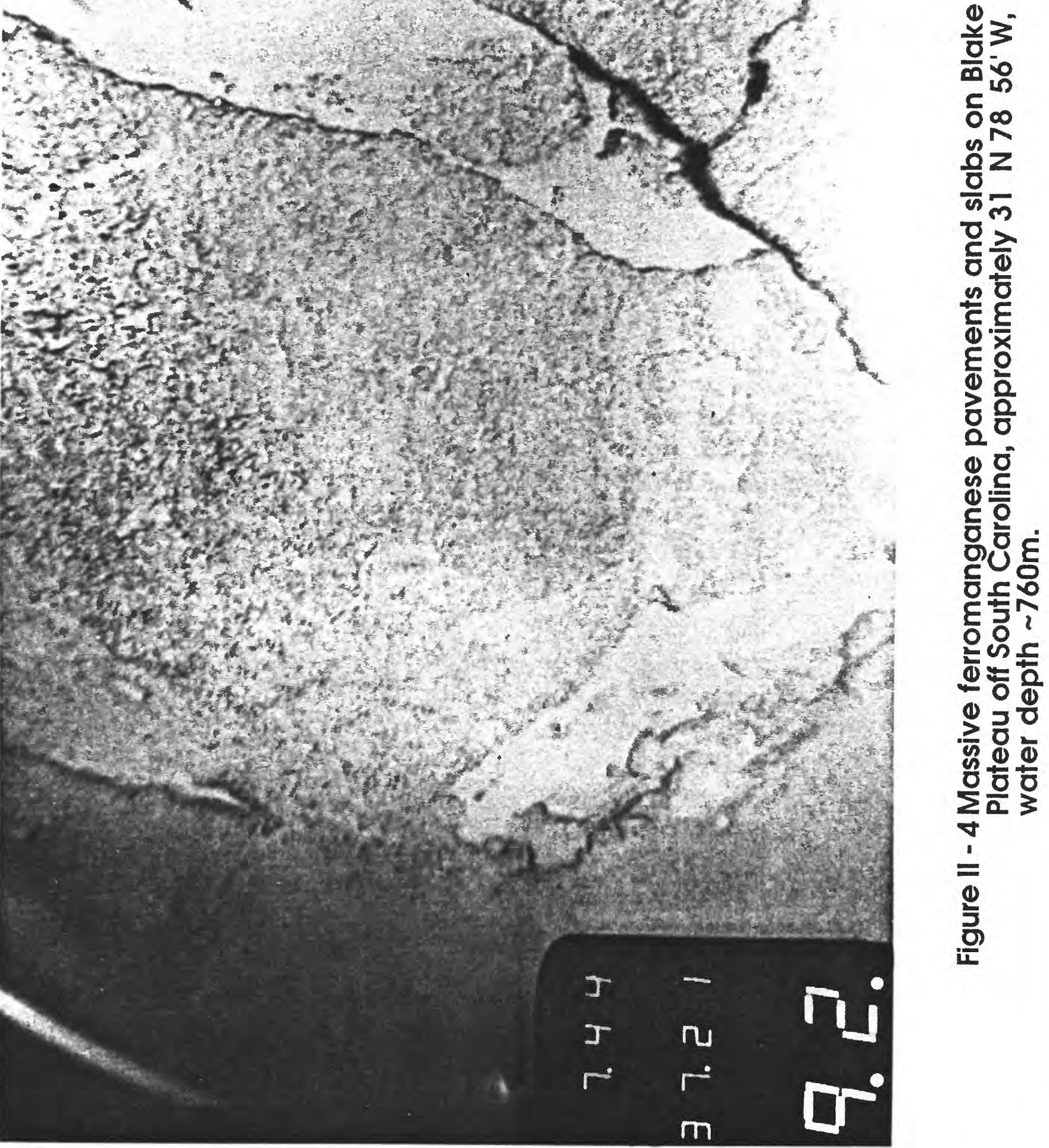
Shallow marine oxides

The Challenger Expedition was not, as commonly assumed, the discovery voyage for marine ferromanganese concretions. That distinction seems to belong to a little-known and, from the scientific point of view, poorly documented Sofia Expedition of 1868, under leadership of the Swedish explorer, A.E. Nordenskiold (Manheim, 1978). The Sofia recovered nodules east of Novaya Zemlya in the Kara Sea, in less than $200 \mathrm{~m}$ water depth. These were later analyzed chemically and reported by Lindstrom (1884).

The extensive historical literature on shallow marine oxides ( $<200 \mathrm{~m}$ water depth) was first retrieved from diverse sources by Manheim (1965), and later reviewed by Calvert and Price (1977). Recently, work by J. Ingri has brought the subject up to date, while providing detailed documentation of the formation of ferromanganese concretions and crusts in the Gulf of Bothnia, between Sweden and Finland (Ingri, 1985). The Baltic and Bothnian concretions are rapidly-formed, sometimes on partly-reducing glacio-marine sediments, are dominated by iron, and have cobalt, nickel and copper concentrations on the order of 100-256 ppm, 50-750 $\mathrm{ppm}$, and 10-90 ppm, respectively.

Post - World War II advances: Mero's nodule mining initiative

After World War II, E.D. Goldberg and his colleagues at the Scripps Institution of Oceanography began a series of studies (Goldberg, 1954; Goldberg and Arrhenius, 1958) that established the concept of metal scavanging on the active catalytic surfaces of manganese oxides on the sea floor. Most of the "nodules" reported by Goldberg (1954) were from abyssal depths (5000m) and had . 30 percent Co content or less, but a few specimens from the Cape Johnson and Sylvania seamounts had cobalt content as high as .70 percent. As discussed more fully later, seamount and other ferromanganese oxide crusts were normally not distinguished from nodules in most scientific literature until the 1980's, and therefore in the following narrative "nodules" in papers before this period will include diverse oxide phases.

Interestingly, some "nodules" reported from ocean ridge areas in Goldberg's pioneering paper showed very low cobalt, on the order of .03 percent Co or less. We now know that these samples, from elevated rift zones off the coast of Mexico in the east Pacific margin, were formed rapidly under the influence of hydrothermally discharging fluids. The importance of hydrothermal phenomena was not suspected until the paper of Bostrom and Peterson (1966), and was not fully confirmed until the shipboard studies of the late 1970's (e.g. Moore and Vogt, 1976), and the direct observations by submersibles of the early $80^{\prime} \mathrm{s}$ (Rona and others, 1983, and references cited therein). These revealed the now famous hot $\left(350^{\circ} \mathrm{C}\right)$ fluid vents, unique benthic organism communities such as foot-long clams and tubeworms, and both sulfide and oxide deposits.

Interest in the economic potential of deep ocean (abyssal) manganese nodules was ignited by the work of John Mero in the early 60's (Mero, 1959-1962). Mero not only provided the first set of extensive minor metal analyses on "nodules", but aggressively advocated commercial exploitation of nodules. The first focused Russian studies of Pacific Ocean manganese nodules commenced about the same time (Skornyakova, 1960). Major Russian research studies from 1960 through 1975 by 
the Institute of Oceanology (Bezrukov, 1976) were undoubtedly stimulated by the perceived economic potential of nodules.

Association between higher cobalt (up to 1.6 percent) content and manganese oxide phases from shallower water sites especially on seamounts was probably first recognized in print by Menard (1964). Mero's book of the next year (Mero, 1965) established that the " $D$ " region centering on topographic highs in the central Pacific Ocean had the highest average cobalt concentrations (mean of 1.2 percent ) for "nodules" of any region in the world oceans. Mero speculated that either the basaltic rocks of the seamounts were enriched in cobalt, or that the strong currents known around the seamounts contributed to more oxidizing conditions. Whereas we now understand that hydrothermal fluids and their associated oxide precipitates have low cobalt and trace element content (Manheim, 1986 and references cited), in the $1960^{\prime}$ 's quite an opposite relationship between cobalt and vulcanism was postulated by Arrhenius and coworkers (Arrhenius, Mero and Korkisch, 1964, and Arrhenius and Bonatti, 1965). Cobalt-rich seamount nodules were ascribed to rapidly formed vulcanogenic origin, whereas the low-cobalt continental margin phases (now known to be rapidly formed under influence of submarine vulcanism) were thought to be slowly accumulated. British investigator D.S. Cronan drew attention to the correlations between water depth and cobalt content of the ferromanganese phases, based on existing data and new analyses performed as a part of his $\mathrm{PhD}$ dissertation at Imperial College, University of London (Cronan, 1967; Cronan and Tooms, 1969; Cronan, 1977). He has continued world-wide research on manganese phases (Cronan, 1980, 1986).

The 1970's: the Scripps Institution Nodule Data Bank (SNDB)

Searching for explanations to the anomalous cobalt content of seamount nodules, Cronan (1977) expanded on the earlier idea of Mero (1965) that enrichment in cobalt might be aided by higher oxidizing conditions on seamounts. This concept was encouraged by the studies of Burns (1965, 1976), who reported that cobalt is oxidized to the Co (III) state and substitutes for Mn (IV), a reaction that can form the basis of highly efficient coprecipitation of cobalt from solution. The trivalent behavior of cobalt was later documented by Murray and Dillard (1979), Varentsov and others. (1980) and Dillard and others (1982) by photoelectron spectroscopy, though the "oxidizing environment" hypothesis was later criticized by Halbach and Manheim (1984). Two other United Kingdom specialists on ferromanganese oxide phases, S.E. Calvert and N.B. Price, had emphasized two separate metal sources for abyssal nodules: one from ambient water (hydrogenetic), and another from metals released by diagenetic alteration from bottom sediments (Price and Calvert, 1970). The former type of deposition was empirically observed to produce vernadite $\left(\delta \mathrm{MnO}_{2}\right.$; Chukhrov and others, 1978), an iron-rich, poorly crystalline mineral form that dominates crusts. The latter often produced todorokite, a more manganese-rich $\mathrm{MnO}_{2}$ mineral that is often found in nodules as well as in hydrothermal manganese oxides (Turner and Buseck, 1981).

Chemical and mineralogical partitioning systems to explain chemical composition in ferromanganese phases generally were elaborated from the $1970^{\prime} \mathrm{s}$. These and other chemical models that partially treat crust phases are documented in books on ferromanganese oxides edited by Glasby (1977), Bischoff and Piper (1979) and Baturin (1986), as well as in results from several National Science Foundation programs; Ferromanganese Nodules, Nazca Plate, and MANOP (see Heath, 1981 and Dymond and others, 1984 for extensive references). 
The general linkage between cobalt content and ferromanganese oxide phases associated with raised areas of the ocean was documented in statistical summaries of chemical data from the Scripps Institution Nodule Data Bank, initiated and led from 1972 to 1980 by J. Frazer and M. Fisk (1980). A summary of variability of cobalt content by environment and water depth (Table II-2, Frazer and Fisk, 1981) provided a statement of relationships that have been confirmed and refined by the present study. A source of confusion common to all studies up to recent time is the fact that no distinction between nodules and crusts was made in Frazer and Fisk's statistical summaries and most other work prior to 1982. Table II-2 shows clearly that there is a systematic inverse relationship between cobalt and water depth for oxides samples from seamounts of all oceans, not merely the Central Pacific, though the latter area exhibits the highest cobalt concentrations and the sharpest increase in cobalt content with decreasing water depth. The present studies suggest that virtually the entire cobalt anomaly is due to presence of crusts and their related phases, as distinguished from sediment-hosted nodules.

Though the existence of cobalt-enriched ferromanganese phases associated with seamounts had been noted and discussed, as indicated above, frequent exceptions to the cobalt-water depth relationships were observed, as for example, around the Hawaiian Islands (Frank and others, 1976; Craig and others, 1982). Moreover, although the origin of $>1$ percent nickel and copper in high-value abyssal nodules from the Clarion-Clipperton zone was explained by their relationship to plankton productivity and mobilization of metals from host sediments (Horn and others, 1972; Heath, 1981 and publications cited), no convincing explanation of cobalt distributions in "seamount nodules" were found. 
Table II-2. Cobalt concentration in "nodules" from various geographic regions and water depths (Frazer and Fisk, 1980).

Water Depth (thousands of meters)

\begin{tabular}{|c|c|c|c|c|c|c|c|c|}
\hline Environment & $<1$ & $1-2$ & $2-3$ & $3-4$ & $4-5$ & $5-6$ & $>6$ & $\begin{array}{l}\text { A11 } \\
\text { Depths }\end{array}$ \\
\hline $\begin{array}{l}\text { Mid Pacific } \\
\text { Abyssal }\end{array}$ & - & - & - & $\begin{array}{l}0.39 \\
(27)\end{array}$ & $\begin{array}{l}0.36 \\
(158)\end{array}$ & $\begin{array}{l}0.27 \\
(354)\end{array}$ & $\begin{array}{l}0.24 \\
(30)\end{array}$ & $\begin{array}{l}0.30 \\
(569)\end{array}$ \\
\hline $\begin{array}{l}\text { CC Zone } \\
\text { Abyssa1 }\end{array}$ & - & - & - & $\begin{array}{r}0.20 \\
(9)\end{array}$ & $\begin{array}{l}0.26 \\
(540)\end{array}$ & $\begin{array}{l}0.23 \\
(223)\end{array}$ & $\begin{array}{l}0.37 \\
(1)\end{array}$ & $\begin{array}{l}0.25 \\
(773)\end{array}$ \\
\hline $\begin{array}{l}\text { Other } \\
\text { Abyssal }\end{array}$ & - & - & - & $\begin{array}{r}0.22 \\
(156)\end{array}$ & $\begin{array}{l}0.20 \\
(617)\end{array}$ & $\begin{array}{l}0.21 \\
(348)\end{array}$ & $\begin{array}{l}0.24 \\
(10)\end{array}$ & $\begin{array}{l}0.21 \\
(1131)\end{array}$ \\
\hline $\begin{array}{l}\text { Mid-Pacific } \\
\text { Seamounts }\end{array}$ & $\begin{array}{l}1.34 \\
(6)\end{array}$ & $\begin{array}{l}0.79 \\
(89)\end{array}$ & $\begin{array}{l}0.59 \\
(25)\end{array}$ & $\begin{array}{l}0.49 \\
(17)\end{array}$ & - & - & - & $\begin{array}{l}0.74 \\
(137)\end{array}$ \\
\hline $\begin{array}{l}\text { Other } \\
\text { Seamounts }\end{array}$ & $\begin{array}{l}0.64 \\
(6)\end{array}$ & $\begin{array}{l}0.57 \\
(37)\end{array}$ & $\begin{array}{l}0.43 \\
(48)\end{array}$ & $\begin{array}{l}0.22 \\
(111)\end{array}$ & - & - & - & $\begin{array}{l}0.34 \\
(202)\end{array}$ \\
\hline Ridges & $\begin{array}{l}0.40 \\
(30)\end{array}$ & $\begin{array}{l}0.45 \\
(13)\end{array}$ & $\begin{array}{l}0.36 \\
(27)\end{array}$ & $\begin{array}{l}0.33 \\
(35)\end{array}$ & - & - & - & $\begin{array}{l}0.37 \\
(105)\end{array}$ \\
\hline $\begin{array}{l}\text { Active } \\
\text { Spreading } \\
\text { Centers }\end{array}$ & - & $\begin{array}{l}0.38 \\
(3)\end{array}$ & $\begin{array}{l}0.33 \\
(33)\end{array}$ & $\begin{array}{r}0.25 \\
(151)\end{array}$ & - & - & - & $\begin{array}{l}0.26 \\
(187)\end{array}$ \\
\hline $\begin{array}{l}\text { Marginal } \\
\text { Highs }\end{array}$ & $\begin{array}{l}0.14 \\
(14)\end{array}$ & $\begin{array}{l}0.34 \\
(42)\end{array}$ & $\begin{array}{l}0.19 \\
(70)\end{array}$ & $\begin{array}{l}0.20 \\
(31)\end{array}$ & - & - & - & $\begin{array}{l}0.23 \\
(157)\end{array}$ \\
\hline $\begin{array}{l}\text { Al1 } \\
\text { Environments }\end{array}$ & $\begin{array}{l}0.46 \\
(56)\end{array}$ & $\begin{array}{r}0.61 \\
(184)\end{array}$ & $\begin{array}{r}0.34 \\
(203)\end{array}$ & $\begin{array}{r}0.25 \\
(537)\end{array}$ & $\begin{array}{c}0.24 \\
(1315)\end{array}$ & $\begin{array}{l}0.24 \\
(925)\end{array}$ & $\begin{array}{l}0.25 \\
(41)\end{array}$ & $\begin{array}{c}0.27 \\
(3261)\end{array}$ \\
\hline
\end{tabular}

() = number of samples 
National studies:

Japanese studies

The first significant Japanese investigations of deep ocean nodules were carried out by the Japanese Geological Survey from 1969 through 1972 (Mizuno, 1974 and references cited). Construction of the oceanographic vessels, Hakurei Maru I in 1974 and Hakurei Maru II in 1980 largely for ocean minerals investigations, greatly increased research capability and led to series of reports on nodule areas (see references in Nakao, 1986 and SIO references, Chapter XI). The narrow focus of these cruises on abyssal nodules almost completely excluded recovery of crusts on adjacent seamounts. However, Japanese university cruises recovered crusts on seamounts around the Japanese islands (e.g., Harada and others, 1985; Usui, 1985; Usui and others, 1987).

On the industrial front a number of Japanese companies collectively identified as the "DOMCO" group had participated in the OMI (Ocean Management Inc.) consortium along with INCO of Canada, SEDCO (U.S.), and AMR of Germany (Preussag, A.G., Metallgesellschaft, and Salzgitter, A.G.) in the 1970's. It appears that except for experimental cruises and engineering studies on the Continuous Line Bucket Dredge system by Y. Masuda (e.g., Masuda and others, 1971; Masuda, 1972) and preliminary processing and mineral assessment studies, independent Japanese studies of ocean minerals were limited.

A national Japanese large-scale project directed toward achieving feasibility of a manganese nodule mining system began in 1981, after most international consortia had greatly reduced or eliminated research efforts (Broadus, 1987). Interest in crusts began in Japan in 1984 and 1985 with the formation of study committees and task forces. One effort is sponsored by the Ministry of International Trade and Industry (MITI) and its associated agencies, Metal Mining Agency of Japan (MMAJ), Deep Ocean Resarch and Development Co. (DORD), and DOMA, a trade association of non-ferrous metal mining companies. The Japan Research Association, a consortium of mining companies associated with the Japanese Center for Marine Science and Technology (JAMSTEC) has begun an independent effort. $\mathrm{Y}$. Masuda is currently associated with this group, and participated in a recent USGS-Bureau of Mines cruise (November, 1986; Hein and others, 1988). First significant government-supported field studies of crusts are scheduled in 1987-88.

French studies

French efforts under the leadership of CNEXO (Centre National pour 1'Exploitation des Oceans) and other organizations coalesced in 1974-1976 to form AFERNOD (Association Francaise pour 1'Etude et la Recherche des Nodules polymetalliques) (Bastien-Thiry, 1978). This government-sponsored organization conducted extensive seagoing investigations, later supported by use of the Seabeam swath-mapping system.

One notable exception to the paucity of French published literature has been the proceedings of a major conference on ferromanganese "nodules" held at Gif sur Yvette, France, edited by C. Lalou (1979). A famous "battle" between protagonists of extremely slow deposition of ferromanganese crusts (T.L. Ku from 
the University of Southern California) and those favoring rapid precipitation (C. Lalou and her colleagues at the Centre des Faibles Radioactivites, C.N.R.S., Gif sur Yvette, France) was fought on the "turf" of the notable "Techno" crust, found at $4000 \mathrm{~m}$ water depth at the base of a volcanic ridge north of the Tuamotu Ridge in the south Pacific (French Polynesia) and reported in this volume (Lalou and others, 1979a and 1979b). Since then, the radiogenic and other evidence has become overwhelming in favor of the concept that normal deep ocean crusts form very slowly, on the order of a few millimeters per million years (Halbach and others, 1984; Manheim, 1986 and references cited), as was already suggested by Bender and others, (1970) and Bhat and others, (1973).

\section{Russian studies}

Soviet studies have been extensive since the early 1960's (Skornyakova, 1960) although with the exception of selected translated articles they are often not well-known or cited in Western literature. Soviet work by the Institute of Oceanology in Moscow under the leadership of P.L. Bezrukov during the 1960's through middle 1970's was characterized by geographic breadth and often solid quality. It culminated in a monograph on Pacific Ocean ferromanganese oxide concretions, edited by Bezrukov (1976). An English translation as prepared under the auspices of the Canadian Department of Energy, Mines, and Resources in 1978, and has been duplicated informally in the U.S..

Some of the contributions of the Soviet studies include the first contoured nodule metal maps including cobalt (Skornyakova and Andrushchenko, 1972), maps of frequency (density) of nodule occurrence, and early emphasis on rarer constituents, including components like tungsten, available oxygen, and sulfur (Isaeva, 1967). The metallogenic maps revealed two large cobalt anomaly areas, one in the north Central Pacific and another in the French Polynesia area, which were outlined in greater detail in later years. It should be emphasized, however, that in the light of present knowledge the cobalt anomalies on "nodule" maps by all authors to date appear to be not due to nodules, but rather are a mixed signal whose high cobalt values are almost entirely due to seamount crusts. The apparent "nodule" relationship was created by the practice of lumping true nodules with crusts. In fact, no true nodule map has been available to date, a circumstance which precludes any valid conclusions about nodule variability.

V. Chukhrov has been among the leaders in study of nodule and crust mineralogy, including use of electron microscopy and diffraction proposed the widely accepted term "vernadite" for the " $\delta \mathrm{MnO}_{2}$ " phase that dominates crusts (Chukhrov and others, 1978a). Soviet workers were among the earliest workers to publish "polygon" studies, including detailed maps of nodule frequency (density) and chemical composition in small areas (N.S. Skornyakova and coworkers, in Bezrukov, 1976). An exceptionally detailed update of Soviet and Western nodule work is available in new books by Murdmaa and Skornyakova, 1986 and especially Baturin, 1986, and a new book on crusts, edited by A.P. Lisitsin, is said to be in press.

After 1976, field investigation of nodules appears to have been taken over by the Ministry of Geology, which utilized its own prospecting vessels for the purpose, maintained little communication with the Institute of Oceanology. This work, along with the Institute of Oceanology studies, was also used as support for a Soviet "pioneering investor" claim with the United Nations Preparatory 
Commission for the Law of the Sea Agreement. The claim, filed in 1984, coincides with that of the Deepsea Ventures Co. claim in the Clarion-Clipperton Zone (R. Kaufman, Deepsea Ventures Co., 1986, and P. Hoagland, Woods Hole Oceanographic Institution, oral communications).

Head of the ocean prospecting work for the Soviet Ministry of Geology is Y.B. Kazmin, resident in the "Sevmorgeologiya", or Northern Enterprise for Offshore Geological Exploration, in Leningrad. Most ship deployment is from Vladivostok in the Soviet Far East. Two volumes of short summaries (Egiazarov, 1980, 1985) and a small monograph by Kazmin (1984) are available on the work of the Ministry with regard to the ocean manganese phases. However, though Kazmin's book has an unusually global approach to concretions (in contrast with most studies focusing on the Pacific Ocean), it provides little substantive new data. It does offer some new interpretive approaches, including factor analysis studies (otherwise little used in Soviet work to date), generalized or schematic maps, often using a $z$ statistic or standard deviation-based contour interval of metal concentration in concretions. Most interesting to scientists it reports perhaps the first global geochemical budget for metal sources in total ocean concretions. Only a brief discussion of crusts as distinguished from nodules, incidental to some regional summaries, is presented in the volume. Moreover, an erroneous linkage of hydrothermal manganese to cobalt-rich crusts suggests that these phases have not been intensively studied or well-understood in the Ministry work.

\section{German studies and the 1981 Midpac I Expedition}

German field research on nodules began in 1972 with cruises on the R/V Valdivia. The studies involved cooperation between industrial groups (Arbeitsgemeinschaft Meerestechnisch gewinnbare Rohstoffe, or AMR), which later joined the international consortium, Ocean Management Inc. (OMI), The German Geological Survey (BGR), and academic scientists. Although some detailed site data and technical developments were held confidential, a great deal of information has been published on the wide-ranging German and cooperative studies (see Halbach and Fellerer, 1980; Glasby, 1982).

The breakthrough development for crust research was the German Midpac I" voyage of 1981, headed by Professor Peter Halbach of the Technical University, Clausthal-Zellerfeld (Federal Republic of Germany) (Figs. II-5, II-6). The results of this work (Halbach and others, 1982, Halbach, 1982, Halbach and Manheim, 1984) concluded the following:

1. The dominant ferromanganese oxide phase on seamounts is crusts which form on or envelope hard substrates like altered basalts, hyaloclastites (volcanic breccias) and phosphatized limestones (Fig.II-7). Nodules, or round, concretionary forms resembling the abyssal "nodules" are also found in a great variety of shapes and sizes, but these are chemically unlike the abyssal types, having higher cobalt and iron like their associated crusts.

2. Crusts vary in cobalt concentration from $<.4$ percent at water depths of $>4000 \mathrm{~m}$, and often exceed 1.0 percent Co at depths $<2000 \mathrm{~m}$ to the shallowest sites at $1100 \mathrm{~m}$ (Fig. II-8). Nickel showed a roughly similar trend, reaching to. 7 percent $\mathrm{Ni}$. On the other hand, copper concentrations are no higher than. 10 percent, much lower than for abyssal nodules. 
3. Crusts increased in thickness toward the upper flanks of seamounts (were sparse on summits which often were characterized by sediment caps) and frequently exceeded $2 \mathrm{~cm}$ thickness.

4. Enrichment in cobalt and nickel was observed in the uppermost millimeters of crusts, whereas these elements were depleted in a lower, phosphate-enriched layer.

5. Well-crystallized hydrothermal oxides with very low concentrations of trace constituents were occasionally observed. These appeared to be remnants of hydrothermal precipitation probably associated with early seamount development, and not linked with normal crust development.

6. Based on the preliminary data, seamount slopes having water depth less than $2500 \mathrm{~m}$ had metal weight and value (for cobalt, nickel, manganese, as well as molybdenum) per square meter significantly greater than a corresponding areas of abyssal nodules in the Clarion Clipperton Zone (Table II-3).

7. Unlike abyssal nodules, crusts occurred extensively within 200 miles of the U.S. territory, presumably placing them within national jurisdiction for mineral recovery.

8. The preliminary findings warranted the suggestion that seamount crusts might become viable economic mineral targets if suitable technology for recovering crusts was developed.

The cruise results attracted wide attention, especially in the United States. New cruises by the $R / V$ Sonne, the vessel used in the Midpac I expedition, were launched in 1984, 1985 and 1986, summarized by Halbach (1986), with cooperating American and other foreign scientists. 

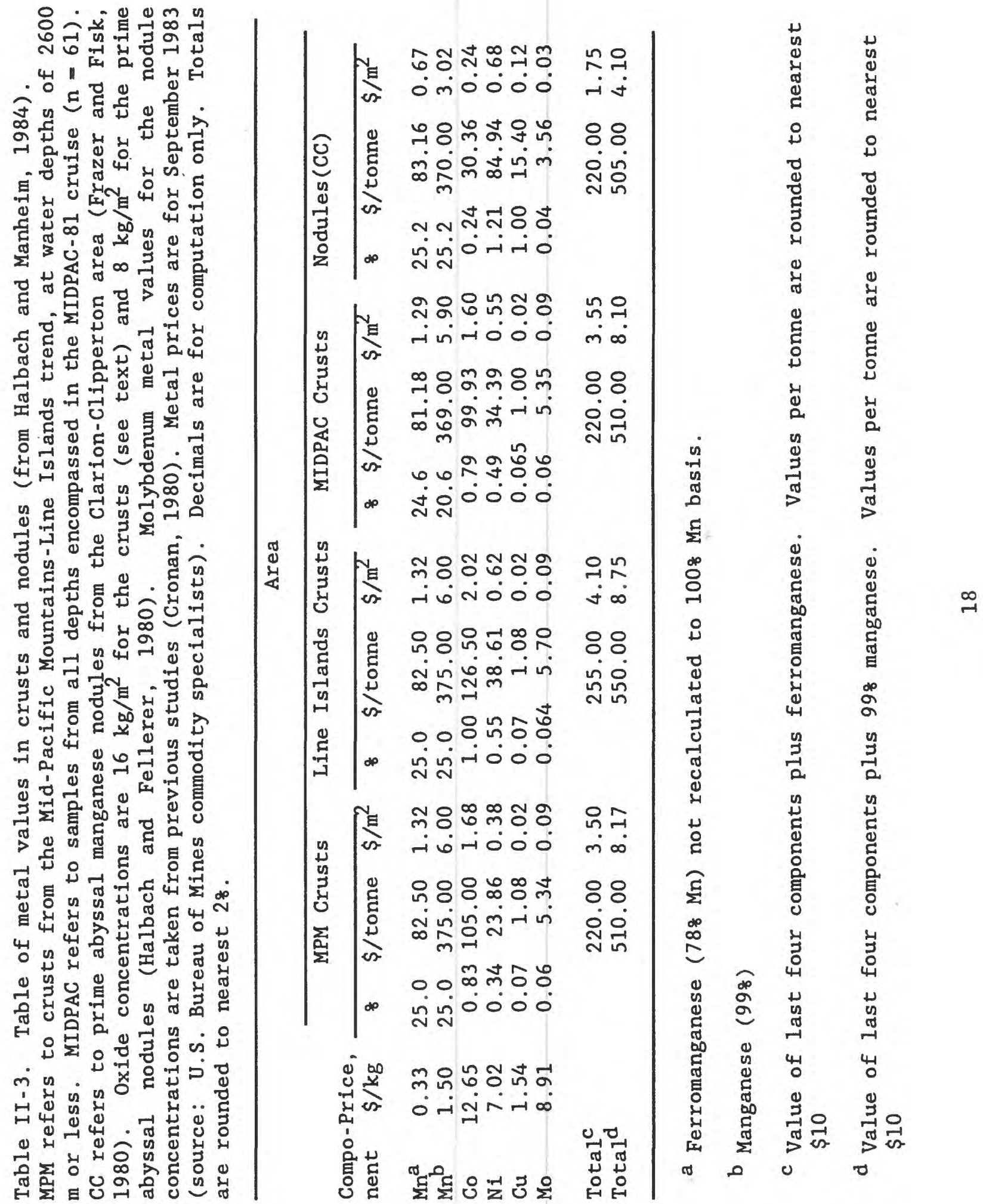


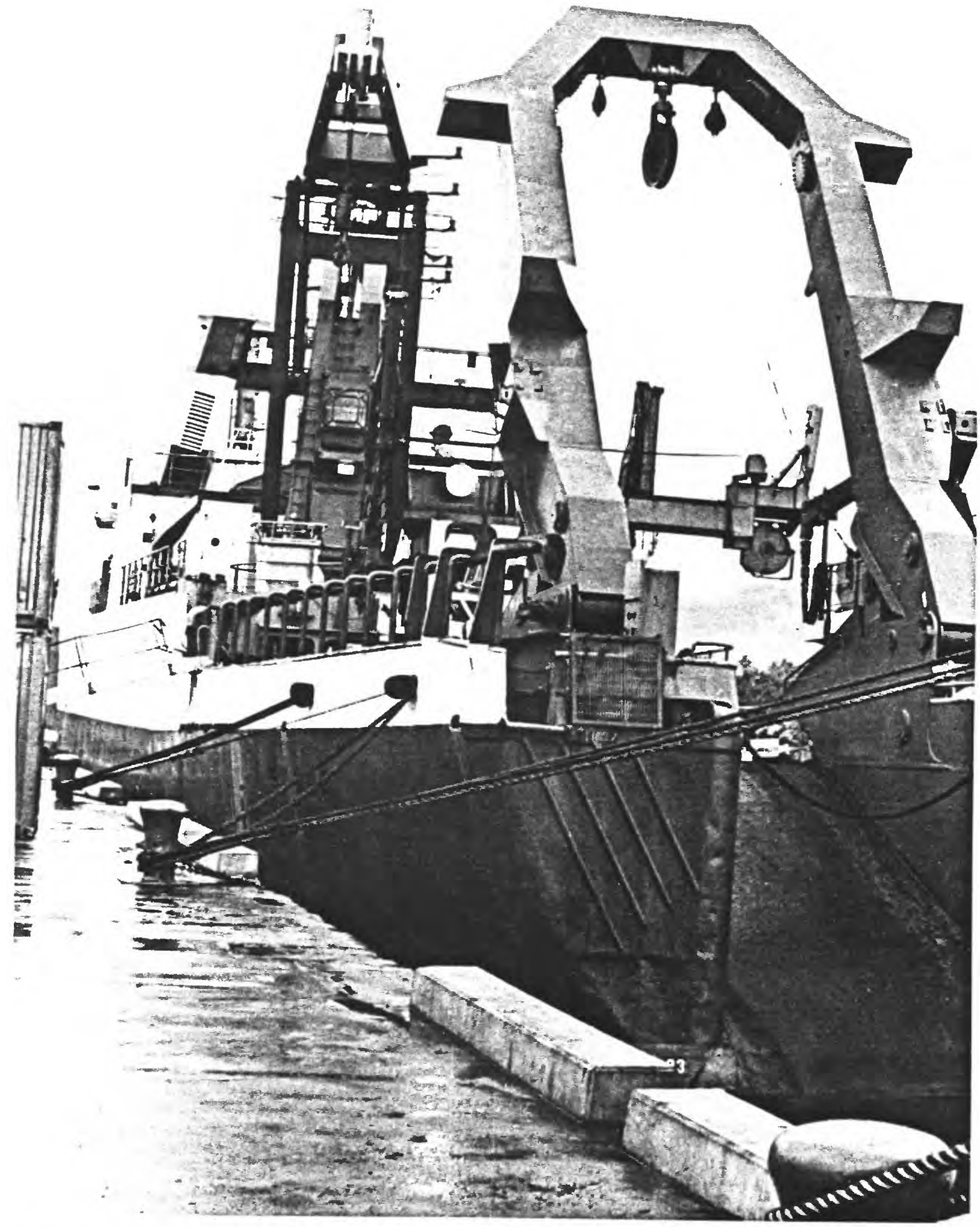

Figure II - 5R/V Sonne, docking in Western Samoa at beginning of Midpac I expedition. 


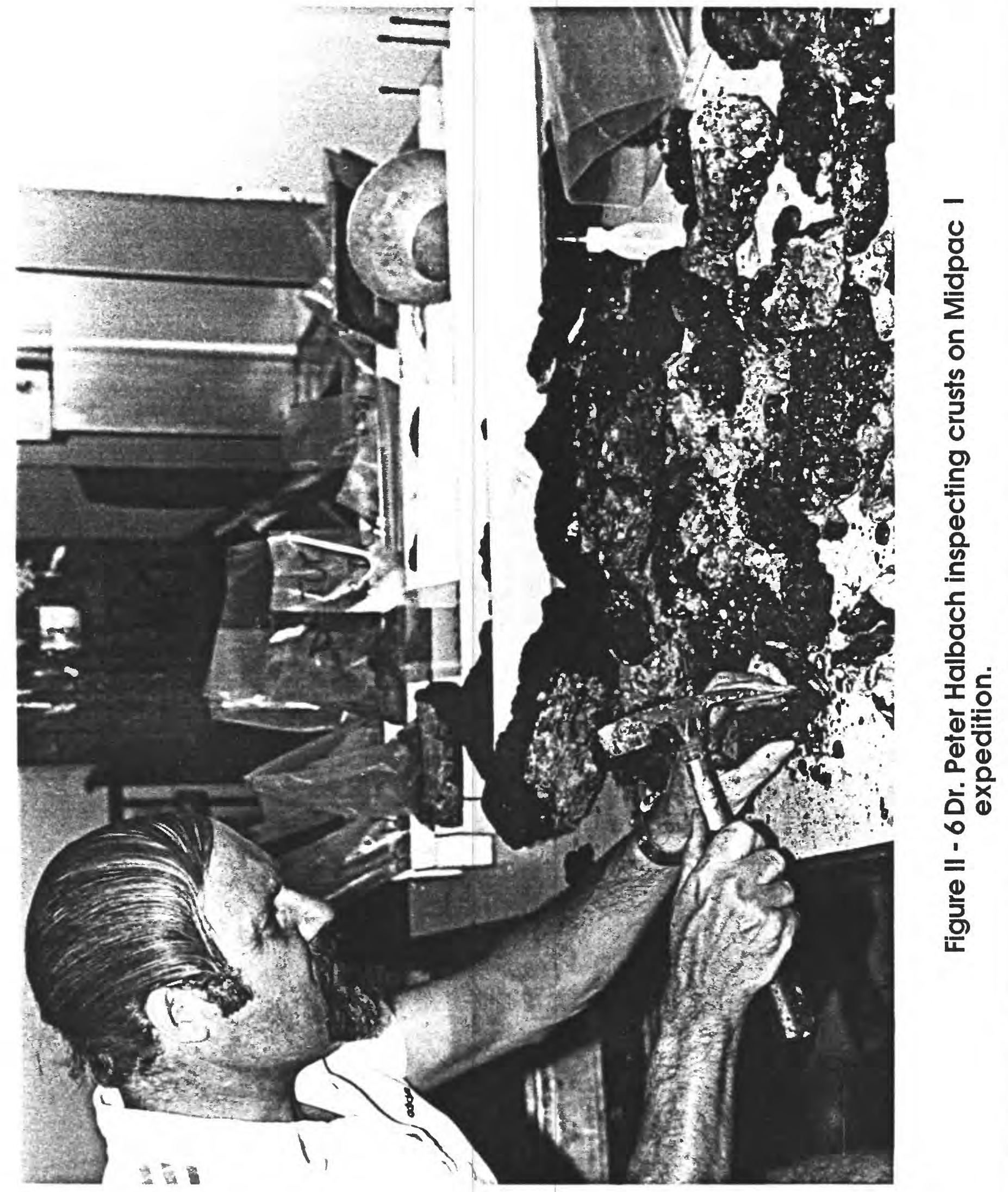




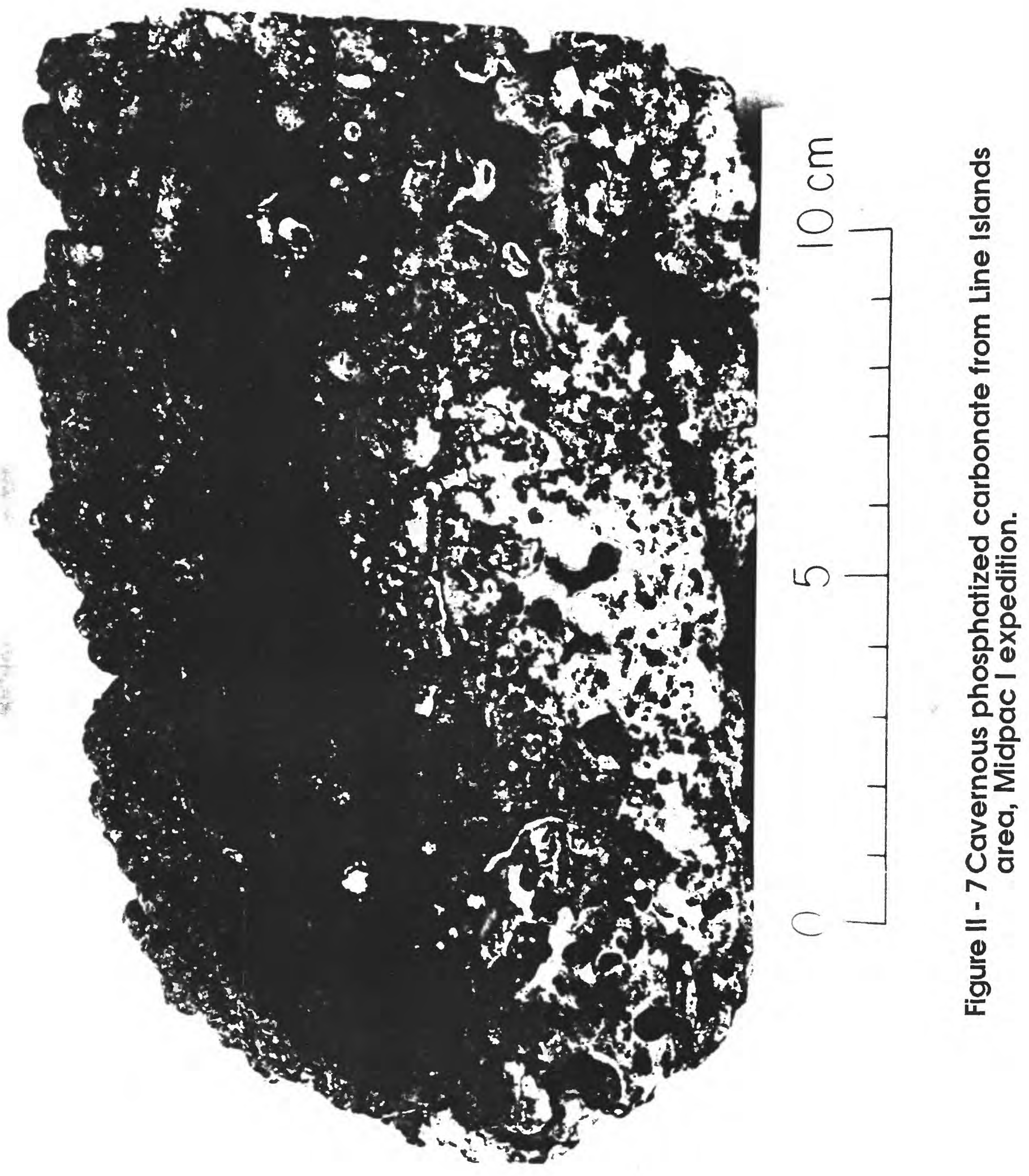




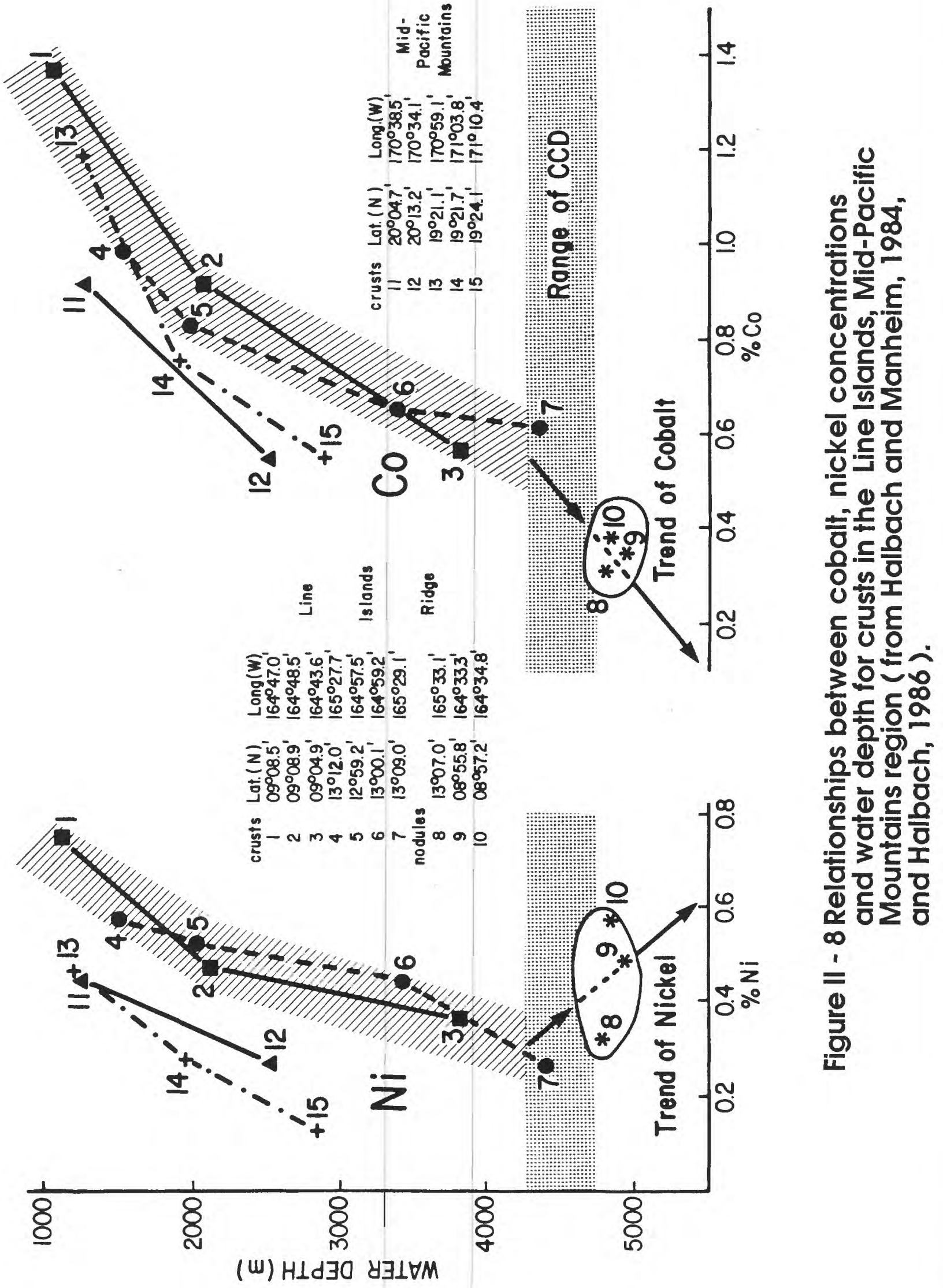


The earlier studies led to awareness that cobalt-rich crusts were included among potential economic resources off U.S. coasts. Mention of this potential was included in the background documentation released with the Declaration by President Reagan of a 200-nautical mile U.S. Exclusive Economic Zone in March, 1983. In that year the first of several U.S. Geological Survey cruises focused on crusts confirmed the results of the German survey on $R / V$ Sonne, but found marked regional differences in crust development and metal content from Necker Ridge, south of the Hawaiian Archipelago, to the giant Horizon Tablemount, and a large seamount in the Line Islands trend (Hein and others, 1985). The USGS vessel, S.P. Lee, also undertook cruises in 1984 (Marshall Islands, Schwab and others, 1985; Hein and others, in press), and in the Johnston Island area in 1987 (Figs. 9 and 10). The latter cruise, with support by the U.S. Bureau of Mines and additional support by Minerals Management Service and Japan Resources Association, discovered crusts as thick as $15 \mathrm{~cm}$ crusts (J.R. Hein and others, 1988, oral communication) in the course of obtaining quantities of crusts for a processing study. Meanwhile, the U.S. Minerals Management Service had initiated a State - Federal Task Force study program that included extensive crust exploration by the University of Hawaii vessels within the U.S. EEZ areas in the Hawaiian archipelago, Johnston Island and Marshall Islands sectors and shipboard work on the German R/V Sonne. Geochemical aspects of these studies are documented in DeCarlo and others, 1987; Chave and others, 1986.

After several years' study a final draft Environmental Impact Statements and a companion volume were released in early 1987 (MMS, 1987; Dept. Economic Development, 1987 and in 1988). These are the first comprehensive Federal documents detailing all of the complex elements of an offshore mineral leasing program: resource evaluation, possible mining technology, transport and processing, and environment assessment and demographic studies. Although the outcome of leasing or other economic outcomes will not be known for some time, it is likely that this complex document will serve as a model for subsequent U.S. mineral leasing activities within the EEZ (Morgan, 1986).

A major compilation of papers relating to Pacific ocean mineral resources includes several chapters specifically relating to the Central Pacific crust studies (Johnson and Clark, 1986). This document summarizes not only exploration data and resource assessment, potential mining technology, transport, and processing, but a host of potential multiple use conflicts and legal and societal issues that would be related to any offshore mineral recovery effort. In an earlier parallel study, Clark and others. (1985) presented a resource assessment for the Minerals Management Service, offering preliminary estimates on favorable coast prospecting sites on a number of U.S. Trust and affiliated territories.

Ferromanganese crusts are by no means limited to the Central Pacific ocean. A USGS cruise to the Blake Plateau (Manheim and others, 1982; Commeau and others, 1984) had revealed that a large portion of the extensive plateau off Florida-North Carolina was covered by a hard phosphorite pavement. This area was already known to be the site of extensive ferromanganese deposits (Manheim and others, 1980), and leases were sought by the Reynolds International Co. in 1978, presumably seeking oxides for petroleum catalyst material and secondary metal recovery. USGS cruises (Schwab and others, 1985; Hein and others, 1987, 
1988) found significant metal potential in the Marshall Islands area, as mentioned earlier. Utilizing archived materials, Aplin and Cronan (1985) published new studies of crusts in the Line Islands sector, including parts of South Pacific areas around the northern part of French Polynesia, and surveys were performed in the Gilbert Islands and other new political areas of the Pacific Ocean (De Carlo and others, in press).

\section{Concluding comments}

This summary will not review in detail the current status of ferromanganese crust studies. Recent reviews in several areas may be consulted as follows: geological and geochemical background (Manheim, 1986; Halbach, 1986; Hein and others, 1987, De Carlo and others, 1987); Several papers on crusts and their economic potential are included in a recent compiled volume on sea floor resource assessment (Teleki and others, 1987). Valuable scientific information relating to the German studies of crusts are incorporated in a $1985 \mathrm{PhD}$ dissertation on the Central Pacific crust deposits by D. Puteanus at the Technical University, Clausthal-Zellerfeld, Federal Republic of Germany (Puteanus, 1986). Environmental, demographic, economic, and marine policy background related to potential economic development of cobalt-rich crusts is given by Morgan, 1986; Wiltshire, 1984; Department of Economic Planning, 1987, Clark and Clark, 1986; Johnson and Otto, 1986; and other papers in Johnson and Clark, 1986. Two student-directed reports provide economic assessments of crust recovery: Carnegie-Mellon, 1985; and Bourne and others, 1987.

Finally, much information is lacking upon which to make meaningful resource and economic evaluations of potential crust resources. Chief among missing information elements are the microtopography, thickness and continuity of crusts in prime crust target areas of the sea floor. Surface-towed swath-mapping techniques such as Sea Beam cannot effectively reflect high-angle rugged topography at depth. Recovery of crusts to date has been largely by 19 th century technology (rock dredges) and an in situ "ore" survey probably cannot be performed without new equipment permitting accurate mapping of sea floor relief and recovery of a significant amount of intact sea floor material from precisely located sites. 

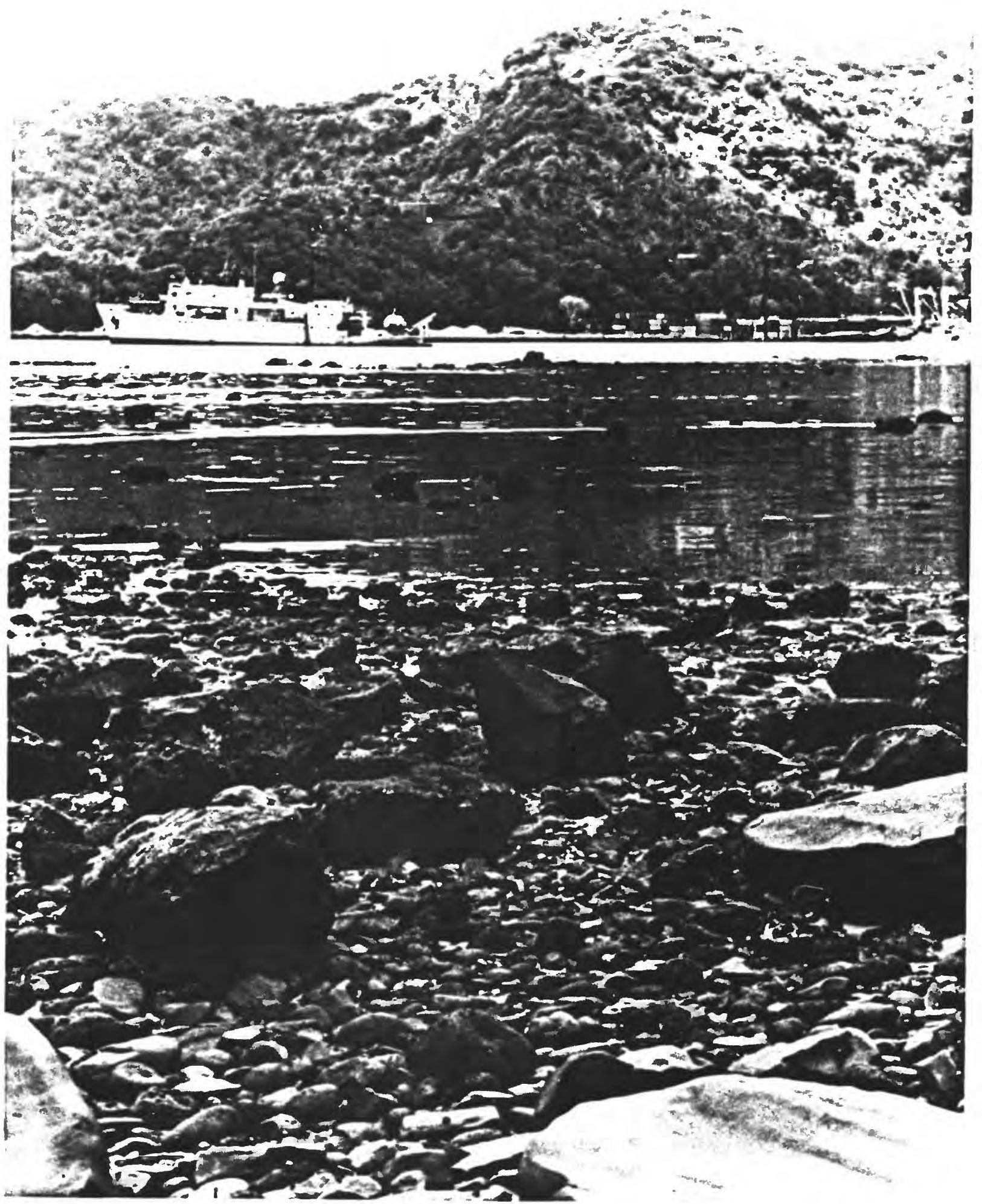

Figure II - 9R/V S.P. Lee at anchor in Pago Pago harbor, Tutuila, American Samoa, on completion of cruise to line Islands, 1983. 


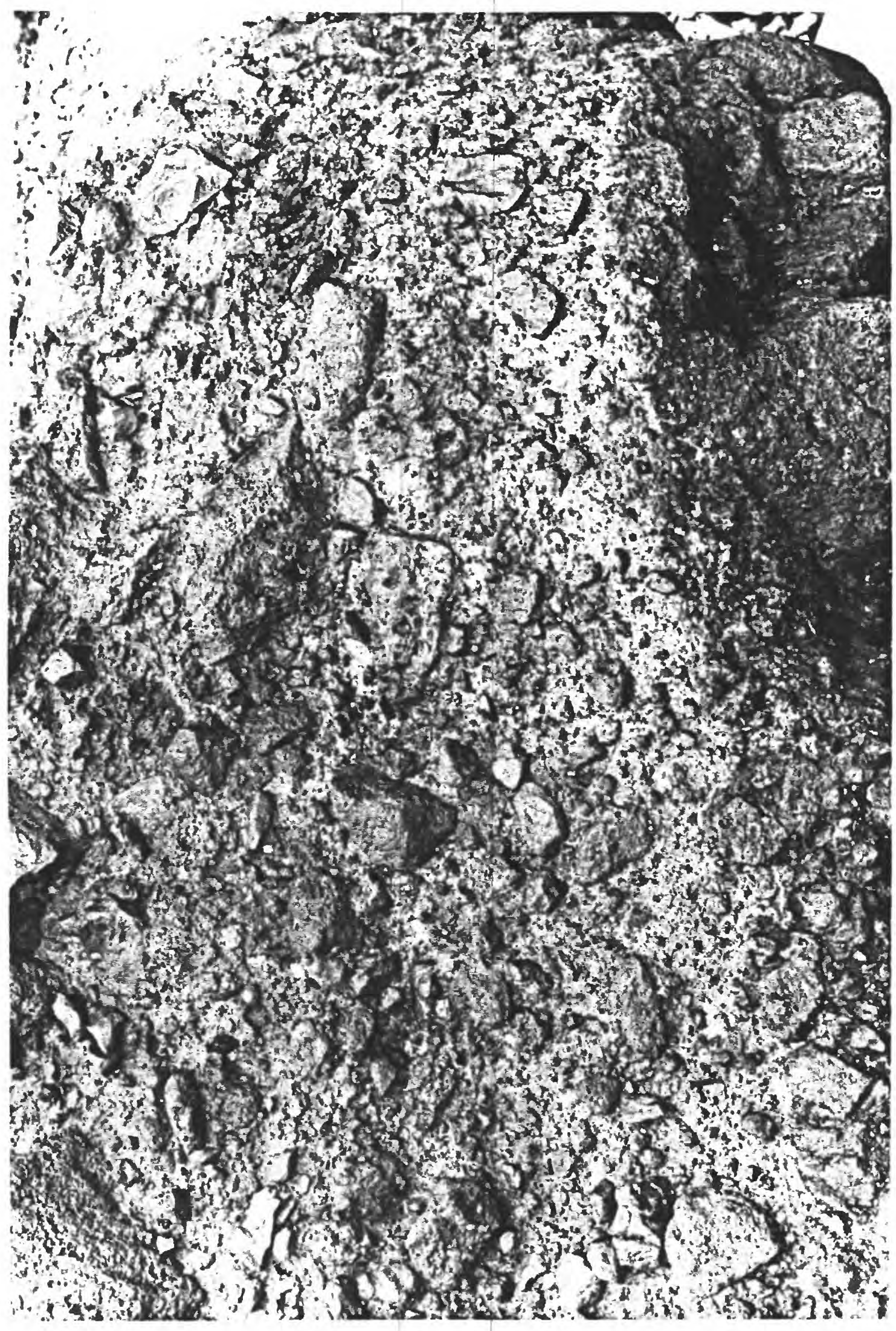

Figure II-10 Boulder of volcanic mudstone-breccia. This material is similar to crust substrates found in altered state throughout Line Islands and Mid-Pacific Mountains. 


\section{ARCHIVE COLLECTIONS by T.H. Ling}

Archive and data search

Beginning in 1983 the USGS searched major oceanographic institution dredge archives for samples and data relevant to the Ferromanganese Crust Data Base. With the cooperation of the institutions more than 600 samples were collectd from twelve institutions and brought to the U.S. Geological Survey Branch of Atlantic Marine Geology in Woods Hole, Mass. for geochemical analysis. This section of the report describes the general sample collection procedure and problems associated with the procedure. All cooperating institutions are listed with quantities and types of samples taken from each facility.

\section{Sampling procedure}

Preparation for a sampling visit began with dredge catalogs and sample descriptions sent by the institutions. Where the dredge collections were well organized, catalogued and described, a collection strategy was devised before arriving at the facility. Specific dredges known to contain crust occurrences, favorable geographic location, or a combination of these were targeted for collection. This reduced the tedious and time-consuming process of trial and error search and sample collection. In practice, most of the institution archives were not catalogued and organized sufficiently to avoid at least some trial and error sample selection.

In order not to deplete valuable dredge materials, samples were kept as small as possible yet still consistent with optimum analytical quantities $(<50 \mathrm{~g})$. The best way to collect valid samples for chemical analysis was to see the entire contents of the original dredge and then choose a representative sample. However, this was not always possible, due mainly to the large size of some of the dredges and their method of storage at the various archive facilities. Due to time and constraints, it was often impossible or impractical to search entire dredge hauls, some of which were as large as seven hundred pounds. Many were stored in facilities with inadequate lighting.

A few of the institutions visited had their dredge collections well organized and stored in neat bins or storage drawers. Where this was the case, a survey of the entire dredge was facilitated and a good representative sample was easily obtained. These cases were more the exception than the rule. Many dredges were stored in canvas sacks, and these could be spread among the entire collection (Fig. III-1). Whereas complete inspection of this type of dredge was impractical, a roughly representative sampling was attempted by means of a systematic search through a reasonable portion of the dredge. Often more than one type of sample was taken.

The amount of sample taken depended in part on the size of the dredge haul, and the amount of Mn crust on the sample. To get the required amount of crust needed for analysis, more substrate and crust had to be taken from samples which had thin manganese encrustations than from samples which had thicker encrustations. In some cases, guidelines were given as to the maximum allowable amount of sample that could be collected, regardless of the size of the dredge or the type of dredge material contained in the dredge. 

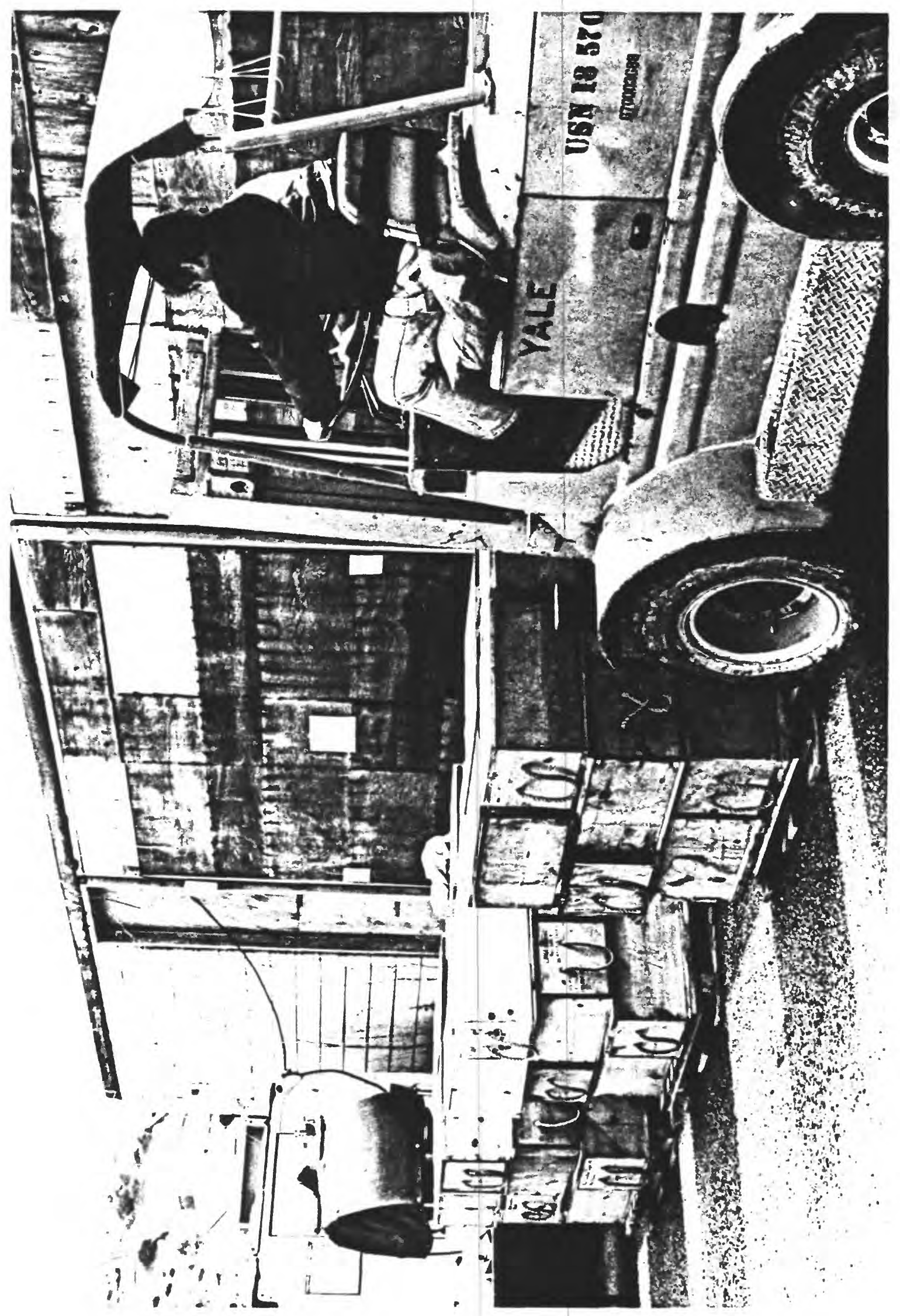

오

눙을

$>$

ơ

을등

앵

옫흔

든 앙으

후응

능호음

노응온

등

는

o

인은홍

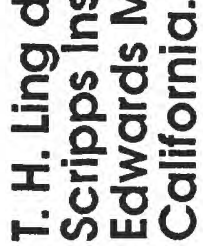

홍 
Mn crusts present and collected ranged in thickness from thin stains to crusts up to 10 centimeters thick. Thin crusts generally occurred on fresh volcanic substrates, whereas thicker crusts generally occurred over older, more weathered substrates. Care was taken to prevent a mixing of the two types of crusts during collection or transit. Fairly thin crusts tended to adhere well to fresh substrates. A sample with a thin crust was usually packed intact for transit without any great concern for separation or mixing of crust and substrate. Thicker crusts over more weathered substrated posed a problem in preventing mixing during transit. Thick crusts tend to be friable and brittle and often were easily pulverized. Weathered volcanic substrates and volcano-clastic substrates were often punky and tended to distintegrate. Packing this type of crust and substrate together for transit risked mixing and contamination. For this reason, thick crusts over weathered substrates were separated at the time of collection and packed separately for transit.

Before any separation was done, samples to be collected were observed, described, and photographed. Noted characteristics included crust thickness, substrate, surficial crust morphology, and distinguishing features. Crust thickness was measured at several points. Where there was significant variability, a range was recorded. The dark color of the crusts made it imperative to make special exposure tests if detail was to be seen on each photo.

Much care was taken to prevent metal contamination during the collection, separation and preparation procedures. At no time during any of these procedures were metal objects allowed to touch either the crusts or the substrates. Plastic spatulas and picks were used to separate crusts from substrates. It is, however, recognized that most samples were collected with metal dredges and also might have been contaminated during shipboard operations. Thicker crusts were often easy to separate, cleaving at the weathered zone. Some substrate might adhere to the base of the crust after separation. This was carefully scraped off before preparation for analysis. Thin crusts were generally harder to separate from substrates. A good deal of scraping and picking was done to assure that all bits of substrate adhering to pieces of crust were scraped off. 
Table III-1. Individual institution listings

INSTITUTION:

DATE OF VISIT:

SAMPLES TAKEN:

GENERAL SAMPLE LOCATION: COOPERATING PERSONNEL:

CURATION FACILITY:

COMMENTS :
Woods Hole Oceanographic Institution $2 / 20 / 83$ - 3/1/83 (Includes collection of Branch of Atlantic \& Gulf of Mexico, U.S. Geological Survey Materials)

51

Eastern North Atlantic

James Broda

One large warehouse. Samples stored in drawers by dredge numbers.

This collection is completely catalogued including cataloguing for manganese crust occurrence. This facilitated the collection process greatly. Dredge hauls are stored separately in drawers, making it relatively easy to select a sample which is representative of the entire dredge.

INSTITUTION:

Smithsonian Institute

DATE OF VISIT:

SAMPLES TAKEN:

GENERAL SAMPLE LOCATION: COOPERATING PERSONNEL: CURATION FACILITY:

COMMENTS :

INSTITUTION:

DATE OF VISIT:

SAMPLES TAKEN:

GENERAL SAMPLE LOCATION: COOPERATING PERSONNEL:

CURATION FACILITY:

PROBLEMS :
$2 / 14 / 83-2 / 17 / 83$

25

Various locations

William Melson, Timothy O'Hearn

One large section of the Mineralogy

Department in the Natural History Building.

All dredge material at this facility is well catalogued, described and curated. The quantity of stored dredges is small. We were given guidelines as far as how much sample material we could take. All material taken from this institution is considered to be on loan.

Scripps institute of oceanography $3 / 21 / 83-3 / 25 / 83,4 / 7 / 83-4 / 10 / 83$ 156

Central and Eastern Pacific

Thomas Walsh, Gurator, Core and Dredge Facility

Dredges are stored at two locations: Seaweed Canyon on the campus, a small garage-like facility where dredge contents are stored in individual drawers by cruise, and Camp Edwards Military Reservation in Clairmont Mesa, where dredges are stored in crates on pallots in an old U.S. Army warehouse.

Catalogued descriptions of this dredge collection are not complete. Many dredges 


\section{COMMENTS :}

INSTITUTION:

DATE OF VISIT:

SAMPLES TAKEN:

GENERAL SAMPLE LOCATION:

COOPERATING PERSONNEL:

CURATION FACILITY:

COMMENTS :

INSTITUTION:

DATE OF VISIT:

SAMPLES TAKEN :

COOPERATING PERSONNEL:

GENERAL SAMPLE LOCATION:

CURATION FACILITY:

COMMENTS : in Camp Edwards location were not open. Time-consuming trial and error search and sampling was necessary. Some samples did not have location data readily available. Most dredges were stored in their original canvas sacks in unopened crates stacked three high (Fig. III-1.) Some of the dredges were stored in numerous crates. In these cases, time considerations made it impossible to search the entire dredge contents. Camp Edwards facility did not have functioning lighting or electricity, and this also made the collection process more time-consuming. Some of the samples did not have location data available at the time of collection. Despite the conditions, well over 100 good samples were obtained with the constant help of the facility curator, Mr. Thomas Walsh.

University of Southern California

$3 / 26 / 83$

3

Gulf of California

Suzanne Reynolds

One fairly small storage room located

under tennis pavilion

Dredges stored here are mostly from shallow-water locations.

University of Miami

$5 / 2 / 83-5 / 6 / 83$

22

Kjell Hayling

Eastern North Atlantic, Carribean, Bahama Slope

Dredged samples were stored in canvas sacks on wooden shelves on the walls of the marine facility building.

This collection was well catalogued, including manganese occurrence. However, the dredges themselves are not organized in any order relating to the catalog. Entire dredge contents were usually stored together, so a search through the entire dredge was facilitated and a representative sample could be gotten. We were unable to locate five samples which were in the catalog. 
INSTITUTION:

DATE OF VISIT:

SAMPLES TAKEN:

GENERAL SAMPLE LOCATION:

COOPERATING PERSONNEL:

CURATION FACILITY:

COMMENTS :

\section{INSTITUTION :}

DATE OF VISIT:

SAMPLES TAKEN :

GENERAL SAMPLE LOCATION:

COOPERATING PERSONNEL:

CURATION FACILITY:
Florida State University

$5 / 9 / 83-5 / 13 / 83$

66

Southern Oceans, Antarctic locations

Dennis Cassidy

Large refrigerated core and dredge facility

Dredge contents, or partialrepresentative portions were neatly stored in small bins on stacking shelves. This facility is immaculately maintained by $\mathrm{Mr}$. Cassidy, and this made the collection effort very easy and time-efficient. Samples open to observation in individual bins made it easy to pick a sample representative of the entire dredge.

Hawaii Institute of Geophysics, University of Hawaii

$7 / 8 / 83-7 / 22 / 83$

125

Central Pacific

Chris Mato, Loren Kroenke, Frisbee Campbell, Fritz Theyer, Don Hussong, Barbara Sinton

Because of problems in completing a new storage facility at Snug Harbor, there was no systematic storage for dredged materials. Some are stored in a trailer van parked at the Snug Harbor Marine Facility, and the rest is stored in the basement, in the compressor room, and on the catwalks throughout the Hawaii Insitute of Geophysics. Sampling was time consuming. Location data were not available for a portion of samples.

COMMENTS : cataloging project, at the time of collection most of the dredges and cruises we were interested in were not yet entered. Most dredges are stored in canvas sacks. Lack of electricity and other problems hindered collection in most locations. Some dredges were quite large and took up as many as 15 canvas sacks. Time constraints made complete searches through these dredges impractical, so some of our samples may unrepresentative of the dredge. In spite of the difficulties, The University of Hawaii collections represent a key resource for crust study in the Pacific ocean, and approximately two weeks were spent at the facility. 
INSTITUTION:

DATE OF VISIT:

SAMPLES TAKEN:

GENERAL SAMPLE LOCATION:

COOPERATING PERSONNEL:

CURATION PERSONNEL:

COMMENTS :

INSTITUTION :

DATE OF VISIT:

SAMPLES TAKEN:

GENERAL SAMPLE LOCATION:

COOPERATING PERSONNEL:

CURATION FACILITY:

COMMENTS :

INSTITUTION :

DATE OF VISIT:

SAMPLES TAKEN:

GENERAL SAMPLE LOCATION:

COOPERATING PERSONNEL:

CURATION FACILITY:

COMMENTS :
University of Washington

$8 / 15 / 83-8 / 16 / 83$

30

Western Pacific

Debby Kelly, Paul Johnson

Samples were already collected prior to my arrival and I did not get to work in the curation facility.

The collection process was nearly completed ahead of time, but there may be other samples of interest.

Oregon State University

$8 / 17 / 83-8 / 19 / 83$

15

Western Pacific

Elizabeth Asbury

Roofed, open-air garage like facility. Most samples are stored in bins on shelves, but some larger dredge contents are stored in sealed crates.

Dredges were catalogued for availability, but not for Mn occurrence, making a trial and error search necessary. Collection did not contain much manganese-encrusted material. The dredge collection was being reorganized and recatalogued at the time of visit. Time limited the number of dredges sampled.

U.S. Geological Survey, Branch of Pacific Marine Geology, Menlo Park, CA.

8/29/83-8/30/83, 6/4/84-6/7/84

40

Central and Western Pacific

Dave Clague, Bob Boyer

Many dredges are stored in sacks on shelves in the flume room, building B, Deer Creek Marine Building, Palo Alto. Others are stored in USGS. warehouses on $0^{\prime}$ Brien $\mathrm{Rd}$. in Redwood City.

The collection process was time consuming due to the lack of systematic cataloguing of the dredges. There may be more of interest. 
INSTITUTION:

DATE OF VISIT:

SAMPLES TAKEN :

GENERAL SAMPLE LOCATION: COOPERATING PERSONNEL: CURATION FACILITY:

COMMENTS :

INSTITUTION:

DATE OF VISIT:

SAMPLES TAKEN:

GENERAL SAMPLE LOCATION: COOPERATING PERSONNEL:

CURATION FACILITY:

COMMENTS :

INSTITUTION :

DATE OF VISIT:

SAMPLES TAKEN :

GENERAL SAMPLE LOCATION: COOPERATING PERSONNEL: CURATION FACILITY:

COMMENTS :

SPECIAL NOTE:
Naval Oceanographic Research and

Development Activity (NORDA), Bay st. Louis, Miss.

$6 / 25 / 83-6 / 28 / 83$

18

Eastern Central Atlantic

Julius Egloff

Dredges are stored in steel drawers by cruise in a utility building at the facility.

Dredges at this facility are not catalogued. It was not too difficult or time consuming to search through the entire dredges in the storage bins for a good representative samples. We do not have location and depth data for a number of NORDA samples.

Lamont-Doherty Geological Observatory

$6 / 18 / 84-6 / 21 / 84$

80

Atlantic

Floyd McCoy

Dredges are stored in pine boxes stacked on storage shelves in a warehouse room.

Lamont has original cruise dredge descriptions available, and they also catalog their dredges for manganese occurrence. This speeded the collection process greatly, since we knew which samples we wanted to collect before arriving. However we could not find a number of targeted dredges.

University of Alaska

$8 / 25 / 84-8 / 26 / 84$

8

Gulf of Alaska

Donald Taylor

Samples stored in trays in storage cabinets located in laboratory.

Most of the samples available had only manganese stains. None had thick, separable crusts. Took eight samples for archives.

The U.S. Geological Survey office, Anchorage Alaska indicated that no offshore dredgings or other prospectively useful materials were held at their facilities. 
INSTITUTION :

DATE OF VISIT:

SAMPLES TAKEN:

COOPERATING PERSONNEL:

COMMENTS :
Graduate School of Oceanography, University of Rhode Island, Narragansett

Various times during 1983

None

Jean-Guy Schilling

The GSO has a number of crusts from dredgings in Atlantic ridge and seamount areas, but permission was not given to sample during the collection period. 
IV COMPUTER DATABASE DEVELOPMENT by C.M. Lane-Bostwick and F.T. Manheim

\section{Introduction and early development}

In December 1982, the U.S. Geological Survey in Woods Hole established a central repository of data specifically related to ferromanganese crusts and their chemical composition (Manheim and others, 1983). While field collections were in process (see Chapter III), data encoded in the Scripps Institute of Oceanography Nodule Data Bank (SNDB) were downloaded for incorporation into the database. We utilized the SNDB's format as a model, although a few of their attributes were modified and new attributes were added to reflect the nature of the current data. This modified data format is detailed in Lane and others. (1986).

New crust sample data collected from repositories and on recent cruises and their subsequent chemical analyses were entered on microprocessors using a data entry program, Datastar. Datastar allows a form to be generated to facilitate data entry by inexperienced computer users. These data and data obtained from SNDB were then converted into the format required by the Geological Retrieval and Synopsis Program (GRASP) (Bowen and Botbol, 1975). GRASP was designed to function primarily as a data retrieval system. Its structure is relational allowing the user to join datafiles that have a common key field. This feature was particularly useful for retrieving crust sample data from the SNDB. GRASP also permits basic statistical computations, output to graphics, and creation of subsets of large datafiles based on logical conditions.

\section{SIO Nodule Databank}

The SIO data (SNDB) were received in 1983 from the National Geophysical Data Center (NGDC) as two separate files, one for station data and one for chemical data. Each record in the chemical analysis file is linked to a corresponding record in the station data file by a field common to both, the sequence number (SEQNO). This is a unique 7-digit number that incorporates a 10-degree numbered grid system ("Frazer" square) as the first 3 digits with the last 4 digits representing an assigned serial number. We retained this concept in the USGS ferromanganese crust datafiles. However to distinguish our entries from those downloaded from the SNDB, we use a 6 digit number where the first 3 digits are based on the more widely known "Marsden square" system and the last 3 digits are an assigned serial number for each sample within that 10-degree grid segment. The maps of Lane and others. (1986) incorporate both for user convenience.

The SNDB station datafile has over 10,000 records of manganese deposits ("nodules") from institutional archives and literature. The chemical datafile has 6,832 records, each representing a chemical analysis, often with more than one analysis for the same sample. After uploading the total SIO databank sample file from tape, we incorporated those station records that were coded to indicate that chemical data were available. This subset resulted in a datafile that contained 2,842 station records.

In the attributes of the chemical analyses data set, Frazer and others (1978) define 10 separate types of ferromanganese oxide samples that were analyzed, 5 of which pertain or may pertain to ferromanganese encrustations. The next step was to set conditions to search for only those analyses performed on samples defined as 1.) Mn-encrusted sediment, 2.) Mn-coated rock, 3.) crust or pavement, 
4.) Mn-coated organic material or 5.) several crusts. This subset resulted in 825 analyses. In addition, observing that samples having cobalt greater than 0.5 percent nearly always occurred on seamounts or other sites suggesting a probable crust environment, samples having Co $\geq 0.50$ percent were added to the previous subset. Nodule-like seamount crusts were shown on the 1981 Midpac cruise to have similar chemical composition to slab crusts. This addition brought the total number of extracted analyses to 1,095. These analyses were linked to the station datafile and found to be associated with 622 sample-stations (Table IV-1). The SIO station and chemical data extracted as described above are listed in Appendix B and Table 9 of Appendix C, respectively. Trace analyses for rare earth elements are listed separately in Table 10 of Appendix C.

\section{UNIX and PC hardware}

During the development of the database, technology was progressing rapidly. The implementation of a Unix based computer system and the improvement of software available for MSDOS IBM-PC compatibles enhanced are graphic and typesetting capabilities. Central to the graphic display of our database have been MAPGEN, PLOTGEN, GRIDS and CONTOUR (Evenden, 1986, Botbol, J., and Evendon, G., in preparation). Table displays were improved by using UNIX troff and tbl software programs. In the PC environment detailed statistical operations were accomplished using Northwest Analytical STATPAK software, data entry with DATASTAR and general computations with LOTUS 1-2-3. All of the above were output on various peripheral devices which have also improved over the years; the most recent output being the gray scale maps of gridded elemental data as shown in Chapter IX.

Table IV-1. Sources of crust samples from SNDB.

\begin{tabular}{lccc} 
Institution & Samples & $\begin{array}{c}\text { Major chem. } \\
\text { analyses }\end{array}$ & $\begin{array}{c}\text { Trace chem. } \\
\text { analyses }\end{array}$ \\
\hline HIG & 48 & 80 & 1 \\
SIO & 176 & 320 & 52 \\
LDGO & 109 & 134 & 5 \\
WHOI & 9 & 11 & 3 \\
FSU & 43 & 54 & 21 \\
USSR & 66 & 87 & 4 \\
Unknown & 171 & 409 & 53 \\
Total & 622 & 1095 & 139
\end{tabular}

\section{New data acquisitions (USGS Woods Hole)}

New data acquisitions include material collected from institutional archives as enumerated in Chapter III, shipboard samplings from 1981 onward and from miscellaneous smaller collections and literature references. A total of about 2000 analyses from about 1500 stations is incorporated. The total list is included in the data tables. The enumeration of complete sources is shown in Table IV-2. No further attempt has been made to maintain comprehensive support 
of this database after April 1988, however selected data sets can be added on a discretionary basis. The arrangement of data categories is displayed and discussed in Chapter VIII (Data), and further explanation and commentary is provided in Lane and others (1986).

Table IV-2. Status of crusts collected by USGS Woods Hole by institution.

\begin{tabular}{lrr} 
Institution & Samples & $\begin{array}{c}\text { Major and minor } \\
\text { chemical analyses }\end{array}$ \\
\hline HIG & 402 & 429 \\
SIO* & 159 & 100 \\
LDGO & 76 & 35 \\
WHOI & 81 & 27 \\
FSU & 64 & 60 \\
UWASH & 31 & 28 \\
NOAA & 22 & 22 \\
UMIAMI & 18 & 26 \\
SMIT & 13 & 8 \\
TUCLZ & 306 & 324 \\
USGS-WH & 117 & 271 \\
USGS -MENLO & 106 & 121 \\
OSU & 3 & 0 \\
NORDA & 18 & 0 \\
UALASKA & 8 & 0 \\
GSJ & 15 & 24 \\
NGDC & 288 & 460 \\
Other & 22 & 22
\end{tabular}

* Additional samples are available but have not been entered pending receipt of station data

Although the database's primary objective has been to permit computer retrieval and manipulation of data, this output incorporates features intended to facilitate use of a hard paper copy. All chemical data are reported in widely used rock chemical/geochemical tabular format rather than computer format. The first three digits in the sequence number (SEQNO) identify the $10^{\circ}$ latitude-longitude block in the station maps (Lane et al, 1986) or any other Marsden-indexed map without having to scale off latitudes and longitudes. Therefore, chemical samples for any area on a map can be located by the first three digits of the sequence numbers by which all chemical tables are listed. Chemical tables are also prefaced by latitude, longitude, depth and lab number to aid in identifications in the hard-copy printout. The SNDB data retain their sequence numbers as assigned by Frazer and Fisk. Maps available in Lane and others, 1986 aid in using their $10^{\circ}$ grid numbering system. NGDC data are listed in Appendix $A$ and Table 11 of Appendix $C$ and are prefaced by their 'MMBIB' identifier; a unique field that incorporates author and date of publication of reference where data were obtained. 
by J.A. Commeau and F.T. Manheim

Sample preparation technique is critical for valid chemical analyses, as some of the comparisons in Chapter VII show. Analytical data from the general literature is particularly prone to errors of various kinds. Some have been discussed by Frazer and Fisk, 1979, who made a careful evaluation of the data they culled from the literature. Many errors in the Scripps Institution Nodule Data Bank (SNDB) remain; some are definable, whereas others are suggested mainly by internal inconsistencies, or marked deviations when data from a given region are compared with more carefully controlled data.

Principal sources of error are 1) the great specific surface area of crusts and hence their tendency to retain or attract hygroscopic moisture; variable treatment of this moisture can in extreme cases lead to analytical value differences as great as 40-50 percent, as demonstrated in Table V-1, 2) inhomogeneities in samples, especially where segregated mineral phases have differing composition, 3) contamination of samples, either through handling with metal-containing equipment, or by contamination of samples in grinding vessels previously used to prepare samples having a different composition, and 4) by analytical or computational errors. Ferromanganese oxides, with their associated metals, have been a traditionally difficult material to analyze, depending on methods. The iron and manganese can potentially create matrix effects or spectral line influence in emission spectrometric, atomic absorption and $\mathrm{X}$-ray fluorescence techniques; many elements can offer interferences with spectrophotometric techniques. Inductively coupled plasma spectrometry has reduced though not eliminated matrix influences. However, properly controlled methods of any type may yield quality data.

\section{Sample selection and preparation}

The basic procedure for selecting crusts was to choose a complete crust thickness, excluding substrate as much as possible. A section that would yield from 5 to 25 grams of sample was sought, but many samples yielded less material. Subsamples had to be scaled to accomodate all analytical uses and duplications, where desired. In a few areas, and where the amount of crust permitted, separate subsampling of crust intervals, especially the top millimeter, was performed. Given the variability of crust thickness and chemical variability with depth in the crust, a top sample would provide a more synoptic analytical base over broad ocean areas than total crust thickness. Normally, macrosampling was limited to. $5 \mathrm{~cm}$ horizons, and no finely detailed subsampling such as was performed using dental drills (Halbach and Puteanus, 1984) was attempted. For thin crusts, large areas were scraped to obtain the required volume of material. These thin crusts provide representative material for geographic areas that otherwise would not yield ferromanganese oxide material, or fill in a range of water depths. Thinner crusts are often found on deeper water flanks of seamounts and sometimes on the top surface of seamounts. They may, of course, also occur in any other position on younger substrates.

Segregation of crusts from underlying substrate was necessary in order to achieve a basis for comparison of crusts. Because crusts, unlike abyssal nodules, adhere to hard substrates, the proportion of crust in recovered rock samples is highly 
variable and arbitrary, depending on the type of recovery device, size of material recovered, physical preservation, etc. The chemical analyses show that a few samples from the USGS data set are dominated or strongly influenced by substrate rock. The material in these cases was often (probably dark) basalt, and may have also entered the samples when thin oxide crusts were scraped from surfaces. Much greater substrate contamination is encountered in the data from the SNDB. Samples with large proportions of substrate contaminant have been useful in one respect. They serve in plots of the kind, Si vs $\mathrm{Fe}+\mathrm{Mn}$ (Chapter IX, Fig. 13) as end member phases, and indirectly help elucidate the chemical influence of less obvious quantities of unavoidable detrital matter in the crusts. Some types of markedly contaminated samples are indicated by special symbols in the chemical tables (Appendix $C$ ) and are discussed in Chapter VIII.

\section{Preparation of bowl for grinding}

Our grinding was performed in a Phoenix Siebtechnik automatic grinding machine, using agate bowls, lids, and pucks. Several days prior to beginning the powdering operation the metal lip of the bowl and lid is lightly coated with epoxy resin. This prevents contamination of the sample by contact with the steel-encased gate lip during sample handling. Acetone removes unwanted wet epoxy from the agate surface. Prior to grinding crust, crushed glass is ground for 10 minutes to clean off the agate grinding surface. The polish of this surface must be preserved to ensure the most efficient powdering of the sample.

\section{Preparation of crusts for grinding}

Substrate is carefully removed and discarded or saved for analysis in special cases (i.e. phosphorite). A mini-slab less than $2 \mathrm{~cm}$ thick is cut from the crust perpendicular to the surface and substrate. This slab is used for total analysis. Other sections may be separated into horizons or layers as appropriate. Each section to be powdered is stored in labeled polyethylene or polystyrene vials or bags until processed. Material for SEM or microprobe analysis is taken from a section adjacent and parallel to the section for bulk analysis.

\section{Grinding and sieving}

The samples for bulk chemcal analyses (XRF, AA, ICP, etc.) are ground in the described automatic mortar and subsequently sieved to $<105$ microns. This size fraction is a compromise between an optimally desirable finer size, such as the $74 \mathrm{u}$ sieve generally used in Scripps Institution analytical work, and consideration of time and tedium in preparation. Commercial sieve and sizing experts were unable to inform us of any metal-free, automated pulverizing equipment.

1) Grinding. The standard grinding time is 8 minutes. Samples with high silica content may take longer. Approximately 5-25g of sample are powdered per run.

2) Sieving. A 105 micron nylon mesh is secured between two plastic rings and set in a glass baking dish which prevents sample loss due to spillage. A secretarial eraser/brush is used to push the powder through the sieve. The powder is homogenized before splitting into 
subsamples, and stored in properly labelled polyethylene or other plastic vials, and lids are sealed with cellophane tape.

3) Cleaning. All parts (bowl, puck, lid, sieve rings, mesh, brushes, glass dish) are thoroughly cleaned between each sample to avoid cross contamination of samples. Loose particles are removed by washing in hot, soapy water with a sponge. Remaining ferromanganese material is removed by washing with 10 percent hydroxylamine hydrochloride ( $\mathrm{NH}_{2} \mathrm{OH} . \mathrm{HCL}$ ) and 1 percent oxalic acid. The use of rubber gloves during cleaning is recommended. Care should be taken to avoid prolonged contact of the oxalic acid with organic compounds. When no trace of ferromanganese stain remains on a surface it is rinsed with distilled water and then with methanol which speeds drying. The next sample may be started as soon as equipment is dry.

Processing time per sample is approximately 45 minutes with practice. Speed should not be obtained at the expense of contamination. Many sieve meshes and brushes are used so that one is always dry and ready to use. The use of two grinding bowls, pucks, and lids eliminates waiting for drying. Minimum and maximum capacities of grinding apparatus are carefully observed. Overloading results in poor grinding and underloading may damage the bowl interiors. Repairs are expensive and time-consuming.

\section{Water content}

Table V-1. Example of differences in reportable manganese concentration, depending on sample treatment. Sample is a ferromanganese oxide-phosphorite pavement from the Blake Plateau. Values are in weight percent. Wt. loss = cumulative loss from original weight.

Reporting basis

A. Powdered samples, with natural
hygroscopic moisture

B. Same, after heating at $110^{\circ} \mathrm{C}$ overnight

C. Same, after heating at $500^{\circ} \mathrm{C}$ one hour

D. Same, after igniting at $1000^{\circ} \mathrm{C}$ one hour
Wt. loss

6.11

14.09

28.87
$\mathrm{MnO}_{2}$

(total)

17.53

18.67

20.40

24.64

In the above sample, the $\mathrm{MnO}_{2}$ value on an ignited basis is 40 percent higher than $\mathrm{MnO}_{2}$ on an air-dry basis. Some samples have much higher ( $>20$ percent) hygroscopic moisture even if they appear dry. Moreover, still other reporting bases than those mentioned above have been used. The standard reporting basis for this report, as well as for the Scripps Institution Data Bank is oven-dry, heated at $110^{\circ} \mathrm{C}$ overnight or the equivalent.

Hygroscopic water content on crusts can be determined in two ways. Analytical portions of powder can be dried at $110^{\circ} \mathrm{C}$ overnight, and kept in desiccators prior to weighing out for analytical purposes. However, the hygroscopic nature of the crust is so great that it can actually draw water from some desiccants, such as 
silica gel. In addition, with frequent opening of desiccators it is nearly impossible to avoid rapid water uptake in samples. We therefore prefer to use a well-homogenized powder sample that has been equilibrated with ambient air, and weigh out this material for analytical purposes. Another sample, weighed out at the same time is used for hygroscopic moisture analysis.

About one gram of manganese crust powder is spread evenly in the bottom of evaporating dishes or preferably weighing bottles with well-fitting stoppers. The bottles are placed open in a regular convection oven at $110^{\circ} \mathrm{C}$ overnight or for an equivalent period of time. Tops are emplaced while the bottles are still hot before containers are moved to a desiccator with a powerful desiccant like fresh $\mathrm{P}_{2} \mathrm{O}_{5}$ in an aluminum foil dish beneath the sample rack. Desiccant is replaced or hydrous surfaces are peeled off regularly. Weighing before and after heating is performed with a single-pan semi-micro analytical balance having a resolution of. $1 \mathrm{mg}$.

All raw analytical data based on air-dry samples are corrected for hygroscopic moistures as in equation 1 , where $V$ (corr) is the corrected analytical value, $\mathrm{V}$ is the uncorrected value, and $\mathrm{H}$ is the percent hygroscopic water content in the sample split used for hygroscopic moisture analyses.

$$
\mathrm{V}(\operatorname{corr})=\mathrm{V} \times 100 /(100-\mathrm{H})
$$

\section{$\underline{X-r a y}$ fluorescence spectrometry}

The X-ray fluorescence analysis of major and minor elements of ferromanganese crusts was performed on an automated, software-controlled X-ray spectrometer (Diano XRD8300). All samples and standards were prepared following the methods of Rose and others (1963).

\section{Sample preparation}

Sample powders and reference standards were fused using anhydrous lithium tetraborate along with the addition of lanthanum oxide. The fusion process eliminates the effect of mineral and physical properties of samples, including grain size, and addition of lanthanum as $\mathrm{La}_{2} \mathrm{O}_{3}$, a high-atomic number element, diminishes absorption or enhancement effects.

A crust sample weighing $0.125 \mathrm{~g}$ is prepared with $.125 \mathrm{~g}$ of high-purity $\mathrm{La}_{2} \mathrm{O}_{3}$ and 1.00 grams of $\mathrm{Li}_{2} \mathrm{~B}_{4} \mathrm{O}_{7}$. The components are pre-mixed by hand by rolling on a weighing paper, and then transferred to a graphite crucible for fusion. Because of the hygroscopic nature of ferromanganese materials the samples are pre-ignited at $500^{\circ} \mathrm{C}$ for 15 minutes prior to fusion. This is done to prevent the samples from splattering at the higher fusion temperature.

Fusion is then completed at $1100^{\circ} \mathrm{C}$ for 15 minutes. The fused samples are allowed to cool and the resultant bead is weighed. Fine-grained chromatographic cellulose is added to the bead weight to bring the total weight to $1.500 \mathrm{~g}$. This is to prevent differences from sample to sample in final weight owing to ignition loss and the cellulose also acts as a binder when the glass bead is cracked,

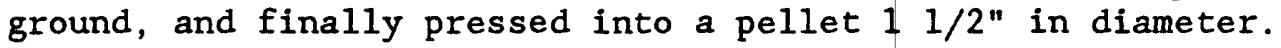




\section{Analytical conditions}

The analyses were performed on an automated vacuum spectrometer system with a ten-position sample carousel, dual-target $\mathrm{X}$-ray tube ( $\mathrm{W}$ and $\mathrm{Cr}$ ) with a front flow proportional counter and a rear sealed proportional counter. All instrument junctions and all software for data-handling, manipulation and storage are performed by the XRD0780 microprocessor board installed on the machine.

Intensity ratios of reference materials are used in establishing calibration curves for each element. A series of multiple linear regression equations is available in the software to provide a means to correct for absorption, enhancement, line interferences from other elements and background variations. Each equation has a specific application for one or a combination of effects. Once the slope, intercept and coefficient of determination has been established for each element from the appropriate calibration curve, the data are stored in tabular form in the Z-80 software. The actual samples are then run, ratioing a11 analyses to a reference sample or drift monitor as a check on instrument stability and precision for repeat analyses over extended periods of time.

Information on the reproducibility and accuracy of the basic X-ray fluorescence method is given in Table V-2, which gives data on the ferromangese crust standard, MCC, a composite of samples from the Line-Islands Midpac area, recovered in the Midpac I cruise. Each of the analyses shown was separately prepared at various times over a period of a year. See also discussion in Chapter VII.

Table V-2. Detection limits and error distribution for elements determined by $X$-ray fluorescence. $n$ refers to number of samples, S.D. is standard deviation, and $C V$ refers to coefficient of variation.

$\begin{array}{lcccc}\text { Element } & \begin{array}{c}\text { Reston } \\ \mathrm{x}(\mathrm{n}-2)\end{array} & \begin{array}{c}\text { Woods Hole } \\ \mathrm{x}(\mathrm{n}-5)\end{array} & \mathrm{SD} & \begin{array}{l}\mathrm{CV} \\ (8)\end{array} \\ \mathrm{Si} & 4.75 & 4.58 & 0.055 & 1.2 \\ \mathrm{Al} & 1.3 & 1.53 & 0.054 & 3.8 \\ \mathrm{Mg} & 1.27 & 1.23 & 0.075 & 6.1 \\ \mathrm{Cu} & 0.071 & - & - & - \\ \mathrm{Ni} & 0.45 & 0.45 & 0.0045 & 1.0 \\ \mathrm{Co} & 0.68 & 0.73 & 0.0217 & 3.0 \\ \mathrm{Fe} & 13.9 & 14.42 & 0.063 & 0.44 \\ \mathrm{Ti} & 1.07 & 1.02 & 0.0045 & 0.44 \\ \mathrm{Mn} & 20.45 & 20.94 & 0.125 & 0.59 \\ \mathrm{Ca} & 3.1 & 2.96 & 0.022 & 0.73 \\ \mathrm{~K} & 0.70 & 0.70 & 0.0084 & 1.2 \\ \mathrm{P} & 0.63 & 0.68 & 0.0184 & 2.2\end{array}$




\section{ANALYTICAL METHODS: THE DETERMINATION OF 27 ELEMENTS IN FERROMANGANESE MATERIALS}

by P.J. Aruscavage, H. Kirschenbaum and F. Brown

\section{Introduction}

Assessment of the geochemistry and economic potential of marine ferromanganese oxides requires a large chemical data base. The challenge is to process large numbers of samples for many elements, from trace levels to major concentrations, with precision and accuracy in complex polymetallic deposits. Along with the manganese, iron, nickel and cobalt, less common elements like cerium, cadmium, vanadium, and molybdenum must be analyzed in complex polymetallic deposits, with concentrations and combinations unlike those in many land deposits and ores.

Comparative studies on the utility of ICP, atomic absorption, and other techniques for the elemental analysis of geological samples has been published (Thompson, 1985; Kane and Dorrzapf, 1987; Willis, 1986). Books and articles describing the theory and use of ICP and AA techniques are abundant (Fassel, V.A., 1978; Thompson and Walsh, 1983; Varma, A., 1984; Slavin, W., 1982; Koirtyohann, S.R., and Kaiser, M.L., 1982; Slavin, W., 1984). With the requirements in mind, we decided to pursue a scheme of analysis based primarily around the induction coupled plasma atomic emission spectrometry (ICPAES) technique because of its simultaneous multielement capability and its relatively large linear concentration range. Atomic absorption techniques are used only for those elements where the sensitivity, precision, or accuracy of the ICPAES technique are not sufficient for the determination.

\section{Instrumentation}

\section{FAA Spectrometry}

A Perkin-Elmer model 5000 atomic absorption spectrometer equipped with an automatic burner control, a deuterium background correction system, and a pre mix burner assembly consisting of an adjustable platinum/rhodium nebulizer, glass impact bead or plastic flow spoiler, and $10 \mathrm{~cm}$ single slot burner head was used for all determinations of $\mathrm{Pb}, \mathrm{Zn}$ and $\mathrm{K}$. The analytical conditions are given in Table VI-1.

\section{GFAA Spectrometry}

A Perkin-Elmer model 5000 Zeeman atomic absorption spectrometer equipped with a model HGA 500 graphite furnace controller, model AS-40 autosampler, dual lamp EDL power supply and a data system 10 computer with high speed graphics capability was used for the determination of $\mathrm{As}, \mathrm{Cd}, \mathrm{Cr}, \mathrm{Pt}, \mathrm{Pd}$ and $\mathrm{Rh}$. The analytical conditions are given in Table VI- 2 .

\section{ICP Spectrometry}

A computer controlled simultaneous ICP spectrometer system was used for all determinations except $\mathrm{Pt}, \mathrm{Pd}, \mathrm{Rh}, \mathrm{Cd}, \mathrm{Cr}, \mathrm{As}, \mathrm{Zn}, \mathrm{K}$, and $\mathrm{Pb}$. The system is composed of: 
1. Two-Jarrel1-Ash Mark III mode1 1160 direct-reader polychromators optically linked to an inductively coupled argon plasma source with 63 preset emission lines for determining 56 different elements with a computer controlled background correction capability.

2. Digital Equipment Corporation (DEC) Model PDP-11/34 minicomputer system with dual RL01 hard disk storage devices.

3. Jarrel1-Ash RF generator with automatic impedance matching network.

4. VT 100 computer interface.

5. Fixed cross flow nebulizer.

6. Carter Fone 5130 printer system.

7. Gilson minipulse peristaltic pump.

\section{$\underline{\text { Reagents }}$}

The chemicals mentioned in the procedures are all of reagent grade quality. National Bureau of Standards (NBS) Standard Reference Materials (SRM) were used to make working standards for ICP, FAA. and GFAA measurements. Doubly distilled water was used throughout the procedures.

\section{Procedures}

\section{Digestion \#1 (see figure 1)}

1. Weigh $100 \mathrm{mg}$ sample ( -100 mesh or finer) into a $30-\mathrm{mL}$ Teflon container.

2. Add $3 \mathrm{~mL} \mathrm{HNO3,} 1 \mathrm{~mL} \mathrm{HC104,} \mathrm{and} 10 \mathrm{~mL} \mathrm{HF}$.

3. Cover and digest sample for $2 \mathrm{hr}$. at $150^{\circ} \mathrm{C}$.

4. Cool; open container and evaporate to dryness.

5. Add $25 \mathrm{~mL} 3 \mathrm{~N} \mathrm{HCl}$ and $0.1 \mathrm{~mL} 308 \mathrm{H}_{2} \mathrm{O}_{2}$. Heat gently on a hot plate at $120^{\circ} \mathrm{C}$ to dissolve $\mathrm{MnO}_{2}$.

6. Transfer the solution to a tared $50-\mathrm{mL}$ disposable polypropylene centrifuge tube.

7. Adjust the weight of solution to 52.5 grams ( $50 \mathrm{~mL}$ ) with $3 \mathrm{~N} \mathrm{HCl}$. The dilution factor of this solution is 500 .

8. Pipet a $1.0 \mathrm{~mL}$ aliquot and transfer to a $15-\mathrm{mL}$ disposable polypropylene centrifuge tube.

9. Add $9.0 \mathrm{ml} \mathrm{H} \mathrm{H}_{2} \mathrm{O}$. The dilution factor of this solution is 5000 .

As shown in figure 1 , the elements $\mathrm{Cu}, \mathrm{Ni}, \mathrm{Mo}, \mathrm{Sr}, \mathrm{V}, \mathrm{Y}, \mathrm{Cr}, \mathrm{Ba}, \mathrm{Co}$, and $\mathrm{P}$ are determined by ICP spectrometry, $\mathrm{K}, \mathrm{Pb}$, and $\mathrm{Zn}$ by FAA spectrometry and $\mathrm{Cr}$ by Zeeman GFAA spectrometry on the concentrated solution. $\mathrm{Mn}, \mathrm{Ti}, \mathrm{Al}, \mathrm{Fe}, \mathrm{mg}$ and $\mathrm{Ca}$ is determined by ICP spectrometry and $\mathrm{Cd}$ by Zeeman GFAA spectrometry on the diluted solution. The instrumental conditions are shown in Tables VI-l through VI -3 .

\section{Digestion \#2 (see figure 1)}

1. Weigh $100 \mathrm{mg}$ sample ( -100 mesh or finer) into a $15-\mathrm{mL}$ polypropylene centrifuge tube.

2. Pipet $5.0 \mathrm{~mL}(1+1) \mathrm{HCl}$ into the tube and cap tightly. Mix sample with the acid.

3. Place in oven at $90^{\circ} \mathrm{C}$ for $2 \mathrm{hr}$.

4. Remove from oven, $\operatorname{cool}$ and pipet $5.0 \mathrm{~mL}$ water into the tube and $\mathrm{mix}$. 


\section{Digestion \# 1}

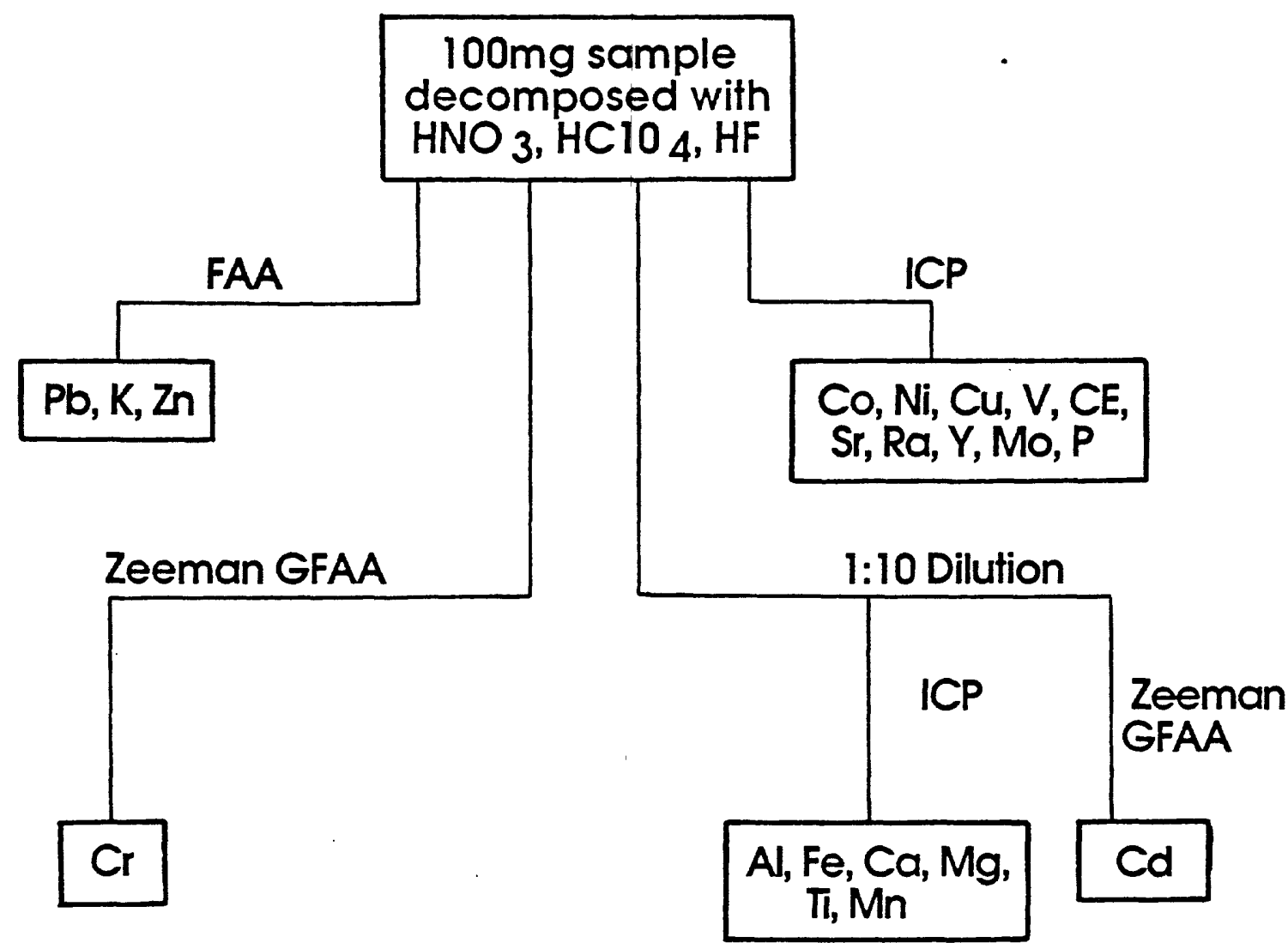

Digestion \#2

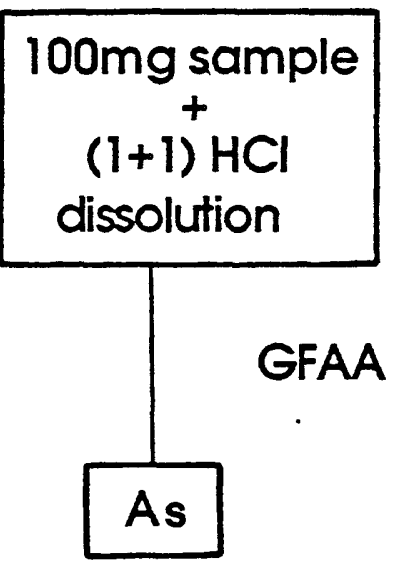

Digestion \#3

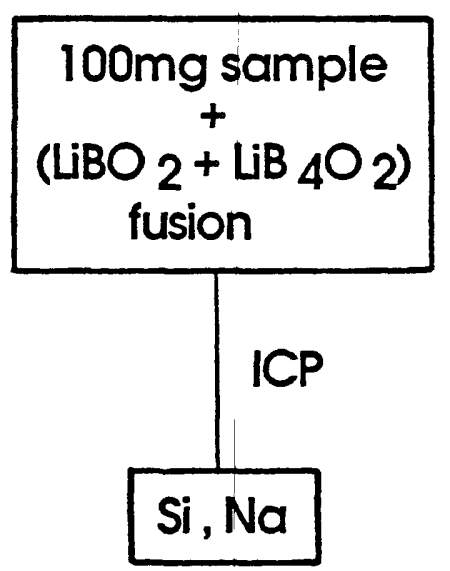

Digestion \#4

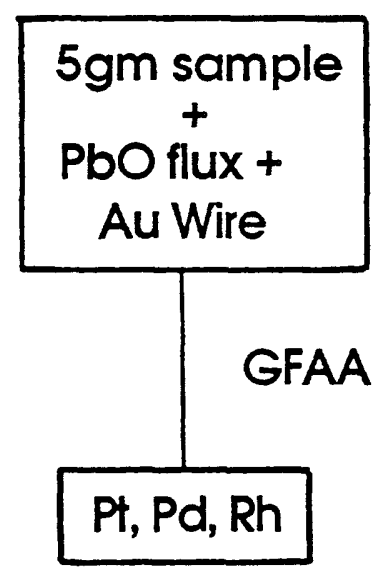

Figure IV-1 Analytical scheme for the determination of 27 elements in Ferromanganese materials. 
5. Centrifuge for $5 \mathrm{~min}$.

6. Pipet $.05 \mathrm{~mL}$ of sample solution into a $5 \mathrm{~mL}$ disposal polystyrene

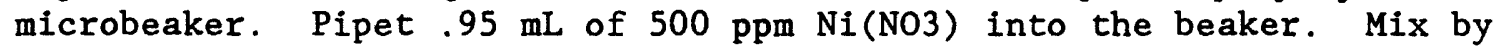
swirling beaker. As shown in figure 1, arsenic is determined by Zeeman GFAA spectrometry. The dilution factor for the solution is equal to 2000 .

\section{Digestion \#3 (see figure 1)}

1. Prepare flux mixture of 1 part powdered lithium metaborate $\left(\mathrm{LiBO}_{2}\right)$ and 2 parts anhydrous lithium tetraborate $\left(\mathrm{LiB}_{4} \mathrm{O}_{7}\right)$.

2. Weigh $100 \mathrm{mg}$ of sample ( -100 mesh or finer) on a glassine powder paper.

3. Mix 0.9 gms of flux with the sample and carefully transfer the mixture to a graphite crucible. Lightly tamp down crucible several times.

4. Dry the mixture in oven at $105^{\circ} \mathrm{C}$ for $60 \mathrm{~min}$.

5. Fuse the mixture in on electric furnace at $1000^{\circ} \mathrm{C}$ for $30 \mathrm{~min}$.

6. Remove the crucibles from the furnace and allow to cool. When cool, the beads produed should be easily dislodged with the touch of a spatula.

7. To a $250-\mathrm{mL}$ plastic bottle add a Teflon covered magnetic stir bar, the sample bead, $50 \mathrm{~mL}$ boiling water, and $5 \mathrm{~mL}(1+1) \mathrm{HNO}_{3}$.

8. Stir the mixture rapidly for $60 \mathrm{~min}$. on magnetic stirrer.

9. Add about $100 \mathrm{~mL}$ water to bottle and transfer the solution to a $200-\mathrm{mL}$ volumetric flask. Rinse the bottle with $\mathrm{H}_{2} \mathrm{O}$ and add to contents in flask.

10. Pipet $5.0 \mathrm{~mL}$ of $1000 \mathrm{~g} / \mathrm{mL}$ indium solution, for internal standardization, into the volumetric flask. Fill to volume with $\mathrm{H} 2 \mathrm{O}$, mix, and pour the solution back into a dry plastic bottle.

$\mathrm{Si}$ and $\mathrm{Na}$ are determined by ICP spectrometry as shown in figure 1 . The dilution factor of the solution is equal to 2000. The instrumental conditions are shown in Tables VI-3a, $3 b$.

\section{The Determination of $\mathrm{Pt}, \mathrm{Pd}$ and $\mathrm{Rh}$}

The elements Pt, Pd and $\mathrm{Rh}$ are determined by GFAA Spectrometry after separation by classical fire-assay methods and collection in a gold bead (Aruscavage and others., 1984). Briefly, the sample (typically 5 grams) is mixed in a clay crucible with a reducing flux consisting of $30 \mathrm{~g} \mathrm{Na}_{2} \mathrm{CO}_{3}, 35 \mathrm{~g} \mathrm{PbO}, 10 \mathrm{~g} \mathrm{SiO}, 35 \mathrm{~g}$ $\mathrm{Na}_{2} \mathrm{~B}_{4} \mathrm{O}_{7}, 3.5 \mathrm{~g}$ flour, $1 \mathrm{~g} \mathrm{CaF}$ and $2 \mathrm{mg}$ gold wire. The sample is fused for approx. $1 \mathrm{hr}$ at $1000^{\circ}$, poured into iron molds and the reduced lead metal containing $\mathrm{Au}$, $\mathrm{Pt}, \mathrm{Pd}$, and $\mathrm{Rh}$ is then oxidized in a bone ash cupel at $850^{\circ} \mathrm{C}$. The gold bead that remains is then dissolved in aqua-regia and subsequently the solution is analyzed for $\mathrm{Pt}, \mathrm{Pd}$ and $\mathrm{Rh}$ by the GFAA technique. Table VI-2 shows the operating conditions for each of the elements.

Results and Discussion

\section{Decomposition of the ferromanganese materials}

After testing various procedures for decomposing these materials, we found that the best results were obtained using three separate and different methods for various elements. Because of the volatility of silicon tetrafluoride, a fusion of the sample with a lithium metaborate/tetraborate mixture similar to that used for the routine determination of rock forming major elements in silicates gave 
the best data for silica and sodium. However because of the large blanks and high dilutions required to lower the salt content from the fusion, an acid decomposition was most useful for the trace and minor elements. The decomposition for arsenic presented a special problem in that its fluoride is also volatile and special precautions must be taken when using a hydrofluoric acid digestion. However we found that greater than 958 of the arsenic was leached from a test set of nodules with a hydrochloric acid digestion when compared with a total decomposition of the sample with hydrofluoric acid in a teflon lined bomb, thus making sample processing much easier.

\section{ICP Determinations}

Multielement capability is a main advantage of the ICP method, compared to atomic absorption methods. However, this advantage is often offset by spectral interferences common to optical emission techniques (A good guide to the selection of proper wavelengths and possible interferences in ICP atomic emission spectroscopy is the publication by Winge and others, 1985). Using standard procedures for investigating potential interferences in these high $\mathrm{Mn}-\mathrm{Fe}$ matrices we found significant spectral interferences for the elements $\mathrm{Mg}$, $\mathrm{P}$, Co, Mo, $\mathrm{V}, \mathrm{Ce}, \mathrm{Zn}, \mathrm{Pb}$, As, and $\mathrm{Cd}$. The extent of these interferences, shown in Table VI-5, is based on the background correction position given in Table VI$3 \mathrm{~b}$ for the standard nodules $\mathrm{A}-1$ and $\mathrm{P}-1$. These interference corrections are applied automatically by computer algorithms (i.e. $\mathrm{Pb}$ (corr) $=\mathrm{Pb}$ (uncorr) + Co (corr) $\times \mathrm{K}$ factor where $\mathrm{K}$ factor is the correction coefficient per concentration value $C_{0}$ in the sample. Because of the large corrections required or the poor sensitivity for the determination, the elements $\mathrm{Zn}, \mathrm{Pb}, \mathrm{Cr}, \mathrm{As}, \mathrm{Cd}$, $\mathrm{K}, \mathrm{Pt}, \mathrm{Pd}$ and $\mathrm{Rh}$ are determined by atomic absorption techniques.

\section{Atomic absorption determinations}

Although atomic absorption techniques are largely free of spectral interferences, interferences caused by differing chemical matrices are common, especially in the graphite furnace technique. Possible interferences on $\mathrm{Pt}, \mathrm{Pd}$ and $\mathrm{Rh}$ were tested previously (Aruscavage and others., 1984) by spiking $10 \mathrm{ng}$ of the individual analyte elements ( $\mathrm{Pt}, \mathrm{Pd}$, and $\mathrm{Rh}$ ) with up to $1000 \mathrm{ng}$ of interferent ( $P t, P d, R h$, and $\mathrm{Ag}$ ). We found insignificant interferences ( $<5 \%$ depression) in all cases except for the depressing effect of $R h$ on the $P d$ signal which amounted to 88 depression. The flame atomic absorption technique was found to be relatively free of interference except in the case of potassium. Because of its low ionization potential in the air-acetylene flame, potassium is ionized to a greater extent in pure solutions of the element than in digested samples which contain large amounts of easily ionizable elements such as sodium. This difference can cause an error in the results towards too high of values if calibration of sensitivity is made using these pure standard solutions. This interference can be corrected by adding a high concentration of ionizable salt such as $\mathrm{NaCl}$ to both samples and standards. We found, however that using a manganese nodule standard $(\mathrm{P}-1)$ for calibration eleminated the need for addition of salt and accurate results were obtained.

In the graphite furnace determination of $\mathrm{Cd}, \mathrm{Cr}$, and As we found significant signal supression caused by $\mathrm{Fe}$ and $\mathrm{Mn}$. Fortunately, the suppression on $\mathrm{Cd}$ leveled off very quickly at about $25 \%$ supression for $10 \mathrm{ppm} \mathrm{Fe}$ or $25 \mathrm{ppm} \mathrm{Mn}$. For $\mathrm{Cr}$ the supression (15\%) leveled off at about $100 \mathrm{ppm} \mathrm{Fe}$ or Mn. By adding appropriate amounts of $\mathrm{Fe}$ to the calibration solutions for $\mathrm{Cd}$ and $\mathrm{Cr}$, accurate results were obtained. For the determination of arsenic, nickel nitrate was 
added to the solutions to allow higher charring temperatures and to provide a more stable matrix during atomization. Under these conditions no interference was observed.

\section{Precision and Accuracy}

The results for replicate analysis of the two USGS manganese nodule standards are shown in Table VI-4. The precision for most elements is better than 108 RSD except for Arsenic.

The accuracy of the procedure as shown by a comparison to literature values is also good and normally within 1 standard deviation from the published values. Exceptions are $\mathrm{Ca}, \mathrm{Ni}, \mathrm{Mo}, \mathrm{Ba}, \mathrm{Sr}$, and $\mathrm{Cr}$ in nodule $\mathrm{A}-1$ and $\mathrm{Mo}, \mathrm{V}$ and $\mathrm{Ba}$ in nodule P-1. The determination limits are based on signals that are approximately 2 times that of the variation in the blanks or low standards are shown in Table VI-6.

Table VI-1. Operating conditions for FAAS determinations

\section{Element}

Wavelength ( $\mathrm{nm})$

Band-pass (nm)

Flow Spoiler

Flame

Flame conditions

High standard

Standard sensitivity (absorbance)

Linear range

Background correction
$\underline{\mathrm{Pb}}$

283.3

0.7

Glass bead

Air/Acetylene oxidizing-blue

$6 \mathrm{ppm}$

0.10

$0-12 \mathrm{ppm}$

deuterium
$\underline{\mathrm{Zn}}$

219.9

0.7

plastic insert

Air/Ace tylene

oxidizing-blue

$2 \mathrm{ppm}$

0.20

0-2 ppm

deuterium

\section{$\underline{\mathrm{K}}$}

766.5

$$
1.4
$$

plastic insert

Air/Acetylene

oxidizing-blue

$21 \mathrm{ppm}(1)$

0.20

$0-21 \mathrm{ppm}$

none

Notes: ( 1 ) = a 500 dilution of the USGS standard Nod-P-1 is used for calibration purposes. The sensitivity for this solution is achieved by rotation of the burner head to a $45^{\circ}$ angle to the light path. 
Table VI-2. Operating conditions for GFAAS determinations

\begin{tabular}{|c|c|c|c|c|c|c|}
\hline Element & $\underline{\mathrm{Cd}}$ & $\underline{\mathrm{Cr}}$ & $\underline{\text { As }}$ & $\underline{P t}$ & $\underline{\mathrm{Pd}}$ & $\underline{\mathrm{Rh}}$ \\
\hline $\begin{array}{l}\text { Wavelength (nm) } \\
\text { Bandpass (nm) } \\
\text { Light source } \\
\text { Current/watts } \\
\text { Bkg. correction } \\
\text { Mode }\end{array}$ & $\begin{array}{c}228.8 \\
0.7 \\
\text { H.C. } \\
6 \text { ma } \\
\text { Zeeman } \\
\text { Peak } \\
\text { Area }\end{array}$ & $\begin{array}{c}357.9 \\
0.7 \\
\text { H.C. } \\
25 \text { ma } \\
\text { Zeeman } \\
\text { Peak } \\
\text { Area }\end{array}$ & $\begin{array}{c}193.7 \\
0.7 \\
\text { EDL } \\
8 \mathrm{w} \\
\text { Zeeman } \\
\text { Peak } \\
\text { Area }\end{array}$ & $\begin{array}{r}265.9 \\
0.7 \\
\text { H.C. } \\
30 \text { ma } \\
\text { Zeeman } \\
\text { Peak } \\
\text { Height }\end{array}$ & $\begin{array}{r}340.5 \\
0.7 \\
\text { H.C. } \\
30 \text { ma } \\
\text { Zeeman } \\
\text { Peak } \\
\text { Height }\end{array}$ & $\begin{array}{r}343.5 \\
0.7 \\
\text { H.C. } \\
30 \text { ma } \\
\text { Zeeman } \\
\text { Peak } \\
\text { Height }\end{array}$ \\
\hline $\begin{array}{c}\text { Integration } \\
\text { time }(\mathrm{sec})\end{array}$ & 4 & 6 & 5 & 4 & 4 & 4 \\
\hline $\begin{array}{l}\text { High calibration } \\
\text { standard ( } p p b)\end{array}$ & $2^{(1)}$ & $200^{(2)}$ & 100 & $200^{(3)}$ & $100^{(3)}$ & $20^{(3)}$ \\
\hline Standard absorbance & .12 & .30 & .38 & .16 & .19 & \\
\hline Dry temp. $\left({ }^{\circ} \mathrm{C}\right)$ & 120 & 120 & 120 & 130 & 130 & 130 \\
\hline Char Temp. $\left({ }^{\circ} \mathrm{C}\right)$ & 250 & 1000 & 500 & 1600 & 1100 & 1250 \\
\hline Atom. Temp. $\left({ }^{\circ} \mathrm{C}\right)$ & 2100 & 2700 & 2700 & 2700 & 2700 & 2700 \\
\hline Purge Gas & Argon & Argon & Argon & Argon & Argon & Argon \\
\hline Gas flow at atom. & 0 & 200 & 0 & 0 & 0 & 0 \\
\hline Ramp to atom. (sec.) & 1 & 1 & 1 & 0 & 0 & 0 \\
\hline Graphite tube type & $\begin{array}{l}\text { non- } \\
\text { pyrolytic }\end{array}$ & $\begin{array}{l}\text { non- } \\
\text { pyrolytic }\end{array}$ & $\begin{array}{l}\text { non- } \\
\text { pyrolytic }\end{array}$ & $\begin{array}{l}\text { pyrolytic } \\
\text { c }\end{array}$ & pyrolytic & pyrolyti \\
\hline atomization & off-wall & off-wall & off-wall & off-wall & off-wall & off-wall \\
\hline $\begin{array}{l}\text { sample aliquot }() 1) \\
\text { modifier }\end{array}$ & $\begin{array}{c}20 \\
\text { none }\end{array}$ & $\begin{array}{l}10 \\
\mathrm{Mg}\left(\mathrm{NO}_{3}\right)_{2}\end{array}$ & $\begin{array}{c}20 \\
\mathrm{Ni}\left(\mathrm{NO}_{3}\right)_{2}\end{array}$ & $\begin{array}{r}20 \\
\text { none }\end{array}$ & $\begin{array}{r}20 \\
\text { none }\end{array}$ & $\begin{array}{r}20 \\
\text { none }\end{array}$ \\
\hline
\end{tabular}

Notes:

(1) solution contains $10 \mathrm{ppm} \mathrm{Fe}$

(2) solution contains $100 \mathrm{ppm} \mathrm{Fe}$

(3) solutions contain $2 \mathrm{Mg} / \mathrm{ml} \mathrm{Au}$

(4) H.C. = Hollow Cathode

(5) EDL = Electrodeless Discharge Lamp 
Table VI-3a. Operating conditions for ICP determinations

$\begin{array}{lll}\text { Forward power } & =1.1 \mathrm{kw} \\ \text { Reflected power } & =<5 \text { watts } \\ \text { Coolant gas flow } & =171 / \mathrm{m} \text { Argon } \\ \text { Nebulizer gas flow } & =0.551 / \mathrm{m} \text { Argon } \\ \text { Signal integration time } & =3 \mathrm{sec} \\ \text { Sample uptake } & =0.9 \mathrm{ml} / \mathrm{minute} \\ \text { pump } & =\text { peristaltic } \\ \text { Observation height above } & =16 \mathrm{~mm} \\ \quad \text { load coil } & & \\ \text { Internal reference channels } & = & \text { low voltage card } \\ & & =\text { fixed cross flow } \\ \text { Nebulizer } & =5 \text { turn copper, water-cooled } \\ \text { Inductance coil } & =27.12 \mathrm{mHz} \\ \text { Power frequency } & \end{array}$

Table VI-3b. Operating conditions for ICP determinations

$\begin{array}{lllll}\text { Wave - } & \text { Background } & \text { High conc- } & \text { Inter-element } & \begin{array}{c}\text { Correction } \\ \text { factor(ppm) }\end{array}\end{array}$

\begin{tabular}{|c|c|c|c|c|c|}
\hline Al & 3082 & +0.48 & 10 & - & - \\
\hline $\mathrm{Fe}$ & 2599 & +0.48 & 25 & - & - \\
\hline $\mathrm{Ca}$ & 3158 & +0.48 & 100 & - & - \\
\hline $\mathrm{Mg}$ & 2795 & +0.48 & 10 & $\mathrm{Mn}$ & -.00100 \\
\hline $\mathrm{P}$ & 2149 & +0.48 & 10 & $\mathrm{Mn} ; \mathrm{Cu}$ & $-.00020 ;+.00200$ \\
\hline $\mathrm{T} i$ & 3349 & +0.48 & 10 & - & - \\
\hline Mn & 2576 & +0.48 & 100 & - & - \\
\hline Co & 2286 & +0.48 & 10 & $\mathrm{Fe} ; \mathrm{Ti}$ & $+.00040 ;+.00180$ \\
\hline $\mathrm{Ni}$ & 2316 & +0.48 & 10 & - & - \\
\hline $\mathrm{Cu}$ & 3247 & -0.48 & 10 & - & - \\
\hline Mo & 2020 & -0.48 & 10 & $\mathrm{Mn} ; \mathrm{Fe}$ & $+.00004 ;+.00010$ \\
\hline $\mathrm{V}$ & 2924 & -0.48 & 10 & $\mathrm{Fe}$ & -.00030 \\
\hline $\mathrm{Ba}$ & 4554 & +0.48 & 10 & - & - \\
\hline $\mathrm{Ce}$ & 4186 & -0.48 & 10 & $\mathrm{Ti}$ & -.00800 \\
\hline $\mathrm{Sr}$ & 4077 & +0.48 & 5 & - & - \\
\hline $\mathrm{Y}$ & 3710 & +0.48 & 5 & - & - \\
\hline Si & 2516 & +0.48 & 10 & - & - \\
\hline $\mathrm{Na}$ & 5890 & +0.48 & 10 & - & - \\
\hline In & 4511 & +0.48 & 10 & - & - \\
\hline
\end{tabular}




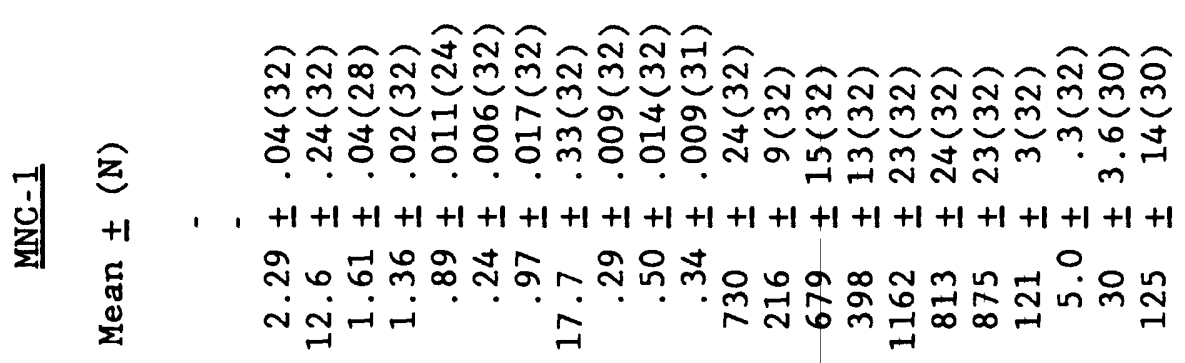

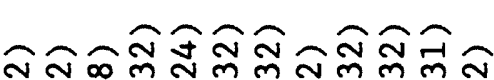

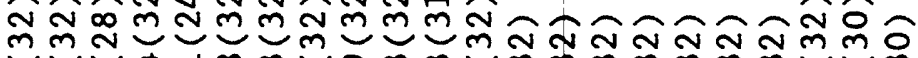

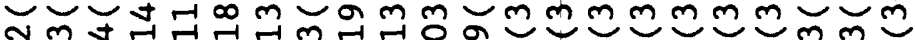

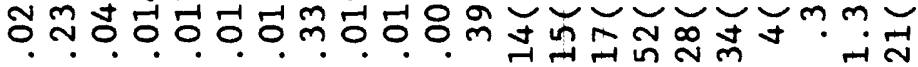
$+1,1+1+1+1+1+1+1+1+1+1+1+1+1+1+1+1+1+1+1+1+1+1$ సึ. नiंतं

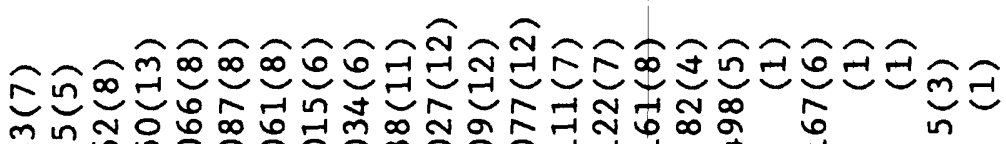

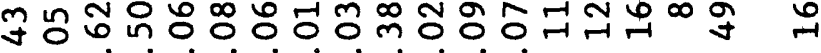

$\stackrel{4}{-3}$ $+1+1+1+1+1+1+1+1+1+1+1+1+1+1+1+1+1+1+1+1$

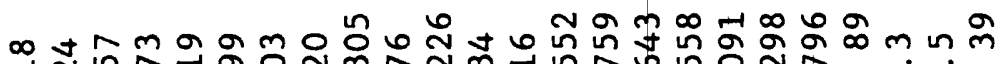

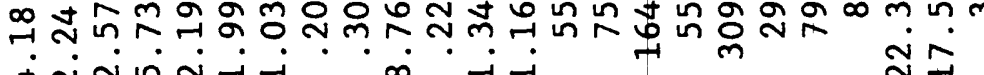
ปั่ง

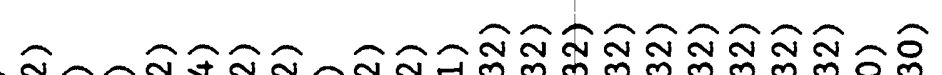
โิกิกิ

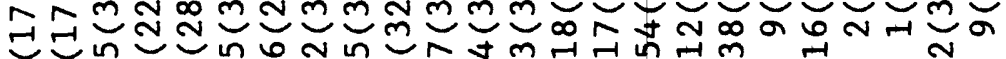

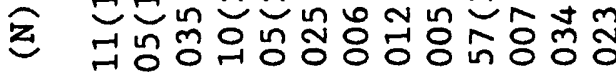

$n$

$+1$

ฮี

$\sum$

ลิ

$\underset{\sim}{+1}$

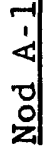
$+1+1+1+1+1+1+1+1+1+1+1+1+1+1+1+1+1+1+1+1+1+1+1+1$

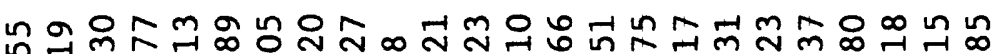
次 $\rightarrow$

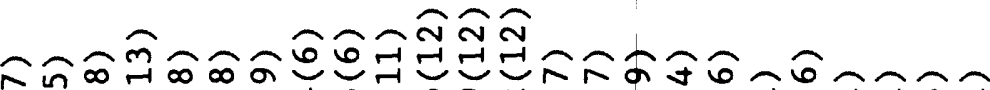

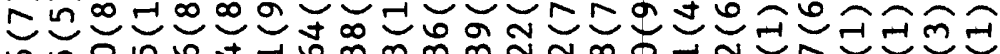

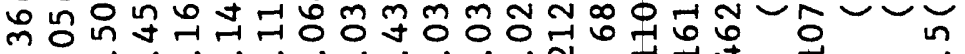

$+1+1+1+1+1+1+1+1+1+1+1+1+1+1+1+1+1+1+1+1$

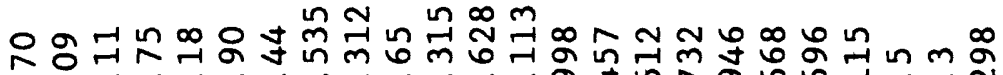

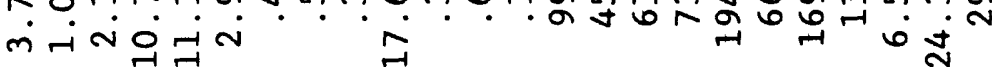
든웅

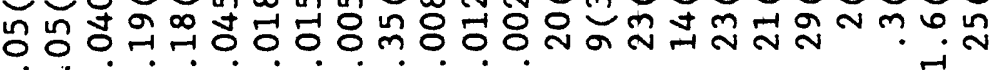

$+1+1+1+1+1+1+1+1+1+1+1+1+1+1+1+1+1+1+1+1+1+1+1+1$

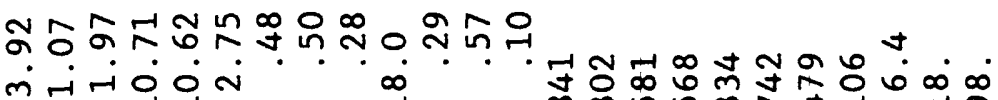

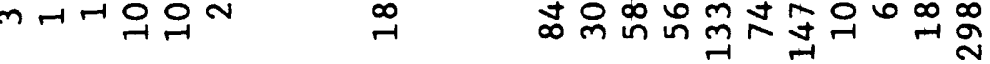

No

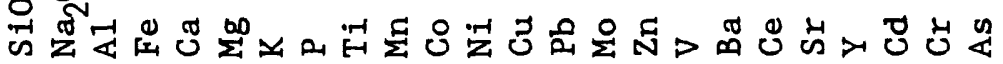

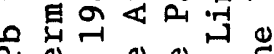

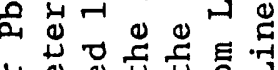

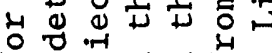

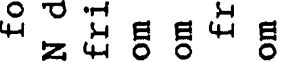

的出员点。

ఖ

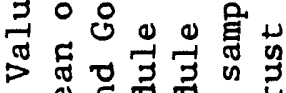

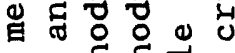

$\infty$ 医

工

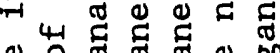

¿ 0 \%

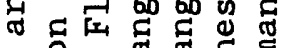

ว

羊话

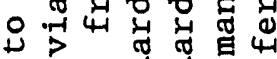

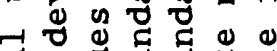

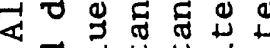

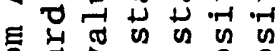

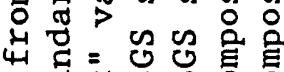

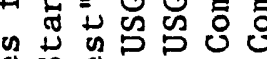

め क ळ || ||

元 $\|=-1$ 白甶山

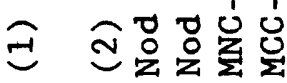


Table VI-5. ICP spectral interferences, see text for discussion

\begin{tabular}{cccrr} 
Wavelength $(/)$ & Analyte Element & Interference & \multicolumn{2}{c}{ Interference } \\
\hline & & & & $\mathrm{A}-1$ \\
2203 & $\mathrm{~Pb}$ & $\mathrm{Mn}$ & 3 & 6 \\
2203 & $\mathrm{~Pb}$ & $\mathrm{Co}$ & 15 & 15 \\
2203 & $\mathrm{~Pb}$ & $\mathrm{Al}$ & 4 & 7 \\
2020 & $\mathrm{Mo}$ & $\mathrm{Mn}$ & 2 & 2 \\
2020 & $\mathrm{Mo}$ & $\mathrm{Fe}$ & 3 & 1 \\
2138 & $\mathrm{Mn}$ & $\mathrm{Fe}$ & 13 & 8 \\
2286 & $\mathrm{Co}$ & $\mathrm{Ti}$ & 2 & 1 \\
2286 & $\mathrm{Co}$ & $\mathrm{Fe}$ & 2 & $<1$ \\
2924 & $\mathrm{~V}$ & $\mathrm{Ti}$ & 6 & 4 \\
4186 & $\mathrm{Me}$ & $\mathrm{Mn}$ & 4 & 9 \\
2149 & $\mathrm{P}$ & $\mathrm{Cu}$ & $<1$ & 3 \\
2149 & $\mathrm{P}$ & $\mathrm{Mn}$ & $<1$ & 7 \\
2795 & $\mathrm{Mg}$ & & $<1$ & 2 \\
& & &
\end{tabular}

Table VI-6. Determination Limits

$\begin{array}{ccc}\text { Element } & \text { Instrument } & \text { Ppm } \\ \mathrm{Cu} & \text { ICP } & 5 \\ \mathrm{Ni} & \text { ICP } & 5 \\ \mathrm{Mo} & \text { ICP } & 5 \\ \mathrm{Sr} & \text { ICP } & 2.5 \\ \mathrm{~V} & \text { ICP } & 5 \\ \mathrm{Zn} & \text { FAA } & 15 \\ \mathrm{Y} & \text { ICP } & 5 \\ \mathrm{Ce} & \text { ICP } & 10 \\ \mathrm{Ba} & \text { ICP } & 2.5 \\ \mathrm{Co} & \text { ICP } & 5 \\ \mathrm{~Pb} & \text { FAA } & 10 \\ \mathrm{Mn} & \text { ICP } & 25 \\ \mathrm{Ti} & \text { ICP } & 10 \\ \mathrm{A1} & \text { ICP } & 250 \\ \mathrm{Fe} & \text { ICP } & 250 \\ \mathrm{~K} & \text { FAA } & 1250 \\ \mathrm{mg} & \text { ICP } & 25 \\ \mathrm{P} & \text { ICP } & 250 \\ \mathrm{Ca} & \text { ICP } & 250 \\ \mathrm{As} & \text { Zeeman GFAA } & 2 \\ \mathrm{Cd} & \text { Zeeman GFAA } & 0.1 \\ \mathrm{Cr} & \text { Zeeman GFAA } & 1 \\ \mathrm{SiO} 2 & \text { ICP } & 500 \\ \mathrm{NaO} & \text { ICP } & 500 \\ & & \end{array}$




\title{
VII INTERLABORATORY CALIBRATIONS AND OTHER ANALYTICAL CHECKS
}

\author{
by F.T. Manheim and J.A. Commeau \\ with contributions by
}

B.J. Haynes, D. Root, V. Marchig, P. Halbach, and D. Puteanus

Several types of analytical problems were studied with the help of different laboratories and analytical methods: influence of grinding and sieving (homogeneity), water content, and systematic error in elemental analysis.

\section{Influence of grinding and sieving on analytical values}

Intercomparison of analytical determinations on ground, unsieved samples from the "Midpac I" cruise (Halbach and others, 1982) showed significant differences, depending on sample treatment. For example, analyses of cobalt and titanium by lithium borate flux X-ray fluorescence spectrometry and DC-plasma spectrometry, performed in the Woods Hole USGS laboratories yielded a pooled coefficient of variation for 10 pairs of cobalt determinations of 6.9 percent, expressed as percent of moisture-free weight. The corresponding value for titanium was still larger, at 19.1 percent. Inhomogeneities were suspected.

Further study showed that titanium tended to occur preferentially in more resistant coarser grains, along with iron and other detrital elements such as aluminum and silica, whereas cobalt was more homogeneously distributed in finer fractions along with manganese, nickel, copper, molybdenum, lead, and zinc. A second set of comparisons was performed on ground and sieved (100 mesh) samples from the R/V S.P. Lee cruise (Hein and others, 1985). In this case data of three different laboratories, including atomic-absorption analyses performed at the Technical University, Clausthal-Zellerfeld, agreed to within much closer limits: 4.3 percent and 5.8 percent pooled coefficient of variation for cobalt and titanium, respectively. As discussed in Chapter $V$, the basic grinding and sieving procedure was followed for all samples analyzed by us. It should be noted that the sieving through 200 mesh screen would have perhaps yielded even better sample homogeneity than sieving to 200 mesh (M. Fisk, United Nations, 1985, written communication). However, avoidance of biasing the results requires repeated regrinding of residual fractions until all fractions pass through the mesh. Time and availability of assistance to process the many samples did not permit this additional precaution.

Interlaboratory calibration of $\mathrm{R} / \mathrm{V}$ S.P. Lee cruise data

The cooperative cruise of the R/V S.P. Lee in 1983 provided an excellent opportunity for a wide-range interlaboratory study on samples of crusts ground and sieved aboard the vessel. Analytical data on splits of approximately 56 samples were performed by the U.S. Geological Survey laboratories in Reston Virginia and Woods Hole Massachusetts, (see Chapter V), the Bureau of Mines laboratory in Avondale, Maryland, and the laboratory of the German Geological Survey in Hannover, Federal Republic of Germany. In addition, shipboard analyses were performed on a small, portable X-ray fluorescence unit (Portaspec, made 
by the Hankinson Corp.) for six fixed elements ( $\mathrm{Mn}, \mathrm{Fe}, \mathrm{Ni}, \mathrm{Co}, \mathrm{Cu}, \mathrm{plus}$ semi-quantitative estimations on $\mathrm{Zn}$ and $\mathrm{Pb}$ ). All the analyses except the shipboard and those of the German Geological Survey had accompanying hygroscopic moisture determinations, permitting recalculation to a moisture-free basis. Because hygroscopic moisture was significant in the "air-dry" powder (to 20 percent) the German data were recalculated to a moisture-free basis based on water values from the USGS-Reston data set. This correction was justified by comparison between water content for the Woods Hole and the Reston splits (Table VII-1). With two exceptions, the samples show remarkably good agreement, considering the expected changes due to sample equilibration with different laboratory environments. The data in Table VII-1 show that the pooled error for sample pairs (coefficient of variation) was only 1.66 percent of the analytical values, considerably less than deviations for elemental determinations, reported above and in Table VII-3.

Table VII-1. Hygroscopic water determinations for S.P. Lee Cruise L5-83-HW, USGS Reston VA(R) and Woods Hole MA (W), data. K = mean correction factor, given by $100 /\left(100-\mathrm{H}_{2} \mathrm{O}^{-}\right)$. CV $=$difference between correction factors for the two sets of data, as a percentage of the corrected sample value.

\begin{tabular}{lrrrlrrrrr} 
SEQNO & $\mathrm{H}_{2} \mathrm{O}^{-}(\mathrm{R})$ & \multicolumn{1}{c}{$\mathrm{H}_{2} \mathrm{O}^{-}(\mathrm{W})$} & $\mathrm{K}$ & $\mathrm{CV}$ & SEQNO & $\mathrm{H}_{2} \mathrm{O}^{-}(\mathrm{R})$ & $\mathrm{H}_{2} \mathrm{O}^{-}(\mathrm{W})$ & $\mathrm{K}$ & $\mathrm{CV}$ \\
& & & & & & & & & \\
17006 & 17.0 & 18.4 & 1.215 & 1.41 & 53029 & 13.0 & 14.4 & 1.158 & 1.34 \\
17008 & 10.4 & 11.3 & 1.121 & 0.78 & 53030 & 8.7 & 6.8 & 1.084 & 1.56 \\
17010 & 5.9 & 5.3 & 1.059 & 0.49 & 53032 & 10.3 & 10.2 & 1.115 & 0.07 \\
17012 & 4.0 & 12.0 & 1.089 & 6.64 & 89013 & 7.5 & 5.5 & 1.069 & 1.61 \\
17014 & 9.7 & 10.3 & 1.113 & 0.28 & 89017 & 17.6 & 19.8 & 1.231 & 2.33 \\
17016 & 9.3 & 13.9 & 1.132 & 4.14 & 89020 & 5.6 & 5.6 & 1.059 & 0.00 \\
17018 & 8.8 & 9.4 & 1.100 & 0.56 & 89022 & 7.2 & 9.3 & 1.090 & 1.77 \\
53020 & 16.4 & 16.7 & 1.198 & 0.28 & 89025 & 6.2 & 5.7 & 1.088 & 0.39 \\
53026 & 15.3 & 14.3 & 1.184 & 2.33 & 89026 & 4.9 & 10.0 & 1.081 & 4.21 \\
53027 & 11.0 & 13.8 & 1.142 & 2.62 & 89031 & 14.7 & 15.0 & 1.176 & 0.28
\end{tabular}

Grand mean, K (correction factor) $=1.125$.

Pooled CV (grand mean of CV values) $=1.66$ (percent) 


\section{$\underline{\mathrm{H}}_{2} \mathrm{O}^{+}$values}

$\mathrm{H}_{2} \mathrm{O}+$ is obtained by subtracting $\mathrm{H}_{2} \mathrm{O}^{-}$from total water obtained by the Penfield method (Shapiro, 1975). $\mathrm{H}_{2} \mathrm{O}^{-}$is determined on a split of powder taken at the same time as splits taken for many other elements (Ch. VI). Unfortunately, time delay between the $\mathrm{H}_{2} \mathrm{O}^{+}$and $\mathrm{H}_{2} \mathrm{O}^{-}$determination permitted change in total water content, owing to gain or loss from atmospheric moisture, thus affecting the $\mathrm{H}_{2} \mathrm{O}^{+}$ values. This problem was recognized early in examining summation values, but because of the large number of samples to be run and frequent shortage of material, it was impractical to perform additional separate $\mathrm{H}_{2} \mathrm{O}^{-}$measurements for the $\mathrm{H}_{2} \mathrm{O}$ determinations. Errors from this source are believed to account for most of the deviation of the summation value from the expected value.

A two-way analysis of variance was performed on the three to five sets of elemental data by the different laboratories to determine possible presence of systematic bias in the analyses. An example of one data set is given in Table VII-2. A summary of all elements studied is provided in Table VII-3. The simplest description of systematic bias of data by the different laboratories is given by the lab effect, or differences of the column means, representing data by a given laboratory for a given element, from the grand mean for all laboratories. Total of all lab effects were equal to zero.

One may observe that the largest (+) lab effect for iron is obtained from the shipboard analyses, as might be expected. These average about 5 percent higher than the mean of data from all laboratories. The F statistic is 14.4 , much greater than the value of 2.9, expected for 4 degrees of freedom for the columns and 18 degrees of freedom for the rows ( 95 percent confidence level). This means that we reject the null hypothesis that the differences between the laboratories' data are not statistically significant.

In nearly every case except for cobalt Table VII-3 suggests that there are statistically significant differences in data from the participating laboratories. However, with some exceptions, these are probably not large enough to be of practical significance. We have included the shipboard measurements in the set to permit comparative evaluations, but a more judicious approach toward estimating true values would exclude these data, which are subject to fewer analytical controls than the laboratory determinations. A more subjective effort to arrive at "true" values might choose the mean of three out of the four, or two of three sets of data for each element on the basis of pairwise comparisons among the sets. There seems to be no concensus on this important problem, as a recent debate on the merits of various approaches to "best values" estimation suggests (Abbey and Rousseau, 1985). 
Table VII-2. Comparison among five data sets for $F e$ analyses on ground and sieved crust samples from S.P.Lee Cruise L5-HW-83. RES = Reston USGS laboratories, WH - Woods Hole USGS laboratories, GERM - German Geological Survey, SH - shipboard analyses, and BURM - Bureau of Mines (Avondale) analyses. $L E$ - lab effect, DF - degrees of freedom, SS - mean square due to regression, and MS mean square due to residual variation (error). F or "F statistic" = SS/MS.

\begin{tabular}{lrrrrrrr} 
SEQNO & \multicolumn{1}{l}{ RES } & WH & GERM & SHIP & BURM & \multicolumn{1}{l}{ Row } \\
& & & & & & \multicolumn{1}{l}{$\begin{array}{l}\text { Row } \\
\text { SD }\end{array}$} \\
\hline 17006 & 16.86 & 16.28 & 16.02 & 19.03 & 18.42 & 17.32 & 1.334 \\
17008 & 19.41 & 18.89 & 18.74 & 20.98 & 19.35 & 19.47 & 0.890 \\
17010 & 18.80 & 17.61 & 18.38 & 21.25 & 18.67 & 18.94 & 1.370 \\
17012 & 8.85 & 9.13 & 8.46 & 8.64 & 9.97 & 9.01 & 0.592 \\
17014 & 13.28 & 13.38 & 13.84 & 13.95 & 14.96 & 13.88 & 0.668 \\
17016 & 12.45 & 12.69 & 12.05 & 13.23 & 13.84 & 12.85 & 0.699 \\
53018 & 17.54 & 17.01 & 16.72 & 18.64 & 18.43 & 17.67 & 0.848 \\
53020 & 19.61 & 19.09 & 18.60 & 20.81 & 18.91 & 19.40 & 0.867 \\
53026 & 19.95 & 19.05 & 19.18 & 22.07 & 18.36 & 19.72 & 1.421 \\
53027 & 19.32 & 18.94 & 18.96 & 19.99 & 19.21 & 19.28 & 0.427 \\
53029 & 16.32 & 15.82 & 15.28 & 16.55 & 17.08 & 16.21 & 0.689 \\
53030 & 14.89 & 14.03 & 14.84 & 15.11 & 16.33 & 15.04 & 0.829 \\
53032 & 13.60 & 13.22 & 13.48 & 13.71 & 14.43 & 13.69 & 0.453 \\
89013 & 18.91 & 18.32 & 18.70 & 19.45 & 20.82 & 19.24 & 0.973 \\
89017 & 17.47 & 16.89 & 16.54 & 18.44 & 18.98 & 17.66 & 1.029 \\
89022 & 22.41 & 21.70 & 21.62 & 24.89 & 22.01 & 22.53 & 1.358 \\
89025 & 22.17 & 20.85 & 22.79 & 21.74 & 20.86 & 21.68 & 0.842 \\
89026 & 19.03 & 18.89 & 18.87 & 19.55 & 18.21 & 18.91 & 0.479 \\
89031 & 19.34 & 18.74 & 17.62 & 19.69 & 19.87 & 19.05 & 0.909 \\
& & & & & & & \\
Column & 17.38 & 16.87 & 16.88 & 18.30 & 17.83 & 17.45 & \\
mean & & & & & & & \\
LE & -0.07 & -0.58 & -0.57 & +0.85 & +0.38 & &
\end{tabular}

DF SS MS

Columns

$\begin{array}{rrc}4 & 29.15 & 7.29 \\ 18 & 984.79 & 74.71 \\ 72 & 36.48 & 0.507 \\ 94 & 1050.42 & \end{array}$

Total $\quad 94 \quad 1050.42$

$F=14.38$ 


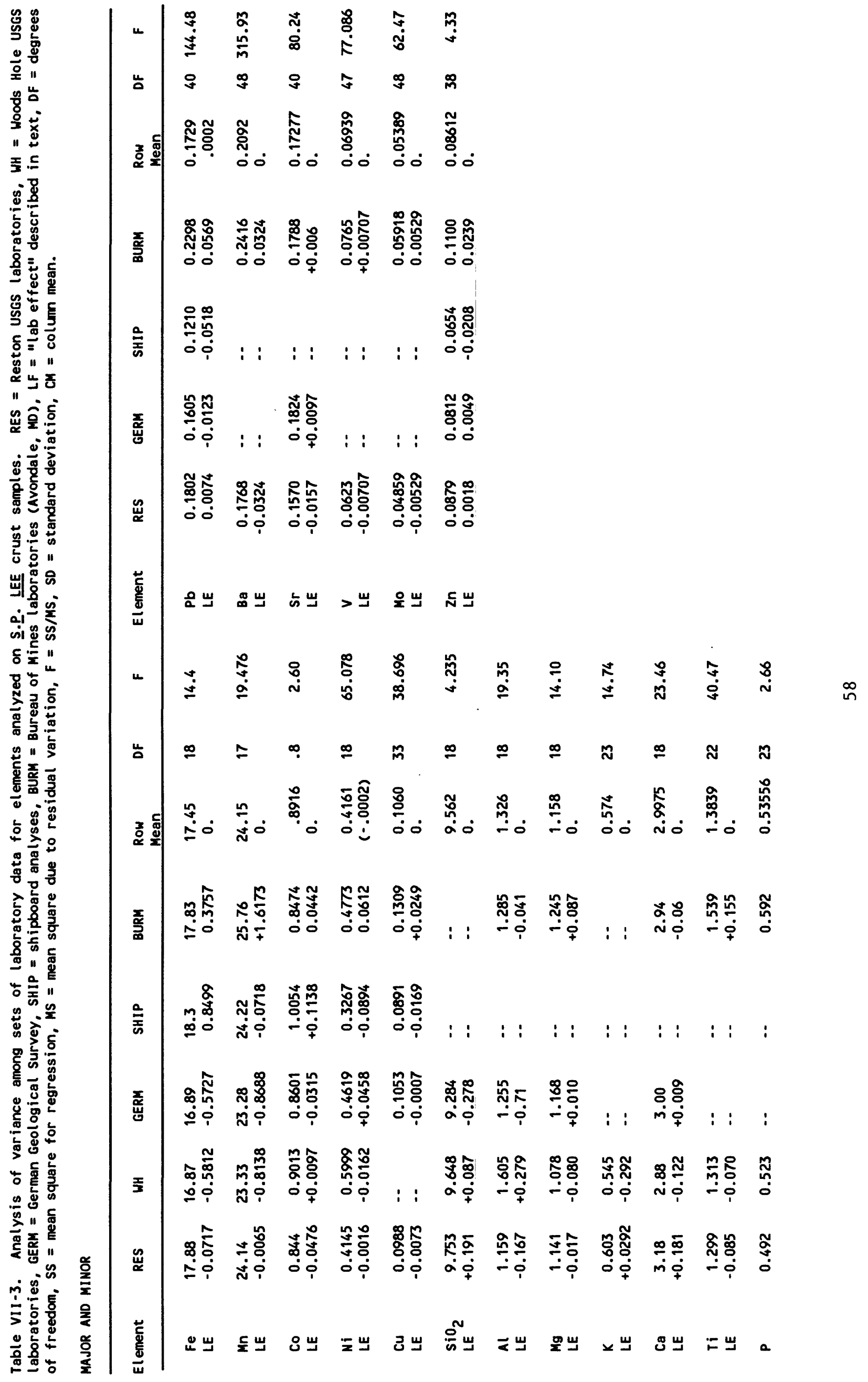


VIII DATA By C.M. Lane and F.T. Manheim

\section{Geological and Station Data}

The geological and station data for ferromanganese crust samples collected by the USGS summarized in this report consist of the geographic location in decimal degrees, water depth, cruise identification, average crust thickness within dredge recoveries, sampling device, institution of origin, and brief description of the nature of the dredge or other sampler recovery. An example of the station data, as it is reported in Appendix $A$, is given in Table VIII-1. We have reported some stations where chemical analyses are not available. These fall into the following categories: 1) crusts were observed at a particular station but there was not enough sample for analysis, 2) absence of crusts at a particular site was regarded as significant or 3 ) sites were camera stations TV grab stations or hydrographic stations. Key attributes such as depth may be missing for a few samples. Further research (e.g., for missing cruise reports) may fill in these gaps in the station data. Locations of principal sites are shown in Fig. VIII-1.

The geological and station data for ferromanganese crusts acquired from SNDB is reported in Appendix B. Similar attributes are represented, however crust thicknesses and description of dredge content were not reported by SNDB. When available length of core, surface lithology and estimated coverage with source of estimate are reported. To facilitate further research original SNDB reference numbers to data sources are given in Appendix B.

Both Appendix A and Appendix B are indexed by geographic area as indicated below. Data in Appendix $A$ have been sorted by latitude because there were often many samples per station and samples were not always consecutively assigned sequence numbers whereas Appendix B is sorted on sequence number. In the tables latitudes are negative south of the Equator, and longitudes are negative west of Greenwich, England. Map insets are shown in Fig. VIII-I.

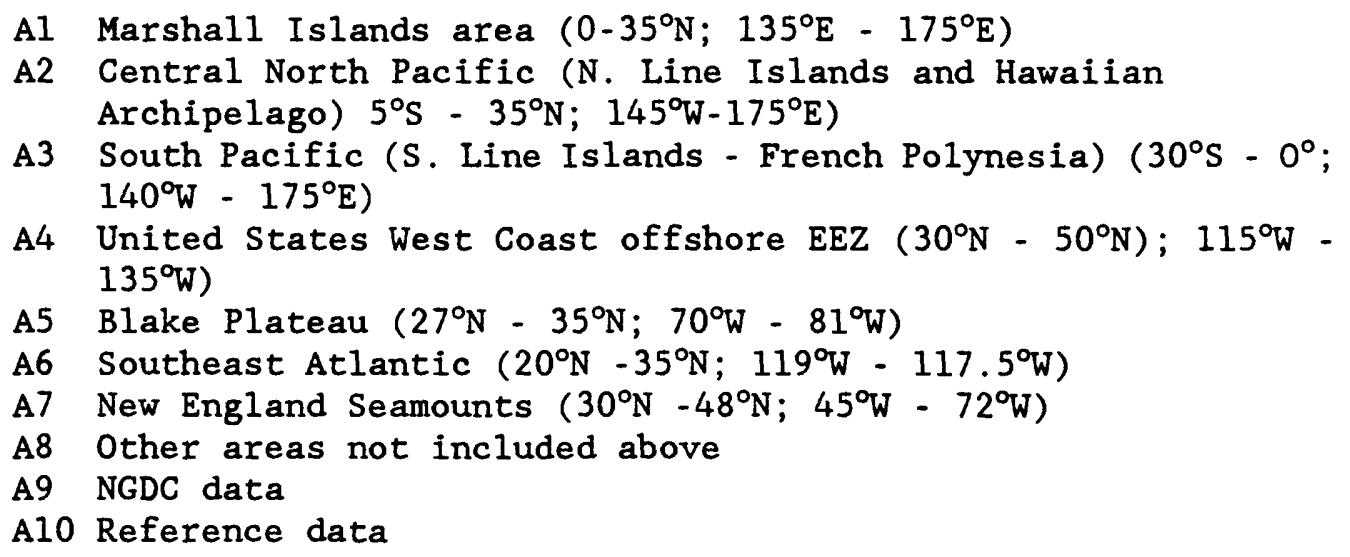




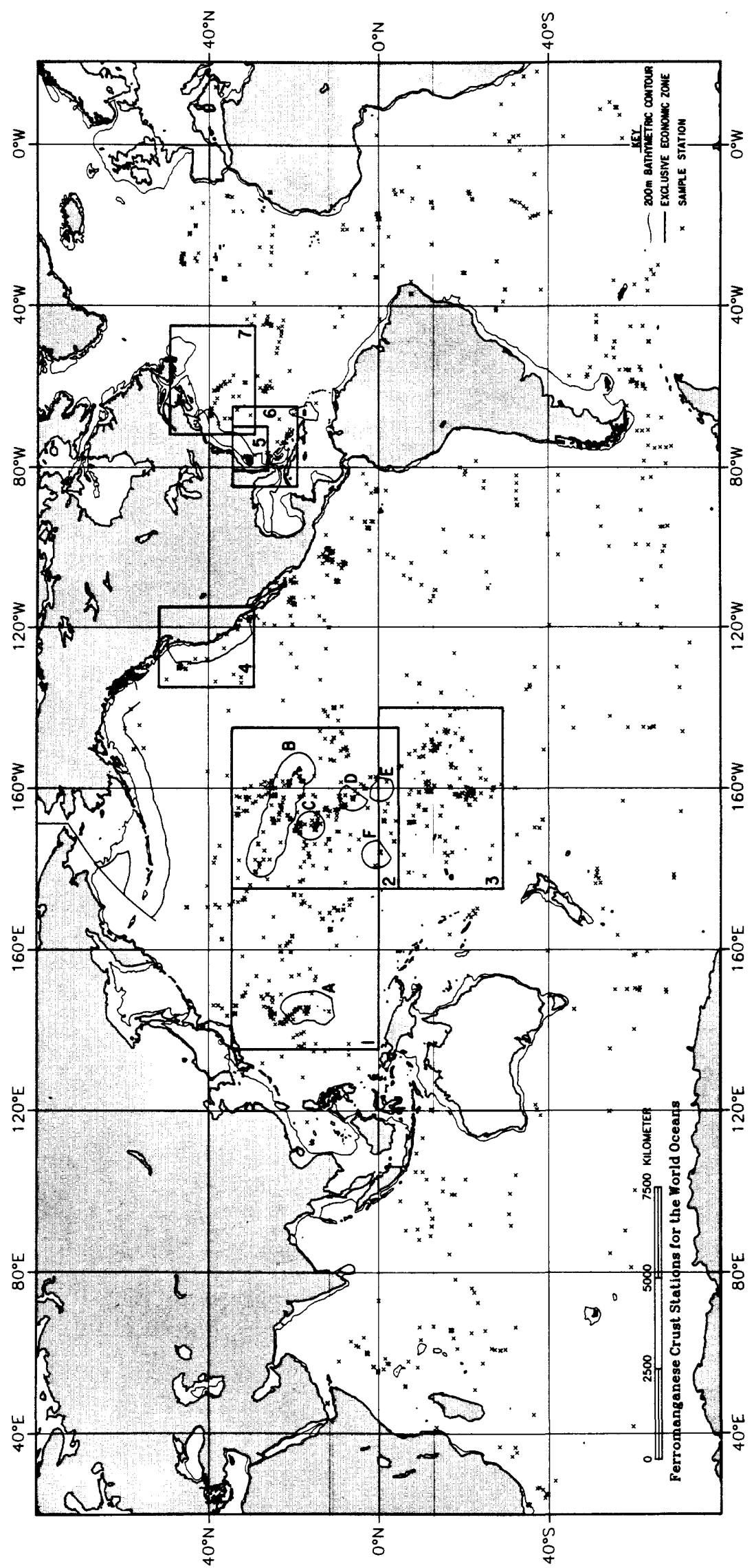

$\frac{2}{0}$

을

$\frac{5}{3}$

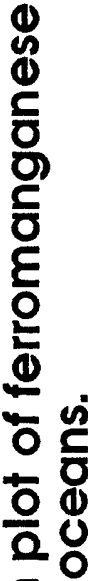

응응

$\frac{1}{5}$
$\frac{0}{5}$
은 


\section{Explanation of column headings}

In the following section each of the column headings for Appendix $A$ will be briefly commented on and qualified. Latitudes and longitudes are given in decimal degrees. "Depth interval" is given in meters. Where available both the start and end positions and corresponding depth for a dredge are reported, however only the position and depth for the beginning of a dredge is used for graphic output.

"Sample" refers to a concatenated designation which includes the original cruise identification, followed by a slash and a sample designation, for example "/D4 - 4" for Dredge No 4, sample 4. There is no uniform procedure amongst organizations for labeling dredge material or subsamples of these dredges. In some cases the only feature that differentiates between subsamples may be different thickness entries for the crust. These differences are also reflected in the assignment of a new sequence number (SEQNO). The sequence number has been described in Chapter IV and is a unique sample identifier.

"Thickness" refers to thickness in millimeters of the sample or subsample. Thickness ranges for the dredge or other gross sampling are given under "Description". Following "sample device", "Inst" refers to institution of origin. The coding for abbreviations is as follows: TUCLZ Technical University, Clausthal-Zellerfeld, Federal Republic of Germany; SIO, Scripps Institution of Oceanography; HIG Hawaii Institute of Geophysics; OSU Oregon State University; FSU Florida State University; LDGO Lamont Doherty Geological Observatory; UWASH University of Washington; SMIT Smithsonian Institution; NOAA National Oceanic and Atmospheric Administration; WHOI Woods Hole Oceanographic Institute, USGSWH U.S. Geological Survey Woods Hole MA, and USGS-MEN U.S. Geological Survey Menlo Park CA.

Explanations of variables in the USGS file of station data are listed below. The number following the variable name refers to the type of variable (1 = integer, 2 - real number, 3 -dictionary, 4 - code, and 6 - character string).

$\begin{array}{lll}\text { SEQNO } & 1 & \text { Sequence number } \\ \text { LAT1 } & 1 & \text { Latitude on bottom (decimal degrees) } \\ \text { LAT2 } & 2 & \text { Latitude off bottom (decimal degrees) } \\ \text { LON1 } & 1 & \text { Longitude on bottom (decimal degrees) } \\ \text { LON2 } & 2 & \text { Longitude off bottom (decimal degrees) } \\ \text { DEPTH1 } & 2 & \text { Depth on bottom (meters) } \\ \text { DEPTH2 } & 2 & \text { Depth off bottom (meters) } \\ \text { CRUISE } & 6 & \text { Cruise identification } \\ \text { STATIO } & 6 & \text { Station number } \\ \text { SAMP\# } & 6 & \text { Sample number } \\ \text { MONCOI } & 1 & \text { Month collected } \\ \text { DAYCOL } & 1 & \text { Day collected } \\ \text { YRCOL } & 1 & \text { Year collected (2 digits) } \\ \text { HOUR } & 1 & \text { Time collected } \\ \text { SEC } & 1 & \text { Time collected } \\ \text { PHASE1 } & 6 & \text { Phases collected } \\ \text { SMPDEV } & 3 & \text { Sampling device } \\ \text { AMTCOI } & 2 & \text { Amount collected }\end{array}$




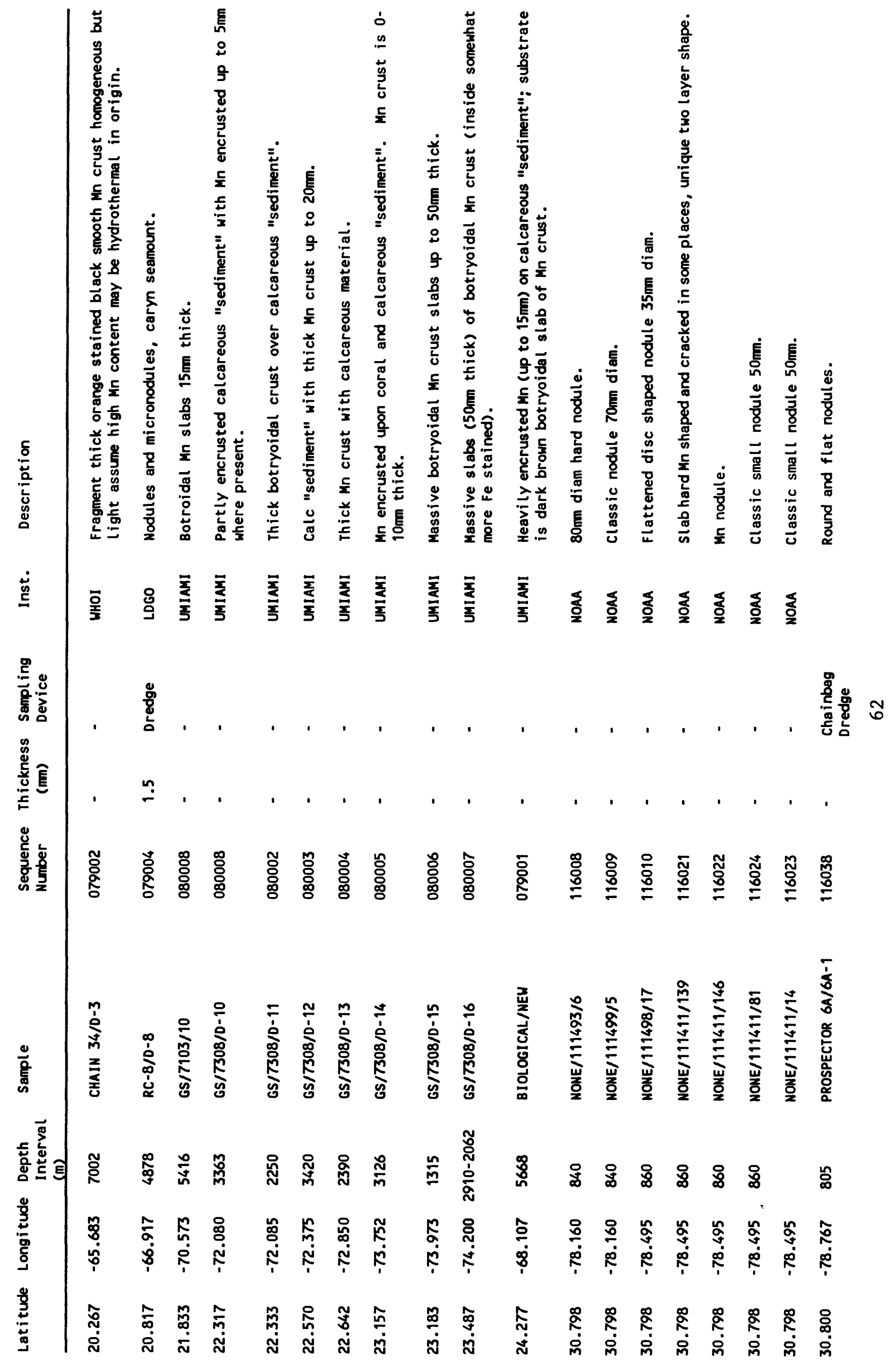




$\begin{array}{lll}\text { UNITSC } & 6 & \text { Units of amount collected } \\ \text { INST } & 3 & \text { Institution affiliated with the sample } \\ \text { PHOTO } & 4 & \text { Photo available (Y or } \mathrm{N} \text { ) } \\ \text { PHASE2 } & 6 & \text { Phase analyzed } \\ \text { THICK } & 2 & \text { Thickness of crust (mm) } \\ \text { MORPH } & 3 & \text { Morphology of crust } \\ \text { REFID } & 6 & \text { Reference number to link with reference file } \\ \text { NAVCDE } & 4 & \text { Navigation quality code } \\ \text { COMMNT } & 6 & \text { Comments }\end{array}$

\section{Chemical data}

Chemical data are provided in two basic modes; oxides and elements. Examples of these are given in Tables VIII-2 and VIII-3. First, where sufficient data are available to permit a chemical summation, i.e. the U.S. Geological Survey (Reston) data, concentrations are presented in oxide form corresponding to the oxidation state nominally assumed for the crusts. The second mode reports elemental concentrations, which include a basic assay of at least five elements: $\mathrm{Mn}, \mathrm{Fe}, \mathrm{Co}, \mathrm{Ni}, \mathrm{Cu}$. The SNDB falls into this category (Appendix C, Table 9). A third mode, normalized data, is not given in tabular form, but is utilized in the chemical maps and selected diagrams. Normalizations procedures are discussed later.

Complete data sets are reported in Appendix C, as itemized below. All Tables (CO1-C11) represent hygroscopic water-free data.

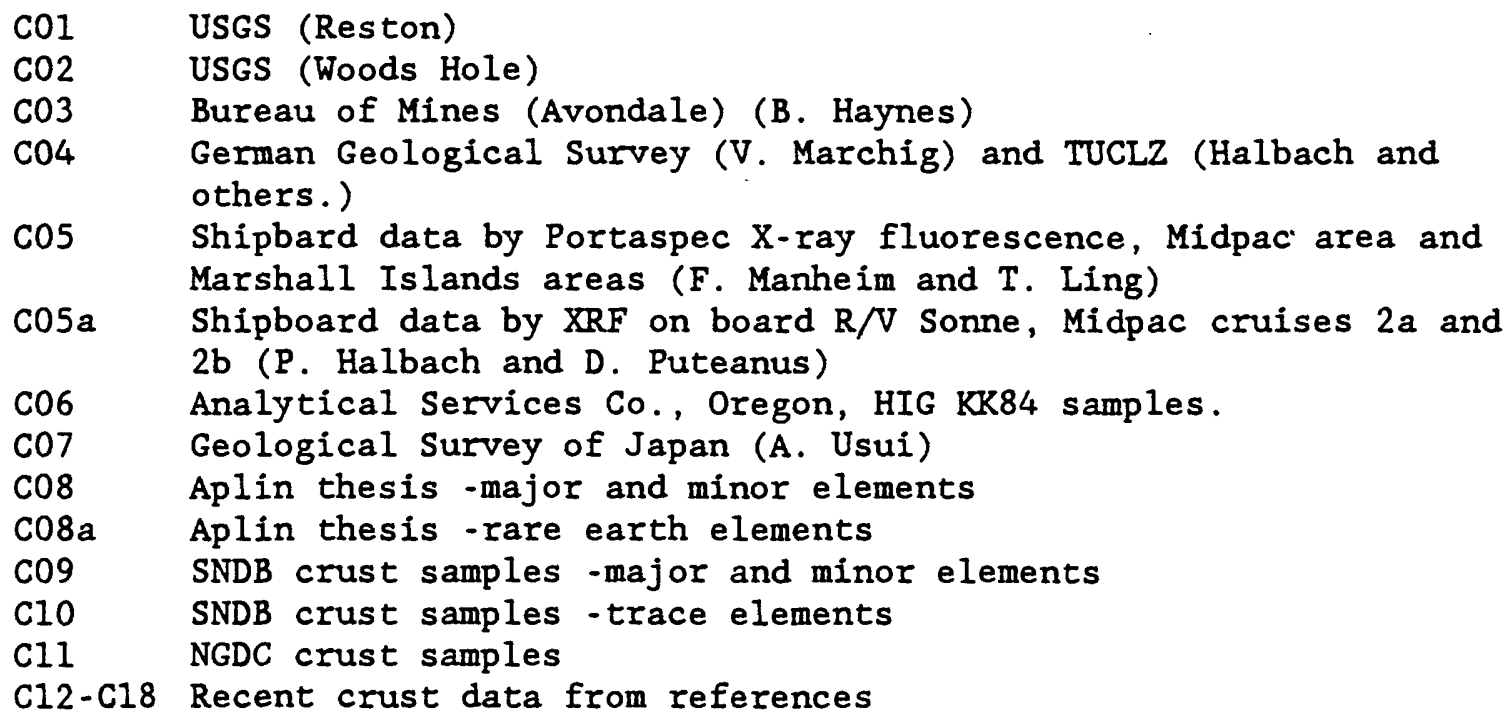

Some special remarks are appropriate regarding the unmodified, water-corrected data and derivation of the oxide sum. All data are presented on a hygroscopic moisture-free basis. Therefore, the $\mathrm{H}_{2} \mathrm{O}^{-}$value is not included in the summation. The summation does not include $\mathrm{Cl}$ and $\mathrm{SO}_{4}$ values (mainly due to dried sea water in pore fluid) nor trace metals. We have found the sum of the majors to have a normal distribution with a mean value 98.6 , rather than 100.0. This is in reasonable agreement with the estimated contributions from 
the above sources. As has been discussed under analytical chemistry, Chapter VII, scatter about the expected sum is believed to be mainly due to unavoidable inaccuracy in determination of $\mathrm{H}_{2} \mathrm{O}^{+}$. This is reflected in Tables VI-1 to VI-3 under discussion of the interlaboratory calibrations. $\tau$ (gamma) refers to the correction factor that would be needed to bring the observed sum to the theoretical sum of 98.6. Table VIII-2 is an example of summed data as reported in Table 01 of Appendix C. Laboratory number refers to the sample number assigned by the performing laboratory, enabling recheck of data by analysts. Sample interval refers to the portion of the crust taken for analysis. Where no description is given one can generally assume that a bulk sample was used. However, one should recognize that many, especially older SNDB materials may represent indeterminate samplings of crusts, and even for newer material, surfaces of crusts may be ground off by unprotected chain dredge bags during recovery.

Other data sets are reported in terms of elements. Where $\mathrm{H}_{2} \mathrm{O}$-data are available they are reported, although the data are already corrected for hygroscopic moisture. For the SNDB data shown in Table VIII-3 the

reference number refers to the cross-reference list provided in Chapter XIII (Frazer and Fisk, 1980).

Crust sample and analytical material are highly heterogeneous. They present some problems common to the study of abyssal manganese nodules, and additional sources of error or complicating factors. These can render data interpretation difficult or misleading. The main sources of uncertainty include high hygroscopic water content of crusts, as discussed in Chapter V, contamination with substrate, poor selection of samples (i.e. not crusts at all), and analytical and other factors.

Total water (hygroscopic and bound) may exceed 40 percent, and the way analyses are presented is therefore critical. For example, some of the early data of both J. Mero and D.S. Cronan (see Table XIII-1) referred to an ignited (about $800^{\circ} \mathrm{C}$ ) basis, thus maximizing reported metal content. On the other end of the spectrum, some workers have reported data on an air-dry basis, and some even on a partly sea-water-wet basis (!). Still other literature data report acid-soluble or reducible metal concentrations. We have chosen a $105-110^{\circ} \mathrm{C}$, oven-dried basis both on grounds of established use (SNDB and most Russian data) as well as on the basis of convenience and reproducibility (see Table V-1). For other data culled from the literature or unpublished sources the leaders of the Scripps study frequently applied discretionary corrections (Frazer and others., 1978). Spot checks revealed inconsistencies in some of the revised data. Similar problems were reported to us by J.R. Hein and L. Morgenson (oral communication, 1986).

\section{Normalized data}

An even more serious problem than water content is that of contamination. Unlike abyssal nodules, most crusts form layers on tabular substrates or envelope large talus fragments. In removing crust for analysis many older samplings incorporated large amounts of substrate material into the analyzed sample, so that "crusts" at the far end of this spectrum can have a nearly pure basaltic, hyaloclastite, volcanic ash, phosphorite or carbonate composition. These 
Table 1. Composition of crusts analyzed by USGS Reston Labs (cont'd).

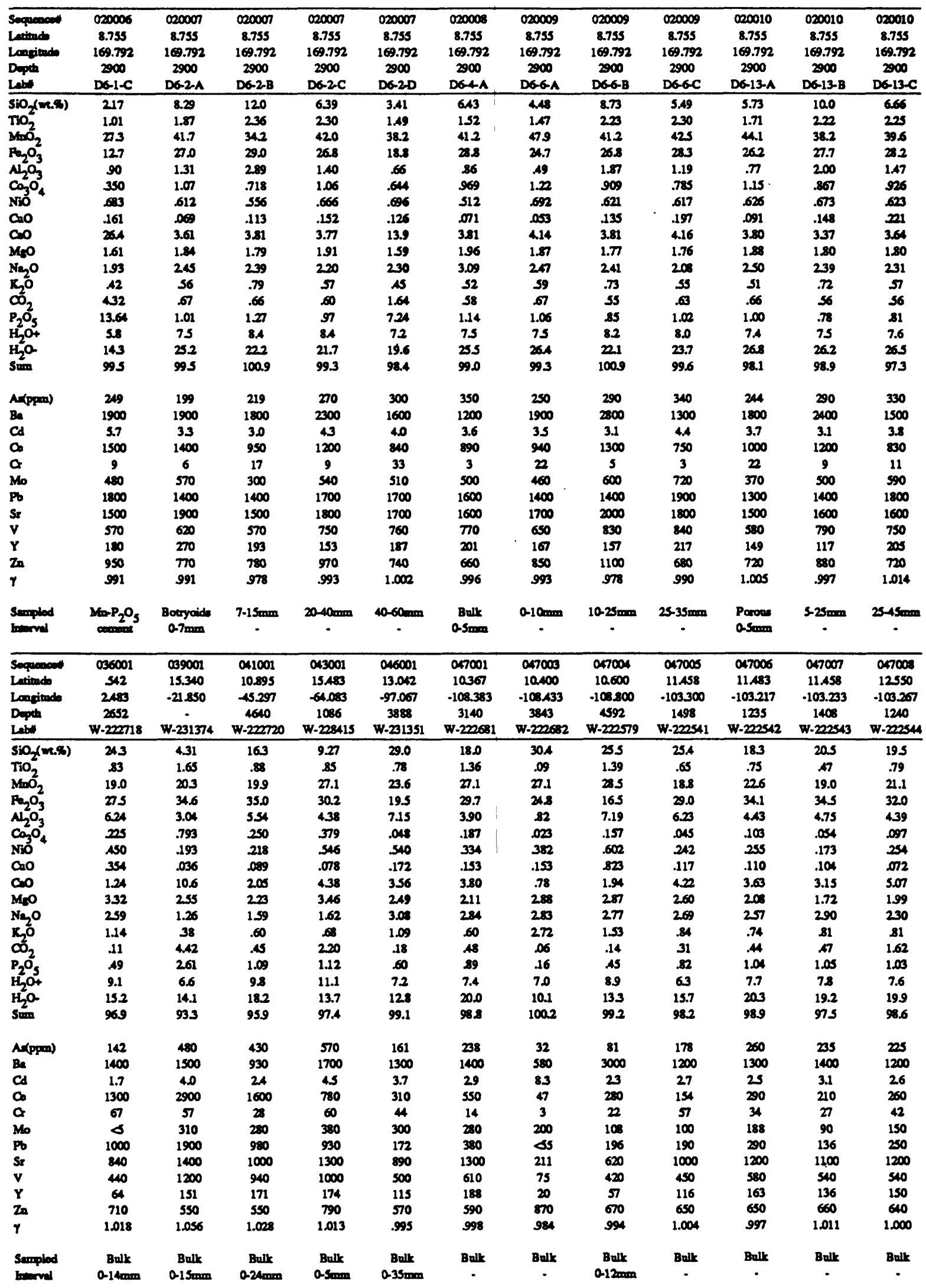
in oxide form, as in Appendix $\mathrm{Cl}$. 
admixtures can seriously bias both regional elemental variability as well as statistical studies involving histograms, correlation coefficients, and factor analysis.

Contamination was present even in newer samples from dedicated crust cruises. It is recognized that for mining engineering, processing and other purposes variable amounts of crust admixture may be taken into consideration, but it is clearly necessary from the point of view of establishing the nature and regional variability of the crust phases to limit the arbitrary admixture of extraneous material.

We therefore created a normalized data set from the total data population. Crusts consist basically of two major phases, a metal oxide phase, itself often composed of several potential subphases, and a detrital phase, also potentially containing subphases. At the simplest level, most of the heavy metal and transition metals that are of both economic and special geochemical interest are associated with the former phase. After careful consideration of alternatives and cross-plotting of chemical information, we concluded that the most stable and workable normalization technique would utilize the sum of iron and manganese as the normalizing factor. This choice also reflects past observations that when influence of diluting (non-crust) substances is minimized, $\mathrm{Fe}$ and $\mathrm{Mn}$ ferromanganese phases show an inversely correlated behavior, with a relatively constant sum.

As noted in Fig. IX-7, for the well-controlled USGS data set, the bulk of the data asympototically approach a maximum $F e+M n$ value representing a hypothetical "pure crust" material when $\mathrm{Mn}$ is plotted against $\mathrm{Mn}+\mathrm{Fe}$. A similar relationship is obtained when $\mathrm{Fe}$ is plotted against Fe+Mn. Based on the histogram in Fig. VIII-2 and other studies we chose a value of 50.00 to represent the "pure crust" end member. The end member composition retains about 22 percent of other constituents including $\mathrm{H}_{2} \mathrm{O}^{+}, \mathrm{Si}, \mathrm{Al}, \mathrm{Mg}, \mathrm{K}$, etc. Some of these are incorporated in the manganese mineral structure.

A set of data wherein the normalized value of each of the "metalliferous" components assumed to be associated with the $\mathrm{Mn}-\mathrm{Fe}$ oxide phase, $\mathrm{M}^{*}$, is obtained by the relationship:

$$
M *=50.00 \times \mathrm{M} /(\mathrm{Fe}+\mathrm{Mn})
$$

where $M$ is the concentration of a given element in the group, $\mathrm{Mn}, \mathrm{Fe}, \mathrm{Co}, \mathrm{Ni}$, $\mathrm{Cu}, \mathrm{Zn}, \mathrm{Cd}, \mathrm{Pb}, \mathrm{Mo}, \mathrm{V}, \mathrm{As}$, and $\mathrm{Ce}$. Thus, values for most samples with ( $\mathrm{Fe}+\mathrm{Mn}$ ) $<50.00$ will be increased in concentration. An exclusion before normalization is applied for samples meeting the test, $\mathrm{Mn} / \mathrm{Fe}>5$. This exclusion eliminates some hydrothermal oxide samples which typically have very high $\mathrm{Mn} / \mathrm{Fe}$ ratios (Toth, 1980).

Where Fe+Mn values are very low, the normalization process may sometimes create unreasonably high (apparent) minor metal concentration, e.g. in SEQNO 127006, values of $5000 \mathrm{ppm} \mathrm{Zn}$ and $497 \mathrm{ppm} \mathrm{Cd}$ were obtained for original Fe+Mn of only 0.21 percent in a nearly pure carbonate rock. However, such outliers are few and are excluded for mapping. 
Table 9. Composition of crusts from the Scripps Nodule Databank (cont'd).

\begin{tabular}{|c|c|c|c|c|c|c|c|c|c|c|c|}
\hline $\begin{array}{l}\text { Sequencen } \\
\text { Letiende } \\
\text { Leneinde } \\
\text { Depth }\end{array}$ & $\begin{array}{c}0000024-01 \\
-62.850 \\
-74.758 \\
4005\end{array}$ & $\begin{array}{c}0570004-00 \\
-64517 \\
-145967 \\
3775\end{array}$ & $\begin{array}{c}0890009-\infty 0 \\
-64.183 \\
-165.933 \\
2932\end{array}$ & $\begin{array}{c}0910002-00 \\
-63.007 \\
178.483 \\
3583\end{array}$ & $\begin{array}{c}0910005-01 \\
-63.100 \\
177.617 \\
3383\end{array}$ & $\begin{array}{c}0910005.02 \\
-63.100 \\
17.617 \\
3383\end{array}$ & $\begin{array}{c}1120020.00 \\
-56.617 \\
-34.800 \\
3696\end{array}$ & $\begin{array}{c}1130048-00 \\
-55.933 \\
-45.202 \\
3740\end{array}$ & $\begin{array}{c}1130061-00 \\
-53.042 \\
48.883 \\
3118 \\
\end{array}$ & $\begin{array}{c}1130052-01 \\
-59.142 \\
48.883 \\
3850 \\
\end{array}$ & $\begin{array}{c}1130063-01 \\
-53.058 \\
-48.950 \\
3255 \\
\end{array}$ \\
\hline$S i(w t s)$ & - & $\cdot$ & 11.3 & 15.5 & $\cdot$ & $\cdot$ & $\cdot$ & $\cdot$ & $\cdot$ & - & $\cdot$ \\
\hline $\mathbf{T i}$ & • & .91 & .03 & 46 &.$x$ & 1.16 & • & • & 53 & $\cdot$ & • \\
\hline $\mathbf{M n}$ & 10.3 & 18.1 & 14.8 & 8.80 & 13.5 & 121 & 220 & 266 & 710 & 123 & 27.7 \\
\hline $\mathbf{F e}_{0}$ & 25.7 & 17.3 & 11.0 & 124 & 224 & 23.0 & 19.2 & 13.0 & 19.9 & 23.6 & 14.5 \\
\hline $\mathbf{N}$ & • & • & 240 & 3.90 & • & • & • & • & - & • & • \\
\hline Co & .140 & 570 & .170 & .140 & 330 & .350 & $A B O$ & .120 & .340 & .150 & 210 \\
\hline $\mathbf{N i}$ & .100 & 200 & .700 & 270 & 200 & .130 & 200 & 990 & .060 & 200 & .770 \\
\hline Ca & 100 & .090 & .200 & .130 & .000 & .000 &.$\infty 60$ & .410 & .090 & .100 & 160 \\
\hline c. & 228 & • & 1.52 & 1.79 & • & - & 224 & 200 & • & 2.14 & 1.65 \\
\hline Ms & 299 & - & • & • & - & - & 96 & 190 & - & 1.82 & 1.63 \\
\hline $\mathrm{Na}$ & 216 & - & - & - & - & - & 1.57 & 357 & - & 297 & $2 \pi$ \\
\hline $\mathbf{K}$ & 98 & - & - & - & - & - & 34 & 1.07 & - & 99 & 1.07 \\
\hline $\mathbf{P}$ & - & - & • & - & - & - & - & - & • & • & - \\
\hline $\mathrm{H}_{2} \mathrm{O}$ & $\cdot$ & 17.1 & 17.4 & 12.2 & 19.1 & 20.4 & $\cdot$ & - & 15.0 & $\cdot$ & $\cdot$ \\
\hline Ax(ppm) & - & • & • & • & • & • & - & - & - & - & - \\
\hline Ba & - & 1200 & 3900 & 2500 & 1700 & 2800 & - & $\cdot$ & 1100 & $\cdot$ & - \\
\hline Cd & - & • & • & - & - & • & - & $\cdot$ & - & - & - \\
\hline Co & - & - & - & - & - & - & - & - & - & $\cdot$ & - \\
\hline$c$ & - & - & - & . & - & - & - & - & - & - & - \\
\hline Mo & • & 1300 & 110 & so & 1100 & 620 & • & • & 510 & • & - \\
\hline Pb & 2000 & - & 1500 & 1400 & $\cdot$ & - & 3100 & 900 & $\cdot$ & 2600 & 800 \\
\hline St & - & 1300 & 1000 & 1100 & 1300 & 1300 & • & - & 860 & • & • \\
\hline $\mathbf{v}$ & - & 560 & • & • & 1200 & 920 & - & - & 490 & - & - \\
\hline $\mathbf{Y}$ & • & • & - & • & • & • & • & $\cdot$ & $\cdot$ & • & $\cdot$ \\
\hline $\mathrm{Zn}$ & 640 & 400 & 1000 & 580 & 600 & 500 & 1800 & 2700 & 300 & 870 & 1600 \\
\hline Sempio & Man-oonend & Noctuls & Cone & Crus & Crus & Cour & Cros & Morooned & Soveral & Ore & Crant \\
\hline type & rock & • & • & - & - & - & - & rock & couts & • & • \\
\hline Portion & unlonown & withour & 0000 & 0 & $\infty$ & onter & unlonown & uniknown & mbows & uxborown & unkrowa \\
\hline ampled & $\bullet$ & melows & rection & antion & cont & $\cos x$ & • & - & • & - & - \\
\hline Noclow & - & Cry & $\cdot$ & • & • & - & - & - & • & - & • \\
\hline Refereaces & A368 & 1220 & A197 & A197 & $A 20$ & $A 22$ & A368 & 136 & A220 & A368 & 136 \\
\hline Sequencill & $1130065-00$ & $1140011-01$ & $1140029-01$ & $1140010-05$ & $1140051-\infty$ & $1140081+01$ & $1140001-02$ & $1140038-01$ & $1140119-00$ & $1140127-\infty$ & $1140127-01$ \\
\hline Letinde & -59.003 & .53 .000 & .57 .538 & -55.100 & -55.733 & .56207 & .562207 & .56 .233 & .55 .642 & .52833 & -52833 \\
\hline Longinde & -48.978 & -52500 & .55 .138 & -55.833 & -56.060 & -58.275 & -58.275 & .58 .700 & .55 .912 & -50.500 & .50500 \\
\hline Dopth & 3862 & 3101 & 4075 & 2871 & 3914 & 4087 & 4087 & 4133 & 4085 & 2842 & 2842 \\
\hline$S(m s)$ & - & 138 & - & - & $\cdot$ & • & $\cdot$ & $\cdot$ & $\cdot$ & $\cdot$ & $\cdot$ \\
\hline $\mathrm{Ti}$ & • & .25 & - & • & .37 & 10 & 47 & • & • & • & - \\
\hline $\mathbf{M n}$ & 18.4 & 10.8 & 11.3 & 14.0 & 3.90 & 1.00 & 1.00 & 156 & 6.20 & 950 & 9.20 \\
\hline $\mathbf{F e}_{\mathbf{e}}$ & 21.1 & 158 & 17.9 & 282 & 192 & 14.5 & 168 & 40.3 & 20.8 & 14.7 & 189 \\
\hline $\mathbf{N}$ & - & 270 & • & - & - & • & - & • & • & 357 & 3.00 \\
\hline Co & .160 & .150 & .160 & 200 & .120 & .130 & .150 & .020 & .100 & .100 & .140 \\
\hline $\mathbf{N i}$ & 500 & 390 & .240 & 230 & .120 & .200 & .130 & .030 & .110 & 240 & .070 \\
\hline $\mathrm{Cn}$ & 250 &.$\infty 0$ &.$\infty 0$ & .070 & .090 & .160 & .160 & .060 & .040 & .100 & .090 \\
\hline C. & 165 & 1.19 & 1.66 & 1.35 & - & • & - & 151 & • & • & • \\
\hline $\mathbf{M}$ & 1.70 & • & 252 & 1.54 & - & - & - & 1.75 & - & - & - \\
\hline $\mathrm{Na}$ & 3.08 & - & 4.07 & 209 & - & - & - & 2.19 & - & - & - \\
\hline $\mathbf{K}$ & 96 & - & 259 & $\pi 7$ & - & . & - & .91 & - & - & $\cdot$ \\
\hline $\mathbf{P}$ & $\cdot$ & - & • & - & - & - & - & - & - & - & - \\
\hline $\mathrm{H}_{2} \mathrm{O}$ & - & 13.0 & - & - & 78 & 10.1 & 7.7 & - & - & - & - \\
\hline Ax(ppon) & - & - & - & - & • & • & • & - & - & $\because$ & - \\
\hline Ba & - & 3000 & - & - & 580 & 400 & 510 & $\cdot$ & $\cdot$ & - & - \\
\hline$c d$ & - & - & - & $\cdot$ & • & $\cdot$ & - & - & - & $\cdot$ & - \\
\hline co & - & - & - & - & - & - & - & - & - & - & $\cdot$ \\
\hline$a$ & - & • & - & - & • & • & • & - & - & 52 & 14 \\
\hline Mo & • & 140 & • & - & 510 & 260 & 200 & - & - & 160 & 230 \\
\hline $\mathbf{P b}$ & 2100. & 1200 & 1100 & 3200 & - & - & • & 2400 & - & 600 & 1100 \\
\hline Sr & • & 800 & $\cdot$ & - & 760 & 480 & 520 & - & - & • & $\cdot$ \\
\hline $\mathbf{v}$ & - & - & - & - & 710 & 230 & 660 & - & - & 350 & 570 \\
\hline $\mathbf{Y}$ & - & - & - & - & - & • & . & • & - & • & - \\
\hline $\mathbf{Z n}$ & 1100 & 670 & 520 & 900 & 300 & 460 & 450 & 660 & $\bullet$ & 720 & 1700 \\
\hline Samplo & Crus & Crus & Crut & Crus & Con & Cont & Con & Mn-conted & Cont & Crus & Crus \\
\hline type & • & • & • & • & • & • & - & roct & $\cdot$ & • & • \\
\hline Partion & unlenowa & crows & untonown & unloown & 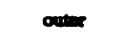 & unlerown & unlonown & unlonown & monknown & untorown & mibnown \\
\hline ampled & • & nection & - & $\cdot$ & $\cos$ & • & - & - & - & • & - \\
\hline Neclowe & - & - & • & • & - & - & - & - & - & - & $\cdot$ \\
\hline Rafirencos & $A 368$ & A197 & $A 368$ & A368 & 1220 & 1227 & 1227 & $A 368$ & $\mathbf{A 3 3 3}$ & A333 & $A 333$ \\
\hline
\end{tabular}


A slightly different normalization factor (51.3) was used in Figs. IX-1c and IX2c, from a prior report (Manheim and Lane-Bostwick, 1987). Use of the new normalization factor does not change the principal features.

\section{Exclusion data set}

This data set removes samples with large amounts of dilutants like silicates and phosphorite, which can yield spurious correlation coefficients. Dilutants also create a spurious low-end tail in frequency plots of elements in the main ferromanganese oxide group ( $\mathrm{Fe}, \mathrm{Mn}, \mathrm{Cu}, \mathrm{Ni}, \mathrm{Co}, \mathrm{Zn}$, etc.) (e.g. Fig. VIII-3).

The special "exclusion" data set is not given in tabular form, but was utilized in descriptive output (Tables IX-1 thru IX-4) and Co histograms (Figs. IX-1B, IX-2B). This set was employed in Manheim (1986) to assess regional means for the world oceans and parts of the Pacific Ocean. The qualifying values are those passing all of the following tests (in percent, on a hygroscopic water water free basis):

$$
\begin{aligned}
& \mathrm{Mn}>15 \quad \mathrm{Fe}<23 \quad \mathrm{P}<3 \\
& \mathrm{Mn} / \mathrm{Fe}<5 \text { Al }<2.5 \\
& \mathrm{Si}<7 \quad \mathrm{Ca}<6
\end{aligned}
$$




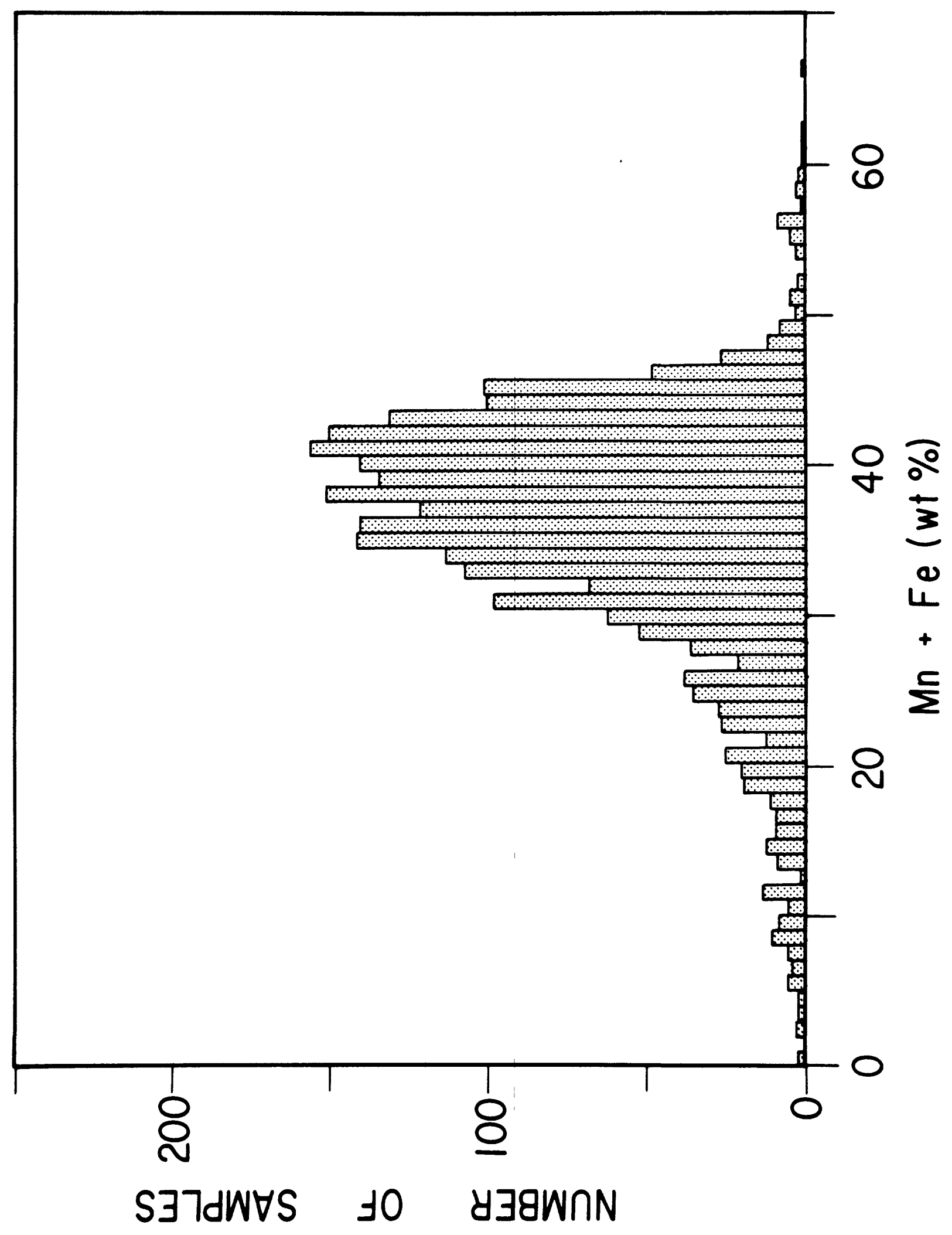


IX Data Interpetation and Mapping by F.T. Manheim and C.M. Lane

\section{Frequency descriptions and regional means}

The various data sets incorporated in the database presented herein offer a large variety of material for display of frequency distributions. These demonstrate both the distribution of chemical values and some of the complicating factors like contamination, mentioned earlier. Figs. IX-1A through IX-1C show histograms for cobalt in the SNDB. The number of class intervals in the histogram was chosen to display maximum coherent detail on modes and submodes. The SNDB data set has complex and irregular features. The new USGS data set (Figure IX-2A) has a smoother lognormal character and suggests a trimodal distribution: a lognormal population with a cobalt mode just under 1.0 percent, a secondary mode at approximately 0.1 percent $C o$, and a weak scattering of data with a mode less than 0.01 percent. The main mode is attributable to hydrogenetic, high-cobalt crusts (see Chapter II), the secondary mode to phases partly influenced by hydrothermal manganese deposits, and the last mode to a combination of highly substrate-contaminated samples, and hydrothermal phases. Use of the exclusion data set (Fig. IX-2B) goes farthest toward delimiting the hydrogenetic mode, which is the critical population for economic interest. The exclusion data set has a scattering of low-cobalt values, which have not yet been investigated in detail. Data normalized to the sum, $F e+M n$, is less effective in removing the tail of low values (Figs. IX-lc and IX-2c) but retains more samples that would be eliminated in the exclusion set.

If means are calculated using the raw (water-corrected) or normalized data, the central value will be shifted toward lower concentrations

because of the low-Co tail. This reflects "dirty samples" (contaminants and extraneous phases, including hydrothermally influenced compositions). Medians are a better measure of central tendency, but are likewise influenced by the tail. The most consistent population measures involve the "exclusion data set" discussed in Chapter VIII. It carries the disadvantage that some analyses are sacrificed. However, it has been chosen as the most consistent basis for regional means. Means for the total Pacific Ocean, Marshall Islands area, Atlantic and Indian oceans are presented in Tables IX-1 to IX-4. 

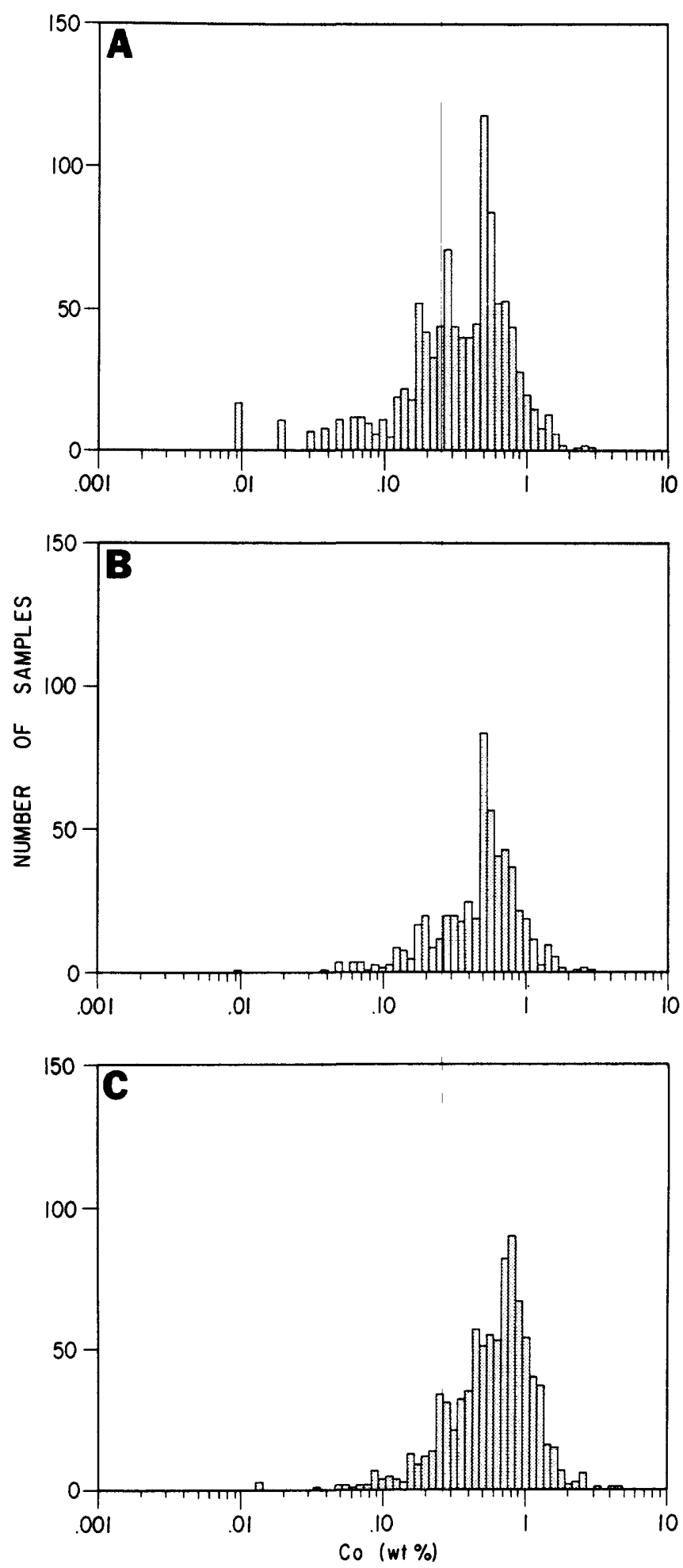

Figure IX-1 Histogram of Co values for USGS data set: a) raw data b) exclusion data set, and c) normalized data set. 

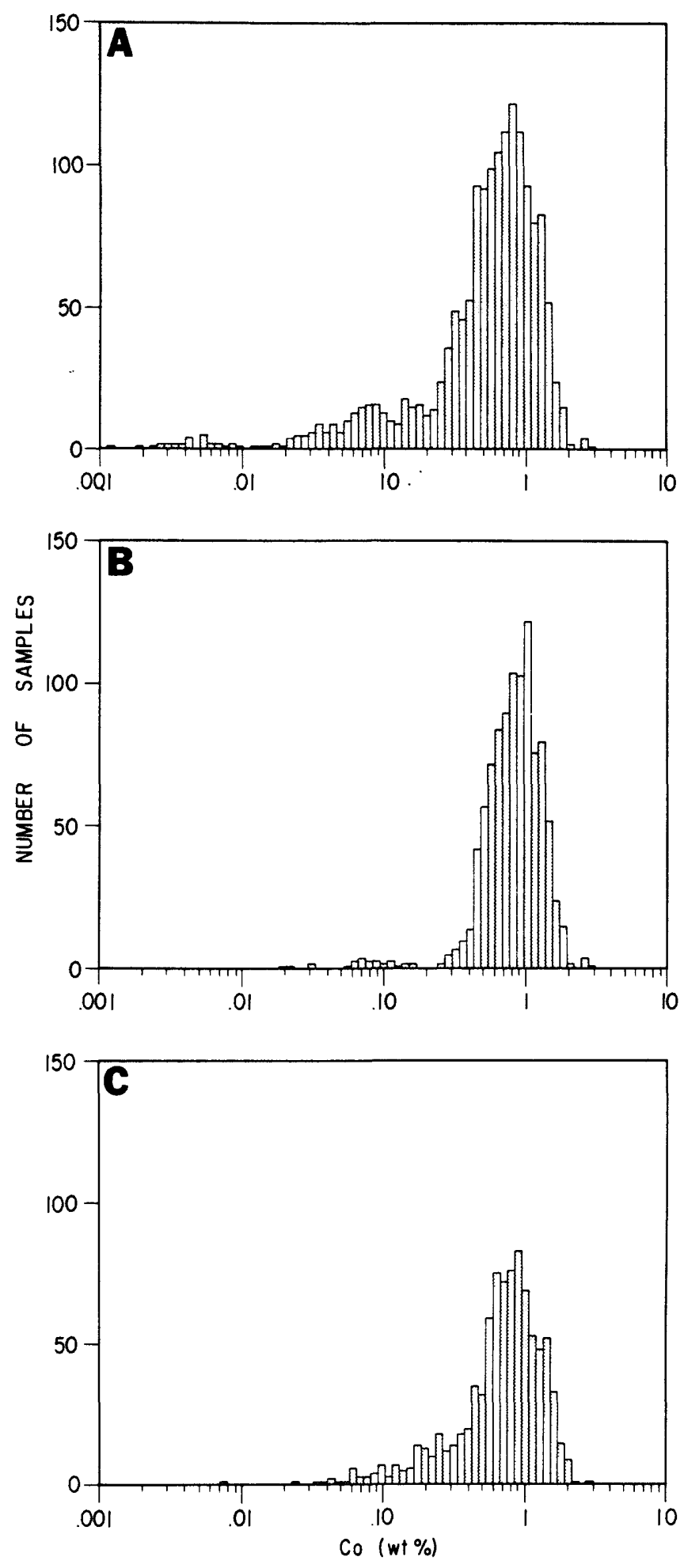

Figure IX-2 Histogram of Co values for SNDB data set: a) raw data b) exclusion data set, and c) normalized data set. 
Table IX-1. Descriptive statistics for the Total Pacific Ocean (exclusion data set: see Chapter VIII discussion). Values for minimum, maximum, mean and SD in percent moisture-free material, except Pt(ppm). Note, however, that the Pacific mean is biased by a large data population in the central, cobalt-rich seamount areas.

\begin{tabular}{|c|c|c|c|c|c|c|}
\hline Variable & $\begin{array}{l}\text { Number } \\
\text { samples }\end{array}$ & Minimum & Maximum & Mean & SD & $\mathrm{CV}$ \\
\hline & - & - & $\cdots \cdots$ & - - - - - & - & $\ldots$ \\
\hline Lat & 834 & -43.000 & 55.127 & 19.0769 & 10.3106 & 54.0 \\
\hline Lon & 834 & -179.745 & 179.288 & -102.8786 & 130.4872 & -126.8 \\
\hline Depth & 819 & 598. & 6890 & 2011.80 & 721. & 35.9 \\
\hline $\mathrm{Mn}$ & 773 & 15.100 & 38.79 & 25.02 & 4.14 & 16.6 \\
\hline $\mathrm{Fe}$ & 802 & 5.980 & 22.95 & 16.89 & 2.93 & 17.4 \\
\hline $\mathrm{Ni}$ & 773 & 0.120 & 1.536 & 0.46 & 0.17 & 36.6 \\
\hline Co & 805 & 0.029 & 3.02 & 0.87 & 0.37 & 42.5 \\
\hline $\mathrm{Cu}$ & 603 & 0.013 & 0.55 & 0.08 & 0.05 & 69.4 \\
\hline $\mathrm{mg}$ & 632 & 0.710 & 2.39 & 1.16 & 0.20 & 17.6 \\
\hline $\mathrm{Ca}$ & 655 & 1.410 & 5.97 & 2.83 & 0.78 & 27.8 \\
\hline A1 & 746 & 0.064 & 2.50 & 0.95 & 0.51 & 54.1 \\
\hline $\mathrm{Ti}$ & 675 & 0.102 & 2.46 & 1.12 & 0.28 & 25.4 \\
\hline $\mathrm{K}$ & 443 & 0.064 & 1.01 & 0.54 & 0.10 & 20.1 \\
\hline $\mathrm{P}$ & 653 & 0.026 & 1.88 & 0.51 & 0.25 & 49.3 \\
\hline $\mathrm{Si}$ & 542 & 0.610 & 6.99 & 3.42 & 1.64 & 48.1 \\
\hline $\mathrm{Na}$ & 293 & 0.207 & 3.32 & 1.74 & 0.48 & 27.8 \\
\hline $\mathrm{CO}_{2}$ & 63 & 0.086 & 2.21 & 0.54 & 0.21 & 40.0 \\
\hline $\mathrm{Ba}$ & 401 & 0.0842 & 0.4300 & 0.1850 & 0.0598 & 32.3 \\
\hline Mo & 399 & 0.0171 & 0.1493 & 0.0569 & 0.0207 & 36.3 \\
\hline $\mathrm{Pb}$ & 476 & 0.0154 & 0.3700 & 0.1921 & 0.0559 & 29.1 \\
\hline Sr & 444 & 0.0827 & 0.2453 & 0.1671 & 0.0226 & 13.5 \\
\hline V & 399 & 0.0249 & 0.1600 & 0.0695 & 0.0129 & 18.6 \\
\hline $\mathrm{Zn}$ & 460 & 0.0100 & 0.6712 & 0.0734 & 0.0393 & 53.6 \\
\hline $\mathrm{Y}$ & 264 & 0.0083 & 0.0501 & 0.0188 & 0.0044 & 23.6 \\
\hline $\mathrm{Ce}$ & 264 & 0.0061 & 0.2849 & 0.1232 & 0.0369 & 29.9 \\
\hline As & 265 & 0.0140 & 0.3430 & 0.0292 & 0.0216 & 74.1 \\
\hline $\mathrm{Cd}$ & 271 & $<0.0007$ & 0.0014 & 0.0004 & 0.0002 & 62.4 \\
\hline $\mathrm{H}_{2} \mathrm{O}^{-}$ & 563 & 4.00 & 37.93 & 16.45 & 6.67 & 40.6 \\
\hline $\mathrm{H}_{2} \mathrm{O}^{+}$ & 281 & 3.78 & 17.59 & 8.73 & 1.67 & 19.2 \\
\hline $\mathrm{Pt}(\mathrm{ppm})$ & 8 & 0.1639 & 1.2935 & 0.4840 & 0.3934 & 81.3 \\
\hline
\end{tabular}

*Decimals are a function of software output and do not reflect level of accuracy. 
Table IX-2. Descriptive statistics for the Marshall Islands area. Values for minimum, maximum, mean and SD in percent moisture-free material, except Pt(ppm).

\begin{tabular}{|c|c|c|c|c|c|c|}
\hline Variable & $\begin{array}{l}\text { Number } \\
\text { samples }\end{array}$ & Minimum & Maximum & Mean & SD & $\mathrm{CV}$ \\
\hline & $\cdots$ & $\cdots$ & - - - - - - - & $\cdots$ & $\cdots$ & \\
\hline Lat & 100 & 6.667 & 33.666 & 16.961 & 7.442 & 43.9 \\
\hline Lon & 100 & 138.968 & 174.325 & 162.523 & 10.709 & 6.6 \\
\hline Depth & 100 & 905 . & 3835 . & 1944. & 680 & 35.0 \\
\hline $\mathrm{Mn}$ & 100 & 15.87 & 32.51 & 25.95 & 3.48 & 13.4 \\
\hline $\mathrm{Fe}$ & 100 & 5.98 & 21.02 & 16.31 & 2.68 & 16.4 \\
\hline $\mathrm{Ni}$ & 100 & 0.26 & 1.05 & 0.52 & 0.14 & 28.4 \\
\hline Co & 100 & 0.07 & 1.96 & 0.85 & 0.33 & 39.1 \\
\hline $\mathrm{Cu}$ & 86 & 0.022 & 0.264 & 0.08 & 0.047 & 56.3 \\
\hline $\mathrm{mg}$ & 82 & 0.84 & 1.72 & 1.16 & 0.16 & 14.5 \\
\hline $\mathrm{Ca}$ & 82 & 1.72 & 5.70 & 2.88 & 0.62 & 21.6 \\
\hline Al & 100 & 0.12 & 2.37 & 0.80 & 0.48 & 59.8 \\
\hline $\mathrm{Ti}$ & 80 & 0.56 & 1.57 & 1.09 & 0.21 & 19.2 \\
\hline $\mathrm{K}$ & 80 & 0.32 & 1.00 & 0.54 & 0.11 & 22.1 \\
\hline $\mathrm{P}$ & 80 & 0.27 & 1.74 & 0.51 & 0.24 & 47.1 \\
\hline Si & 98 & 0.61 & 6.91 & 3.16 & 1.54 & 48.8 \\
\hline $\mathrm{Na}$ & 66 & 1.19 & 2.49 & 1.90 & 0.21 & 11.4 \\
\hline $\mathrm{CO}_{2}$ & 66 & 0.44 & 1.20 & 0.62 & 0.16 & 26.2 \\
\hline $\mathrm{Ba}^{2}$ & 68 & 0.1091 & 0.3609 & 0.1705 & 0.0504 & 29.5 \\
\hline Mo & 66 & 0.0195 & 0.1117 & 0.0541 & 0.0159 & 29.4 \\
\hline $\mathrm{Pb}$ & 84 & 0.1340 & 0.3000 & 0.1924 & 0.0395 & 20.5 \\
\hline $\mathrm{Sr}$ & 68 & 0.1152 & 0.2453 & 0.1622 & 0.0174 & 10.7 \\
\hline $\mathrm{V}$ & 66 & 0.0442 & 0.0842 & 0.0679 & 0.0082 & 12.1 \\
\hline $\mathrm{Zn}$ & 86 & 0.0400 & 0.6712 & 0.0813 & 0.0661 & 81.4 \\
\hline $\mathrm{Y}$ & 66 & 0.0099 & 0.0501 & 0.0194 & 0.0058 & 29.9 \\
\hline $\mathrm{Ce}$ & 66 & 0.0747 & 0.2143 & 0.1156 & 0.0286 & 24.8 \\
\hline As & 66 & 0.0140 & 0.0383 & 0.0282 & 0.0044 & 15.7 \\
\hline $\mathrm{Cd}$ & 66 & 0.0002 & 0.0006 & 0.0004 & 0.0001 & 22.5 \\
\hline $\mathrm{H}_{2} \mathrm{O}^{-}$ & 80 & 11.16 & 31.40 & 23.70 & 4.37 & 18.5 \\
\hline $\mathrm{H}_{2} \mathrm{O}^{+}$ & 83 & 3.78 & 11.95 & 8.10 & 1.54 & 19.0 \\
\hline $\mathrm{Pt}(\mathrm{ppm})$ & 8 & 0.1639 & 1.2935 & 0.4840 & 0.3934 & 81.3 \\
\hline
\end{tabular}


Table IX-3. Descriptive statistics for the Atlantic Ocean. Values for minimum, maximum, mean and SD in percent moisture-free material, except Pt(ppm).

\begin{tabular}{|c|c|c|c|c|c|c|}
\hline Variable & $\begin{array}{l}\text { Number } \\
\text { samples }\end{array}$ & Minimum & Maximum & Mean & SD & $\mathrm{CV}$ \\
\hline$\cdots-\cdots$ & - & $-\ldots$ & 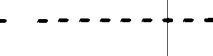 & 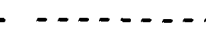 & $-\ldots$ & $\ldots$ \\
\hline Lat & 89 & -59.033 & 45.800 & 4.556 & 36.094 & 792.2 \\
\hline Lon & 89 & -86.548 & 22.383 & -31.637 & 28.624 & -90.5 \\
\hline Depth & 89 & 649 & 5842. & 2594 . & 1250. & 48.2 \\
\hline $\mathrm{Mn}$ & 82 & 15.02 & 42.50 & 20.27 & 4.98 & 24.6 \\
\hline $\mathrm{Fe}$ & 83 & 7.51 & 22.90 & 18.63 & 3.37 & 18.1 \\
\hline $\mathrm{Ni}$ & 83 & 0.02 & 1.56 & 0.39 & 0.24 & 61.2 \\
\hline Co & 83 & 0.01 & 3.00 & 0.54 & 0.40 & 73.2 \\
\hline $\mathrm{Cu}$ & 83 & .010 & 0.800 & 0.107 & 0.113 & 105.3 \\
\hline $\mathrm{mg}$ & 57 & 0.93 & 4.17 & 1.64 & 0.61 & 37.2 \\
\hline $\mathrm{Ca}$ & 61 & 0.16 & 5.30 & 2.54 & 0.96 & 37.9 \\
\hline $\mathrm{Al}$ & 16 & 0.40 & 2.31 & 1.32 & 0.55 & 41.8 \\
\hline $\mathrm{Ti}$ & 15 & 0.02 & 1.37 & 0.70 & 0.38 & 54.9 \\
\hline $\mathrm{K}$ & 58 & 0.18 & 1.69 & 0.45 & 0.28 & 62.7 \\
\hline $\mathrm{P}$ & 12 & 0.16 & 1.80 & 0.62 & 0.48 & 77.4 \\
\hline Si & 19 & 0.50 & 6.96 & 3.22 & 1.84 & 57.3 \\
\hline $\mathrm{Na}$ & 59 & 0.21 & 3.96 & 1.66 & 0.70 & 42.2 \\
\hline $\mathrm{Ba}$ & 13 & 0.0703 & 0.7100 & 0.2071 & 0.17 & 83.6 \\
\hline Mo & 17 & 0.0160 & 0.0680 & 0.0385 & 0.0132 & 34.2 \\
\hline $\mathrm{Pb}$ & 63 & 0.0106 & 0.3500 & 0.1647 & 0.0803 & 48.8 \\
\hline Sr & 15 & 0.0615 & 0.2043 & 0.1394 & 0.0390 & 28.0 \\
\hline $\mathrm{V}$ & 13 & 0.0108 & 0.1500 & 0.0934 & 0.0367 & 39.3 \\
\hline $\mathrm{Zn}$ & 62 & 0.0040 & 0.2730 & 0.0809 & 0.0472 & 58.4 \\
\hline$Y$ & 13 & 0.0057 & 0.0250 & 0.0170 & 0.0066 & 39.0 \\
\hline $\mathrm{Ce}$ & 9 & 0.0127 & 0.2519 & 0.1687 & 0.0820 & 48.6 \\
\hline As & 13 & 0.0095 & 0.0602 & 0.0362 & 0.0148 & 40.9 \\
\hline $\mathrm{Cd}$ & 9 & 0.0003 & 0.0014 & 0.0005 & 0.0004 & 75.1 \\
\hline $\mathrm{H}_{2} \mathrm{O}^{-}$ & 13 & 5.30 & 21.70 & 16.32 & 5.7502 & 35.2 \\
\hline
\end{tabular}


Table IX-4. Descriptive statistics for the Indian Ocean. Values for minimum, maximum, mean and SD in percent moisture-free matieral, except Pt(ppm).

\begin{tabular}{|c|c|c|c|c|c|c|}
\hline Variable & $\begin{array}{l}\text { Number } \\
\text { samples }\end{array}$ & Minimum & Maximum & Mean & SD & CV \\
\hline$\cdots$ & $\ldots$ & $\ldots$ & & $\cdots$ & & -- \\
\hline $\begin{array}{l}\text { Lat } \\
\text { Lon }\end{array}$ & $\begin{array}{l}129 \\
129\end{array}$ & $\begin{array}{r}-52.083 \\
25.050\end{array}$ & $\begin{array}{r}12.728 \\
118.675\end{array}$ & $\begin{array}{l}-4.286 \\
64.478\end{array}$ & $\begin{array}{l}12.571 \\
17.058\end{array}$ & $\begin{array}{r}-293.3 \\
26.5\end{array}$ \\
\hline Depth & 129 & 1170 . & 5579 . & 3402 . & 1044 . & 30.7 \\
\hline $\mathrm{Mn}$ & 128 & 15.00 & 38.30 & 18.15 & 3.17 & 17.5 \\
\hline $\mathrm{Fe}$ & 128 & 7.20 & 23.00 & 16.68 & 3.44 & 20.6 \\
\hline $\mathrm{Ni}$ & 128 & 0.08 & 1.17 & 0.39 & 0.20 & 51.4 \\
\hline Co & 129 & 0.06 & 1.22 & 0.37 & 0.23 & 61.5 \\
\hline $\mathrm{Cu}$ & 129 & 0.020 & 0.830 & 0.129 & 0.12 & 95.0 \\
\hline mg & 59 & 0.94 & 2.04 & 1.22 & 0.30 & 24.4 \\
\hline $\mathrm{Ca}$ & 80 & 0.99 & 5.98 & 2.03 & 0.87 & 43.2 \\
\hline $\mathrm{A} 1$ & 72 & 0.37 & 2.35 & 1.32 & 0.50 & 38.1 \\
\hline $\mathrm{Ti}$ & 84 & 0.27 & 1.76 & 0.70 & 0.26 & 37.3 \\
\hline $\mathrm{K}$ & 3 & 0.51 & 0.62 & 0.56 & 0.05 & 9.5 \\
\hline $\mathbf{P}$ & 7 & 0.23 & 1.65 & 0.59 & 0.53 & 90.1 \\
\hline Si & 23 & 1.60 & 6.90 & 5.35 & 1.27 & 23.8 \\
\hline $\mathrm{Na}$ & 3 & 1.60 & 2.31 & 1.92 & 0.36 & 18.8 \\
\hline $\mathrm{Ba}$ & 10 & 0.1102 & 0.3990 & 0.1652 & 0.0847 & 51.3 \\
\hline Mo & 32 & 0.0280 & 0.0717 & 0.0440 & 0.0110 & 25.0 \\
\hline $\mathrm{Pb}$ & 107 & 0.0200 & 1.3700 & 0.1494 & 0.1741 & 116.5 \\
\hline $\mathrm{Sr}$ & 7 & 0.0810 & 0.1675 & 0.1271 & 0.0278 & 21.9 \\
\hline $\mathrm{V}$ & 27 & 0.0430 & 0.1166 & 0.0767 & 0.0168 & 21.9 \\
\hline $\mathrm{Zn}$ & 102 & 0.0110 & 0.1100 & 0.0564 & 0.0171 & 30.4 \\
\hline $\mathrm{Y}$ & 7 & 0.0112 & 0.0510 & 0.0207 & 0.0137 & 66.0 \\
\hline $\mathrm{Ce}$ & 3 & 0.1284 & 0.2036 & 0.1769 & 0.0421 & 23.8 \\
\hline As & 6 & 0.0236 & 0.0359 & 0.0283 & 0.0047 & 16.8 \\
\hline $\mathrm{Cd}$ & 6 & 0.0003 & 0.0011 & 0.0006 & 0.0002 & 29.6 \\
\hline $\mathrm{H}_{2} \mathrm{O}^{-}$ & 6 & 19.50 & 32.30 & 25.70 & 5.32 & 20.7 \\
\hline
\end{tabular}


The data in all depth figures represent area 2 in Fig. VIII-1 (Mid Pacific area) without duplicate analyses. The inverse relationship between cobalt and water depth of crust occurrence is demonstrated in the Mid-Pacific data set, Figure IX-3. This diagram shows much scatter and much more diffuse trends than studies limited to smaller geographic areas (e.g. Halbach and others, 1983; Halbach and Manheim, 1984). Mn and $\mathrm{Ni}$ likewise show highest values in the shallowest occurrences. A plot of depth vs. Co/Mn (Fig. IX-4) reveals that removing the effect of the Mn relationship still leaves a marginal inverse Co-depth function. In contrast, $\mathrm{Cu}$ is well known to have direct correlation with depth (Fig. IX-5). Consequently, the ratio of $\mathrm{Co}$ to $\mathrm{Cu}(\mathrm{Co} / \mathrm{Cu})$ would be expected to be a sensitive discriminator for water depth of origin for crusts. As we see in Fig. IX-3, a trend is found, but much scatter remains. Some of this scatter can be explained by uncertainty in establishing the depth of crust occurence, because sample sites comprised dredge hauls that covered significant vertical ranges; other factors contributing scatter include crusts on talus that has slumped to a present position deeper than where they formed, and changing depth with time for some seamounts.

Fe is illustrated in Fig. IX-5, which shows a rapid increase in Fe concentration from 1000 to $2000 \mathrm{~m}$, followed by a much more gentle increase concentration with depth. Halbach and Puteanus(1984) suggest that the increase in iron concentrations with depth may be associated with debris from foraminifera that decompose in and beneath the oxygen-minimum zone in the North-Central Pacific equatorial area. The aluminosilicate group of elements tend to increase in concentration with depth in a linear fashion.

\section{Interelement plots}

The varied nature of interelement relationships calls for many types of plots. Fig. IX-7 displays interelement plots using the full USGS data set, whereas remaining plots display the smaller Mid Pacific set utilized for element/depth plots discussed earlier. Several of the sample populations included in the USGS data set can be seen in figure IX-7 ( $\mathrm{Mn} / \mathrm{Fe}$ vs. $\mathrm{Mn}+\mathrm{Fe}$ ). The main hydrogenetic crust population is represented by the dense clustering from 30 to 50 percent $\mathrm{Mn}+\mathrm{Fe}$, and from .5 to $5 \mathrm{Mn} / \mathrm{Fe}$. Samples with greater $\mathrm{Mn} / \mathrm{Fe}$ are often hydrothermally influenced, whereas samples with $\mathrm{Mn}+\mathrm{Fe}<30$ percent are appreciably admixed with substrate material. Substrates having $>8 \%$ iron content, like basalt and altered basalt tend toward lower $\mathrm{Mn} / \mathrm{Fe}$ values.

A large increase in the number of analyses for non-oxide elements is provided by the new data. This is illustrated by comparing Figs. IX-8 (new data) and IX-8b (SNDB) (Al vs. Si). At low $\mathrm{Si}$ values in the main hydrogenetic crust a $\mathrm{Si} / \mathrm{Al}$ ratio of about 5.0 is found. A population of samples with very low Si/A1 ratios implies mineral phases richer in $A 1$ than $S i$ and include recently discovered lithiophorite-like manganese minerals (Manheim and others, 1988; 01son and others, 1988).

A plot of $\mathrm{Si}$ against $\mathrm{Mn}+\mathrm{Fe}$ (Fig. IX-8c) shows the expected inverse relationship for samples in the main crust mode. A smaller low-Si population is associated with phosphatic substrate as displayed when we plot detrital Al against Mn (Fig. IX-8d). The portion of Al that forms part of the Mn mineral structure, 

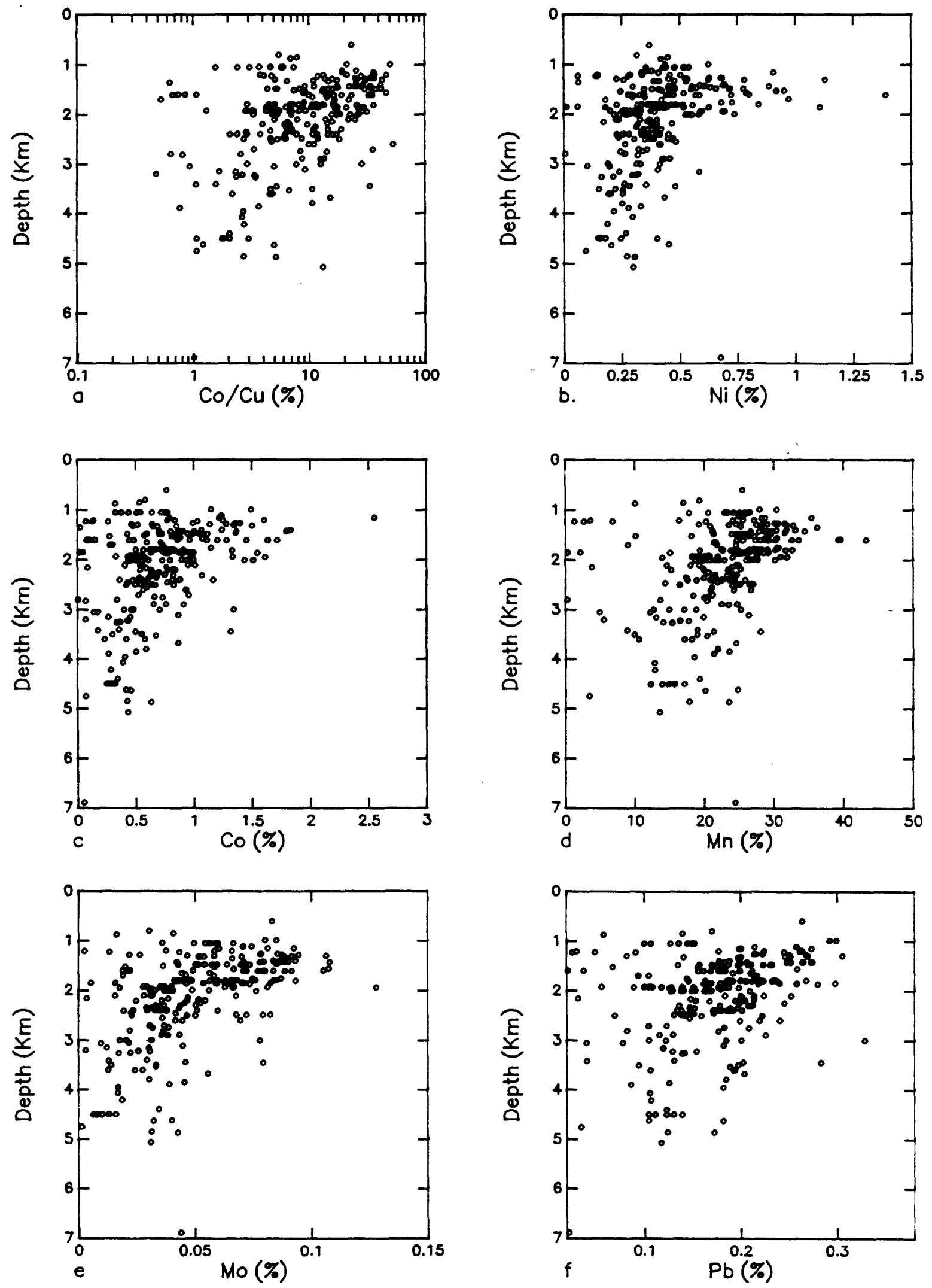

Figure IX-3 Plots of water depth vs. $\mathrm{Co} / \mathrm{Cu}, \mathrm{Ni}, \mathrm{Co}, \mathrm{Mn}, \mathrm{Mo}, \mathrm{Pb}$ for Mid-PacificOcean. 

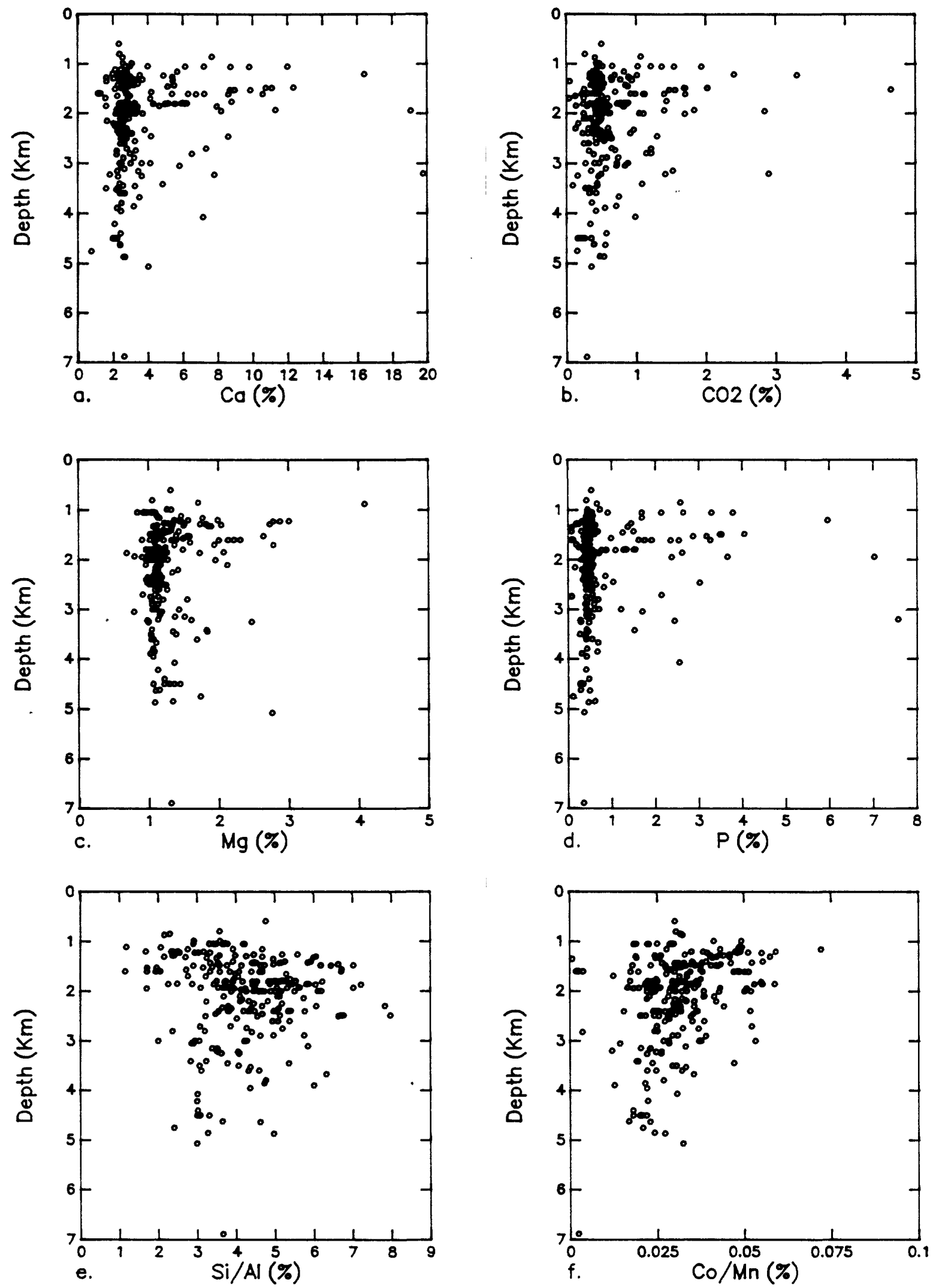

Figure IX-4 Plots of water depth vs. $\mathrm{Ca}, \mathrm{CO}_{2}, \mathrm{Mg}, \mathrm{P}, \mathrm{Si} / \mathrm{Al}, \mathrm{Co} / \mathrm{Mn}$ for Mid-PacificOcean. 

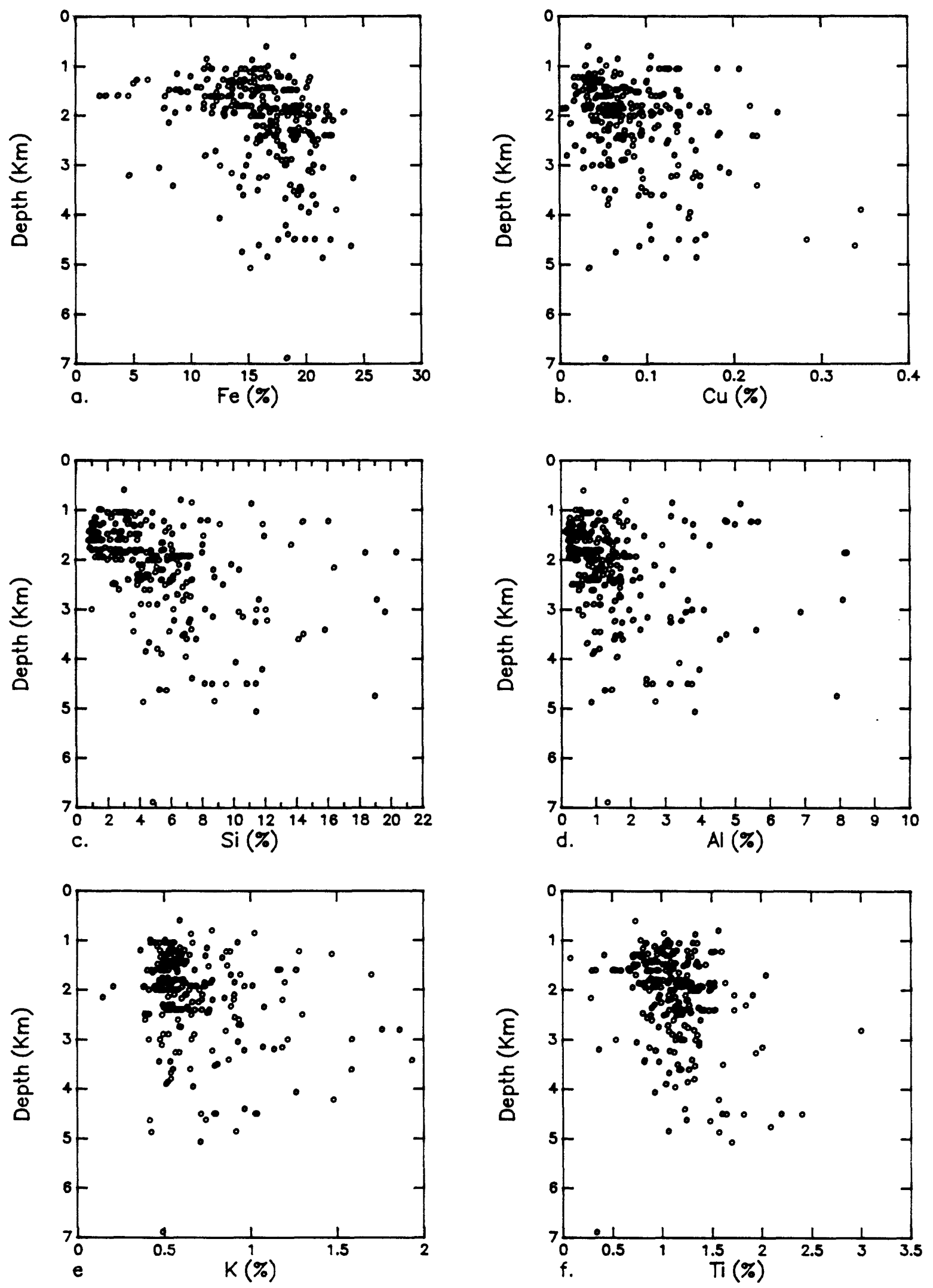

Figure IX-5 Plots of water depth vs. Fe, $\mathrm{Cu}, \mathrm{Si}, \mathrm{Al}, \mathrm{K}, \mathrm{Ti}$ for Mid-PacificOcean. 

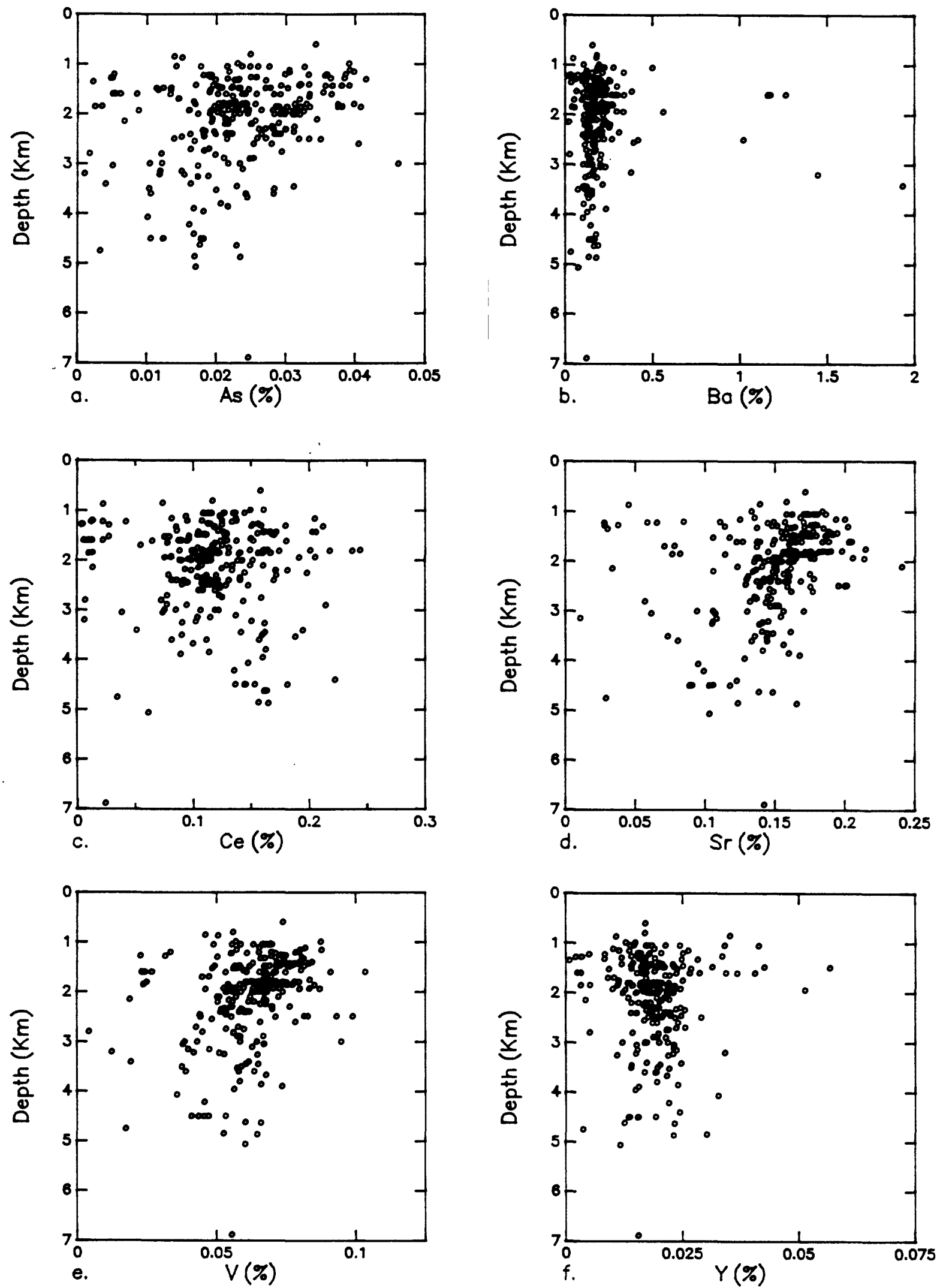

Figure IX-6 Plots of water depth vs. As, Ba, Ce, Sr, V, Y for Mid-PacificOcean. 
delineated as $A 1 *$, is given by $A 1 *=0.016 \times M n$ on the basis of additional data. The plot of Al corrected for this fraction intersects 0.0 percent $A 1$ at about 32 percent $\mathrm{Mn}$ (Fig. IX-8d). An attempt to relate the corrected $\mathrm{Al} / \mathrm{H}_{2} \mathrm{O}^{+}$ratio and Si (Fig. IX-8f) gives a strong divergence for increasingly impure crusts (see Figs. IX-7 and IX-8f). The nearly vertical trend, best shown in the larger population (Fig. IX-7) is related to unweathered basalt admixtures at about 46 percent $\mathrm{SiO}_{2}$, whereas the upcurving trend to the highest $\mathrm{Si}$ values is thought to represent altered basaltic materials.

Various elemental plots against $M n$ are depicted in Figs. IX-9 and IX-10. The larger data set for $C_{0}$ and $\mathrm{Mn}$ data is also shown in the earlier IX-7 plot. For all elements except $\mathrm{Fe}$ and $\mathrm{Cu}$ a positive correlation is obtained. However, outliers are often found at Mn values of about 40 percent or higher, which usually represent hydrothermal oxides. For $\mathrm{Pb}, \mathrm{Co}, \mathrm{Ce}, \mathrm{As}$, and Mo the outliers tend to be on the low side, whereas for $\mathrm{Cd}, \mathrm{Zn}$ and $\mathrm{Ba}$ the outliers tend toward higher values. This is consistent with the finding of these elements in polymetallic sulfides deposits. Ni concentrations for high-Mn outliers are well below the envelope of more normal Mn values, but are subsequentially higher than in the case of Co.

The behavior of elements such asmg and $\mathrm{Ti}$ in crusts is still poorly understood (Fig. IX-11), much of the scatter being due to admixture in substrate. Note the very small spread ofmg values between 1.0 and 1.5 percent for the main envelope of purer crust data, in contrast to much wider dispersion in contaminated samples $(I X-11 e)$.

In Fig. IX-12 Co and $\mathrm{Ni}$ show linear correlation within the main body of purer crusts, whereas an arcuate envelope for the Fe and Si plot (Fig. IX-12d). The large scatter is due to dilution with a variety of phases including basalt and smectite formed from weathering of basalt. As expected, redox-active $\mathrm{Ce}$, which is precipitated with the Mn element group, is poorly correlated with $Y$, whose behavior is generally linked with the rare earth element group (Fig. IX-12c). A distinctive envelope of $\mathrm{Sr}$ values between .1 and .2 percent is obtained for the pure crusts (Fig. IX-12e).

\section{Histograms}

Figs. IX-13 through IX-16 depict normal histograms for 20 constituents in the USGS data set, whereas Figs. IX-17 through IX-19 show paired normal and lognormal histograms for nin additional constituents. The distribution may be grouped roughly as follows:

$\begin{array}{lcc}\text { Normal } & \text { Broad, irregular } & \text { Lognormal } \\ \mathrm{Fe}, \mathrm{Ti} & \mathrm{Ce}, \mathrm{Mo} & \mathrm{Al}, \mathrm{P} \\ \mathrm{Na}, \mathrm{H}_{2} \mathrm{O}^{-} & \mathrm{Pb}, \mathrm{Y} & \mathrm{Si}, \mathrm{Cu} \\ \left(\mathrm{H}_{2} \mathrm{O}^{+}\right), \mathrm{Zn} * & & \mathrm{Mn} / \mathrm{Fe}, \mathrm{Ca}) \\ \mathrm{Cd}, \mathrm{Ba} * & & \mathrm{Ni**}, \mathrm{Mn} * *\end{array}$

where parenthesis refers to slightly skewed distributions, * refers to small tail toward higher values, attributed to hydrothermal samples, and $* *$ refers to small tail toward lower values, probably linked to hydrothermal samples, especially those with higher iron content in the case of $\mathrm{Mn}$. Co remains skewed toward lower 

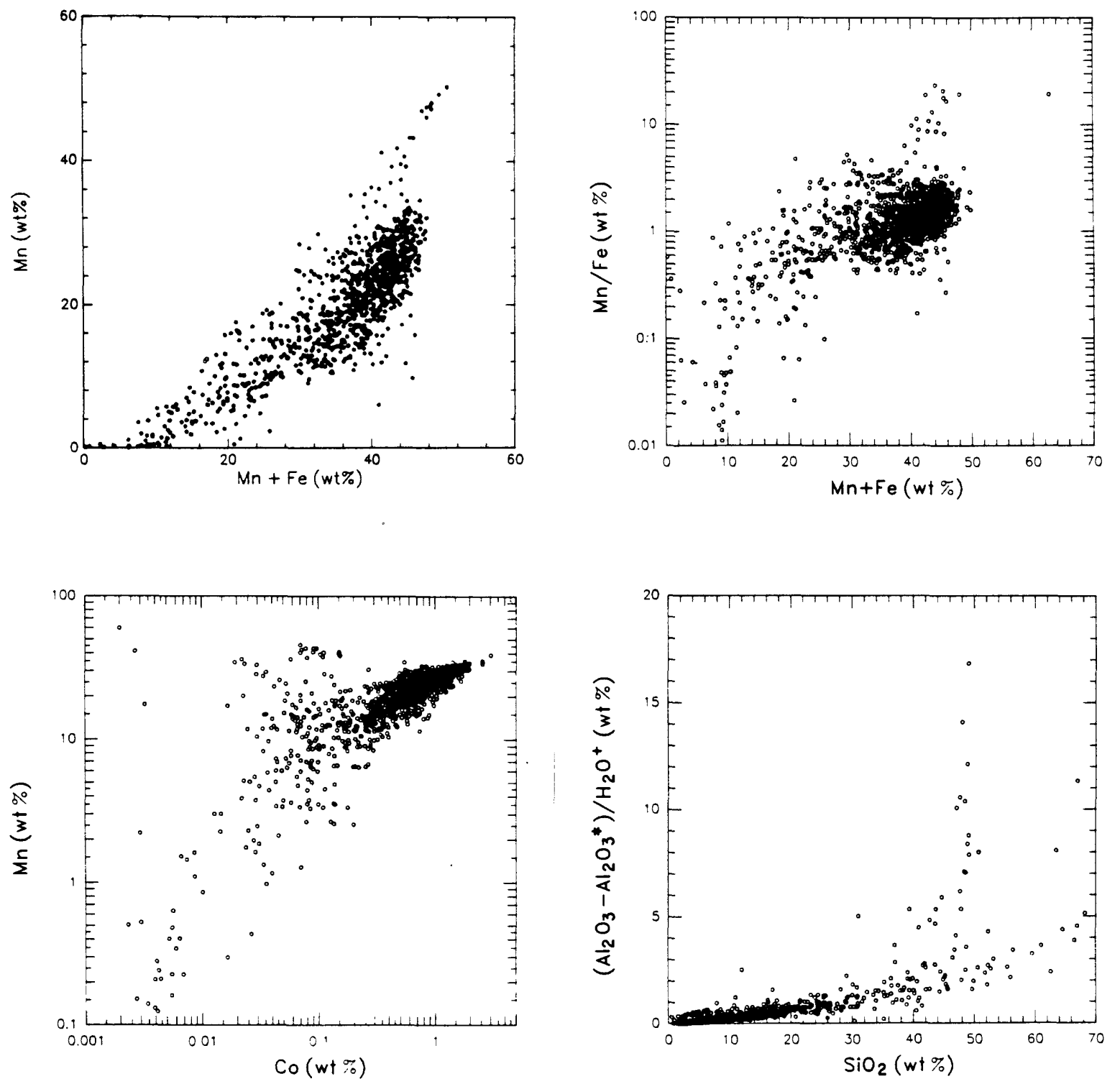

Figure IX-7 Plots of $\mathrm{Mn}$ vs., $\mathrm{Mn}+\mathrm{Fe}, \mathrm{Mn} / \mathrm{Fe}$ vs. $\mathrm{Mn}+\mathrm{Fe}, \mathrm{Mn}$ vs. Co, $\left(\mathrm{Al}_{2} \mathrm{O}_{3}-\mathrm{Al}_{2} \mathrm{O}_{3}{ }^{*}\right) / \mathrm{H}_{2} \mathrm{O}+$ vs. $\mathrm{SiO}_{2}$ for complete USGS data set. 

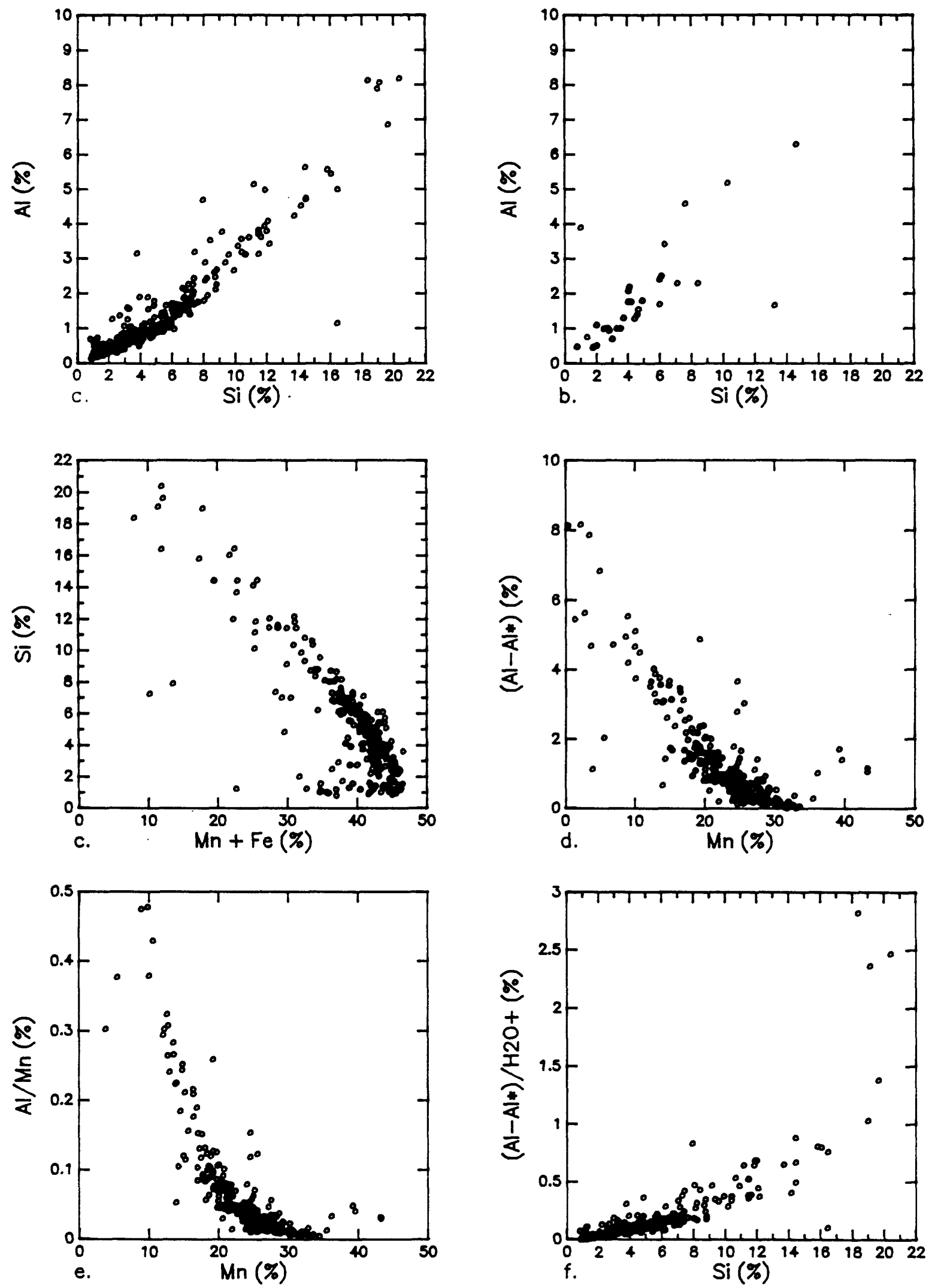

Figure IX-8 Plot of Al vs. Si (USGS), Al vs. Si (SNDB), Si vs. Mn/Fe, $\left(\mathrm{Al}-\mathrm{Al}^{*}\right.$ ) vs. Mn, Al/Mn vs. Mn, (Al-Al*)/ $/ \mathrm{H}_{2} \mathrm{O}+$ vs. Si. 

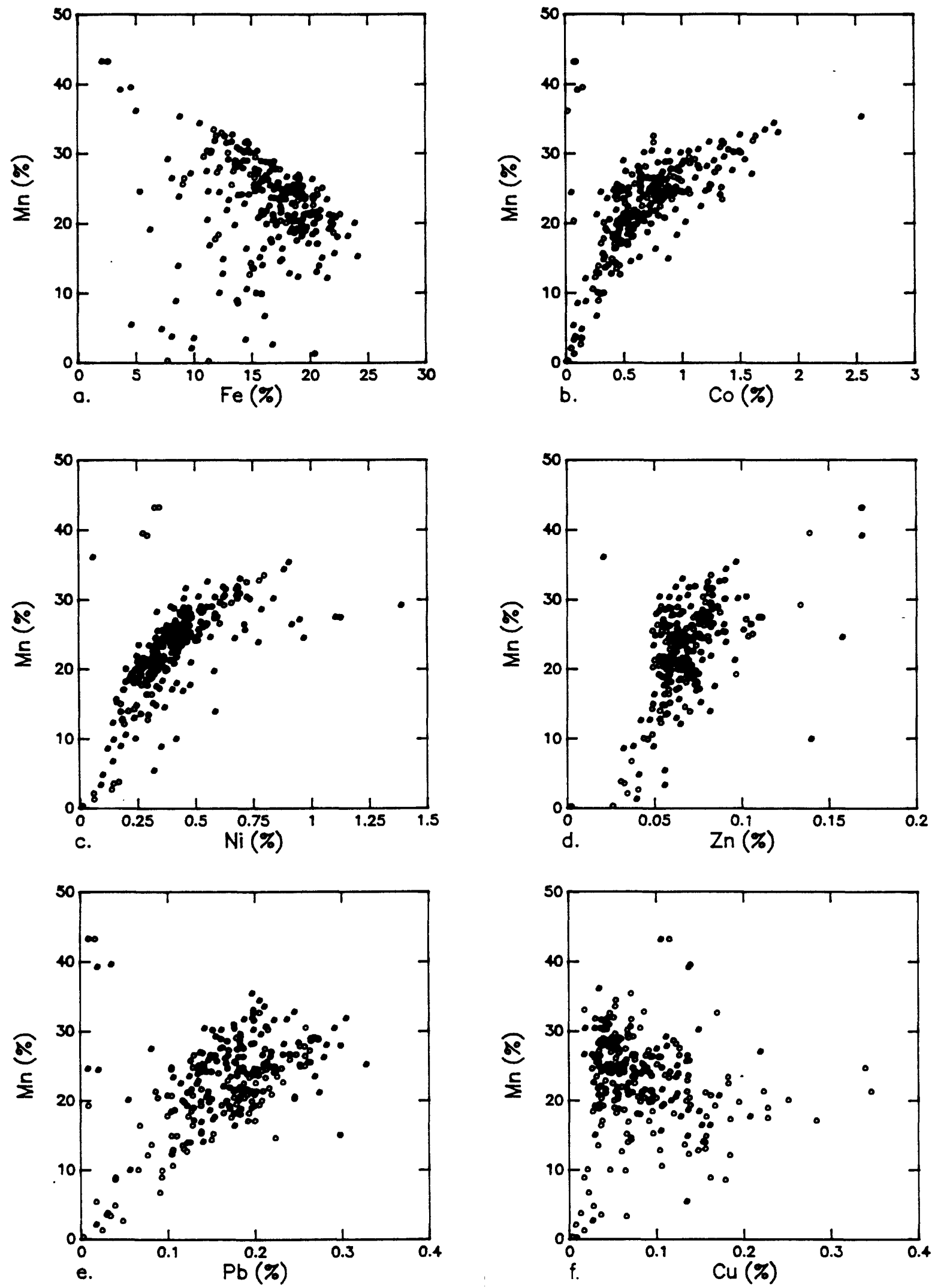

Figure IX-9 Plot of Mn vs. Fe, Co, Ni, Zn, Pb, Cu for Mid-Pacific Ocean. 

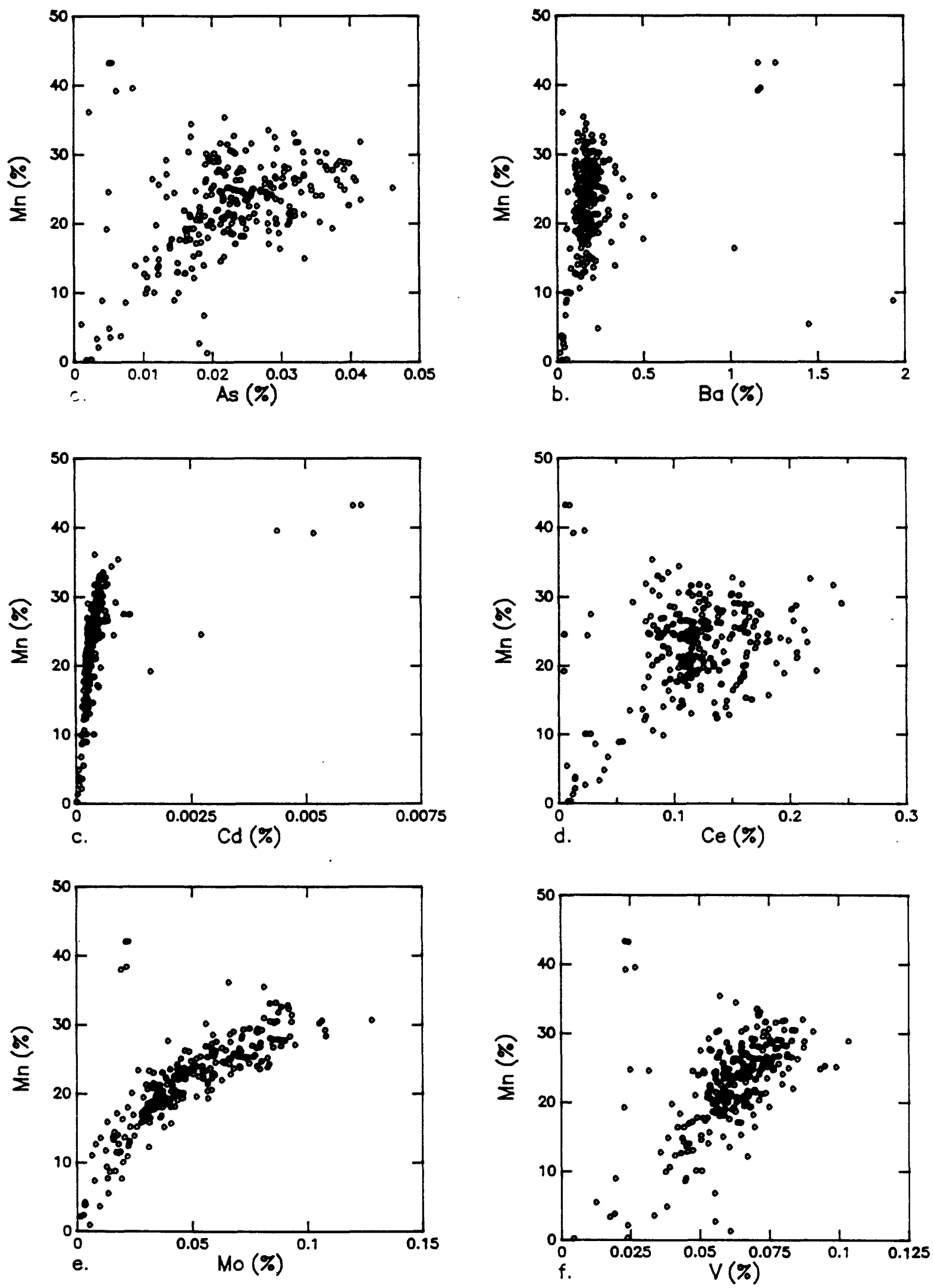

FigurelX-10 Plot of Mn vs. As, Ba, Cd, Ce, Mo, V, for Mid-Pacific Ocean. 

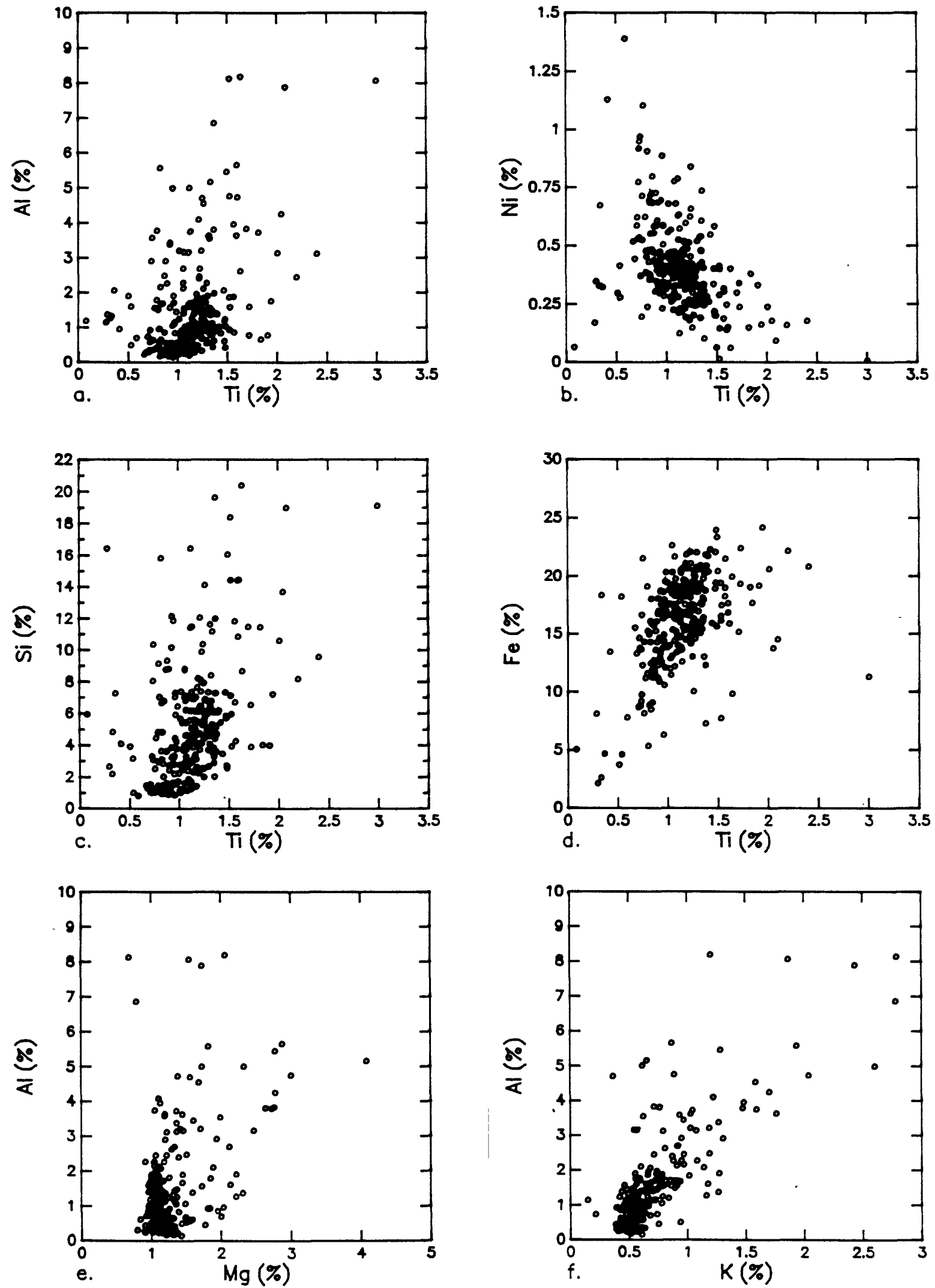

Figure IX-1 I Plot Al, Ni, Si, Fe vs. Ti, Al vs. Mg, Al vs. K for Mid-Pacific Ocean. 

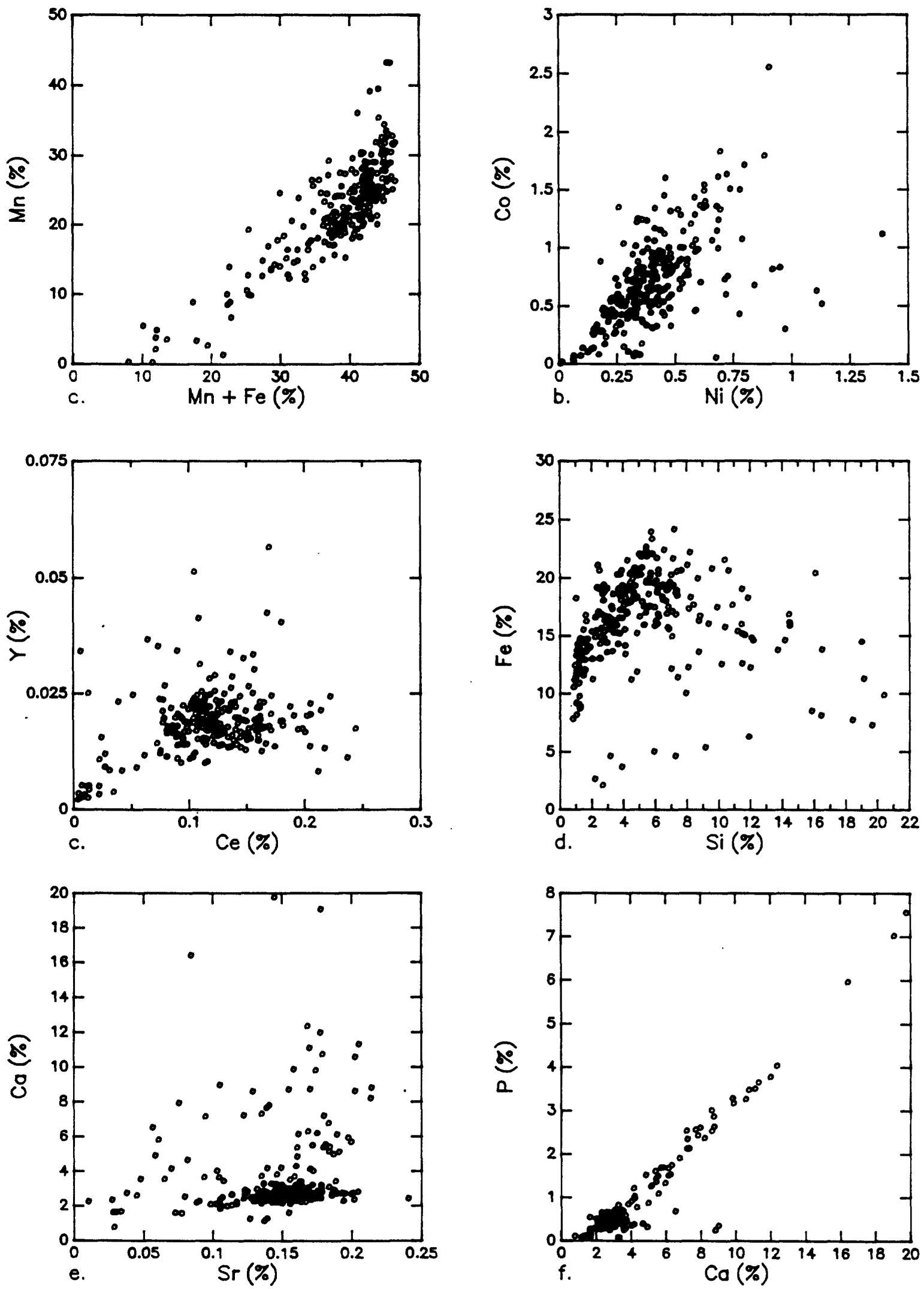

Figure IX-12 Plot of Mn vs. Mn +Fe, Co vs. Ni, Y vs. Ce, Fe vs. Si, Ca vs. Sr, $P$ vs. Ca for Mid-Pacific Ocean. 

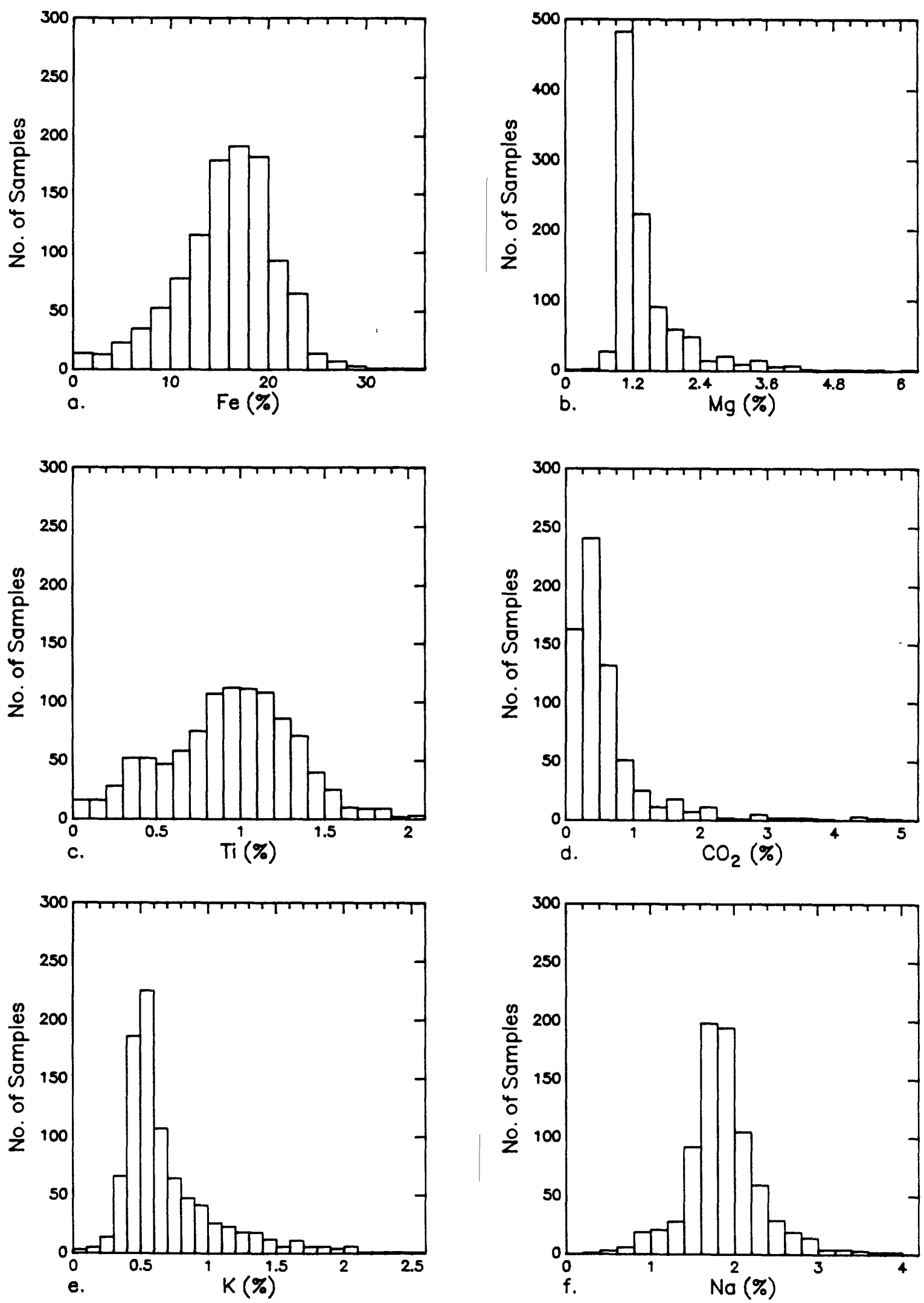

Figure IX-13 Histograms of $\mathrm{Fe}, \mathrm{Mg}, \mathrm{Ti}, \mathrm{CO}_{2}, \mathrm{~K}, \mathrm{Na}$. 

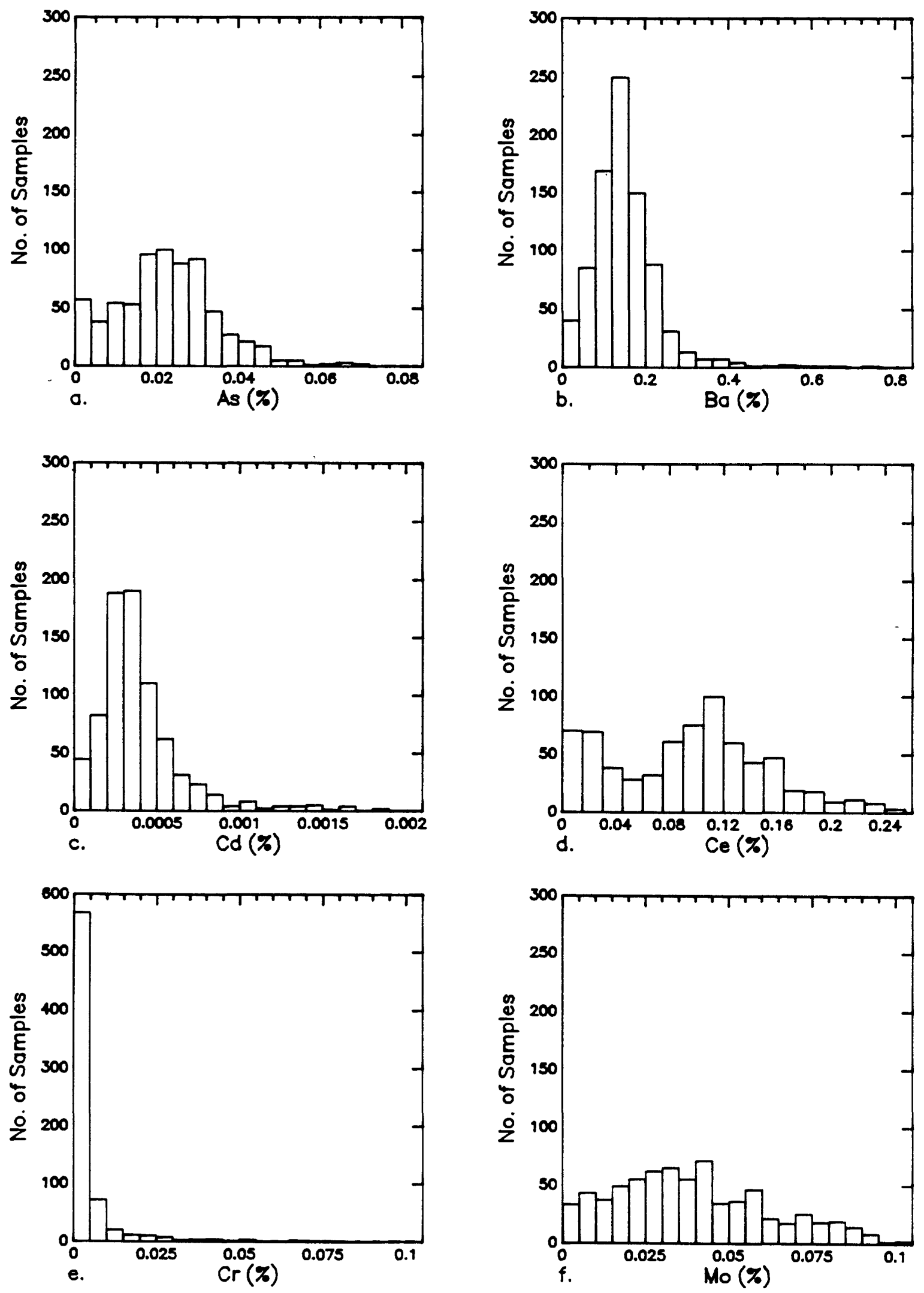

Figure IX-14 Histograms of As, Ba, Cd, $\mathrm{Ce}, \mathrm{Cr}, \mathrm{Mo}$. 

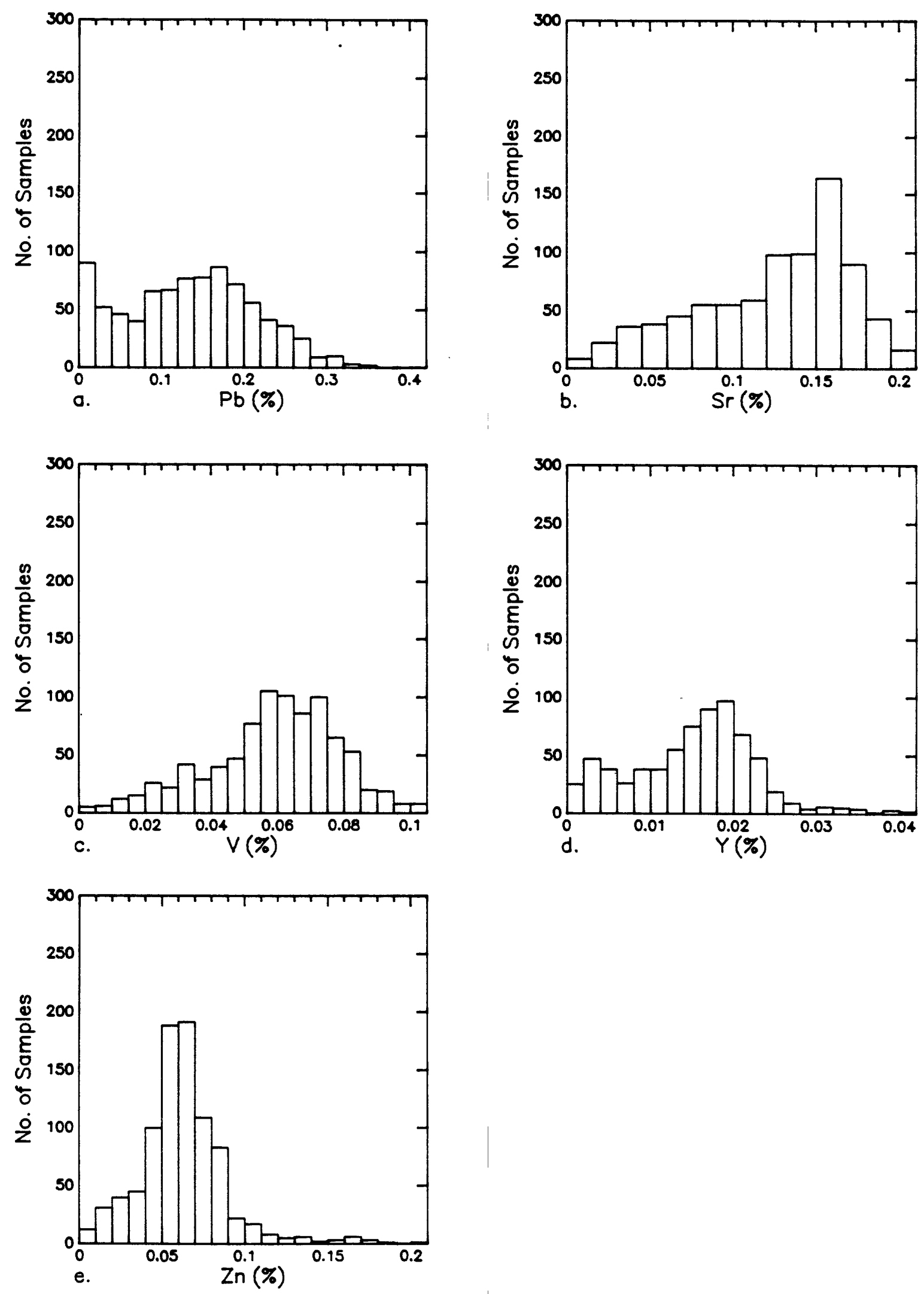

Figure IX-15 Histograms of Pb, Sr, V, Y, Zn. 

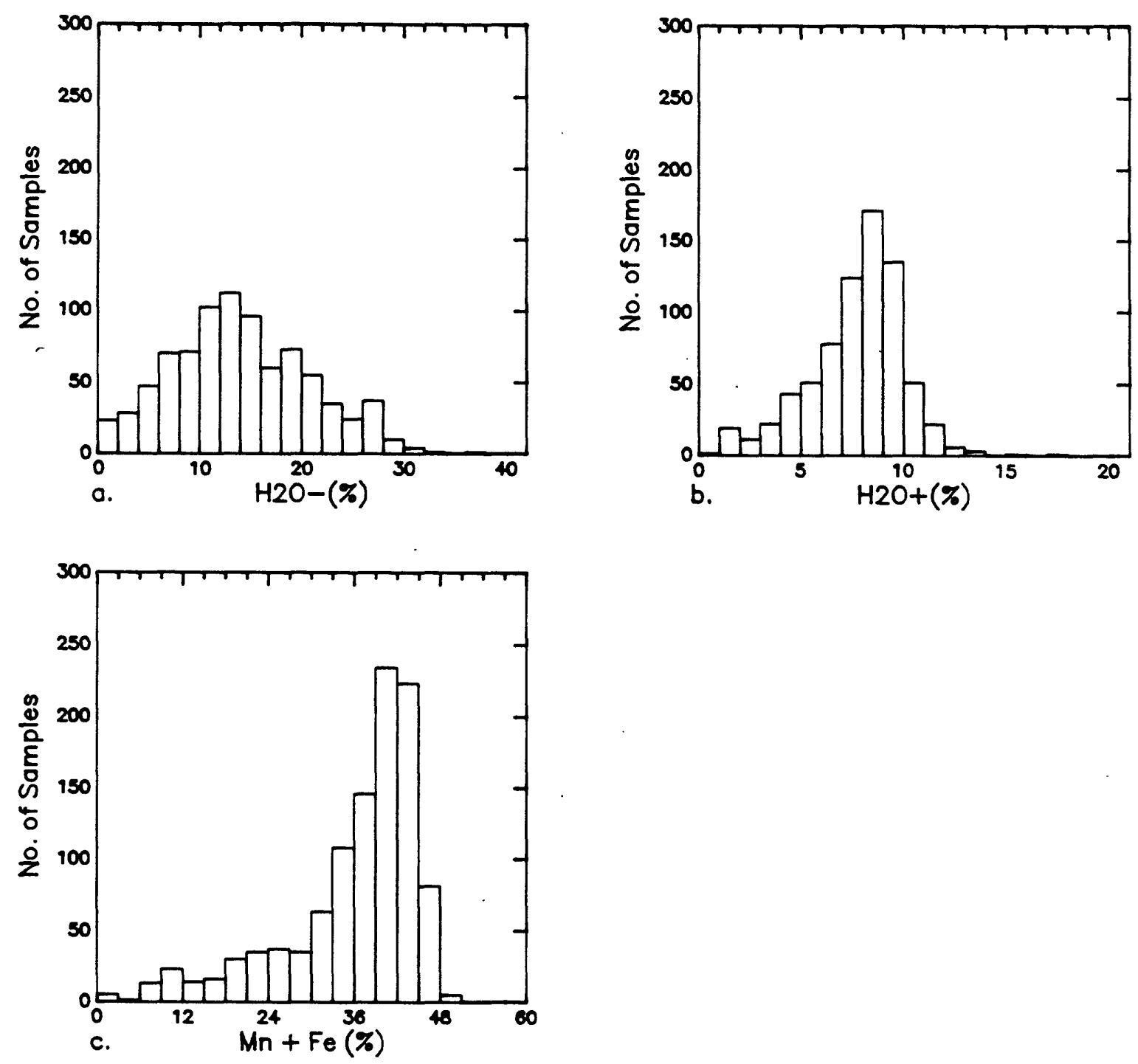

Figure IX-16 Histograms of $\mathrm{H}_{2} \mathrm{O}, \mathrm{H}_{2} \mathrm{O}+, \mathrm{Mn}+\mathrm{Fe}$. 

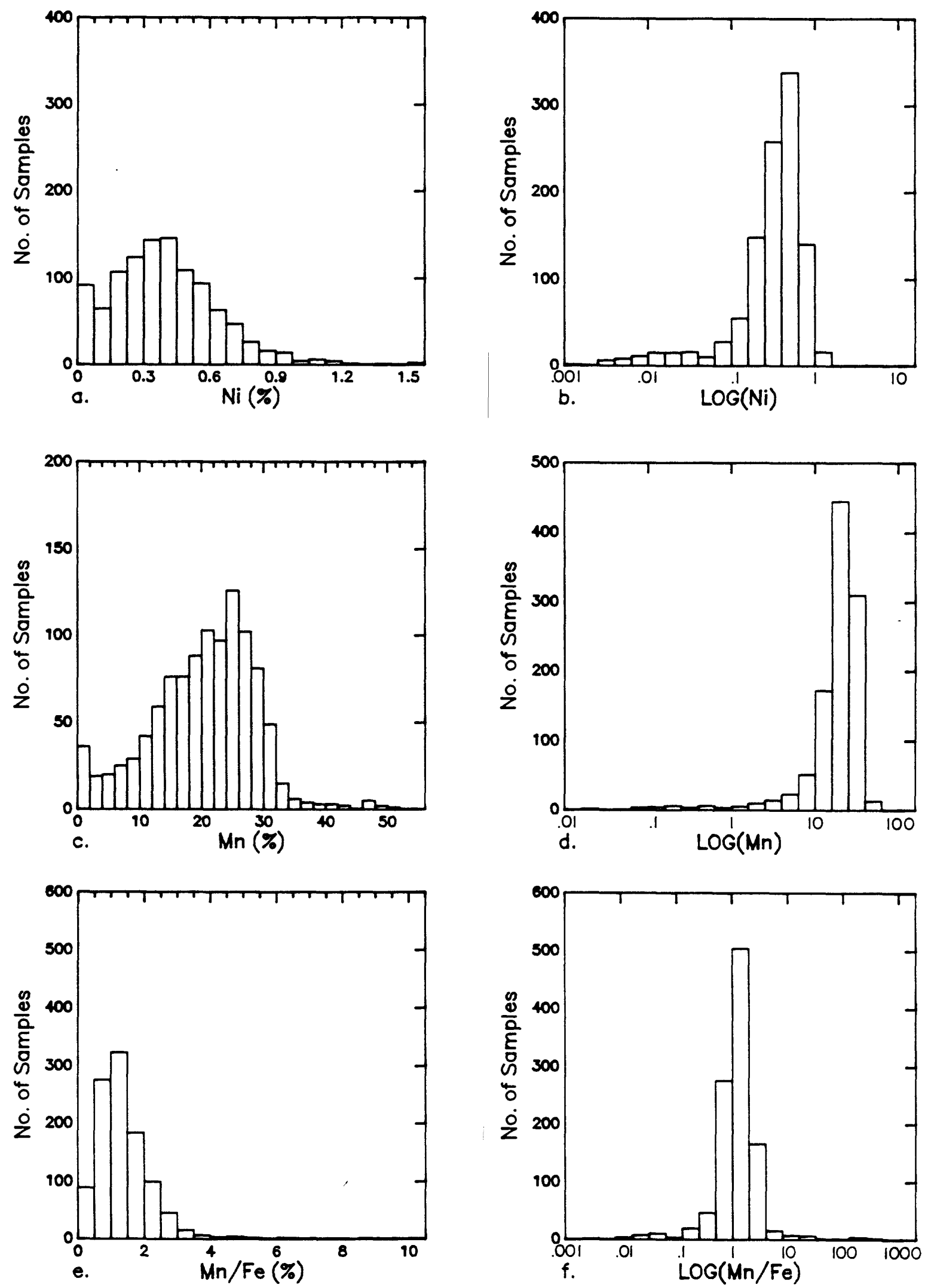

Figure IX-17 Histograms of Ni, log ( Ni), Mn, log (Mn), Mn/ Fe, log ( $\mathrm{Mn} / \mathrm{Fe})$. 

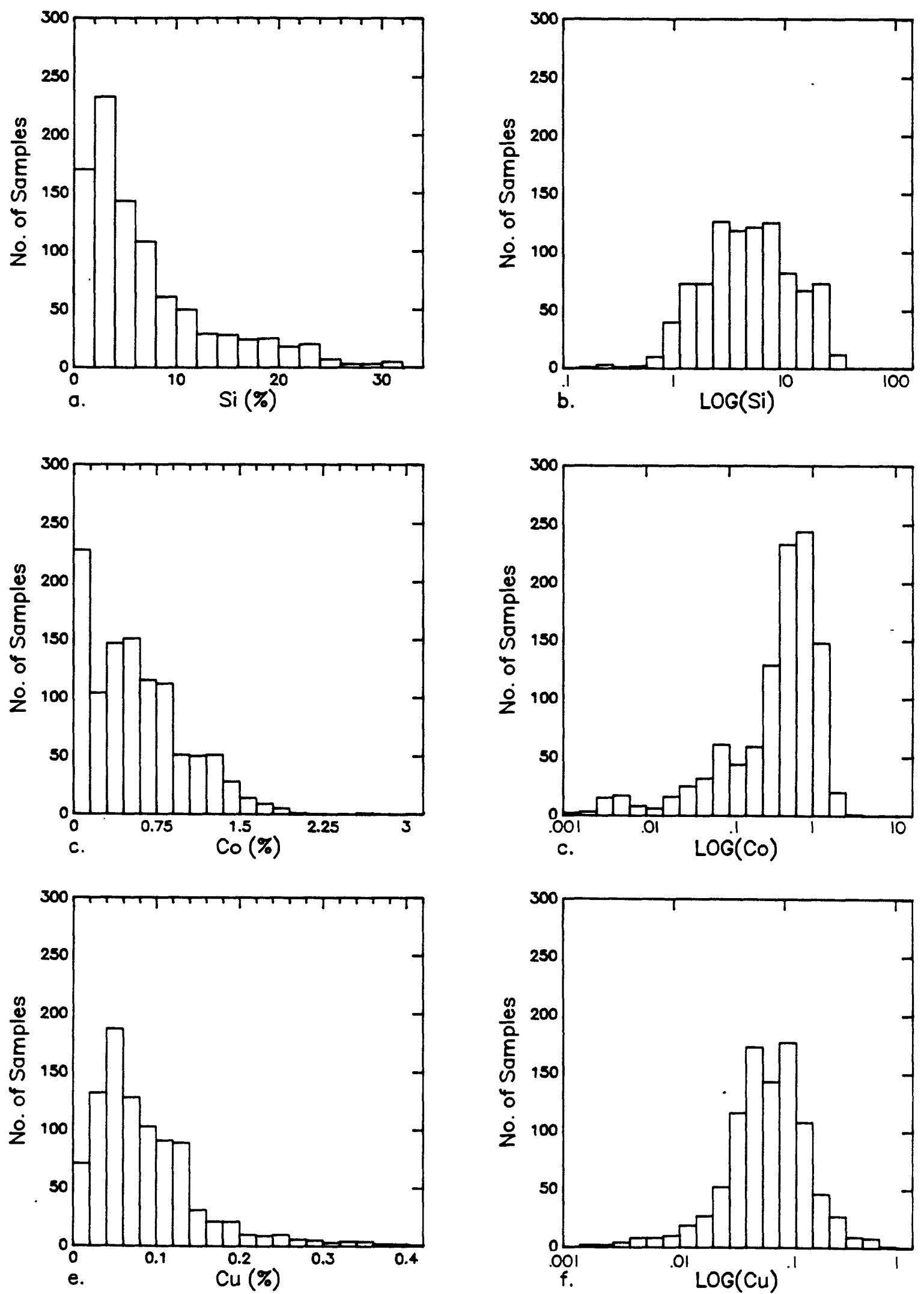

Figure IX-18 Histograms of Si, $\log (\mathrm{Si}), \mathrm{Co}, \log (\mathrm{Co}), \mathrm{Cu}, \log$ (Cu). 

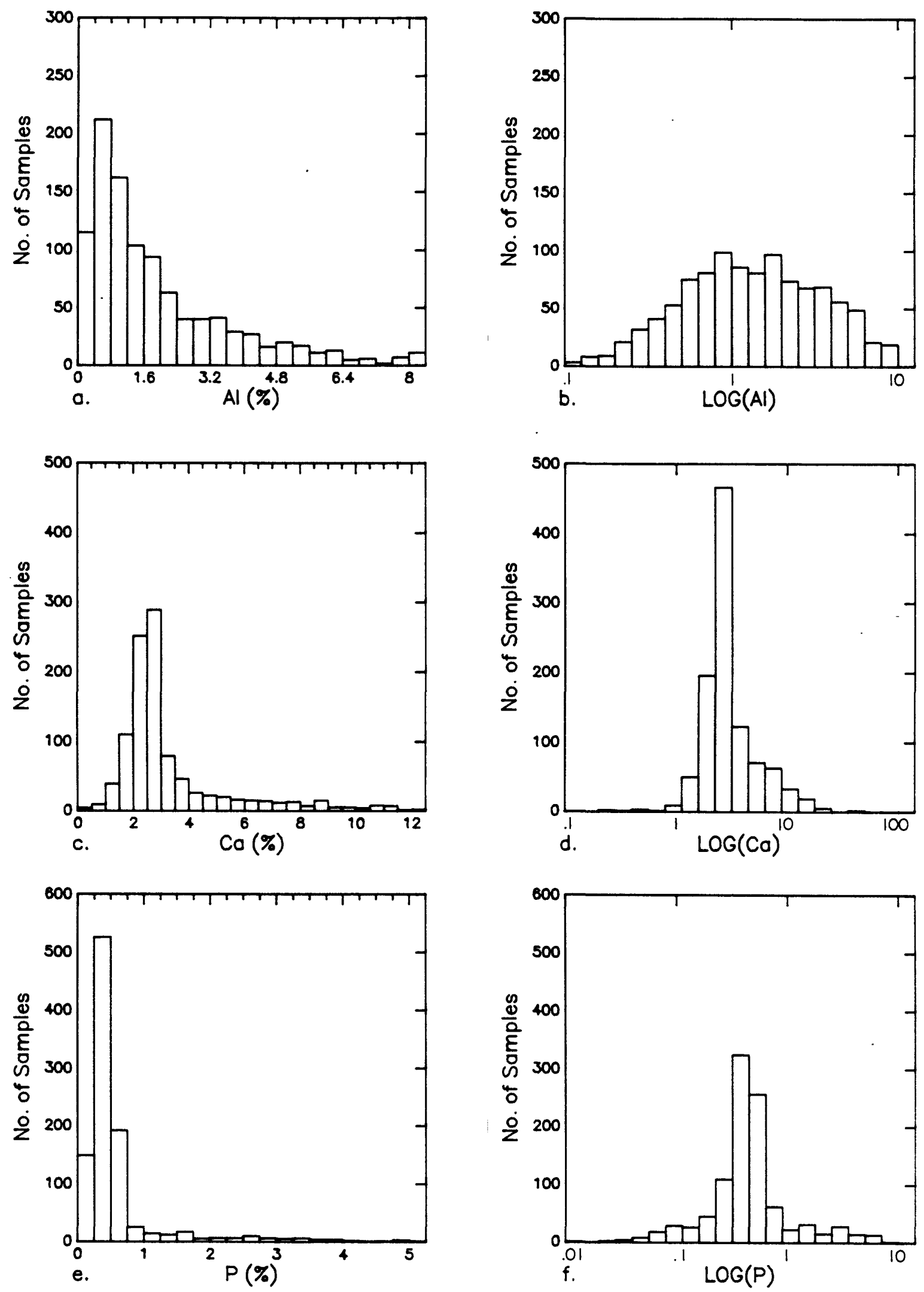

Figure IX-19 Histograms of Al, $\log (A \mid), C a, \log (C a), P, \log$ (P). 
values even in a lognormal plot, which is attributed largely to hydrothermally affected samples.

\section{Correlation coefficients}

Correlation matrices for the Mid-Pacific, southern Line Islands, Marshall Islands, Indian Ocean, Atlantic Ocean and Pacific Ocean areas are presented in Tables IX-5 to IX-9, using the Pearson-Product-Moment statistic. In general, the principal correlation groups include the Mn oxides, silicates, phosphates, and $\mathrm{Fe}$ - depth-latitude. We recognize that dilution by silicates, phosphates, or other substrate materials will tend to create false correlations among elements within the oxide group.

One method of clarifying the influence of dilutants on correlation matrices is to compare correlation coefficients for the raw data sets and exclusion sets for major oceans and ocean areas. A summary table of some of the principal associations for the Mid-Pacific area is given in Table IX-10, including a small, homogeneous data set from the top $5 \mathrm{~mm}$ of crusts from Necker Ridge, Horizon Guyot, and S.P. Lee Guyot (data reported in Hein and others, 1985).

Four principal geochemical associations are examined in Table IX-10: Depth, Mn oxides, silicates (divided into two subcategories $\mathrm{Si}$ and $\mathrm{Al}$ ), and $\mathrm{Ca}$. Table IX10 shows that in most cases the exclusion data set has stronger statistical affinities than the unmodified sets. The correlation levels increase with decreasing sample size and reduction of contaminants and extraneous phases. Exceptions occur for hygroscopic moisture $\mathrm{H}_{2} \mathrm{O}^{-}: \mathrm{Mn}, \mathrm{CO}_{2}: \mathrm{Ca}$ and $\mathrm{K}: \mathrm{Al}$. The manganese phases have great surface area, and therefore greater tendency toward higher hygroscopic moisture. This relationship, though at a low level, is more pronounced in the most heterogeneous data set. Likewise, correlation between elements associated with carbonates and aluminosilicates is greater in this set. Dilution by silicates, phosphates, or other substrate materials that create outliers not only tend to create false correlations among elements within the oxide group. False correlations may also be induced among constituents making up dilutants; i.e. a group of crust samples contaminated with phosphates and silicates may tend to show false correlations between elements in phosphates and silicates, because both phases may change in concentration in reverse proportion to the amount of "dilution" by $\mathrm{Mn}-\mathrm{Fe}$ oxide phase that may be present. One means of minimizing these problems is referred to below.

The Pearson-Product-Moment ( $P-P$ ) correlation coefficient presumes a normally distributed population even though it has been and remains widely used in geochemical studies on which non-normally distributed populations are present. A correlation method that does not depend on frequency distribution is rank correlation $(S-R)$. A new program was developed in house to perform both the $P$ $P$ and the S-R correlations. The results are in Table IX-11 and IX-12 and used the smaller data set in the Mid Pacific area to be consistent with majority of the interelement plots. The numbers for the bottom matrix refer to number of sample points used for each correlation. The S-R data set displays much lower correlations for elements where a tail or a few outliers affect the Pearson Product Moment matrices. For example, the P-P correlation for $\mathrm{Ca}: \mathrm{P}$ is .95, whereas the $S-R$ correlation yields only .59. The $P-P$ index is strongly influenced by a few samples which have high apatite content. On the other hand, the correlations $\mathrm{Mn}: \mathrm{Co}, \mathrm{Mn}: \mathrm{Ni}, \mathrm{Co}: \mathrm{Ni}, \mathrm{Pb}: \mathrm{Co}, \mathrm{Mo}: \mathrm{Co}$, and As:Co are all higher for the $S R$ procedure. 


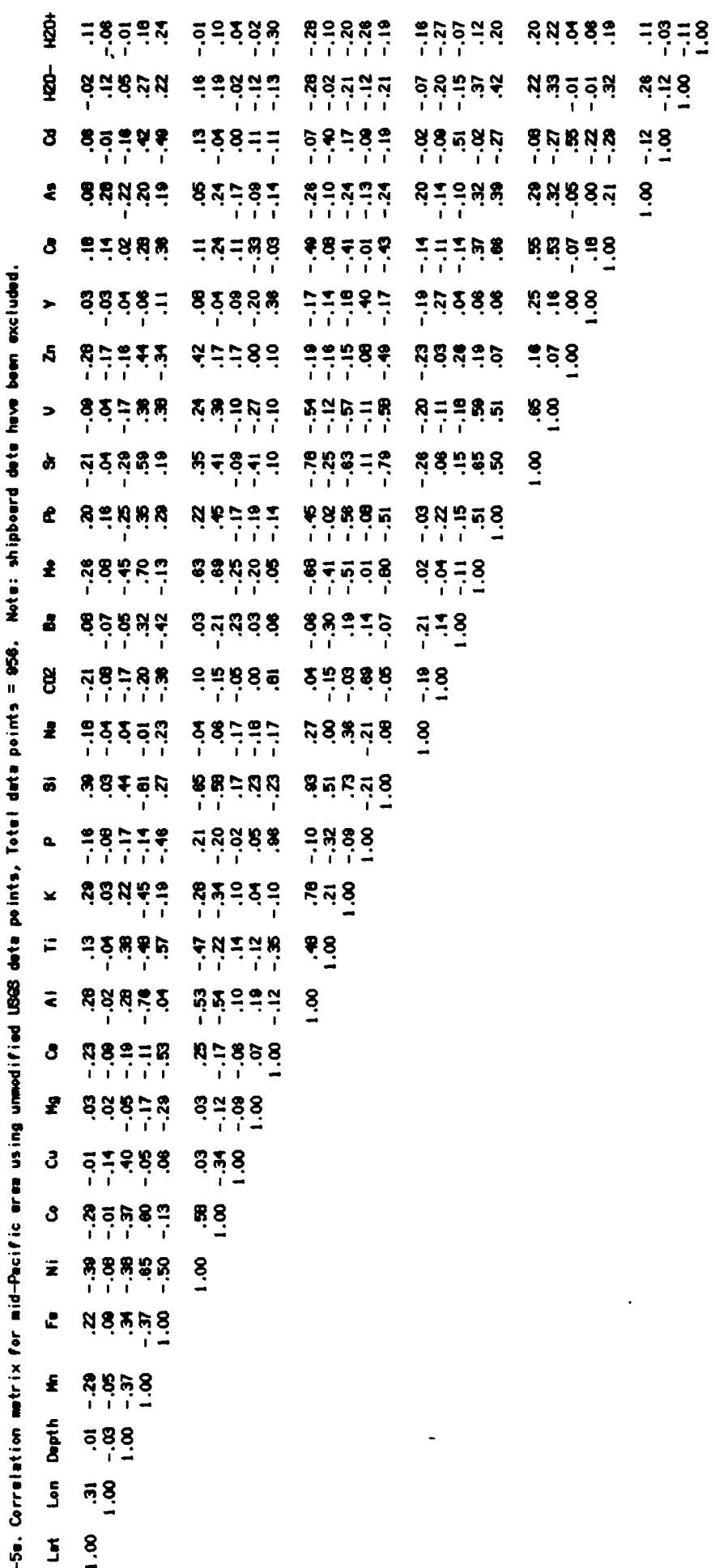

萑 58

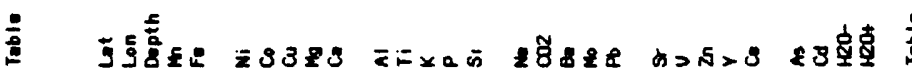

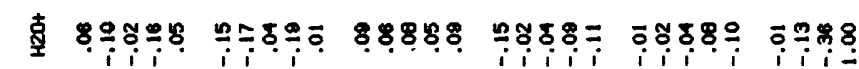

\$ิ

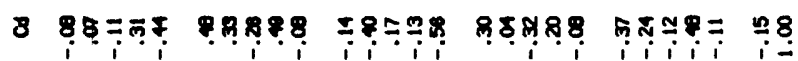

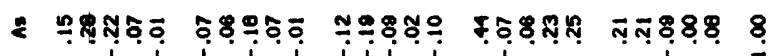

8 พ

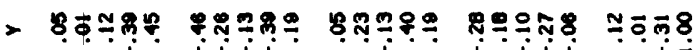

ง

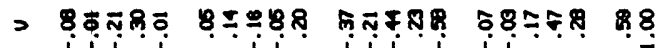

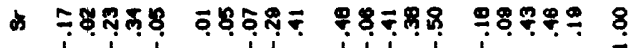

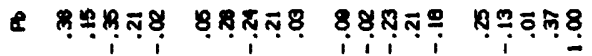

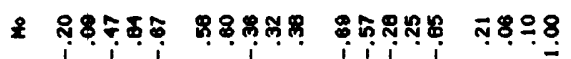

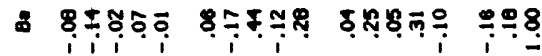

8

กิ่

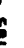

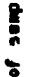

- a 5 OQNA

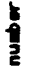

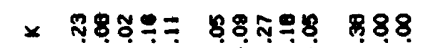

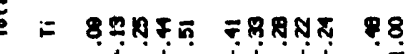

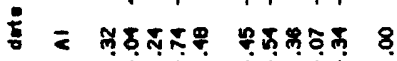

ร

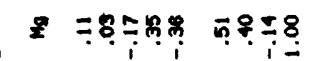

3 ำำำำ

s $98 \% 28 \$ 8$

- भ०की 8

i.

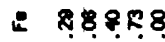

5 ₹ $78 \% 8$

音 8

ต के

58

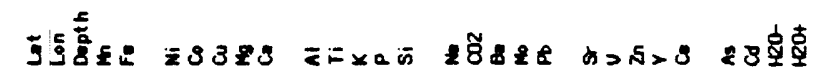




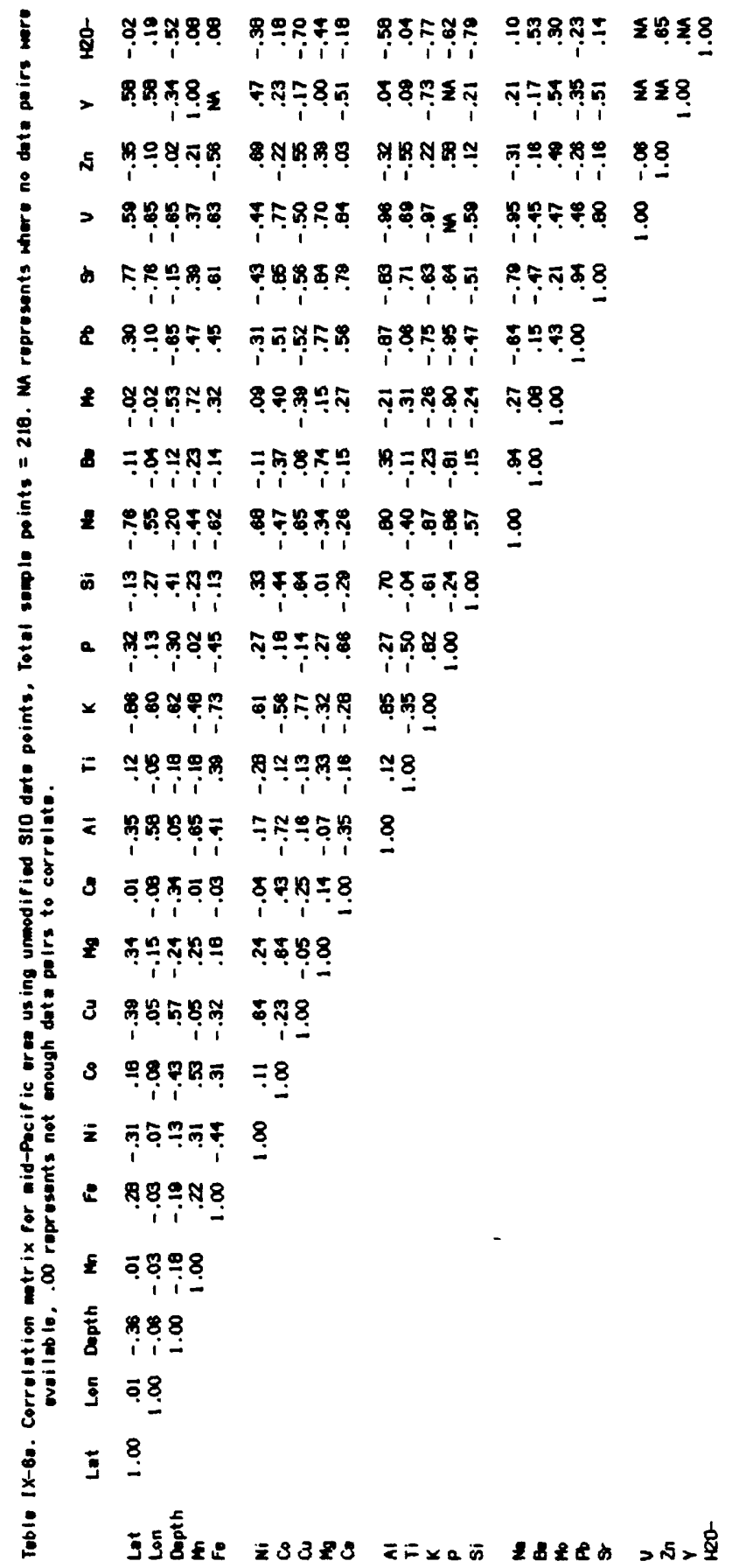

卞

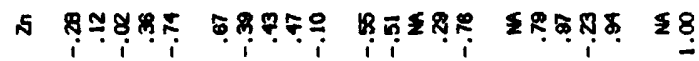

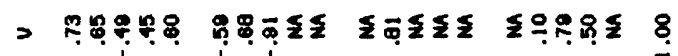

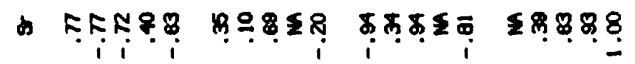

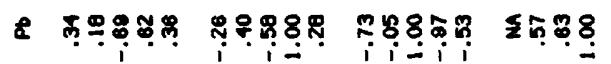

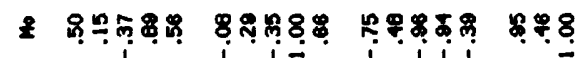

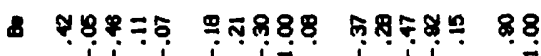

28 8ㅜำ

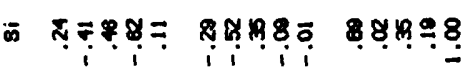

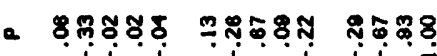

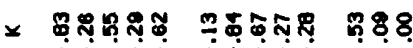

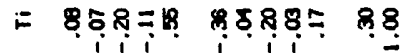

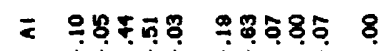

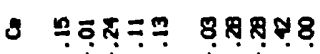

z

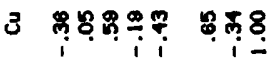

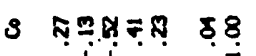

₹ จุษกำกุด

- หุ8 898

5 ำㅇำ

है

58

58

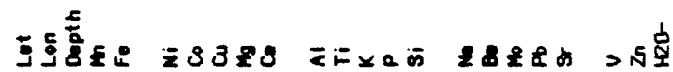




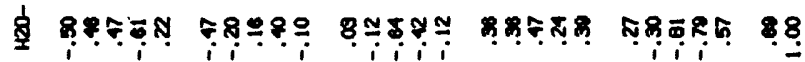

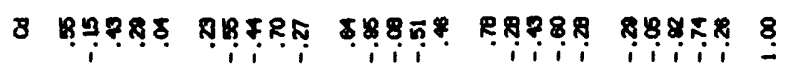

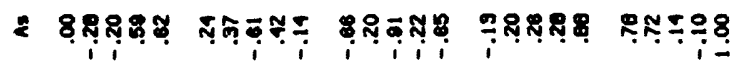

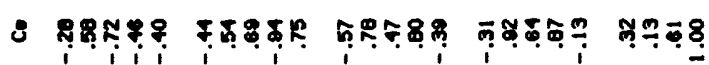

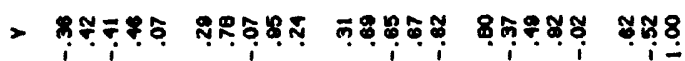

5 588

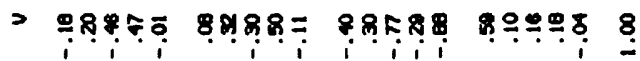

*

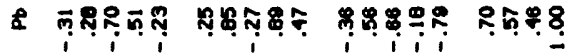

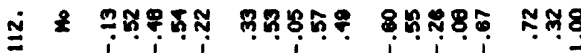

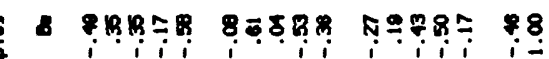

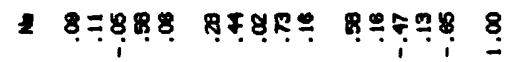

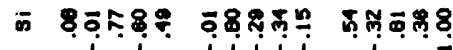

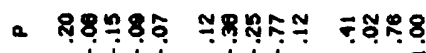

× หุดุ

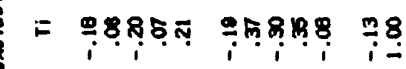

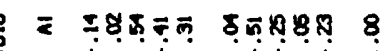

8 8

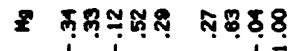

उ

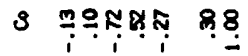

$=8 \div 8 \div 8$

$\therefore 898 \div$

E ตฺุุ

ะิำ

$\xi=8$

58

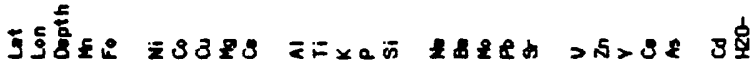

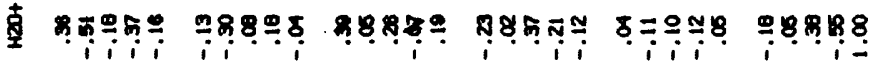

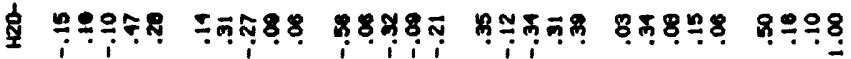

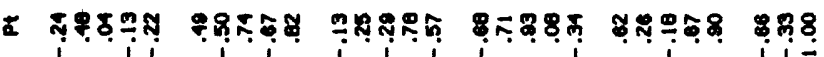

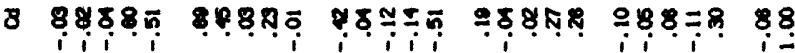

- 88ำ

-

> 车

ง

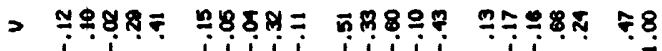

๙

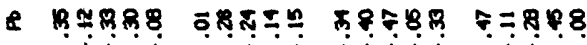

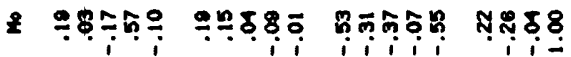

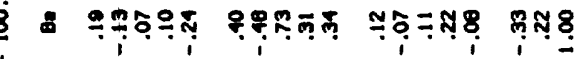

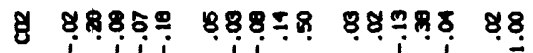

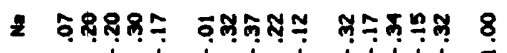

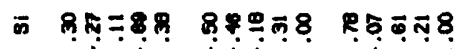

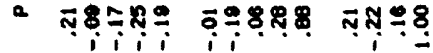

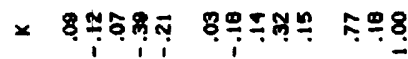

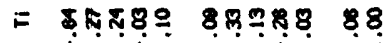

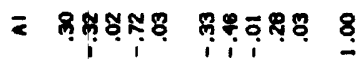

क 58

₹

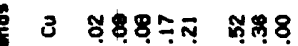

$3 \% 75888$

z 58989

- จุลุตุด 8

I 8988

喜

g. 8

宅

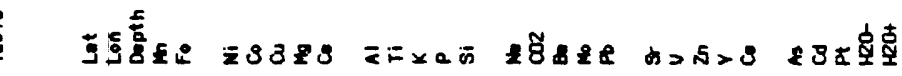




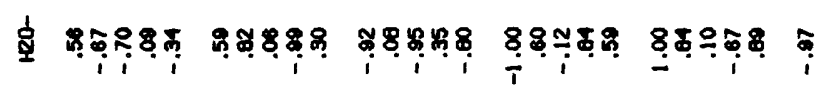

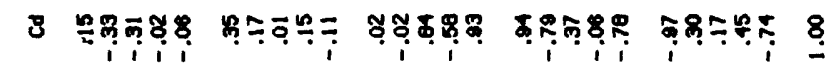

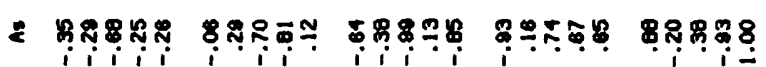

8

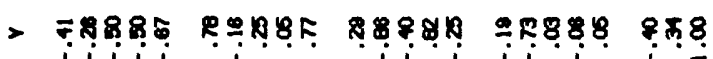

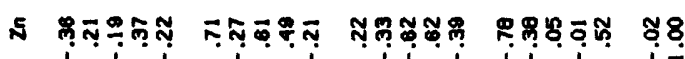

> ํํำํำ

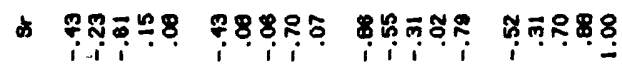

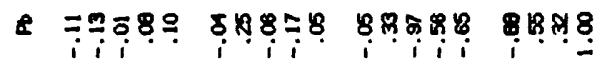

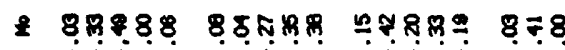

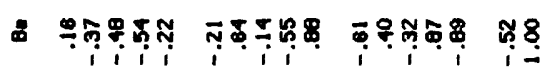

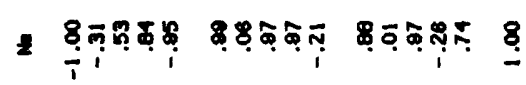

-

-

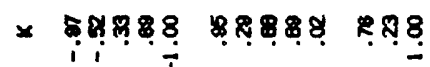

= ติำฺำ

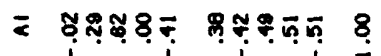

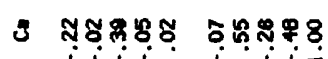

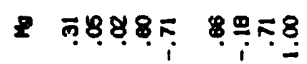

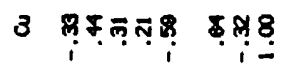

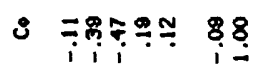

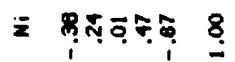

น กุ๊ตฺำ

f 워요

产 8

9

$5 \stackrel{8}{-}$

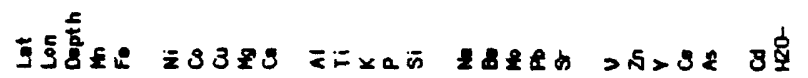

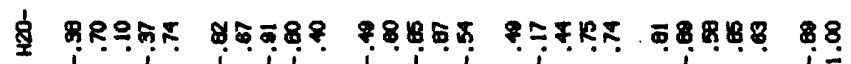

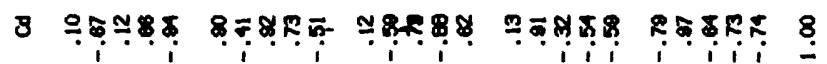

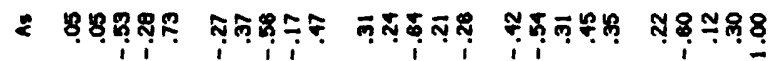

- ํํำ

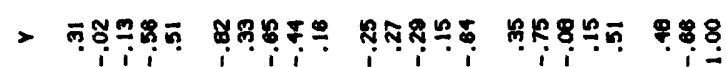

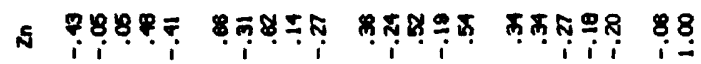

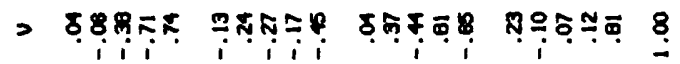

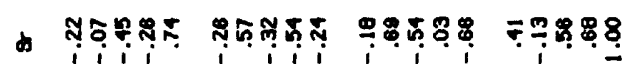

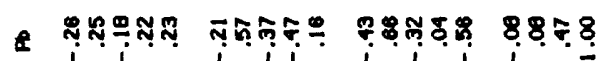

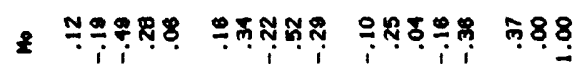

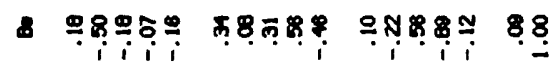

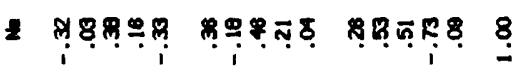

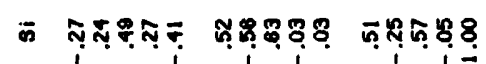

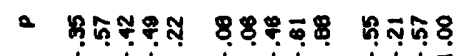

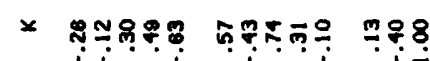

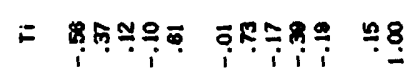

= ํㅜㅇํำ

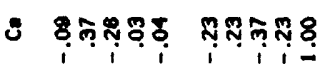

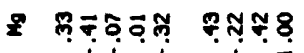

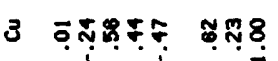

\&

z

-

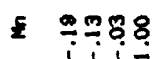

ह

5 p

I8

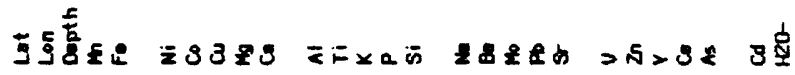




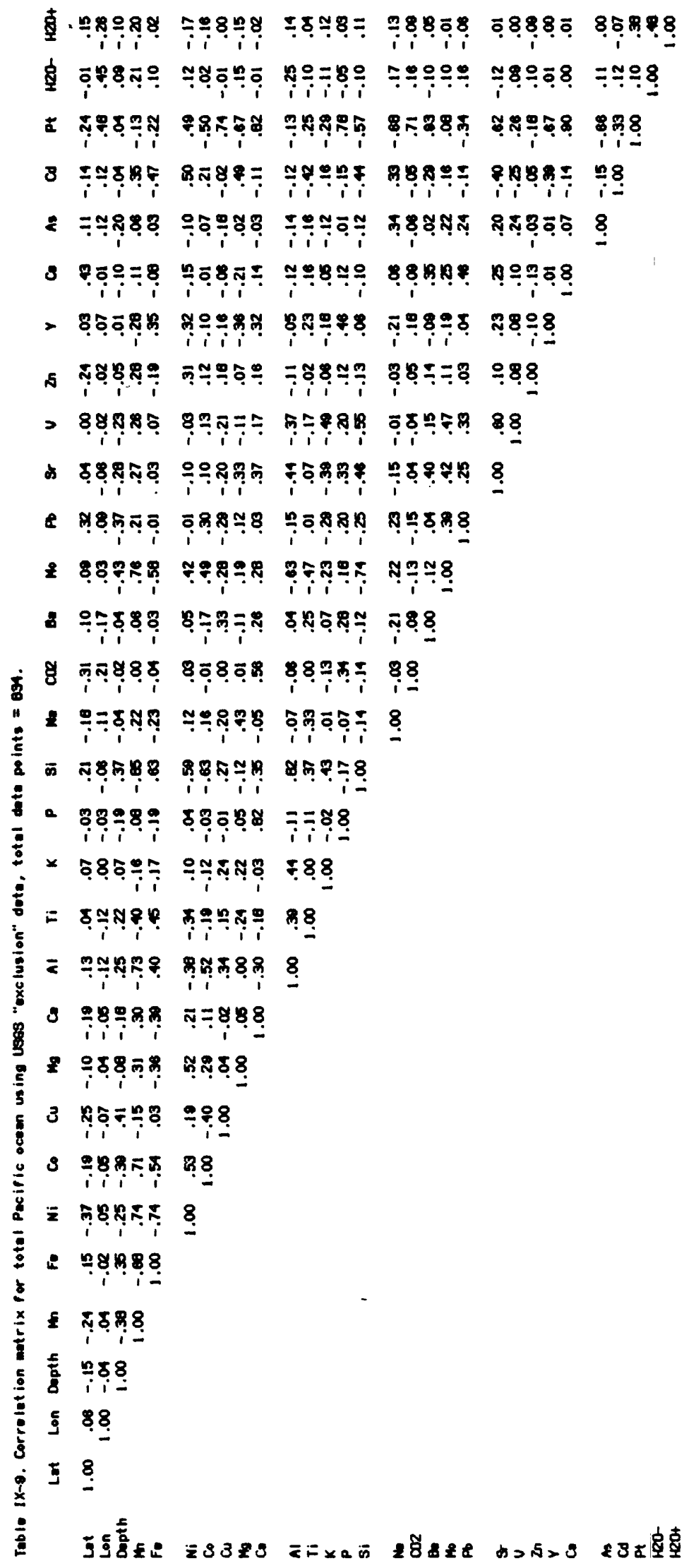




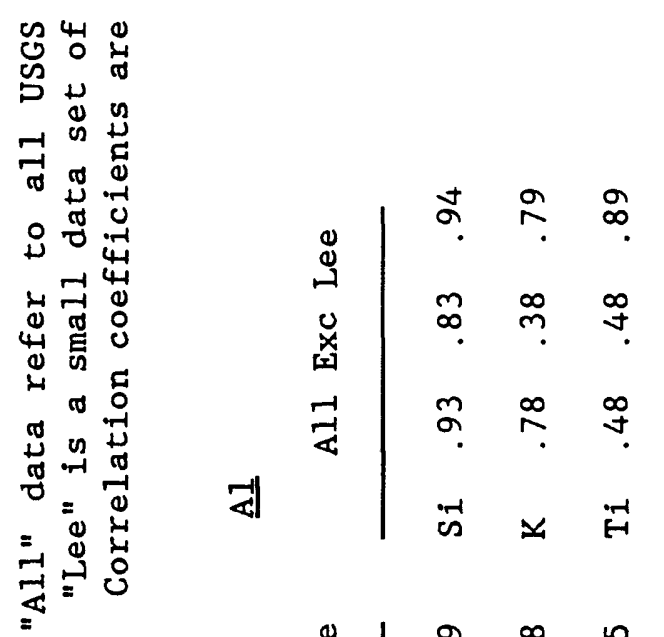

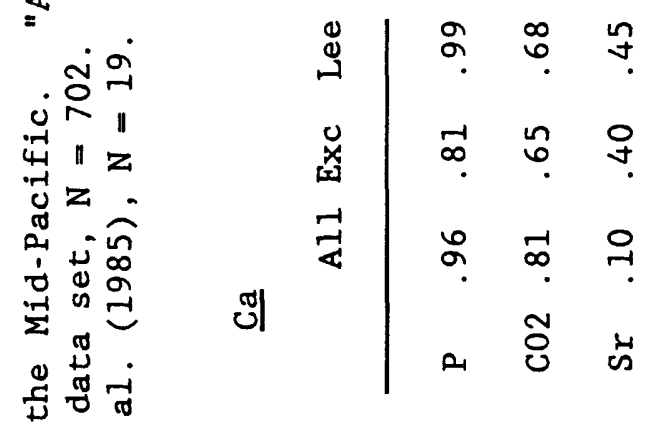

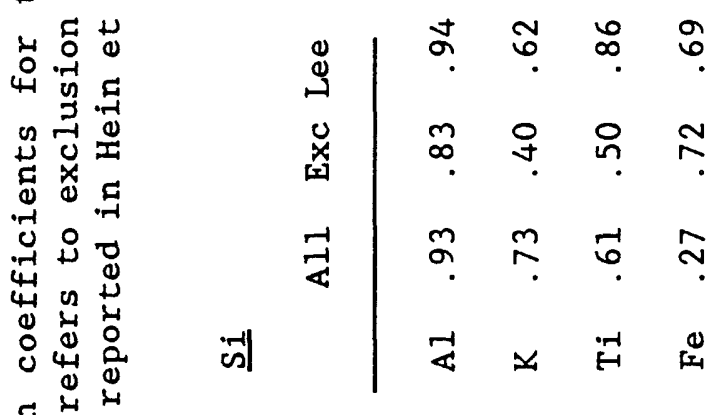

吾记

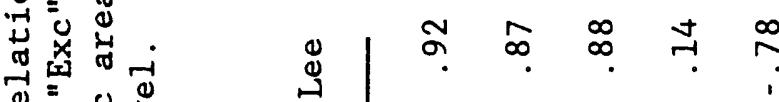

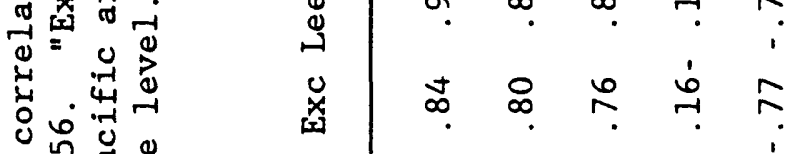

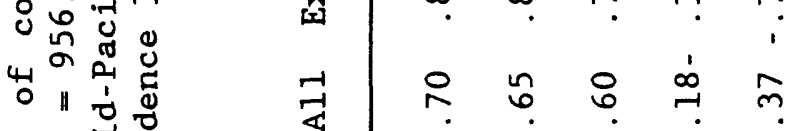

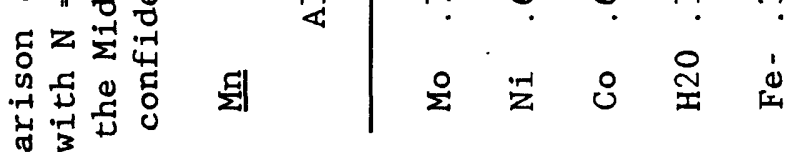

官 퉁

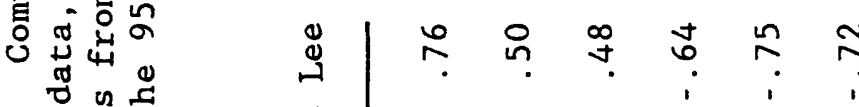

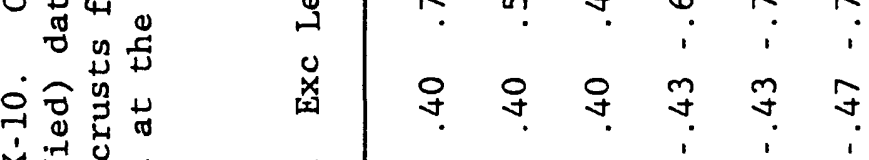

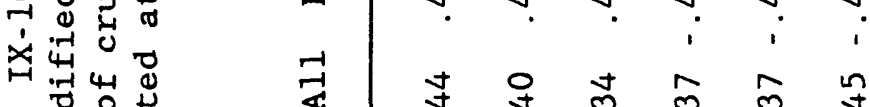

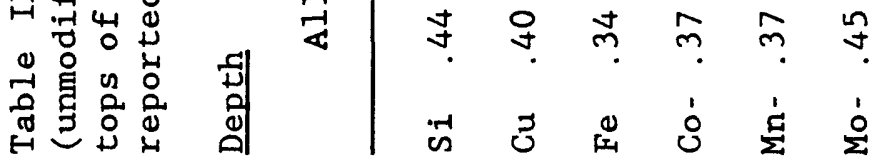




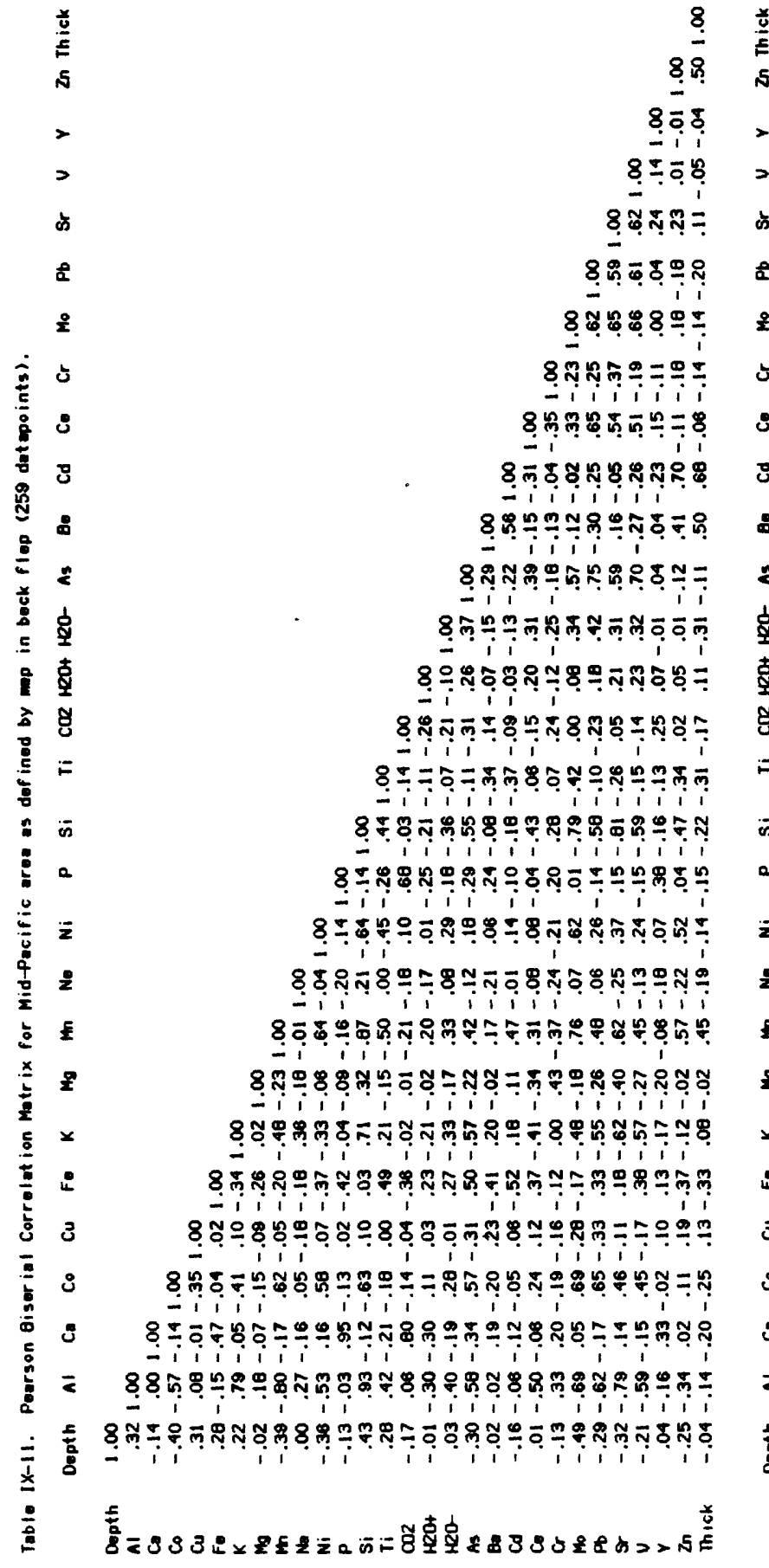

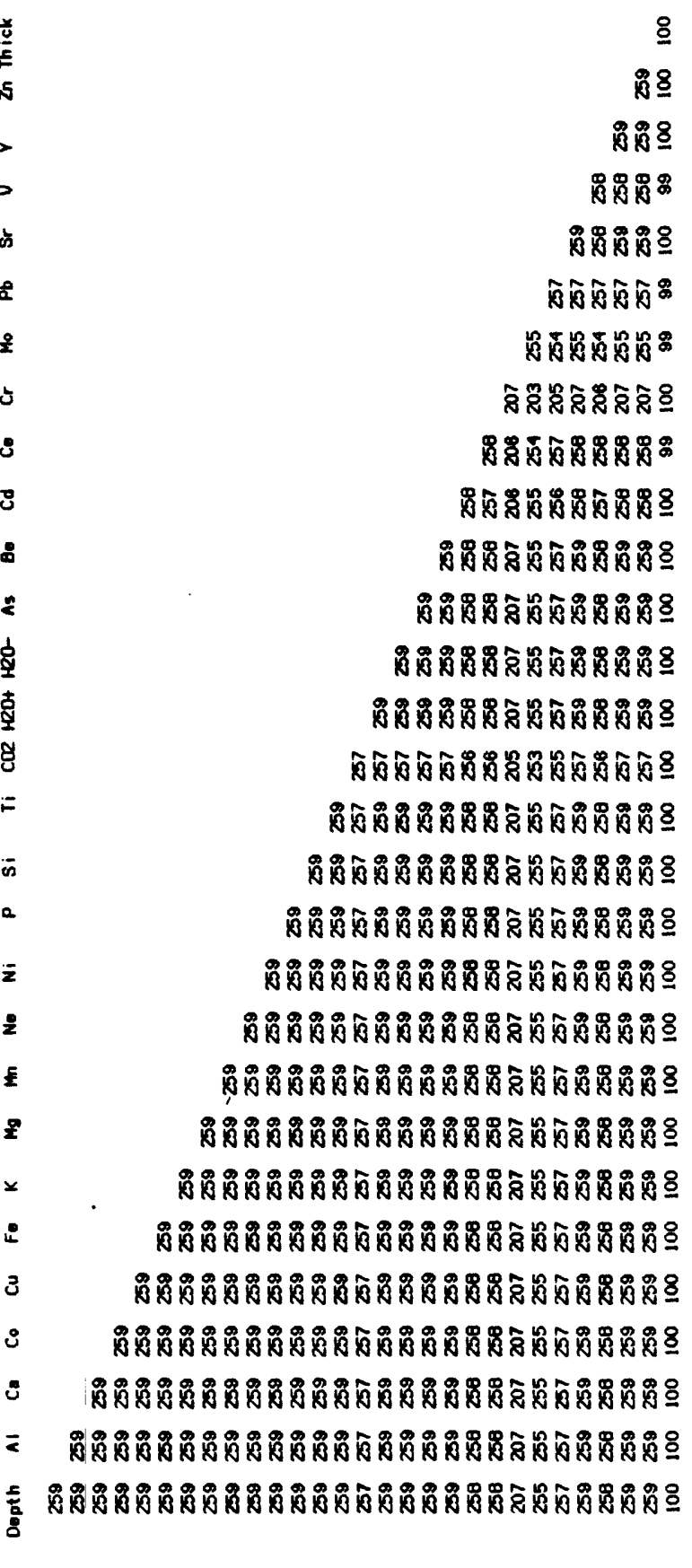

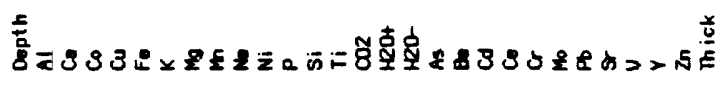




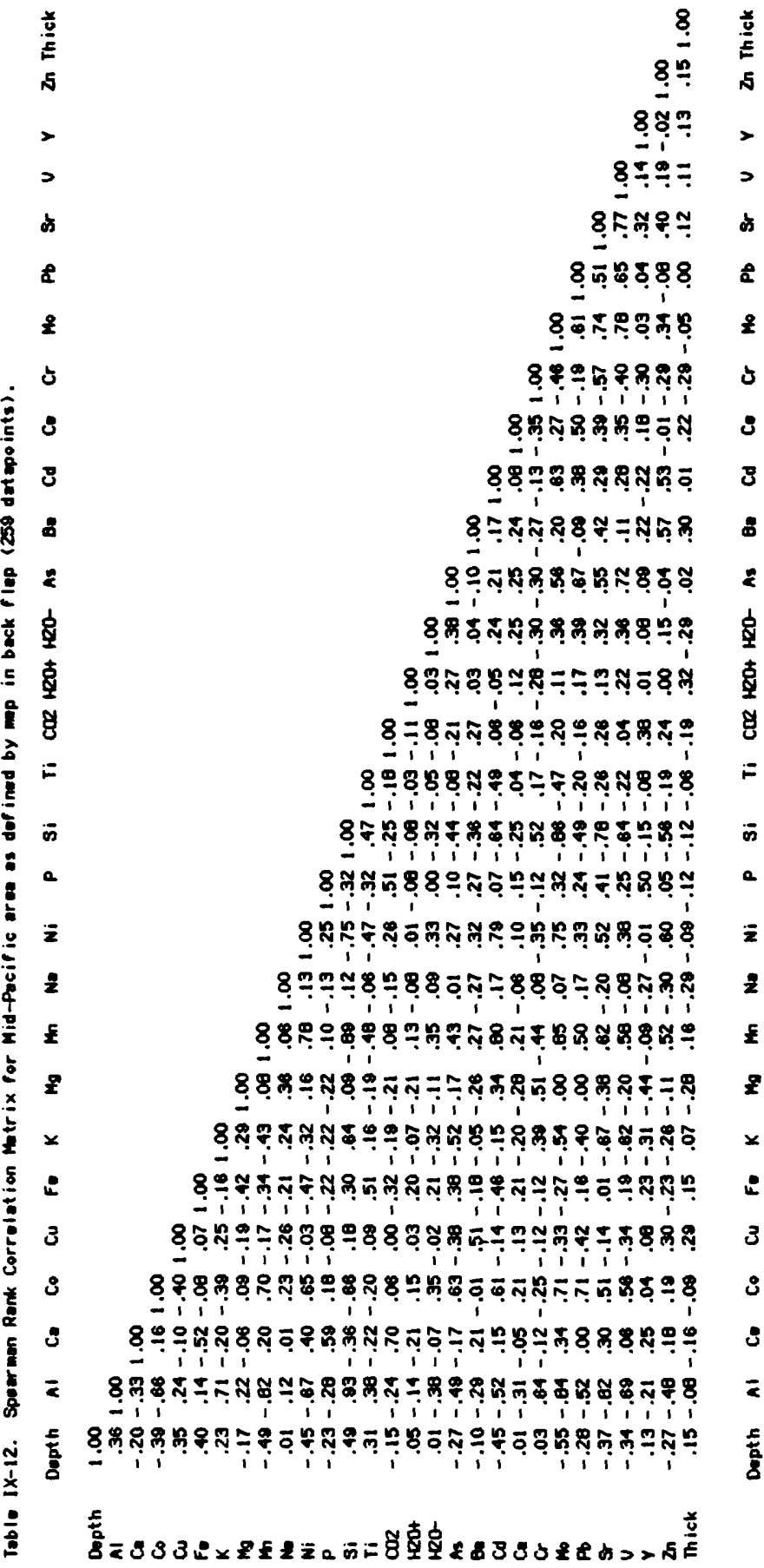

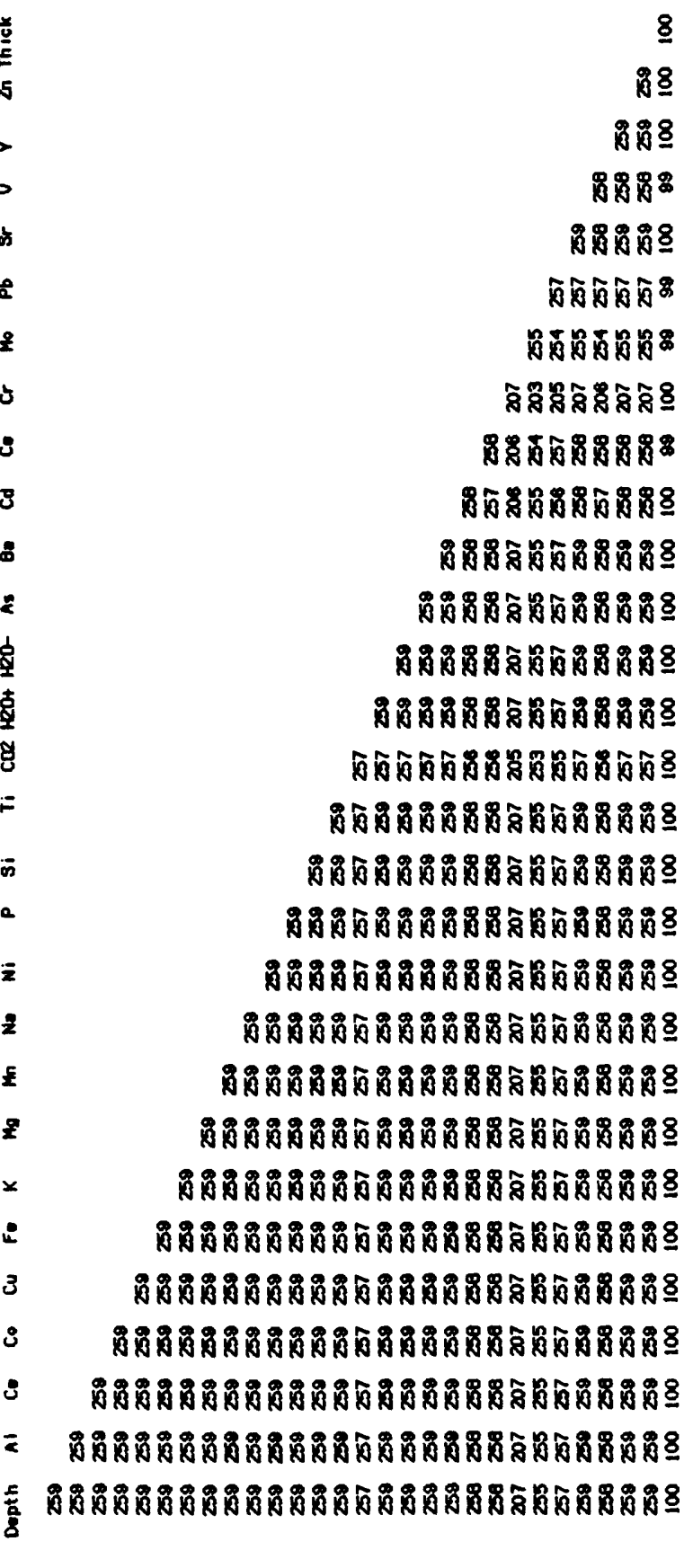

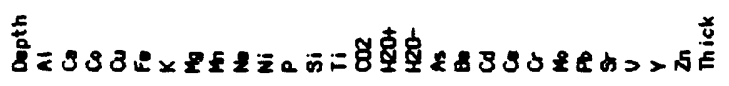




\section{Principal components analysis}

Q-mode factor analysis was undertaken on an earlier data set, using standard methodologies as discussed in Harman (1967) and Davis (1986). Partial results were reported in Manheim and Lane (1987). Since the results yield little difference from relationships already noted from plots and the correlation matrices we offer only highlights here. For large data sets a "Mn oxide" factor includes $\mathrm{Mo}, \mathrm{Ni}, \mathrm{Mn}, \mathrm{Sr}, \mathrm{Pb}, \mathrm{Co}$, and $\mathrm{V}$; a factor described as "Fe-Lat" includes Fe, latitude and $\mathrm{Pb}$; and a "aluminosilicate phase" factor includes $\mathrm{K}, \mathrm{Al}$, mg and depth. A "depth" factor includes $\mathrm{Na}, \mathrm{Fe}$, depth and $\mathrm{Cu}$, and a "phosphate" factor selected $\mathrm{Ca}, \mathrm{Y}, \mathrm{P}, \mathrm{Ba}, \mathrm{CO}_{2}$, and $\mathrm{Sr}$. Finally, with a small data set consisiting of tops of seamount crusts collected by the "S.P. Lee" cruise in 1983 an additional $\mathrm{Ba}-\mathrm{Zn}-\mathrm{Cd}$ factor not previously discerned was suggested.

\section{Bathymetric base map}

Because crusts are closely related, both by habitat and chemistry, to distinct topographic zones we have prepared a bathymetric base map for active investigation areas in the Central Pacific Ocean. Bathymetric data for the Central Pacific map in the pocket are plotted on a spheroidal Mercator projection using the National Geophysical Data Center gridded bathymetric data set, DBDB5. Computer contouring was performed by Peter Sloss at NGDC, Boulder, with subsequent modifications at Woods Hole. The computer contours have been manually smoothed in drafting, and obviously improper or erratic features such as line crossings removed or modified. A subprogram has been used to identify ambiguous contours and permit additional labelling.

The basemap is of importance because navigation problems have led to conflicting and questionable locations for given seamounts. The DBDB5 database, referred to in earlier versions as the U.S. Navy SYNBAPS, is a subset of the NGDC ETOPO5 database which is the most carefully controlled and readily available gridded global elevation data set known to us. It is stored as 360-degree longitude circles for each 5 minutes of latitude starting at the North pole and stepping southward. Each logical record contains 4320 ( $360 \times 12) 5$-minute grid points, and there are 2160 logical records in the file. Data values are positive above sea level and negative below and are given to the nearest whole meter. Following general oceanographic practice, the tape is "uncorrected" for local sound velocity, which permits intercorrelation with most bathymetric data bases of large scale.

Coastlines and land areas associated with ETOPO5 tend to be schematic and are often inaccurate. Hence, we have utilized the World Data Bank II (1984) data set for coastlines and land areas. We have added to the Central Pacific base map station locations and contours for normalized cobalt concentration for all depths.

\section{Maps of elements}

Graphic displays of chemical elements and components in map format offer many striking relationships. In this section we use two alternative display modes. The first, is computer gridding and contouring, and the second replaces contouring by gray scale maps. Computer gridding, interpolation and contouring are procedures that can yield very different final products, depending on the parameters utilized. The following briefly describes the parameters used in 
this study. The GRIDS and CONTOUR subroutines were written by G. Evenden of this Branch.

GRIDS superimposes a rectangular grid on the area desired to be mapped, bounded by lat1, lat2, long1, and long2. A cell size of $1 \mathrm{~cm}$ for the original map size of $1: 5,000,000$ is arbitrarily chosen to correspond to an apparent sample density ranging from 0 to 10 points per cell. The interpolation method fits a least squares polynomial surface to the data points. The intersection of the surface with the grid establishes nodal values, which can then be contoured. In our gridding we arbitrarily chose a search radius of $5 \mathrm{~cm}$. This ensures continuous interpolation in areas such as the northeast Pacific and Southeast Pacific, where relatively few data points are available, but where it is geologically reasonable to estimate data values.

The gridding option we chose divided points within the search radius into octants or pie slices, with no more than 10 points per octant. Cells that have more than 3 conterminous empty octants will be rejected, yielding "white space" on the map for these areas.

After examining 0 th, 1 st and 2nd degree polynomial surface options, we chose the first degree surface to establish nodal grid values for most maps. This permits relatively high-resolution mapping, and closer conformity of contours to actual data points, especially in the Central Pacific where the greatest data density is available. The computer derived contours, slightly smoothed manually in drafting, have been modified to remove some closely spaced contours in areas of sparse data coverage, where this degree of detail is not justified, to connect contours where continuity appears reasonable and to terminate contours at the coasts where needed. The influence of the change in elemental values that is often obtained due to the influence of the continental margin, could not be incorporated into the gridding software. For example, cobalt values often decrease to about the 0.1 percent level at or near the shelf break. Thus, unrealistic values may be projected by contours in close proximity to land areas.

The normalized cobalt map at a $>4000 \mathrm{~m}$ depth horizon (Figs. IX-20) is gridded with fewer data points. For this figure we have utilized the zero-degree polynomial surface function. This yields smoother, more generalized contours whose nodal values may not "honor the data points"; i.e. contours drawn through the nodal values do not necessarily agree with the data points themselves, but project the influence of data values to greater distances from these points. It should be noted that this map and a large base map were drawn on the database at an earlier date (early 1986) than the other maps. However regional anomalies depicted by the contours are little affected.

The regional base map in the pocket of the first production of this report also excluded points having $\mathrm{Mn} / \mathrm{Fe}$ values of $<.5$ and $>5$ in order to emphasize "normal" hydrogenetic crust distribution. In the Central Pacific area, occasional older generation of hydrothermal crusts have been found (Manheim and Halbach, 1984; DeCarlo and others, 1987). The recent maps located at the end of this chapter eliminate these restrictions, which permits more detailed control in the more peripheral areas of the Pacific ocean. 


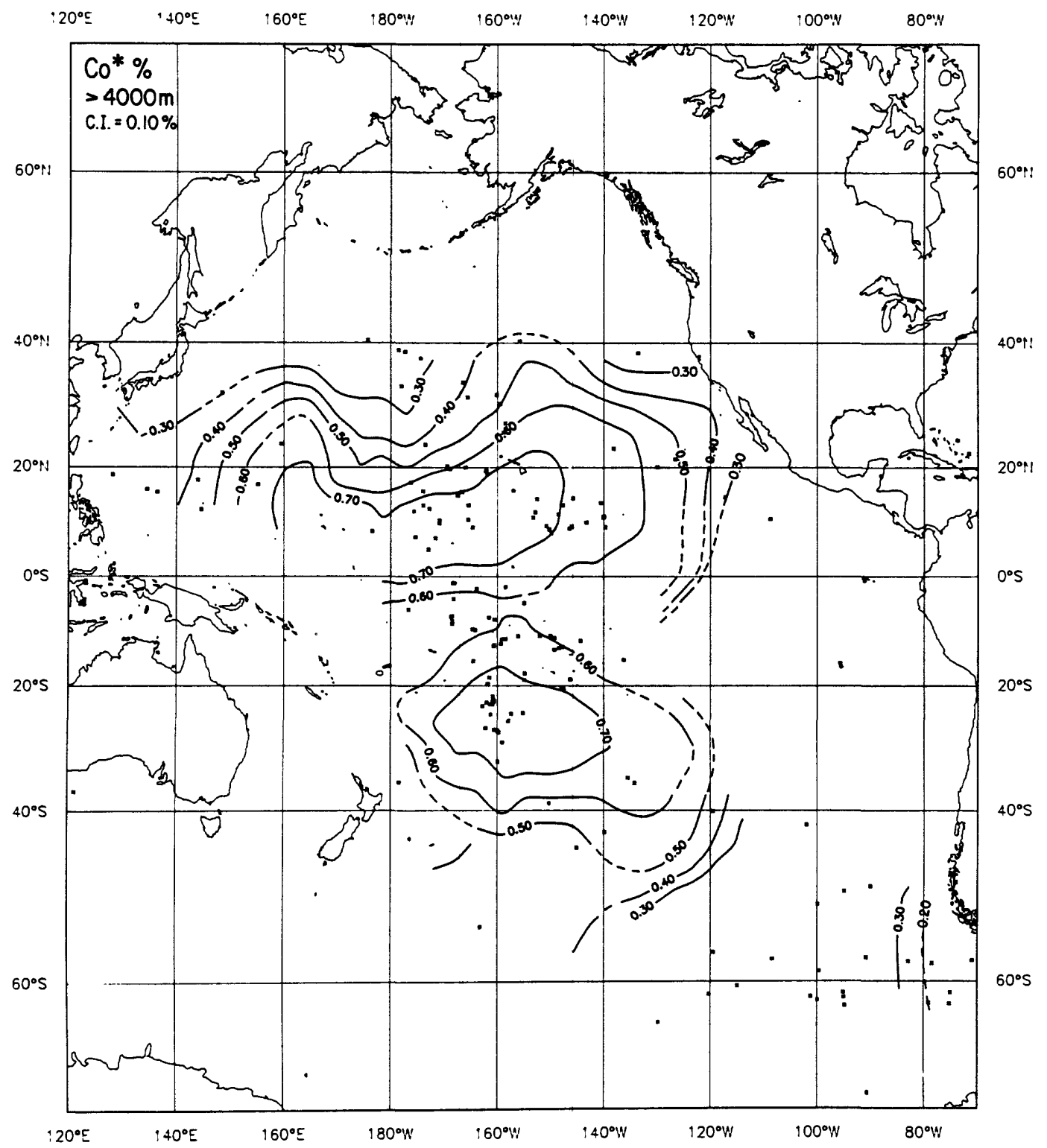

Figure IX-20 Elemental concentration of normalized cobalt in the Pacific Ocean, water depths $>4000 \mathrm{~m}$. 
Where appropriate we have displayed both the contoured and gray scale maps for a particular element (Figs. IX-21 to IX-65). Most of the trace elements are shown only in the gray scale map output. Both contour and gray-scale maps are based on a grid cell size of $1 \mathrm{~cm}$ for a 1:50 million map. Contoured and gray scale maps each have their advantages and disadvantages. Contours offer greater detail, but also have greater potential to mislead, if not carefully controlled and checked. The gray scale maps are particularly effective and convenient for the minor constituents that have lesser sample coverage.

Within the main Central Pacific seamount crust areas manganese oxides of hydrothermal origin are rare, and when found usually can be shown to be formed early in seamount history (Halbach and Manheim, 1984). On the other hand, in the eastern Pacific large regional areas are characterized by modern and subrecent hydrothermal influence (Manheim, Lane-Bostwick, 1988). This means that for insight into the distribution of hydrothermal phases, maps of normalized but unqualified data provide a better indicator of the extent of chemical influence of hydrothermally discharged water on the Pacific Ocean floor. These relationships indicate that no one data set can be used for all purposes; one must be prepared to explore a variety of data combinations, manipulations, and presentation modes to realize the full value of available information.

In Table IX-13 we give a brief discussion of some major features of crust composition and their relationships, as depicted in (Figs. IX-21 to IX-65). It is noteworthy that in virtually all cases large regional features of composition are the rule, not scattered local patterns. We believe that these large features support the hypothesis (Manheim, 1986, and references cited) that metals in crusts come mainly from ambient water rather than from underlying sediment and rock substrates. Abyssal manganese nodules, in contrast, get much of their metal content from host sediments (Dymond and others, 1984, and references cited).

Cobalt is probably the most distinctive representative of hydrogenetic metals in crusts, and its distribution is particularly informative of processes taking place in the bottom water-crust interface. From the regional point of view cobalt-enriched areas occupy the central parts of the Pacific ocean, far from continental influence or sources of $\mathrm{Fe}$ and $\mathrm{Mn}$, such as the vent zones that discharge fluids at temperaturs over $350^{\circ} \mathrm{C}$ (Von Damm and others, 1985). A recent article suggests that the isolation and selective loss of $\mathrm{Fe}$ and $\mathrm{Mn}$ from water masses in the central area, not special sources of Co, cause enrichment of Co as well as other metals such as $\mathrm{Pb}$ and $\mathrm{Ce}$ in these areas (Manheim and LaneBostwick, 1988).

From the geochemical point of view the cobalt-depleted areas are of equal interest as the cobalt-enriched ones. We have pointed out that major submarine hydrothermal discharge areas in the Pacific: the Galapagos, Juan de Fuca, and the Tonga-Lau rift zones, and the Marianas back-arc area all are characterized by halos of cobalt-depleted crusts (Manheim and Lane-Bostwick, 1988), and that cobalt (Fig. IX-21, IX-22) serves as a monitor of such discharge in the ocean in the present and the past.

The rate of accumulation of crusts was demonstrated by Halbach and others., 1983 to be inversely proportional to their cobalt content. This relationship was further extended by Manheim (1986) to a variety of marine manganese-rich 
sedimentary phases, and suggested to be described by the algorithm $R=6.8 \mathrm{x}$ $10 / \mathrm{Co}^{1.67}$. A preliminary map of accumulation rate based on this algorithm is included in this report (Fig. IX-37, IX-38). The age/Co relationship was analyzed in more detail by F.T. Manheim and D.E. Olson (unpublished data) recently, refining the formula but in general terms confirming the inverse loglinear relationship.

Recent studies have enhanced this data base. Several offer additional discussion of discrete areas in the Pacific Ocean, for which only sparse information existed before: the maps are discussed in more detail by recent field studies of discrete areas, such as those of Chave and others, 1986, DeCar10 and others, 1987 (Circum-Hawaii area); Halbach, 1986, Hein and others, 1985a, 1987 (Mid Pacific Mountains area), Hein and others, 1988 (Marshall Islands area), and Usui (1987) seamount crusts from the Izu-Bonin area, and the De Carlo and others (1988) (Kiribati area). Most recent mean chemical values for discrete areas in the Pacific are given by Hein and others., 1988.

Data from other areas of the world oceans are much sparser than for the Pacific. They were summarized briefly in Manheim (1986), and descriptive statistics are listed in Tables IX-1 to IX-4. In the Atlantic Ocean a large collection of data are available for the Blake Plateau, where pavements having up to $5 \mathrm{~cm}$-thick oxide layers encrust phosphorite substrates under the Gulf Stream. Similar substrates and encrustations are present in the Caribbean (Kang et), 1986, and the Ceira platform off Brazil, and, in the New England Seamounts.

In the Mid-Atlantic Ridge new data by Goddard and others (1987) have obtained compositions that tend to be either $\mathrm{Mn}$ or Fe-rich and impoverished in trace metals when they are of hydrothermal origin, or higher in $\mathrm{Co}, \mathrm{Pb}$, etc. when they are hydrogenetic. Co values approaching 1.0 percent are obtained in the Sierra Leone Rise area, but reach very low values in the Mid Atlantic Ridge (Goddard and others, 1987) and in the New England Seamount crusts (<.1 percent Co). Values in our data base for the New England Seamount are closer to normal hydrogenetic Atlantic types, and this discrepancy has not been resolved yet.

Synthesis of the Atlantic and Indian crusts has not been undertaken. A greater geographic distribution of crust material needs to be recovered to prepare meaningful regional maps. 
Table IX-13. Some principal features of chemical constituents are enumerated below. Areas in the third column refer to enrichments except where noted.

Elements with

Component similar patterns

A1203 Si(limited)

As

$\mathrm{Ba}$

$\mathrm{Ca}$

$\mathrm{Cd}$

$\mathrm{Ce}$

Cr

Co

$\mathrm{Cu}$

$\mathrm{Fe}$

mg

Mn

$\mathrm{Mn} / \mathrm{Fe}$

Mo

$\mathrm{Ni}$

$\mathbf{P}$

$\mathrm{Pb}$

$\mathrm{Si} / \mathrm{Al}$
$\mathrm{Ce}, \mathrm{Pb}, \mathrm{Co}$

$\mathrm{Cu}, \mathrm{Zn}, \mathrm{Ni}$

P

$\mathrm{Zn}$

Co

A1

$\mathrm{Ce}, \mathrm{Pb}, \mathrm{Ni}$

$\mathrm{Zn}, \mathrm{Ni}, \mathrm{Cd}$

$\mathrm{V}, \mathrm{Ti}$

$\mathrm{Cr}$

$\mathrm{Cu}$

$\mathrm{Cu}$

$\mathrm{Cu}, \mathrm{Zn}$

$\mathrm{Ca}$

Ce, Co
Regional distributions $\underline{\text { d notes }}$

Lowest in Galapagos rift, Chatham and Shatskiy rise area; high toward polar regions

Highest in the Tonga-Lau-Fiji area, off southern South America

Highest in the Clarion-Clipperton fracture zone \& Cook Is. area

Wide N-S band in E. Central Pacific; also Peru Basin; probably due mainly to physical phosphorite admixture

High in Clarion Clipperton zone but lacks sample distribution

Low in hydrothermal areas, highest values unclear affinity

Tonga-Lau-Fiji

Marshall Is., French Polynesia, $140^{\circ} \mathrm{W}$, Northern Line Islands, S. Central Pacific

Clarion-Clipperton zone

$10^{\circ} \mathrm{S}, 110^{\circ} \mathrm{W}$, Juan de Fuca, SW of

Tasmania, Tonga-Lau-Fiji

Juan de Fuca fracture zone

NW Pacific, SE Pacific, Clarion

Galapagos, Clarion-Clipp., erton zone, Tonga Lau-Fiji, Marianas

Juan de Fuca, NW Pacific

Clarion-Clipperton Zone, off New Zealand, SE Pacific

E. Central Pacific, Peru Trench

Enriched areas roughly similar to those of Co

Low near New Zealand 
Southern Marshall Is.; $40^{\circ} \mathrm{S}, 130^{\circ} \mathrm{W}$ Hawaiian Islands

$\mathrm{Ti} /(\mathrm{Mn}+\mathrm{Fe})$ Co

V

$\mathrm{Zn}$
$\mathrm{Fe}$

$\mathrm{Cu}, \mathrm{Ni}, \mathrm{Cd}$
Hydrothermal indicator less precise than Co

SE South America, Juan de Fuca (+)

Clarion Clipperton zone, S. Marshalls, Micronesia(+) 
$120^{\circ} \mathrm{E} \quad 140^{\circ} \mathrm{E} \quad 160^{\circ} \mathrm{E} \quad 180^{\circ} \mathrm{W} \quad 160^{\circ} \mathrm{W} \quad 140^{\circ} \mathrm{W} \quad 120^{\circ} \mathrm{W} \quad 100^{\circ} \mathrm{W} \quad 80^{\circ} \mathrm{W}$

$60^{\circ} \mathrm{N}$

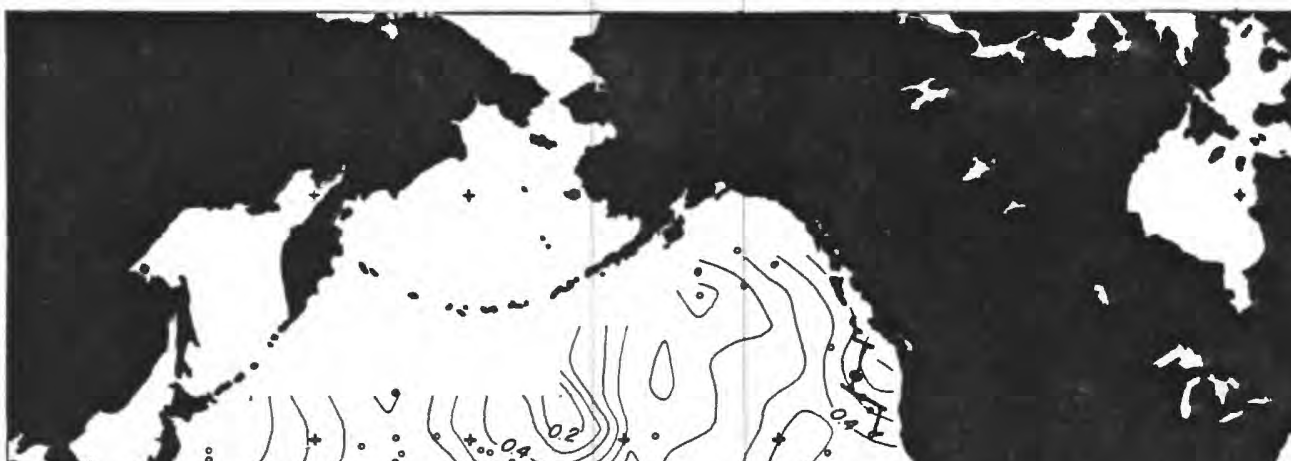

$60^{\circ} \mathrm{N}$

$40^{\circ} \mathrm{N}$

$0 \cdot 5$

$20^{\circ} \mathrm{N}$

$20^{\circ} \mathrm{S}$

$40^{\circ} \mathrm{S}$

$60^{\circ} \mathrm{S}$
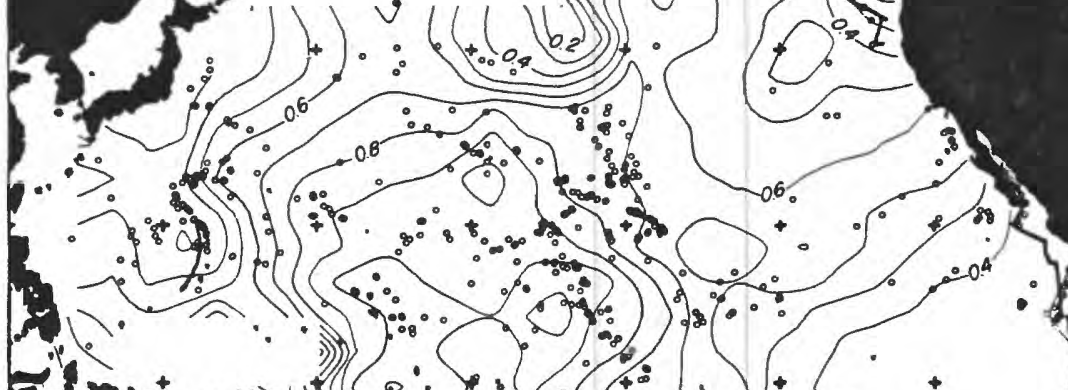

(i)) 0
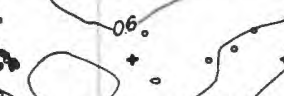

5े:
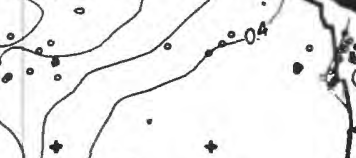
$120^{\circ} \mathrm{E} \quad 140^{\circ} \mathrm{E} \quad 160^{\circ} \mathrm{E} \quad 180^{\circ} \mathrm{W} \quad 160^{\circ} \mathrm{W} \quad 140^{\circ} \mathrm{W} \quad 120^{\circ} \mathrm{W} \quad 100^{\circ} \mathrm{W} \quad 80^{\circ} \mathrm{W}$

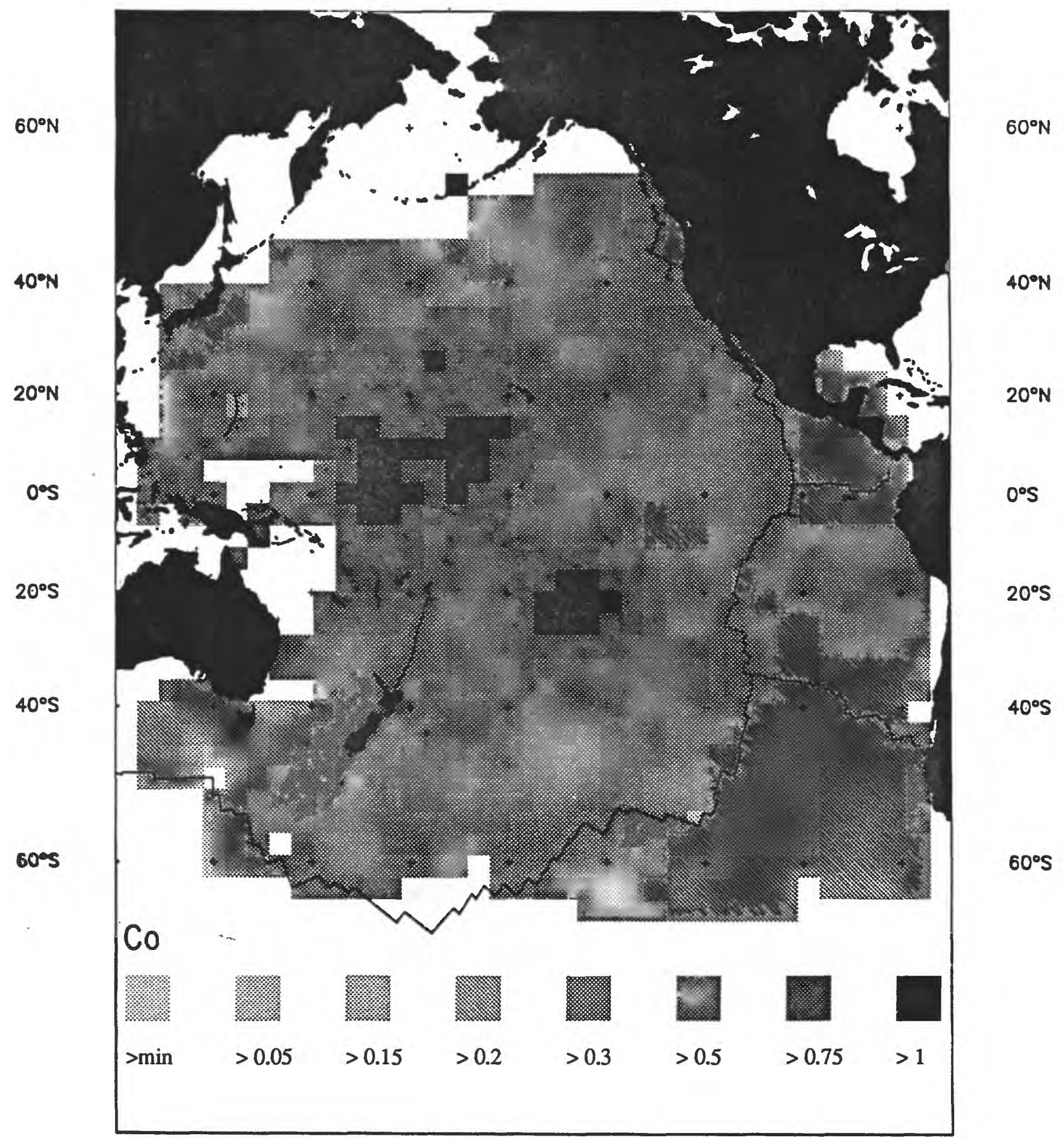

Figure IX-22 Gray scale map of Co\% in the Pacific Ocean. 
$120^{\circ} \mathrm{E} \quad 140^{\circ} \mathrm{E} \quad 160^{\circ} \mathrm{E} \quad 180^{\circ} \mathrm{W} \quad 160^{\circ} \mathrm{W} \quad 140^{\circ} \mathrm{W} \quad 120 \% \mathrm{~W} 100^{\circ} \mathrm{W} \quad 80^{\circ} \mathrm{W}$

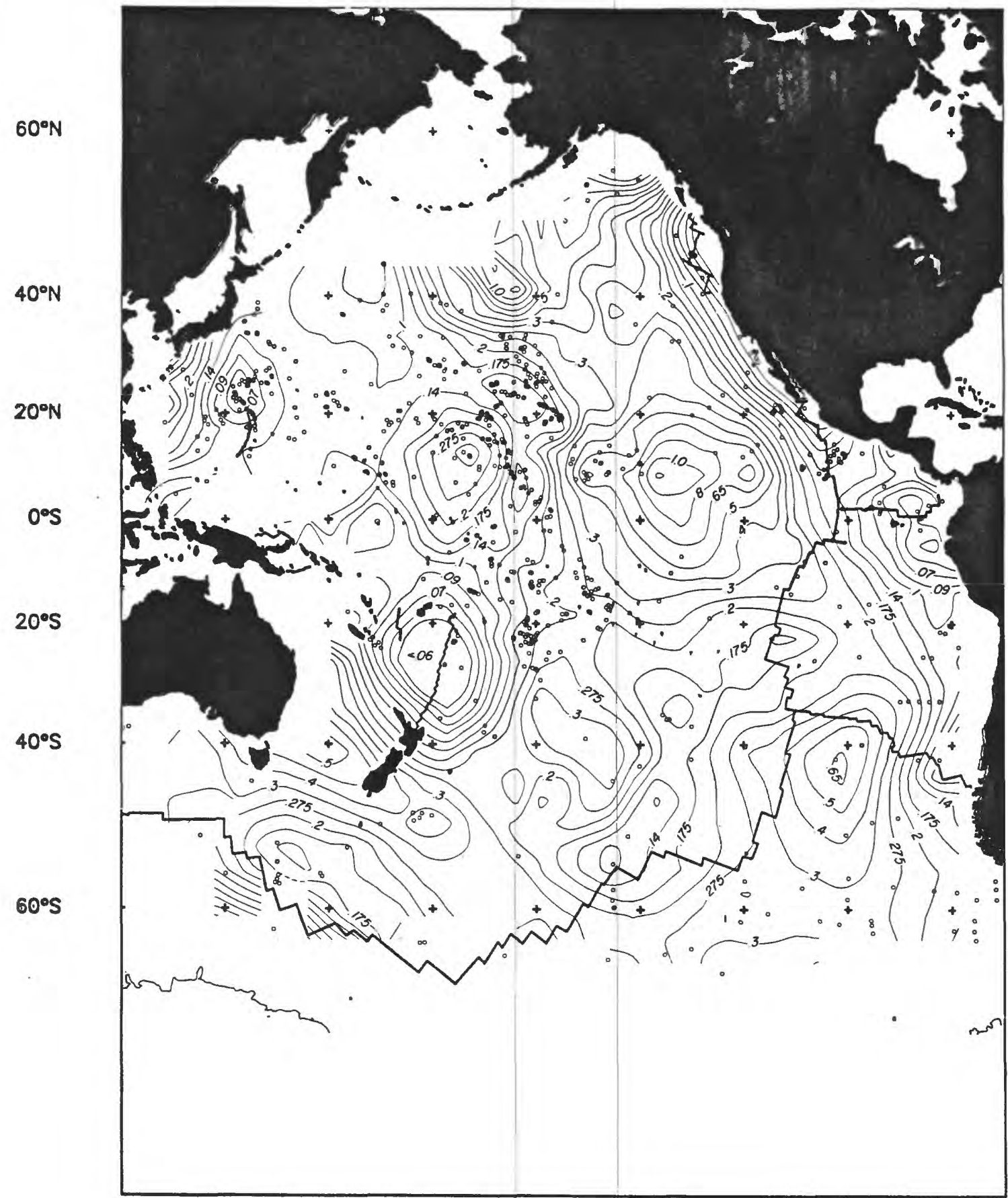

$60^{\circ} \mathrm{N}$

$40^{\circ} \mathrm{N}$

$20^{\circ} \mathrm{N}$

$0^{\circ} \mathrm{S}$

$20^{\circ} \mathrm{S}$

$40^{\circ} \mathrm{S}$

$60^{\circ} \mathrm{S}$

Figure IX-23 Elemental concentration of Cu\% in the Pacific Ocean; c.i. $n=.2$ for $\mathrm{Cu} \%=3 n$. 
$120^{\circ} \mathrm{E} \quad 140^{\circ} \mathrm{E} \quad 160^{\circ} \mathrm{E} \quad 180^{\circ} \mathrm{W} \quad 160^{\circ} \mathrm{W} \quad 140^{\circ} \mathrm{W} \quad 120^{\circ} \mathrm{W} \quad 100^{\circ} \mathrm{W} \quad 80^{\circ} \mathrm{W}$

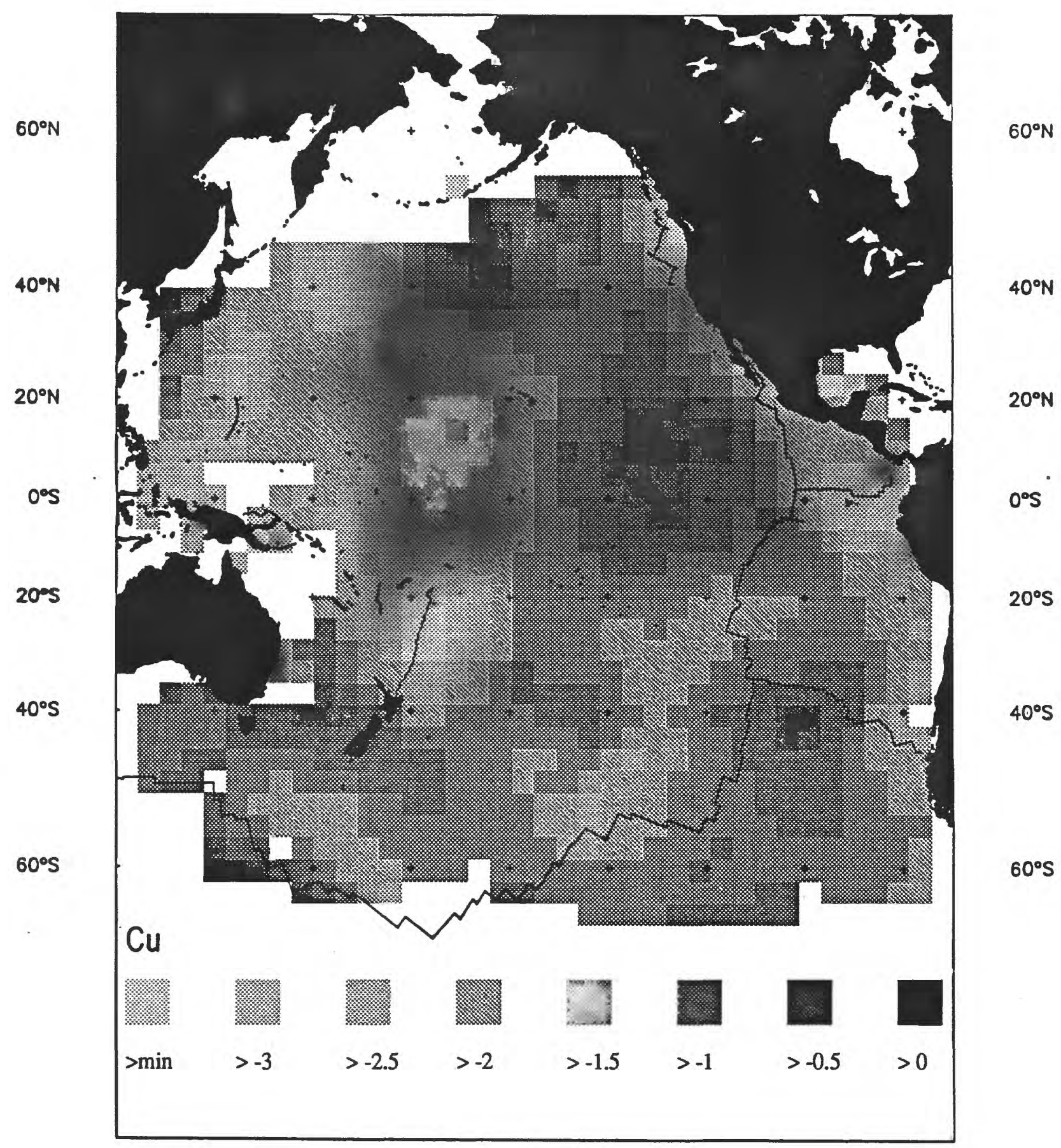

Figure IX-24 Gray scale map of LOG3Cu\% in the Pacific Ocean. 
$120^{\circ} \mathrm{E} \quad 140^{\circ} \mathrm{E} \quad 160^{\circ} \mathrm{E} \quad 180^{\circ} \mathrm{W} \quad 160^{\circ} \mathrm{W} \quad 140^{\circ} \mathrm{W} \quad 1200^{\circ} \mathrm{W} \quad 100^{\circ} \mathrm{W} \quad 80^{\circ} \mathrm{W}$

$60^{\circ} \mathrm{N}$

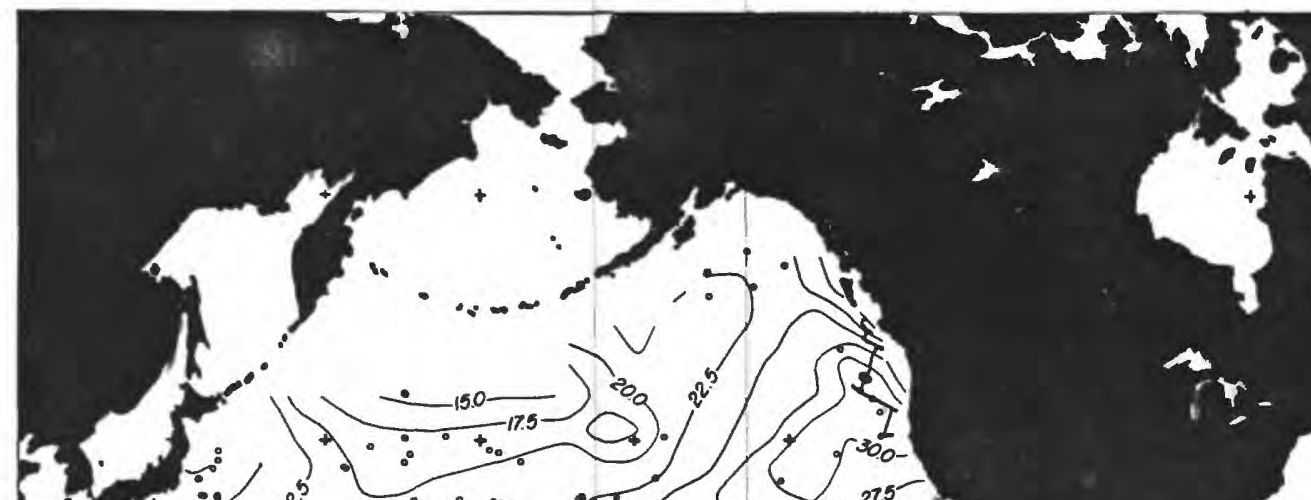

$60^{\circ} \mathrm{N}$

$40^{\circ} \mathrm{N}$

$40^{\circ} \mathrm{N}$

o's

$20^{\circ} \mathrm{N}$

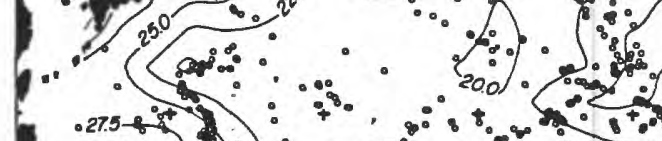

.
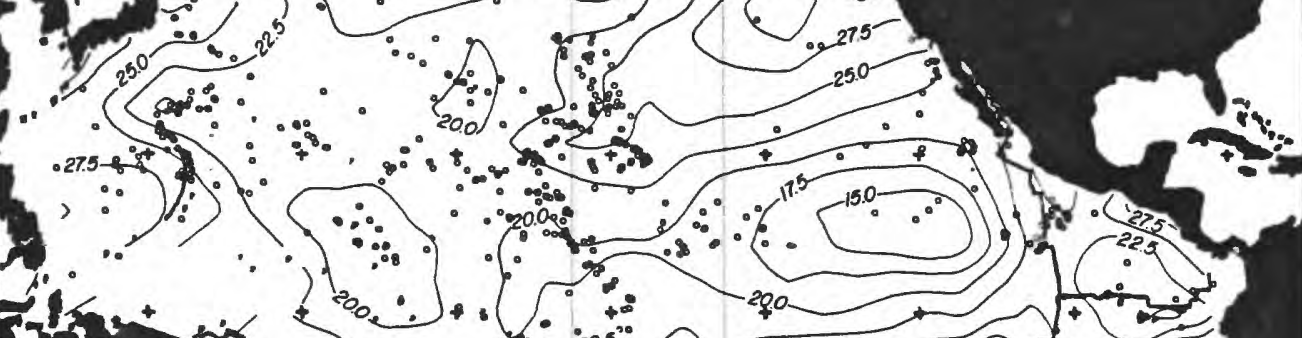

-20.050

$\therefore$
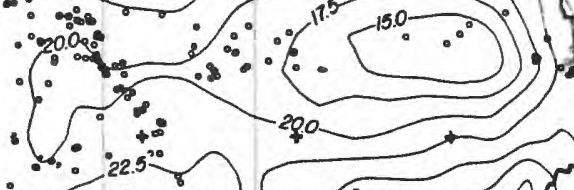

8

$20^{\circ} \mathrm{S}$

$40^{\circ} \mathrm{S}$

$60^{\circ} \mathrm{S}$

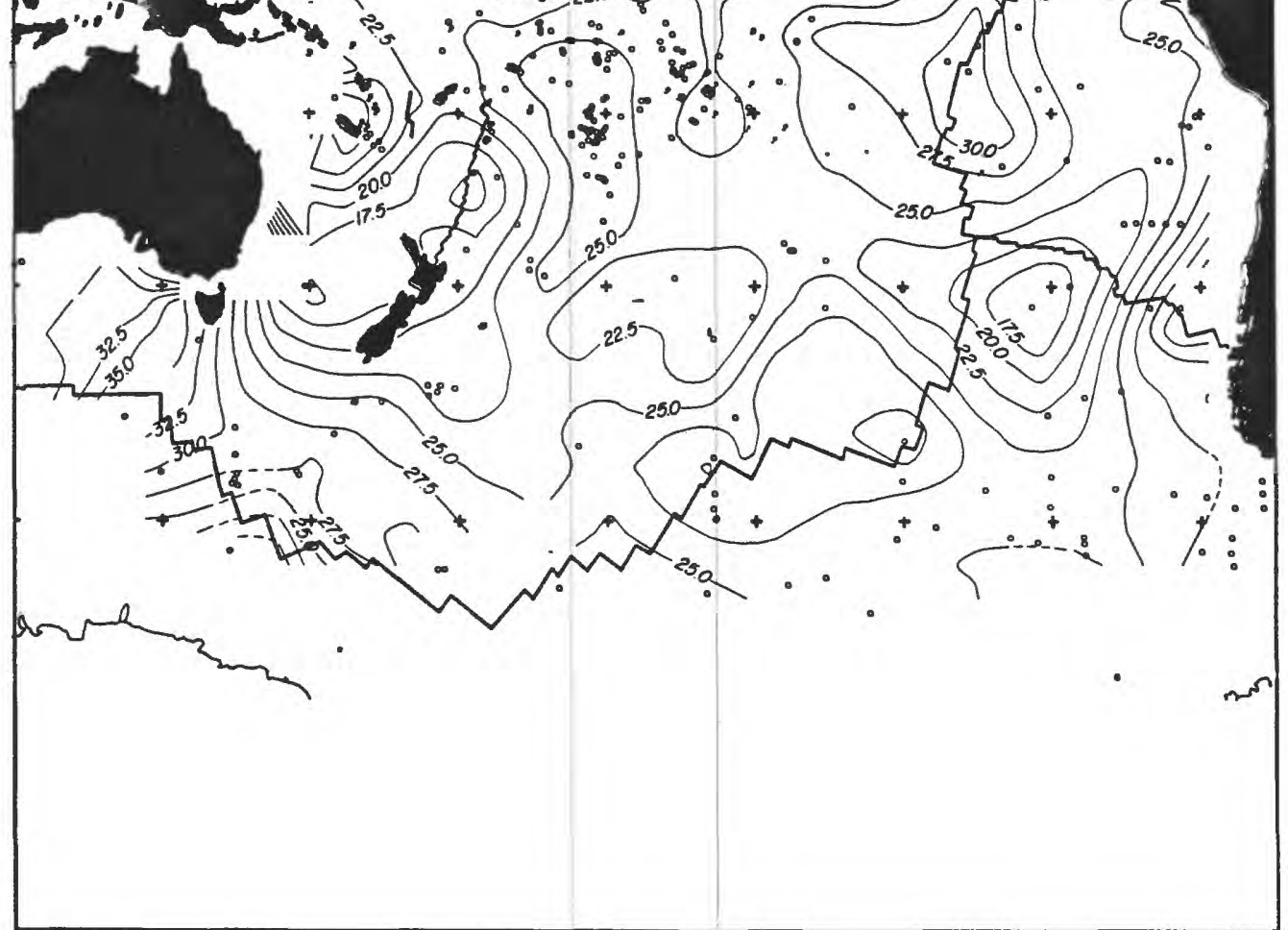

$40^{\circ} \mathrm{S}$

$60^{\circ} \mathrm{S}$

Figure IX-25 Elemental concentration of Fe\% in the Pacific 
$120^{\circ} \mathrm{E} \quad 140^{\circ} \mathrm{E} \quad 160^{\circ} \mathrm{E} \quad 180^{\circ} \mathrm{W} \quad 160^{\circ} \mathrm{W} \quad 140^{\circ} \mathrm{W} \quad 120^{\circ} \mathrm{W} \quad 100^{\circ} \mathrm{W}$ 80 W

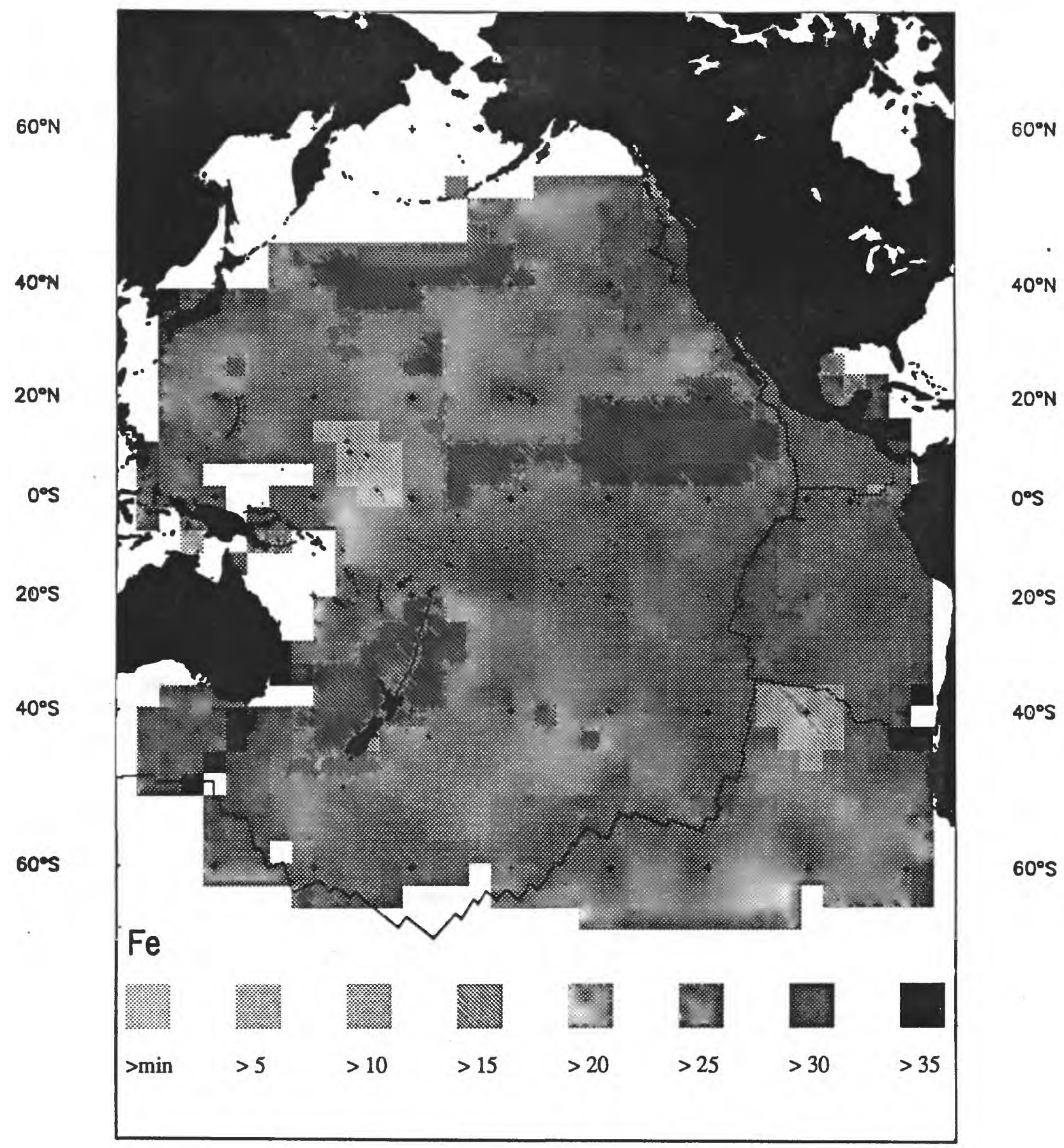

Figure IX-26 Gray scale map of $\mathrm{Fe} \%$ in the Pacific Ocean. 
$120^{\circ} \mathrm{E} \quad 140^{\circ} \mathrm{E} \quad 160^{\circ} \mathrm{E} \quad 180^{\circ} \mathrm{W} \quad 160^{\circ} \mathrm{W} \quad 140^{\circ} \mathrm{W} \quad 120^{\circ} \mathrm{W} \quad 100^{\circ} \mathrm{W} \quad 80^{\circ} \mathrm{W}$

$60^{\circ} \mathrm{N}$

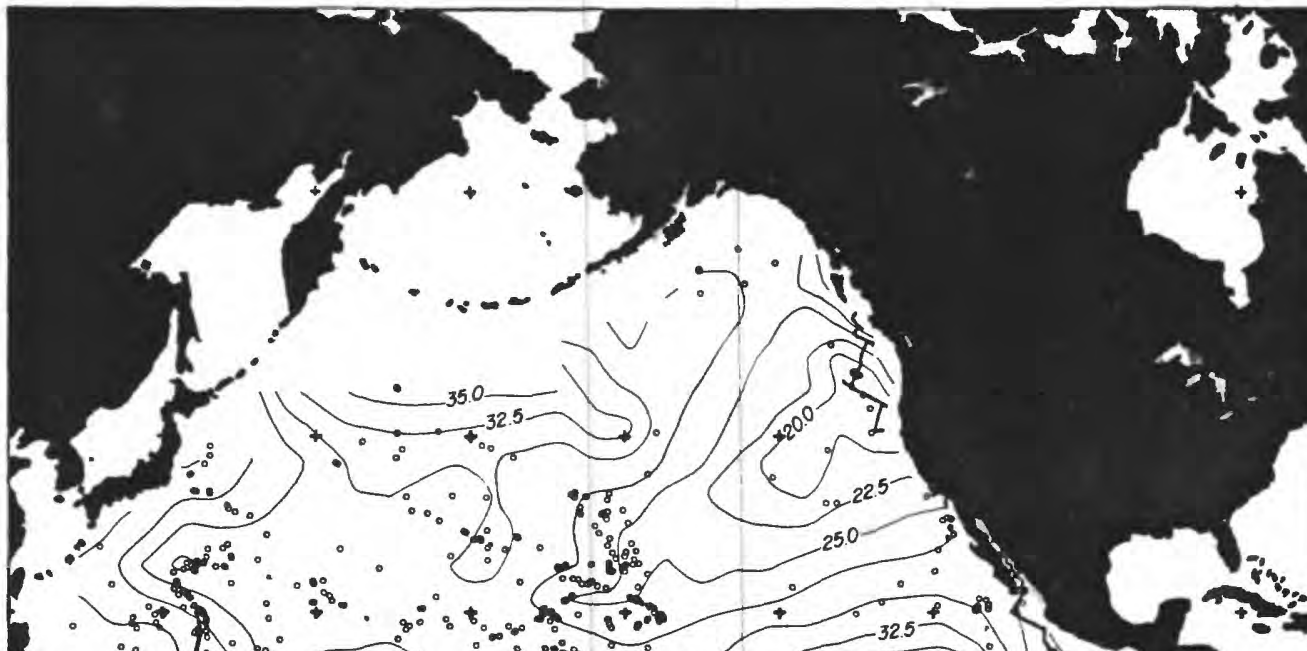

$60^{\circ} \mathrm{N}$

$40^{\circ} \mathrm{N}$

$20^{\circ} \mathrm{N}$

ors

$20^{\circ} \mathrm{S}$

$40^{\circ} \mathrm{S}$

$60^{\circ} \mathrm{S}$
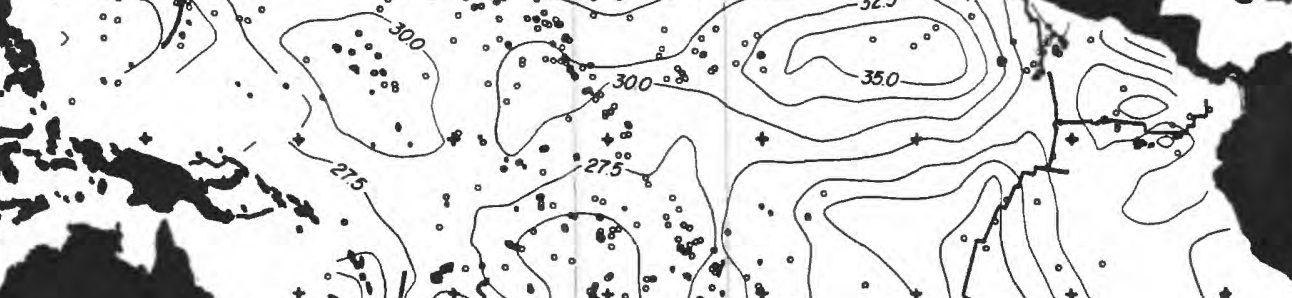

$0^{\circ}$

$40^{\circ} \mathrm{N}$

$20^{\circ} \mathrm{N}$

$20^{\circ} \mathrm{S}$

$40^{\circ} \mathrm{S}$

$60^{\circ} \mathrm{S}$

Figure IX-27 Elemental concentration of $\mathrm{Mn} \%$ in the Pacific Ocean, c.i. $=2.5 \%$ 


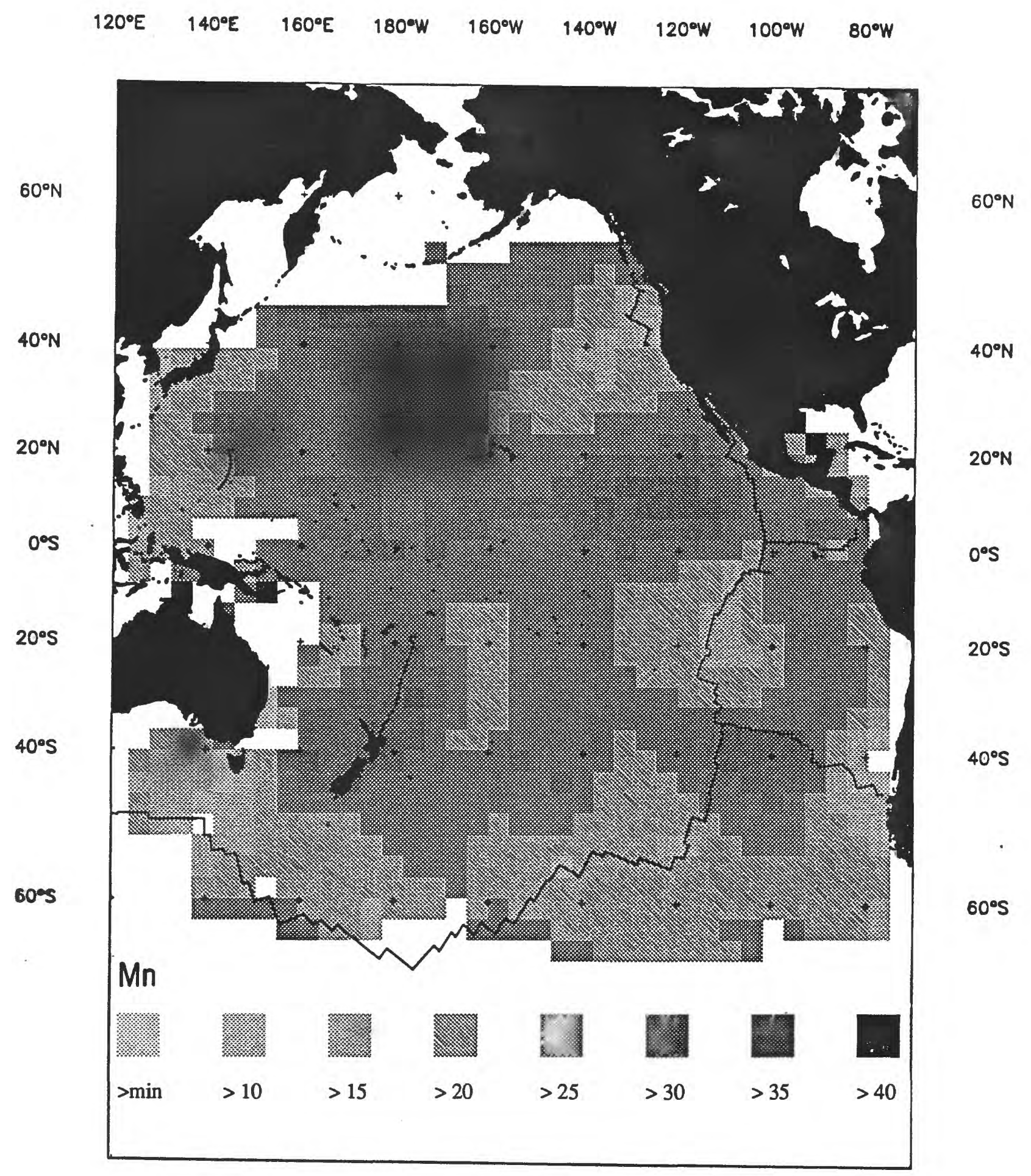

Figure IX-28 Gray scale map of $\mathrm{Mn} \%$ in the Pacific Ocean. 
$120^{\circ} \mathrm{E} \quad 140^{\circ} \mathrm{E} \quad 160^{\circ} \mathrm{E} \quad 180^{\circ} \mathrm{W} \quad 160^{\circ} \mathrm{W} \quad 140 \% \mathrm{~W} \quad 120^{\circ} \mathrm{W} \quad 100^{\circ} \mathrm{W} \quad 80^{\circ} \mathrm{W}$

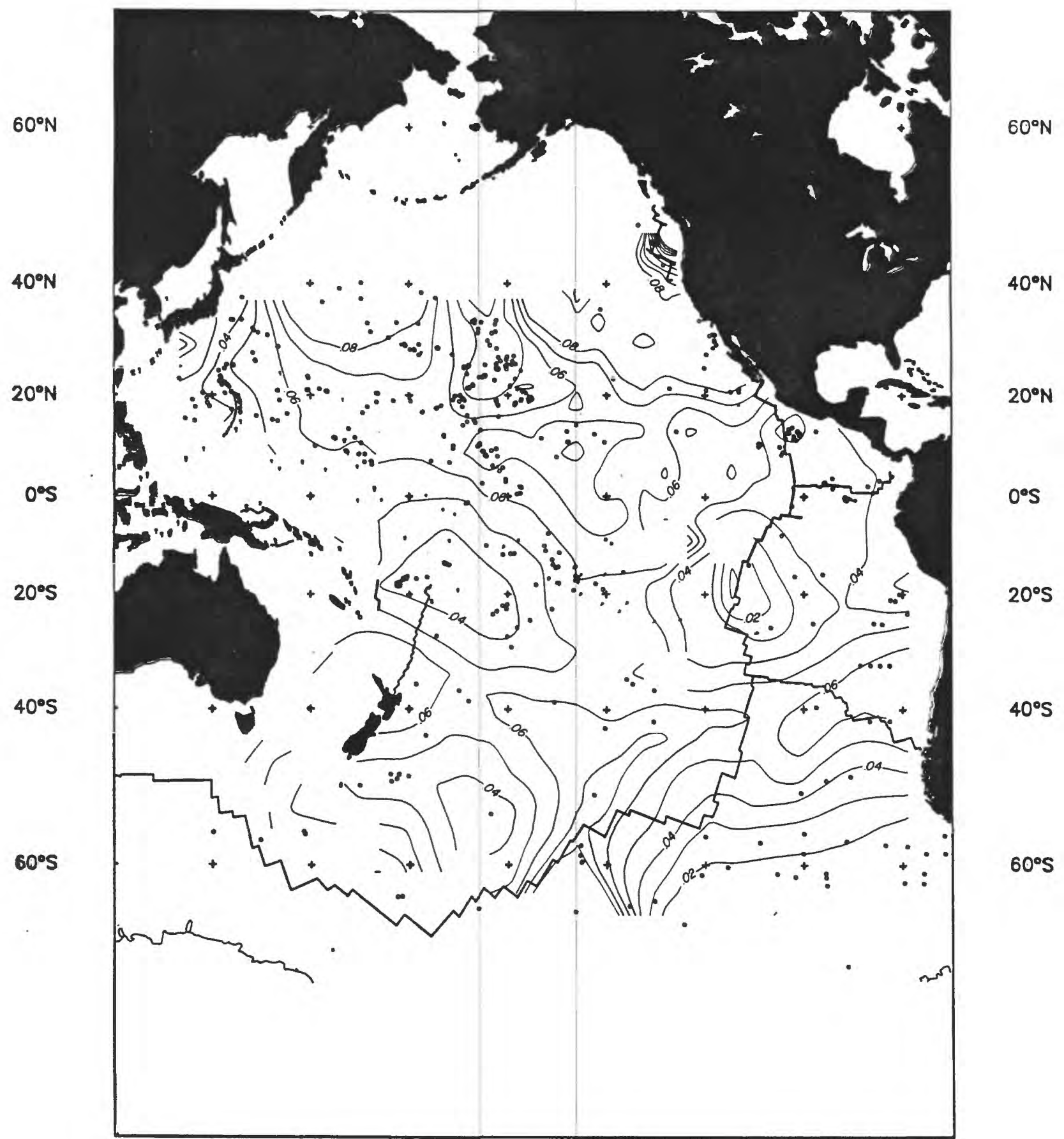

Figure IX-29 Elemental concentration of Mo\% in the Pacific 
$120^{\circ} \mathrm{E} \quad 140^{\circ} \mathrm{E} \quad 160^{\circ} \mathrm{E} \quad 180^{\circ} \mathrm{W} \quad 160^{\circ} \mathrm{W} \quad 140^{\circ} \mathrm{W} \quad 120^{\circ} \mathrm{W} \quad 100^{\circ} \mathrm{W} \quad 80^{\circ} \mathrm{W}$

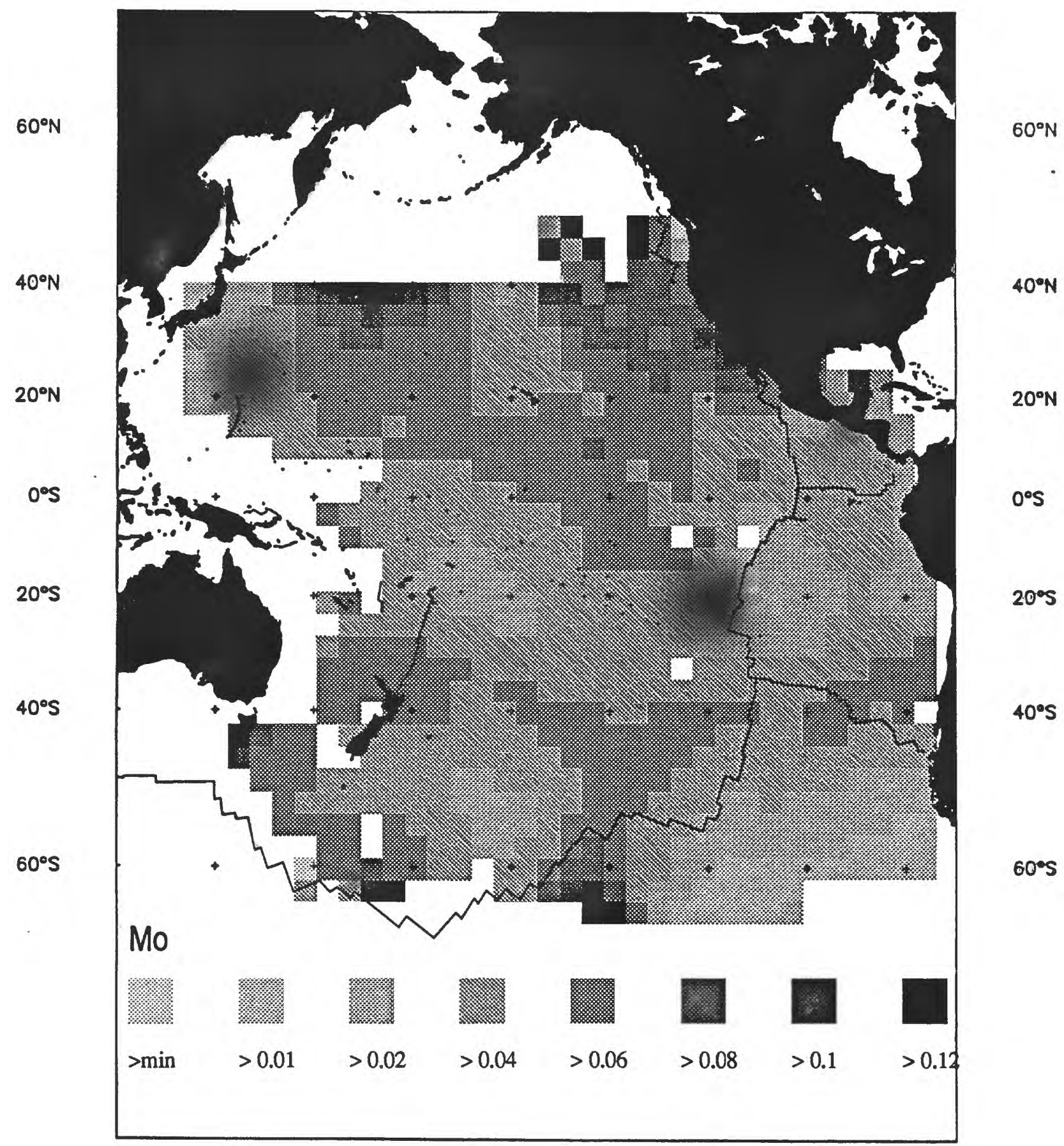

Figure IX-30 Gray scale map of Mo\% in the Pacific Ocean. 
$120^{\circ} \mathrm{E} \quad 140^{\circ} \mathrm{E} \quad 160^{\circ} \mathrm{E} \quad 180^{\circ} \mathrm{W} \quad 160^{\circ} \mathrm{W} \quad 140^{\circ} \mathrm{W} \quad 1200^{\circ} \mathrm{W} \quad 100^{\circ} \mathrm{W} \quad 80^{\circ} \mathrm{W}$

$60^{\circ} \mathrm{N}$

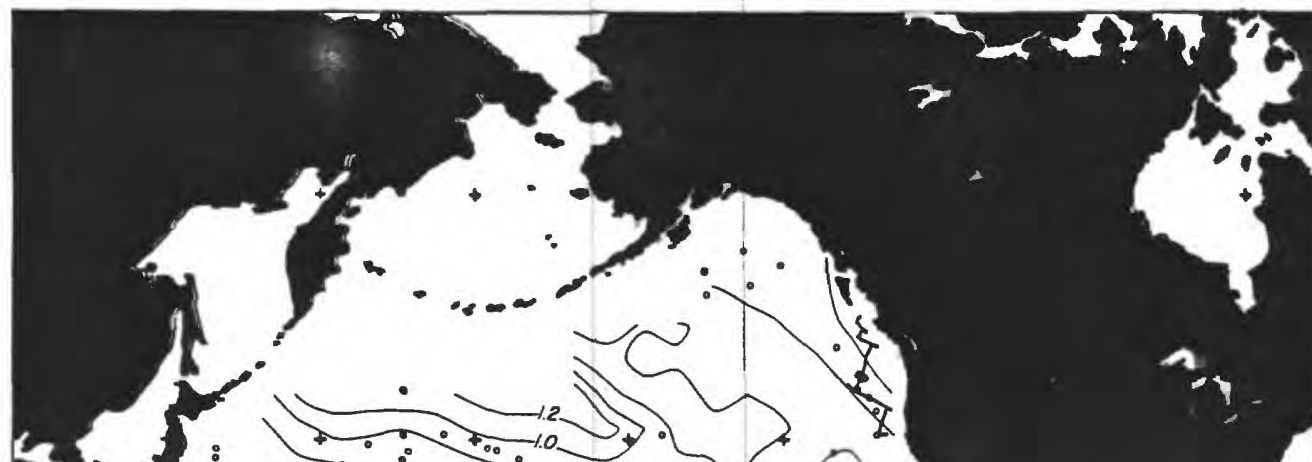

$60^{\circ} \mathrm{N}$

$40^{\circ} \mathrm{N}$

$20^{\circ} \mathrm{N}$

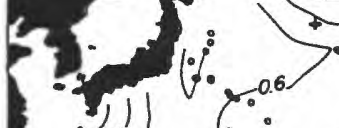

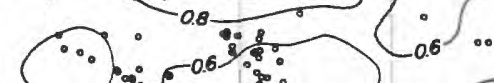

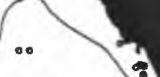

0

$20^{\circ} \mathrm{N}$

os

$20^{\circ} \mathrm{S}$
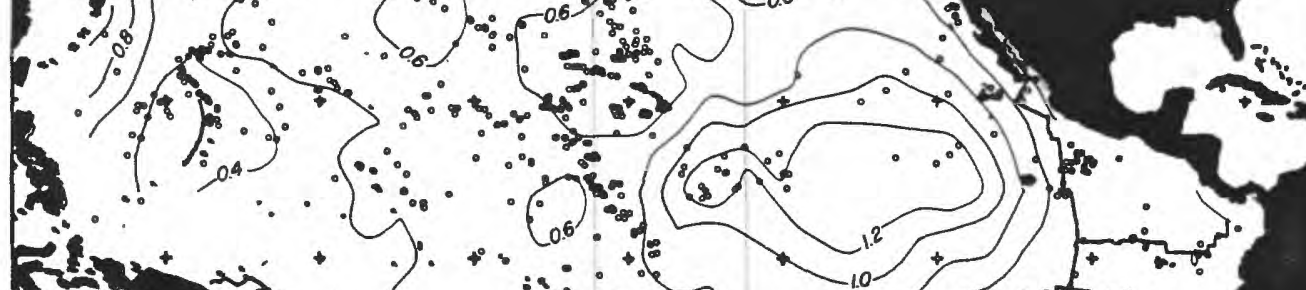

$40^{\circ} \mathrm{N}$

$0^{\circ} 5$

$40^{\circ} \mathrm{S}$

$60^{\circ} \mathrm{S}$

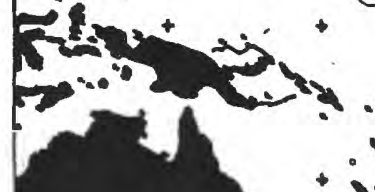

.

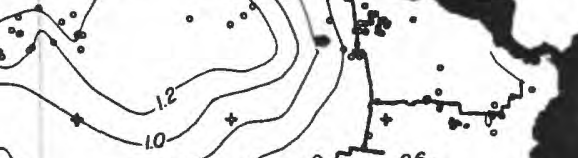


$120^{\circ} \mathrm{E} \quad 140^{\circ} \mathrm{E} \quad 160^{\circ} \mathrm{E} \quad 180^{\circ} \mathrm{W} \quad 160^{\circ} \mathrm{W} \quad 140^{\circ} \mathrm{W} \quad 120^{\circ} \mathrm{W} \quad 100^{\circ} \mathrm{W} \quad 80^{\circ} \mathrm{W}$

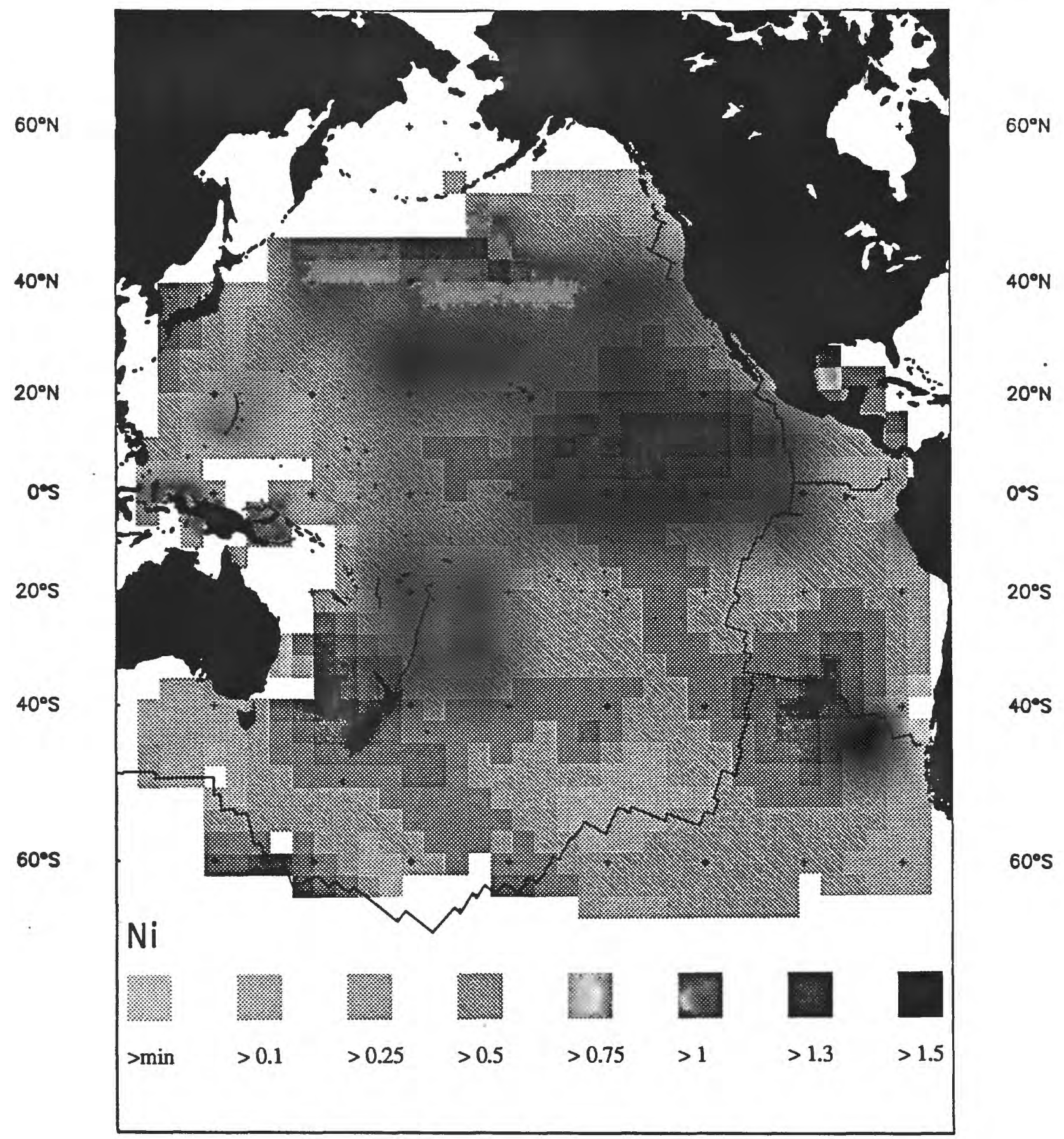

Figure IX-32 Gray scale map of Ni\% in the Pacific Ocean. 
$120^{\circ} \mathrm{E} \quad 140^{\circ} \mathrm{E} \quad 160^{\circ} \mathrm{E}$ 180 W $160^{\circ} \mathrm{W} \quad 140^{\circ} \mathrm{W} \quad 120^{\circ} \mathrm{W} \quad 100^{\circ} \mathrm{W} \quad 80^{\circ} \mathrm{W}$

$60^{\circ} \mathrm{N}$

$40^{\circ} \mathrm{N}$

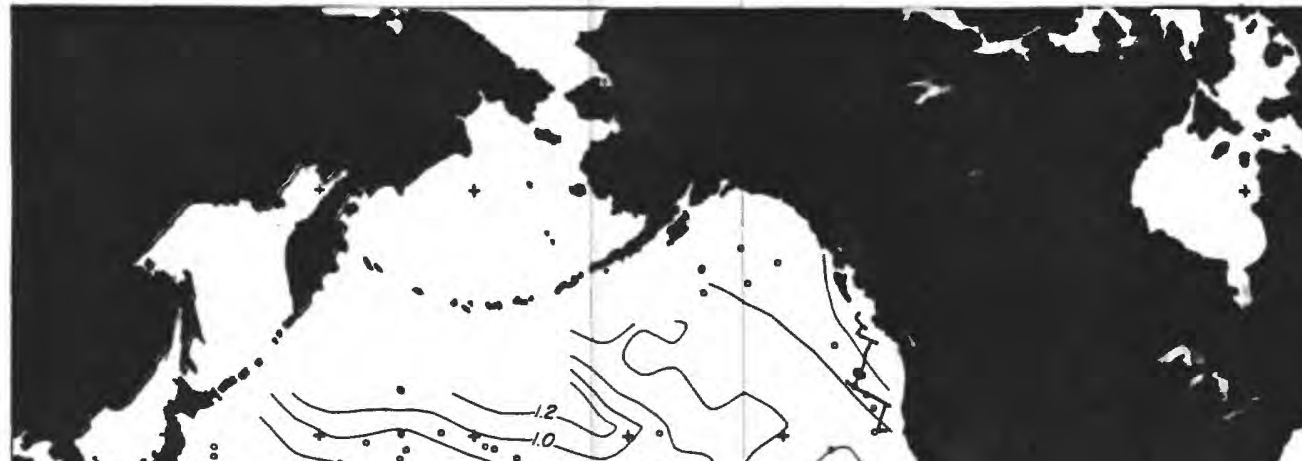

$60^{\circ} \mathrm{N}$

$40^{\circ} \mathrm{N}$

$20 \%$

o's
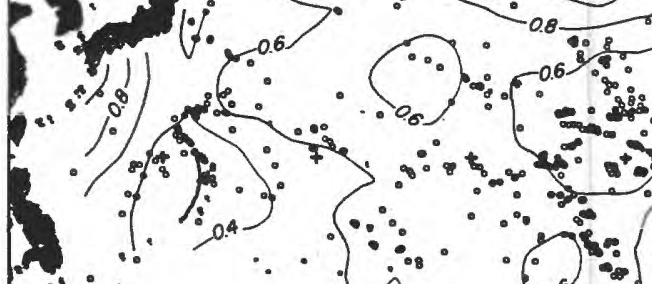

$20^{\circ} \mathrm{S}$
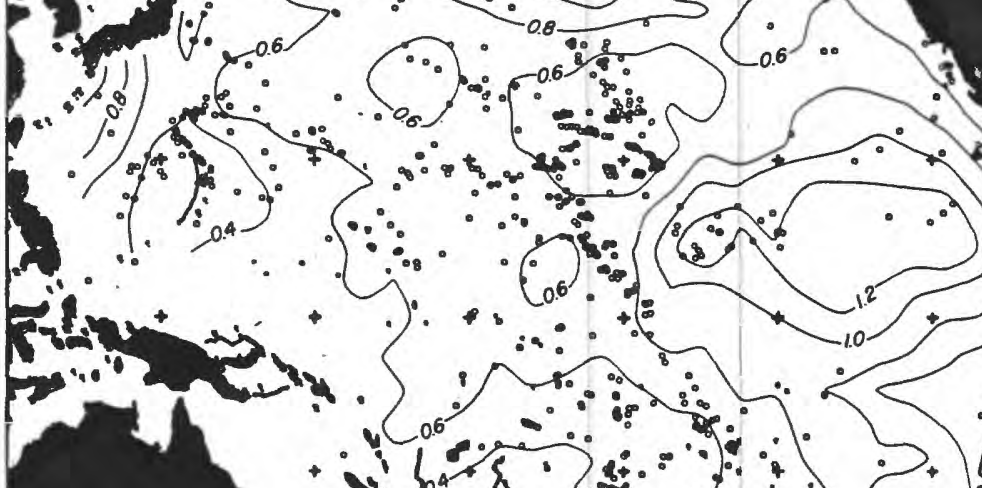

10:
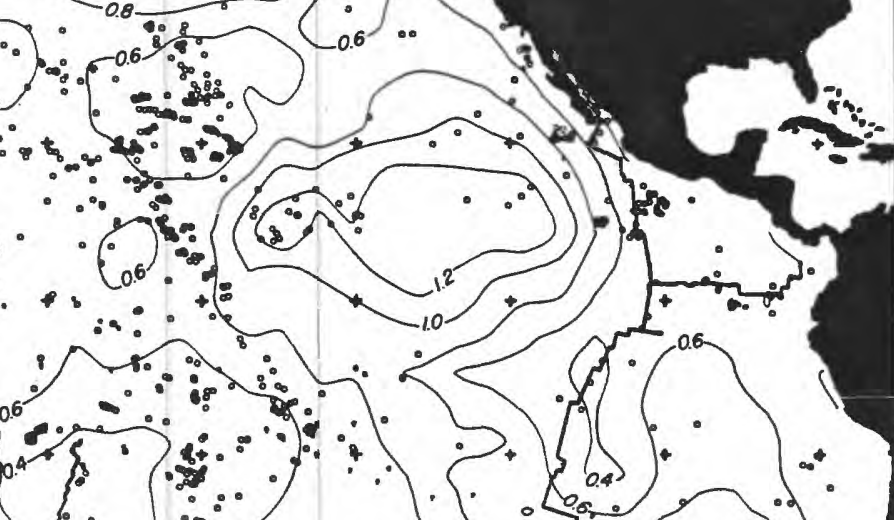

$20^{\circ} \%$

$40^{\circ} \mathrm{S}$

$60^{\circ} \mathrm{S}$

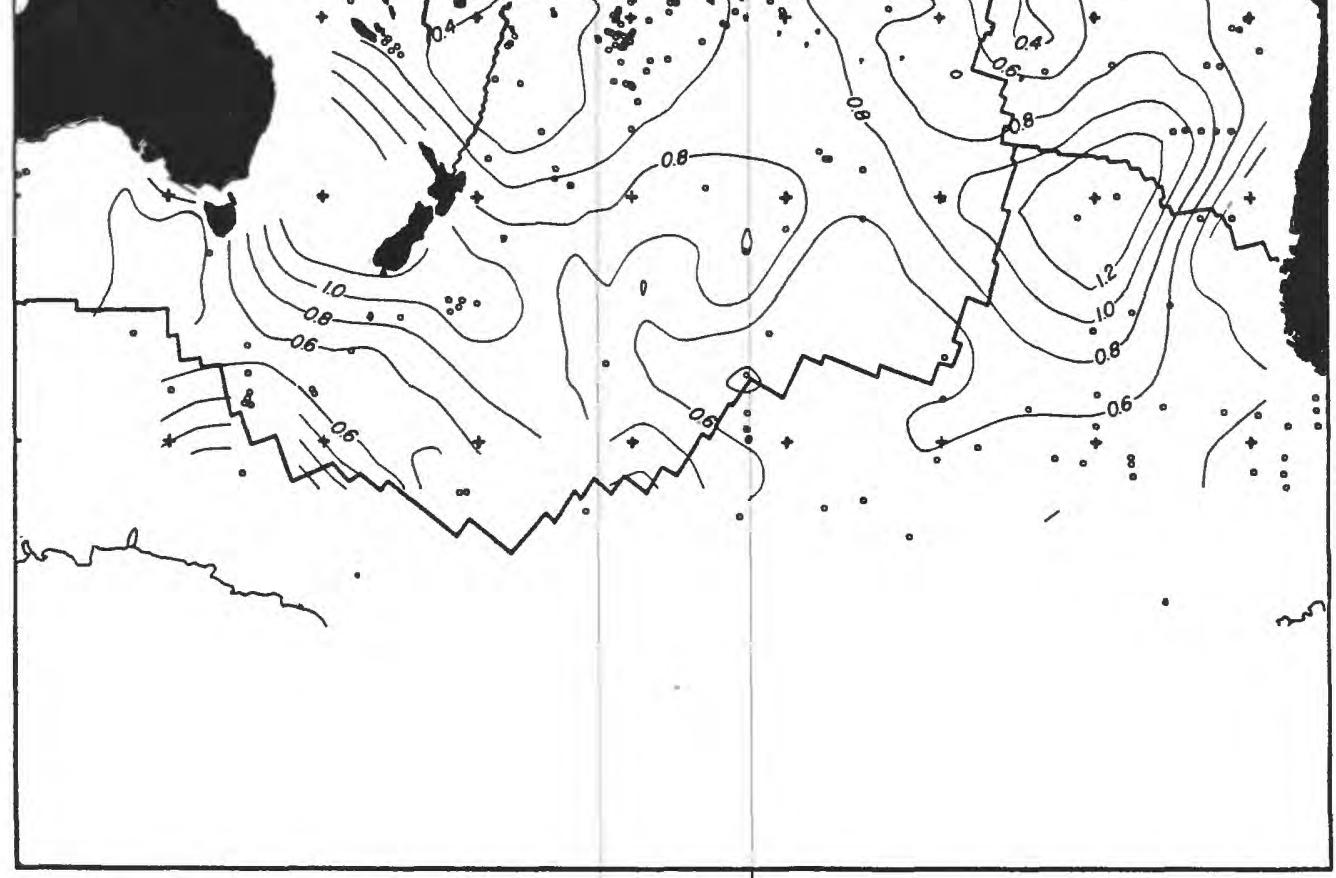

$40^{\circ} \mathrm{S}$

$60^{\circ} \mathrm{S}$

Figure IX-33 Elemental concentration of Ni\% in the Pacific Ocean; c.i. $n=.2$ for $\mathrm{Ni} \%-3 n$. 
$120^{\circ} \mathrm{E} \quad 140^{\circ} \mathrm{E} \quad 160^{\circ} \mathrm{E} \quad 180^{\circ} \mathrm{W} \quad 160^{\circ} \mathrm{W} \quad 140^{\circ} \mathrm{W} \quad 120^{\circ} \mathrm{W} \quad 100^{\circ} \mathrm{W} \quad 80^{\circ} \mathrm{W}$

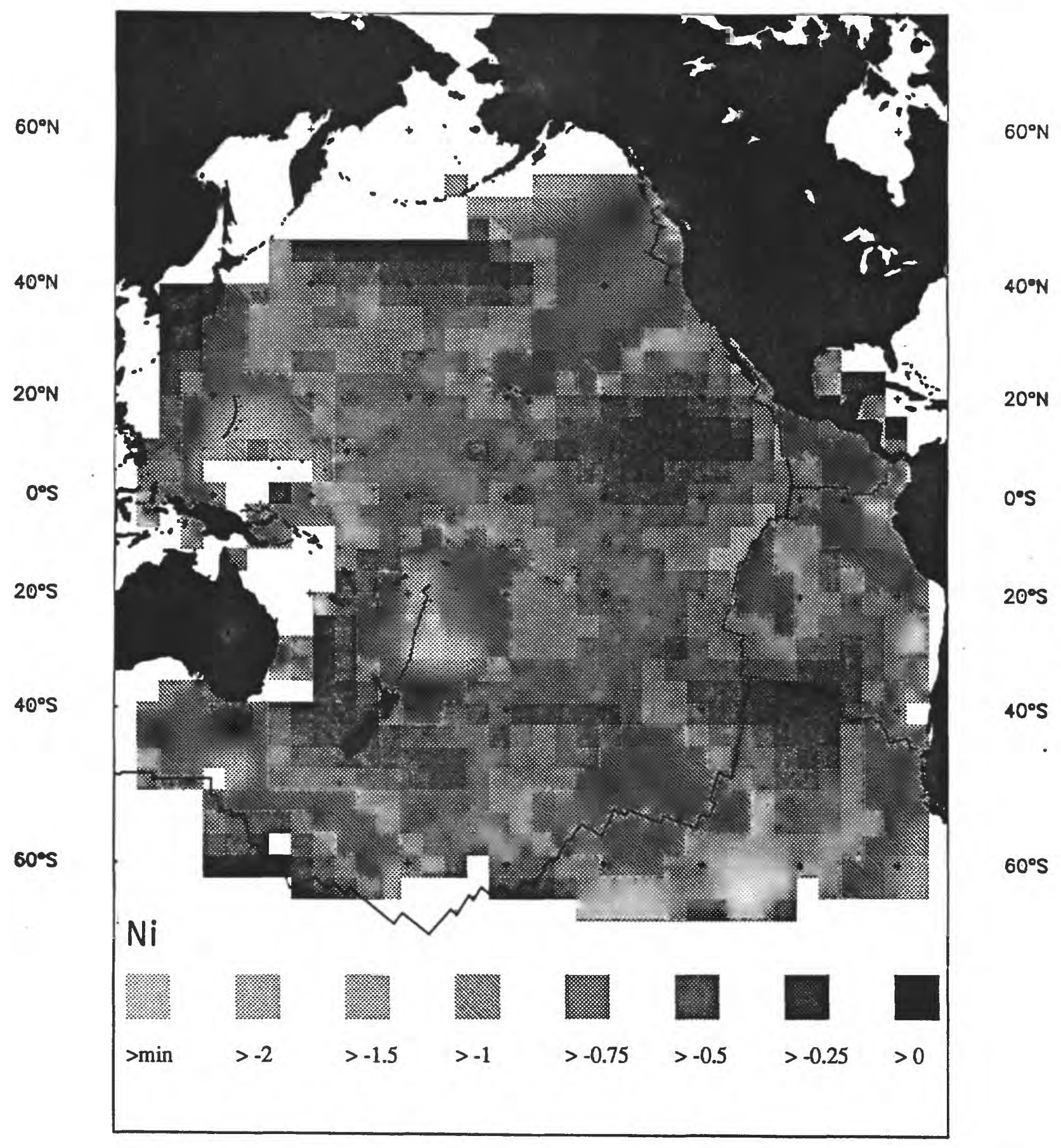

Figure IX-34 Gray scale map of LOG3Ni\% in the Pacific Ocean. 
$120^{\circ} \mathrm{E} \quad 140^{\circ} \mathrm{E} \quad 160^{\circ} \mathrm{E} \quad 180^{\circ} \mathrm{W} \quad 160^{\circ} \mathrm{W} \quad 140^{\circ} \mathrm{W} \quad 120^{\circ} \mathrm{W} \quad 100^{\circ} \mathrm{W} \quad 80^{\circ} \mathrm{W}$

$60^{\circ} \mathrm{N}$

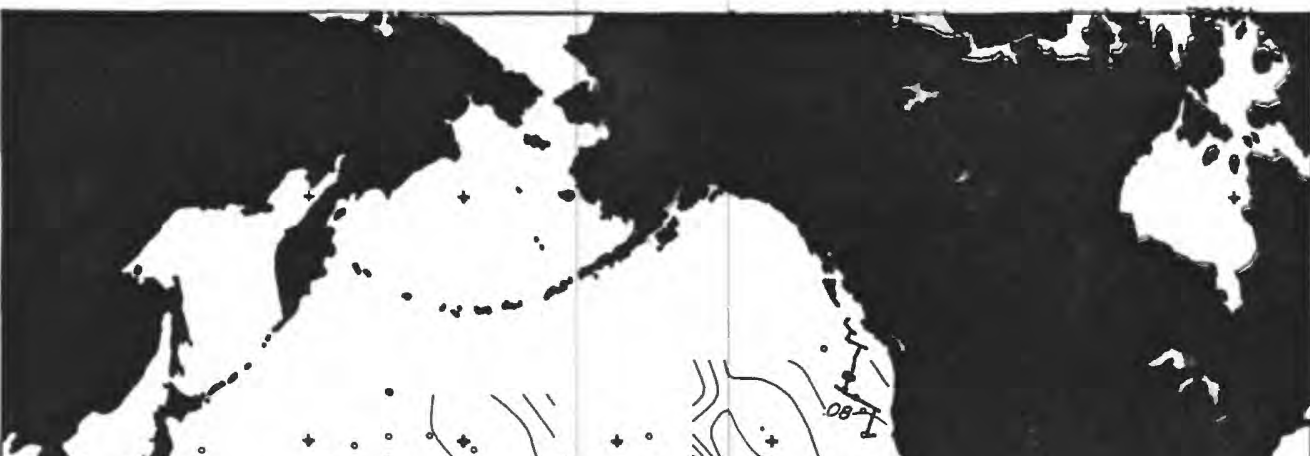

$60^{\circ} \mathrm{N}$

$40^{\circ} \mathrm{N}$

$40^{\circ} \mathrm{N}$

ors

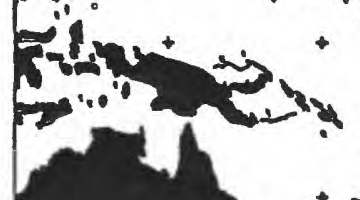

$20^{\circ} \mathrm{S}$

$40^{\circ} \mathrm{S}$

$60^{\circ} \mathrm{S}$

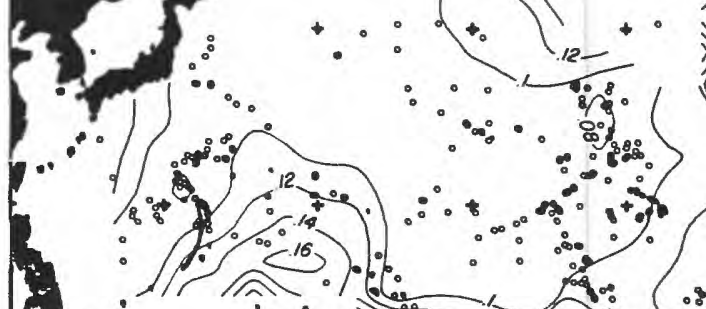

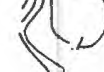

$20^{\circ} \mathrm{N}$
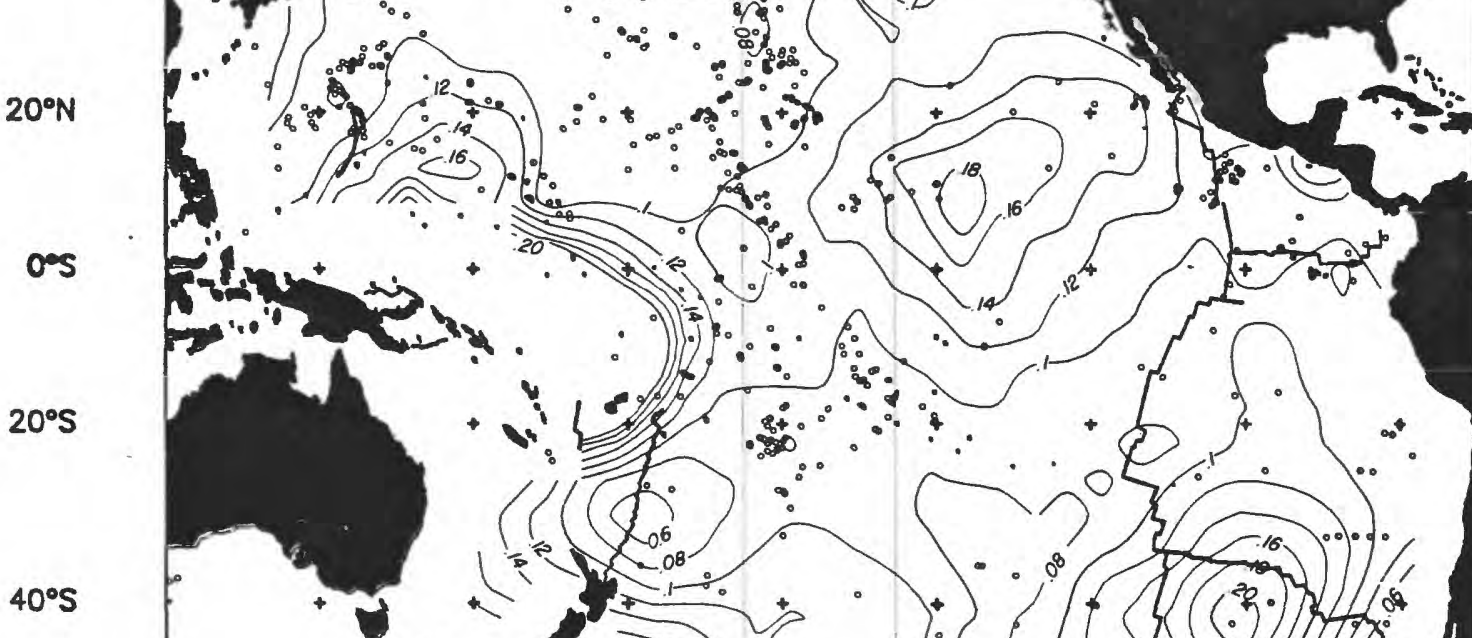

$20^{\circ} N$

$0^{\circ} \mathrm{S}$

$20^{\circ} \mathrm{S}$

$40^{\circ} \mathrm{S}$

$60^{\circ} \mathrm{S}$

Figure IX-35 Elemental concentration of $\mathrm{Zn} \%$ in the Pacific 
$120^{\circ} \mathrm{E} \quad 140^{\circ} \mathrm{E} \quad 160^{\circ} \mathrm{E} \quad 180^{\circ} \mathrm{W} \quad 160^{\circ} \mathrm{W} \quad 140^{\circ} \mathrm{W} \quad 120 \% \mathrm{~W} 100^{\circ} \mathrm{W} \quad 80^{\circ} \mathrm{W}$

$60^{\circ} \mathrm{N}$
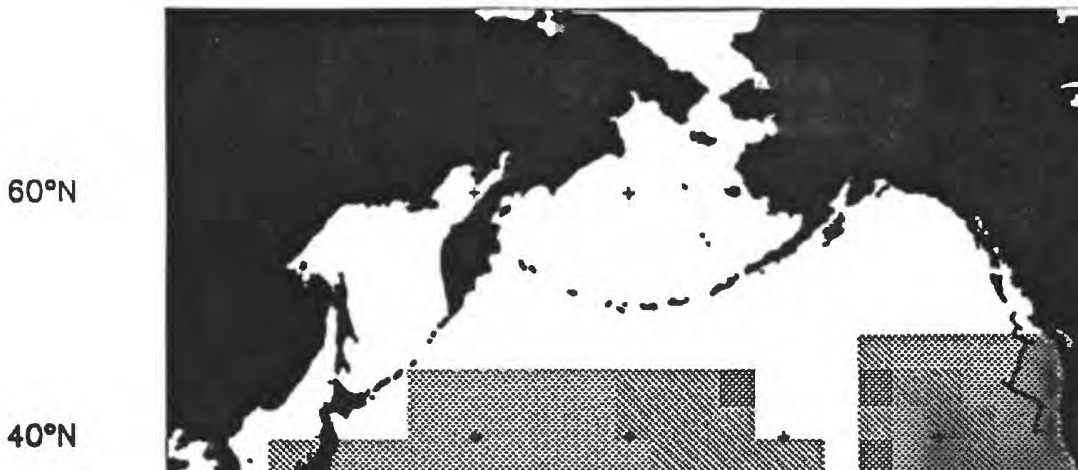

$40^{\circ} \mathrm{N}$

$20^{\circ} \mathrm{N}$

$0^{\circ} \mathrm{S}$

$20^{\circ} \mathrm{S}$

$40^{\circ} \mathrm{S}$
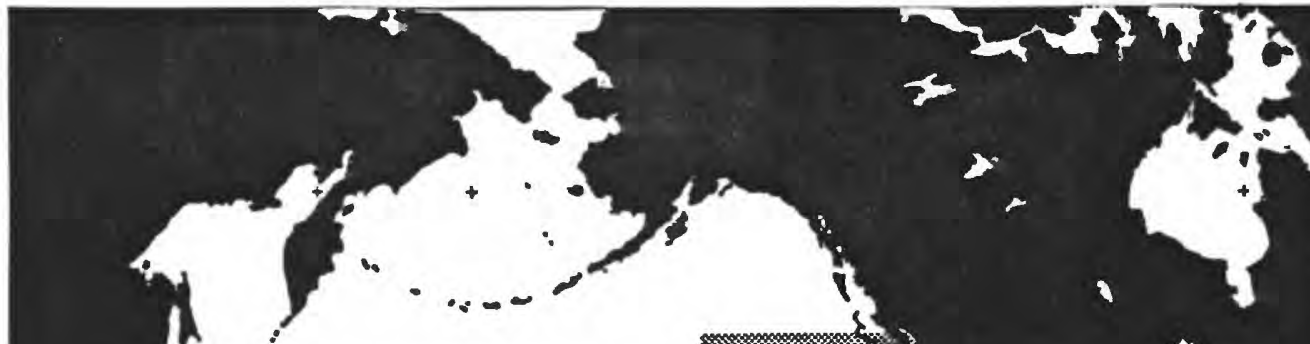

$60^{\circ} \mathrm{N}$

$40^{\circ} \mathrm{N}$
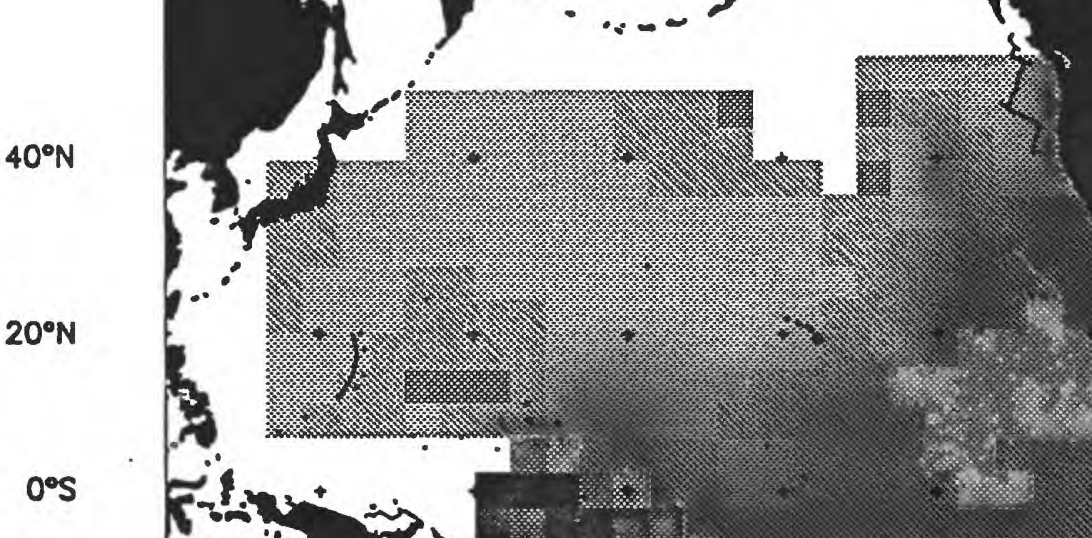
$120^{\circ} \mathrm{E} \quad 140^{\circ} \mathrm{E} \quad 160^{\circ} \mathrm{E} \quad 180^{\circ} \mathrm{W} \quad 160^{\circ} \mathrm{W} \quad 140^{\circ} \mathrm{W} \quad 120^{\circ} \mathrm{W} \quad 100^{\circ} \mathrm{W} \quad 80^{\circ} \mathrm{W}$

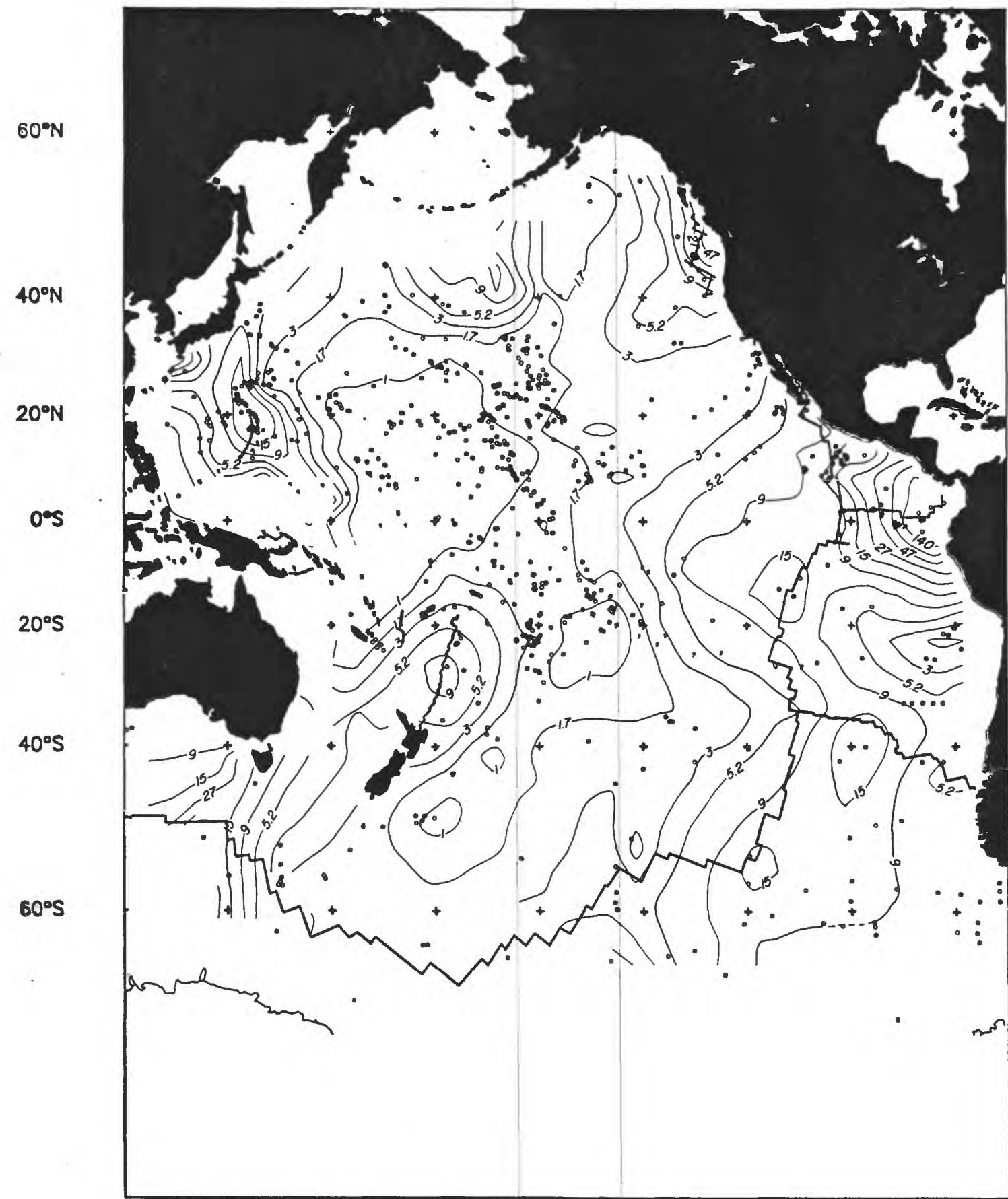

$60^{\circ} \mathrm{N}$

$40^{\circ} \mathrm{N}$

$20^{\circ} \mathrm{N}$

$0^{\circ}$

$20^{\circ} \mathrm{S}$

$40^{\circ} \mathrm{S}$

$60^{\circ} \mathrm{S}$

Figure IX-37 Accumulation rate R(mm/m.y.) in the Pacific Ocean; c.i. $n=.5$ for $R=3 n$. 
$120^{\circ} \mathrm{E} \quad 140^{\circ} \mathrm{E} \quad 160^{\circ} \mathrm{E} \quad 180^{\circ} \mathrm{W} \quad 160^{\circ} \mathrm{W} \quad 140^{\circ} \mathrm{W} \quad 120^{\circ} \mathrm{W} \quad 100^{\circ} \mathrm{W} \quad 80^{\circ} \mathrm{W}$

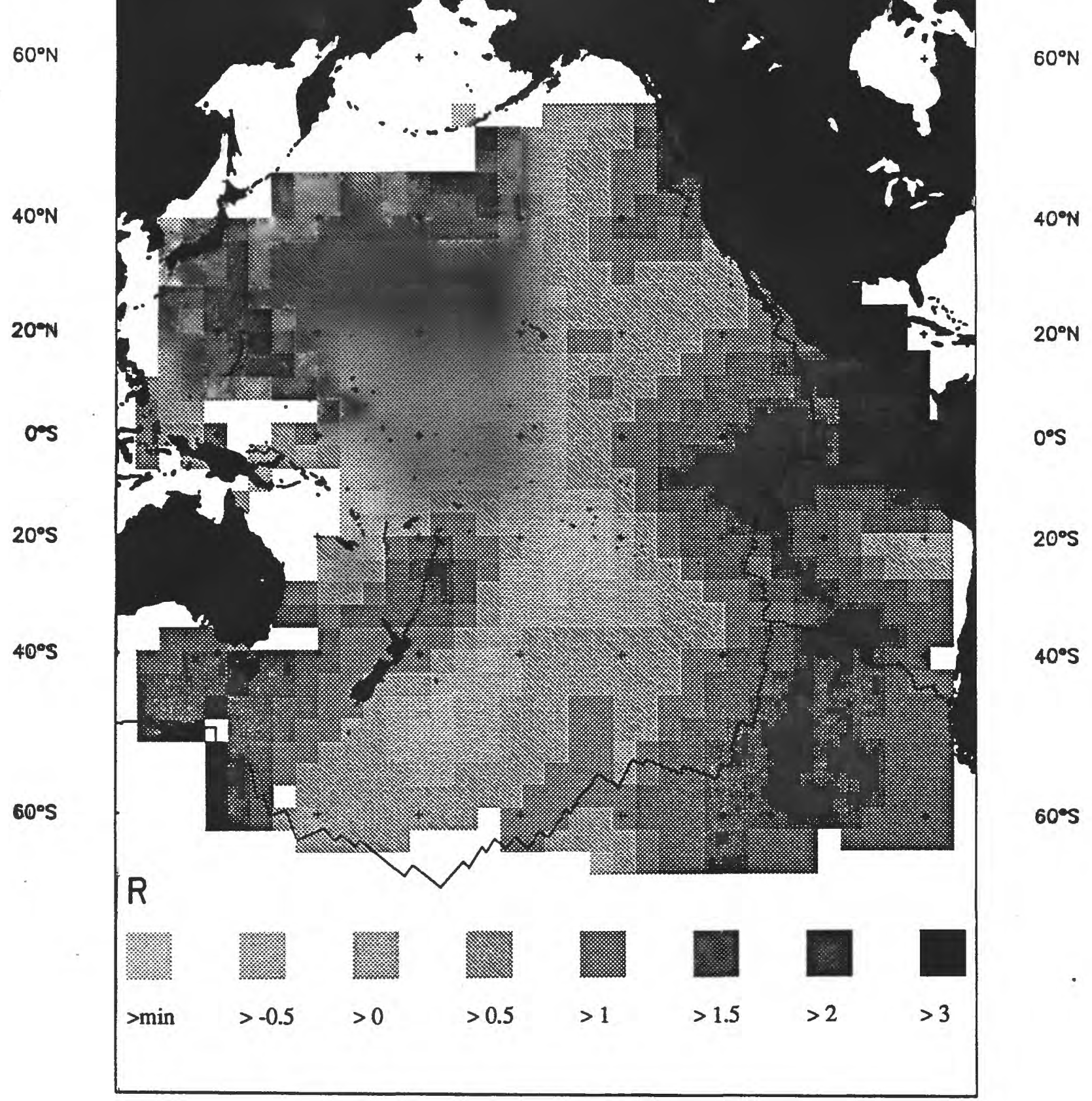

Figure IX-38 Gray scale map of Rmm/m.y. in the Pacific Ocean. 
$120^{\circ} \mathrm{E} \quad 140^{\circ} \mathrm{E} \quad 160^{\circ} \mathrm{E} \quad 180^{\circ} \mathrm{W} \quad 160^{\circ} \mathrm{W} \quad 140^{\circ} \mathrm{W} \quad 120^{\circ} \mathrm{W} \quad 100^{\circ} \mathrm{W} \quad 80^{\circ} \mathrm{W}$

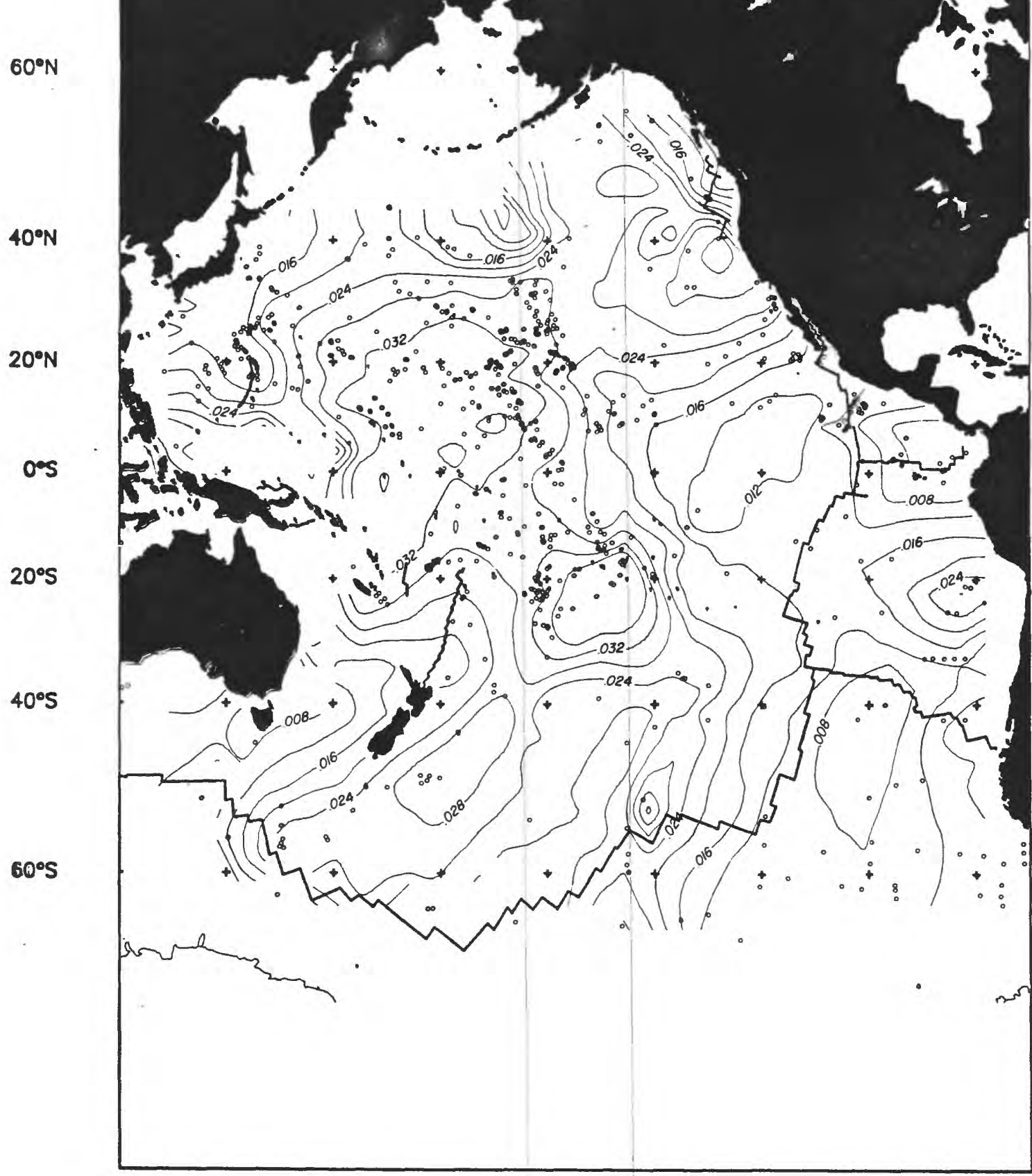

$60^{\circ} \mathrm{N}$

$40^{\circ} \mathrm{N}$

$20^{\circ} \mathrm{N}$

o.s

$20^{\circ} \mathrm{S}$

$40^{\circ} \mathrm{S}$

$60^{\circ} \mathrm{S}$

Figure IX-39 Elemental concentration of Co/Mn\% in the Pacific Ocean, c.i. $=.008 \%$ 
$120^{\circ} \mathrm{E} \quad 140^{\circ} \mathrm{E} \quad 160^{\circ} \mathrm{E} \quad 180^{\circ} \mathrm{W} \quad 160^{\circ} \mathrm{W} \quad 140^{\circ} \mathrm{W} \quad 120^{\circ} \mathrm{W} \quad 100^{\circ} \mathrm{W} \quad 80^{\circ} \mathrm{W}$

$60^{\circ} \mathrm{N}$

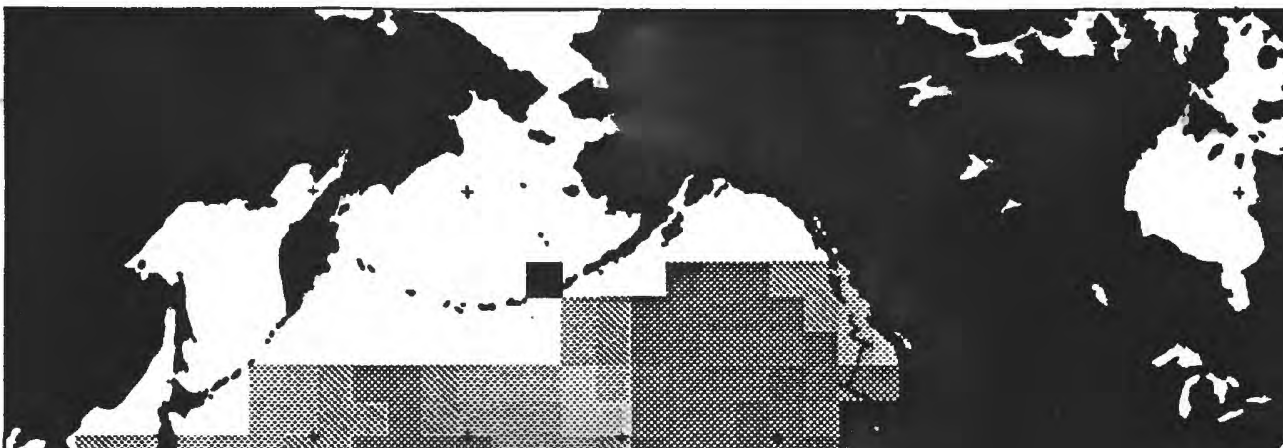

$60^{\circ} \mathrm{N}$

$40^{\circ} \mathrm{N}$

$40^{\circ} \mathrm{N}$

$20^{\circ} \mathrm{N}$

$0^{\circ} \mathrm{S}$

$20^{\circ} \mathrm{S}$

$40^{\circ} \mathrm{S}$

6005
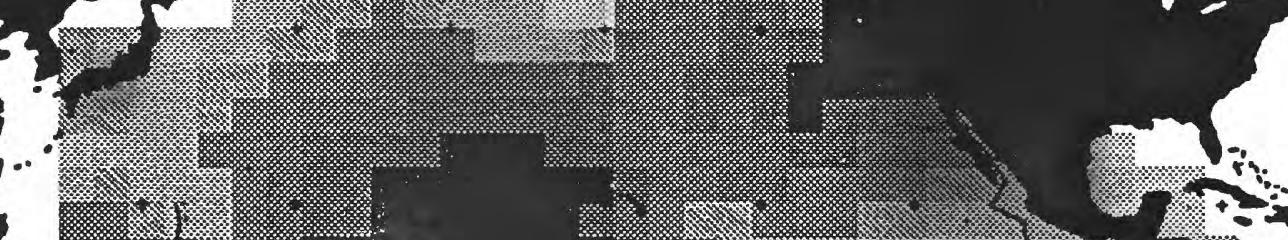

$20^{\circ} \mathrm{N}$

$0^{\circ} 5$

$20^{\circ} \mathrm{S}$

$40^{\circ} \mathrm{S}$

$60^{\circ} \mathrm{S}$

$\mathrm{Co} / \mathrm{Mn}$

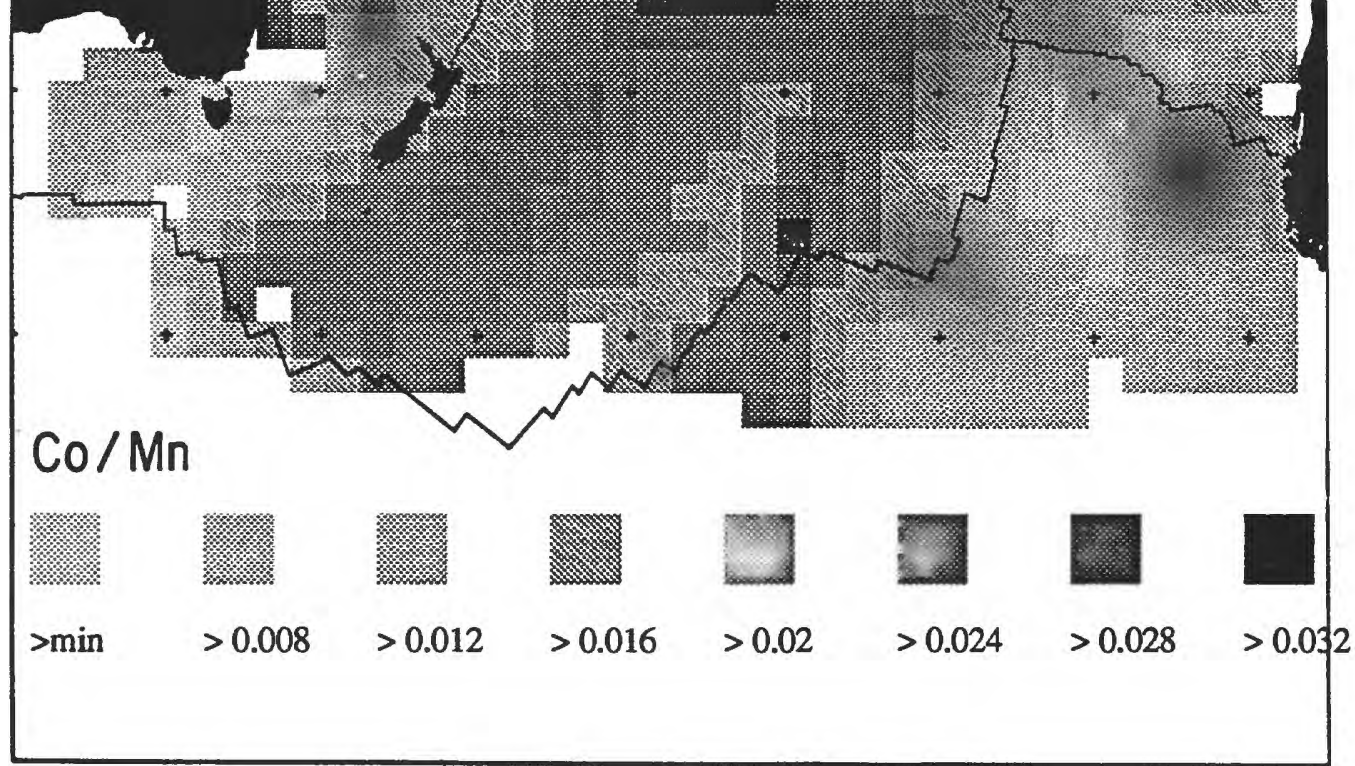

Figure IX-40 Gray scale map of $\mathrm{Co} / \mathrm{Mn} \%$ in the Pacific Ocean. 
$120^{\circ} \mathrm{E} \quad 140^{\circ} \mathrm{E} \quad 160^{\circ} \mathrm{E} \quad 180^{\circ} \mathrm{W} \quad 160^{\circ} \mathrm{W} \quad 1400^{\circ} \mathrm{W} \quad 120^{\circ} \mathrm{W} \quad 100^{\circ} \mathrm{W} \quad 809 \mathrm{~W}$

$60^{\circ} \mathrm{N}$

$40^{\circ} \mathrm{N}$

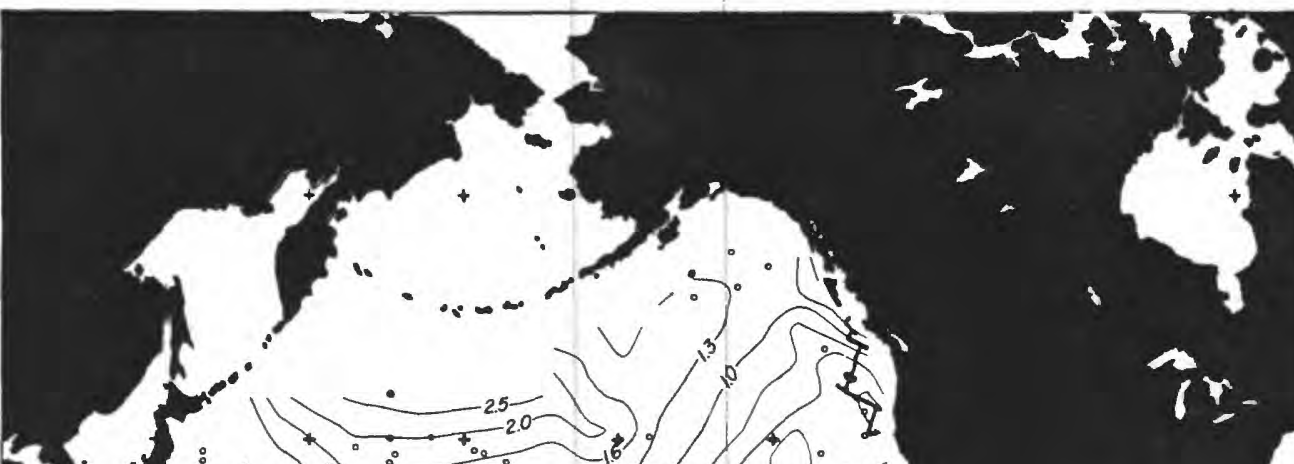

$60^{\circ} \mathrm{N}$

$40^{\circ} \mathrm{N}$

$20^{\circ} N$

$0^{\circ} \mathrm{S}$

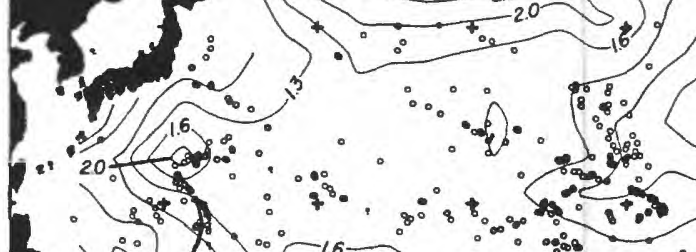

$20^{\circ} \mathrm{N}$

o's

$20^{\circ} \mathrm{S}$

$40^{\circ} \mathrm{S}$

$60^{\circ} \mathrm{S}$

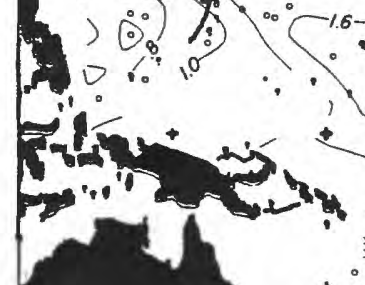


$120^{\circ} \mathrm{E} \quad 140^{\circ} \mathrm{E} \quad 160^{\circ} \mathrm{E} \quad 180^{\circ} \mathrm{W} \quad 160^{\circ} \mathrm{W} \quad 140^{\circ} \mathrm{W} \quad 120 \% \mathrm{~W} \quad 100^{\circ} \mathrm{W} \quad 80^{\circ} \mathrm{W}$

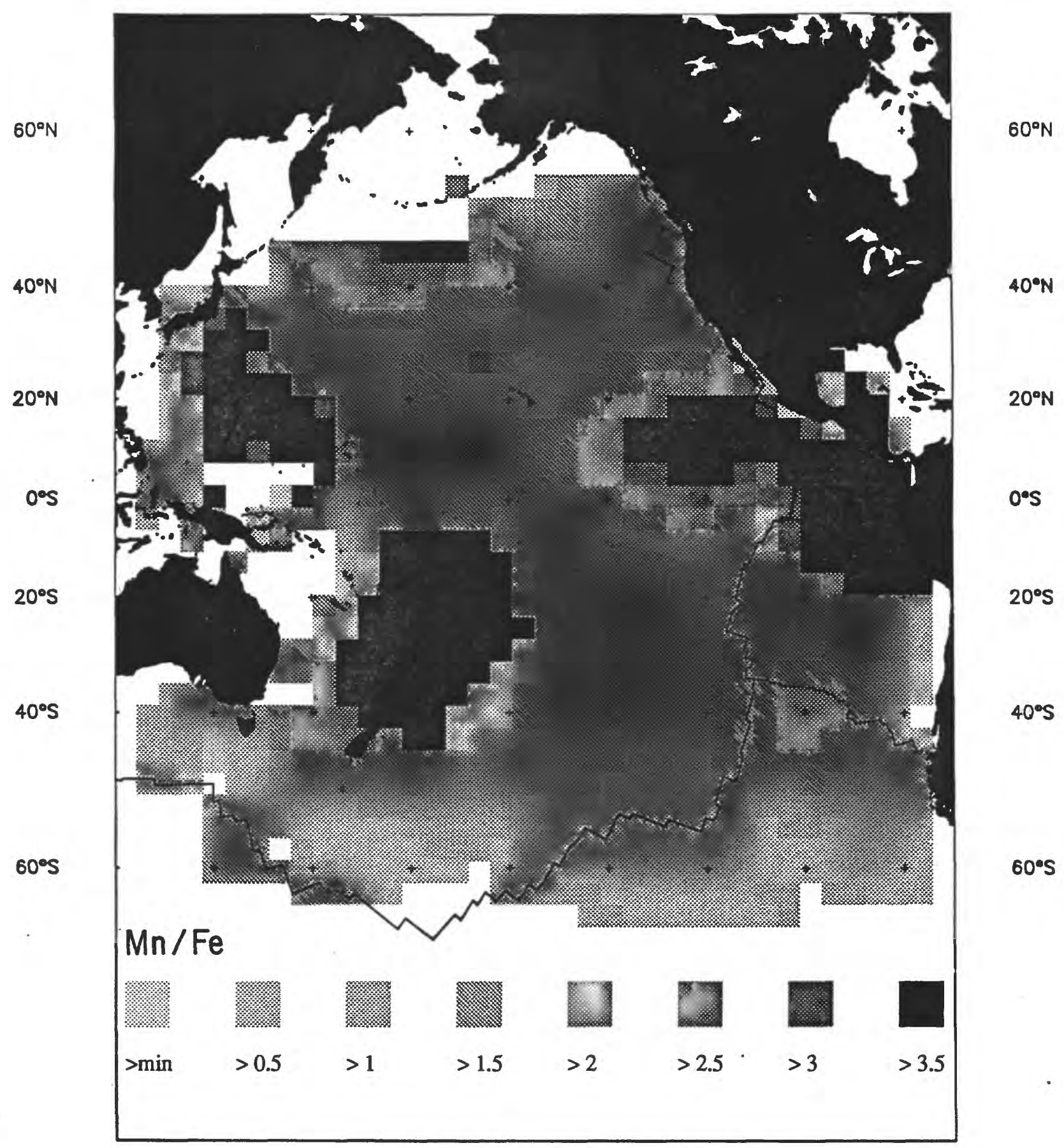

Figure IX-42 Gray scale map of $\mathrm{Mn} / \mathrm{Fe} \%$ in the Pacific Ocean. 
$120^{\circ} \mathrm{E} \quad 140^{\circ} \mathrm{E} \quad 160^{\circ} \mathrm{E} \quad 180^{\circ} \mathrm{W} \quad 160^{\circ} \mathrm{W} \quad 140^{\circ} \mathrm{W} \quad 120^{\circ} \mathrm{W} \quad 100^{\circ} \mathrm{W} \quad 80^{\circ} \mathrm{W}$

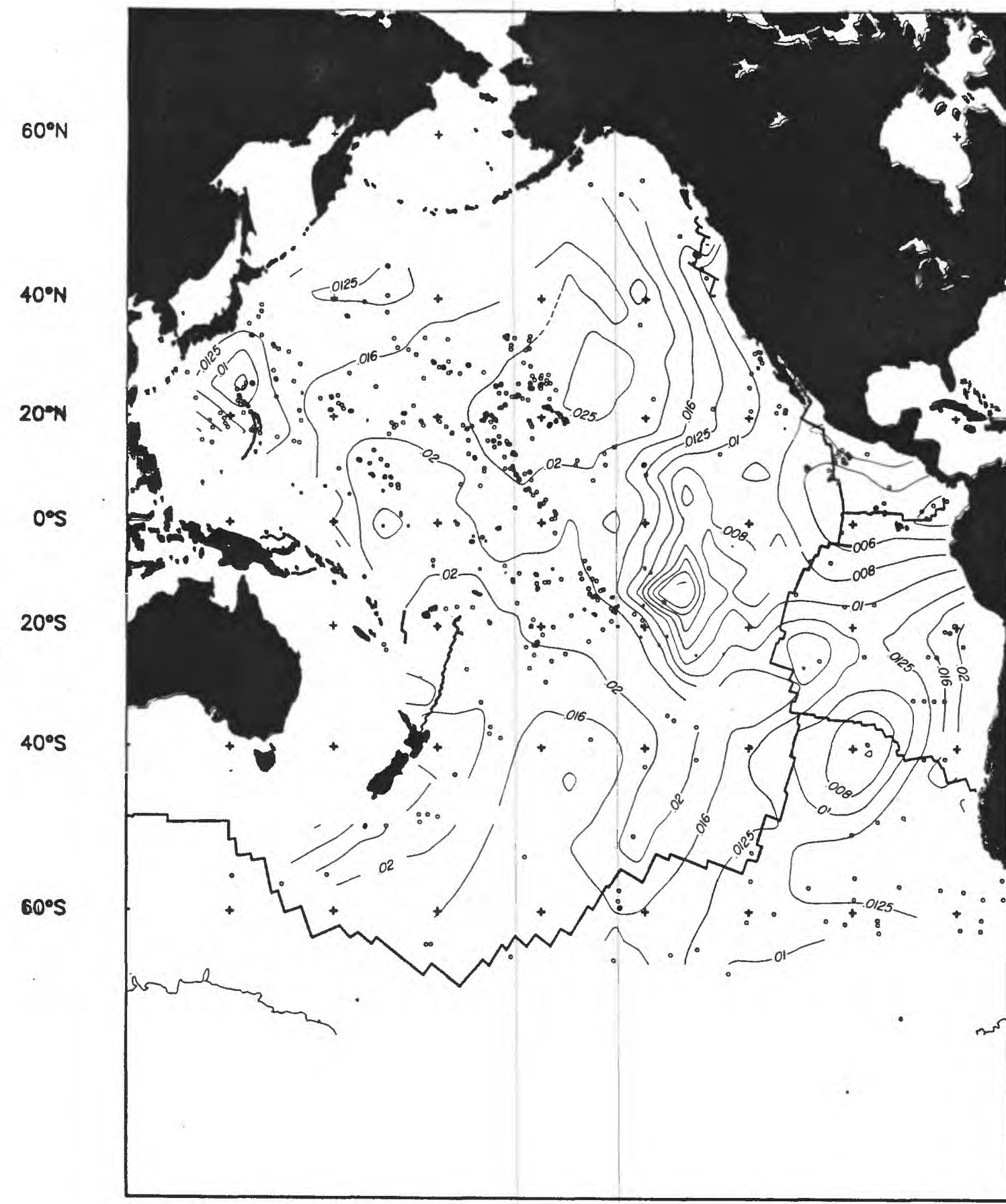

$60^{\circ} \mathrm{N}$

$40^{\circ} \mathrm{N}$

$20^{\circ} \mathrm{N}$

$0^{\circ}$

$20^{\circ} \mathrm{S}$

$40^{\circ} \mathrm{S}$

$60^{\circ} \mathrm{S}$

Figure IX-43 Elemental concentration of Ti/(Mn+Fe) in the Pacific Ocean; c.i. $n=.1$ for $T i /(M n+F e)=10 n$. 
$120^{\circ} \mathrm{E} \quad 140^{\circ} \mathrm{E} \quad 160^{\circ} \mathrm{E} \quad 180^{\circ} \mathrm{W} \quad 160^{\circ} \mathrm{W} \quad 140^{\circ} \mathrm{W} \quad 120^{\circ} \mathrm{W} \quad 100^{\circ} \mathrm{W} \quad 80^{\circ} \mathrm{W}$

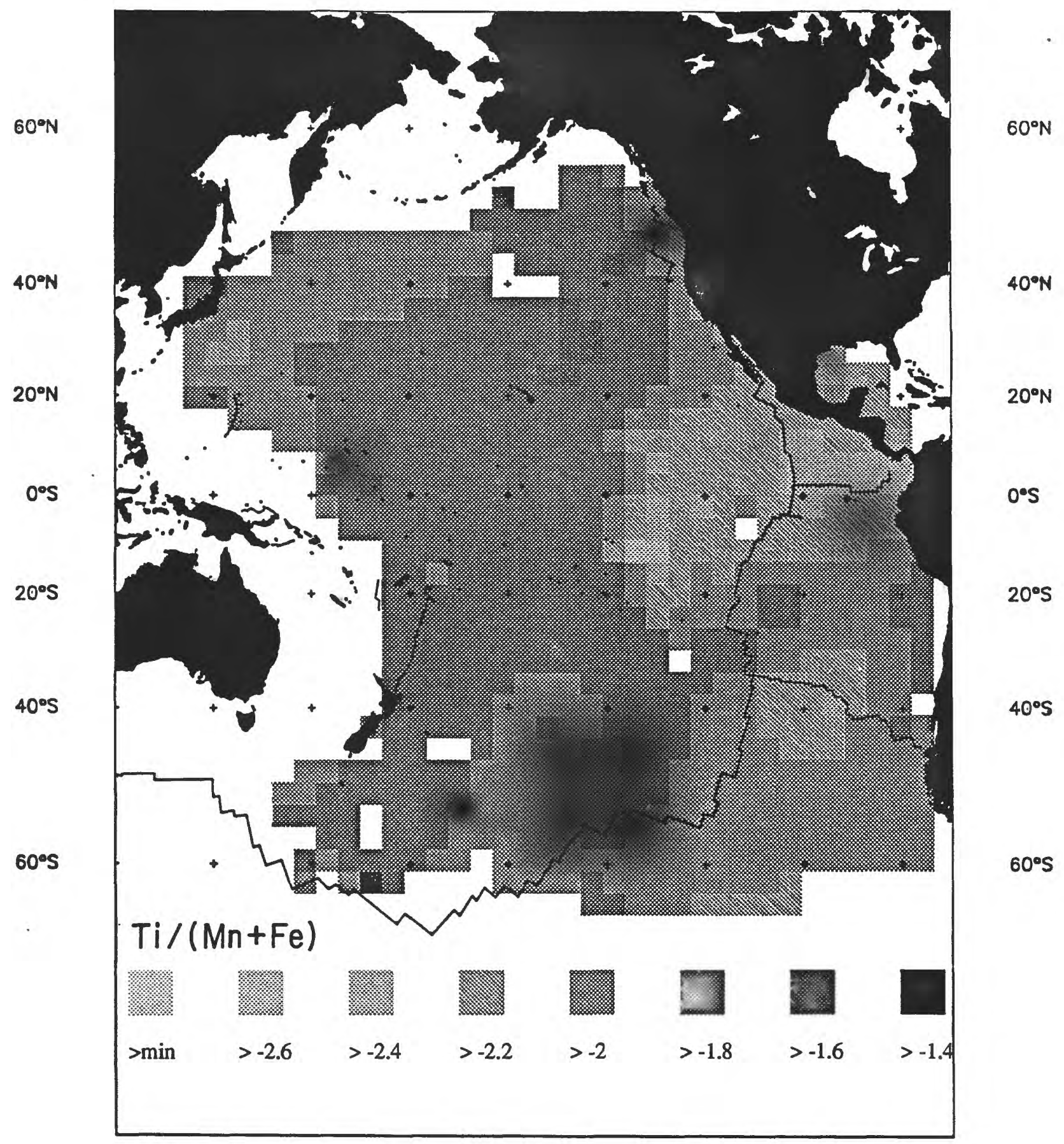

Figure IX-44 Gray scale map of Ti/Mn+Fe(\%) in the Pacific Ocean. 
$120^{\circ} \mathrm{E} \quad 140^{\circ} \mathrm{E} \quad 160^{\circ} \mathrm{E} \quad 180^{\circ} \mathrm{W} \quad 160^{\circ} \mathrm{W} \quad 140^{\circ} \mathrm{W} \quad 120{ }^{\circ} \mathrm{W} \quad 100^{\circ} \mathrm{W} \quad 80^{\circ} \mathrm{W}$

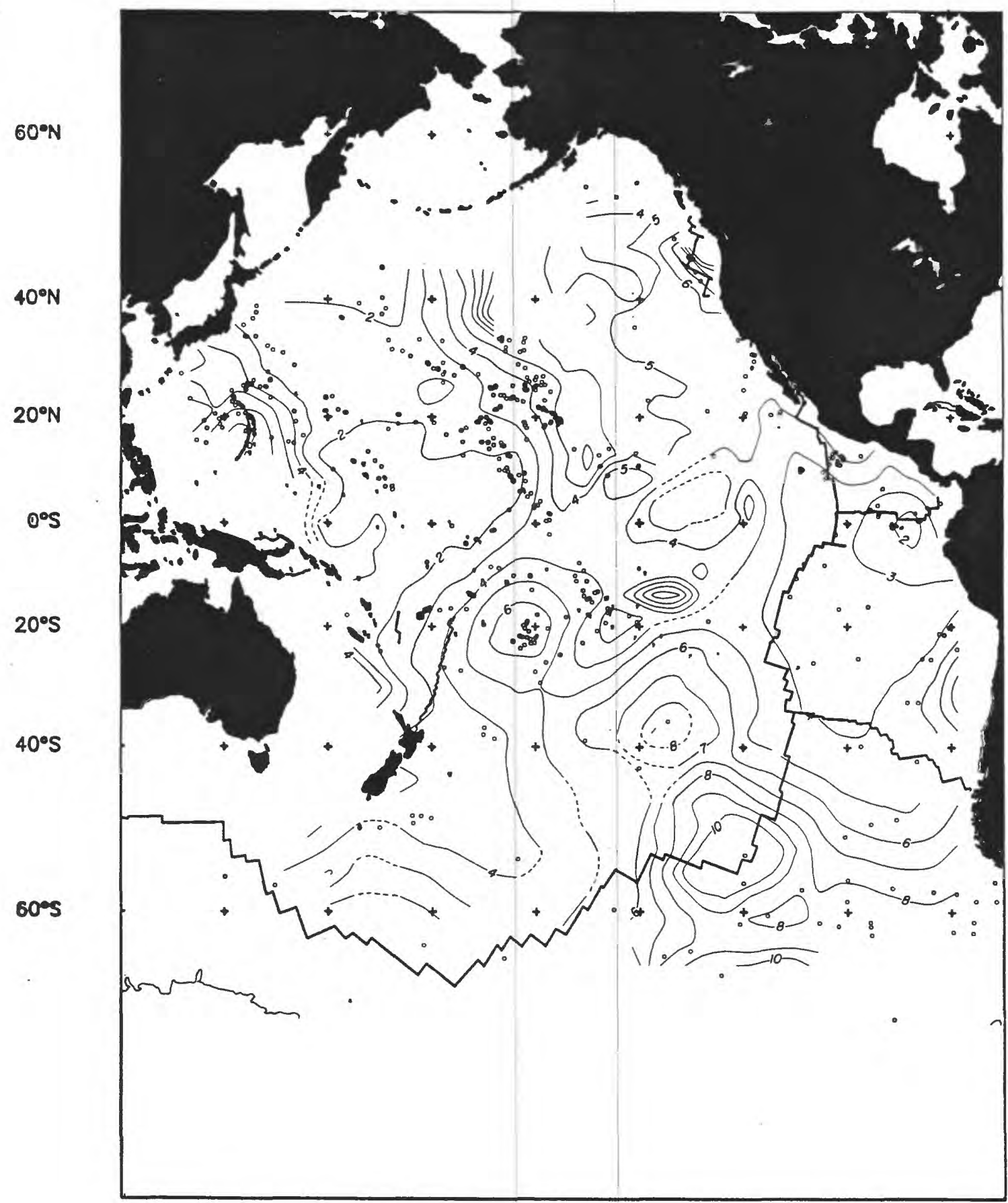

$60^{\circ} \mathrm{N}$

$40^{\circ} \mathrm{N}$

$20^{\circ} \mathrm{N}$

$0^{\circ} \mathrm{S}$

$20^{\circ} 5$

$40^{\circ} \mathrm{S}$

$60^{\circ} \mathrm{S}$

Figure IX-45 Elemental concentration of $\mathrm{Al}_{2} \mathrm{O}_{3} \%$ in the Pacific Ocean, c.i. $=1 \%$ 
$120^{\circ} \mathrm{E} \quad 140^{\circ} \mathrm{E} \quad 160^{\circ} \mathrm{E} \quad 180^{\circ} \mathrm{W} \quad 160^{\circ} \mathrm{W} \quad 140^{\circ} \mathrm{W} \quad 120^{\circ} \mathrm{W} \quad 100^{\circ} \mathrm{W} \quad 80^{\circ} \mathrm{W}$

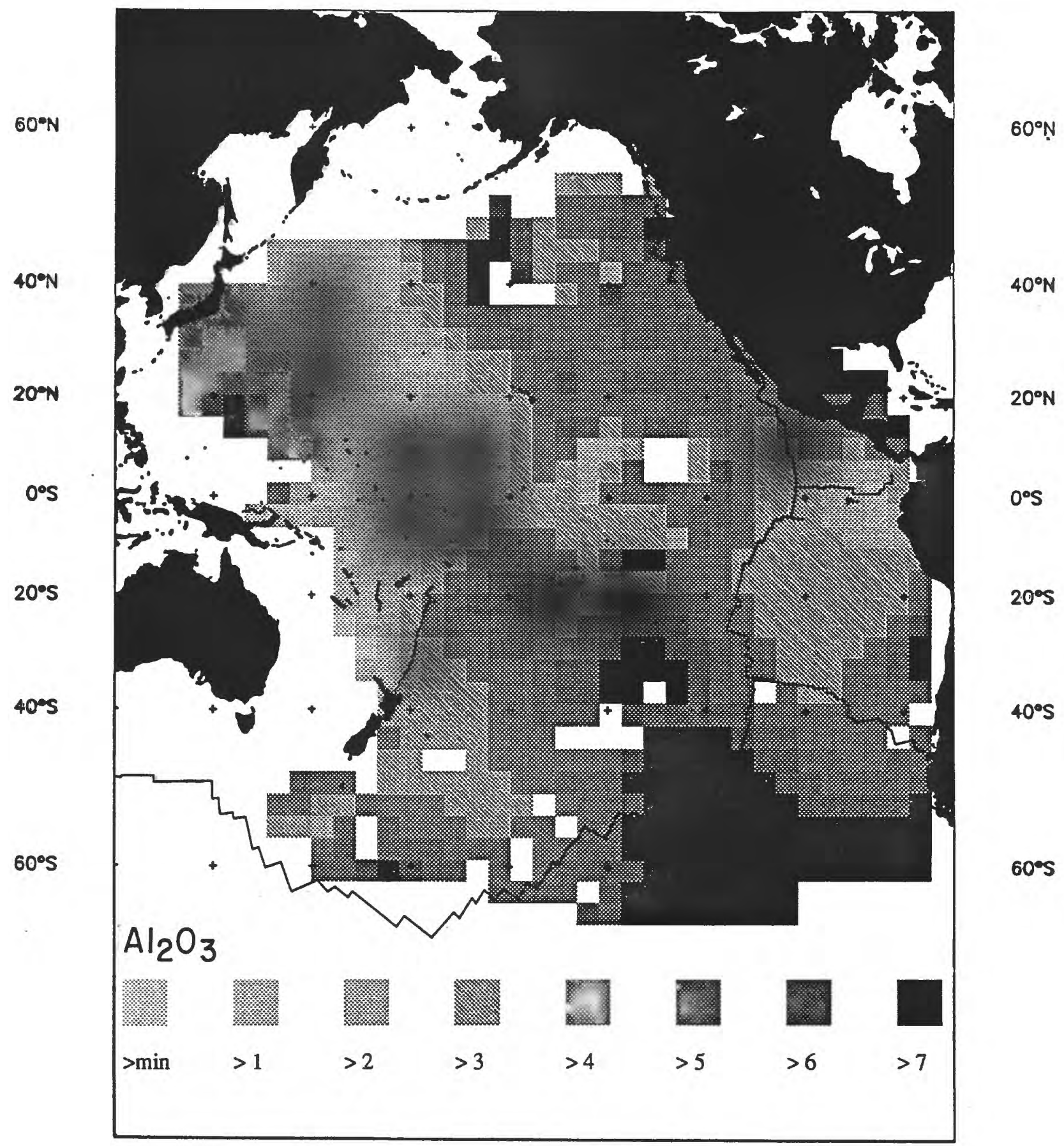

Figure IX-46 Gray scale map of $\mathrm{Al}_{2} \mathrm{O}_{3} \%$ in the Pacific Ocean. 
$120^{\circ} \mathrm{E} \quad 140^{\circ} \mathrm{E} \quad 160^{\circ} \mathrm{E} \quad 180^{\circ} \mathrm{W} \quad 160^{\circ} \mathrm{W} \quad 140^{\circ} \mathrm{W} \quad 120^{\circ} \mathrm{W} \quad 100^{\circ} \mathrm{W} \quad 80^{\circ} \mathrm{W}$

$60^{\circ} \mathrm{N}$

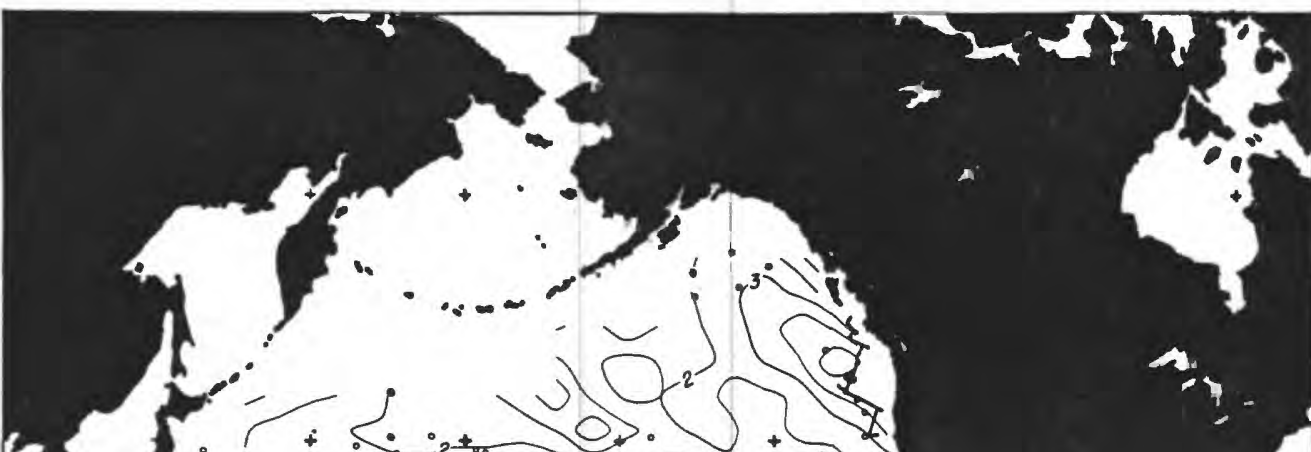

$60^{\circ} \mathrm{N}$

$40^{\circ} \mathrm{N}$

$20^{\circ} \mathrm{N}$

$20^{\circ} \mathrm{N}$

$20^{\circ} \mathrm{S}$

$40^{\circ} \mathrm{S}$

$60{ }^{\circ}$

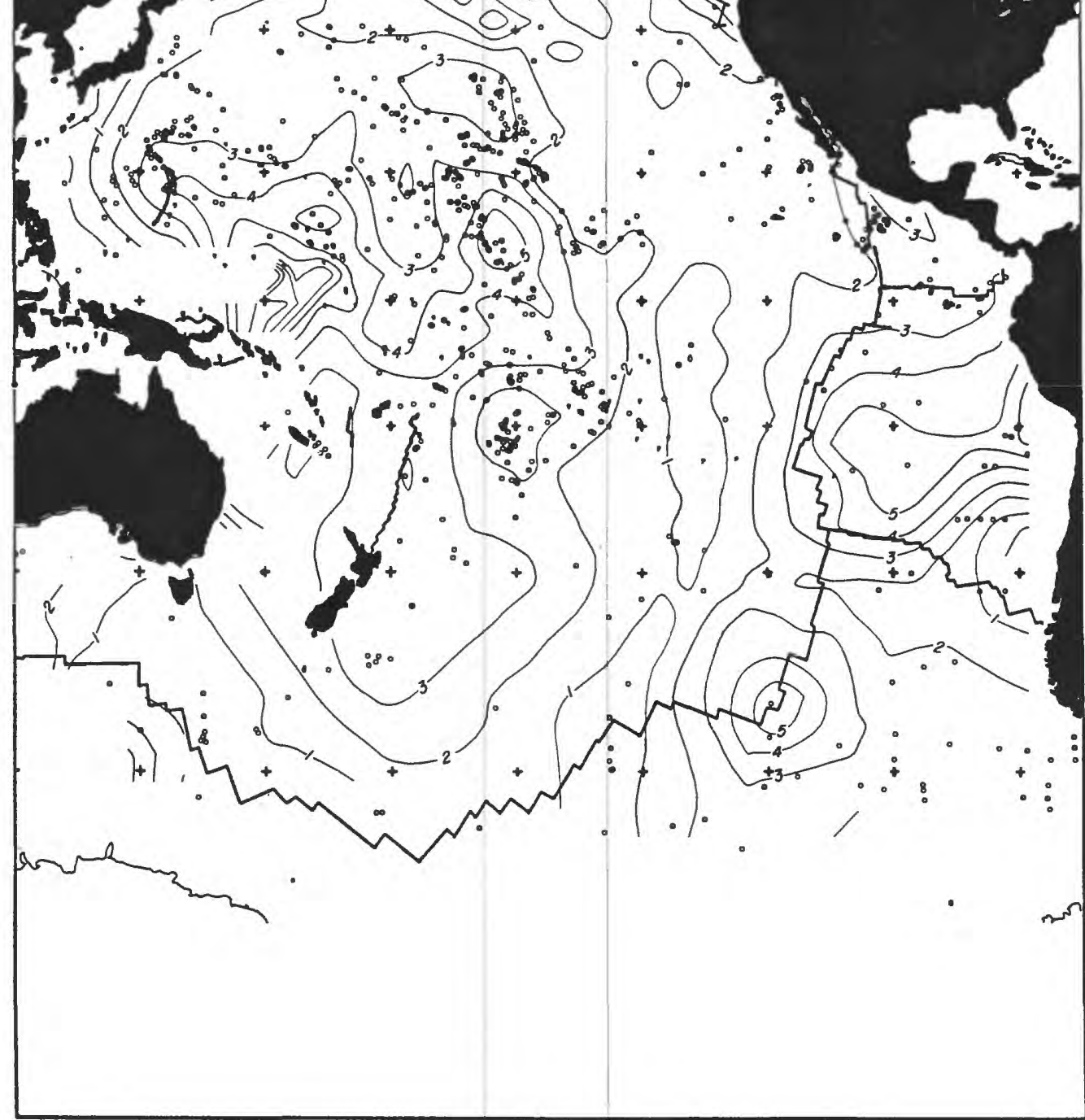

$0^{\circ}$ 
$120^{\circ} \mathrm{E} \quad 140^{\circ} \mathrm{E} \quad 160^{\circ} \mathrm{E} \quad 180^{\circ} \mathrm{W} \quad 160^{\circ} \mathrm{W} \quad 140^{\circ} \mathrm{W} \quad 120^{\circ} \mathrm{W} \quad 100^{\circ} \mathrm{W} \quad 80^{\circ} \mathrm{W}$

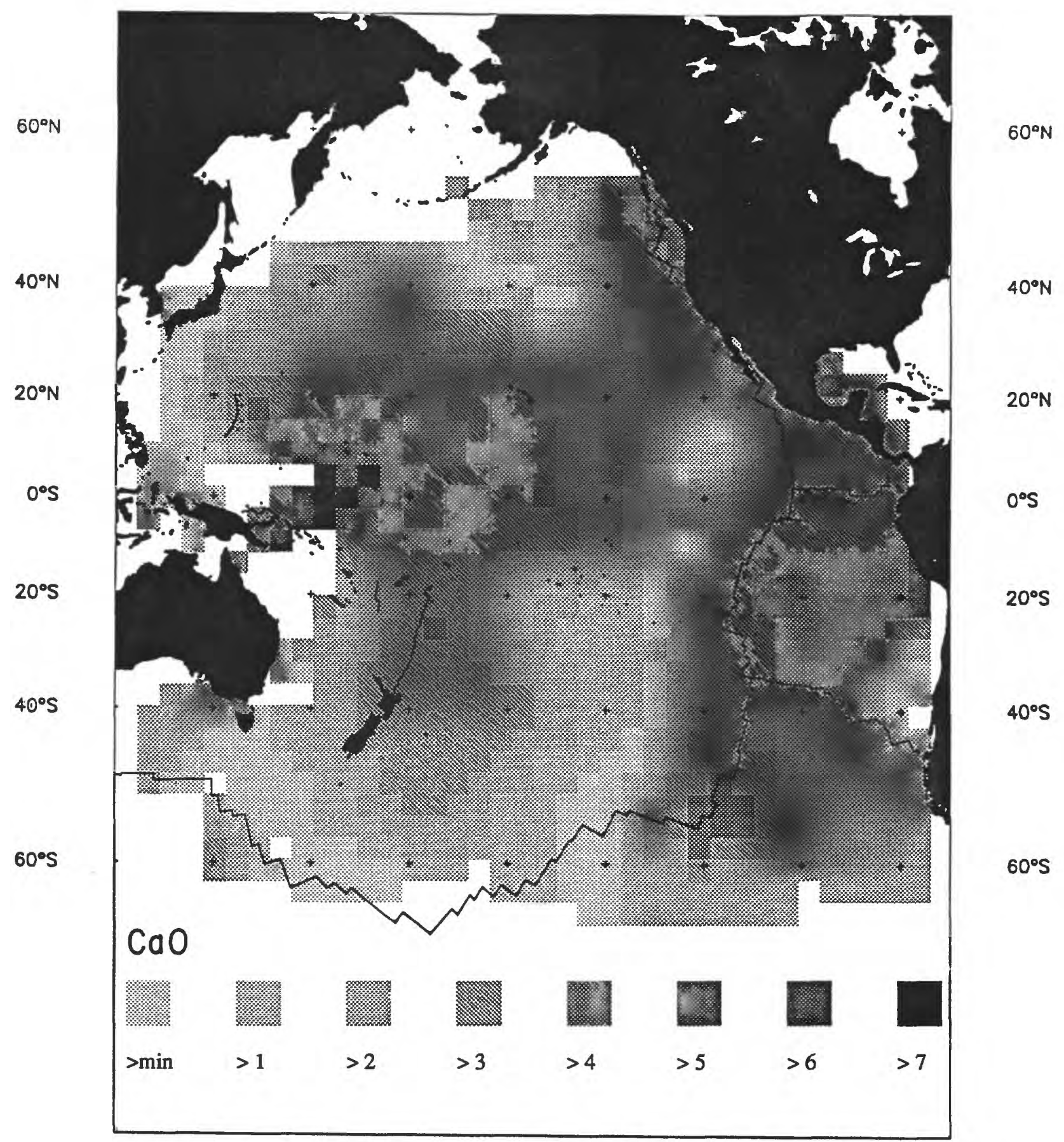

Figure IX-48 Gray scale map of CaO\% in the Pacific Ocean. 
$120^{\circ} \mathrm{E} \quad 140^{\circ} \mathrm{E} \quad 160^{\circ} \mathrm{E} \quad 180^{\circ} \mathrm{W} \quad 160^{\circ} \mathrm{W} \quad 140^{\circ} \mathrm{W} \quad 120^{\circ} \mathrm{W} \quad 100^{\circ} \mathrm{W}$ 80\%

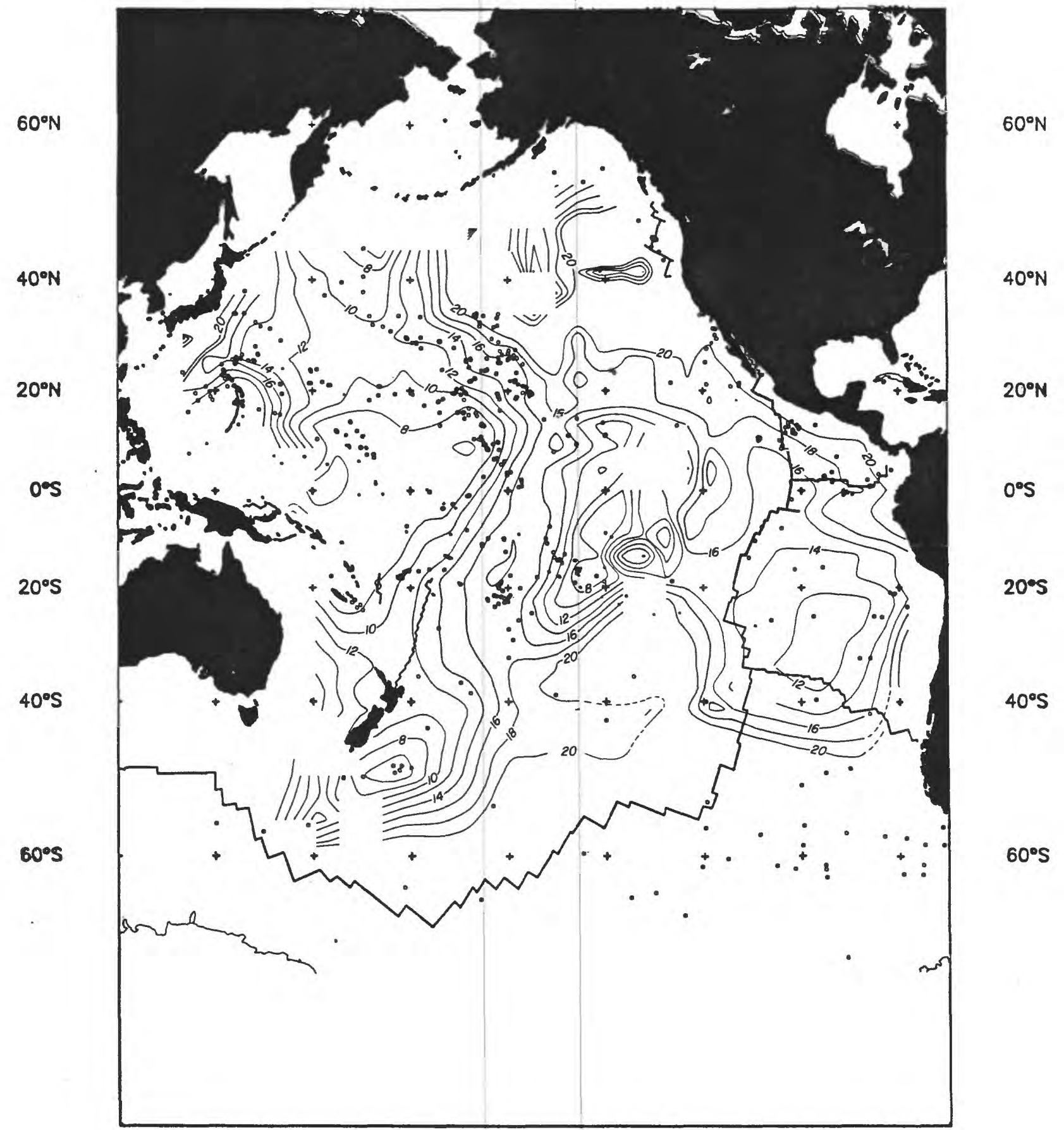

Figure IX-49 Elemental concentration of $\mathrm{SiO}_{2} \%$ in the Pacific Ocean, c.i. $=2 \%$ 
$120^{\circ} \mathrm{E} \quad 140^{\circ} \mathrm{E} \quad 160^{\circ} \mathrm{E} \quad 180^{\circ} \mathrm{W} \quad 160^{\circ} \mathrm{W} \quad 140^{\circ} \mathrm{W} \quad 120^{\circ} \mathrm{W} \quad 100^{\circ} \mathrm{W} \quad 80^{\circ} \mathrm{W}$

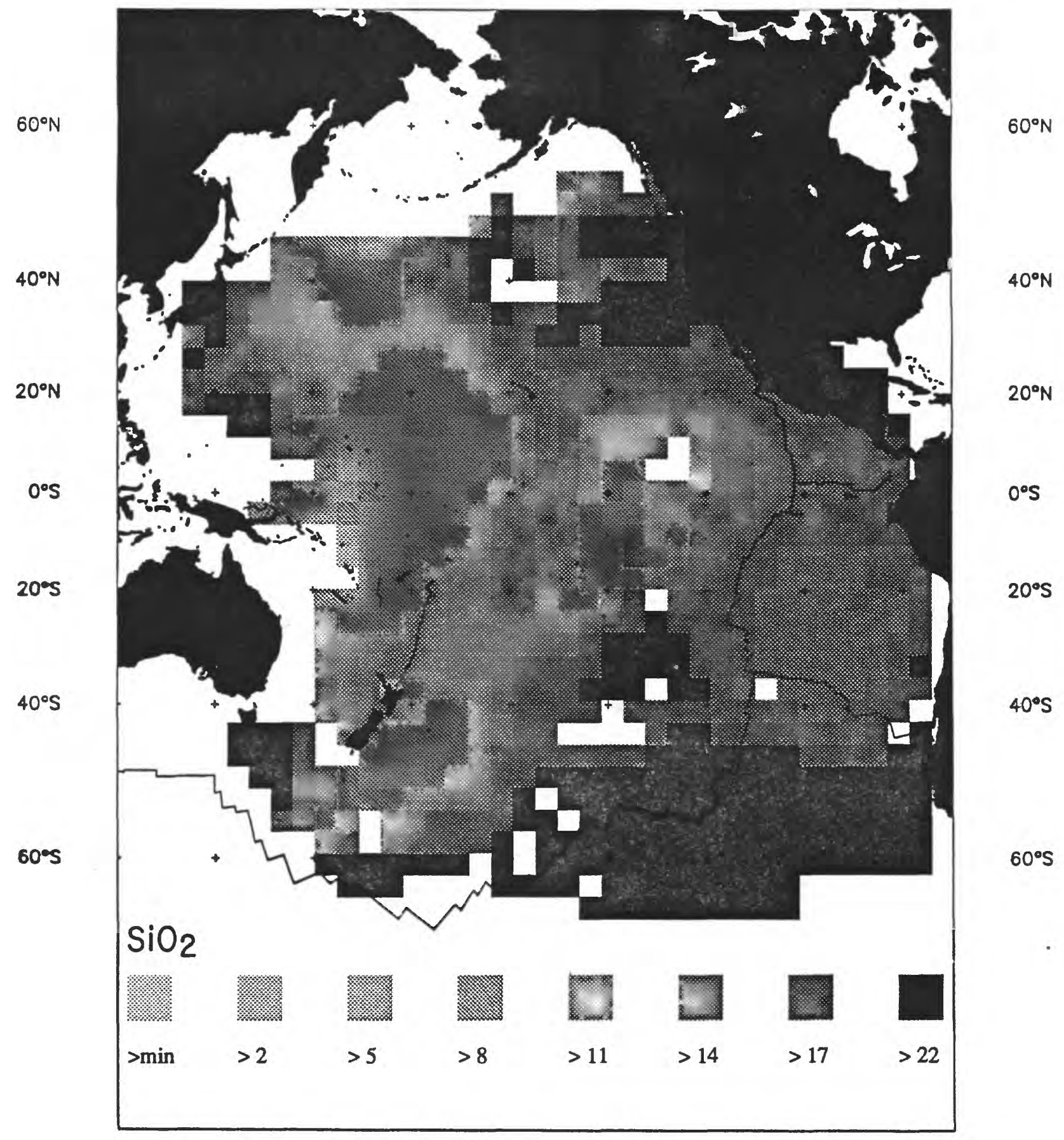

Figure IX-50 Gray scale map of $\mathrm{SiO}_{2} \%$ in the Pacific Ocean. 
$120^{\circ} \mathrm{E} \quad 140^{\circ} \mathrm{E} \quad 160^{\circ} \mathrm{E} \quad 180^{\circ} \mathrm{W} \quad 160^{\circ} \mathrm{W} \quad 140^{\circ} \mathrm{W} \quad 120^{\circ} \mathrm{W} \quad 100^{\circ} \mathrm{W} \quad 80^{\circ} \mathrm{W}$

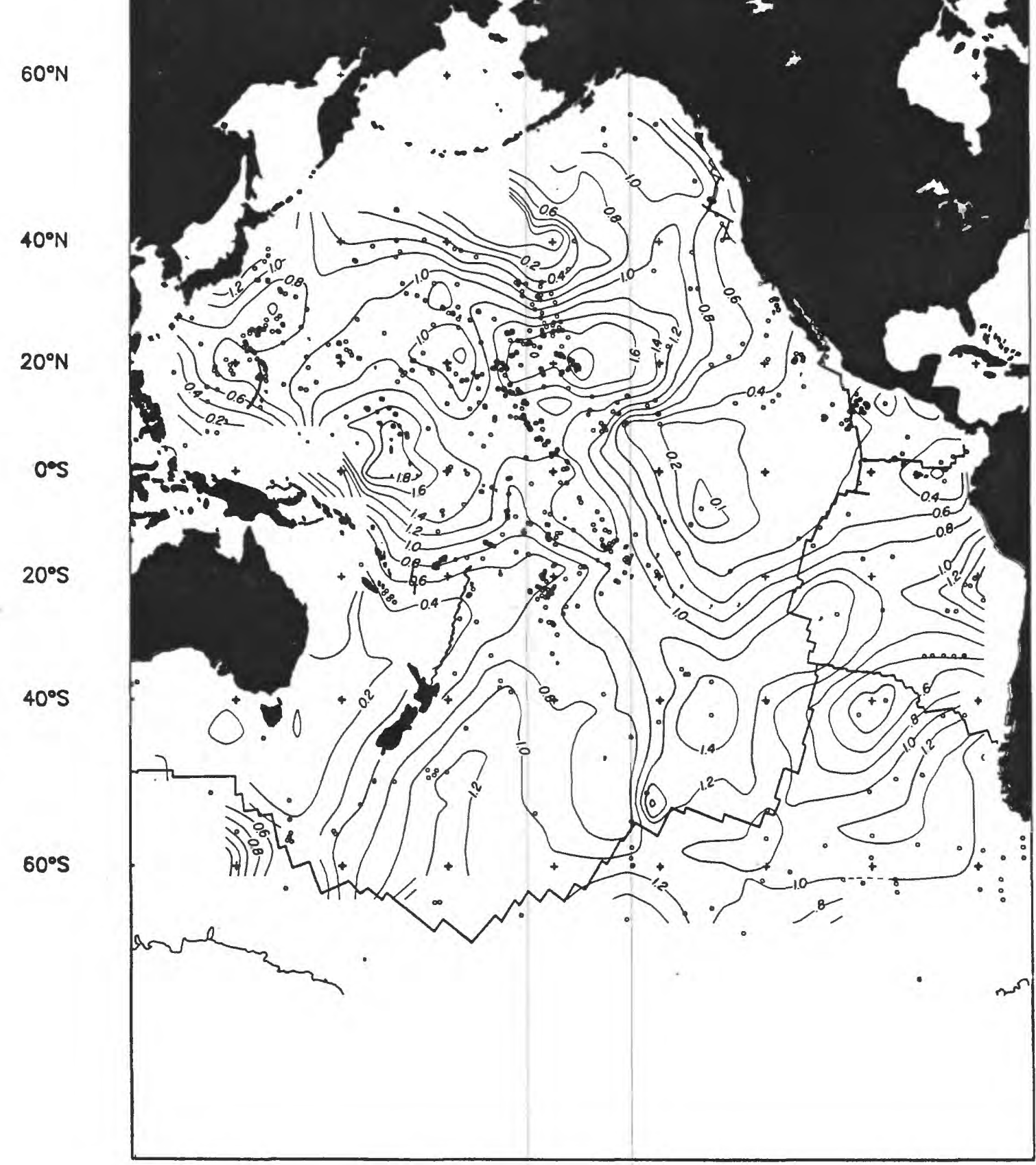

$40^{\circ} \mathrm{N}$

$20^{\circ} \mathrm{N}$

$0^{\circ} \mathrm{S}$

$20^{\circ} \mathrm{S}$

$40^{\circ} \mathrm{S}$

$60^{\circ} \mathrm{S}$

Figure IX-51 Elemental concentration of $\mathrm{TiO}_{2} \%$ in the Pacific Ocean, c.i. $=.2 \%$ 
$120^{\circ} \mathrm{E} \quad 140^{\circ} \mathrm{E} \quad 160^{\circ} \mathrm{E} \quad 180^{\circ} \mathrm{W} \quad 160^{\circ} \mathrm{W} \quad 140^{\circ} \mathrm{W} \quad 120^{\circ} \mathrm{W} \quad 100^{\circ} \mathrm{W} \quad 80^{\circ} \mathrm{W}$

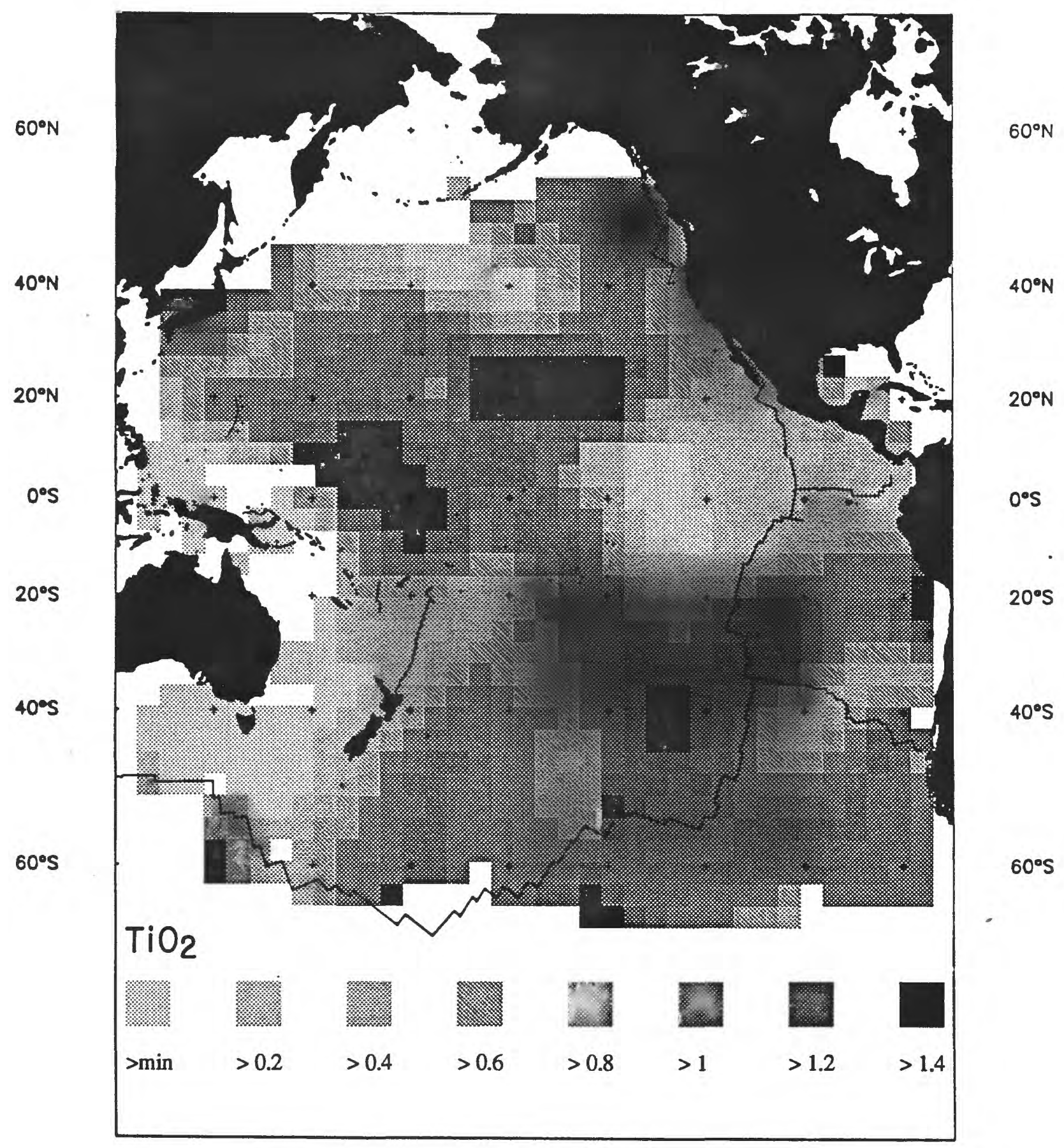

Figure IX-52 Gray scale map of $\mathrm{TiO}_{2} \%$ in the Pacific Ocean. 


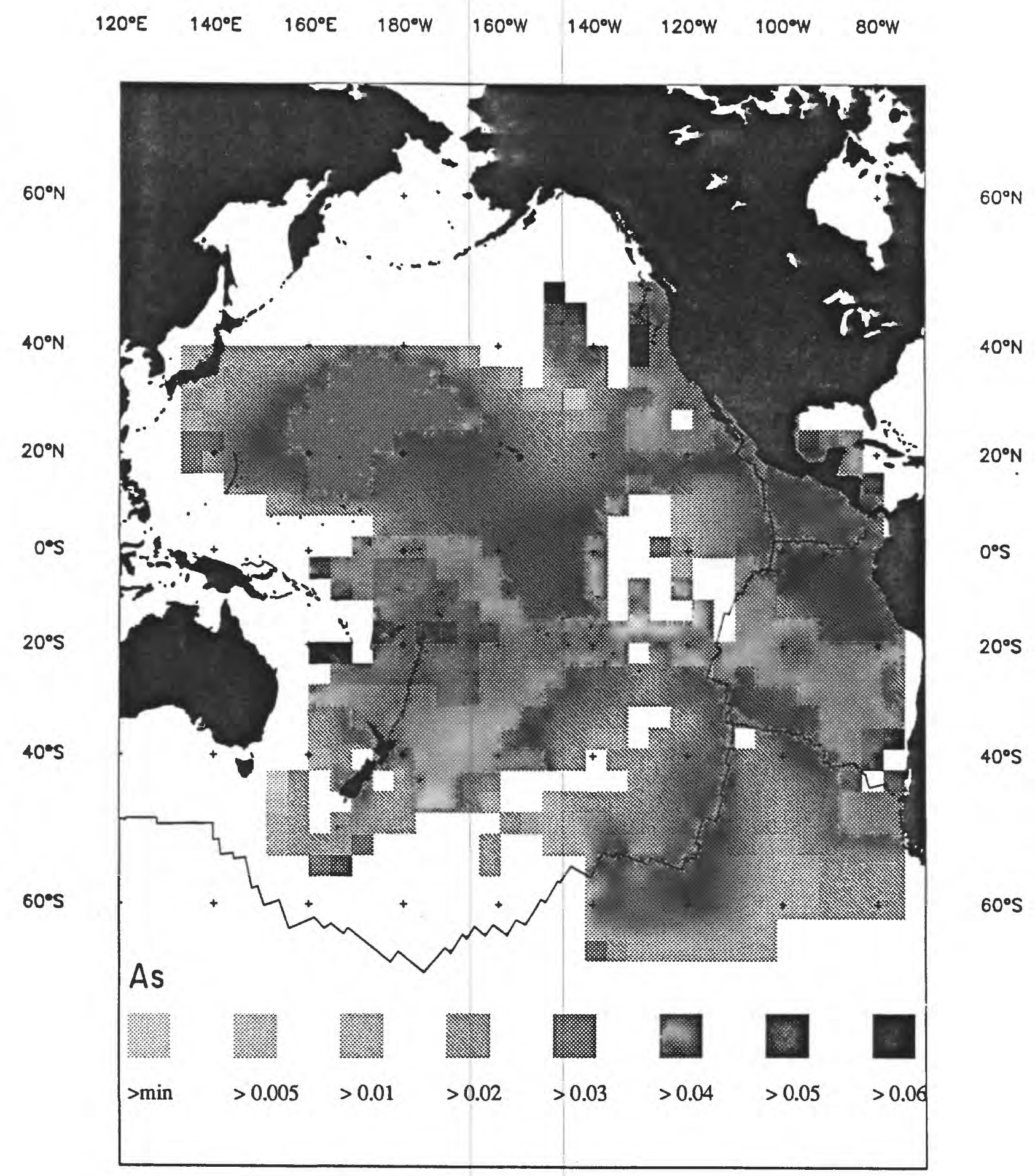

Figure IX-53 Gray scale map of As\% in the Pacific Ocean. 
$120^{\circ} \mathrm{E} \quad 140^{\circ} \mathrm{E} \quad 160^{\circ} \mathrm{E} \quad 180^{\circ} \mathrm{W} \quad 160^{\circ} \mathrm{W} \quad 140^{\circ} \mathrm{W} \quad 120^{\circ} \mathrm{W} \quad 100 \% \mathrm{~W} \quad 80^{\circ} \mathrm{W}$

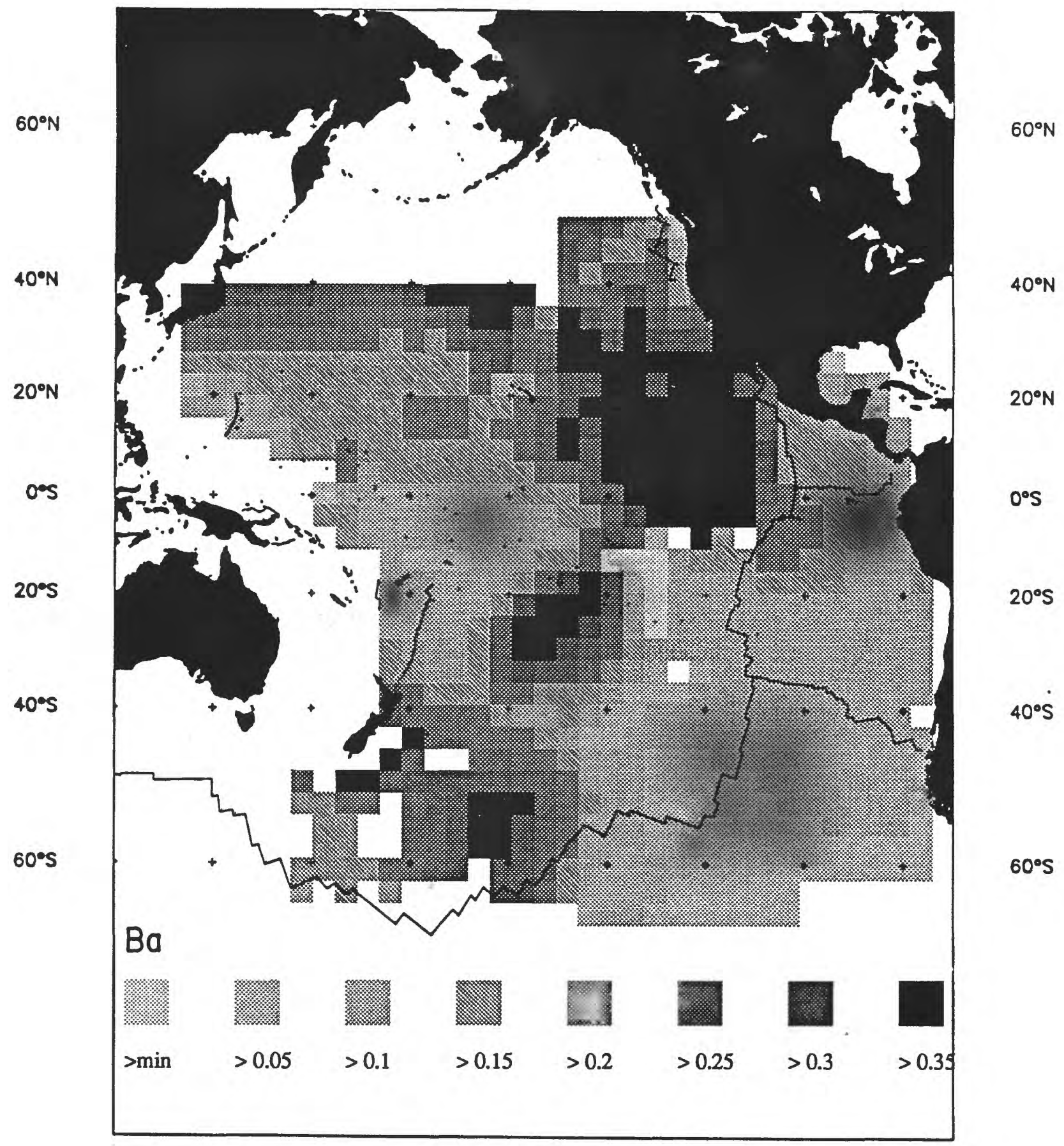

Figure IX-54 Gray scale map of Ba\% in the Pacific Ocean. 


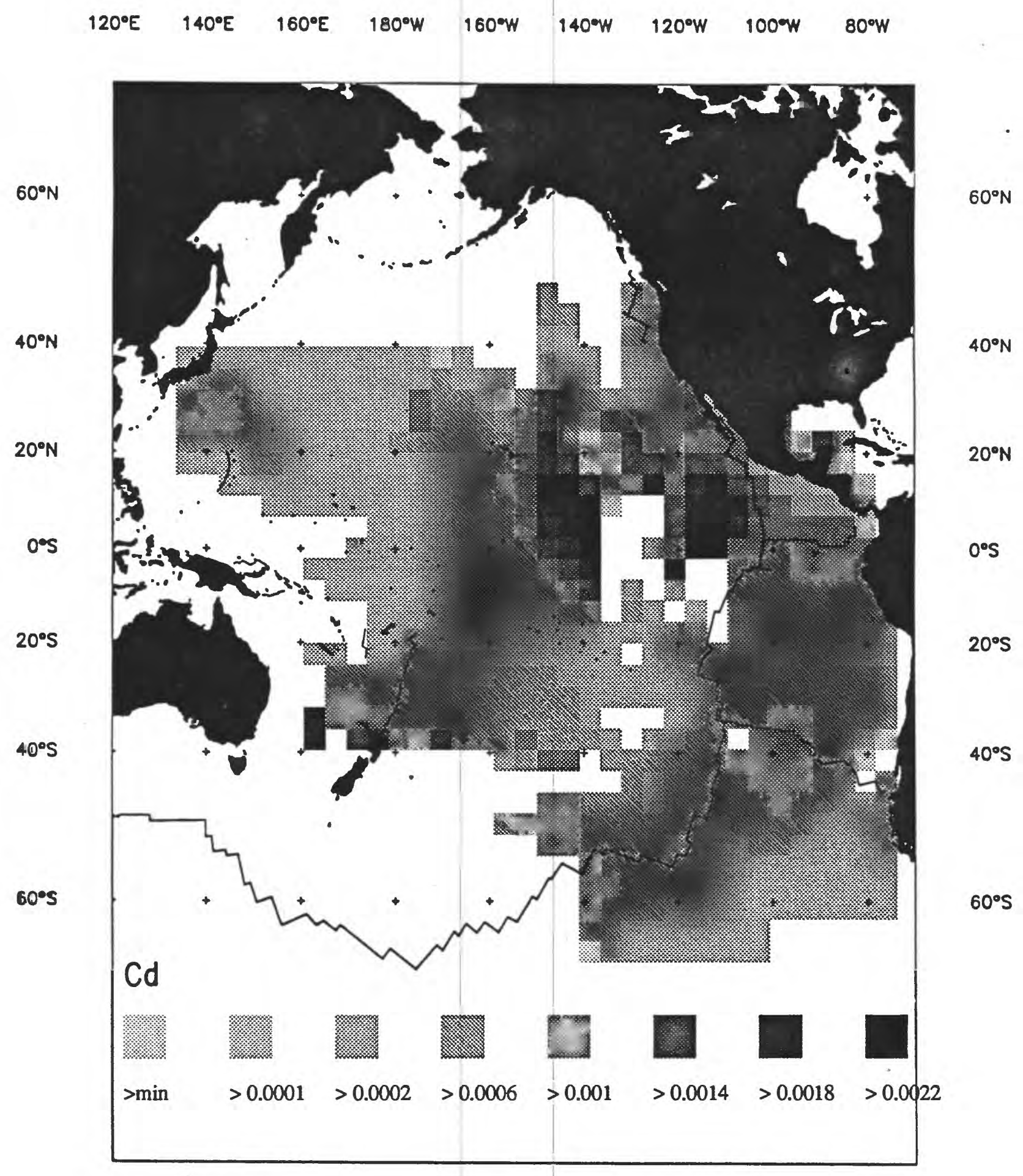

Figure IX-55 Gray scale map of Cd\% in the Pacific Ocean. 
$120^{\circ} \mathrm{E} \quad 140^{\circ} \mathrm{E} \quad 160^{\circ} \mathrm{E} \quad 180^{\circ} \mathrm{W} \quad 160^{\circ} \mathrm{W} \quad 140^{\circ} \mathrm{W} \quad 120^{\circ} \mathrm{W} \quad 100 \%$ W $80^{\circ} \mathrm{W}$

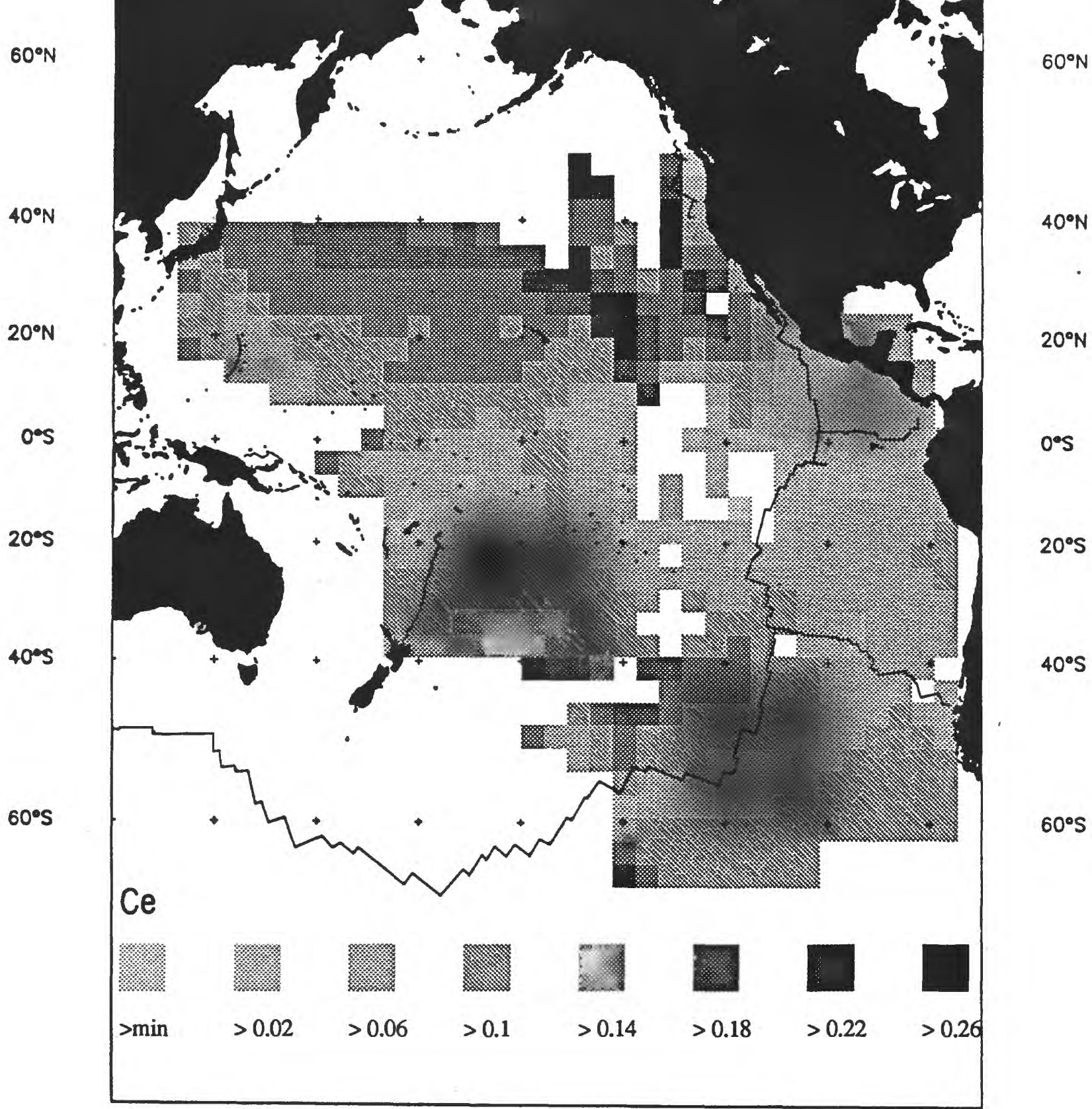

Figure IX-56 Gray scale map of $\mathrm{Ce} \%$ in the Pacific Ocean. 


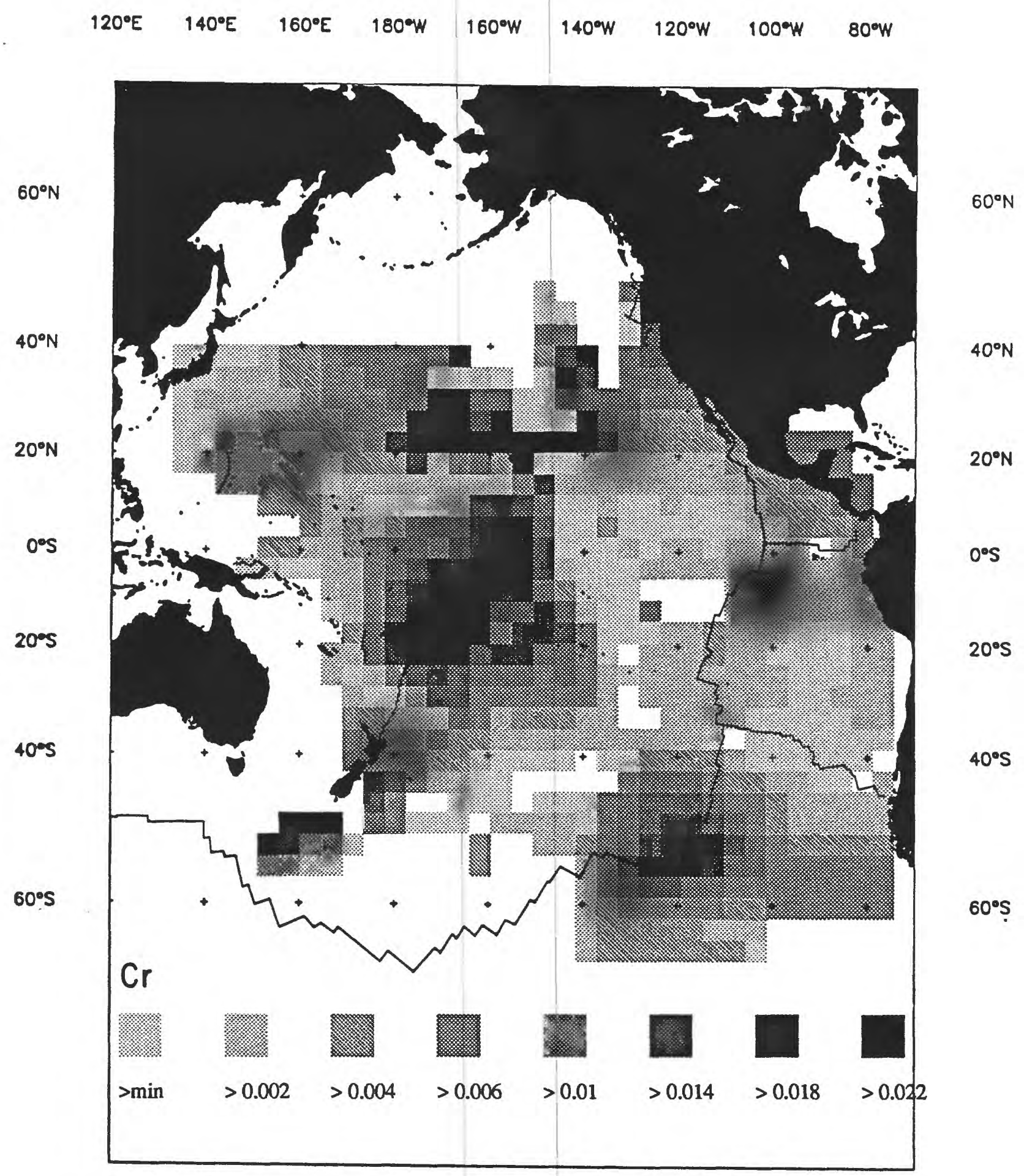

Figure IX-57 Gray scale map of $\mathrm{Cr} \%$ in the Pacific Ocean. 
$120^{\circ} \mathrm{E} \quad 140^{\circ} \mathrm{E} \quad 160^{\circ} \mathrm{E} \quad 180^{\circ} \mathrm{W} \quad 160^{\circ} \mathrm{W} \quad 140^{\circ} \mathrm{W} \quad 120^{\circ} \mathrm{W} \quad 100^{\circ} \mathrm{W} \quad 80^{\circ} \mathrm{W}$

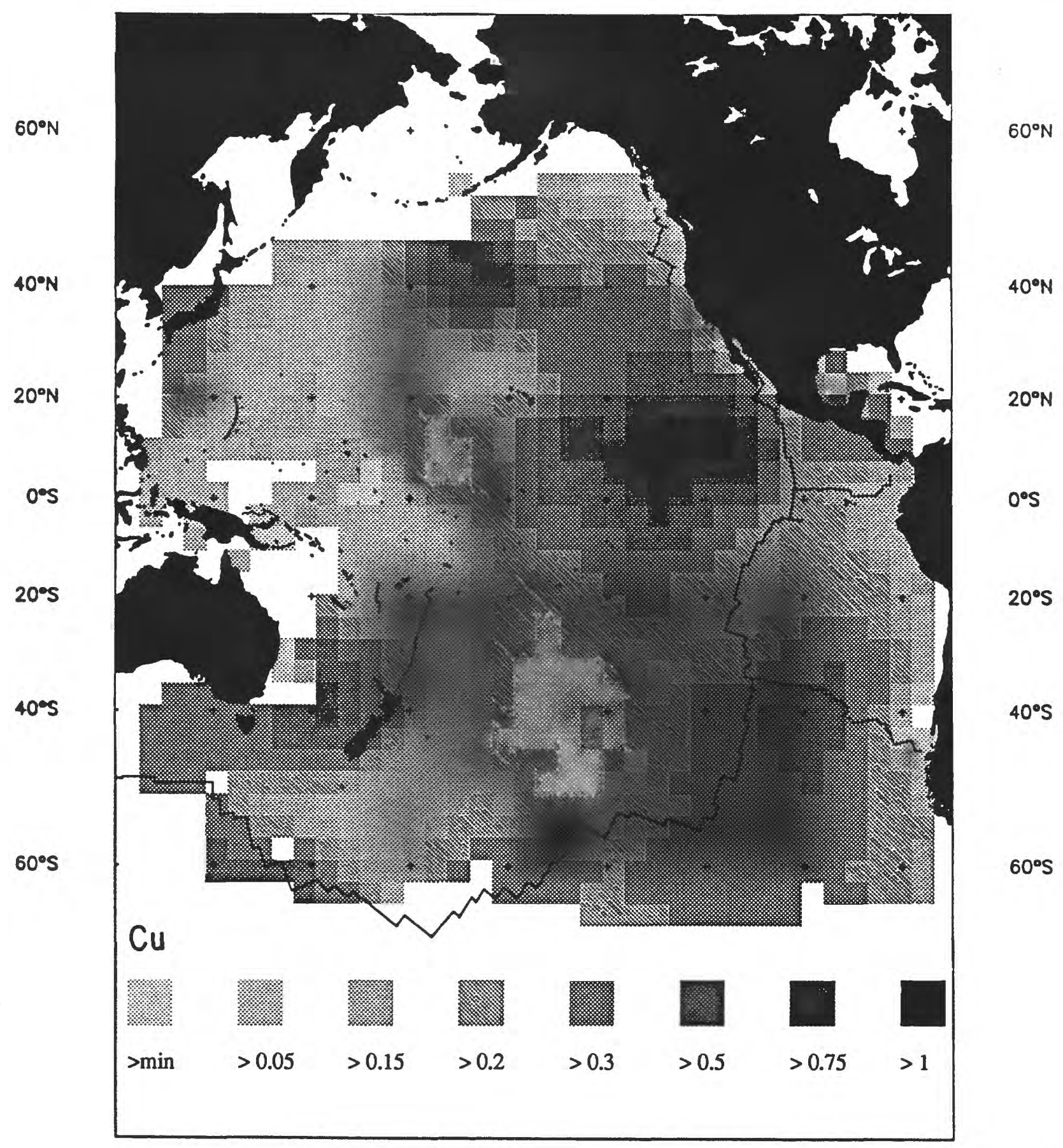

Figure IX-58 Gray scale map of Cu\% in the Pacific Ocean. 


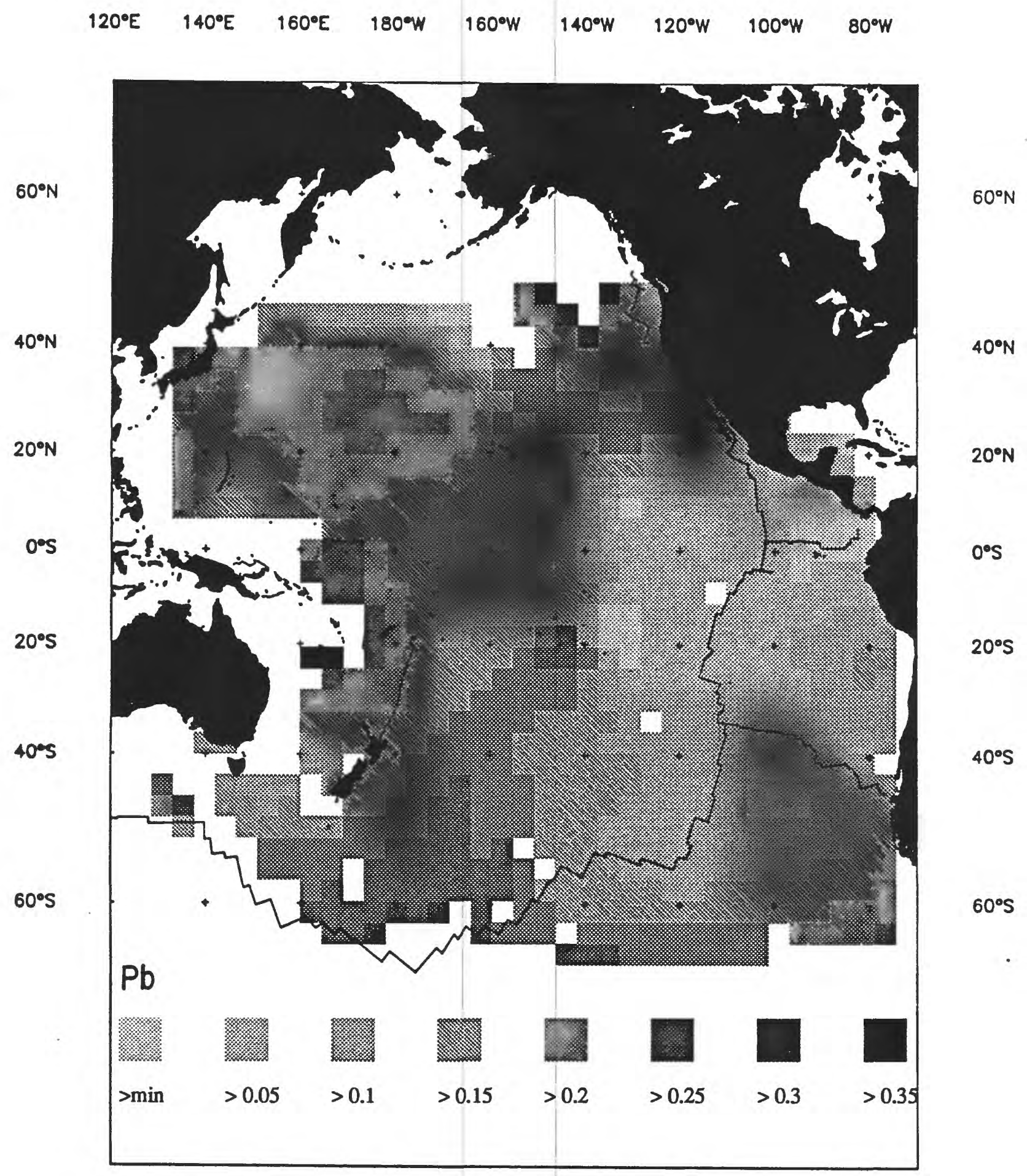

Figure IX-59 Gray scale map of Pb\% in the Pacific Ocean. 
$120^{\circ} \mathrm{E} \quad 140^{\circ} \mathrm{E} \quad 160^{\circ} \mathrm{E} \quad 180^{\circ} \mathrm{W} \quad 160^{\circ} \mathrm{W} \quad 140^{\circ} \mathrm{W} \quad 120^{\circ} \mathrm{W} \quad 100^{\circ} \mathrm{W} \quad 80^{\circ} \mathrm{W}$

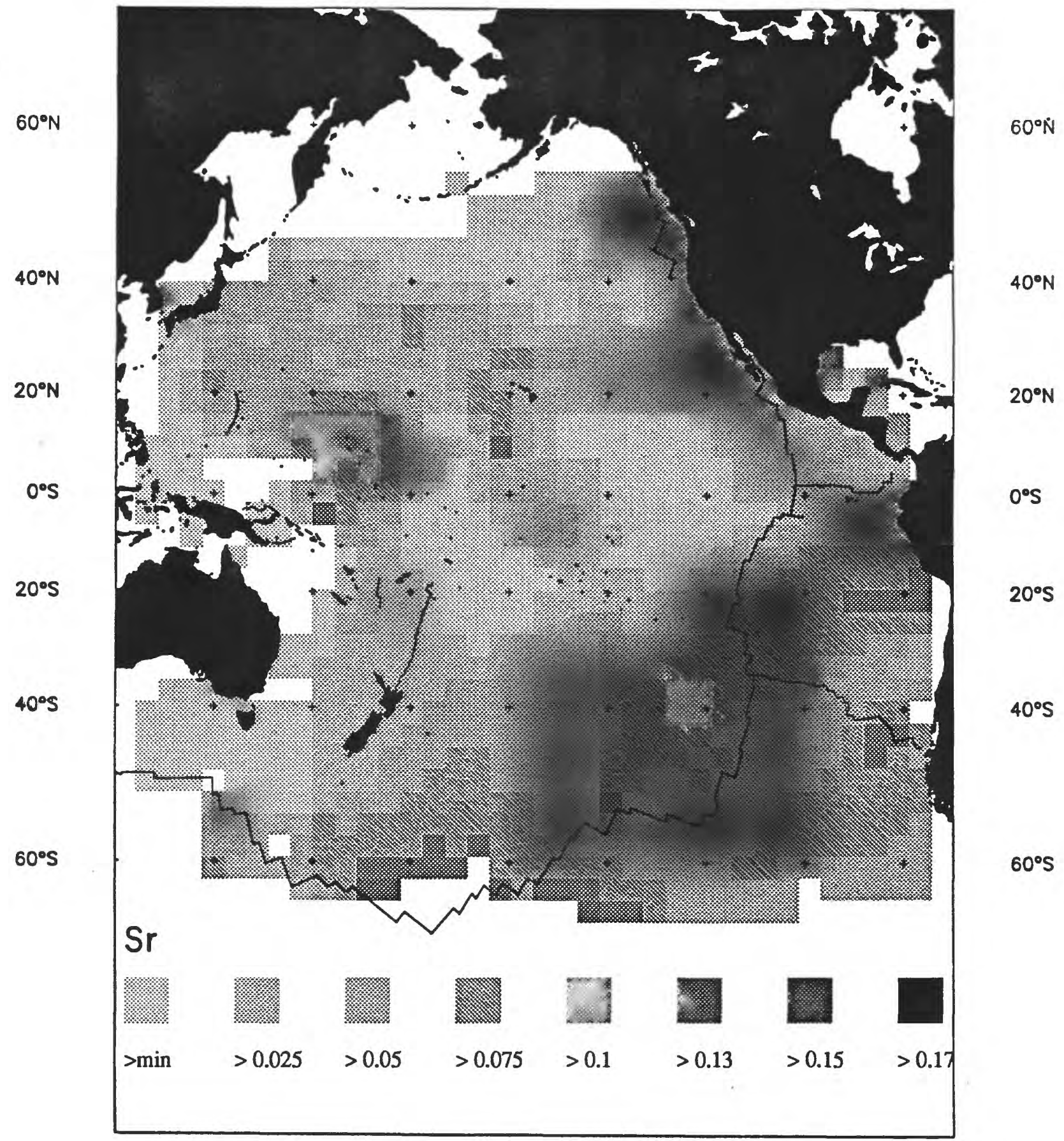

Figure IX-60 Gray scale map of Sr\% in the Pacific Ocean. 


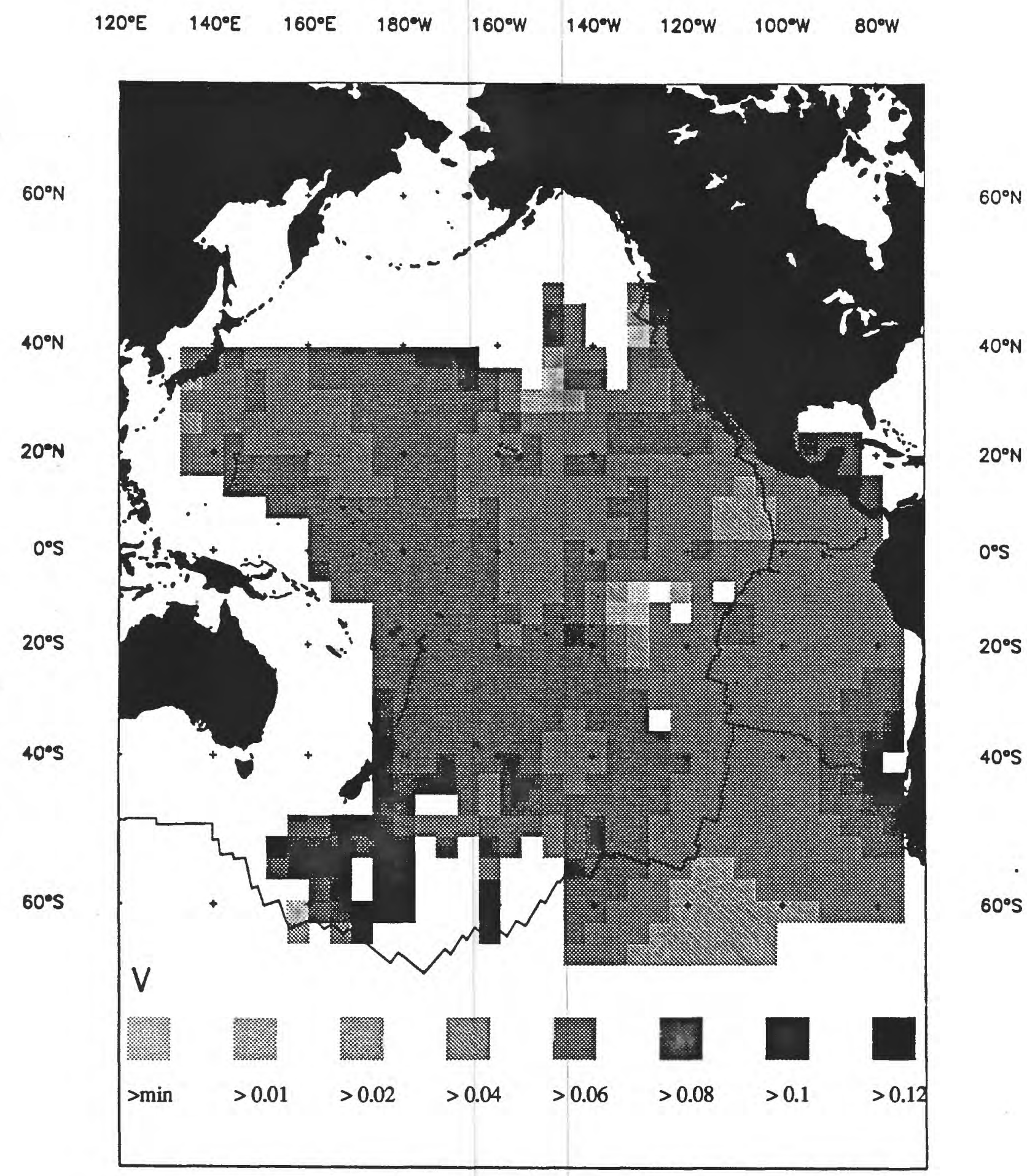

Figure IX-61 Gray scale map of V\% in the Pacific Ocean. 
$120^{\circ} \mathrm{E} \quad 140^{\circ} \mathrm{E} \quad 160^{\circ} \mathrm{E} \quad 180 \% \mathrm{~W} \quad 160^{\circ} \mathrm{W} \quad 140^{\circ} \mathrm{W} \quad 120^{\circ} \mathrm{W} \quad 100^{\circ} \mathrm{W} \quad 80^{\circ} \mathrm{W}$

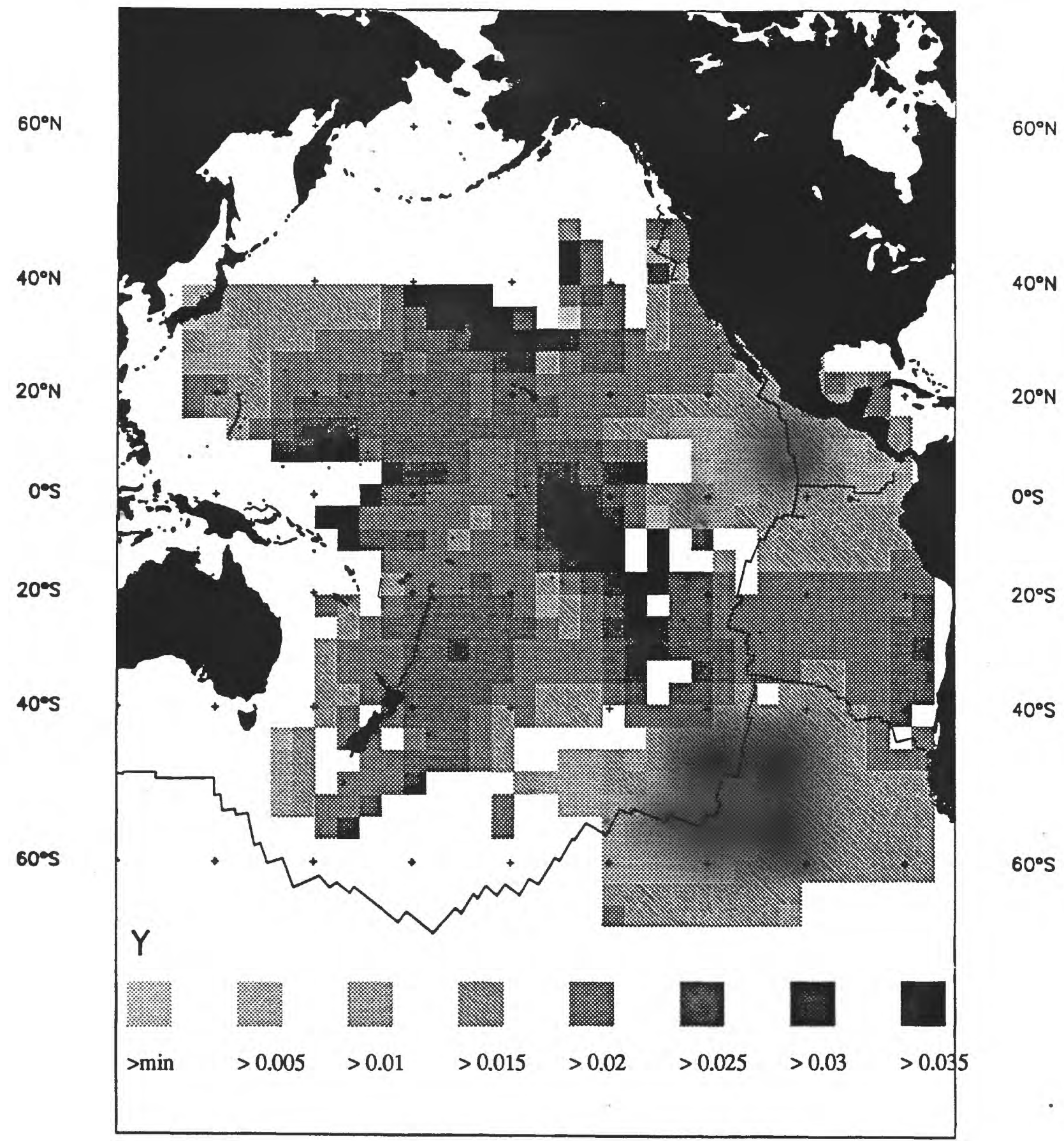

Figure IX-62 Gray scale map of Y\% in the Pacific Ocean. 


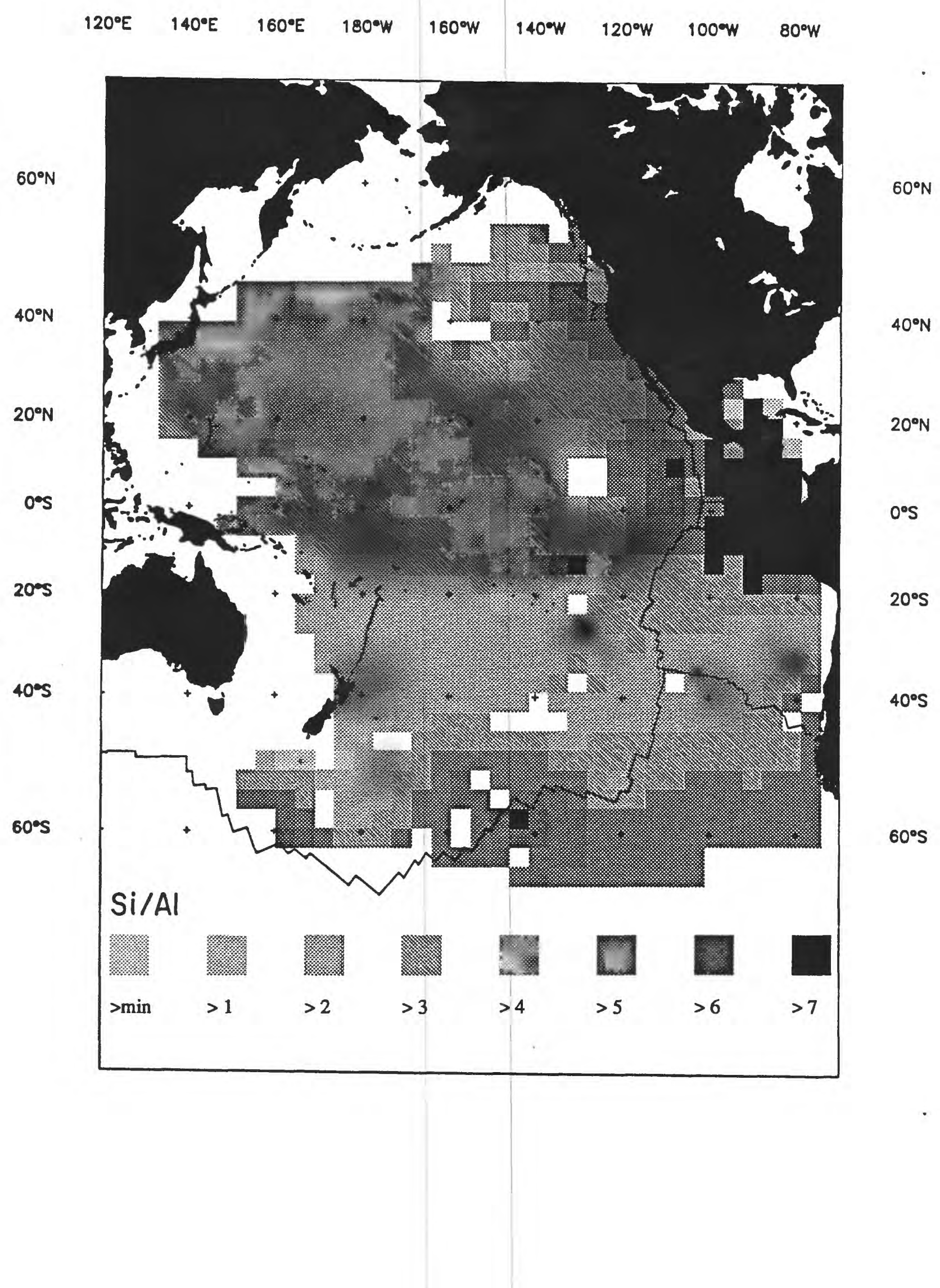

Figure IX-63 Gray scale map of Si/Al\% in the Pacific Ocean. 
$120^{\circ} \mathrm{E} \quad 140^{\circ} \mathrm{E} \quad 160^{\circ} \mathrm{E} \quad 180^{\circ} \mathrm{W} \quad 160^{\circ} \mathrm{W} \quad 140^{\circ} \mathrm{W} \quad 120^{\circ} \mathrm{W} \quad 100^{\circ} \mathrm{W} \quad 80^{\circ} \mathrm{W}$

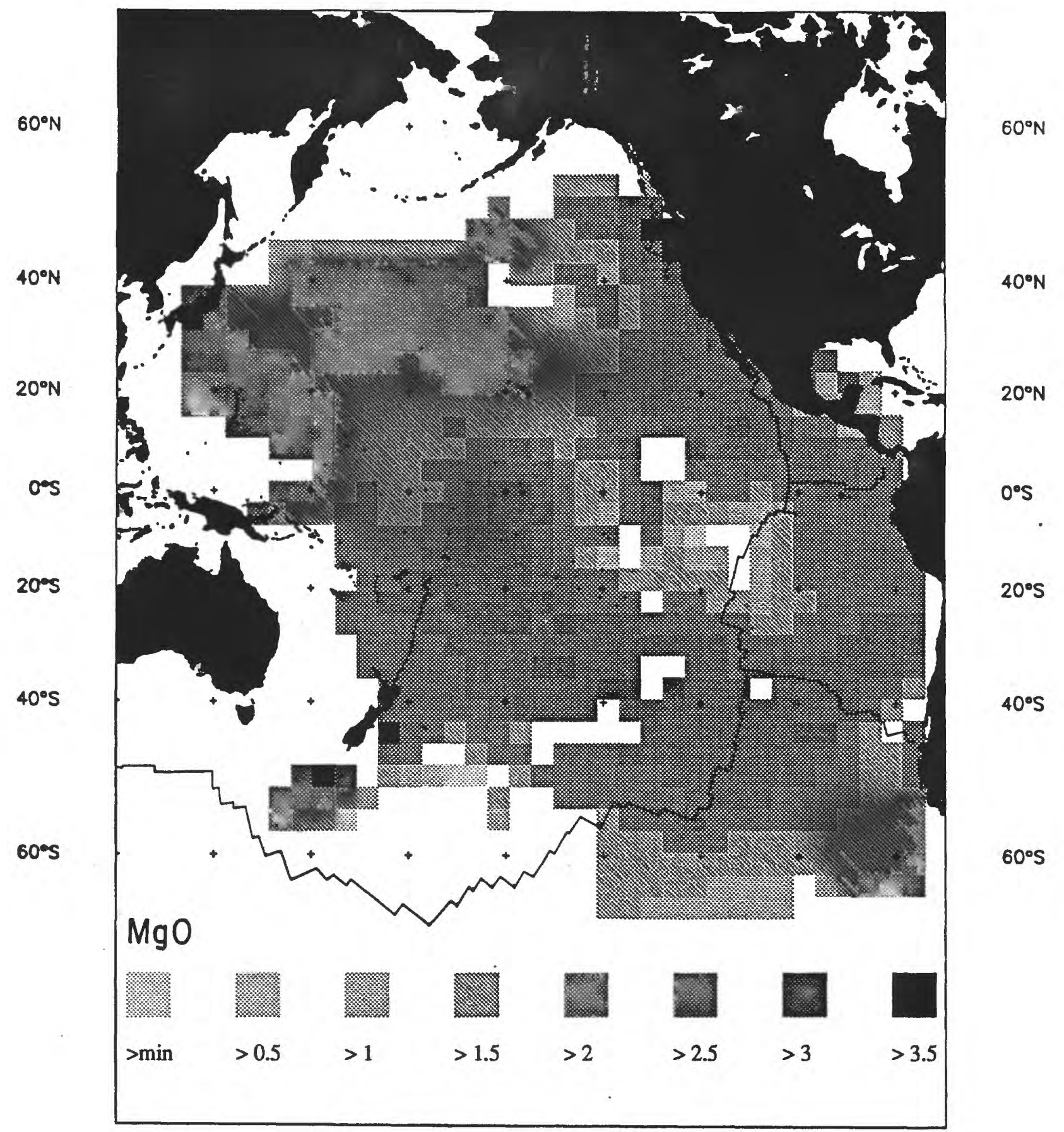

Figure IX-64 Gray scale map of MgO\% in the Pacific Ocean. 


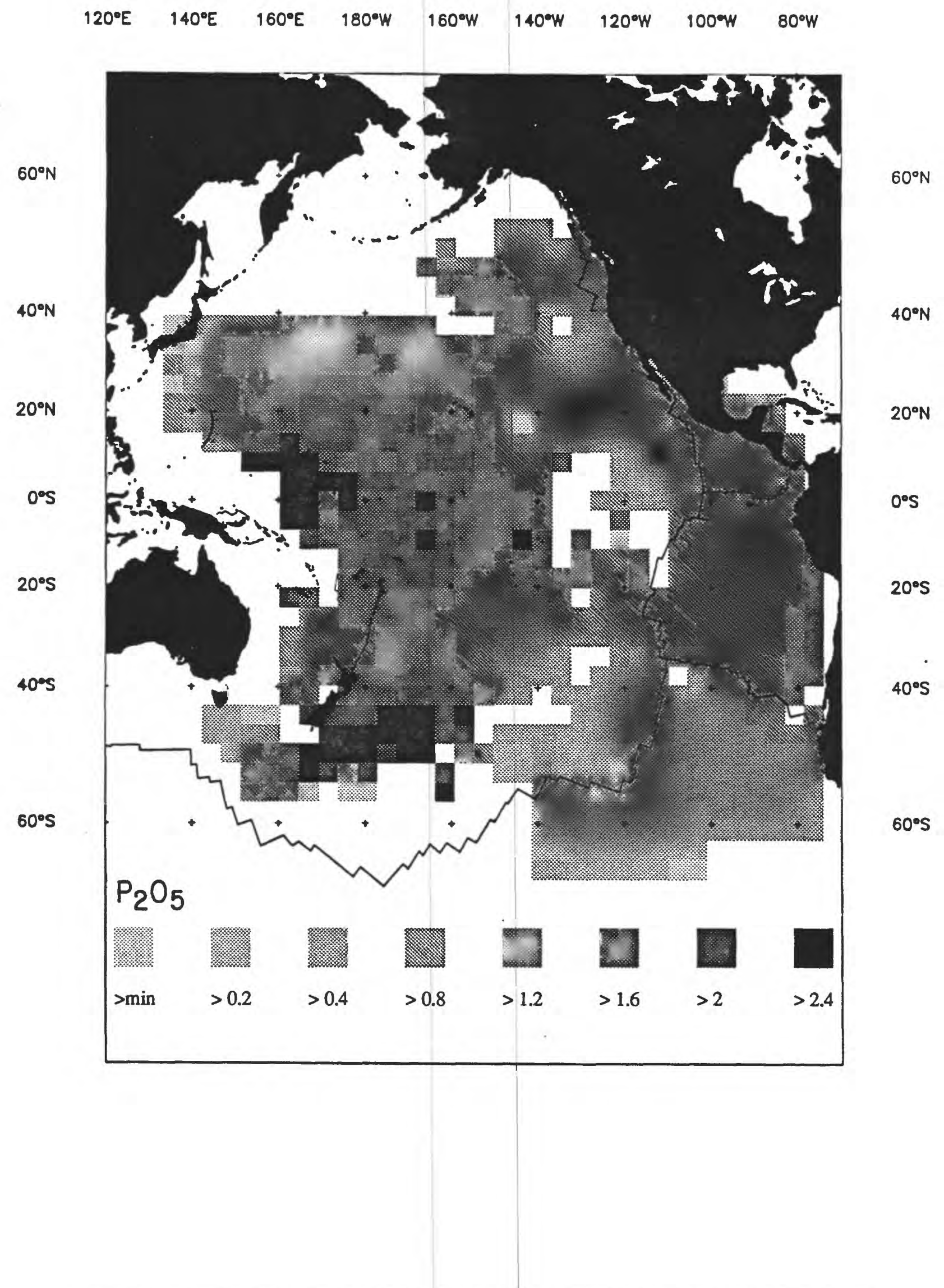

Figure IX-65 Gray scale map of $\mathrm{P}_{2} \mathrm{O}_{5} \%$ in the Pacific Ocean. 


\section{DISCUSSION}

Much of the interest in element mapping of crust composition lies in the postulate that sources of the metalliferous crust components are largely obtained from ambient water, rather than from underlying sediment and rock substrates. Because water masses show coherent chemical character over much larger areas than do bottom sediments and substrates, crust compositions should show a more consistent and and predictable regional chemical composition than do abyssal nodules. The maps (of cobalt distribution) strikingly bear out this expectation, not only showing a series of high-cobalt bullseyes, but also showing distinct regional lows that correlate closely to what is known about the distribution of hydrothermal fluid discharge in the oceans. These patterns also suggest other active hydrothermal areas in parts of the Pacific Ocean that have not yet been explored for polymetallic deposits and discharge zones. The southeast Pacific south of the Peru basin is a case in point.

For application to more local economic targets the cobalt maps reported here appear to reflect real regional variability, although the distribution of shallow seamounts is an ancillary factor that affects cobalt highs on all-depth maps. This conclusion tends to reduce changes that isolated high-cobalt crust regions will be found in peripheral areas of the Pacific ocean.

Second, the maps show several cobalt-enhanced areas for which only sparse sampling exists. Most of these areas are outside the US EEZ zones, but some occur within the EEZ zones of independent countries (Cook and Society Islands, and Tuamotu Archipelago, Marshall Islands), and need to be confirmed by additional sampling.

The data suggests that there is no constant cobalt-depth zonation throughout the oceans, but that the relationships, though remaining inversely correlated, in hydrogenetic areas are quite different in different parts of the ocean. A future task will be to explore details of depth zonation for other elements besides cobalt.

Although the data on crust thickness is far more limited than the chemical information, and some is of very poor quality. We should use what there is to examine regional relationships that will add to the base of scientific and economic knowledge. A current subject of expanding interest is chemical "crust stratigraphy", which promises to shed light on the distribution of seamounts, water masses and hydrothermal activity in earlier oceans. 


\section{SUMMARY AND CONCLUSIONS}

Our database has placed in usable and coherent form more than three times the number of crust analyses available than when we began the work in 1983. The current total is about 2400 analyses, approaching 100,000 individual data items. Many of the new data maintain a higher standard of sampling and analytical quality than preexisting data, and have been preferentially used here to develop normalization and correction algorithms for earlier data.

The Scripps Institution Nodule Data Bank (SNDB), updated by the NGDC, was carefully searched and qualified with respect to crust data. We searched some 13 U.S. repositories for existing seafloor samples containing crusts. We followed this by applications of a carefully-controlled analytical methodology for 27 chemical constitutents, and conducted interlaboratory calibrations of various sampling and analytical procedures. The new shipboard samples and data were added to the database.

The database was implemented on the UNIX system and desktop microcomputers at USGS, Woods Hole, Mass. offices. Mapping and statistical software were applied to data manipulation, samples of which are included in this report, specially designed for hard-copy viewing by persons with a variety of interests: geological/resource analysis, geochemical, analytical, and administrative. All chemical tables in this paper copy are in standard rock and geochemical analysis format, and are preceded by location and depth, as well as sequence number data.

Ferromanganese crusts and their composition are subject to many sources of error, and critical use of data are shown to be even more important than for abyssal nodules. A system of values normalized against Fe+Mn concentrations helps compensate for the serious problem of substrate contamination as well as variable treatments of hygroscopic and bound moisture in past reports.

Our efforts at elemental mapping confirm that ferromanganese crusts have chemical coherence over large geographical regions. Our maps of cobalt concentration show three well-defined high concentration areas. The largest one, in the central-western Pacific is a true regional feature extending deeper than $4000 \mathrm{~m}$; i.e. it is not merely a function of prevalence of shallow seamounts. Regions having low cobalt concentration in crusts are found in the vicinity of submarine hydrothermal vents and characterize a large part of the East Pacific Ocean. Other trace metals show distinctive associations; $\mathrm{Zn}, \mathrm{Ni}$, and $\mathrm{Cu}$ are highly concentrated in the Clarion-Clipperton fracture zone, $\mathrm{Ce}, \mathrm{Pb}$ and $\mathrm{As}$ show some common features with Co, whereas $V$ is enriched along with Fe, for examples in the East Pacific Rise and Juan de Fuca areas.

We hope that this database and updated versions will be useful in evaluating and synthesizing economically useful relationships pertaining to ferromanganese crust composition, as well as offering new scientific knowledge bearing on a variety of ocean geological and geochemical problems. 


\section{ACKNOWLEDGMENTS}

This report could not have been completed without the aid of many persons and institutions. We express gratitude to all those persons and institutions cited in Chapter III for their cooperation and assistance, as well as C. Moore and P. sloss of NGDC, NOAA, Boulder, Colorado and their colleagues for key data transfers and base maps created for us. At the Geological Survey an essential building block for this work has been the first-rate analytical data on crusts determined by the analysts at the Reston analytical laboratories (see Chapter IV). At the Branch of Atlantic-Gulf Marine Geology, J. Commeau contributed significantly to the analytical effort, and in many other ways. We thank our colleagues G. Evenden, J. Botbol, L. North, J. Fredericks and C. Polloni for invaluable computer assistance and advice, P. Forrestel, J. Zwinakis, and D. $B$ lackwood for suggestions and aid regarding drafting and photography, and $W$. Schwab and R. Commeau for discussion and data. Student assistants M. Kashgarian, P. Davis, K. Schmitz, J. DaSilva and M. Bourne aided in the earlier stages of the work. In the Menlo Park office of USGS J. Hein, D. Clague, L. Morgenson, C. Madison and others materially aided sampling, data collections, and data output. D. Clague aided in coordinating the early cooperation with the German effort. M. Cruickshank and J.L. Bischoff provided key stimulus at critical junctures for this work.

T. Hillman of the Bureau of Mines, Spokane, and M. Fisk of the United Nations provided early data and assistance with respect to the Scripps Nodule Data Bank (SNDB). A special debt is owed to. P. Halbach of the Technical University, Clausthal, for his early inspiration, collaboration and information, and to his student D. Puteanus, as well as V. Marchig of the German Geological Survey, and B. Haynes for cooperation in interlaboratory analytical calibrations. D. Root of the USGS Branch of Resource Analysis guided us in aspects of this work. The manuscript was improved by reviews of J. Botbol, J,L. Bischoff and E. Winget. Finally, the Minerals Management Service's support of this work made the present scope and degree of completeness of our effort possible. 


\section{REFERENCES}

The references are provided in two tables. The first (Table XI-1) is an index for references pertaining to both the station and chemical data of the SNDB. Then follows the Scripps Institution reference file, presented as in the original in both a chronological and alphabetical order.

Most of the references in the U.S.G.S. crust reference list are supplementary to the Scripps reference file, although a few duplications of commonly-referred to papers have been retained to permit ready reference to the discussion sections without switching back and forth between the two references lists. Some of the articles in the USGS references pertain primarily to nodules, but have pertinent sections in them relating to crusts. Others that have minor references to crusts have not been included in this list. Finally, a few ancillary references pertaining to subjects mentioned in the text are also included. 
Table X-1. Cross reference table of SIO "REFID" and SIO references to be used in conjunction with station and chemical tables as needed.

\begin{tabular}{|c|c|c|c|}
\hline A005 & $\cdots$ & 1960 & List of cores, Tethys expedition - SIO. \\
\hline A033 & $\cdots$ & 1965 & List of cores, Wahine expedition - SIO. \\
\hline A056 & $-\cdot$ & - & Confidential Source. \\
\hline A098 & $\cdots$ & - & $\begin{array}{l}\text { Joint Oceanographic Institutions for Deep Earth Sampling } \\
\text { (JOIDES), preliminary core reports. }\end{array}$ \\
\hline A105 & $\cdots$ & 1968 & $\begin{array}{l}\text { Preliminary megascopic descriptions of cores taken on } \\
\text { Vema, LDGO. }\end{array}$ \\
\hline Al08 & $\cdots$ & 1968 & Megascopic core descriptions - Vema 22, LDGO. \\
\hline A109 &.- & 1966 & Megascopic core descriptions - Vema 23, LDGO. \\
\hline A110 & $\cdots$ & 1965 & $\begin{array}{l}\text { Preliminary megascopic descriptions of cores taken on } \\
\text { Vema, LDGO. }\end{array}$ \\
\hline A114 & $\cdots$ & 1969 & $\begin{array}{l}\text { Preliminary megascopic core descriptions taken on Robert } \\
\text { D. Conrad, LDGO. }\end{array}$ \\
\hline A115 & $-\cdot$ & 1966 & $\begin{array}{l}\text { Preliminary megascopic descriptions of cores taken on } \\
\text { Robert D. Conrad cruise 10, LDGO. }\end{array}$ \\
\hline A116 & $\cdots$ & 1967 & $\begin{array}{l}\text { Preliminary megascopic core descriptions of cores taken } \\
\text { on Robert D. Conrad cruise } 11 \text {, LDGO. }\end{array}$ \\
\hline A117 & -- & 1970 & SIO list of cores and dredge hauls, Scan expedition. \\
\hline A119 & $-\cdots$ & 1971 & SIO 1ist of Samples, 7-TOW. \\
\hline A121 & -- & 1966 & List of cores and dredge hauls, Zetes expedition, SIO. \\
\hline A123 & $\cdots$ & 1969 & $\begin{array}{l}\text { Preliminary megascopic description of cores taken on } \\
\text { Vema, LDGO. }\end{array}$ \\
\hline A125 & $\cdots$ & 1969 & List of cores, Circe expedition, SIO. \\
\hline A130 & $\cdots$ & - & Trawls, dredges and grab samples, LDGO. \\
\hline A140 & $\cdots$ & 1965 & Akademiia Nauk, SSSR. \\
\hline A145 & $\cdots$ & 1968 & $\begin{array}{l}\text { List of cores and dredge hauls and visual core } \\
\text { description log, Styx expedition. }\end{array}$ \\
\hline A146 & $-\cdot$ & 1966 & List of cores, Tripod expedition - SIO. \\
\hline A150 & $-\cdot$ & - & Florida State University, Core description. \\
\hline A158 & $-\cdot$ & 1970 & $\begin{array}{l}\text { Preliminary megascopic core descriptions of cores taken } \\
\text { on Robert D. Conrad cruise 13, LDGO. }\end{array}$ \\
\hline A167 & $\cdots$ & 1971 & List of cores and dredge hauls, Aries expedition, SIO. \\
\hline A174 & $-\cdots$ & 1971 & $\begin{array}{l}\text { List of cores and dredge hauls, Antipode expedition, } \\
\text { SIO. }\end{array}$ \\
\hline A194 & $-\cdot$ & 1967 & $\begin{array}{l}\text { National Institute of Oceanography, RSS Discovery cruise } \\
16 .\end{array}$ \\
\hline A213 &.- & 1973 & Unidentified Source, coded as Amigo. \\
\hline A221 & -- & 1972 & List of sediment cores, Cato expedition, SIO. \\
\hline A226 & $\cdots$ & 1973 & Florida State University, Eltanin cruise 4-54. \\
\hline A233 & -- & 1973 & $\begin{array}{l}\text { List of cores and dredge hauls, Benthiface } \\
\text { expedition, SIO. }\end{array}$ \\
\hline A241 & $-\cdot$ & 1974 & $\begin{array}{l}\text { Inter-University Program Research on Ferromanganese } \\
\text { Deposits of the Ocean. Station summary and sample logs. }\end{array}$ \\
\hline
\end{tabular}

1975 Inter-University Program of Research on Ferromanganese Deposits of the Ocean. Station summary and sample logs.

1975 Nationa1 Oceanographic Data Center. Bottom Photograph Camera Station File (photo). Note: depth of photo stations may refer to camera depth.

1974 List of cores and dredge hauls. 


\begin{tabular}{|c|c|c|c|}
\hline $\begin{array}{l}\text { A267 } \\
\text { A269 } \\
\text { A290 }\end{array}$ & $\cdots$ & $\begin{array}{l}1972 \\
1975 \\
1965\end{array}$ & $\begin{array}{l}\text { Megascopic core descriptions, Conrad, LDGO. } \\
\text { List of cores and dredge hauls, Eurydice expedition, SIO. } \\
\text { Kennecott Copper Inc., (Special Products). Analyses of } \\
\text { manganese nodules obtained from Scripps Institution of } \\
\text { Oceanography. }\end{array}$ \\
\hline A306 & $-\cdot$ & 1973 & $\begin{array}{l}\text { Megascopic core descriptions (R/V Robert D. Conrad } \\
\text { cruise } 16 \text {, LDGO. }\end{array}$ \\
\hline A308 & - - & 1973 & Megascopic core descriptions. R/V Vema cruise 30 , LDGO. \\
\hline A325 & $-\cdot$ & 1976 & Sample data index, Deepsonde expedition, SIO. \\
\hline A333 & $-\cdot-$ & 1976 & Nodule analyses done by Kennecott Exploration, Inc. \\
\hline A336 & $-\cdot$ & 1975 & $\begin{array}{l}\text { Preliminary megascopic core descriptions taken on Robert } \\
\text { D. Conrad, LDGO. }\end{array}$ \\
\hline A337 & $-\cdot-$ & 1977 & Domes Project. Index of Box Cores. \\
\hline A339 & $-\cdot$ & 1976 & $\begin{array}{l}\text { List of cores and dredge hauls. Pleiades } \\
\text { expedition, SIO. }\end{array}$ \\
\hline A340 & -.. & 1976 & Sample index, Indopac, SIo. \\
\hline A352 & $\cdots$ & 1973 & Kennecott Exploration, Inc. \\
\hline A386 & $\cdots$ & 1977 & Seascope Exploration Data Summary. \\
\hline A392 & $\cdots$ & 1977 & International CLB Group. \\
\hline A393 & $-\cdot-$ & 1977 & International CLB Group. \\
\hline A407 & 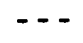 & - & Kennecott Exploration, Inc. \\
\hline A409 &.-- & - & Deepsea Ventures, Inc. \\
\hline A419 & $-\cdot$ & 1978 & Kennecott Copper Corporation. \\
\hline A421 & -- & 1979 & List of cores and dredge hauls Indomed expedition, SIO. \\
\hline A426 & -- & 1979 & Preussag, VA- 07 \\
\hline A430 & -- & 1979 & U.S. Navy Cruises Bartlett (1971) and Kane (1970). \\
\hline B509 &.- & 1958 & $\begin{array}{l}\text { Preliminary megascopic description of cores taken on } \\
\text { Vema, LDGO. }\end{array}$ \\
\hline B511 & -. & 1960 & Vema 16 preliminary description, LDGO. \\
\hline B540 &.- & 1966 & Data file, WHOI ref. no. 66-8. \\
\hline B579 & $-\cdot$ & 1965 & $\begin{array}{l}\text { Preliminary megascopic descriptions of cores taken on } \\
\text { Vema, LDGO. }\end{array}$ \\
\hline B733 & $\cdots$ & 1963 & $\begin{array}{l}\text { Preliminary megascopic descriptions of cores taken on } \\
\text { Vema, LDGO. }\end{array}$ \\
\hline B830 & -- & 1964 & $\begin{array}{l}\text { Preliminary megascopic descriptions of cores taken on } \\
\text { Vema, LDGO. }\end{array}$ \\
\hline M001 & $-\cdot$ & 1961 & Core and dredge lists, Monsoon expedition, SIO. \\
\hline M002 & -- & 1962 & Various logs, Proa expedition. \\
\hline M003 & $\cdots$ & 1963 & Lusiad Expedition core and dredge list, SIO. \\
\hline M004 & $\cdots$ & - & Carrousel II expedition list of cores \\
\hline M009 & -- & 1958 & Downwind expedition core and dredge lists, SIO. \\
\hline M013 & $-\cdot$ & 1964 & Various logs, Dodo expedition, SIO. \\
\hline M015 & $\cdots$ & 1961 & List of cores, Japanyon expedition, SIO. \\
\hline M025 & -- & 1963 & Core and dredge list, Luciad expedition, SIO. \\
\hline M033 & $\cdots$ & 1958 & $\begin{array}{l}\text { Downwind expedition list of cores and dredge } \\
\text { SIO. }\end{array}$ \\
\hline N184 & -- & 1954 & $\begin{array}{l}\text { List of cores collected on Acapulco Trench (Chubasco) } \\
\text { expedition, SIO. }\end{array}$ \\
\hline N684 & $-\cdots$ & 1962 & Akademiia Nauk, SSSR. \\
\hline N729 & $-\cdots$ & 1958 & Akademiia Nauk, SSSR. \\
\hline
\end{tabular}




\begin{tabular}{|c|c|}
\hline ???? & Heezen \\
\hline A006 & Revelle \\
\hline A009 & Murray \\
\hline A010 & Murray \\
\hline A012 & Murray \\
\hline A033 & Moore \\
\hline A068 & Goldberg \\
\hline A069 & Khristianova \\
\hline A077 & Young \\
\hline A082 & Fisher \\
\hline A083 & Bruun \\
\hline A088 & Von Gumbel \\
\hline A117 & Johnson \\
\hline A119 & Johnson \\
\hline A140 & Skornyakova \\
\hline A148 & Bezrukov \\
\hline A165 & Goldberg \\
\hline A166 & Barnes \\
\hline A178 & Skornyakova \\
\hline A179 & Horn \\
\hline A189 & Cronan \\
\hline A194 & Glasby \\
\hline Al95 & Glasby \\
\hline A197 & Mero \\
\hline A199 & Bender \\
\hline A200 & Ahrens \\
\hline A201 & Willis \\
\hline A202 & Grant \\
\hline A203 & Cronan \\
\hline A206 & Tin \\
\hline A217 & Glasby \\
\hline A220 & Isaeva \\
\hline A223 & Glockhoff \\
\hline A227 & Meylan \\
\hline A230 & Bonatti \\
\hline A232 & Burns \\
\hline A240 & Cronan \\
\hline A243 & Ostwald \\
\hline A246 & Bezrukov \\
\hline A247 & Frazer \\
\hline A248 & Shima \\
\hline A249 & Okada \\
\hline A250 & Glasby \\
\hline A251 & Andrews \\
\hline A254 & Fein \\
\hline A255 & Dietz \\
\hline A256 & Yabuki \\
\hline A2 60 & Summerhayes \\
\hline A261 & Menard \\
\hline A262 & Hewett \\
\hline A272 & Van der \\
\hline & Weijden \\
\hline
\end{tabular}

\begin{tabular}{|c|c|c|c|}
\hline A276 & Nikolayev & A413 & Piper \\
\hline A277 & Hubred & A414 & Meylan \\
\hline A280 & Mizuno & A416 & Leclaire \\
\hline A281 & Meylan & A417 & Shepard \\
\hline A283 & Aumento & A418 & Andersen \\
\hline A284 & Lonsdale & A422 & Fisk \\
\hline A285 & Mero & A431 & Batiza \\
\hline A289 & Cassidy & A433 & Colley \\
\hline A291 & Cronan & $A 437$ & Fisk \\
\hline A293 & Backer & A438 & Siegel \\
\hline A298 & Frazer & A439 & Lonsdale \\
\hline A301 & Korkisch & A446 & Andrews \\
\hline A303 & Korkisch & A446 & Bischoff \\
\hline A305 & Gilbert & A448 & Fewkes \\
\hline A309 & Greenslate & A451 & Skornyakova \\
\hline A321 & Fisk & $A 458$ & Campbel1 \\
\hline A323 & Piper & A459 & Frazer \\
\hline A324 & Morgan & A462 & Hoffert \\
\hline A324 & Morgan & A463 & Hoffert \\
\hline A326 & Marchig & A464 & Hoffert \\
\hline A332 & Friedrich & A465 & Korkisch \\
\hline A334 & Greenslate & A466 & Summerhayes \\
\hline A338 & Greenslate & A467 & Cronan \\
\hline A342 & Frazer & A474 & Roonwal \\
\hline A343 & Bezrukov & A475 & Sims \\
\hline A346 & Krishnaswami & A477 & Morten \\
\hline A347 & Walsh & B559 & Goodel1 \\
\hline A349 & Monzier & M005 & Glockhoff \\
\hline A351 & Mueller & M025 & Conolly \\
\hline A354 & Smith & M036 & Krause \\
\hline A360 & Batiza & M038 & Hamilton \\
\hline A361 & Mizuno & $\mathrm{N} 1.70$ & Stetson \\
\hline A361 & Mizuno & N682 & Riedel \\
\hline A363 & Mizuno & & \\
\hline A366 & Nohara & & \\
\hline A367 & Jacobs & & \\
\hline A368 & Cronan & & \\
\hline A369 & Hein & & \\
\hline A371 & Jacobs & & \\
\hline A372 & Jacobs & & \\
\hline A373 & Cronan & & \\
\hline A37.4 & Hathaway & & \\
\hline A376 & Fisk & & \\
\hline A379 & Hoover & & \\
\hline A380 & Frank & & \\
\hline A381 & Fuerstenau & & \\
\hline A383 & Piper & & \\
\hline A388 & Willis & & \\
\hline A389 & Yabuki & & \\
\hline A390 & Andrushchenko & & \\
\hline A395 & Pasho & & \\
\hline A405 & Fisk & & \\
\hline
\end{tabular}




\section{SNDB REFERENCE LIST}

Reference list for Scripps Institution Nodule Data Bank (SNDB). Original Scripps chronological format has been retained. References are as delivered by NGDC in 1983.

Carrousel II expedition list of cores, dredge samples and camera stations. Scripps Institution of Oceanography (unpublished) :M004 Confidential Source. :A056

Deepsea Ventures, Inc. Analyses of SIO Nodules. Personal communication. :A409

Florida State University, core descriptions and locations cruises 16-27, USNS Eltanin. Antarctic Core Facility, Dept. of Geology, Florida State University, NSF, Office of Polar Programs. :Al50

Joint Oceanographic Institutions for Deep Earth Sampling (JOIDES), Preliminary Core Reports (beginning with Site 32), (R/V Glomar Challenger). Unpublished :A098

Kennecott Exploration, Inc. Nodules analyses by AA, dried at 105C for 24 hours: Ocean Mining Lab. Private communication, D. Felix. :A407

National Oceanographic Data Center. Bottom Photograph Camera Station File (photo). Note: depth of photo stations may refer to camera depth. ENDEX/OASIS. Washington, D.C. :A252

Trawls, dredges, and grab samples (R/V Robert D. Conrad, cruises 5-18; R/V Vema, cruises 12-30), Lamont-Doherty Geological Observatory (unpublished) :Al30

1954, List of cores collected on Acapulco Trench (Chubasco) expedition, 1954, (R/V New Horizon), Scripps Institution of Oceanography :N184

1958, Akademiia Nauk, SSSR, Institut Okeanologii, Tablitsa donnykh oBottom Sediment Listings), Cruise 26 (Pacific Ocean) of the Vityaz, 17 Nov. 1957 to 16 February 1958, (unpublished) :N729

1958, Downwind expedition core and dredge lists, smooth plots. (R/V Spencer F. Baird, Oct. 1957-Feb. 1958), Scripps Institution of Oceanography :M009

1958, List of cores and dredge samples, copied from shipboard logs, Downwind expedition, Oct. 1957-Feb. 1958. (R/V Horizon) Scripps Institution of Oceanography (unpublished) :M033

1958, Preliminary megascopic core description taken on Vema, cruise 14, Nov. 1957-Aug. 1958. Prepared by Geology Dept. Lamont-Doherty Geological Observatory (unpublished) :B509

1960, List of cores, Tethys expedition, June-July 1960. (R/V Spencer F. Baird) Scripps Institution of Oceanography (unpublished) :A005

1960, Vema 16 preliminary description (Vema, Oct. 1959-Sept. 1960) Lamont-Doherty Geological Observatory (unpublished manuscript) :B511 
1961, Core and dredge 1ists, Monsoon expedition, Aug. 1960- Apr. 1961. (R/V Argo) Scripps Institution of Oceanography (unpublished) :M001

1961, List of cores, copied from shipboard logs, Japanyon expedition, May 1961-Sept. 1961. (R/V Spencer F. Baird) Scripps Institution of Oceanography (unpublished) :M015

1962, Akademiia Nauk, SSSR, Institut Okeanologii, Tablitsa Donnykh Osadkov (Bottom Sediment Listings), cruise 35 (Indian Ocean) of the Vityaz, 2 July 1962 to 2 Nov 1962. WDC-A 137 B-9: N684

1962, Various logs, Proa expedition March-Oct. 1962. (R/V Spencer F. Baird) Scripps Institution of Oceanography (unpublished) :M002

1963, Core and dredge 1ist, Lusiad expedition, May 1962-Aug. 1963. (R/V Horizon, $\mathrm{R} / \mathrm{V}$ Argo) Scripps Institution of Oceanography. :M025

1963, Lusiad Expedition core and dredge list, camera log. ( $R / V$ Argo, May 1962-Aug. 1963) Scripps Institution of Oceanography (unpublished) :M003

1963, Preliminary megascopic core descriptions taken on Vema, cruise 19, Feb. - Dec. 1963. Lamont-Doherty Geological Observatory (unpublished) :B733

1964, Preliminary megascopic core descriptions taken on Vema, cruise 20, Feb.-Dec. 1964. Lamont-Doherty Geological Observatory (unpublished) :B830 1964, Various logs, Dodo expedition April - Dec. 1964. (R/V Argo) Scripps Institution of Oceanography (unpublished) :M013

1965, Akademiia Nauk, SSSR, Institut Okeanologii. Tablitsa Donnykh Osadkov (Bottom Sediment Listings). cruise 37 (Western Pacific Ocean) of the Vityaz, 3 May to $10 \mathrm{Ju} 1 \mathrm{y}$ 1965. WDC-A No. 137.1 B-11. Unpublished. (Vityaz) :A140

1965, Kennecott Copper Co. (Special Products). Analyses of manganese nodules obtained from Scripps Institution of Oceanography, 1965. Unpublished. :A290

1965, List of cores, Wahine expedition, Feb. - April 1965. (R/V Spencer. Baird) Scripps Institution of Oceanography (unpublished) :A033

1965, Preliminary megascopic core descriptions taken on Vema, cruise 21, Aug. - Dec. 1965. Lamont-Doherty Geological Observatory (unpublished) :A110

1965, Preliminary megascopic core descriptions taken on the Vema, cruise 18, Dec. 1961- Dec. 1962. Revised June 1965. Lamont-Doherty Geological Observatory (unpublished) :B579

1966, Data file, continental margin program, Atlantic coast of the U. S., Vol. 1, Sample collection data. Woods Hole Oceanographic Institution Ref. No. 66-8, March. :B540

1966, List of cores and dredge hauls, Zetes expedition, March- Aug. 1966. (R/V Argo) Scripps Institution of Oceanography (unpublished) :A121

1966, List of cores, Tripod expedition, Nov. - Dec. 1966. (R/V Argo) Scripps Institution of Oceanography (unpublished) :Al46 
1966, Megascopic core descriptions taken on Vema, cruise 23, July 27-Dec. 13, 1966. Revised Apr. 1968. Lamont-Doherty Geological Observatory (unpublished) :A109

1966, Preliminary megascopic core descriptions taken on Robert D. Conrad cruise 10, Jan. 1965 - Oct. 1966. Lamont-Doherty Geological Observatory (unpublished) :A115

1967, National Institute of Oceanography, RSS Discovery cruise 16 report, geology and geophysics in the Red Sea, Gulf of Aden and N. W. Indian Ocean. NIO Cruise Report series, CR-16, Wormley, England, Jan-May. :Al94

1967, Preliminary megascopic core descriptions taken on Robert D. Conrad cruise 11. Nov. 30, 1966- Nov. 11, 1967. Lamont-Doherty Geological Observatory (unpublished) :Al16

1968, List of cores and dredge hauls and visual core description log, Styx expedition, Apr. - Sept. 1968. ( $/ \mathrm{V}$ Alexander Agassiz) Scripps Institution of Oceanography (unpublished) :A145

1968, Megascopic core descriptions taken on Vema cruise 22, Jan. 11-Jun. 12, 1966. Revised April 1968. Lamont-Doherty Geological Observatory (unpublished) :A108

1968, Preliminary megascopic core descriptions taken on Vema, cruise 24, Jan.-Dec. 1967. Revised Oct. 1968. Lamont-Doherty Geological Observatory. (unpublished) :A105

1969, List of cores, Circe expedition, March 1968-Jan. 1969. (R/V Argo) Scripps Institution of Oceanography(unpublished) : A125

1969, Preliminary megascopic core descriptions taken on Robert D. Conrad, Cruise 12, Jan 1968 - April 1969, prepared by the geology dept. Lamont-Doherty Geological Observatory. :Al14

1969, Preliminary megascopic core description taken on Vema, cruise 26, July 1968 to May 1969, prepared by the Geology Deptartment Lamont-Doherty Geological Observatory :A123

1970, Preliminary megascopic core descriptions taken on Robert D. Conrad cruise 13, Sept. 10, 1969 - Jan. 9, 1970. Lamont- Doherty Geological Observatory (unpub1ished) :A158

1970, SIO list of cores and dredge hauls, scan expedition, Mar. 1969-Feb.1970, and from initial reports of the Deep Sea Drilling Project, Vol. V, VI, VIII. :A117

1971, List of cores and dredge hauls, Antipode expedition, June 1970-October 1971. (R/V Melville) Scripps Institution of Oceanography (unpublished) :A174

1971, List of cores and dredge hauls, Aries expedition, Nov. 1970-Oct. 1971. ( $R / N$ Thomas Washington) Scripps Institution of Oceanography (unpublished) :A167 
1971, Megascopic core descriptions taken on Vema, cruise 28, 25 June $1970-14$ Nov. 1971. Lamont-Doherty Geological Observatory :A266

1971, Scripps Institution of Oceanography, List of geological samples, copied from Shipboard Logs, Seven-Tow Expedition, Feb.- Sept. 1970. (R/V Thos. Washington) (unpublished.):A119

1972, List of sediment cores copied from shipboard logs, Cato expedition, Aug. - Sept. 1972. (R/V Melville) Scripps Institution of Oceanography (unpublished) :A221

1972, Megascopic core descriptions taken on Vema, cruise 29, 18 Nov. $1971-7$ Oct. 1972. Lamont-Doherty Geological Observatory (unpublished) :A265

1972, Megascopic core descriptions, taken on Robert D. Conrad cruise 15, 29 Sept. 1971 - 4 Aug. 1972 Lamont-Doherty Geological Observatory 2 (unpublished) :A267

1973, Florida State University, Department of Geology, Antarctic Core Facility, USNS Eltanin core descriptions, Cruises 4-54. Sedimentology research laboratory contribution no. 37, July 1973.:A226

1973, Kennecott Exploration, Inc., San Diego, California. Atomic absorption analyses of nine Aries samples. Communication, David Felix, 1973 :A352

1973, List of cores and dredge hauls copied from shipboard logs, Benthiface expedition, June-July 1973. (R/V Melville) Scripps Institution of Oceanography. :A233

1973, Megascopic core descriptions taken on R/V Robert. D. Conrad cruise 16, 10 Aug. 1972 to 14 Sept. 1973) Lamont-Doherty Geological Observatory (unpublished) :A306

1973, Megascopic core descriptions taken on Vema cruise 30, 22 Nov. 1972 - 29 Dec. 1973. Lamont-Doherty Geological Observatory (unpublished) :A308

1973, Unidentified source, Nov. 1973. Coded as Amigo. :A213

1974, Inter-University Program Research on Ferromanganese Deposits of the Ocean. Station summary and sample logs, cruise MN-7401, July-Aug. 1974. Seabed Assessment Program, IDOE, National Science Foundation, HIG-74-9. Sept. 1974 (R/V Moana Wave) : A241

1974, List of cores and dredge hauls, Tasaday expedition. July 1973-Jan. 1974 (R/V Thomas Washington) Scripps Institution of Oceanography (unpub1ished) :A257

1975, Inter-University Program of Research on Ferromanganese Deposits of the Ocean. Station summary and sample logs, cruise MN-7402, Sept.-Oct. 1974. Seabed Assessment Program, IDOE, National Science Foundation, May 1975. :A242

1975, List of cores and dredge hauls copied from shipboard logs, Eurydice expedition, 4 Sept. 1974-2 May 1975. (R/V Thomas Washington) Scripps Institution of Oceanography (unpublished) : A269 
1975, Preliminary megascopic core descriptions taken on Robert D. Conrad, cruise 17, Oct. 1973-Jan. 1975. Lamont-Doherty Geological Observatory (unpublished) :A336

1976, List of cores and dredge hauls. Pleiades expedition 29 May 1976-23 Sept. 1976. ( $R / V$ Melville) Scripps Institution of Oceanography (unpublished) :A339

1976, Nodule analyses done by Kennecott Exploration, Inc. Samples from Vema 29-32, Robert D. Conrad 15-18, 1973-1976. Lamont-Doherty Geological Observatory (unpublished) :A333

1976, Sample data index, informal report and index of navigation, depth, magnetic, and subbottom profiler data. Deepsonde expedition leg 2, 9 Feb. --9 Mar. 1976 ( $R / V$ Thomas Washington) Scripps Institution of Oceanography (unpublished) : A325

1976, Sample index, core data. Indopac (first half), San Diego, CA 24 March 1976 - Apra, Guam - 29 Sept. 1976. (R/V Thomas Washington) Scripps Institution of Oceanography (unpublished) :A340

1977, Domes Project. Index of Box Cores, Cruise RP-8-OC-75 leg 3. Private communication J. Greenslate, 1977. :A337

1977, International CLB Group. Chemical analyses of manganese nodules from samples gathered on Kyokuyo Maru during 1972 fullscale CLB system test. Private communication, 1977 :A392

1977, International CLB Group. Chemical assay data on manganese nodule samples gathered by CNEXO free-fall samples on Kana Keoki during 1972 full scale CLB system test. Private communication, 1977 :A393

1977, Seascope Exploration Data Summary, Feb.-July 1972 (unpublished). Received June, 1977. :A386

1978, Kennecott Copper Corporation, Deepsea Nodules Analytical Report. Lamont-Doherty nodules and micronodules analyzed by wet chemistry in 1967-68. private communication, S.K. Addy, 1978 : A419

1979, List of cores and dredge hauls copied from shipboard logs. Scripps Institution of Oceanography, Indomed expedition, Sept. 1977-April 1979. (R/V Melville) :A421

1979, Preussag, Aktiengesellschaft. Station locations, sediment descriptions and nodule chemistry for cruise VA-07 Indian Ocean. Private communication, AMR, 1979 :A426

1979, U.S. Navy Cruises Bartlett (1971) and Kane (1970). Station locations for cores and dredges. Private communication, J. Green, Norda. Bay St. Louis, Missouri 1979 :A430

Ahrens, L.H., Willis, J.P., and Oosthuizen, C.0., 1967, Further observations on the composition of manganese nodules with particular reference to some of the rarer elements: Geochimica et Cosmochimica Acta, Vol. 31, pp. 2169-2180 :A200 
Andersen, M.E., 1978, Accumulation rates of manganese nodules and sediments:an alpha track method. MS thesis, USCD. : A418

Andrews, J.E., Landmesser, C.W., and Morgenstein, M., 1973, Hawaii Institute of Geophysics data banks for manganese collections and hydration-rind dating. Seabed Assessment Program, IDOE, National Science Foundation, Tech. Rept. No. 5 HIG-73-5, data rept. no. 23, March 1973. :A251

Andrews, J.E., and Friedrich, G.H.W. 1979, Distribution patterns of manganese nodule deposits in the northeast equatorial Pacific: Marine Mining, Vo1. 2, No. 1-2, pp. 1-43: A446

Andrushchenko, N.F., Gradusov, B.P., Yeroshchev-Shak, V.A., Yanshima, R.S., and Borisovsky, 1975, Composition and structure of metamorphosed ferromanganese nodules, new vein formations of manganese hydroxides, and the surrounding pelagic sediments in the southern basin of the Pacific Ocean floor: International Geology Review, Vo1. 17, No. 12, 1975. pp. 1375-1392.: A390

Aumento, F., Lawrence, D.E., and Plant, A.G., 1968, The ferromanganese pavement on the San Pablo Seamount: Canada Geological Survey Paper 68-32 to 68-39, pp. 1-30.:A283

Backer, H. Glasby, G.P., and Meylan, M.A., 1976, Manganese nodules from the southwestern Pacific basin:NZOI Oceanographic Field Report No. 6, March. :A293

Barnes, S.S., 1967, The Formation of Oceanic Ferromanganese Nodules. Unpublished PhD dissertation, Univ. of Calif., San Diego. :Al66

Batiza, R., 1979, Origin and Evolution of Sma11 Oceanic Volcanoes near Mid-ocean Ridges. Cruise report, rise III, leg 3, Washington University, St. Louis. 29 March to 22 April 1979. (R/V New Horizon) :A431

Batiza, R., Rosendah1, B.R., and Fisher, R.L., 1977, Evolution of oceanic crust w. Petrology and chemistry of basalts from the East Pacific Rise and the Siqueiros Transform Fault. Journal Geophysical Research, Vo1. 82, No. 2, Jan. 1977 :A360

Bender, M.F. and Shultz, C., 1979, The distribution of trace metals in cores from a traverse across the Indian Ocean: Geochimica et Cosmochimica Acta, Vol. 33, pp. 292-297. (Vema- 18):A199

Bezrukov, P. L. (editor) 1970 Osadkoobrazovaniye v Tixom okeane". Kn. 1I2 ("Sedimentation in the Pacific Ocean" part I and II). Tom VI, Tixii Okean. Chief ed. V.G. Kort, Moscow, 1970. :A148

Bezrukov, P. L. (editor) 1976, Ferromanganese nodules of the Pacific Ocean. Transactions of the P. P. Shirshov Institute of Oceanology, Vol. 109, Moscow. [ZHELEZO-MARGANTSEVYE KONKRETSII TIKHOGO OKEANA] : A343

Bezrukov, P.L. and Andrushchenko, P.F., 1974, Geochemistry of iron-manganese nodules from the Indian Ocean: International Geology Review, Vo1. 16, No. 9, pp. 1044-1061. :A246

Bischoff, J.L., and Piper, D.Z. (editors). 1979, Station locations from map in U. F. Stackelberg, sedimentation, hiatuses, and development of manganese nodules: 
Valdivia Site 13/2, northern central Pacific: In Marine Geology and Oceanography of the Pacific Manganese Nodule Province, P1 Plenum Press, New York :A446

Bonatti, E., 1967, Mechanisms of deep-sea volcanism in the South Pacific: Researches in Geochemistry, Philip H. Abelson (ed.), John Wiley Sons, Vol. 2, pp. 453-481. :A230

Bruun, A.F., 1959, General Introduction to the Reports and List of the Deep Sea Stations, Galathea Report: Scientific Results of the Danish Deep Sea Expedition Round the World 1950-1952, Vol. 1, pp. 7-48, Copenhagen. :A083

Burns, R,G., and Fuerstenau, D.W. . 1966, Electron-probe determination of inter-element relationships in manganese nodules:American Min Vol. 51, May-June. : A232

Campbe11, J.F. (chief Sc), 1980, Narrative cruise report of KK- 80-04-14 1eg 2, 18 May-11 June 1980. ( $R / \mathrm{V}$ Kana Keoki) (unpublished.) :A458

Cassidy, D.S., Kahharoeddin, F.A., Zemmels, I., and Knapp, M.B., 1977, USNS Eltanin: An inventory of core location data with core location maps and cruise 55 core descriptions. Antarctic research facility, Florida State Univ., sed. res. lab contrib. no. 44, April.:A289

Colley, N., Cronan, D.S., and Moorby, S., 1978, Some geochemical and mineralogical studies of recently collected ferromanganese oxide deposits from the north-west Indian Ocean: Presented at Colloque International No. 289 Sur 1a Genese des Nodules de Manganese, GIF-SUR-YVETTE, 25-30 Sept. 1978. :A433

Conolly, I.O., Core descriptions (relating to expedition described in 168) (unpublished); SIO core locker :M025

Cronan, D., 1973, Manganese nodules in sediments cored during leg 16, Deep Sea Drilling Project:Initial Reports of the Deep Sea Drilling Project, Vol. XVI, Ansis G. Kaneps (ed), government printing office, Washington, 1973, pp. 605-608. (R/V Glomar Challenger, 1971.) :A291

Cronan, D.S., 1975, Manganese nodules and other ferro-manganese oxide deposits from the Atlantic Ocean:Journal Geophysical Research, Vol. 80, no. 7, appendix I. :A368

Cronan, D.S., 1980, Underwater minerals:Academic Press, 362 p. :A467

Cronan, D.S., and Tooms, J.S., 1967, Geochemistry of manganese nodules from the N. W. Indian Ocean: Deep-Sea Research, Vo1. 14, pp. 239-249. :A203

Cronan, D.S. and Tooms, J.S., 1969, The geochemistry of managanese nodules and associated pelagic deposits from the Pacific and Indian Oceans. Deep Sea Research, Vo1. 16, pp. 335- 359.:A189

Cronan, D.S., 1967, The Geochemistry of some manganese nodules and associated pelagic deposits:PhD thesis, Imperial College, Univ. of London, March 1967. :A240

Cronan, D.S., 1977, Station locations from Shackleton 5/75, Private communication, April 1977 :A373 
Dietz, R.W., 1955, Manganese deposits on the northeast Pacific seafloor: California Journal of Mines and Geology, Vol. 51, pp. 209-220, 1955. :A255

Fein, C.D. and Morgenstein, M., 1973, Microprobe analysis of manganese crusts from the Hawaiian archipelago:Inter-university program of research on ferromanganese deposits on the ocean floor, phase I report, Seabed Assessment Program, IDOE, National Science Foundation, pp. 85-92, April :A254

Fewkes, R.H., McFarland, W.D., Reinhart, W.R., and Sorem, R.K., 1980, Evaluation of Metal Resources at and near Proposed Deep Sea Mine Sites: Final report, Aug. 15, 1978 to Sept. 29, 1979, U.S. Bureau of Mines, Feb. 15, 1980. :A448

Fisher, R.L., Engel, C.G., Hilde, T.W.C., 1968, Basalts dredged from the Admirante Ridge, Western Indian Ocean:Deep-Sea Research, Vol. 15, pp. 521-534 :A082

Fisk, M.B., Fitzgerald, R.W., and Frazer, J.Z., 1976, Pacific manganese nodules and crusts analyzed at Scripps Institution of Oceanography, Aug.-Dec. 1976. Ground to $<74$ microns, pressed into pellets, dried at $110 \mathrm{C}$, and analyzed by $\mathrm{x}$-ray energy spectrometry for $1000 \mathrm{sec}$. (unpublished.) :A321

Fisk, M.B. and Frazer, J.Z., 1978, Manganese nodules and crusts analyzed at Scripps Institution of Oceanography, 1977-1978. Ground to <74 microns, analyzed as pellets by xes for 400 secs, calculated to 110C. Unpublished. :A405

Fisk, M.B. and Frazer, J.Z., 1980, Manganese nodule chemical analyses, June 1979-June 1980: Scripps Institution of Oceanography, sediment data bank laboratory (in preparation) : A437

Fisk, M.B., Frazer, J.Z., Elliott, J.S., and Wilson, L.L., 1979, Availability of copper, nickel, cobalt and manganese from ocean ferromanganese nodules (II). Scripps Institution of Oceanography ref. no. 79-17, Aug. 24, 1979. :A422

Fisk, M.B., Frazer, J.z., and Fitzgerald, R.W., 1977, Scripps Institution of Oceanography manganese nodule analyses done April, 1977; dried at 110C, ground to $<74$ microns, pressed into pellets and analyzed by X-ray energy spectrometry for 400 seconds. :A376

Frank, D.J., Meylan, M.A., Craig, J.D., and Glasby, G.P., 1976, Ferromanganese deposits of the Hawaiian archipelago. UNIHI- SEAGRANT-CR-77-01, HIG 76-14, Hawaii Inst. Geophysics, Univ. of Hawaii, Dec. :A380

Frazer, F.W., and Ostwald, J., 1970, Chemical and Mineralogical investigations on a suite of deep-sea manganese nodules:Central Research Laboratories, Broken Hill Proprietary Co.,Ltd. and the Bureau of Mineral Resources, Shortland, Australia, CRL/TC/7/70:A247

Frazer, J., Fisk, M., Fitzgerald, R., and Guy, J., 1976, Chemical analyses of manganese nodules, 1975-1976, Scripps Institution of Oceanography. Nodules ground to < 74 microns, analyzed as pellets by $x$-ray energy spectrometry for $1000 \mathrm{sec}$, calculated to 110C. (unpublished.) :A298

Frazer, J., Fisk, M.B., and Fitzgerald, R., 1977, Manganese nodules and crusts analyzed at Scripps Institution of Oceanography, Jan.-Feb. 1977. Ground to < 74 microns, pressed into pellets. Dried at $110 \mathrm{C}$ and analyzed by $\mathrm{x}$-ray energy 
spectrometry for 1000 seconds. (unpublished.) :A342

Frazer, J.Z., 1980, Scripps Institution of Oceanography; Manganese Nodules Analyzed Sept. 1980. Samples dried at 110C, ground to $<74$ microns, pressed into pellets and analyzed by $x$ - ray energy spectrometry. :A459

Friedrich, G.H.W., Kunzendorf, H., and Pluger, W.L., 1974, Ship- borne geochemical investigations of deep-sea-manganese nodule deposits in the Pacific using a radio-isotope energy-dispersive $x$-ray system:Journal of Geochemical Exploration, Vol. 3, pp. 303-317. :A332

Fuerstenau, D.W., 1966, Metal recovery. from manganese nodules: Office of Research Services, College of Engineering, Univ. of Calif., Berkeley, June 1966. :A381

Gilbert, R.L.G., 1967, Cruise report no. 180, CSS Hudson. Bedford Inst. of Oceanography. :A305

Glasby, G.P., Influence of manganiferous fragments on the trace element geochemistry of pelagic sediments, N. W. Indian Ocean: New Zealand Journal of Geology and Geophysics, Vol. 15, No. 13, pp. 451-464. :Al94

Glasby, G.P., 1973, Manganese deposits in the southwest Pacific: Inter-university program of research on ferro-manganese deposits of the ocean floor, Phase I report, Seabed Assessment Program, IDOE, National Science Foundation, pp. 137-169, April 1973. :A250

Glasby, G.P., Tooms, J.S., and Cann, J.R., 1971, The geochemistry of manganese encrustations from the Gulf of Aden: Deep-Sea Res., Vol. 18, pp. 1179-87. :A195

Glasby, G.P., 1970, The geochemistry of manganese nodules and associated pelagic sediments from the Indian Ocean:Unpublished $\mathrm{PhD}$ thesis, University of London :A217

Glockhoff, C., 1973, Descriptions of cores from the Pacific Ocean taken on South Tow expedition: SIO ref. no. 76-21, Dec. 1976. (R/V Thomas Washington). Additional information from list of cores and dredge hauls copied from shipboard logs, Southtow Expedition, July 1972-January 1973. :A223

Glockhoff, C., and Helms, P.B., 1975, Descriptions of cores from the Tropical Central Pacific taken on Amphitrite Expedition. SIO ref. 75-29. Oct. 1975. Also SIO core logs, Amphitrite expedition. Unpublished. (R/V Argo) :M005

Goldberg, E.D., 1954, Marine geochemistry I. Chemical scavengers of the sea: Journal of Geology, Vol. 62, No. 3, pp. 249-265. :A165

Goldberg, E.D., and Koide, M., 1962, Geochronological studies of deep sea sediments of the ionium-thorium method:Geochimica et Cosmochimica Acta, vol. 26. :A068

Goodell, H.G.,, Marine geology of the Drake Passage, Scotia Sea, and South Sandwich Trench. Sedimentology Research Lab, Dept. of Geology, Florida State 19, Eltanin cruises 1-8.:B559

Grant, J.B., 1967, A comparison of the chemistry and mineralogy with the distribution and physical aspects of marine manganese concretions of the 
southern oceans:MS thesis, Sedimentological Research Lab, Dept. of Geology, Florida State Univ. :A202

Greenslate, J. and Gerard, R., 1976, Bomdrop Expedition (MN7503), R/V Kana Keoki, Honolulu to Honolulu, 20 Nov.-10 Dec. 1975. Technical report no. 13, Seabed Assessment Program, IDOE, National Science Foundation, (unpublished.) :A309

Greenslate, J, and Spiess, F.N., 1976, Pleiades Expedition, leg 4, R/V Melville; Honolulu, Hawaii to San Diego, California, 20 August-23 September 1976. Tech report no. 15, Seabed Assessment Program, IDOE, NSF, Washington, D.C., Dec. 1976. (unpublished.) :A338

Greenslate, J., Fisk, M., and Felix, D., 1978, Systematics in manganese concretion composition in the northeastern equatorial Pacific: Colloq. on the genesis of manganese nodules, GIF-SUR- YVETTE. :A334

Hamilton, E.L., 1956, Sunken Islands of the Mid-Pacific Mountains: Geol. Soc. Am. Memoir 64, 1956. : M038

Hathaway, J.C., 1971, Data file, Continental margin program, Atlantic coast of the United States Vol. 2. Sample collection and analytical data: Woods Hole Oceanographic Institution Ref. No. 71-15, Feb. 1971. (unpublished.) :A374

Heezen, B.C., Glass, B., and Menard, H.W., 1966, The Manihiki Plateaux, twenty cores, two dredge hauls, four bottom trawls and fifteen camera stations from the Vema cruise 18 Deep Sea Research, vol. 13, pp. 455-458:

Hein, P., 1977, CNEXO Station Locations, Grab Samples. Private communication, 1977. :A369

Hewett, D.F., Fleisher, M., and Conklin, N., 1963, Deposits of the manganese oxides:Economic Geology 58, pp. 1-51. : A262

Hoffert, M., Karpoff, A.M., Clauer, N., Schaff, A., Courtois, C., and Pautot, G. 1970, Neoformations and others terations dans trois facies volcano sedimentaires du Pacific Sud Oceanologica Acta, Vol. 1, No. 2, pp. 187-202. :A463

Hoffert, M., Perseil, A., Hekinian, R., Chourkroune, P., Needham, H.D., Francheteau, J., and LePichon, X., 1978, Hydrothermal deposits sampled by diving saucer in transform fault A near $37 \mathrm{~N}$ on the mid-Atlantic ridge, Famous area: Oceanologica Acta, Vo1.1, No. 1, pp. 73-86.:A464

Hoffert, M., 1980, Les argiles rouges des grands fonds" dans le Pacific centre-est: authigenese, transport, diagenese. PhD dissertation, L'Universite Louis Pasteur, Strasbourg, France. : A462

Hoover, P., 1967, Studies on the Dissolution of $\mathrm{Cu}$, Ni, and Co from Ocean Mn Nodules. Thesis, Univ. of Calif., Berkeley. :A379

Horn, D.R., Delach, N.N., and Horn, B.M., 1973, Metal content of ferromanganese deposits of the oceans: Office for the International Decade of Ocean Exploration, Tech. Rept. No. 3, NSF GX-33616, National Science Foundation, 1973 :A179 
Hubred, G.L., 1970, Relationship of morphology and transition metal content of manganese nodules to an abyssal hill Hawaii Inst. of Geophysics, HIG-70-18, Sea Grant 70-5, Sept. 1970. : A277

Isaeva, A.B., 1967, Khimicheskii sostav zhelezo-margantsovykh konkretsii indiiskogo okeana (Chemical composition of iron-manganese concretions of the Indian Ocean): Litologia i Poleznye Iskopaemye,No. 3, pp. 43-56. [Lith. Min. Res., No. 3, pp. 310-320.] :A220

Jacobs, S.S., Bruchhausen, P.M., and Bauer, E.B., 1970, Eltanin Reports, cruises 32-36, 1968. Hydroscopic stations, bottom photographs, current measurements. Lamont-Doherty Geological Observatory. :A371

Jacobs, S.S., Bruchhausen, P.M., Rosselot, F.L., Gordon, A.L., Amos, A.F., and Belliard, M., 1972, Eltanin Reports, cruises 37-39, 1969; 42-46, 1970. Hydrographic stations, bottom photographs, current measurements, nephelometer profiles. Lamont-Doherty Geological Observatory, TR 1-CU1-72, 1972 :A372

Jacobs, S.S., Bauer, E.B., Bruchhausen, P.M., Gordon, A.L., Root, T.A., and Rosselot, F.L., 1974, Eltanin Reports, cruises 47-50, 1971; 52-55, 1972. TR CU-2-74, Lamont-Doherty Geological Observatory. :A367

Johnson, D.A., 1971, Studies of deep sea erosion using deep-towed instrumentation: PhD thesis, Univ. of Calif., San Diego : Al19

Johnson, T.C. and Glockhoff, C., 1974, Description of Cores from the Pacific Ocean taken on Scan Expedition: Scripps Institution of Oceanography, SIO ref. 74-22, June 1974. ( $R / V$ Argo). : A117

Khristianova, L.A. and Romankevich, E.A., 1968, Rate of accumulation of deep sea sediments: Byulleten Komissii Po Izucheniyu Chetvertichnogo Perioda (Bulletin of the Commission on the Study of the Quarternary Period), Akademiia Nauk, SSSR, pp. 20-30, U. S. Naval Oceano. Office Trans. No. 458 :A069

Korkisch, J., 1976, Elemental analyses of manganese nodules by atomic absorption: Private communication, 1976 : A303

Korkisch, J., 1980, Analyses of $\mathrm{Ti}, \mathrm{Li}, \mathrm{Na}, \mathrm{Mg}, \mathrm{Al}, \mathrm{Ba}, \mathrm{Cd}, \mathrm{Mn}, \mathrm{Mo}, \mathrm{Fe}, \mathrm{Cu}, \mathrm{Ni}$, Co in Mn nodules by atomic absorption: Personal communication, 1980 :A465

Korkisch, J., Hubner, H., Steffan, I., Arrhenius, G., Fisk, M., and Frazer, J, 1976 Part I. Determination of seven main and trace constituents after anion-exchange separation:Anal. Chim. Acta,, Vo1. 83, pp. 83-92:A301

Krause, D.C., 1961, Geology of the Southern Continental Borderland West of Baja California, Mexico: PhD thesis, Univ. of Calif., San Diego, May 1961. Sediment lithology and geochemistry from thesis published in D.C.

Krause, Lithology and Sedimentation in the Southern Continental Borderland, in Papers in Marine Geology (ed. Robert L. Miller), pp. 274-318, Macmillan Co., New York, 1964. :M036

Krishnaswami, S., 1976, Authigenic transition elements in Pacific pelagic clays:Geochimica et Cosmochimica Acta, Vo1. 40. :A346 
Leclaire, L., and Perseil, E.A., 1978, Mineralogie, Composition Chimique et Milieux de Sedimentation de Concretions Polymetalliques dans l'Ocean Indien:Colloque sur la Genese des Nodules de Manganese:GIF-SUR-YVETTE, 25-30 Sept. 1978. : A416

Lonsdale, P., 1980, Locations of Turtle Dives on East Pacific Rise: Private communication, Jan. 1980 : A439

Lonsdale, P., 1971, Manganese Nodule analyses by International Nickel Company (Chain Cruise 100, 1971) Scripps Institution of Oceanography:Private communication. : A284

Marchig, V. and Gundlach, H., 1976, Zur Geochemie von Manganknollen aus den Zentralpazifik und Ihrer Sedimentunterlage I. Manganknollen: Geol. J.B. I, D 16, 8ABB, Hanover, 1976. : A326

Menard, H.W., Goldberg, E.D., and Hawkes, H.E., Composition of Pacific sea-floor manganese nodules: SIO unpublished data. : A261

Mero, J.L., 1965, The Mineral resources of the sea:Elsevier Pub. Co., Amsterdam. :A197

Mero, J., 1975, Nodule product, Private communication :A285

Meylan, M.A., Backer, H., and Glasby, G.P, 1975, Manganese nodule investigations in the southwestern Pacific basin, 1974: New Zealand Oceanographic Institution Field Report No. 4, Jan. 1975. (Tangaroa) :A281

Meylan, M.A., Glasby, G.P., McDougall, J.C., and Singleton, R.J., 1978, Manganese nodules and associated sediments from the Samoan Basin and Passage: NZOI Oceanographic Field Report, NO. 11, Feb. 1978. :A414

Meylan, M.A. and Goode11, H.G., 1976, Chemical composition of manganese nodules from the Pacific-Antarctic Ocean, Drake Passage and Scotia Sea: relation to ferromanganese oxide mineralogy and nucleus type: In G.P. Glasby and H.R. Katz (eds), Marine Geological Investigations in the Southwest Pacific and Adjacent Areas, CCOP/SOPAC Tech. Bull No. 2, papers presented at the IDOE workshop, Suva, Fiji 9/75,76:A227

Mizuno, A., 1977, Analyses and sample data from GH74-5. Private communication, $1977:$ :A363

Mizuno, A. and Chujo, J. (eds.)., 1975, Deep sea mineral resources investigation in the eastern Central Pacific basin, Aug.-Oct. 1974 (GH74-5 Cruise). Cruise report no. 4, Geological Survey of Japan. :A280

Mizuno, A. and Moritani, T. (eds.), 1977, Deep sea mineral resources investigation in the central-eastern part of the central Pacific Basin, Jan. -Mar. 1976 (GH76-1). Geological Survey of Japan, Cruise report no. 8. :A361

Mizuno, A., and Moritani, T., (eds.), 1977, Deep sea mineral resources investigation in the central-eastern part of the central Pacific Basin, Jan. -Mar. 1976 (GH76-1). Geological Survey of Japan, Cruise report no. 8. :A361 
Monzier, M., 1976, Manganese nodules and encrustations in the vicinity of New Caledonia and the Loyalty Islands: In papers presented at IDOE Workshop, Suva, Fiji, 1-6 Sept. 1975, G. P. Glasby and H. R. Katz (eds.), tech. bull. no. 2, U.N. Economic and Social Commission for Asia and the Pacific, CCOP/SOPAC. : A349

Moore, H., 1966, Mn nodules, topography and thickness of quaternary sediments in the Central Pacific: Nature, Vo1. 212, Dec. 3, 1966 :A033

Morgan, C.L., 1975, Nucleation and accumulation of marine ferromanganese deposits: PhD thesis, Univ. of Wisconsin, May 9, 1975 :A324

Morgan, C.L., and Moore, J.R., 1975, Role of the nucleus in formation of ferromanganese nodules:Processing Guidelines for the Marine Miner. Offshore Technology Conference Paper No. OTC- 2243, May 5, 1975. :A324

Morten, L., Landini, F., Bocchi, G., Mottana, A., and Brunfelt, A.0., 1980, Fe-Mn crusts from the southern Tyrrhenian Sea: Chemical Geology 1980, Vo1. 28, pp. 261-278.:A477

Mueller, D., 1977, Station data for manganese nodule samples sent from Metallgesellschaft a.g.: Private communication, Jan. 1977 :A351

Murray, J. and Lee, G.V., 1909, The Depth and Marine Deposits of the Pacific. Reports on the Scientific Results of the Expedition to the Tropical Pacific on U.S. Fish Commission Steamer Albatross, Aug. 1899-March 1900. No. XII, Memoirs of the Museum of Comparative Zoology, Harvard College, Vol. XXXVIII, No. 1, Cambridge, 1909. (Albatross) :A010

Murray, J. and Lee, G.V., 1909, The Depth and Marine Deposits of the Pacific. Report on the Scientific Results of the Expedition to the Eastern Tropical Pacific... by the... Albatross from October 1904 to March 1905... No. XVII, Memoirs of the Museum of Comparative Zoology at Harvard College, Vol. XXXVIII, No. 1, Cambridge, 1909. (Albatross) :A009

Murray, J. and Renard, A.F., 1891, Report on Deep-Sea Deposits Based on the Specimens Collected in the Years 1872 to 1876 in Report on the Scientific Results of the Voyage of HMS Challenger during the Years 1873-1876 Longmans Co., London, 1891. (HMS Challenger 1873-1876) :A012

Nikolayev, D.S. and Yefimova, E.I., 1963, On the age of iron-manganese concretions from the Indian and Pacific Oceans: Geochemistry, No. 7, pp. 703-714 :A276

Nohara, M., 1976, Geochemical Characteristics in Pacific Ocean manganese nodules and its controlling factors:Marine Sciences Monthly, Vol. 8, No. 11, 1976 :A366

Okada, A. and Shima, M., 1970, Study on the manganese nodule: Journal of the Oceanographical Society of Japan, Vol. 26, No. 3, pp. 151-158, June. :A249

Ostwald, W. Frazer, J.F., 1973, chemical and mineralogical investigations on deep sea manganese nodules from the Southern ocean: Mineral. Deposita (Ber1.) 8, pp. 303-311. (USNS E1tanin.) :A243

Pasho, D., 1977, Confidential reference, 1977 :A395 
Piper, D.Z., 1974, Rare earth elements in ferromanganese nodules and other marine phases: Geochimica et Cosmochimica Acta, Vo1. 38, pp. 1007-1022. :A413

Piper, D.Z., 1977, Nodule analyses of seascope samples, done by atomic absorption: USGS Menlo Park, Feb. 10, 1977. Private communication. :A383

Piper, D.Z. and Williamson, M., 1977, Composition of Pacific Ocean Ferromanganese nodules:Marine Geology, Vol. 23, pp. 285- 303, 1977. :A323

Revelle, R.D., 1944, Marine bottom samples collected in the Pacific Ocean by the Carnegie on its 7 th cruise: Carnegie Inst., Washington, Publication 556, Oceanography-II, Pt. 1, PP. 1-180, Dec. 30, 1944.:A006

Riedel, W.R., and Funnel1, B.M., 1964, Tertiary sediment cores and microfossils from the Pacific Ocean floor:Quarterly Journal of the Geological Society of London, Vol. 120. :N682

Roonwal, G.W., and Friedrich, G.H., 1980, Chemical variation and element correlation in manganese nodules from. a dredge haul in the central Pacific:Indian Journal of Marine Sciences, Dec., Vol. 9, pp. 235-239. :A474

Shepard, F.P., 1964, Sea-floor valleys of Gulf of California: In Marine Geology of the Gulf of California, eds. T.H. Van Andel and G.G. Shor, Jr. Mem. 3, 1964, pp. 157-192. : A417

Shima, Makoto, and Okada, Akihiko, 1974, Study on the manganese nodule (VIII), unhomogenous chemical distribution of manganese nodule: Scientific Report of the Inst. of Physical and Chemical Research, Vol. 50, No. 4, July, 1974, pp. 155-164. :A248

Siegel, M. and Fisk, M.B., Geochemical studies of manganese nodules from the northeastern equatorial Pacific (in preparation) Confidential until publication :A438

Sims, R., 1981, Methods and results of analysis of trace metal levels in selected ferromanganese nodules: Science Applications, Inc., Report to U.S. Bureau of Mines, March, 1981. (unpublished.) :A475

Skornyakova, N.C., Bezrukov, P.L., Vasilevskaya, E.C., and Gordeev, V.V., 1979, Ferro-manganese Concretions from the Eastern Indian Ocean (in Russian): Lithology and Mineral Resources, No. 3, 1979. :A451

Skornyakova, N.S. and Andruschenko, P.F., 1970, Zhelezo-margantseviye konkretsii $v$ Tikhom Okeane. (Ferro-manganese nodules in the Pacific Ocean.): in Osadkoobrazovaniye $v$ Tikhom Okeane, (Sedimentation in the Pacific Ocean), P.L. Bezrukov (ed.-in-chief), Vol. VI, parts 1 2,ch. 17, pp. 203-268, Akademiia Nauk, SSSR, P.P. Shirskov Inst. O.Moscow :A178

Skornyakova, N.S. and Petelin, V.P., 1967, Sediments of the central region of the southern part of the Pacific Ocean (in Russian):Okeanologiya, Vol. 7, pp. 1005-1019:A140

Smith, J.D. and Burton, J.D., 1972, The occurrence and distribution of tin with particular reference to marine environments:Geochimica et Cosmochimica Acta, 
Vol. 36, pp. 621- 629.:A354

Stetson, T.R., 1961, Report on Atlantis cruise no. 266, June- July 1961. Woods Hole Oceanographic Institution Ref. No. 61-35, Oct. :N170

Summerhayes, C.P., 1967, Manganese nodules from the southwestern Pacific.: NZOI Journal of Geology and Geophysics, Vol. 10, No. 6, pp. 1372-81. :A260

Summerhayes, C.P. and Willis, J.P., 1973, Manganese encrustations from the Agulhas Bank. Chapter XI in joint Geological Survey-Univ. of Cape Town Marine Geology Program Technical Report 5, pp. 121-126. :A466

Tin, M.T., Suttle, A.D. and Sackett, W.M., 1973, Uranium concentrations in marine sediments:Geochimica et Cosmochimica Acta, Vo1. 37, pp. 35-51. :A206

Van der Weijden, C.H., 1975, Sorption experiments relevant to the geochemistry of manganese nodules: Dept. of Geochemistry, Univ. of Utrecht, May 6, 1975. :A272

Von Gumbe1, A., 1888, Die Mineralogisch-Geologische Beschaffenheit der Auf der Forschungsreise S.M.S, Gazelle" Gesammelten Meersgrundablagerungen. (The geological- mineralogical analysis...sediments...S.M.S. "Gazelle".) Die Forschungreise S.M.S. "Gaze1le" in den Jahren 1874 bis 1876. Hydrographischen Amt der Admiralitat, , Berlin, pp. 69-116. :A088

Walsh, T., 1977, Station data for Dodo dredges 6-16 and misc. dredge locations: Scripps Institution of Oceanography. Private communication, 1977 :A347

Willis, J.P., 1970, Investigations on the composition of manganese nodules with particular reference to certain trace elements:MS thesis, Dept. of Geochemistry, Univ. of Cape Town, 1970. :A388

Willis, J.P., and Ahrens, L.H., 1962, Some investigations on the composition of manganese nodules, with particular reference to certain trace elements:Geochimica et Cosmochimica Acta, Vol. 26, pp. 751-764. :A201

Yabuki, H., 1971, Fission track analysis of uranium in manganese nodules collected from Pacific Ocean floor: SCI Papers, Inst. of Physical and Chemical Research, Vo1. 65, No. 3- 4. :A389

Yabuki, H. and Shima, M., 1973, Uranium and other heavy elements in deep sea sediments coexisting with manganese nodules: Scientific Papers, Inst. of Physical and Chemical Research, Vo1. 67,No. 3, pp. 155-56. :A256

Young, E.J., 1968, Spectographic data on cores from the Pacific Ocean and the Gulf of Mexico:Geochimica et Cosmochimica Acta, Vo1. 32, No. 4, pp. 466-471, Apri1. :A077 


\section{USGS SUPPLEMENTARY REFERENCE LIST}

Abbey, S. and Rousseau, R.M., 1985, Pragmatism vs. rigour: A debate on the resolution of disparate analytical data on four Canadian iron-formation reference samples, Geostandards Newsletter, v. 9, p. 1-16.

Aplin, A.C., 1983, The geochemistry and environment of deposition of some ferromanganese oxide deposits from the south equatorial Pacific, D.Sc. dissertation, Royal School of Mines, Imperial College, London, 347p.

Aplin, A.C., 1984, Rare earth element geochemistry of Central Pacific ferromanganese encrustations, Earth and Planetary science letters, v. 71, p. 13-22.

Aplin, A.C., and Cronan, D.S.,1985, Ferromanganese oxide deposits from the Central Pacific Ocean, Encrustations from the Line Islands Archipelago, Geochimica et Cosmochimica Acta, v. 49, no. 2, p. 437-451.

Arrhenius, G., Cheung, S., Crane, K., Frazer, Z., Korkisch, T., and Mellin, T., 1979, Couterions in marine manganese minerals, in Lalou, C. (Ed.), La genese de nodules de manganese, Coloque 289 du CNRS, p. 237-358.

Arrhenius, G.O.S., Mero, J., and Korkisch, J., 1964. Origin of oceanic manganese minerals, Science, v. 144, p. 170-173.

Arrhenius, G.O.S. and Bonatti, E., 1965. Neptunism and vulcanism in the ocean, Progr. Oceanography, v. 3, p. 7-22.

Arrhenius, 0., 1959, Die Grundlagen unserer älteren Eisenherstellung. Kung1. Vitterhets Historie och Antikvitetsakad. Handlingar, Stockholm, , v. 13, p. 1-46.

Aruscavage, P. J., Simon, F.O. and Moore, R., 1984, Flameless Atomic Absorption Determination of Platinum, Palladium, and Rhodium in Geologic Materials; Geostandards Newsletter, v. 8, 1, 6 p.

Aruscavage, P.J., Simon, F.0., and Moore, R., 1984, Flameless atomic absorption determination of $\mathrm{Pt}, \mathrm{Pd}$, and $\mathrm{Rh}$ in geologic material, Geostandards Newsletter, v. 8 p. $3-6$.

Aumento, F., 1969, The Mid-Atlantic-Ridge near $45^{\circ} \mathrm{N}$, V. fission track and ferro-manganese chronology, Canadian Journal of Earth Sciences, v. 6, p. $1432-1440$.

Aumento, F., Lawrence, D.E., and Plant, A.G., 1968, "The ferromanganese pavement on San Pablo seamount, Geological Survey Canada Paper 68-32, 30p.

Barnes, S.S., 1967. Minor element composition of feromanganese nodules. Science, v. 157, p. $63-65$.

Bastien-Thiry, H., 1978. Le point des activites nodules. In Resources Minerales sous-marines, Comptes Rendus, Document 7, BRGM, Germanal, Orleans, p. 381-390. Baturin, G.N., 1986, Geokhimiya Zhelezomargantsevykh Konkretsii Okeana (Geochemistry of iron-manganese concretions in the ocean), Nauka, Moscow, 328p. 
Bazilevskaya, E.S., 1976. Fiziko-mineralogicheskoe issledovaniye margantsevykh rud. (Nikopol'skoii bassein i konkretsii Tikhogo okeana) (Physico-mineralogical studies on manganese ores (Nikopol Basin and concretions of the Pacific Ocean). Trudy Geologicheskii Institut Akademi Nauk, SSSR, v. 287, 143p.

Bender, M.L., Ku, T.L., and Broecker, W.S., 1970. Accumulation rates of manganese in pelagic sediments and nodules. Earth and Planetary Science Letters, v. 8, p143-148.

Bender, M.,, Ku, T.L., and Broecker, W.S., 1970, Manganese nodules: their evolution, Science, v. 151, p. 325-328.

Bezrukov, P.L. and Andruschenko, P.F., 1973, Iron-manganese concretions of the Indian Ocean, International Geology Review, v. 15, p. 342-356.

Bezrukov, P.L., 1976, (ed.), Zhelezomargantsevye konkretsii Tikhogo okeana, Trudy Institituta Okeanologii, Akademii Nauk, SSSR,, Moscow, 301p.

Bhat, S.G., Krishnaswamy, S., Lal, D., Rama, and Somayajulu, B.L.K., 1973, Radiometric and trace elemental studies of ferromanganese nodules, Proceedings Symposium on Hydrogeochemistry and Biogeochemistry, Washington D.C., p. 443-462.

Bignot, G., and Lamboy, M., 1980. Les Foramineferes epibiontes a test calcaire hyalin des encroutements polymetalliques de la marge continental au Nord-Ouest de la Peninsule iberique, Revue Micropaleontologie, v. 23, p. 3-16.

Bischoff, J.L., and Piper, D.Z., 1979. Marine geology and oceanography of the Pacific Manganese Nodule Province, Plenum Press, New York and London, 842p.

Bogdanov, Yu. A., Zonenshain, L.P., Lisitsin, A.P., Podrazhanskii, A.M., Sagalevich, A.M., and Sorokhtin, O.G., 1987, Ferromanganese crusts on oceanic seamounts (Russian), Izvestiya Akademii Nauk, SSSR, Seriya Geologicheskaya, 1987:7, p. 103-120.

Bonatti, E., Kraemer, T., and Ryde11, H., 1972, Classification and genesis of submarine iron-manganese deposits: In Ferromanganese Deposits on the Ocean Floor, NSF-IDOE, Washington D.C., p. 149-166.

Bostroem, K., and Peterson, 1966, Precipitates from hydrothermal exhalations on the East Pacific Rise, Economic Geology, v. 61, p. 1258-1265.

Botbol, J.M., 1986, Some basic concepts useful in initiating and implementing marine geologic databases in Johnson, C.J. and Clark, A.L., editors, Pacific marine mineral resources, physical economic and legal issues; Proceedings of the 1985 Pacific mineral resources training course, Resources Systems Institute, East-West Center, Honolulu, Hawaii, p. 389-422.

Bourne, M.A., Tavares, G.L. Jr., and Whipple, M.R., 1987, An economic analysis of factors influencing the mining of ferromanganese deposits for the purpose of strategic metals recovery, Interactive Qualifying Project, Worcester Polytechnic Institute, Worcester, MA., 109p.

Boussingault, M., 1882, Sur 1'apparition du manganese a la surface des roches, Annales de Chimie et le Physique 5th Ser. T. xxv, p. 289-311. 
Bowen, R. W. and Botbol, J.M., 1975, Geological retrieval and synopsis program (GRASP), U.S. Geological Survey Professional Paper 960, 87p.

Broadus, J.M., 1987, Seabed materials, Science, v. 235, p. 853- 859.

Buchanan, J.Y., 1876, On chemical and geological work done on board H.M.S. Challenger: Proceedings of the Royal Society of London, v. 24, p. 593-623.

Buchanan, J.Y., 1891, On the composition of oceanic and 1ittoral manganese nodules, Transactions of the Royal Society of Edinburgh v. 36, p 459-484.

Burnette, W.C., and Piper, D.Z, 1977, Rapidly-formed ferromanganese deposit from the eastern Pacific Hess Deep, Nature, v. 265, no. 5595, p. 596-600.

Burnette, W.C., and Morgenstein, M., 1976, Growth rates of Pacific manganese nodules as deduced by uranium series and hydrationb-rind dating techniques, Earth and Planetary Science Letters, v. 33, p. 208-218.

Burns, R.G., 1965, Formation of cobalt (III) in the amorphous $\mathrm{FeOOH} . \mathrm{nH}_{2} \mathrm{O}$ phase of manganese nodules, Nature, v. 205, p. 999-1002.

Burns, R.G., 1976, The uptake of cobalt into ferromanganese nodules, soils, and synthetic manganese (IV) oxides, Geochimica et Cosmochimica Acta, v. 40, p. 95-102.

Burns, R.G., and Burns, V.M., 1979, Manganese oxides, in Burns, R.G. (ed.) Marine Minerals, Reviews in Mineralogy, v. 6, p. 1-46.

Callendar, E., and Bowser, C.J., 1976, Freshwater ferromanganese deposits, In Wolf, K.H. (ed.), Handbook of strata-bound and stratiform ore deposits, Elsevier Scientific Publishing Company, Amsterdam, p. 341-394.

Calvert, S.E., 1978, Geochemistry of oceanic ferromanganese deposits: Philosophical Transactions of the Royal Society of London, v. 290A, p. 43-73.

Calvert, S.E., and Price, N.B., 1977, Geochemical variation in ferromanganese nodules and associated sediments from the Pacific Ocean: Marine Chemistry, v. 5, p. $43-74$.

Calvert, S.E., and Piper, D.Z., 1984, Geochemistry of ferromanganese nodules from Domes Site A, northern equatorial Pacific; multiple diagenetic source sin the deep sea, Geochimica et Cosmochimica Acta, v. 48, p. 1913-1928.

Campbel1, F., 1981. Narrative Cruise Report, KK81-06-26 Leg 2, (Hawaii Institute of Geophysics), Majuro, Marshall Islands to Truk, Caroline Islands. 9p

Cann, J. R., and others, 1977, A hydrothermal deposit from the floor of the Gulf of Aden: Mineralogical Magazine, London, v. 41, p. 193-199.

Carnegie-Mellon University, 1985, Seabed mining of cobalt-rich manganese crusts in the Pacific United States Exclusive Economic Zone, Department of Engineering and Public Policy, School of Urban and Public Affairs and Department of Social Sciences Project Report, 209p. 
Chave, K.E., Morgan, C.L., and Green, W.J., 1986. A geochemical comparison of manganese oxide deposits of the Hawaiian Archipelago and the Deep Sea, Applied Geochemistry, v. 1 p. $233-240$

Chukhrov, F.V., Gorshkov, A.I., Rudnitskaya, E.S., Berezovskaya, V.V., and Sivtsov, A.V., 1978, On vernadite (in Russian), Izvestiya Akademii Nauk, SSSR, Seriya geologicheskaya, No. 6, p. 5-19.

Chukhrov, F.V., Gorshkov, A.I., Rudnitskaya, E.S., and Sivtsov, A.V., 1978, On the characteristics of birnessite (in Russian), Izvestiya Akademii Nauk, SSSR, Seriya geologischeskaya, No. 9, p.67-76.

Chukhrov, F.V., Gorshkov, Berezovskya, V.V., and Sivtsov, A.V., 1979, Contributions to the mineralogy of authigenic manganese phases from marine manganese deposits, Mineralium deposita, v. 14, p. 2249-261.

Chukhrov, F.V., Gorskhkov, A.I., Drits, V.A., Shterenbeerg, L.Y, Sivtsov, A.V., and Sakharov, B.A., 1983, Mixed-layer asbolite-buserite minerals and asbolites in oceanic iron-manganese nodules, International Geology Review, v. , p. 838-847.

Clark, A.L., 1986, Basic data problems inherent in cobalt-rich manganese crust research, in Johnson, C.J. and Clark, A.L., (eds.), Pacific marine mineral resources, physical economic and legal issues; Proceedings of the 1985 Pacific mineral resources training course, Resources Systems Institute, East-West Center, Honolulu, Hawaii, p.423-432.

Clark, A.L., and Clark, J.C., 1986, Elements of a rational marine mineral policy within national exclusive economic zones, in Johnson, C.J. and

Clark, A.L., (eds.), Pacific marine mineral resources, physical economic and legal issues; Proceedings of the 1985 Pacific Mineral Resources Training Course, Resources Systems Institute, East-West Center, Honolulu, Hawaii, p.485-497.

Clark, A.L., Humphrey, P., Johnson, C.J., and Pak, D.K., 1985, Cobalt-rich manganese crust potential, U.S. department of the Interior Minerals Management Service OCS Study MMS 85-0006, 35 p. plus maps.

Clark, A., Johnson, C. and Chinn, P. 1984 Assessment of cobalt- rich manganese crusts in the Hawaiian, Johnston and Palmyra Islands' Exclusive Economic Zones, Natural Resources Forum, v. 8, p. 163-174.

Colley, N.M., Cronan, D.S., and Moorby, S.A., 1979 In Lalou, C. (editor) La genese des nodules de manganese, Colloques Internationaux du C.N.R.S., No. 289, p. $13-22$.

Commeau, R.F., Clark, A., Johnson, C. Manheim, F.T., Aruscavage, P.J., and Lane, C.M., 1984. Ferromanganese crust resources in the Pacific and Atlantic Oceans: Proceedings of Oceans'84, P. 421-430.

Commeau, R.F., Commeau, J.A., Brown, F.W., and Manheim, F.T., 1985, Energy dispersive $\mathrm{X}$-ray analysis of ocean ferromanganese crusts using conventional ZAF corrections, Scanning electron microscopy, 1985/1, p. 143-149. 
Corliss, J. B., and others, 1978, The chemistry of hydrothermal mounds near the Galapagos Rift: Earth and Planetary Science Letters, v. 40, no. 1, p. 12-24.

Craig, J.D. and Andrews, I.E., 1978, A factor analysis study of deep-sea ferromanganese deposits in the equatorial North Pacific Ocean, Marine Mining, v. 2, No. 14, p. 305-326.

Craig, J.D., Andrews, J.E., and Meylan, M.A., 1982, Ferromanganese deposits in the Hawaiian Archipelago, Marine Geology, v. 45, p. 127-157.

Cronan, D.S., 1967, The geochemistry of some manganese nodules and associated pelagic deposits: PhD thesis, Imperial College, University of London, March 1967.

Cronan, D. S., and others, 1982, A submarine hydrothermal manganese deposit from the South-west Pacific island arc: Nature, v. 298, no. 5873, p. 456-458.

Cronan, D.S., 1975, Manganese nodules and other ferromanganese oxide deposits from the Atlantic Ocean, Journal of Geophysical Research, v. 80, p. 3831-3837.

Cronan, D.S., 1977, Deep-sea nodules: distribution and geochemistry, in Glasby, J.P. (ed.) Marine manganese deposits, Elsevier Publishing Co., Amsterdam, p. $11-44$.

Cronan, D.S., 1980, Underwater Minerals, Academic Press, London -New York, 362 p.

Cronan, D.S., 1984, Criteria for the recognition of areas of potentially economic manganese nodules and encrustations in the CCOP/SOPAC region of the Central and Southwestern tropical Pacific, South Pacific Marine Geological Notes, Technical Secretariat CCOP-SOPAC, ESCAP, v. 3, p. 1-17.

Cronan, D.S., 1986, editor, Sedimentation and mineral deposits in the Southwestern Pacific Ocean, Academic Press, London - Orlando, 344 p.

Cronan, D.S., and Tooms, J., 1969, Geochemistry of manganese nodules and associated pelagic deposits from the Pacific and Indian Oceans, Deep-Sea Reseearch, v.16, p. 335-359.

Cronan, D.S., and Moorby, S.A., 1981, Manganese nodules and other ferromanganese oxide deposits from the Indian Ocean, Journal Geological Society of London, v. 138 , p. $527-539$.

Cruickshank, M.J., Bailey, A.V., and Kesterke, D.E., 1985, (eds) EEZ Mineral Notes, U.S. Department of the Interior (Newsletter), 12 issues.

Davis, J.C., 1986, Statistics and data analysis in geology, 2nd ed., John Wiley \& Sons, New York, 646 p.

DeCarlo, E.H., McMurtry, G.M., and Kim, K.H., 1987, Geochemistry of ferromanganese crusts from the Hawaiian Archipelago Exclusive Economic Zone: 
Northern survey areas, Deep-Sea Research, v. 34, p. 441-467.

DeCarlo, E.H., Penniwell, P.A., and Fraley, C.M., 1988, Geochemistry of ferromanganese deposits from the Kiribati and Tuvalu region of the West-Cental Pacific Ocean, Marine Mining, v. 6, p. 301-321.

Defossez, M., Monget, J.M., and Roux, P., 1980, Contribution of statistical methods to the study of some south-Pacific sediments in relation with manganese nodule deposits, in Varentsov, I.M. and Grasselly, G. (eds.), Geology and geochemistry of manganese, v.1, Schweizerbartsche Verlagsbuchhandlung, Stuttgart, p. 413- 442 .

Department of Planning and Economic Development, 1987, Mining Development scenario for cobalt-rich manganese crusts in the exclusive economic zones of the Hawaiian Archipelago and Johnston Islands. State of Hawaii Department of Planning and Economic Development and U.S. Department of the Interior, Minerals Management Service, Department of Planning and Econmomic Development, Ocean Resources Branch Contribution No. 38, Honolulu, Division of Ocean Resources, 323p.

Dillard, J. R., and Crowther, D. L., 1984, X-ray photoelectron spectroscopic study of ferromanganese nodules: Chemical speciation for selected transition metals: Geochimica et Cosmochimica Acta, v. 48, no. 5, p. 1565-1569.

Dillard, J.D., Crowther, D.L., and Murray, J.W., 1982, The oxidation states of cobalt and selected metals in Pacific Ocean ferromanganese nodules, Geochimica et Cosmochimica Acta, v. 46, p. 755-759.

Dugolinsky, B.K., Margolis, S.V., and Dudley, W.S., 1977, Biogenic influence in growth of manganese noduels, Journal of Sedimentary Petrology, v. 47, p. 428445 .

Dymond, J., Lyle, M., Finney, B., Piper, D.Z., Murphy, K., Conard, R., and Pisia, N., 1984, Ferromanganese nodules from MANOP Sites H, S, and R - Control of mineralogical and chemical composition by multiple accretionary processes, Geochimica et Cosmochimica Acta, v. 48, p. 931-949.

Ehrlich, A. M., 1968, Rare earths in Manganese-Iron concretions (Ph.d. Dissertation): Cambridge Massachusetts Institute of Technology, $225 \mathrm{p}$.

Egiazarov, B.Kh., 1980 Geologiya i Tverdye Poleznye Iskopaemye Mirovogo Okeana (Geology and hard mineral resources in the world oceans), Sbornik, Nauchno-Issledovatel'skii Institut Geologii Arktiki, Ministerstva Geologii, SSSR, Leningrad, 145p.

Evenden, G.I., 1986, The MAPGEN cartographic system, in Stagger, D. (ed.), Proceedings of the 1986 workshop and symposium on oceanographic data systems, Publication of the Computer Service Press, Institute of Electrical and Electronic Engineers, p. $239-245$.

Ewing, M., Shipley, T.H., and Connary, S.D., 1973, In Inter- University Program of research on ferromanganese deposits of the ocean floor, Phase I Report, p. $187-215$. 
Exon, N. F., 1982, Offshore sediments, phosphorite and manganese noduels in the Samoan region, southwest pacific: Southwest Pacific Marine Geological Notes, v. 2 , no. 7, p. 103-120.

Fasse1, V. A., 1978, Quantitative Elemental Analysis by plasma emission spectroscopy, Science, V. 202, No. 13, p. 183-191.

Flanagan, F. J. and Gottfried, David, 1980, USGS Rock Standards, III: Manganese Nodule Reference Samples USGS-NOD-A-1 and USGS-NOD-P-1; U.S. Geological Survey Professional Paper 1155, 39 p.

Frank, D.J., Meyland, M.A., Craig, J.D., and Glasby, G.P., 1976, Ferromanganese deposits of the Hawaiian Archipelago, Hawaii Inst. of Geophysics Report, No. HIG $76-14, .71 \mathrm{pp}$.

Frazer, J.Z., Freelander, N.A., and Hawkins, D.L., 1978, Sediment databank coding instructions, Scripps Institution of Oceanography SIO reference 78-9, 27p.

Frazer, J.Z, and Fisk, M.B., 1980, Availability of copper, nickel, cobalt and manganese from ocean ferromanganese nodules (III), Scripps Institution of Oceanography Report SIO 80-16, 117p.

Frazer, J.Z., and Fisk, M.B. 1980, Geological factors related to characteristics of seafloor manganese nodule deposits, Report prepared for U.S. Department of Interior, Bureau of Mines, SIO Reference 79-19, 41p.

Frazer, J.Z. and Fisk, M.B., 1981, Geological factors related to chearacteristics of sea-floor manganese nodule deposits. Deepsea Research, v. 28A, p. 1533-1551. Friedrich, G. and Schmitz-Wiechowski, A., 1980, Mineralogy and chemistry of a ferromanganese crust from a deep-sea hill, Central Pacific, "Valdivia" cruise VA $13 / 2$. Marine Geology, v 37, p. 71- 90.

Futa, K., Peterman, Z.E., and Hein, J.R., 1988, Sr and Nd isotopic variations in ferromanganese crusts from the Central Pacific: implications for age and source provenance, Geochimica et Cosmochimica Acta, v. 52, p. 2229-2235.

Giovanoli, R., 1980, On natural and synthetic manganese nodules, In Varentsov, I.M. (ed.) Geology and geochemistry of manganese, v. 1, Schweizerbartsche Verlagsbuchhandlung, Stuttgart, p. 159- 202.

Glasby, G. P., Keays, R. R., and Rankin P. C., 1978, The distribution of rare earth, precious metal and other trace elements in recent and fossil deep-sea manganese nodules, Geochemical Journal, v. 12, pp. 229-243.

Glasby, G.P., 1972, Manganese deposits in the southwest Pacific, in Andrews, J.E., Moregenstein, M., Fein, C.D., Meyland, M.A., Margolis, S.V., Andermann, G., Glasby, G.P., Investigations of ferromanganese deposits of the Central Pacific, Hawaii Institute of Geophysics Report H16-72-23, 133p.

Glasby, G. P., 1972, Indian/Antarctic nodules, in Symposium Proceedings, Manganese nodule deposits in the Pacific: Honolulu, Department of Planning and 
Economic Development, State of Hawaii, p. 73-82.

Glasby, G. P., and others 1974, Geochemistry of manganese concretions from the northwest Indian ocean: New Zealand Journal of Science, v. 17, p. 387-407.

Glasby, G. P., and others, 1975, Metal contents of Manganese nodules from the southwestern Pacific basin: Erzmetall, v. 28, no. 7/8, p. 340-342.

Glasby, G.P., 1977, Marine manganese deposits, Elsevier Scientific Publishing Co., Amsterdam-New York., 523p.

Glasby, G.P., 1982, Marine mining and mineral research activities in Europe, Marine Mining, v. 3, p. 379-409.

Glasby, G.P., 1986, Marine minerals in the Pacific, Oceanographic and Marine Biology Annual Reviews, v. 24, p. 11-64.

Glasby, G.P., and Andrews, J.E., 1978, Manganese crusts and nodules from the Hawailan Ridge, Pacific Science, v. 31, p. 363-379.

Glasby, G. P., and Thijssen, T., 1982, The nature and composition of the acidinsoluble residue and hydrosalate fraction of manganese nodules from selected areas in the equatorial and southwest Pacific: T.M.P.M. Tschermaks Mineralogische und Petrographische Mitteilungen, v. 30, no. 4, p. 205-225.

Glasby, G.P., Friedrich, G., Thijssen, T., Plueger, W.L., Kunzendorf, H., Ghosh, A.K., and Roonwal, G.S., 1982, Distribution, morphology and geochemistry of manganese nodules from the Valdivia $13 / 2$ area, equatorial North Pacific, Pacific Science, v. 36, p. 241-263.

Glasby, G. P., and others, 1983, Manganese nodule distribution mineralogy and geochemistry and relation to sediment type in the Aitutaki Passage, S.W. Pacific: Hawaii Institute of Geophysics, HIG-83-1, 112 p.

Goddard, D.A., Thompson, G., Jones, E.J.W., and Okada, H., 1987, The chemistry and mineralogy of ferromanganese encrustations on rocks from the Sierra Leone Rise, the Equatorial Mid-Atlantic Ridge, and the New England Seamount Chain, in press.

Goldberg, E.D., 1954. Marine geochemistry, Chemical scavengers of the sea, Journal of Geology, v. 62, p. 249-265.

Goldberg, E.D., and Arrhenius, G.O.S., 1958. Chemistry of Pacific pelagic sediments, Geochimica et Cosmochimica Acta, v. 26, p. 417-450.

Goldberg, E.D., Hodge, V., Kay, P., Stallard, M., and Koide, M., 1986, Some comparative marine chemistries of platinum and iridium, Applied Geochemistry, v. 1, p. 227-232.

Goodel1, H.G., Meylan, M.A., and Grant, B., 1971, Ferromanganese deposits of the South Pacific Ocean, Drake Passage and Scotia Sea, in Reid, J.I. (editor) Antarctic Oceanology, Antarctic Research Series, American Geophysical Union, 
Baltimore, p. 27-92.

Greenslate, J., Fisk, M., and Felix, D., 1980, Systematics in manganese concretion composition in the Northeastern Equatorial Pacific, in Lalou, C. (ed.), La genese des nodules de manganese, Colloques Internationaux, No. 289, p. 39-54.

Guichard, F., Reyss, J.L., and Yokoyama, Y., 1985, Growth rate of manganese nodules measured with ${ }^{10} \mathrm{Be}$ and ${ }^{26} \mathrm{Al}$, Nature, v. 272, p. 155-156.

Halbach, P., 1982, Co-rich ferromanganese seamount deposits of the Central Pacific Basin, In Halbach, P. and Winter P. (editors), Marine Mineral Deposits, Marine Rohstoffe und Meerestechnik, v. 6, p. 60-85.

Halbach, P., 1986, Cobalt-rich and platinum-bearing manganese crusts - nature, occurrence and formation, in Johnson, C.J., and Clark, A.L. (eds.), Pacific mineral resources physical, economic, and legal issues, Proceedings of the Pacific Marine Mineral Resources Training Course, East-West Center, Honolulu, p. $137-160$.

Halbach, P., Manheim, F.T., and Otten, P., 1982 Co-rich ferromanganese deposits in the marginal seamount regions of the Central Pacific Basin - results of the Midpac '81. Erzmetall., 35, 9, p. 447-453.

Halbach, P., and Puteanus, D., 1983, Lagerstaettenkundlich-geochemische Untersuchungen zur Verbreitung und Genese Co-reicher Erzvorkommen auf dem Tiefseeboden im östlichen und nordlichen Teil des Zentralpazifischen Beckens, Zwischenbericht zum Vorschungsvorhaben Ha 563/21, Midpac '81, Technical University Clausthal-Zellerfeld, $141 \mathrm{p}$. with plates and maps.

Halbach, P. and Manheim, F.T., 1984, Potential of cobalt and other metals in ferromanganese crusts on seamounts of the Central Pacific Basin, Marine Mining, v. 4 , p. $319-336$.

Halbach, P., Hebisch, U., and Scherhag, C., 1981, Geochemical variation of ferromanganese nodules and crusts from different provinces of the Pacific Ocean and their genetic control, Chemical Geology, v. 34, p. 3-17.

Halbach, P., and Fellerer, R., 1980, The metallic minerals of the Pacific seafloor, Geo Journal, v. 4, p.407-422.

Halbach, P., Seg1, M. Puteanus, D., and Mangini, A., 1983, Relationships between Co-fluxes and growth rates in ferromanganese deposits from central Pacific seamount areas, Nature v. 304, p. 716-719.

Halbach, P., and Puteanus, D., 1984, The influence of the carbonate dissolution rate on the growth and composition of Co-rich ferromanganese crusts from Central Pacific seamounts areas, Earth and Plantetary Science Letters, v. 68, p. 73-87

Halbach, P., Puteanus, D., and Manheim, F., 1984, Platinum concentration in ferromanganese seamounts crusts from the Central Pacific, Naturwissenschaften, v. 71 , p. 577-579. 
Halbach, P., 1986, Processes controlling the heavy metal distribution of Pacific ferromanganese nodules and crusts. Geologische Rundschau, v. 75, p. 235-247.

Hamilton, E.L., 1956, Horizon Guyot, Geologic Society of America Memoir 64, p. $5-9$ and $36-38$.

Han, K. N., and Fuerstenam, D. W., 1980, Extraction behavior of metal elements from deep-sea manganese nodules in reducing media: Marine Mining, v. 2, no. 3, p. $155-169$.

Harada, K., 1977, Selected bibliography of marine manganese nodules, Department of Geology, Kyoto University, unpublished manuscript, 7p.

Harada, K., 1978, Micropaleontologic investigation of Pacific Ocean nodules, Memoires Faculte Science Kyoto University, Serie Geologique et Mienralogique, v. 45, p. $111-132$.

Harada, K.I., 1984, Bibliography for deep-sea polymetallic resources compiled by Japanese researchers, Study of deep-sea polymetallic hard mineral resources; their mineralogy and origin, Ministry of Education, Japan Data Report, Yamagata City, $106 \mathrm{p}$.

Harada, K., Nishida, S., and Usui, A., 1985, Geologic significance of manganese nodules from seamounts and ridges in the Northern Philippine Sea (Japanese), Journal of the Japanese Geological Society, v. 94, p. 30-40.

Harman, H.H., 1967, Modern factor analysis, University of Chicago Press, Chicago, 474p.

Harris, D.P., 1987, Thoughts on appraising marine mineral resources, in Teleki, P.G., Dobson, M.R., Moore, J.R., and von Stackelberg, U. (editors), Marine Minerals, Advances in research, resource assessment, D. Reidel Publishing Co., Dordrecht/Boston, p. 433-466.

Haynes, B.W., Law, S.L., and Barron, D.C., 1982, Mineralogical and elemental description of Pacific magnanese nodules, Bureau of Mines Information Circular IC $8906,60 p$.

Haynes, B.W., and Magyar, M.J., 1987, Analysis and metallurgy of manganese nodules and crusts in Teleki, P.G., Dobson, M.R., Moore, J.R., and von Stackelberg, U.(editors), Marine Minerals, Advances in research, p. 235-246.

Heath, G.R., 1981, Ferromanganese nodules of the Deep Sea, Economic Geology 75th Anniversary Volume, p. 736-765.

Hein, J.R., Manheim, F.T., Schwab, W.C., and Davis, A.S., 1985, Geology of cobalt-rich ferromangnaese crusts from Necker Ridge and seamounts in the Line Islands, Marine Geology, v. 69, p. 25- 54.

Hein, J.R., Manheim, F.T., Schwab, W.C., Davis, A.S., Daniel, C.L., Bouse, R.M., Morgenson, L.A., Sliney, R.E., Clague, D., Tate, G.B., and Cacchione, D.A., 1985, Geological and geochemical data for seamounts and associated ferromanganese 
crusts in and near the Hawaiian, Johnnston Island, and Palmyra Island Exclusive Economic Zones. U.S., Geological Survey Open-File Report 85-292, 129 p.

Hein, J.R., Manheim, F.T., Schwab, W.C., and Clague, D.A., 1986, Cobalt-rich ferromanganese crusts from the Central Pacific, Proceedings of the Offshore Technology Conference 'Richardson TX OTC 5234, p. 119-126.

Hein, J.R., Morgenson, L.A., Clague, D., and Koski, R.A., 1987, Cobalt-rich ferromangnese crusts from the Exclusive Economic Zone of the United States and nodules from the oceanic Pacific. In Scholl, D., Grantz, A., and Vedder, J. (editors), Geology and resource potential of the continental margin of western North America and adjacent ocean basins: Beaufort Sea to Baja, California, American Association of Petroleum Geologists Memoir (in press).

Hein, J.R., Fleishman, C.L., Morgenson, L.A., Bloomer, S.H., and Stern, R.J., 1987, Submarine ferromanganese deposits from the Mariana and Volcano volcanic arcs, Geological Survey Open File Report 87-281, 9 p. with tables and figures.

Hein, J.R., Schwab, W.C., and Davis, A.S., 1987, Cobalt- and Platinum-rich ferromanganese crusts and associated substrate rocks from the Marshall Islands (in press).

Hekinian, C.R., and Hoffert, M., 1975, Rate of palagonitization and manganese coating on basaltic rocks fromn the rift valley in the Atlantic Ocean near $36050^{\prime}$, Marine Geology, v. 19, p. 91- 109.

Heye, D., 1978, Changes in the growth rate of manganese nodules from the Central Pacific in the area of a seamount as shown by the Io method, Marine Geology, v. 28 , p. $59-65$.

Heye, D., and Marchig, V., 1977, Relationship between the growth rate of manganese nodules from the Central Pacific and their chemical constituients.

Horn, D.R., Horn, B.M., Delach, M.N., 1972, Ferromanganese deposits of the North Pacific Ocean, Technical Report No. 1, NSF-GX 33616, 78p.

Hubred, G.I., 1981, Bibliography of references to manganese nodule processing and patents, Unpublished reference file, $43 p$.

Ingri, J., 1985, Geochemistry of ferromanganese concretions and associated sediments in the Gulf of Bothnia, PhD Dissertation published by Department of Economic Geology, University of Lulea, Lulea, Sweden, $-300 p$.

Isaeva, A.B., 1967, Chemical composition of iron-manganese concretions in the Indian Ocean (Russian), Litologiya i Poleznye Iskopaemye, No. 3, p. 43-56.

Iskowitz, J. M., and others, 1982, Determination of thallium in deep-sea ferromanganese nodules: Marine Mining, v. 3, no. 3-4, p. 285-295.

Jannin, M.C., 1981, Essai de datation de concretions polymetalliques et evolution quaternaire du coccolithe Cyclococcolithus leptoporus-macintyrei, Bulletin Societe geologique France, v. 23, p. 287-296. 
Japan Resources Institute, 1986, Co-rich manganese crust deposits: development of new seabed resources (in Japanese), Technical Report, Resources Institute, Japan Science and Technology Agency, Tokyo, 230p.

Japan Resources Association, 1986, Report on crust ore (II) (In Japanese), Marine Resources Committee, Japan Resources Association, Tokyo, 59p.

Johnson, C.J., and Clark, A.L. (eds.), 1986, Pacific Mineral Resources: Physical, Economic, and Legal Issues, Proceedings of Pacific Marine Mineral resources training course, East-West Center, Honolulu, Hawaii, 639p.

Johnson, C.J., and Otto, J.M., 1986, Thinking about nodule economics. In Johnson, C.J., and Clark, A.L., editors, Pacific mineral resources: physical, economic and legal issues, East-West Center, Honolulu, p. 501-520.

Jones, E.J.W. and Goddard, D.A., 1979, Deep-sea phosphorite of Tertiary age from Annan Seamount, eastern equatorial Atlantic, Deep-Sea Research, v. 26A, p. $13634-1379$.

Jones, H. A., 1980, Deep-sea manganese nodules in the Australian region -A review: Australian Mineral Industry Quarterly, v. 33, p. 10-23.

Kane, J. S., and Dorrzapf, A. F., 1987, Current atomic absorption and inductively coupled optical emission methods for Geochemical Investigations, Geoexpo ' 86 Symposium, Volume, In press.

Kang, J.K., and Kosakevitch, 1984, Etude texturale des encroutements ferro-manganesiferes de l'est Caraibe, Bureau de Recherche Geologiques et Minieres, Service Geologique National, Rapport 84 SGN 328 MGS, 76 p. plus tables, maps and plates.

Kazmin, Yu. B., 1984. Zhelezomargantsevye konkretsiii mirovogo okeana (Iron-manganese concretions of the world ocean), Trudy Vsesoyuznyi Nauchno-issledovatel'skii Institut Geologii i Mineral'nykh Resursov Mirovogo Okeana, v. 192, 175p.

Keays, R.R., and Scott, R.B., 1976, Precious metals in ocean- ridge basalts: implications for basalts and source rocks for gold mineralization, Economic Geology, v. 71, p. 705-720.

Kirschenbaum, H., 1983, The classical chemical analysis of silicate rocks - the old and the new, Geological Survey Bulletin 1547, 55p.

Kobayashi, K., editor, Preliminary report of the Hakuho Maru cruise KH84-1 -Geological and geophysical investigation of seafloor east of Ogasawara (Bonin) islands, northern Mariana trough, southern Mariana, Yap and Paleau trench and arcs: WESTPAC, ODP, University of Tokyo, 305 p.

Koirtyohann, S. R., and Kaiser, M. L., 1982, Furnace Atomic absorption -A mthod approaching maturity, Anal. chem., v. 54, no. 14, pp. 1515A-1524A. 
Koski, Randolph A., 1988, Ferromanganese deposits from the Gulf of Alaska Seamount Province: mineralogy, chemistry, and origin. Canadian Journal of Earth Sciences, v. 25, p. 116-133.

Koski, R.A., Hein, J.R., Bouse, R.M., and Sliney, R.E., 1985, Composition and origin of ferromanganese crusts from Tonga platform, southwest Pacific, in Scholl, D.W., and Vallier, T.L., (eds.), Geology and offshore resources of Pacific island arcs - Tonga region: Circum-Pacific Council for Energy and Mineral Resources, Earth Science Series, Houston, Texas, v. 2, p. $179-186$.

Kraemer, T., and Schornick, J. C., 1974, Comparison of elemental accumulation rates between ferromanganese deposits and sediments in the south Pacific ocean: Chemical Geology, v. 13, no. 3, p. 187-196.

Krishnaswami, S., and Lal, D., 1972, Manganese nodules and budget of trace solubles in the oceans, Proceedings of the Nobel Symposium, 20th, p. 307-320.

Krishnaswami, S., 1976, Authigenic transition elements in Pacific pelagic clays, Geochimica et Cosmochimica Acta, v. 40, p. 425-434.

$\mathrm{Ku}$, T.L., 1977, Rates of accretion, in Glasby, G.P. (ed.), Marine manganese deposits, Elsevier Publishing Co., Amsterdam, p. 249- 267.

Ku, T.L., Kusakabe, M., Nelson, D.E., Southon, J.R., Korteling, R.G., Vogel, J., and Nowikow, I., 1982, Constancy of oceanic deposition of ${ }^{10} \mathrm{Be}$ as recorded in manganese crusts, Nature, v. 299, p. 240-242.

$\mathrm{Ku}$, T.L., Omura, A., and Chen, P.S., 1979, ${ }^{10} \mathrm{Be}$ and U-series isotopes in manganese nodules from the Central North Pacific. In Bischoff, J.L., and Piper, D.Z., (editors)., Marine geology and oceanography of the Pacific nodule province, Plenum Press, N.Y. and London, p. 791-814.

Kunzendorf, H., 1987, Geochemical methods in manganese nodule exploration, in Teleki, P.G., Dobson, M.R., Moore, J.R., and von Stackelberg, U., (editors), Marine Minerals, Advances in research and resource assessment, $D$. Reidel Publishing Co., Dordrecht/Boston, p. 221-234.

Kusakabe, M., and Ku, T.L., 1984, Incorporation of Be isotopes and other trace metals into marine ferromanganese deposits, Geochimica et Cosmochimica Acta, v. 48 , p. $2187-2193$.

Lalou, C., (ed), 1979, La genese des nodules de manganese, Colloques Internationaux du Centre National de la Recherche Scientifique (CNRS), No. 289, p. 262-269.

Lalou, C., Ku, T.L., Brichet, E., Poupeau, G., and Romary, P., 1979a, Techno encrustation, Part I: Radiometric studies, in Lalou, C.(ed.) La genese des nodules de manganese, Colloques Internationaux du Centre National de la Recherche Scientifique (CNRS), No. 289, p. 261-270.

Lalou, C., Brichet, E., and Jehanno, C., 1979b, Techno Encrustation, Part II: Structural and chemical study, in Lalou, C. (ed.) La genese des nodules de 
manganese, Colloques Internationaux du Centre National de la Recherche Scientifique (CNRS) No. 289, p. 271-280.

Lalou, C., Thompson, G., Rona, P.A., Brichet, E., and Jehanno, C., 1986, Chronology of selected hydrothermal Mn oxide deposits from the transatlantic geotraverse "TAG" area, Mid-Atlantic Ridge, $26^{\circ} \mathrm{N}$, Geochimica et Cosmochimica Acta, v. 50, p. $1737-1743$.

Landmesser, C.W., and Morgenstein, M.E., 1973, Survey and mapping of manganese deposits in the Hawailan Archipelago, In Morgenstein, M. (ed.) Papers on the origin and distribution of manganese nodules in the Pacific and Prospects for exploration, Honolulu, Hawaii, p. 93-101.

Lane, C.M., Manheim, F.T., Hathaway, J.C. and Ling, T.H., 1986, Station maps of the world ocean-ferromanganese-crust database, US Geological Survey Miscellaneous Field Studies Map 1869.

Latimer, J.P., and Kaufman, R., 1985, Preliminary considerations for the design of cobalt crust mining systems in the U.S. EEZ, Oceans '85, p. 378-399.

Lavrov, V. M., and others, 1973, Titanium in the Quarterly deposits of the Atlantic Ocean: Oceanology, v. 13, no. 2, p. 231-237.

Lindstrom, G., 1884, Analyser af bergarter och bottenprof fran Ishafvet, Asiens nordkust och Japan. Stockholm.

Lonsdale, P. F., and others, 1980, Nodules of hydrothermal birnessite in the caldera of a young seamount: Journal of Geology, v. 88, no. 5, p. 611-618.

Lyle, M., Dymond, J., and Heath, G.R., 1977, Copper-nicke1- enriched ferromanganese nodules and associated crusts from the Bauer Basin, Northwest Nazca Plate, Earth and Planetary Science Letters, v. 35, p. 55-64.

Mangini, A., Halbach, P., Puteanus, D., and Seg1, M., 1987, Chemistry and growth history of Central Pacific Mn-crusts and their economic importance, in Teleki, P.G., Dobson, M.R., Moore, J.R., and von Stackelberg, U. (editors), Marine Minerals, Advances in Research and resource assessment, D. Reidel Pub1. Co., Dordrecht/Boston, p. 205-220.

Manheim, 1965, Manganese-iron accumulations in the shallow marine environment, In Schink, D.R., and Corless, J.T., editors, Symposium on marine geochemistry: University of Rhode Island, Narragansett Marine Laboratory Occasional Publication 3 , p. $217-276$.

Manheim, F.T., 1978, Marine Manganese Deposits, ed. by G.P. Glasby, E1sevier Scientific Publishing Co. (Book Review), Geochimica et Cosmochimica Acta, v. 42, p. $541-542$.

Manheim, F.T., 1986, Marine cobalt resources, Science, v. 232, p. 601-611.

Manheim, F.T., Pratt, R.M., and McFarlin, P.F., 1980, Composition and origin of phosphorite deposits of the Blake Plateau, Society of Economic Paleontologists 
and Mineralogists, Special Publication No. 29, p.117-137.

Manheim, F.T., Popenoe, P., Siapno, W., and Lane, C.M., 1982, Manganese deposits on the Blake Plateau, in Halbach, P. and Winter, P. (editors), Marine mineral deposits - New research results and economnic prospects, Marine Rohstoff und Meerestechnik, Verlag Glueckauf GmbbH Essen, p. 9-44.

Manheim, F.T., Ling, T.H., and Lane, C.M., 1983, An extensive data base for cobalt-rich ferromanganese crusts from the Worlds Oceans, Proceedings of Oceans '83, p. $828-830$.

Manheim, F.T. and Hein, J.R., 1986, Ferromanganese crusts, in Lockwood, M., and Hill, G. (editors), Proceedings of Exclusive Economic Zone Symposium, Exploring the New Ocean Frontier, U.S. Department of Commerce, Washington D.C., p. 79-95.

Manheim, F. T., Aruscavage, P. J., Simon, F. O., and Woo, C. C., 1979, Composition and Mineralogy of Western Atlantic ferromanganese nodules, with special emphasis on platinum metals: Third International Symposium on Geology and Geochemistry of Manganese, Paris, France.

Manheim, F.T., and Lane-Bostwick, C.M., 1988, Cobalt in ferromanganese crusts as a monitor of hydrothermal discharge on the Pacific Ocean seafloor: Nature, v. 335 , p. 59-62.

Masuda, Y., 1972, Japanese programs for manganese nodule exploitation, in Morgenstern, M., (ed.) Proceedings of the symposium on manganese deposits in the Pacific, Honolulu, Hawaii, p. 107-112.

Masuda, Y., Cruickshank, M.J., and Mero, J.L., 1971, Continuous bucket-line dredging at 12,000 feet, Preprints Offshore Technology Conference, v. 1, p. $1837-1858$.

McGoldrick, P. J., and others, 1979, Thallium -A sensitive indicator of rock/seawater interaction and of sulfur saturation of silicate melts: Geochimica et Cosmochimica Acta, v. 43, no. 8, p. 1303-1311.

McGregor, B.A., and Offield, T.W., 1984, The exclusive economic zone: an exciting new frontier, U.S. Department of Interior, Geological Survey, Washington D.C., 20p.

McKelvey, V.E., Wright, N.A., and Bowen, R.W., 1983, Analysis of the world distribution of metal-rich subsea manganese nodules, Geological Survey Circular $886,55 \mathrm{p}$.

McKelvey, V.E., 1986, Subsea mineral resources, U.S. Geological Survey Bulletin $1689-\mathrm{A}, 106 \mathrm{p}$.

McMurtry, G.M., and Sinton, J.M., 1986, Geochemical methods for the determination of marine mineral resources, p. 227-272.

Menard, H.W., 1964, Marine Geology of the Pacific, McGraw-Hil1, N.Y., N.Y. 27lp. 
Mero, J.L., 1959, The mining and processing of deep-sea manganese nodules, University of California, Institute of Marine Resources, 96p.

Mero, J.L., 1960, Minerals on the ocean floor, Scientific American, v. 203, p. $64-72$.

Mero, J.L., 1962, Ocean-floor manganese nodules, Economic Geology, v. 57, p. $747-767$.

Mero, J.L., 1965, The Mineral resources of the Sea, Elsevier Publishing Co., Amsterdam-London, 312p.

Meylan, M. A., 1978, Marine sedimentation and manganese nodule formation in the southwestern Pacific ocean (Ph.d thesis): University of Hawaii, $312 \mathrm{p}$.

Meylan, M.A., Glasby, G.P., and Fortin, L., 1981, Bibliography and index to literature on manganese nodules (1861-1979), Department of Planning and Economic Development, State of Hawaii, 530p.

Minerals Management Service, 1987, Draft Environmental Impact Statement, Proposed Marine Mineral Lease Sale in the Hawaiian Archipelago and Johnston Island Exclusive Economic Zones, U.S. Department of Interior, Minerals Management Service and State of Hawaii, Department of Planning and Economic Development, OCS EIS/EA MMS 87-0004, 343p. plus appendices.

Mizuno, A., and Chujo, J., 1975 (eds.), Deep sea mineral resources investigation in the eastern Central Pacific basin, August-October, 1974 (GH74-5 Cruise), Geological Survey of Japan Cruise Report No. 4, 103p.

Mizuno, A. (editor), 1981, Deep sea mineral resources investigation in the northern part of central Pacific basin, January-March 1979 (GH79-1 cruise): Geological Survey of Japan cruise report no. 15, 297 p.

Mizuno, A., and Nakao, S. (eds.), 1982, Regional data of marine geology, geophysics and manganese nodules - the Wake -Tahiti transect in the central Pacific, January-March 1980 (GH80-1 cruise): Geological Survey of Japan, Cruise Report no. $18,399 \mathrm{p}$.

Monget, I.M., Murray, J.W., and Mascle, J., 1976, A world-wide compilation of published, multicomponent analyses of ferromanganese concretions, NSF-IDOE Manganese Nodule Project, Technical Report No. 12, CNEXO-BREST, 205p.

Moore, W.S., 1973, Accumulation rates of manganese crusts on rocks exposed on the sea floor, In Inter-university program of research on ferromanganese deposits of the ocean floor, Phase I report, p. 93-98.

Moore, W.S., and Vogt, P.R., 1976, Hydrothermal manganese crusts from two sites near the Galapagos spreading axis, Earth and Planetary Science Letters, v. 29, p. 349-356.

Moore, W. S., and others, 1981, Fluxes of metals to a manganese nodule radiochemical, chemical, structural and mineralogical studies: Earth and Planetary Science Letters, v. 52, p. 151-171. 
Moore, W. S., 1984, Thorium and radium isotopic relationships in Mn nodules and sediments at MANOP Site S: Geochimica et Cosmochimica Acta, v. 48, no. 5, p. 987-992.

Moorby, S.A., Cronan, D.S., and Glasby, G.P., 1984, Geochemistry of hydrothermal Mn-oxide deposits from the S.W. Pacific island arc, Geochimica et Cosmochimica Acta, v. 48 , p. 433-441.

Morgan, C.L., 1986, The Hawaii Manganese Crust Environmental Impact Statement: an example of modern environmental analysis, p. 449-458.

Moritani, T., and Nakao, S. (eds.), 1981, Deep sea mineral resources investigation in the Western part of the Central Pacific Basin, Cruise Report No. 17, Geological Survey of Japan, 281p.

Murdmaa, I.O., and Skornyakova, N.S.(eds), 1986, Zhelezo-margantsevye konkretsii tsentral'noi chasti Tikhogo okeana (Ferromanganese nodules of the Central Pacific Ocean), Trudy Instituta Okeanologii, v. 122, 344p

Murray, J., 1876, Preliminary report on specimens of the sea bottom obtained in soundings, dredgings, and trawlings of H.M.S. Challenger in the years 1873-1875 between England and Valparaiso, Proceedings of the Royal Society, v. 24, p. 471-547.

Murray, J., and Renard, A.F., 1891, Report on deep sea deposits, Report on the Scientific Results of the Voyage of H.M.S. Challenger, Deep Sea Deposits, p. $1-525$.

Murray, J., and Irvine, R., 1894, On the manganese oxides and manganese nodules in marine deposits, Transactions of the Royal Society of Edinburgh, v. $37, \mathrm{p}$. $721-742$.

Murray, J.W., 1975, The interaction of cobalt with hydrous manganese dioxide, Geochimica et Cosmochimicat Acta, v. 39, p. 635-647.

Murray, J.W., and Dillard, 1979, The oxidation of cobalt(II) adsorbed on manganese dioxide, Geochimica et Cosmochimica Acta, v. 43, p. 781-787.

Nakao, S., (Ed.), 1986, Marine geology, geophysics, and manganese nodules around deep-sea hills in the Central Pacific Basin, August-Ocxtober 1981 (GH81-4 Cruise), Cruise Report Geological Survey of Japan, no. 21, 257p.

Naumann, E., 1922, Soedra och mellersta Sveriges sjö-och myrmallmer. Sveriges geologiska undersökning, Ser. C., 297, p. 1-194.

Noakes, L. C., and Jones, H. A., 1975, Mineral resources offshore, in Knight, C. L., (editor), Economic geology of Australia and Papau, New Guinea: Australian Inst. Min. and Met., Victoria Australia, p. 1093-1109.

Nohara, M., 1976, The geochemistry of manganese nodules from the Pacific ocean: Journal of the Geological Society of Japan, v. 83, no. 5, p. 267-276. 
Pichocki, C., and Hoffert, M., 1987, Quelques characteristiques de 1'environment geologique de nodules et d'encroutements cobaltifere preleves en Polynesie francaise, Marine Geology, (in press).

Piper, D.Z., Basler, J.R., and Bischoff, J.L., 1988, Oxidation state of marine manganese nodules, Geochimica et Cosmochimica Acta, v. 48, p. 2347-2355.

Pratt, R.M., and McFarlin, P.F., 1966, Manganese pavement on the Blake Plateau, Science, v. 151, p. 1080-1082.

Price, N.B., and Calvert, S.E., 1970, Compositional variation in Pacific Ocean ferromanganese nodules and its relationship to sediment accumulation rates, Marine Geology, v. 9, p. 145-171.

Ritchey, J.L., 1987, Assessment of cobalt-rich manganese crust resources on Horizon and S.P. Lee Guyots, U.S. Exclusive Economic Zone, Marine Mining (in press).

Rona, P.A., Bostrom, K., Laubier, L., and Smith, K.L. Jr., 1983, Hydrothermal processes at seafloor spreading centers, Plenum Press, N.Y. and London, 796 p.

Rona, P.A., Thompson, G., Mott1, M.J. Karson, J.A., Jenkins, W.J., Graham, D., Mallette, M., Von Damm, K., and Edmond. J.M. Rona, P.A., 1984, Hydrothermal activity at the Transatlantic Geotraverse Hydrothermal Field, Mid-Atlantic ridge crest at $26^{\circ} \mathrm{N}$. Journal of Geophysical Research, v. 89, p. 11365-11377.

Roonwal, G. S., and Friedrich, G. H., 1980, Chemical variation and element correlation in manganese nodules from a dredge haul in the central Pacific: Indian Journal of Marine Sciences, v. 9, no. 4, p. 235-239.

Rose, H.J., Adler, I., and Flanagan, F.J., 1963, X-ray fluorescence analysis of the 1ight elements in rocks and minerals, Applied Spectroscopy, v. 17, p. 81-85. Savenko, V.S., 1981, The stability of manganese oxide compounds in ocean water and some questions of the origin of iron-manganese concretions (Russian), Doklady Akademii Nauk, SSSR, no. 5, p 1217- 1220.

Schwab, W.C., Davis, A.S., Haggerty, J.A., Ling, T.H., and Commeau, J.A., 1985, Geologic reconnaissance and geochemical analysis of ferromanganese crusts of the Ratak Chain, Marshall Islands, U.S. Geological Survey Open File Report 85-18, $6 \mathrm{p}$.

Schwab, W.C., Hein, J.R., Davis, A.S., Morgenson, L.A., Daniel, C.L., and Haggerty, J.A., 1986, Geological and geochemical data for seamounts and associated ferromanganese crusts in the Ratak Chain, Marshall Islands, U.S. Geological Survey Open-File Report 86-338, 36p.

Scott, M.R., Scott, R.B., Rona, P.A., Butler, L.W., and Nalwalk, A.J., 1974, Rapidly accumulating manganese deposit from the median valley of the Mid-Atlantic Ridge, Geophysical Research Letters, v. 1, p. 355-358.

Scott, R.B., Rona, P.A., Butler, L.W., Nalwalk, A.J., Scott, M.R., 1972, Manganese crusts of the Atlantis Fracture Zone, Nature, v. 239, p. 77-79. 
Seg1, M., Mangini, A., Bonani, G., Hofmann, H.J., Nessi, M., Suter, M., Woelfli, W., Friedrich, G., Plueger, W.L., Wiechowski, A., and Beer, J., 1984, ${ }^{10} \mathrm{Be}$-dating of a manganese crust from Central North Pacific and implications for ocean palaeocirculation, Nature, v. 309, p. 540-542.

Shapiro, L., 1975, Rapid Analysis of Silicate, Carbonate, and Phosphate Rocks Revised Edition, Geological Survey Bulletin 1401, 76 p.

Shnyukov, E.F. and Orlovskii, G.N., 1980, Iron-manganese concretions of the Indian ocean (Russian), Geologicheskii Zhurnal, v. 40, p. 46-64.

Shnyukov, E.F. and Orlovskii, G.N., 1982, Iron-manganese concretions in the Indian Ocean (in Russian), in Smirnov, V.I., (ed.), Geologiya I Geokhimiya Margantsa, Nauka, Moscow, p. 210-227.

Siegel, M. D., 1981, Studies of the mineralogy, chemical composition, texture and distribution of manganese nodules at a site in the north equatorial Pacific ocean (Ph.D thesis): Cambridge, Harvard University, 274 p.

Skornyakova, N.S., 1960, Manganese concretions in sediments of the northeastern part of the Pacific Ocean (in Russian), Doklady Akademii Nauk, SSSR, v. 130, p. 653-656.

Skornyakova, N.S., 1976, Chemical composition of ferromanganese nodules of the Pacific, in Bezrukov, P.L. (ed.), Zhelezo- margentsevye konkretsii Tikhogo okeana, Trudy Instituta Okeanologii, Akademii Nauk, v. 109, p. 190-240.

Skornyakova, N.S., and Andrushchenko. P.F., 1972, Iron-manganese concretions in the Pacific Ocean, International Geology Review, v. 6, p. 863-919.

Skornyakova, N. S., and others, 1975, Mineralogy and geochemistry of ferromanganese nodules in the Pacific ocean: Geochemistry International, v. 12, no. 4, p. 83-95.

Skornyakova, N.S. and Zenkevich, N.S., 1976, Regularities of the spatial distribution of ferromanganese nodules (in Russian), in Bezrukov, P.L. (ed.) Zhelezo-margantsevye konkretsii Tikhogo okeana, Trudy Instituta Okeanologii, v. 109 , p. $37-75$.

Slavin, W., 1982, Atomic absorption spectroscopy - The present and future, Anal. Chem., v. 54, no. 6, p. 685A-694A.

Slavin, W., 1984, Graphite Furnace AAS -A source book, the Perkin Elmer Corp., Spectroscopy Division, Ridgefield, CT, No. 0993-8139, 230 p.

Smith, J.B., Holt, B.R., and Paul, R.G., 1985, Current status of leasing proposals for the U.S. Exclusive Economic Zone, Offshore Technology Conference paper OTC 4899 , p. $9-17$.

Stoffers, P., and others, 1981, The geochemistry of coexisting manganese nodules, micronodules, sediments and pore waters from five areas in the equatorial and SW Pacific: Chemie der Erde, v. 40, p. 273-297.

Suess, E., and Ojafari, D., 1977, Trace metal distribution of Baltic Sea ferromanganese concretions - Inferences on accretion rates: Earth and Planetary 
Science Letters, v. 35, no. 1, p. 49-54.

Swedenborg, E., 1734, De Ferro (Opera Philosophica et Mineralia), Friedrich Haeckel, Dresden and Leipzig, Quarto 455 p., Facsimile edition in Swedish translation with glossary of chemical and mineralogical terms (N.Zenzen) edited by S. Arrhenius, V. Carlheim-Gyllensköd and H.J. Sjögren, Stockholm; Wahlström and Widstrand, and Marcus Boktryckeri, Stockholm 1923.

Takeda, H., (Ed.), 1974, Deep sea mineral resources: Investigations in Northwest Pacific, Nov.-Dec. 1972. Cruise Report Geologizal Survey of Japan, no. 1, 42p.

Takematsu, N., 1979, The incorporation of minor transition metals into marine manganese nodules: Journal of the Oceanographical Society of Japan, v. 35, p. $191-198$.

Teleki, P.G., Dobson, M.R., Moore, J.R., and von Stackelberg, U., 1987, (eds.) Marine minerals, D. Reidel Publishing Co., Dordrecht, 588p.

Thijo, A., and others, 1981, Mössbauer effect studies of iron in manganese nodules and associated marine sediments in five areas in the equatorial S.W. Pacific: Geochemical Journal, v. 15, p. 25-37.

Thompson, G., Mottl, M.J., and Rona, P.A., 1985, Morphology, mineralogy and chemistry of hydrothermal deposits from the TAG area, $26^{\circ} \mathrm{N}$, Mid-Atlantic Ridge, Chemical Geology, v. 49, p. 243- 257.

Thompson, M., 1985, The capabilities of inductively coupled plasma atomic emission spectrometry -Some conjectures and refutations, Analyst, v. 110, pp. 443-449.

Thompson, M., and Walsh, J. N., 1983, A Handbook of Inductively Coupled Plasma Spectrometry; Chapman and Hall, New York, 273 p.

Toth, J.R., 1980, Deposition of submarine crusts rich in manganese and iron, Geological Society of America Bulletin, v. 91, p. 44-54.

Turner, S., and Buseck, P.R., 1981, Todorokites: a new family of naturally occurring manganese oxides, Science, v. 212, p. 1024- 1027.

Turner, S., Siegel, M.D., and Buseck, P.R., 1982, Structural features of todorokite intergrowth in manganese nodules, Nature, v. 296, p. 841-842.

Tylecote, R.F., 1962, Metallurgy in Archaeology, London.

Uchio, T., Studies of deep-sea manganese nodules (part 1): Journal of the faculty of Engineering, The University of Tokyo, v. XXXIII, no. 4, p. 611-625.

Usui, A., 1985, Mineralogical and geochemical characteristics of manganese nodules and crusts collected during GDPO-5 and GDPO-8 Cruises in the Western Pacific, In Shiki, T., (editor), Geology of the Northern Phillipine Sea, Tokai University Press, Tokai, p. 179-201. 
Usui, A., Yuasa, M. Yokota, S., Nohara, M., Nishimura, A., and Murakami, F., 1987, Submarine hydrothermal manganese deposits from the Ogasawarea (Bonin) arc, off the Japan Islands: Marine Geology, v. 73, p. 311-322.

Vaasjoki, 0., 1956, On the natural occurrence of manganese in Finland, Symposium Sobre Yacimiento de Manganeso-20th International Geologic Congress, Mexico, v. 5, p. 51-62.

Varentsov, I.M., Dikov, Yu.P., and Bakova, N.V., 1980, The study of Fe-Mn ore formation in recent basins: experiments on the synthesis of $\mathrm{Mn}, \mathrm{Fe}, \mathrm{Ni}$, Co hydroxide on iron hydroxides (gamma FeOOH), In Varentsov, I.M., and Grasselly, eds., 1980, Geology and Geochemistry of Manganese, v. III, Publishing House of the Hungarian Academy of Sciences, Budapest, 1980, p. 91-104.

Varentsov, I.M., and Grasselly, G., (eds.), 1980, Geology and Geochemistry of Manganese, v. I-III, E. Schweitzerbart'sche Verlagsbuchhandlung, stuttgart.

Van der Weijden, C.H., 1976, Some geochemical controls on $\mathrm{Ni}$ and Co concentrations in marine ferromanganese deposits, Chemical Geology, v. 18, p. $65-80$.

Van Heck, M.S., and Barlett, R.W., 1973, Kinetics of sulfation of Atlantic Ocean manganese nodules, Metallurgical Transactions No. 4, Apri1, p. 941-947.

Varma, A., Ed., 1984, CRC Handbook of atomic absorption analysis, CRC press: Boca Raton, FL. vol. 1 and 2.

Volkov, I.I., Fomina, L.S., and Yagodinskaya, T.A., 1976, Chemical composition of iron-manganese concretions of the Pacific Ocean on the transect from Wake Ato11 to the Mexican coast (in Russian), in Volkov, I.I. (ed.), Biokhimiya Diageneza Osadkov Okeana, Nauka, Moscow, p. 186-204.

Volkov, I.I., Ferromanganese concretions (Russian), Okeanologiya, Geokhimiya donnykh osadkov, Nauka, Moscow, pp. 414-467.

Von Stackelberg, U., 1978, A polygenetic manganese nodule from the Scott plateau off northwest Australia: Journal of Australian Geology and Geophysics, v. 3, p. 349-351.

Von Stackleberg, U., Kunzendorf, H., Marchig, V., and Gwozdz, R., 1984, Growth history of a large ferromanganese crust from the equatorial north Pacific nodule be1t: Geologisches tahrbuch, v. 75, p. 213-235.

Willis, J. P., 1986, Instrumental analytical techniques in Geochemistry: Requirements and applications, Fresenius Anal. Chem., v. 324, no. 8, pp. 485494.

Winge, R. K., Fasse1, V. A., Peterson, V. J., and Floyd, M. A., 1985, Inductively coupled plasma -Atomic Emission Spectroscopy, Atlas of Spectral Information, E1sevier, New York, 584 p. 
World Data Bank II, 1984, World land area and coastline map 1:250,000, U.S. Department of Commerce, National Technical Information Service, v. 1 PB-271 $871 / 2$, v. $4 \mathrm{~PB}-271872 / 2$, and v. $5 \mathrm{~PB}-271873 / 2$. 
APPENDIX A 


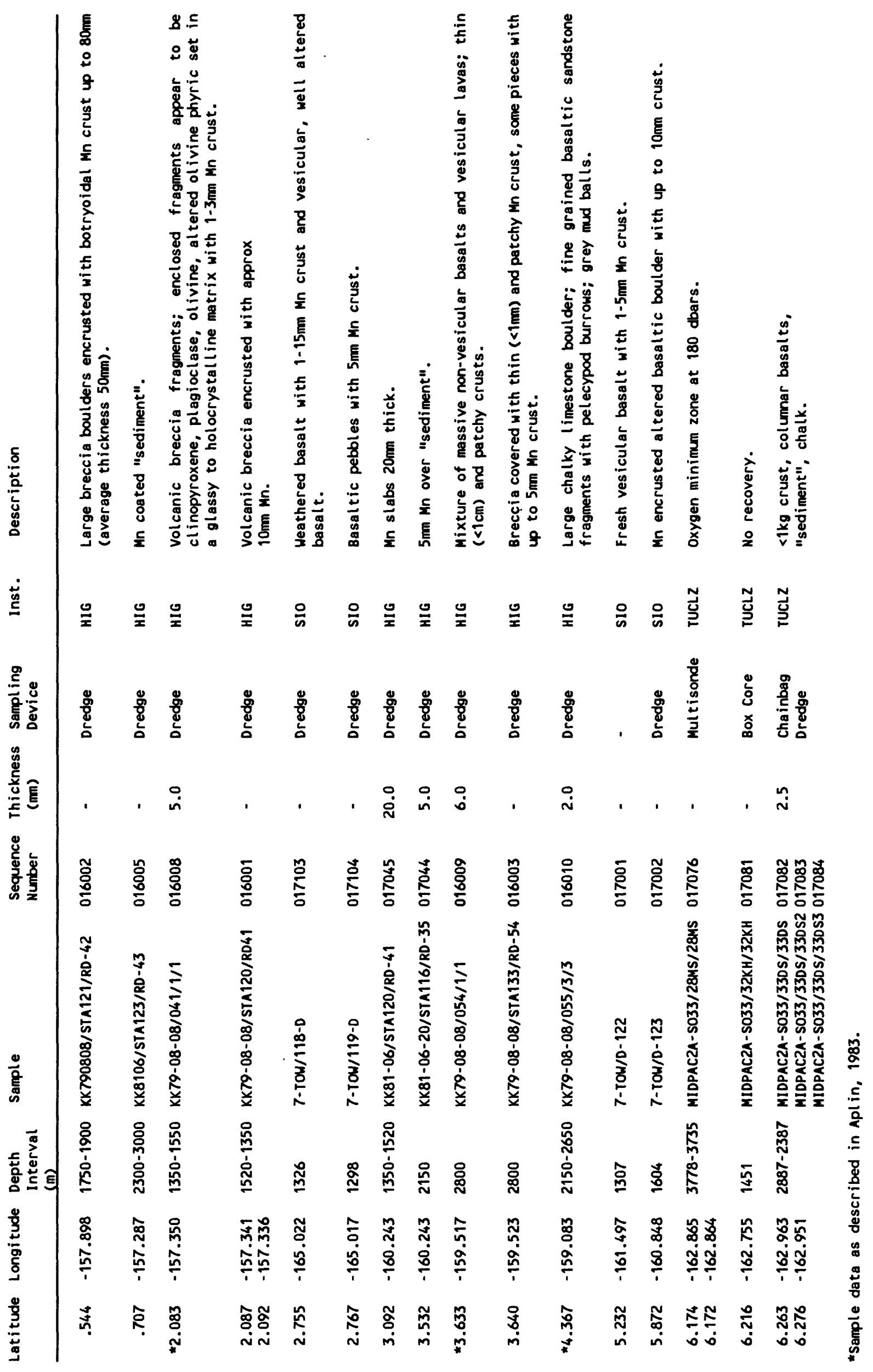




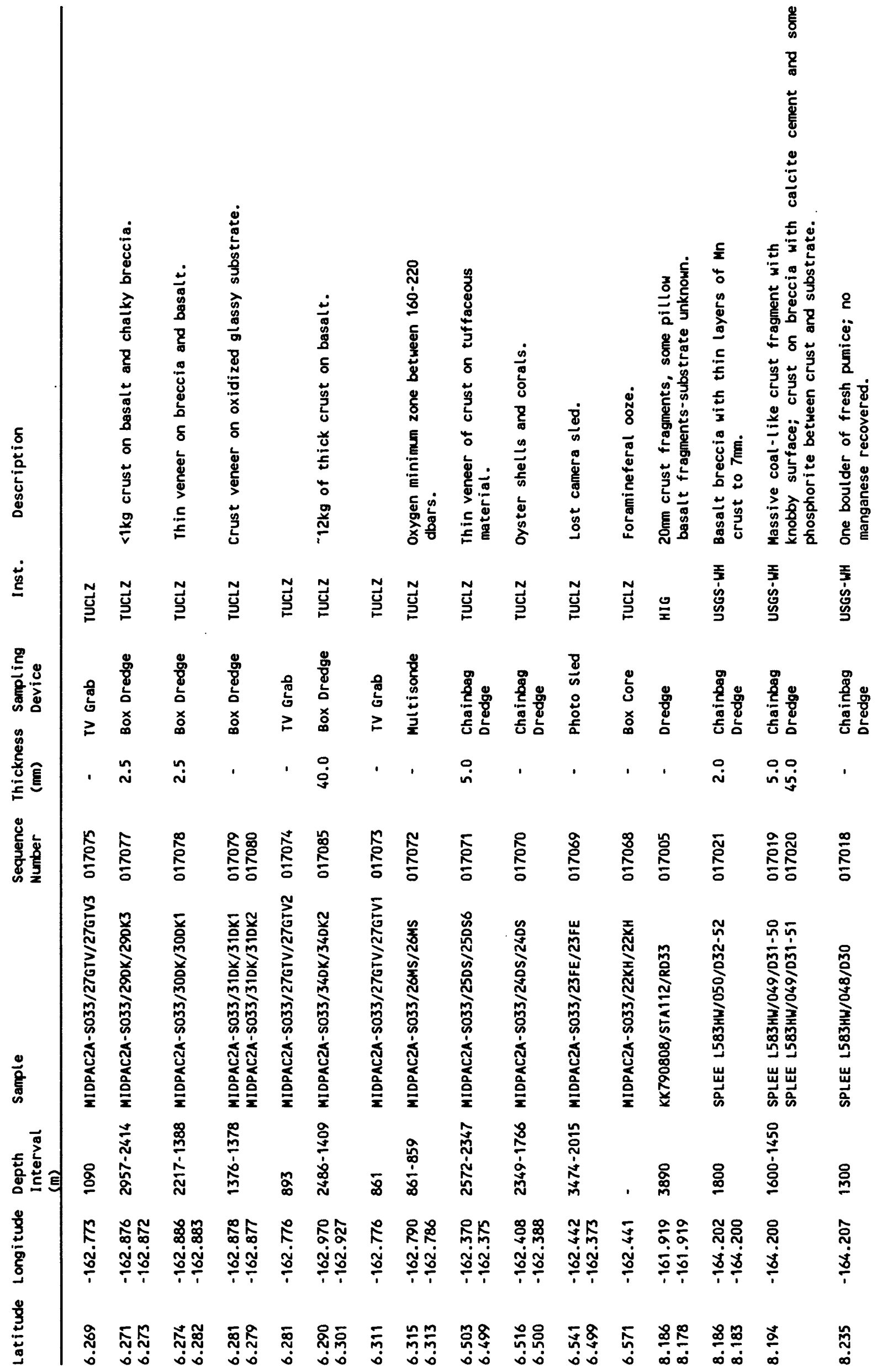




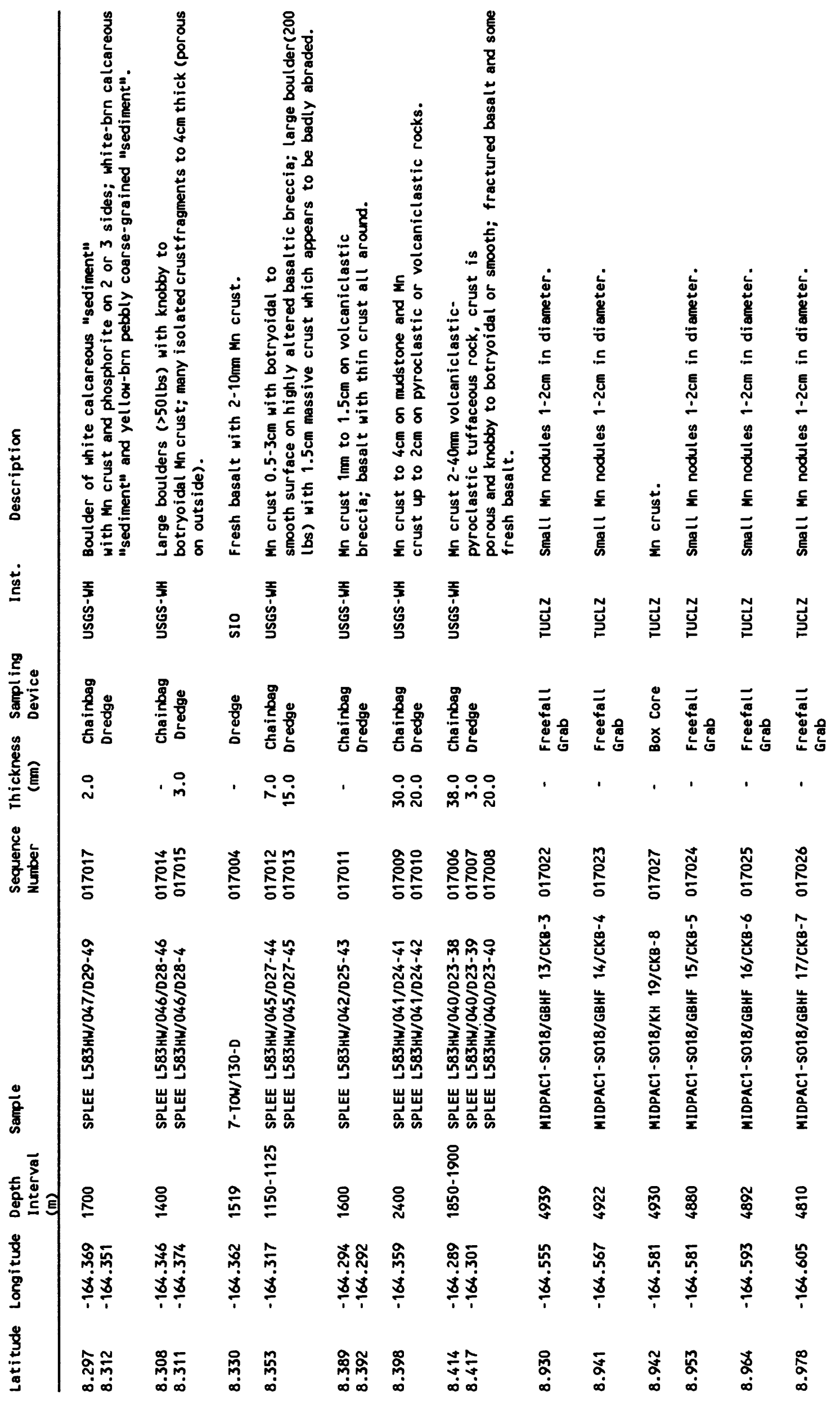




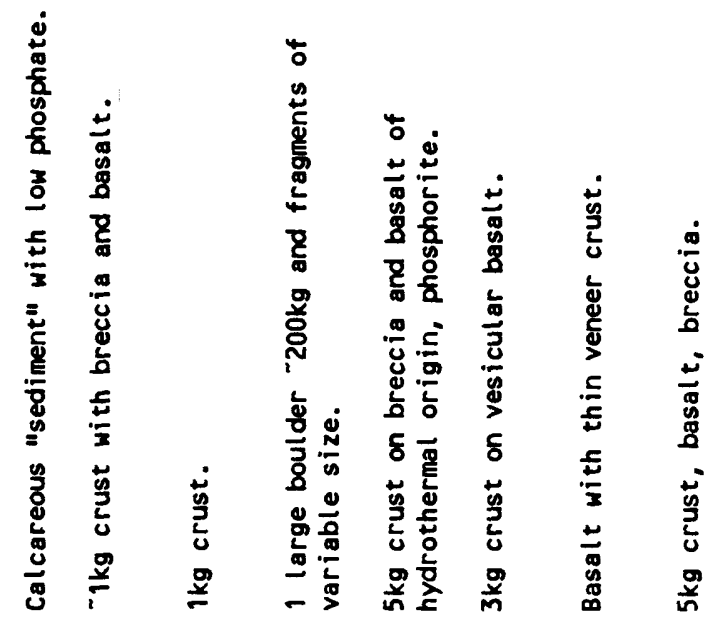

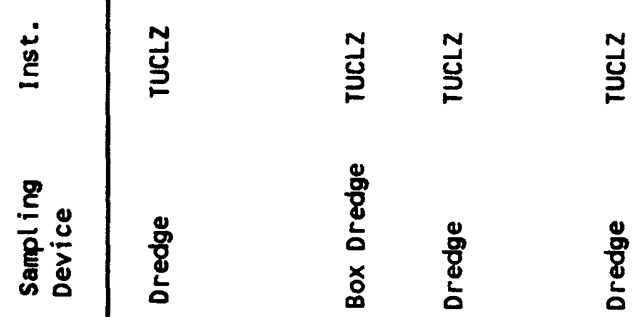

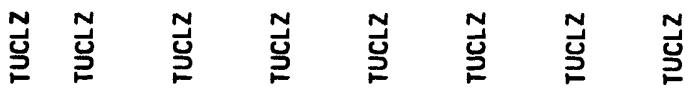

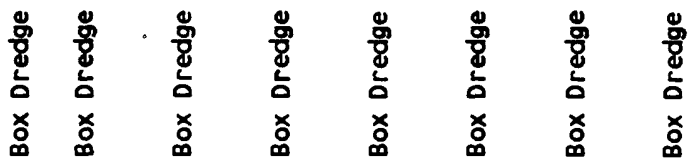
递囟

\section{尺ิ์}

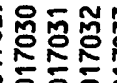

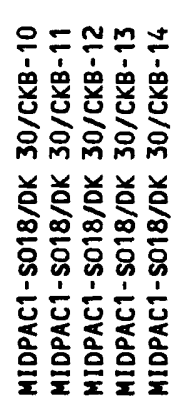

के

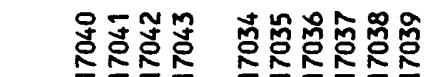

$\frac{0}{0}$

$\frac{0}{\circ}$

8

$\frac{9}{0}$

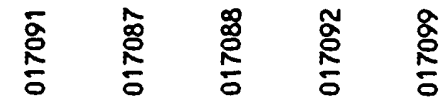

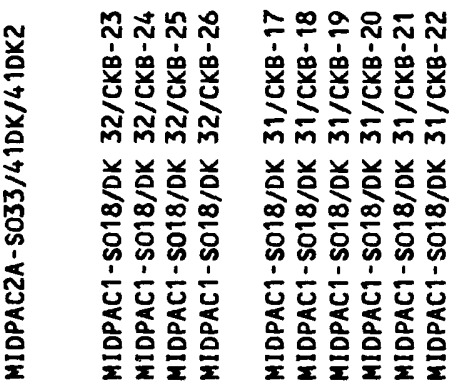

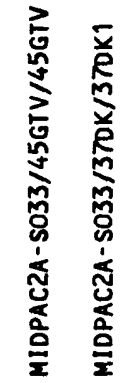

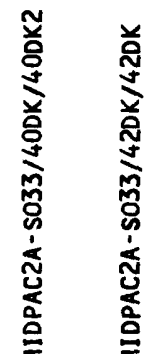

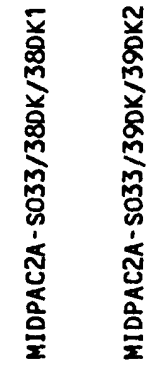

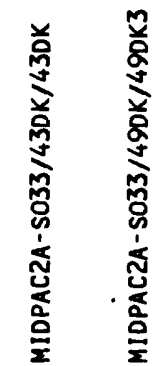

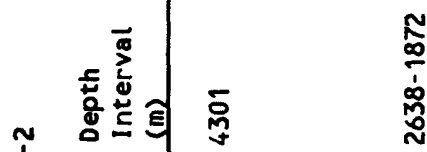

萬

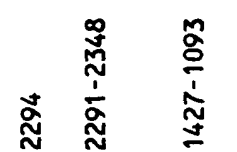

$\stackrel{\text { लิ }}{\grave{N}}$

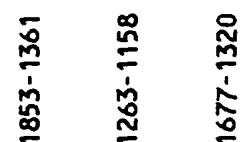

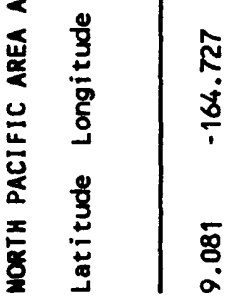

बi.

ㅎำ

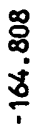

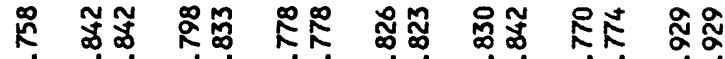

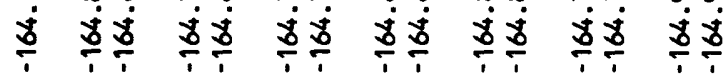

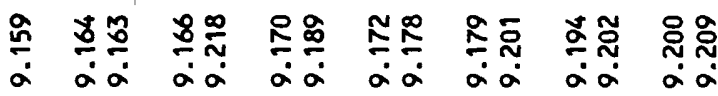




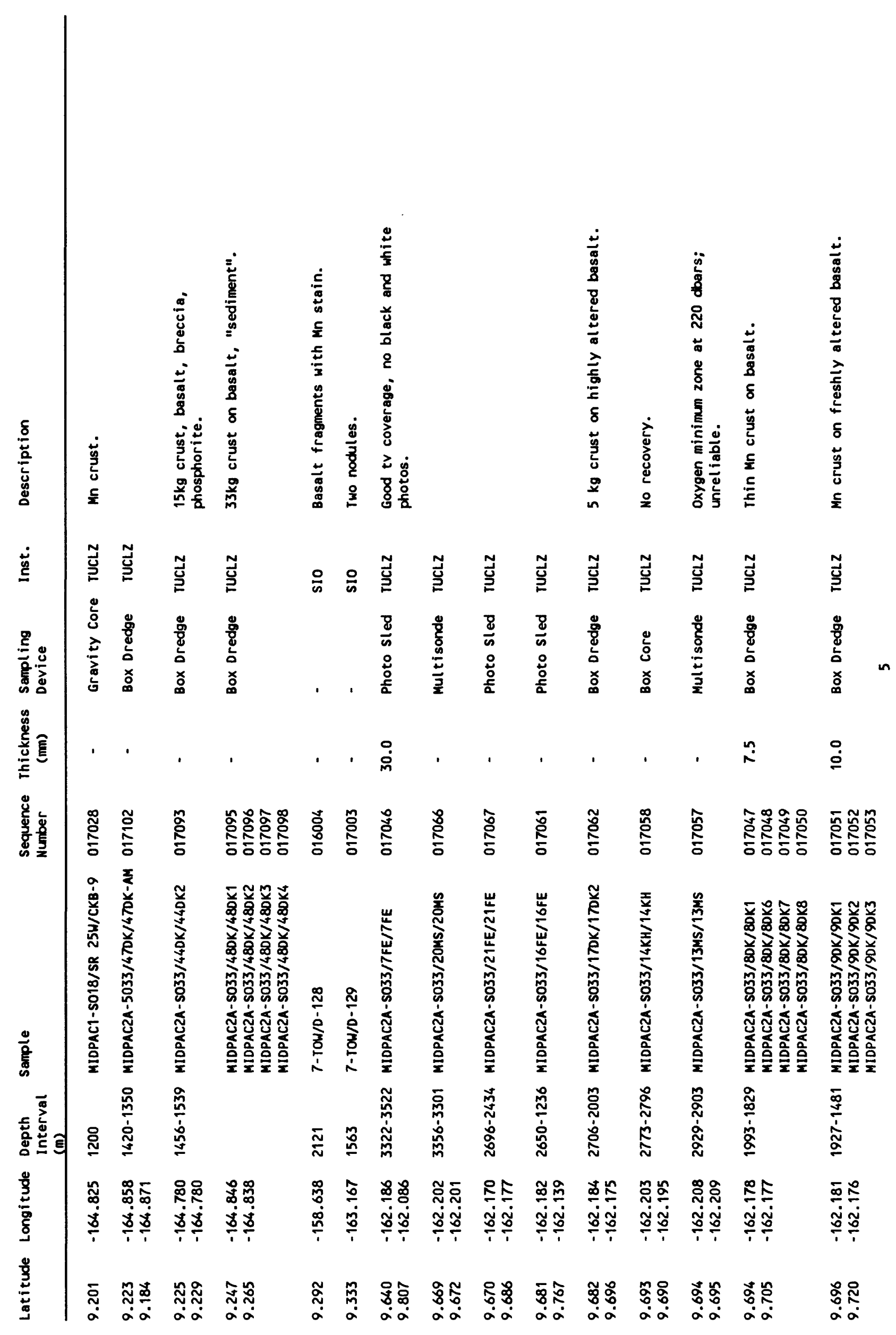




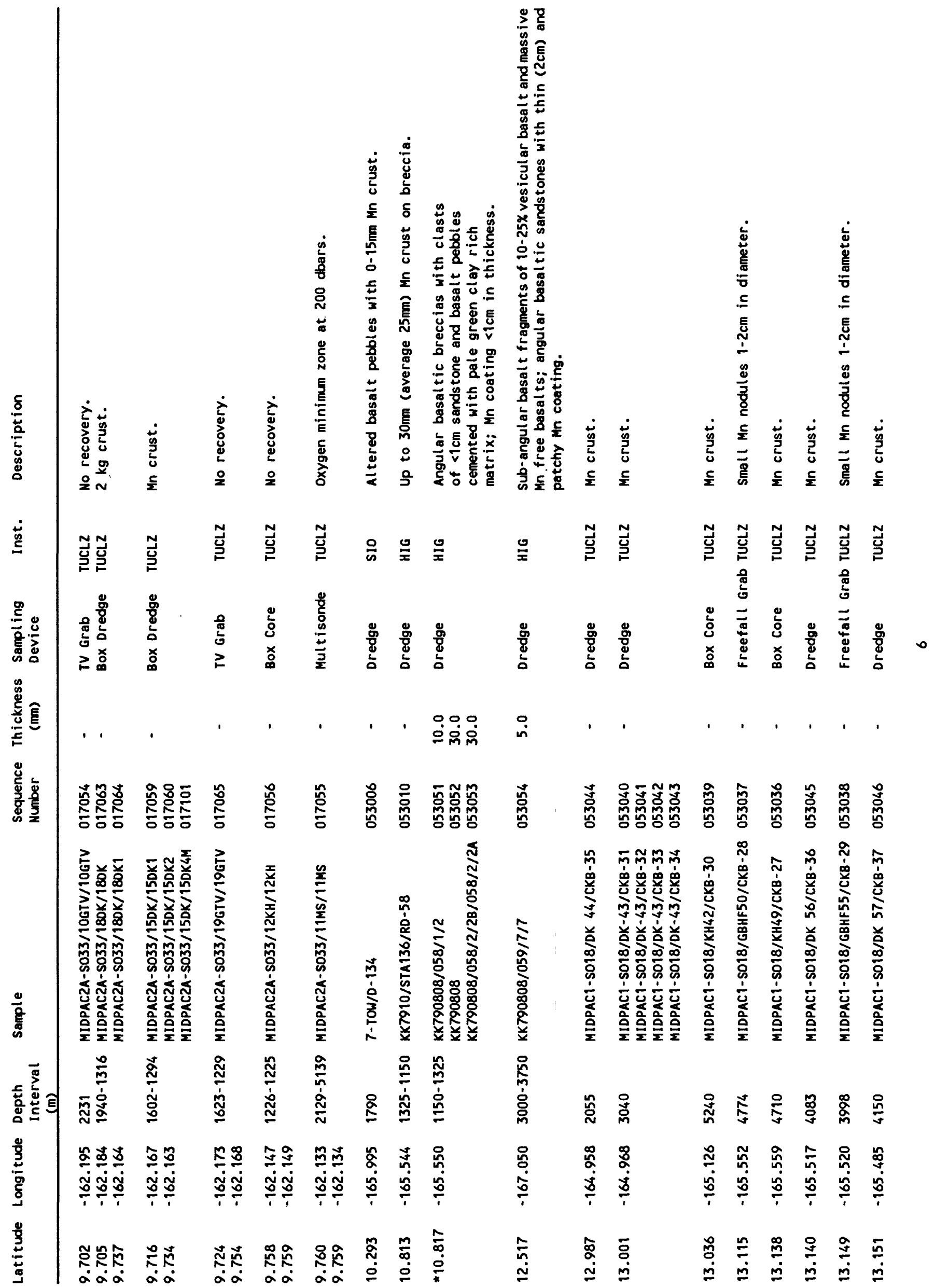




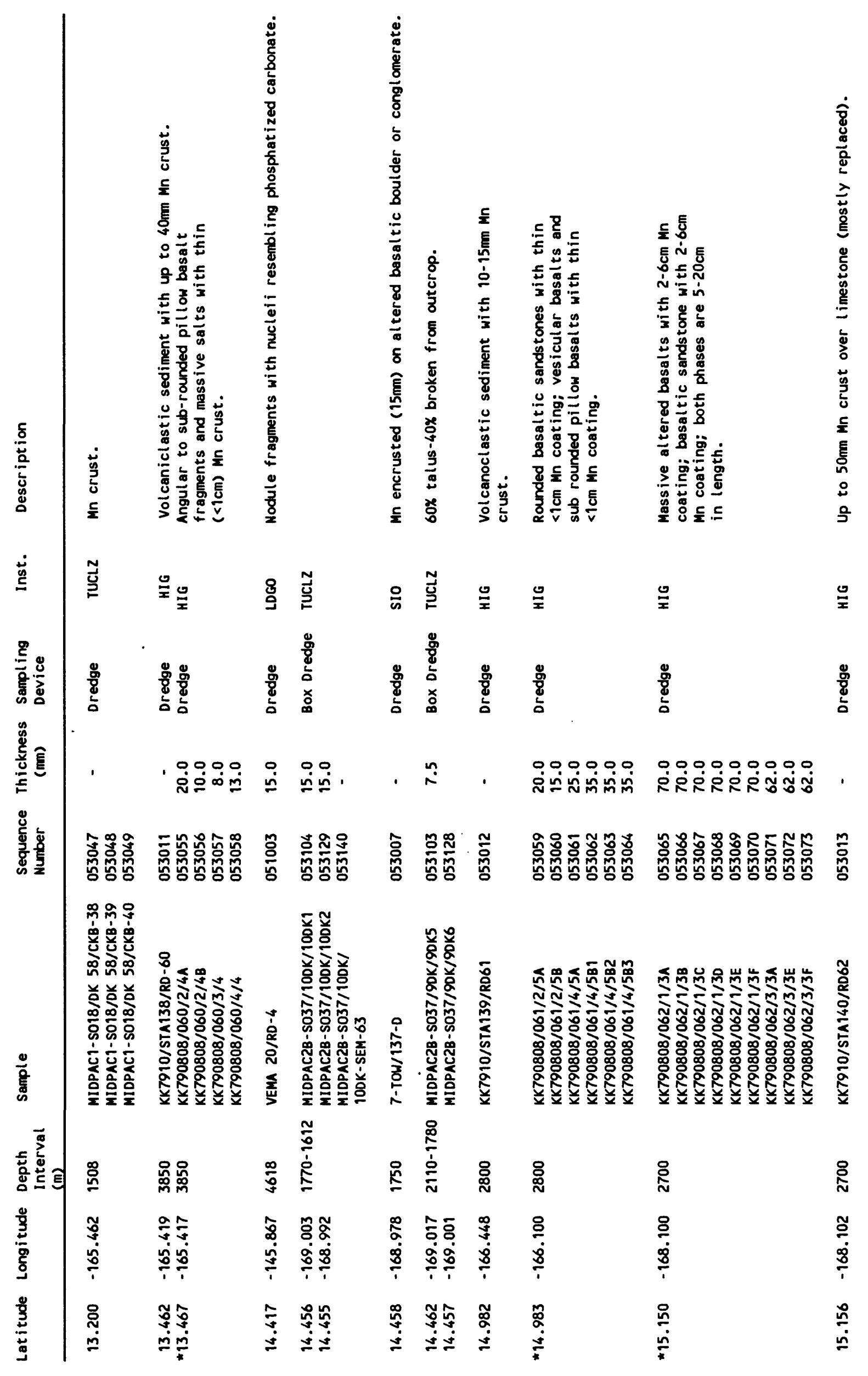




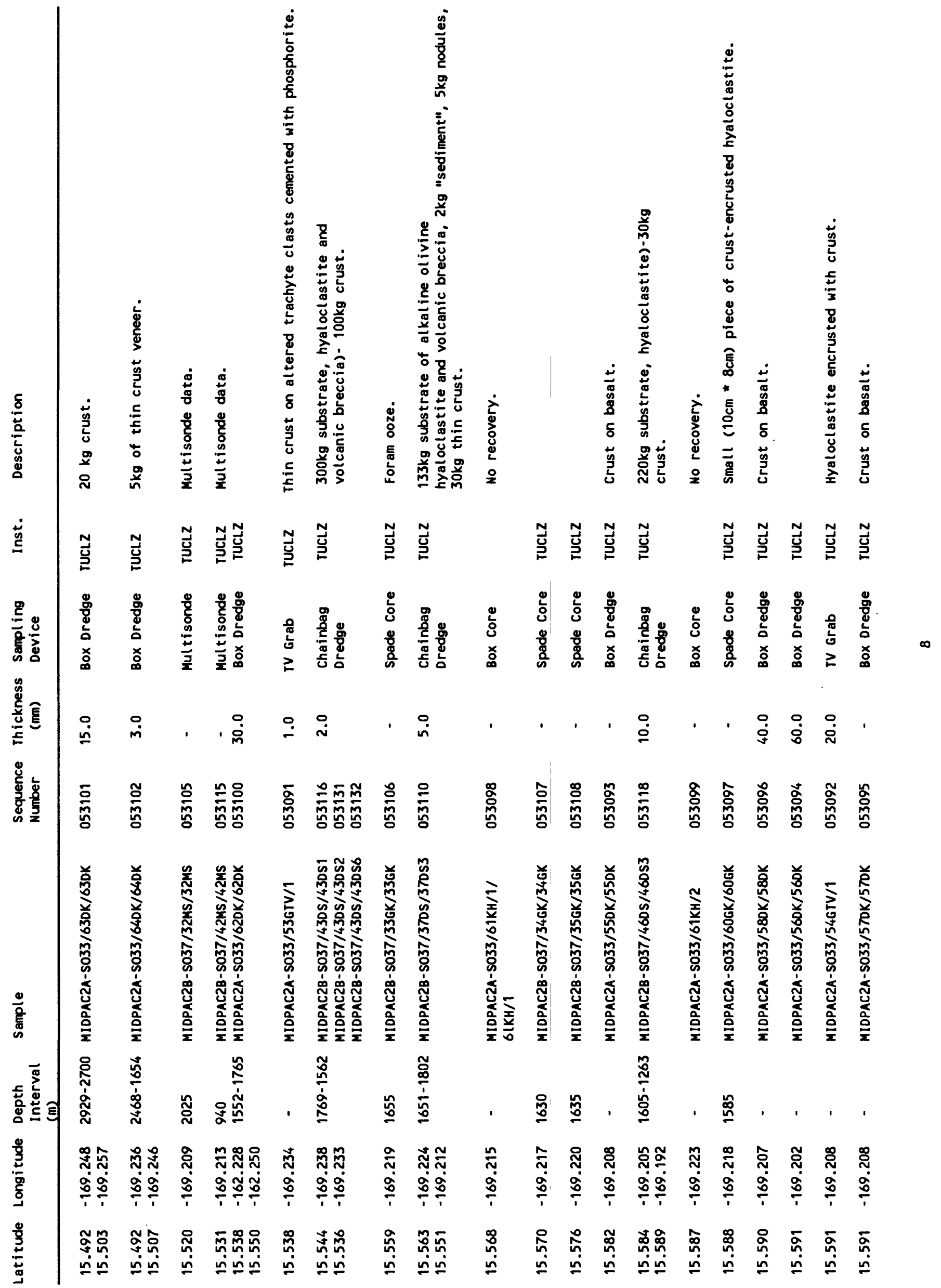




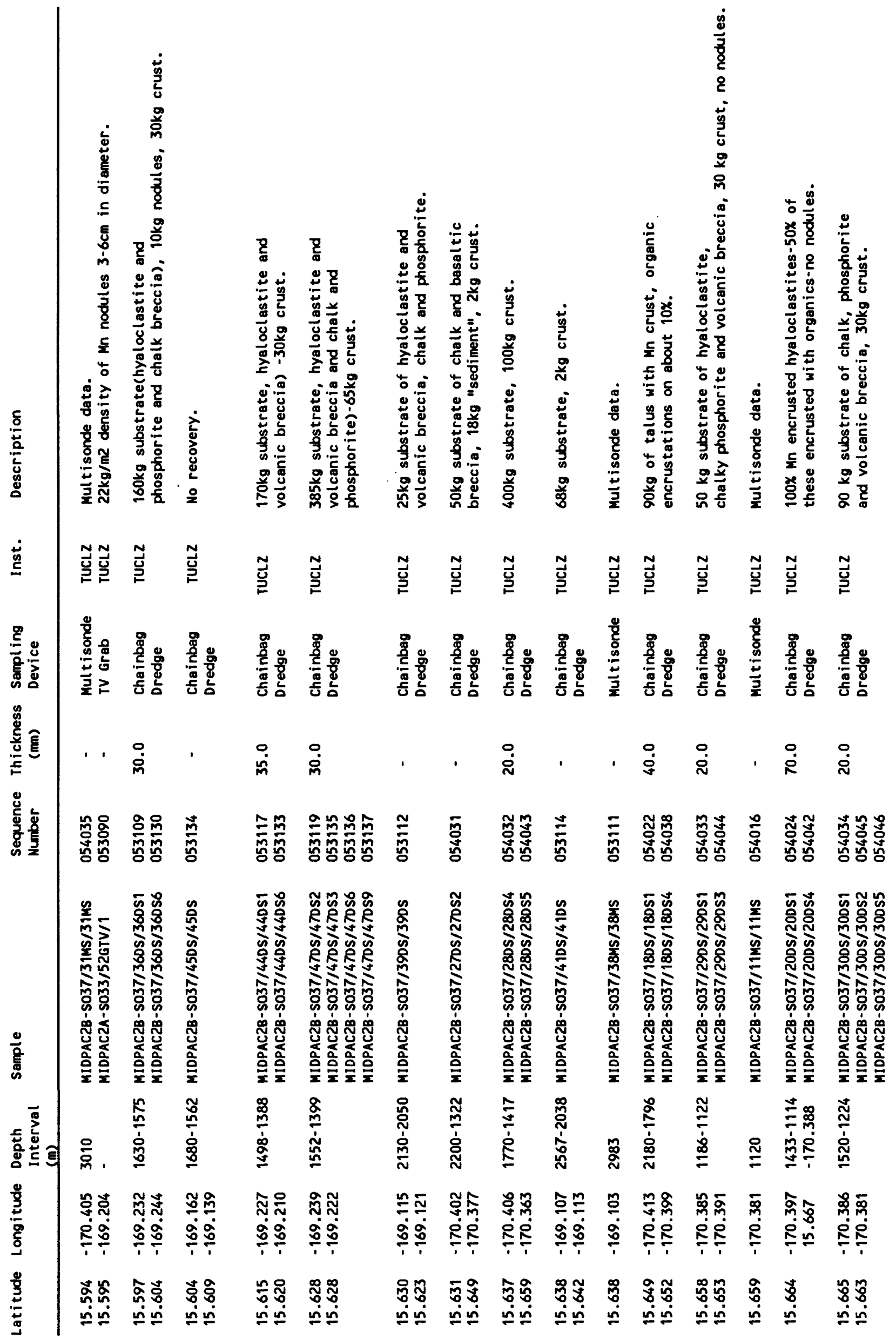




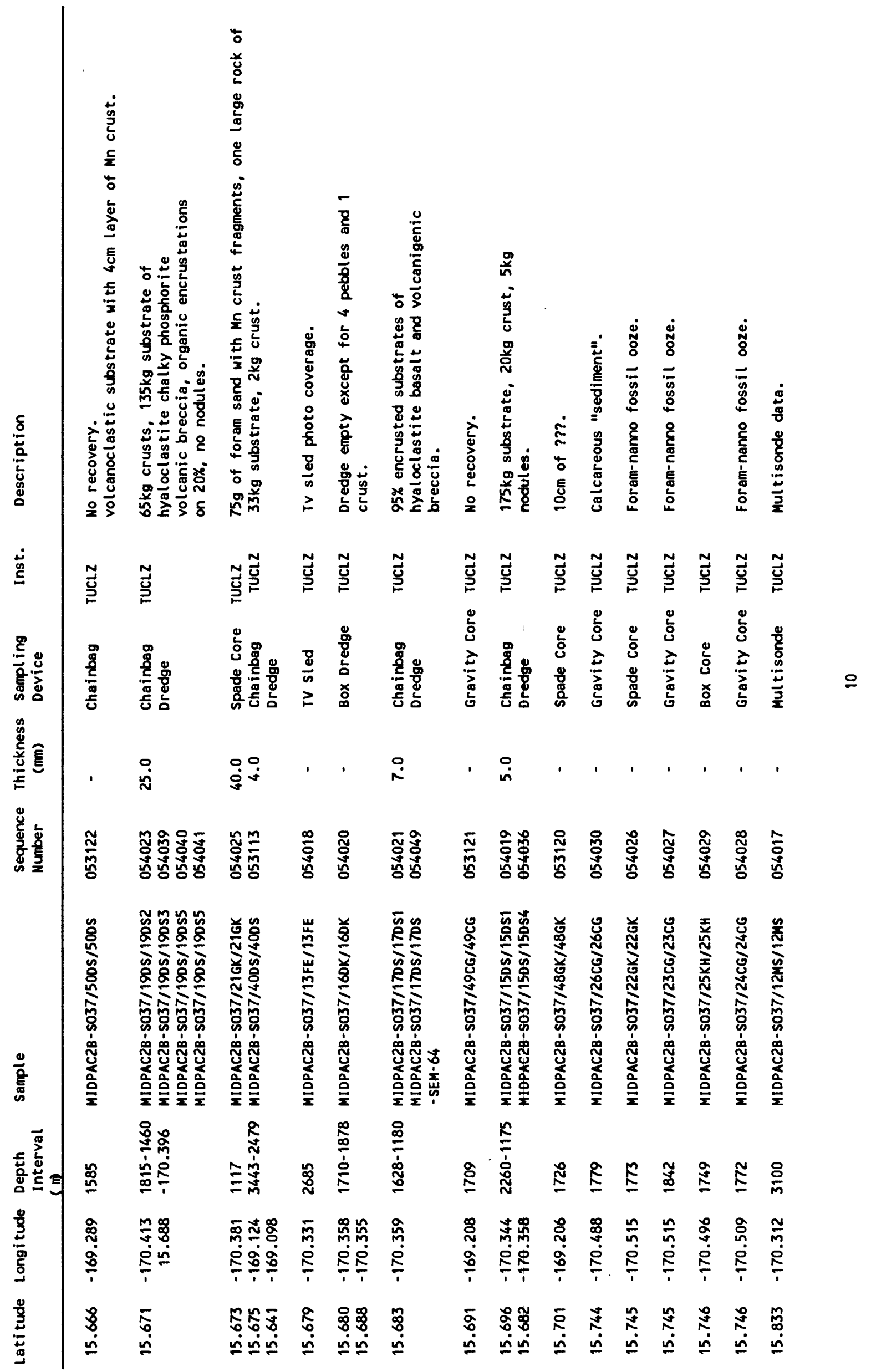




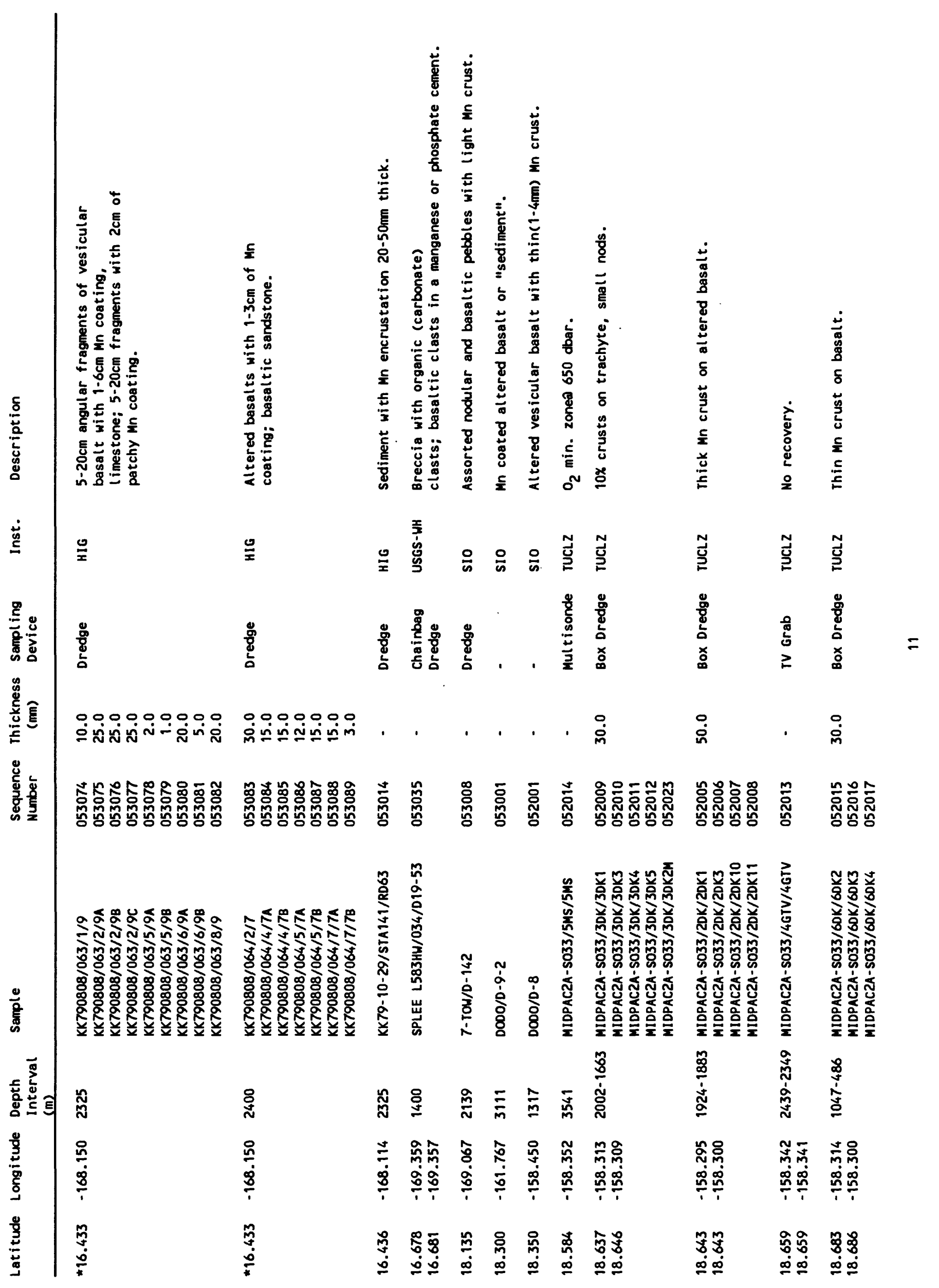




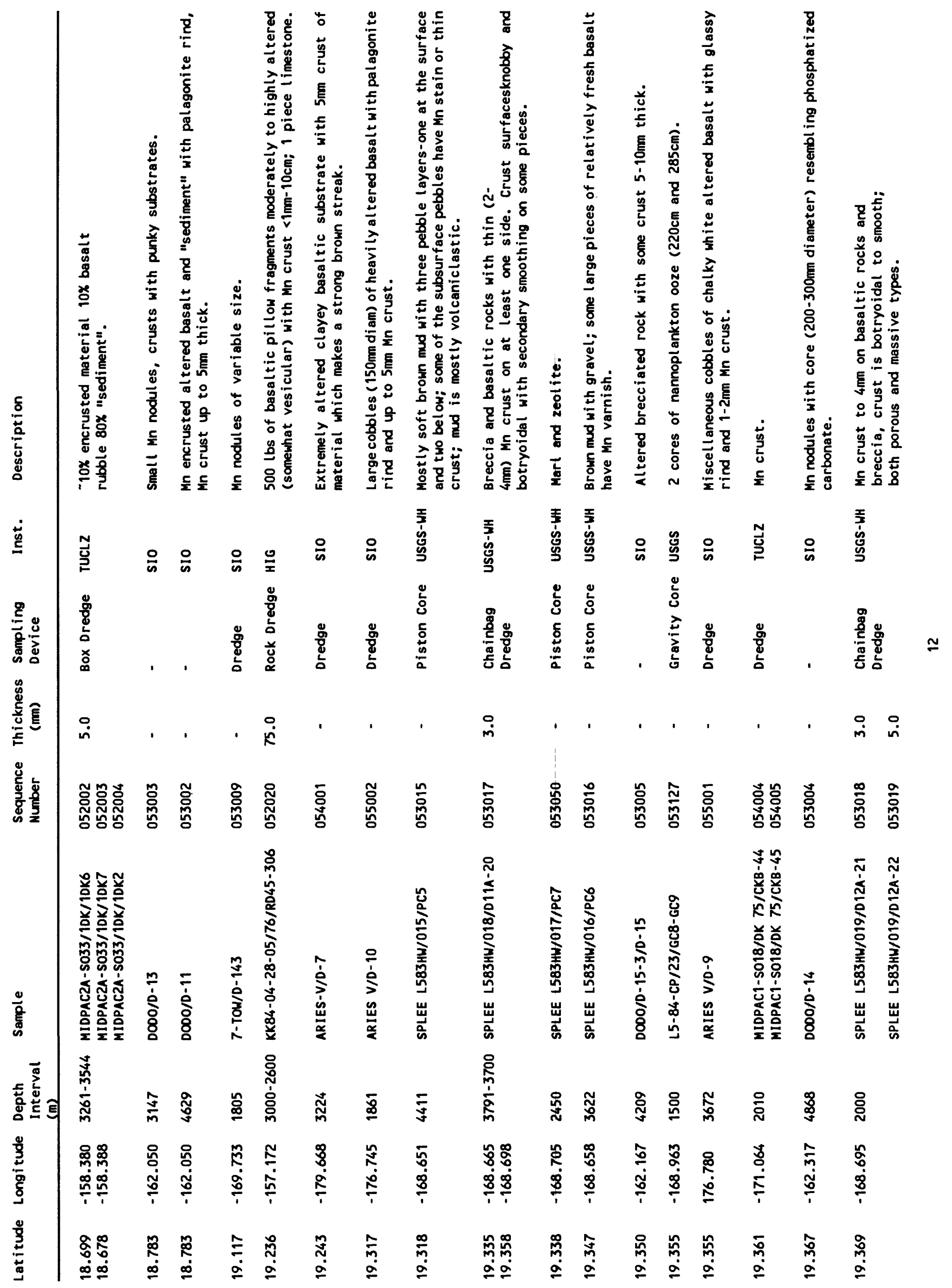




\begin{tabular}{|c|c|c|c|c|c|c|c|c|c|c|c|c|}
\hline & $\begin{array}{l}\dot{0} \\
\sum_{0}^{0} \\
0 \\
\frac{c}{2}\end{array}$ & 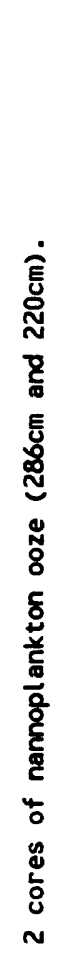 & 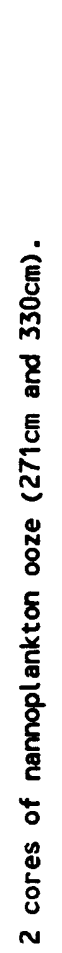 & $\begin{array}{l}\dot{0} \\
\stackrel{0}{2} \\
\dot{2} \\
\frac{c}{2}\end{array}$ & 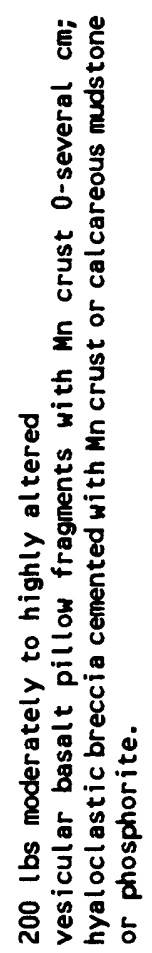 & $\begin{array}{l}\dot{0} \\
\stackrel{n}{3} \\
\dot{2} \\
\frac{c}{2}\end{array}$ & 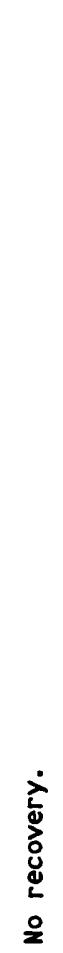 & 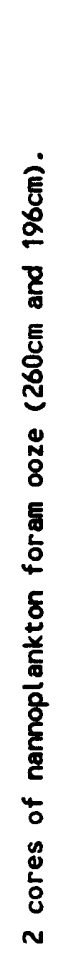 & 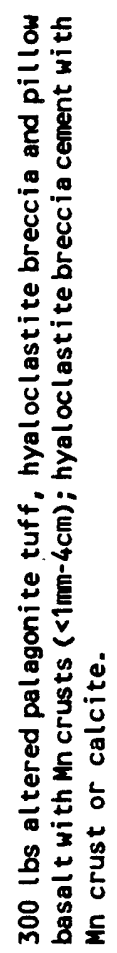 & 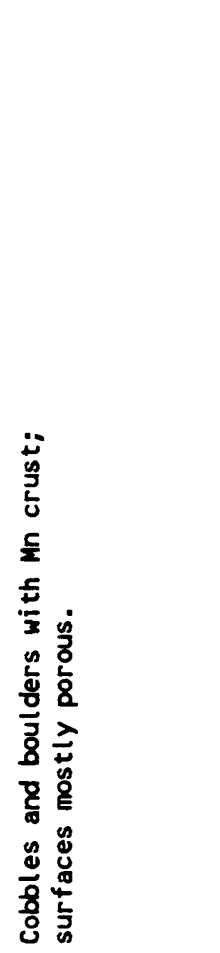 & 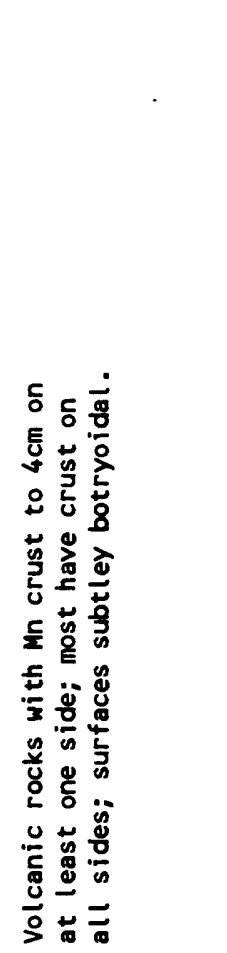 & 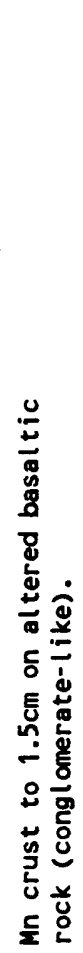 \\
\hline 造 & 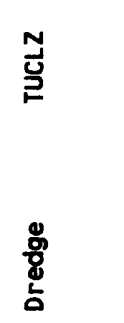 & 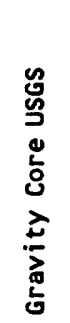 & 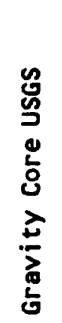 & $\begin{array}{l}\text { : } \\
\frac{0}{d} \\
\text { L }\end{array}$ & 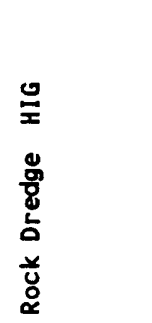 & 旁 & 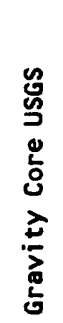 & 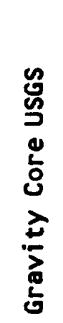 & 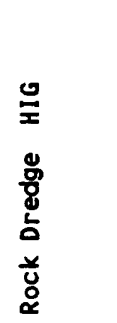 & 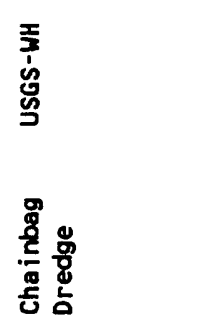 & 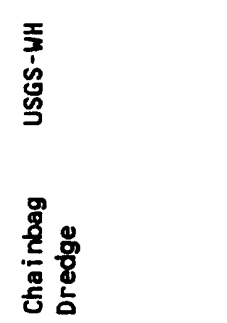 & 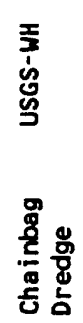 \\
\hline 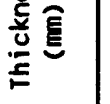 & ' & ' & ' & ' & ํํำ & ' & ' & ' & $\stackrel{\circ}{0}$ & 웅용요 & 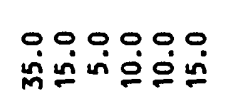 & $\ddot{0}$ \\
\hline 娄离 & 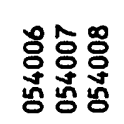 & 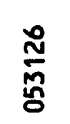 & $\frac{n}{\frac{n}{n}}$ & 颉 & 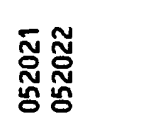 & 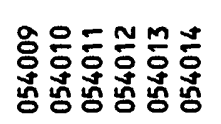 & $\frac{N}{\frac{N}{n}}$ & $\frac{n}{n}$ & 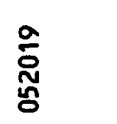 & 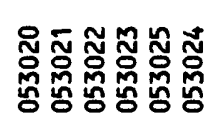 & 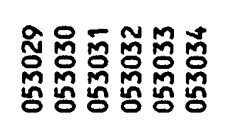 & กิ̃ \\
\hline & 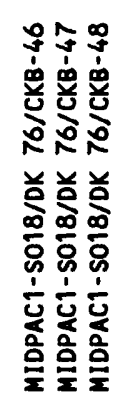 & 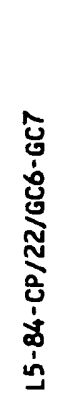 & 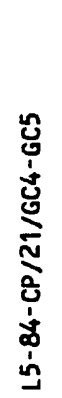 & 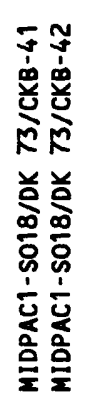 & 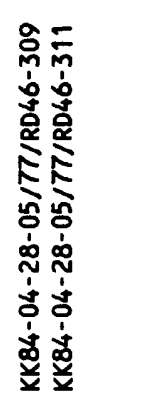 & 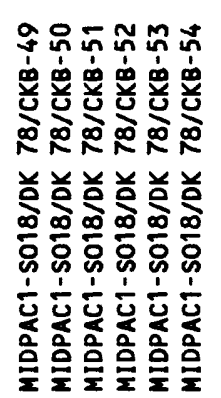 & 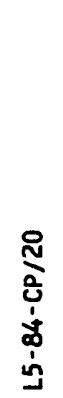 & 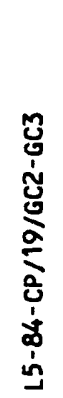 & 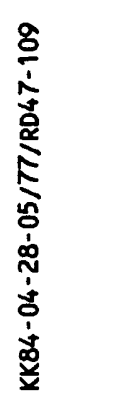 & 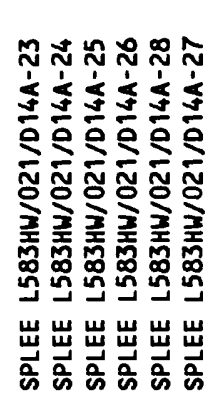 & 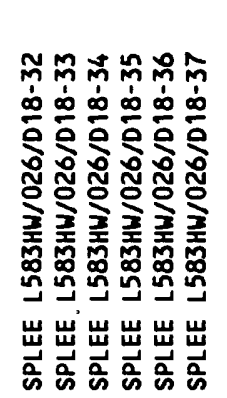 & 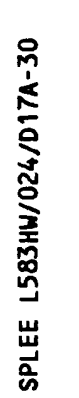 \\
\hline 总 & $\stackrel{\varrho}{\varrho}$ & ณี & 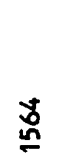 & 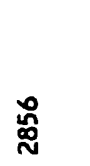 & 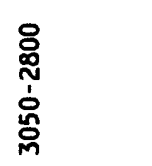 & 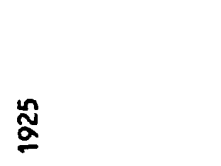 & 응 & $\underline{8}$ & 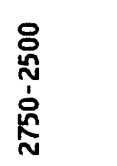 & 莫 & $\stackrel{8}{\stackrel{8}{2}}$ & ষ্ণ \\
\hline$\frac{1}{8}$ & \begin{tabular}{l}
$\overline{8}$ \\
$\stackrel{0}{0}$ \\
\hdashline
\end{tabular} & $\begin{array}{l}\hat{8} \\
8 \\
8 \\
\div\end{array}$ & \begin{tabular}{l}
$n$ \\
$\infty$ \\
0 \\
\hdashline \\
$\square$
\end{tabular} & $\frac{K}{\frac{K}{5}}$ & 苂 & $\frac{\bar{a}}{\stackrel{m}{\vdots}}$ & 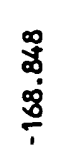 & \begin{tabular}{l}
$\infty$ \\
$\$$ \\
0 \\
8 \\
\hdashline
\end{tabular} & 雚 & 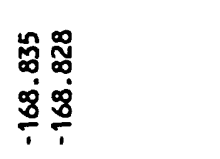 & 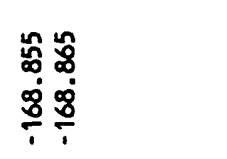 & 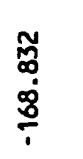 \\
\hline & $\underset{c}{\kappa}$ & $\begin{array}{c}\stackrel{0}{0} \\
\stackrel{m}{a} \\
\alpha\end{array}$ & $\underset{\tilde{a}}{\stackrel{\tilde{\alpha}}{\alpha}}$ & $\begin{array}{l}\tilde{Q} \\
\stackrel{0}{0} \\
\underline{q}\end{array}$ & 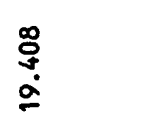 & 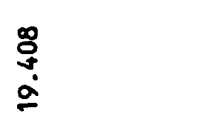 & $\underset{m}{m}$ & $\begin{array}{l}\text { fọ } \\
\text { å }\end{array}$ & $\begin{array}{l}\bar{g} \\
a \\
a\end{array}$ & 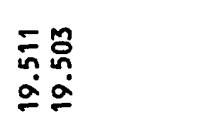 & 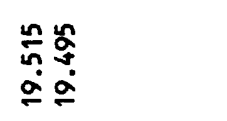 & $\underline{n}$ \\
\hline
\end{tabular}




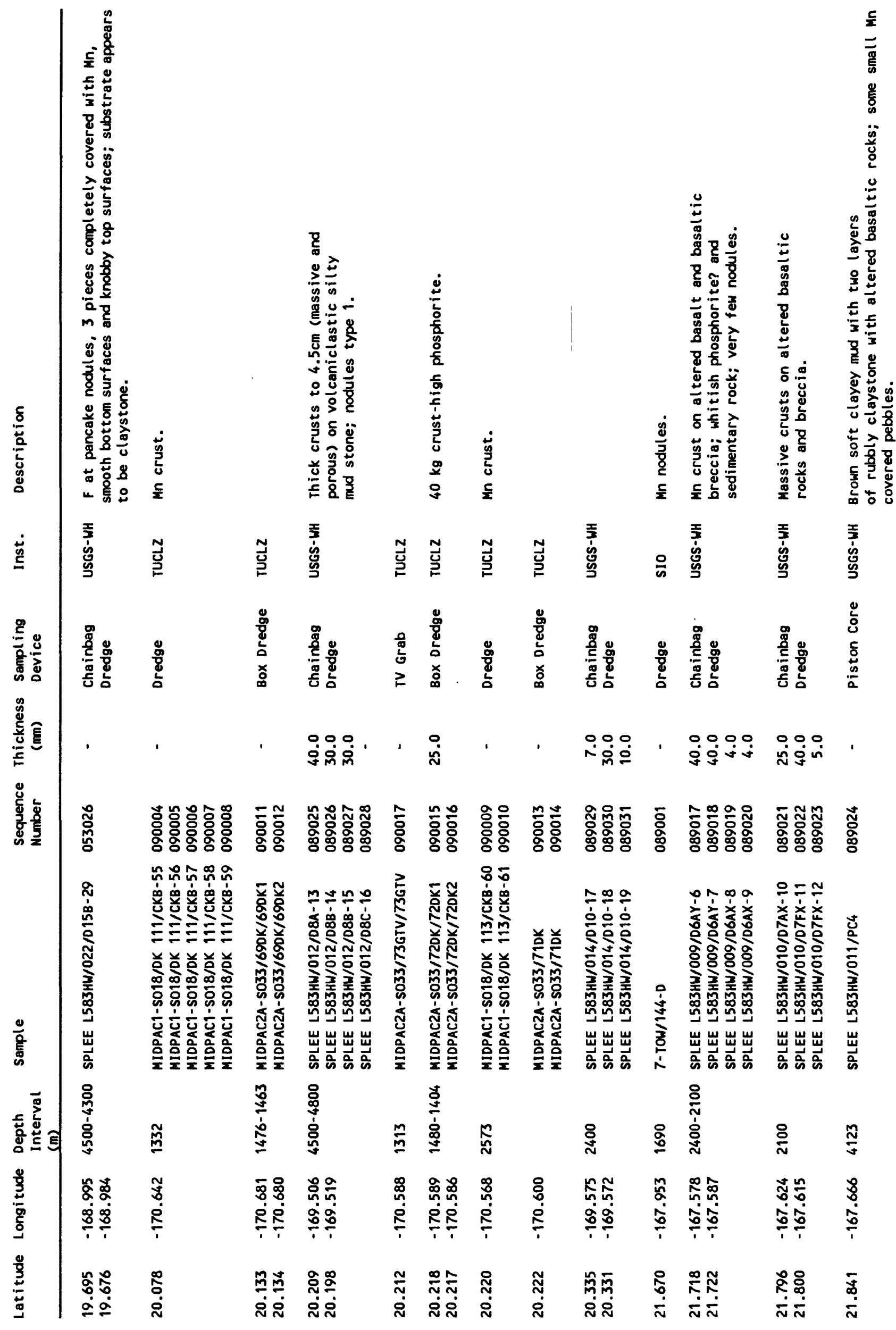




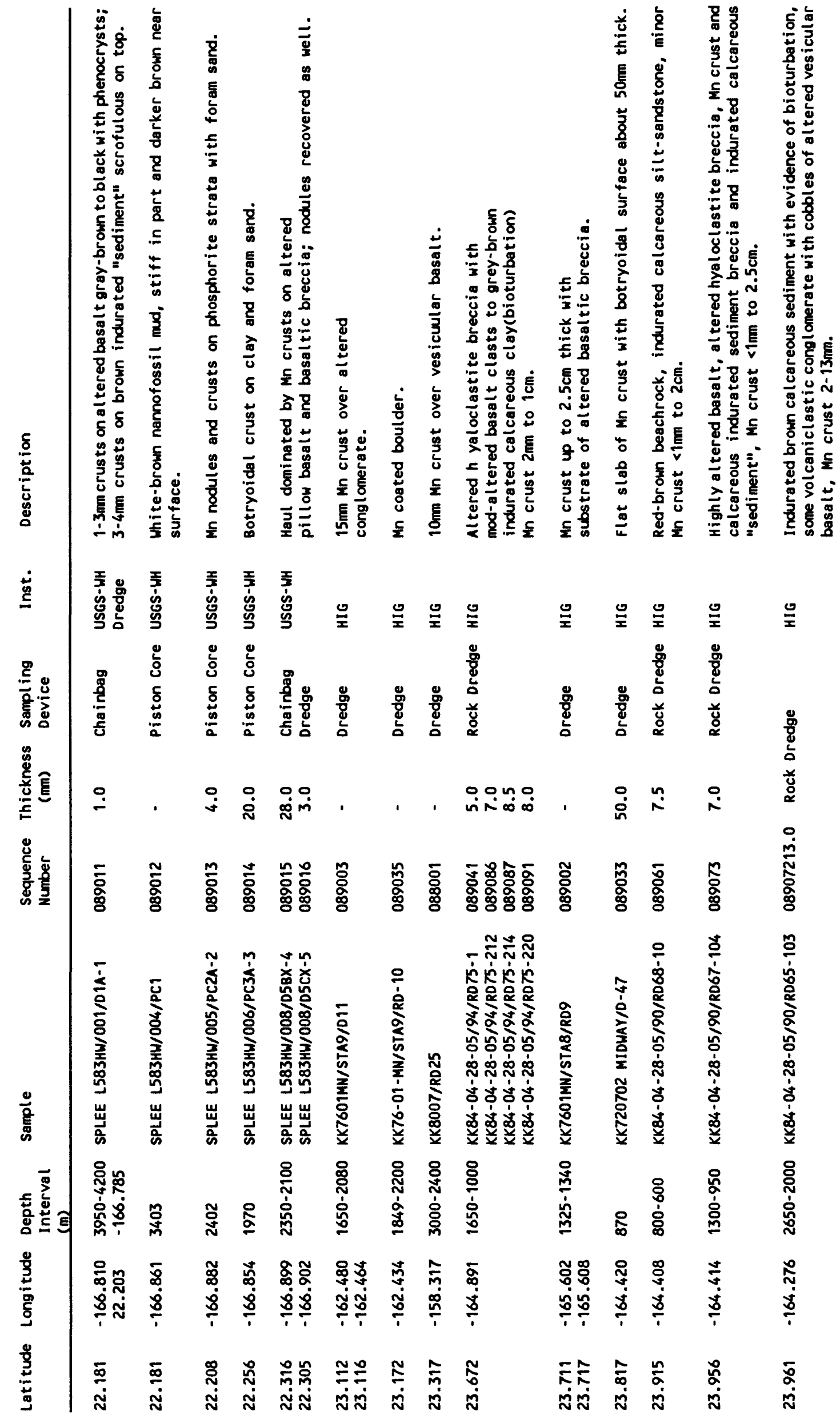




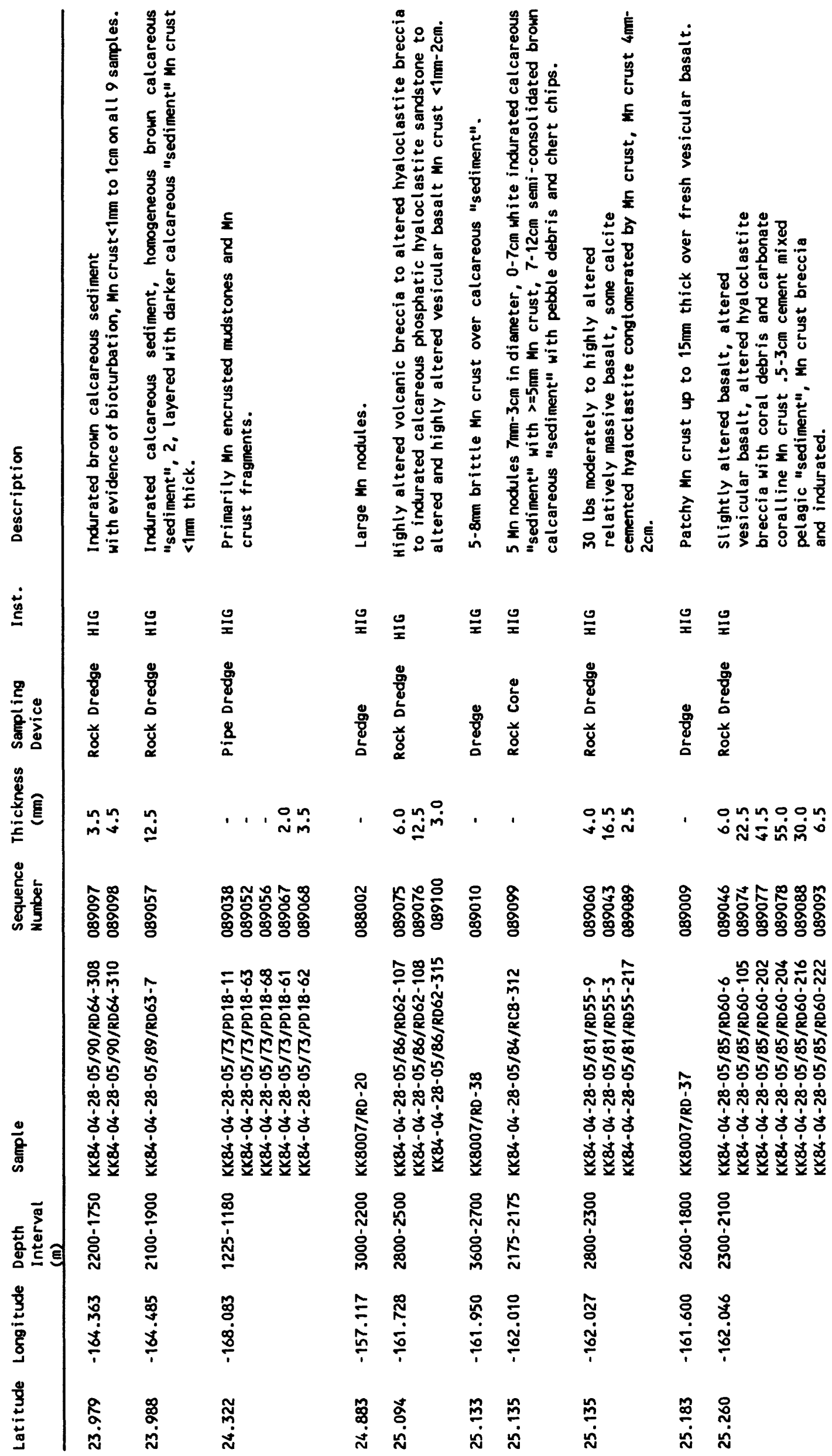




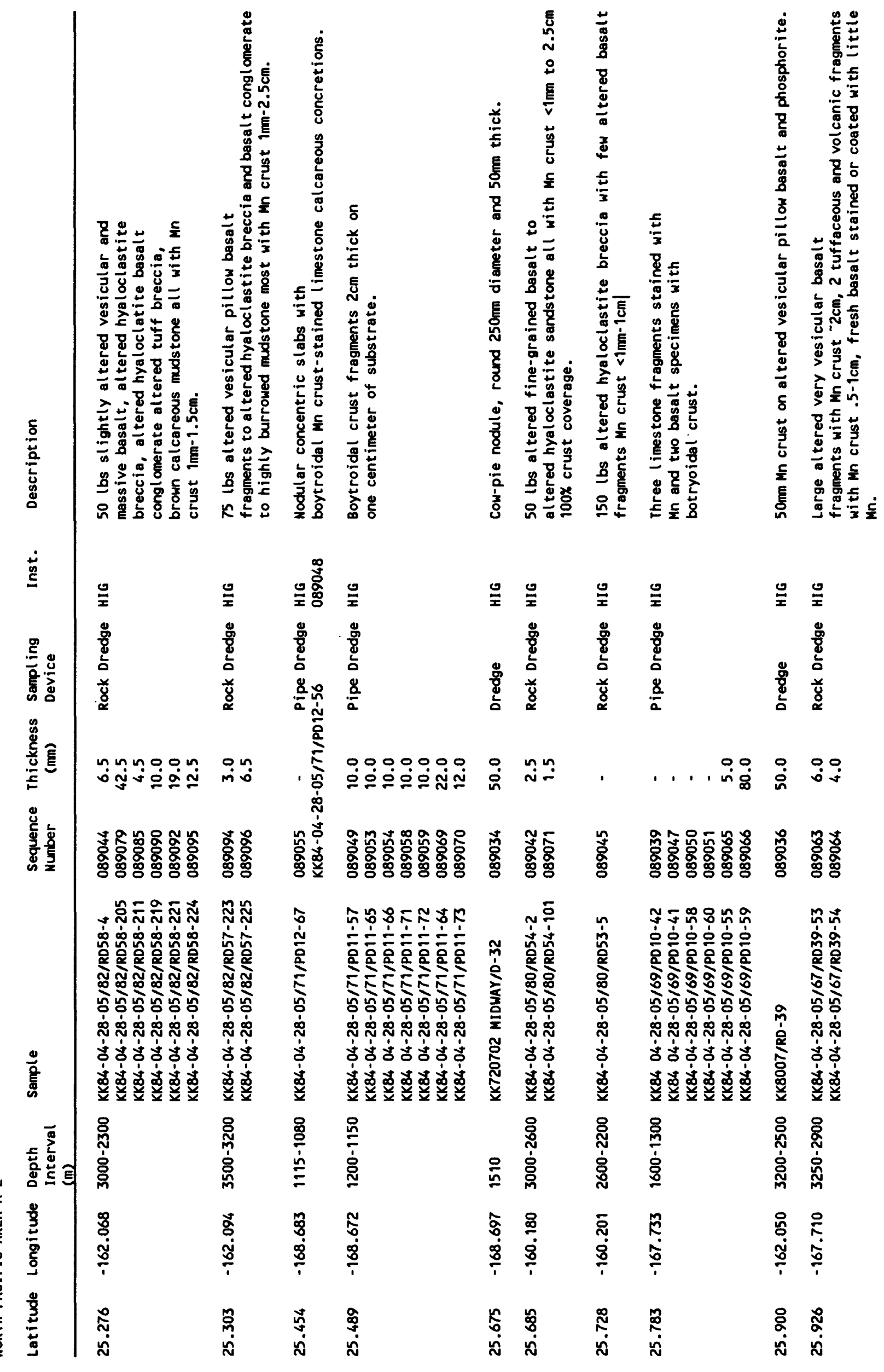




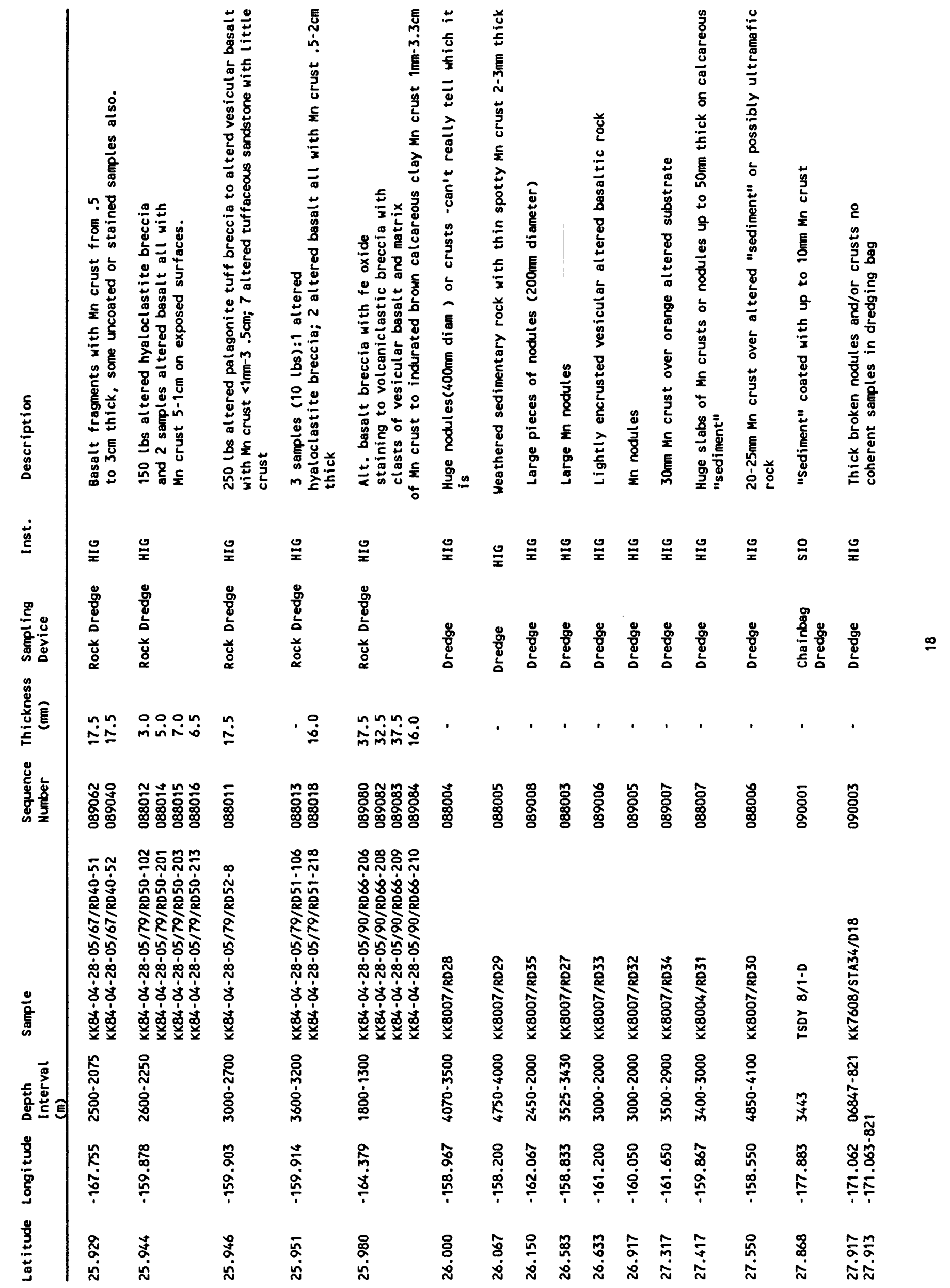




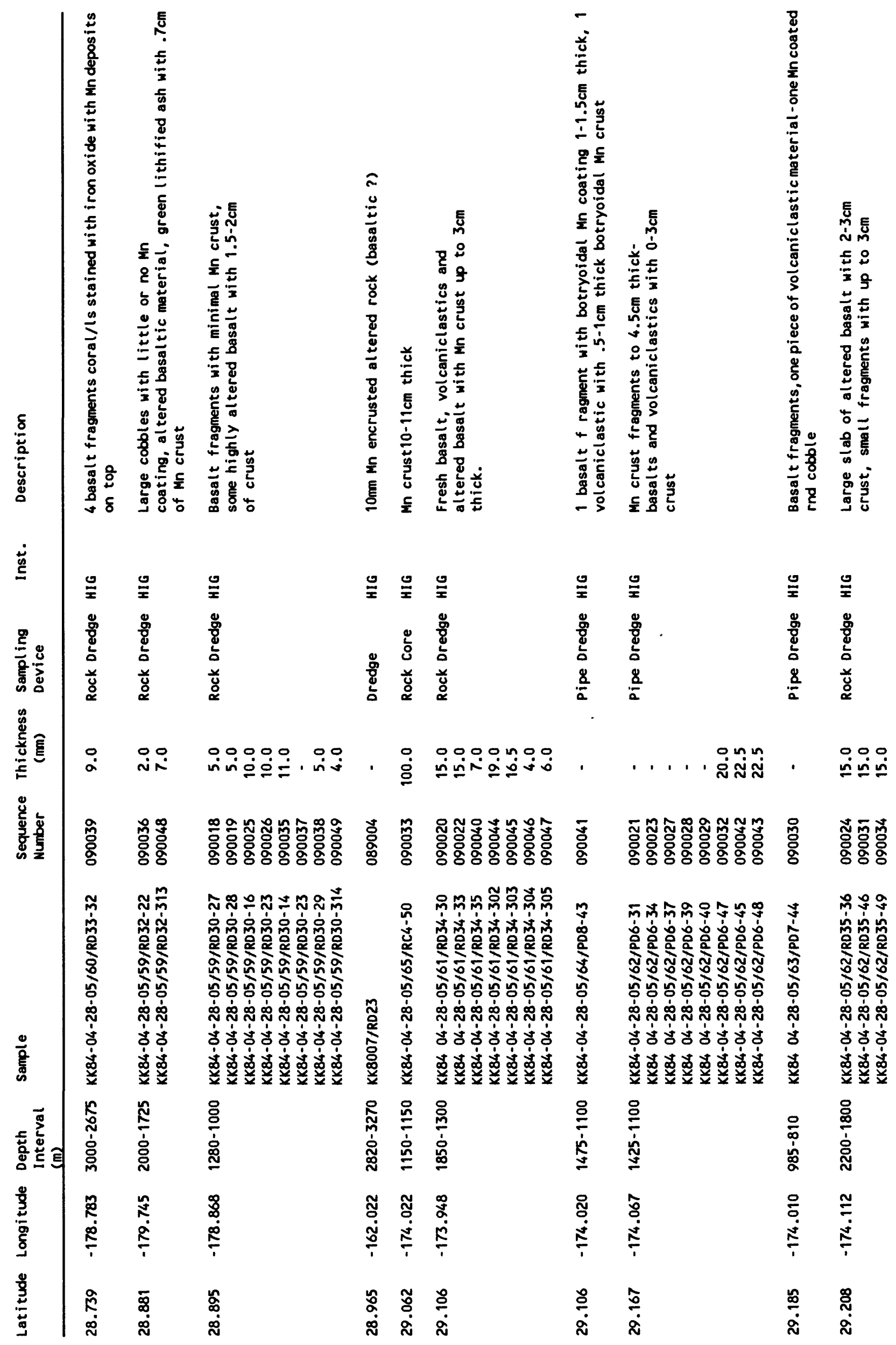




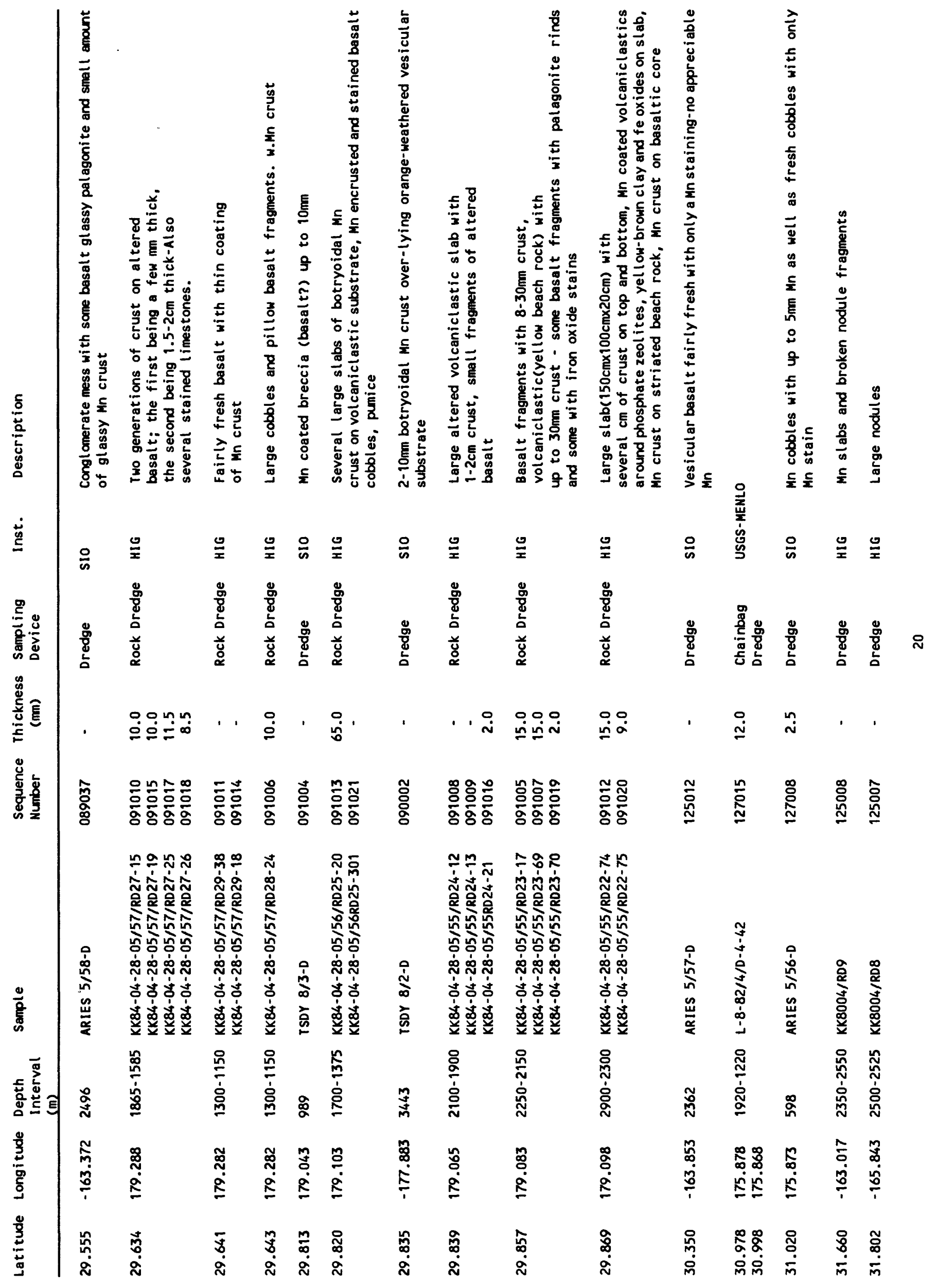




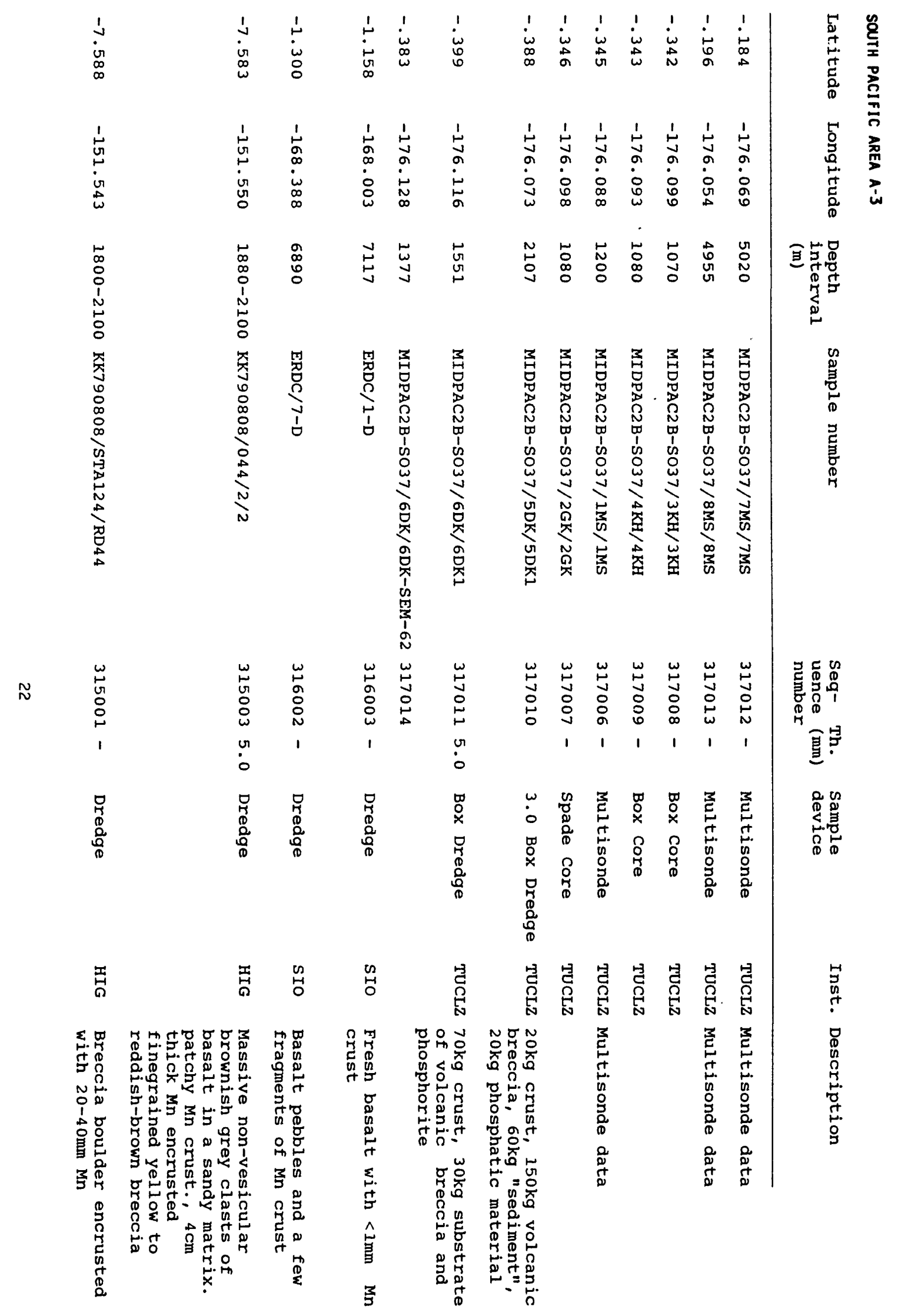




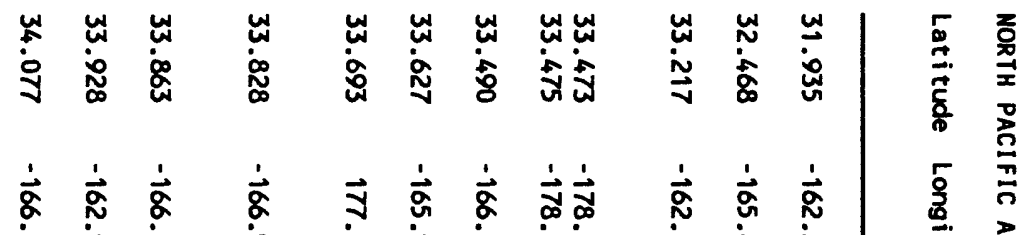

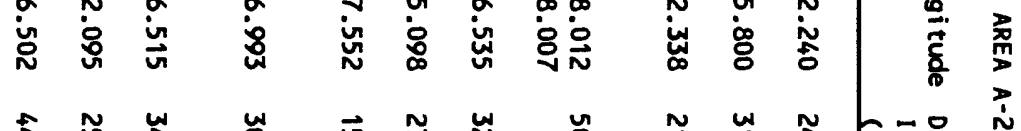

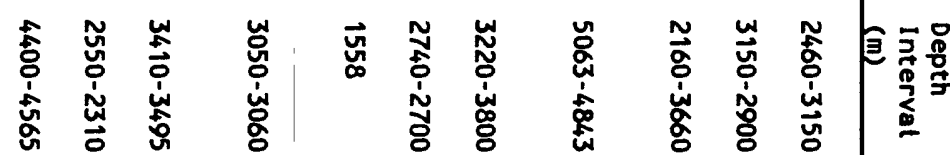

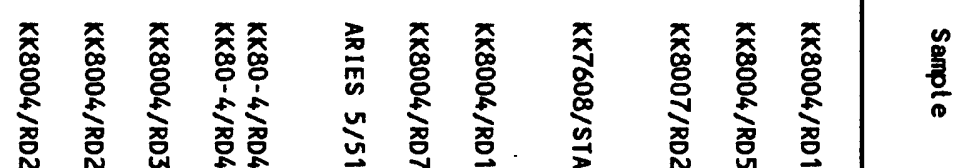

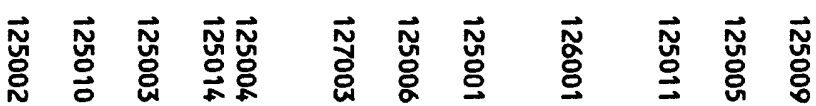




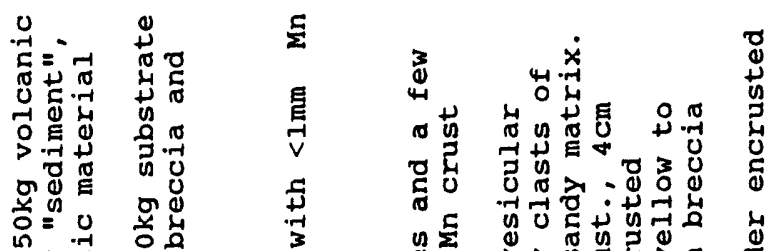

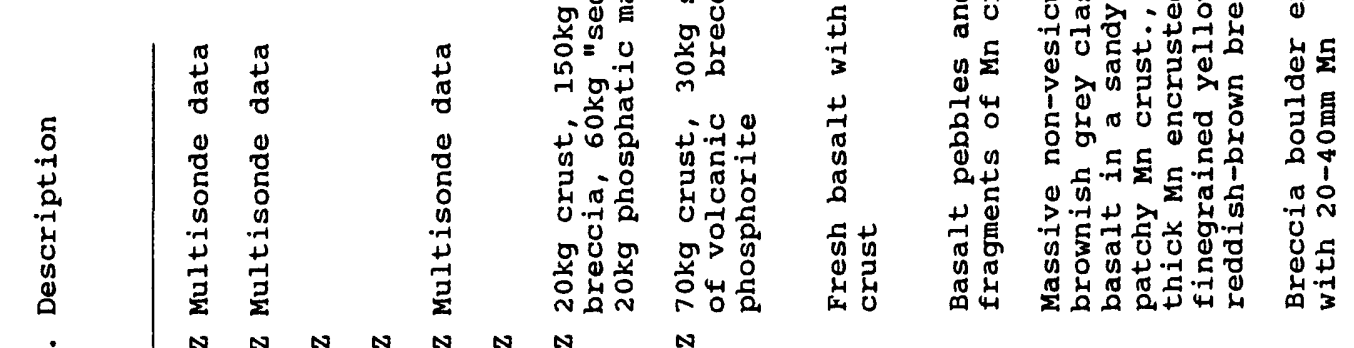

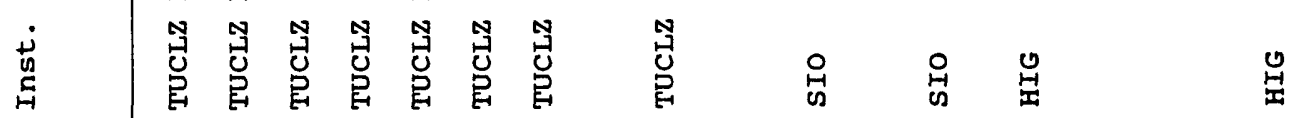

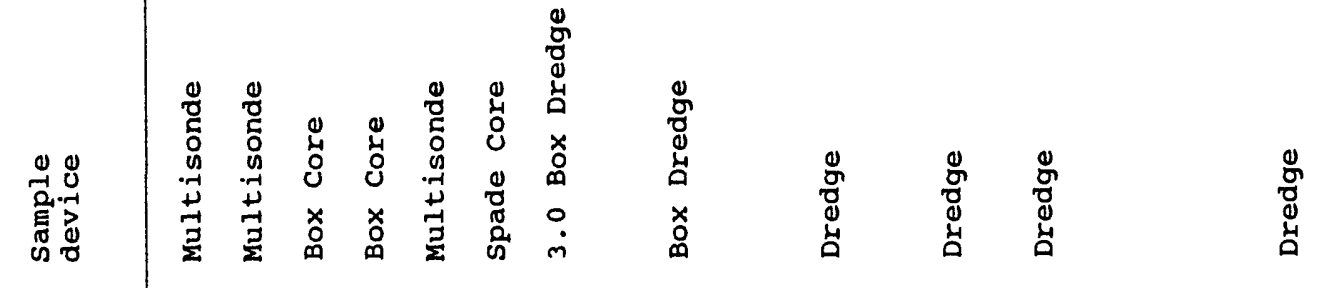

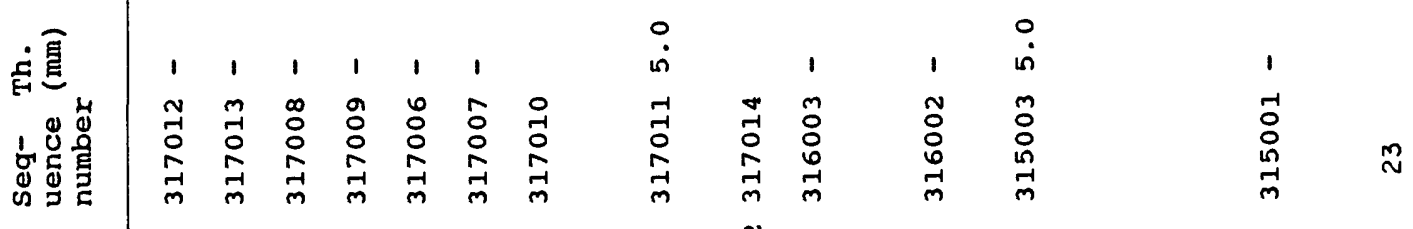

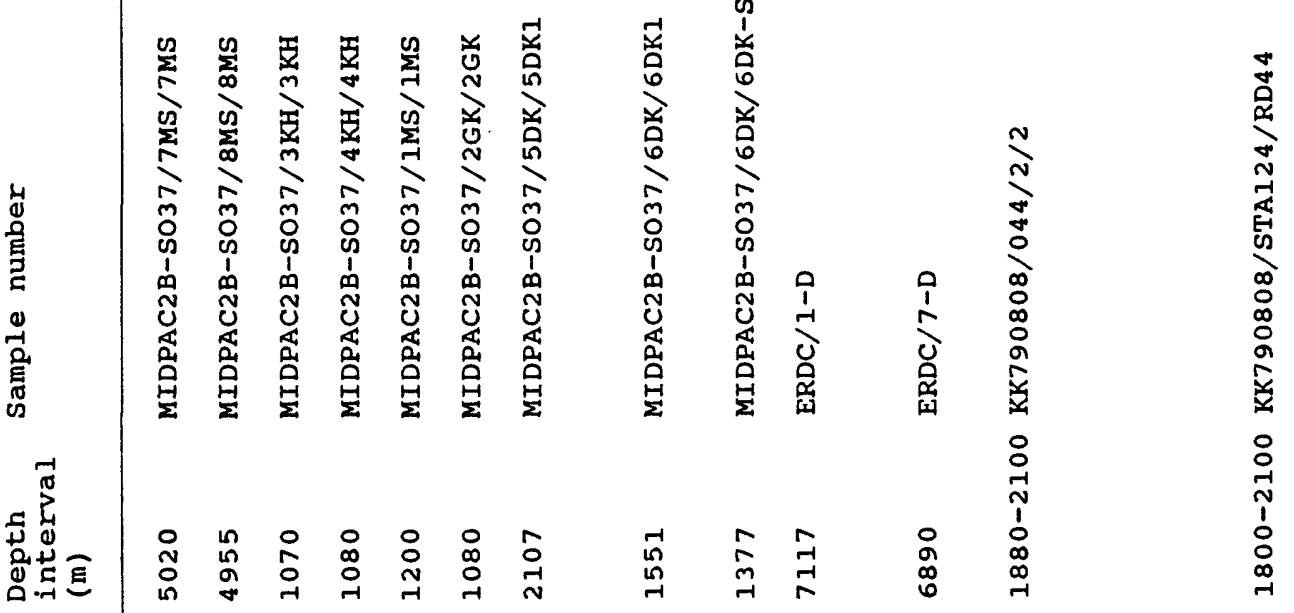

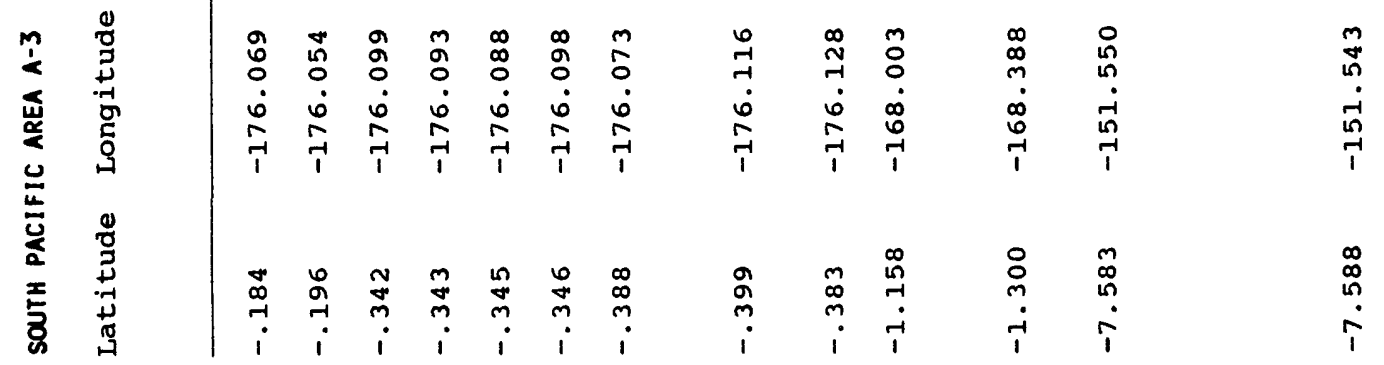




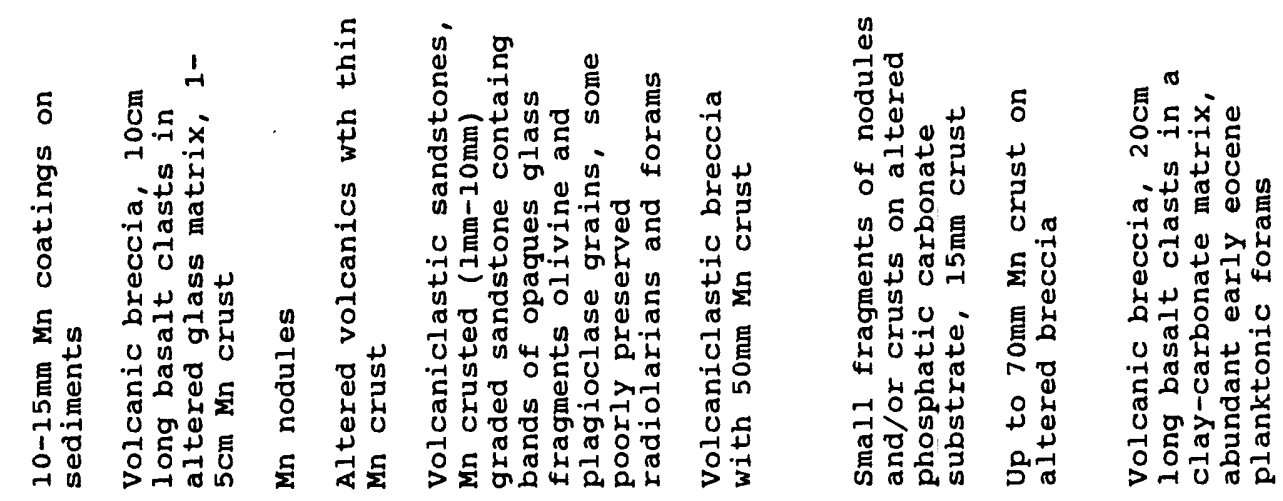

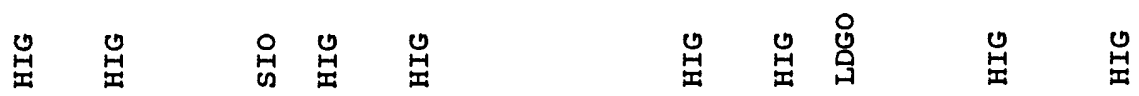

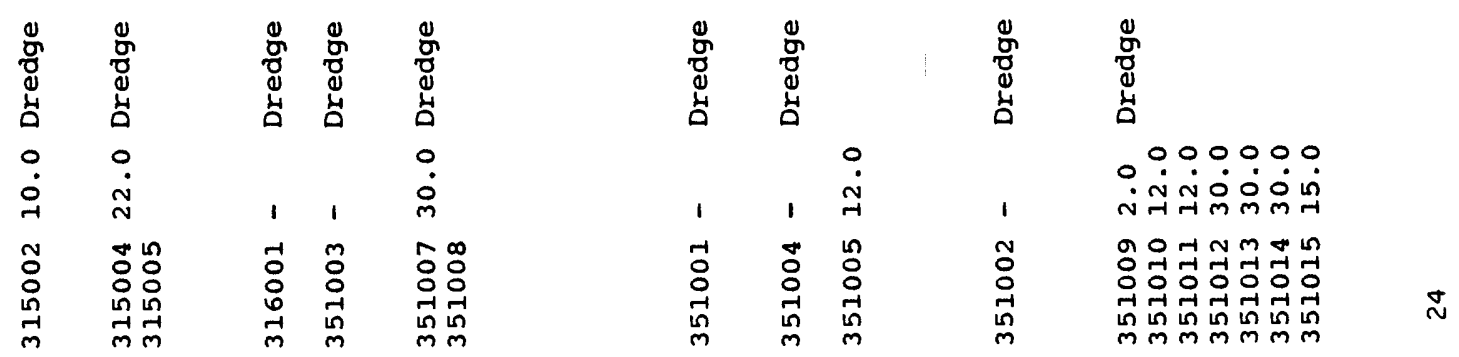

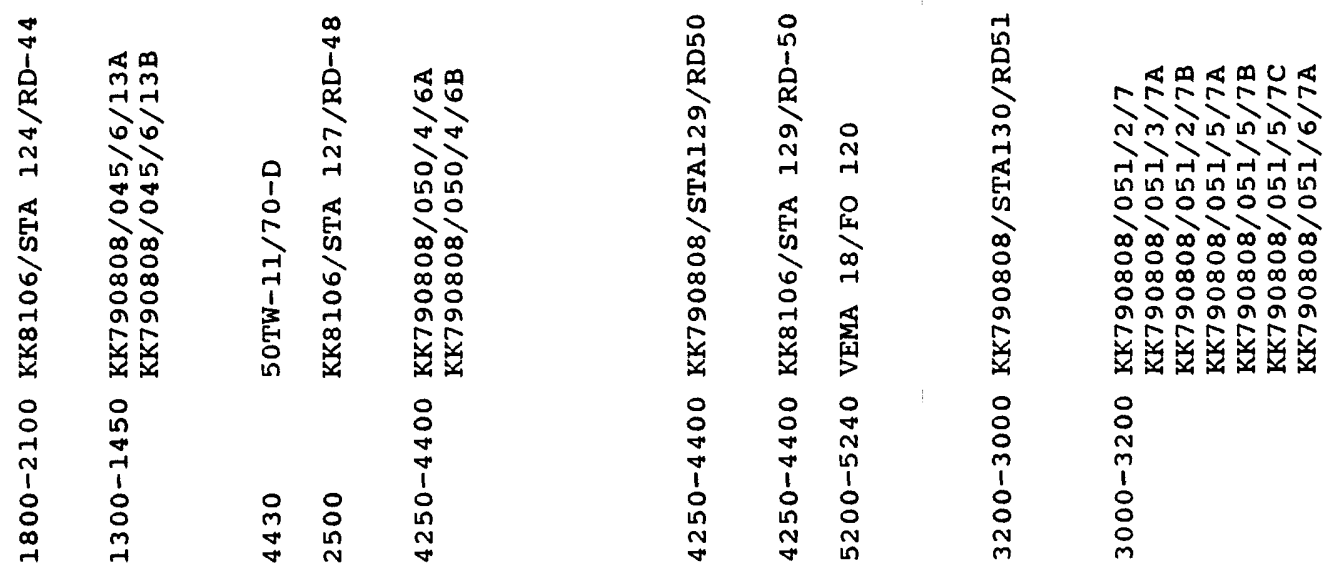

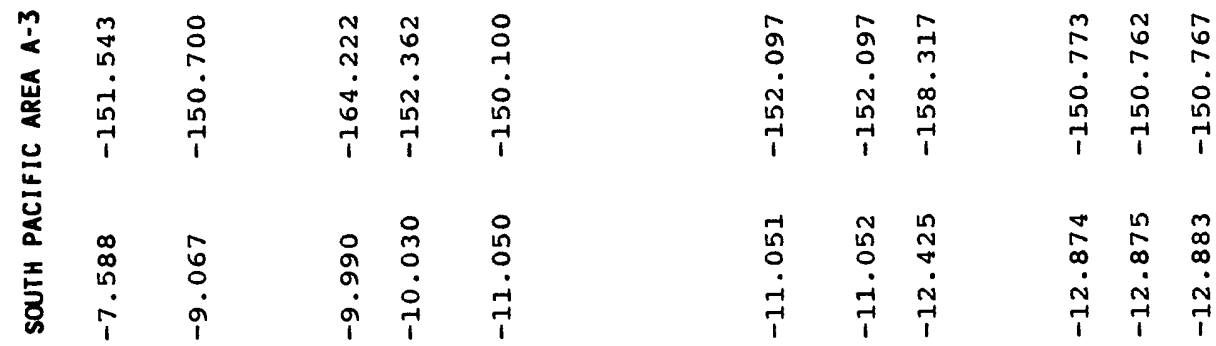



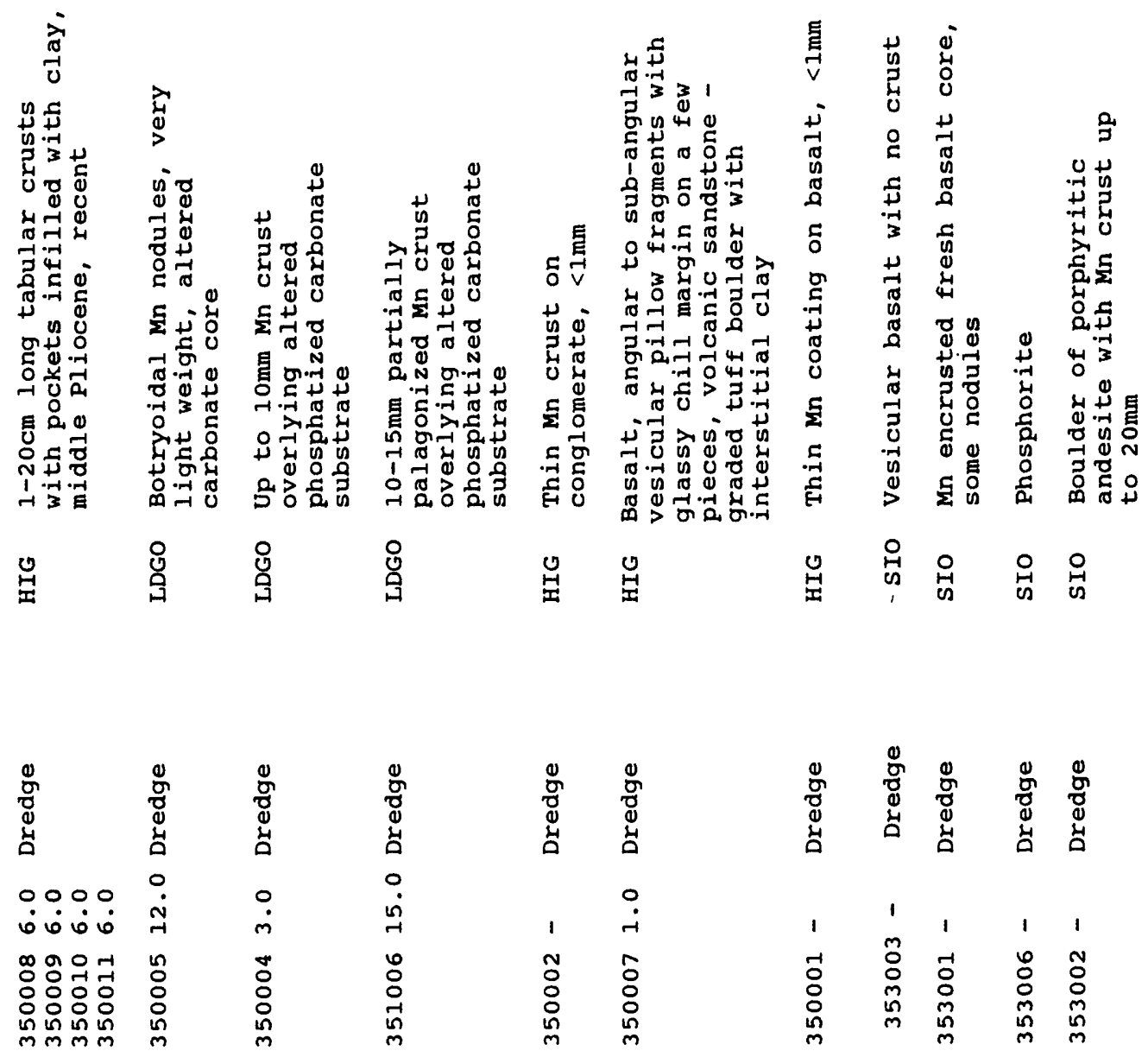

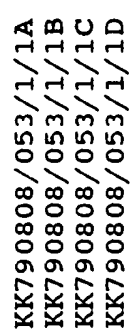

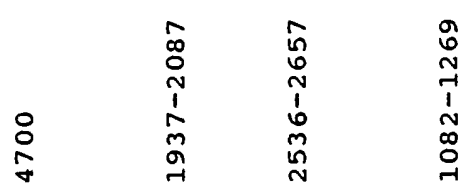

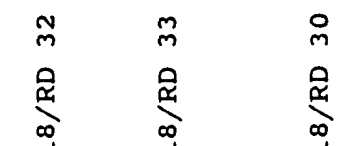

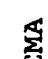

的

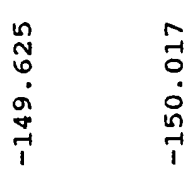

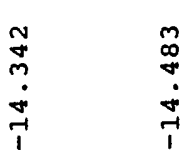

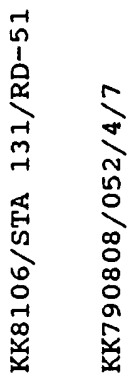

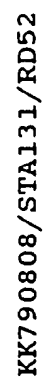

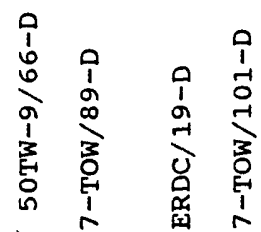

品

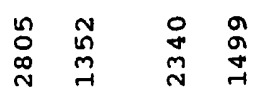

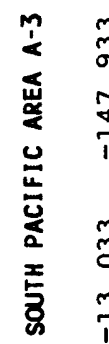

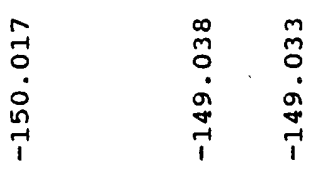

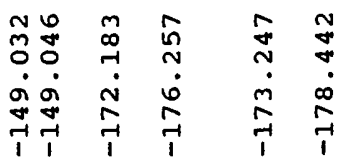

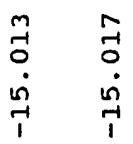

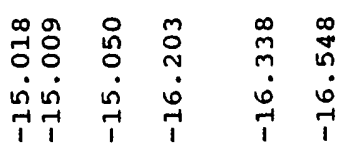




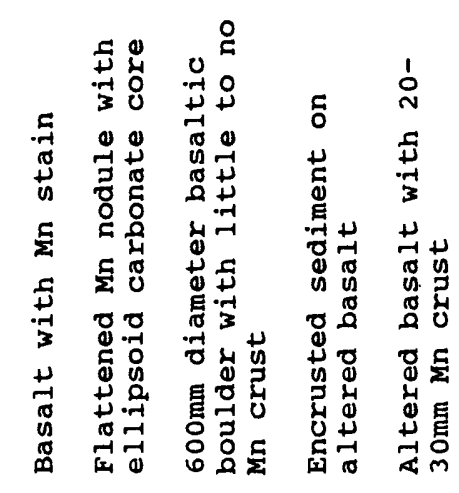

움욜음

min

$\begin{array}{llll}0 & & 0 \\ 0 & 1 & \text { n } \\ 1 & 0 & 1 & \text { n }\end{array}$

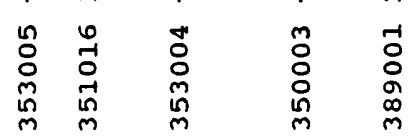

Hin

章

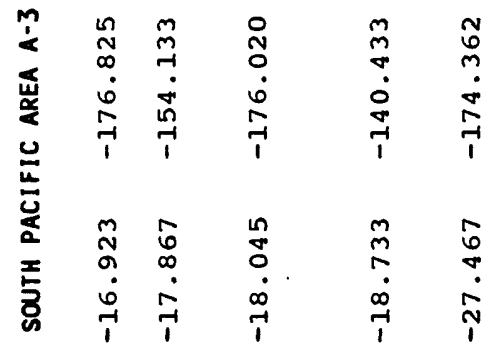



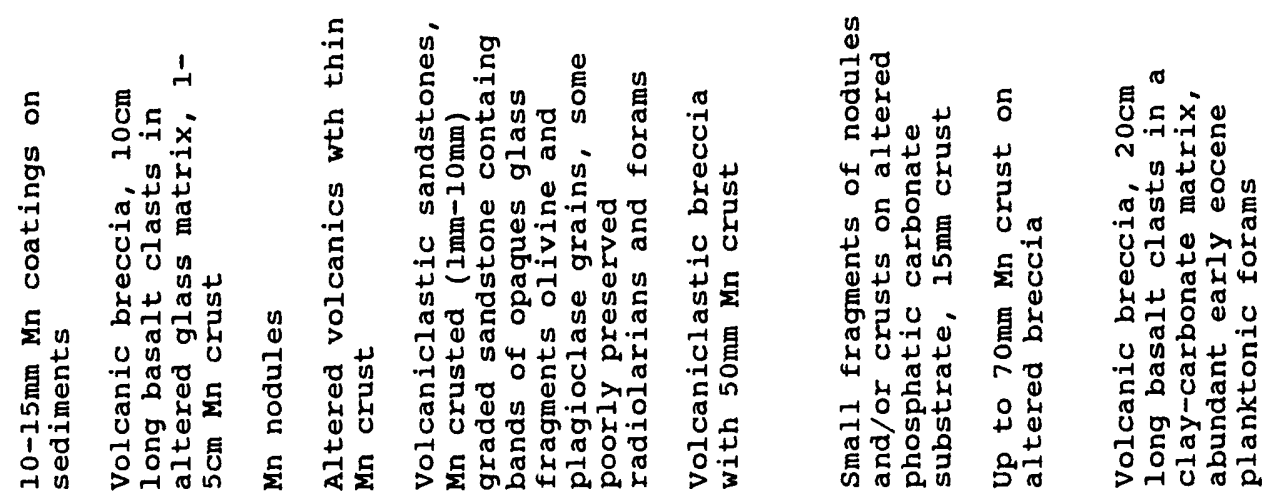

$\begin{array}{lllll}0 & 0 & 0 & 0 & 0 \\ H & y & H & H\end{array}$

㝵䓌导

$\underset{H}{U}$

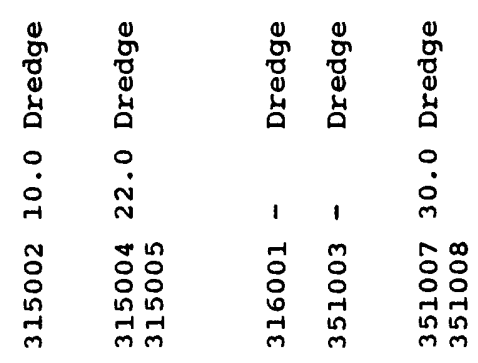

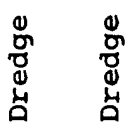

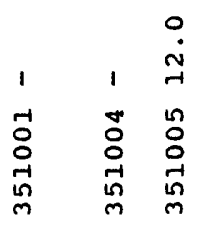

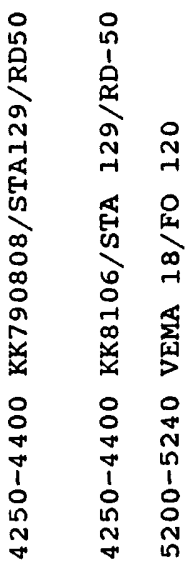

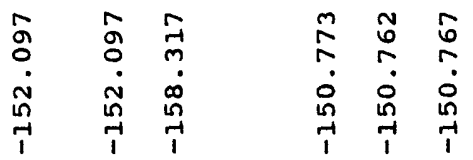

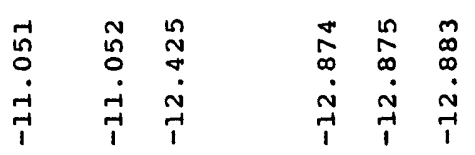

0.0000

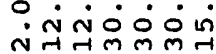

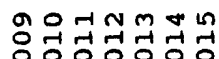
南 ๓ก๊

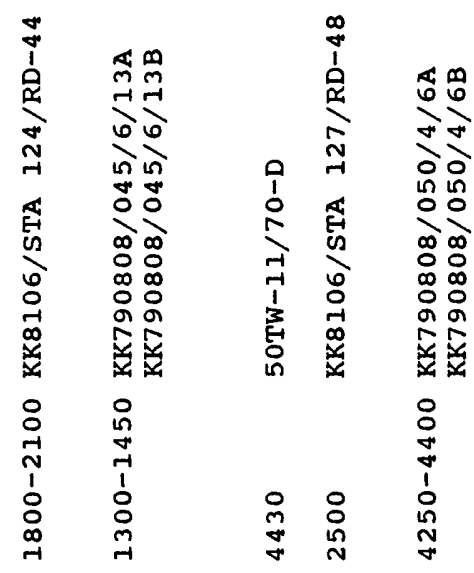

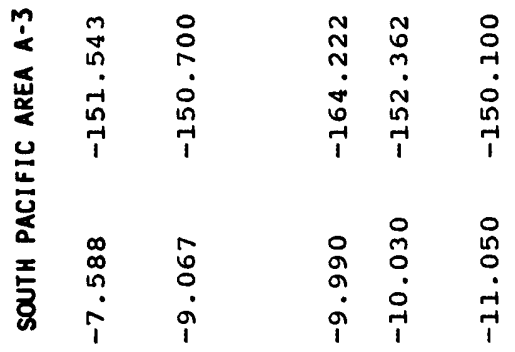

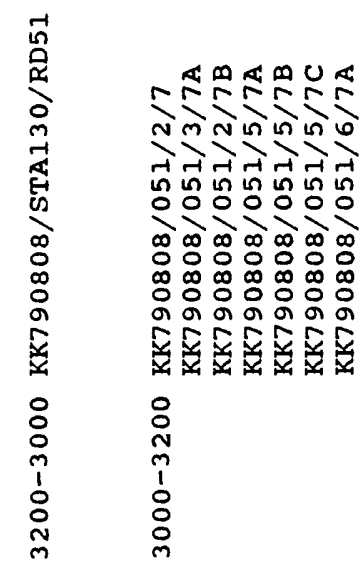




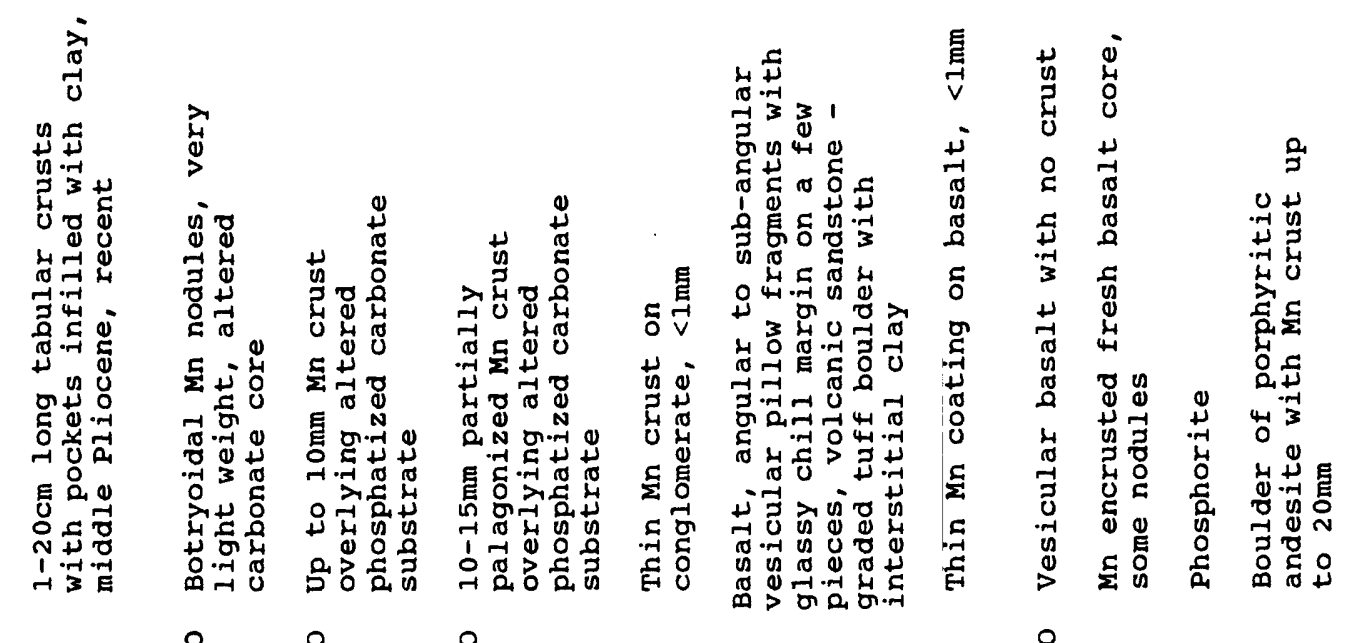

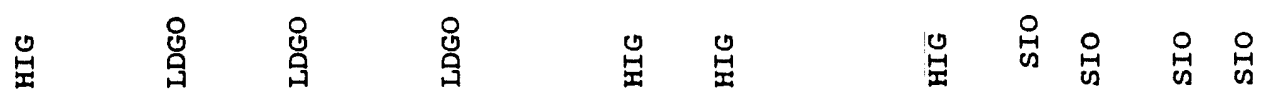

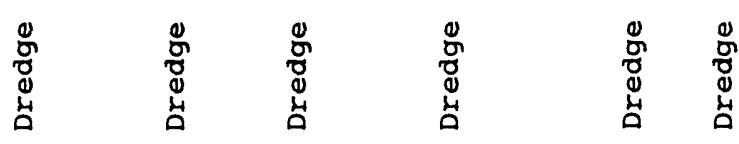

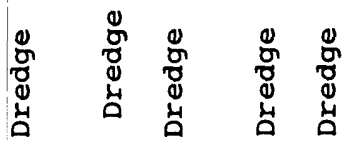

$\dot{0} \dot{0} \dot{0} \dot{0} \dot{0} \stackrel{\dot{y}}{\stackrel{m}{m}} \dot{m}$

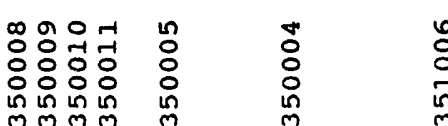

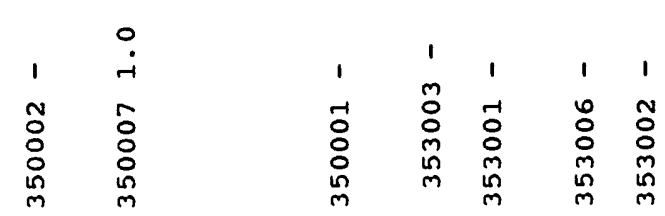

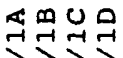

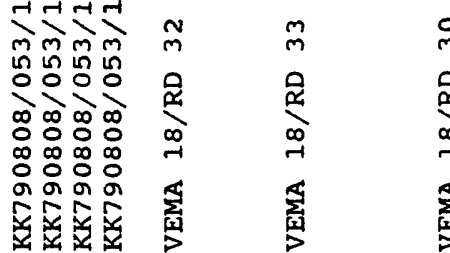

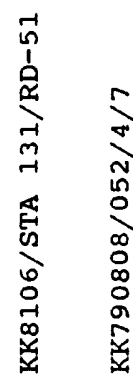

㖞

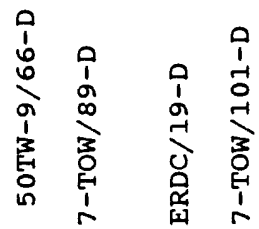

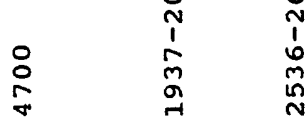

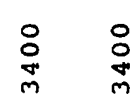

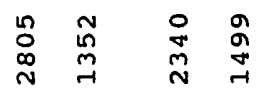

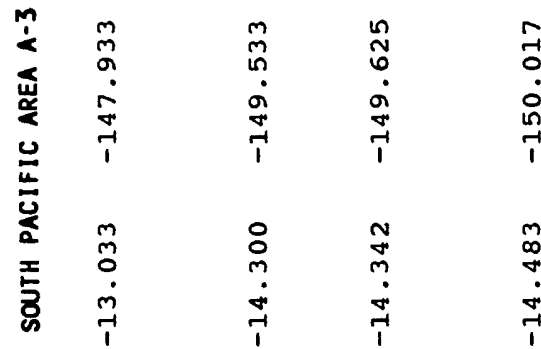

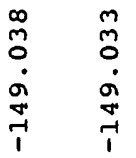

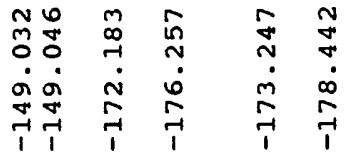

\begin{tabular}{ll}
$m$ & \multirow{2}{7}{} \\
0 & 0 \\
$\dot{1}$ & $\dot{n}$ \\
1 & 1
\end{tabular}

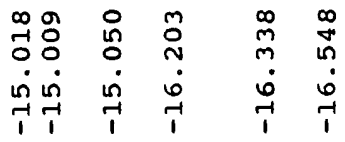




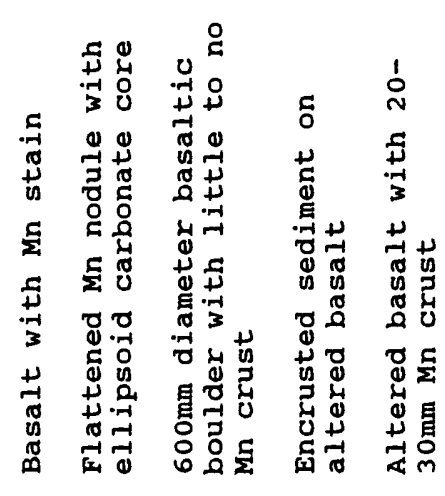

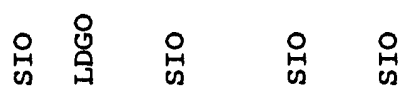

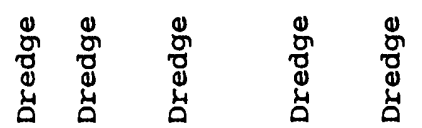

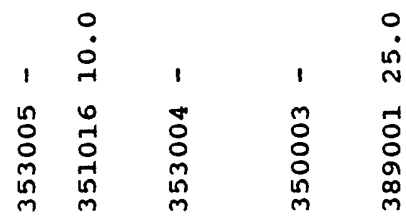

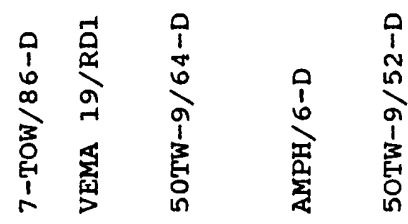

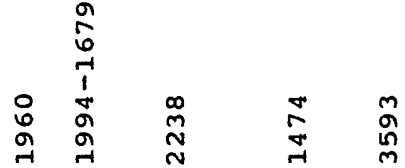

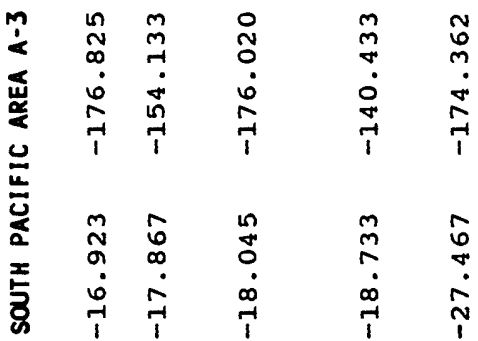




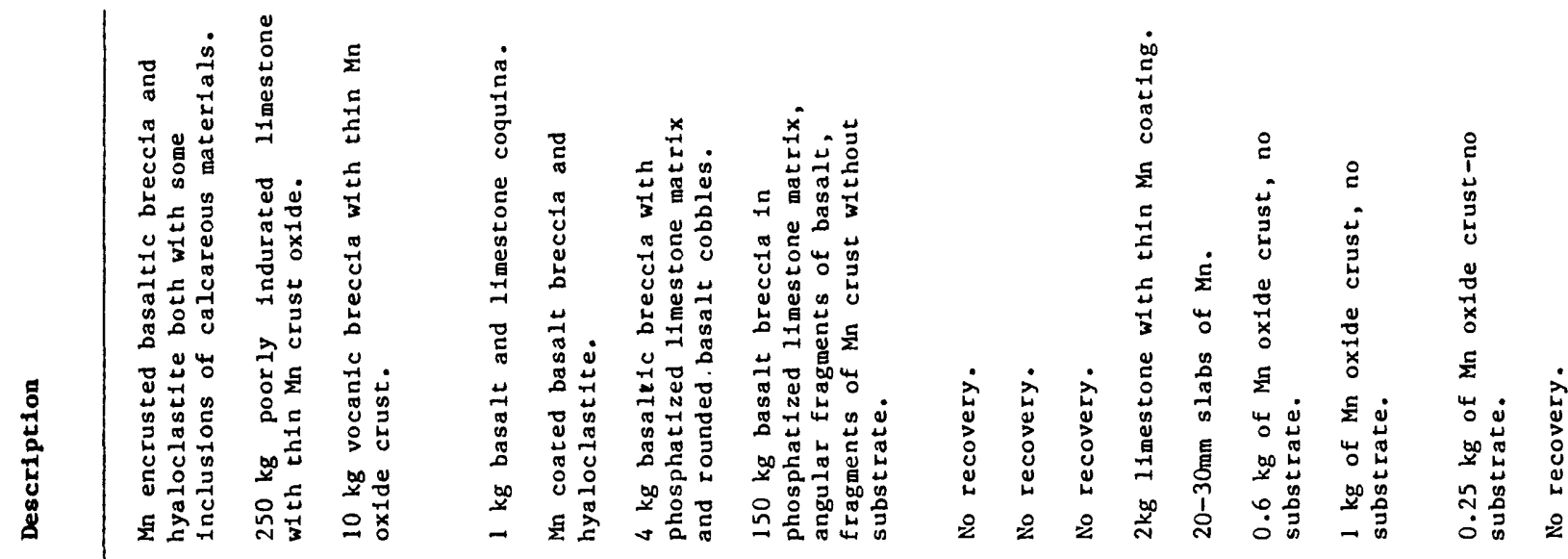

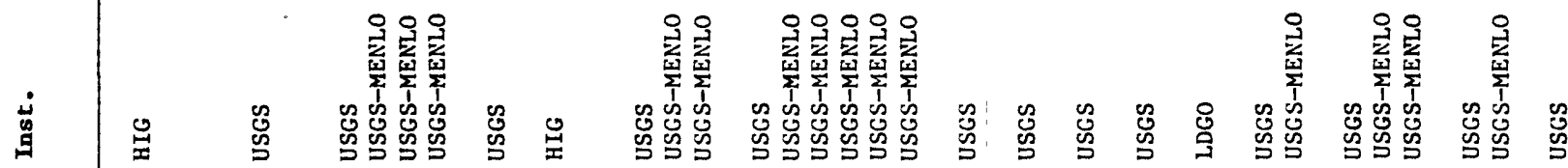

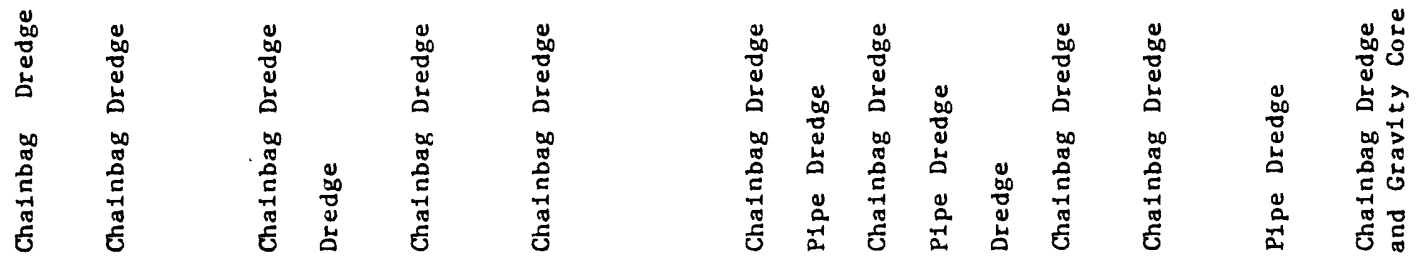

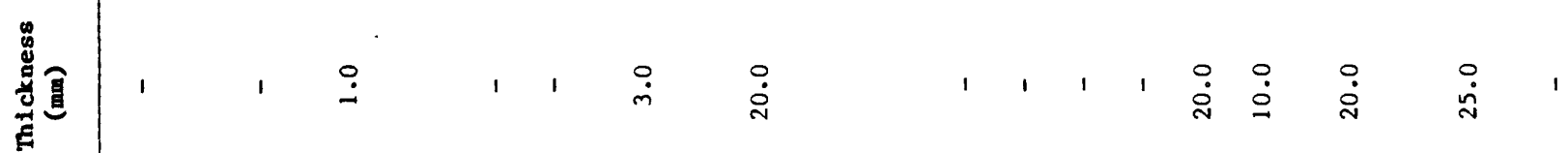

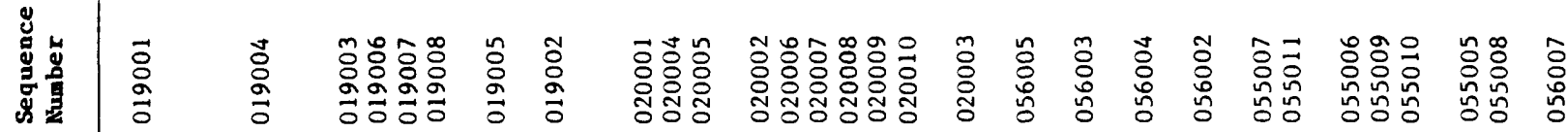

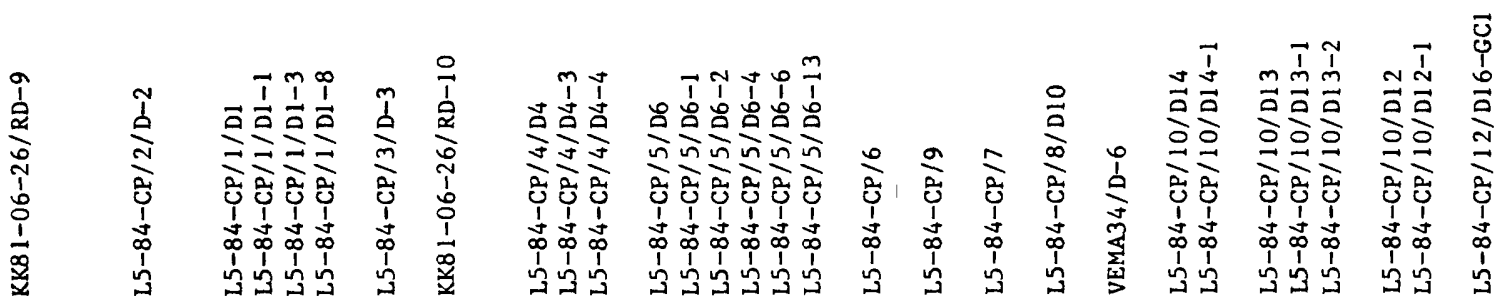

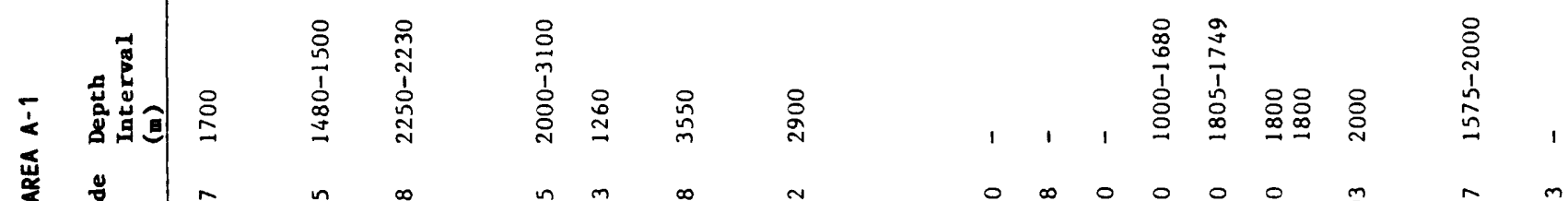

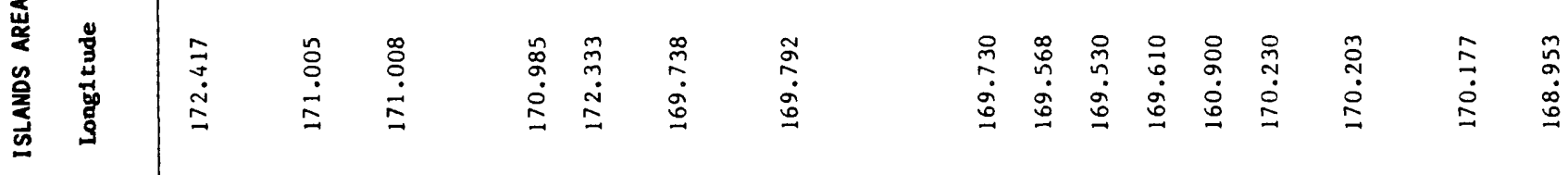

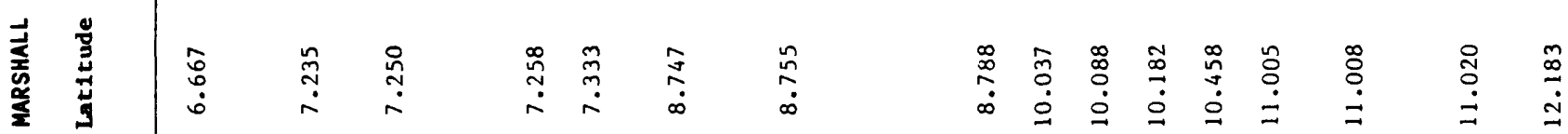




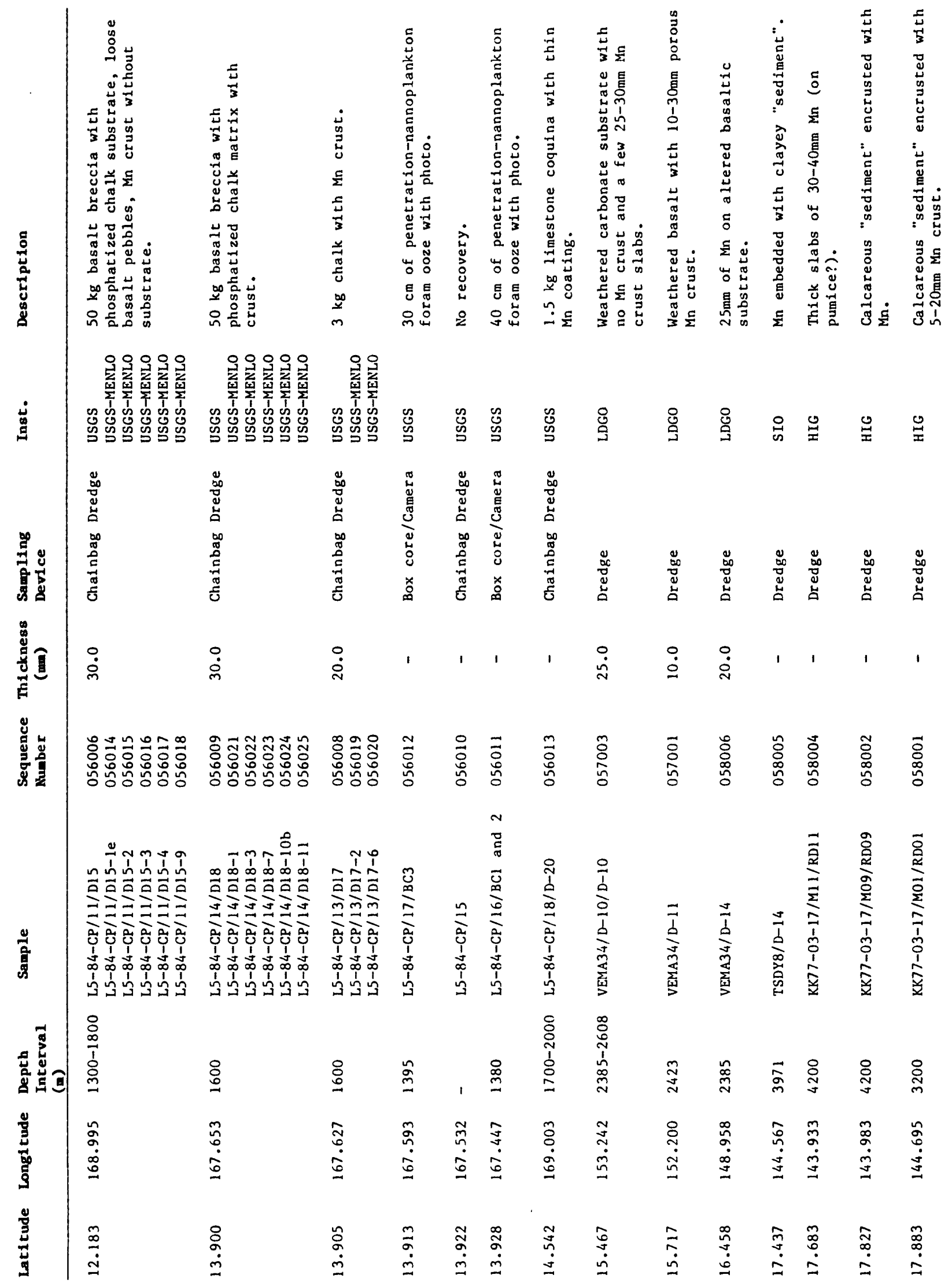




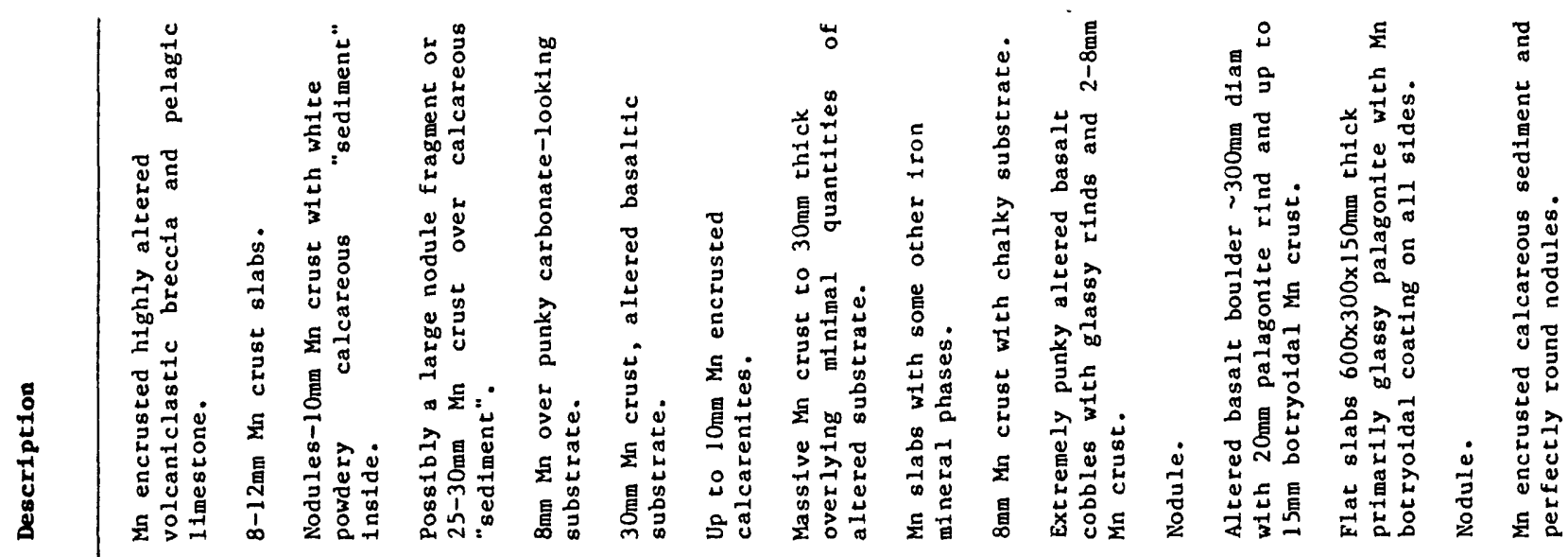

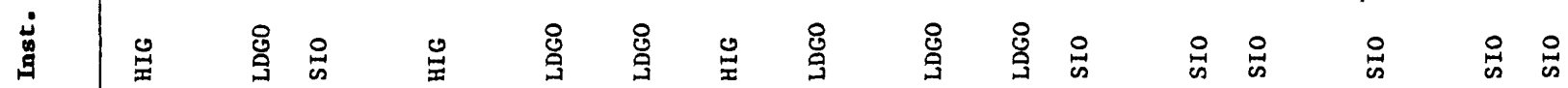

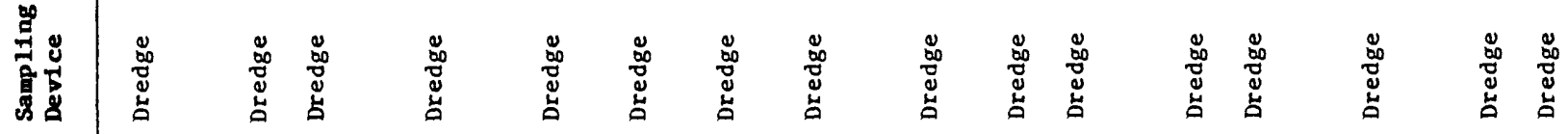

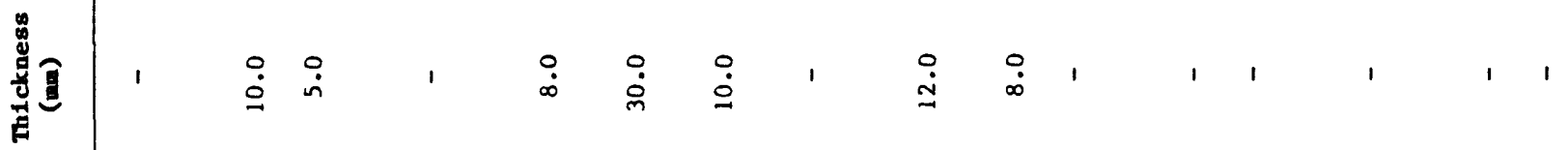

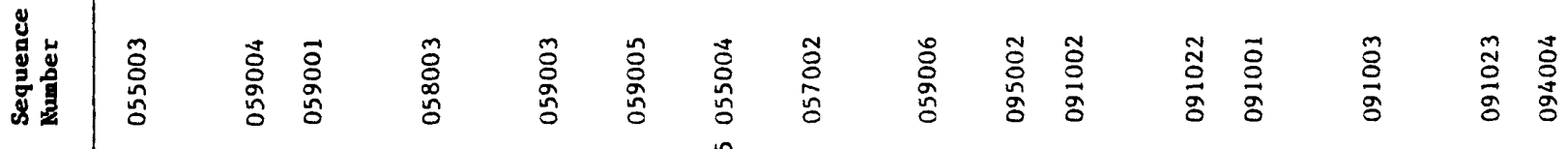

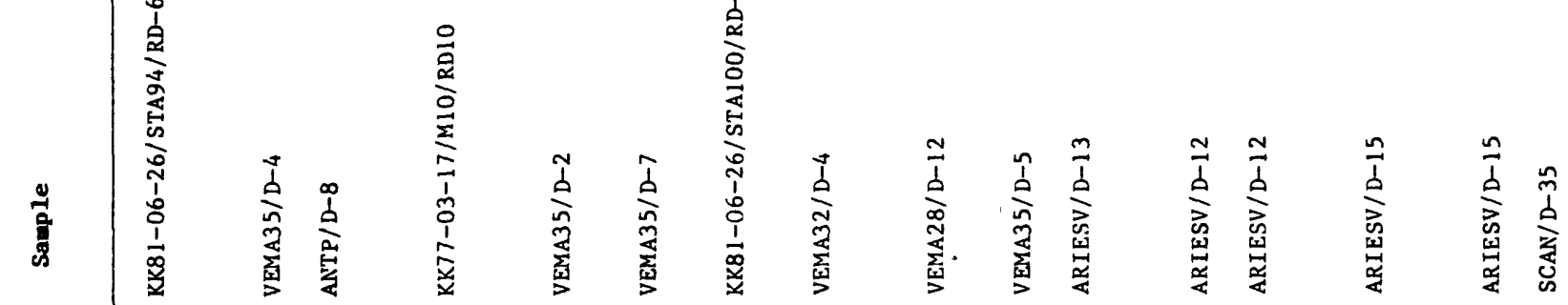

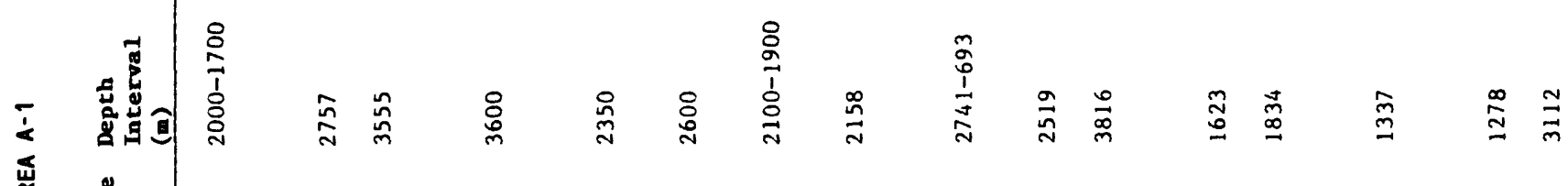

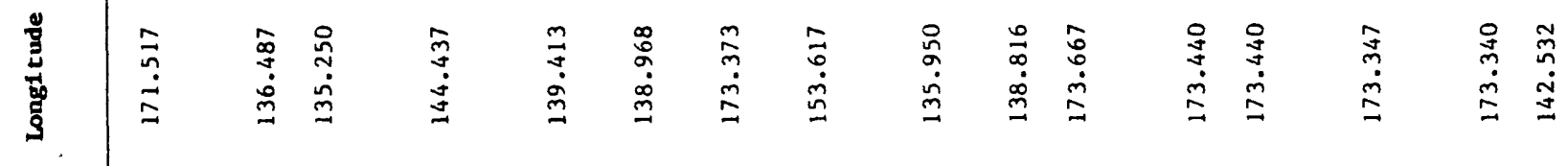

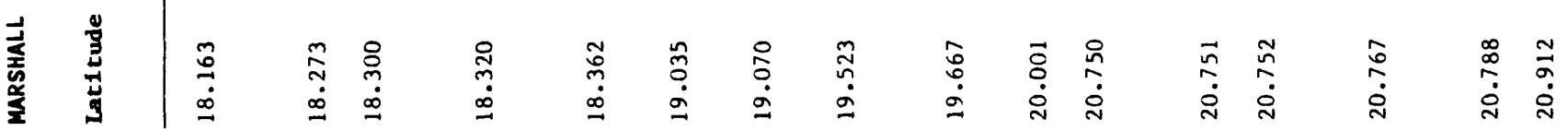




$$
\begin{array}{llll}
\mid & \mid
\end{array}
$$




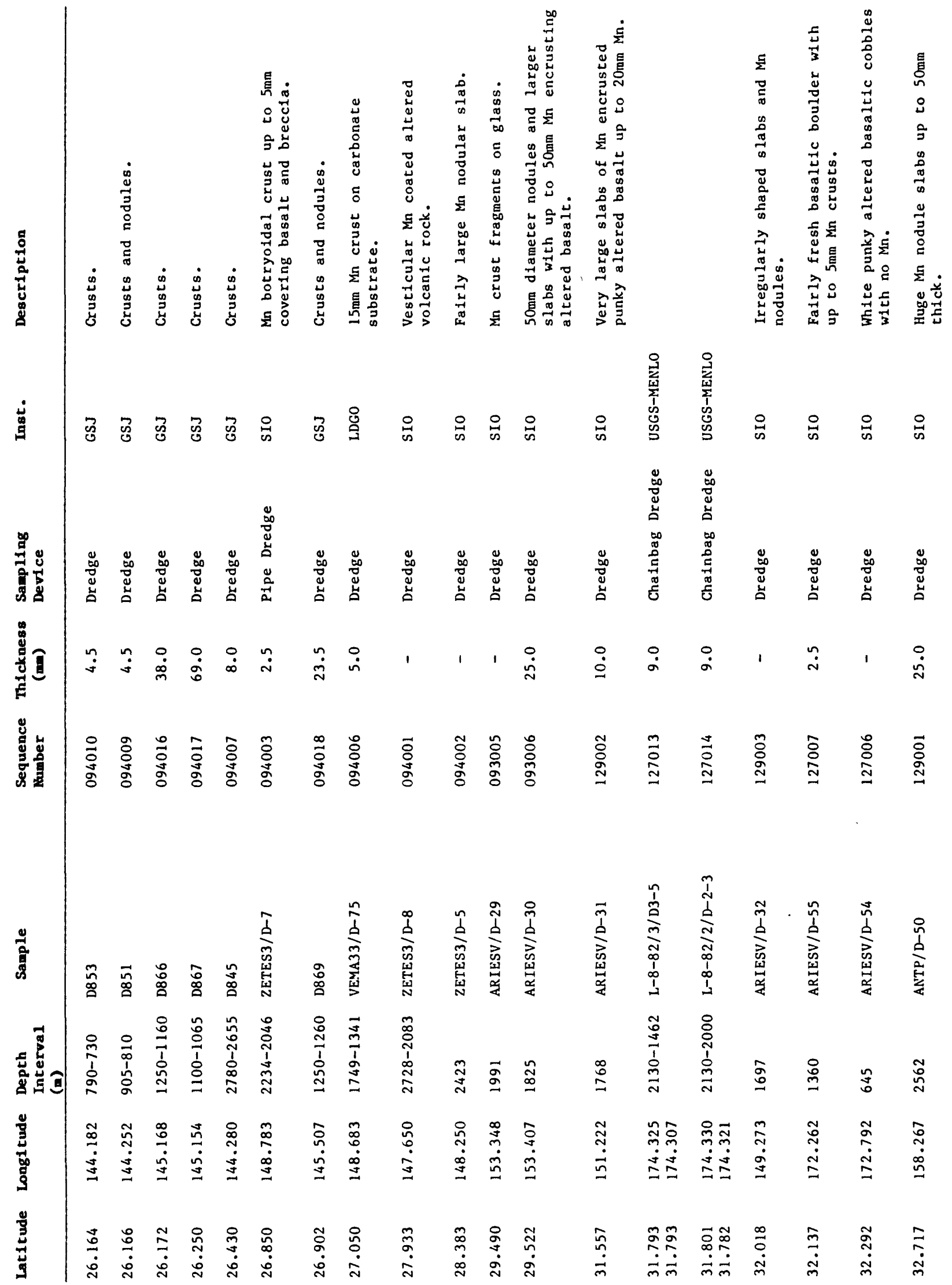




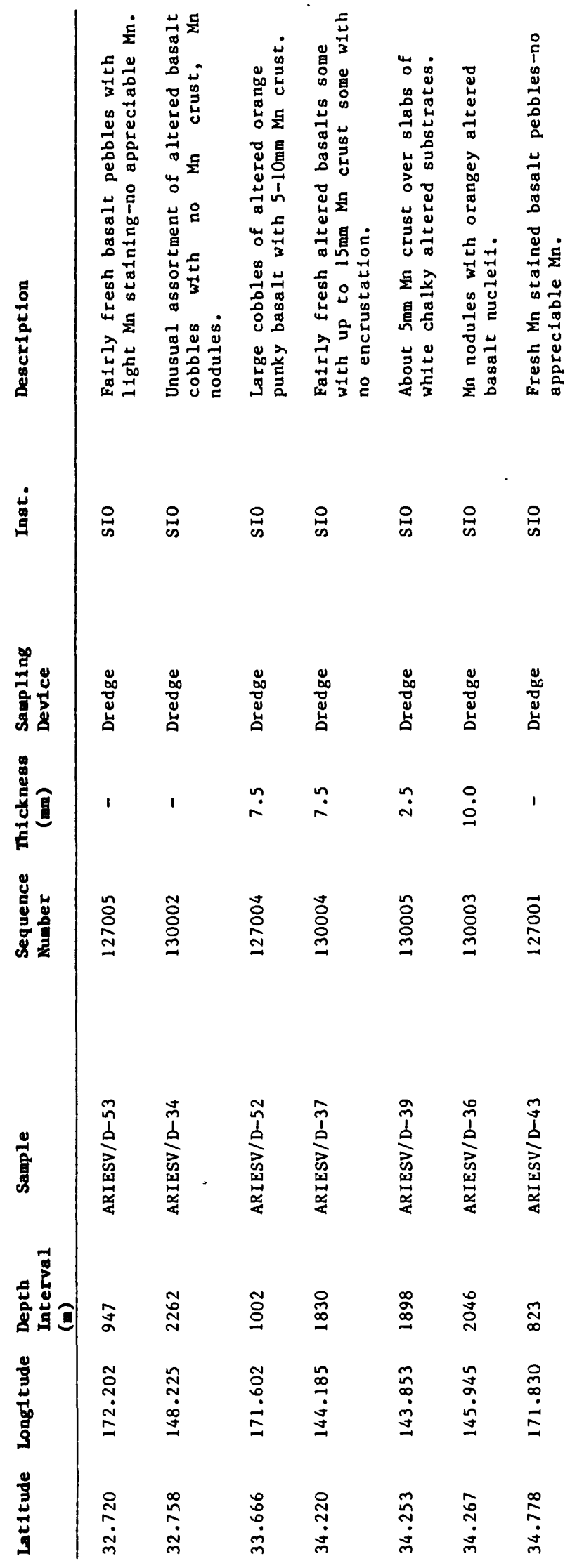




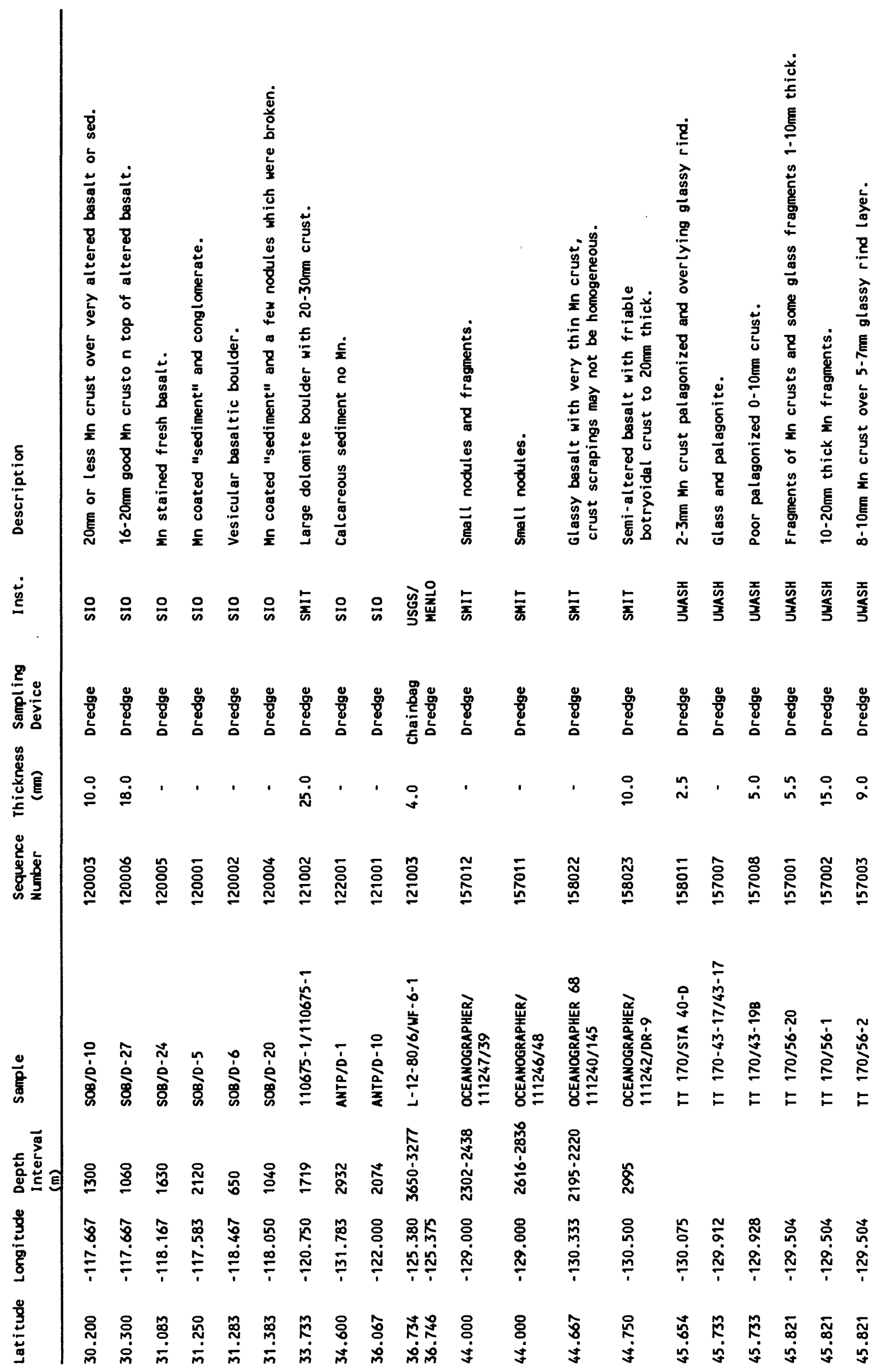




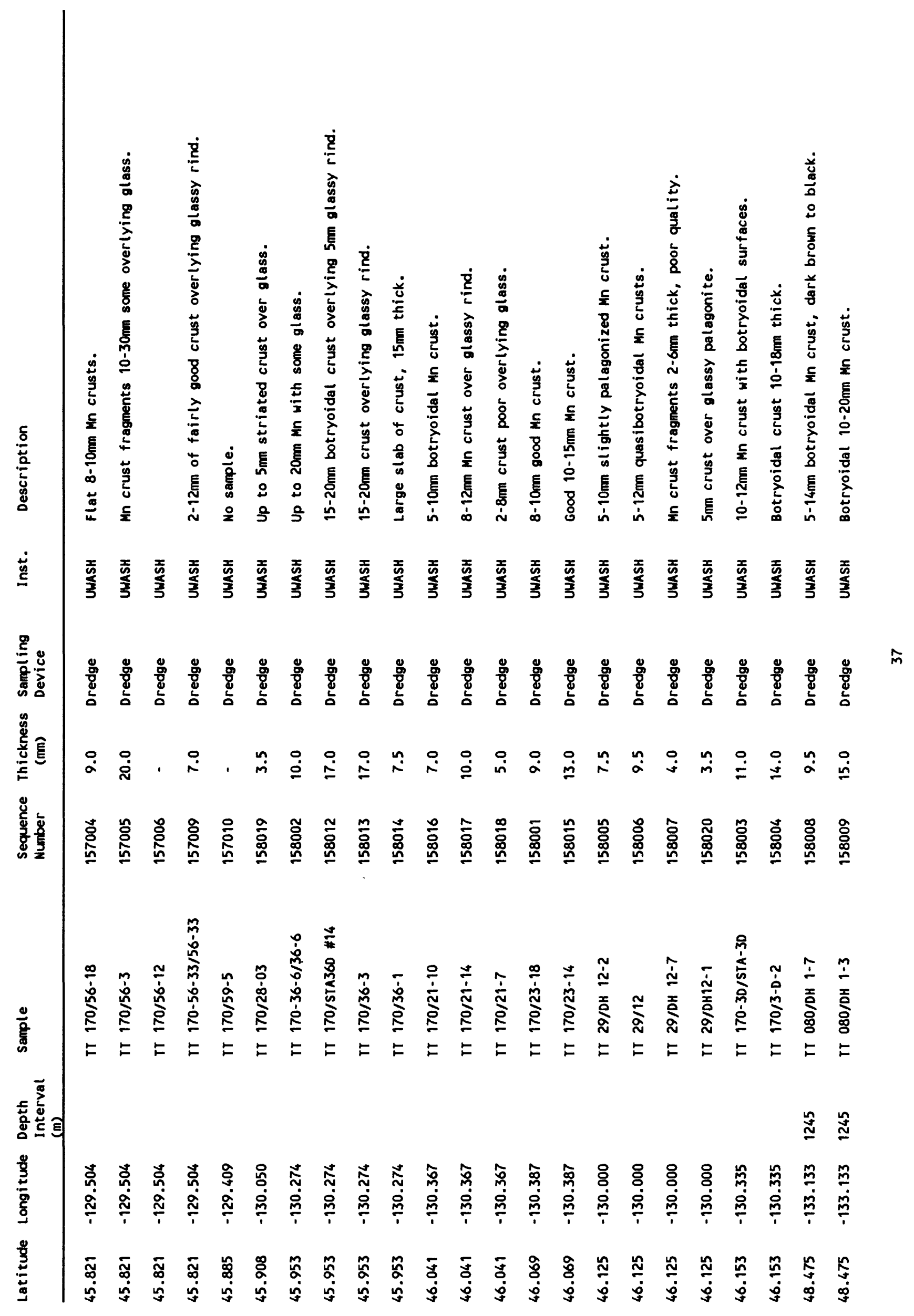




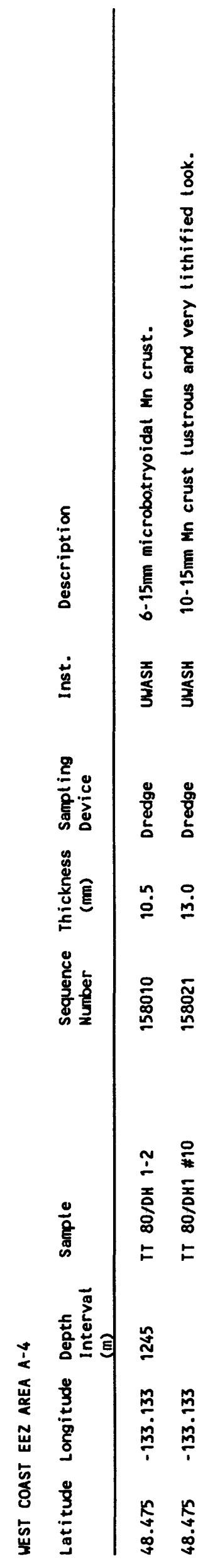




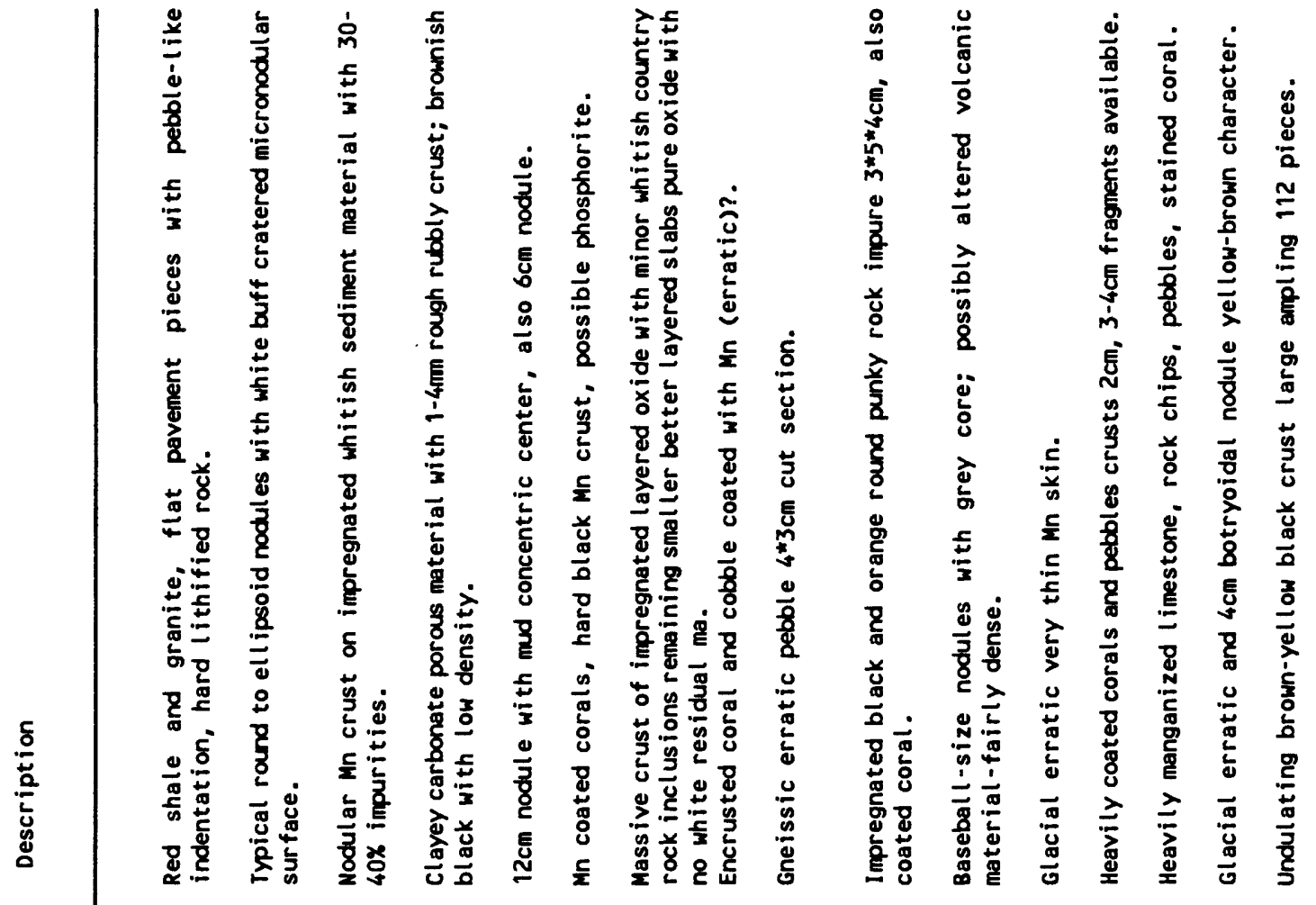

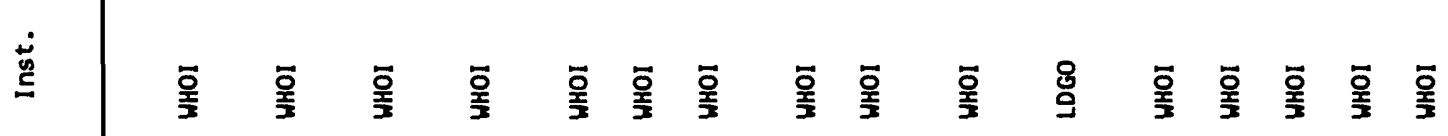

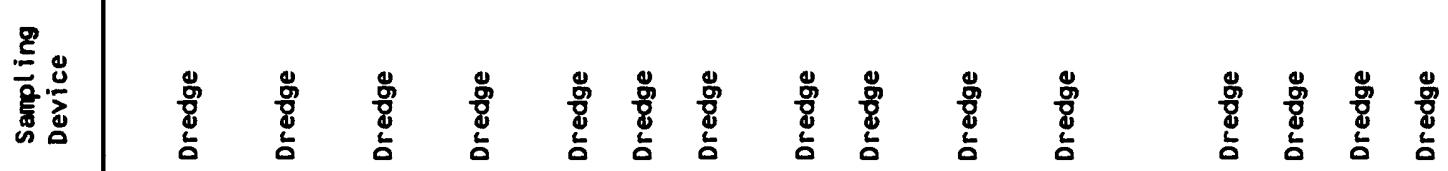

$$
\begin{aligned}
& \text { 总 }
\end{aligned}
$$

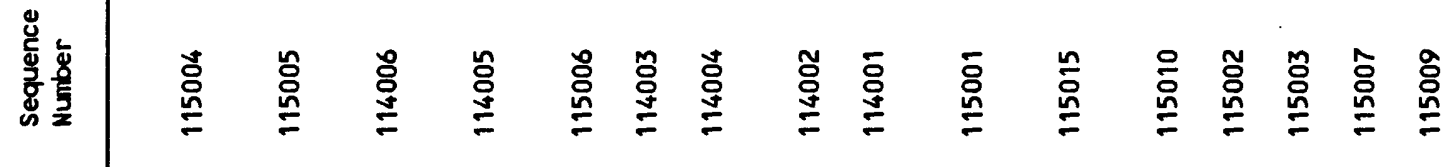

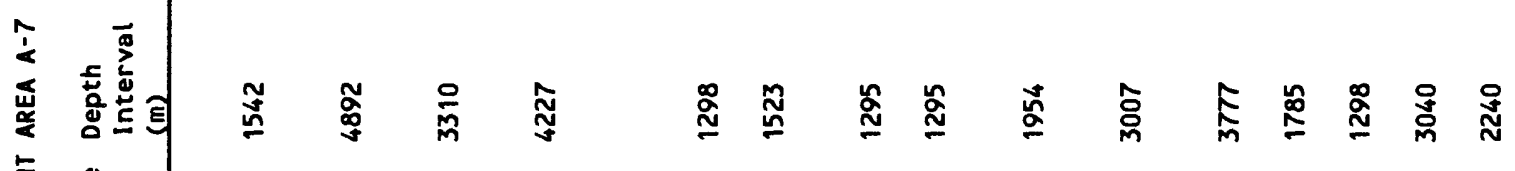

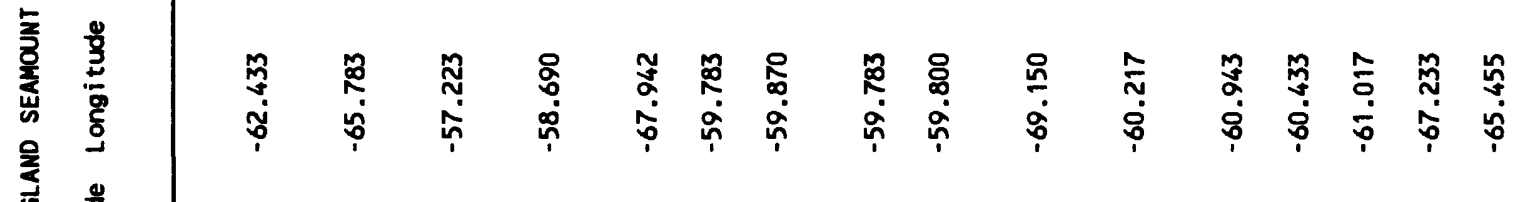

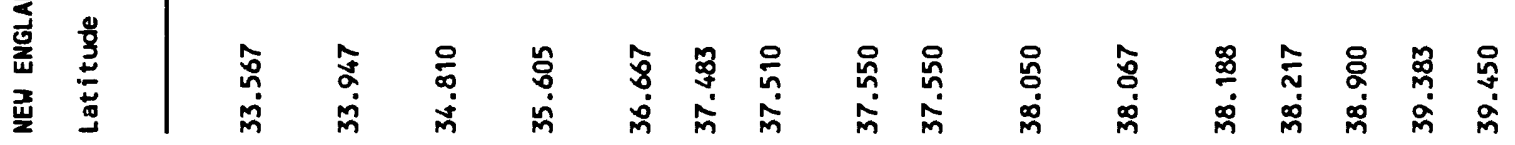




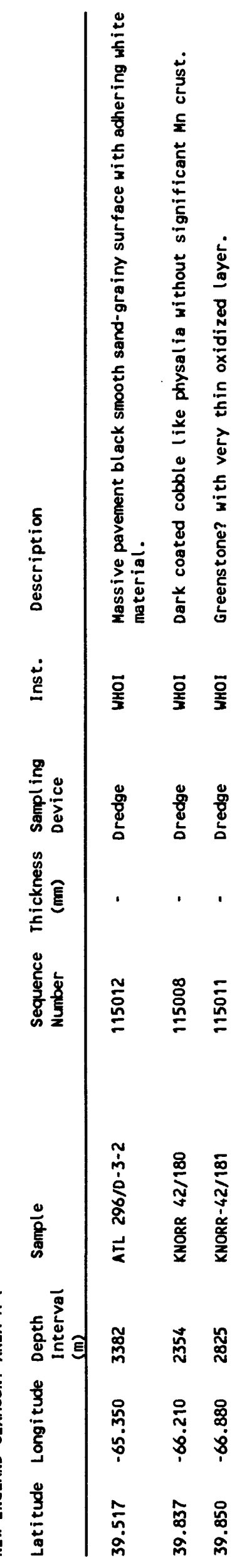




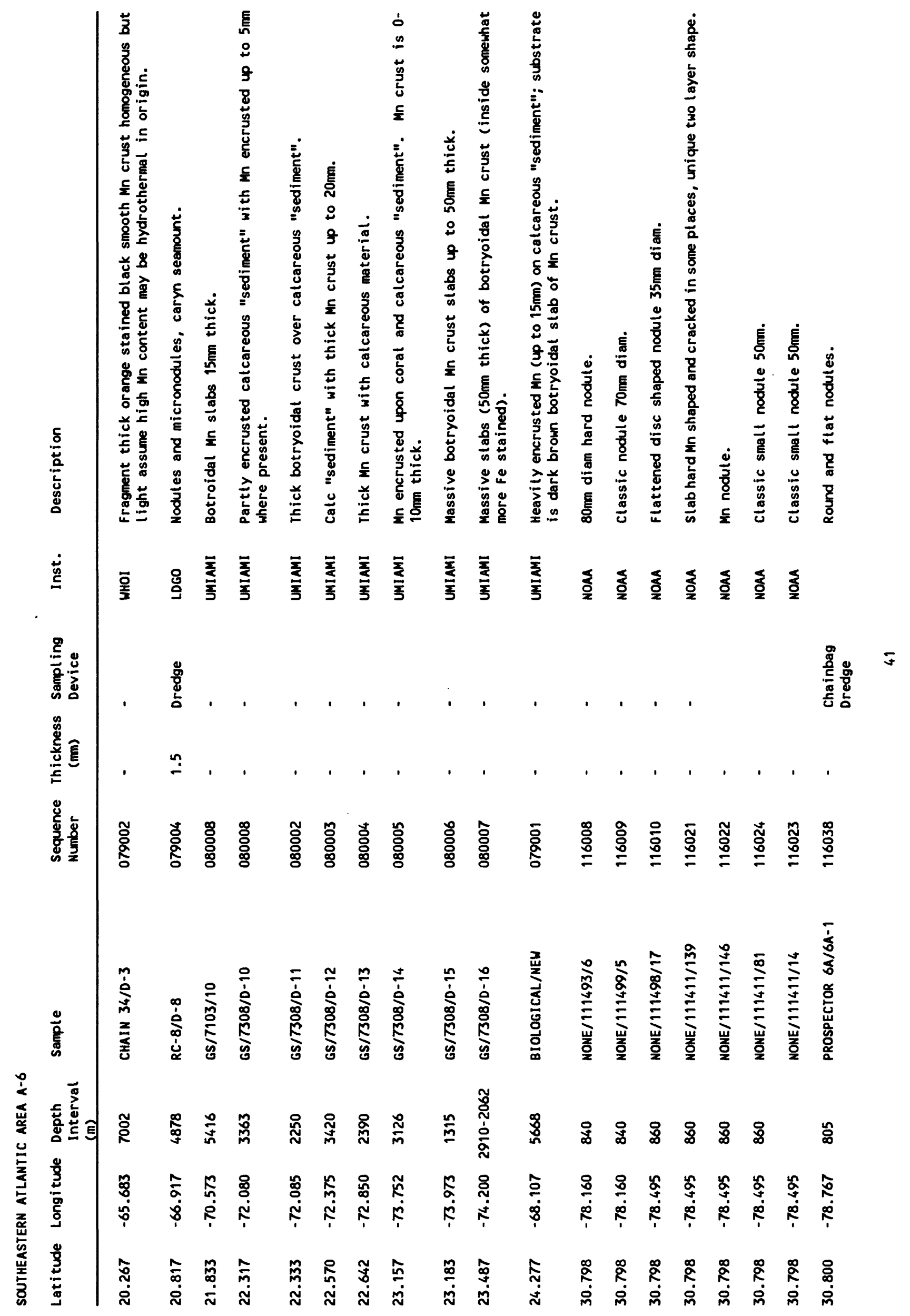




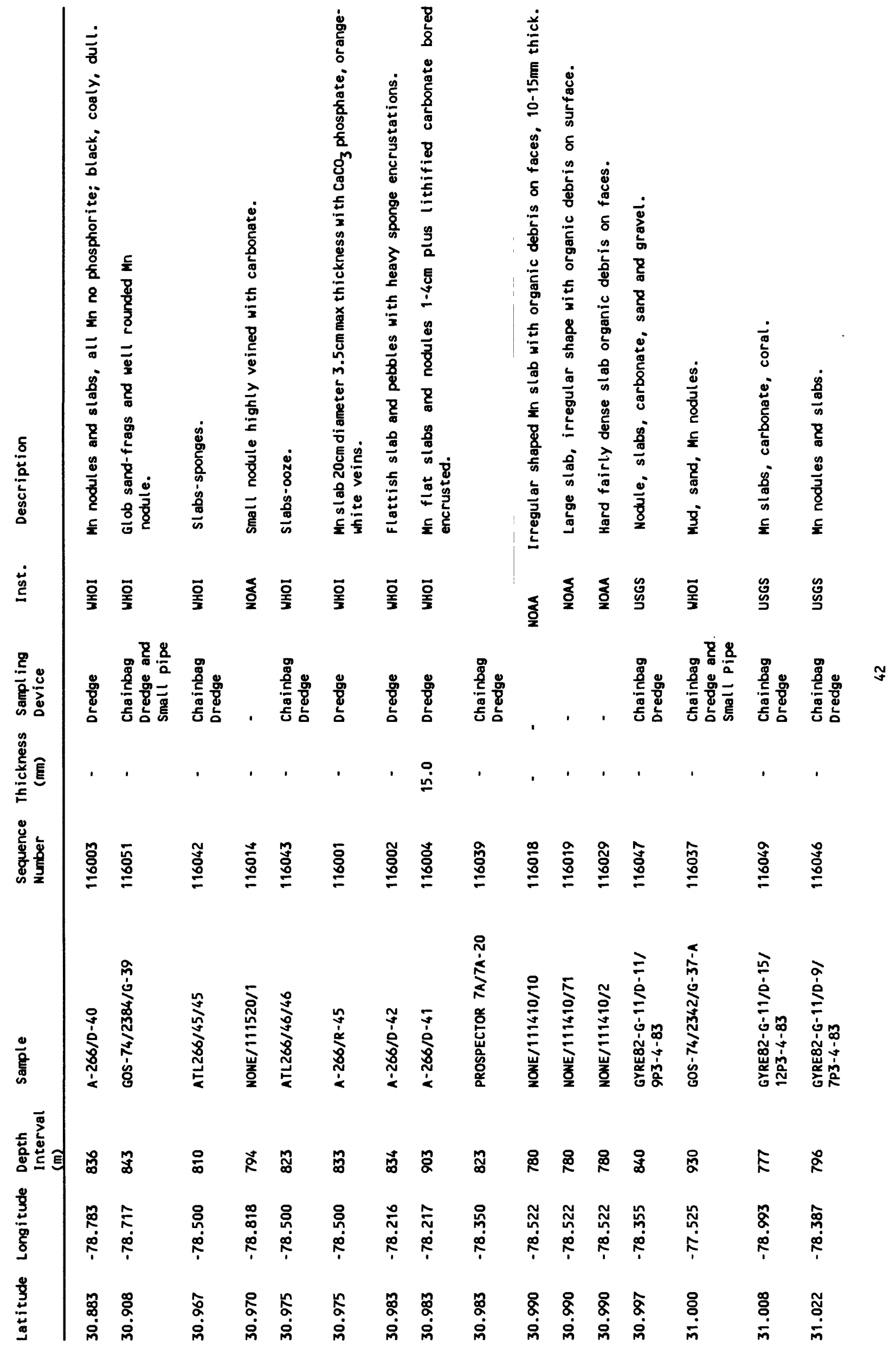




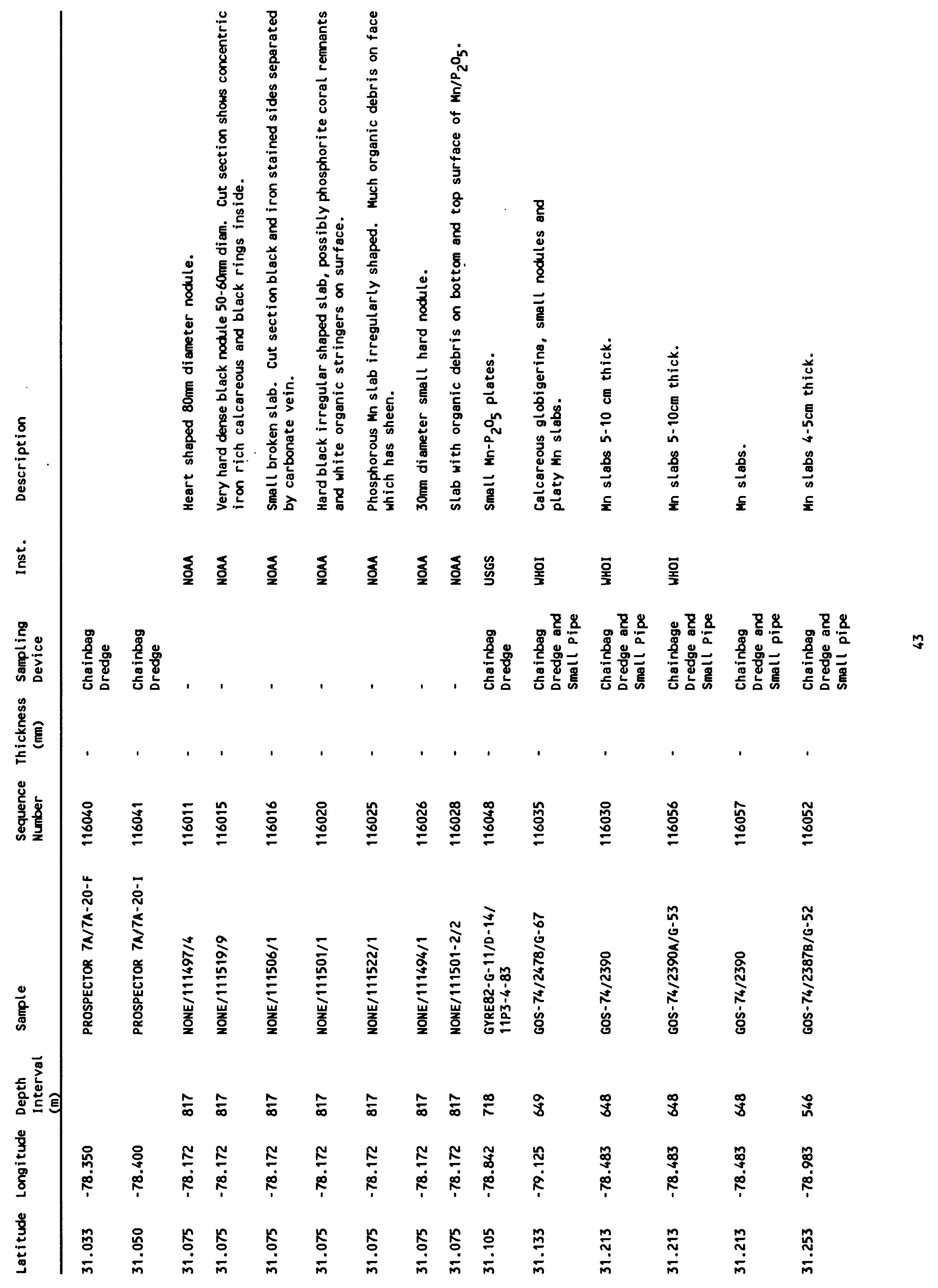




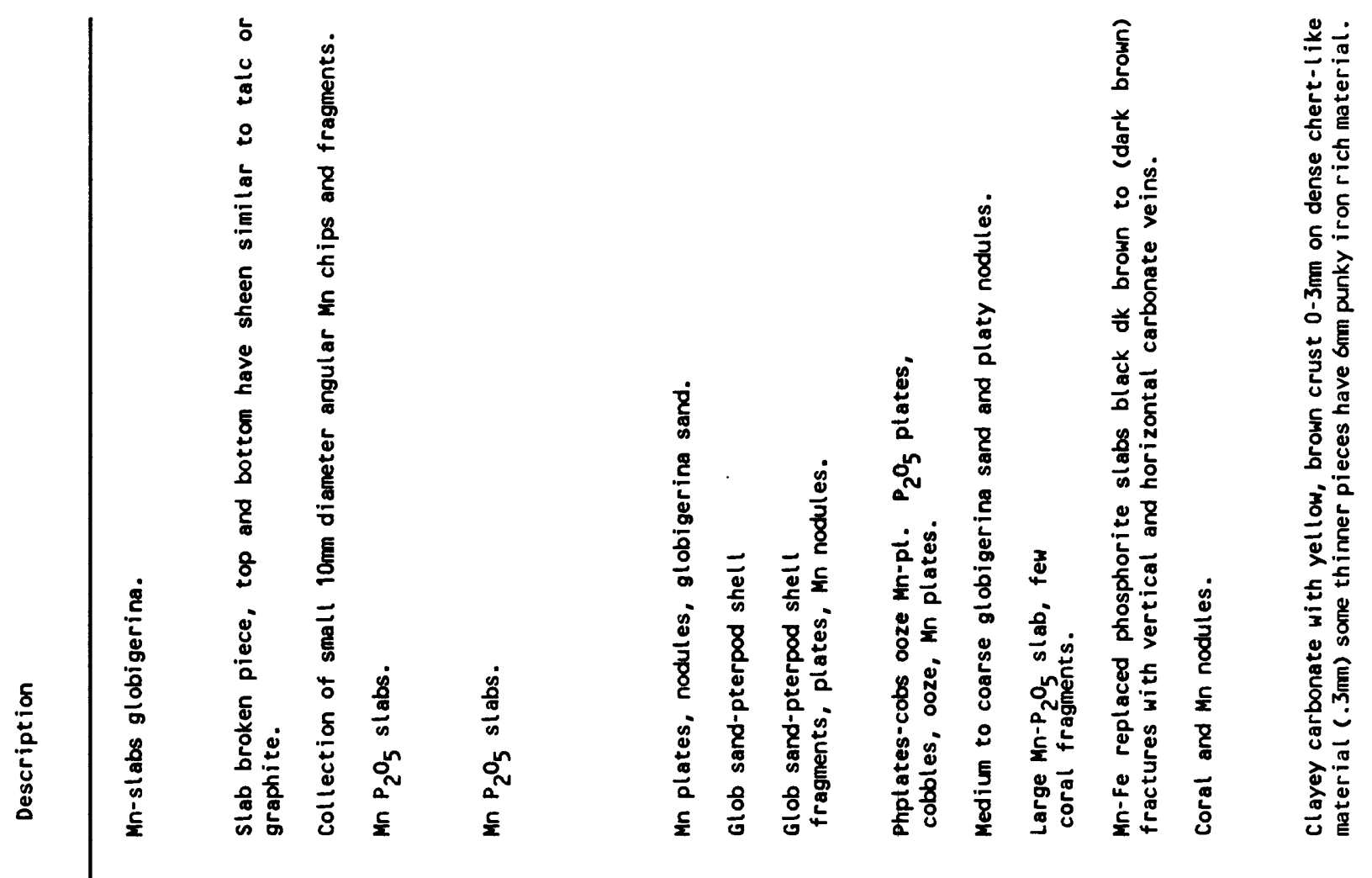

茴

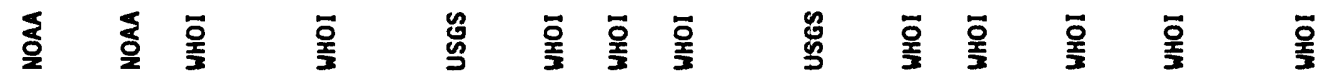

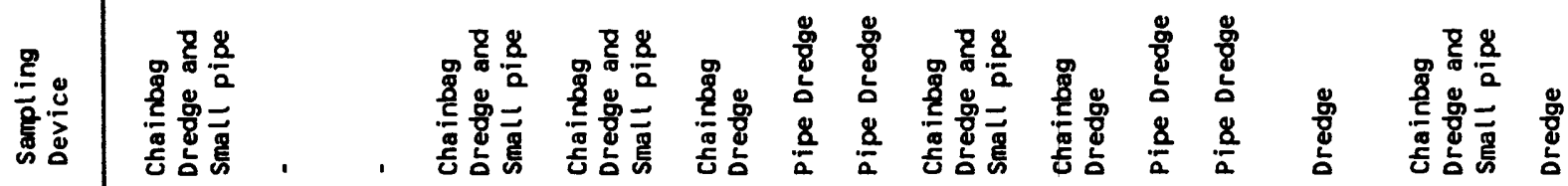

㪣

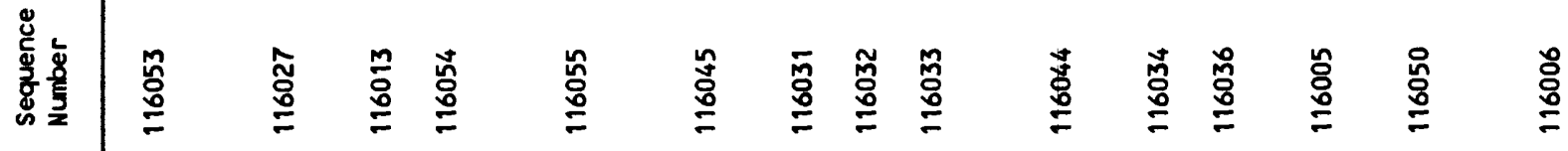

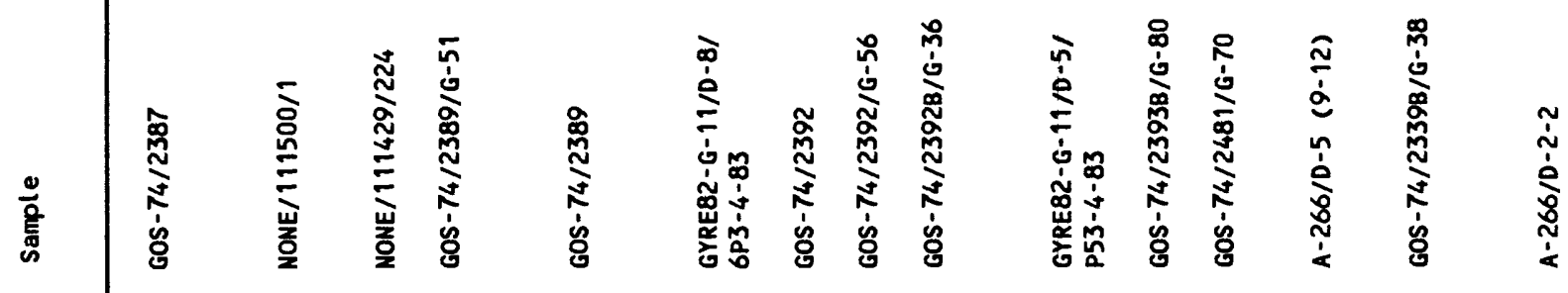

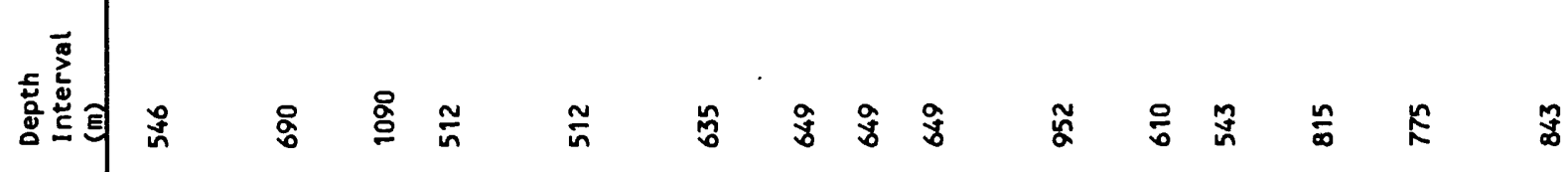

(1)

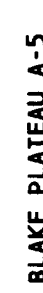

(1)

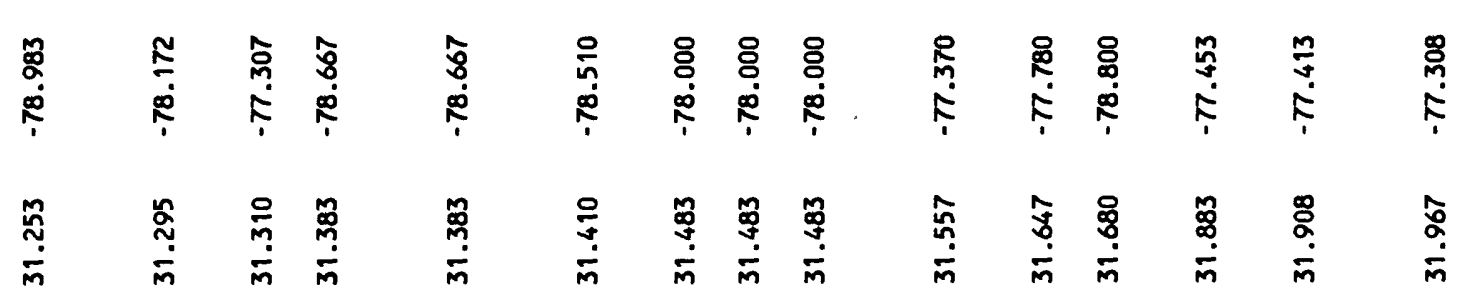




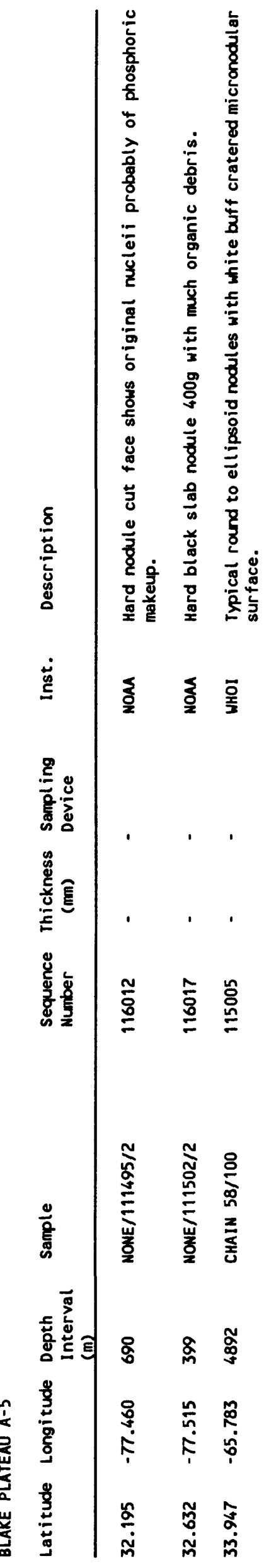




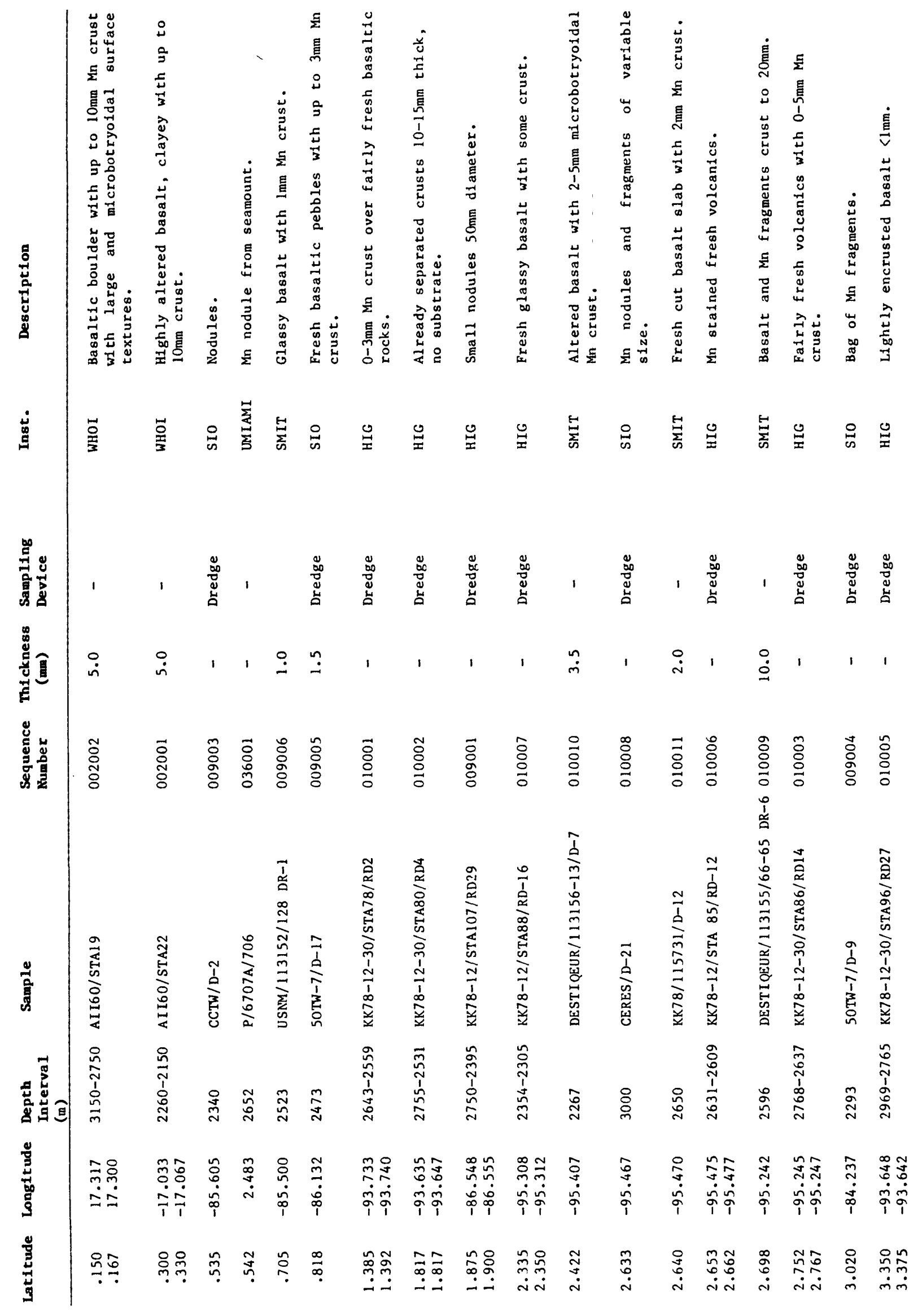




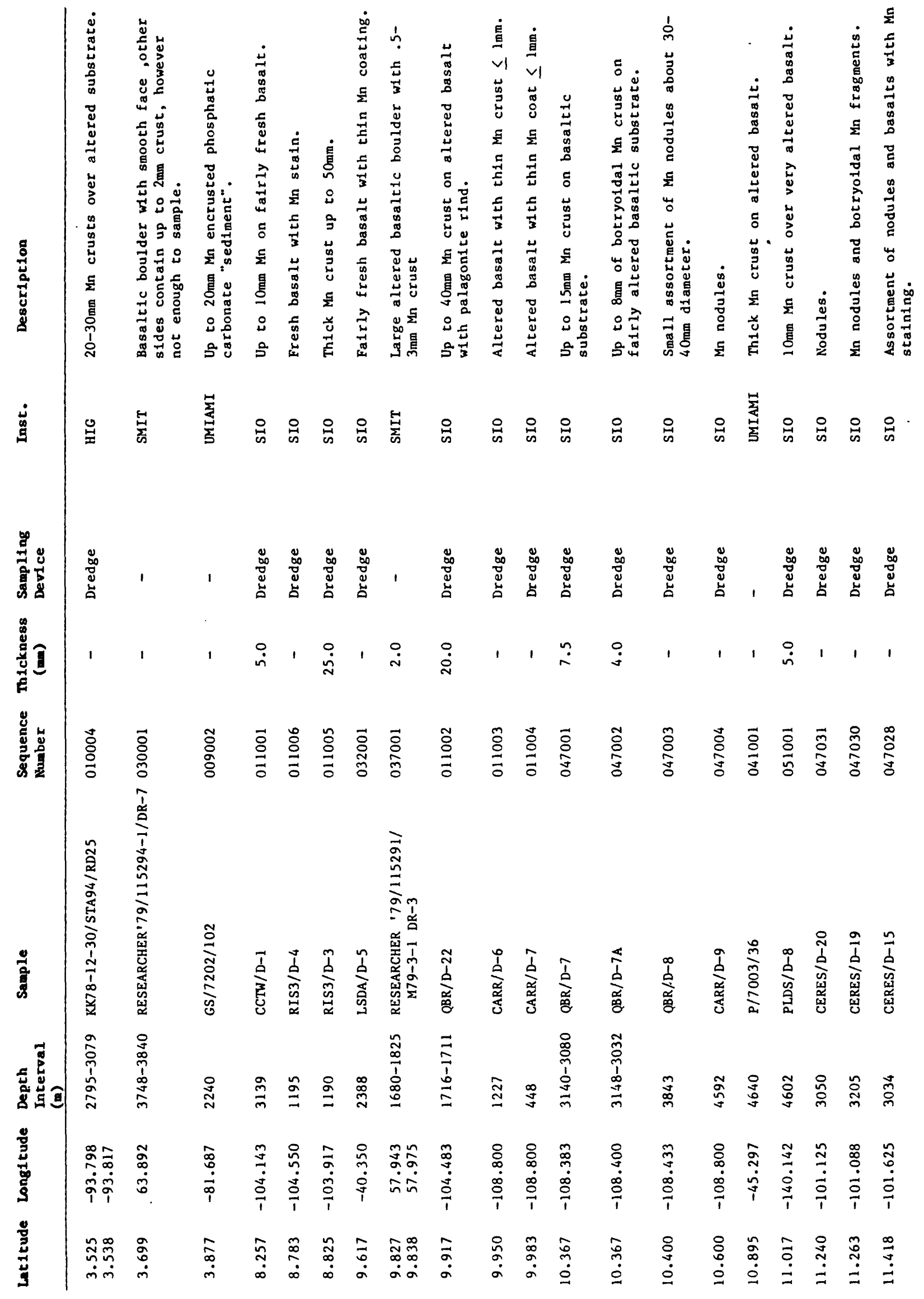




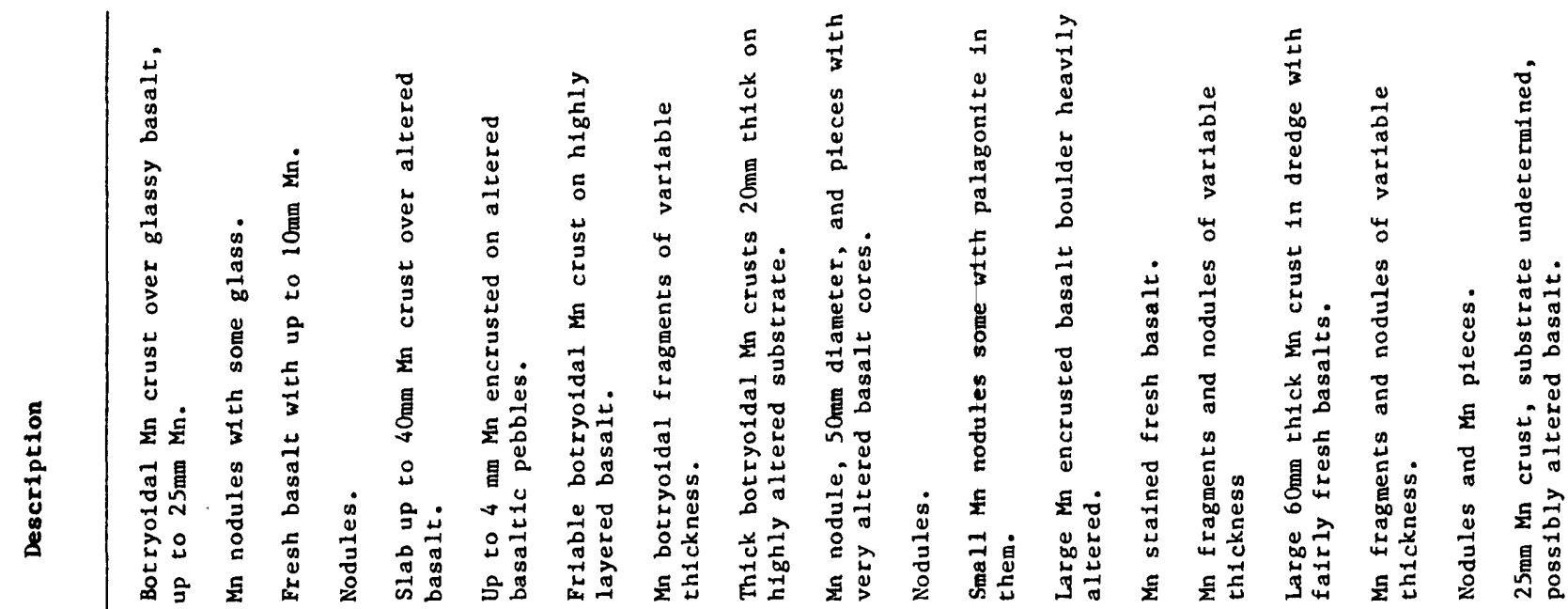

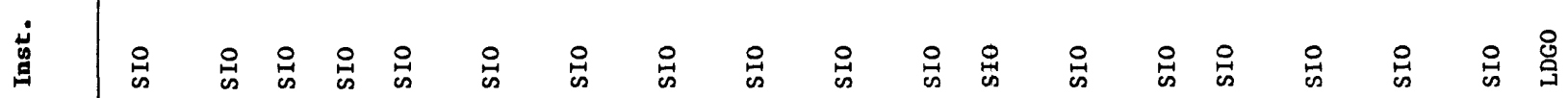

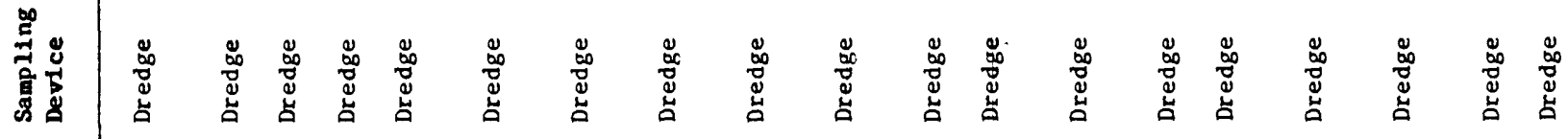

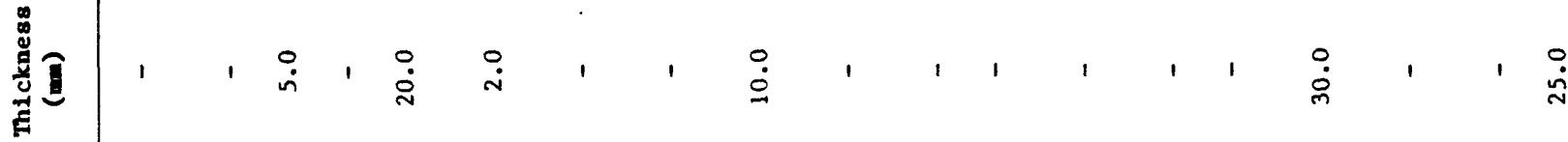

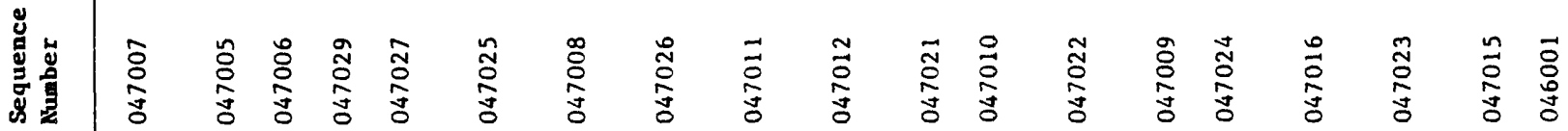

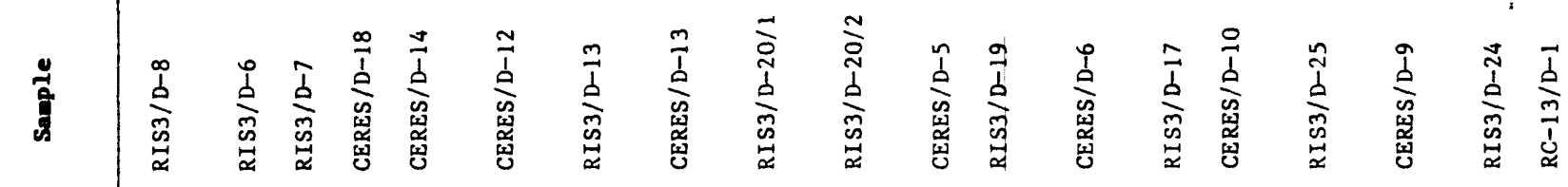

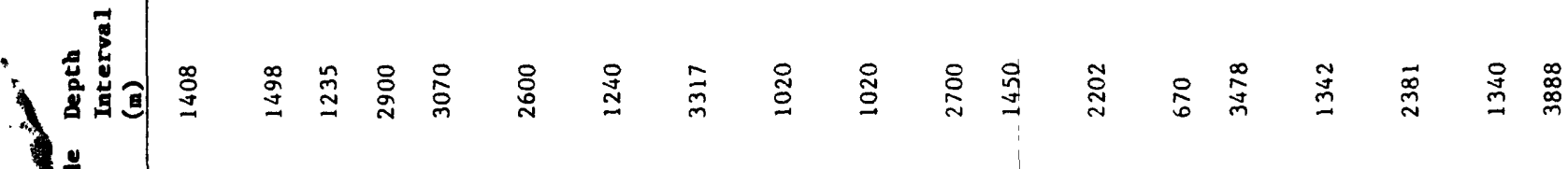

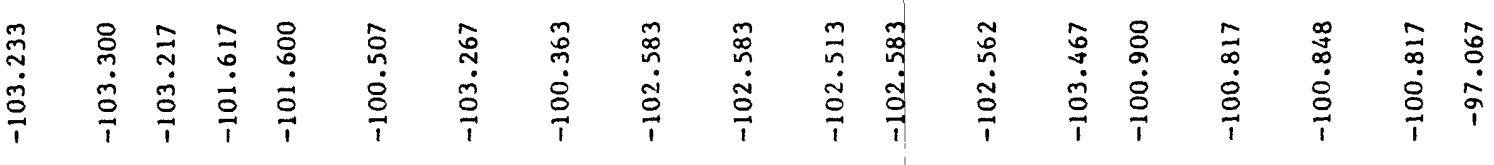
䍃

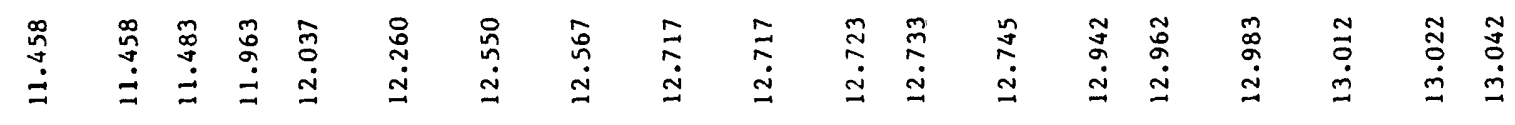




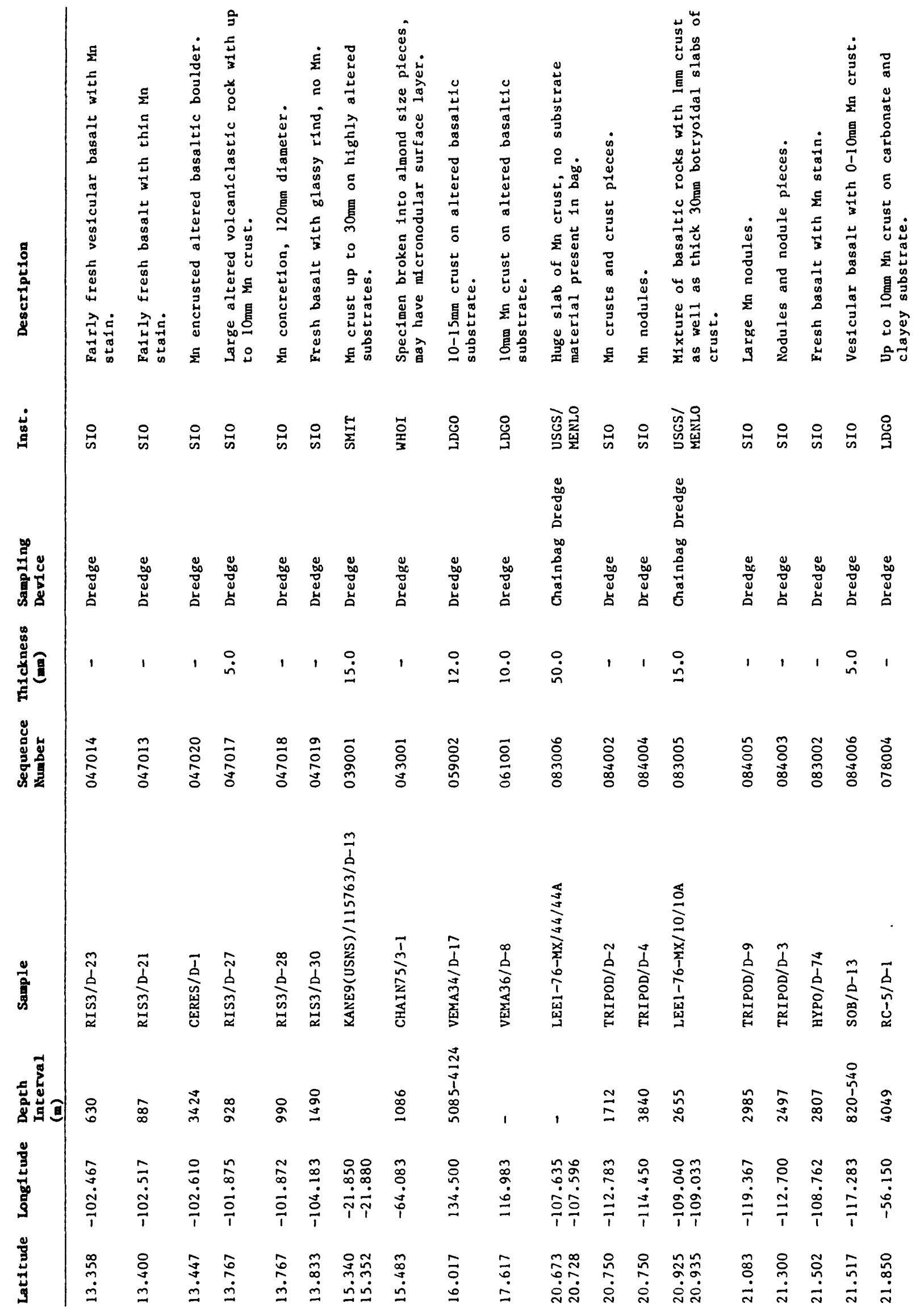




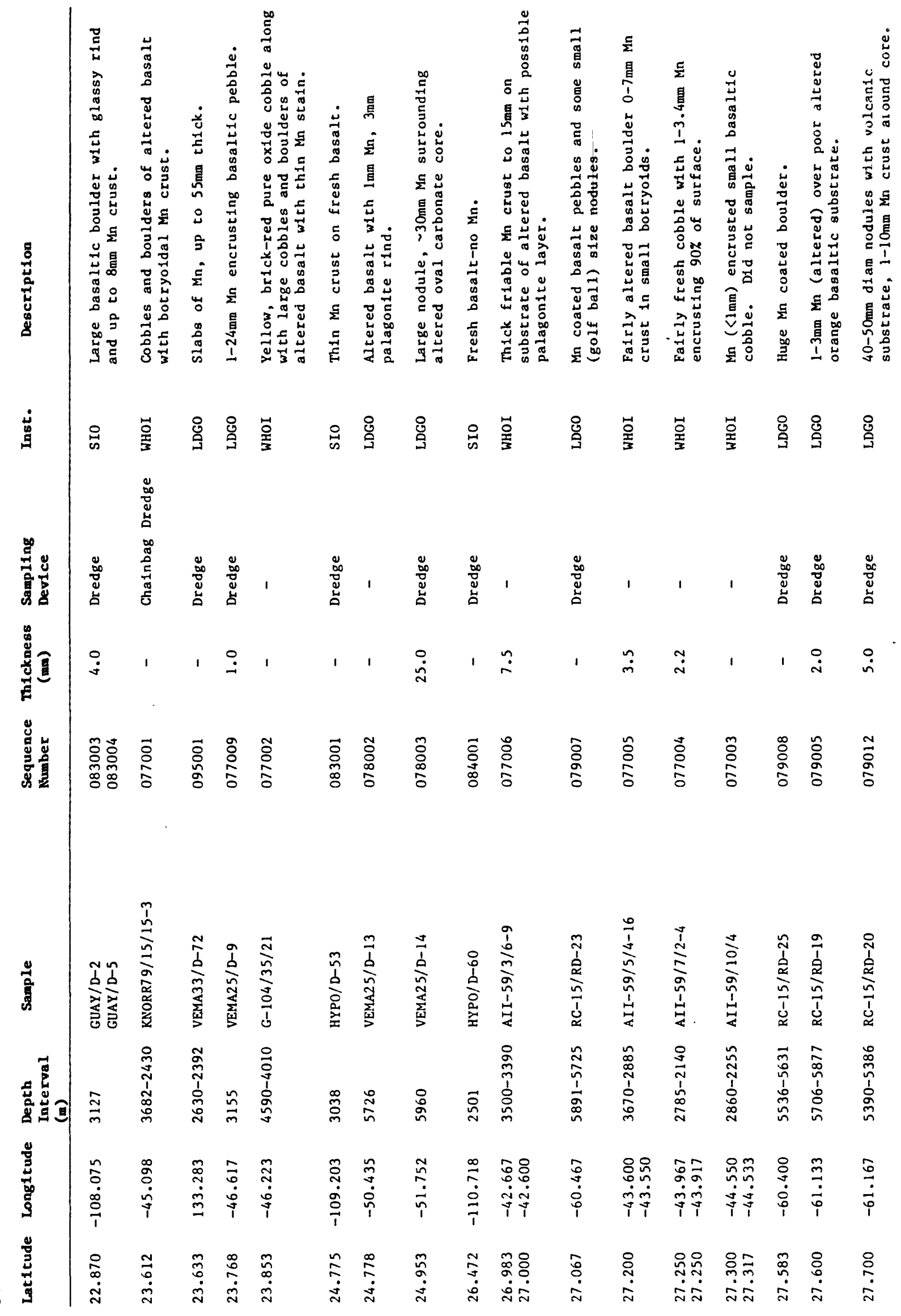




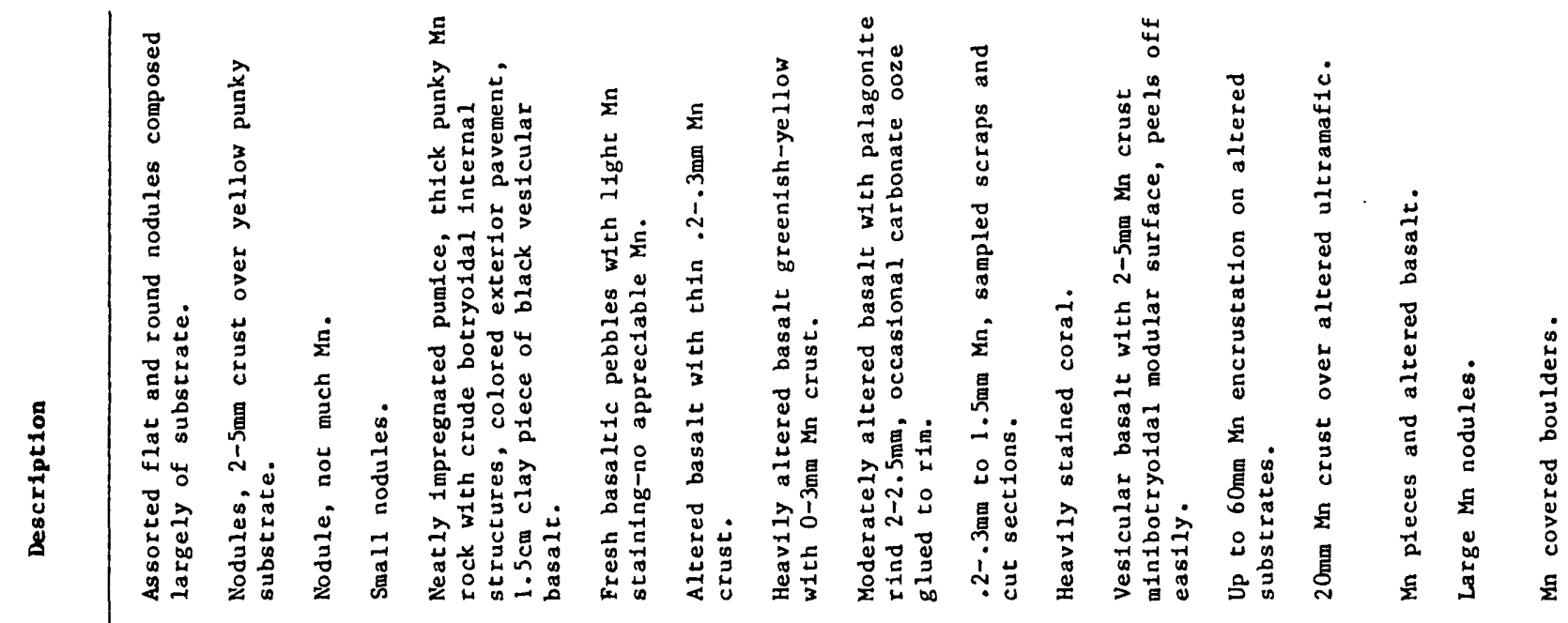

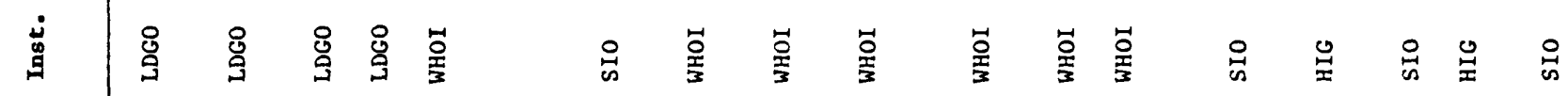

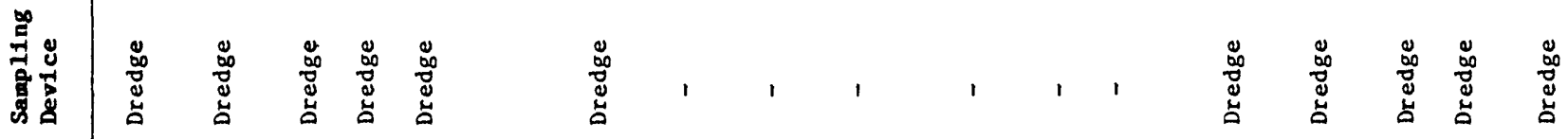

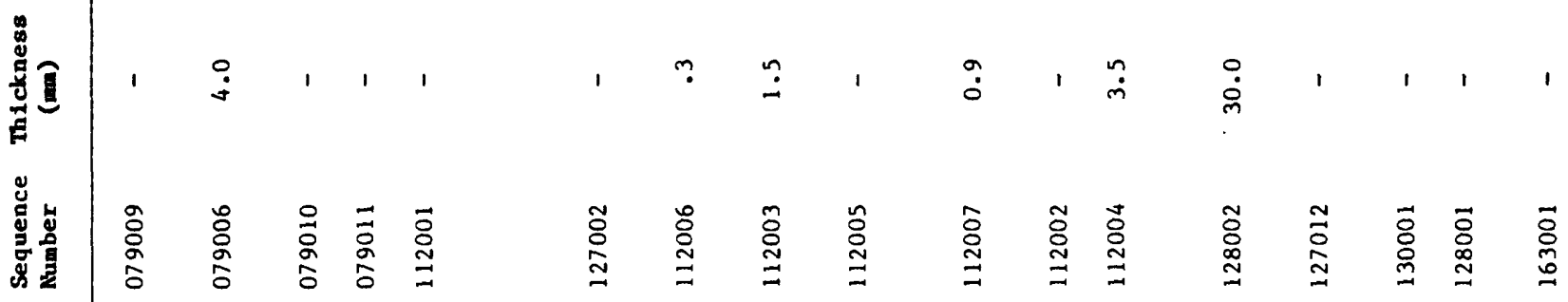

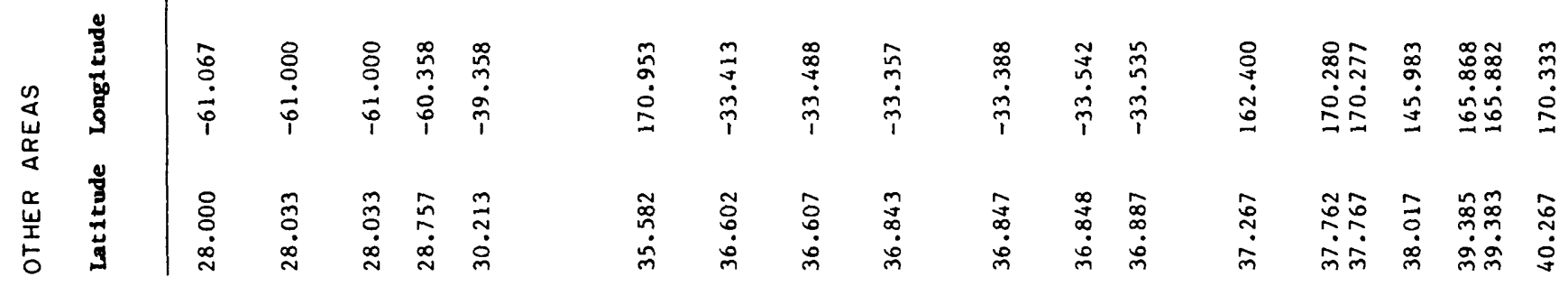




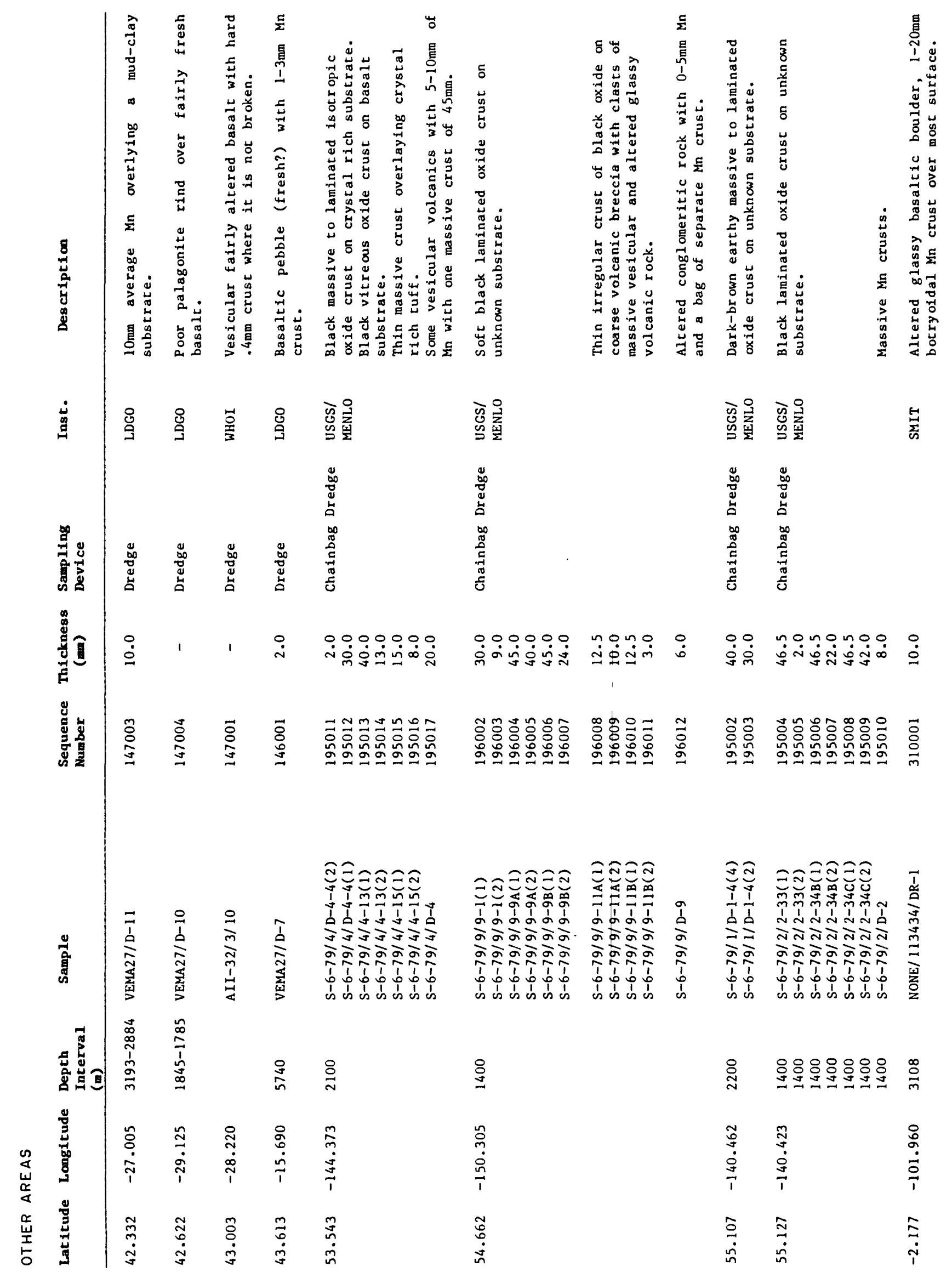




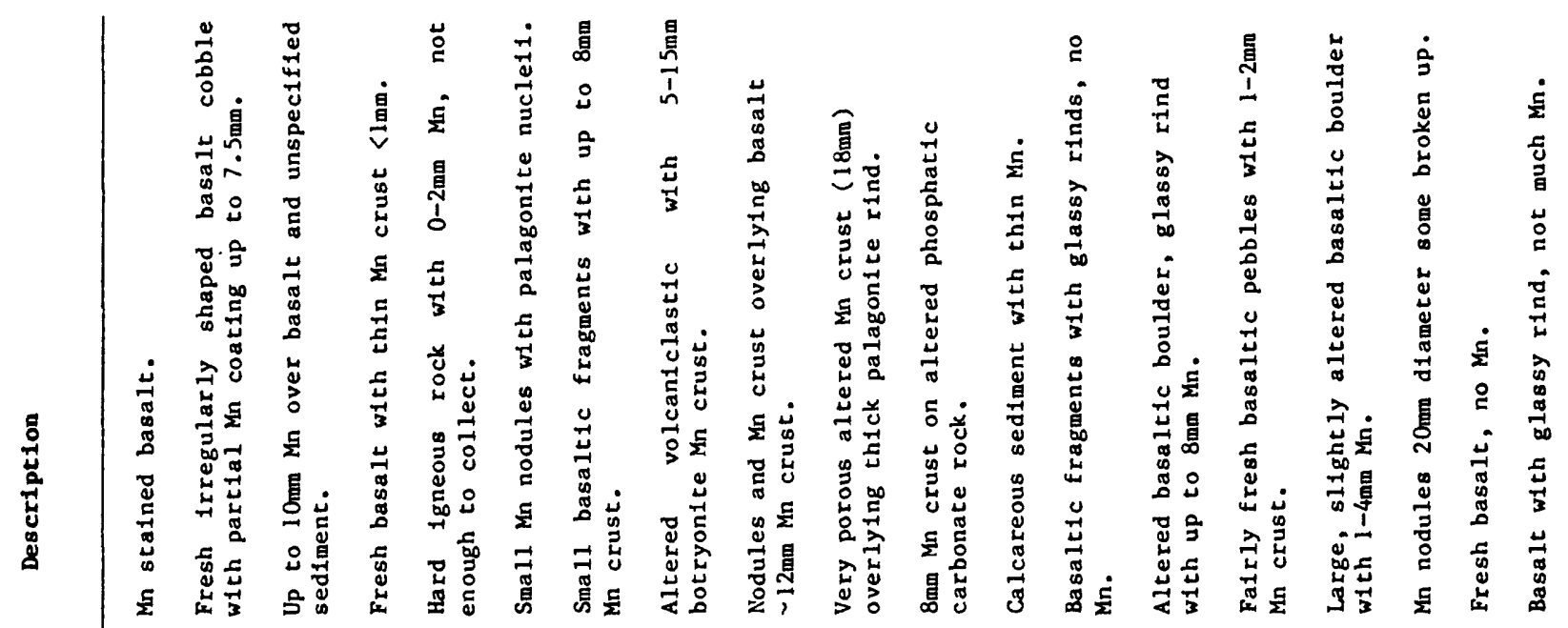

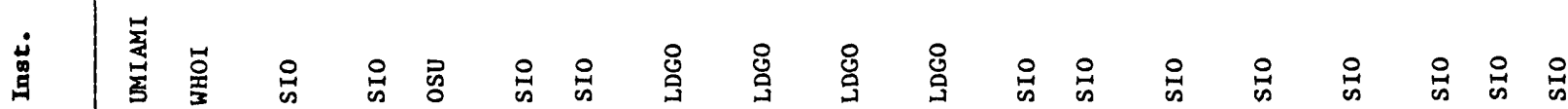

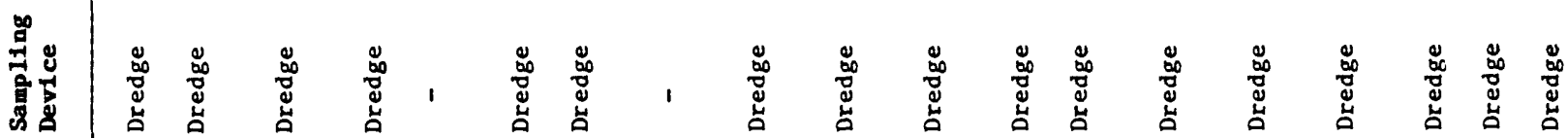

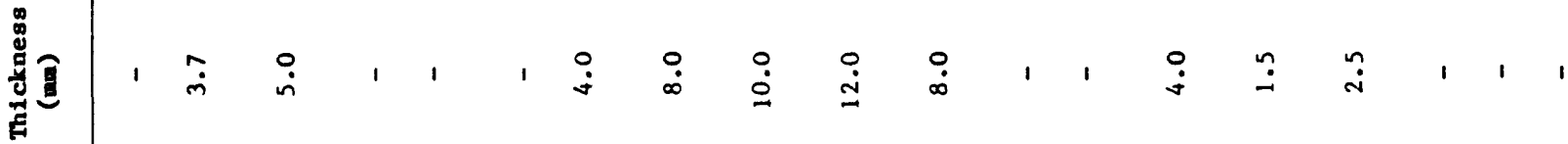

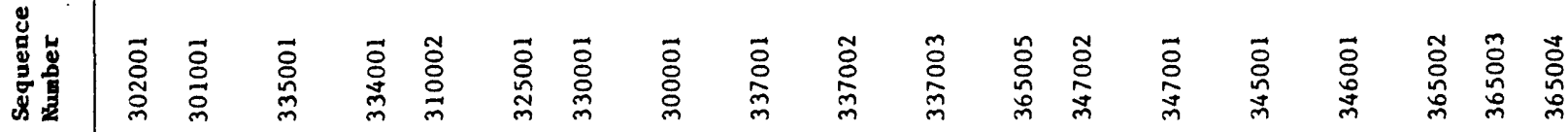

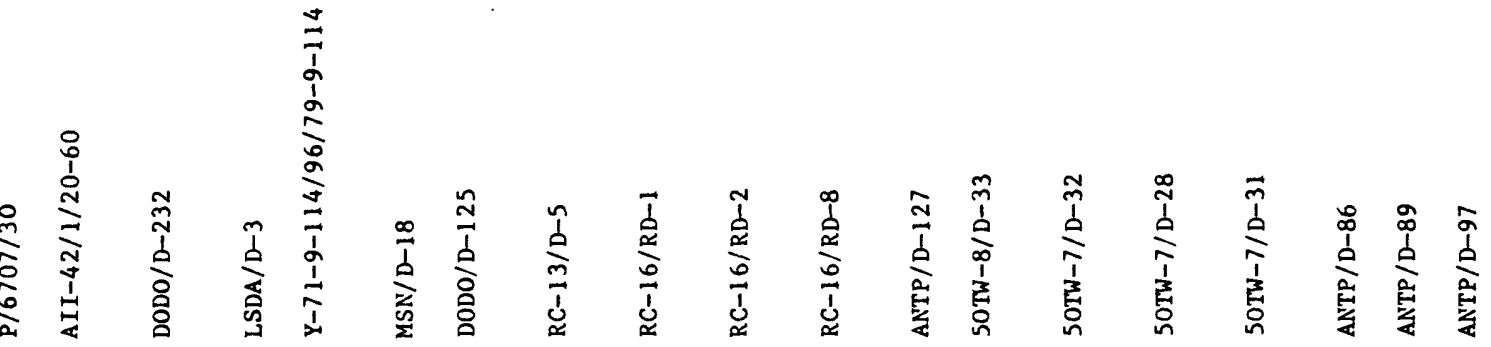

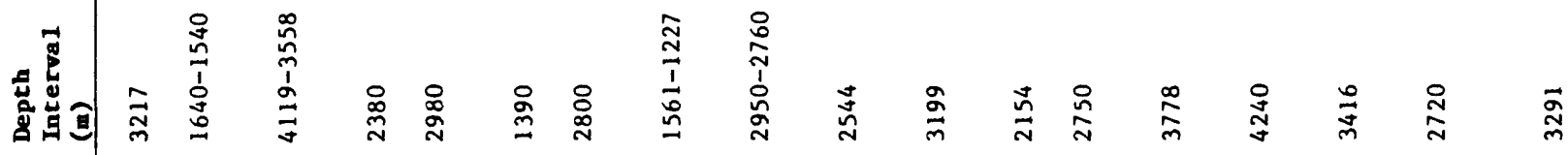

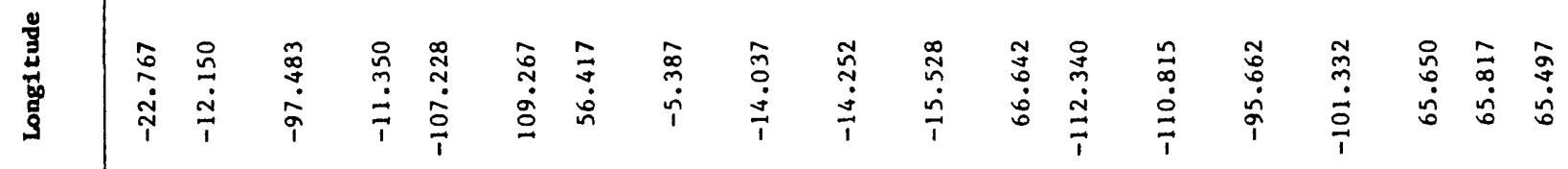

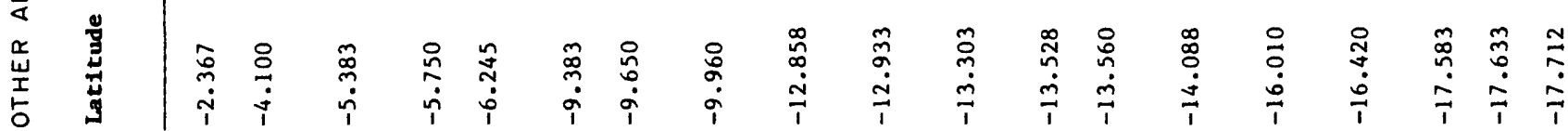




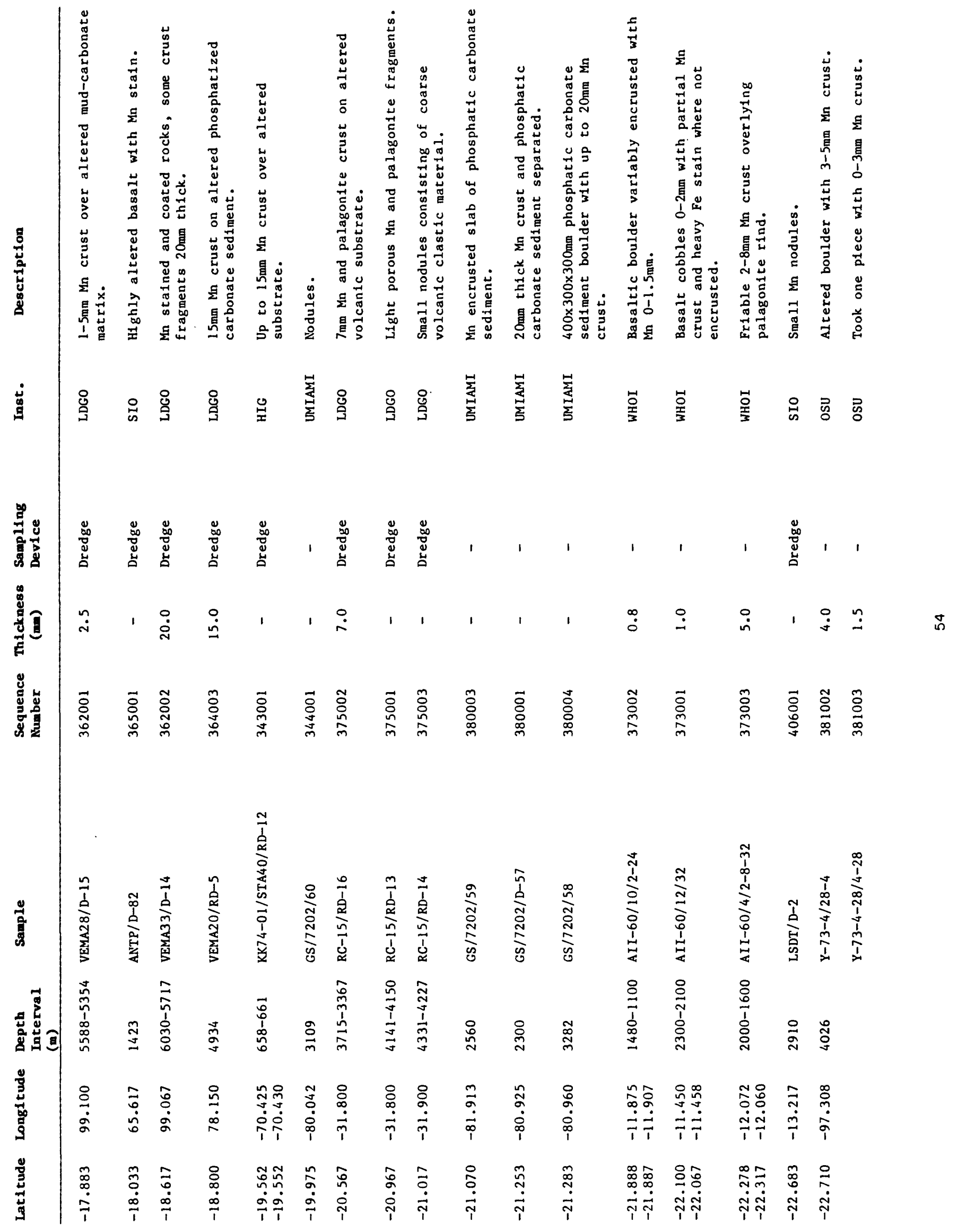




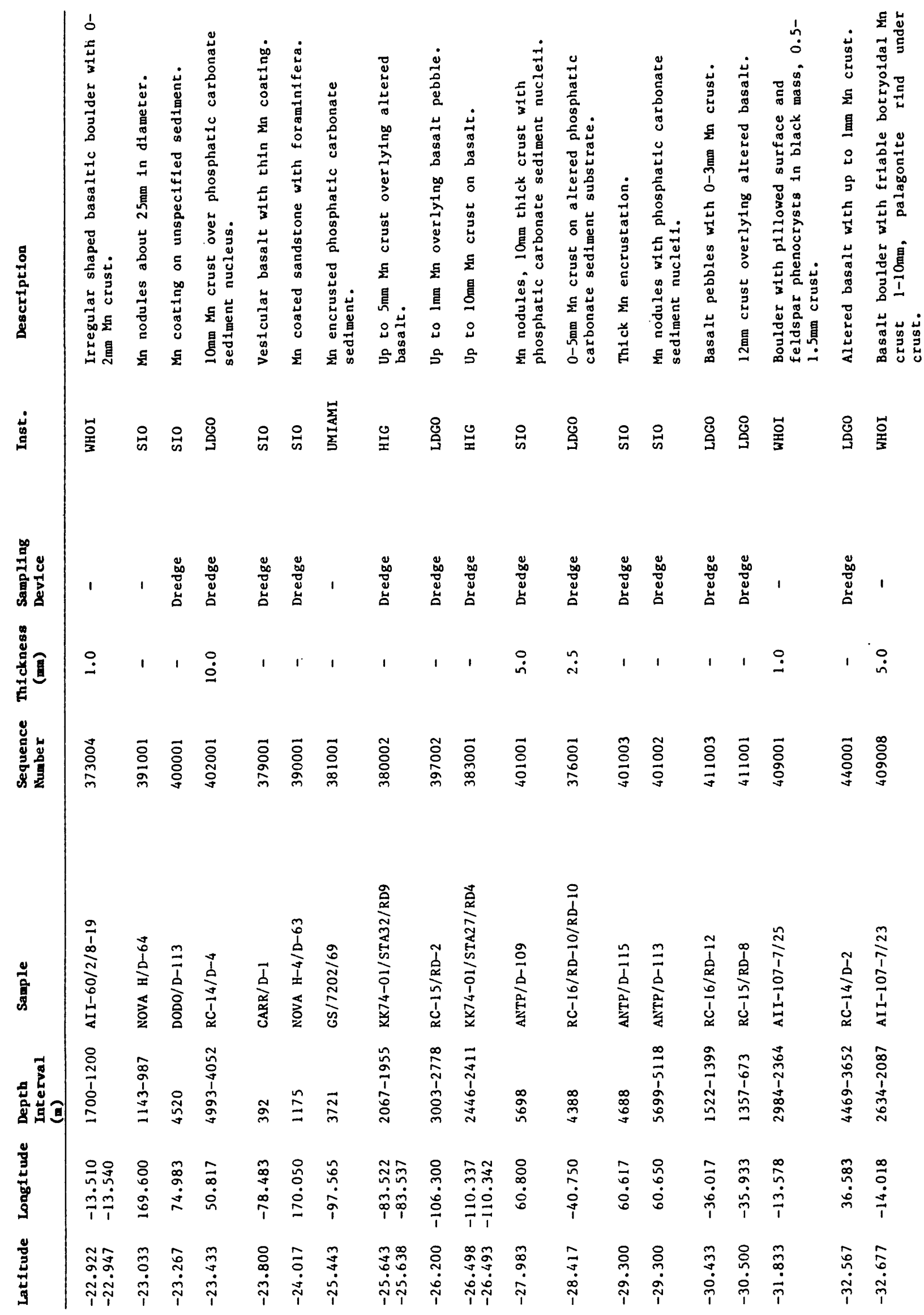




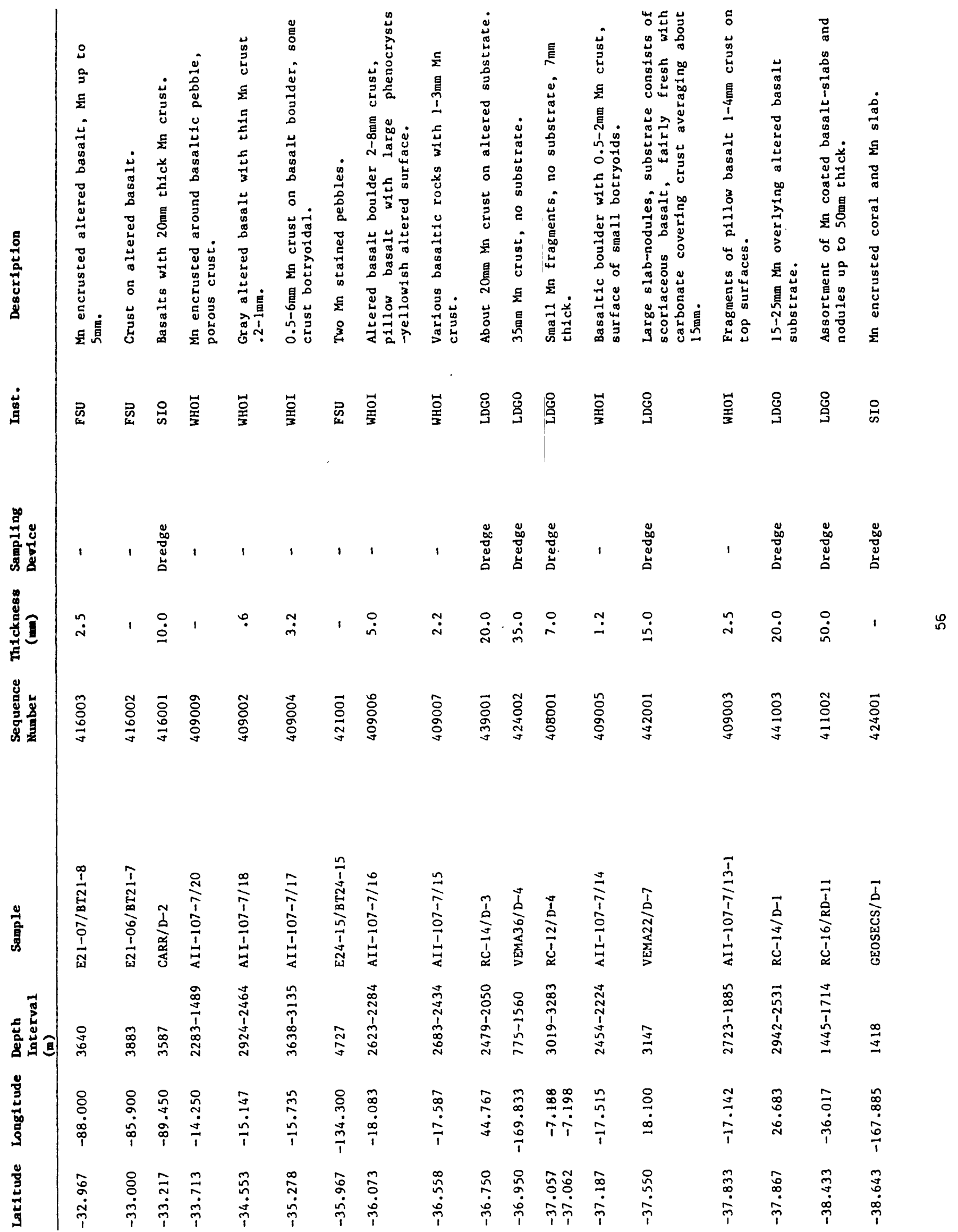




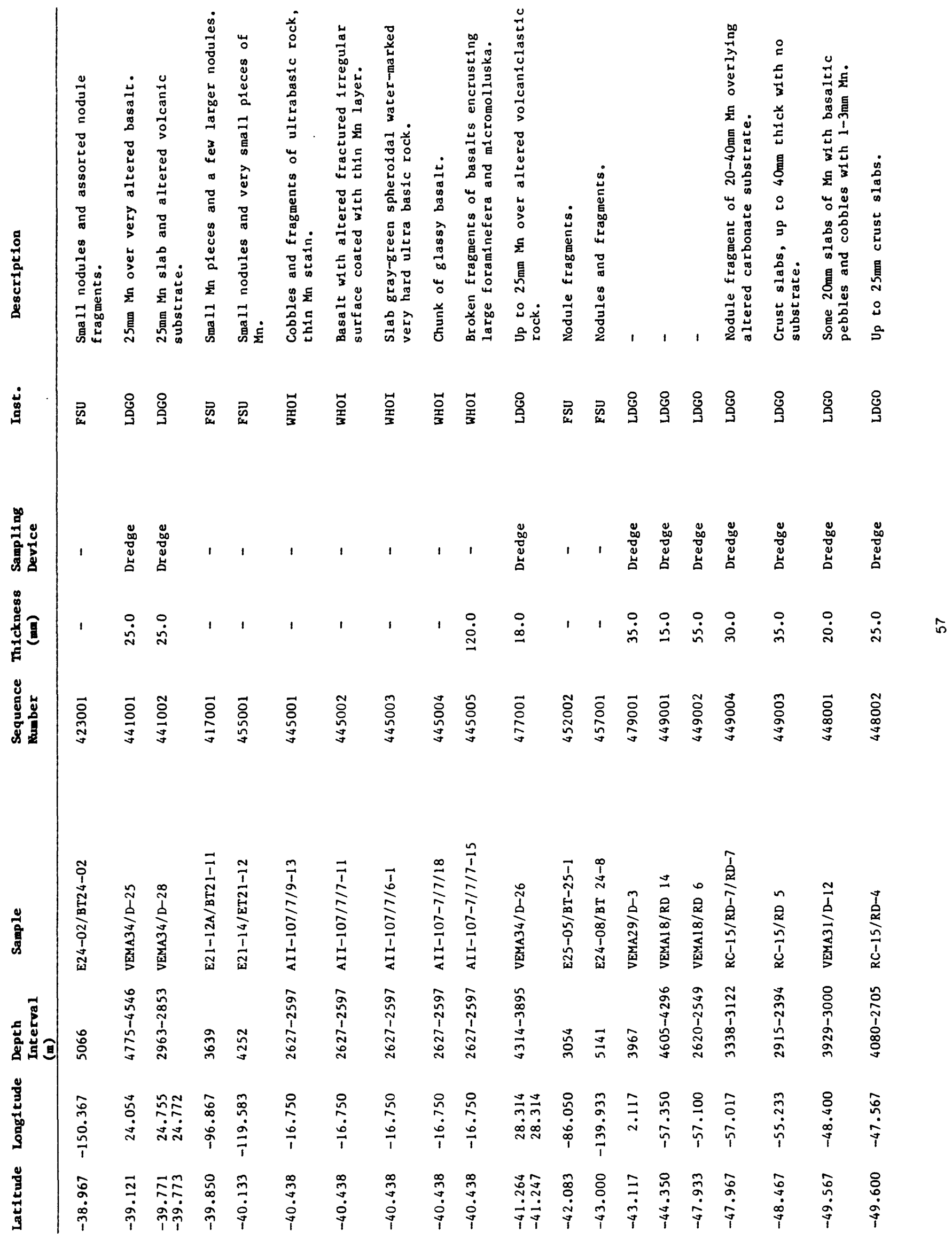




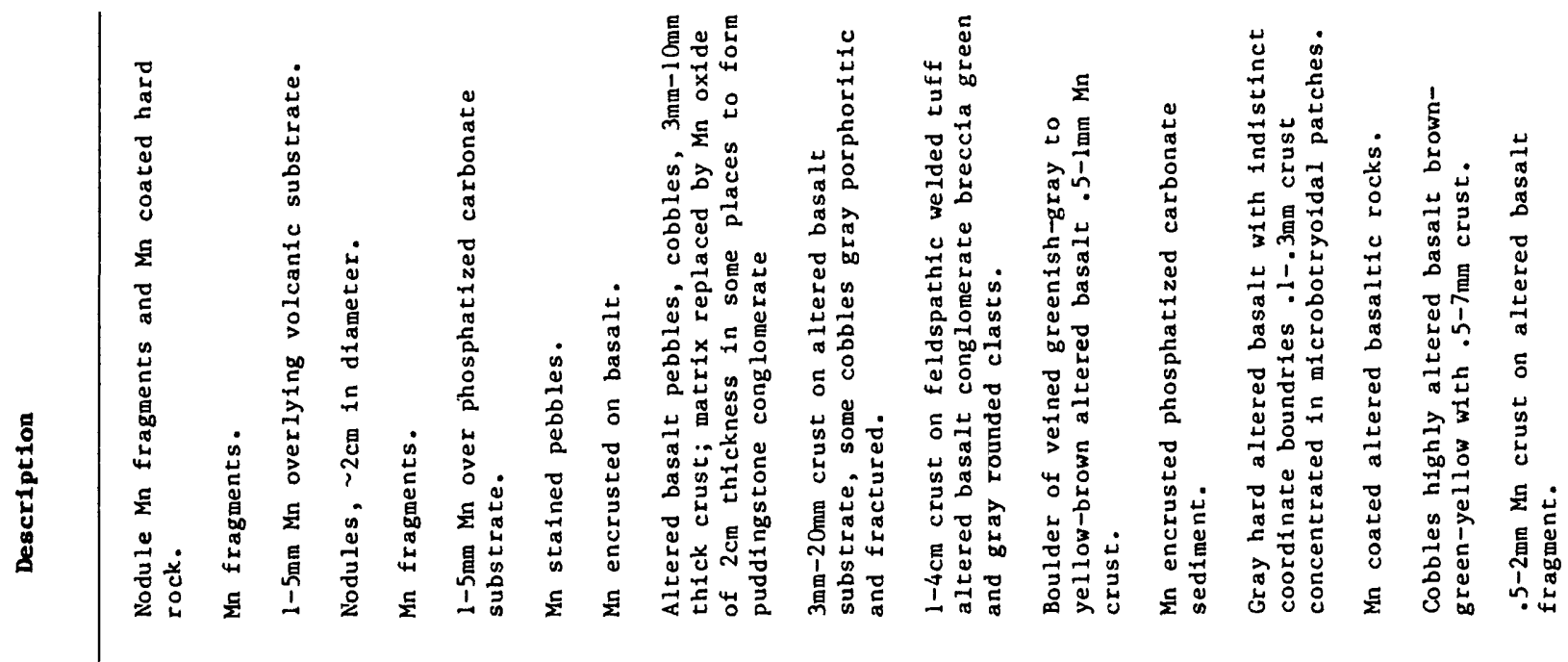

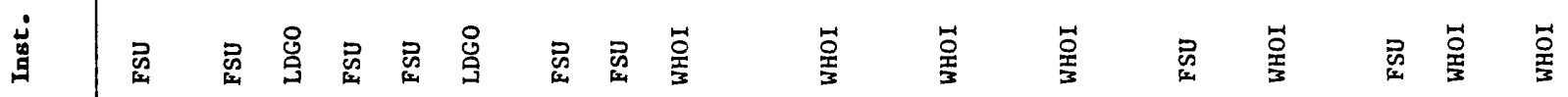

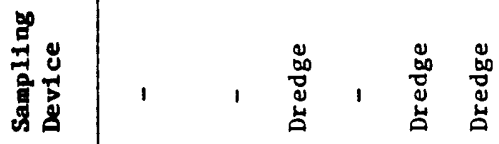

lo

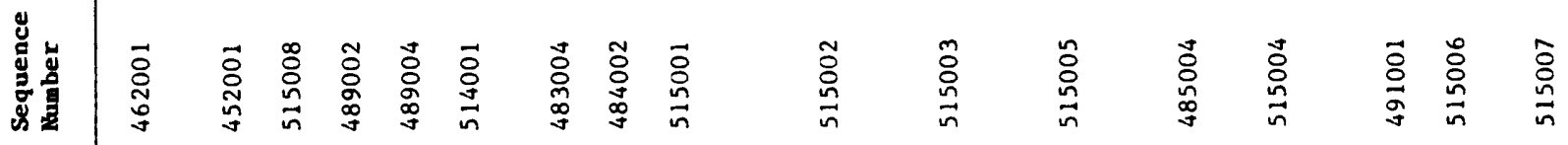

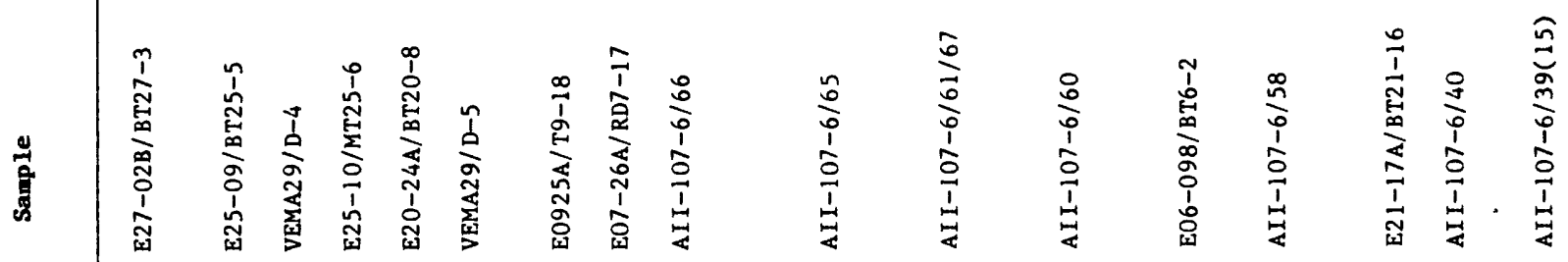

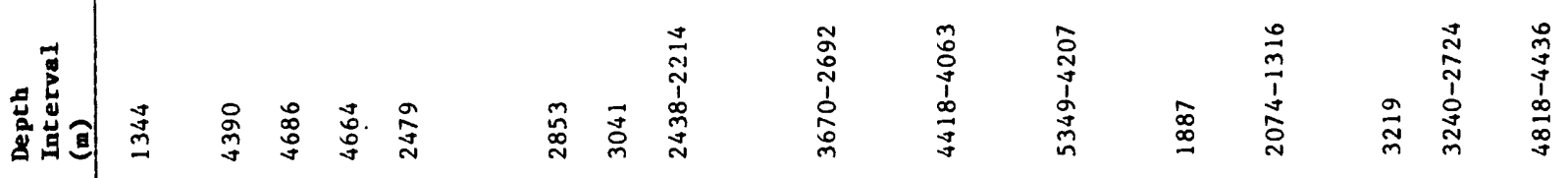

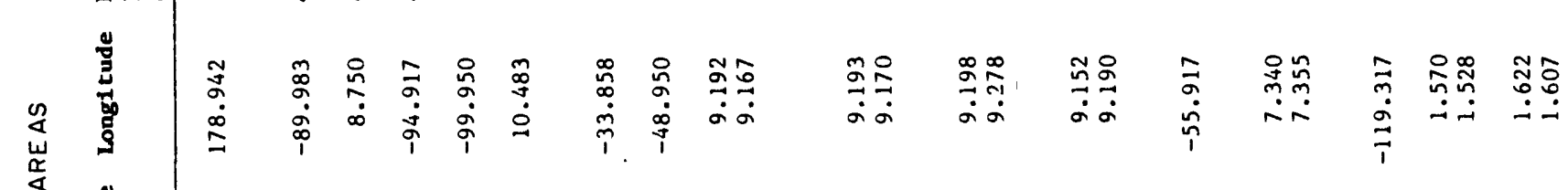

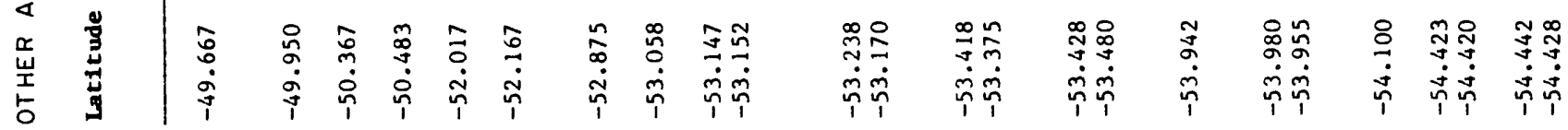




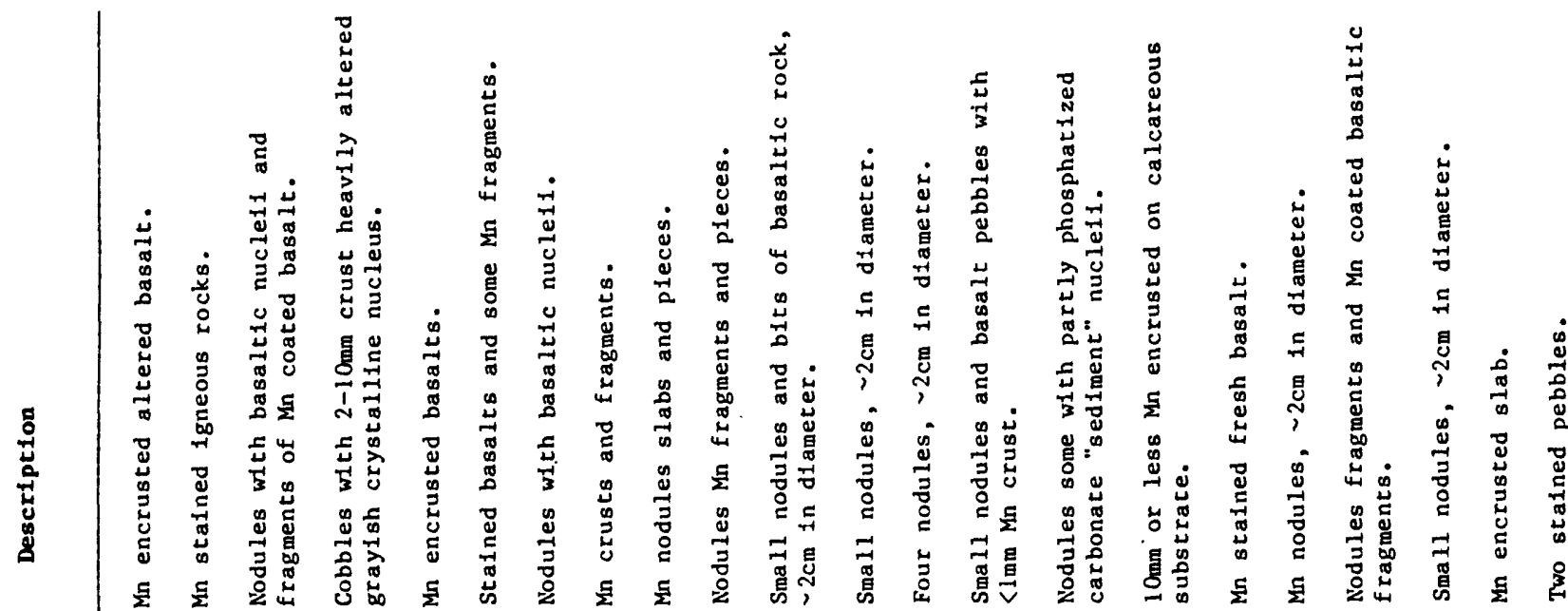

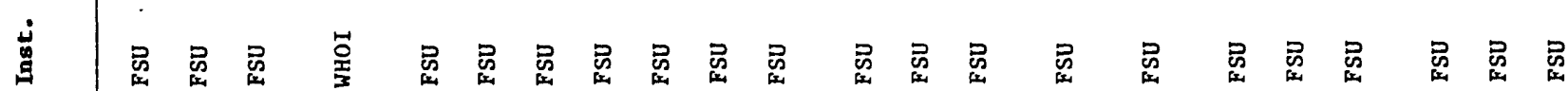

芴

害

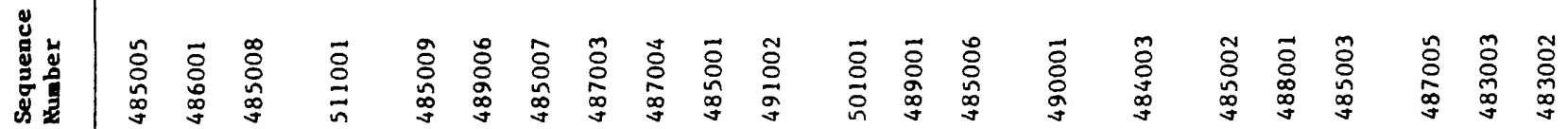

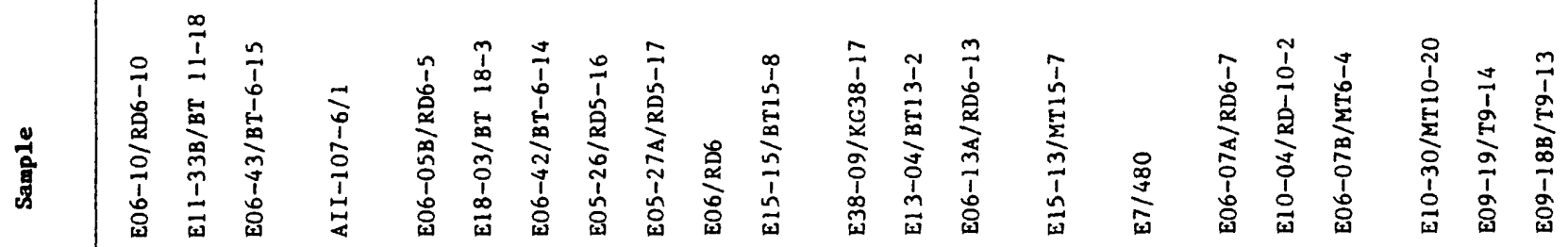

苔䓫四

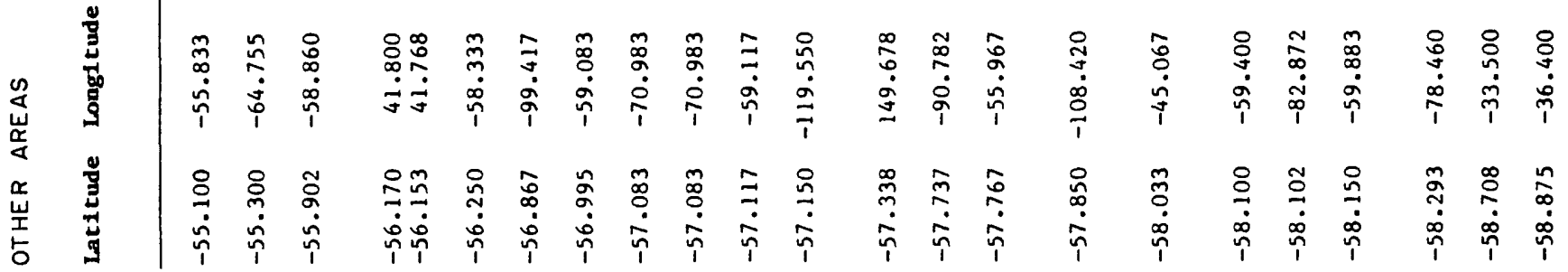




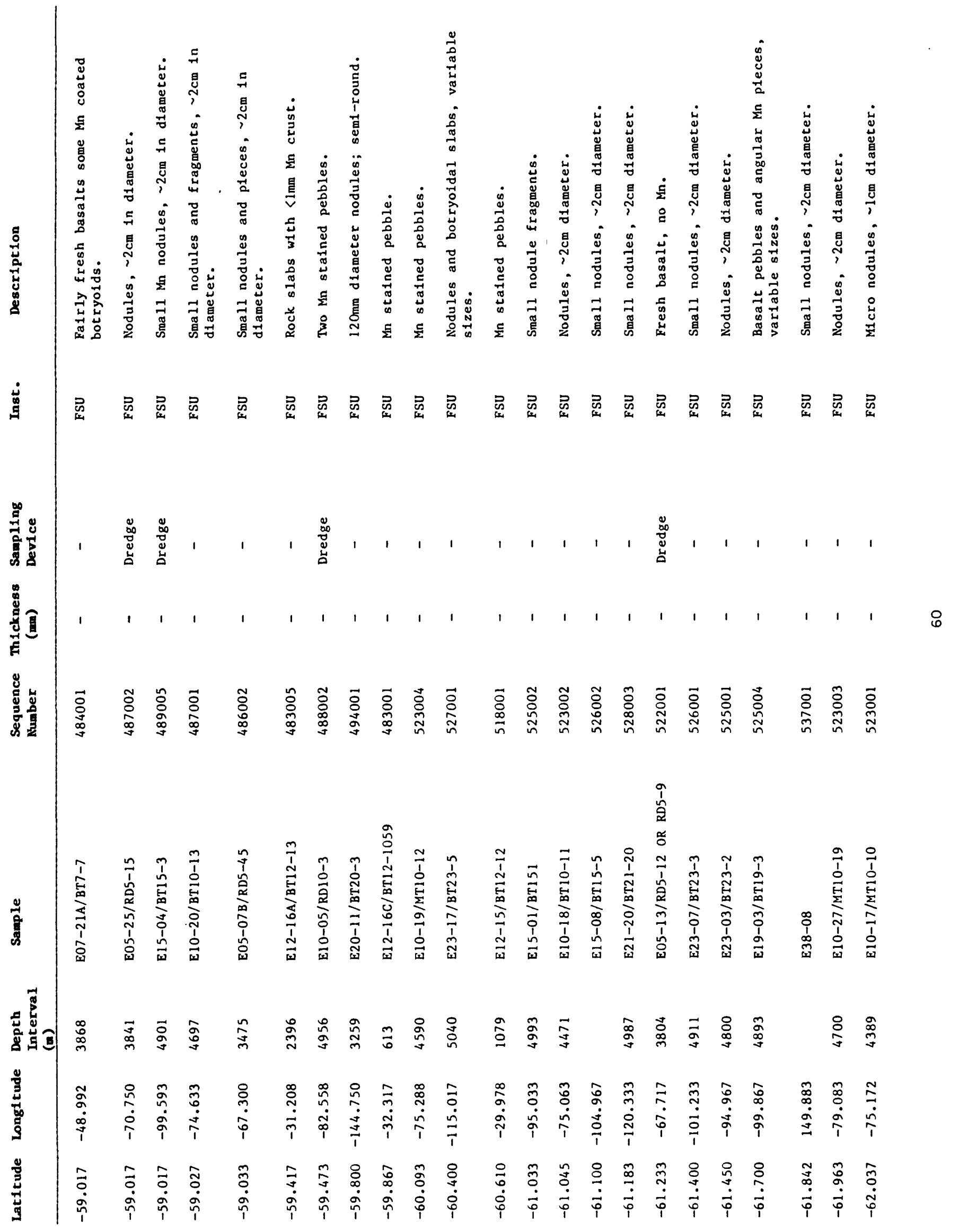




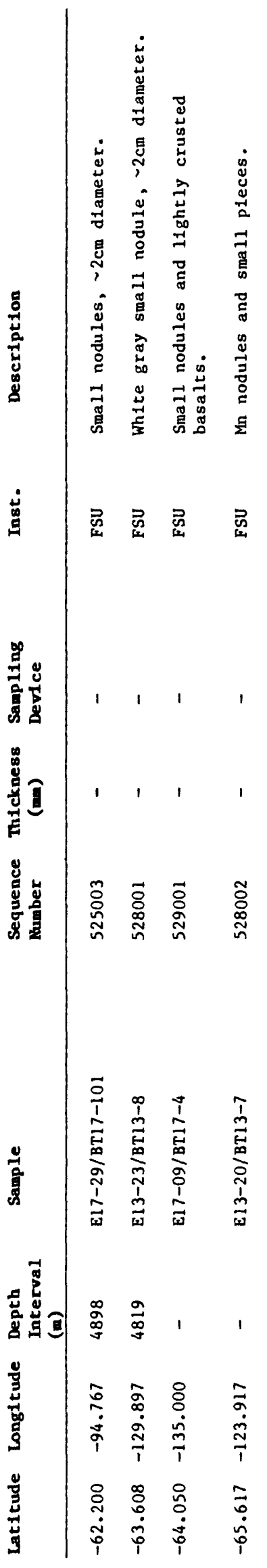




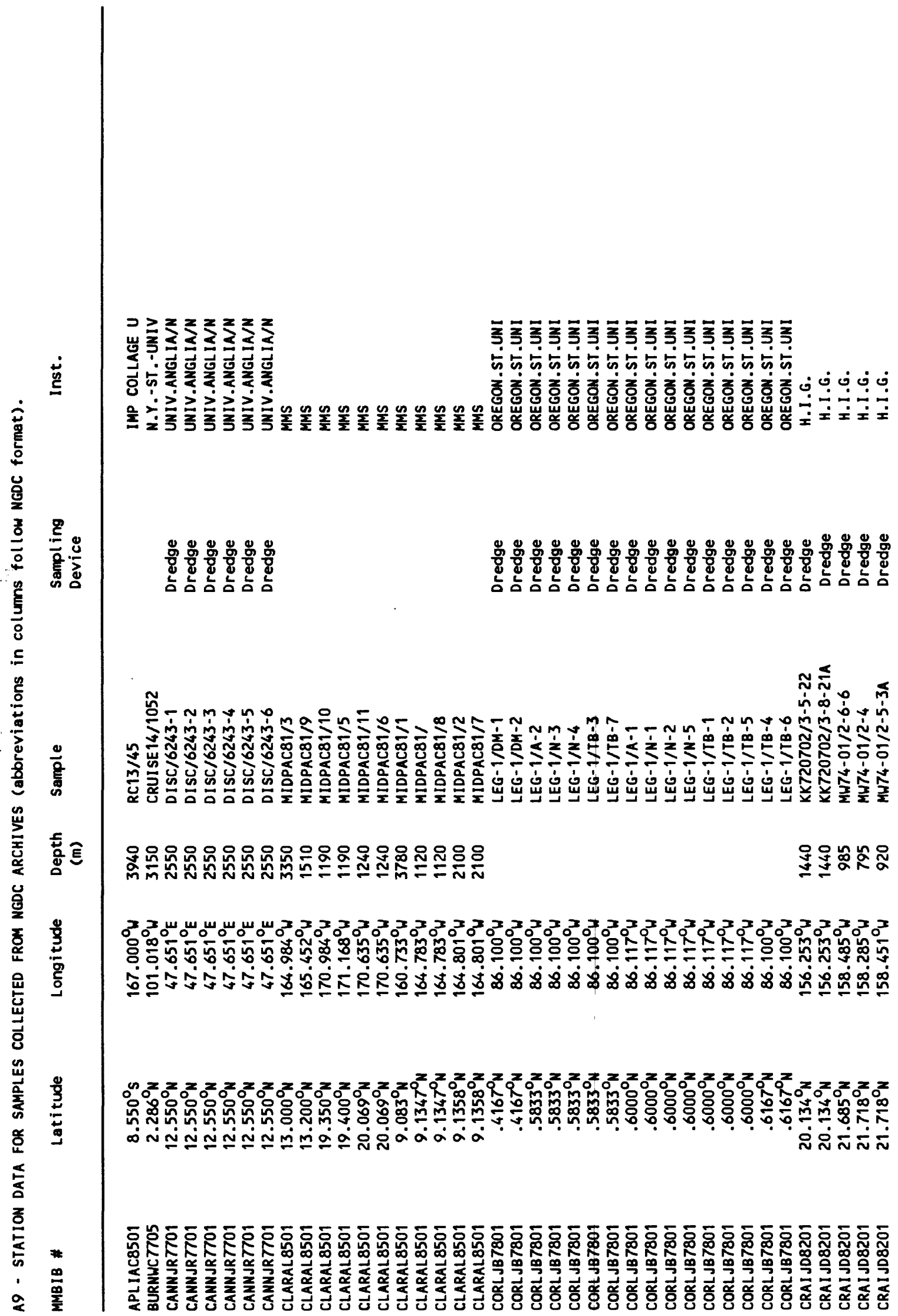




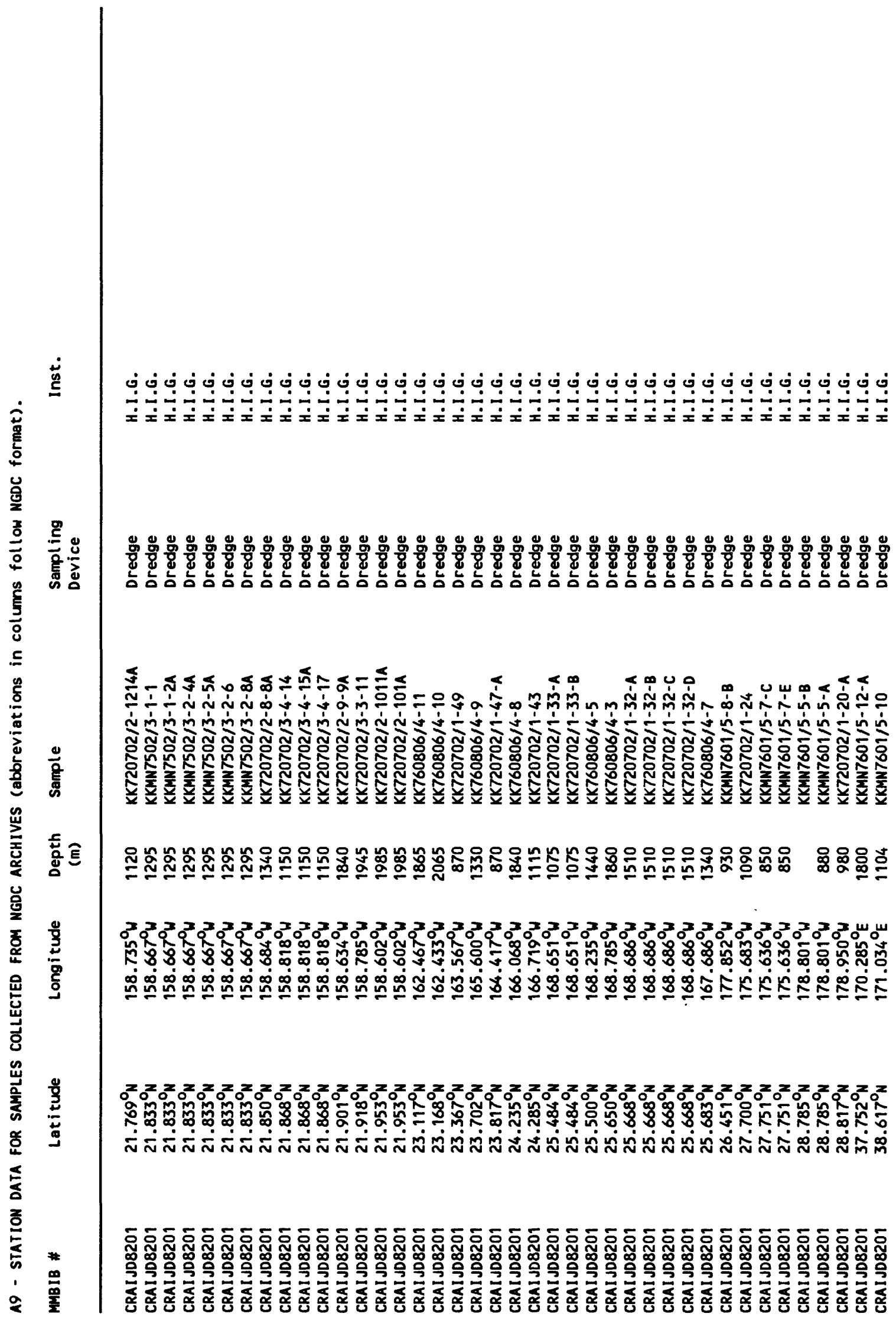




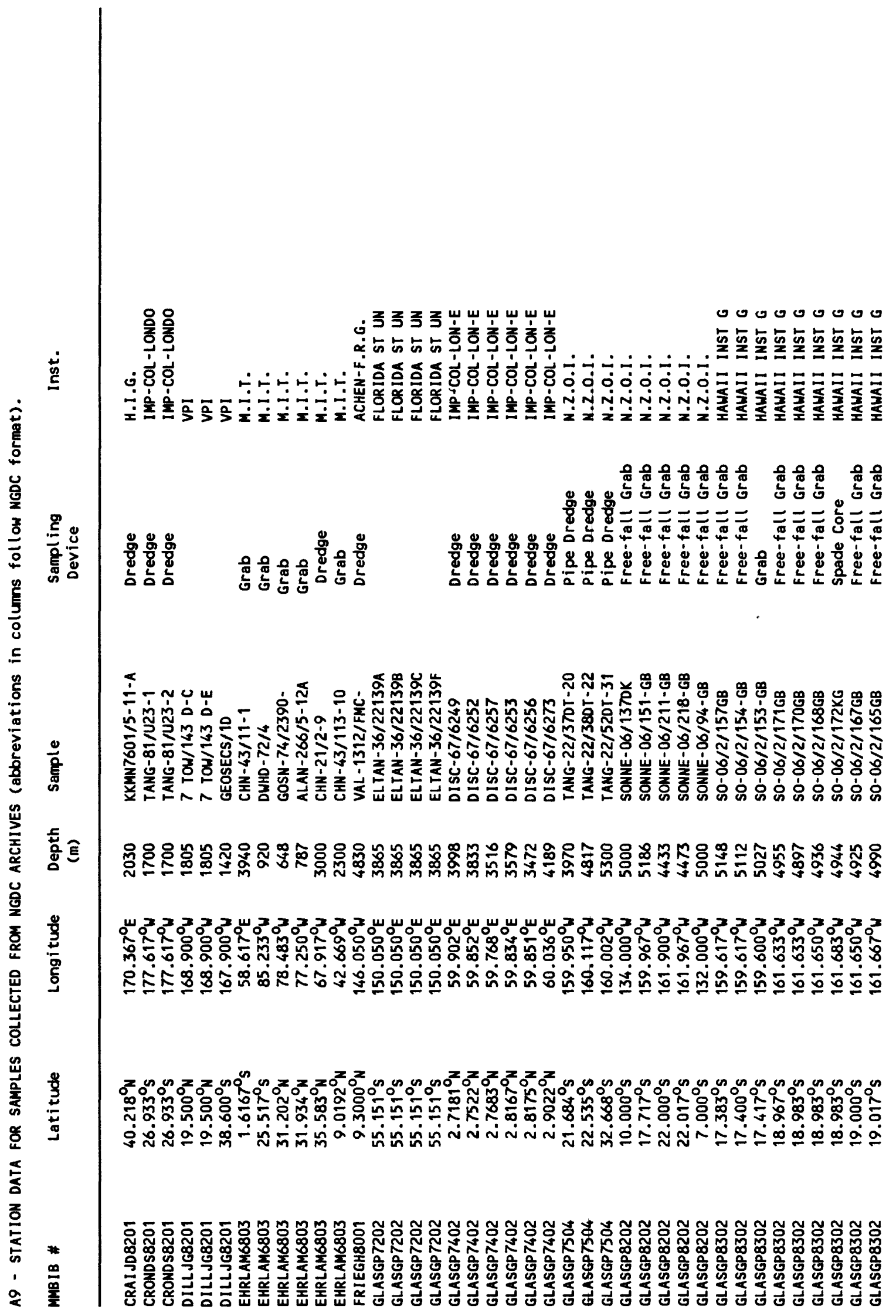




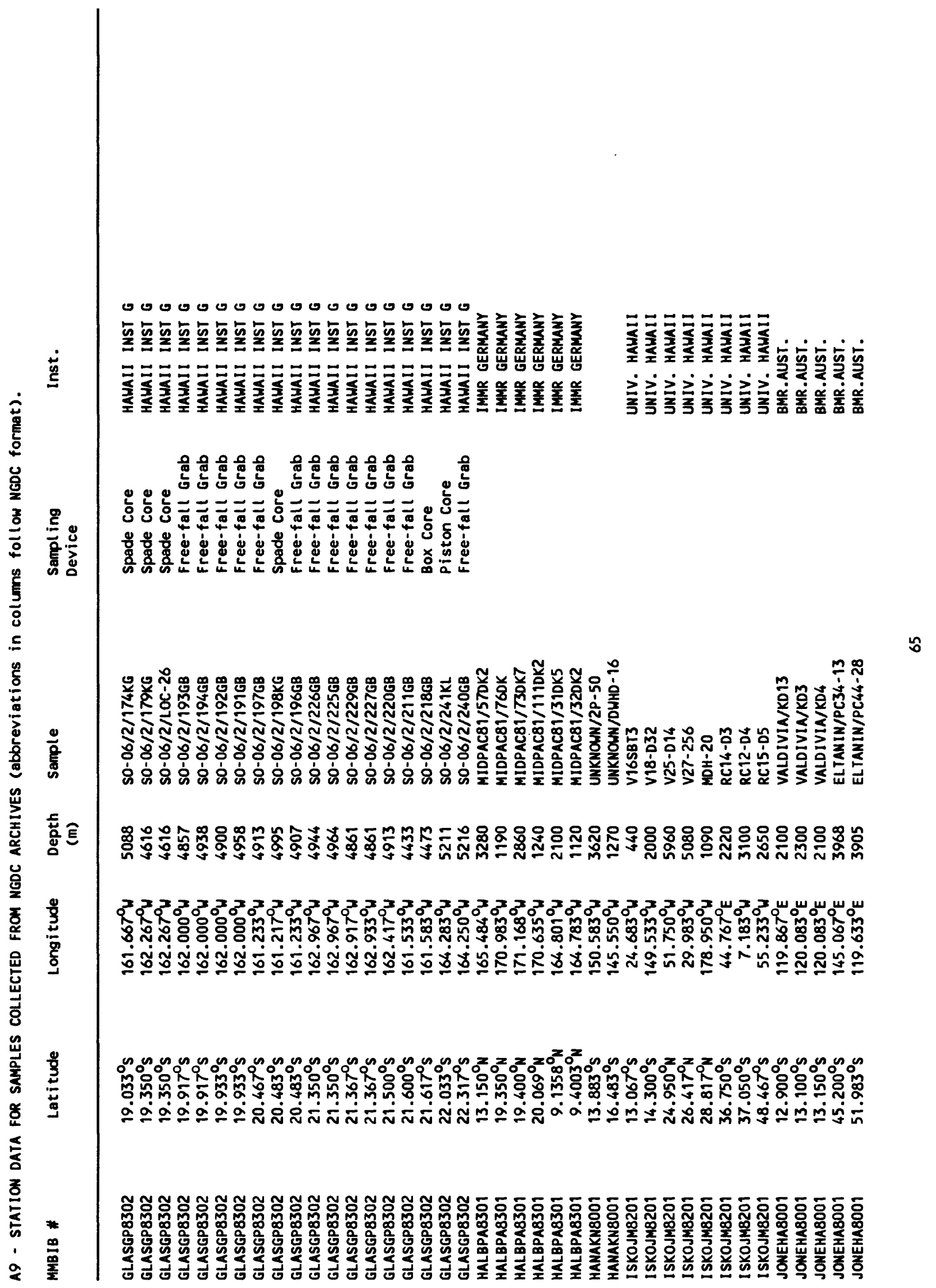




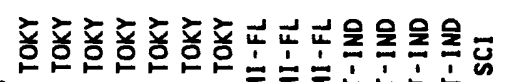

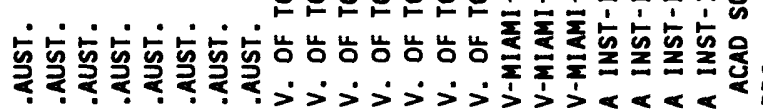

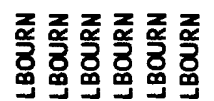

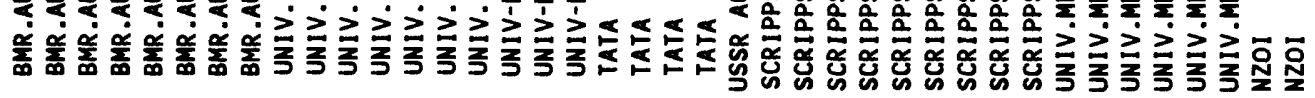

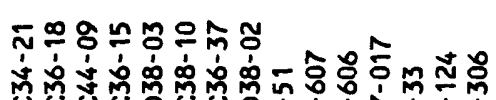

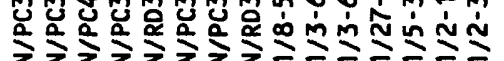

高

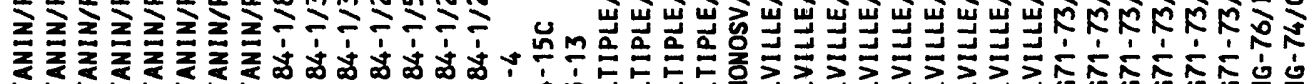

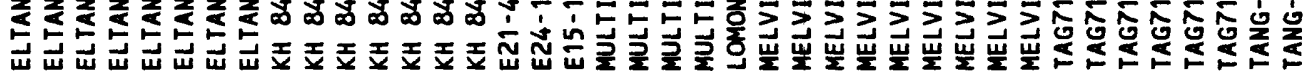

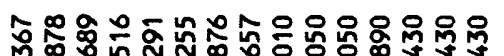

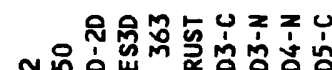

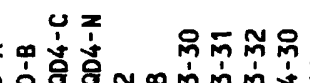

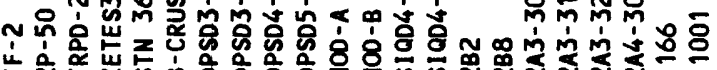

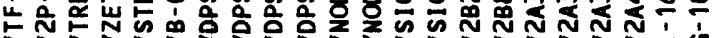

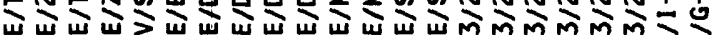

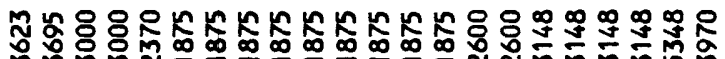

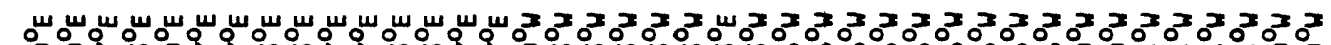

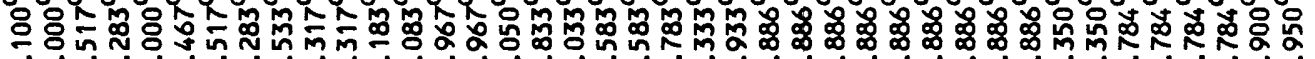

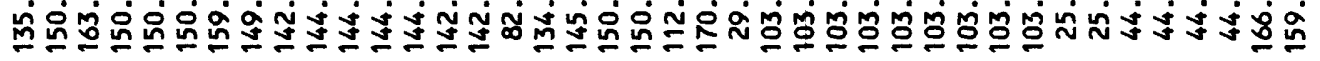

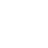

年

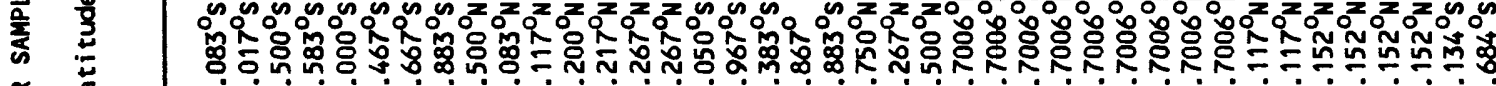

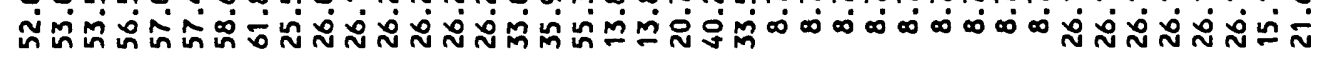




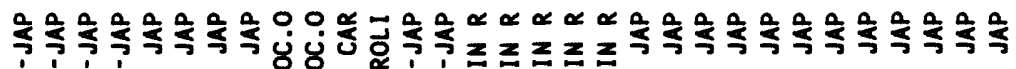

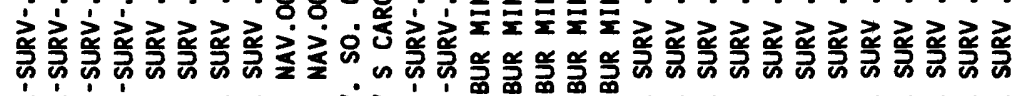

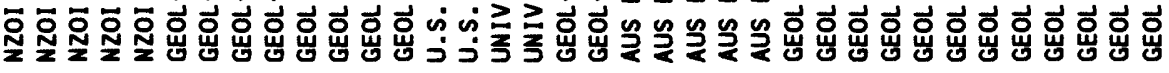

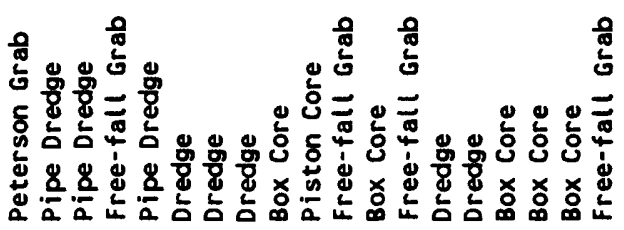

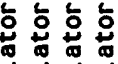

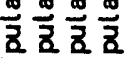

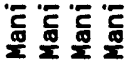

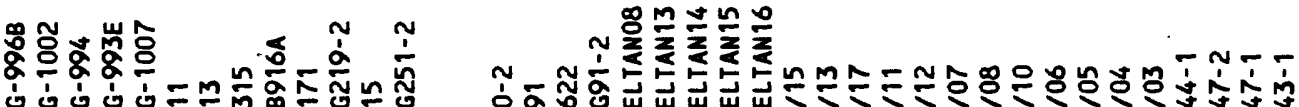

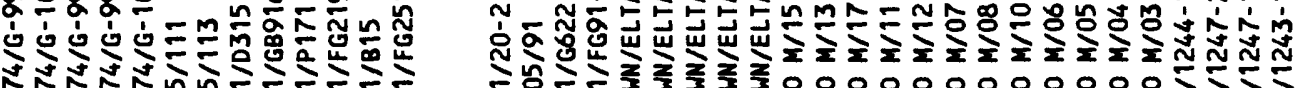

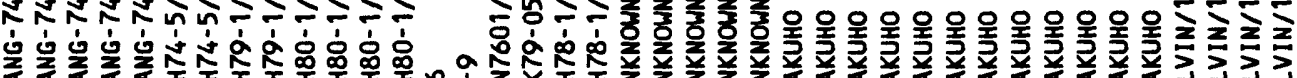

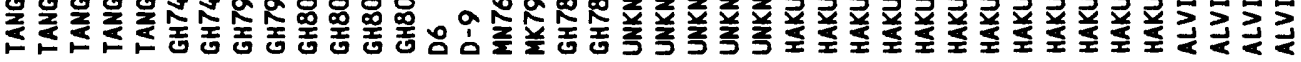

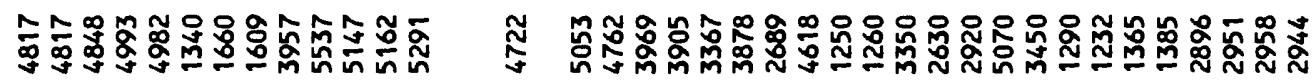

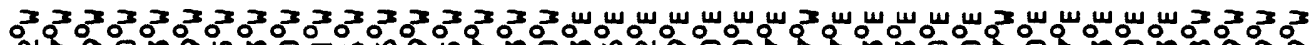

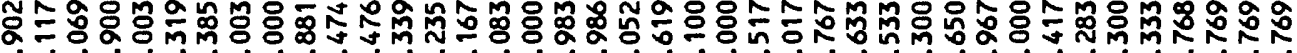

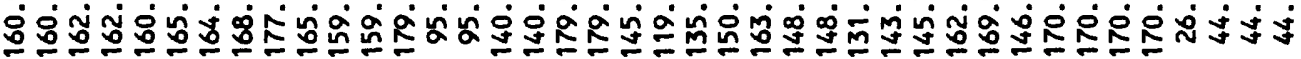

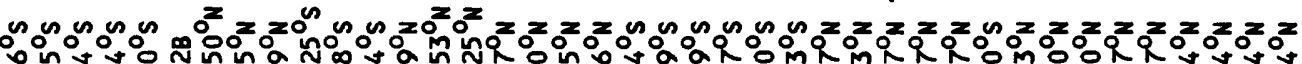

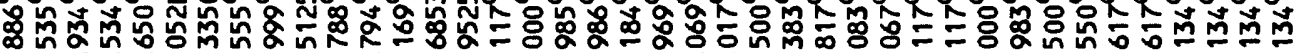

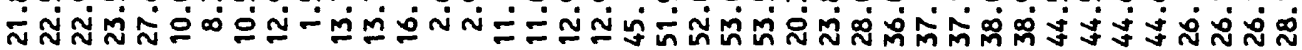

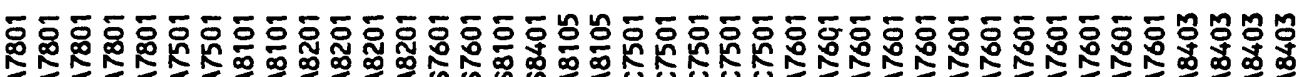

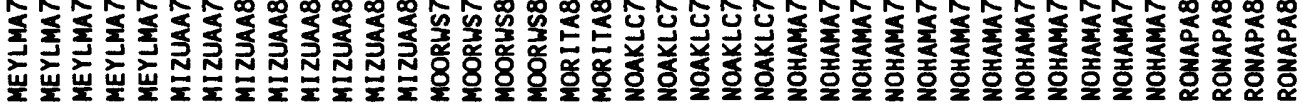




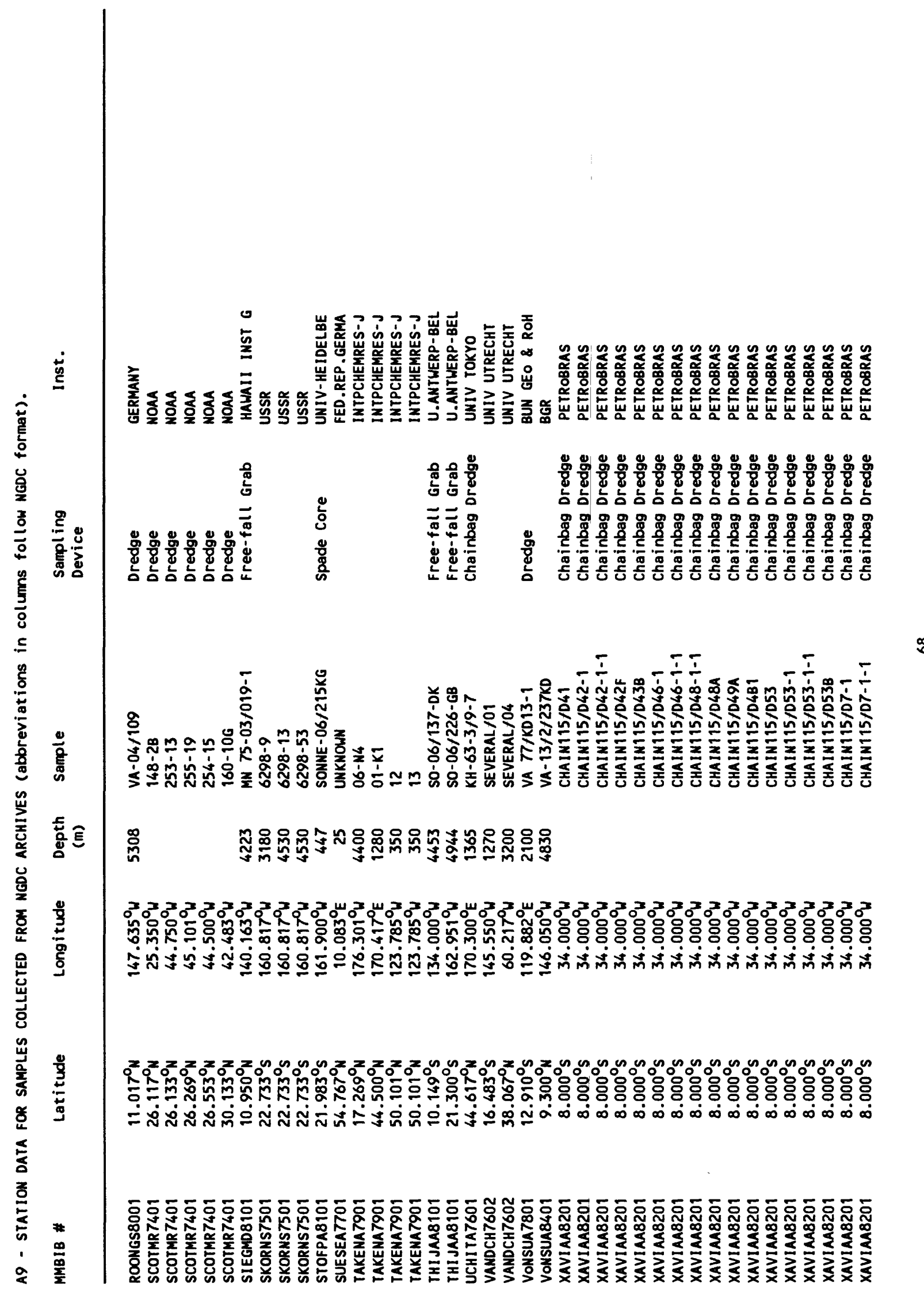




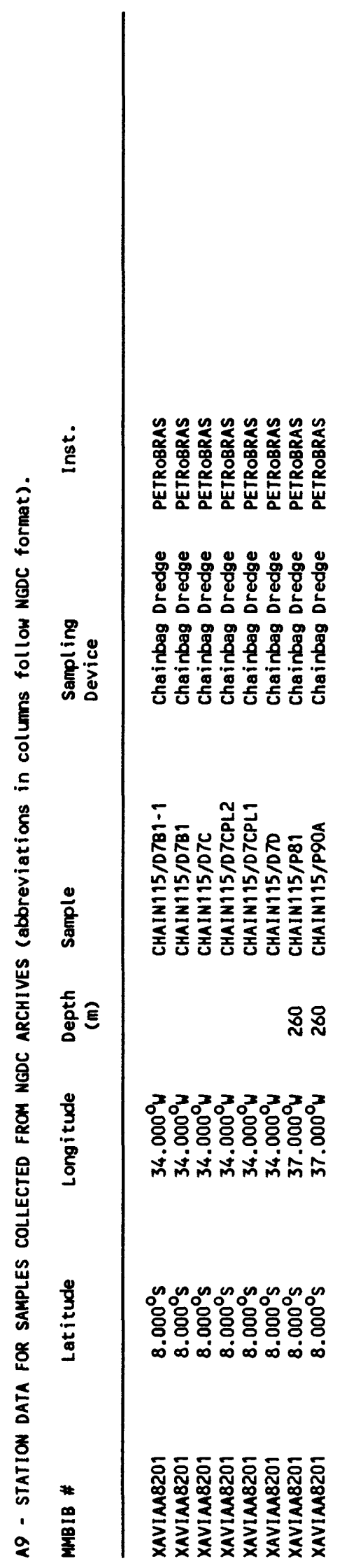




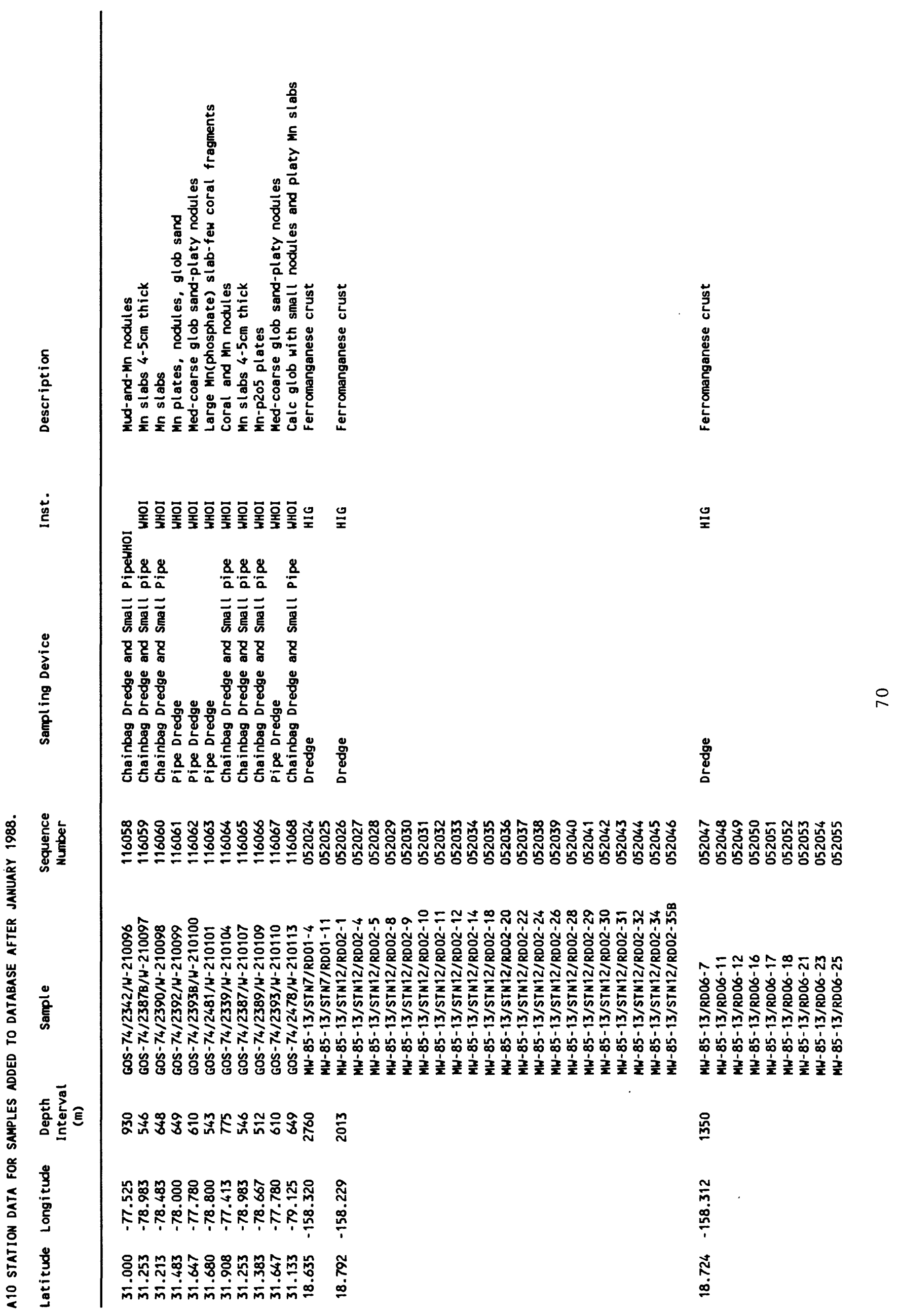




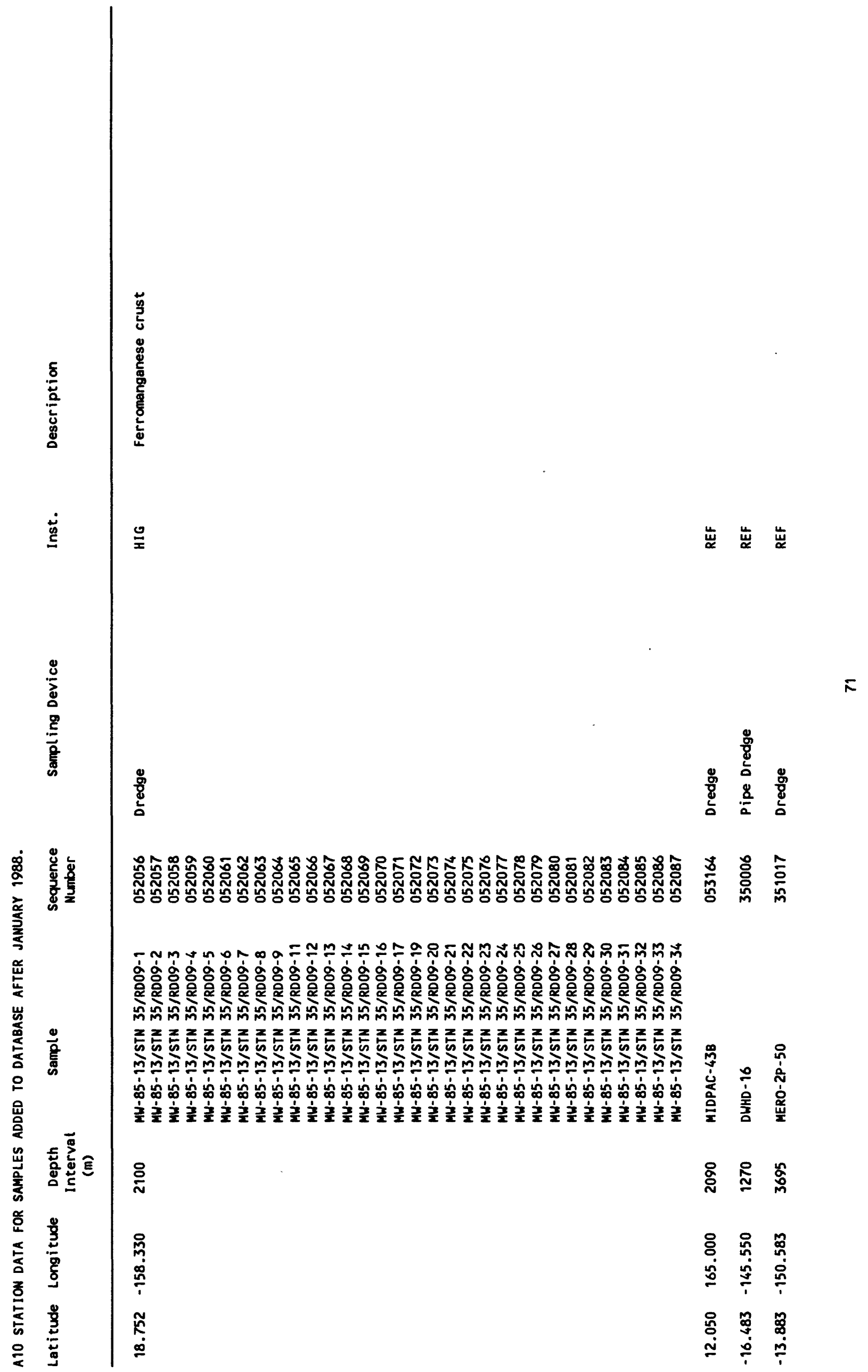




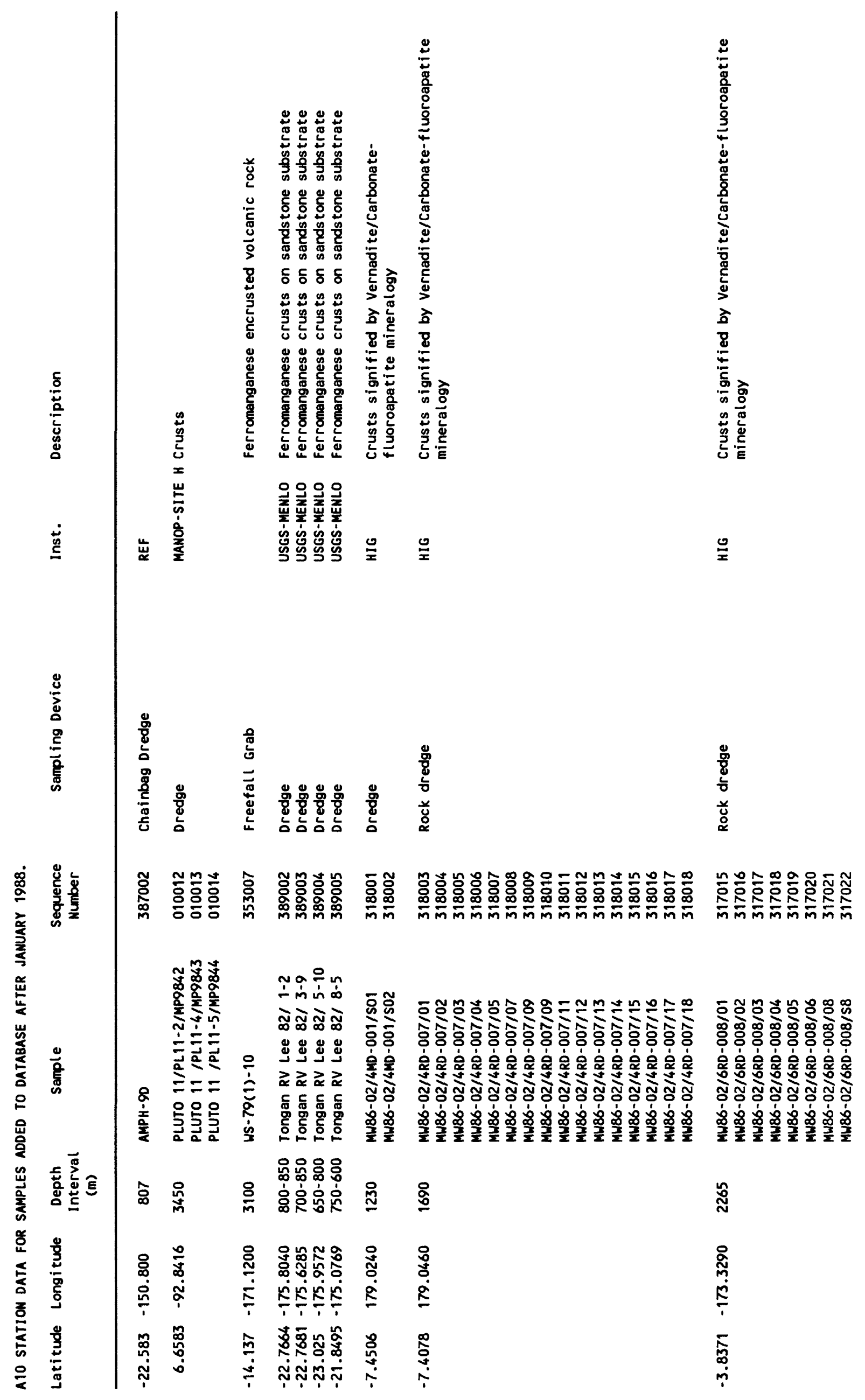




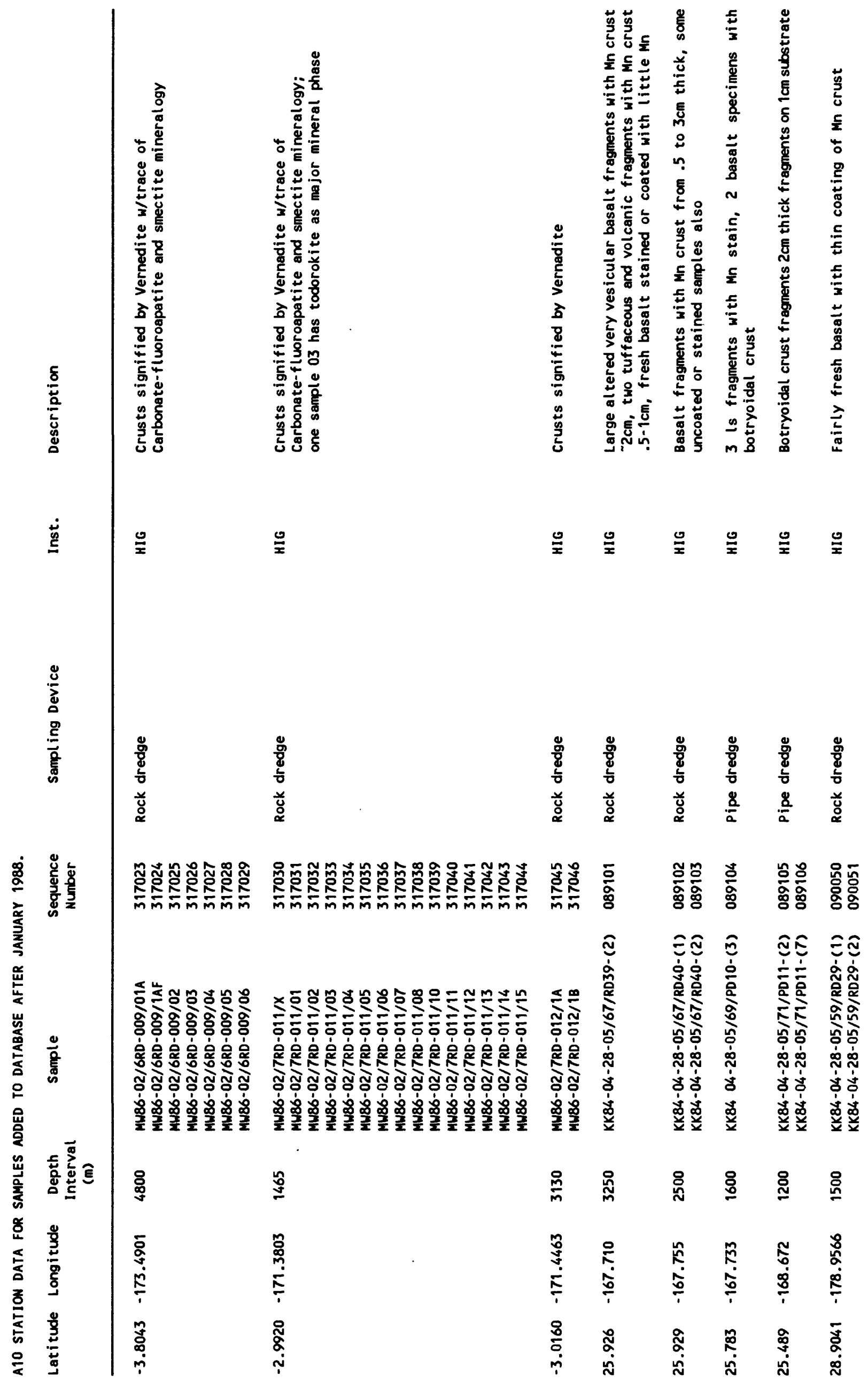




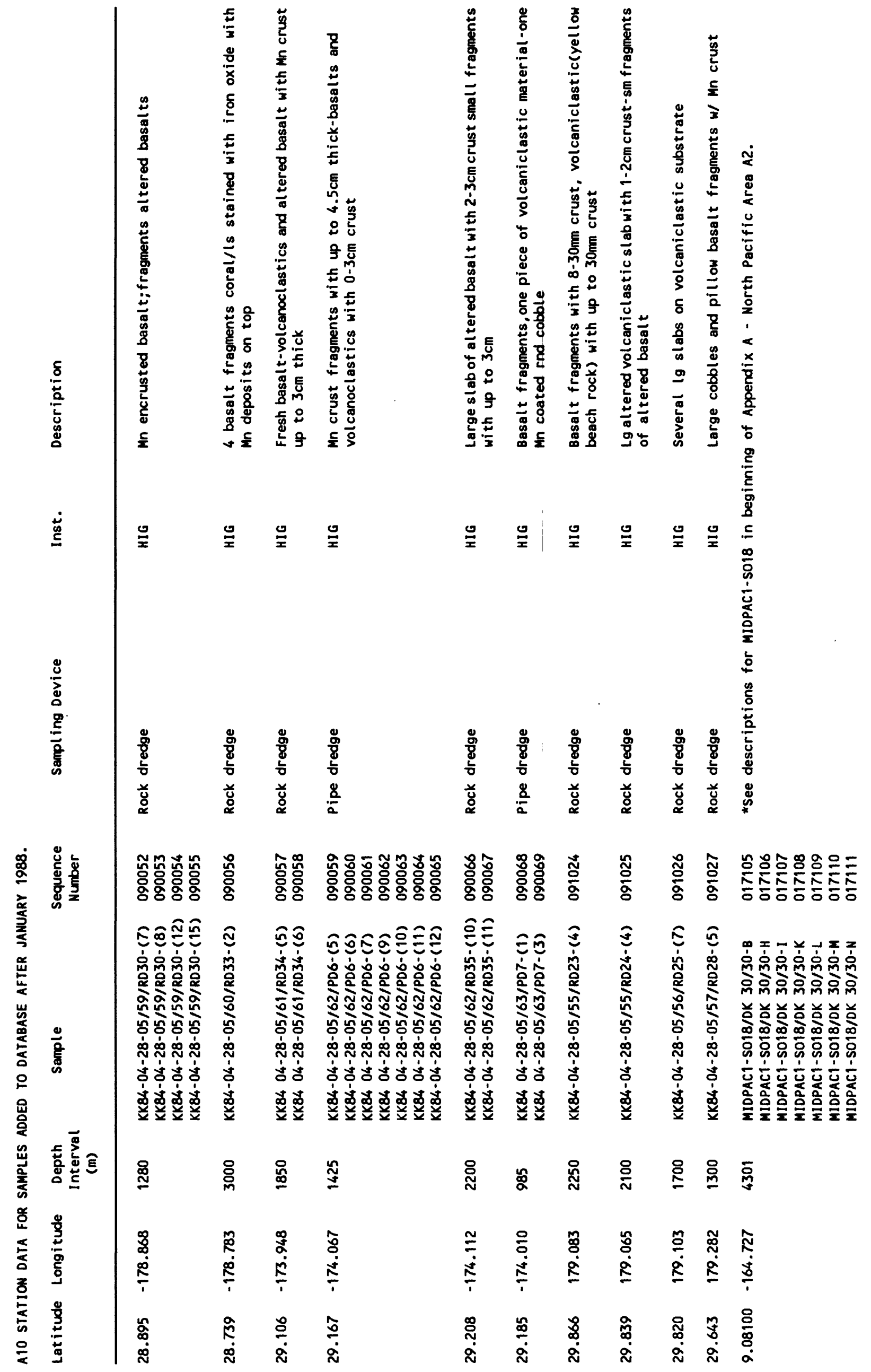




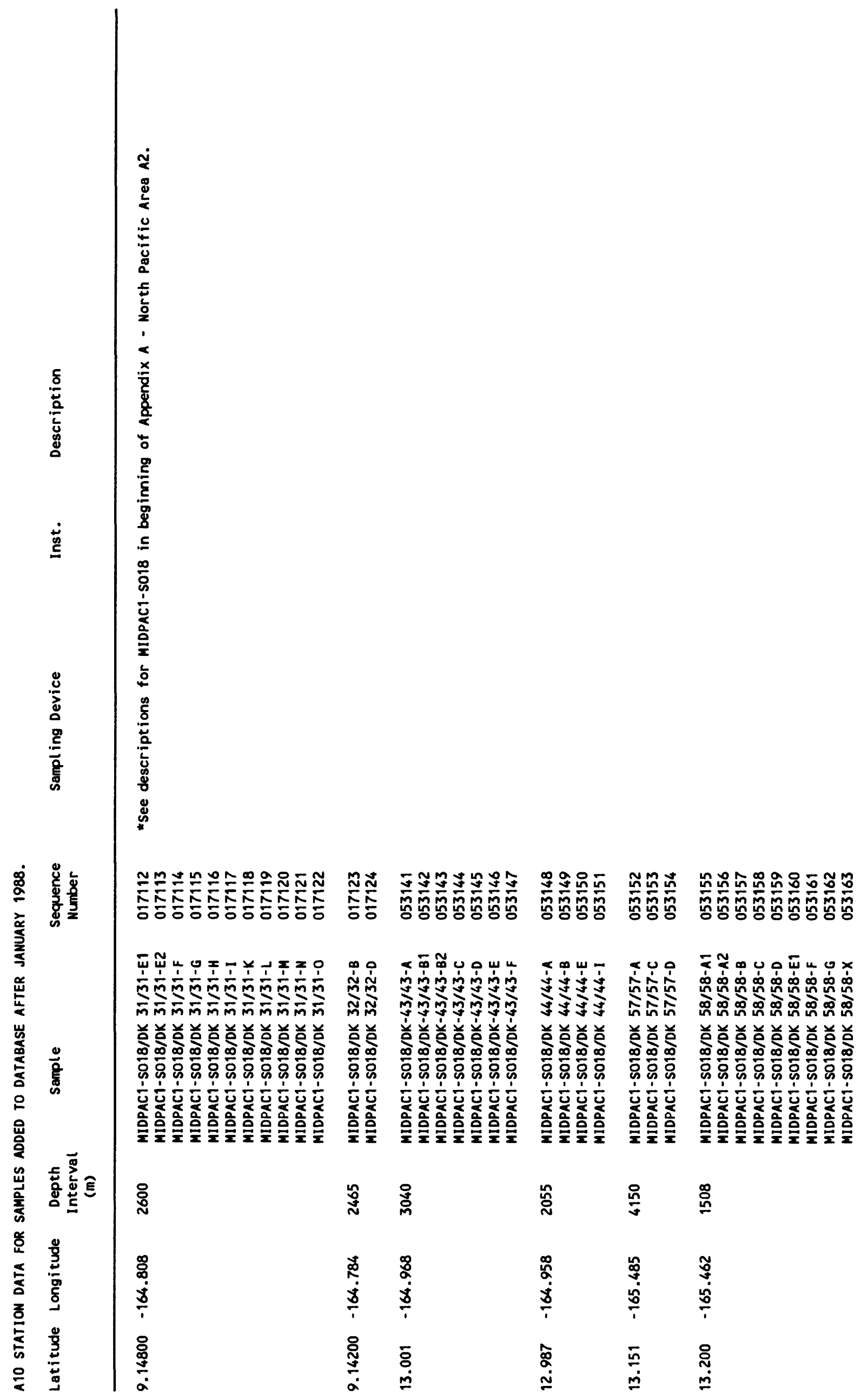




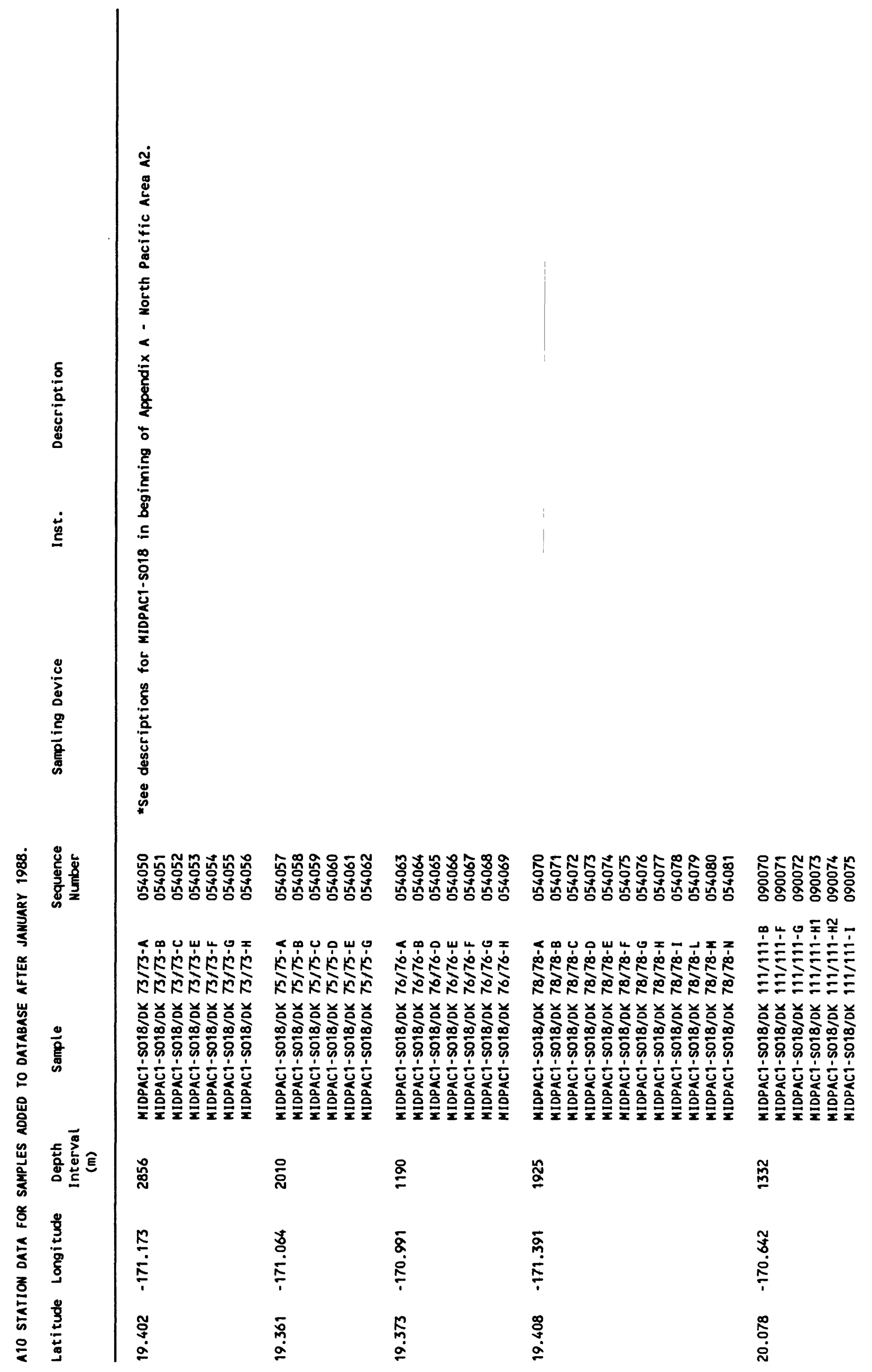




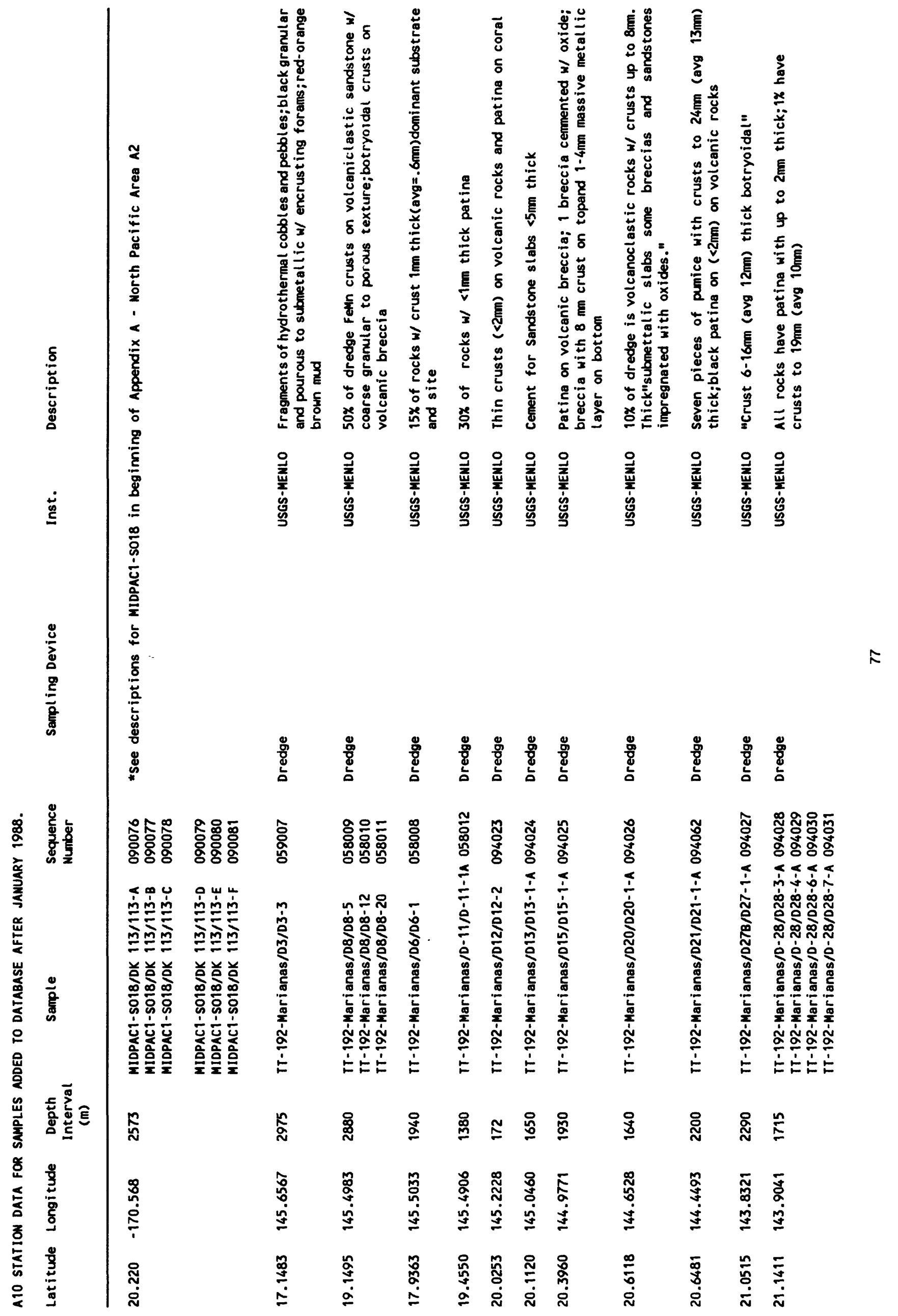




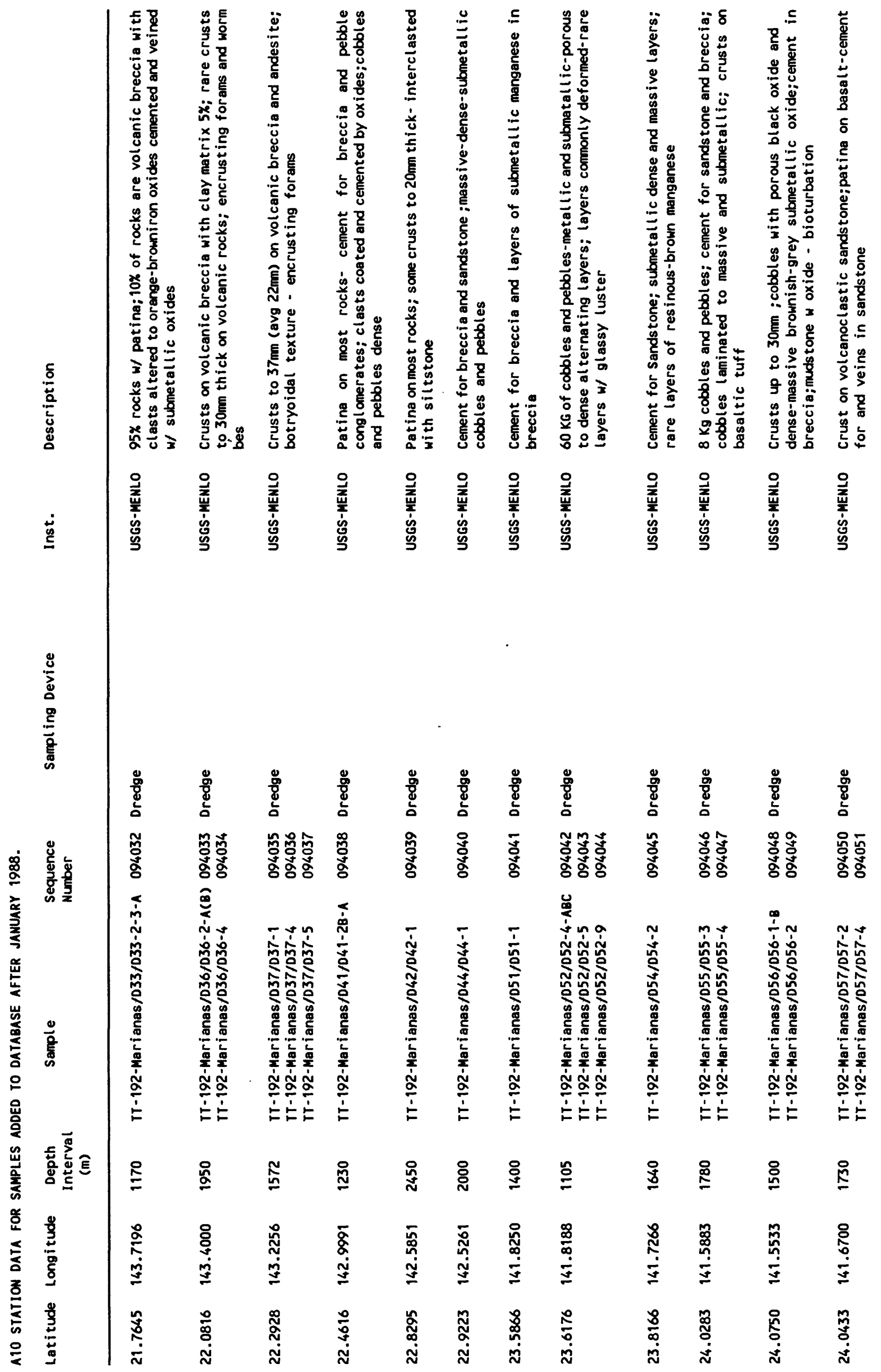




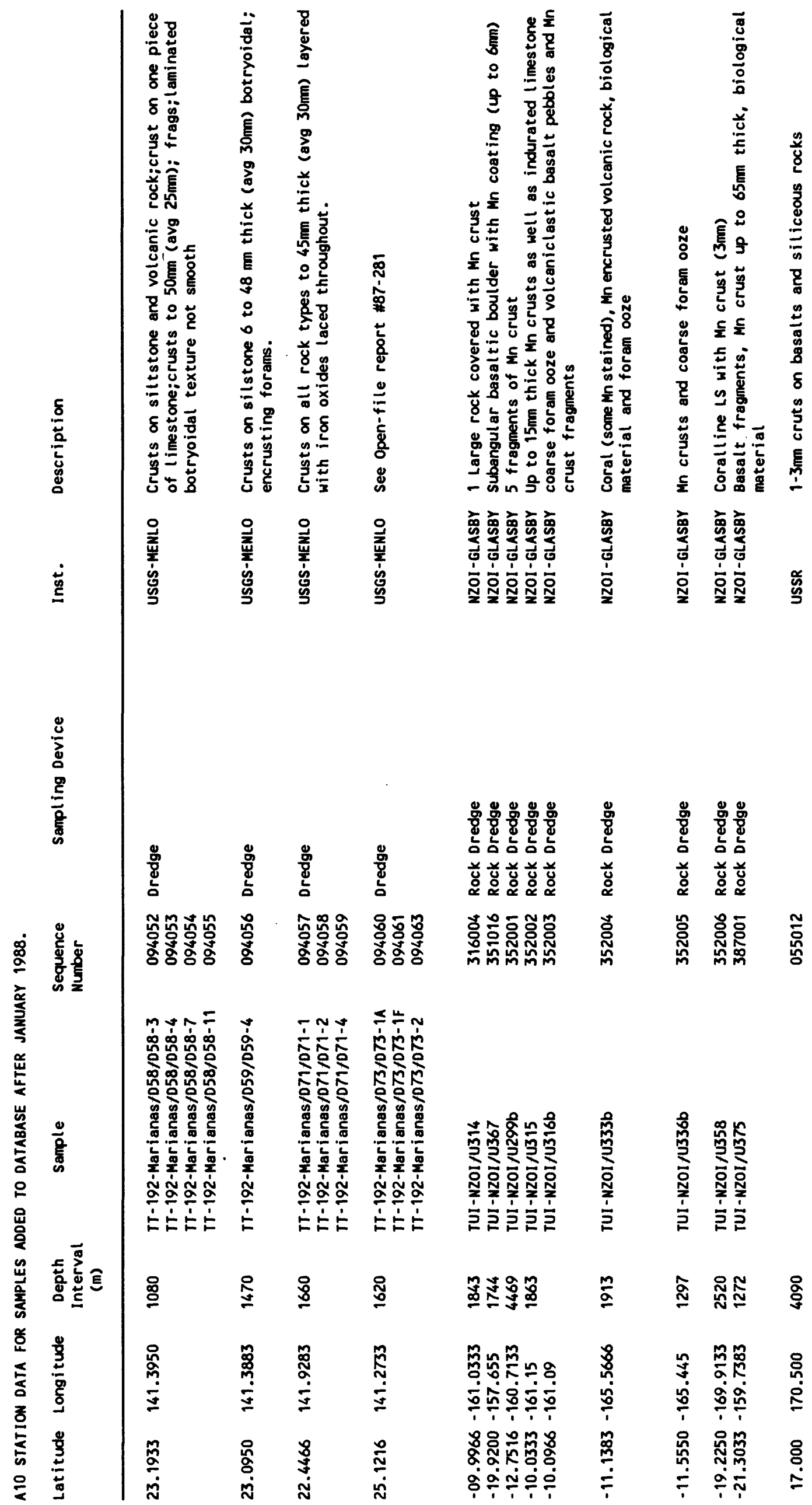


APPENDIX B 
1

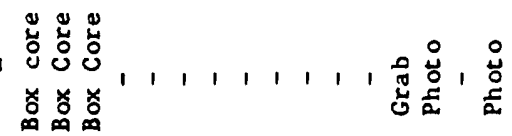

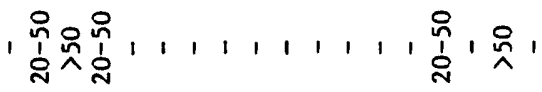

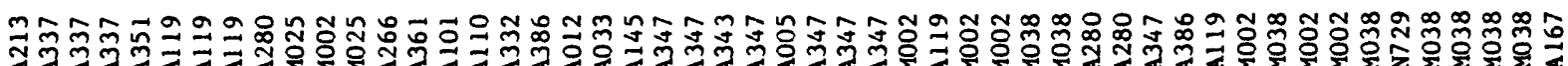

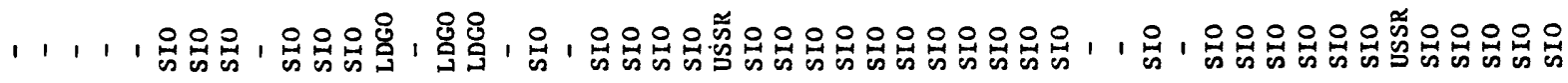

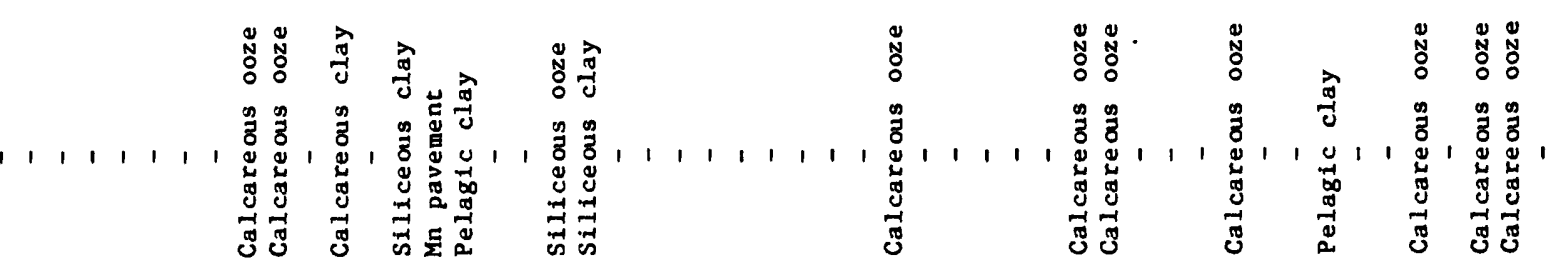

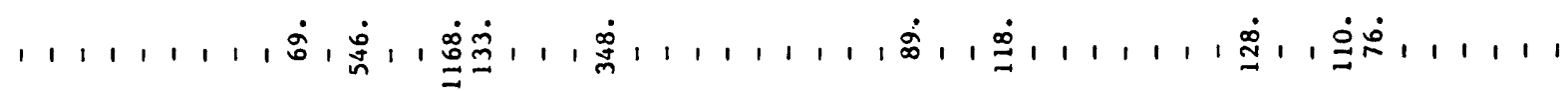

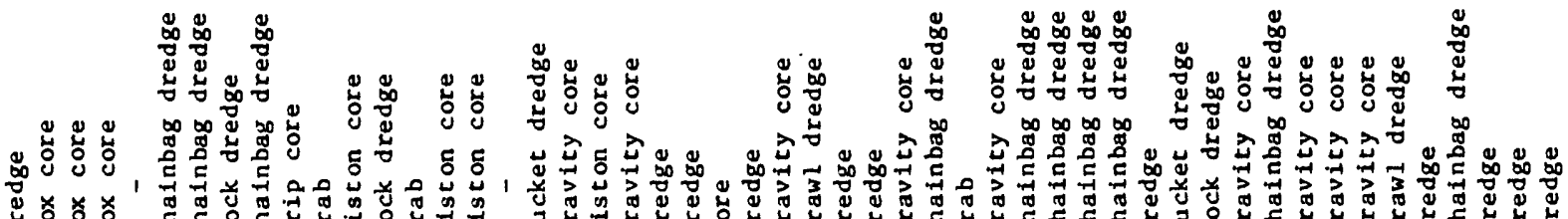

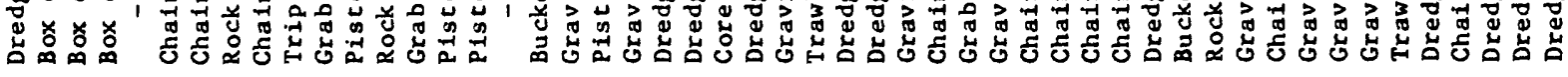

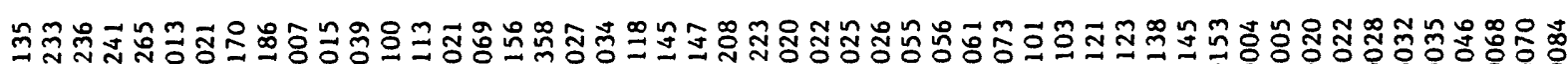

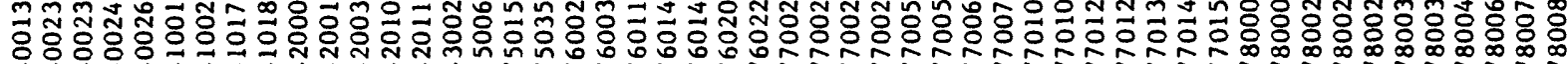

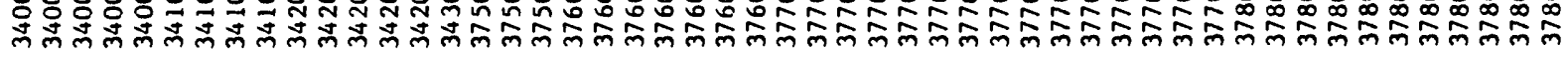

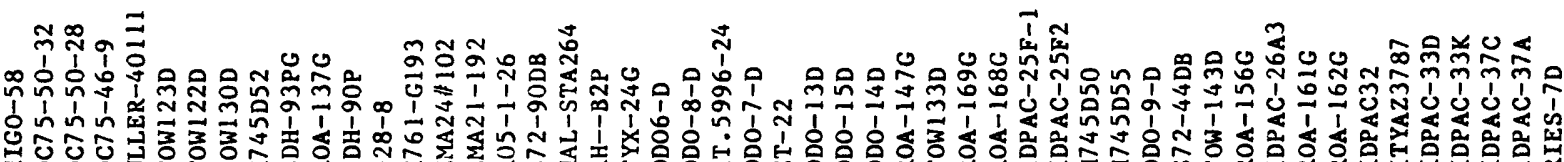

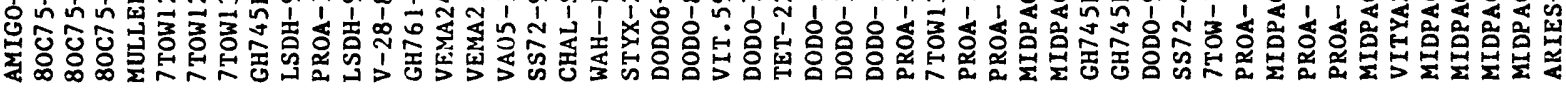

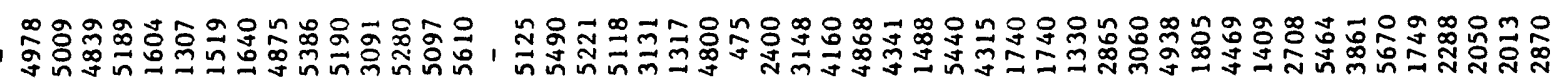

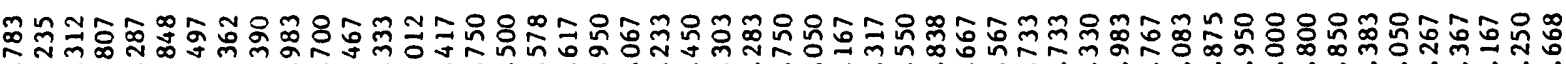

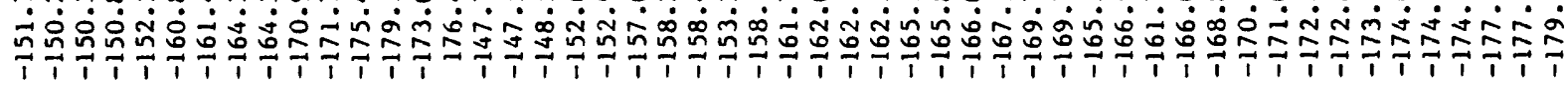

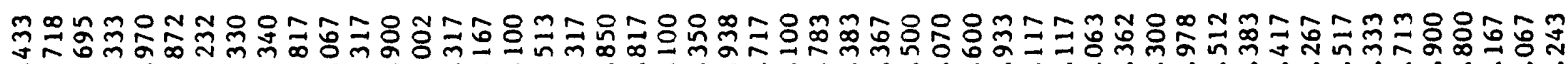

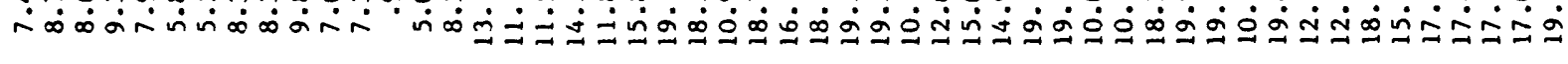




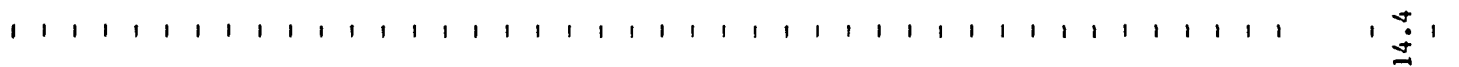

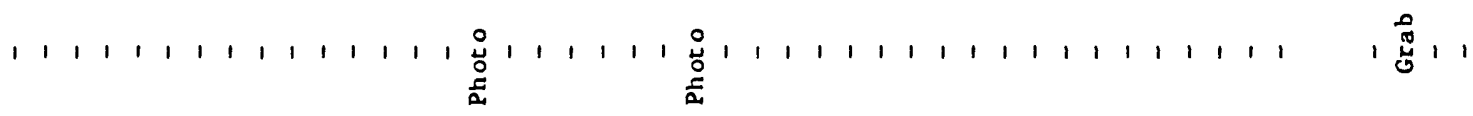

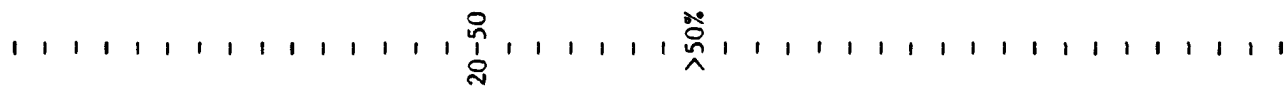

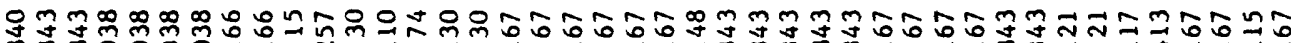

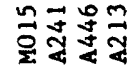

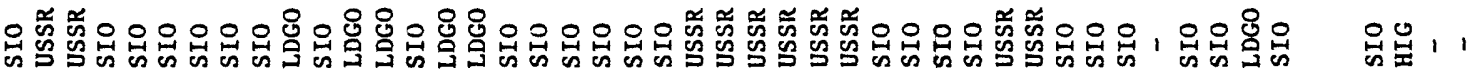

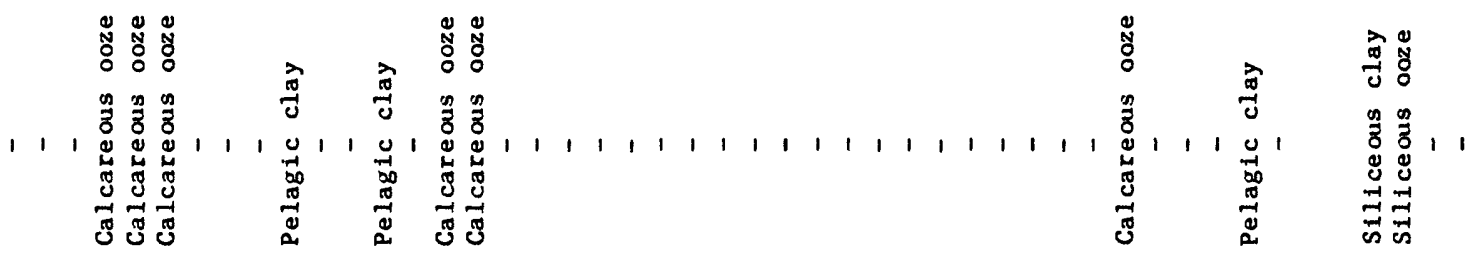

:

$\stackrel{\dot{\infty}}{=}, \stackrel{\dot{n}}{=}$

苛

$\dot{\infty}, 1$

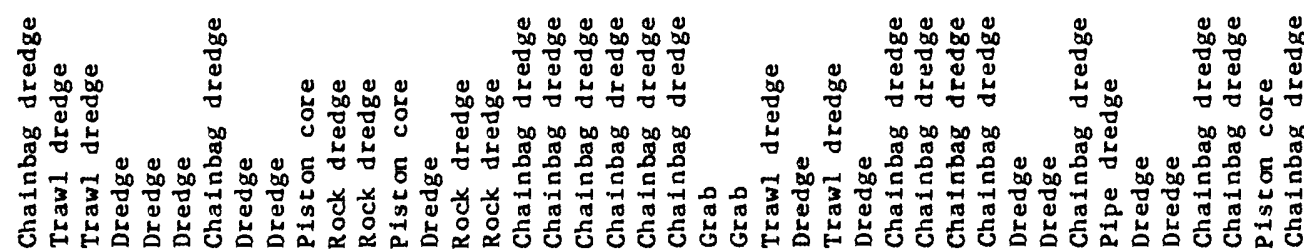

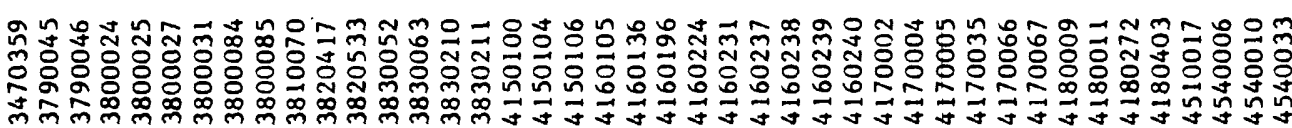

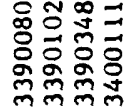

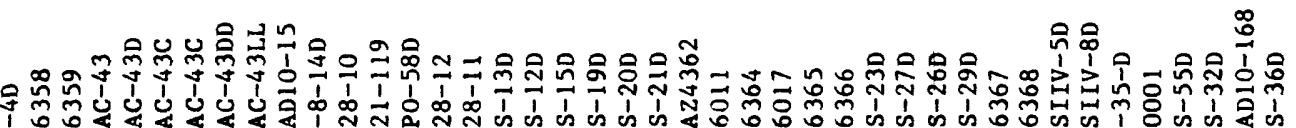

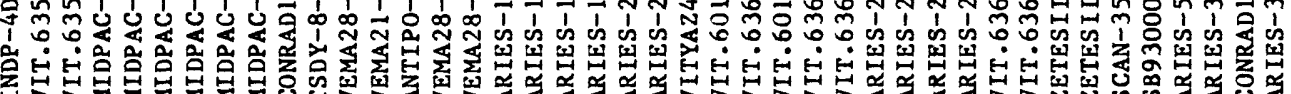

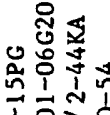

17施

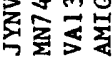

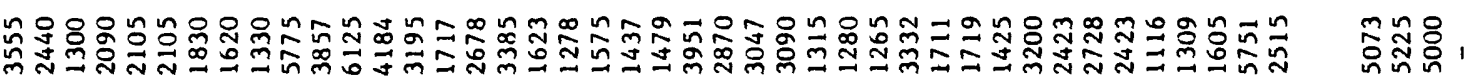

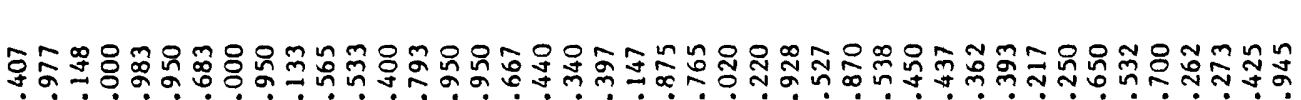

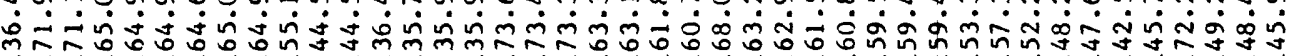

พั่

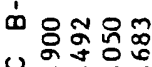

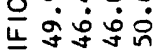

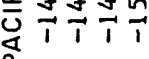

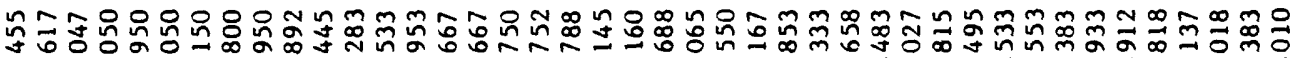

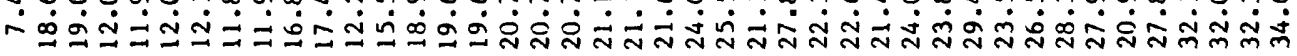

工 


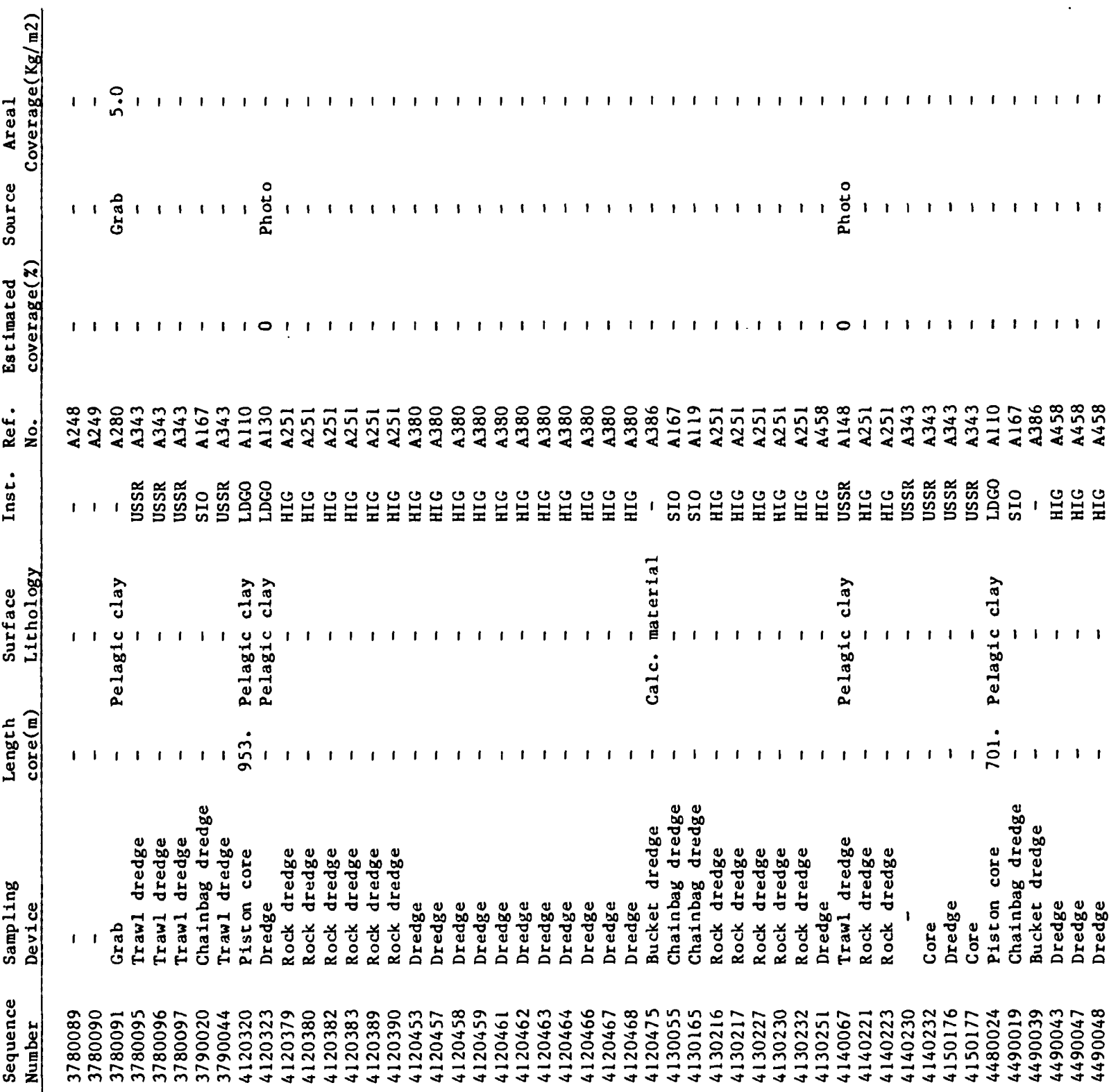

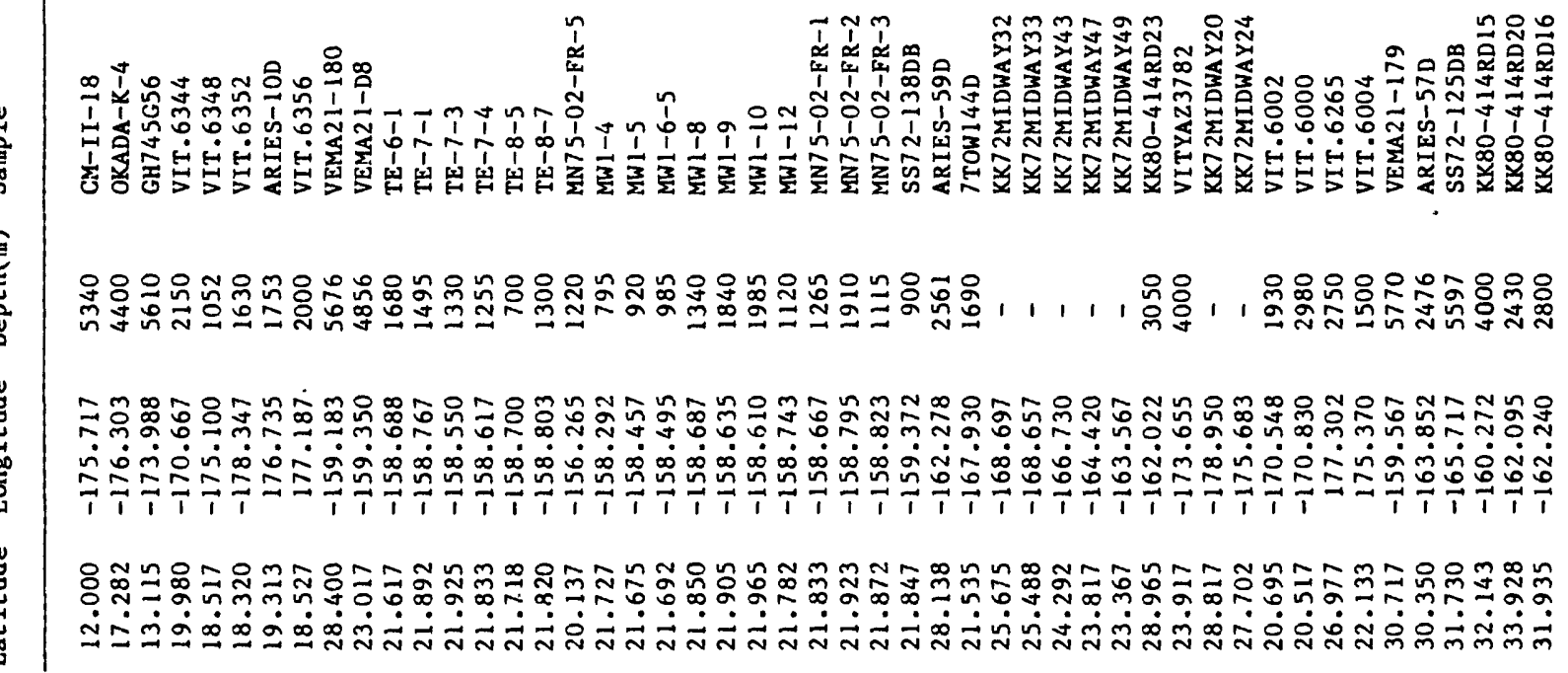




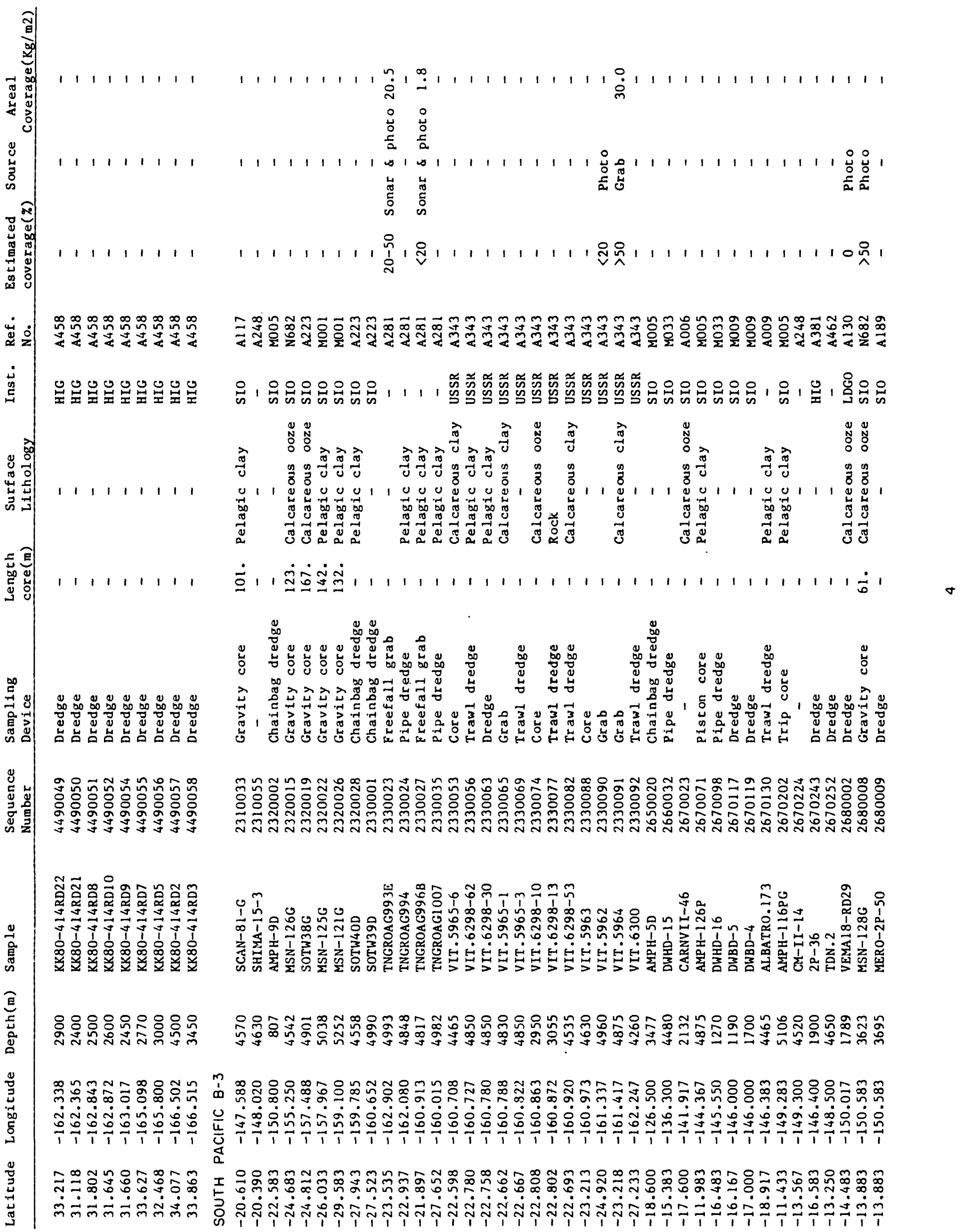




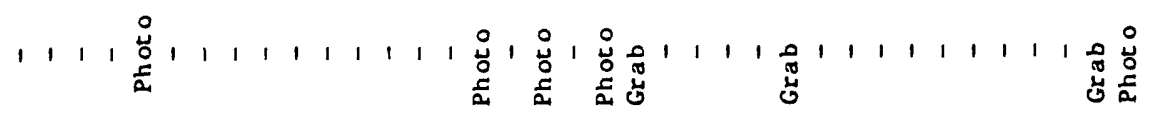

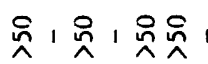

: 귱

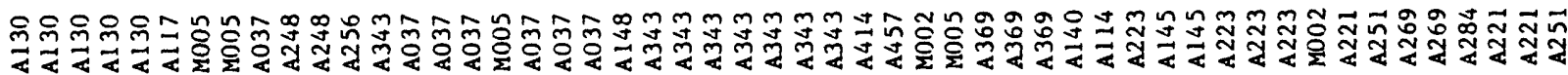

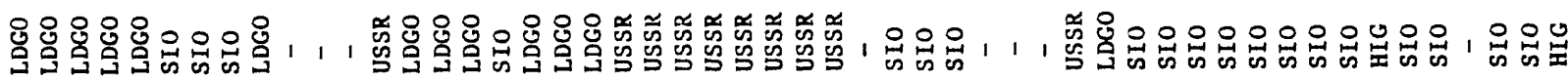

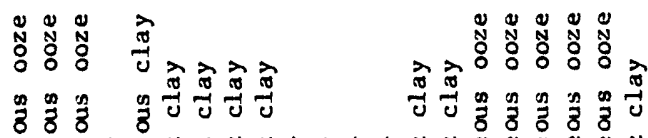

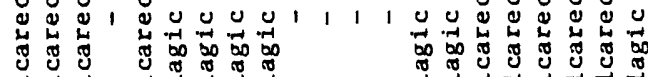

ภु

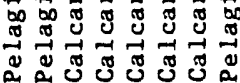

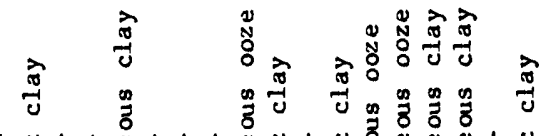

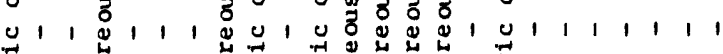

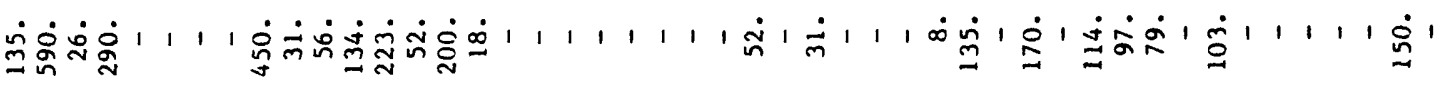

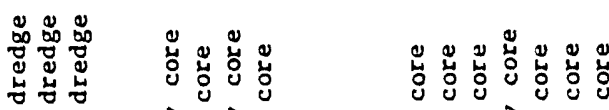

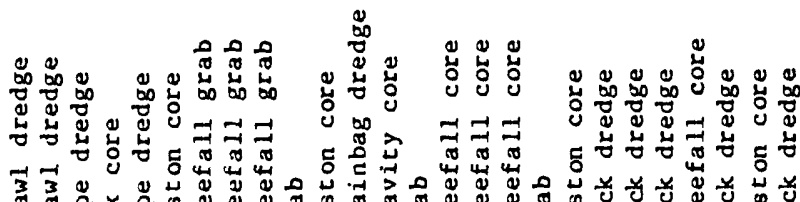

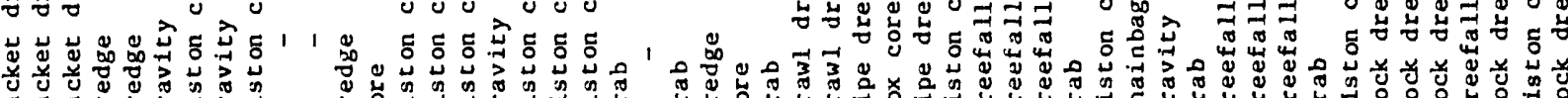

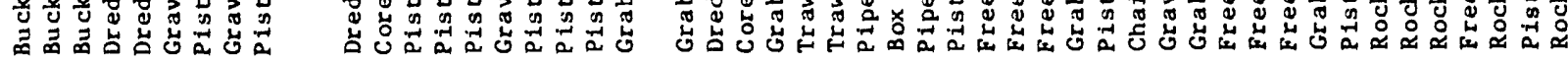

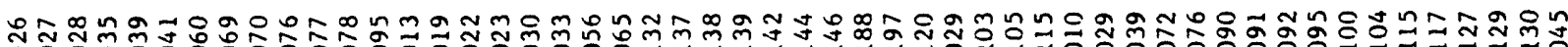

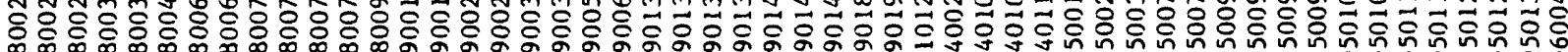

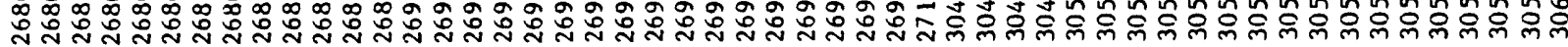

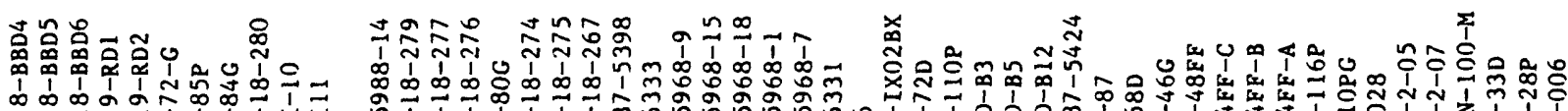

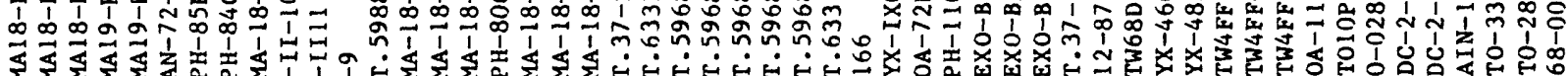

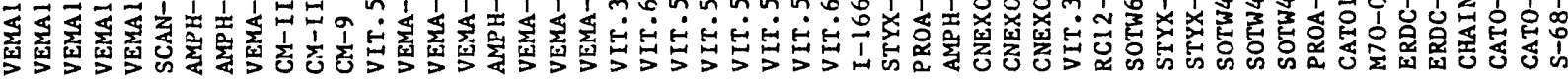

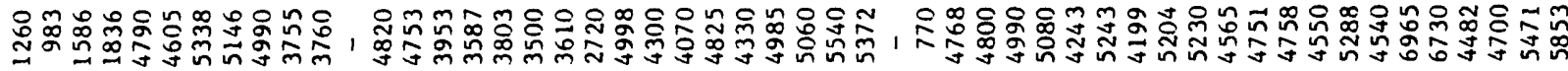

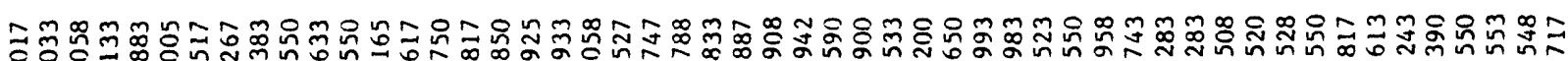

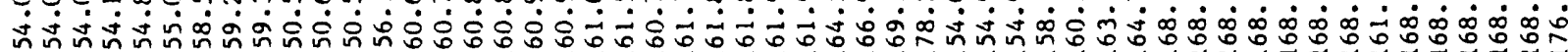

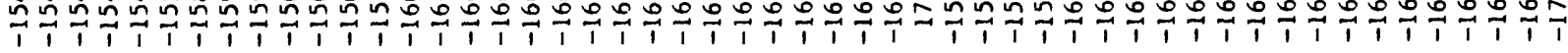

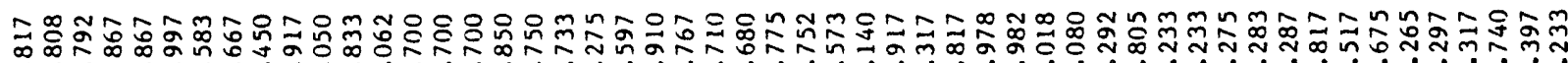

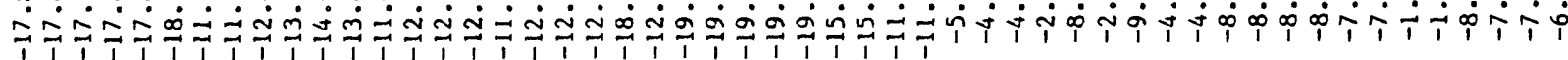




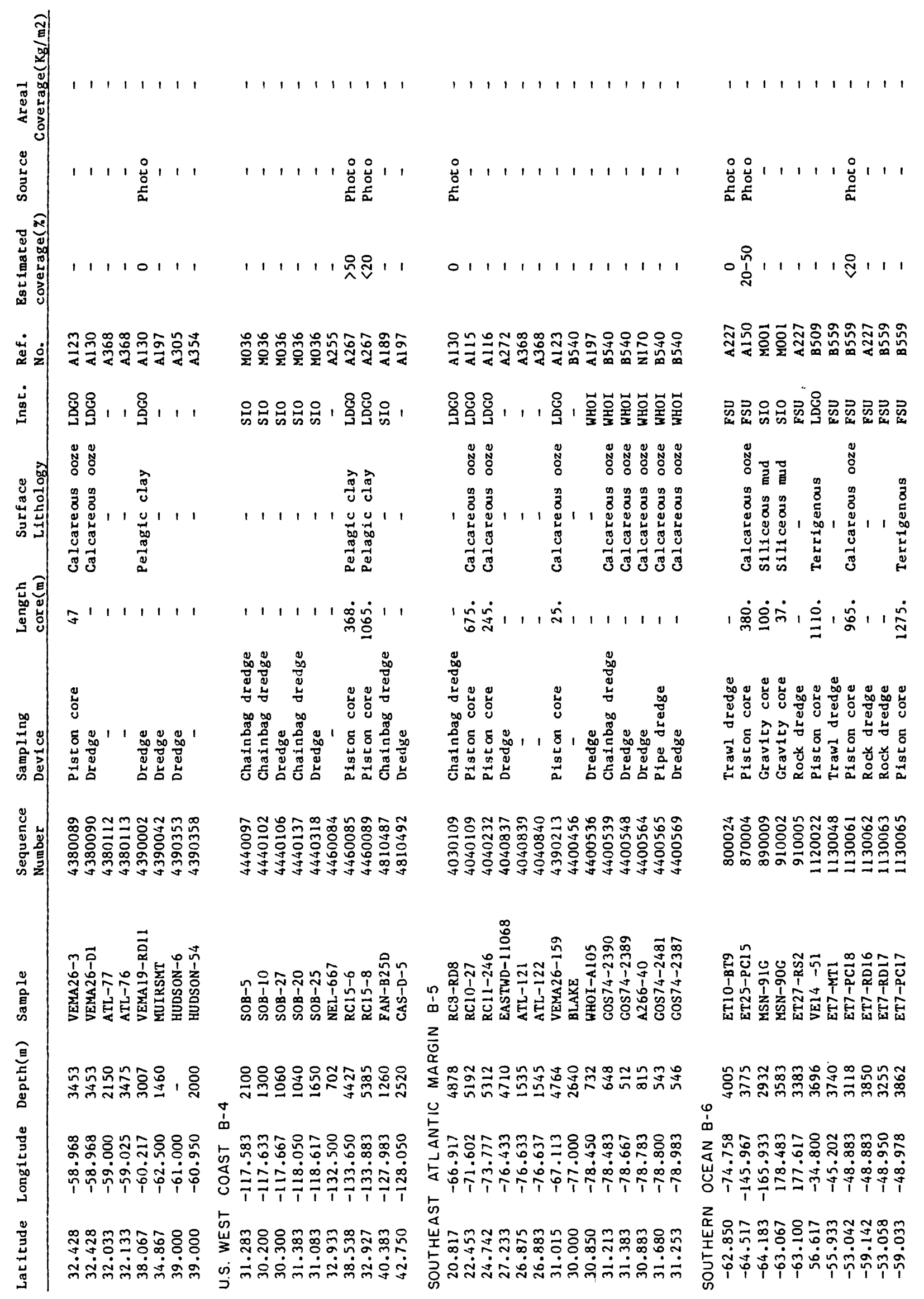




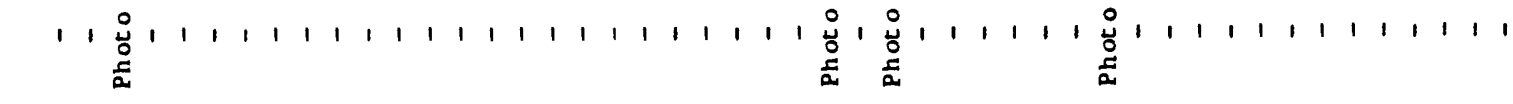

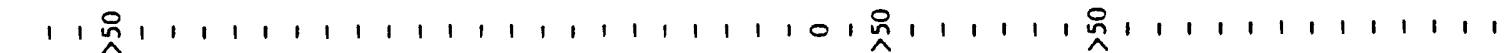
:

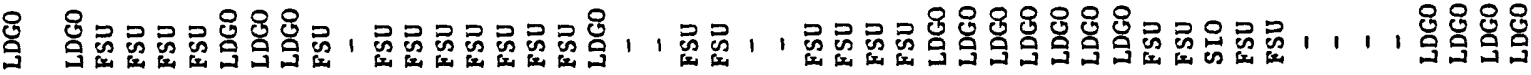

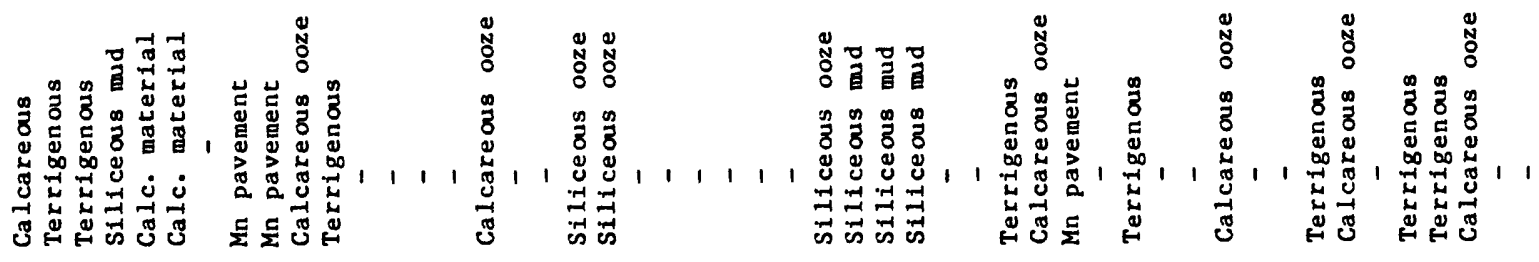

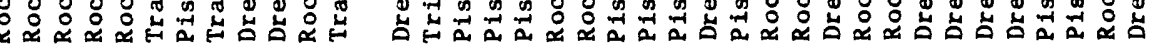

בิ

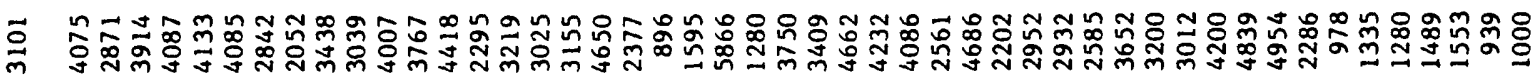

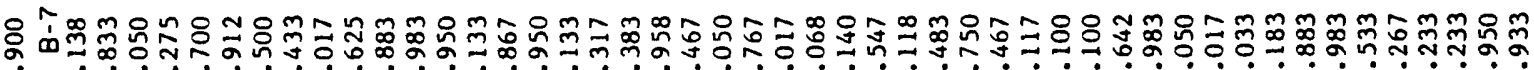

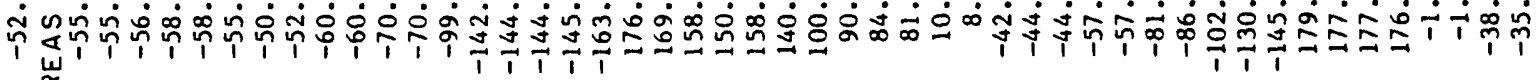

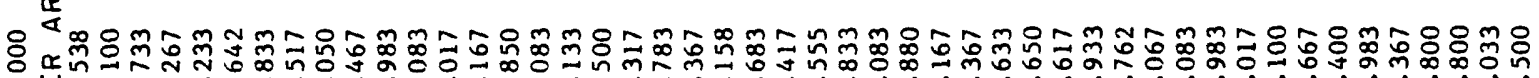

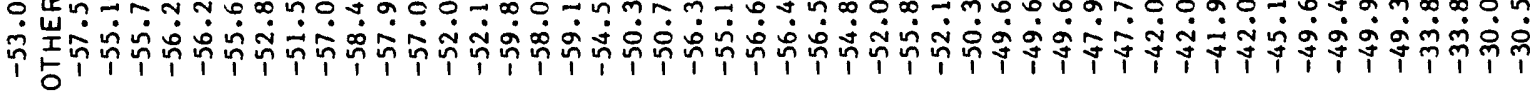




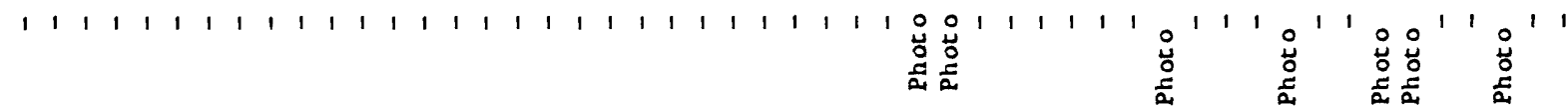

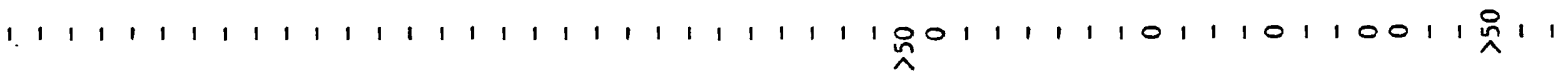

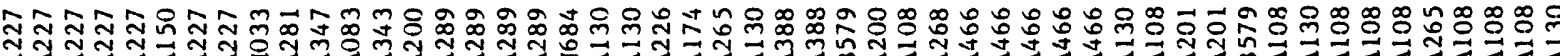

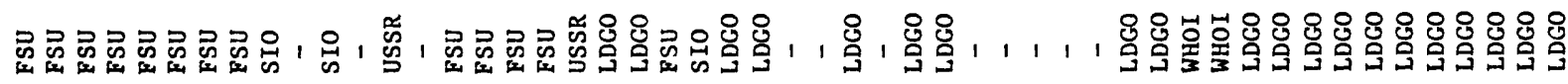

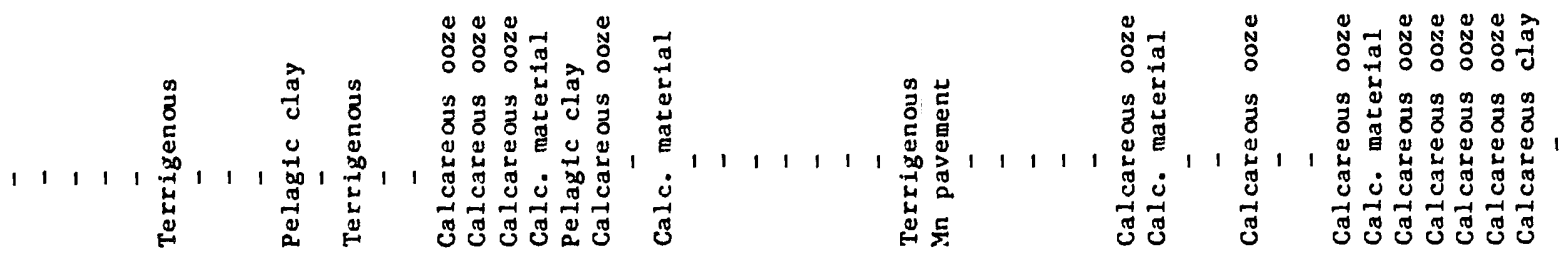

sं

,

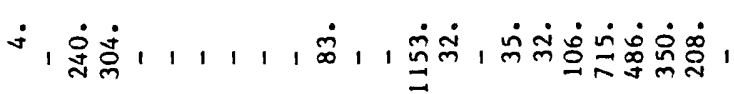

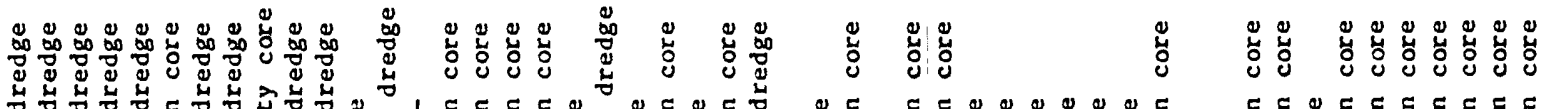

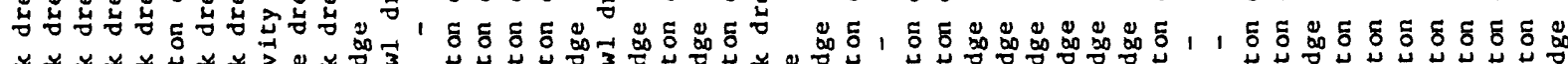

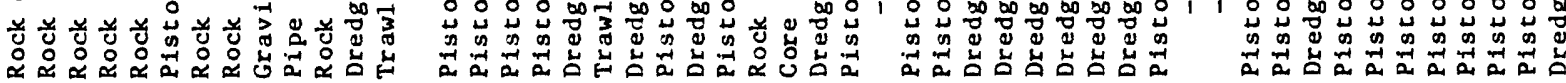

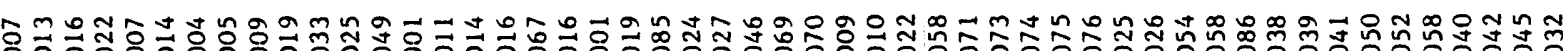

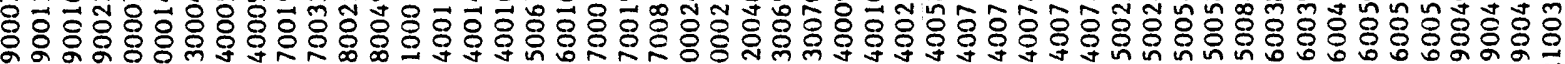

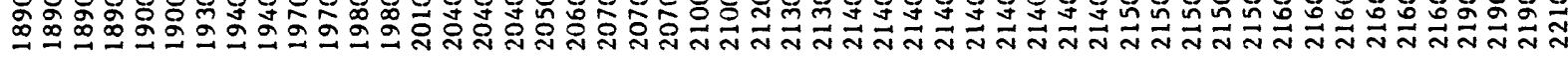

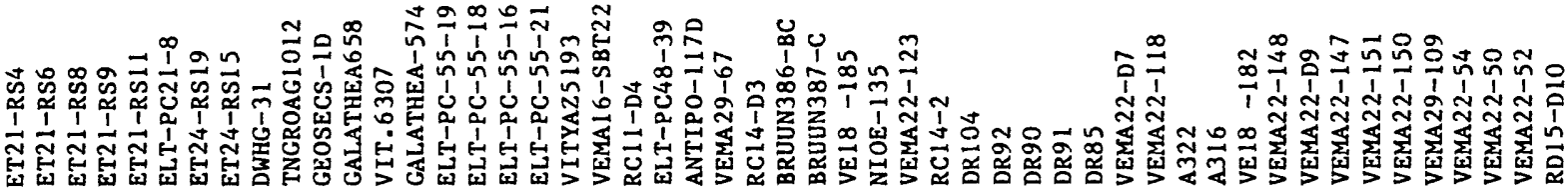

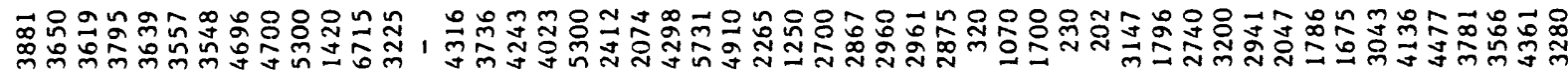

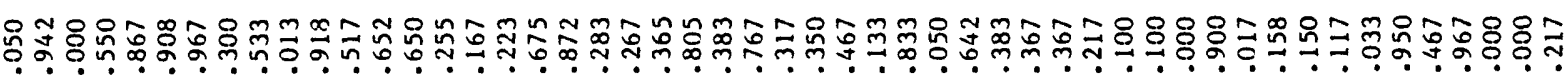

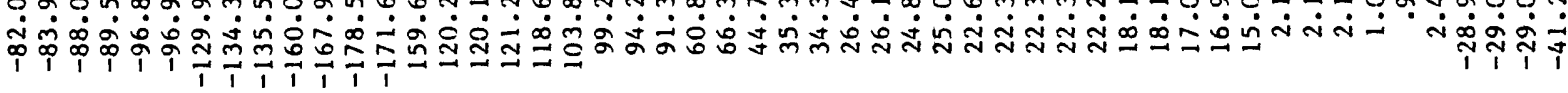

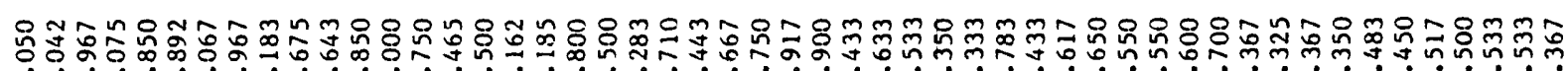

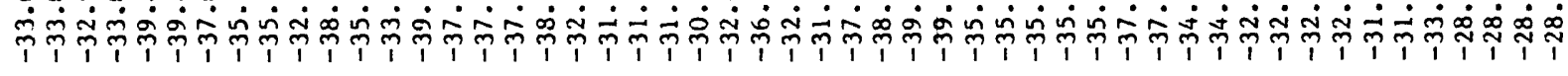




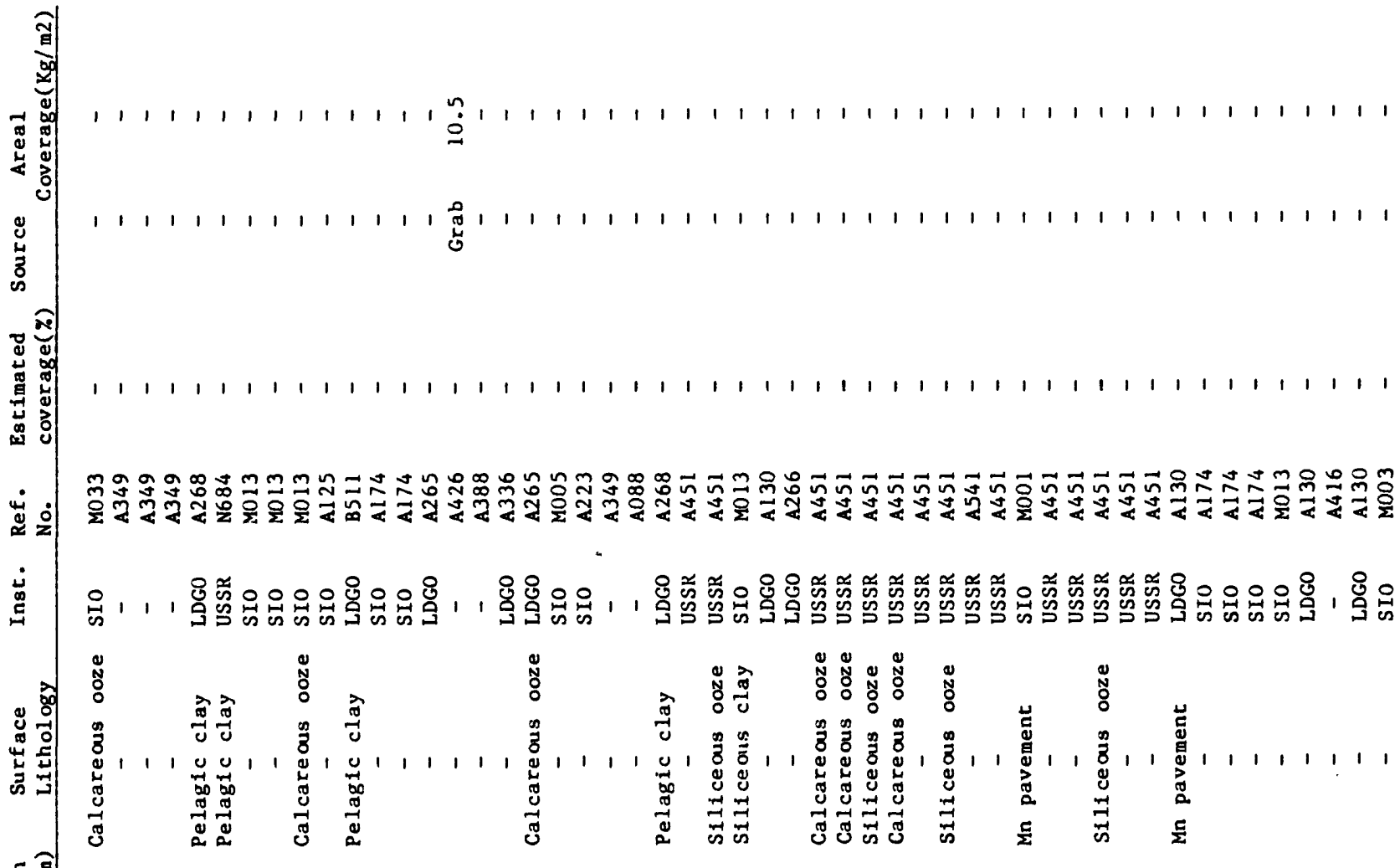

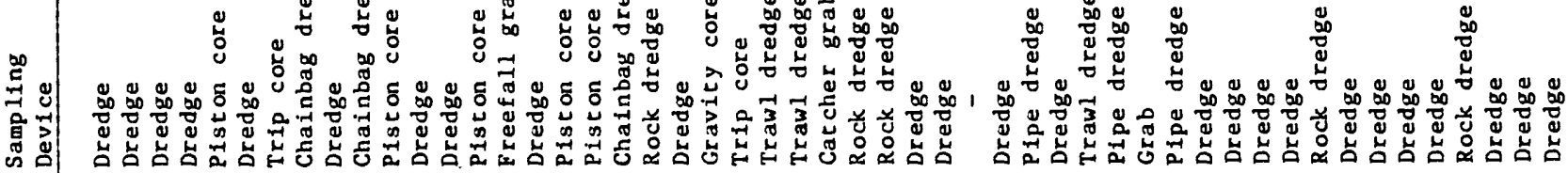

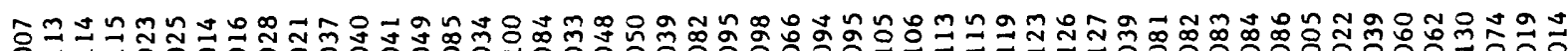

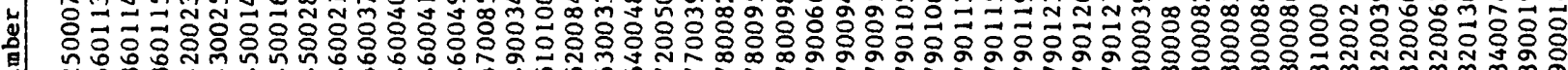

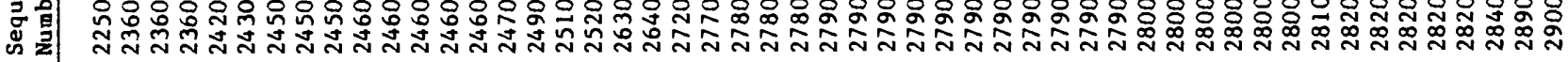

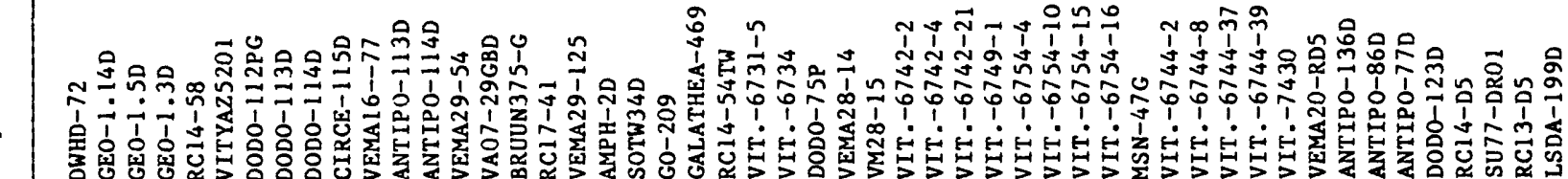

$\widehat{\theta}$

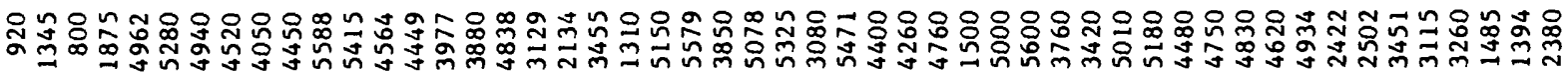

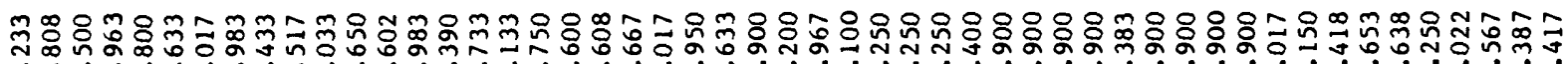
की

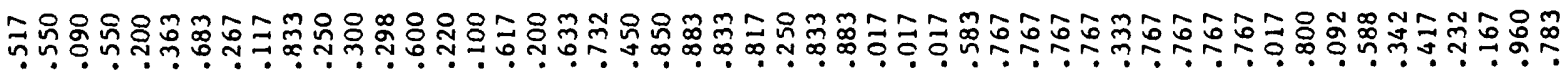
जิ 


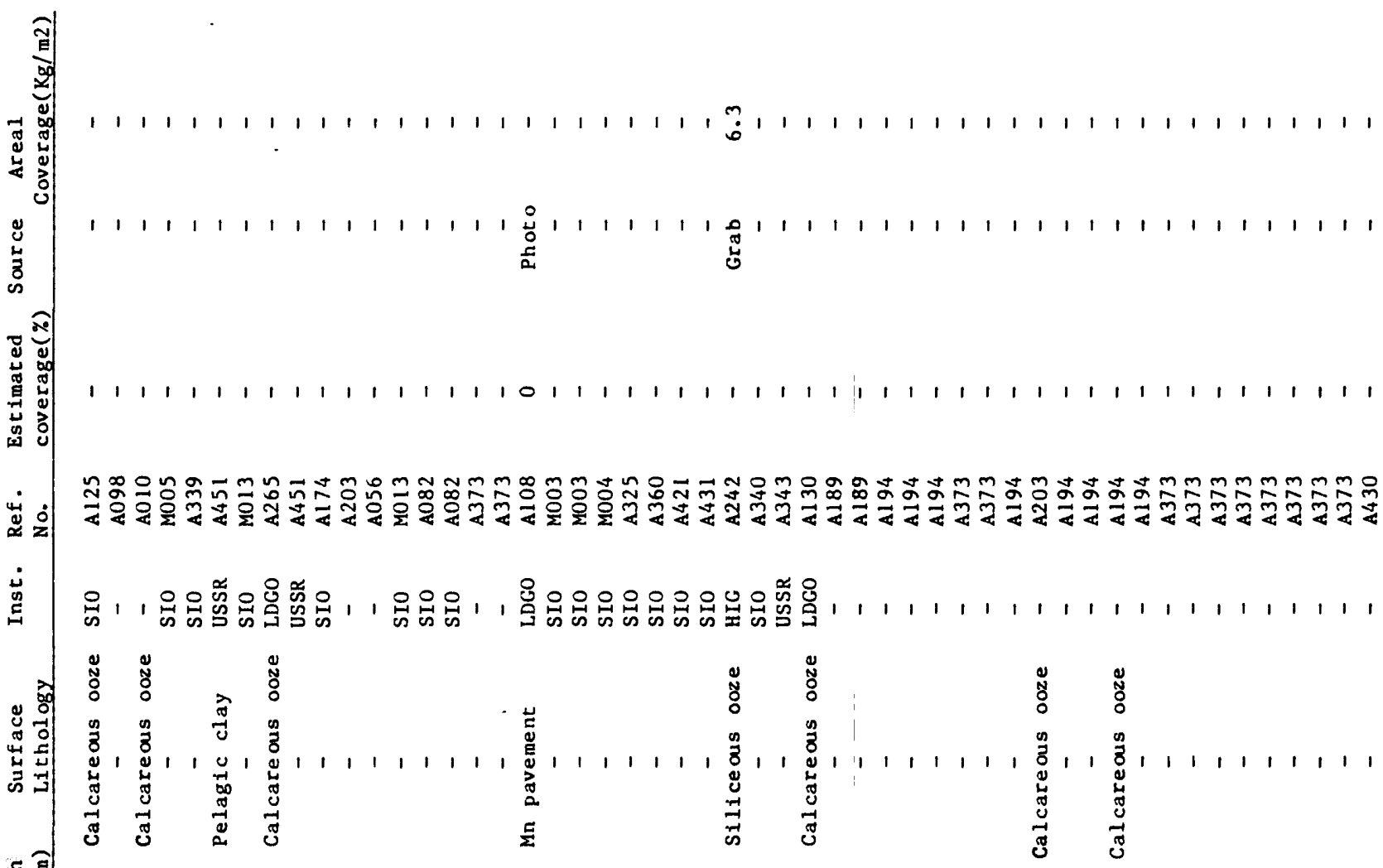

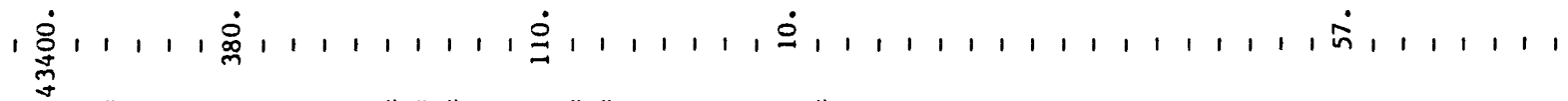

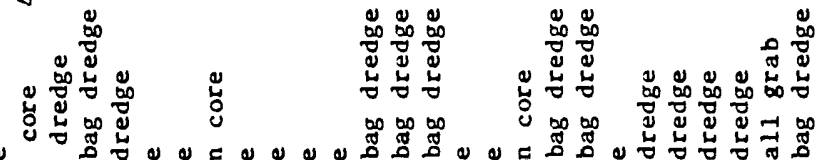

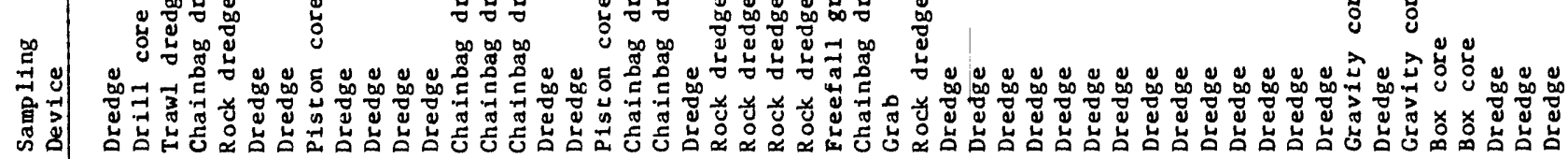

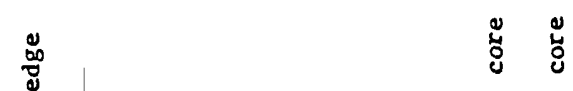

ป

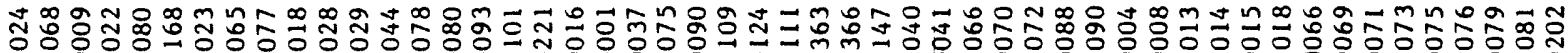

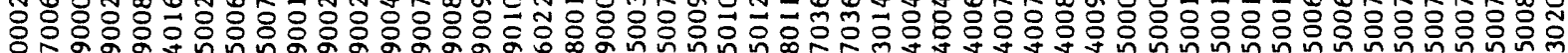

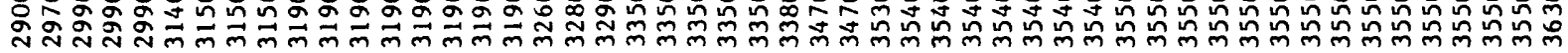

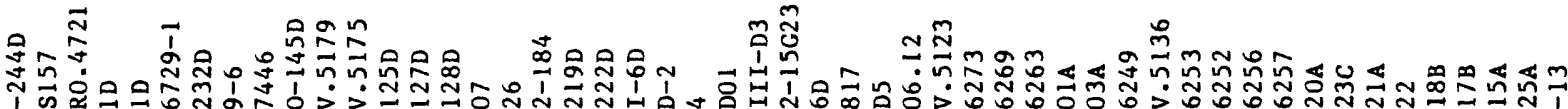

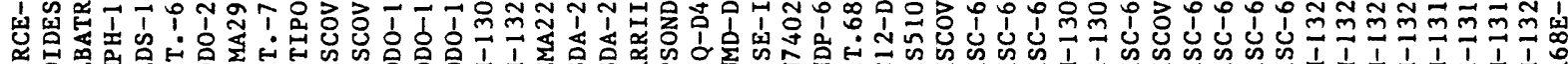

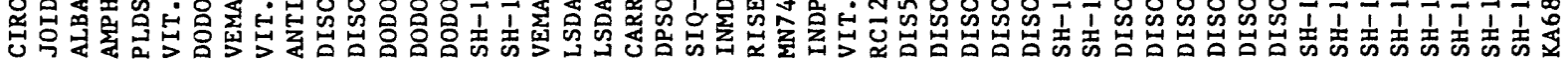

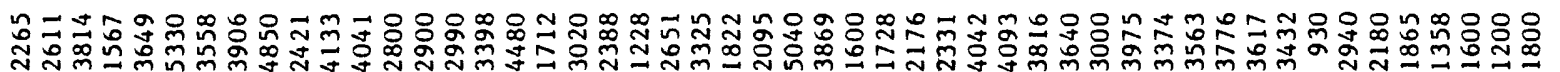

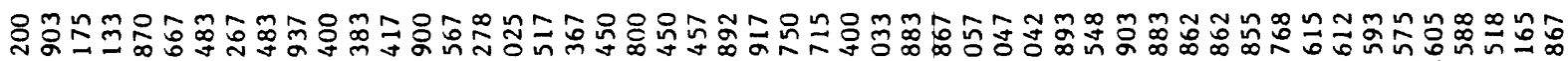

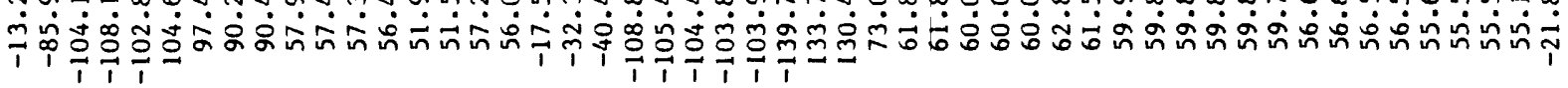

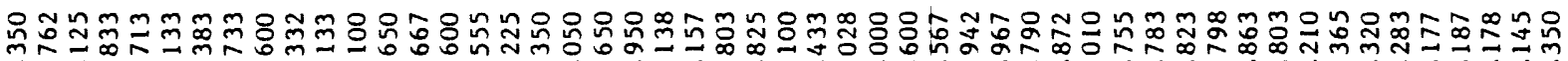

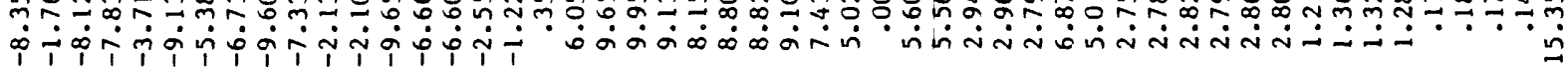




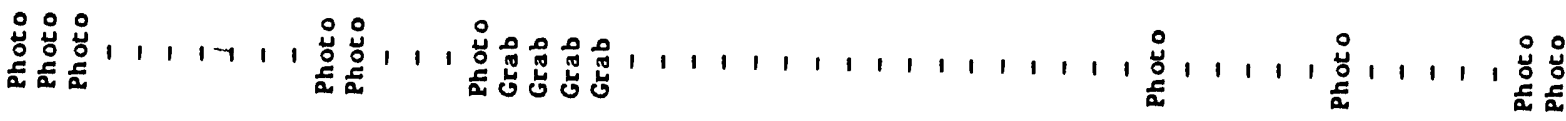

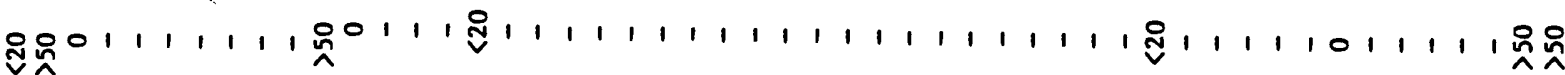

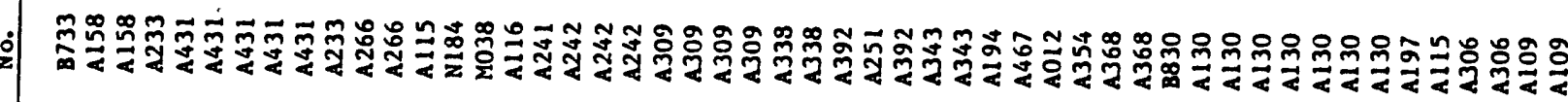

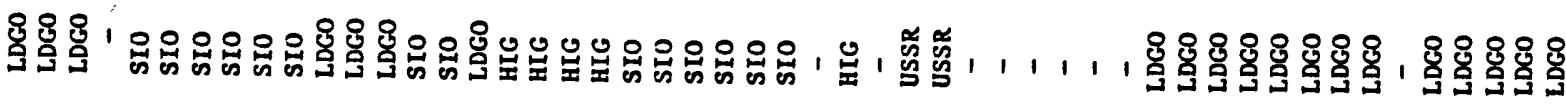

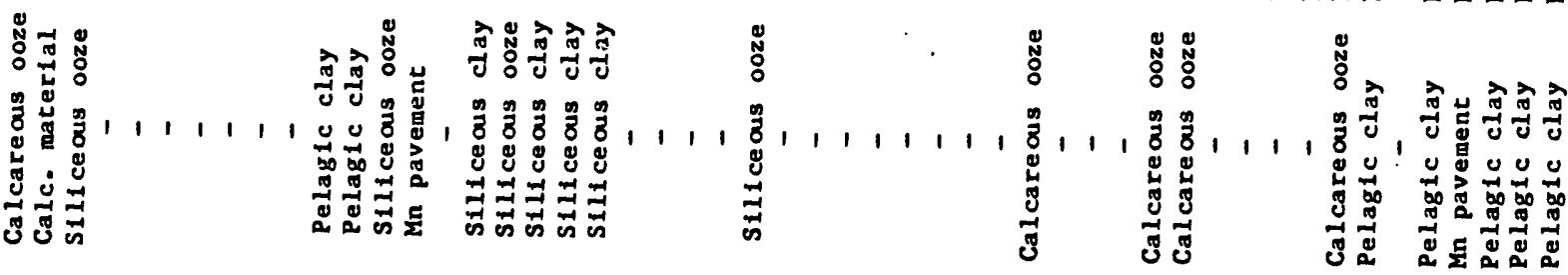

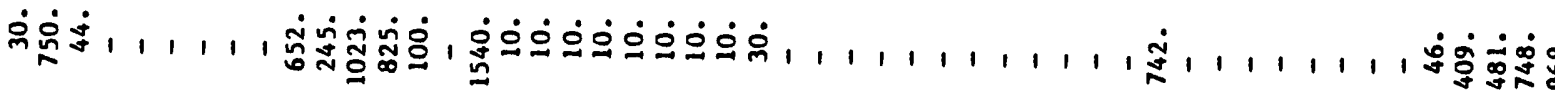

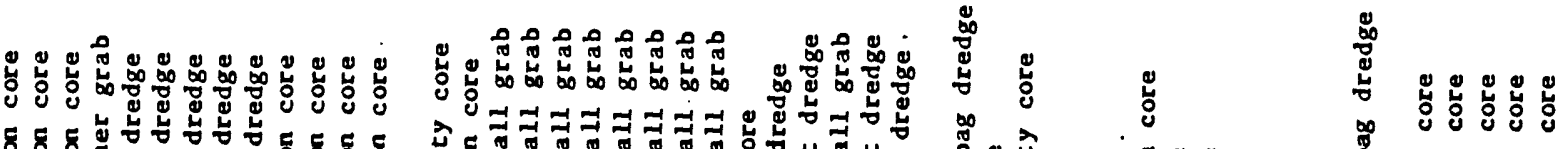

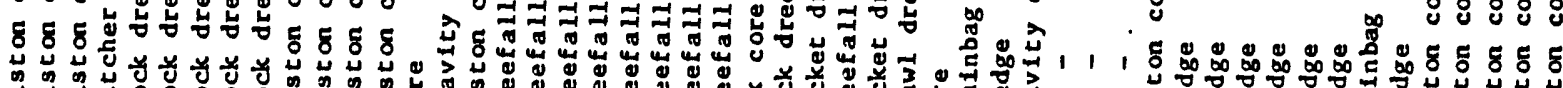

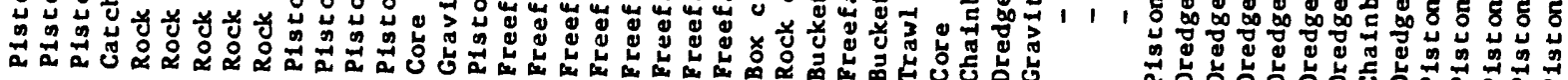

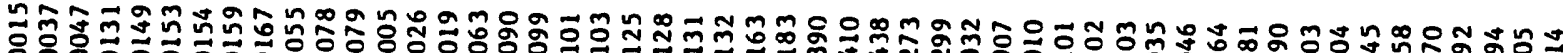

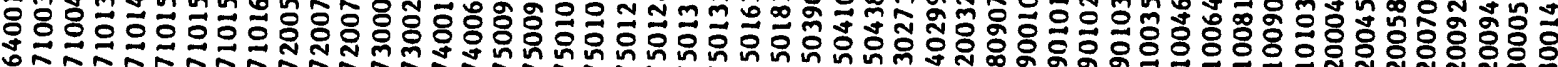

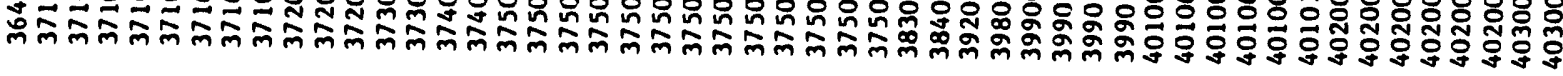

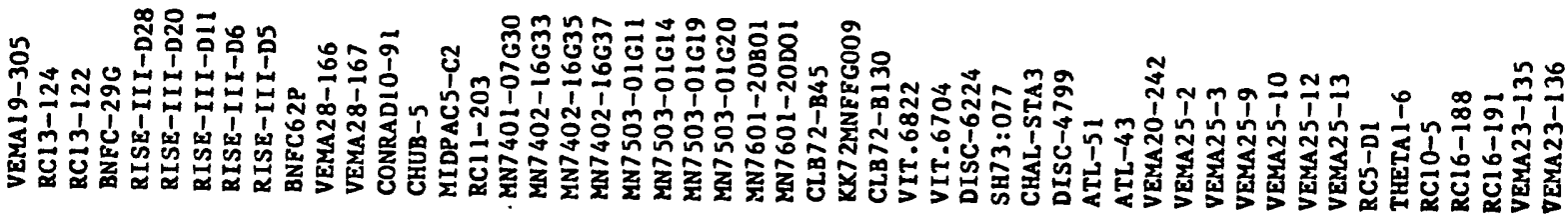

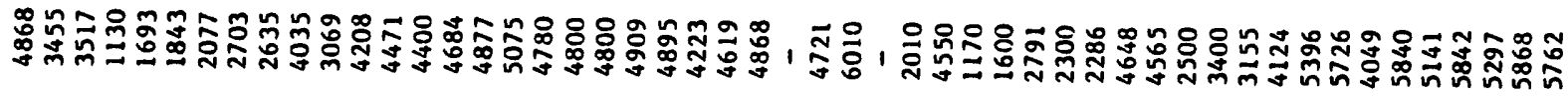

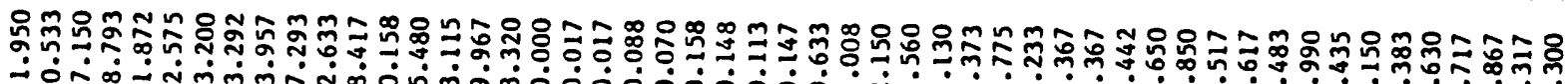

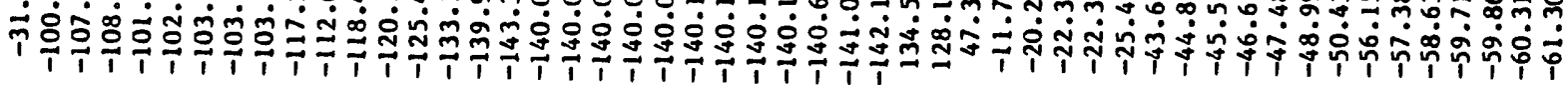

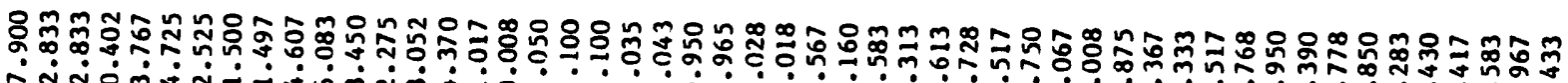

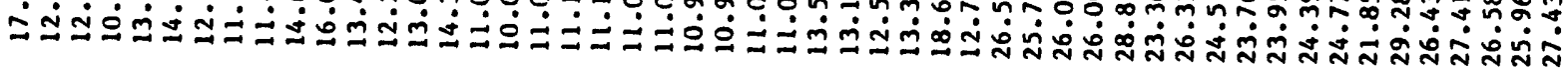




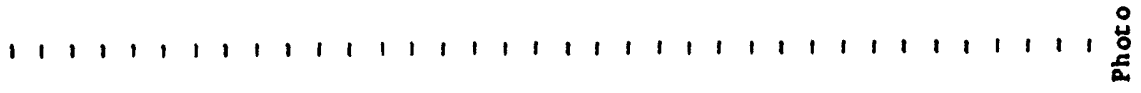

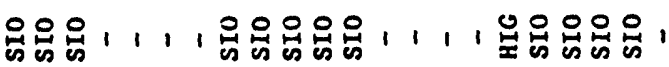

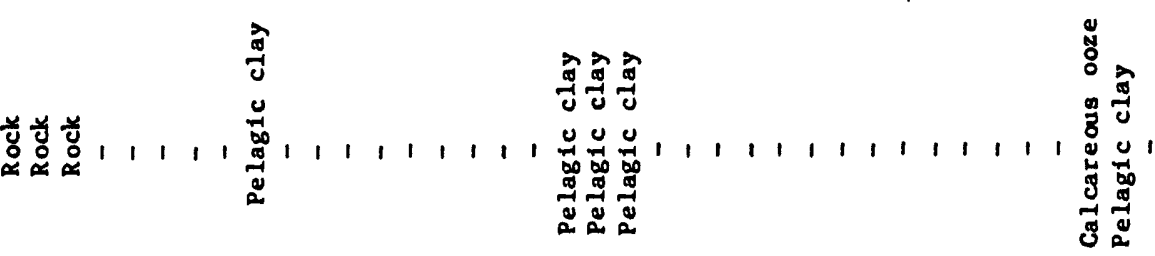

11111111111111111111111118

¿

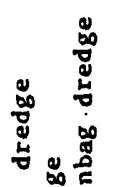

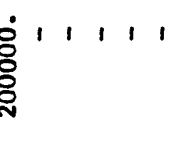

i

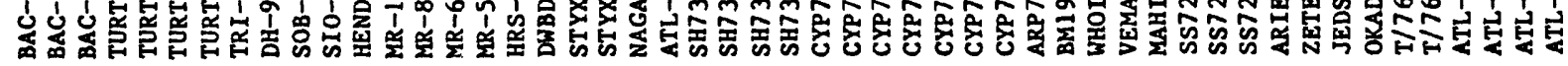

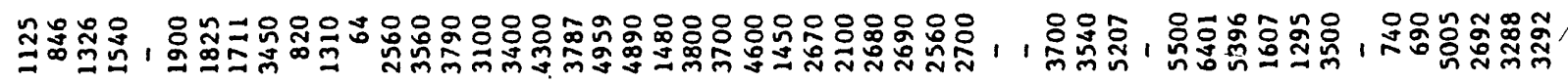

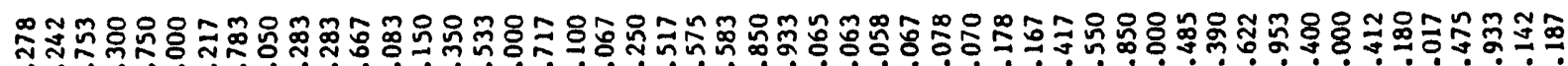

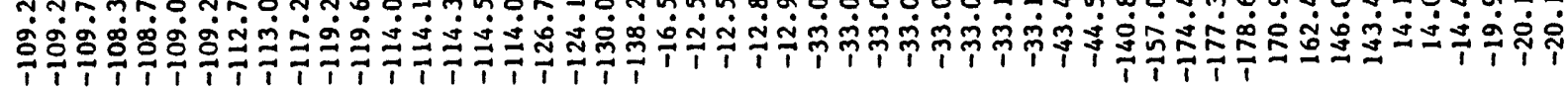

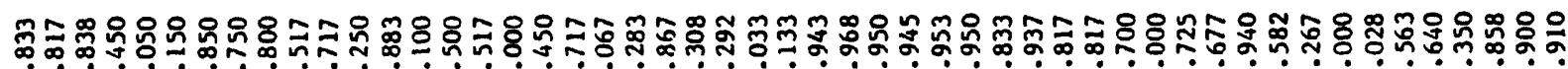
ล் సं் 


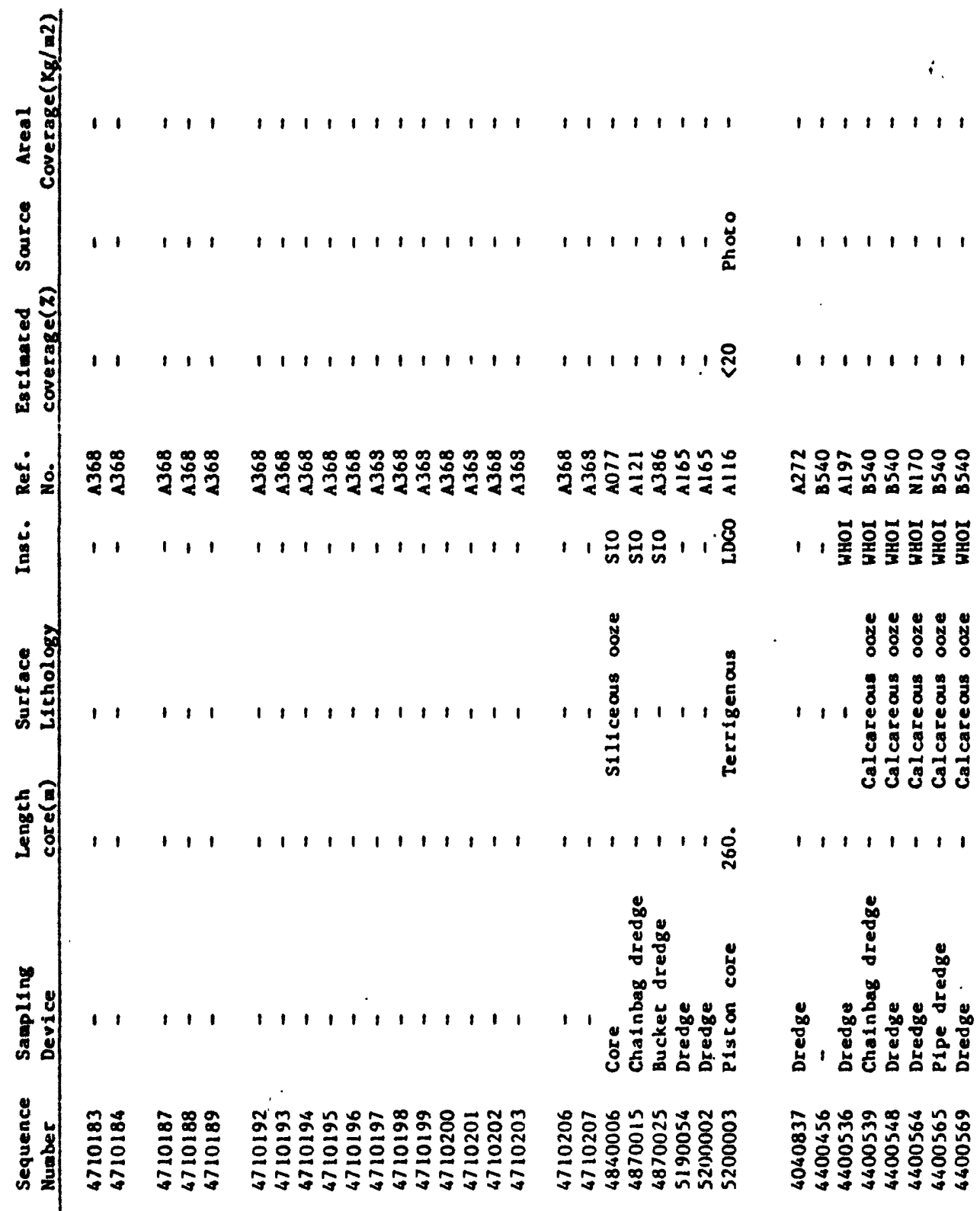

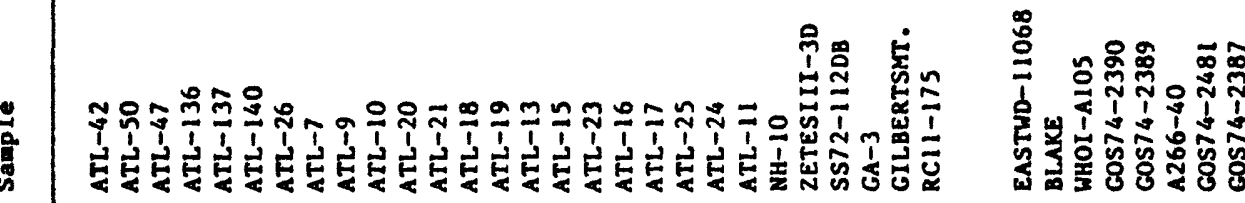

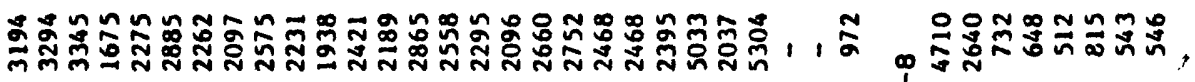

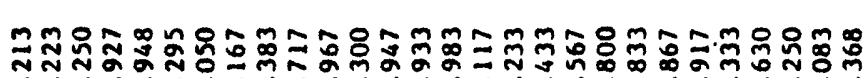

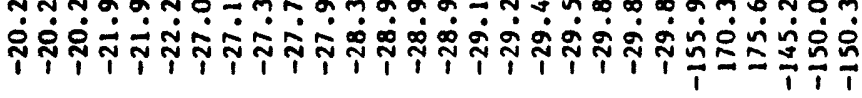

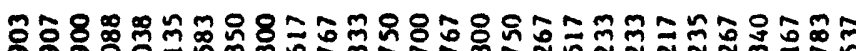

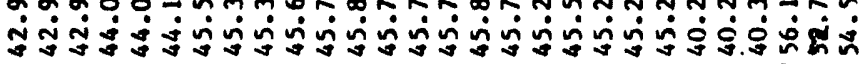
官

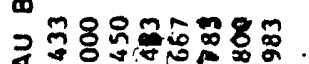
क

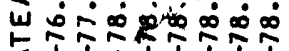
a. w

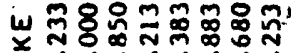

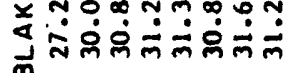


APPENDIX C 
Table 1. Composition of crusts analyzed by USGS Reston Labs

\begin{tabular}{|c|c|c|c|c|c|c|c|c|c|c|c|c|}
\hline $\begin{array}{l}\text { Soquencell } \\
\text { Lationds } \\
\text { Longitude } \\
\text { Depth } \\
\text { Labl }\end{array}$ & $\begin{array}{c}002002 \\
.150 \\
-17.317 \\
3150 \\
\text { W.2222728 }\end{array}$ & $\begin{array}{c}009001 \\
1.875 \\
-86548 \\
2750 \\
W-224394\end{array}$ & $\begin{array}{c}\text { cosc02 } \\
3.877 \\
-81.687 \\
2240 \\
\text { w-222717 }\end{array}$ & $\begin{array}{c}009003 \\
535 \\
-85.605 \\
2340 \\
\text { w.222577 }\end{array}$ & $\begin{array}{c}009004 \\
3.020 \\
84.237 \\
2203 \\
\text { w-222321 }\end{array}$ & $\begin{array}{c}010001 \\
1.385 \\
-93.733 \\
2643 \\
\text { W-224389 }\end{array}$ & $\begin{array}{c}010002 \\
1.817 \\
-93.635 \\
2255 \\
w-224390\end{array}$ & $\begin{array}{c}010003 \\
2752 \\
-95.245 \\
2168 \\
w-224391\end{array}$ & $\begin{array}{c}010004 \\
3.525 \\
-93.798 \\
2795 \\
w-224392 \\
\end{array}$ & $\begin{array}{c}010005+ \\
3.350 \\
-93.648 \\
2969 \\
\text { W-224393 }\end{array}$ & $\begin{array}{c}010008 \\
2.033 \\
-95.467 \\
3000 \\
w-222518\end{array}$ & $\begin{array}{c}010009 \\
2.698 \\
-95.242 \\
2596 \\
\text { w-231372 }\end{array}$ \\
\hline $\mathrm{SiO}_{2}$ (wt.\%) & 28.5 & 14.9 & 27.6 & 16.1 & 13.1 & 25.9 & 19.0 & 25.1 & 208 & 47.6 & 15.3 & $\mu$ \\
\hline & .74 & 49 & .85 & $A 3$ & .08 & .72 & 1.24 & 1.21 & 1.02 & 200 & 24 & .02 \\
\hline & 145 & 449 & 235 & 46.7 & 18.6 & 205 & 28.3 & 215 & 264 & 241 & 53.2 & 75.1 \\
\hline & 20.7 & 16.2 & 15.8 & 121 & 47.1 & 204 & 27.1 & 24.8 & 252 & 115 & 7.62 & 37 \\
\hline & 7.28 & 3.39 & 7.19 & 3.38 & 1.62 & 220 & 4.02 & 6.31 & 5.35 & 16.1 & 4.49 & 20 \\
\hline & .137 & .040 & .047 & .049 & .034 & .096 & .125 & .086 & .089 & .009 & .032 & $<.001$ \\
\hline Nó & .159 & .954 & .711 & .960 & .117 & 941 & .434 & 311 & 374 & .052 & .805 & .089 \\
\hline Co & .057 & .330 & .092 & .204 & .098 & .166 & .084 & .061 & .085 & .025 & .584 & .034 \\
\hline $\mathrm{CaO}$ & 3.72 & 222 & 3.83 & 2.68 & 2.69 & 278 & 4.92 & 5.45 & 5.20 & 9.10 & 155 & 1.95 \\
\hline MoO & 9.01 & 3.68 & 3.35 & 2.97 & 1.71 & 4.57 & 3.00 & 3.50 & 3.31 & 590 & 3.85 & 3.31 \\
\hline $\mathrm{Na}_{2} \mathrm{O}$ & 289 & 2.53 & 3.33 & 3.20 & 2.06 & 254 & 2.45 & 3.11 & 252 & 3.35 & 249 & 4.37 \\
\hline & .60 & 98 & .87 & 1.06 & .48 & 1.33 & 54 & .74 & .54 & 1.35 & 1.43 & 1.42 \\
\hline $\mathrm{CO}_{2}$ & .22 & 22 & .45 & 12 & 50 & 49 & so & 58 & .39 & .06 & .10 & .02 \\
\hline & .62 & 39 & .68 & 38 & 1.39 & .48 & .76 & .66 & .72 & .40 & 28 & $<06$ \\
\hline $\mathrm{H}_{2} \mathrm{O}+$ & 9.6 & 10.2 & 7.8 & 5.9 & 8.7 & 85 & 9.1 & 6.9 & 7.7 & 15 & 73 & 9.3 \\
\hline $\mathrm{H}_{2} \mathrm{O}$ & 11.7 & 5.3 & 15.9 & 21.8 & 19.3 & 9.4 & 6.1 & 10.1 & 4.7 & 1.6 & 9.9 & 6.9 \\
\hline sum & 98.8 & 1015 & 96.2 & 96.6 & 98.3 & 100.7 & 1015 & 1003 & 99.7 & 101.4 & 99.3 & 96.6 \\
\hline$A \times p p(n)$ & 159 & 95 & 119 & 105 & 360 & 121 & 181 & 145 & 199 & 20 & 49 & 22 \\
\hline $\mathrm{Ba}$ & 430 & 4100 & 980 & 1900 & 1200 & 1300 & 1300 & 930 & 1200 & 380 & 4600 & 690 \\
\hline Cd & 1.7 & 14.0 & 4.4 & 14.1 & 19 & 9.1 & 34 & 3.7 & 26 & 3 & 18.9 & 24.7 \\
\hline co & 860 & 127 & 260 & 107 & 81 & 121 & 224 & 145 & 220 & 59 & 79 & $<10$ \\
\hline$C$ & 540 & 25 & $\pi$ & 2 & 17 & 25 & 25 & 49 & 81 & 152 & 22 & 0 \\
\hline Mo & 88 & 290 & 260 & 370 & 211 & 232 & 270 & 134 & 360 & 4 & 320 & 1300 \\
\hline Pb & 410 & 106 & 190 & 109 & 47 & 55 & 280 & 122 & 199 & 4 & 39 & $<15$ \\
\hline Sr & 590 & 950 & 770 & 770 & 1200 & 790 & 1300 & 970 & 1200 & 440 & 540 & 410 \\
\hline $\mathbf{v}$ & 500 & 410 & 430 & 410 & 830 & 380 & 580 & 480 & 550 & 244 & 570 & 4 \\
\hline $\mathbf{Y}$ & 83 & 57 & 81 & 51 & 174 & 85 & 149 & 122 & 157 & 32 & 43 & 10 \\
\hline $\mathbf{z n}$ & 1400 & 2400 & 830 & 1800 & 740 & 1200 & 710 & 670 & 610 & 203 & 1800 & 1600 \\
\hline$\gamma$ & 998 & 972 & 1.025 & 1.021 & 1.003 & 979 & 971 & 983 & 989 & 973 & .991 & 1.020 \\
\hline $\begin{array}{l}\text { Sermplod } \\
\text { brtarval }\end{array}$ & Bulk & $\begin{array}{c}\text { Bulk } \\
\text { O-15rmm }\end{array}$ & $\begin{array}{c}\text { Bulk } \\
0-8 \mathrm{~mm}\end{array}$ & $\begin{array}{c}\text { Bulk } \\
0-12 \text { man }\end{array}$ & Bulk & $\begin{array}{c}\text { Bulk } \\
\text { a-1mm }\end{array}$ & $\begin{array}{c}\text { Bulk } \\
0-11 \text { mm }\end{array}$ & $\begin{array}{c}\text { Bulk } \\
\text { O-Imm }\end{array}$ & $\begin{array}{c}\text { Bulk } \\
0-28 \mathrm{~mm}\end{array}$ & $\begin{array}{c}\text { Bulk } \\
\text { <lmm }\end{array}$ & Bulk & $\begin{array}{c}\text { Bulk } \\
0-10 \mathrm{~mm}\end{array}$ \\
\hline $\begin{array}{l}\text { Soquenoull } \\
\text { Latitude }\end{array}$ & $\begin{array}{c}010010 \\
2422\end{array}$ & $\begin{array}{c}021001 \\
8.257\end{array}$ & $\begin{array}{c}011002 \\
9.917\end{array}$ & $\begin{array}{c}011003 \\
9.950\end{array}$ & $\begin{array}{c}011005 \\
8.825\end{array}$ & $\begin{array}{c}016001 \\
2.087\end{array}$ & $\begin{array}{c}016002 \\
544\end{array}$ & $\begin{array}{c}016003 \uparrow \\
3.640\end{array}$ & $\begin{array}{c}016005 \\
.707\end{array}$ & $\begin{array}{c}017001 \\
5.232\end{array}$ & $\begin{array}{c}017002 \\
5.872\end{array}$ & $\begin{array}{c}017003 \\
9.333\end{array}$ \\
\hline Longitudo & -95.407 & -104.143 & -104483 & -108.800 & -103.917 & -157.341 & -157.898 & -159.523 & -157.287 & -161.497 & -160.848 & -163.167 \\
\hline Depth & 2207 & 3139 & 1716 & 1227 & 1190 & 1520 & 1750 & 2800 & 2300 & 1307 & 1604 & 1563 \\
\hline Lab" & W-231369 & W-222576 & W-222083 & W-222578 & $w-222540$ & W-224396 & W-224397 & W-224402 & W.231394 & W.222563 & W.222564 & w-222565 \\
\hline $\mathrm{sio}_{2}(w \mathrm{x} . \%)$ & 17.3 & 16.9 & 6.05 & 5.33 & 82 & 25.7 & 1.90 & 40.9 & 9.73 & 2.46 & 2.78 & 6.25 \\
\hline & 98 & 52 & .17 & .64 & .03 & 229 & 1.49 & 5.01 & 2.12 & 1.80 & 1.14 & 1.86 \\
\hline & 25.9 & 20.0 & 520 & 57.5 & 65.2 & 15.9 & 48.2 & 36 & 34.0 & 51.9 & 40.5 & 45.4 \\
\hline & 264 & 35.1 & 175 & 5.36 & 12 & 175 & 16.0 & 16.1 & 285 & 19.1 & 19.0 & 194 \\
\hline $\mathrm{Al}_{2}^{2} \mathrm{O}_{3}^{3}$ & 4.18 & 3.46 & .76 & 4.93 & 55 & 7.18 & 43 & 15.3 & 2.26 & 1.01 & 53 & 203 \\
\hline $\mathrm{CO}_{3}^{2} \mathrm{O}_{4}^{3}$ & .138 & .084 & .040 & .079 & .004 & 374 & 1.02 & .001 & 921 & 2.05 & .603 & 1.46 \\
\hline $\mathrm{NiO}^{4}$ & 359 & 216 & 332 & .419 & .048 & .309 & 924 & .009 & 438 & 991 & .564 & 1.003 \\
\hline Co & .059 & .149 & .031 & .058 & .011 & .026 & .103 & .010 & .119 & .108 & .040 & .150 \\
\hline $\mathrm{CaO}$ & 4.95 & 2.74 & 3.24 & 254 & 12.6 & 12.6 & 123 & 9.13 & 3.17 & 4.40 & 12.0 & 589 \\
\hline $\mathrm{M}_{8} \mathrm{O}$ & 289 & 1.73 & 287 & 6.40 & 1.93 & 438 & 1.89 & 2.57 & 1.93 & 2.49 & 151 & 248 \\
\hline $\mathrm{Ne}_{2} \mathrm{O}$ & 260 & 275 & 4.03 & 238 & 4.30 & 2.43 & 238 & 3.83 & 2.75 & 3.28 & 241 & 2.72 \\
\hline $\mathbf{K}_{2}^{2} \mathbf{O}$ & 52 & .66 & 1.00 & 1.07 & .62 & 92 & .63 & 2.24 & .66 & .61 & 52 & .69 \\
\hline $\mathrm{CO}_{2}$ & .34 & 33 & .53 & 56 & 8.06 & 4.65 & 1.43 & 2.14 & .13 & .51 & 1.39 & .69 \\
\hline $\mathrm{P}_{2} \mathrm{O}_{5}$ & 1.02 & .96 & .84 & .73 & $<06$ & .77 & 55 & 1.57 & .84 & 125 & 5.80 & 224 \\
\hline $\mathrm{H}_{2}^{2} \mathrm{O}+$ & 6.8 & 9.9 & 85 & 10.8 & 6.6 & 5.5 & 9.6 & 3.4 & 75 & 53 & 9.2 & 5.0 \\
\hline $\mathrm{H}_{2} \mathrm{O}$ & 15.0 & 235 & 15.7 & 11.9 & 7.0 & 5.3 & 16.0 & $3 A$ & 15.7 & 268 & 21.0 & 264 \\
\hline $\sin$ & 945 & 985 & 97.9 & 98.8 & 101.1 & 100.4 & 98.9 & 101.6 & 95.0 & 973 & 98.0 & 973 \\
\hline$A x$ (ppon) & 235 & 260 & 202 & 80 & 17 & 216 & 167 & 18 & 260 & 232 & 203 & 190 \\
\hline $\mathrm{Be}$ & 1100 & 1800 & 840 & 940 & 910 & 650 & 2100 & 290 & 1300 & 2000 & 1900 & 1900 \\
\hline Cd & 3.1 & 24 & 14.2 & 60.2 & 3.3 & 23 & 55 & .1 & 2.9 & 6.7 & 29 & 45 \\
\hline$c_{0}$ & 247 & 235 & 61 & 109 & $<10$ & 270 & 2300 & 74 & 1100 & 1500 & 2500 & 960 \\
\hline$a$ & 66 & 12 & 11 & 19 & 13 & 270 & 11 & 25 & 6 & 22 & 19 & 31 \\
\hline Mo & 224 & 248 & 390 & 440 & 204 & 201 & 870 & $<4$ & 330 & 920 & 770 & 670 \\
\hline Pb & 340 & 196 & 154 & 97 & $<15$ & 670 & 2400 & 44 & 1800 & 2500 & 1800 & 1800 \\
\hline Sr & 1300 & 1400 & 970 & 600 & 550 & 1100 & 2100 & 570 & 1300 & 1600 & 2000 & 1500 \\
\hline $\mathbf{v}$ & 600 & 590 & 450 & 320 & 83 & 490 & 830 & 42 & 560 & 710 & 750 & 600 \\
\hline $\mathbf{Y}$ & 177 & 157 & 95 & 83 & 4 & 92 & 250 & 52 & 190 & 157 & 270 & 290 \\
\hline $\mathbf{z a}$ & 660 & 730 & 1100 & 1500 & 151 & 440 & 1000 & 21 & 530 & 900 & 630 & 830 \\
\hline$\gamma$ & 1.044 & 1.001 & 1.007 & .998 & .975 & .982 & 997 & 971 & 1.038 & 1.013 & 1.006 & 1.014 \\
\hline $\begin{array}{l}\text { Sampled } \\
\text { betorval }\end{array}$ & $\begin{array}{c}\text { Bulk } \\
0-3.5 \mathrm{mmm}\end{array}$ & $\begin{array}{c}\text { Bulk } \\
0-10 \operatorname{mon}\end{array}$ & $\begin{array}{c}\text { Bulk } \\
\text { - }\end{array}$ & $\begin{array}{l}\text { Bulk } \\
\text { O-3anm }\end{array}$ & $\begin{array}{c}\text { Bulk } \\
\text { - }\end{array}$ & $\begin{array}{c}\text { Bulk } \\
\text { Q-9mman }\end{array}$ & $\begin{array}{c}\text { Bulk } \\
0-12 \mathrm{mmm}\end{array}$ & $\begin{array}{l}\text { Bulk } \\
\text { <lmm }\end{array}$ & $\begin{array}{c}\text { Bulk } \\
\text { - }\end{array}$ & $\begin{array}{c}\text { Bulk } \\
\text { a-2mm }\end{array}$ & $\underset{\text { O-8mm }}{\text { Bulk }}$ & $\underset{0-4 m \min }{\text { Bulk }}$ \\
\hline
\end{tabular}


Table 1. Composition of crusts analyzed by USGS Reston Labs (cont'd).

\begin{tabular}{|c|c|c|c|c|c|c|c|c|c|c|c|c|}
\hline $\begin{array}{l}\text { Sequencell } \\
\text { Letitude } \\
\text { Longitude } \\
\text { Depth } \\
\text { Labl }\end{array}$ & $\begin{array}{c}017004 \\
8330 \\
-164362 \\
1519 \\
W-222566 \\
\end{array}$ & $\begin{array}{c}017004 \\
8330 \\
-164.362 \\
1519 \\
W-222566 \\
\end{array}$ & $\begin{array}{c}017005 \\
8.186 \\
-161.919 \\
3890 \\
W-224395 \\
\end{array}$ & $\begin{array}{c}017006 \\
8.414 \\
-164.289 \\
1850 \\
W-223766 \\
\end{array}$ & $\begin{array}{c}017007 \\
8.414 \\
-164.289 \\
1850 \\
w-203767 \\
\end{array}$ & $\begin{array}{c}017008 \\
8.414 \\
-164.289 \\
1850 \\
\text { w-223768 } \\
\end{array}$ & $\begin{array}{c}017009 \\
8.398 \\
-164.359 \\
2400 \\
W-223769 \\
\end{array}$ & $\begin{array}{c}017010 \\
8.398 \\
-164.359 \\
2400 \\
W-223770 \\
\end{array}$ & $\begin{array}{c}017011 \\
8.389 \\
-164.294 \\
1600 \\
w-223771 \\
\end{array}$ & $\begin{array}{c}017012 \\
8.353 \\
-164317 \\
1150 \\
w-223772 \\
\end{array}$ & $\begin{array}{c}017013 \\
8.353 \\
-164.317 \\
1150 \\
w-223773 \\
\end{array}$ & $\begin{array}{c}017014 \\
8.308 \\
.164346 \\
1400 \\
W-223774 \\
\end{array}$ \\
\hline $\mathrm{SiO}_{2}(\mathrm{w. \%})$ & 2.15 & 256 & 11.6 & 5.54 & 5.70 & 5.80 & 7.05 & 8.82 & 590 & 271 & 252 & 210 \\
\hline & 1.21 & 1.22 & 1.73 & 197 & 2.16 & 2.46 & 1.79 & 1.97 & 1.74 & 1.36 & 1.68 & 1.53 \\
\hline & 41.9 & 43.0 & 33.7 & 45.2 & 40.2 & 40.1 & 40.9 & 38.2 & 39.1 & 56.0 & 44.8 & 503 \\
\hline & 13.1 & 13.9 & 324 & 24.1 & 25.7 & 27.8 & 24.8 & 26.9 & 21.9 & 127 & 20.0 & 19.0 \\
\hline & 1.10 & 1.15 & 1.71 & .84 & 1.03 & 1.14 & 1.15 & 1.57 & 156 & 87 & .48 & 31 \\
\hline & 1.11 & 1.13 & 365 & 1.36 & 1.02 & 1.20 & 1.12 & 1.20 & 1.64 & 3.48 & 1.66 & 1.69 \\
\hline Nió & 1.169 & 1.211 & 355 & .659 & 514 & $s 11$ & 575 & 527 & .724 & 1.153 & .428 & 874 \\
\hline Co & .111 & .114 & .433 & .07 & .079 & .105 & .095 & .094 & .091 & .089 & .042 & .037 \\
\hline $\mathrm{CaO}$ & 122 & 13.8 & 3.12 & 4.05 & 4.49 & 3.90 & 3.97 & 4.31 & 751 & 3.94 & 794 & 4.03 \\
\hline Migo & 257 & 2.63 & 1.70 & 1.86 & 1.69 & 1.74 & 1.74 & 194 & 231 & 294 & 1.92 & 2.02 \\
\hline $\mathrm{N}_{2} \mathrm{O}$ & 1.91 & 2.07 & 2.12 & 220 & 2.02 & 2.01 & 2.21 & 223 & 2.25 & 281 & 231 & 233 \\
\hline & .66 & .68 & .62 & .65 & .57 & .62 & .68 & .73 & .61 & 90 & .66 & .61 \\
\hline & 1.55 & 1.46 & .55 & .55 & .61 & $A 0$ & 38 & $s 1$ & 91 & 41 & .82 & .50 \\
\hline $\mathrm{P}_{2} \mathrm{O}_{5}$ & 6.56 & 7.27 & .69 & 1.13 & 144 & .95 & 1.06 & 1.36 & 3.69 & 98 & 386 & 1.02 \\
\hline $\mathrm{H}_{2} \mathrm{O}+$ & 9.4 & 45 & 11.7 & 10.7 & 10.3 & 95 & 8.0 & 8.0 & 8.4 & 7.6 & 8.7 & 11.3 \\
\hline $\mathbf{H}_{2}^{2} \mathrm{O}$ & 16.2 & -18.0 & 10.4 & 17.0 & 15.8 & 10.4 & 4.9 & 5.9 & 6.8 & 4.0 & 4.9 & 9.7 \\
\hline Sum & 96.8 & 96.8 & 1028 & 101.0 & 985 & 98.2 & 95.6 & 98.3 & 98.3 & 97.9 & 97.8 & 97.6 \\
\hline Axppon) & 134 & 191 & 167 & 290 & 290 & 300 & 280 & 290 & 260 & 219 & 300 & 320 \\
\hline $\mathrm{Ba}$ & 2200 & 3800 & 2300 & 1800 & $\mathbf{2 3 0 0}$ & 2300 & 1900 & 2100 & 1600 & 1600 & 3400 & 1800 \\
\hline Cd & 6.8 & 6.4 & 23 & 35 & 26 & 27 & 28 & 3.2 & 4.2 & 9.4 & 35 & 4.3 \\
\hline$\infty$ & 790 & 750 & 890 & 1000 & 1300 & 1600 & 820 & 860 & 900 & 810 & 1400 & 1200 \\
\hline$\alpha$ & 21 & 7 & 10 & • & - & - & • & - & • & - & • & • \\
\hline Mo & 590 & 580 & 390 & 590 & 590 & 540 & 600 & 510 & 490 & 810 & 930 & 870 \\
\hline Pb & 1700 & 1600 & 860 & 1700 & 1800 & 1700 & 1500 & 1400 & 1800 & 2000 & 2000 & 1900 \\
\hline Sr & 1600 & 1600 & 1700 & 1700 & 1800 & 1800 & 1700 & 1600 & 1600 & 1300 & 2000 & 1800 \\
\hline V & 570 & 560 & 740 & 650 & 720 & 740 & 620 & 580 & 650 & 570 & 800 & 800 \\
\hline $\mathbf{Y}$ & 270 & 239 & 156 & 169 & 166 & 179 & 179 & 181 & 340 & 115 & 260 & 144 \\
\hline $\mathbf{z n}$ & 1000 & 1100 & 960 & 770 & 890 & 900 & 680 & 710 & 800 & 970 & 660 & 700 \\
\hline$\gamma$ & 1.019 & 1.018 & 959 & 976 & 1.001 & 1.004 & 1.032 & 1.003 & 1.003 & 1.007 & 1.008 & 1.010 \\
\hline Samplod & Bulk & Bulk & Bulk & Top & Bulk & Bulk & Bulk & Bulk & Bnlk & Top & Bulk & Bulk \\
\hline lonervil & $0-4 \mathrm{~mm}$ & $0-4 \mathrm{~mm}$ & $0.20 \mathrm{~mm}$ & • & • & - & - & • & $0.15 \mathrm{~mm}$ & $\bullet$ & - & • \\
\hline Sequenoes" & $\begin{array}{l}017015 \\
8308\end{array}$ & $\begin{array}{l}017016 \\
8.308\end{array}$ & 017019 & $\begin{array}{l}017020 \\
8.194\end{array}$ & $\begin{array}{l}017021 \\
8.186\end{array}$ & $017044^{*}$ & $\begin{array}{l}017045 \\
3.092\end{array}$ & $\begin{array}{l}017047 \\
9.694\end{array}$ & $\begin{array}{c}017048 \\
9.694\end{array}$ & $\begin{array}{l}017049 \\
9.694\end{array}$ & $\begin{array}{c}017050 \\
9.694\end{array}$ & $\begin{array}{c}017051 \\
9.696\end{array}$ \\
\hline Longitude & -164.346 & -164.346 & -164200 & -164.200 & -164202 & -160243 & -160.243 & .162 .178 & -162.178 & -162.178 & -162178 & -162.181 \\
\hline $\begin{array}{l}\text { Depth } \\
\text { Lebul }\end{array}$ & $\begin{array}{c}1400 \\
W-223775\end{array}$ & $\begin{array}{c}1400 \\
W-223776\end{array}$ & $\begin{array}{c}1600 \\
w-223778\end{array}$ & $\begin{array}{c}1600 \\
w-223777\end{array}$ & $\begin{array}{c}1800 \\
W-223779\end{array}$ & $\begin{array}{c}2150 \\
w-231376\end{array}$ & $\begin{array}{c}1350 \\
w-231393\end{array}$ & $\begin{array}{c}1993 \\
W-229261\end{array}$ & $\begin{array}{c}1993 \\
w-229262\end{array}$ & $\begin{array}{c}1993 \\
\text { W-228955 }\end{array}$ & $\begin{array}{c}1993 \\
W-220281\end{array}$ & $\begin{array}{c}1927 \\
\text { w-228992 }\end{array}$ \\
\hline $\mathrm{SiO}_{2}(\mathrm{w} . \%)$ & 233 & 1.98 & 3.32 & 3.55 & 8.81 & 35.1 & 127 & 6.18 & 4.33 & 7.62 & 5,48 & 3.33 \\
\hline & 1.48 & 1.38 & 1.59 & 1.80 & 205 & .48 & .13 & 1.87 & 2.26 & 222 & 2.47 & 1.52 \\
\hline & 48.1 & 52.3 & 47.8 & 45.7 & 383 & 6.01 & 57.2 & 425 & 47.8 & 41.3 & 43.8 & 34.7 \\
\hline & 20.4 & 17.8 & 20.6 & 23.2 & 20.2 & 11.6 & 7.19 & 23.2 & 18.6 & 228 & 224 & 18.4 \\
\hline $\mathrm{Al}_{2}^{2} \mathrm{O}_{3}$ & 52 & 33 & .63 & .47 & 1.98 & 2.17 & 226 & 88 & 82 & 1.51 & .79 & .58 \\
\hline $\mathrm{Ca}_{3} \mathrm{O}_{4}$ & 1.45 & 2.49 & 1.98 & 1.17 & 1.81 & .113 & .030 & 1.22 & 2.05 & 1.36 & 1.95 & 591 \\
\hline $\mathrm{NiO}^{4}$ & .748 & 884 & 578 & .603 & .653 & .215 & .080 & .668 & .938 & .682 & .742 & $A 21$ \\
\hline Cro & .046 & .022 & .054 & .074 & .046 & .016 & .043 & .072 & .083 & .007 & .067 & .076 \\
\hline $\mathrm{CaO}$ & 4.81 & 3.86 & 3.87 & 3.92 & 4.06 & 2.33 & 2.29 & 4.31 & 3.99 & 3.93 & 4.28 & 15.8 \\
\hline $\mathrm{MgO}$ & 1.84 & 219 & 1.84 & 1.70 & 1.85 & 26.93 & 2.20 & 1.74 & 2.11 & 1.88 & 1.90 & 1.32 \\
\hline $\mathrm{Ne}_{2} \mathrm{O}$ & 233 & 254 & 255 & 2.15 & 208 & 57 & 5.24 & 233 & 278 & 243 & 270 & 1.89 \\
\hline $\mathrm{K}_{2} \mathrm{O}$ & .60 & .61 & .69 & .64 & .94 & .18 & 1.01 & .55 & .63 & .70 & 59 & 47 \\
\hline$\infty_{2}$ & .53 & 43 & .47 & .37 & .72 & 46 & .04 & .62 & 51 & .61 & 1.04 & 1.83 \\
\hline $\mathrm{P}_{2} \mathrm{O}_{5}$ & 1.35 & 1.04 & 1.14 & 94 & 1.18 & 34 & .17 & 1.23 & .70 & 1.06 & .72 & 8.37 \\
\hline $\mathrm{H}_{2} \mathrm{O}$ & 10.8 & 11.0 & 9.4 & 10.4 & 93 & 11.2 & 8.1 & 8.3 & 8.5 & 8.2 & 9.2 & 7.1 \\
\hline $\mathrm{H}_{2} \mathrm{O}$ & 9.8 & 9.3 & 9.7 & 7.1 & 103 & 5.2 & 4.5 & 14.3 & 14.5 & 16.0 & 143 & 124 \\
\hline Sum & 973 & 98.9 & 96.5 & 96.7 & 94.5 & 97.7 & 98.6 & 95.6 & 96.1 & 964 & 98.1 & 964 \\
\hline Axppon) & 330 & 320 & 370 & 330 & 260 & 68 & 22 & 290 & 234 & 191 & 245 & 160 \\
\hline Ba & 2000 & 1200 & 2300 & 2700 & 1200 & 243 & 360 & 1500 & 1600 & 1500 & 1600 & 3000 \\
\hline Cd & 4.7 & 5.4 & 4.7 & 3.7 & 4.0 & .6 & 43 & 3.9 & 0.0 & 5.0 & 4.6 & 23 \\
\hline C. & 1200 & 860 & 870 & 1300 & 880 & 137 & $<10$ & 890 & 1200 & 990 & 1500 & 2100 \\
\hline$\alpha$ & $\cdot$ & $\cdot$ & • & - & $\cdot$ & 1600 & 5 & 8 & 11 & 18 & 12 & 14 \\
\hline Mo & 930 & 840 & 1100 & 790 & 430 & 34 & 660 & 560 & 560 & 490 & 400 & 630 \\
\hline Po & 1800 & 2000 & 2000 & 1600 & 1400 & 310 & $<15$ & 1400 & 1500 & 1700 & 1600 & 1800 \\
\hline Sr & 1900 & 1500 & 1900 & 2000 & 1400 & 340 & 300 & 1600 & 1500 & 1500 & 1500 & 2100 \\
\hline $\mathbf{v}$ & 840 & 720 & 910 & 1000 & 640 & 190 & $<4$ & 650 & 560 & 640 & 540 & 830 \\
\hline $\mathbf{Y}$ & 200 & 165 & 166 & 172 & 178 & 43 & 8 & 198 & 152 & 179 & 175 & 228 \\
\hline $\mathbf{Z n}$ & 780 & 660 & 640 & 860 & 670 & 310 & 209 & 760 & 900 & 820 & 810 & 820 \\
\hline $\boldsymbol{\gamma}$ & 1.013 & 997 & 1.021 & 1.020 & 1.043 & 1.009 & 1.000 & 1.031 & 1.026 & 1.023 & 1.005 & 1.022 \\
\hline Samplod & Bottom & Top & Top & Bulk-minns & Bulk & Bulk & Bulk & Bulk & Top & Bulk & Pocous & Old \\
\hline htarval & - & 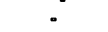 & $\cdot$ & Top & - & $0.5 \mathrm{~mm}$ & $0-20 \mathrm{~mm}$ & - & - & • & - & - \\
\hline
\end{tabular}


Table 1. Composition of crusts analyzed by USGS Reston Labs (cont'd).

\begin{tabular}{|c|c|c|c|c|c|c|c|c|c|c|c|c|}
\hline Sequenceit & 017051 & 017053 & 017059 & 017059 & 017059 & 017059 & 017060 & 017062 & 017063 & 017064 & 017064 & 017083 \\
\hline Latitude & 9.696 & 9.696 & 9.716 & 9.716 & 9.716 & 9.716 & 9.716 & 9.682 & 9.705 & 9.705 & 9.705 & 6.263 \\
\hline Longituds & .162181 & -162181 & -162167 & -162167 & -162167 & -162167 & -162.167 & -162.184 & -162184 & -162.184 & -162.184 & -162.963 \\
\hline Depeh & 1927 & 1927 & 1602 & 1602 & 1602 & 1602 & 1602 & 2706 & 1940 & 1940 & 1940 & 2887 \\
\hline Lab: & W-2229263 & W-220264 & W-2228956 & W-229265 & W-2229266 & $W-220207$ & W-220268 & W-220269 & W-220270 & W-229271 & W-229272 & W-228959 \\
\hline $\mathrm{SiO}_{2}$ (wt.S) & 3.53 & 4.80 & 1.70 & 253 & 204 & 250 & 211 & 15.0 & 3.71 & 2.67 & 3.16 & 8.80 \\
\hline & 1.57 & 1.99 & .98 & 1.42 & 1.45 & 1.49 & 1.43 & 1.77 & 1.65 & 1.17 & 1.39 & 1.84 \\
\hline $\mathrm{MnO}_{2}$ & 49.0 & 435 & 46.3 & 51.5 & 39.0 & 44.3 & 53.1 & 29.1 & 38.1 & 22.1 & 50.5 & 39.2 \\
\hline $\mathrm{Pe}_{2} \mathrm{O}_{3}^{2}$ & 19.7 & 22.1 & 11.1 & 18.2 & 16.1 & 17.6 & 16.8 & 17.4 & 19.8 & 124 & 16.9 & 26.2 \\
\hline & $\mu$ & 80 & 1.30 & 49 & 47 & 54 & 12 & 4.30 & .83 & 1.39 & .66 & 1.35 \\
\hline $\mathrm{CO}_{3} \mathrm{O}^{\circ}$ & 1.81 & 1.34 & 1.51 & 2.22 & .659 & 1.31 & 2.34 & 1.31 & 597 & .628 & 220 & 1.04 \\
\hline $\mathrm{NiO}$ & .833 & .760 & 1.769 & .921 & .602 & .745 & 1.018 & 459 & 501 & .747 & .872 & 583 \\
\hline Co & .057 & .070 & .139 & .058 & .061 & .063 & .066 & .034 & .115 & .084 & .064 & .071 \\
\hline $\mathrm{ClO}$ & 3.85 & 4.00 & 10.1 & 3.97 & 14.8 & 9.48 & 3.92 & 10.3 & 115 & 26.7 & 4.75 & 3.65 \\
\hline $\mathrm{M} 8 \mathrm{O}$ & 1.99 & 1.86 & 3.32 & 2.21 & 151 & 1.83 & 228 & 1.51 & 151 & 2.00 & 1.98 & 1.89 \\
\hline $\mathrm{Na}_{2} \mathrm{O}$ & 251 & 245 & 2.25 & 265 & 209 & 227 & 265 & 3.07 & 1.92 & 3.31 & 2.54 & 232 \\
\hline $\mathbf{K}_{2} \mathbf{O}$ & 58 & 54 & .82 & .60 & .49 & 34 & .61 & 1.14 & .46 & .25 & .73 & .64 \\
\hline$\infty_{2}$ & 49 & so & 1.11 & .46 & 1.70 & 1.10 & $A 1$ & 1.21 & 1.39 & 2.84 & 53 & -58 \\
\hline $\mathrm{P}_{2} \mathrm{O}_{5}$ & 84 & 1.10 & 5.35 & 94 & 7.46 & 4.37 & 86 & 4.88 & 5.41 & 16.10 & 1.30 & 89 \\
\hline $\mathrm{H}_{2} \mathrm{O}+$ & 8.8 & 8.9 & 78 & 9.5 & 7.9 & 8.4 & 8.8 & 6.5 & 7.9 & 6.2 & 8.6 & 8.5 \\
\hline $\mathrm{H}_{2} \mathrm{O}$ & 15.0 & 14.6 & 10.1 & 14.3 & 11.2 & 129 & 15.0 & 11.3 & 11.1 & 4.6 & 13.9 & 14.8 \\
\hline Sum & 96.0 & 948 & 95.6 & 97.6 & 96.4 & 96.5 & 96.8 & 98.0 & 95.4 & 98.6 & 96.3 & 975 \\
\hline Ax(ppon) & 290 & 300 & 134 & 214 & 241 & 200 & 280 & 164 & 191 & 330 & 88 & 211 \\
\hline Be & 1500 & 1900 & 3000 & 2800 & 2200 & 1500 & 1600 & 1200 & 5600 & 2100 & 3400 & 1400 \\
\hline Cd & 5.4 & 4.0 & 8.8 & 26 & 4.0 & 5.8 & 6.1 & 4.1 & 3.0 & 4.9 & 28 & 4.2 \\
\hline$\infty$ & 810 & 1000 & 650 & 1800 & 1400 & 900 & 950 & 780 & 1900 & 750 & 1000 & no \\
\hline$a$ & 4 & 8 & 31 & 3 & 4 & 6 & 3 & 21 & 17 & 10 & 23 & 21 \\
\hline Mo & 810 & 670 & 720 & 800 & 840 & 890 & 920 & 300 & 660 & 1300 & 178 & 430 \\
\hline $\mathbf{P b}$ & 1800 & 1900 & 1600 & 1700 & 1800 & 2000 & 2100 & 1000 & 1800 & 2000 & 1300 & 1300 \\
\hline S? & 1600 & 1800 & 1200 & 2000 & 1800 & 1600 & 1600 & 1400 & 2100 & 1600 & 1800 & 1500 \\
\hline v & 710 & 730 & 530 & 680 & 710 & 710 & 710 & 430 & 800 & 870 & 460 & 670 \\
\hline $\mathbf{Y}$ & 165 & 187 & 370 & 410 & 290 & 163. & 153 & 237 & 203 & 128 & 510 & 188 \\
\hline $\mathbf{Z n}$ & 810 & 820 & 1300 & 770 & 770 & $810^{\circ}$ & 820 & 580 & 810 & 730 & 820 & 740 \\
\hline$\gamma$ & 1.027 & 1.041 & 1.031 & 1.010 & 1.023 & 1.021 & 1.018 & 1.000 & 1.034 & 1.000 & 1.024 & 1.012 \\
\hline Semplod & Youns & Bulk & Bulk & Young & Old & Bulk & Top & Top & Bulk & ad & Young & Bulk \\
\hline fortoind & - & • & - & - & - & - & - & $\cdot$ & • & • & • & • \\
\hline $\begin{array}{l}\text { Soquencell } \\
\text { Letitude }\end{array}$ & $\begin{array}{c}017084 \\
6.203\end{array}$ & $\begin{array}{c}017085 \\
6.290\end{array}$ & $\begin{array}{c}017085 \\
6.290\end{array}$ & $\begin{array}{c}017085 \\
6.290\end{array}$ & $\begin{array}{c}017085 \\
6.290\end{array}$ & $\begin{array}{c}017086 \\
9.164\end{array}$ & $\begin{array}{c}017087 \dagger \\
9.172\end{array}$ & $\begin{array}{c}017087 \\
9.172\end{array}$ & $\begin{array}{c}017088 \\
9.179\end{array}$ & $\begin{array}{c}017008 \\
9.179\end{array}$ & $\begin{array}{c}017088 \\
9.179\end{array}$ & $\begin{array}{c}017089 \\
9.166\end{array}$ \\
\hline Longitade & -162.963 & -162.970 & -162970 & -162970 & -162970 & -164.842 & -164.826 & -164.826 & -164830 & -164830 & -164.830 & -164.798 \\
\hline Depth & 2887 & 2486 & 2486 & 2486 & 2486 & 2291 & 1853 & 1853 & 1263 & 1263 & 1263 & 1427 \\
\hline Labl & W-2229274 & W-228972 & W-228973 & w-229275 & W-229279 & W-228968 & W-228963 & W-228971 & W.228962 & W-228965 & W-228980 & W-228961 \\
\hline $\mathrm{SiO}_{2}$ (wt.\%) & 9.91 & 5.31 & 5.06 & 5.28 & 4.96 & 8.25 & 39.3 & 5.40 & 2.60 & 206 & 3.68 & 252 \\
\hline $\mathrm{TOO}_{2}^{2-}$ & 1.96 & 201 & 1.95 & 1.74 & 1.91 & 1.54 & 254 & 207 & 1.70 & 1.44 & 186 & 151 \\
\hline $\mathrm{MnO}_{2}$ & 35.6 & 39.2 & 39.7 & 427 & 41.9 & 39.0 & 47 & 46.2 & 50.0 & 47.8 & 48.5 & 46.8 \\
\hline & 26.9 & 295 & 30.1 & 27.3 & 27.4 & 268 & 11.0 & 18.6 & 18.4 & 16.5 & 21.1 & 15.6 \\
\hline $\mathrm{AL}_{2}^{2} \mathrm{O}_{3}^{3}$ & 1.76 & 91 & .67 & 59 & .65 & 93 & 15.4 & 1.39 & .44 & .41 & 58 & .63 \\
\hline $\mathrm{CO}_{3} \mathrm{O}_{4}$ & .894 & .674 & .738 & .782 & .869 & 1.02 & .022 & 211 & 1.85 & 1.36 & 1.91 & 1.86 \\
\hline $\mathrm{NiO}^{4}$ & 542 & .495 & $A 80$ & .599 & .572 & 533 & .016 & .796 & 864 & 870 & .801 & .773 \\
\hline Co & .094 & .133 & .108 & .060 & .092 & .065 & .005 & .092 & .047 & .050 & .052 & .066 \\
\hline $\mathrm{CaO}$ & 450 & 3.78 & 3.74 & 3.78 & 3.80 & 3.69 & 11.1 & 4.19 & 3.93 & 7.55 & 4.15 & 7.70 \\
\hline $\mathrm{M} 8 \mathrm{O}$ & 1.74 & 1.70 & 1.68 & 1.75 & 1.70 & 1.80 & 1.14 & 203 & 2.12 & 2.03 & 205 & 215 \\
\hline $\mathrm{N}_{2} \mathrm{O}$ & 232 & 208 & 210 & 239 & 2.28 & 251 & 4.02 & 2.69 & 2.53 & 2.36 & 2.53 & 244 \\
\hline$k_{2}^{2}$ & .60 & so & .47 & 47 & .47 & 59 & 3.36 & 89 & .65 & .64 & .64 & .76 \\
\hline$\infty_{2}$ & .74 & 53 & .54 & 52 & 59 & 59 & 80 & 49 & .49 & .89 & 50 & 84 \\
\hline $\mathrm{P}_{2} \mathrm{O}_{5}$ & 1.35 & 1.00 & 1.03 & .95 & .89 & 1.19 & 5.97 & 1.10 & .94 & 3.27 & 1.14 & 3.42 \\
\hline $\mathrm{H}_{2}^{2} \mathrm{O}+$ & 8.4 & 8.6 & 9.0 & 10.0 & 8.9 & 8.0 & 29 & 9.8 & 8.4 & 8.0 & 8.2 & 8.3 \\
\hline $\mathrm{H}_{2}^{2} \mathrm{O}$ & 13.2 & 15.2 & 15.1 & 129 & 15.4 & 18.8 & 2.9 & 18.5 & 14.6 & 16.6 & 15.8 & 12.8 \\
\hline Sum & 97.2 & 96.4 & 97.4 & 98.8 & 96.9 & 96.5 & 98.1 & 97.9 & 95.0 & 95.2 & 97.6 & 95.3 \\
\hline$A=(p p o n)$ & 250 & 260 & 270 & 320 & 330 & 270 & 196 & 25 & 192 & 249 & 260 & 195 \\
\hline $\mathrm{Ba}$ & 1500 & 2700 & 2700 & 2200 & 2000 & 1400 & 1700 & 550 & 1900 & 1700 & 1600 & 2300 \\
\hline Cd & 3.0 & 27 & 2.8 & 3.1 & 26 & 3.4 & 5.9 & $<1$ & 5.9 & 5.6 & 6.6 & 4.7 \\
\hline$\omega_{0}$ & 940 & 1200 & 1200 & 1100 & 910 & 790 & 1200 & 97 & 1600 & 1100 & 1100 & 1300 \\
\hline$a$ & 42 & 6 & 11 & 7 & 5 & 15 & 15 & 87 & 7 & 14 & 12 & 52 \\
\hline Mo & 360 & 820 & 790 & 720 & 680 & 500 & 710 & $<4$ & 780 & 780 & 850 & 1100 \\
\hline $\mathrm{Pb}_{\mathbf{b}}$ & 1200 & 1400 & 1400 & 1300 & 1400 & 1400 & 1800 & 24 & 2200 & 2100 & 2000 & 1800 \\
\hline St & 1500 & 2000 & 2000 & 2000 & 2000 & 1600 & 1600 & 760 & 1800 & 1800 & 1800 & 1700 \\
\hline $\mathbf{v}$ & 580 & 990 & 930 & 830 & 820 & 760 & 690 & 237 & 730 & 750 & 740 & 750 \\
\hline $\mathbf{Y}$ & 196 & 200 & 189 & 177 & 207 & 197 & 160 & 32 & 340 & 154 & 141 & 229 \\
\hline $\mathbf{Z n}$ & 690 & 1100 & 1000 & 900 & 790 & 730 & 820 & 260 & 900 & 830 & 830 & 790 \\
\hline$\gamma$ & 1.014 & 1.023 & 1.013 & .998 & 1.017 & 1.022 & 1.005 & 1.008 & 1.037 & 1.035 & 1.010 & 1.034 \\
\hline Sempled & Bulk & Bottom & Middle & Top & Bulk & Bulk & Bulk & Bulk & Bulk & Young & old & Bulk \\
\hline Interval & - & - & - & $\therefore$ & - & • & • & • & • & - & - & - \\
\hline
\end{tabular}


Table 1. Composition of crusts analyzed by USGS Reston Labs (cont'd).

\begin{tabular}{|c|c|c|c|c|c|c|c|c|c|c|c|c|}
\hline Sequencer" & 017093 & 017093 & 017095 & 017095 & 017096 & 017096 & 017097 & 017098 & 017098 & 017099 & 017099 & 017101 \\
\hline Latitude & 9.225 & 9.225 & 9.247 & 9.247 & 9.267 & 9.247 & 9.247 & 9.247 & 9.247 & 9.200 & 9.200 & 9.716 \\
\hline Langinude & -164.780 & -164.780 & -164846 & -164.846 & -164846 & -164.846 & -164846 & -164.846 & -164.846 & -164.929 & -164.929 & -162.167 \\
\hline Depth & 1456 & 1456 & - & - & • & • & • & • & - & $=$ & • & 1602 \\
\hline Lab* & W-228974 & W-2289883 & W-228964 & W-228970 & W-228966 & W-228967 & W-228979 & W-228969 & W.228975 & W-228976 & W-228978 & W-229273 \\
\hline $\mathrm{SiO}_{2}(\mathrm{~m} \cdot \%)$ & 3.05 & 2.66 & 2.75 & 279 & 257 & 205 & 4.08 & 200 & 3.20 & 1.60 & 213 & 3.38 \\
\hline & 1.89 & 1.88 & 1.76 & 1.75 & 1.69 & 1.50 & 1.46 & 1.39 & 1.99 & 1.23 & 1.65 & 1.62 \\
\hline $\mathrm{MmO}_{2}$ & 45.9 & 49.9 & 41.0 & 49.6 & 50.0 & 40.1 & 50.4 & 41.1 & 47.9 & 47.4 & 52.8 & 43.3 \\
\hline $\mathrm{Fo}_{2} \mathrm{O}_{3}$ & 19.5 & 21.1 & 18.2 & 19.0 & 19.1 & 16.2 & 16.8 & 16.6 & 20.7 & 15.1 & 16.7 & 17.2 \\
\hline & 54 & 35 & 56 & .49 & 34 & 30 & 1.01 & 42 & .49 & .20 & 34 & .83 \\
\hline $\mathrm{CO}_{3} \mathrm{O}_{4}$ & 1.38 & 1.85 & 1.11 & 1.91 & 1.95 & 1.08 & 252 & .880 & 1.80 & 1.02 & 251 & 1.75 \\
\hline $\mathrm{NiO}^{4}$ & .730 & .806 & .651 & .869 & .835 & .651 & 934 & .718 & .804 & 954 & 1.019 & .745 \\
\hline Co & .072 & .050 & .048 & .047 & .045 & .038 & .051 & .049 & .059 & .044 & .058 & .069 \\
\hline $\mathrm{CaO}$ & 7.19 & 4.08 & 10.8 & 4.14 & 3.86 & 124 & 3.75 & 123 & 3.89 & 10.2 & 3.74 & 8.82 \\
\hline $\mathrm{M}_{8} \mathrm{O}$ & 2.86 & 2.04 & 1.81 & 217 & 2.10 & 1.66 & 276 & 1.73 & 2.04 & 1.90 & 2.28 & 202 \\
\hline $\mathrm{N}_{2} \mathrm{O}$ & 2.33 & 247 & 2.16 & 249 & 255 & 216 & 268 & 2.10 & 249 & 2.26 & 274 & 231 \\
\hline & 59 & .62 & .53 & 64 & .62 & 51 & .73 & 52 & .65 & .63 & .71 & 58 \\
\hline क & .87 & 47 & 1.14 & 48 & .47 & 1.59 & 37 & 1.53 & .39 & 1.15 & 41 & 98 \\
\hline $\mathrm{P}_{2} \mathrm{O}$ & 285 & 94 & 5.42 & 1.13 & 93 & 6.30 & .83 & 6.17 & .99 & 4.64 & .79 & 4.00 \\
\hline $\mathrm{H}_{2}^{2} \mathrm{O}+$ & 7.9 & 9.1 & 7.7 & 8.5 & 8.6 & 8.3 & 8.4 & 6.6 & 8.4 & 7.7 & 8.0 & 8.2 \\
\hline $\mathrm{H}_{2} \mathrm{O}$ & 16.3 & 14.7 & 12.0 & 15.1 & 16.2 & 12.0 & 14.2 & 14.9 & 14.5 & 14.6 & 16.3 & 11.2 \\
\hline Sum & 96.7 & 98.4 & 95.7 & 96.0 & 95.7 & 94.9 & 96.8 & 94.2 & 95.9 & 96.1 & 95.9 & 95.9 \\
\hline$A x$ (ppm) & 203 & 223 & 182 & 260 & 182 & 227 & 233 & 176 & 222 & 176 & 203 & 237 \\
\hline $\mathrm{Ba}$ & 2000 & 1800 & 1900 & 1600 & 1800 & 1600 & 1400 & 2000 & 1900 & 2100 & 1600 & 3400 \\
\hline Cd & 4.4 & 5.0 & 4.0 & 6.2 & 3.7 & 5.7 & 6.9 & 3.8 & 5.3 & 5.6 & 7.0 & 4.4 \\
\hline ce & 1600 & 1300 & 1400 & 1200 & 1500 & 1200 & 890 & 1400 & 1300 & 1800 & 1200 & 1200 \\
\hline$a$ & 13 & 8 & 17 & 8 & 7 & 6 & 34 & 5 & 13 & 5 & 8 & 8 \\
\hline Mo & 850 & 860 & 700 & 780 & 720 & 800 & 800 & 800 & 770 & 1000 & 810 & 710 \\
\hline$P_{0}$ & 1800 & 2000 & 1700 & 2100 & 1600 & 2100 & 2100 & 1500 & 2100 & 1900 & 2200 & 1800 \\
\hline Sz & 1900 & 1900 & 1900 & 1600 & 1900 & 1800 & 1500 & 1900 & 1900 & 1900 & 1600 & 1700 \\
\hline $\mathrm{v}$ & 750 & 770 & 680 & 720 & 660 & 740 & 650 & 710 & 760 & 750 & 660 & 610 \\
\hline $\mathbf{Y}$ & 250 & 141 & 310 & 141 & 250 & 131 & 140 & 400 & 140 & 390 & 143 & 237 \\
\hline $\mathrm{Zn}$ & 860 & 810 & 820 & 820 & 810 & 780 & 800 & 880 & 870 & 1100 & 900 & 780 \\
\hline$\gamma$ & 1.020 & 1.002 & 1.031 & 1.027 & 1.030 & 1.039 & 1.019 & 1.047 & 1.028 & 1.026 & 1.029 & 1.020 \\
\hline Samplod & Old & Young & Old & Young & Young & Old & Top & Old & Young & ad & Young & - \\
\hline Imorvil & - & - & $\cdot$ & - & - & $\cdot$ & - & - & - & - & - & $\cdot$ \\
\hline Sequenceill & 017102 & 017103 & 017104 & 019001 & 019002 & 019006 & 019007 & 019008 & 020004 & 020005 & 020006 & 020006 \\
\hline Latimads & 9.223 & 2755 & 2.767 & 6.667 & 7.333 & 7.250 & 7.250 & 7.250 & 8.747 & 8.747 & 8.755 & 8.755 \\
\hline Longibude & -164.858 & .165 .022 & -165.017 & 172417 & 172333 & 171.008 & 171.008 & 171.008 & 169.738 & 169.738 & 169.792 & 169.792 \\
\hline Depth & 1420 & 1326 & 1298 & 1700 & 1260 & 2250 & 2250 & 2250 & 3550 & 3550 & 2900 & 2900 \\
\hline Lab" & W-228977 & W-222561 & w-222562 & W-231387 & W-231388 & D1-1 & D1-3 & D1-8 & D4-3 & D4-4 & D6-1-A & D6-1-B \\
\hline $\mathrm{SiO}_{2}(w t . \%)$ & 1.82 & 2.21 & 8.76 & 14.0 & 4.06 & 7.12 & 7.38 & 4.40 & 37.1 & 14.8 & 4.70 & 3.18 \\
\hline & 1.60 & 1.19 & .70 & 255 & 1.50 & 1.83 & 1.77 & 1.97 & 2.80 & 243 & 2.49 & 1.45 \\
\hline $\mathrm{MmO}_{2}^{2}$ & 54.5 & 44.6 & 43.5 & 30.7 & 40.7 & 44.9 & 43.5 & 50.1 & 10.3 & 33.9 & 46.6 & 34.3 \\
\hline $\mathrm{Po}_{2} \mathrm{O}_{3}^{2}$ & 15.1 & 19.7 & 19.2 & 19.9 & 16.8 & 225 & 25.3 & 20.8 & 19.1 & 23.1 & 22.3 & 20.9 \\
\hline $\mathrm{Al}_{2}^{2} \mathrm{O}_{3}^{3}$ & 28 & 56 & 1.81 & 4.48 & 1.17 & 1.48 & 1.24 & .72 & 11.3 & 3.90 & 99 & .76 \\
\hline $\mathrm{CO}_{3}^{2} \mathrm{O}_{4}^{3}$ & 245 & .938 & .703 & 1.24 & 1.09 & 1.87 & 1.54 & 231 & .184 & 1.22 & 1.69 & .447 \\
\hline $\mathrm{NiO}^{4}$ & 1.127 & .673 & 1.436 & .535 & .674 & .715 & .649 & .854 & .113 & A75 & .984 & .674 \\
\hline coo & .067 & .059 & .126 & .059 & .089 & .034 & .037 & .036 & .031 & .073 & .121 & .126 \\
\hline $\mathrm{CaO}$ & 3.68 & 7.57 & 2.86 & 5.62 & 10.7 & 4.31 & 4.11 & 4.26 & 5.19 & 4.09 & 4.29 & 15.4 \\
\hline $\mathrm{MgO}$ & 237 & 1.84 & 338 & 2.25 & 1.94 & 200 & 1.91 & 2.13 & 2.31 & 2.21 & 2.20 & 1.59 \\
\hline $\mathrm{Ne}_{2} \mathrm{O}$ & 2.74 & 209 & 2.64 & 2.27 & 1.88 & 270 & 259 & 290 & 299 & 2.87 & 242 & 2.21 \\
\hline $\mathbf{K}_{2} \mathbf{O}$ & .73 & 58 & .78 & 1.21 & .76 & 81 & .71 & 56 & 2.43 & 1.15 & .68 & 47 \\
\hline$\infty_{2}^{6}$ & 38 & 93 & .46 & 56 & 1.04 & .84 & .79 & .73 & .80 & .51 & .91 & 1.99 \\
\hline $\mathrm{P}_{2} \mathrm{O}_{5}$ & .70 & 3.10 & .94 & 2.28 & 5.43 & 1.07 & 1.14 & 97 & .98 & .97 & .79 & 7.92 \\
\hline $\mathrm{H}_{2}^{2} \mathrm{O}+$ & 8.4 & 13.5 & 11.0 & 8.8 & 8.5 & 7.4 & 8.9 & 8.2 & 3.9 & - & 7.3 & 6.1 \\
\hline $\mathrm{H}_{2} \mathrm{O}$ & 17.6 & 18.7 & 16.7 & 14.4 & 11.3 & 27.0 & 25.5 & 27.0 & 11.1 & 19.6 & 27.6 & 20.7 \\
\hline Sum & 96.0 & 99.5 & 98.4 & 96.4 & 96.3 & 99.6 & 101.6 & 100.9 & 99.5 & • & 98.5 & 97.5 \\
\hline$A x$ (ppen) & 170 & 270 & 216 & 175 & 226 & 280 & 310 & 270 & 98 & 224 & 109 & 202 \\
\hline $\mathrm{Ba}$ & 1700 & 2100 & 2500 & 1200 & 3800 & 1100 & 1200 & 1200 & 470 & 1200 & 1900 & 2100 \\
\hline Cd & 7.9 & 4.8 & 11.8 & 3.4 & 5.1 & 5.6 & 5.0 & 2.6 & .6 & 3.2 & 26 & 29 \\
\hline$\infty$ & 1000 & 1200 & 280 & 840 & 1700 & 1000 & 980 & 1100 & 300 & 1100 & 900 & 1400 \\
\hline$a$ & 0 & 5 & 30 & 75 & 23 & 5 & 8 & 3 & 79 & 57 & 7 & 7 \\
\hline Mo & 860 & 890 & 470 & 370 & 990 & 420 & 430 & 510 & 82 & 350 & 320 & 380 \\
\hline Pb & 2100 & 2000 & 820 & 1400 & 2300 & 1600 & 1600 & 1600 & 440 & 1400 & 850 & 1400 \\
\hline St & 1600 & 1800 & 1100 & 2500 & 1700 & $15 \infty$ & 1600 & 1500 & 730 & 1200 & 1500 & 1800 \\
\hline $\mathbf{v}$ & 630 & 810 & 500 & 570 & 700 & 640 & 710 & 630 & 400 & 560 & 400 & 570 \\
\hline $\mathbf{Y}$ & 120 & 280 & 120 & 260 & 430 & 192 & 215 & 192 & 75 & 162 & 330 & 790 \\
\hline $\mathrm{Zn}$ & 910 & 870 & 1100 & 630 & 870 & 820 & 770 & 850 & 330 & 610 & 840 & 760 \\
\hline $\bar{\gamma}$ & 1.028 & .990 & 1.002 & 1.023 & 1.024 & .990 & .970 & .977 & 991 & $\cdot$ & 1.001 & 1.012 \\
\hline Sampled & - & Bulk & Bulk & Bulk & Bulk & Bulk & Bulk & Bulk & Bulk & Bulk & $0.10 \mathrm{~mm}$ & $10-20 \mathrm{~mm}$ \\
\hline Interval & - & $0.5 \mathrm{~mm}$ & $0-2 \mathrm{~mm}$ &. &. & $0-2 \mathrm{~mm}$ & $0-2 \mathrm{~mm}$ & $0-2 \mathrm{~mm}$ & $0-2 \mathrm{~mm}$ & $0.5 \mathrm{~mm}$ & 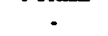 & 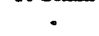 \\
\hline
\end{tabular}


Table 1. Composition of crusts analyzed by USGS Reston Labs (cont'd).

\begin{tabular}{|c|c|c|c|c|c|c|c|c|c|c|c|c|}
\hline $\begin{array}{l}\text { Soquenoell } \\
\text { Latitudo }\end{array}$ & $\begin{array}{c}020006 \\
8.755\end{array}$ & $\begin{array}{c}020007 \\
8.755\end{array}$ & $\begin{array}{c}020007 \\
8.755\end{array}$ & $\begin{array}{c}020007 \\
8.755\end{array}$ & $\begin{array}{c}020007 \\
8.755\end{array}$ & $\begin{array}{c}020008 \\
8.755\end{array}$ & $\begin{array}{c}020009 \\
8.755\end{array}$ & $\begin{array}{c}020009 \\
8.755\end{array}$ & $\begin{array}{c}020009 \\
8.755\end{array}$ & $\begin{array}{c}020010 \\
8.755\end{array}$ & $\begin{array}{c}020010 \\
8.755\end{array}$ & $\begin{array}{c}020010 \\
8.755\end{array}$ \\
\hline Langitade & 169.792 & 169.792 & 169.792 & 169.792 & 169.792 & 169.792 & 169.792 & 169.792 & 169.792 & 169.792 & 169.792 & 169.792 \\
\hline Depth & 2000 & 2000 & 2900 & 2900 & 2000 & 2900 & 2000 & 2000 & 2900 & 2900 & 2900 & 2000 \\
\hline Lab: & D6-1-C & D6-2-A & DG-2-B & D6-2-C & D6-2-D & D6-4-A & DG-6-A & D6-6-B & $D 6-6-C$ & D6-13-A & D6-13-B & D6-13-C \\
\hline $\mathrm{SiO}_{2}$ (wt.\%) & 2.17 & 8.29 & 120 & 6.39 & 3.41 & 6.43 & 4.48 & 8.73 & 5.49 & 5.73 & 10.0 & 6.66 \\
\hline $\mathrm{TO}_{2}$ & 1.01 & 1.87 & 2.36 & 2.30 & 1.49 & 1.52 & 1.47 & 223 & 2.30 & 1.71 & 2.22 & 2.25 \\
\hline $\mathrm{MmO}_{2}$ & 273 & 41.7 & 34.2 & 42.0 & 38.2 & 41.2 & 47.9 & 41.2 & 425 & 44.1 & 38.2 & 39.6 \\
\hline & 12.7 & 27.0 & 29.0 & 26.8 & 18.8 & 28.8 & 24.7 & 26.8 & 283 & 26.2 & 27.7 & 28.2 \\
\hline & 90 & 131 & 289 & 1.40 & .66 & .86 & 49 & 1.87 & 1.19 & .77 & 200 & 1.47 \\
\hline $\mathrm{Co}_{3}^{\circ} \mathrm{O}$ & 350 & 1.07 & .718 & 1.06 & .644 & 969 & 1.22 & 909 & .785 & 1.15 & .867 & 926 \\
\hline NiO & .683 & .612 & 556 & .666 & .696 & 512 & .692 & .621 & .617 & .626 & .673 & .623 \\
\hline$\infty$ & .161 & .069 & .113 & .152 & .126 & .071 & .053 & .135 & .197 & .091 & .148 & .221 \\
\hline $\mathrm{CnO}$ & 26.4 & 3.61 & 3.81 & 3.77 & 13.9 & 3.81 & 4.14 & 3.81 & 4.16 & 3.80 & 3.37 & 3.64 \\
\hline MgO & 1.61 & 1.84 & 1.79 & 1.91 & 159 & 1.96 & 1.87 & 1.77 & 1.76 & 1.88 & 1.80 & 1.80 \\
\hline $\mathrm{N}_{2} \mathrm{O}$ & 1.93 & 245 & 2.39 & 2.20 & 2.30 & 3.09 & 2.47 & 241 & 208 & 2.50 & 2.39 & 2.31 \\
\hline $\mathbf{K}_{2} \mathbf{0}$ & .42 & 56 & .79 & 57 & AS & 52 & 59 & .73 & .55 & 51 & .72 & 57 \\
\hline $\mathrm{Co}_{2}$ & 4.32 & .67 & .66 & .60 & 1.64 & 58 & .67 & 35 & .63 & .66 & .56 & 56 \\
\hline $\mathrm{P}_{2} \mathrm{O}_{5}$ & 13.64 & 1.01 & 1.27 & 97 & 7.24 & 1.14 & 1.06 & .85 & 1.02 & 1.00 & .78 & .81 \\
\hline $\mathrm{H}_{2} \mathrm{O}+$ & 5.8 & 75 & 8.4 & 8.4 & 7.2 & 7.5 & 75 & 8.2 & 8.0 & 7.4 & 75 & 7.6 \\
\hline $\mathrm{H}_{2}^{2} \mathrm{O}$ & 24.3 & 25.2 & 222 & 21.7 & 19.6 & 25.5 & 26.4 & 22.1 & 23.7 & 26.8 & 26.2 & 26.5 \\
\hline Sum & 995 & 995 & 100.9 & 99.3 & 98.4 & 99.0 & 99.3 & 100.9 & 99.6 & 98.1 & 98.9 & 97.3 \\
\hline$A($ ppon) & 249 & 199 & 219 & 270 & 300 & 350 & 250 & 290 & 340 & 244 & 290 & 330 \\
\hline Ba & 1900 & 1900 & 1800 & 2300 & 1600 & 1200 & 1900 & 2800 & 1300 & 1800 & 2400 & 1500 \\
\hline Cd & 5.7 & 3.3 & 3.0 & 4.3 & 4.0 & 3.6 & 3.5 & 3.1 & 4.4 & 3.7 & 3.1 & 3.8 \\
\hline $\mathbf{C o}$ & 1500 & 1400 & 950 & 1200 & 840 & 890 & 940 & 1300 & 750 & 1000 & 1200 & 830 \\
\hline$\alpha$ & 9 & 6 & 17 & 9 & 33 & 3 & 22 & 5 & 3 & 22 & 9 & 11 \\
\hline Mo & 480 & 570 & 300 & 540 & 510 & 500 & 460 & 600 & 720 & 370 & 500 & 590 \\
\hline Pb & 1800 & 1400 & 1400 & 1700 & 1700 & 1600 & 1400 & 1400 & 1900 & 1300 & 1400 & 1800 \\
\hline Sr & 1500 & 1900 & 1500 & 1800 & 1700 & 1600 & 1700 & 2000 & 1800 & 1500 & 1600 & 1600 \\
\hline $\mathbf{v}$ & 570 & 620 & 570 & 750 & 760 & 770 & 650 & 830 & 840 & 580 & 790 & 750 \\
\hline $\mathbf{Y}$ & 180 & 270 & 193 & 153 & 187 & 201 & 167 & 157 & 217 & 149 & 117 & 205 \\
\hline $\mathbf{Z n}$ & 950 & 770 & 780 & 970 & 740 & 660 & 850 & 1100 & 680 & 720 & 880 & 720 \\
\hline$\gamma$ & 991 & .991 & 978 & .993 & 1.002 & 996 & 993 & 978 & 990 & 1.005 & .997 & 1.014 \\
\hline Sampled & $\mathrm{Mm}-\mathrm{P}_{2} \mathrm{O}_{5}$ & Botryoids & $7-15 \sin$ & $20-40 \mathrm{mom}$ & $40-60 \mathrm{~mm}$ & Bulk & $0.10 \mathrm{~mm}$ & $10-25 \mathrm{~mm}$ & $25.35 \mathrm{tmm}$ & Parou: & $5-25 \mathrm{~mm}$ & $25-45 \mathrm{~mm}$ \\
\hline Intival & comont & $0.7 \mathrm{~mm}$ & $\cdot$ & - & - & $0-5 \mathrm{men}$ & - & 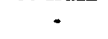 & 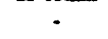 & $0.5 \mathrm{~mm}$ & - & - \\
\hline Soquencolt & 036001 & 039001 & 0.1001 & 043001 & 046001 & 047001 & 047003 & 047004 & 047000 & 047006 & 047007 & 047008 \\
\hline Letituds & 542 & 15.340 & 10.895 & 15.483 & 13.042 & 10.367 & 10.400 & 10.600 & 11458 & 11.483 & 11.458 & 12550 \\
\hline Langitude & 2.483 & -21.850 & -45.297 & -64.083 & -97.067 & -108.383 & -108.433 & -108.800 & -103.300 & -103.217 & -103.233 & -103.267 \\
\hline Depth & 2652 & - & 4640 & 1086 & 3888 & 3140 & 3843 & 4592 & 1498 & 1235 & 1408 & 1240 \\
\hline Labil & W-222718 & W-231374 & W-222720 & W-228415 & W-231351 & W-222681 & W-222682 & W-222579 & W-222541 & W-222542 & W-222543 & W-222544 \\
\hline $\mathrm{SiO}_{2}$ (wt.s) & 24.3 & 4.31 & 16.3 & 9.27 & 29.0 & 18.0 & 30.4 & 255 & 254 & 18.3 & 20.5 & 195 \\
\hline & 83 & 1.65 & .88 & 85 & .78 & 1.36 &.$\infty$ & 1.39 & .65 & .75 & $A 7$ & .79 \\
\hline $\mathrm{MnO}_{2}$ & 19.0 & 20.3 & 19.9 & 27.1 & 23.6 & 27.1 & 27.1 & 285 & 18.8 & 22.6 & 19.0 & 21.1 \\
\hline $\mathrm{Fo}_{2} \mathrm{O}_{3}$ & 275 & 34.6 & 35.0 & 30.2 & 19.5 & 29.7 & 248 & 163 & $\boldsymbol{2 0 . 0}$ & 34.1 & 34.5 & 32.0 \\
\hline $\mathrm{Al}_{2}^{2} \mathrm{O}_{3}^{3}$ & 6.24 & 3.04 & 5.54 & 4.38 & 7.15 & 3.90 & 82 & 7.19 & 6.23 & 4,43 & 4.75 & 4.39 \\
\hline $\mathrm{CO}_{3}^{2} \mathrm{O}$ & 225 & .793 & 250 & .379 & .048 & .187 & .023 & .157 & .045 & .103 & .054 & .097 \\
\hline $\mathrm{NiO}$ & .450 & .193 & 218 & .546 & .540 & 334 & 382 & .602 & 242 & .255 & .173 & 254 \\
\hline$\infty 0$ & 354 & .036 & .089 & .078 & .172 & .153 & .153 & .823 & .117 & .110 & .104 & .072 \\
\hline $\mathrm{CaO}$ & 1.24 & 10.6 & 205 & 4.38 & 3.56 & 3.80 & .78 & 1.94 & 4.22 & 3.63 & 3.15 & 5.07 \\
\hline $\mathrm{MgO}$ & 3.32 & 2.55 & 2.23 & 3.46 & 249 & 2.11 & 288 & 2.87 & 260 & 2.08 & 1.72 & 1.99 \\
\hline $\mathrm{N}_{2} \mathrm{O}$ & 259 & 1.26 & 1.59 & 1.62 & 3.08 & 284 & 283 & 277 & 269 & 2.57 & 290 & 230 \\
\hline $\mathrm{K}_{2}{ }^{2} \mathrm{O}$ & 1.14 & 38 & .60 & .68 & 1.09 & .60 & 2.72 & 1.53 & .84 & .74 & 81 & .81 \\
\hline$\infty_{2}$ & .11 & 4.42 & .45 & 2.20 & .18 & .48 & .06 & .14 & 31 & .44 & .47 & 1.62 \\
\hline $\mathrm{P}_{2} \mathrm{O}_{5}$ & 49 & 261 & 1.09 & 1.12 & .60 & 89 & .16 & .45 & .82 & 1.04 & 1.05 & 1.03 \\
\hline $\mathrm{H}_{2}^{2} \mathrm{O}+$ & 9.1 & 6.6 & 9.8 & 11.1 & 7.2 & 7.4 & 7.0 & 8.9 & 6.3 & 7.7 & 7.8 & 7.6 \\
\hline $\mathrm{H}_{2}^{6} \mathrm{O}$ & 15.2 & 14.1 & 18.2 & 13.7 & 12.8 & 20.0 & 10.1 & 13.3 & 15.7 & 20.3 & 19.2 & 19.9 \\
\hline Sum & 96.9 & 93.3 & 95.9 & 97.4 & 99.1 & 98.8 & 100.2 & 99.2 & 98.2 & 98.9 & 97.5 & 98.6 \\
\hline Axppon) & 142 & 480 & 430 & 570 & 161 & 238 & 32 & 81 & 178 & 260 & 235 & 225 \\
\hline Ba & 2400 & 1500 & 930 & 1700 & 1300 & 1400 & 580 & 3000 & 1200 & 1300 & 1400 & 1200 \\
\hline Cd & 1.7 & 4.0 & 24 & 45 & 3.7 & 29 & 8.3 & 23 & 27 & 25 & 3.1 & 26 \\
\hline Co & 1300 & 2900 & 1600 & 780 & 310 & 550 & 47 & 280 & 154 & 200 & 210 & 260 \\
\hline$a$ & 67 & 57 & 28 & 60 & 44 & 14 & 3 & 22 & 57 & 34 & 27 & 42 \\
\hline Mo & $\delta$ & 310 & 280 & 380 & 300 & 280 & 200 & 108 & 100 & 188 & 90 & 150 \\
\hline$P_{0}$ & 1000 & 1900 & 980 & 930 & 172 & 380 & $\delta 5$ & 196 & 190 & 290 & 136 & 250 \\
\hline Sr & 840 & 1400 & 1000 & 1300 & 890 & 1300 & 211 & 620 & 1000 & 1200 & 1100 & 1200 \\
\hline $\mathbf{v}$ & 440 & 1200 & 940 & 1000 & 500 & 610 & 75 & 420 & 450 & 580 & 540 & 540 \\
\hline $\mathbf{Y}$ & 64 & 151 & 171 & 274 & 115 & 188 & 20 & 57 & 116 & 163 & 136 & 150 \\
\hline $\mathbf{Z n}$ & 710 & 550 & 550 & 790 & 570 & 590 & 870 & 670 & 650 & 650 & 660 & 640 \\
\hline$\gamma$ & 1.018 & 1.056 & 1.028 & 1.013 & .995 & 998 & 984 & .994 & 1.004 & 997 & 1.011 & 1.000 \\
\hline Sampled & Bulk & Bulk & Bulk & Bulk & Bulk & Bulk & Bulk & Bulk & Bulk & Bulk & Bulk & Bulk \\
\hline Intorval & $0-14 \mathrm{~mm}$ & $0-15 \mathrm{~mm}$ & $0.24 \mathrm{~mm}$ & $0.50 \mathrm{~mm}$ & $0-35 \mathrm{~mm}$ & - & - & $0.12 \mathrm{anm}$ & - & - & - & - \\
\hline
\end{tabular}


Table 1. Composition of crusts analyzed by USGS Reston Labs (cont'd).

\begin{tabular}{|c|c|c|c|c|c|c|c|c|c|c|c|c|}
\hline Sequencell & 047010 & 047011 & $047013 \uparrow$ & $047014 \uparrow$ & 047015 & 047016 & 047017 & 047018 & 047020 & 047021 & $047022 t$ & 047023 \\
\hline Lationde & 12.733 & 12.717 & 13.400 & 13.358 & 13.022 & 12983 & 13.767 & 13.767 & 13,447 & 12.723 & 12.745 & 13.012 \\
\hline Longitude & -102583 & -102583 & -102517 & -102.467 & -100.817 & -100.817 & -101.875 & -101.872 & -102.610 & -102513 & .102 .562 & -100.848 \\
\hline Depth & 1450 & 1020 & 887 & 630 & 1340 & 1342 & 928 & 990 & 3424 & 2700 & 2202 & 2381 \\
\hline Labl & W-222545 & W-222546 & W-222547 & W-222548 & W-2202549 & W-222550 & W-220551 & W-222552 & W-222506 & $w-222507$ & W-222508 & W-222509 \\
\hline $\mathrm{SiO}_{2}$ (m.s) & 19.3 & 195 & 47.1 & 48.8 & 164 & 21.6 & 13.9 & 9.76 & 23.4 & 24.4 & 49.0 & 16.1 \\
\hline $\mathrm{TiO}_{2}^{2}$ & 89 & 1.28 & 1.32 & 2.20 & 35 & .63 & 1.47 & 1.34 & .44 & .58 & 1.23 & 80 \\
\hline $\mathrm{MnO}_{2}^{2}$ & 23.8 & 29.3 & 1.55 & .24 & 41.0 & 22.1 & 28.1 & 35.3 & 165 & 18.2 & .26 & 23.1 \\
\hline $\mathrm{Fo}_{2} \mathrm{O}_{3}^{2}$ & 29.9 & 222 & 109 & 13.0 & 20.2 & 29.0 & 33.2 & 20.2 & 345 & 32.7 & 10.6 & 30.8 \\
\hline & 5.15 & 6.06 & 15.4 & 14.7 & 3.97 & 594 & 2.65 & 2.14 & 5.97 & 5.67 & 17.0 & 3.71 \\
\hline & .141 & .332 & .049 & .004 & .064 & .082 & .608 & .737 & .041 & on & .007 & .190 \\
\hline $\mathrm{NIO}^{4}$ & 314 & $A 41$ & .026 & .004 & .669 & 272 & 220 & 520 & .158 & .211 & .011 & 323 \\
\hline CuO & .078 & .068 & .007 & .002 & 322 & .134 & .021 & .040 & .171 & .136 & .016 & .122 \\
\hline $\mathrm{CaO}$ & 3.81 & 269 & 115 & 10.6 & 1.80 & 3.34 & 3.03 & 3.14 & 2.24 & 2.88 & 12.9 & 3.26 \\
\hline $\mathrm{M}_{8} \mathrm{O}$ & 2.06 & 3.83 & 6.41 & 4.70 & 2.91 & 1.79 & 1.86 & 1.92 & 1.81 & 1.97 & 553 & 1.68 \\
\hline $\mathrm{N}_{2} \mathrm{O}$ & 233 & 2.44 & 2.75 & 3.55 & 234 & 226 & 2.17 & 251 & 282 & 254 & 2.99 & 255 \\
\hline $\mathbf{k}_{2}^{2} \mathbf{O}$ & 92 & 1.18 & .34 & .62 & 1.14 & .10 & .78 & .60 & 1.02 & .89 & .32 & 82 \\
\hline $\mathrm{CO}_{2}$ & 99 & 53 & - & .01 & 41 & .88 & .42 & $A 6$ & .20 & .20 & .01 & 51 \\
\hline $\mathrm{P}_{2} \mathrm{O}_{5}$ & 92 & .71 & .19 & 30 & .48 & 89 & 1.20 & 1.24 & 1.00 & .88 & .10 & 1.07 \\
\hline $\mathrm{H}_{2}^{2} \mathrm{O}+$ & 95 & 8.0 & 15 & 1.2 & 98 & 10.9 & 9.6 & 10.3 & 7.0 & 6.1 & 1.0 & 7.5 \\
\hline $\mathrm{H}_{2}^{2} \mathrm{O}$ & 229 & 22.1 & 1.7 & 1.3 & 14.4 & 205 & 21.6 & 24.2 & 19.6 & 21.7 & 1.1 & 21.1 \\
\hline Sum & 100.1 & 98.5 & - & 100.1 & 101.9 & 100.0 & 99.2 & 99.2 & 97.3 & 97.4 & 101.0 & 975 \\
\hline$A(p p m)$ & 182 & 128 & 14 & 16 & 11 & 189 & 310 & 290 & 224 & 217 & 21 & 280 \\
\hline Ba & 1100 & 950 & 45 & 36 & 4200 & 1400 & 1300 & 1300 & 1400 & 1500 & 28 & 1500 \\
\hline Cd & 1.8 & 12.5 & $<.1$ & $<1$ & 6.0 & 2.1 & 2.2 & 3.7 & 2.6 & 2.4 & 4 & 28 \\
\hline$\infty$ & 380 & 580 & 59 & 20 & 175 & 280 & 1300 & 1300 & 174 & 230 & $\theta$ & 480 \\
\hline$a$ & 40 & 57 & 370 & 213 & 18 & 18 & 55 & 19 & 25 & 47 & 350 & 13 \\
\hline Mo & 221 & 200 & $<4$ & $<4$ & 210 & 189 & 340 & 440 & 93 & 54 & $<4$ & 390 \\
\hline $\mathbf{P b}$ & 320 & 630 & 36 & $\theta$ & 70 & 189 & 1400 & 1300 & 224 & 270 & 44 & 380 \\
\hline Sz & 1200 & 960 & 224 & 213 & 960 & 1100 & 1500 & 1600 & 920 & 1200 & 121 & 1500 \\
\hline $\mathbf{v}$ & 520 & 420 & 300 & 400 & 560 & 530 & 730 & 740 & 550 & 520 & 310 & 670 \\
\hline $\mathbf{Y}$ & 156 & 103 & 32 & 38 & 82 & 138 & 204 & 185 & 124 & 111 & 28 & 203 \\
\hline $\mathbf{z}$ & 610 & 760 & 112 & 122 & 1100 & 590 & 590 & 650 & 610 & 730 & 152 & 650 \\
\hline$\gamma$ & 985 & 1.001 & $\cdot$ & .985 & .968 & 986 & 993 & 994 & 1.014 & 1.012 & .977 & 1.011 \\
\hline Samplod & Bulk & Bulk & Bulk & Bulk & Bulk & Bulk & Bulk & Bulk & Bulk & Bulk & Bulk & Bulk \\
\hline Interval & $0-22 \mathrm{~mm}$ & $0-9$ mm & - & $\cdot$ & $0-16 \mathrm{am}$ & $0-11 \mathrm{~mm}$ & O-Sinm & $0-7 \mathrm{~mm}$ & - & • & $\cdot$ & - \\
\hline Sequencell & 047024 & 047025 & 047026 & 047022 & 047028 & 047029 & 047030 & 047031 & 051001 & 051003 & 052001 & 052003 \\
\hline Latitude & 12.962 & 12.260 & 12567 & 12.037 & 11.418 & 11.963 & 11.263 & 11.240 & 11.017 & 14.417 & 18.350 & 18.699 \\
\hline Longitude & -100.900 & -100507 & -100.363 & -101.600 & -101.625 & -101.617 & -101.088 & -101.125 & -140.142 & -145.867 & -158.450 & -158.380 \\
\hline Depth & 3478 & 2600 & 3317 & 3070 & 3034 & 2000 & 3205 & 3050 & 4602 & 4618 & 1317 & 3261 \\
\hline Lab* & W-222510 & W.222511 & W-222512 & W-222513 & $w-222514$ & W-222515 & W-222516 & W-222517 & W. 222700 & W-229850 & W-222531 & W-228990 \\
\hline $\mathrm{SiO}_{2}(\mathrm{w} . \%)$ & 18.3 & 36.4 & 15.1 & 18.4 & 220 & 21.8 & 18.8 & 11.8 & 11.0 & 11.3 & 10.3 & 15.4 \\
\hline $\mathrm{TiO}_{2}^{2}$ & $s 1$ & 1.56 & .38 & .49 & 44 & 41 & 52 & 30 & 1.64 & 2.07 & 1.33 & 3.24 \\
\hline $\mathrm{MnO}_{2}$ & 24.2 & 10.9 & 43.6 & 22.6 & 29.5 & 20.7 & 36.9 & 54.4 & 35.8 & 39.2 & 39.8 & 24.1 \\
\hline $\mathrm{Pe}_{2} \mathrm{O}_{3}^{2}$ & 33.3 & 18.7 & 18.0 & 34.3 & 22.6 & 27.0 & 179 & 9.05 & 28.2 & 22.8 & 21.8 & 345 \\
\hline $\mathrm{Al}_{2} \mathrm{O}_{3}$ & 4.38 & 11.9 & 4.20 & 3.99 & 6.01 & 4.81 & 5.46 & 4.36 & 251 & 2.73 & 3.38 & 3.32 \\
\hline $\mathrm{CO}_{3}^{2} \mathrm{O}_{4}^{3}$ & .069 & .077 & .046 & .090 & .071 & .082 & .057 & .026 & .448 & 565 & .649 & .457 \\
\hline Nล0 4 & 259 & .143 & .738 & .281 & .706 & .623 & 858 & 1.007 & .611 & 576 & .607 & 206 \\
\hline cio & .135 & .052 & 378 & .215 & .318 & 352 & 407 & .637 & .269 & .425 & .055 & .120 \\
\hline $\mathrm{CaO}$ & 2.65 & 5.82 & 2.35 & 2.27 & 2.12 & 194 & 2.36 & 1.93 & 3.06 & 3.37 & 5.19 & 3.22 \\
\hline $\mathrm{M} 8 \mathrm{O}$ & 1.62 & 4.79 & 2.18 & 1.87 & 3.07 & 266 & 2.87 & 3.50 & 1.91 & 1.92 & 3.07 & 1.67 \\
\hline $\mathrm{N}_{2} \mathrm{O}$ & 258 & 3.13 & 3.50 & 2.73 & 2.67 & 256 & 3.04 & 259 & 1.88 & 247 & 3.84 & 208 \\
\hline $\mathbf{K}_{2} \mathbf{O}$ & 84 & 1.14 & .93 & .75 & 1.07 & 1.03 & 1.06 & 1.23 & .69 & .89 & 89 & .71 \\
\hline $\mathrm{CO}_{2}$ & 34 & .18 & .23 & .18 & .10 & .11 & .13 & .11 & .43 & .39 & .77 & 45 \\
\hline $\mathrm{P}_{2} \mathrm{O}_{5}$ & 96 & 59 & .55 & .93 & $A B$ & 59 & 55 & 31 & .78 & .63 & 1.09 & 1.09 \\
\hline $\mathrm{H}_{2}^{2} \mathrm{O}+$ & 6.9 & 6.7 & 7.1 & 95 & 7.6 & 6.6 & 7.6 & 7.7 & 73 & 8.7 & 4.6 & 8.1 \\
\hline $\mathrm{H}_{2} \mathrm{O}$ & 21.4 & 11.1 & 17.2 & 18.6 & 13.5 & 18.3 & 16.9 & 115 & 20.9 & 20.4 & 24.5 & 135 \\
\hline Sum & 97.0 & 102.1 & 99.3 & 98.5 & 98.8 & 98.2 & 98.5 & 98.8 & 96.6 & 98.0 & 97.4 & 98.7 \\
\hline Ax(ppon) & 270 & 97 & 145 & 246 & 101 & 147 & 132 & 76 & 240 & 176 & 199 & 173 \\
\hline Ba & 1400 & 600 & 1400 & 2200 & 2300 & 2200 & 2000 & 3300 & 1500 & 1900 & 2600 & 1900 \\
\hline Cd & 25 & 1.7 & 8.7 & 3.6 & 4.6 & 5.6 & 10.6 & 12.4 & 3.9 & 3.6 & 4.4 & 2.1 \\
\hline$\infty$ & 280 & 203 & 169 & 270 & 162 & 184 & 205 & 113 & 1100 & 1600 & 2100 & 1600 \\
\hline$a$ & 12 & 124 & 9 & 10 & 37 & 21 & 25 & 10 & 16 & 3 & 20 & 29 \\
\hline Mo & 280 & 350 & 350 & 184 & 98 & 135 & 320 & 340 & 390 & 400 & 700 & 173 \\
\hline $\mathbf{P b}$ & 153 & 169 & 112 & 233 & 113 & 171 & 156 & 78 & 1100 & 1000 & 2500 & 1400 \\
\hline Sr & 1200 & 670 & 820 & 1400 & 880 & 920 & 870 & 680 & 1300 & 1400 & 1600 & 1400 \\
\hline $\mathbf{v}$ & 600 & 320 & 430 & 640 & 460 & 470 & 420 & 410 & 660 & 600 & 740 & 650 \\
\hline $\mathbf{Y}$ & 165 & 81 & 89 & 147 & 65 & 70 & 77 & 45 & 177 & 126 & 83 & 220 \\
\hline $\mathbf{z n}$ & 620 & 380 & 1600 & 850 & 980 & 950 & 1600 & 1800 & 1100 & 680 & 860 & 760 \\
\hline$\gamma$ & 1.017 & 966 & 993 & 1.001 & .998 & 1.004 & 1.001 & 998 & 1.021 & 1.006 & 1.012 & .999 \\
\hline Sempled & Bulk & Bulk & Bulk & Bulk & Bulk & Bulk & Bulk & Bulk & Bulk & Bull & Bulk & Bulk \\
\hline Interval & 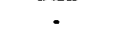 & - & - & - & $\cdot$ & . & - & - & - & • & $0-1 \mathrm{~mm}$ & - \\
\hline
\end{tabular}


Table 1. Composition of crusts analyzed by USGS Reston Labs (cont'd).

\begin{tabular}{|c|c|c|c|c|c|c|c|c|c|c|c|c|}
\hline Sequencoll & 052005 & 052005 & 052005 & 052005 & 052006 & 052006 & 052006 & 052007 & 052000 & 052008 & 052009 & 052010 \\
\hline Letionde & 18.643 & 18.643 & 18.643 & 18.643 & 18.643 & 18.643 & 18.643 & 18.643 & 18.643 & 18.643 & 18.637 & 18.637 \\
\hline Longitude & -158.295 & -158.295 & -158.295 & -158.295 & -158.295 & -158.295 & -158.295 & -158.295 & -158.295 & -158.295 & -158.313 & -158.313 \\
\hline Depth & 1924 & 1924 & 1924 & 1924 & 1924 & 1924 & 1924 & 1924 & 1924 & 1924 & 2002 & 2002 \\
\hline Leby & W-2283954 & $w-229243$ & W-229244 & W.2229245 & W-229246 & $w \cdot 229247$ & $W-2229280$ & W-223991 & W-229248 & W-2229249 & W-229250 & W-229251 \\
\hline$\overline{\mathrm{SiO}_{2}(\mathrm{~m} . \%)}$ & 124 & 15.2 & 15.6 & 13.8 & 13.1 & 14.3 & 13.2 & 14.7 & 11.0 & 144 & 10.7 & 10.7 \\
\hline $\mathrm{TiO}_{2}^{25}$ & 248 & 254 & 220 & 202 & 1.98 & 1.39 & 2.34 & 211 & 200 & 218 & 214 & 215 \\
\hline $\mathrm{MmO}_{2}$ & 28.8 & 202 & 29.5 & 327 & 32.2 & 31.8 & 31.7 & 304 & 320 & 328 & 31.4 & 328 \\
\hline & 33.4 & 20.7 & 268 & 26.3 & 27.1 & 25.8 & 29.1 & 27.7 & 31.2 & 24.2 & 31.5 & 29.6 \\
\hline $\mathrm{Al}_{2}^{2} \mathrm{O}_{3}$ & 1.95 & 3.51 & 3.73 & 3.10 & 3.13 & 4.07 & 261 & 3.46 & 1.72 & 3.59 & 1.71 & 1.84 \\
\hline $\mathrm{CO}_{3}^{2} \mathrm{O}$ & .607 & .723 & .618 & .646 & .611 & ASO & .685 & .769 & .708 & .670 & .594 & 686 \\
\hline $\mathrm{NiO}$ & 254 & 367 & 390 & .412 & .395 & 479 & 342 & $A 11$ & 323 & $A 2$ & .307 & 343 \\
\hline Co & 068 & .119 & .171 & .203 & .173 & .314 & .146 & .133 & .069 & .215 & .102 & .120 \\
\hline $\mathrm{CaO}$ & 3.10 & 3.44 & 3.37 & 3.38 & 3.46 & 3.48 & 347 & 3.22 & 3.33 & 3.79 & 3.24 & 3.31 \\
\hline $\mathrm{MgO}_{8}$ & 1.63 & 1.84 & 1.75 & 1.63 & 1.70 & 1.74 & 1.67 & 1.66 & 1.65 & 1.67 & 1.60 & 1.61 \\
\hline $\mathrm{Ne}_{2} \mathrm{O}$ & 212 & 232 & 237 & 240 & 2.38 & 247 & 240 & 2.51 & 2.21 & 2.47 & 210 & 214 \\
\hline $\mathrm{k}_{2} \mathrm{O}$ & 55 & .79 & .89 & 82 & .83 & 1.17 & .65 & 1.22 & .54 & .88 & 50 & 58 \\
\hline$\infty_{2}$ & 18 & .44 & .43 & 46 & $A 7$ & 41 & so & 47 & .45 & .54 & 52 & 52 \\
\hline $\mathrm{P}_{2} \mathrm{O}^{2}$ & 1.10 & 95 & .94 & 85 & 92 & 91 & 99 & 1.03 & 1.01 & .84 & 95 & 94 \\
\hline $\mathrm{H}_{2} \mathrm{O}+$ & 8.6 & 6.4 & 8.6 & 8.3 & 8.9 & 9.2 & 9.0 & 9.4 & 8.0 & 8.1 & 8.8 & 9.3 \\
\hline $\mathrm{HO}$ & 14.7 & 13.4 & 119 & 13.5 & 13.1 & 123 & 14.5 & 13.2 & 134 & 126 & 129 & 14.6 \\
\hline $\operatorname{sen}$ & $97 A$ & 95.6 & 974 & 97.0 & 97.4 & 97.9 & 98.8 & 993 & 964 & 96.8 & 96.2 & 96.6 \\
\hline$A x(p p m)$ & 227 & 231 & 243 & 246 & 245 & 260 & 280 & 161 & 310 & 217 & 310 & 280 \\
\hline $\mathrm{Be}$ & 2400 & 2100 & 1900 & 1800 & 1900 & 2000 & 2000 & 2200 & 1500 & 1800 & 1600 & 1500 \\
\hline Cd & 2.5 & 25 & 2.5 & 25 & 23 & 22 & 23 & 25 & 24 & 29 & 23 & 29 \\
\hline 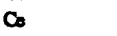 & 1100 & 1100 & 1200 & 1100 & 810 & 1100 & 1100 & 1100 & 1100 & 1100 & 1100 & 1100 \\
\hline$a$ & 25 & 38 & 13 & 16 & 6 & 13 & 16 & 14 & 14 & 14 & 10 & 10 \\
\hline Mo & 280 & 280 & 360 & 290 & 400 & 370 & 350 & 370 & 370 & 290 & 390 & 370 \\
\hline $\mathrm{Pb}$ & 1100 & 1300 & 1000 & 1600 & 550 & 890 & 1200 & 1400 & 1500 & 1000 & 1400 & 1400 \\
\hline Sr & 1400 & 1400 & 1500 & 1600 & 1400 & 1500 & 1600 & 1600 & 1500 & 1500 & 1600 & 1500 \\
\hline v & 540 & 550 & 570 & 690 & 550 & 600 & 630 & 680 & 660 & 530 & 670 & 590 \\
\hline $\mathbf{Y}$ & 227 & 231 & 220 & 246 & 171 & 219 & 234 & 219 & 231 & 101 & 230 & 231 \\
\hline $\mathbf{Z n}$ & 650 & 650 & 660 & 740 & 700 & 660 & 630 & 690 & 670 & 650 & 710 & 660 \\
\hline $\bar{\gamma}$ & 1.012 & 1.031 & 1.013 & 1.016 & 1.012 & 1.007 & 998 & .993 & 1.023 & 1.019 & 1.025 & 1.021 \\
\hline Sampled & Top & Bulk & Bulk & Bottom & Bulk & Bottom & Top & - & Bulk & Top & Bulk & Bulk \\
\hline Intervil & $\cdot$ & - & - & - & - & - & $\bullet$ & - & . & 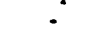 & $\cdot$ & . \\
\hline Sequencoll & 052010 & 052011 & 052012 & 052015 & 052015 & 052015 & 052016 & 052016 & 052016 & 052017 & 052017 & 052017 \\
\hline Latitude & 18.637 & 18.637 & 18.637 & 18.683 & 18.683 & 18.683 & 18.683 & 18.683 & 18.683 & 18.683 & 18.683 & 18.683 \\
\hline Lonpitude & -158.313 & -158.313 & -158.313 & -158.314 & -158.314 & .158 .314 & -158.314 & -158.314 & -158.314 & -158.314 & -158.314 & -158.314 \\
\hline Depth & 2002 & 2002 & 2002 & 1047 & 1047 & 1047 & 1047 & 1047 & 1047 & 1047 & 1047 & 1047 \\
\hline Lab* & W-229252 & W-229253 & W-229254 & W-229255 & W-2229256 & W-229257 & W-220258 & W-220276 & W-229277 & W-229259 & W-2229260 & W-229278 \\
\hline $\mathrm{siO}_{2}(\mathrm{wt} . \%)$ & 10.4 & 125 & 9.83 & 5.90 & 6.98 & 5.33 & 8.69 & 7.47 & 103 & 6.67 & 4.33 & 6.31 \\
\hline $\mathrm{TiO}_{2}$ & 228 & 246 & 2.18 & 1.81 & 2.25 & 1.72 & 2.18 & 224 & 1.40 & 1.96 & 1.41 & 1.77 \\
\hline $\mathrm{MmO}_{2}$ & 34.2 & 205 & 34.2 & 38.2 & 41.6 & 37.0 & 39.7 & 420 & 28.1 & 40.7 & 325 & 36.2 \\
\hline $\mathrm{Pa}_{2} \mathrm{O}_{3}$ & 28.9 & 31.5 & 294 & 21.2 & 23.5 & 18.7 & 22.3 & 23.0 & 17.0 & 224 & 16.0 & 20.6 \\
\hline $\mathrm{Al}_{2}^{2} \mathrm{O}_{3}$ & 1.84 & 225 & 1.68 & 1.38 & 1.47 & 1.37 & 207 & 1.56 & 3.15 & 1.61 & 1.15 & 1.60 \\
\hline $\mathrm{CO}_{3}^{2} \mathrm{O}_{4}$ & 834 & .607 & .708 & .740 & 1.06 & .603 & .984 & 1.03 & A45 & .877 & 514 & .690 \\
\hline $\mathrm{NiO}^{4}$ & 412 & 276 & 353 & .544 & .60 & 563 & .661 & .687 & .610 & .658 & .452 & 545 \\
\hline$\infty$ & .109 & .079 & .133 & .145 & .131 & 227 & .155 & .158 & .259 & .173 & .153 & .169 \\
\hline $\mathrm{CaO}$ & 3.38 & 3.28 & 3.33 & 8.57 & 3.84 & 12.2 & 4.18 & 3.80 & 13.7 & 5.63 & 16.8 & 10.1 \\
\hline $\mathrm{MgO}_{8}$ & 1.72 & 1.63 & 1.61 & 159 & 1.76 & 1.54 & 1.82 & 1.77 & 1.63 & 1.72 & 1.40 & 1.59 \\
\hline $\mathrm{N} 2 \mathrm{O}$ & 218 & 2.11 & 2.14 & 215 & 231 & 2.15 & 244 & 252 & 222 & 2.37 & 1.81 & 229 \\
\hline K్20 & .60 & 59 & 57 & 57 & .67 & .62 & 80 & .65 & 1.12 & .65 & 51 & 54 \\
\hline$\infty_{2}$ & AS & 43 & .47 & 1.02 & .51 & 1.36 & .44 & .46 & 1.54 & .66 & 1.93 & 1.20 \\
\hline $\mathrm{P}_{2} \mathrm{O}_{5}^{2}$ & 95 & 97 & .90 & 3.84 & 1.23 & 6.04 & 1.22 & 1.00 & 752 & 208 & 8.65 & 488 \\
\hline $\mathrm{H}_{2} \mathrm{O}+$ & 8.9 & 89 & 94 & 9.0 & 10.0 & 7.9 & 8.6 & 9.0 & 7.8 & 92 & 7.7 & 8.0 \\
\hline H, & 135 & 125 & 13.5 & 13.5 & 14.1 & 11.9 & 114 & 13.0 & 8.2 & 13.0 & 9.9 & 11.2 \\
\hline sum & 97.2 & 97.1 & 96.9 & 96.7 & 97.9 & 97.4 & 96.1 & 97.3 & 96.9 & 97.3 & 95.3 & 964 \\
\hline$A \times(p p m)$ & 290 & 300 & 310 & 216 & 249 & 330 & 142 & 260 & 280 & 178 & 237 & 280 \\
\hline Be & 1600 & 1600 & 1600 & 2300 & 2000 & 1900 & 5000 & 1900 & 2000 & 2800 & 2100 & 2000 \\
\hline$\overline{C d}$ & 23 & 2.6 & 25 & 27 & 3.1 & 3.5 & 2.1 & 34 & 29 & 24 & 3.0 & 3.3 \\
\hline$c_{0}$ & 1100 & 1100 & 1100 & 1400 & 1300 & 1200 & 1100 & 1100 & 1300 & 1400 & 1400 & 1100 \\
\hline$a$ & 8 & 19 & 6 & 4 & 5 & 8 & 9 & 7 & 6 & 28 & 11 & 6 \\
\hline Mo & 370 & 310 & 400 & 540 & 590 & 660 & 360 & 500 & 560 & 570 & 560 & 600 \\
\hline Po & 1300 & 1500 & 1300 & 1100 & 1500 & 1500 & 990 & 1400 & 1500 & 1400 & 1400 & 1300 \\
\hline St & 1500 & 1500 & 1600 & 1700 & 1600 & 1900 & 1700 & 1600 & 1700 & 1800 & 1800 & 1700 \\
\hline V & 630 & 630 & 670 & 560 & 670 & 700 & 490 & 590 & 680 & 630 & 660 & 690 \\
\hline $\mathbf{Y}$ & 223 & 217 & 220 & 340 & 185 & 175 & 410 & 147 & 138 & 244 & 214 & 172 \\
\hline $\mathbf{Z n}$ & 690 & 720 & 680 & 740 & 720 & 710 & 730 & 760 & 760 & 710 & 690 & 720 \\
\hline $\bar{\gamma}$ & 1.014 & 1.016 & 1.017 & 1.020 & 1.007 & 1.013 & 1.026 & 1.013 & 1.018 & 1.013 & 1.035 & 1.022 \\
\hline Samplod & Bulk & Bulk & Bulk & Bulk & Young & Old & Bulk & Young & Old & Young & Old & Bulk \\
\hline Interval &. &. &. &. & - & - & 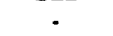 & . & - & 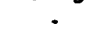 & 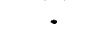 & 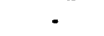 \\
\hline
\end{tabular}


Table 1. Composition of crusts analyzed by USGS Reston Labs (cont'd).

\begin{tabular}{|c|c|c|c|c|c|c|c|c|c|c|c|c|}
\hline Sequencelt & 052019 & 052023 & 052024 & 052025 & 052026 & 052027 & 052028 & 052029 & 052030 & 052031 & 052032 & 052033 \\
\hline Letitude & 19.481 & 18.637 & 18.635 & 18.635 & 18.792 & 18.792 & 18.792 & 18.792 & 18.792 & 18.792 & 18.792 & 18.792 \\
\hline Longitude & -157.054 & -158.313 & -158.320 & -158.320 & -158.229 & -158.229 & -158.229 & -158.220 & -158.220 & -158.220 & -158.229 & -158.229 \\
\hline Depth & 2750 & 2002 & 2760 & 2760 & 2013 & 2013 & 2013 & 2013 & 2013 & 2013 & 2013 & 2013 \\
\hline Leb* & W-234337 & W-228958 & W-235995 & W-235996 & w-230057 & W-235997 & W-235998 & W-235999 & W-236000 & W-236001 & W-236002 & W. 236003 \\
\hline $\mathrm{SiO}_{2}(w t . \%)$ & 12.2 & 13.8 & 19.7 & 25.0 & 17.5 & 17.7 & 14.1 & 13.7 & 21.8 & 27.9 & 17.2 & 28.0 \\
\hline & 210 & 220 & 1.50 & 1.53 & 2.43 & 1.97 & 1.98 & 1.89 & 1.48 & .86 & 1.94 & .78 \\
\hline $\mathrm{MmO}_{2}$ & 31.6 & 30.6 & 28.8 & 22.6 & 28.1 & 28.7 & 35.0 & 25.1 & 25.8 & $20 \mathrm{~A}$ & 28.4 & 213 \\
\hline & 29.2 & 27.6 & 223 & 19.9 & 25.6 & 24.7 & 244 & 21.5 & 20.8 & 18.0 & 25.8 & 16.7 \\
\hline & 2.08 & 264 & 5.82 & 7.68 & 4.28 & 4.77 & 3.18 & 3.75 & 6.56 & 9.11 & 4.65 & 9.07 \\
\hline $\mathrm{Co}_{3}^{2} \mathrm{O}_{4}$ & 1.00 & .630 & .482 & .395 & .813 & .633 & .752 & .466 & 160 & .275 & .591 & 296 \\
\hline NiO & 312 & 412 & 436 & .355 & .334 & 394 & .458 & 378 & 475 & 499 & .418 & $A 07$ \\
\hline CuO & .064 & .174 & .192 & .233 & .052 & .146 & .143 & .200 & .190 & 337 & .161 & 243 \\
\hline $\mathrm{CaO}$ & 3.12 & 3.01 & 2.91 & 4.42 & 3.04 & 3.10 & 3.70 & 10.7 & 2.64 & 3.07 & 3.17 & 3.34 \\
\hline MgO & 1.70 & 1.76 & 1.57 & 1.66 & 1.72 & 1.68 & 1.67 & 1.51 & 1.60 & 1.60 & 1.87 & 136 \\
\hline $\mathrm{Ne}_{2} \mathrm{O}$ & 230 & 2.25 & 299 & 3.01 & 3.01 & 272 & 2.48 & 2.23 & 3.31 & 3.64 & 2.53 & 3.74 \\
\hline $\mathbf{k}_{2}^{2} \mathbf{O}$ & .72 & .85 & 2.13 & 1.86 & 1.35 & 1.55 & 1.08 & 1.13 & 237 & 3.44 & 1.21 & 3.28 \\
\hline $\mathrm{C}_{2}^{2}$ & 32 & 42 & .33 & .40 & 31 & 34 & 32 & 1.14 & .35 & .22 & 31 & .81 \\
\hline $\mathrm{P}_{2} \mathrm{O}_{5}$ & 93 & .85 & .70 & 1.52 & 96 & 85 & 1.10 & 5.49 & .67 & 1.23 & 86 & 55 \\
\hline $\mathrm{H}_{2}^{2} \mathrm{O}+$ & 10.0 & 8.2 & 43 & 5.1 & 5.5 & 6.1 & 5.7 & 2.2 & 5.8 & 4.6 & 5.9 & 6.2 \\
\hline $\mathrm{H}_{2}^{2} \mathrm{O}$ & 183 & 13.5 & 15.3 & 13.9 & 16.2 & 16.1 & 16.7 & 124 & 14.2 & 10.8 & 14.7 & 125 \\
\hline Sum & 97.7 & 953 & 94.1 & 95.7 & 95.1 & 95.4 & 96.0 & 91.4 & 944 & 95.2 & 95.0 & 96.1 \\
\hline$A(p p m)$ & 290 & 173 & 189 & 151 & 227 & 203 & 234 & 137 & 163 & 135 & 188 & 126 \\
\hline Ba & 1500 & 2200 & 1900 & 2000 & 1400 & 1800 & 2000 & 5700 & 2100 & 2100 & 2200 & 1400 \\
\hline Cd & 24 & 2.9 & 3.0 & 20 & 27 & 3.6 & 3.0 & 1.8 & 26 & 2.0 & 23 & 1.7 \\
\hline$\infty$ & 1600 & 1400 & 810 & 720 & 920 & 920 & 1300 & 960 & 680 & 620 & 900 & 580 \\
\hline$a$ & 15 & 19 & 25 & 10 & 20 & 24 & 11 & 9 & 14 & 4 & 23 & 5 \\
\hline Mo & 320 & 400 & 320 & 221 & 270 & 310 & 500 & 270 & 270 & 235 & 246 & 229 \\
\hline$P_{b}$ & 2100 & 1600 & 760 & 600 & 1600 & 1100 & 1700 & 1000 & 700 & 570 & 880 & 470 \\
\hline St & 1500 & 1500 & 1300 & 1300 & 1300 & 1300 & 1600 & 1600 & 1100 & 920 & 1300 & 960 \\
\hline $\mathbf{v}$ & 600 & 680 & 530 & 460 & 570 & 570 & 680 & 530 & 480 & 400 & 530 & 350 \\
\hline $\mathbf{Y}$ & 220 & 150 & 105 & 174 & 179 & 143 & 132 & 194 & 101 & 101 & 188 & 70 \\
\hline $\mathbf{Z n}$ & 590 & 750 & 610 & 550 & 600 & 600 & 650 & 610 & 620 & 610 & 620 & 530 \\
\hline$\gamma$ & 1.009 & 1.034 & 1.047 & 1.031 & 1.037 & 1.034 & 1.027 & 1.078 & 1.045 & 1.036 & 1.038 & 1.026 \\
\hline Sampled & Bulk & Bulk & Bulk & Bulk & Bulk & Bulk & Balk & Balk & Bulk & Bulk & Bulk & Balk \\
\hline loverval & $7-11 \mathrm{~mm}$ & $\cdot$ & $\cdot$ & - & $\cdot$ & $=$ & $\cdot$ & • & - & $\cdot$ & - & • \\
\hline Sequencer\% & 052034 & 052035 & 052036 & 052037 & 052038 & 052039 & 052040 & 052041 & 052042 & 052043 & 052044 & 052045 \\
\hline Latitude & 18.792 & 18.792 & 18.792 & 18.792 & 18.792 & 18.792 & 18.792 & 18.792 & 18.792 & 18.792 & 18.792 & 18.792 \\
\hline Longituds & -158.229 & -158.220 & -158.220 & -158.229 & -158.209 & -158.229 & -158.229 & -158.220 & -158.229 & -158.220 & -158.229 & -158.229 \\
\hline Depth & 2013 & 2013 & 2013 & 2013 & 2013 & 2013 & 2013 & 2013 & 2013 & 2013 & 2013 & 2013 \\
\hline Lab: & W-236004 & W-236005 & W-236006 & W-236007 & W-236008 & W-236009 & W-236010 & W-236011 & W-236012 & W-236013 & W-236014 & W-236015 \\
\hline $\mathrm{SiO}_{2}$ (wt.\%) & 24.0 & 16.2 & 203 & 19.1 & 21.3 & 21.6 & 10.7 & 8.84 & 16.9 & 225 & 17.3 & 11.0 \\
\hline $\mathrm{TiO}_{2}^{2}$ & 1.01 & 2.15 & 1.50 & 209 & 1.13 & 2.23 & 1.63 & 1.89 & 1.64 & 1.33 & 1.36 & 1.85 \\
\hline $\mathrm{Mmo}_{2}$ & 20.8 & 30.2 & 27.7 & 28.0 & 24.9 & 25.1 & 35.4 & 36.9 & 29.1 & 23.6 & 27.9 & 38.6 \\
\hline $\mathrm{Fe}_{2} \mathrm{O}_{3}^{2}$ & 17.5 & 21.9 & 22.1 & 22.6 & 23.7 & 22.2 & 27.2 & 28.0 & 24.1 & 21.8 & 25.7 & 21.0 \\
\hline $\mathrm{Al}_{2} \mathrm{O}_{3}^{3}$ & 7.01 & 4.38 & 6.03 & 5.38 & 637 & 639 & 254 & 1.51 & 4.62 & 6.95 & 4.78 & 246 \\
\hline $\mathrm{CO}_{3}^{2} \mathrm{O}_{4}^{3}$ & 322 & .702 & .486 & .558 & .360 & .543 & .535 & .882 & .622 & .402 & .427 & 1.07 \\
\hline $\mathrm{NiO}^{4}$ & 387 & .462 & .469 & .447 & .351 & 373 & A39 & .444 & .432 & $A 70$ & .399 & .645 \\
\hline $\mathrm{CuO}$ & .225 & .114 & .187 & .176 & .187 & .132 & .253 & .065 & .191 & 277 & .247 & .151 \\
\hline $\mathrm{CaO}$ & 7.55 & 4.73 & 2.76 & 2.97 & 2.67 & 2.61 & 3.40 & 3.47 & 3.19 & 3.12 & 2.93 & 4.03 \\
\hline $\mathrm{MgO}_{8}$ & 142 & 1.67 & 1.74 & 1.71 & 1.56 & 1.65 & 1.62 & 1.71 & 1.65 & 1.69 & 1.69 & 1.72 \\
\hline $\mathrm{Ne}_{2} \mathrm{O}$ & 3.21 & 271 & 3.06 & 278 & 2.98 & 294 & 234 & 2.33 & 271 & 3.27 & 2.61 & 260 \\
\hline $\mathrm{K}_{2} \mathrm{O}$ & 252 & 1.52 & 200 & 1.62 & 2.16 & 2.09 & .82 & .71 & 1.61 & 2.61 & 1.47 & 1.15 \\
\hline$\infty_{2}$ & 1.68 & .44 & .28 & .20 & .28 & .25 & .40 & 40 & .30 & .51 & 30 & .25 \\
\hline $\mathrm{P}_{2} \mathrm{O}_{5}$ & 281 & 1.83 & .69 & .72 & .76 & .78 & .76 & 1.03 & 1.02 & .85 & $n$ & 1.22 \\
\hline $\mathrm{H}_{2}^{2} \mathrm{O}+$ & 5.0 & 6.6 & 6.9 & 6.2 & 63 & 6.4 & 6.4 & 6.4 & 6.5 & 5.9 & 5.9 & 5.9 \\
\hline $\mathrm{H}_{2}^{2} \mathrm{O}$ & 11.1 & 14.6 & 13.2 & 14.6 & 13.1 & 14.8 & 16.0 & 19.7 & 14.6 & 18.7 & 13.9 & 17.1 \\
\hline Sum & 95.5 & 95.6 & 96.2 & 94.6 & 95.1 & 95.2 & 94.5 & 94.5 & 94.6 & 953 & 93.9 & 93.6 \\
\hline Ax(ppm) & 124 & 187 & 173 & 176 & 184 & 153 & 238 & 300 & 199 & 160 & 197 & 205 \\
\hline Ba & 1600 & 1900 & 1800 & 4900 & 1800 & 1800 & 2000 & 1500 & 2000 & 2000 & 2100 & 1600 \\
\hline Cd & 21 & 27 & 25 & 26 & 1.8 & 28 & 2.4 & 3.0 & 23 & 1.8 & 20 & 3.4 \\
\hline$\infty$ & 510 & 1100 & 880 & 850 & 780 & 800 & 1000 & 910 & 970 & 800 & 930 & 940 \\
\hline$a$ & 15 & 22 & 17 & 19 & 8 & 19 & 5 & 9 & 11 & 11 & 8 & 8 \\
\hline Mo & 214 & 320 & 280 & 246 & 260 & 200 & 420 & 520 & 330 & 221 & 300 & 480 \\
\hline Pb & 460 & 1500 & 850 & 1000 & 660 & 1200 & 770 & 1900 & 970 & 810 & 660 & 1300 \\
\hline sr & 1000 & 1300 & 1200 & 1400 & 1200 & 1200 & 1500 & 1600 & 1300 & 1000 & 1300 & 1400 \\
\hline $\mathbf{v}$ & 370 & 540 & 470 & 490 & 490 & 520 & 650 & 720 & 550 & 500 & 570 & 590 \\
\hline $\mathbf{Y}$ & 180 & 222 & 127 & 141 & 107 & 141 & 101 & 237 & 234 & 113 & 114 & 193 \\
\hline $\mathbf{Z n}$ & 530 & 610 & 630 & 660 & 560 & 620 & 700 & 610 & 610 & 690 & 690 & 660 \\
\hline$\gamma$ & 1.033 & 1.031 & 1.025 & 1.042 & 1.037 & 1.036 & 1.043 & 1.044 & 1.043 & 1.035 & 1.051 & 1.053 \\
\hline Sampled & Bulk & Bulk & Bulk & Bulk & Bulk & Bulk & Bulk & Bulk & Bulk & Bulk & Bulk & Bulk \\
\hline hotorval & • & • & - & - & - & - & • & $\cdot$ & $\cdot$ & - & - & - \\
\hline
\end{tabular}


Table 1. Composition of crusts analyzed by USGS Reston Labs (cont'd).

\begin{tabular}{|c|c|c|c|c|c|c|c|c|c|c|c|c|}
\hline Sequencell & 052046 & 052047 & 052048 & 052049 & 052050 & 052051 & 052052 & 052053 & 052054 & 052055 & 052056 & 052057 \\
\hline Latimds & 18.792 & 18.723 & 18.723 & 18.723 & 18.723 & 18.723 & 18.723 & 18.723 & 18.723 & 18.723 & 18.752 & 18.752 \\
\hline Langitude & -158.229 & -158.312 & -158.312 & -158.312 & -158.312 & -158.312 & -158.312 & -158.312 & -158.312 & -158.312 & -158.330 & -158.330 \\
\hline Depth & 2013 & 1350 & 1350 & 1350 & 1350 & 1350 & 1350 & 1350 & 1350 & 1350 & 2100 & 2100 \\
\hline Lab" & W-236016 & W-236017 & W-236018 & W-236019 & W.236020 & $w-236021$ & W-236022 & W-236023 & W-236024 & W-236025 & W-236026 & W.236027 \\
\hline $\mathrm{SiO}_{2}(\mathrm{mt} . \%)$ & 135 & 10.6 & 122 & 9.74 & 19.1 & 13.7 & 102 & 14.5 & 8.64 & 6.72 & 8.99 & 124 \\
\hline $\mathrm{TiO}_{2}^{2}$ & 220 & 247 & 282 & 2.15 & 1.87 & 2.22 & 2.26 & 295 & 227 & 2.18 & 1.99 & 241 \\
\hline & 31.8 & 33.8 & 28.1 & 37.7 & 30.7 & 323 & 35.1 & 20.0 & 372 & 39.3 & 34.8 & 320 \\
\hline & 26.5 & 27.6 & 32.1 & 23.9 & 18.2 & 228 & 275 & 27.7 & 25.7 & 26.8 & 30.3 & 28.8 \\
\hline & 2.91 & 202 & 2.42 & 2.36 & 5.59 & 3.46 & 1.77 & 3.46 & 1.79 & 1.00 & 1.32 & 259 \\
\hline $\mathrm{Co}_{3} \mathrm{O}_{4}$ & .726 & 859 & .789 & .949 & .749 & 890 & 920 & 912 & 928 & 1.09 & .806 & 242 \\
\hline Nió 7 & 101 & $A 02$ & 292 & 505 & .625 & 520 & .434 & .365 & .433 & 526 & .377 & .355 \\
\hline $\mathrm{CuO}$ & .138 & .064 & .026 & .116 & .205 & .131 & .061 & .054 & .090 & .055 & .055 & on \\
\hline $\mathrm{CaO}$ & 3.34 & 3.51 & 3.48 & 3.50 & 3.11 & 3.69 & 3.39 & 3.43 & 3.57 & 3.51 & 3.36 & 3.39 \\
\hline $\mathrm{M}_{8} \mathrm{O}$ & 1.69 & 1.71 & 1.68 & 1.81 & 1.98 & 1.81 & 1.66 & 1.94 & 1.73 & 1.78 & 1.69 & 1.85 \\
\hline $\mathrm{Ne}_{2} \mathrm{O}$ & 261 & 232 & 2.23 & 2.35 & 2.85 & 234 & 2.28 & 232 & 2.20 & 2.26 & 2.22 & 232 \\
\hline $\mathrm{K}_{2} \mathrm{O}$ & .96 & .64 & .64 & .72 & 1.93 & .93 & .67 & 89 & .03 & .59 & .60 & 80 \\
\hline$\infty$ & 31 & .19 & .37 & 35 & .24 & .20 & .19 & 24 & .40 & .22 & 31 & 32 \\
\hline $\mathrm{P}_{2} \mathrm{O}_{5}$ & 1.00 & 1.11 & 138 & 1.02 & .83 & 1.17 & 1.06 & 1.12 & 1.14 & 1.07 & 1.08 & 1.00 \\
\hline $\mathrm{H}_{2} \mathrm{O}^{3}$ & 6.0 & 6.4 & 6.1 & 5.7 & 3.6 & 5.6 & 6.3 & 5.8 & 7.0 & 6.2 & 6.9 & 5.9 \\
\hline $\mathrm{H}_{2}^{2} \mathrm{O}$ & 175 & 17.6 & 172 & 16.8 & 14.5 & 15.8 & 179 & 16.4 & 17.8 & 22.6 & 15.5 & 17.5 \\
\hline Sum & 94.1 & 93.7 & 94.6 & 92.8 & 91.6 & 91.7 & 93.8 & 94.6 & 93.7 & 93.2 & 94.8 & 95.0 \\
\hline Auppon) & 242 & 300 & 310 & 280 & 175 & 190 & 280 & 245 & 300 & 340 & 300 & 270 \\
\hline Be & 1900 & 1600 & 1600 & 1800 & 2900 & 14000 & 1600 & 1900 & 4000 & 1600 & 1500 & 1500 \\
\hline Cd & 27 & 2.9 & 24 & 4.2 & 4.4 & 3.7 & 3.0 & 29 & 3.6 & 3.2 & 2.8 & 3.0 \\
\hline Co & 1100 & 1100 & 1200 & 950 & 700 & 900 & 1100 & 1100 & 970 & 1000 & 1100 & 1100 \\
\hline$c$ & 12 & 17 & $\mathbf{2 8}$ & 14 & 20 & 20 & 16 & 43 & 9 & 10 & 8 & 17 \\
\hline Mo & 360 & 420 & 300 & 520 & 360 & 340 & 430 & 280 & 470 & 530 & 410 & 330 \\
\hline $\mathbf{P b}$ & 1300 & 1700 & 1900 & 1600 & 1100 & 1300 & 1700 & 1600 & 1800 & 1900 & 1900 & 1700 \\
\hline Sx & 1500 & 1600 & 1600 & 1700 & 1300 & 2300 & 1600 & 1400 & 1700 & 1700 & 1700 & 1500 \\
\hline $\mathbf{v}$ & 630 & 700 & 740 & 730 & 510 & 590 & 670 & 560 & 770 & 700 & 700 & 610 \\
\hline $\mathbf{Y}$ & 230 & 206 & 217 & 144 & 90 & 154 & 219 & 200 & 170 & 233 & 249 & 206 \\
\hline $\mathbf{Z n}$ & 630 & 590 & 600 & 620 & 640 & 610 & 580 & 590 & 620 & 590 & 690 & 630 \\
\hline$\gamma$ & 1.048 & 1.053 & 1.042 & 1.062 & 1.076 & 1.075 & 1.051 & 1.042 & 1.052 & 1.058 & 1.040 & 1.038 \\
\hline Sermpled & Bulk & Buik & Bulk & Bulk & Bulk & Bulk & Bulk & Bulk & Bulk & Bulk & Bulk & Bulk \\
\hline loterval & - & - & - & - & $\cdot$ & - & • & - & • & • & • & - \\
\hline Sequencell & 052058 & 052059 & 052060 & 052061 & 052062 & 052063 & 052064 & 052065 & 052066 & 052067 & 052068 & 052069 \\
\hline Latitude & 18.752 & 18.752 & 18.752 & 18.752 & 18.752 & 18.752 & 18.752 & 18.752 & 18.752 & 18.752 & 18.752 & 18.752 \\
\hline Longitude & -158.330 & -158.330 & -158.330 & -158330 & -158.330 & -158.330 & -158.330 & -158.330 & -158.330 & -158.330 & -158.330 & -158.330 \\
\hline Depth & 2100 & 2100 & 2100 & 2100 & 2100 & 2100 & 2100 & 2100 & 2100 & 2100 & 2100 & 2100 \\
\hline Lab" & W-236028 & W-236029 & W-236030 & W-236031 & W-236032 & W-236033 & W-236034 & W-236036 & W-236037 & W-235994 & W-236038 & W-236039 \\
\hline $\mathrm{SiO}_{2}(w t . \%)$ & 9.22 & 12.0 & 14.4 & 8.52 & 6.81 & 13.9 & 13.8 & 8.49 & 10.4 & 13.4 & 12.2 & 10.4 \\
\hline $\mathrm{TiO}_{2}^{2}$ & 251 & 236 & 2.28 & 1.97 & 249 & 273 & 212 & 204 & 2.14 & 2.46 & 2.26 & 203 \\
\hline $\mathrm{MmO}_{2}$ & 35.3 & 29.8 & 28.8 & 35.3 & 39.0 & 30.0 & 20.6 & 35.7 & 349 & 28.5 & 29.5 & 36.8 \\
\hline & 273 & 32.9 & 28.4 & 29.7 & 26.0 & 29.6 & 20.9 & 28.3 & 27.2 & 32.2 & 320 & 24.6 \\
\hline $\mathrm{N}_{2}^{2} \mathrm{O}_{3}^{3}$ & 1.57 & 205 & 3.21 & 1.19 & .95 & 263 & 293 & 1.26 & 2.01 & 2.65 & 209 & 247 \\
\hline $\mathrm{CO}_{3}^{2} \mathrm{O}_{4}^{3}$ & 1.08 & .600 & .636 & .811 & 1.30 & 818 & 577 & .892 & 930 & .684 & .546 & 1.05 \\
\hline $\mathrm{NiO}^{4}$ & .413 & .258 & .290 & .387 & .472 & 345 & .299 & 386 & .427 & .282 & .255 & .491 \\
\hline Cuo & .075 & .057 & .067 & .051 & .063 & .077 & .047 & .040 & .090 & .075 & .063 & .105 \\
\hline $\mathrm{CaO}$ & 3.71 & 3.28 & 3.52 & 3.37 & 3.67 & 3.23 & 3.37 & 3.58 & 3.65 & 3.20 & 3.38 & 3.86 \\
\hline $\mathrm{M}_{8} \mathrm{O}$ & 1.85 & 1.68 & 1.75 & 1.70 & 184 & 1.89 & 1.70 & 1.77 & 1.77 & 1.70 & 1.66 & 1.82 \\
\hline $\mathrm{Ne}_{2} \mathrm{O}$ & 2.43 & 2.15 & 2.37 & 2.28 & 264 & 232 & 2.30 & 242 & 242 & 2.16 & 2.21 & 255 \\
\hline $\mathrm{K}_{2} \mathrm{O}$ & .70 & .62 & .76 & 58 & .62 & 81 & .68 & .66 & .69 & .72 & .60 & .72 \\
\hline$\infty_{2}$ & 32 & 33 & .34 & 35 & 38 & 32 & 37 & .45 & .39 & .20 & 35 & 37 \\
\hline $\mathrm{P}_{2} \mathrm{O}_{5}$ & 1.10 & 1.12 & 1.07 & 1.07 & 99 & 1.00 & 1.08 & 1.22 & 1.13 & 1.07 & 1.19 & 1.10 \\
\hline $\mathrm{H}_{2}^{2} \mathrm{O}+$ & 5.9 & 5.2 & 6.1 & 7.5 & 7.7 & 13.5 & 6.6 & 6.8 & 7.1 & 6.3 & 7.4 & 6.6 \\
\hline $\mathrm{H}_{2}^{2} \mathrm{O}$ & 23.0 & 16.0 & 165 & 14.4 & 16.4 & 15.1 & 15.0 & 17.6 & 16.5 & 14.4 & 15.2 & 14.4 \\
\hline Sum & 93.5 & 94.3 & 94.0 & 94.8 & 94.9 & 1032 & 95.4 & 94.0 & 95.3 & 95.7 & 95.7 & 949 \\
\hline$A \in(p p m)$ & 247 & 300 & 260 & 300 & 280 & 250 & 280 & 330 & 260 & 270 & 290 & 245 \\
\hline $\mathrm{Ba}$ & 1800 & 1700 & 1400 & 1500 & 1700 & 1500 & 1500 & 1300 & 1400 & 1600 & 1800 & 1400 \\
\hline Cd & 3.0 & 24 & 1.9 & 26 & 3.6 & 27 & 2.6 & 3.0 & 3.2 & 2.7 & 27 & 3.5 \\
\hline$\infty$ & 1400 & 1100 & 980 & 1100 & 1400 & 1200 & 990 & 1100 & 1100 & 1200 & 1000 & 1000 \\
\hline$c$ & 19 & 10 & 9 & 7 & 11 & 24 & 14 & 7 & 12 & 13 & 9 & 10 \\
\hline Mo & 340 & 330 & 350 & 430 & 380 & 260 & 340 & 400 & 410 & 290 & 370 & 460 \\
\hline $\mathrm{Pb}$ & 1700 & 1800 & 1600 & 1900 & 1800 & 1600 & 1600 & 1800 & 1700 & 1800 & 1700 & 1600 \\
\hline St & 1600 & 1700 & 1600 & 1600 & 1700 & 1400 & 1500 & 1700 & 1600 & 1500 & 1700 & 1600 \\
\hline $\mathbf{v}$ & 600 & 710 & 650 & 700 & 590 & 590 & 660 & 690 & 650 & 670 & 740 & 610 \\
\hline $\mathbf{Y}$ & 260 & 250 & 216 & 245 & 227 & 224 & 235 & 243 & 228 & 234 & 248 & 222 \\
\hline $\mathrm{Zn}$ & 600 & 670 & 590 & 620 & 600 & 640 & 620 & 580 & 590 & 630 & 660 & 550 \\
\hline$\gamma$ & 1.054 & 1.045 & 1.049 & 1.040 & 1.039 & .956 & 1.034 & 1.049 & 1.035 & 1.030 & 1.030 & 1.039 \\
\hline Samplod & Bulk & Bulk & Bulk & Bulk & Bulk & Bulk & Bulk & Bulk & Bulk & Bulk & Bulk & Bulk \\
\hline Interval & • & • & - & • & • & • & • & • & • & • & - & - \\
\hline
\end{tabular}


Table 1. Composition of crusts analyzed by USGS Reston Labs (cont'd).

\begin{tabular}{|c|c|c|c|c|c|c|c|c|c|c|c|c|}
\hline Soguencent & 052070 & 052001 & 052072 & 052073 & 052074 & 052075 & 052076 & 052077 & 052078 & 052079 & 052080 & 052081 \\
\hline Latimde & 18.752 & 18.752 & 18.752 & 18.752 & 18.752 & 18.752 & 18.752 & 18.752 & 18.752 & 18.752 & 18.752 & 18.752 \\
\hline Longitude & -158.330 & -158.330 & -158.330 & -158.330 & -158.330 & -158.330 & -158.330 & -158.330 & -158.330 & -158.330 & -158.330 & -158.330 \\
\hline Depth & 2100 & 2100 & 2100 & 2100 & 2100 & 2100 & 2100 & 2100 & 2100 & 2100 & 2100 & 2100 \\
\hline Lab* & W-236040 & W-236041 & W-236042 & W-236043 & W-236044 & W.236045 & W-236046 & W.236047 & W-236048 & W-236049 & W-236050 & W-236051 \\
\hline $\mathrm{SiO}_{2}(\mathrm{~m} . \%)$ & 12.6 & 11.8 & 8.97 & 13.3 & 125 & 14.4 & 13.0 & 120 & 13.7 & 9.79 & 122 & 11.7 \\
\hline $\mathrm{TiO}_{2}^{2}$ & 231 & 2.26 & 200 & 2.29 & 253 & 2.61 & 243 & 2.24 & 204 & 2.35 & 231 & 232 \\
\hline $\mathrm{MnO}_{2}$ & 29.0 & 31.0 & 33.0 & 29.1 & 29.6 & 27.1 & 28.1 & 30.0 & 29.4 & 35.7 & 29.8 & 30.4 \\
\hline $\mathrm{Fe}_{2} \mathrm{O}_{3}$ & 327 & 30.4 & 31.7 & 31.1 & 31.0 & 31.0 & 32.9 & 31.7 & 28.1 & 26.6 & 32.5 & 32.2 \\
\hline $\mathrm{Al}_{2} \mathrm{O}_{3}$ & 206 & 207 & 1.05 & 2.64 & 234 & 281 & 233 & 200 & 294 & 1.74 & 2.04 & 1.95 \\
\hline $\mathrm{CO}_{3} \mathrm{O}_{4}^{3}$ & .607 & .745 & .710 & .602 & .751 & .742 & .629 & .665 & .713 & 1.04 & .678 & .579 \\
\hline Nió & .269 & .333 & 355 & .303 & .321 & 260 & .256 & .208 & 318 & $A 71$ & .286 & .263 \\
\hline $\mathrm{CuO}$ & .053 & .071 & .052 & .064 & .073 & .080 & .059 & .066 & .057 & .103 & .064 & .065 \\
\hline $\mathrm{CaO}$ & 3.17 & 3.29 & 3.20 & 3.41 & 3.20 & 3.11 & 3.22 & 3.31 & 4.53 & 3.51 & 3.23 & 3.39 \\
\hline $\mathrm{MgO}$ & 1.65 & 1.70 & 1.63 & 1.71 & 1.79 & 1.75 & 1.63 & 1.66 & 1.62 & 1.82 & 1.67 & 1.64 \\
\hline $\mathrm{Ne}_{2} \mathrm{O}$ & 218 & 223 & 214 & 2.25 & 2.29 & 231 & 220 & 228 & 250 & 2.51 & 2.27 & 2.18 \\
\hline $\mathbf{k}_{2} \mathbf{O}$ & .62 & .63 & .51 & .68 & .71 & .77 & .63 & .60 & .74 & .73 & .61 & .61 \\
\hline $\mathrm{CO}_{2}$ & .35 & 35 & .42 & 34 & .34 & 31 & .33 & 36 & .41 & .34 & 35 & .23 \\
\hline $\mathrm{P}_{2} \mathrm{O}_{5}$ & 1.16 & 1.09 & 1.17 & 1.17 & 1.08 & 1.04 & 1.11 & 1.12 & 1.80 & 1.04 & 1.11 & 1.16 \\
\hline $\mathrm{H}_{2}^{2} \mathrm{O}+$ & 7.2 & 6.9 & 7.1 & 7.9 & 6.7 & 7.2 & 6.3 & 6.9 & 6.7 & 6.6 & 6.7 & 7.4 \\
\hline $\mathrm{H}_{2}^{2} \mathrm{O}$ & 148 & 15.9 & 175 & 11.8 & 129 & 11.9 & 15.6 & 16.0 & 15.9 & 163 & 15.6 & 13.0 \\
\hline sum & 95.9 & 94.9 & 94.1 & 96.8 & 95.3 & 95.5 & 95.1 & 95.2 & 955 & 94.4 & 95.9 & 96.1 \\
\hline Ax(ppm) & 280 & 270 & 300 & 270 & 250 & 238 & 270 & 290 & 250 & 239 & 280 & 300 \\
\hline $\mathrm{Ba}$ & 1800 & 1700 & 1700 & 1600 & 1600 & 1600 & 1800 & 1700 & 1400 & 1700 & 1700 & 1700 \\
\hline Cd & 2.6 & 27 & 29 & 26 & 29 & 23 & 24 & 26 & 24 & 3.6 & 25 & 25 \\
\hline$c_{0}$ & 1200 & 1200 & 1200 & 1100 & 1100 & 1200 & 1200 & 1100 & 1100 & 1200 & 1200 & 1000 \\
\hline$c$ & 12 & 11 & 9 & 16 & 18 & 20 & 13 & 13 & 12 & 19 & 14 & 9 \\
\hline Mo & 320 & 330 & 410 & 320 & 260 & 227 & 270 & 330 & 330 & 380 & 300 & 380 \\
\hline$P_{b}$ & 1600 & 1700 & 1800 & 1600 & 1700 & 1600 & 1700 & 1700 & 1500 & 1700 & 1800 & 1600 \\
\hline St & 1600 & 1500 & 1700 & 1600 & 1500 & 1500 & 1500 & 1500 & 1500 & 1600 & 1500 & 1600 \\
\hline $\mathbf{v}$ & 690 & 670 & 740 & 680 & 620 & 590 & 660 & 680 & 620 & 590 & 660 & 740 \\
\hline $\mathbf{Y}$ & 246 & 238 & 250 & 238 & 230 & 216 & 237 & 238 & 226 & 239 & 237 & 250 \\
\hline $\mathbf{Z n}$ & 650 & 630 & 650 & 650 & 640 & 600 & 650 & 630 & 580 & 600 & 640 & 670 \\
\hline$\gamma$ & 1.028 & 1.039 & 1.048 & 1.018 & 1.035 & 1.032 & 1.036 & 1.036 & 1.033 & 1.044 & 1.028 & 1.026 \\
\hline Sempled & Bulk & Bulk & Bulk & Bulk & Bulk & Bulk & Bulk & Bulk & Bulk & Bulk & Bulk & Bulk \\
\hline Intervil & - & - & - & - & - & - & - & - & - & - & - & - \\
\hline Sequence:" & 052082 & 052083 & 052084 & 052085 & 052086 & 052087 & 053001 & 053002 & 053003 & 053004 & 053005 & 053006 \\
\hline Latitude & 18.752 & 18.752 & 18.752 & 18.752 & 18.752 & 18.752 & 18.300 & 18.783 & 18.783 & 19.367 & 19.350 & 10.293 \\
\hline Longitude & -158.330 & -158.330 & -158.330 & -158.330 & -158.330 & -158.330 & -161.767 & -162.050 & -162050 & -162317 & -162.167 & -165.995 \\
\hline Depth & 2100 & 2100 & 2100 & 2100 & 2100 & 2100 & 3111 & 4629 & 3147 & 4868 & 4209 & 1790 \\
\hline Lab* & W-236052 & W-236035 & W-236053 & W-236054 & W-236055 & W-236056 & W-222532 & W-222533 & W-222534 & W-222535 & W-222536 & W-222567 \\
\hline $\mathrm{SiO}_{2}(w t . \%)$ & 123 & 10.2 & 10.7 & 128 & 10.1 & 185 & 7.69 & 12.2 & 227 & 9.09 & 25.3 & 5.42 \\
\hline $\mathrm{TiO}_{2}^{2}$ & 213 & 2.14 & 2.42 & 2.35 & 1.98 & 207 & 220 & 2.47 & 3.35 & 2.62 & 2.62 & 208 \\
\hline $\mathrm{MnO}_{2}^{2}$ & 30.4 & 31.1 & 34.0 & 28.5 & 322 & 24.6 & 41.6 & 31.7 & 205 & 37.1 & 20.3 & 47.8 \\
\hline & 31.4 & 33.2 & 27.7 & 328 & 31.9 & 25.0 & 29.0 & 34.3 & 295 & 30.7 & 26.1 & 21.3 \\
\hline $\mathrm{Al}_{2}{ }_{2} \mathrm{O}_{3}$ & 219 & 1.30 & 2.14 & 2.21 & 1.40 & 482 & 1.16 & 234 & 5.92 & 1.61 & 7.47 & 1.28 \\
\hline $\mathrm{Co}_{3}^{2} \mathrm{O}_{4}^{2}$ & .666 & .648 & .933 & .655 & .640 & .615 & 1.18 & .623 & .357 & 863 & .387 & .923 \\
\hline $\mathrm{NiO}^{4}$ & .319 & .303 & .407 & .262 & .314 & .280 & 516 & .257 & .302 & .386 & .236 & 1.069 \\
\hline$\infty 0$ & .057 & .040 & .070 & .059 & .051 & .055 & .117 & .115 & .195 & .153 & .130 & .187 \\
\hline $\mathrm{CaO}$ & 3.36 & 3.22 & 3.56 & 3.14 & 3.30 & $5 s 1$ & 3.72 & 3.39 & 3.14 & 3.66 & 294 & 4.55 \\
\hline $\mathrm{MgO}$ & 1.70 & 1.64 & 1.89 & 1.67 & 1.66 & 1.61 & 1.92 & 1.83 & 2.28 & 1.80 & 1.88 & 247 \\
\hline $\mathrm{N}_{2} \mathrm{O}$ & 2.25 & 2.12 & 2.40 & 2.29 & 2.23 & 287 & 252 & 2.42 & 2.37 & 248 & 2.84 & 271 \\
\hline $\mathrm{K}_{2} \mathrm{O}$ & .65 & 53 & .73 & .63 & 54 & .98 & 59 & 50 & 1.29 & .51 & 1.78 & .69 \\
\hline$\infty_{2}$ & 37 & .40 & .23 & .18 & .22 & 21 & 56 & 55 & .35 & .54 & 33 & $A 3$ \\
\hline $\mathrm{P}_{2} \mathrm{O}_{5}$ & 1.18 & 1.15 & 1.02 & 1.10 & 1.21 & 231 & 93 & 1.11 & $1 . \infty$ & 1.04 & .91 & 1.12 \\
\hline $\mathrm{H}_{2} \mathrm{O}+$ & 6.7 & 6.1 & 7.6 & 7.1 & 6.9 & 5.5 & 6.0 & 55 & 5.7 & 6.9 & 6.0 & 6.1 \\
\hline $\mathrm{H}_{2} \mathrm{O}$ & 16.2 & 24.3 & 124 & 127 & 14.9 & 13.7 & 28.5 & 25.7 & 19.9 & 27.4 & 19.0 & 26.2 \\
\hline Sum & 95.7 & 94.1 & 958 & 95.7 & 94.6 & 95.0 & 99.8 & 99.4 & 99.0 & 99.4 & 99.3 & 98.2 \\
\hline$A \simeq(p p m)$ & 290 & 320 & 250 & 270 & 310 & 197 & 238 & 229 & 150 & 234 & 161 & 203 \\
\hline $\mathrm{Ba}$ & 1600 & 1700 & 1500 & 1700 & 1600 & 1400 & 1700 & 1700 & 1600 & 1800 & 1500 & 2400 \\
\hline Cd & 26 & 2.6 & 3.1 & 29 & 26 & 2.1 & 3.1 & 3.0 & 3.0 & 2.6 & 22 & 5.0 \\
\hline Co & 1100 & 1200 & 1100 & 1100 & 1100 & 940 & 1400 & 1600 & 1100 & 1700 & 1400 & 1500 \\
\hline$a$ & 11 & 8 & 17 & 16 & 6 & 17 & 11 & 19 & 84 & 17 & 36 & 22 \\
\hline Mo & 350 & 360 & 340 & 290 & 420 & 270 & 450 & 320 & 121 & 430 & 185 & 680 \\
\hline$P_{b}$ & 1700 & 2000 & 1600 & 1700 & 1800 & 1300 & 1800 & 1800 & 1200 & 1700 & 1100 & 1600 \\
\hline Sr & 1600 & 1700 & 1500 & 1500 & 1600 & 1400 & 1500 & 1500 & 106 & 1700 & 990 & 1600 \\
\hline $\mathbf{v}$ & 690 & 730 & 580 & 650 & 740 & 530 & 630 & 660 & 470 & 650 & 460 & 650 \\
\hline $\mathbf{Y}$ & 239 & 250 & 217 & 241 & 247 & 243 & 231 & 233 & 154 & 231 & 221 & 163 \\
\hline $\mathbf{z}_{\mathbf{n}}$ & 640 & 670 & 610 & 640 & 650 & 530 & 590 & 570 & 620 & 620 & 530 & 980 \\
\hline$\gamma$ & 1.031 & 1.047 & 1.029 & 1.030 & 1.042 & 1.038 & 988 & .992 & .996 & 992 & .993 & 1.005 \\
\hline Sampled & Bulk & Bulk & Bulk & Bulk & Bulk & Bulk & Bulk & Bulk & Bulk & Bulk & Bulk & Bulk \\
\hline Interval & 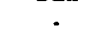 & - & . & . & - & . & - & $0-10 \mathrm{~mm}$ & $0.7 \mathrm{~mm}$ & $0-5 \mathrm{~mm}$ & $0-12 \mathrm{~mm}$ & $0-7 \mathrm{~mm}$ \\
\hline
\end{tabular}


Table 1. Composition of crusts analyzed by USGS Reston Labs (cont'd).

\begin{tabular}{|c|c|c|c|c|c|c|c|c|c|c|c|c|}
\hline Sequencest & 053007 & 053008 & 053009 & 053010 & 053011 & 053012 & 053013 & 053014 & 053017 & 053018 & 053019 & 053020 \\
\hline Latinde & 14.458 & 18.135 & 19.117 & 10.813 & 13.462 & 14.982 & 15.156 & 16.436 & 19.335 & 19.369 & 19.369 & 19511 \\
\hline Longitude & -168.978 & -169.007 & -169.733 & -165544 & -165.419 & -166.448 & -168.102 & .168 .114 & -168.665 & .168 .695 & -168.695 & -168.835 \\
\hline Depth & 1750 & 2139 & 1805 & 1325 & 3850 & 2800 & 2700 & 2325 & 3791 & 2000 & 2000 & 1800 \\
\hline Lab: & W-222568 & W-220569 & W-222570 & W. 224403 & W-224404 & W-224405 & W-224406 & $W-224407$ & W-223748 & W-223749 & $w-223750$ & W-223751 \\
\hline $\mathrm{SiO}_{2}$ (wt.\%) & 4.00 & 10.9 & 10.1 & 7.87 & 9.46 & 24.9 & 138 & 13.2 & 11.1 & 113 & 9.92 & 11.6 \\
\hline $\mathrm{TOO}_{2}$ & 1.82 & 1.88 & 1.96 & 2.16 & 2.09 & 220 & 2.04 & 1.88 & 2.20 & 223 & 1.81 & 1.96 \\
\hline $\mathrm{MmO}_{2}$ & 485 & 37.9 & 39.1 & 39.9 & 37.2 & 21.6 & 33.5 & 33.7 & 34.6 & 34.9 & 36.2 & 37.7 \\
\hline & 20.0 & 29.0 & 26.1 & 27.3 & 28.0 & 21.6 & 26.0 & 24.2 & 299 & 25.1 & 26.4 & 28.0 \\
\hline & .66 & 1.68 & 2.15 & 1.16 & 1.77 & 6.86 & 3.16 & 3.19 & 2.05 & 2.57 & 1.85 & 1.85 \\
\hline & 1.56 & 1.04 & 1.09 & .881 & .683 & .464 & .713 & 859 & .797 & 1.28 & .998 & 1.06 \\
\hline NiO & 865 & 462 & .619 & .494 & .421 & .336 & $\$ 11$ & 502 & 317 & .405 & .416 & .426 \\
\hline Cno & .088 & .001 & .112 & .126 & .171 & .165 & .195 & .169 & .069 & .045 & .052 & .072 \\
\hline $\mathrm{CaO}$ & 3.92 & 3.57 & 3.78 & 3.77 & 4.47 & 4.16 & 3.90 & 5.36 & 3.49 & 4.60 & 5.21 & 3.68 \\
\hline $\mathrm{M} 8 \mathrm{O}$ & 206 & 1.83 & 1.99 & 1.77 & 180 & 201 & 1.81 & 1.87 & 1.76 & 200 & 1.72 & 1.75 \\
\hline $\mathrm{Ne}_{2} \mathrm{O}$ & 253 & 255 & 2.43 & 248 & 2.39 & 2.75 & 2.34 & 2.70 & 2.17 & 263 & 237 & 239 \\
\hline $\mathrm{R}_{2} \mathrm{O}$ & 59 & .63 & .57 & .61 & .63 & 2.12 & 1.13 & 1.07 & .65 & .78 & .68 & .69 \\
\hline & $A 4$ & .48 & .39 & .65 & .71 & 1.21 & .65 & 96 & .36 & 1.10 & 1.69 & $A 4$ \\
\hline $\mathrm{P}_{2} \mathrm{O}_{5}$ & .70 & 1.02 & 1.12 & 94 & 152 & 1.36 & 1.25 & 1.94 & .97 & .90 & 1.01 & .90 \\
\hline $\mathrm{H}_{2} \mathrm{O}+$ & 9.6 & 58 & 5.3 & 11.1 & 9.7 & 9.2 & 98 & 10.7 & 88 & 8.7 & 96 & 8.7 \\
\hline $\mathrm{H}_{2} \mathrm{O}$ & 25.0 & 25.6 & 26.0 & 73 & 12.3 & 9.1 & 10.2 & 11.2 & 7.7 & 8.8 & 113 & 16.4 \\
\hline Sum & 97.4 & 988 & 96.8 & 1013 & 101.0 & 100.9 & 100.6 & 1022 & 99.2 & 98.5 & 100.0 & 1013 \\
\hline A-ppon) & 227 & 260 & 243 & 227 & 217 & 121 & 189 & 180 & 206 & 208 & 226 & 227 \\
\hline Be & 1600 & 1400 & 1600 & 1700 & 1600 & 2100 & 1800 & 2100 & 1100 & 1100 & 1100 & 1400 \\
\hline Cd & 4.7 & 3.0 & 3.9 & 29 & 2.7 & 2.0 & 2.7 & 29 & 3.3 & 3.4 & 38 & 3.5 \\
\hline c & 1000 & 1000 & 990 & 1200 & 1100 & 730 & 1200 & 1000 & 1600 & 1400 & 900 & 1100 \\
\hline$a$ & 9 & 18 & 62 & 8 & 11 & 41 & 15 & 14 & - & • & $\cdot$ & - \\
\hline Mo & 750 & 470 & 490 & 540 & 460 & 220 & 370 & 350 & 300 & 330 & 410 & 420 \\
\hline $\mathbf{P b}_{\mathbf{b}}$ & 2100 & 2000 & 2200 & 1400 & 1300 & 810 & 1200 & 1500 & 1800 & 1600 & 1700 & 1900 \\
\hline Sr & 1600 & 1600 & 1600 & 1600 & 1600 & 1300 & 1400 & 1500 & 1400 & 1400 & 1400 & 1600 \\
\hline $\mathbf{v}$ & 670 & 670 & 690 & 660 & 660 & 450 & 580 & 530 & 590 & 540 & 560 & 60 \\
\hline $\mathbf{Y}$ & 113 & 222 & 189 & 248 & 240 & 143 & 260 & 237 & 195 & 186 & 180 & 191 \\
\hline $\mathbf{Z n}$ & 690 & 560 & 730 & 630 & 590 & 570 & 590 & 640 & 610 & 590 & 590 & 620 \\
\hline$\gamma$ & 1.012 & 998 & 1.018 & .974 & .976 & 977 & .980 & .965 & 994 & 1.001 & .986 & 974 \\
\hline Sampled & Bulk & Bulk & Bulk & Bulk & Bulk & Bulk & Bulk & Bulk & Bulk & Bulk & Bulk & Top \\
\hline Interval & $0-9 \operatorname{man}$ & $0-14 \mathrm{~mm}$ & $0-10 \mathrm{~mm}$ & $0-8 \mathrm{~mm}$ & $0-13 \operatorname{mon}$ & $0-8 \mathrm{~mm}$ & $0-20 \mathrm{~mm}$ & $0-10 \mathrm{~mm}$ & - & - & - & $\bullet$ \\
\hline Sequencell & 0.53021 & 053022 & 053023 & 053024 & 053025 & 053026 & 053027 & 053028 & 053029 & 053030 & 053031 & 053032 \\
\hline Latimde & 19511 & 19.511 & 19511 & 19511 & 19s11 & 19.695 & 19.553 & 19.553 & 19.515 & 19.515 & 19515 & 19515 \\
\hline Longitudo & -168.835 & -168835 & -168.835 & -168.835 & -168.835 & -168.995 & -168.832 & .168832 & -168.855 & -168.855 & -168.855 & -168.855 \\
\hline Depth & 1800 & 1800 & 1800 & 1800 & 1800 & 4500 & 2400 & 2400 & 1790 & 1790 & 1790 & 1790 \\
\hline Lebl & W-223752 & W-223753 & W-223754 & W-22375s & $w-223756$ & W-223757 & W-223758 & W.223759 & W-223760 & W-223761 & W-223762 & W.223763 \\
\hline $\mathrm{SiO}_{2}(w \cdot \%)$ & 2.18 & 7.27 & 13.1 & 3.00 & 7.98 & 185 & 10.1 & 13.1 & 5.98 & 4.71 & 8.70 & 3.46 \\
\hline $\mathrm{THO}_{2}$ & 1.69 & 1.93 & 1.99 & 1.96 & 2.02 & 274 & 2.02 & 2.13 & 1.59 & 1.59 & 180 & 1 AS \\
\hline $\mathrm{MnO}_{2}$ & 51.7 & 44.1 & 39.2 & 50.2 & 43.4 & 27.1 & 37.3 & 33.8 & 41.7 & 428 & 38.9 & 44.6 \\
\hline & 17.1 & 21.9 & 27.1 & 20.7 & 23.7 & 285 & 20.6 & 23.0 & 23.3 & 21.3 & 27.4 & 19.4 \\
\hline & .43 & 1.33 & 186 & 52 & 1.44 & 4.95 & 2.00 & 3.03 & .96 & .81 & 1.47 & .61 \\
\hline & 1.04 & 1.24 & 1.24 & 1.04 & 1.14 & 418 & .490 & .734 & 892 & 880 & .976 & 850 \\
\hline $\mathrm{NiO}^{4}$ & .708 & .612 & $s 0 n$ & .588 & .619 & 511 & .458 & 462 & 483 & 530 & .464 & 553 \\
\hline $\mathrm{CuO}$ & .212 & .140 & .069 & .089 & .081 & .355 & .118 & .279 & .119 & .274 & .078 & .154 \\
\hline $\mathrm{CaO}$ & 7.78 & 6.04 & 3.66 & 6.99 & 4.54 & 281 & 3.62 & 3.61 & 7.24 & 8.74 & 3.78 & 858 \\
\hline $\mathrm{MgO}_{8}$ & 1.84 & 1.82 & 1.78 & 1.78 & 1.80 & 2.15 & 1.79 & 1.83 & 1.64 & 1.03 & 1.75 & 1.63 \\
\hline $\mathrm{N}_{2} \mathrm{O}$ & 230 & 234 & 2.24 & 2.37 & 2.37 & 2.24 & 2.36 & 2.23 & 2.18 & 2.19 & 2.23 & 2.12 \\
\hline $\mathbf{k}_{2} \mathbf{O}$ & .68 & .73 & .74 & .65 & .72 & .97 & .70 & .78 & .60 & .61 & .62 & 59 \\
\hline$\infty_{2}$ & .79 & .73 & .47 & .76 & 56 & 35 & 38 & 52 & .77 & .82 & 52 & 86 \\
\hline $\mathrm{P}_{2} \mathrm{O}_{5}$ & 247 & 1.75 & .80 & 1.97 & 94 & .73 & 1.03 & .91 & 2.90 & 351 & 1.16 & 3.45 \\
\hline $\mathrm{H}_{2}^{6} \mathrm{O}+$ & 9.6 & 9.1 & 9.6 & 10.6 & 9.4 & 8.5 & 8.9 & 10.3 & 93 & 78 & 10.2 & 9.3 \\
\hline $\mathrm{H}_{2}^{2} \mathrm{O}$ & 17.3 & 18.9 & 19.7 & 19.9 & 19.8 & 15.3 & 11.0 & 14.7 & 13.0 & 8.7 & 14.9 & 10.3 \\
\hline sum & 100.5 & 101.0 & 104.3 & 103.2 & 100.7 & 100.9 & $989^{\circ}$ & 96.7 & 99.6 & 98.2 & 100.0 & 97.6 \\
\hline Ax(ppm) & 169 & 210 & 237 & 212 & 237 & 177 & 290 & 223 & 241 & 219 & 330 & 223 \\
\hline $\mathrm{Ba}$ & 2700 & 2000 & 1500 & 2700 & 2100 & 1500 & 1300 & 1500 & 2100 & 2100 & 1500 & 2300 \\
\hline Cd & 5.2 & 4.9 & 3.9 & 5.0 & 4.5 & 4.8 & 3.3 & 3.2 & 3.7 & 4.1 & 3.8 & 4.2 \\
\hline c & 2200 & 1600 & 1200 & 2400 & 1700 & 1500 & 1100 & $11 \infty$ & 1600 & 1600 & 1100 & 2000 \\
\hline$a$ & - & - & • & - & - & - & - & • & - & - & • & • \\
\hline Mo & 930 & 630 & 450 & 840 & 600 & 130 & 380 & 235 & 660 & 680 & 480 & 760 \\
\hline $\mathbf{P B}$ & 2100 & 1900 & 1900 & 2400 & 2100 & 1300 & 1800 & 1800 & 2200 & 2200 & 2000 & 2300 \\
\hline Sr & 1800 & 1600 & 1600 & 1900 & 1600 & 1100 & 1600 & 1400 & 1800 & 1800 & 1600 & 1900 \\
\hline $\mathbf{v}$ & 710 & 600 & 249 & 740 & 650 & 470 & 610 & 530 & 710 & 670 & 680 & 690 \\
\hline $\mathbf{Y}$ & 133 & 160 & 187 & 112 & 137 & 154 & 214 & 152 & 195 & 175 & 200 & 167 \\
\hline $\mathrm{Zn}$ & 870 & 740 & 650 & 800 & 720 & 760 & 640 & 770 & 710 & 760 & 690 & 800 \\
\hline$\gamma$ & .982 & .976 & 945 & .955 & .979 & 977 & 997 & 1.020 & .990 & 1.004 & .986 & 1.010 \\
\hline Samplod & Bottom & Bulk & Top & Bottom & Bulk & Top & Bulk & Bulk & Bulk & Top & Top & Bottom \\
\hline Interval & • & • & $\because$ & - & • & - & • & - & - & - & - & • \\
\hline
\end{tabular}


Table 1. Composition of crusts analyzed by USGS Reston Labs (cont'd).

\begin{tabular}{|c|c|c|c|c|c|c|c|c|c|c|c|c|}
\hline Sequenow & 053033 & 053034 & 054001 & 055001 & 055002 & 055003 & 055004 & 055008 & 055008 & 055008 & 055009 & 055009 \\
\hline Latinedo & 19515 & 19.515 & 19.243 & 19.355 & 19.317 & 18.163 & 19.070 & 11.020 & 11.020 & 11.020 & 11.008 & 11.008 \\
\hline Langitude & -168.855 & -168.855 & -179.668 & 176.780 & 176.745 & 171.517 & 173.373 & 170.177 & 170.177 & 170.177 & 170.203 & 170.203 \\
\hline Dopth & 1790 & 1790 & 3224 & 3672 & 1861 & 2000 & 2100 & 1575 & 1575 & 1575 & 2000 & 2000 \\
\hline Leb: & W-223764 & W-223765 & W-222588 & W-222589 & W-222590 & W.23140s & W-231406 & D12-1-A & D12-1-B & D12-1-C & D13-1-A & D13-1-B \\
\hline $\mathrm{SiO}_{2}$ (wt.s) & 120 & 279 & 13.3 & 9.89 & 10.2 & 30.0 & 7.08 & 6.00 & 8.77 & 3.14 & 4.23 & 2.62 \\
\hline & 1.94 & 1.51 & 1.79 & 1.79 & 1.91 & 1.76 & 1.12 & 1.65 & 214 & 1.69 & 2.07 & 1.94 \\
\hline $\mathrm{MnO}_{2}$ & 35.6 & 46.0 & 28.0 & 38.8 & 39.5 & 13.7 & 23.9 & 45.4 & 40.0 & 50.9 & 48.6 & 51.2 \\
\hline & 28.0 & 20.9 & 23.8 & 26.2 & 26.1 & 15.7 & 15.4 & 25.6 & 25.9 & 223 & 20.7 & 20.8 \\
\hline $\mathrm{Al}_{2}^{2}$ & 205 & .70 & 2.90 & 1.38 & 1.49 & 8.80 & 1.38 & 1.03 & 211 & .60 & 54 & .29 \\
\hline $\mathrm{CO}_{3}^{2}$ & 1.11 & .681 & 593 & 1.18 & 1.24 & 362 & .774 & 1.38 & 933 & 1.20 & 247 & 1.58 \\
\hline Niô & .429 & 503 & .375 & .552 & .555 & .212 & 384 & .680 & .759 & 899 & 816 & 948 \\
\hline Cuo & .063 & .086 & .160 & .071 & .068 & .046 & .052 & .082 & .121 & .142 & .048 & .138 \\
\hline $\mathrm{CnO}$ & 3.49 & 8.30 & 109 & 4.93 & 4.77 & 7.76 & 195 & 3.96 & 4.35 & 4.44 & 3.72 & 4.33 \\
\hline $\mathrm{MgO}$ & 1.72 & 1.60 & 1.63 & 1.84 & 1.83 & 283 & 1.44 & 1.95 & 1.89 & 1.92 & 206 & 201 \\
\hline $\mathrm{N}_{2} \mathrm{O}$ & 237 & 1.98 & 2.18 & 2.71 & 2.72 & 2.15 & 291 & 2.45 & 2.28 & 2.46 & 2.72 & 2.52 \\
\hline $\mathrm{K}_{2} \mathrm{O}$ & .74 & .60 & .94 & .65 & .75 & 230 & 57 & .63 & .75 & .66 & .58 & .59 \\
\hline & ss & 87 & 1.41 & .75 & 82 & .61 & 1.97 & .67 & 1.21 & .73 & .64 & 54 \\
\hline $\mathrm{P}_{2} \mathrm{O}_{5}$ & 92 & 3.04 & 5.58 & 155 & 1.62 & 4.04 & 11,43 & 1.10 & 90 & .83 & .84 & .81 \\
\hline $\mathrm{H}_{2}^{2} \mathrm{O}+$ & 10.1 & 9.8 & 5.1 & 5.0 & 5.0 & 6.7 & 6.3 & 8.3 & 8.5 & 8.7 & 5.6 & 8.2 \\
\hline $\mathrm{H}_{2} \mathrm{O}$ & 199 & 14.0 & 21.9 & 26.2 & 26.6 & 9.8 & 13.8 & 25.1 & 21.2 & 25.0 & 267 & 20.8 \\
\hline Sum & 101.1 & 99.4 & 98.7 & 97.3 & 98.6 & 96.9 & 94.2 & 1009 & 100.6 & 100.6 & 95.6 & 98.4 \\
\hline$A \propto$ (ppen) & 310 & 209 & 154 & 244 & 232 & 109 & 151 & 250 & 290 & 330 & 250 & 260 \\
\hline $\mathrm{Ba}$ & 1500 & 2700 & 1700 & 1400 & 1400 & 630 & 2200 & 1900 & 2300 & 1300 & 2100 & 1400 \\
\hline Cd & 25 & 2.8 & 3.1 & 3.4 & 3.4 & 9 & 20 & 3.9 & 4.3 & 4.0 & 5.4 & 5.9 \\
\hline C. & 1100 & 2400 & 1000 & 1000 & 1100 & 420 & 650 & 1000 & 1200 & 890 & 1400 & 1200 \\
\hline$a$ & - & - & 12 & 19 & 12 & 189 & 4 & 11 & 1 & 5 & 7 & 7 \\
\hline Mo & 410 & 850 & 310 & 560 & 460 & 166 & 250 & 440 & 870 & 610 & 860 & 570 \\
\hline $\mathbf{P b}$ & 1900 & 2700 & 1500 & 2000 & 2200 & 850 & 1300 & 1700 & 1600 & 2000 & 1800 & 2000 \\
\hline Sr & 1600 & 2000 & 1400 & 1600 & 1600 & 800 & 1500 & 1500 & 1700 & 1700 & 1800 & 1600 \\
\hline $\mathbf{v}$ & 620 & 780 & 510 & 680 & 680 & 320 & 410 & 610 & 800 & 790 & 760 & 640 \\
\hline $\mathbf{Y}$ & 187 & 174 & 231 & 217 & 218 & 189 & 480 & 140 & 129 & 200 & 139 & 191 \\
\hline $2 n$ & 620 & 710 & 550 & 580 & 600 & 470 & 440 & 840 & 950 & 770 & 1000 & 800 \\
\hline$y$ & .976 & 992 & 999 & 1.025 & 1.000 & 1.017 & 1.047 & 978 & .980 & .980 & 1.032 & 1.002 \\
\hline Sampled & Bulk & Bulk & Bulk & Bulk & Bulk & Bulk & Bulk & a.5mm & $5-15 \mathrm{~mm}$ & $15-25 \mathrm{~mm}$ & $0.8 \mathrm{~mm}$ & $10-20 \mathrm{~mm}$ \\
\hline Interval & - & - & $0.8 \mathrm{~mm}$ & $0.2 \mathrm{~mm}$ & $0-4 x m$ & - & - & - & $\cdot$ & • & - & - \\
\hline Sequenco: & 055009 & 055010 & 055010 & 055010 & 055011 & 055011 & 056002 & 056014 & 056015 & 056015 & 056015 & 056016 \\
\hline Latimds & 11.008 & 11.008 & 11.008 & 11.008 & 11.006 & 11.005 & 10.458 & 12.183 & 12183 & 12.183 & 12.183 & 12.183 \\
\hline Longibude & 170.203 & 170.203 & 170.203 & 170.203 & 170.230 & 170.230 & 160.900 & 168.995 & 168.995 & 168.995 & 168.995 & 168.995 \\
\hline Depth & 2000 & 2000 & 2000 & 2000 & 1800 & 1800 & 1805 & 1300 & 1300 & 1300 & 1300 & 1300 \\
\hline Lib* & D13-1-C & D13-2-A & D13-2-B & D13-2-C & D14-1-A & D14-1-B & W-2229861 & D15-10-A & D15-2-A & D15-2-B & D15-2-C & D15-3-A \\
\hline $\mathrm{SiO}_{2}$ (wt.\$) & 3.15 & 4.02 & 284 & 2.63 & 5.36 & 3.21 & 276 & 4.08 & 5.56 & 8.53 & 3.06 & 5.50 \\
\hline $\mathrm{TrO}_{2}$ & 1.82 & 2.40 & 252 & 1.91 & 2.18 & 1.83 & 1.00 & 1.68 & 1.53 & 2.16 & 1.77 & 2.14 \\
\hline & 46.9 & 49.0 & 51.4 & 50.4 & 45.2 & 42.7 & 28.7 & 47.2 & 45.3 & 39.9 & 49.9 & 44.3 \\
\hline & 24.0 & 21.1 & 20.5 & 21.2 & 227 & 223 & 18.2 & 21.6 & 25.7 & 27.1 & 221 & 20.9 \\
\hline & 52 & .65 & .44 & 49 & .72 & .60 & 56 & 1.00 & .76 & 2.04 & .73 & 1.54 \\
\hline & .769 & 2.67 & 1.81 & 1.15 & 2.13 & 909 & 297 & 1.82 & 1.40 & 817 & 1.16 & 1.44 \\
\hline Nio ${ }^{4}$ & .876 & .703 & 960 & .909 & .698 & .743 & $A 68$ & .798 & .637 & .628 & 982 & 1.175 \\
\hline $\mathrm{CuO}$ & .172 & .041 & .142 & .172 & .046 & .164 & .128 & .123 & .038 & .159 & .293 & .201 \\
\hline $\mathrm{CaO}$ & 4.28 & 3.92 & 3.95 & 4.64 & 3.78 & 7.98 & 22.2 & 3.89 & 3.87 & 3.81 & 449 & 4.16 \\
\hline $\mathrm{MgO}$ & 1.92 & 204 & 2.05 & 1.99 & 1.91 & 1.78 & 1.28 & 2.17 & 1.89 & 1.95 & 2.10 & 2.62 \\
\hline $\mathrm{N}_{2} \mathrm{O}$ & 2.71 & 274 & 2.61 & 2.64 & 2.54 & 240 & 1.36 & 3.36 & 2.46 & 2.43 & 256 & 2.71 \\
\hline $\mathrm{K}_{2} \mathbf{O}$ & 51 & .65 & .70 & .65 & 58 & 50 & 36 & .62 & 59 & .79 & .66 & .65 \\
\hline $\mathrm{CO}_{2}$ & 56 & .65 & .48 & 54 & .62 & .97 & 2.53 & so & 45 & .55 & .54 & .68 \\
\hline $\mathrm{P}_{2} \mathrm{O}_{5}$ & 85 & 85 & .63 & .92 & 90 & 3.35 & 1238 & 1.00 & 1.12 & 1.01 & .98 & 1.26 \\
\hline $\mathrm{H}_{2}^{2} \mathrm{O}+$ & 6.9 & 8.7 & 8.7 & 7.7 & 7.6 & 7.5 & 7.1 & 75 & 7.2 & 7.3 & 7.4 & 7.1 \\
\hline $\mathrm{H}_{2} \mathrm{O}$ & 27.4 & 27.6 & 23.1 & 30.0 & 28.9 & 28.1 & 13.0 & 26.6 & 28.1 & 25.0 & 27.4 & 25.3 \\
\hline sum & 95.9 & 100.1 & 99.7 & 98.0 & 97.0 & 96.9 & 99.4 & 97.4 & 985 & 99.1 & 98.7 & 963 \\
\hline$A \times(p p m)$ & 260 & 234 & 243 & 260 & 250 & 300 & 195 & 300 & 280 & 280 & 350 & 228 \\
\hline Ba & 2500 & 2200 & 2700 & 1300 & 2500 & 1400 & 1700 & 1400 & 1900 & 2300 & 1200 & $\overline{1700}$ \\
\hline cd & 4.7 & 6.1 & 5.1 & 5.7 & 3.8 & 4.9 & 2.0 & 4.9 & 2.9 & 4.5 & 4.3 & 6.4 \\
\hline co & 1800 & 1800 & 2100 & 1400 & 1800 & 1200 & 1100 & 820 & 1000 & 1100 & 820 & 1100 \\
\hline$a$ & 5 & 3 & 4 & 3 & 9 & 10 & 4 & 5 & 9 & 4 & 6 & 21 \\
\hline Mo & 540 & 480 & 590 & 460 & 560 & 460 & 430 & 630 & 410 & 810 & 580 & 500 \\
\hline Po & 1800 & 2200 & 2400 & 2100 & 1800 & 2000 & 1300 & 2200 & 1600 & 1500 & 2100 & 1700 \\
\hline St & 1800 & 1600 & 1700 & 1500 & 1800 & 1500 & 1800 & 1500 & 1600 & 1800 & 1500 & 1500 \\
\hline $\mathbf{v}$ & 690 & 530 & 630 & 580 & 710 & 650 & 570 & 690 & 640 & 760 & 740 & 600 \\
\hline $\mathbf{Y}$ & 207 & 143 & 220 & 193 & 500 & 197 & 570 & 150 & 187 & 134 & 209 & 147 \\
\hline $\mathbf{Z n}$ & 880 & 960 & 940 & 760 & 860 & 760 & 640 & 760 & 750 & 1000 & 720 & 1100 \\
\hline$\gamma$ & 1.028 & .985 & .989 & 1.006 & 1.017 & 1.017 & 992 & 1.013 & 1.001 & .995 & .999 & 1.024 \\
\hline Sermplod & $20-25 \mathrm{~mm}$ & $0.3 \mathrm{~mm}$ & $5-16 \mathrm{~mm}$ & $17-20 \mathrm{mmm}$ & Porous & $20-25 \mathrm{~mm}$ & Bulk & Bulk & $0.5 \mathrm{~mm}$ & $5-25 \mathrm{~mm}$ & $25.55 \mathrm{~mm}$ & a-smm \\
\hline & & - & - & . & $0-5 \mathrm{~mm}$ & 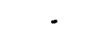 & - & $0-5 \sin n$ & & & & - \\
\hline
\end{tabular}


Table 1. Composition of crusts analyzed by USGS Reston Labs (cont'd).

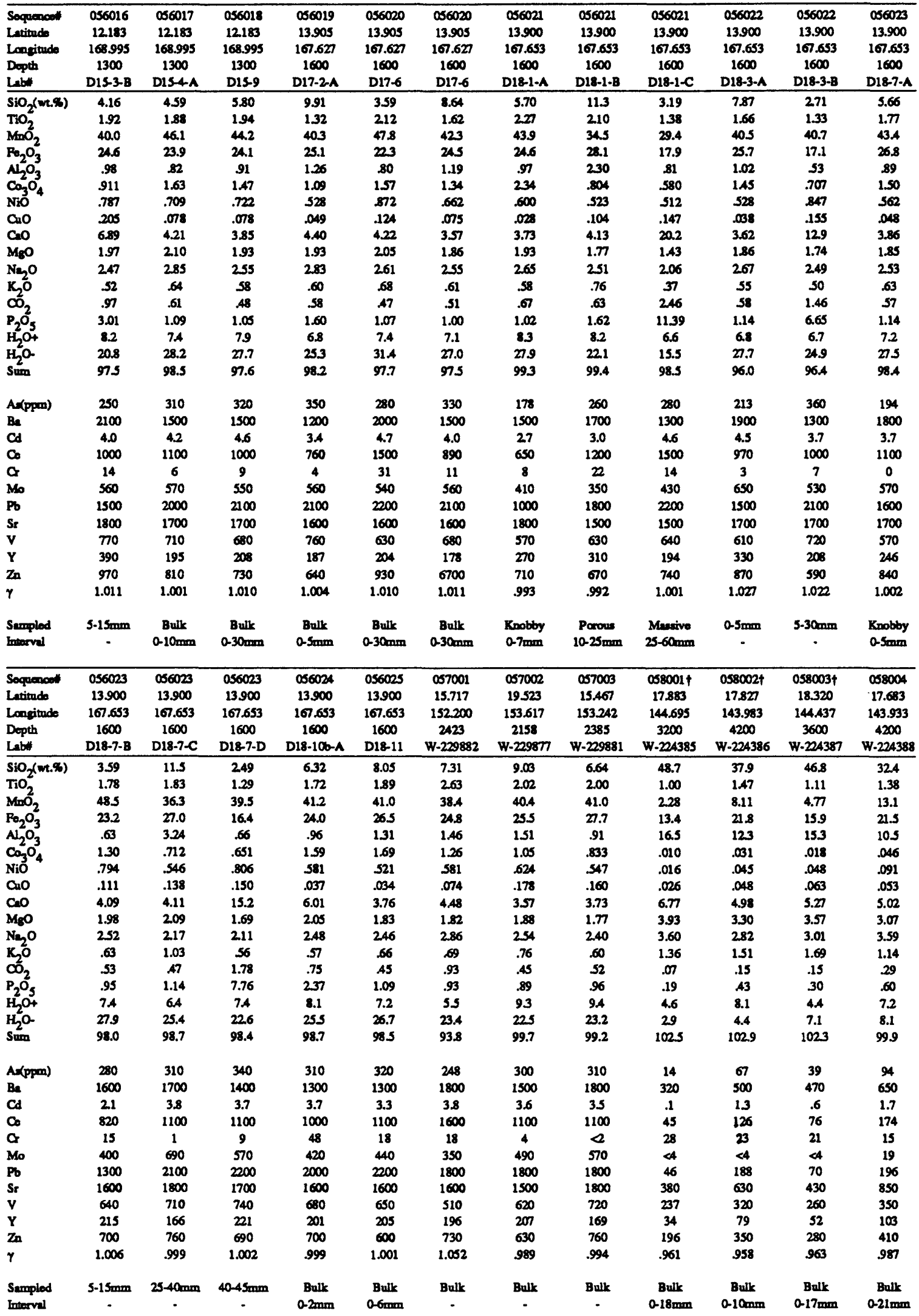


Table 1. Composition of crusts analyzed by USGS Reston Labs (cont'd).

\begin{tabular}{|c|c|c|c|c|c|c|c|c|c|c|c|c|}
\hline Sequencell & $058005 t$ & 058006 & 059002 & 059003 & 059004 & 059005 & 059006 & 061001 & 077004 & 077005 & 077006 & 078003 \\
\hline Letitude & 17.437 & 16.458 & 16.017 & 18.362 & 18.273 & 19.035 & 19.607 & 17.617 & 27.250 & 27.200 & 26.983 & 24.953 \\
\hline Longitude & 144.567 & 148.958 & 134.500 & 139.413 & 136.487 & 138.968 & 135.950 & 116.983 & -43.967 & -43.600 & -42.667 & -51.752 \\
\hline Depth & 3971 & 2385 & 5085 & 2350 & 2757 & 2600 & 2741 & - & 2785 & 3670 & 3500 & 5960 \\
\hline Lub" & W-222575 & W-229884 & W-229862 & W-229888 & W-229860 & W-229859 & W.231343 & W-229887 & W-222732 & $w-222730$ & W-222731 & W-229869 \\
\hline $\mathrm{SiO}_{2}$ (m.s) & 46.5 & 10.3 & 16.9 & 23.1 & 19.0 & 6.38 & 14.3 & 18.3 & 7.00 & 16.5 & 7.74 & 17.1 \\
\hline & 1.13 & 1.88 & 1.84 & 1.62 & 1.69 & 1.40 & 1.12 & 1.68 & .33 & 1.90 & 1.97 & 1.01 \\
\hline $\mathrm{MnO}_{2}$ & 256 & 36.7 & 262 & 17.9 & 20.8 & 40.2 & 229 & 18.4 & 25.5 & 21.4 & 28.2 & 24.6 \\
\hline $\mathrm{Fo}, \mathrm{O}_{3}^{2}$ & 154 & 30.1 & 324 & 31.8 & 37.0 & 27.5 & 26.8 & 37.3 & 33.7 & 27.4 & 31.4 & 30.2 \\
\hline $\mathrm{Al}_{2}^{2} \mathrm{O}_{3}^{3}$ & 15.3 & 1.67 & 4.81 & 5.95 & 4.75 & 1.78 & 3.32 & 4.70 & 3.48 & 5.84 & 3.92 & 7.55 \\
\hline $\mathrm{CO}_{3}^{2} \mathrm{O}_{4}^{3}$ & .012 & .786 & .400 & .248 & .223 & .903 & .475 & .198 & 920 & .783 & 1.10 & 389 \\
\hline $\mathrm{NiO}^{4}$ & .022 & 457 & 296 & .141 & .129 & .666 & 291 & .103 & .235 & .183 & 313 & 538 \\
\hline CoO & .028 & .122 & .184 & .111 & .125 & .109 & .051 & .112 & .062 & .057 & .108 & .257 \\
\hline $\mathrm{COO}$ & 6.19 & 3.44 & 2.74 & 2.60 & 237 & 3.62 & 6.73 & 237 & 5.33 & 5.36 & 5.09 & 1.82 \\
\hline $\mathrm{M}_{8} \mathrm{O}$ & 3.93 & 1.79 & 1.81 & 210 & 184 & 241 & 223 & 1.91 & 240 & 4.84 & 2.26 & 3.01 \\
\hline $\mathrm{Ne} O$ & 237 & 226 & 1.49 & 3.17 & 212 & 268 & 4.69 & 3.09 & 269 & 2.24 & 2.29 & 201 \\
\hline Kुo & 1.30 & .60 & .69 & 1.08 & 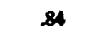 & 54 & .77 & .73 & .47 & .55 & 53 & .94 \\
\hline $\mathrm{C}_{2}$ & .06 & 51 & .32 & .22 & 32 & 52 & 3.25 & .19 & 209 & 1.56 & 209 & .22 \\
\hline $\mathrm{P}_{2} \mathrm{O}_{5}$ & .20 & $1 . \infty$ & .81 & 81 & 92 & 1.17 & 1.19 & 92 & 1.32 & .96 & 1.13 & .92 \\
\hline $\mathrm{H}_{2}^{2} \mathrm{O}+$ & 5.0 & 9.1 & 9.3 & 7.7 & 8.7 & 9.1 & 6.5 & 9.1 & 10.9 & 9.6 & 11.1 & 9.5 \\
\hline $\mathrm{H}_{2} \mathrm{O}$ & 7.3 & 22.0 & 18.3 & 17.7 & 18.0 & 21.6 & 16.9 & 17.5 & 18.6 & 16.5 & 18.6 & 12.4 \\
\hline Sum & 100.0 & 100.7 & 100.1 & 98.5 & 100.8 & 98.9 & 94.6 & 99.1 & 96.5 & 99.2 & 99.2 & 100.0 \\
\hline A_(ppon) & 30 & 290 & 233 & 260 & 290 & 380 & 200 & 300 & 540 & 360 & 410 & 340 \\
\hline $\mathrm{Ba}$ & 260 & 1700 & 1600 & 1200 & 1500 & 1700 & 910 & 1300 & 1100 & 770 & 960 & 780 \\
\hline Cd & 8 & 3.1 & 2.8 & 2.2 & 22 & 5.5 & 23 & 1.9 & 3.1 & 20 & 3.2 & 4.1 \\
\hline Ce & 49 & 940 & 1300 & 920 & 1200 & 1000 & 690 & 990 & 1800 & 1700 & 2200 & 1600 \\
\hline$\alpha$ & 24 & 6 & 9 & 11 & 9 & 5 & 18 & 9 & 20 & 59 & 17 & 27 \\
\hline Mo & $<4$ & 500 & 270 & 122 & 183 & 560 & 300 & 158 & 184 & 168 & 233 & 270 \\
\hline $\mathrm{Pb}$ & 54 & 1800 & 1600 & 1100 & 1300 & 2700 & 1700 & 1200 & 1600 & 1300 & 1600 & 710 \\
\hline Sr & 350 & 1700 & 1200 & 980 & 1100 & 1700 & 1200 & 1000 & 1500 & 1100 & 1200 & 670 \\
\hline $\mathbf{v}$ & 300 & 710 & 590 & 520 & 620 & 820 & 540 & 630 & 1200 & 870 & 950 & 740 \\
\hline $\mathbf{Y}$ & 36 & 180 & 196 & 146 & 171 & 179 & 181 & 158 & 233 & 168 & 197 & 104 \\
\hline $\mathbf{Z n}$ & 205 & 620 & 600 & 580 & 520 & 870 & 460 & 550 & 630 & 490 & 590 & 570 \\
\hline$\gamma$ & 986 & 980 & 985 & 1.001 & .978 & 996 & 1.042 & 995 & 1.022 & 994 & .994 & 986 \\
\hline Samplod & Bulk & Bulk & Bulk & Bulk & Bulk & Bulk & Bulk & Bulk & Bulk & Bulk & Bulk & Bulk \\
\hline Interval & $04 \operatorname{mm}$ & - & - & . & - & - & $0-12 \mathrm{~mm}$ & - & - & - & - & $\because$ \\
\hline Sequencell & 078004 & 079001 & 079005 & 079006 & 079007 & 079008 & 079010 & 079011 & 079012 & 080001 & 080002 & 080003 \\
\hline Latitude & 21.850 & 24.277 & 27.600 & 28.033 & 27.067 & 27.583 & 28.033 & 28.757 & 27.700 & 22317 & 22.333 & 22570 \\
\hline Longitude & -56.150 & -68.107 & -61.133 & -61.000 & -60.467 & -60.400 & -61.000 & -60.358 & .61 .167 & -72.080 & -72085 & .72375 \\
\hline Depth & 4049 & 5668 & 5706 & 5028 & 5891 & 5536 & 5105 & 5338 & 5390 & 3363 & 2250 & 3420 \\
\hline Labi & W.231344 & W-222722 & W-231352 & W-231349 & W-231359 & W.231361 & W-231362 & W-229863 & W-231348 & $W-222702$ & W-222703 & W-222704 \\
\hline $\mathrm{SiO}_{2}(\mathrm{w}, \mathrm{s})$ & 13.8 & 30.1 & 13.0 & 20.5 & 13.7 & 15.5 & 16.6 & 127 & 19.5 & 6.30 & 5.84 & 10.7 \\
\hline $\mathrm{TiO}_{2}$ & 1.16 & 89 & 248 & 1.01 & 1.27 & 1.04 & 1.02 & 1.15 & .92 & 1.78 & 1.95 & 1.52 \\
\hline $\mathrm{MmO}_{2}$ & 24.8 & 15.5 & 19.3 & 229 & 26.1 & 24.9 & 25.0 & 20.8 & 24.1 & 16.5 & 24.9 & 17.1 \\
\hline $\mathrm{Pe}_{2} \mathrm{O}_{3}$ & 27.6 & 25.5 & 323 & 26.1 & 29.4 & 27.2 & 26.4 & 30.7 & 22.9 & 34.1 & 43.4 & 38.1 \\
\hline $\mathrm{Al}_{2}^{2} \mathrm{O}_{3}^{3}$ & 6.20 & 10.2 & 5.41 & 7.53 & 6.84 & 8.29 & 7.84 & 6.11 & 8.54 & 4.63 & 4.17 & 6.50 \\
\hline $\mathrm{Co}_{3}^{2} \mathrm{O}_{4}^{3}$ & 433 & .219 & .644 & .388 & .477 & 425 & 439 & .484 & 385 & 545 & .916 & .468 \\
\hline $\mathrm{NIO}^{4}$ & 449 & .173 & .220 & .348 & .461 & A85 & 528 & .482 & .647 & .216 & .275 & .250 \\
\hline $\mathrm{CoO}$ & .147 & .138 & .088 & .138 & .190 & .159 & .216 & .223 & .325 & .091 & .081 & .135 \\
\hline $\mathrm{CaO}$ & 288 & 1.58 & 5.00 & 2.47 & 2.29 & 207 & 2.11 & 249 & 1.71 & 8.82 & 3.37 & 6.53 \\
\hline $\mathrm{M}_{8} \mathrm{O}$ & 3.55 & 3.08 & 2.91 & 270 & 2.61 & 2.64 & 273 & 2.67 & 3.69 & 3.01 & 2.74 & 285 \\
\hline $\mathrm{N}_{2} \mathrm{O}$ & 3.22 & 211 & 220 & 2.22 & 1.82 & 201 & 206 & 1.86 & 260 & 4.85 & 1.78 & 1.60 \\
\hline $\mathbf{k}_{2} \mathbf{O}$ & .67 & 1.36 & .82 & 1.32 & .79 & 1.03 & 1.05 & .77 & 1.16 & .61 & 55 & .75 \\
\hline $\mathrm{C}_{2}$ & 34 & 33 & 1.96 & 39 & .28 & 24 & 30 & .40 & .15 & - & 1.12 & 3.69 \\
\hline $\mathrm{P}_{2} \mathrm{O}_{5}$ & 1.27 & 80 & 1.22 & 86 & 1.07 & .95 & 92 & 1.03 & .83 & 1.69 & 1.45 & 1.52 \\
\hline $\mathrm{H}_{2}^{2} \mathrm{O}+$ & 9.4 & 5.6 & 10.3 & 10.3 & 8.8 & 9.1 & 9.9 & 9.6 & 9.5 & 6.9 & 6.5 & 7.5 \\
\hline $\mathrm{H}_{2} \mathrm{O}$ & 15.0 & 19.3 & 13.3 & 122 & 14.4 & 13.4 & 13.2 & 15.6 & 11.5 & 17.5 & 21.2 & 18.6 \\
\hline sum & 95.8 & 97.6 & 97.9 & 99.1 & 96.0 & 96.0 & 97.1 & 100.4 & 97.1 & - & 99.0 & 99.2 \\
\hline Ax(ppm) & 340 & 235 & 440 & 280 & 330 & 290 & 300 & 340 & 260 & 158 & 660 & 540 \\
\hline $\mathrm{Ba}$ & 960 & 500 & 970 & 640 & 740 & 690 & 790 & 850 & 850 & 740 & 1300 & 770 \\
\hline Cd & 4.1 & 2.4 & 20 & 28 & 4.0 & 3.7 & 45 & 4.9 & 4.9 & 23 & 25 & 3.6 \\
\hline Co & 1900 & 1500 & 1600 & 2200 & 2300 & 2200 & 2200 & 2300 & 1500 & 1600 & 2300 & 1600 \\
\hline$a$ & 47 & 71 & 127 & 57 & 32 & 29 & 23 & 23 & 40 & 102 & 79 & 112 \\
\hline Mo & 310 & 198 & 219 & 250 & 300 & 280 & 310 & 360 & 290 & 206 & 370 & 233 \\
\hline $\mathrm{Pb}$ & 840 & 580 & 980 & 690 & 970 & 800 & 840 & 1000 & 700 & 840 & 1400 & 880 \\
\hline Sr & 950 & 500 & 1200 & 740 & 820 & 750 & 770 & 940 & 600 & 860 & 1400 & 960 \\
\hline v & 780 & 690 & 1000 & 670 & 790 & 680 & 670 & 830 & 660 & 1100 & 1500 & 1100 \\
\hline $\mathbf{Y}$ & 153 & 74 & 185 & 105 & 164 & 150 & 150 & 178 & 102 & 170 & 222 & 154 \\
\hline $\mathbf{Z n}$ & 560 & 410 & 480 & 430 & 500 & 490 & 530 & 590 & 640 & 450 & 560 & 520 \\
\hline$\gamma$ & 1.029 & 1.011 & 1.007 & .995 & 1.027 & 1.027 & 1.016 & 982 & 1.016 & $\cdot$ & .996 & .994 \\
\hline $\begin{array}{l}\text { Sampled } \\
\text { forterval }\end{array}$ & $\begin{array}{c}\text { Bulk } \\
0-10 \mathrm{~mm}\end{array}$ & $\begin{array}{c}\text { Bulk } \\
0-12 \mathrm{~mm}\end{array}$ & Bulk & $\underset{\text { O-Amm }}{\text { Bulk }}$ & $\begin{array}{c}\text { Bulk } \\
0-8 \mathrm{mmm}\end{array}$ & $\begin{array}{c}\text { Bulk } \\
0-40 \mathrm{~mm}\end{array}$ & $\underset{0-10 \mathrm{~mm}}{\text { Bulk }}$ & Bulk & $\begin{array}{c}\text { Bulk } \\
\text { O-Gmm }\end{array}$ & $\underset{0-2 m m}{\text { Bulk }}$ & $\begin{array}{c}\text { Bulk } \\
\text { a-17mm }\end{array}$ & $\underset{\text { O-4min }}{\text { Bulk }}$ \\
\hline
\end{tabular}


Table 1. Composition of crusts analyzed by USGS Reston Labs (cont'd).

\begin{tabular}{|c|c|c|c|c|c|c|c|c|c|c|c|c|}
\hline Sequencell & 080004 & 080005 & 080006 & 080007 & 080008 & 083001 & 083003 & 083004 & $084001 \dagger$ & 084002 & 084003 & 084004 \\
\hline Latitado & 22.642 & 23.157 & 23.183 & 23.487 & 21.833 & 24.775 & 22870 & 22870 & 26,472 & 20.750 & 21.300 & 20.750 \\
\hline Longitude & -72850 & -73.752 & -73.973 & -74.200 & -70.573 & -109.203 & -108.075 & -108.075 & -110.718 & -112783 & -112700 & -114.450 \\
\hline Depth & 2390 & 3126 & 1315 & 2910 & 5416 & 3038 & 3127 & 3127 & 2501 & 1712 & 2497 & 3840 \\
\hline Labu & w-22270s & W-222706 & W-222707 & W-222708 & W-222719 & W-222615 & W-222586 & W-222587 & W-222616 & W-222581 & W-222582 & W-222583 \\
\hline \multirow{7}{*}{$\begin{array}{l}\mathrm{SiO}_{2}(\mathrm{wt} . \%) \\
\mathrm{TiO}_{2} \\
\mathrm{MnO}_{2} \\
\mathrm{Pe}_{2} \mathrm{O}_{3} \\
\mathrm{Al}_{2} \mathrm{O}_{3} \\
\mathrm{CO}_{3} \mathrm{O}_{4} \\
\mathrm{NiO}^{2}\end{array}$} & 109 & 7.84 & 4.90 & 3.93 & 8.49 & 39.1 & 30.9 & 269 & 47.7 & 11.4 & 19.0 & 15.5 \\
\hline & 96 & 1.12 & 1.64 & 1.64 & 1.56 & 81 & 31 & 47 & 209 & 1.13 & .47 & 39 \\
\hline & 14.2 & 10.2 & 27.2 & 29.2 & 20.2 & 27.7 & 31.8 & 220 & .33 & 31.4 & 35.0 & 43.6 \\
\hline & 31.7 & 245 & 38.7 & 38.6 & 41.9 & 5.30 & 10.5 & 25.7 & 125 & 31.6 & 20.2 & 129 \\
\hline & 7.24 & 4.19 & 5.19 & 4.46 & 4.88 & 11.2 & 6.05 & 4.38 & 14.2 & 1.89 & 3.56 & 4.47 \\
\hline & 310 & .279 & 931 & .946 & .486 & .004 & .031 & .055 & .005 & .478 & .257 & .177 \\
\hline & 229 & .133 & 394 & .434 & .219 & .027 & .698 & 218 & .006 & 331 & .839 & 1.431 \\
\hline Cuo & .165 & .007 & .162 & .128 & .094 & .006 & .112 & .035 & .006 & .036 & .221 & 993 \\
\hline $\mathrm{CaO}$ & 11.6 & 24.5 & 3.97 & 3.30 & 2.93 & 4.15 & 1.92 & 256 & 8.46 & 2.91 & 2.14 & 232 \\
\hline $\mathrm{M}_{\mathrm{g} O} \mathrm{O}$ & 278 & 1.88 & 299 & 3.26 & 2.45 & 3.34 & 246 & 1.58 & 6.74 & 1.72 & 2.54 & 275 \\
\hline $\mathrm{N}_{2} \mathrm{O}$ & 1.44 & 1.25 & 1.42 & 1.44 & 1.48 & 4.13 & 297 & 281 & 4.59 & 2.21 & 2.24 & 272 \\
\hline & .72 & 53 & .48 & 47 & 58 & 1.38 & 234 & 1.18 & .21 & .70 & 1.35 & 1.25 \\
\hline & 8.26 & 18.2 & 1.55 & 93 & 1.09 & .21 & 32 & 50 & 1.56 & .53 & 58 & .78 \\
\hline $\mathrm{P}_{2} \mathrm{O}_{5}$ & 1.26 & 89 & 1.36 & 1.38 & 1.44 & .19 & 37 & 98 & .22 & 1.31 & .65 & $A 6$ \\
\hline $\mathrm{H}_{2} \mathrm{O}+$ & 5.9 & 4.3 & 8.8 & 8.1 & 7.1 & 4.7 & 73 & 9.2 & 23 & 9.9 & 6.5 & 9.1 \\
\hline $\mathrm{H}_{2} \mathrm{O}$ & 16.5 & 120 & 225 & 23.7 & 18.7 & 5.6 & 125 & 18.1 & 4.1 & 23.0 & 15.1 & 155 \\
\hline Sum & 97.6 & 99.9 & 99.7 & 98.3 & 94.9 & 102.2 & 98.0 & 98.5 & 100.9 & 97.6 & 955 & 98.9 \\
\hline Ax(ppm) & 420 & 380 & 440 & 620 & 690 & 21 & 105 & 260 & 2 & 390 & 189 & 154 \\
\hline Ba & 730 & 490 & 1500 & 1400 & 1000 & 1200 & 2200 & 1000 & 38 & 1400 & 7300 & 2000 \\
\hline cd & 3.4 & 20 & 4.5 & 4.1 & 25 & 23 & 16.0 & 29 & $<.1$ & 3.0 & 14.1 & 15.4 \\
\hline$c_{0}$ & 1400 & 1000 & 2300 & 2200 & 2200 & 39 & 330 & 660 & 18 & 1400 & 480 & 450 \\
\hline$a$ & 77 & 63 & 74 & 56 & 44 & 40 & 91 & 34 & 167 & 21 & 28 & 13 \\
\hline Mo & 216 & 136 & 440 & 430 & 300 & 280 & 410 & 171 & $<4$ & 470 & 310 & 580 \\
\hline$P_{0}$ & 800 & 610 & 1500 & 1500 & 1100 & $<10$ & 206 & 570 & $\theta$ & 1400 & 440 & 380 \\
\hline St & 1200 & 680 & 1300 & 1400 & 1200 & 510 & 570 & 1100 & 167 & 1600 & 1400 & 720 \\
\hline v & 780 & 750 & 1200 & 1200 & 1500 & 233 & 380 & 530 & 380 & 810 & 540 & 450 \\
\hline $\mathbf{Y}$ & 78 & 114 & 136 & 190 & 200 & 19 & 57 & 159 & 40 & 208 & 100 & 85 \\
\hline $\mathbf{z a}$ & 500 & 350 & 770 & 730 & 600 & 300 & 1700 & 560 & 89 & 640 & 1100 & 1700 \\
\hline$r$ & 1.010 & .987 & 989 & 1.003 & 1.039 & .965 & 1.006 & 1.001 & 977 & 1.011 & 1.032 & 997 \\
\hline $\begin{array}{l}\text { Samplod } \\
\text { Intorval }\end{array}$ & $\begin{array}{c}\text { Bulk } \\
\text { a-28mm }\end{array}$ & $\begin{array}{c}\text { Bulk } \\
\text { a-Sinm }\end{array}$ & $\begin{array}{c}\text { Bulk } \\
\text { 0-30mm }\end{array}$ & $\begin{array}{c}\text { Bulk } \\
\text { O-40mm }\end{array}$ & $\begin{array}{c}\text { Bulk } \\
\text { O-19rmm }\end{array}$ & $\begin{array}{c}\text { Bulk } \\
0-8 \mathrm{cmm}\end{array}$ & $\begin{array}{c}\text { Bulk } \\
0-20 \mathrm{~mm}\end{array}$ & $\begin{array}{c}\text { Bulk } \\
0-6 \mathrm{~mm}\end{array}$ & Bulk & $\begin{array}{c}\text { Bulk } \\
0-22 \text { mmm }\end{array}$ & $\begin{array}{c}\text { Bulk } \\
0-20 \mathrm{~mm}\end{array}$ & $\begin{array}{c}\text { Bulk } \\
0-11 \text { mm }\end{array}$ \\
\hline Sequencoll & 084005 & 088001 & 088002 & 088003 & 088004 & 088005 & 088006 & 088007 & 088013 & 088015 & 088018 & 089001 \\
\hline Latitude & 21.083 & 23.317 & 24.883 & 26.583 & 26.000 & 26.067 & 27.550 & 27.417 & 25.951 & 25.944 & 25.951 & 21.670 \\
\hline Lagitude & -119.367 & -158.317 & -157.117 & -158.833 & -158.967 & -158.200 & -158.550 & -159.867 & -159.914 & -159.878 & -159.914 & .167 .953 \\
\hline Depth & 2985 & 3000 & 3000 & 3525 & 4070 & 4750 & 4850 & 3400 & 3600 & 2600 & 3600 & 1690 \\
\hline Lab: & W-222584 & W-224422 & W-224423 & W-224424 & W-224425 & $w-224426$ & W-224427 & W-224428 & W-234336 & W-234339 & W-234342 & W-222571 \\
\hline $\mathrm{SiO}_{2}$ (wt.\%) & 18.2 & 13.3 & 24.5 & 14.5 & 21.7 & 40.6 & 18.8 & 15.7 & 16.3 & 9.31 & 15.0 & 17.2 \\
\hline $\mathrm{TiO}_{2}^{2-}$ & 1.86 & 2.23 & 1.89 & 2.21 & 1.55 & 3.48 & 1.78 & 216 & 1.98 & 1.86 & 1.96 & 1.23 \\
\hline $\mathrm{MmO}_{2}^{2}$ & 28.0 & 27.0 & 23.5 & 32.2 & 20.2 & 5.32 & 28.0 & 29.9 & 27.0 & 38.3 & 28.7 & 38.8 \\
\hline & 25.9 & 29.6 & 18.0 & 27.2 & 17.9 & 20.7 & 23.9 & 26.7 & 29.5 & 25.5 & 27.7 & 17.5 \\
\hline $\mathrm{Al}_{2}^{2} \mathrm{O}_{3}$ & 3.88 & 274 & 7.08 & 2.93 & 6.38 & 14.9 & 5.08 & 4.29 & 3.33 & 1.66 & 2.88 & 5.50 \\
\hline $\mathrm{CO}_{3}^{2} \mathrm{O}_{4}^{3}$ & 542 & .652 & 541 & .919 & .532 & .095 & 580 & .486 & 572 & 1.27 & .790 & .413 \\
\hline $\mathrm{NiO}^{4}$ & 376 & .241 & .419 & .324 & .376 & .117 & .342 & 330 & 244 & 521 & .323 & 1.236 \\
\hline Cuo & .127 & .047 & .171 & .125 & .185 & .081 & .196 & 284 & .114 & .106 & .150 & .714 \\
\hline $\mathrm{CaO}$ & 287 & 4.21 & 3.34 & 3.10 & 10.0 & 1.06 & 3.76 & 3.33 & 3.34 & 3.39 & 3.76 & 2.21 \\
\hline $\mathrm{M}_{8} \mathrm{O}$ & 1.98 & 1.85 & 1.75 & 1.72 & 2.27 & 288 & 223 & 1.74 & 1.77 & 1.81 & 1.72 & 3.22 \\
\hline $\mathrm{N}_{2} \mathrm{O}$ & 244 & 3.34 & 3.63 & 2.54 & 2.22 & 244 & 235 & 2.38 & 2.24 & 2.47 & 2.24 & 243 \\
\hline $\mathrm{K}_{2} \mathrm{O}$ & 1.13 & 58 & 1.91 & .96 & 1.52 & 293 & 1.11 & 1.05 & .67 & .70 & .79 & 1.14 \\
\hline $\mathrm{CO}_{2}$ & .14 & .40 & .27 & 39 & .98 & .15 & .47 & .43 & .32 & .25 & 42 & .24 \\
\hline $\mathrm{P}_{2} \mathrm{O}_{5}$ & .71 & 1.61 & .91 & .89 & 5.80 & 24 & 1.39 & 94 & .94 & .91 & 1.25 & 42 \\
\hline $\mathrm{H}_{2}^{2} \mathrm{O}+$ & 9.0 & 17.6 & 10.3 & 121 & 11.7 & 7.7 & 12.9 & 13.1 & 9.0 & 9.8 & 9.1 & 5.9 \\
\hline $\mathrm{H}_{2}^{2} \mathrm{O}$ & 22.1 & 10.2 & 11.9 & 9.6 & 5.2 & 13.8 & 10.8 & 75 & 16.7 & 19.4 & 17.3 & 17.6 \\
\hline sum & 97.2 & 1053 & 98.3 & 102.1 & 103,4 & 102.7 & 1029 & 1029 & 97.4 & 97.9 & 96.7 & 983 \\
\hline$A x$ (ppom) & 231 & 234 & 104 & 199 & 101 & 33 & 168 & 162 & 280 & 280 & 242 & 146 \\
\hline Be & 1300 & 1400 & 2000 & 1500 & 1000 & 310 & 1300 & 2200 & 1600 & 1700 & 1500 & 1700 \\
\hline cd & 27 & 22 & 23 & 25 & 22 & .7 & 1.8 & 2.4 & 22 & 3.6 & 23 & 8.4 \\
\hline$\infty$ & 1700 & 1200 & 1100 & 1900 & 1500 & 350 & 1600 & 1900 & 1100 & 1200 & 1600 & 1200 \\
\hline$c$ & 28 & 12 & 28 & 16 & 25 & 280 & 12 & 13 & 56 & 11 & 19 & 22 \\
\hline Mo & 260 & 310 & 204 & 330 & 169 & 13 & 310 & 290 & 280 & 450 & 242 & 460 \\
\hline Po & 1100 & 2000 & 1000 & 1900 & 1100 & 340 & 1200 & 1300 & 1900 & 2000 & 1900 & 1000 \\
\hline St & 1200 & 1600 & 1100 & 1400 & 950 & 200 & 1200 & 1400 & 1400 & 1500 & 1300 & 780 \\
\hline v & 460 & 650 & 390 & 580 & 360 & 174 & 530 & 620 & 640 & 660 & 580 & 470 \\
\hline $\mathbf{Y}$ & 180 & 200 & 170 & 221 & 330 & 37 & 300 & 173 & 192 & 186 & 194 & 103 \\
\hline $\mathbf{Z n}$ & 580 & 580 & 540 & 550 & 470 & 560 & 560 & 690 & 620 & 610 & 590 & 760 \\
\hline $\bar{r}$ & 1.015 & .936 & 1.003 & .966 & .953 & 960 & .958 & .959 & 1.013 & 1.007 & 1.020 & 1.003 \\
\hline Samplod & Bulk & Bulk & Bulk & Bulk & Bulk & Bulk & Bulk & Bulk & Bulk & Bulk & Bulk & Bulk \\
\hline Interval & $0-10 \mathrm{~mm}$ & $0-8 \mathrm{~mm}$ & $0-4 \mathrm{~mm}$ & $0-24 \mathrm{~mm}$ & $0-28 \mathrm{~mm}$ & $0-2 \mathrm{~mm}$ & $0.30 \mathrm{~mm}$ & $0-32 \mathrm{~mm}$ & - & 6-8mm & $7-25 \mathrm{~mm}$ & $0-3 \mathrm{~mm}$ \\
\hline
\end{tabular}


Table 1. Composition of crusts analyzed by USGS Reston Labs (cont'd).

\begin{tabular}{|c|c|c|c|c|c|c|c|c|c|c|c|c|}
\hline Sequencell & 089002 & 089003 & 089004 & 089005 & 089006 & 089007 & 0089008 & 0.89009 & 089010 & 089011 & 089013 & 089014 \\
\hline Letitude & 23.711 & 23.112 & 28.965 & 26.917 & 26.633 & 27.317 & 26.150 & 25.183 & 25.133 & 22.181 & 22.208 & 22.256 \\
\hline Langitude & -165.602 & -162.480 & -162.022 & -160.050 & -161.200 & -161.650 & -162.067 & -161.600 & -161.950 & -166.810 & -166.882 & -166854 \\
\hline Depth & 1325 & 1650 & 2820 & 3000 & 3000 & 3500 & 2450 & 2600 & 3600 & 3950 & 2402 & 1970 \\
\hline Lebl" & W-224379 & W-224380 & W-224421 & W-224429 & $w-224430$ & W.224431 & W-224432 & W-224433 & W-224434 & W-223731 & W-2223732 & W.223733 \\
\hline $\mathrm{SiO}_{2}$ (wt.\%) & 14.6 & 11.8 & 14.6 & 17.6 & 25.8 & 30.9 & 12.8 & 10.0 & 30.2 & 14.9 & 15.7 & 13.0 \\
\hline $\mathrm{TiO}_{2}^{2}$ & 2.11 & 1.95 & 1.78 & 1.99 & 2.03 & 268 & 2.15 & 231 & 2.12 & 1.88 & 2.45 & 226 \\
\hline $\mathrm{MnO}_{2}$ & 33.0 & 34.2 & 323 & $\boldsymbol{2 9 . 7}$ & 20.0 & 15.6 & 34.6 & 33.0 & 16.7 & 29.2 & 27.7 & 30.8 \\
\hline $\mathrm{Pe}_{2} \mathrm{O}_{3}^{2}$ & 223 & 25.1 & 25.1 & 26.2 & 212 & 22.7 & 22.7 & 29.9 & 20.9 & 29.0 & 27.1 & 28.3 \\
\hline $\mathrm{Al}_{2}^{2} \mathrm{O}_{3}$ & 3.98 & 2.61 & 2.92 & 3.69 & 7.74 & 8.92 & 3.24 & 1.74 & 8.59 & 3.02 & 3.90 & 27 \\
\hline $\mathrm{CO}_{3}^{2} \mathrm{O}_{4}^{3}$ & 1.16 & .811 & .096 & .960 & .633 & $A 09$ & 1.05 & .770 & .317 & 552 & .648 & .791 \\
\hline $\mathrm{NiO}^{4}$ & 522 & .47 & $A 07$ & .400 & .240 & .191 & .464 & .338 & .256 & .271 & .426 & 384 \\
\hline Co & .042 & .052 & .108 & .071 & .075 & .081 & .082 & .071 & .132 & .187 & .284 & .136 \\
\hline $\mathrm{CaO}$ & 4.57 & 4.17 & 3.09 & 3.49 & 5.11 & 225 & 5.85 & 3.39 & 3.55 & 3.43 & 3.63 & 3.60 \\
\hline $\mathrm{MgO}_{8}$ & 3.14 & 2.65 & 1.74 & 1.80 & 1.84 & 231 & 1.95 & 2.10 & 2.80 & 1.77 & 1.79 & 1.63 \\
\hline $\mathrm{Ne}_{2} \mathrm{O}$ & 274 & 3.40 & 2.76 & 2.49 & 288 & 236 & 257 & 3.11 & 296 & 2.24 & 2.27 & 223 \\
\hline $\mathrm{K}_{2} \mathrm{O}$ & .72 & 53 & 1.04 & .80 & 1.47 & 246 & 92 & .47 & 1.91 & .81 & .82 & .65 \\
\hline$\infty_{2}$ & 44 & so & .39 & .47 & .87 & 27 & .62 & 13 & .33 & .42 & 53 & 54 \\
\hline $\mathrm{P}_{2} \mathrm{O}_{5}$ & 1.01 & .98 & .99 & 97 & 1.35 & 59 & 2.33 & 1.08 & .92 & .95 & .87 & 87 \\
\hline $\mathrm{H}_{2}^{2} \mathrm{O}+$ & 10.9 & 11.4 & 120 & 11.2 & 9.1 & 9.5 & 15.8 & 13.6 & 11.2 & 7.6 & 9.3 & 10.4 \\
\hline $\mathrm{H}_{2} \mathrm{O}$ & 5.0 & 6.0 & 9.3 & 7.8 & 9.7 & 6.8 & 6.7 & 13.3 & 5.4 & 6.2 & 7.5 & 10.5 \\
\hline Sum & 101.3 & 100.6 & 99.2 & 101.8 & 100.3 & 1013 & 107.0 & 1024 & 102.9 & 96.2 & 97.4 & 98.2 \\
\hline$A(p p m)$ & 232 & 223 & 199 & 217 & 122 & 103 & 182 & 250 & 106 & 181 & 162 & 201 \\
\hline $\mathbf{B a}_{\mathbf{a}}$ & 1000 & 1100 & 1300 & 1300 & 1100 & 750 & 1200 & 1600 & 1300 & 1300 & 1700 & 1700 \\
\hline$\overline{c d}$ & 3.6 & 29 & 2.6 & 2.6 & 1.9 & 1.2 & 3.1 & 2.2 & 1.8 & 3.3 & 27 & 2.5 \\
\hline c. & 850 & 780 & 1400 & 1200 & 750 & 900 & 950 & 1100 & 810 & 1600 & 920 & 960 \\
\hline$a$ & 116 & 117 & 7 & 59 & 48 & 20 & 15 & 7 & 99 & - & - & $\cdot$ \\
\hline Mo & 520 & 560 & 360 & 310 & 177 & 140 & 4200 & 420 & 127 & 171 & 195 & 310 \\
\hline $\mathrm{Pb}$ & 2000 & 1900 & 1800 & 1800 & 1200 & 930 & 1700 & 2200 & 1100 & 1800 & 1700 & 1800 \\
\hline Sr & 1400 & 1600 & 1400 & 1300 & 940 & 730 & 1400 & 1600 & 800 & 1300 & 1400 & 1500 \\
\hline v & 670 & 670 & 560 & 590 & 430 & 380 & 540 & 660 & 390 & 570 & 610 & 660 \\
\hline $\mathbf{Y}$ & 168 & 213 & 199 & 174 & 122 & 140 & 225 & 196 & 169 & 149 & 141 & 168 \\
\hline $\mathbf{z a}$ & 570 & 560 & 550 & 570 & 420 & 460 & 530 & 610 & 490 & 640 & 840 & 730 \\
\hline $\boldsymbol{\gamma}$ & .974 & .981 & .994 & .969 & .983 & .973 & .921 & .963 & .958 & 1.025 & 1.013 & 1.004 \\
\hline Sempled & Bulk & Bulk & Bulk & Bulk & Bulk & Bulk & Balk & Bulk & Bulk & Balk & Bulk & Top \\
\hline Interval & $0-18 \mathrm{~mm}$ & $0-11 \mathrm{~mm}$ & $0-14 \mathrm{~mm}$ & $0-8 \mathrm{~mm}$ & $0-2 \mathrm{~mm}$ & $0.100 \mathrm{mon}$ & $0-8 \mathrm{~mm}$ & $0-6 \mathrm{~mm}$ & $0-4 \mathrm{~mm}$ & - & • & <lmm \\
\hline Sequencell & 089015 & 089016 & 089017 & 089018 & 089019 & 089020 & 089021 & 0899022 & 089023 & 089025 & 089026 & 089027 \\
\hline Letitude & 22316 & 22316 & 21.718 & 21.718 & 21.718 & 21.718 & 21.796 & 21.796 & 21.796 & 20.209 & 20.209 & 20.209 \\
\hline Longimde & -166.899 & -166.899 & -167.578 & -167.578 & -167.578 & -167.578 & -167.624 & -167.624 & -167.624 & -169.506 & -169.506 & -169.506 \\
\hline Depth & 2350 & 2350 & 2400 & 2400 & 2400 & 2400 & 2100 & 2100 & 2100 & 4500 & 4500 & 4500 \\
\hline Leb* & W-223734 & W-223780 & W-223735 & W-2223736 & W-223737 & W-223781 & W-223738 & W-223739 & W-223740 & W-223741 & W-223742 & w-223743 \\
\hline $\mathrm{SiO}_{2}(w t . \%)$ & 13.1 & 9.45 & 127 & 13.0 & 15.8 & 8.37 & 8.54 & 14.0 & 15.2 & 175 & 24.5 & 23.2 \\
\hline $\mathrm{TiO}_{2}^{2}$ & 1.95 & 228 & 255 & 2.33 & 1.89 & 254 & 3.19 & 288 & 1.78 & 3.66 & 3.03 & 2.67 \\
\hline $\mathrm{MnO}_{2}^{2}$ & 31.4 & 34.0 & 35.5 & 33.6 & 29.8 & 35.7 & 38.8 & 28.5 & 30.4 & 24.8 & 19.5 & 23.5 \\
\hline $\mathrm{Fe}_{2} \mathrm{O}_{3}$ & 27.6 & 29.1 & 25.0 & 31.0 & 29.4 & 23.6 & 27.5 & 32.0 & 31.0 & 31.7 & 27.2 & 25.2 \\
\hline $\mathrm{Al}_{2}^{2} \mathrm{O}_{3}^{2}$ & 2.64 & 1.64 & 2.96 & 2.64 & 3.21 & 1.76 & 1.47 & 3.01 & 2.66 & 4.63 & 7.03 & 6.84 \\
\hline $\mathrm{CO}_{3}^{2} \mathrm{O}_{4}^{2}$ & .833 & .924 & .727 & .703 & .746 & 1.59 & 1.38 & 558 & .734 & .436 & .344 & .441 \\
\hline $\mathrm{NIO}^{4}$ & .431 & $A 25$ & 510 & .358 & .291 & 526 & .421 & 302 & .283 & .204 & .187 & 313 \\
\hline Co & .119 & .117 & .228 & .104 & .043 & .085 & .051 & .101 & .048 & .132 & .171 & .196 \\
\hline $\mathrm{CaO}$ & 3.67 & 3.42 & 3.74 & 3.78 & 3.83 & 4.00 & 3.58 & 3.77 & 3.44 & 3.13 & 3.09 & 3.13 \\
\hline $\mathrm{MeO}$ & 1.76 & 1.64 & 1.69 & 1.71 & 1.67 & 1.76 & 1.95 & 1.59 & 1.59 & 1.77 & 2.27 & 241 \\
\hline $\mathrm{N}_{2} \mathrm{O}$ & 2.18 & 222 & 231 & 2.23 & 2.40 & 275 & 2.67 & 2.16 & 2.23 & 2.13 & 2.21 & 2.23 \\
\hline$k_{2} 0$ & .76 & .60 & .92 & .72 & .87 & .68 & .76 & .68 & .75 & .86 & 1.25 & 1.24 \\
\hline$\infty_{2}$ & .37 & .48 & .50 & 38 & 51 & .91 & 46 & 29 & .49 & .17 & .16 & 26 \\
\hline $\mathrm{P}_{2} \mathrm{O}_{5}$ & 1.18 & 1.05 & .75 & 1.05 & 1.13 & 1.02 & 91 & 99 & 1.05 & .76 & .63 & 59 \\
\hline $\mathrm{H}_{2}^{2} \mathrm{O}+$ & 9.5 & 10.2 & 127 & 9.0 & 10.6 & 6.9 & 7.8 & 8.0 & 10.4 & 7.6 & 6.9 & 7.7 \\
\hline H. & 8.4 & 10.1 & 17.6 & 14.8 & 12.4 & 5.6 & 6.3 & 7.2 & 14.7 & 6.2 & 4.9 & 10.5 \\
\hline Sum & 97.6 & 97.6 & 1029 & 102.7 & 102.1 & 92.2 & 99.5 & 98.8 & 102.2 & 99.4 & 98.5 & 100.0 \\
\hline Ax(ppon) & 197 & 310 & 182 & 223 & 217 & 260 & 213 & 194 & 223 & 181 & 105 & 123 \\
\hline $\mathrm{Ba}$ & 1400 & 1800 & 1900 & 1600 & 1100 & 1500 & 1600 & 1800 & 1300 & 1700 & 1400 & 1500 \\
\hline $\mathrm{Cd}$ & 3.1 & 2.1 & 25 & 25 & 23 & 3.1 & 3.3 & 2.2 & 2.6 & 23 & 2.2 & 27 \\
\hline 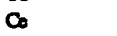 & 1100 & 1100 & 1200 & 1200 & 1100 & 1700 & 1500 & 1100 & 1300 & 1800 & 1400 & 1500 \\
\hline$a$ & 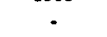 & - & 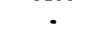 & - & - & 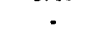 & 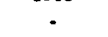 & 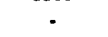 & $\cdot$ & $\cdot$ & 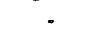 & $\cdot$ \\
\hline Mo & 320 & 430 & 350 & 350 & 300 & 300 & 310 & 280 & 320 & 160 & 63 & 101 \\
\hline Pb & 2000 & 1900 & 1300 & 1900 & 1700 & 2000 & 2100 & 1800 & 2000 & 1400 & 1000 & 1100 \\
\hline Sr & 1400 & 1800 & 1600 & 1500 & 1400 & 1600 & 1500 & 1500 & 1400 & 1200 & 880 & 910 \\
\hline $\mathbf{v}$ & 590 & 700 & 530 & 610 & 580 & 600 & 510 & 570 & 620 & 530 & 410 & 40 \\
\hline $\mathbf{Y}$ & 186 & 260 & 182 & 211 & 194 & 180 & 213 & 216 & 211 & 192 & 137 & 134 \\
\hline $\mathbf{z n}$ & 660 & 670 & 760 & 690 & 550 & 720 & 640 & 740 & 590 & 640 & 540 & 560 \\
\hline$\gamma$ & 1.011 & 1.011 & 958 & .960 & .965 & 1.069 & .991 & .998 & .965 & .991 & 1.001 & 986 \\
\hline Semplod & Bulk & Bulk & Botam & Top & Top & Top & Bulk & Bulk & Top & Bulk & Top & Bottom \\
\hline Interval &. & $=$ & - & $\cdot$ & - & $<\operatorname{lmm}$ & - & . & 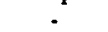 & 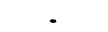 & - & - \\
\hline
\end{tabular}


Table 1. Composition of crusts analyzed by USGS Reston Labs (cont'd).

\begin{tabular}{|c|c|c|c|c|c|c|c|c|c|c|c|c|}
\hline Seqpencoll & 089028 & 089029 & 089030 & 089031 & 089033 & 089034 & 089035 & 089036 & 089037 & 089038 & 089039 & 089040 \\
\hline Letitude & 20.209 & 20.335 & 20.335 & 20.335 & 23.817 & 25.675 & 23.172 & 25.900 & 29.555 & 24.322 & 25.783 & 25.929 \\
\hline Longitude & -169.506 & -169.575 & -169.575 & -169.575 & -164.420 & -168.697 & -162434 & -162050 & -163.372 & -168.083 & -167.733 & .167 .755 \\
\hline Depth & 4500 & 2400 & 2400 & 2400 & 870 & 1510 & 1849 & 3200 & 2496 & 1225 & 1600 & 2500 \\
\hline Lab" & W-223744 & W-223745 & W-223746 & $W-223747$ & W-231381 & W-231380 & W-231375 & W-224419 & W-222614 & W.228552 & W-228573 & W-228579 \\
\hline \multirow{7}{*}{$\begin{array}{l}\mathrm{SiO}_{2}(w t . \%) \\
\mathrm{TiO}_{2}\end{array}$} & 20.5 & 11.6 & 11.6 & 8.32 & 23.9 & $17 A$ & 43.6 & 155 & 20.0 & 30.8 & 5.68 & 8.29 \\
\hline & 4.01 & 204 & 237 & 287 & 2.23 & 203 & 2.73 & .60 & 1.48 & 2.67 & .49 & 1.91 \\
\hline & 220 & 33.3 & 33.0 & 38.4 & 15.8 & 28.6 & 3.36 & 8.68 & 26.0 & 4.22 & 68.5 & 38.1 \\
\hline & 29.7 & 31.5 & 31.9 & 27.7 & 22.0 & 24.8 & 14.1 & 6.66 & 23.0 & 24.1 & 3.01 & 29.6 \\
\hline & 589 & 1.7 & 1.92 & 146 & 9.76 & 452 & 15.5 & 3.91 & 5.49 & 10.7 & 259 & 1.10 \\
\hline & 379 & 818 & .721 & 1.20 & .440 & .782 & .062 & .089 & 568 & .174 & .106 & 1.13 \\
\hline & .227 & 320 & 330 & .433 & 534 & .537 & .076 & .408 & 386 & .176 & .442 & 146 \\
\hline $\mathrm{CnO}$ & .195 & .052 & .085 & .004 & .058 & .097 & .009 & .268 & .190 & .033 & .145 & .057 \\
\hline $\mathrm{CaO}$ & 296 & 3.36 & 3.31 & 3.94 & 3.61 & 297 & 6.52 & 27.7 & 3.89 & 6.86 & 1.62 & 3.43 \\
\hline $\mathrm{M}_{8} \mathrm{O}$ & 203 & 1.67 & 1.61 & 1.75 & 6.78 & 231 & 3.44 & 1.89 & 1.99 & 4.77 & 3.84 & 1.82 \\
\hline $\mathrm{NeO}$ & 211 & 229 & 2.14 & 234 & 2.03 & 225 & 4.04 & 1.24 & 291 & 2.24 & 2.11 & 234. \\
\hline & .95 & .64 & .02 & .68 & .79 & 1.05 & 1.45 & 1.37 & 1.57 & 1.04 & 1.52 & 59 \\
\hline क. & .21 & .23 & .30 & .60 & 41 & .25 & .13 & 290 & .42 & 3.30 & 31 & .48 \\
\hline $\mathrm{P}_{2} \mathrm{O}_{5}^{2}$ & .76 & .98 & .96 & 1.13 & 150 & 99 & 93 & 17.32 & 1.45 & .71 & .17 & 1.07 \\
\hline $150+$ & 9.3 & 84 & 9.3 & 9.5 & 8.0 & 8.4 & 3.3 & 5.4 & 8.2 & 64 & 8.6 & 9.0 \\
\hline Ho & 10.1 & 84 & 11.2 & 14.7 & 7.1 & 14.7 & 3.5 & 3.4 & 20.9 & 6.2 & 5.0 & 144 \\
\hline sum & 101.3 & 99.0 & 100.1 & $100 A$ & 97.9 & 97.0 & 99.2 & 93.9 & 97.5 & 98.2 & 99.1 & 99.3 \\
\hline Au(ppon) & 122 & 218 & 214 & 199 & 151 & 246 & 35 & 10 & 139 & 181 & S5 & 350 \\
\hline $\mathrm{Ba}$ & 1600 & 1200 & 1600 & 1500 & 470 & 1400 & 400 & 14000 & 10000 & 330 & 13000 & 1400 \\
\hline cd & 2.8 & 2.6 & 29 & 3.5 & 3.9 & 3.2 & 1.2 & 1.6 & 1.8 & .6 & 62.1 & 27 \\
\hline 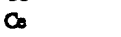 & 1400 & 1300 & 1100 & 1400 & 226 & 960 & 135 & 65 & 1200 & 224 & 60 & 1300 \\
\hline$a$ & . & 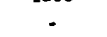 & 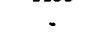 & 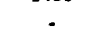 & 550 & 106 & 30 & 37 & 23 & 1100 & 43 & 8 \\
\hline Mo & 78 & 340 & 370 & 330 & 162 & 360 & 51 & 30 & 228 & $<4$ & 211 & 500 \\
\hline Pb & 1200 & 1900 & 1900 & 1900 & 570 & 1800 & 176 & 176 & 680 & 480 & 95 & 2200 \\
\hline Sr & 1000 & 1400 & 1500 & 1500 & 450 & 1200 & 820 & 1400 & 1400 & 590 & 1400 & 1800 \\
\hline$v$ & 460 & 600 & 610 & 520 & 510 & 590 & 238 & 134 & 440 & 550 & 232 & 710 \\
\hline $\mathbf{Y}$ & 156 & 218 & 214 & 223 & 108 & 141 & 52 & 340 & 290 & 50 & 24 & 234 \\
\hline $\mathbf{Z n}$ & 700 & 610 & 680 & 640 & 1400 & 680 & 340 & 560 & 560 & 410 & $17 \infty 0$ & 600 \\
\hline$\gamma$ & .974 & 996 & 985 & .982 & 1.007 & 2.017 & 994 & 1.050 & 1.011 & 1.004 & .995 & 993 \\
\hline Sumpled & Bulk & Top & Bulk & Bulk & Bulk & Bulk & Bulk & Bulk & Bulk & Bulk & Bulk & Bulk \\
\hline Intorval & - & - & - & - & - & - & $\therefore$ & . & 0.5 inm & - & $75-85 \mathrm{mmon}$ & $10-1$ Smm \\
\hline Soquenoull & 089041 & 089045 & 089046 & 089047 & 089048 & 089049 & 089050 & 089051 & 089052 & 089053 & 089054 & 089055 \\
\hline Latimas & 23.672 & 25.728 & 25.260 & 25.783 & 25.454 & 25.489 & 25.783 & 25.783 & 24322 & 25.489 & 25.489 & 25.454 \\
\hline Longitude & -164891 & -160.201 & -162.046 & -167.733 & -168.683 & -168.672 & -167.733 & -167.733 & -168.083 & -168.672 & -168.672 & -168.683 \\
\hline Depth & 1650 & 2600 & 2300 & 1600 & 1115 & 1200 & 1600 & 1600 & 1225 & 1200 & 1200 & 1115 \\
\hline Leb* & W-234354 & W-234329 & $W-234330$ & $W-228572$ & W-228580 & W-228581 & W-2288582 & W-228583 & W-228584 & W-228585 & W-2228586 & W-228587 \\
\hline $\mathrm{SiO}_{2}(\mathrm{~m} . \%)$ & 11.6 & 5.82 & 9.50 & 6.74 & 8.00 & 6.76 & 8.35 & 4.65 & 34.3 & 7.15 & 16.9 & 6.94 \\
\hline & 1.79 & 1.50 & 1.92 & 89 & 1.79 & 1.91 & 85 & 55 & 2.50 & 159 & 2.09 & 1.55 \\
\hline $\mathrm{MnO}_{2}$ & 33.8 & 42.2 & 36.8 & 62.6 & 40.5 & 43.0 & 62.1 & 68.4 & 203 & 40.4 & 5.62 & 39.5 \\
\hline & 28.2 & 25.8 & 27.2 & 6.58 & 21.8 & 223 & 5.29 & 3.78 & 29.2 & 26.6 & 14.3 & 25.1 \\
\hline $\mathrm{Al}_{2}^{2} \mathrm{O}_{3}^{3}$ & 1.94 & 96 & 1.80 & 3.03 & 5.97 & 238 & 3.60 & 240 & 10.3 & 1.99 & 8.89 & 2.96 \\
\hline $\mathrm{CO}_{3}^{2} \mathrm{O}_{4}$ & .975 & 1.27 & 1.12 & .204 & 1.69 & 2.18 & .141 & .124 & .096 & 1.70 & .185 & 1.68 \\
\hline $\mathrm{NiO}$ & 377 & 531 & .461 & .354 & .459 & 583 & $3 n$ & 417 & .078 & .433 & .186 & .485 \\
\hline CuO & .083 & .022 & .094 & .174 & .080 & .043 & .172 & .132 & .021 & .043 & .044 & .060 \\
\hline $\mathrm{CaO}$ & 3.14 & 3.70 & 3.27 & 1.80 & 3.00 & 3.37 & 1.77 & 1.63 & 3.29 & 3.34 & 23.0 & 3.45 \\
\hline $\mathrm{M}_{80}$ & 1.80 & 1.91 & 1.78 & 3.55 & 2.43 & 2.28 & 3.68 & 3.68 & 4.61 & 2.26 & 2.60 & 1.86 \\
\hline $\mathrm{N}_{2} \mathrm{O}$ & 226 & 240 & 243 & 2.14 & 2.25 & 2.63 & 2.22 & 2.11 & 2.03 & 2.50 & 1.46 & 246 \\
\hline $\mathrm{K}_{2} \mathrm{O}$ & .70 & 1.13 & .65 & 1.42 & .65 & .63 & 1.53 & 1.40 & 155 & 57 & .44 & 55 \\
\hline $\mathrm{C}_{2}$ & .12 & 32 & .31 & 30 & .42 & 33 & .24 & 22 & 1.01 & .39 & 240 & 43 \\
\hline $\mathrm{P}_{2} \mathrm{O}_{5}$ & 1.05 & 1.25 & .97 & .25 & 93 & 1.00 & .22 & .19 & .78 & 1.25 & 13.65 & 1.28 \\
\hline $\mathrm{H}_{2}^{2} \mathrm{O}+$ & 9.8 & 10.0 & 10.1 & 84 & 98 & 8.9 & 84 & 8.2 & 6.8 & 89 & 5.6 & 9.5 \\
\hline $\mathrm{H}_{2} \mathrm{O}$ & 19.0 & 20.9 & 20.0 & 6.5 & .11 .3 & 12.7 & 5.4 & 5.4 & 6.5 & 11.9 & 4.3 & 10.7 \\
\hline Sum & 97.6 & 98.8 & 98.4 & 98.5 & 99.7 & 98.3 & 98.9 & 98.0 & 98.7 & 99.1 & 97.4 & 97.9 \\
\hline$A \subseteq$ (ppon) & 330 & 400 & 300 & 86 & 300 & 330 & 61 & 51 & 193 & 390 & 52 & 390 \\
\hline Ba & 1400 & 1200 & 1400 & 12000 & 1800 & 1100 & 12000 & 12000 & 139 & 1100 & 320 & 1200 \\
\hline Cd & 2.2 & 3.7 & 3.1 & 43.9 & 4.6 & 4.6 & 51.8 & 60.3 & 3 & 3.3 & 1.3 & 3.6 \\
\hline 0 & 1200 & 1000 & 1200 & 225 & 940 & 1400 & 127 & 93 & 118 & 1200 & 136 & 1200 \\
\hline$a$ & 16 & 6 & 14 & 54 & 670 & 138 & 70 & so & 1100 & 81 & $3800^{\circ}$ & 224 \\
\hline Mo & 440 & 700 & 440 & 214 & 740 & 580 & 190 & 222 & $<4$ & 660 & 25 & 710 \\
\hline $\mathrm{Pb}$ & 2100 & 2400 & 2100 & 360 & 2100 & 2600 & 201 & 169 & 246 & 2600 & 290 & 2600 \\
\hline St & 1500 & 1800 & 1500 & 1400 & 1400 & 1500 & 1300 & 1400 & 280 & 1700 & 850 & 1700 \\
\hline $\mathbf{v}$ & 680 & 780 & 700 & 270 & 650 & 650 & 233 & 243 & 610 & 780 & 330 & 820 \\
\hline $\mathbf{Y}$ & 222 & 240 & 200 & 32 & 102 & 160 & 25 & 28 & so & 204 & 250 & 190 \\
\hline $\mathbf{z n}$ & 570 & 560 & 630 & 1400 & 540 & 550 & 1700 & 1700 & 400 & 490 & 320 & 540 \\
\hline$\gamma$ & 1.010 & .998 & 1.002 & 1.001 & .989 & 1.003 & 997 & 1.007 & 999 & 995 & 1.012 & 1.007 \\
\hline Sampled & Bulk & Bulk & Bulk & Bulk & Bulk & Bulk & Bulk & Bulk & Bulk & Balk & Bulk & Bulk \\
\hline Interval & $4-6 \mathrm{~mm}$ & . & $5-7 \mathrm{~mm}$ & $75-85 \mathrm{~mm}$ & $8-10 \mathrm{~mm}$ & $2.22 \mathrm{~mm}$ & 75-85.mm & $75-85 \mathrm{~mm}$ & $0-1 \mathrm{~mm}$ & $3-18 \mathrm{~mm}$ & $1.2 \mathrm{~mm}$ & $10-15 \mathrm{mom}$ \\
\hline
\end{tabular}


Table 1. Composition of crusts analyzed by USGS Reston Labs (cont'd).

\begin{tabular}{|c|c|c|c|c|c|c|c|c|c|c|c|c|}
\hline Sequencell & 089056 & 089058 & 089059 & 089061 & 089063 & 089067 & 089071 & 089077 & 089082 & 089083 & 089096 & 089097 \\
\hline Latitude & 24.322 & 25.489 & 25.489 & 23.915 & 25.926 & 24.322 & 25.685 & 25.260 & 25.980 & 25.980 & 25.303 & 23.979 \\
\hline Longitude & -168.083 & -168.672 & -168.672 & $-164,408$ & -167.710 & -168.083 & -160.180 & -162.046 & -164.379 & -164.379 & -162.094 & -164.363 \\
\hline Depth & 1225 & 1200 & 1200 & 800 & 3250 & 1225 & 3000 & 2300 & 1800 & 1800 & 3500 & 2200 \\
\hline Labu & W-228588 & W-228590 & W-228591 & W-234331 & W-234333 & W-234334 & W-234335 & W-234338 & W-234340 & W-234341 & W-234343 & W-234347 \\
\hline $\mathrm{SiO}_{2}(\mathrm{~m} . \%)$ & 11.9 & 9.53 & 17.9 & 14.4 & 24.5 & 30.9 & 2.14 & 8.59 & 11.7 & 8.08 & 14.7 & 222 \\
\hline $\mathrm{TiO}_{2}^{2}$ & 1.86 & 1.61 & 2.21 & 2.62 & 1.86 & 255 & 89 & 3.07 & 1.97 & 1.63 & 1.93 & 208 \\
\hline $\mathrm{MnO}_{2}$ & 32.1 & 38.1 & 25.9 & 30.4 & 22.2 & 10.7 & 39.9 & 38.4 & 30.6 & 35.8 & 29.9 & 24.0 \\
\hline $\mathrm{Fe}_{2} \mathrm{O}_{3}^{2}$ & 26.6 & 25.6 & 25.3 & 27.1 & 22.9 & 23.1 & 26.1 & 253 & 31.3 & 29.1 & 28.1 & 22.5 \\
\hline $\mathrm{Al}_{2}^{2} \mathrm{O}_{3}^{3}$ & 3.16 & 3.60 & 6.69 & 3.54 & 5.96 & 8.99 & 94 & 1.25 & 278 & 157 & 3.20 & 6.06 \\
\hline $\mathrm{Co}_{3}^{2} \mathrm{O}_{4}$ & 1.41 & 1.15 & 1.05 & .795 & .497 & .362 & 1.82 & 1.46 & .773 & .983 & .753 & 864 \\
\hline $\mathrm{NiO}^{4}$ & 354 & .642 & 366 & .403 & .269 & .183 & .526 & $A 84$ & 292 & 405 & .281 & 404 \\
\hline Cuo & .046 & .079 & .046 & .132 & .191 & .028 & .059 & .071 & .042 & .035 & .065 & .082 \\
\hline $\mathrm{CaO}$ & 3.43 & 3.14 & 279 & 3.35 & 5.10 & 4.95 & 5.79 & 3.88 & 3.36 & 3.31 & 3.18 & 286 \\
\hline MgO & 240 & 242 & 3.30 & 1.75 & 4.10 & 4.99 & 2.38 & 1.91 & 1.82 & 1.83 & 1.71 & 236 \\
\hline $\mathrm{Ne}_{2} \mathrm{O}$ & 256 & 2.24 & 2.21 & 247 & 2.47 & 242 & 2.20 & 1.77 & 2.31 & 2.30 & 2.24 & 3.13 \\
\hline $\mathbf{r}_{2} \mathbf{O}$ & .68 & .66 & .75 & 94 & .68 & 1.07 & so & .64 & .64 & .60 & .98 & 1.43 \\
\hline & AO & .47 & .38 & 27 & .15 & .69 & .72 & .43 & .24 & .33 & 32 & .16 \\
\hline $\mathrm{P}_{2} \mathrm{O}_{5}$ & 1.17 & 1.18 & 1.14 & .95 & .65 & .71 & 2.76 & 93 & 1.25 & 1.23 & 90 & .73 \\
\hline $\mathrm{H}_{2} \mathrm{O}+$ & 7.9 & 8.6 & 8.1 & 8.9 & 8.1 & 7.1 & 7.7 & 9.4 & 9.9 & 9.4 & 9.5 & 8.5 \\
\hline $\mathrm{H}_{2}^{2} \mathrm{O}$ & 102 & 10.8 & 9.6 & 17.8 & 15.0 & 9.6 & 17.8 & 18.5 & 17.2 & 18.3 & 18.6 & 14.9 \\
\hline Sum & 96.0 & 99.1 & 98.1 & 98.0 & 99.6 & 98.6 & 94.3 & 97.6 & 98.9 & 96.6 & 97.8 & 97.3 \\
\hline$A(p p m)$ & 360 & 360 & 300 & 249 & 188 & 188 & 460 & 280 & 370 & 400 & 280 & 217 \\
\hline Ba & 1100 & 1300 & 750 & 1800 & 1200 & 450 & 1600 & 2000 & 1600 & 1600 & 1200 & 1200 \\
\hline Cd & 29 & 5.2 & 28 & 24 & 15 & 1.1 & 49 & 3.2 & 2.1 & 3.2 & 20 & 2.6 \\
\hline$\infty$ & 1300 & 1100 & 950 & 1200 & 910 & 420 & 850 & 1700 & 1200 & 1200 & 1600 & 990 \\
\hline$a$ & 167 & 660 & 1300 & 22 & 247 & 750 & 29 & 37 & 41 & 23 & 11 & 103 \\
\hline Mo & 430 & 580 & 380 & 300 & 224 & 133 & 780 & 370 & 420 & 560 & 330 & 247 \\
\hline$P_{b}$ & 2500 & 2200 & 1800 & 1700 & 1400 & 910 & 3300 & 2100 & 2100 & 2300 & 2000 & 1500 \\
\hline St & 1400 & 1600 & 1100 & 1600 & 1000 & 650 & 1700 & 1600 & 1600 & 1700 & 1400 & 1100 \\
\hline $\mathbf{v}$ & 710 & 800 & 700 & 560 & 530 & 550 & 950 & 550 & 750 & 800 & 590 & 510 \\
\hline $\mathbf{Y}$ & 178 & 179 & 155 & 170 & 111 & 84 & 219 & 245 & 205 & 220 & 197 & 141 \\
\hline $\mathbf{Z n}$ & 490 & 650 & 500 & 680 & 530 & 370 & 670 & 610 & 580 & 610 & 540 & 590 \\
\hline$\gamma$ & 1.028 & .995 & 1.005 & 1.006 & .990 & 1.000 & 1.045 & 1.010 & 997 & 1.020 & 1.008 & 1.013 \\
\hline $\begin{array}{l}\text { Sampled } \\
\text { Imoerval }\end{array}$ & $\begin{array}{c}\text { Bulk } \\
1-10 \text { mm }\end{array}$ & $\underset{15-20 \mathrm{~mm}}{\text { Bulk }}$ & $\underset{1-5 \mathrm{~mm}}{\text { Bulk }}$ & $\begin{array}{c}\text { Bulk } \\
3-12 \mathrm{~mm}\end{array}$ & $\begin{array}{c}\text { Bulk } \\
\text { 4-8mm }\end{array}$ & $\begin{array}{c}\text { Bulk } \\
1.3 \mathrm{mmm}\end{array}$ & $\begin{array}{c}\text { Bulk } \\
1-2 \mathrm{mmm}\end{array}$ & $\underset{38-45 \mathrm{~mm}}{\text { Bulk }}$ & $\begin{array}{c}\text { Bulk } \\
\text { 30-35mm }\end{array}$ & $\begin{array}{c}\text { Bulk } \\
35-40 \mathrm{~mm}\end{array}$ & $\begin{array}{l}\text { Bulk } \\
\text { 5-8xmm }\end{array}$ & $\begin{array}{c}\text { Bulk } \\
2-5 \mathrm{~mm}\end{array}$ \\
\hline Sequencell & 089099 & 090001 & 090002 & 090003 & 090011 & 090011 & 090011 & 090012 & 090012 & 090012 & 090013 & 090014 \\
\hline Latitude & 25.135 & 27.868 & 29.835 & 27.917 & 20.133 & 20.133 & 20.133 & 20.133 & 20.133 & 20.133 & 20.222 & 20.222 \\
\hline Longitude & -162.010 & -177.883 & -177.883 & -171.062 & -170.681 & -170.681 & -170.681 & -170.681 & -170.681 & -170.681 & -170.600 & -170.600 \\
\hline Depth & 2175 & 3443 & 3443 & 847 & 1476 & 1476 & 1476 & 1476 & 1476 & 1476 & - & - \\
\hline Lab* & W-234348 & W-2225572 & W-222573 & W-224384 & W-2228984 & W-228986 & W-2289987 & W-228953 & W-228985 & W-228993 & W-2228989 & W-228957 \\
\hline $\mathrm{SiO}_{2}(\mathrm{w}, \%)$ & 8.93 & 12.7 & 7.78 & 15.8 & 5.93 & 2.67 & 4.27 & 8.08 & 8.19 & 2.44 & 10.2 & 9.84 \\
\hline $\mathrm{TiO}_{2}$ & 1.83 & 1.62 & 1.37 & 1.71 & 1.69 & 1.20 & 208 & 1.73 & 2.24 & 1.40 & 206 & 2.18 \\
\hline $\mathrm{MnO}_{2}$ & 39.8 & 33.7 & 44.3 & 26.7 & 42.1 & 37.8 & 46.8 & 38.9 & 40.0 & 40.6 & 36.3 & 35.5 \\
\hline $\mathrm{Fo}_{2} \mathrm{O}_{3}$ & 24.5 & 27.9 & 20.4 & 16.3 & 26.9 & 125 & 22.0 & 26.4 & 25.9 & 13.0 & 27.4 & 27.8 \\
\hline $\mathrm{Al}_{2} \mathrm{O}_{3}$ & 1.90 & 209 & 1.82 & 6.06 & .75 & .66 & .61 & 1.16 & 1.52 & 57 & 1.87 & 1.42 \\
\hline $\mathrm{CO}_{3} \mathrm{O}_{4}$ & 1.13 & .679 & 1.79 & .727 & 1.52 & 581 & 1.45 & 1.35 & 1.29 & 809 & .982 & .914 \\
\hline $\mathrm{NiO}{ }^{4}$ & 592 & 360 & .612 & .571 & .577 & 986 & 838 & .485 & .610 & .913 & 592 & .442 \\
\hline $\mathrm{CuO}$ & .072 & .118 & .050 & .084 & .033 & 103 & .084 & .033 & .081 & .168 & .247 & .070 \\
\hline $\mathrm{CaO}$ & 3.57 & 3.58 & 4.55 & 10.8 & 3.63 & 17.3 & 3.73 & 3.44 & 3.52 & 15.1 & 3.61 & 3.35 \\
\hline $\mathrm{M}_{8} \mathrm{O}$ & 1.88 & 2.23 & 3.05 & 283 & 1.87 & 1.75 & 1.97 & 1.84 & 1.84 & 1.77 & 1.85 & 1.71 \\
\hline $\mathrm{Ne}_{2} \mathrm{O}$ & 2.43 & 3.64 & 3.39 & 2.35 & 250 & 221 & 2.54 & 2.48 & 251 & 2.19 & 2.26 & 230 \\
\hline $\mathbf{r}_{2}^{2} \mathrm{O}$ & .73 & 57 & .65 & 1.24 & 56 & .61 & .65 & .60 & .70 & .63 & .63 & .63 \\
\hline$\omega_{2}$ & - & .09 & .44 & 1.07 & .49 & 202 & A7 & .40 & .47 & 1.68 & 59 & 54 \\
\hline $\mathrm{P}_{2} \mathrm{O}_{5}$ & 1.01 & 1.02 & .97 & 5.88 & 1.23 & 9.26 & .93 & 1.19 & 1.02 & 7.97 & 1.17 & .98 \\
\hline $\mathrm{H}_{2} \mathrm{O}+$ & 10.4 & 5.9 & 5.7 & 7.5 & 8.6 & 7.1 & 9.1 & 8.3 & 8.5 & 6.8 & 9.7 & 9.7 \\
\hline $\mathrm{H}_{2}^{2} \mathrm{O}$ & 20.5 & 25.8 & 29.3 & 6.4 & 14.0 & 10.9 & 13.4 & 13.4 & 145 & 10.8 & 14.0 & 13.6 \\
\hline Sum & - & 96.2 & 96.9 & 99.6 & 98.4 & 96.7 & 97.5 & 96.5 & 98.4 & 96.1 & 99.4 & 97.4 \\
\hline$A \simeq$ (ppm) & 290 & 220 & 310 & 139 & 135 & 208 & 300 & 123 & 222 & 280 & 244 & 243 \\
\hline $\mathrm{Ba}$ & 1300 & 1200 & 1000 & 1800 & 1800 & 1700 & 1300 & 2200 & 1600 & 1300 & 1500 & 1600 \\
\hline Cd & 3.5 & 2.3 & 65 & 5.1 & 4.3 & 4.5 & 3.5 & 4.3 & 3.6 & 3.3 & 3.1 & 3.1 \\
\hline 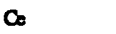 & 930 & 1600 & 1400 & 740 & 1100 & 1300 & 1100 & 1700 & 1300 & 1100 & 1100 & 1100 \\
\hline$a$ & 21 & 20 & 177 & 290 & 6 & 8 & 13 & 6 & 20 & 16 & 15 & 13 \\
\hline Mo & 520 & 460 & 790 & 410 & 590 & 720 & 700 & 640 & 500 & 540 & 400 & 440 \\
\hline $\mathrm{Pb}_{\mathbf{b}}$ & 2100 & 2000 & 2800 & 1400 & 1300 & 2100 & 2300 & 1600 & 2100 & 2300 & 2000 & 2000 \\
\hline St & 1500 & 1500 & 1400 & 1400 & $17 \infty 0$ & 1700 & 1900 & 1800 & 1600 & 1700 & 1600 & 1600 \\
\hline $\mathbf{v}$ & 680 & 610 & 650 & 460 & 490 & 730 & 810 & 580 & 690 & 720 & 670 & 680 \\
\hline $\mathbf{Y}$ & 201 & 202 & 141 & 350 & 310 & 162 & 233 & 430 & 175 & 208 & 151 & 232 \\
\hline $\mathbf{Z n}$ & 650 & 500 & 510 & 740 & 910 & 820 & 630 & 1000 & 660 & 590 & 800 & 640 \\
\hline $\bar{\gamma}$ & - & 1.025 & 1.018 & .990 & 1.002 & 1.019 & 1.011 & 1.022 & 1.002 & 1.027 & .992 & 1.012 \\
\hline Sampled & nochule & Bulk & Bulk & Bulk & Young & Old & Middle & Young & Middle & ad & - & - \\
\hline Interval & - & $0.6 \mathrm{~mm}$ & Q-5mm & $0.9 \mathrm{~mm}$ & - & - & - & - & . & - & - & - \\
\hline
\end{tabular}


Table 1. Composition of crusts analyzed by USGS Reston Labs (cont'd).

\begin{tabular}{|c|c|c|c|c|c|c|c|c|c|c|c|c|}
\hline Sequencell & 0,0015 & 090016 & 090016 & 090016 & 090018 & 090019 & 090020 & 090021 & 090022 & 090023 & 090024 & 090025 \\
\hline Letitude & 20.218 & 20.218 & 20.218 & 20.218 & 28.895 & 28.895 & 29.106 & 29.167 & 29.106 & 29.167 & 29.208 & 28.895 \\
\hline Longitndo & -170.589 & -170.589 & -170589 & -170589 & -178.868 & -178.868 & -173.948 & -174.067 & -173.948 & -174.067 & -174.112 & -178.868 \\
\hline Depth & 1480 & 1480 & 1480 & 1480 & 1280 & 1280 & 1850 & 1425 & 1850 & 1425 & 2000 & 1280 \\
\hline Lab: & W-228981 & W-223960 & W-228982 & W-2228988 & W-228561 & W-2285562 & W-228563 & W-228564 & W-228556 & W-228566 & W-2285567 & W-228556 \\
\hline $\mathrm{SiO}_{2}(\mathrm{~m} . \%)$ & 13.1 & 7.37 & 2.17 & 8.57 & 19.6 & 25.4 & 8.12 & 5.22 & 691 & 6.19 & 11.6 & 7.05 \\
\hline & 209 & 2.40 & 1.27 & 1.76 & 1.33 & 1.59 & 1.7 & 1.50 & 1.58 & 2.25 & 208 & 1.21 \\
\hline $\mathrm{MmO}_{2}$ & 33.7 & 41.6 & 419 & 39.4 & 38.9 & 30.4 & 40.9 & 45.8 & 43.9 & 42.1 & 37.4 & 448 \\
\hline & 26.8 & 22.7 & 11.7 & 27.5 & 7.67 & 8.99 & 24.7 & 243 & 24.9 & 23.3 & 243 & 19.5 \\
\hline & 249 & 1.57 & .66 & 1.18 & 7.15 & 9.43 & 1.32 & 1.03 & 1.04 & 1.64 & 236 & 1.74 \\
\hline $\mathrm{CO}_{3}^{2} \mathrm{O}$ & 961 & 1.16 & 987 & 14 & .874 & .035 & 1.08 & 1.18 & 1.31 & 951 & 1.14 & 1.55 \\
\hline Nió & .442 & .700 & 908 & 523 & .575 & 200 & 524 & 574 & .566 & .774 & .506 & .680 \\
\hline CuO & .067 & .120 & .170 & .044 & .058 & .037 & .082 & .049 & .057 & .094 & .171 & .047 \\
\hline $\mathrm{ClO}$ & 3.89 & 3.60 & 15.5 & 3.51 & 3.83 & 2.28 & 3.52 & 3.82 & 3.60 & 4.25 & 3.49 & 4.14 \\
\hline $\mathrm{MgO}_{8}$ & 1.69 & 1.96 & 1.86 & 1.83 & 4.53 & 288 & 1.78 & 191 & 190 & 2.05 & 1.88 & 3.02 \\
\hline $\mathrm{Ne}_{2} \mathrm{O}$ & 243 & 2.50 & 2.20 & 239 & 3.15 & 4.23 & 2.40 & 2.49 & 246 & 236 & 250 & 2.73 \\
\hline & .70 & .75 & .68 & 59 & 1.7 & 3.14 & .66 & .63 & .63 & .73 & 94 & .60 \\
\hline$\infty$ & 51 & .44 & 1.70 & 49 & .89 & .64 & $A 2$ & .42 & 43 & .42 & 39 & 33 \\
\hline $\mathrm{P}_{2} \mathrm{O}$ & 1.38 & .94 & 8.05 & 1.21 & 55 & .40 & 1.15 & 1.20 & 1.15 & 1.55 & 1.01 & 1.04 \\
\hline $\mathrm{H}_{2}^{2} \mathrm{O}+$ & 8.1 & 8.7 & 6.8 & 79 & 78 & 7.2 & 8.6 & 7.5 & 8.4 & 9.1 & 8.7 & 6.8 \\
\hline $\mathrm{H}_{2} \mathrm{O}$ & 13.6 & 14.5 & 11.7 & 14.8 & 49 & 7.8 & 12.6 & 15.7 & 14.6 & 11.2 & 11.9 & 12.1 \\
\hline Sum & 983 & 96.6 & 96.6 & 983 & 98.7 & 975 & 97.1 & 97.5 & 98.9 & 97.8 & 98.5 & 953 \\
\hline$A \propto(p p a n)$ & 232 & 113 & 234 & 270 & 51 & 48 & 320 & 390 & 370 & 290 & 250 & 360 \\
\hline $\mathrm{Ba}$ & 1600 & 2400 & 1800 & 1300 & 630 & 560 & 1600 & 1500 & 1300 & 2100 & 2600 & 1000 \\
\hline $\mathrm{Cd}$ & 2.9 & 5.0 & $4 A$ & 29 & 27.3 & 163 & 3.5 & 4.4 & 4.2 & 5.7 & 3.5 & 5.6 \\
\hline C. & 1000 & 1700 & 1300 & 1100 & 44 & 43 & 1600 & 1700 & 1400 & 2000 & 1800 & 1300 \\
\hline$c$ & 21 & 14 & 15 & 15 & 350 & 163 & 11 & 5 & 12 & 34 & 23 & 87 \\
\hline Mo & 430 & 740 & 510 & 580 & 260 & 217 & 630 & 900 & 700 & 710 & 540 & 940 \\
\hline $\mathbf{P b}$ & 2000 & 1700 & 1900 & 2200 & 84 & 87 & 2400 & 2700 & 2600 & 2500 & 2000 & 2500 \\
\hline st & 1600 & 1700 & 1600 & 1800 & 380 & 280 & 1700 & 1900 & 1600 & 1700 & 1600 & 1500 \\
\hline $\mathbf{V}$ & 670 & 570 & 620 & 760 & 320 & 228 & 760 & 830 & 770 & $\pi 0$ & 640 & 740 \\
\hline $\mathbf{Y}$ & 197 & 570 & 164 & 211 & 35 & 22 & 183 & 178 & 199 & 203 & 182 & 159 \\
\hline $\mathbf{Z n}$ & 650 & 1100 & 780 & 620 & 1600 & 970 & 630 & 580 & 600 & 820 & 620 & 520 \\
\hline$\gamma$ & 1.003 & 1.021 & 1.021 & 1.003 & .999 & 1.012 & 1.016 & 1.011 & .997 & 1.008 & 1.001 & 1.034 \\
\hline Semplod & Bulk & Middle & Old & Youns & Bulk & Bulk & Bulk & Bulk & Bulk & Bulk & Bulk & Bulk \\
\hline Imorvil & - & - & - & - & $3-5 m m$ & $1-3 \mathrm{~mm}$ & $10-20 \mathrm{~mm}$ & $14-40 \mathrm{cmm}$ & $25-33 \mathrm{cmm}$ & $15-20 \mathrm{~mm}$ & $15-20 \mathrm{~mm}$ & $1-2 m m$ \\
\hline Sequencoll & 090026 & 090027 & 090028 & 090029 & 090030 & 090031 & 090032 & 090033 & 090034 & 090040 & 090046 & 090047 \\
\hline Latitude & 28.895 & 29.167 & 29.167 & 29.167 & 29.185 & 29.208 & 29.167 & 29.062 & 29.208 & 29.106 & 29.106 & 29.106 \\
\hline Longitude & -178.868 & -174.067 & -174.067 & -174.067 & -174.010 & -174.112 & -174.067 & -174.022 & -174.112 & -173.948 & -173.948 & -173.948 \\
\hline Depth & 1280 & 1425 & 1425 & 1425 & 985 & 2200 & 1425 & 1150 & 2200 & 1850 & 1850 & 1850 \\
\hline Lab* & W-228559 & W-2223568 & W-2285570 & W-2285571 & W-228574 & W-2228575 & W-228576 & W-2228578 & w-228577 & W-234332 & W-234345 & W-234346 \\
\hline $\mathrm{SiO}_{2}(w t . \%)$ & 536 & 12.1 & 6.09 & 6.11 & 3.45 & 11.8 & 5.15 & 4.78 & 18.7 & 17.2 & 6.63 & 9.56 \\
\hline $\mathrm{TiO}_{2}$ & 1.26 & 2.10 & 1.40 & 1.56 & 1.76 & 2.09 & 1.82 & 1.67 & 1.77 & 2.05 & 1.37 & 1.20 \\
\hline $\mathrm{MnO}_{2}$ & 46.1 & 33.5 & 45.8 & 45.2 & 44.2 & 36.9 & 44.0 & 45.5 & 31.5 & 23.8 & 41.8 & 43.6 \\
\hline $\mathrm{Pa}_{2} \mathrm{O}_{3}^{2}$ & 20.5 & 28.8 & 227 & 23.9 & 24.0 & 25.0 & 24.4 & 23.7 & 23.4 & 30.2 & 23.0 & 16.0 \\
\hline $\mathrm{N}_{2}^{2} \mathrm{O}_{3}^{3}$ & 1.19 & 2.38 & 1.22 & 1.04 & 1.04 & 2.16 & 89 & 1.07 & 4.02 & 3.42 & 1.09 & 2.94 \\
\hline $\mathrm{CO}_{3}^{2} \mathrm{O}_{4}^{3}$ & 1.74 & 1.09 & 1.22 & 1.36 & 1.57 & 1.06 & 1.23 & 1.08 & .731 & 1.20 & 1.32 & .863 \\
\hline $\mathrm{NiO}^{4}$ & .667 & 378 & .702 & .601 & .556 & 508 & 583 & 521 & .484 & .228 & 547 & 1.407 \\
\hline Co & .061 & .090 & .065 & .045 & .068 & .143 & .046 & .051 & .168 & .037 & .045 & .159 \\
\hline $\mathrm{CaO}$ & 3.58 & 3.20 & 3.70 & 3.71 & 4.34 & 3.35 & 4.49 & 3.82 & 3.60 & 225 & 3.92 & 3.72 \\
\hline MgO & 251 & 1.89 & 2.10 & 1.91 & 2.10 & 1.82 & 1.90 & 1.89 & 1.85 & 2.09 & 1.87 & 2.87 \\
\hline $\mathrm{N}_{2} \mathrm{O}$ & 279 & 2.40 & 2.41 & 2.30 & 2.41 & 240 & 2.52 & 262 & 257 & 247 & 294 & 282 \\
\hline $\mathbf{k}_{2} \mathbf{O}$ & .73 & .69 & .66 & .64 & .61 & 87 & .61 & .71 & 1.09 & 93 & .74 & 1.10 \\
\hline$\infty_{2}$ & .47 & 40 & .38 & 35 & .47 & $A 1$ & 49 & 38 & 39 & - & .29 & 51 \\
\hline $\mathrm{P}_{2} \mathrm{O}_{5}$ & 1.09 & 1.47 & 1.16 & 1.19 & 1.66 & 92 & .17 & 1.23 & 1.08 & 1.23 & 1.41 & 1.06 \\
\hline $\mathrm{H}_{2}^{2} \mathrm{O}+$ & 8.4 & 8.5 & 9.5 & 93 & 9.2 & 9.0 & 9.4 & 9.0 & 8.4 & 9.7 & 9.6 & 9.8 \\
\hline $\mathrm{H}_{2}^{2} \mathrm{O}$ & 14.1 & 125 & 13.0 & 13.2 & 13.0 & 12.4 & 12.7 & 12.1 & 10.6 & 16.1 & 18.6 & 13.2 \\
\hline Sum & 96.4 & 98.9 & 99.2 & 99.2 & 97.4 & 98.3 & 97.7 & 98.1 & 99.7 & • & 96.6 & 97.6 \\
\hline$A \times(p p m)$ & 380 & 320 & 360 & 380 & 390 & 250 & 370 & 400 & 213 & 330 & 380 & 200 \\
\hline $\mathrm{Ba}$ & 1100 & 3000 & 1600 & 1400 & 2000 & 2200 & 1500 & 2200 & 2500 & 1900 & 2100 & 2400 \\
\hline Cd & 5.5 & 3.2 & 53 & 4.1 & 4.5 & 3.8 & 3.9 & 3.6 & 3.4 & 1.8 & 3.5 & 10.5 \\
\hline$\infty$ & 1500 & 2100 & 1600 & 1600 & 1600 & 1700 & 1700 & 2000 & 1300 & 1700 & 1200 & 1000 \\
\hline$a$ & 30 & 16 & 9 & 7 & 13 & 23 & 13 & 14 & 20 & 20 & 8 & 31 \\
\hline Mo & 870 & 420 & 890 & 790 & 850 & 470 & 780 & 600 & 360 & 155 & 820 & 650 \\
\hline $\mathbf{P b}$ & 2700 & 2700 & 2600 & 2700 & 3000 & 1900 & 2500 & 2700 & 1700 & 3000 & 2600 & 1600 \\
\hline Sr & 1500 & 1900 & 1700 & 1700 & 1800 & 1500 & 1700 & 1900 & 1300 & 1500 & 1600 & 1100 \\
\hline $\mathbf{v}$ & 700 & 720 & 790 & 760 & 870 & 620 & 780 & 880 & 560 & 580 & 740 & 540 \\
\hline $\mathbf{Y}$ & 163 & 206 & 172 & 184 & 149 & 171 & 206 & 137 & 157 & 143 & 197 & 111 \\
\hline $\mathbf{Z n}$ & 540 & 620 & 630 & 560 & 640 & 640 & 610 & 590 & 640 & 490 & 520 & 1100 \\
\hline$\gamma$ & 1.023 & .997 & .994 & .994 & 1.012 & 1.003 & 1.009 & 1.005 & .989 & • & 1.021 & 1.010 \\
\hline Stampled & Bulk & Bulk & Bulk & Bulk & Bulk & Bulk & Bulk & Bulk & Bulk & Bulk & Bulk & Bulk \\
\hline Interval & 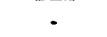 & $10-25 \mathrm{~mm}$ & $25-35 \mathrm{~mm}$ & $15-20 \mathrm{~mm}$ & - & $15-25 \mathrm{~mm}$ & $4-12 \mathrm{~mm}$ & $90-110 \mathrm{~mm}$ & $20-25 \mathrm{~mm}$ & 4-10mm & 3-5mm & $48 \mathrm{~mm}$ \\
\hline
\end{tabular}


Table 1. Composition of crusts analyzed by USGS Reston Labs (cont'd).

\begin{tabular}{|c|c|c|c|c|c|c|c|c|c|c|c|c|}
\hline Sequencoul & 090048 & 091001 & 091002 & 091003 & 091004 & 091005 & 091006 & 091007 & 091008 & 091009 & 091010 & 091011 \\
\hline Latiude & 28.881 & 20.752 & 20.750 & 20.767 & 29.813 & 29.857 & 29.643 & 29.857 & 29.839 & 29.839 & 29.634 & 29.641 \\
\hline Langitude & -179.745 & 173.440 & 173.667 & 173.347 & 179.043 & 179.083 & 179.282 & 179.083 & 179.065 & 179.065 & 179.288 & 179.282 \\
\hline Depth & 2000 & 1834 & 3816 & 1337 & 989 & 2250 & 1300 & 2250 & 2100 & 2100 & 1865 & 1300 \\
\hline Lab: & W.234349 & W-222591 & W-220592 & W-222593 & W-2222574 & W-228555 & W.228560 & W-228589 & W-228553 & W-228554 & W-228555 & W-2228569 \\
\hline $\mathrm{SiO}_{2}$ (wt.\%) & 7.75 & 6.01 & 5.68 & 6.91 & 3.12 & 10.7 & 3.30 & 13.8 & 21.2 & 9.06 & 6.29 & 786 \\
\hline & 1.46 & 1.62 & 1.59 & 1.82 & 1.31 & 1.95 & 1.18 & 1.66 & 2.05 & 1.03 & 1.32 & 1.74 \\
\hline $\mathrm{MnO}_{2}$ & 42.0 & 44.1 & 47.3 & 43.3 & 48.1 & 37.5 & 50.4 & 325 & 23.0 & 39.4 & 41.5 & 37.1 \\
\hline & 21.8 & 24.2 & 22.6 & 24.7 & 16.5 & 23.9 & 20.9 & 27.8 & 25.0 & 24.3 & 27.3 & 29.0 \\
\hline & 1.60 & 1.14 & 1.15 & 1.20 & $n$ & 1.83 & .62 & 274 & 5.08 & 2.17 & $n$ & 1.15 \\
\hline $\mathrm{Co}_{3} \mathrm{O}_{4}$ & 1.09 & 1.30 & 1.24 & 1.88 & 203 & 953 & 1.83 & .866 & .763 & 1.30 & 1.05 & 1.83 \\
\hline $\mathrm{NIO}^{4}$ & .704 & .678 & .899 & .598 & .795 & 505 & .792 & 398 & $\$ 70$ & 511 & .489 & 320 \\
\hline CuO & .091 & .109 & .127 & .033 & .037 & .137 & .055 & .070 & .088 & .055 & .036 & .039 \\
\hline Cno & 3.41 & 4.21 & 4.65 & 4.64 & 3.61 & 3.43 & 3.59 & 297 & 4.07 & 3.37 & 3.59 & 3.30 \\
\hline M8O & 3.24 & 2.11 & 2.20 & 192 & 2.21 & 1.82 & 223 & 2.22 & 3.53 & 1.90 & 1.82 & 1.86 \\
\hline $\mathrm{Ne}_{2} \mathrm{O}$ & 240 & 3.28 & 2.49 & 2.62 & 2.85 & 257 & 293 & 246 & 2.69 & 2.64 & 2.45 & 247 \\
\hline $\mathrm{K}_{2}^{c} \mathrm{O}$ & .67 & .67 & .60 & .60 & 51 & 80 & .68 & .85 & 1.09 & .87 & 58 & .61 \\
\hline$\infty_{2}$ & .25 & .55 & .57 & 86 & 42 & 42 & 49 & $A 1$ & .25 & .34 & 50 & 36 \\
\hline $\mathrm{P}_{2} \mathrm{O}_{5}$ & 1.04 & 1.38 & 1.49 & 1.71 & 1.00 & 1.07 & 1.20 & 1.13 & 1.03 & 1.31 & 1.26 & 1.41 \\
\hline $\mathrm{H}_{2} \mathrm{O}+$ & 9.5 & 6.6 & 5.7 & 4.7 & 10.5 & 8.4 & 95 & 8.8 & 7.1 & 8.4 & 9.0 & 9.0 \\
\hline $\mathrm{H}_{2} \mathrm{O}$ & 18.7 & 26.8 & 27.8 & 27.6 & 26.4 & 143 & 18.1 & 10.4 & 10.7 & 12.8 & 14.2 & 10.9 \\
\hline Sum & 97.0 & 97.9 & 98.3 & 97.4 & 93.8 & 96.0 & 99.8 & 98.7 & 97.3 & 972 & 97.9 & 98.1 \\
\hline$A \times$ (ppen) & 320 & 270 & 249 & 280 & 231 & 280 & 420 & 310 & 213 & 340 & 410 & 420 \\
\hline $\mathrm{Be}$ & 1600 & 1400 & 1500 & 1100 & 1100 & 2600 & 1200 & 1500 & 2200 & 1100 & 1300 & 2000 \\
\hline Cd & 7.0 & 4.8 & 5.1 & 4.0 & 5.4 & 4.3 & 7.0 & 3.2 & 3.8 & 3.8 & 35 & 3.4 \\
\hline C. & 1100 & 890 & 1100 & 1300 & 1500 & 2000 & 1600 & 1500 & 1300 & 1600 & 1400 & 1800 \\
\hline$a$ & 37 & 14 & 15 & 11 & 14 & 19 & 5 & 28 & 168 & 24 & 5 & 10 \\
\hline Mo & 760 & 740 & 710 & 540 & 800 & 530 & 1100 & 460 & 157 & 680 & 730 & 580 \\
\hline $\mathrm{Pb}$ & 2100 & 2300 & 2400 & 2600 & 2900 & 2000 & 3100 & 2500 & 2200 & 2500 & 2800 & 2700 \\
\hline St & 1600 & 1600 & 1500 & 1500 & 1300 & 1800 & 1700 & 1600 & 1200 & 2400 & 1700 & 2000 \\
\hline $\mathbf{v}$ & 740 & 750 & 690 & 680 & 570 & 680 & 810 & 660 & 500 & 720 & 850 & 740 \\
\hline $\mathbf{Y}$ & 123 & 164 & 249 & 221 & 129 & 175 & 171 & 16 & 134 & 195 & 233 & 191 \\
\hline $\mathbf{Z n}$ & 840 & 680 & 800 & 620 & 580 & 680 & 620 & 590 & 670 & 500 & 560 & 490 \\
\hline$\gamma$ & 1.016 & 1.007 & 1.003 & 1.012 & 1.051 & 1.028 & 988 & 999 & 1.013 & 1.015 & 1.007 & 1.005 \\
\hline Sumpled & Balk & Bulk & Bulk & Bulk & Bulk & Balk & Bulk & Bulk & Bulk & Bulk & Bulk & Bulk \\
\hline Intarval & $6-8 \mathrm{~mm}$ & $0.3 \mathrm{~mm}$ & 0.5mm & $0.5 \mathrm{~mm}$ & $0.7 \mathrm{~mm}$ & - & $2-12 \mathrm{~mm}$ & $1.6 \mathrm{~mm}$ & $2-6 \mathrm{~mm}$ & $5-15 \mathrm{~mm}$ & $7-8 \mathrm{~mm}$ & - \\
\hline Sequencoll & 091012 & 091013 & 091021 & 092001 & 092002 & 092003 & 093001 & 093002 & 093003 & 093004 & 093005 & 093006 \\
\hline Latitude & 29.869 & 29.820 & 29.820 & 21.145 & 21.160 & 21.678 & 21.483 & 21.480 & 23.703 & 24.017 & 29.490 & 29.522 \\
\hline Langitude & 179.098 & 179.103 & 179.104 & 163.397 & 163.147 & 161.870 & 159.538 & 159.248 & 159.547 & 159.450 & 153.348 & 153.407 \\
\hline Depth & 2900 & 1700 & 1700 & 177 & 1468 & 1715 & 1273 & 1602 & 2812 & 4079 & 1991 & 1825 \\
\hline Lab" & W-228592 & W-228558 & W-234344 & W.222594 & W-222595 & W-222596 & W-222597 & W-222598 & W-222599 & W- 222600 & W-222601 & W-222602 \\
\hline $\mathrm{SiO}_{2}$ (w.\%) & 11.1 & 12.9 & 29.3 & 21.5 & 5.12 & 8.64 & 17.0 & 12.2 & 20.0 & 19.2 & 7.95 & 142 \\
\hline $\mathrm{TiO}_{2}$ & 2.26 & 209 & 3.41 & 1.78 & 1.61 & 1.53 & 1.65 & 1.53 & 1.80 & 1.46 & 1.42 & 95 \\
\hline $\mathrm{MmO}_{2}$ & 372 & 345 & 142 & 27.5 & 47.3 & 41.0 & 30.1 & 37.7 & 28.4 & 33.4 & 422 & 39.7 \\
\hline $\mathrm{Pe}_{2} \mathrm{O}_{3}$ & 25.7 & 233 & 19.7 & 23.4 & 24.8 & 24.9 & 25.0 & 282 & 27.1 & 25.4 & 27.3 & 21.5 \\
\hline $\mathrm{AL}_{2} \mathrm{O}_{3}$ & 2.11 & 280 & 8.03 & 6.57 & .81 & 1.63 & 3.40 & 1.82 & 3.69 & 3.70 & 1.19 & 3.50 \\
\hline $\mathrm{CO}_{3} \mathrm{O}_{4}$ & 1.25 & 994 & 381 & .877 & 1.45 & 1.19 & .753 & .97 & .602 & .728 & .684 & 521 \\
\hline $\mathrm{NiO}{ }^{4}$ & 546 & 597 & 228 & .361 & .670 & .601 & 393 & 430 & 348 & 398 & .568 & .990 \\
\hline CuO & .090 & .041 & .021 & .037 & .055 & .068 & .077 & .047 & .150 & .075 & .052 & .155 \\
\hline $\mathrm{CaO}$ & 3.63 & 3.99 & 5.79 & 3.79 & 4.18 & 4.91 & 5.93 & 3.79 & 3.28 & 3.10 & 3.71 & 242 \\
\hline $\mathrm{M}: \mathrm{O}$ & 1.87 & 227 & 4.62 & 201 & 205 & 224 & 1.75 & 1.84 & 1.83 & 1.90 & 1.87 & 287 \\
\hline $\mathrm{Ne}_{2} \mathrm{O}$ & 248 & 262 & 3.20 & 2.45 & 256 & 297 & 2.70 & 271 & 2.86 & 2.61 & 251 & 222 \\
\hline $\mathbf{k}_{2}^{2} \mathbf{O}$ & .82 & 1.07 & 2.05 & 1.13 & .55 & 39 & 1.05 & .60 & .70 & .82 & 59 & .94 \\
\hline$\infty_{2}$ & 51 & .58 & .03 & .43 & .57 & .65 & 1.17 & .62 & .43 & .43 & .61 & .46 \\
\hline $\mathrm{P}_{2} \mathrm{O}_{5}$ & .96 & 1.12 & .85 & 1.36 & 1.34 & 204 & 245 & 1.36 & 1.07 & .96 & 1.21 & .82 \\
\hline $\mathrm{H}_{2} \mathrm{O}+$ & 8.6 & 92 & 6.5 & 5.9 & 6.0 & 5.0 & 4.4 & 3.8 & 4.9 & 42 & 4.7 & 85 \\
\hline $\mathrm{H}_{2}^{2} \mathrm{O}$ & 11.4 & 12.3 & 10.6 & 22.4 & 29.7 & 25.9 & 2.2 & 26.1 & 23.1 & 23.3 & 28.3 & 19.0 \\
\hline Sum & 99.0 & 98.0 & 98.2 & 99.2 & 99.0 & 97.7 & 97.8 & 97.6 & 97.3 & 98.3 & 96.6 & 99.7 \\
\hline Axppm) & 248 & 320 & 145 & 206 & 310 & 280 & 206 & 310 & 195 & 248 & 320 & 198 \\
\hline Ba & 2000 & 970 & 540 & 910 & 1300 & 1200 & 1200 & 1200 & 1400 & 1100 & 1400 & 2300 \\
\hline Cd & 3.5 & 4.3 & 1.6 & 3.4 & 3.8 & 3.8 & 24 & 3.0 & 3.8 & 3.0 & 3.6 & 4.2 \\
\hline Co & 2100 & 1300 & 550 & 820 & . 970 & 970 & 1100 & 1000 & 1200 & 1300 & 1700 & 1100 \\
\hline 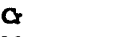 & 31 & 81 & 340 & 55 & 6 & 19 & 30 & 15 & 33 & 35 & 11 & 17 \\
\hline Mo & 380 & 580 & 190 & 370 & 770 & 670 & 310 & 570 & 300 & 460 & 820 & 210 \\
\hline $\mathrm{Pb}$ & 2300 & 2200 & 930 & 1700 & 2500 & 2400 & 2000 & 2400 & 1900 & 1600 & 2700 & 1600 \\
\hline St & 1500 & 1400 & 700 & 1500 & 1700 & 1600 & 1300 & 1600 & 1200 & 1300 & 1700 & 1200 \\
\hline V & 590 & 700 & 450 & 520 & 730 & 700 & 570 & 700 & 520 & 630 & 740 & 540 \\
\hline $\mathbf{Y}$ & 214 & 171 & 91 & 142 & 213 & 216 & 193 & 162 & 176 & 170 & 195 & 99 \\
\hline $\mathrm{Zn}$ & 650 & 550 & 380 & 460 & 630 & 650 & 690 & 530 & 550 & 460 & 590 & 860 \\
\hline$\gamma$ & 996 & 1.015 & 1.004 & .994 & .996 & 1.009 & 1.009 & 1.010 & 1.014 & 1.003 & 1.021 & .989 \\
\hline $\begin{array}{l}\text { Sumpled } \\
\text { Interval }\end{array}$ & $\begin{array}{c}\text { Bulk } \\
\text { - }\end{array}$ & $\begin{array}{l}\text { Bulk } \\
\text { S-Gmm }\end{array}$ & $\begin{array}{c}\text { Bulk } \\
\text {. }\end{array}$ & $\underset{0-4 m m}{\text { Bulk }}$ & $\begin{array}{c}\text { Bulk } \\
0-9 \mathrm{mmn}\end{array}$ & $\begin{array}{l}\text { Bulk } \\
0-4 \mathrm{~mm} m\end{array}$ & $\begin{array}{l}\text { Bulk } \\
0-2 \mathrm{~mm}\end{array}$ & $\begin{array}{c}\text { Bulk } \\
0-12 \mathrm{mmn}\end{array}$ & $\begin{array}{c}\text { Bulk } \\
\text { O-10mm }\end{array}$ & $\begin{array}{l}\text { Bulk } \\
0-6 \mathrm{cmm}\end{array}$ & $\begin{array}{c}\text { Bulk } \\
\text { 0-8mm }\end{array}$ & $\begin{array}{c}\text { Bulk } \\
0.12 \mathrm{~mm}\end{array}$ \\
\hline
\end{tabular}


Table 1. Composition of crusts analyzed by USGS Reston Labs (cont'd).

\begin{tabular}{|c|c|c|c|c|c|c|c|c|c|c|c|c|}
\hline Sequence"l & 093007 & 093008 & 093009 & 094002 & 094003 & 094004 & 094006 & 095001 & 095002 & 095003 & 114004 & 114006 \\
\hline Latinde & 21.367 & 21.375 & 21.350 & 23383 & 26.850 & 20.912 & 27.050 & 23.633 & 20.001 & 20.938 & 37.510 & 34.810 \\
\hline Longitude & 153.287 & 153.272 & 153.333 & 148.250 & 148.783 & 142532 & 148.683 & 133.283 & 138.816 & 138.033 & -59.870 & -57.223 \\
\hline Depth & 2083 & 1787 & 3835 & 2423 & 2234 & 3112 & 1749 & 2630 & 2519 & 2508 & 1523 & 3310 \\
\hline Lebü & W-2229875 & W-229876 & W-2229878 & W-222686 & W-222687 & W-222585 & W-229871 & W-229880 & W-229858 & W-2229857 & W-228418 & w-228417 \\
\hline $\mathrm{siO}_{2}(\mathrm{wt.} . \%)$ & 14.6 & 5.24 & 10.2 & 6.25 & 17.3 & 25.5 & 15.1 & 24.3 & 232 & 224 & 8.40 & 242 \\
\hline $\mathrm{TiO}_{2}^{2}$ & 1.89 & 1.66 & 1.95 & 1.44 & 1.67 & 1.56 & 1.06 & .79 & 1.64 & 1.80 & 90 & .62 \\
\hline & 37.4 & 45.9 & 38.6 & 44.6 & 28.1 & 16.7 & 26.7 & 29.6 & 18.0 & 19.5 & 30.6 & 20.1 \\
\hline & 24.9 & 22.7 & 25.1 & 23.2 & 19.2 & 23.0 & 18.4 & 16.0 & 31.0 & 320 & 31.2 & 17.1 \\
\hline & 2.75 & 80 & 235 & 95 & 5.24 & 7.70 & 4.25 & 8.69 & 6.49 & 6.22 & 3.79 & 9.46 \\
\hline & .781 & 1.04 & 599 & .706 & .722 & .108 & .623 & .214 & .228 & 203 & .512 & 396 \\
\hline Nió & .628 & .830 & 576 & .914 & .534 & .145 & .552 & 1.141 & .198 & .167 & .511 & AS9 \\
\hline Cuo & .187 & .093 & .189 & .097 & .059 & .057 & .100 & .074 & .147 & .109 & .100 & 233 \\
\hline $\mathrm{CnO}$ & 3.63 & 4.12 & 4.34 & 3.61 & 6.90 & 5.87 & 120 & 2.27 & 261 & 2.69 & 2.98 & 3.58 \\
\hline $\mathrm{M}_{8} \mathrm{O}$ & 1.88 & 206 & 1.85 & 1.92 & 2.47 & 219 & 2.20 & 3.31 & 2.26 & 2.10 & 2.70 & 2.70 \\
\hline $\mathrm{Ne}_{2} \mathrm{O}$ & 1.62 & 3.08 & 2.35 & 253 & 2.61 & 288 & 3.87 & 244 & 3.43 & 287 & 1.63 & 291 \\
\hline$k_{2} 0$ & 87 & .71 & .83 & .72 & 89 & 1.21 & .79 & 220 & 1.02 & .85 & 50 & 258 \\
\hline & 49 & 49 & .49 & 51 & .69 & 2.04 & 1.17 & 20 & .19 & .23 & 83 & 92 \\
\hline $\mathrm{P}_{2} \mathrm{O}_{5}$ & 95 & 1.17 & 1.32 & 91 & 3.48 & 80 & 6.26 & 49 & .77 & $\pi$ & 98 & 1.41 \\
\hline $\mathrm{H}_{2} \mathrm{O}^{3}$ & 9.4 & 83 & 8.5 & 9.0 & 7.8 & 7.9 & 63 & 93 & 7.8 & 8.1 & 11.3 & 9.1 \\
\hline Ho & 128 & 21.8 & 20.4 & 24.8 & 18.9 & 16.6 & 14.7 & 10.8 & 16.5 & 16.4 & 20.3 & 14.1 \\
\hline $\operatorname{sam}$ & 1020 & 98.2 & 993 & 97.4 & 97.6 & 97.7 & 99.5 & 101.7 & 99.0 & 100.1 & 96.9 & 95.8 \\
\hline$A-(p p m)$ & 250 & 310 & 239 & 280 & 210 & 168 & 188 & 123 & 192 & 227 & 600 & 221 \\
\hline $\mathrm{Ba}$ & 1700 & 1700 & 2000 & 1700 & 880 & 1000 & 1100 & 1500 & 1200 & 1200 & 1600 & 1000 \\
\hline Cd & 4.5 & 5.8 & 3.4 & 5.5 & 3.6 & 25 & 5.2 & 8.1 & 29 & 23 & 3.1 & 2.3 \\
\hline$\infty$ & 1300 & 1400 & 1600 & 1300 & 950 & 310 & 860 & 360 & 900 & 1100 & 1600 & 1200 \\
\hline a & 9 & $<$ & 28 & 3 & 58 & 17 & 14 & 7 & 14 & 11 & 20 & 16 \\
\hline Mo & 450 & 730 & 460 & 740 & 410 & 22 & 290 & 224 & 97 & 144 & 440 & 210 \\
\hline $\mathbf{P b}$ & 1600 & 2200 & 1600 & 2000 & 1600 & 470. & 1400 & 660 & 970 & 1200 & 1400 & 470 \\
\hline St & 1500 & 1800 & 1600 & 1600 & 1200 & 1100 & 1100 & 620 & 930 & 970 & 1500 & 690 \\
\hline v & 600 & 700 & 620 & 720 & 530 & 350 & 470 & 360 & 490 & 530 & 110 & 520 \\
\hline $\mathbf{Y}$ & 184 & 192 & 239 & 186 & 185 & 12 & 116 & 75 & 144 & 156 & 125 & 90 \\
\hline $\mathbf{Z n}$ & 610 & 720 & 690 & 840 & 580 & 460 & 590 & 1300 & 540 & 510 & 880 & 630 \\
\hline$\gamma$ & .967 & 1.004 & .993 & 1.012 & 1.010 & 1.009 & 991 & 969 & 996 & 985 & 1.017 & 1.029 \\
\hline Sempled & Bulk & Bulk & Bulk & Bulk & Bulk & Bulk & Bulk & Bulk & Bulk & Bulk & Bulk & Bulk \\
\hline Interval & - & • & - & - & • & $0.7 \mathrm{~mm}$ & • & • & $\cdot$ & $\cdot$ & $0.25 \mathrm{~mm}$ & $0.11 \mathrm{~mm}$ \\
\hline Sequencell & 115005 & 115007 & 115009 & 115015 & 116008 & 116009 & 116010 & 116011 & 116012 & 116013 & 116014 & 116015 \\
\hline Latitude & 33.947 & 39.383 & 39.450 & 38.067 & 30.798 & 30.798 & 30.798 & 31.075 & 32.195 & 31310 & 30.970 & 31.075 \\
\hline Longitude & -65.783 & -67.233 & -65.455 & -60.217 & .78 .160 & -78.160 & -78.495 & -78.172 & -77.460 & -77.307 & -78.818 & -78.172 \\
\hline Depth & 4892 & 3040 & 2240 & 3007 & 840 & 840 & 860 & 817 & 690 & 1090 & 794 & 817 \\
\hline Lab" & W-228416 & W.228419 & W-228420 & W-229864 & W-229289 & W-229287 & W-229298 & W-229300 & W-229291 & W-229293 & W-229286 & W-229296 \\
\hline$\overline{\mathrm{siO}_{2}(w t . \%)}$ & 22.3 & 38.1 & 831 & 21.2 & 256 & 4.52 & 5.78 & 4.85 & 6.89 & 1.59 & 6.42 & 8.65 \\
\hline & 83 & .48 & 1.13 & 1.59 & .78 & .66 & .62 & 45 & .56 & .64 & 59 & .22 \\
\hline & 19.2 & 14.0 & 229 & 18.1 & 326 & 30.1 & 33.4 & 37.8 & 29.6 & 23.9 & 22.1 & 7.99 \\
\hline & 283 & 23.8 & 38.0 & 30.0 & 20.5 & 21.4 & 22.4 & 16.4 & 20.8 & 16.7 & 21.2 & 14.6 \\
\hline $\mathrm{Al}_{2}^{2} \mathrm{O}_{3}^{3}$ & 7.34 & 6.08 & 3.14 & 7.05 & 3.62 & 5.43 & 6.66 & 5.73 & 4.56 & 285 & 5.83 & 3.68 \\
\hline $\mathrm{CO}_{3}^{2} \mathrm{O}_{4}^{3}$ & 225 & .115 & $\$ 70$ & .413 & .717 & .615 & 587 & .553 & 594 & .417 & .437 & .119 \\
\hline $\mathrm{NiO}^{4}$ & 285 & .310 & .211 & .297 & .807 & .826 & 1.183 & 1.248 & .789 & 571 & .701 & 328 \\
\hline CuO & .177 & 250 & .032 & .190 & .093 & .145 & .170 & .183 & .158 & .051 & .113 & .052 \\
\hline $\mathrm{CaO}$ & 280 & 1.16 & 3.76 & 3.85 & 10.2 & 8.76 & 1.84 & 6.47 & 10.8 & 20.4 & 13.2 & 20.5 \\
\hline $\mathrm{M}_{80}$ & 234 & 2.76 & 212 & 2.78 & 4.90 & 5.61 & 6.39 & 6.54 & 5.23 & 4.15 & 4.91 & 3.19 \\
\hline $\mathrm{N}_{2} \mathrm{O}$ & 1.88 & 1.66 & 1.66 & 1.81 & 1.56 & 1.26 & 1.59 & 1.33 & 1.26 & 1.16 & 1.07 & 91 \\
\hline $\mathbf{k}_{2} \mathbf{o}$ & 1.01 & 1.60 & .60 & 86 & .40 & $A 7$ & 55 & .64 & .54 & .26 & 54 & 83 \\
\hline$\infty_{2}$ & 1.18 & $A 1$ & 1.53 & 51 & 7.89 & 8.57 & 5.78 & 4.40 & 7.58 & 7.22 & 4.70 & 5.66 \\
\hline $\mathrm{P}_{2} \mathrm{O}_{5}$ & 94 & .61 & 1.20 & 2.06 & 1.01 & 1.09 & 1.09 & 85 & $1 . \infty$ & 8.53 & 5.97 & 15.58 \\
\hline $\mathrm{H}_{2} \mathrm{O}+$ & 7.1 & 5.7 & 10.4 & 8.9 & 8.9 & 9.6 & 11.0 & 10.5 & 8.8 & 7.7 & 9.6 & 6.9 \\
\hline $\mathrm{H}_{2} \mathrm{O}$ & 15.1 & 9.8 & 21.8 & 14.2 & 16.4 & 13.7 & 11.8 & 113 & 129 & 8.6 & 12.8 & 29 \\
\hline Sum & 95.8 & 97.0 & 95.4 & 99.5 & 96.5 & 99.0 & 99.0 & 98.0 & 99.2 & 96.1 & 97.4 & 96.2 \\
\hline Axppon) & 340 & 260 & 690 & 330 & 410 & 450 & 480 & 340 & 460 & 300 & 310 & 185 \\
\hline $\mathrm{Ba}$ & 680 & 1100 & 1200 & 970 & 1100 & 1900 & 1900 & 2600 & 2000 & 1900 & 1500 & 310 \\
\hline $\mathrm{Cd}$ & 3.7 & 1.3 & 1.9 & 3.8 & 7.2 & 7.1 & 6.2 & 7.1 & 5.1 & 3.4 & 5.0 & 1.8 \\
\hline$\infty$ & 1600 & 520 & 1500 & 1300 & 1100 & 1100 & $11 \infty$ & 720 & 940 & 1100 & 870 & 270 \\
\hline$a$ & 38 & 36 & 41 & 37 & 19 & 21 & 28 & 23 & 26 & 12 & 29 & 38 \\
\hline Mo & 189 & 67 & 330 & 187 & 370 & 390 & 340 & 360 & 300 & 270 & 206 & 134 \\
\hline Pb & 920 & 370 & 1100 & 820 & 1200 & $11 \infty$ & 1200 & 790 & 940 & 1300 & 880 & 270 \\
\hline Sr & 750 & 410 & 1400 & 860 & 1400 & 1300 & 950 & 1000 & 1100 & 1600 & 1100 & 950 \\
\hline v & 790 & 620 & 1400 & 830 & 770 & 790 & 850 & 730 & 760 & 660 & 710 & 430 \\
\hline$Y$ & 130 & 44 & 230 & 152 & 179 & 139 & 113 & 99 & 109 & 219 & 241 & 460 \\
\hline $\mathbf{Z n}$ & 2600 & 450 & 540 & 590 & 660 & 650 & 690 & 710 & 640 & 510 & 630 & 320 \\
\hline$\gamma$ & 1.020 & 1.016 & 1.033 & .990 & 1.022 & .996 & 996 & 1.006 & 994 & 1.026 & 1.013 & 1.025 \\
\hline $\begin{array}{l}\text { Sampled } \\
\text { Inturval }\end{array}$ & $\begin{array}{c}\text { Bulk } \\
0-12 \mathrm{~mm}\end{array}$ & $\begin{array}{c}\text { Bulk } \\
0-4 m m\end{array}$ & $\begin{array}{c}\text { Bulk } \\
0-25 \mathrm{~mm}\end{array}$ & $\begin{array}{c}\text { Bulk } \\
-\end{array}$ & $\begin{array}{c}\text { Bulk } \\
0-18 \mathrm{rmm}\end{array}$ & $\begin{array}{c}\text { Bulk } \\
0-25 \mathrm{~mm}\end{array}$ & $\begin{array}{c}\text { Bulk } \\
0-17 \mathrm{~mm}\end{array}$ & $\begin{array}{c}\text { Bulk } \\
0-17 \mathrm{~mm}\end{array}$ & $\begin{array}{c}\text { Bulk } \\
0-15 \mathrm{~mm}\end{array}$ & $\begin{array}{c}\text { Bulk } \\
\text { O-4mm }\end{array}$ & $\begin{array}{c}\text { Bulk } \\
0-14 m m\end{array}$ & $\underset{0-3 \operatorname{mm} m}{\text { Bulk }}$ \\
\hline
\end{tabular}


Table 1. Composition of crusts analyzed by USGS Reston Labs (cont'd).

\begin{tabular}{|c|c|c|c|c|c|c|c|c|c|c|c|c|}
\hline Soquencell & 116016 & 116017 & 116018 & 116019 & 116020 & 116021 & 116022 & 116023 & 116024 & 116025 & 116027 & 116028 \\
\hline Latitude & 31.075 & 32.632 & 30990 & 30.990 & 31.075 & 30.798 & 30.798 & 30.798 & 30.798 & 31.075 & 31.295 & 31.075 \\
\hline Longitude & -78.172 & -77515 & -78.522 & .78 .522 & -78.172 & -78.495 & -78.495 & -78.495 & .78 .495 & .78 .172 & .78 .172 & -78.172 \\
\hline Depth & 817 & 399 & 780 & 780 & 817 & 860 & 860 & 860 & 860 & 817 & 690 & 817 \\
\hline Labil & W-229290 & W-229283 & W-229288 & W-229297 & W-229304 & W-229295 & W-229302 & w-22030s & W-229284 & W-229285 & W-2229294 & W.229292 \\
\hline $\mathrm{SiO}_{2}$ (wt.\%) & 6.32 & 6.09 & 4.70 & 276 & 2.26 & 250 & 3.13 & 4.12 & 3.10 & 7.85 & 3.77 & 279 \\
\hline & 51 & 55 & .59 & 58 & 53 & 58 & .63 & .67 & .65 & .30 & 51 & 52 \\
\hline & 12.2 & 35.4 & 223 & 26.3 & 26.3 & 279 & 33.0 & 30.0 & 33.1 & 18.5 & 22.4 & 229 \\
\hline & 17.5 & 21.1 & 27.5 & 23.0 & 20.5 & 22.2 & 19.2 & 21.5 & 20.6 & 13.0 & 20.4 & 21.2 \\
\hline $\mathrm{Al}_{2}^{2}$ & 4.02 & 5.91 & 4.36 & 3.23 & 3.74 & 289 & 4.28 & 4.67 & 4.43 & 4.41 & 3.09 & 3.89 \\
\hline $\mathrm{CO}_{3}^{2} \mathrm{O}$ & .186 & 523 & 406 & .472 & .402 & .489 & 546 & .609 & .612 & 274 & .327 & 331 \\
\hline Nố & 509 & 1.077 & .496 & .54 & .665 & .546 & 810 & .734 & 813 & .715 & 436 & $s 77$ \\
\hline CuO & .057 & .155 & .093 & .064 & .063 & .057 & .162 & .146 & .136 & .090 & .057 & .062 \\
\hline $\mathrm{CrO}$ & 23.6 & 4.90 & 114 & 14.1 & 14.3 & 13.8 & 11.1 & 10.7 & 9.27 & 21.7 & 17.7 & 15.9 \\
\hline $\mathrm{M}_{8} \mathrm{O}$ & 3.39 & 5.99 & 4.35 & 4.31 & 5.52 & 4.09 & 5.04 & 4.90 & 5.06 & 4.71 & 3.85 & 5.48 \\
\hline $\mathrm{NaO}$ & 1.18 & 1.42 & 1.08 & 1.21 & 1.00 & 1.25 & 139 & 1.22 & 1.38 & 128 & 1.23 & 86 \\
\hline $\mathrm{K}_{2} \mathrm{O}$ & 53 & .72 & .44 & 39 & 30 & 39 & 53 & 51 & .53 & .65 & .43 & 35 \\
\hline$\infty$ & 3.37 & 3.84 & 9.17 & 11.3 & 11.5 & 10.4 & 6.84 & 7.53 & 6.51 & 2.76 & 143 & 133 \\
\hline $\mathbf{P}_{2}$ & 14.72 & 1.01 & 1.20 & 1.03 & 91 & 1.01 & 89 & 1.02 & .98 & 12.15 & 1.02 & .94 \\
\hline $\mathrm{H}_{2} \mathrm{O}+$ & 7.9 & 9.6 & 8.5 & 8.3 & 10.5 & 8.3 & 8.6 & 8.9 & 9.6 & 8.0 & 7.4 & 8.8 \\
\hline $\mathrm{H}_{2} \mathrm{O}$ & 5.0 & 11.4 & 12.8 & 13.4 & 12.0 & 13.7 & 15.2 & 15.0 & 15.5 & 5.7 & 12.5 & 9.6 \\
\hline Sum & 95.9 & 98.3 & 96.7 & 973 & 98.4 & 96.4 & 96.2 & 973 & 96.9 & 963 & 97.0 & 98.0 \\
\hline$A \times$ (ppm) & 250 & 440 & 540 & 490 & 450 & 480 & 390 & 440 & 410 & 223 & 410 & 460 \\
\hline $\mathrm{Ba}$ & 490 & 2600 & 1700 & 1300 & 1400 & 1500 & 2200 & 1800 & 2400 & 730 & 880 & 1200 \\
\hline Cd & 23 & 5.8 & 4.9 & 4.6 & 5.2 & 4.9 & 5.9 & 5.9 & 6.5 & 3.4 & 4.0 & 5.0 \\
\hline $\bar{c}$ & 860 & 860 & 950 & 1000 & 990 & 950 & 960 & 1000 & 1000 & 420 & 890 & 1000 \\
\hline$a$ & 97 & 31 & 34 & 32 & 27 & 20 & 20 & 22 & 21 & 79 & 31 & 37 \\
\hline Mo & 158 & 360 & 310 & 330 & 310 & 390 & 410 & 360 & 450 & 180 & 320 & 250 \\
\hline $\mathbf{P b}$ & 470 & 990 & 960 & 1200 & 1100 & 1200 & 1200 & 1200 & 1200 & 450 & 940 & 980 \\
\hline St & 1100 & 1000 & 1300 & 1500 & 1600 & 1700 & 1400 & 1600 & 1400 & 1000 & 1300 & 1300 \\
\hline $\mathbf{v}$ & 480 & 820 & 970 & 850 & 820 & 870 & 750 & 800 & 850 & 450 & 720 & 800 \\
\hline $\mathbf{Y}$ & 590 & 93 & 161 & 185 & 216 & 209 & 142 & 141 & 166 & 320 & 183 & 188 \\
\hline $\mathbf{z n}$ & 350 & 690 & 600 & 470 & 520 & 500 & 670 & 620 & 690 & 450 & 430 & 510 \\
\hline$\gamma$ & 1.029 & 1.003 & 1.019 & 1.011 & 1.002 & 1.022 & 1.025 & 1.014 & 1.018 & 1.024 & 1.016 & 1.006 \\
\hline Sempled & Bulk & Bulk & Bulk & Bulk & Bulk & Bulk & Balk & Bulk & Bulk & Bulk & Bulk & Bulk \\
\hline Interval & $0-2 \mathrm{~mm}$ & $0-15 \mathrm{~mm}$ & $0-14 \mathrm{~mm}$ & $0-16 \mathrm{~mm}$ & $0-9 m m$ & $0-8 \mathrm{~mm}$ & $0-15 \mathrm{~mm}$ & $0.22 \mathrm{~mm}$ & $0-18 \mathrm{~mm}$ & $0-2 \mathrm{~mm}$ & $0-1 \mathrm{~mm}$ & $0.4 \mathrm{~mm}$ \\
\hline Soquenoell & 116029 & 120001 & $120002 \dagger$ & 121002 & 125001 & 125002 & 125003 & 125004 & 125005 & 125006 & 125007 & 125008 \\
\hline Letituds & 30.990 & 31.250 & 31.283 & 33.733 & 33.490 & 34.07 & 33.863 & 33.828 & 32.468 & 33.627 & 31.802 & 31.660 \\
\hline Longitude & -78.522 & -117.583 & -118.467 & -120.750 & -166.535 & -166.502 & -166.515 & -166.993 & -165.800 & -165.098 & -165.843 & -163.017 \\
\hline Depth & 780 & 2120 & 650 & 1719 & 32200 & 4400 & 3410 & 3050 & 3150 & 2740 & 2500 & 2350 \\
\hline Lab: & W-220301 & W-222553 & W-222554 & W-231371 & W-224408 & W-224409 & W-224410 & W-224411 & W-224413 & W-224414 & W-224415 & W.224416 \\
\hline $\mathrm{SiO}_{2}(\mathrm{wt} . \%)$ & 252 & 33.6 & 50.7 & 13.4 & 26.0 & 15.8 & 33.8 & 22.2 & 18.7 & 15.6 & 12.1 & 18.8 \\
\hline $\mathrm{TiO}_{2}$ & .62 & 50 & 1.48 & .63 & 1.56 & 204 & 1.38 & 1.25 & 1.46 & 1.61 & 1.70 & 1.50 \\
\hline $\mathrm{MmO}_{2}$ & 28.5 & 178 & .36 & 22.1 & 26.0 & 30.5 & 14.1 & 19.2 & 31.3 & 324 & 37.9 & 27.4 \\
\hline & 24.9 & 22.6 & 8.68 & 22.1 & 20.9 & 26.4 & 12.1 & 30.8 & 19.5 & 24.1 & 25.1 & 24.0 \\
\hline $\mathrm{Al}_{2} \mathrm{O}_{3}^{3}$ & 297 & 5.48 & 17.3 & 209 & 6.50 & 4.62 & 10.6 & 6.75 & 4.69 & 3.23 & 2.36 & 4.29 \\
\hline $\mathrm{CO}_{3}^{2} \mathrm{O}_{4}$ & 554 & .088 & .009 & .341 & .585 & $\mathbf{A 7 0}$ & 234 & 236 & .618 & 900 & .911 & .721 \\
\hline $\mathrm{NiO}^{4}$ & 562 & 399 & .007 & .261 & .396 & 340 & $A 51$ & 248 & .742 & 435 & .592 & .440 \\
\hline $\mathrm{CuO}$ & .060 & .023 & .001 & .023 & .202 & 209 & 202 & .230 & 243 & .098 & .156 & 230 \\
\hline $\mathrm{CaO}$ & 124 & 2.11 & 10.1 & 9.55 & 255 & 3.42 & 6.76 & 3.48 & 4.84 & 4.62 & 3.49 & 3.18 \\
\hline $\mathrm{M}_{8} \mathrm{O}$ & 4.14 & 250 & 4.78 & 5.68 & 2.67 & 203 & 3.03 & 1.97 & 251 & 1.89 & 2.07 & 1.97 \\
\hline $\mathrm{N}_{2} \mathrm{O}$ & 1.19 & 3.60 & 3.81 & 1.77 & 2.79 & 222 & 2.15 & 249 & 259 & 2.85 & 2.61 & 281 \\
\hline $\mathbf{K}_{2} \mathbf{O}$ & 38 & 1.24 & .71 & .62 & 1.16 & 1.17 & 2.33 & 1.12 & 1.43 & .71 & .79 & 130 \\
\hline$\infty_{2}$ & 9.07 & 30 & .06 & 11.3 & .49 & .57 & 1.07 & .71 & 1.51 & .69 & .65 & 42 \\
\hline $\mathrm{P}_{2} \mathrm{O}_{5}$ & 1.12 & .69 & .50 & 1.04 & .62 & 1.07 & 3.45 & 1.07 & 1.24 & .17 & 88 & 1.14 \\
\hline $\mathrm{H}_{2}^{2} \mathrm{O}+$ & 8.5 & 8.1 & 2.2 & 73 & 9.0 & 9.8 & 6.9 & 10.5 & 6.9 & 83 & 5.1 & 125 \\
\hline $\mathrm{H}_{2}^{2} \mathrm{O}$ & 14.0 & 13.8 & 2.8 & 12.1 & 6.9 & 10.1 & 6.9 & 7.6 & 7.4 & 122 & 11.8 & 75 \\
\hline Sum & 97.5 & 99.1 & 100.6 & 98.2 & 101.4 & 100.6 & 98.6 & 1022 & 98.2 & 97.7 & 96.4 & 100.7 \\
\hline$A(p p m)$ & 530 & 174 & 17 & 250 & 118 & 167 & 41 & 173 & 119 & 182 & 215 & 184 \\
\hline $\mathrm{Ba}$ & 1400 & 1300 & 103 & 1100 & 1400 & 1800 & 19000 & 2100 & 3800 & 1700 & 4200 & 3100 \\
\hline Cd & 5.0 & 3.7 & $<.1$ & 15 & 29 & 22 & 23 & 1.7 & 5.5 & 3.2 & 3.6 & 3.1 \\
\hline$c$ & 990 & 600 & 23 & 980 & 1500 & 2200 & 520 & 740 & 1000 & 1300 & 1500 & 1400 \\
\hline$a$ & 30 & 74 & 206 & 47 & 16 & 15 & 64 & 15 & 30 & 14 & 13 & 15 \\
\hline Mo & 380 & 174 & $<4$ & 480 & 260 & 340 & 129 & 217 & 300 & 390 & 460 & 200 \\
\hline $\mathrm{Pb}$ & 1300 & 570 & $\Leftrightarrow$ & 1600 & 1300 & 1200 & 400 & 770 & 1200 & 1800 & 1600 & 1500 \\
\hline Sr & 1700 & 750 & 247 & 1300 & 1100 & 1200 & 1600 & 1100 & 1100 & 1400 & 1600 & 1300 \\
\hline v & 970 & 450 & 247 & 580 & 420 & 1800 & 193 & 670 & 400 & 540 & 560 & 520 \\
\hline $\mathbf{Y}$ & 221 & 93 & 35 & 159 & 150 & 245 & 247 & 151 & 238 & 216 & 193 & 173 \\
\hline $\mathbf{Z n}$ & $\mathbf{s 5 0}$ & 630 & 82 & 430 & 580 & 520 & 490 & 650 & 730 & 520 & 620 & 640 \\
\hline$\gamma$ & 1.011 & 995 & .980 & 1.004 & .972 & .980 & 1.000 & .965 & 1.004 & 1.010 & 1.023 & 979 \\
\hline $\begin{array}{l}\text { Semplod } \\
\text { Interval }\end{array}$ & $\begin{array}{c}\text { Bulk } \\
0-9 \mathrm{mmm}\end{array}$ & $\begin{array}{c}\text { Bulk } \\
0-10 \mathrm{~mm}\end{array}$ & Bulk & $\begin{array}{c}\text { Bulk } \\
\text { 0-25mm }\end{array}$ & $\begin{array}{c}\text { Bulk } \\
\text { 0-6mm }\end{array}$ & $\begin{array}{c}\text { Bulk } \\
\text { 0-38mm }\end{array}$ & $\underset{\text { O-4mm }}{\text { Balk }}$ & $\begin{array}{c}\text { Bulk } \\
\text { 0-32mm }\end{array}$ & $\begin{array}{c}\text { Bulk } \\
0-14 \mathrm{~mm}\end{array}$ & $\begin{array}{c}\text { Bulk } \\
\text { a-10mm }\end{array}$ & $\begin{array}{c}\text { Bulk } \\
0-20 \mathrm{~mm}\end{array}$ & $\begin{array}{c}\text { Bulk } \\
\text { a-11mm }\end{array}$ \\
\hline
\end{tabular}


Table 1. Composition of crusts analyzed by USGS Reston Labs (cont'd).

\begin{tabular}{|c|c|c|c|c|c|c|c|c|c|c|c|c|}
\hline Soquenoent: & 125009 & 125010 & 125011 & 125014 & 126001 & 127003 & 127004 & $127006 \$$ & 127007 & 127008 & 127012 & 128001 \\
\hline Letitude & 31.935 & 33.928 & 33.217 & 33.828 & 33.473 & 33.693 & 33.666 & 32292 & 32.137 & 31.020 & 37.762 & 39.385 \\
\hline Longindo & -162.240 & -162.095 & -162338 & -166.993 & -178.012 & 177.552 & 171.602 & 172792 & 172.262 & 175.873 & 170.280 & 165.868 \\
\hline Dopth & 2460 & 2550 & 2160 & 3050 & 5063 & 1558 & 1002 & 645 & 1360 & 598 & 1620 & 3537 \\
\hline Lab" & W-224417 & W-224418 & W-224420 & W-224412 & W-224381 & W-222609 & W-222610 & W-222611 & W-222612 & W-222613 & W-224382 & W-224383 \\
\hline $\mathrm{SiO}_{2}(\mathrm{w}, \%)$ & 15.1 & 14.5 & 15.1 & 420 & 24.5 & 3.13 & 6.47 & 56 & 10.7 & 6.60 & 18.6 & 26.4 \\
\hline & 1.35 & 1.42 & 1.61 & 2.29 & 2.84 & 1.11 & 1.16 & .01 & 1.10 & 1.23 & 1.58 & 1.44 \\
\hline $\mathrm{MnO}_{2}$ & 22.6 & 33.3 & 31.3 & 7.69 & 21.4 & 48.2 & 44.8 & .12 & 40.0 & 40.3 & 25.4 & 26.1 \\
\hline $\mathrm{Fe}_{2} \mathrm{O}_{3}^{2}$ & 21.4 & 22.5 & 24.5 & 10.4 & 21.7 & 22.2 & 21.4 & .19 & 21.7 & 23.8 & 21.8 & 21.1 \\
\hline & 284 & 3.18 & 3.22 & 13.0 & 7.23 & $\$ 1$ & 1.92 & 27 & 253 & 1.22 & 4.76 & 6.23 \\
\hline $\mathrm{Co}_{3}^{2} \mathrm{O}_{4}^{3}$ & 586 & 811 & .804 & .187 & .593 & 1.20 & 1.38 & .002 & .106 & 1.04 & 529 & .217 \\
\hline No & 301 & .615 & 477 & .128 & .379 & .658 & 484 & .003 & 511 & .470 & .204 & .076 \\
\hline CuO & .089 & .155 & .115 & .034 & .041 & .024 & .081 & $<001$ & .079 & .041 & .045 & 266 \\
\hline Cno & 120 & 4.56 & 3.65 & 8.13 & 5.65 & 3.81 & 3.55 & 51.5 & 3.74 & 3.32 & 8.08 & 268 \\
\hline M\&O & 1.62 & 1.86 & 1.88 & 1.31 & 4.58 & 1.94 & 252 & 1.58 & 282 & 219 & 2.44 & 264 \\
\hline $\mathrm{Na}_{2} \mathrm{O}$ & 258 & 2.58 & 2.61 & 3.17 & 2.76 & 286 & 292 & .66 & 3.16 & 4.49 & 2.21 & 244 \\
\hline & .91 & 1.10 & 1.03 & 3.35 & 86 & .57 & .72 & .06 & .50 & .72 & 1.10 & 154 \\
\hline & 1.29 & 54 & .47 & 83 & 35 & AS & 49 & 429 & 1.16 & .50 & 99 & 23 \\
\hline $\mathrm{P}_{2} \mathrm{C}$ & 6.90 & 1.88 & 1.22 & 3.87 & 80 & 1.12 & 1.19 & .14 & .98 & 1.21 & 3.85 & .66 \\
\hline $\mathrm{H}_{2}^{2} \mathrm{O}+$ & 9.2 & 10.2 & 11.5 & 5.0 & 7.2 & 9.0 & 9.0 & .8 & 10.3 & 8.8 & 8.8 & 9.0 \\
\hline $\mathrm{H}_{2} \mathrm{O}-$ & 2.0 & 11.0 & 11.9 & 53 & 5.9 & 26.5 & 21.2 & .5 & 17.8 & 24.2 & 4.8 & 5.9 \\
\hline Sum & 98.8 & 99.2 & 99.4 & 1014 & 100.9 & 96.7 & 98.1 & 98.9 & 99.5 & 95.9 & 100.5 & 101.6 \\
\hline Ax(ppm) & 151 & 169 & 193 & 51 & 170 & 350 & 330 & 3 & 231 & 340 & 158 & 117 \\
\hline Ba & 1700 & 3900 & 2400 & 2300 & 770 & 1000 & 2000 & 13 & 2700 & 1600 & 6500 & 1400 \\
\hline Cd & 20 & 4.4 & 2.6 & .6 & 23 & 4.2 & 4.1 & 2.0 & 27 & 4.4 & 3.2 & 4.5 \\
\hline$\infty$ & 1100 & 1200 & 1200 & 390 & 620 & 1500 & 1300 & $\theta$ & 1300 & 1600 & 1100 & 1000 \\
\hline$\alpha$ & 12 & 9 & 27 & 41 & 270 & 8 & 33 & 3 & 39 & 16 & 280 & 28 \\
\hline Mo & 226 & 360 & 390 & 94 & 310 & 1100 & 1100 & $<4$ & 195 & 830 & 250 & 290 \\
\hline $\mathbf{P O}_{0}$ & 1500 & 1500 & 1500 & 390 & 1200 & 2600 & 2700 & $\theta$ & 2300 & 2600 & 1700 & 1200 \\
\hline Sr & 1300 & 1500 & 1400 & 610 & 1000 & 1600 & 1700 & 930 & 1600 & 1700 & 1600 & 910 \\
\hline $\mathbf{v}$ & 440 & 480 & 540 & 380 & 610 & 800 & 760 & 8 & 610 & 740 & 540 & 440 \\
\hline $\mathbf{Y}$ & 247 & 169 & 204 & 232 & 117 & 191 & 127 & 6 & 146 & 172 & 168 & 128 \\
\hline $\mathbf{Z n}$ & 480 & 660 & 590 & 410 & 560 & 530 & 650 & 20 & 730 & 570 & 550 & 970 \\
\hline$\varphi$ & 998 & 994 & .992 & .973 & .977 & 1.020 & 1.005 & .997 & 991 & 1.028 & .981 & 971 \\
\hline Sempied & Bulk & Bulk & Bulk & Bulk & Bulk & Bulk & Bulk & Bulk & Bulk & Bulk & Bulk & Bulk \\
\hline Interval & $0-23 \mathrm{~mm}$ & $0.15 \mathrm{~mm}$ & $0-14 \mathrm{~mm}$ & . & $0-14 \mathrm{~mm}$ & $0-10 \mathrm{~mm}$ & $0.6 \mathrm{~mm}$ & - & $0.2 \mathrm{~mm}$ & $0-3 \mathrm{~mm}$ & - & $0-11 \mathrm{~mm}$ \\
\hline Sequencoll & 128002 & 129002 & 129003 & 130001 & 130002 & 130003 & 130004 & 130005 & 146001 & 147001 & 147003 & 157001 \\
\hline Letituds & 37.267 & 31.557 & 32.018 & 38.017 & 32758 & 34.267 & 34.220 & 34.253 & 43.613 & 43.003 & 42.332 & 45.821 \\
\hline Longiude & 162.400 & 151.222 & 149.273 & 145.983 & 148.225 & 145.945 & 144.185 & 143.853 & -15.690 & .28 .220 & -27.005 & .129 .504 \\
\hline Depth & 1110 & 1768 & 1697 & 2785 & 2262 & 2046 & 1830 & 1898 & 5740 & • & 3193 & • \\
\hline Lab" & W-222684 & W-222603 & W.2222604 & W-222695 & W-222605 & W-222606 & W-222607 & W-222608 & W.229853 & W-222734 & W-229854 & W-230116 \\
\hline $\mathrm{SiO}_{2}$ (wt.\%) & 3.09 & 12.2 & 15.0 & 24.2 & 11.6 & 17.2 & 23.3 & 18.8 & 18.7 & 11.2 & 9.66 & 35.3 \\
\hline & 1.18 & 1.03 & 1.23 & 86 & 20 & 1.21 & 1.30 & 1.35 & 1.83 & 2.26 & 2.42 & 82 \\
\hline $\mathrm{MnO}_{2}$ & 54.8 & 38.0 & 325 & 22.1 & 15.3 & 31.1 & 22.2 & 29.6 & 24.4 & 19.5 & 24.1 & 14.0 \\
\hline $\mathrm{Fe}_{2} \mathrm{O}_{3}^{2}$ & 17.5 & 15.1 & 24.9 & 31.7 & 51.5 & 27.2 & 28.5 & 22.5 & 264 & 321 & 33.8 & 22.7 \\
\hline $\mathrm{Al}_{2}^{2} \mathrm{O}_{3}^{3}$ & $A 1$ & 3.52 & 4.34 & 3.21 & 1.78 & 3.32 & 4.54 & 3.72 & 8.27 & 6.28 & 5.59 & 8.04 \\
\hline $\mathrm{CO}_{3}^{2} \mathrm{O}_{4}$ & .739 & 571 & .675 & .180 & .051 & 307 & .294 & 268 & 565 & 501 & .740 & .133 \\
\hline $\mathrm{NiO}{ }^{4}$ & .639 & .859 & .831 & .230 & .131 & 542 & 336 & 595 & 352 & 227 & .202 & .110 \\
\hline co & .053 & .175 & .151 & .050 & .233 & .188 & .071 & .185 & .187 & .048 & .138 & .046 \\
\hline $\mathrm{CaO}$ & 3.85 & 7.66 & 2.03 & 2.46 & 209 & 281 & 3.02 & 4.13 & 3.32 & 4.52 & 3.77 & 5.17 \\
\hline $\mathrm{MgO}$ & 1.71 & 2.70 & 3.21 & 1.48 & 1.73 & 202 & 1.87 & 224 & 3.32 & 4.57 & 3.62 & 3.30 \\
\hline $\mathrm{Ne}_{2} \mathrm{O}$ & 280 & 1.98 & 2.30 & 231 & 1.61 & 226 & 228 & 2.71 & 1.91 & 3.18 & 214 & 278 \\
\hline $\mathbf{K}_{2} \mathbf{O}$ & 83 & 1.12 & .82 & .73 & 34 & 59 & .90 & 1.05 & .92 & .73 & .65 & 90 \\
\hline$\infty_{2}$ & 37 & .92 & .21 & 25 & 41 & .40 & 32 & .65 & .46 & .62 & 82 & .17 \\
\hline $\mathrm{P}_{2} \mathrm{O}_{5}$ & 96 & 4.00 & .94 & 86 & 1.25 & .83 & 1.24 & 1.55 & .92 & 1.55 & 1.25 & 80 \\
\hline $\mathrm{H}_{2} \mathrm{O}+$ & 8.1 & 8.6 & 104 & 7.0 & 10.3 & 8.9 & 8.9 & 8.0 & 10.6 & 9.0 & 9.5 & 6.4 \\
\hline $\mathrm{H}_{2}^{2} \mathrm{O}$ & 263 & 14.1 & 17.3 & 17.0 & 19.5 & 20.2 & 16.7 & 18.7 & 13.2 & 15.8 & 17.2 & 10.7 \\
\hline Sum & 97.0 & 98.5 & 99.5 & 97.6 & 98.6 & 98.9 & 99.1 & 97.4 & 1021 & 963 & 98.6 & 100.7 \\
\hline Ax(ppm) & 310 & 140 & 169 & 229 & 390 & 175 & 132 & 160 & 310 & 550 & 470 & 168 \\
\hline Be & 3400 & 3600 & 2500 & 1300 & 1200 & 1600 & 1900 & 3000 & 820 & 1100 & 1200 & 1000 \\
\hline Cd & 4.2 & 4.3 & 2.9 & 27 & 1.5 & 4.1 & 2.3 & 2.6 & 7.0 & 5.1 & 5.8 & 1.5 \\
\hline co & 2800 & 1300 & 1500 & 1000 & 174 & 1100 & $11 \infty$ & 1600 & 1600 & 1200 & 1700 & 310 \\
\hline$a$ & 3 & 34 & 12 & 11 & 8 & 14 & 17 & 15 & 31 & 105 & 79 & 71 \\
\hline Mo & 1500 & 360 & 73 & 250 & 174 & 330 & 72 & 340 & 161 & 214 & 280 & 157 \\
\hline Pb & 2400 & 1400 & 2200 & 1700 & 174 & 1800 & 1700 & 1700 & 1300 & 1100 & 1100 & 350 \\
\hline Sr & 1900 & 1200 & 1200 & 1100 & 1000 & 1400 & 1200 & 1400 & 880 & 1800 & 1300 & 850 \\
\hline v & 790 & 440 & 460 & 600 & 1000 & 600 & 520 & 500 & 700 & 1100 & 1000 & 460 \\
\hline $\mathbf{Y}$ & 107 & 140 & 85 & 157 & 137 & 125 & 132 & 62 & 184 & 260 & 230 & 123 \\
\hline $\mathbf{Z n}$ & 680 & 830 & 850 & 720 & 820 & 830 & 660 & 840 & 500 & 640 & 620 & 400 \\
\hline$\gamma$ & 1.017 & 1.001 & .990 & 1.010 & 1.000 & .997 & .995 & 1.013 & .966 & 1.024 & 1.000 & 980 \\
\hline Sempled & Bulk & Bulk & Bulk & Bulk & Bulk & Bulk & Bulk & Bulk & Bulk & Bulk & Bulk & Bulk \\
\hline haterval & 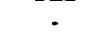 & $0-8 \mathrm{~mm}$ & $0-7 \mathrm{~mm}$ & . & $0-3 \mathrm{~mm}$ & $0.10 \mathrm{~mm}$ & $0-10 \mathrm{~cm}$ & $0-5 \mathrm{~mm}$ & - & • & - & - \\
\hline
\end{tabular}


Table 1. Composition of crusts analyzed by USGS Reston Labs (cont'd).

\begin{tabular}{|c|c|c|c|c|c|c|c|c|c|c|c|c|}
\hline Sequencos" & 157002 & 157003 & 157004 & 157005 & 157006 & $157007 \uparrow$ & $157008 \uparrow$ & 157009 & $157011+$ & 157012 & 158001 & 158003 \\
\hline Letituds & 45.821 & 45.821 & 45.821 & 45.821 & 45.821 & 45.733 & 45.733 & 45.821 & 44,000 & 44.000 & 46.069 & 46.153 \\
\hline Langitude & -129.504 & -129.504 & -129.504 & -129504 & -129.504 & -129.912 & -129.928 & -129.504 & -129.000 & -129.000 & $-130,387$ & -130.335 \\
\hline Depth & - & $\cdot$ & - & - & • & - & • & • & 2616 & 2302 & • & $\cdot$ \\
\hline Lab: & W-230106 & W-230107 & W-230111 & w-230091 & W-230115 & W-230102 & W-230101 & w-230099 & W-231368 & W-231367 & W-230110 & W-230109 \\
\hline $\mathrm{SiO}_{2}$ (wt.\%) & 32.0 & 31.6 & 43.6 & 27.3 & 37.5 & 49.1 & 47.9 & 33.4 & 41.4 & 38.9 & 36.3 & 41.6 \\
\hline & .76 & .75 & 1.03 & .68 & .92 & 1.38 & .96 & .74 & .49 & .65 & 87 & 1.29 \\
\hline & 15.6 & 16.2 & 5.90 & 20.0 & 11.3 & 1.00 & 3.50 & 135 & 3.64 & 7.56 & 11.6 & 6.97 \\
\hline & $23 A$ & 23.5 & 16.9 & 26.8 & 20.1 & 13.8 & 23.1 & 22.7 & 33.6 & 28.1 & 19.5 & 16.9 \\
\hline $\mathrm{Al}_{2}^{2} \mathrm{O}_{3}^{3}$ & 7.58 & 7.39 & 11.5 & 5.86 & 9.56 & 13.5 & 9.36 & 8.61 & 5.42 & 6.07 & 120 & 11.4 \\
\hline $\mathrm{CO}_{3}^{2} \mathrm{O}_{4}^{3}$ & .128 & .132 & .068 & .163 & .096 & .008 & .004 & .112 & .034 & .091 & .059 & .051 \\
\hline NiO & .136 & .146 & .043 & .152 & .099 & 011 & .024 & .122 & .091 & .141 & .122 & .068 \\
\hline Co & .004 & .058 & .043 & .057 & .044 & .011 & .013 & .040 & .034 & .029 & .022 & .016 \\
\hline $\mathrm{ClO}_{2}$ & 5.61 & 5.47 & 8.95 & 3.91 & 7.70 & 12.0 & 7.86 & 6.37 & 1.22 & 1.63 & 7.46 & 8.12 \\
\hline MEO & 3.63 & 3.59 & 5.83 & 2.55 & 4.87 & 7.01 & 5.66 & 4.49 & 2.19 & 2.71 & 3.59 & 5.32 \\
\hline $\mathrm{Ne}_{2} \mathrm{O}$ & 266 & 278 & 2.56 & 280 & 2.62 & 268 & 251 & 248 & 2.37 & 252 & 276 & 3.01 \\
\hline $\mathbf{k}_{2} 0$ & .75 & .78 & .63 & 91 & .65 & .21 & 1.66 & .67 & 1.00 & 1.46 & .78 & .75 \\
\hline$\infty_{2}$ & .21 & .21 & .06 & .26 & .13 & .04 & .04 & .19 & .04 & .07 & .17 & .09 \\
\hline $\mathrm{P}_{2} \mathrm{O}_{5}$ & .84 & .87 & .39 & 1.04 & .63 & .16 & .20 & .81 & .63 & .66 & .66 & 52 \\
\hline $\mathrm{H}_{2} \mathrm{O}+$ & 6.4 & 7.2 & 4.2 & 8.5 & 5.4 & 1.7 & 4.6 & 6.4 & 6.8 & 7.0 & 5.7 & 4.2 \\
\hline $\mathrm{H}_{2} \mathrm{O}$ & 127 & 13.1 & 6.2 & 16.2 & 9.1 & .7 & 9.2 & 10.0 & 8.6 & 10.0 & 10.0 & 6.9 \\
\hline $\sin$ & 99.7 & 100.8 & 101.7 & 101.0 & 101.7 & 102.6 & 1075 & 100.8 & 989 & 97.6 & 101.6 & 100.2 \\
\hline Axppm) & 195 & 196 & 63 & 239 & 132 & 9 & 20 & 178 & 164 & 167 & 133 & 84 \\
\hline Ba & 870 & 930 & 430 & 1000 & 610 & 111 & 121 & 790 & 800 & 1100 & 740 & 630 \\
\hline Cd & 1.6 & 2.0 & 1.0 & 1.8 & 1.9 & 5 & 21 & 1.3 & $A$ & 1.1 & 1.2 & 1.0 \\
\hline c. & 270 & 280 & 96 & 380 & 198 & $\theta$ & 12 & 290 & 300 & 360 & 167 & 150 \\
\hline$c$ & 104 & 99 & 203 & 64 & 154 & 59 & 53 & 156 & 270 & 260 & 122 & 183 \\
\hline Mo & 195 & 207 & 35 & 260 & 132 & $<4$ & $\delta$ & 178 & $\delta$ & 38 & 99 & 46 \\
\hline $\mathrm{Pb}$ & 390 & 370 & 171 & 490 & 280 & $<49$ & 84 & 290 & 470 & 430 & 156 & 183 \\
\hline st & 850 & 870 & 410 & 1100 & 640 & 161 & 143 & 800 & 320 & 540 & 720 & 570 \\
\hline $\mathbf{V}$ & 480 & 480 & 350 & 530 & 430 & 350 & 250 & 480 & 490 & 390 & 370 & 340 \\
\hline $\mathbf{Y}$ & 126 & 127 & 64 & 155 & 99 & 34 & $28^{\circ}$ & 122 & 58 & 67 & 98 & 70 \\
\hline $\mathbf{z n}$ & 420 & 410 & 300 & 430 & 350 & 131 & 231 & 340 & 450 & 390 & 200 & 280 \\
\hline$\gamma$ & .989 & .978 & 970 & .976 & .970 & 961 & .917 & 978 & 997 & 1.010 & .970 & .984 \\
\hline Sampled & Bulk & Bulk & Bulk & Bulk & Bulk & Bulk & Bulk & Bulk & Bulk & Bulk & Bulk & Bulk \\
\hline Intorval & - & • & - & - & • & - & $\cdot$ & • & • & • & $\cdot$ & - \\
\hline Sequencoll & 158004 & $158005 t$ & $158006+$ & $158007+$ & 158008 & 158009 & 158010 & 1580114 & 158012 & 158013 & 158014 & 158015 \\
\hline Letinde & 46.153 & 46.125 & 46.125 & 46.125 & 48.475 & 48.475 & 48.475 & 45.654 & 45.953 & 45.953 & 45.953 & 46.069 \\
\hline Longinude & -130.335 & -130.000 & -130.000 & -130.000 & -133.133 & -133.133 & -133.133 & -130.075 & -130.274 & -130.274 & -130.274 & -130.387 \\
\hline Depth & - & $\cdot$ & - & - & 1245 & 1245 & 1245 & - & - & - & • & $\cdot$ \\
\hline Lebi: & W-230108 & W-230103 & W-230091 & W-230104 & W-2301 14 & w.230095 & $w-230092$ & W-230089 & W-230090 & W-230098 & W-230096 & W.230112 \\
\hline $\mathrm{SiO}_{2}$ (wt.\$) & 39.9 & 48.6 & 479 & 44.5 & 6.18 & 5.92 & 4.22 & 48.9 & 26.3 & 224 & 30.8 & 36.4 \\
\hline $\mathrm{TiO}_{2}$ & 1.46 & 1.96 & 1.80 & 1.69 & 1.30 & 1.18 & 1.43 & 1.51 & .64 & .47 & .71 & .71 \\
\hline $\mathrm{MmO}_{2}$ & 9.56 & .76 & 1.36 & 3.58 & 427 & 43.8 & 47.3 & .44 & 20.2 & 24.9 & 16.5 & 13.2 \\
\hline $\mathrm{Pe}_{2} \mathrm{O}_{3}$ & 19.0 & 14.2 & 15.0 & 17.1 & 24.7 & 25.9 & 20.8 & 129 & 25.9 & 27.8 & 24.3 & 22.1 \\
\hline $\mathrm{Al}_{2} \mathrm{O}_{3}^{3}$ & 10.2 & 13.5 & 125 & 11.5 & 1.12 & 93 & .87 & 13.5 & 7.07 & 4.69 & 7.86 & 8.88 \\
\hline $\mathrm{CO}_{3} \mathrm{O}_{4}$ & .059 & .008 & .014 & .020 & 1.22 & 1.17 & 1.49 & .006 & .091 & .114 & .099 & .080 \\
\hline $\mathrm{NiO}{ }^{4}$ & .114 & .012 & .014 & .035 & .497 & 449 & .632 & .008 & .193 & 219 & .137 & .131 \\
\hline CuO & .028 & .009 & .013 & .014 & .043 & .035 & .061 & .011 & .020 & .051 & .039 & .032 \\
\hline $\mathrm{CaO}$ & 7.40 & $11 A$ & 10.3 & 9.24 & 3.48 & 3.63 & 3.68 & 12.2 & 4.65 & 3.21 & 5.01 & 452 \\
\hline MgO & 4.60 & 6.50 & 5.92 & 5.30 & 1.88 & 1.80 & 1.95 & 6.99 & 225 & 1.87 & 3.39 & 266 \\
\hline $\mathrm{Ne}_{2} \mathrm{O}$ & 3.12 & 3.01 & 3.16 & 2.86 & 3.40 & 3.16 & 3.64 & 290 & 2.62 & 2.89 & 2.92 & 292 \\
\hline $\mathbf{k}_{2} \mathbf{O}$ & .79 & 39 & .55 & 58 & .65 & .62 & .69 & .21 & .79 & .99 & .81 & 97 \\
\hline$\infty_{2}$ & .11 & .01 & .03 & .04 & 37 & .45 & 37 & .02 & .26 & .34 & 23 & 21 \\
\hline $\mathrm{P}_{2} \mathrm{O}_{5}$ & .57 & .23 & .23 & 35 & 1.30 & 1.39 & 1.11 & .16 & 1.10 & 1.27 & .90 & .81 \\
\hline $\mathrm{H}_{2}^{2} \mathrm{O}+$ & 4.9 & 19 & 23 & 4.7 & 93 & 10.2 & 12.4 & 1.6 & 8.0 & 9.0 & 6.5 & 6.4 \\
\hline $\mathrm{H}_{2}^{2} \mathrm{O}$ & 7.3 & .6 & 2.0 & 3.1 & 20.7 & 20.6 & 19.4 & .4 & 14.5 & 18.6 & 13.5 & 12.8 \\
\hline Sum & 101.7 & 1025 & 101.1 & 101.6 & 98.2 & 100.7 & 100.6 & 1014 & 100.1 & 100.1 & 100.1 & 100.1 \\
\hline$A \simeq$ (ppm) & 94 & 7 & 17 & 41 & 400 & 420 & 400 & 5 & 260 & 310 & 208 & 172 \\
\hline $\mathrm{Ba}$ & 690 & 64 & 290 & 340 & 1200 & 1400 & 1700 & 47 & 1300 & 1600 & 1400 & 1400 \\
\hline Cd & 1.8 & .3 & .1 & .8 & 4.8 & 4.4 & 5.7 & $<1$ & 2.2 & 2.0 & 1.7 & 1.8 \\
\hline$\infty$ & 162 & 14 & $\ddot{2}$ & 78 & 1900 & 1900 & 2000 & $\theta$ & 260 & 310 & 250 & 280 \\
\hline$a$ & 82 & 131 & 122 & 114 & 6 & 6 & 8 & 241 & 59 & 30 & 86 & 83 \\
\hline Mo & 78 & $<4$ & $<4$ & $<4$ & 790 & 880 & 1400 & $<4$ & 234 & 450 & 173 & 95 \\
\hline $\mathbf{P b}$ & 173 & $<49$ & $\infty 0$ & 62 & 2300 & 2400 & 2400 & $<49$ & 290 & 330 & 300 & 201 \\
\hline Sr & 550 & 201 & 270 & 350 & 1500 & 1800 & 1500 & 161 & 1100 & 1500 & $11 \infty$ & 860 \\
\hline v & 400 & 360 & 360 & 360 & 720 & 780 & 710 & 340 & 530 & 600 & 460 & 410 \\
\hline $\mathbf{Y}$ & 80 & 37 & 40 & 55 & 189 & 202 & 149 & 31 & 152 & 184 & 139 & 109 \\
\hline $\mathbf{Z n}$ & 350 & 151 & 163 & 227 & 540 & 490 & 580 & 110 & 430 & 450 & 430 & 390 \\
\hline$\gamma$ & .969 & .962 & 975 & .971 & 1.004 & 979 & .980 & .973 & 985 & .985 & .985 & 985 \\
\hline Sampled & Bulk & Bulk & Bulk & Bulk & Bulk & Bulk & Bulk & Bulk & Bulk & Bulk & Bulk & Bulk \\
\hline luterval & - & - & . & $\cdot$ & - & - & - & • & - & - & - & - \\
\hline
\end{tabular}


Table 1. Composition of crusts analyzed by USGS Reston Labs (cont'd).

\begin{tabular}{|c|c|c|c|c|c|c|c|c|c|c|c|c|}
\hline Sequencell & 158016 & 158017 & 158018 & 158020 & 158021 & 158023 & 163001 & 300001 & 301001 & $302001+$ & 310001 & 315001 \\
\hline Latitude & 46.041 & 46.041 & 46.041 & 46.125 & 48.475 & 44.750 & 40.207 & -9.960 & 4.100 & -2367 & -2.177 & -7.588 \\
\hline Langinde & -130.367 & -130.367 & .130367 & -130.000 & -133.133 & -130.500 & 170.333 & -5.387 & -12.150 & -22.76 & -101.960 & -151.543 \\
\hline Depth & - & - & . & - & - & 2995 & 2037 & 1561 & 1640 & 3217 & 3108 & 1800 \\
\hline Leb: & w-230093 & W-230105 & W-230100 & W-230088 & W-230113 & W-231370 & W-222085 & W-231346 & W-222733 & W-222711 & W-231373 & W-224398 \\
\hline $\mathrm{SiO}_{2}$ (wt.\%) & 39.1 & 39.5 & 41.9 & 39.4 & 5.19 & 283 & 4.58 & 5.04 & 12.7 & 49.1 & 8.43 & 9.65 \\
\hline $\mathrm{TiO}_{2}^{2}$ & .74 & .75 & .84 & 1.17 & 1.10 & 59 & 1.08 & 1.85 & .80 & 1.59 & .43 & 1.63 \\
\hline $\mathrm{MnO}_{2}$ & 8.01 & 8.65 & 6.14 & 9.24 & 43.8 & 19.7 & 46.9 & 37.3 & 9.AS & .64 & 33.1 & 22.6 \\
\hline & 192 & 19.1 & 14.9 & 21.3 & 25.1 & 25.4 & 23.7 & 258 & 50.1 & 12.2 & 33.5 & 172 \\
\hline & 11.4 & 10.9 & 120 & 8.99 & .79 & 5.51 & .61 & 2.74 & 4.81 & 153 & .77 & 257 \\
\hline & .035 & .039 & .030 & .050 & 1.13 & .097 & 843 & 1.89 & .133 & .009 & .074 & 371 \\
\hline $\mathrm{NiO}^{4}$ & .078 & .085 & .087 & .081 & .479 & .233 & .651 & 513 & .102 & .022 & .315 & 390 \\
\hline $\mathrm{CoO}$ & .018 & .022 & .014 & .030 & .041 & .064 & .045 & .055 & .073 & .018 & .126 & .135 \\
\hline $\mathrm{CaO}$ & 7.68 & 6.88 & 8.96 & 6.05 & 3.63 & 2.43 & 3.84 & 3.74 & 2.37 & 7.69 & 3.69 & 21.3 \\
\hline $\mathrm{M}_{8} \mathrm{O}$ & 5.53 & 5.18 & 6.78 & 3.57 & 1.77 & 1.88 & 1.72 & 2.67 & 2.50 & 6.36 & 1.75 & 1.54 \\
\hline $\mathrm{Ne}_{2} \mathrm{O}$ & 298 & 3.05 & 2.85 & 2.81 & 3.27 & 3.01 & 2.53 & 3.11 & 241 & 3.53 & 2.12 & 1.93 \\
\hline$k_{2} 0$ & .62 & .71 & .43 & 81 & .61 & 1.06 & .65 & 49 & 39 & 1.25 & 40 & .74 \\
\hline $\mathrm{C}_{2}$ & 46 & .16 & .12 & .13 & .45 & .09 & so & 30 & .88 & .02 & AO & 284 \\
\hline $\mathrm{P}_{2} \mathrm{O}_{5}$ & .57 & 53 & .36 & .62 & 1.37 & .84 & 1.17 & 1.15 & 1.64 & .19 & 1.33 & 11.71 \\
\hline $\mathrm{H}_{2}^{2} \mathrm{O}+$ & 5.5 & 5.4 & 4.6 & 5.8 & 9.7 & 7.2 & 8.3 & 8.9 & 10.6 & 1.8 & 9.5 & 6.1 \\
\hline $\mathrm{H}_{2} \mathrm{O}$ & 7.1 & 85 & 4.7 & 75 & 23.0 & 12.6 & 25.7 & 20.6 & 16.3 & 3.6 & 19.3 & 11.9 \\
\hline Sum & 101.9 & 100.9 & 100.8 & 100.1 & 98.4 & 96.4 & 97.1 & 95.7 & 98.9 & 99.8 & 96.0 & 100.7 \\
\hline Ax(ppm) & 103 & 103 & 68 & 119 & 420 & 195 & 360 & 400 & 650 & 6 & 330 & 100 \\
\hline $\mathrm{Ba}$ & 1100 & 840 & 410 & 890 & 1300 & 1300 & 1500 & 1200 & 880 & 187 & 1200 & 2300 \\
\hline Cd & 9 & 1.1 & .7 & 1.3 & 4.7 & 2.2 & 3.9 & 3.7 & 18 & .6 & 1.7 & 27 \\
\hline co & 162 & 153 & 91 & 184 & 1800 & 390 & 1900 & 2500 & 930 & 35 & 186 & 830 \\
\hline$a$ & 120 & 131 & 189 & 71 & 5 & 23 & 2 & 33 & 30 & 239 & 0 & 64 \\
\hline Mo & 23 & 38 & 28 & 24 & 940 & 183 & 1200 & 500 & $\delta$ & $<4$ & 470 & 260 \\
\hline $\mathrm{PO}$ & 194 & 175 & 100 & 141 & 2500 & 310 & 2600 & 2000 & 440 & 57 & 200 & 810 \\
\hline St & 580 & 560 & 410 & 640 & 1700 & 970 & 1800 & 1600 & 740 & 249 & 1700 & 1500 \\
\hline v & 320 & 320 & 270 & 390 & 820 & 430 & 850 & 930 & 1200 & 228 & 760 & 490 \\
\hline $\mathbf{Y}$ & 80 & 79 & 59 & 91 & 195 & 149 & 202 & 239 & 143 & 25 & 235 & 790 \\
\hline $\mathbf{Z n}$ & 310 & 280 & 220 & 360 & 560 & 470 & 610 & 540 & 670 & 239 & 590 & 700 \\
\hline$\gamma$ & 907 & 977 & 978 & .985 & 1.002 & 1.023 & 1.016 & 1.030 & 997 & .988 & 1.027 & 979 \\
\hline Sampled & Bulk & Bulk & Bulk & Bulk & Bulk & Bulk & Bulk & Bulk & Bulk & Bulk & Bulk & Bulk \\
\hline Intorval & - & - & - & - & - & - & - & $0.8 \mathrm{~mm}$ & - & $<1 \mathrm{~mm}$ & $0-10 \mathrm{~mm}$ & $0-30 \mathrm{~mm}$ \\
\hline Sequencell & 315002 & 316001 & 316002 & 316003 & 325001 & 330001 & 334001 & 335001 & 337002 & 337003 & 343001 & 344001 \\
\hline Latitude & -7588 & -9.990 & -1.300 & -1.158 & -9.383 & .9 .650 & -5.750 & -5.383 & -12.933 & .13303 & -19562 & -19.975 \\
\hline Longitudo & -151.543 & -164.222 & -168.388 & -168.003 & 109.267 & 56417 & -11.350 & 97.483 & -14.252 & -15528 & -70.425 & -80.042 \\
\hline Depth & 1800 & 4430 & 6890 & 7117 & 1390 & 2800 & 2380 & 4119 & 2544 & 3199 & 658 & 3109 \\
\hline Leb* & W-231399 & W-222526 & W-222520 & W-222528 & W-222701 & W-222538 & w.222530 & W-222539 & W-231366 & W-231364 & W-224378 & W-222715 \\
\hline $\mathrm{SiO}_{2}(\mathrm{wt} . \mathrm{s})$ & 8.91 & 15.3 & 10.3 & 35.2 & 10.4 & 8.78 & 7.94 & 105 & 8.95 & 6.42 & 18.1 & 9.77 \\
\hline $\mathrm{TiO}_{2}^{2}$ & 1.92 & 1.58 & .56 & 1.88 & .82 & 1.89 & 1.66 & .94 & 1.09 & 1.25 & 2.01 & 1.74 \\
\hline $\mathrm{MnO}_{2}$ & 36.8 & 33.4 & 38.7 & 13.6 & 23.1 & 33.6 & 245 & 34.1 & 18.8 & 24.7 & 25.9 & 26.0 \\
\hline $\mathrm{Fe}_{2} \mathrm{O}_{3}^{2}$ & 21.1 & 23.1 & 26.3 & 19.7 & 33.2 & 28.0 & 342 & 32.7 & 31.6 & 37.1 & 19.3 & 31.8 \\
\hline $\mathrm{Al}_{2} \mathrm{O}_{3}$ & 200 & 4.89 & 2.49 & 9.47 & 2.13 & 2.21 & 4.34 & 2.43 & 3.91 & 2.77 & 4.98 & 2.71 \\
\hline $\mathrm{Co}_{3}^{2} \mathrm{O}_{4}$ & .695 & .537 & .072 & .138 & .224 & .953 & 811 & 210 & 348 & 495 & .568 & .638 \\
\hline $\mathrm{NHO}{ }^{*}$ & .680 & 581 & 856 & .156 & .226 & .453 & .221 & 392 & .192 & 216 & .503 & .298 \\
\hline $\mathrm{CuO}$ & 238 & .463 & .065 & .223 & .127 & .145 & .043 & .135 & .079 & .105 & .247 & .158 \\
\hline $\mathrm{CaO}$ & 6.81 & 3.55 & 3.73 & 4.99 & 8.51 & 5.87 & 5.73 & 3.05 & 112 & 4.82 & 8.29 & 5.28 \\
\hline $\mathrm{MgO}_{8}$ & 1.89 & 209 & 2.19 & 3.88 & 1.66 & 1.84 & 280 & 1.79 & 2.27 & 2.13 & 2.18 & 1.77 \\
\hline $\mathrm{Ne}_{2} \mathrm{O}$ & 247 & 2.56 & 2.88 & 2.92 & 2.56 & 249 & 2.80 & 2.16 & 1.81 & 2.40 & 2.85 & 221 \\
\hline $\mathrm{K}_{2} \mathbf{O}$ & .79 & 1.25 & .59 & .74 & 53 & .69 & .49 & .62 & .32 & .35 & 1.45 & .60 \\
\hline $\mathrm{Co}_{2}$ & 52 & 35 & .28 & .09 & 4.82 & .67 & 2.11 & .45 & 6.86 & 1.70 & 121 & 221 \\
\hline $\mathrm{P}_{2} \mathrm{O}_{5}$ & 2.91 & .93 & .80 & .56 & 1.05 & 236 & 1.39 & 91 & 1.25 & 1.36 & 4.03 & 90 \\
\hline $\mathrm{H}_{2} \mathrm{O}+$ & 9.0 & 9.1 & 8.0 & 6.6 & 7.5 & 8.4 & 73 & 8.5 & 8.8 & 8.6 & 9.3 & 10.2 \\
\hline $\mathrm{H}_{2}^{2} \mathrm{O}$ & 15.8 & 18.9 & 22.7 & 10.2 & 21.1 & 21.4 & 19.4 & 22.1 & 14.0 & 17.5 & 8.9 & 232 \\
\hline Sum & 96.8 & 99.7 & 97.9 & 100.1 & 96.8 & 98.4 & 96.4 & 98.9 & 975 & 94.5 & 101.0 & 963 \\
\hline$A(p p o n)$ & 190 & 160 & 246 & 75 & 200 & 250 & 670 & 300 & 400 & 470 & 121 & 290 \\
\hline $\mathrm{Ba}$ & 1900 & 1700 & 1200 & 490 & 1100 & 1500 & 1000 & 1300 & 980 & 1200 & 2700 & 1400 \\
\hline Cd & 3.1 & 65 & 58 & 13 & 4.7 & 3.2 & 3.6 & 32 & 3.0 & 24 & 25 & 1.8 \\
\hline co & 1200 & 860 & 246 & 310 & 580 & 2000 & 1600 & 1300 & 830 & 1300 & 990 & 1100 \\
\hline$a$ & 7 & 26 & 14 & 78 & 10 & 11 & 53 & 7 & 22 & 4 & 35 & 4 \\
\hline Mo & 510 & 280 & 440 & 74 & 250 & 340 & 260 & 440 & 244 & 330 & 250 & 182 \\
\hline $\mathrm{Pb}$ & 1500 & 790 & 200 & 400 & 620 & 1700 & 1500 & 1800 & 630 & 870 & 1000 & 1000 \\
\hline Sr & 1300 & 1200 & 1400 & 480 & 1500 & 1500 & 1500 & 1300 & 1500 & 1600 & 1200 & 1400 \\
\hline v & 570 & 510 & 560 & 450 & 610 & 690 & 1200 & 730 & 800 & 990 & 420 & 590 \\
\hline $\mathbf{Y}$ & 214 & 148 & 155 & 85 & 165 & 140 & 260 & 193 & 198 & 218 & 198 & 182 \\
\hline $\mathrm{Zn}$ & 710 & 760 & 780 & 320 & 620 & 700 & 670 & 650 & 550 & 560 & 660 & 660 \\
\hline$\gamma$ & 1.019 & .989 & 1.008 & .985 & 1.019 & 1.002 & 1.022 & .997 & 1.011 & 1.044 & .977 & 1.024 \\
\hline Sumpled & Bulk & Bulk & Bulk & Bulk & Bulk & Bulk & Bulk & Bulk & Bulk & Bulk & Bulk & Bulk \\
\hline Interval & • & - & • & • & • & • & • & • & $0-10 \mathrm{~mm}$ & $0-8 \mathrm{~mm}$ & $0.7 \mathrm{~mm}$ & $0-10 \mathrm{~mm}$ \\
\hline
\end{tabular}


Table 1. Composition of crusts analyzed by USGS Reston Labs (cont'd).

\begin{tabular}{|c|c|c|c|c|c|c|c|c|c|c|c|c|}
\hline Sequencoll & 345001 & 346001 & 347001 & $350001 \dagger$ & 350003 & 350004 & 350005 & 351001 & 351002 & 351003 & $351004 t$ & 351006 \\
\hline Latitude & -16.010 & -16.420 & -14.088 & -15.018 & -18.733 & -14.342 & -14.300 & -11.051 & .12 .874 & -10.030 & -11.052 & -14.483 \\
\hline Langitude & -95.662 & -101.332 & -110.815 & -149.032 & -140.433 & -149.625 & -149.533 & -152.097 & -150.773 & -152362 & -152.097 & -150.017 \\
\hline Depth & 4240 & 3416 & 3778 & 3350 & 1474 & 2536 & 1937 & 4250 & 3200 & 2500 & 4250 & 1082 \\
\hline Leb: & W-222522 & W-222523 & W-222524 & $W-224401$ & W.222696 & W-229848 & W-220849 & W-224399 & $W-224400$ & W-231403 & W-231401 & W-229866 \\
\hline $\mathrm{SiO}_{2}(w . \%)$ & 14.5 & 10.9 & 143 & 44.7 & 11.2 & 7.88 & 6.94 & 26.4 & 35.8 & 23.8 & 47.0 & 5.21 \\
\hline & 80 & 86 & .53 & 3.08 & 1.57 & 1.90 & 1.58 & 3.61 & 1.97 & 1.73 & 2.21 & 1.95 \\
\hline $\mathrm{MnO}_{2}$ & 48.5 & 34.6 & 18.8 & 2.11 & 25.2 & 37.0 & 39.3 & 18.2 & 9.86 & 15.7 & 20 & 39.2 \\
\hline & 19.5 & 26.5 & 42.2 & 14.7 & 34.3 & 28.8 & 25.8 & 25.7 & 16.6 & 25.0 & 12.7 & 28.9 \\
\hline $\mathrm{AL}_{2} \mathrm{O}_{3}$ & 5.69 & 2.74 & 3.13 & 15.1 & 3.50 & 1.68 & 200 & 7.37 & 10.4 & 4.82 & 14.5 & 1.34 \\
\hline $\mathrm{CO}_{3}^{2} \mathrm{O}_{4}$ & 364 & 370 & .090 & .046 & 572 & .903 & .910 & 280 & .123 & 246 & .006 & 1.28 \\
\hline $\mathrm{NiO}$ & 1.969 & .724 & 203 & .042 & .378 & A94 & .738 & 248 & 219 & .158 & .010 & .647 \\
\hline Cuo & .757 & .194 & .169 & .019 & .248 & .105 & .189 & .149 & .161 & .044 & .009 & .140 \\
\hline $\mathrm{CaO}$ & 3.44 & 3.08 & 3.50 & 10.3 & 4.58 & 4.27 & 4.43 & 2.43 & 6.31 & 3.03 & 6.09 & 4.05 \\
\hline $\mathrm{M}_{\mathrm{BO}} \mathrm{O}$ & 3.87 & 2.02 & 2.03 & 4.60 & 1.95 & 2.10 & 209 & 2.16 & 2.14 & 10.29 & 9.45 & 2.04 \\
\hline $\mathrm{Ne}_{2} \mathrm{O}$ & 3.64 & 2.61 & 2.19 & 2.67 & 2.37 & 2.28 & 2.26 & 260 & 3.11 & 2.26 & 2.98 & 247 \\
\hline $\mathbf{K}_{2} \mathbf{O}$ & 1.14 & .78 & .55 & .89 & .70 & 50 & .62 & 2.48 & 3.01 & .53 & 97 & 54 \\
\hline$\infty_{2}$ & .27 & 49 & .78 & .22 & 1.36 & .88 & .95 & .24 & 1.06 & .02 & .01 & .60 \\
\hline $\mathrm{P}_{2} \mathrm{O}_{5}$ & .74 & .86 & 1.15 & .64 & 1.27 & 1.06 & 1.01 & .77 & .30 & .80 & 31 & 1.11 \\
\hline $\mathrm{H}_{2} \mathrm{O}+$ & 11.0 & 7.4 & 9.6 & 26 & 8.8 & 8.5 & 8.7 & 8.6 & 8.1 & 8.5 & 35 & 9.0 \\
\hline $\mathbf{H}_{2} \mathrm{O}$ & 28.9 & 22.7 & 18.4 & 26 & 19.1 & 20.1 & 20.7 & 7.7 & 6.9 & 11.4 & 3.5 & 21.3 \\
\hline Sum & 116.2 & 94.0 & 99.3 & 101.7 & 98.1 & 98.4 & 97.5 & 101.2 & 99.0 & 96.9 & 100.0 & 98.5 \\
\hline$A x(p p m)$ & 183 & 270 & 290 & 16 & 300 & 300 & 227 & 65 & 46 & 169 & $<1$ & 320 \\
\hline Be & 1500 & 1100 & 1600 & 123 & 1400 & 1300 & 1400 & 850 & 470 & 850 & 197 & 1800 \\
\hline Cd & 12.8 & 4.9 & 23 & .1 & 25 & 29 & 4.2 & 1.6 & 1.2 & 1.5 & $<1$ & 3.7 \\
\hline Co & 660 & 820 & 208 & 91 & 560 & 630 & 440 & 550 & 172 & 760 & $\theta$ & 950 \\
\hline$a$ & 11 & 13 & 29 & 440 & 21 & 18 & 7 & 430 & 430 & 226 & 85 & 9 \\
\hline Mo & 480 & 360 & 123 & $<4$ & 223 & 400 & 440 & 228 & 76 & 86 & $<4$ & 440 \\
\hline $\mathbf{P b}$ & 870 & 720 & 320 & 51 & 1000 & 910 & 1100 & 420 & 183 & 1100 & 36 & 1800 \\
\hline Sr & 1000 & 1200 & 1100 & 490 & 1400 & 1800 & 1600 & 790 & 530 & 850 & 280 & 1800 \\
\hline V & 520 & 600 & 720 & 350 & 700 & 650 & 630 & 430 & 280 & 470 & 300 & 830 \\
\hline $\mathbf{Y}$ & 141 & 155 & 147 & 49 & 198 & 213 & 189 & 173 & 236 & 124 & 21 & 178 \\
\hline$Z_{n}$ & 1400 & 700 & 740 & 175 & 840 & 680 & 720 & 620 & 370 & 470 & 145 & 880 \\
\hline$\gamma$ & .849 & 1.049 & 993 & .970 & 1.006 & 1.002 & 1.011 & .974 & .996 & 1.017 & .986 & 1.001 \\
\hline Sempled & Bulk & Bulk & Bulk & Bulk & Bulk & Bulk & Bulk & Bulk & Bulk & Bulk & Bulk & Bulk \\
\hline Interval & $\cdot$ & - & $\cdot$ & $<1 \mathrm{~mm}$ & - & - & • & $0-20 \mathrm{mon}$ & $0-25 \mathrm{mmn}$ & - & - & - \\
\hline Sequenoell & 351016 & 353001 & 353002 & 353006 & 362001 & 362002 & 364003 & $365001+7$ & 365002 & $365005 \ddagger$ & 373001 & 373002 \\
\hline Latituds & -17.867 & -16.203 & -16.548 & -16.338 & -17.883 & -18.617 & -18.800 & -18.033 & -17.583 & -13.528 & .22 .100 & -21.888 \\
\hline Longitude & -154.133 & -176.257 & -178.442 & -173.247 & 99.100 & 99.067 & 78.150 & 65.617 & 65.650 & 66.642 & -11.450 & -11.875 \\
\hline Depth & 1994 & 1352 & 1499 & 2340 & 5588 & 6030 & 4934 & 1423 & 2720 & 2154 & $\mathbf{2 3 0 0}$ & 1480 \\
\hline Lab* & W-220868 & W-222559 & $w-222560$ & W-222529 & W-231342 & W-2229879 & W-2229870 & W-222689 & $w-222690$ & W-222694 & W-222729 & W-222726 \\
\hline $\mathrm{SiO}_{2}(w . \%)$ & 16.6 & 16.2 & 16.1 & 5.06 & 38.3 & 26.4 & 13.4 & 37.0 & 11.3 & .95 & 120 & 5.43 \\
\hline $\mathrm{TiO}_{2}^{2}$ & 2.65 & 1.34 & 1.23 & .10 & 1.46 & 1.55 & 1.12 & 1.67 & 1.01 & .02 & 1.96 & 1.41 \\
\hline $\mathrm{MnO}_{2}$ & 25.4 & 21.6 & 19.9 & .83 & 14.2 & 23.9 & 35.8 & .69 & 24.8 & .33 & 18.4 & 29.2 \\
\hline $\mathrm{Fe}_{2} \mathrm{O}_{3}$ & 30.6 & 34.1 & 34.5 & 29.1 & 19.8 & 23.1 & 25.2 & 13.2 & 35.9 & .83 & 40.9 & 32.1 \\
\hline $\mathrm{Al}_{2} \mathrm{O}_{3}$ & 5.23 & 6.32 & 6.19 & 2.65 & 8.60 & 8.12 & 3.71 & 11.7 & 2.37 & .20 & 3.71 & 243 \\
\hline $\mathrm{CO}_{3} \mathrm{O}_{4}$ & .533 & 313 & .171 & .004 & .151 & .269 & 389 & .036 & .262 & .006 & .445 & .671 \\
\hline $\mathrm{NiO}^{7}$ & .296 & 293 & 243 & .012 & .155 & 281 & .632 & .016 & .245 & .008 & .117 & 257 \\
\hline Cuo & .169 & .067 & .091 & .003 & .093 & .189 & .233 & .009 & .143 & .003 & .058 & .032 \\
\hline $\mathrm{CaO}$ & 286 & 4.65 & 5.51 & 24.4 & 2.16 & 2.18 & 2.92 & 15.5 & 5.27 & 53.2 & 2.88 & 6.01 \\
\hline $\mathrm{M}_{8} \mathrm{O}$ & 1.95 & 2.33 & 2.06 & 7.71 & 2.65 & 1.89 & 2.16 & 5.64 & 1.81 & .75 & 2.15 & 2.43 \\
\hline $\mathrm{Na}_{2} \mathrm{O}$ & 257 & 2.81 & 2.54 & 51 & 2.29 & 224 & 3.12 & 3.30 & 2.10 & 1.06 & 4.00 & 3.02 \\
\hline $\mathbf{K}_{2} \mathrm{O}$ & 90 & 94 & .61 & 51 & 1.60 & 1.16 & .75 & 94 & .49 & .08 & 23 & 27 \\
\hline$\infty_{2}$ & .26 & 1.28 & 1.78 & 18.2 & .07 & .14 & 31 & 5.32 & 2.05 & 40.9 & .47 & 2.15 \\
\hline $\mathrm{P}_{2} \mathrm{O}_{5}$ & .87 & 1.23 & 1.14 & 6.56 & 51 & .64 & .74 & 21 & 1.15 & $<.05$ & 1.17 & 1.30 \\
\hline $\mathrm{H}_{2}^{2} \mathrm{O}+$ & 8.7 & 6.3 & 5.6 & 6.1 & 5.5 & 8.5 & 8.7 & 3.2 & 9.2 & 1.7 & 10.0 & 10.5 \\
\hline $\mathrm{H}_{2} \mathrm{O}$ & 18.3 & 21.7 & 21.3 & 32 & 9.9 & 13.9 & 19.5 & 6.0 & 220 & .6 & 17.4 & 20.8 \\
\hline Sum & 99.6 & 99.8 & 97.7 & 1018 & 97.6 & 100.5 & 99.2 & 98.4 & 98.1 & 100.1 & 98.5 & 97.2 \\
\hline Ax(ppm) & 220 & 310 & 290 & 420 & 144 & 186 & 236 & 20 & 330 & 8 & 480 & 470 \\
\hline Ba & 1600 & 980 & 1000 & 54 & 1400 & 1400 & 1100 & 69 & 1200 & 28 & 1200 & 900 \\
\hline Cd & 3.3 & 4.5 & 4.8 & 1.5 & 8 & 24 & 4.6 & .2 & 1.9 & 23 & 1.9 & 3.4 \\
\hline Co & 1300 & 260 & 241 & 44 & 2300 & 1400 & 2000 & 25 & 600 & $\theta$ & 1500 & 1300 \\
\hline$c$ & 20 & 31 & 32 & 720 & 86 & 14 & 9 & 138 & 22 & 12 & 18 & 6 \\
\hline Mo & 208 & 217 & 191 & $<4$ & 260 & 340 & 420 & $<4$ & 270 & $<4$ & 206 & 250 \\
\hline $\mathbf{P b}$ & 1200 & 1100 & 750 & 186 & 830 & 590 & 1600 & $<52$ & 630 & $<49$ & 1000 & 970 \\
\hline Sx & 1200 & 1300 & 1300 & 490 & 640 & 820 & 1100 & 390 & 1500 & 800 & 1100 & 1600 \\
\hline $\mathbf{v}$ & 600 & 660 & 600 & 500 & 550 & 580 & 560 & 234 & 670 & 11 & 1000 & 1000 \\
\hline $\mathbf{Y}$ & 171 & 195 & 178 & 33 & 37 & 64 & 174 & 32 & 180 & 15 & 182 & 215 \\
\hline $\mathbf{Z n}$ & 580 & 690 & 670 & 155 & 420 & 550 & 560 & 181 & 590 & 63 & 580 & 560 \\
\hline$\gamma$ & .990 & .988 & 1.009 & .969 & 1.010 & .981 & 994 & 1.002 & 1.005 & .985 & 1.001 & 1.014 \\
\hline Sampled & Bulk & Bulk & Bulk & Bulk & Bulk & Bulk & Bulk & Bulk & Bulk & Bulk & Balk & Bulk \\
\hline Interval & - & $0-1 \mathrm{~mm}$ & $0-10 \mathrm{~mm}$ & - & $0-25 \mathrm{~mm}$ & - & - & • & • & - & • & $\cdot$ \\
\hline
\end{tabular}


Table 1. Composition of crusts analyzed by USGS Reston Labs (cont'd).

\begin{tabular}{|c|c|c|c|c|c|c|c|c|c|c|c|c|}
\hline Sequencell & 373003 & 373004 & 375001 & 375002 & 375003 & 376001 & $379001 *$ & 380001 & 380002 & 3800003 & 380004 & 381001 \\
\hline Latimds & -22278 & .22 .922 & -20.967 & -20.567 & -21.017 & -28.417 & -23.800 & -21.253 & -25.643 & .21 .070 & -21.283 & -25.443 \\
\hline Langitude & -12072 & -13.510 & -31.800 & -31.800 & -31.900 & -40.750 & .78 .483 & -80.925 & -83.522 & -81.913 & -80.960 & -97.565 \\
\hline Dopth & 2000 & 1700 & 4141 & 3715 & 4331 & 4388 & 392 & $\mathbf{2 3 0 0}$ & 2067 & 2560 & 3282 & 3721 \\
\hline Lab* & W-2222727 & W-222725 & W-231347 & W-231363 & W-231360 & W-2229872 & W-222580 & W-2220712 & w-224377 & W. 222714 & W-222713 & W-222716 \\
\hline $\mathrm{SiO}_{2}(w t . \$)$ & 6.28 & 8.10 & 29.1 & 21.4 & 29.2 & 20.9 & 28.1 & 12.8 & 8.99 & 14.2 & 10.4 & 8.21 \\
\hline & 1.39 & 1.43 & 3.15 & 1.02 & 1.84 & 1.59 & 259 & 1.37 & 2.11 & 1.34 & 1.38 & 1.21 \\
\hline & 28.4 & 25.2 & 10.4 & 18.1 & 16.4 & 19.4 & 13.1 & 18.8 & 34.3 & 23.5 & 32.0 & 15.1 \\
\hline & 33.5 & 33.0 & 23.7 & 29.7 & 22.5 & 288 & 15.2 & 30.4 & 27.0 & 30.1 & 27.2 & 31.1 \\
\hline & 1.94 & 3.08 & 9.51 & 9.43 & 7.81 & 6.22 & 10.5 & 3.75 & 283 & 4.04 & 2.36 & 221 \\
\hline & .646 & 535 & .198 & .355 & .203 & .482 & 241 & $A 13$ & 1.54 & 531 & .661 & 230 \\
\hline $\mathrm{NiO}^{4}$ & 228 & .203 & .171 & .260 & .234 & .270 & .211 & 241 & 396 & .304 & .484 & .184 \\
\hline $\mathrm{CuO}$ & .083 & .046 & .073 & .170 & .073 & .103 & .018 & .128 & .026 & .121 & .081 & .113 \\
\hline $\mathrm{CaO}$ & 6.28 & 6.70 & 5.32 & 1.55 & 3.23 & 4.03 & 6.35 & 11.1 & 3.87 & 5.11 & 5.14 & 15.2 \\
\hline MgO & 2.10 & 242 & 4.49 & 2.71 & 3.05 & 227 & 3.67 & 1.80 & 2.10 & 1.67 & 2.83 & 1.50 \\
\hline $\mathrm{Na}_{2} \mathrm{O}$ & 297 & 2.79 & 265 & 1.98 & 294 & 3.54 & 6.53 & 265 & 3.34 & 2.52 & 3.54 & 205 \\
\hline $\mathrm{K}_{2} \mathrm{O}$ & 34 & .35 & 1.63 & 1.07 & 1.62 & 88 & 87 & .87 & .58 & .92 & 55 & 39 \\
\hline & 282 & - & .50 & .17 & .22 & 1.42 & 31 & 6.95 & .50 & 2.14 & 1.25 & 10.7 \\
\hline & 1.20 & 1.21 & .72 & .88 & .68 & 97 & 1.50 & .99 & 1.45 & .92 & 1.02 & .83 \\
\hline & 10.5 & 11.9 & 4.3 & 9.3 & 6.5 & 8.0 & 6.5 & 4.8 & 8.4 & 10.6 & 4.6 & 7.5 \\
\hline $\mathrm{H}_{2}^{2} \mathrm{O}$ & 22.0 & 18.5 & 10.6 & 11.8 & 12.9 & 15.2 & 9.7 & 20.9 & 13.2 & 20.5 & 23.8 & 17.2 \\
\hline Sum & 98.8 & • & 95.9 & 98.1 & 96.4 & 98.9 & 95.8 & 97.1 & 97.4 & 98.0 & 93.4 & 96.6 \\
\hline Aхppm) & 380 & 390 & 168 & 270 & 172 & 340 & 111 & 228 & 310 & 260 & 290 & 270 \\
\hline Ba & 940 & 930 & 590 & 880 & 720 & 870 & 510 & 1300 & 1000 & 1100 & 1000 & 990 \\
\hline Cd & 26 & 3.3 & 1.7 & 22 & 20 & 3.2 & 4.7 & 1.8 & 3.3 & 23 & 3.1 & 1.3 \\
\hline co & 1400 & 1400 & 1000 & 1800 & 1300 & 1400 & 270 & 630 & 1100 & 720 & 710 & 310 \\
\hline$a$ & 5 & 16 & 246 & 24 & 115 & 27 & 7 & 10 & 15 & 13 & 37 & 4 \\
\hline Mo & 244 & 209 & 73 & 270 & 126 & 165 & 133 & 152 & 500 & 176 & 249 & 81 \\
\hline $\mathbf{P b}$ & 960 & 990 & 580 & 670 & 930 & 1100 & 340 & 1000 & 3200 & 1100 & 920 & 540 \\
\hline Sr & 1500 & 1500 & 590 & 600 & no & 980 & 840 & 1300 & 1700 & 1300 & 1400 & 1300 \\
\hline $\mathbf{v}$ & 970 & 910 & 500 & 650 & 460 & 730 & 330 & 520 & 840 & 550 & 600 & 540 \\
\hline $\mathbf{Y}$ & 192 & 196 & 87 & 91 & 106 & 142 & 122 & 139 & 219 & 139 & 171 & 133 \\
\hline $2 n$ & 470 & 560 & 400 & 440 & 400 & 480 & 390 & 560 & 550 & 790 & 660 & 560 \\
\hline$\gamma$ & 998 & - & 1.029 & 1.005 & 1.022 & .997 & 1.030 & 1.016 & 1.012 & 1.006 & 1.055 & 1.021 \\
\hline Sampled & Bulk & Bulk & Porrous & Bulk & Bulk & Bulk & Bulk & Bulk & Bulk & Bulk & Bulk & Bulk \\
\hline homerval & • & - & • & $0-7 \mathrm{~mm}$ & $0-8 \mathrm{~mm}$ & - & $0-1 m=$ & $0.20 \mathrm{~mm}$ & $0.3 \mathrm{~cm}$ & $0.11 \mathrm{~mm}$ & $0.3 \mathrm{~mm}$ & $0-20 \mathrm{~mm}$ \\
\hline Sequencell & $383001 \uparrow$ & 389001 & 390001 & 391001 & 397002 & 400001 & 401001 & $401002 \uparrow$ & 401003 & 402001 & 409003 & 409005 \\
\hline Letitude & -26.498 & -27.467 & .24 .017 & -23.033 & -26.200 & -23.207 & -27.983 & .29 .300 & -29.300 & -23.433 & -37.833 & -37.187 \\
\hline Longimde & -110.337 & -174.362 & 170.050 & 169.600 & -106.300 & 74.983 & 60.800 & 60.650 & 60.617 & 50.817 & -17.142 & -17.515 \\
\hline Depth & 2446 & 3593 & 1175 & 1143 & 3003 & 4520 & 5698 & 5699 & 4688 & 4993 & 2723 & 2454 \\
\hline Lab* & W-224376 & W-222525 & W-222698 & W-222699 & W-231365 & W-222537 & W-222691 & W.222692 & $w-222693$ & W-231354 & W. 222724 & W-222735 \\
\hline $\mathrm{SiO}_{2}$ (wt.S) & 48.5 & 15.5 & 271 & 4.96 & 14.4 & 20.5 & 28.8 & 40.4 & 20.7 & $17 A$ & 17.7 & 10.5 \\
\hline $\mathrm{TOO}_{2}$ & 270 & 1.98 & 1.59 & 1.34 & 2.26 & .66 & 1.73 & $A 1$ & 1.73 & 2.01 & 1.45 & .93 \\
\hline & .64 & 27.9 & 40.9 & 36.4 & 22.8 & 29.8 & 15.1 & 2.58 & 20.4 & 26.2 & 16.8 & 20.8 \\
\hline & 12.9 & 25.8 & 29.6 & 31.1 & 30.5 & 20.6 & 223 & 10.2 & 26.9 & 30.0 & 34.1 & 38.1 \\
\hline $\mathrm{AL}_{2}^{2} \mathrm{O}_{3}$ & 14.7 & 5.36 & 83 & 2.33 & 4.25 & 6.77 & 8.06 & 5.69 & 3.45 & 3.74 & 7.77 & 3.35 \\
\hline $\mathrm{CO}_{3}^{2} \mathrm{O}_{4}$ & .007 & 824 & 1.11 & .849 & .363 & .180 & .298 & .039 & 341 & 575 & .313 & .253 \\
\hline $\mathrm{NiO}^{4}$ & .015 & .321 & 575 & .567 & .340 & 1.007 & .138 & 231 & 243 & .292 & .200 & .189 \\
\hline $\mathrm{CuO}$ & .010 & .158 & .058 & .081 & .099 & .615 & .058 & .063 & .115 & .107 & .044 & .061 \\
\hline $\mathrm{CaO}$ & 9.75 & 4.69 & 4.27 & 4.09 & 4.93 & 223 & 3.82 & 1.36 & 2.25 & 278 & 4.13 & 5.38 \\
\hline $\mathrm{M}_{8} \mathrm{O}$ & 6.70 & 2.11 & 2.16 & 2.53 & 1.87 & 3.32 & 6.31 & 22.82 & 8.50 & 1.68 & 2.59 & 2.20 \\
\hline $\mathrm{Na}_{2} \mathrm{O}$ & 3.13 & 2.57 & 239 & 2.14 & 2.34 & 266 & 2.62 & 2.23 & 1.73 & 205 & 2.21 & 256 \\
\hline$K_{2} \mathrm{O}$ & 50 & 1.25 & .45 & 44 & .73 & 1.40 & .85 & .98 & .53 & .68 & .76 & .49 \\
\hline$\infty_{2}$ & .13 & 50 & .83 & .93 & 1.51 & .18 & .20 & .02 & $\boldsymbol{2}$ & .30 & 39 & 223 \\
\hline $\mathrm{P}_{2} \mathrm{O}_{3}$ & 32 & 1.76 & 1.18 & 1.40 & 90 & .63 & 56 & .15 & .68 & .94 & 1.25 & 1.45 \\
\hline $\mathrm{H}_{2} \mathrm{O}+$ & 1.4 & 8.6 & 9.4 & 8.9 & 8.8 & 8.6 & 7.8 & 9.8 & 11.6 & 8.6 & 7.3 & 9.6 \\
\hline $\mathrm{H}_{2} \mathrm{O}$ & 1.0 & 20.7 & 22.6 & 21.4 & 13.8 & 16.6 & 13.3 & 6.3 & 16.1 & 17.1 & 17.3 & 19.4 \\
\hline Sum & 101.4 & 99.3 & 98.0 & 98.1 & 96.1 & 99.2 & 98.7 & 97.1 & 99.4 & 97.3 & 96.9 & 98.2 \\
\hline Ax(ppm) & $<1$ & 214 & 430 & 420 & 197 & 156 & 150 & 25 & 155 & 270 & 340 & 480 \\
\hline $\mathrm{Ba}$ & 92 & 1200 & 1600 & 1400 & 1100 & 950 & 750 & 117 & 1100 & 1400 & 920 & 1200 \\
\hline Cd & $<1$ & 24 & 3.5 & 4.8 & 3.8 & 8.4 & 1.4 & .2 & 2.7 & 24 & 34 & 2.5 \\
\hline $\mathbf{C o}$ & 36 & 1100 & 790 & 600 & 560 & 670 & 1300 & 117 & 1300 & 2300 & no & 810 \\
\hline$\alpha$ & 212 & 30 & 18 & 18 & 21 & 29 & 400 & 2500 & 540 & 13 & 71 & 19 \\
\hline Mo & $<4$ & 280 & 610 & 430 & 151 & 280 & 127 & $<4$ & 79 & 290 & 133 & 174 \\
\hline Pb & $<4$ & 840 & 2700 & 2700 & 580 & 740 & 910 & 101 & 1200 & 1400 & 1500 & 1000 \\
\hline Sx & 300 & 1200 & 2100 & 1700 & 1200 & 700 & 730 & 117 & 980 & 1300 & 1100 & 1400 \\
\hline $\mathbf{v}$ & 310 & 550 & 960 & 900 & 480 & 400 & 450 & 149 & 490 & 630 & no & 1000 \\
\hline $\mathbf{Y}$ & 35 & 330 & 207 & 204 & 186 & 132 & 99 & 17 & 119 & 157 & 157 & 186 \\
\hline $\mathbf{Z n}$ & 131 & 540 & 890 & 890 & 600 & 820 & 360 & 213 & 560 & 540 & 740 & 770 \\
\hline$\gamma$ & .973 & 993 & 1.006 & 1.005 & 1.026 & 994 & 999 & 1.016 & 991 & 1.014 & 1.017 & 1.005 \\
\hline Sempled & Bulk & Bulk & Bulk & Bulk & Bulk & Bulk & Bulk & Bulk & Bult & Bulk & Bulk & Bulk \\
\hline Intorval & $0-4 m m$ & - & • & • & O-lum & - & • & • & - & $0-10 \mathrm{~mm}$ & • & • \\
\hline
\end{tabular}


Table 1. Composition of crusts analyzed by USGS Reston Labs (cont'd).

\begin{tabular}{|c|c|c|c|c|c|c|c|c|c|c|c|c|}
\hline Soquencoll & 409006 & 411001 & 411002 & 411003 & 416002 & 416003 & 417001 & 421001 & 423001 & 424001 & 424002 & 439001 \\
\hline Latitude & .36 .073 & -30.500 & -38.433 & .30 .433 & -33.000 & -32.967 & .39 .850 & .35 .967 & .38 .967 & .38 .643 & .36 .950 & -36.750 \\
\hline Longinde & -18.083 & -35.933 & -36.017 & -36.017 & -85.900 & -88.000 & -96.867 & -134.300 & -150.367 & -167.885 & -169.833 & 44.767 \\
\hline Depth & 2623 & 1357 & 1445 & 1522 & 3883 & 3640 & 3639 & 4727 & 5066 & 1418 & 775 & 2479 \\
\hline Lab* & W.222723 & W-220865 & W-2229874 & W.229873 & W-2222661 & W.2203662 & W-2220663 & W.222673 & W.222670 & $w-222527$ & W-229856 & W-231358 \\
\hline $\mathrm{SiO}_{2}$ (wt.\%) & 10.2 & 3.45 & 3.54 & 16.5 & 13.3 & 16.5 & 8.63 & 42.7 & 23.3 & 3.79 & 21.2 & 14.6 \\
\hline $\mathrm{TiO}_{2}^{2}$ & 1.20 & 1.77 & 2.30 & 2.18 & 57 & 83 & 32 & 2.99 & .85 & 1.37 & 1.89 & 1.16 \\
\hline $\mathrm{MnO}_{2}^{2}$ & 21.9 & 39.4 & 40.3 & 28.0 & 38.2 & 29.6 & 45.3 & 5.34 & 368 & 36.9 & 23.8 & 30.9 \\
\hline $\mathrm{Fo}_{2} \mathrm{O}_{3}$ & 35.8 & 31.0 & 28.2 & 23.6 & 13.9 & 24.7 & 13.9 & 19.4 & 11.7 & 26.3 & 21.3 & 17.2 \\
\hline $\mathrm{Al}_{2} \mathrm{O}_{3}$ & 3.07 & 1.35 & 1.60 & 6.34 & 6.05 & 5.36 & 3.80 & 11.7 & 7.17 & 1.62 & 7.20 & 5.78 \\
\hline $\mathrm{Co}_{3}^{2} \mathrm{O}_{4}$ & 360 & 1.08 & 1.84 & .882 & .187 & .193 & .155 & .067 & .355 & 962 & .458 & 520 \\
\hline $\mathrm{NiO}^{4}$ & .176 & 455 & 467 & 500 & 1.454 & .782 & 1.956 & .094 & 1.223 & 562 & .713 & 1.315 \\
\hline $\mathrm{CoO}$ & .050 & .070 & .048 & .093 & .730 & 340 & .695 & .055 & .639 & .062 & .140 & 352 \\
\hline $\mathrm{CaO}$ & 6.70 & 3.82 & 3.91 & 4.46 & 4,48 & 2.65 & 281 & 7.22 & 2.16 & 6.18 & 3.81 & 5.66 \\
\hline MEO & 1.90 & 208 & 222 & 3.30 & 3.03 & 274 & 3.53 & 3.32 & 3.15 & 2.36 & 2.94 & 3.91 \\
\hline $\mathrm{N}_{2} \mathrm{O}$ & 215 & 226 & 225 & 246 & 2.06 & 1.89 & 260 & 3.27 & 285 & 3.67 & 2.39 & 2.61 \\
\hline $\mathrm{K}_{2} \mathbf{O}$ & 55 & 54 & .56 & 1.69 & .70 & 1.14 & 84 & 88 & .96 & .53 & 1.74 & 1.07 \\
\hline$\infty_{2}$ & 3.53 & .61 & .59 & 53 & 38 & 35 & 37 & .17 & .30 & .79 & .67 & .49 \\
\hline $\mathrm{P}_{2} \mathrm{O}_{5}^{2}$ & 1.33 & 1.17 & 1.13 & 1.67 & 1.44 & .79 & 54 & 82 & .44 & 2.63 & 1.54 & 2.91 \\
\hline $\mathrm{H}_{2} \mathrm{O}+$ & 8.3 & 8.8 & 9.2 & 8.2 & 9.8 & 9.9 & 11.2 & 24 & 5.2 & 10.2 & 10.4 & 8.0 \\
\hline $\mathrm{H}_{2} \mathrm{O}$ & 20.6 & 21.7 & 21.0 & 13.5 & 125 & 15.4 & 15.4 & 5.1 & 15.7 & 20.8 & 10.8 & 11.0 \\
\hline Sum & 97.3 & 97.9 & 98.2 & 100.5 & 96.2 & 97.8 & 96.5 & 100.5 & 97.0 & 98.0 & 100.2 & 96.5 \\
\hline$A(p p m)$ & 470 & 450 & 390 & 290 & 149 & 236 & 166 & 42 & 116 & 320 & 247 & 180 \\
\hline Be & 1100 & 2200 & 1600 & 1700 & 1300 & 1300 & 1300 & 221 & 900 & 1800 & 1200 & 1200 \\
\hline Cd & 25 & 3.2 & 4.8 & 5.7 & 10.3 & 4.7 & 13.0 & .6 & 7.6 & 4.7 & 63 & 7.1 \\
\hline$\infty$ & 1000 & 2400 & 2300 & 1600 & 370 & 520 & 310 & 300 & 790 & 1600 & 670 & 820 \\
\hline$\alpha$ & 15 & 24 & 14 & 60 & 13 & 23 & 12 & 7 & 8 & 14 & 67 & 49 \\
\hline Mo & 227 & 570 & 490 & 360 & 450 & 380 & 560 & 25 & 450 & 580 & 260 & 310 \\
\hline $\mathbf{P b}$ & 1600 & 3200 & 3200 & 2100 & 410 & 630 & 350 & 200 & 970 & 2800 & 1200 & 1200 \\
\hline Sr & 1500 & 2000 & 1900 & 1400 & 890 & 1000 & 830 & 300 & 640 & 1900 & 900 & 850 \\
\hline$v$ & 940 & 1300 & 940 & 740 & 450 & 570 & 480 & 420 & 390 & 910 & 570 & 480 \\
\hline $\mathbf{Y}$ & 189 & 100 & 203 & 127 & 171 & 166 & 109 & 126 & 96 & 189 & 179 & 191 \\
\hline $\mathrm{Zn}$ & 740 & 720 & 630 & 670 & 1300 & 820 & 1700 & 280 & 950 & 900 & 820 & 1100 \\
\hline$\gamma$ & 1.014 & 1.007 & 1.004 & .981 & 1.025 & 1.008 & 1.021 & .981 & 1.016 & 1.006 & .984 & 1.022 \\
\hline Samplod & Balk & Bulk & Bulk & Bulk & Bulk & Bulk & Bulk & Bulk & Bulk & Bulk & Bulk & Balk \\
\hline Interval & . & 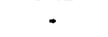 & - & - & $0-9 \operatorname{man}$ & $0-8 \mathrm{~mm}$ & $0-11 \mathrm{~mm}$ & $<1 \mathrm{~mm}$ & - & - & . & $0-15 \mathrm{~mm}$ \\
\hline Soquencoll & 441001 & 441002 & 441003 & 442001 & 448001 & 448002 & 449001 & 449002 & 449003 & 449004 & 452001 & 452002 \\
\hline Latitude & -39.121 & -39.771 & -37.867 & -37.550 & -49.567 & -49.600 & 44.350 & -47.933 & -48.467 & -47.967 & -49.950 & -42083 \\
\hline Longitude & 24.054 & 24.755 & 26.683 & 18.100 & -48.400 & -47.567 & -57.350 & .57 .100 & -55.233 & -57.017 & 89.983 & -86.050 \\
\hline Depth & 4775 & 2963 & 2942 & 3147 & 3929 & 4080 & 4605 & 2620 & 2915 & 3338 & 4390 & 3054 \\
\hline Lab* & W-2229883 & W-220885 & W-231356 & W-229851 & W-231350 & W. 231353 & W-2229855 & W-229867 & W-231355 & W.231357 & W-222675 & W-222674 \\
\hline $\mathrm{SiO}_{2}(\mathrm{wt} . \%)$ & 26.3 & 14.5 & 19.4 & 13.1 & 40.7 & 31.0 & 37.2 & 17.2 & 18.6 & 24.4 & 19.8 & 14.5 \\
\hline & 1.16 & 1.18 & 1.16 & 1.40 & 55 & 1.19 & .48 & 1.12 & 1.25 & .99 & 1.58 & .88 \\
\hline $\mathrm{MnO}_{2}^{2}$ & 29.2 & 28.6 & 24.3 & 28.1 & 19.6 & 19.9 & 19.2 & 29.4 & 23.7 & 27.8 & 224 & 20.8 \\
\hline $\mathrm{Fo}{ }_{2} \mathrm{O}_{3}$ & 21.4 & 33.3 & 26.6 & 34.1 & 16.6 & 22.6 & 194 & 30.9 & 33.9 & 23.1 & 30.8 & 384 \\
\hline $\mathrm{Al}_{2} \mathrm{O}_{3}^{3}$ & 4.30 & 218 & 4.24 & 1.82 & 6.06 & 647 & 7.10 & 242 & 3.27 & 3.70 & 5.22 & 3.24 \\
\hline $\mathrm{Co}_{3} \mathrm{O}_{4}$ & 392 & 557 & 506 & .605 & .057 & 204 & .114 & 399 & .270 & 372 & .246 & 200 \\
\hline $\mathrm{NiO}^{4}$ & .641 & 237 & 517 & .236 & .376 & 220 & .408 & 311 & .297 & 522 & .260 & .203 \\
\hline $\mathrm{CuO}$ & .165 & .062 & .145 & .039 & .096 & .074 & 318 & .070 & .095 & .157 & .150 & .109 \\
\hline co & 230 & 3.50 & 4.29 & 3.59 & 1.99 & 3.05 & 1.79 & 296 & 253 & 2.42 & 2.69 & 257 \\
\hline $\mathrm{MgO}_{8}$ & 2.19 & 1.73 & 2.46 & 2.05 & 2.03 & 1.97 & 275 & 1.80 & 1.65 & 2.40 & 1.71 & 1.79 \\
\hline $\mathrm{N}_{2} \mathrm{O}$ & 2.28 & 1.82 & 2.07 & 238 & 274 & 298 & 280 & 297 & 2.04 & 2.07 & 252 & 2.21 \\
\hline $\mathbf{K}_{2} \mathbf{O}$ & 1.10 & .60 & .90 & .25 & 156 & 1.14 & 1.92 & .63 & .51 & 1.34 & 1.42 & 53 \\
\hline$\infty_{2}$ & .25 & 87 & .69 & .62 & .08 & .21 & .10 & .32 & .28 & .22 & .41 & .37 \\
\hline $\mathrm{P}_{2} \mathrm{O}_{5}$ & 55 & 1.05 & 200 & 1.22 & .33 & .63 & 43 & .95 & .99 & .84 & .91 & 1.15 \\
\hline $\mathrm{H}_{2}^{2} \mathrm{O}+$ & 7.3 & 9.3 & 7.1 & 8.4 & 5.8 & 1.3 & 64 & 8.5 & 7.9 & 8.0 & 7.2 & 10.5 \\
\hline $\mathrm{H}_{2} \mathrm{O}$ & 16.6 & 19.3 & 13.9 & 19.0 & 8.7 & 13.3 & 9.5 & 18.1 & 14.4 & 12.2 & 16.8 & 18.4 \\
\hline Sum & 99.6 & 99.5 & 963 & 97.9 & 98.6 & 92.9 & 100.5 & 100.0 & 97.3 & 98.3 & 97.4 & 97.4 \\
\hline$A x$ (ppm) & 156 & 400 & 290 & 470 & 94 & 196 & 84 & 320 & 330 & 205 & 240 & 390 \\
\hline $\mathrm{Ba}$ & 1200 & 1400 & 1300 & 1400 & 960 & 990 & 1300 & 1600 & 1800 & 1700 & 1100 & 1300 \\
\hline Cd & 5.3 & 3.7 & 3.5 & 3.1 & 8.4 & 20 & 5.1 & 2.6 & 2.2 & 5.7 & 20 & 1.5 \\
\hline c. & 1900 & 1700 & 1300 & 1900 & 270 & 830 & 166 & 1000 & 1000 & 720 & 880 & 540 \\
\hline c & 16 & 11 & 16 & 12 & 19 & 50 & 21 & 7 & 13 & 43 & 13 & 8 \\
\hline Mo & 310 & 480 & 310 & 430 & 208 & 300 & 166 & 550 & 470 & 330 & 144 & 310 \\
\hline $\mathbf{P b}$ & 1300 & 2200 & 1500 & 2200 & 320 & 1300 & 177 & 1800 & 1400 & 1400 & 900 & 560 \\
\hline Sr & 910 & 1500 & 1100 & 1600 & 530 & 990 & 450 & 1500 & 1300 & 990 & 1100 & 1300 \\
\hline v & 500 & 920 & 700 & 960 & 310 & 510 & 280 & 810 & 840 & 540 & 610 & 780 \\
\hline$Y$ & 95 & 198 & 197 & 210 & 54 & 115 & 44 & 183 & 175 & 114 & 156 & 184 \\
\hline $\mathbf{Z n}$ & 650 & 730 & 720 & 680 & 710 & 470 & 630 & 710 & 810 & 880 & 640 & 660 \\
\hline $\bar{\gamma}$ & 990 & 991 & 1.024 & 1.007 & 1.000 & 1.061 & .981 & 986 & 1.014 & 1.003 & 1.013 & 1.013 \\
\hline Samplod & Bulk & Bulk & Bulk & Bulk & Bulk & Bulk & Bulk & Bulk & Bulk & Bulk & Bulk & Bulk \\
\hline Interval & - & . & $0-20 \mathrm{~mm}$ & - & $0-20 \mathrm{~mm}$ & $0.25 \mathrm{~mm}$ & - & - & $0.35 \mathrm{~mm}$ & $0-25 \mathrm{~mm}$ & $0-16 \mathrm{~mm}$ & $0-15 \operatorname{mon}$ \\
\hline
\end{tabular}


Table 1. Composition of crusts analyzed by USGS Reston Labs (cont' $d$ ).

\begin{tabular}{|c|c|c|c|c|c|c|c|c|c|c|c|c|}
\hline Sequancoll & 455001 & 457001 & $462001 t$ & 477001 & 479001 & $483001 \uparrow$ & 483003 & $483004 t$ & $483005 t$ & 484001 & 484002 & 484003 \\
\hline Latimde & -40.133 & -43.000 & -49.667 & -41.264 & -43.117 & -59.867 & -58.708 & -52.875 & -59.417 & .59 .017 & .53 .058 & -58.003 \\
\hline Longitude & -119.583 & -139.933 & 178.942 & 28.314 & 2.117 & .32 .317 & -33.500 & .33 .858 & -31.208 & -48.992 & -48.950 & -45.067 \\
\hline Depth & 4252 & 5141 & 1344 & 4314 & 3967 & 613 & 3328 & 2853 & 2396 & 3868 & 3041 & 2861 \\
\hline Lab" & W-2222664 & W-222071 & W-222077 & W-229886 & W-231345 & W-2222645 & W-222033 & W.2222634 & W-2220644 & W-222630 & W.222631 & W-222632 \\
\hline $\mathrm{SiO}_{2}$ (wt.\$) & 13.6 & 14.5 & 48.0 & 22.1 & 11.9 & 50.6 & 55.9 & 64.6 & 669 & 49.6 & 29.7 & 14.2 \\
\hline $\mathrm{TiO}_{2}$ & 99 & 1.56 & 3.05 & 1.67 & 1.89 & 1.76 & .64 & .69 & .65 & .79 & .79 & 1.37 \\
\hline & 37.3 & 37.4 & 55 & 27.1 & 30.5 & .21 & 7.45 & 80 & 1.74 & 123 & 23.8 & 30.7 \\
\hline $\mathrm{Fo}_{2} \mathrm{O}_{3}^{2}$ & 19.7 & 20.4 & 13.4 & 24.1 & 31.5 & 121 & 14.1 & 258 & 7.28 & 15.4 & 229 & 29.3 \\
\hline $\mathrm{Al}_{2}^{2} \mathrm{O}_{3}$ & 4.27 & 4.72 & 15.7 & 4.55 & 3.25 & 11.0 & 8.20 & 18.2 & 10.2 & 7.95 & 5.08 & 4.75 \\
\hline $\mathrm{CO}_{3} \mathrm{O}$ & 340 & .494 & .008 & 583 & .464 & .005 & .042 & .003 & .012 & .091 & .221 & 200 \\
\hline Nó & 1.165 & 1.002 & .012 & .373 & 326 & .022 & .127 & .019 & .019 & .288 & 501 & 586 \\
\hline Co & .47 & 485 & .015 & .112 & .090 & .006 & .086 & .020 & .020 & .123 & .096 & 280 \\
\hline Co & 266 & 245 & 10.1 & 2.62 & 3.02 & 9.85 & 207 & .68 & 3.70 & 207 & 2.72 & 3.13 \\
\hline $\mathrm{M}_{8 \mathrm{O}}$ & 276 & 269 & 3.87 & 2.05 & 1.90 & 8.00 & 1.63 & 1.33 & 230 & 1.66 & 1.88 & 2.23 \\
\hline $\mathrm{Ne}_{2} \mathrm{O}$ & 2.62 & 250 & 3.05 & 3.02 & 218 & 247 & 273 & 1.85 & 1.26 & 3.03 & 2.92 & 1.82 \\
\hline $\mathrm{K}_{2} \mathrm{O}$ & 1.00 & .75 & 1.35 & 96 & .75 & 38 & 206 & 4.08 & 1.94 & 1.51 & 1.02 & 54 \\
\hline $\mathrm{Co}_{2}$ & $M$ & AS & .02 & 34 & .41 & .05 & .10 & .01 & .04 & .12 & .25 & A1 \\
\hline $\mathrm{P}_{2} \mathrm{O}$ & .63 & .63 & .47 & .70 & .95 & .15 & .29 & $<\infty$ & .54 & .40 & .64 & 97 \\
\hline & 6.9 & 6.5 & 1.1 & 7.9 & 1.2 & 4.2 & 3.9 & 4.1 & 2.2 & 5.0 & 6.5 & 8.5 \\
\hline $\mathrm{H}_{2}^{2} \mathrm{O}$ & 15.9 & 20.0 & 1.5 & 18.2 & 17.9 & 5 & 6.5 & 1.2 & 18 & 7.3 & 13.7 & 19.6 \\
\hline Sum & 94.7 & 96.5 & 100.7 & 98.3 & 90.4 & 1008 & 99.4 & 98.9 & 98.8 & 100.3 & 99.0 & 99.0 \\
\hline Ax(ppm) & 226 & 200 & 2 & 245 & 290 & $<1$ & 60 & $<1$ & 15 & 84 & 197 & 270 \\
\hline Ba & 1200 & 1200 & 370 & 1000 & 1500 & 52 & 920 & 930 & 6000 & 780 & 1400 & 1600 \\
\hline Cd & 6.3 & 6.0 & $<.1$ & 3.1 & 26 & 3 & 21 & 3 & $A$ & 2.3 & 7.4 & 5.5 \\
\hline c. & 1300 & 1600 & 90 & 2800 & 1900 & $\theta$ & 225 & 71 & 83 & 370 & 590 & 920 \\
\hline$a$ & 13 & 6 & 52 & 16 & 9 & 480 & 26 & 37 & 32 & 30 & 16 & 8 \\
\hline Mo & 420 & 390 & $<4$ & 280 & 410 & $<$ & 37 & $<$ & $<4$ & 84 & 250 & 300 \\
\hline $\mathbf{P b}$ & 1000 & 1400 & 74 & 1600 & 1700 & 10 & 400 & 33 & 101 & 680 & 960 & 860 \\
\hline Sr & 1000 & 1000 & 560 & 1100 & 1500 & 88 & 410 & 243 & 183 & 490 & 980 & 1200 \\
\hline $\mathbf{v}$ & 520 & 500 & 310 & 510 & 760 & 320 & 203 & 132 & 92 & 280 & 560 & 630 \\
\hline $\mathbf{Y}$ & 143 & 138 & 31 & 135 & 195 & 24 & 47 & 23 & 28 & 46 & 100 & 199 \\
\hline $\mathbf{Z n}$ & 900 & 750 & 142 & 530 & 550 & 171 & 400 & 122 & 163 & 560 & 850 & 750 \\
\hline$\gamma$ & 1.041 & 1.022 & 979 & 1.004 & 1.091 & .979 & .992 & .997 & .998 & .983 & .996 & 996 \\
\hline $\begin{array}{l}\text { Sempled } \\
\text { Intervel }\end{array}$ & $\begin{array}{c}\text { Bulk } \\
\text { O-10mm }\end{array}$ & $\begin{array}{c}\text { Bulk } \\
\text { O-8mm }\end{array}$ & $\begin{array}{c}\text { Bulk } \\
\text { 0-2mmm }\end{array}$ & $\begin{array}{c}\text { Bulk } \\
\text { - }\end{array}$ & $\begin{array}{c}\text { Bulk } \\
0.35 \mathrm{~mm}\end{array}$ & $\begin{array}{c}\text { Bulk } \\
-\end{array}$ & $\begin{array}{c}\text { Bulk } \\
\text { O-Smm }\end{array}$ & $\begin{array}{c}\text { Bulk } \\
\text { - }\end{array}$ & $\underset{0-7 \mathrm{~mm}}{\text { Bulk }}$ & $\begin{array}{c}\text { Bulk } \\
\text { O-4mm }\end{array}$ & $\begin{array}{c}\text { Bulk } \\
0-9 \mathrm{~mm}\end{array}$ & $\begin{array}{c}\text { Bulk } \\
\text { O-10mm }\end{array}$ \\
\hline Sequencoli & $485001 \uparrow$ & $485002 f$ & 485003 & 485004 & 485005 & $485006 t$ & $485007 f$ & 485008 & 485009 & $486001 \uparrow$ & 486002 & 487001 \\
\hline Letitude & -57.117 & -58.100 & -58.150 & -53.942 & -55.100 & -57.767 & -56.995 & .55 .902 & -56.250 & -55.300 & .59 .033 & -59.027 \\
\hline Longitnde & -59.117 & -59.400 & .59 .883 & -55.917 & -55.833 & -55.967 & -59.083 & -58.860 & -58.333 & -64.755 & -67.300 & -74.633 \\
\hline Depth & 4115 & 4358 & 3917 & 1887 & 2890 & 4084 & 4023 & 4023 & 4115 & 2149 & 3475 & 4697 \\
\hline Lab: & W-222622 & W-222623 & W-222624 & W-222625 & W-2222626 & $w-222627$ & W-222628 & W-222629 & W-222021 & W-2222642 & W-222618 & W-222639 \\
\hline $\mathrm{SiO}_{2}$ (wt.s) & 62.6 & 66.5 & 521 & 36.6 & 28.2 & 61.0 & 55.4 & 45.6 & 527 & 59.6 & 45.1 & 45.5 \\
\hline $\mathrm{TiO}_{2}^{2}$ & .48 & 57 & .98 & 81 & .78 & .75 & .76 & 81 & .82 & .76 & 1.31 & 1.21 \\
\hline $\mathrm{MnO}_{2}$ & 3.12 & 38 & 5.74 & 14.1 & 21.5 & 279 & 295 & 8.38 & 5.39 & .04 & 11.0 & 10.1 \\
\hline $\mathrm{Fe}_{2} \mathrm{O}_{3}^{2}$ & 8.59 & 5.78 & 16.3 & 22.8 & 25.9 & 11.2 & 18.6 & 22.1 & 16.6 & 8.86 & 16.7 & 18.4 \\
\hline $\mathrm{Al}_{2}^{2} \mathrm{O}_{3}$ & 11.0 & 14.5 & 9.27 & 6.06 & 3.96 & 11.0 & 9.27 & 7.92 & 9.86 & 165 & 9.27 & 8.54 \\
\hline $\mathrm{CO}_{3}^{2} \mathrm{O}_{4}^{3}$ & .038 & .006 & .080 & .292 & .255 & .033 & .042 & .111 & .059 & .001 & .135 & .117 \\
\hline NiÓ & .028 & .008 & .023 & .331 & .357 & .029 & .023 & .125 & .068 & .005 & .096 & .084 \\
\hline CuO & .017 & .006 & .028 & .083 & .056 & .025 & .033 & .098 & .052 & .004 & .059 & .059 \\
\hline $\mathrm{CaO}$ & 2.13 & .72 & 2.45 & 1.74 & 250 & 246 & 2.52 & 261 & 2.94 & 1.20 & 3.52 & 298 \\
\hline M8O & 89 & 1.98 & 1.66 & 2.87 & 2.15 & 1.50 & 1.50 & 1.99 & 1.75 & 2.85 & 2.24 & 1.85 \\
\hline $\mathrm{Nz}_{2} \mathrm{O}$ & 3.73 & 1.93 & 3.04 & 268 & 2.77 & 3.23 & 292 & 298 & 3.30 & 2.59 & 2.99 & 2.94 \\
\hline $\mathrm{K}_{2} \mathbf{O}$ & 2,00 & 3.14 & 1.49 & 1.76 & 1.07 & 1.96 & 1.73 & 1.26 & 1.26 & 1.71 & 1.68 & 1.63 \\
\hline$\infty_{2}$ & .08 & .02 & .17 & .19 & 30 & .09 & .14 & .14 & .10 & .38 & .26 & .18 \\
\hline $\mathrm{P}_{2} \mathrm{O}_{5}$ & .19 & .07 & .42 & .07 & .72 & .24 & .34 & A3 & .32 & .10 & AS & 45 \\
\hline $\mathrm{H}_{2} \mathrm{O}+$ & 4.6 & 3.7 & 5.1 & 63 & 64 & 3.0 & 3.5 & 5.0 & 38 & 5.1 & 4.3 & 5.0 \\
\hline $\mathrm{H}_{2}^{2} \mathrm{O}$ & 3.5 & 1.1 & 6.2 & 11.5 & 14.5 & 4.0 & 6.2 & 9.3 & 6.1 & 1.6 & 8.3 & 9.3 \\
\hline $\sin$ & 99.4 & 99.4 & 98.9 & 97.3 & 96.9 & 99.4 & 99.8 & 99.5 & 99.1 & 99.7 & 99.1 & 99.1 \\
\hline Ax(ppm) & 54 & 9 & 107 & 215 & 260 & 50 & 107 & 132 & 117 & 12 & 92 & 110 \\
\hline $\mathrm{Be}$ & 670 & 520 & 630 & 1000 & 1300 & 590 & 590 & 750 & 590 & 450 & 850 & 800 \\
\hline Cd & $A$ & .2 & 1.0 & 3.7 & 6.1 & 4 & .6 & .1 & 23 & .1 & 15 & 1.3 \\
\hline co & 270 & 99 & 510 & 640 & 750 & 250 & 330 & 430 & 330 & so & 490 & 510 \\
\hline$a$ & 12 & 45 & 33 & so & 18 & 21 & 29 & 35 & 30 & 62 & 40 & 34 \\
\hline Mo & $<4$ & $<$ & $<4$ & 158 & 234 & $<4$ & $<4$ & 28 & 20 & $<4$ & 19 & 38 \\
\hline $\mathbf{P b}$ & 390 & 111 & 800 & 1500 & 1400 & 410 & 690 & 840 & 540 & 23 & 800 & 860 \\
\hline Sr & 390 & 95 & 640 & 700 & 1000 & 410 & 440 & 600 & 510 & 183 & 770 & 770 \\
\hline $\mathbf{v}$ & 145 & 142 & 300 & 570 & 600 & 188 & 330 & 400 & 300 & 163 & 290 & 320 \\
\hline $\mathbf{Y}$ & 34 & 26 & 57 & 87 & 109 & 34 & 44 & 54 & 44 & 18 & 68 & 70 \\
\hline $\mathbf{2 n}$ & 197 & 142 & 300 & 660 & 820 & 208 & 260 & 410 & 280 & 122 & 400 & 340 \\
\hline$\gamma$ & .992 & 992 & 997 & 1.013 & 1.017 & $\$ 92$ & .988 & .991 & .995 & 989 & .994 & .995 \\
\hline Sampled & Bulk & Bulk & Bulk & Bulk & Bulk & Bulk & Bulk & Bulk & Bulk & Bulk & Bulk & Bulk \\
\hline Interval & $0-5 \mathrm{~mm}$ & $0-3 \mathrm{~mm}$ & $0-13 \mathrm{~mm}$ & $0-10 \mathrm{~mm}$ & $0-21 \mathrm{~mm}$ & $0-2 m m$ & $0-5 \mathrm{~mm}$ & $0.3 \mathrm{~mm}$ & $0-6 \mathrm{~mm}$ & - & $0-8 \mathrm{~mm}$ & $0-7 \mathrm{~mm}$ \\
\hline
\end{tabular}


Table 1. Composition of crusts analyzed by USGS Reston Labs (cont'd).

\begin{tabular}{|c|c|c|c|c|c|c|c|c|c|c|c|c|}
\hline Sequenoul & 487002 & 487003 & 487004 & 487005 & 488001 & 489001 & 489002 & 489004 & 489005 & 489006 & 490001 & 491001 \\
\hline Latituds & -59.017 & -57.083 & -57.083 & -58.293 & .58 .102 & -57.737 & -50.483 & -52.017 & -59.017 & -56.867 & -57.850 & $-\$ 4.100$ \\
\hline Longitude & -70.750 & -70.983 & -70.983 & $-78,460$ & -82872 & -90.782 & -94.917 & -99.950 & -99.593 & -99.417 & -108.420 & -119.317 \\
\hline Depth & 3841 & 3790 & 3790 & 4938 & 4206 & 5093 & 4664 & 2479 & 4901 & 4737 & 4682 & 3219 \\
\hline Lab" & W-222619 & W-222079 & W-222620 & $w-222641$ & W.222635 & W-220646 & W-222076 & W-222660 & $W-222650$ & W-222657 & W-222652 & W-222665 \\
\hline $\mathrm{SiO}_{2}$ (w.s) & 49.9 & 48.6 & 427 & 51.8 & 24.6 & 35.2 & 18.4 & 30.8 & 522 & 48.4 & 23.9 & 43.7 \\
\hline & 1.00 & 1.00 & .99 & $\pi$ & 1.49 & 1.15 & 1.14 & 99 & .78 & 2.21 & 1.49 & 1.05 \\
\hline & 9.44 & 10.6 & 13.6 & 10.0 & 23.4 & 20.0 & 34.5 & 23.2 & 11.0 & 4.78 & 27.3 & 3.90 \\
\hline $\mathrm{Fe}_{2} \mathrm{O}_{3}^{2}$ & 16.0 & 15.2 & 18.1 & 11.7 & 27.9 & 19.2 & 20.1 & 16.6 & 10.4 & 11.7 & 25.7 & 13.2 \\
\hline $\mathrm{Al}_{2}^{2} \mathrm{O}_{3}^{3}$ & 8.61 & 9.92 & 7.72 & 9.97 & 4.52 & 7.20 & 4.86 & 5.59 & 9.85 & 127 & 3.47 & 16.6 \\
\hline $\mathrm{CO}_{3}^{2} \mathrm{O}$ & .096 & .116 & .122 & .073 & .321 & .148 & 234 & .131 & .061 & .020 & .236 & .041 \\
\hline NIO & .097 & .167 & 309 & .151 & .363 & .288 & 920 & .603 & 221 & .134 & .410 & .038 \\
\hline CuO & .064 & .073 & .105 & .084 & .186 & .164 & 583 & .356 & .121 & .092 & .186 & .043 \\
\hline $\mathrm{ClO}$ & 3.04 & 2.30 & 2.95 & 3.04 & 2.43 & 247 & 2.40 & 248 & 280 & 5.89 & 258 & 8.69 \\
\hline $\mathrm{MgO}_{\mathrm{g}}$ & 1.56 & 1.72 & 1.72 & 152 & 1.65 & 1.80 & 2.44 & 2.55 & 1.53 & 3.07 & 1.58 & 284 \\
\hline $\mathrm{Ne}_{2} \mathrm{O}$ & 3.02 & 3.61 & 3.07 & 3.44 & 2.66 & 3.27 & 270 & 3.20 & 3.80 & 3.47 & 269 & 225 \\
\hline $\mathbf{K}_{2}^{2} \mathbf{O}$ & 1.53 & 250 & 138 & 1.65 & 1.03 & 1.74 & 1.03 & 1.57 & 1.96 & 1.98 & 1.21 & 1.03 \\
\hline$\infty_{2}$ & .04 & .20 & .19 & .13 & 20 & .17 & 34 & 40 & .09 & .68 & 29 & .49 \\
\hline $\mathrm{P}_{2} \mathrm{O}_{5}$ & .37 & .30 & .43 & $2 \pi$ & .65 & .41 & 48 & .49 & .21 & .70 & .60 & .18 \\
\hline $\mathrm{H}_{2} \mathrm{O}+$ & 4.3 & 3.9 & 4.7 & 4.2 & 7.1 & 3.7 & 6.7 & 7.1 & 3.6 & 1.8 & 6.7 & 3.1 \\
\hline $\mathrm{H}_{2} \mathrm{O}$ & 7.8 & 8.6 & 9.4 & 7.1 & 19.4 & 16.0 & 18.4 & 15.5 & 7.9 & 4.9 & 19.3 & 6.6 \\
\hline Sum & 99.1 & 100.2 & 98.2 & 98.8 & 98.4 & 96.9 & 96.9 & 96.1 & 98.6 & 97.6 & 98.3 & 97.1 \\
\hline$A(p p m)$ & 100 & 88 & 110 & 62 & 161 & 131 & 123 & 102 & 48 & 17 & 186 & 58 \\
\hline Ba & 720 & 830 & 940 & 670 & 1500 & 950 & 1200 & 1400 & 710 & 800 & 1200 & 520 \\
\hline Cd & 1.2 & 1.4 & 28 & 1.6 & 3.2 & 26 & 5.9 & 3.9 & 1.1 & 1.1 & 27 & 3 \\
\hline$\omega$ & 430 & 390 & 450 & 300 & 1100 & 610 & 830 & 460 & 270 & 168 & 870 & 171 \\
\hline c & 32 & 23 & 20 & 32 & 10 & 57 & 4 & 19 & 27 & 53 & 11 & 97 \\
\hline Mo & 14 & 56 & 60 & 38 & 107 & 106 & 260 & 142 & 43 & 18 & 149 & $<4$ \\
\hline $\mathrm{Pb}$ & 650 & 680 & 920 & 530 & 1200 & 1000 & 960 & 600 & 460 & 179 & 1200 & 270 \\
\hline Sr & 660 & 690 & 740 & 600 & 1100 & 850 & 980 & 770 & 610 & 770 & 1200 & 400 \\
\hline $\mathbf{V}$ & 200 & 260 & 330 & 205 & 430 & 300 & 380 & 300 & 163 & 200 & 460 & 150 \\
\hline $\mathbf{Y}$ & 58 & 58 & 64 & so & 137 & 87 & 112 & 89 & 45 & 43 & 123 & 28 \\
\hline $\mathbf{z n}$ & 400 & 350 & 470 & 290 & 670 & 460 & 890 & 600 & 330 & 290 & 630 & 290 \\
\hline $\boldsymbol{\gamma}$ & 995 & 984 & 1.004 & .998 & 1.002 & 1.017 & 1.017 & 1.026 & 1.000 & 1.010 & 1.003 & 1.016 \\
\hline Sampled & Bulk & Bulk & Bulk & Bulk & Bulk & Bulk & Bulk & Bulk & Bulk & Top & Bulk & Bulk \\
\hline Intorval & $0-8 \mathrm{mmn}$ & $0.8 \mathrm{~mm}$ & $0.1500 m$ & $0.7 \mathrm{~mm}$ & $0-8 \mathrm{~mm}$ & $0-4 \mathrm{~mm}$ & $0.8 \mathrm{~mm}$ & $0-6 \mathrm{~mm}$ & $0.9 \mathrm{~mm}$ & $0-1 \mathrm{~mm}$ & $0.5 \mathrm{~mm}$ & $0.1 \mathrm{~mm}$ \\
\hline Sequenowill & 491002 & 494001 & 501001 & $518001+$ & 523001 & 523002 & 523003 & $523004 t$ & 525001 & 525002 & 525003 & 525004 \\
\hline Latituds & -57.150 & -59.800 & -57.338 & -60.610 & -62.037 & -61.045 & -61.963 & -60.093 & -61.450 & -61.033 & -62200 & -61.700 \\
\hline Longitude & -119.550 & -144.750 & 149.678 & -29.978 & -75.172 & -75.063 & -79.083 & -75.288 & -94.967 & -95.033 & -94.767 & -99.867 \\
\hline Depth & 4517 & 3259 & 2000 & 1079 & 4389 & 4471 & 4700 & 4590 & 4800 & 4993 & 4898 & 4893 \\
\hline Lab\# & W-2222653 & W-2222659 & W-222078 & W-2222643 & $w-222636$ & W-222037 & W-2222640 & W.222638 & W-222667 & W-222649 & W-222655 & W-222658 \\
\hline $\mathrm{SiO}_{2}(\mathrm{wt} . \%)$ & 44.3 & 40.9 & 28.2 & 68.2 & 420 & 45.2 & 40.2 & 67.0 & 33.9 & 564 & 44.9 & 63.5 \\
\hline $\mathrm{TiO}_{2}^{2}$ & .62 & .72 & 1.39 & .65 & 1.19 & 1.06 & 1.29 & 33 & 1.41 & .73 & .84 & 59 \\
\hline $\mathrm{MmO}_{2}$ & 18.6 & 23.6 & 27.2 & 22 & 13.7 & 11.3 & 14.1 & .11 & 20.6 & 7.99 & 17.5 & 5.94 \\
\hline $\mathrm{Pe}_{2} \mathrm{O}_{3}$ & 10.7 & 12.2 & 21.7 & 3.22 & 17.4 & 16.7 & 18.8 & 3.97 & 21.2 & 9.52 & 11.5 & 7.45 \\
\hline $\mathrm{Al}_{2} \mathrm{O}_{3}$ & 7.18 & 5.47 & 3.56 & 16.5 & 8.30 & 9.05 & 7.86 & 14.8 & 6.14 & 10.4 & 9.33 & 11.8 \\
\hline & .061 & .080 & 209 & .005 & .179 & .120 & 230 & $<\infty 01$ & 221 & .059 & .111 & .040 \\
\hline $\mathrm{NiO}$ & 594 & .863 & .749 & .008 & .224 & .132 & .127 & .001 & 236 & .096 & .419 & .133 \\
\hline $\mathrm{CuO}$ & .435 & .487 & .324 & .004 & .133 & .093 & .058 & .001 & .138 & .082 & .261 & .099 \\
\hline $\mathrm{ClO}$ & 289 & 202 & 264 & 35 & 2.84 & 296 & 242 & .29 & 2.60 & 2.43 & 2.29 & 204 \\
\hline MgO & 2.20 & 1.51 & 1.82 & 1.30 & 1.80 & 1.55 & 1.86 & .12 & 1.52 & 1.39 & 1.44 & 1.18 \\
\hline $\mathrm{N}_{2} \mathrm{O}$ & 3.91 & 3.34 & 271 & 2.66 & 299 & 3.23 & 3.01 & 5.71 & 290 & 3.97 & 3.62 & 282 \\
\hline $\mathbf{k}_{2} \mathbf{O}$ & 1.62 & 214 & 1.19 & 3.33 & 1.57 & 1.68 & 1.78 & 4.71 & 1.40 & 2.19 & 1.96 & 284 \\
\hline$\infty_{2}$ & .14 & .30 & .31 & .04 & .18 & .14 & .26 & .05 & .32 & .10 & .14 & .04 \\
\hline $\mathrm{P}_{2} \mathrm{O}_{5}$ & .15 & .22 & .43 & .11 & .40 & 36 & .49 & .07 & .45 & .21 & .28 & .21 \\
\hline $\mathrm{H}_{2}^{2} \mathrm{O}+$ & 4.8 & 4.6 & 5.4 & 3.2 & 5.3 & 4.0 & 5.6 & 1.3 & 5.8 & 3.0 & 4.5 & 1.5 \\
\hline $\mathrm{H}_{2}^{2} \mathrm{O}$ & 7.9 & 10.1 & 15.1 & 5 & 8.9 & 10.2 & 11.1 & .3 & 13.8 & 6.9 & 8.9 & 4.1 \\
\hline Sum & 98.1 & 98.5 & 97.8 & 99.8 & 98.3 & 97.6 & 98.1 & 98.4 & 98.8 & 98.5 & 99.1 & 100.2 \\
\hline$A x(p p m)$ & 35 & 51 & 108 & 4 & 103 & 94 & 135 & 3 & 128 & 47 & 62 & 22 \\
\hline $\mathrm{Ba}$ & 990 & 1300 & 1200 & 620 & 1100 & 940 & 800 & 610 & 1000 & 780 & 990 & 650 \\
\hline Cd & 4.2 & 5.9 & 4.8 & 2 & 3.7 & 20 & 1.7 & .6 & 1.9 & 9 & 3.7 & 8 \\
\hline$\infty$ & 217 & 380 & 790 & 101 & 590 & 490 & 720 & 201 & 800 & 280 & 400 & 177 \\
\hline$a$ & 21 & 16 & 20 & 52 & 31 & 32 & 56 & 0 & 14 & 23 & 14 & 19 \\
\hline Mo & 96 & 134 & 110 & $<4$ & 44 & 43 & 53 & $<4$ & 87 & 25 & 121 & 16 \\
\hline $\mathbf{P b}$ & 300 & 590 & 1300 & 68 & 970 & 840 & 1100 & 65 & 1300 & 450 & 700 & 330 \\
\hline St & 490 & 600 & 1000 & 89 & 800 & 770 & 780 & 74 & 1000 & 550 & 630 & 440 \\
\hline $\mathbf{v}$ & 174 & 211 & 320 & 111 & 310 & 280 & 350 & 10 & 350 & 140 & 209 & 115 \\
\hline $\mathbf{Y}$ & 36 & 56 & 85 & 15 & 74 & 70 & 81 & 37 & 95 & 42 & 55 & 32 \\
\hline $\mathbf{z n}$ & 650 & 1200 & 1000 & 141 & 440 & 320 & 380 & 80 & 480 & 215 & 440 & 219 \\
\hline$\gamma$ & 1.005 & 1.001 & 1.008 & .987 & 1.003 & 1.010 & 1.005 & 1.002 & 998 & 1.001 & .995 & .984 \\
\hline Sampled & Bulk & Bulk & Bulk & Bulk & Bulk & Bulk & Bulk & Bulk & Bulk & Bulk & Bulk & Bulk \\
\hline Interval & $0.3 \mathrm{~mm}$ & $0-6 \mathrm{cms}$ & $0-7 \mathrm{~mm}$ & - & $0.3 \mathrm{~mm}$ & $0-6 \mathrm{~mm}$ & $0-1 \mathrm{~mm}$ & - & $0.8 \mathrm{~mm}$ & $0.2 \mathrm{~mm}$ & $0-10 \mathrm{~mm}$ & $<1 \mathrm{~mm}$ \\
\hline
\end{tabular}


Table 1. Composition of crusts analyzed by USGS Reston Labs (cont'd).

\begin{tabular}{|c|c|c|c|c|c|c|c|}
\hline Sequencoll & 526001 & 526002 & 527001 & 528001 & 528002 & 528003 & 529001 \\
\hline Latimds & -61.400 & -61.100 & $-60,400$ & -63.608 & -65.617 & -61.183 & -64.050 \\
\hline Longitude & -101.233 & -104.967 & -115.017 & -129.897 & -123.917 & -120.333 & -135.000 \\
\hline Depth & 4911 & - & 5040 & 4819 & - & 4987 & - \\
\hline Leb" & W-2220668 & W-220561 & W-222069 & W-222648 & W-222647 & W-222666 & W-220654 \\
\hline $\mathrm{SiO}_{2}(w t . \%)$ & 52.3 & 45.6 & 38.5 & 45.3 & 53.2 & 40.0 & 39.2 \\
\hline & .77 & 94 & 1.20 & 93 & .71 & 1.13 & 1.36 \\
\hline $\mathrm{MmO}_{2}$ & 122 & 15.1 & 19.5 & 15.5 & 9.54 & 18.6 & 16.3 \\
\hline $\mathrm{Po}_{2} \mathrm{O}_{3}$ & 10.7 & 14.4 & 18.1 & 14.3 & 9.67 & 179 & 19.4 \\
\hline & 10.0 & 7.94 & 6.80 & 8.63 & 11.2 & 6.74 & 7.06 \\
\hline $\mathrm{CO}_{3}^{2} \mathrm{O}_{4}$ & .080 & .117 & .158 & .114 & .066 & .132 & .185 \\
\hline $\mathrm{NBO}{ }^{4}$ & 239 & 239 & 340 & .283 & .215 & .264 & .231 \\
\hline $\mathrm{CaO}$ & .152 & .152 & .160 & .167 & .146 & .158 & .140 \\
\hline $\mathrm{ClO}$ & 232 & 223 & 2.60 & 2.35 & 2.50 & 242 & 206 \\
\hline M\&O & 1.32 & 1.41 & 1.69 & 144 & 1.42 & 1.66 & 1.56 \\
\hline $\mathrm{Ne}, \mathrm{O}$ & 353 & 3.32 & 290 & 3.56 & 3.70 & 3.11 & 3.05 \\
\hline K,o & 226 & 1.88 & 1.82 & 2.20 & 2.22 & 1.66 & 1.80 \\
\hline $\mathrm{CO}_{2}$ & .18 & .15 & .27 & .16 & .07 & .25 & .18 \\
\hline $\mathrm{P}_{2} \mathrm{O}_{5}$ & .20 & 30 & .37 & 28 & .19 & 34 & 14 \\
\hline $\mathrm{H}_{2} \mathrm{O}+$ & 23 & 4.6 & 4.1 & 4.7 & 3.7 & 4.4 & 59 \\
\hline $\mathrm{H}_{2} \mathrm{O}$ & 9.4 & 9.6 & 13.8 & 10.2 & 5.4 & 13.1 & 11.7 \\
\hline Sum & 98.6 & 98.4 & 98.4 & 100.0 & 98.5 & 98.8 & 98.9 \\
\hline Ax(ppen) & 42 & 75 & 95 & 69 & 36 & 85 & 111 \\
\hline $\mathrm{Ba}$ & 760 & 850 & 970 & 870 & 760 & 870 & $11 \infty 0$ \\
\hline Cd & 1.3 & 1.5 & 22 & 21 & 1.4 & 1.7 & 1.7 \\
\hline$c$ & 290 & 440 & 640 & 460 & 250 & 560 & 820 \\
\hline$a$ & 19 & 19 & 19 & 18 & 18 & 27 & 20 \\
\hline Mo & 43 & 68 & 107 & 66 & 38 & 74 & 56 \\
\hline Pb & 620 & 860 & 1100 & 850 & 520 & 990 & 1400 \\
\hline Sr & 600 & 720 & 880 & 710 & 570 & 810 & 880 \\
\hline $\mathbf{v}$ & 155 & 221 & 300 & 200 & 137 & 250 & 290 \\
\hline $\mathbf{Y}$ & 46 & 64 & 77 & 59 & 40 & 74 & 83 \\
\hline$z_{n}$ & 310 & 370 & 460 & 380 & 250 & 440 & 460 \\
\hline$\gamma$ & 999 & 1.002 & 1.002 & .986 & 1.001 & 998 & .997 \\
\hline $\begin{array}{l}\text { Samplod } \\
\text { tnforval }\end{array}$ & $\begin{array}{c}\text { Bulk } \\
0-9 \operatorname{mmn}\end{array}$ & $\underset{0-7 \mathrm{~mm}}{\text { Bulk }}$ & $\begin{array}{c}\text { Bulk } \\
\text { 0-10mm }\end{array}$ & $\underset{\text { O-lmm }}{\text { Bulk }}$ & $\underset{0-3 \mathrm{~mm}}{\text { Bulk }}$ & $\begin{array}{c}\text { Bulk } \\
\text { 0-10mm }\end{array}$ & $\begin{array}{c}\text { Bulk } \\
\text { O-1mmin }\end{array}$ \\
\hline
\end{tabular}


Table 2. Composition of ferromanganese crusts analyzed by USGS Woods Hole analytical laboratories

\begin{tabular}{|c|c|c|c|c|c|c|c|c|c|}
\hline $\begin{array}{l}\text { Soquencoll } \\
\text { Latitude }\end{array}$ & $\begin{array}{c}017002 \\
5872\end{array}$ & $\begin{array}{c}017003 \\
9.333\end{array}$ & $\begin{array}{c}017004 \\
8.330\end{array}$ & $\begin{array}{c}017006 \\
8.414\end{array}$ & $\begin{array}{c}017008 \\
8.414\end{array}$ & $\begin{array}{c}017010 \\
8.398\end{array}$ & $\begin{array}{c}017012 \\
8.353\end{array}$ & $\begin{array}{c}017014 \\
8.308\end{array}$ & $\begin{array}{c}017016 \\
8.308\end{array}$ \\
\hline Longituds & -160.848 & -163.167 & -164.362 & -164.289 & $-164,289$ & -164.359 & -164.317 & -164346 & -164.346 \\
\hline Depth & 1604 & 1563 & 1519 & 1850 & 1850 & 2400 & 1150 & 1400 & 1400 \\
\hline Labu & W-222564 & W-222565 & W-222566 & 38 & 40 & 42 & 44 & 46 & 48 \\
\hline Si(wt.\%) & 1.82 & 3.25 & 1.22 & 2.77 & 3.14 & 4.01 & 1.27 & 1.25 & 1.36 \\
\hline $\mathbf{T i}$ & 1.32 & 1.16 & .74 & 1.12 & 1.40 & 1.07 & 81 & .90 & 1.23 \\
\hline Mn & 27.0 & 29.9 & 27.3 & 27.2 & 24.6 & 22.6 & 35.2 & 31.2 & 32.7 \\
\hline Po & 14.1 & 14.2 & 9.94 & 16.3 & 18.9 & 17.7 & 9.14 & 134 & 12.7 \\
\hline A & 54 & 1.11 & .45 & .59 & 1.15 & 1.01 & $A 7$ & .27 & .49 \\
\hline Co & .496 & 1.19 & .865 & 1.05 & 925 & 993 & 256 & 1.22 & 1.93 \\
\hline $\mathrm{Ni}$ & .482 & 800 & 1.036 & 539 & .384 & .412 & 887 & .691 & .756 \\
\hline $\mathrm{Ca}$ & 8.80 & 4.40 & 9.22 & 2.54 & 2.55 & 271 & 271 & 273 & 2.67 \\
\hline $\mathbf{M g}$ & 1.33 & 1.66 & 1.95 & 1.10 & 1.12 & 1.02 & 1.67 & 1.09 & 1.12 \\
\hline $\mathrm{K}$ & .47 & .69 & 53 & .49 & .49 & .54 & .72 & .49 & 50 \\
\hline $\mathbf{P}$ & 2.61 & 1.08 & 3.17 & .40 & .44 & .56 & .44 & .38 & .40 \\
\hline $\mathrm{H}_{2} \mathrm{O}$ & 25.3 & 28.7 & 17.9 & 18.4 & 11.4 & 5.3 & 120 & 10.3 & 24.0 \\
\hline $50^{\circ} \mathrm{C}$ & - & - & - & - & $10 A$ & 10.8 & 7.2 & 13.9 & 11.1 \\
\hline $1000^{\circ} \mathrm{C}$ & - & - & - & - & 8.9 & 6.9 & 8.0 & 9.8 & 10.1 \\
\hline Sampled & Bulk & Bulk & Bulk & Top & Bulk & Bulk & Top & Bulk & Top \\
\hline Irterval & $0-8 \mathrm{~mm}$ & $0.4 \mathrm{~mm}$ & $0-4 \mathrm{~mm}$ & - & - & • & $\therefore$ & • & 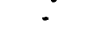 \\
\hline
\end{tabular}

\begin{tabular}{|c|c|c|c|c|c|c|c|c|c|}
\hline Sequenow & 017030 & 017030 & 017035 & 017035 & 017036 & 017036 & 017036 & 017036 & 017037 \\
\hline Latitude & 9.081 & 9.081 & 9.148 & 9.148 & 9.148 & 9.148 & 9.148 & 9.148 & 9.148 \\
\hline Longitude & -164.727 & -164.727 & -164.808 & -164808 & -164808 & -164808 & -164.808 & -164808 & -164.808 \\
\hline Dopth & 4301 & 4301 & 2600 & 2600 & 2600 & 2600 & 2600 & 2600 & 2600 \\
\hline
\end{tabular}

\begin{tabular}{|c|c|c|c|c|c|c|c|c|c|}
\hline Labu & MP1-CKB-11 & MP1-CKB-11* & MP1-CKB-18 & MP1-CKB-18* & MP1-CKB-19 & MP1-CKB-19 & MP1-CKB-19* & MP1-CKB-19* & MP1-CKB-20 \\
\hline Si(wt.\%) & - & - & - & - & - & - & - & - & - \\
\hline
\end{tabular}

\begin{tabular}{|c|c|c|c|c|c|c|c|c|c|}
\hline Si(wt.\%) & $\cdot$ & - & $\cdot$ & - & • & $\cdot$ & - & • & - \\
\hline $\mathrm{Ti}$ & 1.28 & 1.10 & 1.13 & .96 & 1.39 & 1.42 & - & 1.26 & 99 \\
\hline Mn & 24.6 & - & 28.0 & $\cdot$ & 28,2 & 28.8 & - & - & 22.6 \\
\hline$F_{0}$ & 15.5 & - & 17.6 & - & 17.7 & 18.1 & - & - & 14.1 \\
\hline $\mathbf{A}$ & $\cdot$ & - & - & • & - & - & • & $\cdot$ & $\cdot$ \\
\hline$C_{0}$ & .340 & 390 & .920 & 1.000 & 1.29 & 1.09 & 1.26 & 1.13 & .400 \\
\hline $\mathrm{Ni}$ & .530 & $\cdot$ & .540 & - & 550 & .530 & - & - & .350 \\
\hline $\mathrm{Cl}_{2}$ & 1.89 & - & 252 & - & 2.63 & 269 & $\cdot$ & - & 9.94 \\
\hline $\mathbf{M} \mathbf{8}$ & - & - & $\cdot$ & - & - & - & - & - & $\cdot$ \\
\hline $\mathbf{K}$ & . & - & - & - & - & - & - & - & - \\
\hline $\mathbf{P}$ & .23 & - & .38 & - & .31 & .36 & - & $\cdot$ & 3.39 \\
\hline $\mathrm{H}_{2} \mathrm{O}$ & 15.5 & 15.5 & 16.0 & 16.0 & $28 A$ & 30.5 & 28.4 & 30.5 & 24.3 \\
\hline $50^{\circ} \mathrm{C}$ & - & $\cdot$ & - & - & • & - & $\cdot$ & • & • \\
\hline $1000^{\circ} \mathrm{C}$ & - & $\cdot$ & - & - & - & $\cdot$ & - & - & - \\
\hline Sampled & Bulk & Bulk & Bulk & Bulk & Bulk & Bulk & Bulk & Bulk & Bulk \\
\hline Interval & - & - & - & - & - & - & - & - & - \\
\hline
\end{tabular}

\begin{tabular}{|c|c|c|c|c|c|c|c|c|c|}
\hline $\begin{array}{l}\text { Sequencoll } \\
\text { Letitude }\end{array}$ & $\begin{array}{c}017037 \\
9.148\end{array}$ & $\begin{array}{c}017037 \\
9.148\end{array}$ & $\begin{array}{c}017037 \\
9.148\end{array}$ & $\begin{array}{c}017038 \\
9.148\end{array}$ & $\begin{array}{c}017038 \\
9.148\end{array}$ & $\begin{array}{c}017041 \\
9.142\end{array}$ & $\begin{array}{c}017041 \\
9.142\end{array}$ & 017042 & 017042 \\
\hline Longitude & -164.808 & -164.808 & -164.808 & -164.808 & -164.808 & -164.784 & -164.784 & -164.784 & -164.784 \\
\hline $\begin{array}{l}\text { Dapth } \\
\text { Labi }\end{array}$ & $\begin{array}{c}2600 \\
\text { MP1-CKB-20 }\end{array}$ & $\begin{array}{c}2600 \\
\text { MP1 -CKB-20 }\end{array}$ & $\begin{array}{c}2600 \\
\text { MP1-CKB-20* }\end{array}$ & $\begin{array}{c}2600 \\
\text { MP1-CKB-21 }\end{array}$ & $\begin{array}{c}2600 \\
\text { MP1-CKB-21* }\end{array}$ & $\begin{array}{c}2465 \\
\text { MP1-CKB-24 }\end{array}$ & $\begin{array}{c}2465 \\
\text { MP1-CKB-24* }\end{array}$ & $\begin{array}{c}2465 \\
\text { MP1-CKB-25 }\end{array}$ & $\begin{array}{c}2465 \\
\text { MP1-CKB-25* }\end{array}$ \\
\hline Si(wt.\%) & - & - & - & - & - & - & - & - & . \\
\hline $\mathbf{T i}$ & 1.73 & - & .94 & 1.87 & 1.22 & .71 & - & 1.64 & .98 \\
\hline Mn & 23.4 & - & - & 26.5 & - & 30.5 & - & 28.8 & - \\
\hline Fo & 14.6 & - & - & 16.7 & - & 19.3 & - & 18.1 & - \\
\hline $\mathbf{A}$ & $\cdot$ & • & - & - & - & $\cdot$ & - & - & - \\
\hline Co & .440 & .460 & .480 & .780 & .840 & 1.91 & 1.77 & 1.09 & 1.26 \\
\hline $\mathbf{N i}$ & .380 & - & - & .460 & - & .880 & 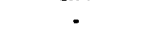 & .760 & - \\
\hline Ce & 9.96 & . & - & 253 & . & 291 & - & 3.46 & - \\
\hline $\mathbf{M g}$ & - & - & - & - & - & - & - & - & - \\
\hline $\mathbf{K}$ & - & . & - & - & - & - & - & - & - \\
\hline $\mathbf{P}$ & 3.57 & - & - & .46 & - & .45 & . & .66 & . \\
\hline $\mathrm{H}_{2} \mathrm{O}$ & 25.8 & 24.3 & 25.8 & 13.5 & 13.5 & 30.4 & 30.4 & 27.9 & 27.9 \\
\hline $50^{\circ} \mathrm{C}$ & - & • & - & - & - & • & • & - & - \\
\hline $1000^{\circ} \mathrm{C}$ & - & - & - & - & . & - & - & - & - \\
\hline Sampled & Bulk & Bulk & Bulk & Bulk & Bulk & Bulk & Bulk & Bulk & Bulk \\
\hline Intorval & . & - & - & - & - & - & - & - & . \\
\hline
\end{tabular}


Table 2. Composition of ferromanganese crusts analyzed by USGS Woods Hole analytical laboratories (cont'd).

\begin{tabular}{|c|c|c|c|c|c|c|c|c|c|c|}
\hline $\begin{array}{l}\text { Soquenco: } \\
\text { Latitude }\end{array}$ & $\begin{array}{c}017043 \\
9.142\end{array}$ & $\begin{array}{c}017043 \\
9.142\end{array}$ & $\begin{array}{c}017047 \\
9.694\end{array}$ & $\begin{array}{c}017048 \\
9.694\end{array}$ & $\begin{array}{c}017049 \\
9.694\end{array}$ & $\begin{array}{c}017049 \\
9.694\end{array}$ & $\begin{array}{c}017050 \\
9.694\end{array}$ & $\begin{array}{c}017051 \\
9.696\end{array}$ & $\begin{array}{c}017051 \\
9.696\end{array}$ & $\begin{array}{c}017051 \\
9.696\end{array}$ \\
\hline Longimde & -164.784 & -164.784 & -162.178 & -162.178 & -162.178 & -162178 & -162.178 & -162.181 & -162181 & .162181 \\
\hline $\begin{array}{l}\text { Depth } \\
\text { Leb. }\end{array}$ & $\begin{array}{c}2465 \\
\text { MP1-CKB-26 }\end{array}$ & $\begin{array}{c}2465 \\
\text { MP1-CKB-26* }\end{array}$ & $\begin{array}{c}1993 \\
\text { CKB-30 }\end{array}$ & $\begin{array}{c}1993 \\
\text { CKB-31 }\end{array}$ & $\begin{array}{c}1993 \\
\text { CKB-32 }\end{array}$ & $\begin{array}{c}1993 \\
\text { CKB-32GR/SVD }\end{array}$ & $\begin{array}{c}1993 \\
\text { CKB-33 }\end{array}$ & $\begin{array}{c}1927 \\
\text { CKB-34 }\end{array}$ & $\begin{array}{c}1927 \\
\text { CKB-34GR/SVD }\end{array}$ & CXB-35 \\
\hline Si(wt.\%) & . & 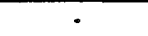 & 287 & 2.18 & 3.36 & 3.12 & 245 & 201 & 1.37 & 1.75 \\
\hline $\mathbf{T i}$ & 12 & .45 & 1.06 & 1.28 & 1.28 & 1.30 & 1.42 & 84 & .83 & .92 \\
\hline Mn & 24.2 & • & 26.4 & 30.3 & 25.7 & 26.7 & 27.3 & 21.2 & 21.5 & 31.3 \\
\hline Pe & 15.2 & - & 16.0 & 13.1 & 16.0 & 164 & 15.4 & 12.3 & 125 & 13.9 \\
\hline Al & - & - & 88 & 1.14 & 1.44 & 1.38 & 1.19 & .06 & .44 & .56 \\
\hline $\mathbf{c o}_{0}$ & .640 & .750 & 900 & 1.49 & 1.01 & 1.05 & 1.40 & .410 & .385 & 1.30 \\
\hline $\mathbf{N i}$ & .990 & $\cdot$ & $\$ 70$ & .690 & A80 & 520 & 540 & 280 & .280 & 590 \\
\hline Ca & 10.0 & - & 282 & 262 & 2.65 & 2.74 & 282 & 10.6 & 10.7 & 2.61 \\
\hline $\mathbf{M}$ & - & - & .92 & 1.02 & 88 & 1.06 & 1.03 & 1.07 & 1.04 & .98 \\
\hline $\mathrm{K}$ & - & - & .47 & 52 & 53 & .55 & 50 & 37 & 36 & .49 \\
\hline $\mathbf{P}$ & 3.82 & • & 49 & 31 & .42 & .43 & 29 & 3.42 & 3.43 & .37 \\
\hline $\mathrm{H}_{2} \mathrm{O}$ & 21.7 & 21.7 & 10.1 & 120 & 11.3 & 16.2 & 112 & 10.0 & 11.9 & 123 \\
\hline $50^{\circ} \mathrm{C}$ & - & - & 14.4 & 13.6 & 127 & 6.7 & 11.8 & 8.6 & 6.0 & 8.2 \\
\hline $1000^{\circ} \mathrm{C}$ & - & - & 5.2 & - & 4.7 & 9.9 & • & 7.7 & 9.4 & 10.7 \\
\hline Sampled & Bulk & Bulk & Bulk & Top & Bulk & Bulk & Porous & Old & Old & Young \\
\hline Interval & - & - & - & - & - & - & - & - & - & - \\
\hline
\end{tabular}

\begin{tabular}{|c|c|c|c|c|c|c|c|c|c|c|}
\hline Sequencoll & 017053 & 017059 & 017059 & 017059 & 017059 & 017059 & 017060 & 017062 & 017063 & 017064 \\
\hline Letitude & 9.696 & 9.716 & 9.716 & 9.716 & 9.716 & 9.716 & 9.716 & 9.682 & 9.705 & 9.705 \\
\hline Longitude & -162181 & -162.167 & -162.167 & -162167 & -162167 & -162167 & -162.167 & -162.184 & .162184 & -162184 \\
\hline Depth & 1927 & 1602 & 1602 & 1602 & 1602 & 1602 & 1602 & 2706 & 1940 & 1940 \\
\hline Leb: & CKB-38 & CKB-40 & CKB-41 & CKB-42 & CKB-43 & CKB-43GR/SVD & CKB-44 & CKB-45 & CKB-46 & CKB-47 \\
\hline Si(wt.\%) & 2.72 & 1.57 & 1.48 & 1.61 & 1.31 & .79 & 1.38 & 6.80 & 1.98 & 1.97 \\
\hline $\mathbf{T i}$ & 1.14 & 81 & .78 & .83 & 55 & .58 & 82 & .93 & .87 & .58 \\
\hline Mn & 28.1 & 32.3 & 23.8 & 27.4 & 28.8 & 30.2 & 33.3 & 173 & 22.6 & 127 \\
\hline Po & 15.8 & 12.6 & 11.1 & 121 & 7.80 & 8.18 & 11.8 & 11.9 & 13.1 & 8.28 \\
\hline Al & 1.01 & .48 & 57 & 56 & .71 & .71 & 12 & 230 & 90 & .92 \\
\hline$C_{0}$ & 1.000 & 1.54 & $\$ 70$ & 960 & 1.07 & 1.11 & 1.65 & .980 & .410 & .460 \\
\hline $\mathbf{N i}$ & .560 & 590 & ASO & 570 & 1.250 & 1.320 & .710 & 350 & .350 & 590 \\
\hline ca & 271 & 261 & 9.72 & 6.29 & 6.64 & 6.93 & 259 & 6.70 & 7.33 & 16.1 \\
\hline $\mathbf{M g}_{\mathbf{8}}$ & 1.08 & 1.04 & 1.12 & 1.05 & 1.55 & 1.64 & 1.07 & 1.02 & 1.04 & 139 \\
\hline $\mathbf{K}$ & .47 & 49 & .41 & .46 & .60 & .62 & 52 & 83 & 39 & .24 \\
\hline $\mathbf{P}$ & 49 & $A 1$ & 3.20 & 1.82 & 217 & 217 & 38 & 209 & 219 & 6.80 \\
\hline $\mathrm{H}_{2} \mathrm{O}$ & 120 & 10.7 & 8.3 & 10.4 & 7.8 & 11.5 & 12.2 & 9.5 & 5.9 & 3.8 \\
\hline $50^{\circ} \mathrm{C}$ & 8.3 & 9.3 & 7.7 & 8.3 & 7.5 & 6.7 & 9.6 & 6.4 & 9.7 & 6.6 \\
\hline $1000^{\circ} \mathrm{C}$ & 9.6 & 9.5 & 8.6 & 9.0 & 10.1 & 9.8 & 10.0 & 7.6 & 8.5 & 6.6 \\
\hline Semplod & Bulk & Youn: & Old & Bulk & Bulk & Bulk & Top & Top & Bulk & Old \\
\hline Interval & - & - & $\cdot$ & - & - & - & $=$ & $\cdot$ & - & - \\
\hline
\end{tabular}

\begin{tabular}{|c|c|c|c|c|c|c|c|c|c|c|}
\hline Sequencell & 017064 & 017083 & 017083 & 017084 & 017085 & 017085 & 017085 & 017085 & 017086 & 017087 \\
\hline Latinude & 9.705 & 6.263 & 6.263 & 6.263 & 6.290 & 6.200 & 6.290 & 6.290 & 9.164 & 9.172 \\
\hline Langitude & -162184 & -162.963 & -162.963 & -162963 & -162970 & -162970 & -162970 & -162.970 & -164.842 & -164.826 \\
\hline $\begin{array}{l}\text { Depth } \\
\text { Leby }\end{array}$ & $\begin{array}{c}1940 \\
\text { CKB-48 }\end{array}$ & $\begin{array}{c}2887 \\
\text { CKB-59 }\end{array}$ & $\begin{array}{c}2887 \\
\text { CKB-59GR/SVD }\end{array}$ & $\begin{array}{c}2887 \\
\text { CKB-60 }\end{array}$ & $\begin{array}{c}2486 \\
\text { CKB-61 }\end{array}$ & $\begin{array}{c}2486 \\
\text { CKB-62 }\end{array}$ & $\begin{array}{c}2486 \\
\text { CKB-63 }\end{array}$ & $\begin{array}{c}2486 \\
\text { CKB-64 }\end{array}$ & $\begin{array}{c}2291 \\
\text { CKB-65 }\end{array}$ & $\begin{array}{c}1853 \\
\text { CKB-66 }\end{array}$ \\
\hline Si(wt.8) & 208 & 3.87 & 3.67 & 4.49 & 264 & 2.37 & 1.20 & 244 & 4.08 & 2.82 \\
\hline $\mathbf{T i}$ & .80 & 1.00 & 1.07 & 1.09 & 1.04 & .96 & 1.07 & 1.10 & .85 & 1.18 \\
\hline Mn & 31.6 & 24.0 & 25.3 & 22.0 & 25.3 & 25.9 & 23.0 & 24.5 & 24.5 & 20.5 \\
\hline Fo & 11.9 & 17.7 & 18.7 & 18.5 & 18.4 & 18.2 & 19.3 & 20.6 & 18.7 & 13.2 \\
\hline $\mathbf{A}$ & .69 & 1.06 & .95 & 1.37 & .92 & .76 & 88 & .79 & 54 & 1.10 \\
\hline Co & 1.55 & .770 & 800 & .680 & .630 & 550 & .470 & 560 & .780 & 1.53 \\
\hline $\mathrm{Ni}$ & .640 & .470 & $A 10$ & .420 & .400 & .420 & 320 & .410 & .380 & 580 \\
\hline $\mathbf{C a}$ & 3.17 & 237 & 250 & 295 & 246 & 241 & 2.39 & 248 & 250 & 2.83 \\
\hline $\mathbf{M}_{\mathbf{8}}$ & 1.08 & .82 & 1.02 & .92 & 98 & .87 & 84 & .95 & .95 & 1.16 \\
\hline 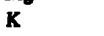 & 59 & .48 & 50 & 52 & 39 & .40 & 35 & .36 & .46 & .66 \\
\hline $\mathbf{P}$ & 59 & 36 & .37 & 58 & .39 & .39 & 39 & .41 & .9 & .45 \\
\hline $\mathrm{H}_{2} \mathrm{O}$ & 11.1 & 10.5 & 15.4 & 11.5 & 8.0 & 9.3 & 14.8 & 13.9 & 18.0 & 18.5 \\
\hline $50^{\circ} \mathrm{C}$ & 11.0 & 11.0 & 6.6 & 9.8 & 11.4 & 10.9 & 120 & 18.6 & 127 & 11.7 \\
\hline $1000^{\circ} \mathrm{C}$ & 8.5 & 9.2 & 8.9 & 9.9 & 11.4 & 10.2 & 7.0 & 6.9 & 6.3 & 7.6 \\
\hline Stroplod & Young & Bulk & Bulk & Bulk & Bulk & Top & Bottom & Middlo & Bulk & Bulk \\
\hline Interval & - & - & - & - & - & $\cdot$ & - & • & - & $\cdot$ \\
\hline
\end{tabular}


Table 2. Composition of ferromanganese crusts analyzed by USGS Woods Hole analytical laboratories (cont'd).

\begin{tabular}{|c|c|c|c|c|c|c|c|c|c|c|}
\hline Sequencoll & 017087 & 017088 & 017088 & 017088 & 017089 & 017093 & 017093 & 017095 & 017095 & 017096 \\
\hline Latimde & 9.172 & 9.179 & 9.179 & 9.179 & 9.166 & 9.225 & 9.225 & 9.247 & 9.247 & 9.247 \\
\hline Longitude & -164.826 & -164.830 & -164.830 & -164.830 & -164.798 & -164.780 & -164.780 & -164.846 & -164.846 & -164.846 \\
\hline Depth & 1853 & 1263 & 1263 & 1263 & 1427 & 1456 & 1456 & $\bullet$ & $\bullet$ & $\cdot$ \\
\hline Lab" & CKB-67 & CKB-69 & CKB-71 & CKB-72 & CKB-73 & CKB-75 & CKB-76 & CKB-79 & CKB-80 & CKB-81 \\
\hline$S i(w t . \%)$ & 20.5 & 1.76 & 1.38 & 1.37 & 1.57 & 1.50 & 1.76 & 1.77 & 1.57 & 1.27 \\
\hline $\mathbf{T i}$ & 136 & 1.04 & .99 & 82 & .65 & 1.05 & 1.00 & 1.01 & 95 & 1.04 \\
\hline Mn & 28 & 29.9 & 32.5 & 30.7 & 20.9 & 31.1 & 28.2 & 31.6 & 24.9 & 33.6 \\
\hline Po & 7.82 & 14.1 & 13.4 & 11.9 & 11.2 & 14.1 & 134 & 13.5 & 124 & 14.2 \\
\hline $\mathbf{A}$ & 9.84 & 59 & .60 & .33 & 97 & 53 & .69 & .62 & 57 & 59 \\
\hline Co & • & 1.34 & 1.38 & 1.02 & 1.37 & 1.30 & 980 & 1.42 & 810 & 1.49 \\
\hline $\mathbf{N i}$ & • & 560 & .740 & .710 & 590 & .530 & 540 & .750 & 540 & .630 \\
\hline Ca & 8.02 & 2.73 & 2.74 & 5.25 & 5.33 & 270 & 4.74 & 282 & 7.25 & 2.83 \\
\hline $\mathbf{M g}_{\mathbf{g}}$ & 1.09 & 1.10 & 1.12 & 1.10 & 1.16 & 1.00 & 1.02 & 1.03 & 1.12 & 1.20 \\
\hline $\mathrm{K}$ & 270 & .48 & so & 50 & 58 & 46 & .46 & .49 & .42 & 51 \\
\hline $\mathbf{P}$ & 247 & .44 & .40 & 1.31 & 1.40 & 38 & 1.11 & .47 & 2.18 & .40 \\
\hline $\mathrm{H}_{2} \mathrm{O}$ & 2.1 & 13.7 & 14.6 & 173 & 13.2 & 13.2 & 16.5 & 14.9 & 11.3 & 18.0 \\
\hline $50^{\circ} \mathrm{C}$ & 3.1 & 23.5 & 9.3 & 9.1 & 6.5 & 11.0 & 9.9 & 9.3 & 8.7 & 8.8 \\
\hline $1000^{\circ} \mathrm{C}$ & 20 & 8.4 & 9.3 & 8.0 & 9.1 & 8.2 & 7.4 & 9.0 & 7.8 & 11.0 \\
\hline Samplod & Bulk & ad & Bulk & Young & Bulk & Young & Old & Young & Old & Youns \\
\hline Inderval & . & . & . & - &. &. & - & 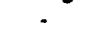 & - & 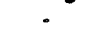 \\
\hline
\end{tabular}

\begin{tabular}{|c|c|c|c|c|c|c|c|c|c|c|}
\hline $\begin{array}{l}\text { Sequencell } \\
\text { Latitude }\end{array}$ & $\begin{array}{c}017096 \\
9.247\end{array}$ & $\begin{array}{c}017097 \\
9.247\end{array}$ & $\begin{array}{c}017098 \\
9.247\end{array}$ & $\begin{array}{c}017098 \\
9.247\end{array}$ & $\begin{array}{c}017099 \\
9.200\end{array}$ & $\begin{array}{c}017099 \\
9.200\end{array}$ & $\begin{array}{c}017101 \\
9.716\end{array}$ & $\begin{array}{c}017102 \\
9.223\end{array}$ & $\begin{array}{c}019001 \\
6.667\end{array}$ & $\begin{array}{c}019002 \\
7.333\end{array}$ \\
\hline Longitude & -164.846 & -164.846 & -164.846 & -164.846 & -164.929 & -164.929 & -162.167 & .164 .858 & 172.417 & 172.333 \\
\hline Depth & crisen & Cं- & cris & CKRos & - & cros & 1602 & 1420 & 1700 & 1260 \\
\hline & CKB-82 & CKB-83 & CKB-84 & CKB-85 & CKB-87 & CKB-88 & CKB-49 & CKB-78 & KK810626-RD9 & KK810626-RD1 \\
\hline Si(wt.\%) & .96 & 1.74 & 1.35 & .92 & 1.39 & .88 & 1.75 & 1.05 & 6.04 & 1.95 \\
\hline $\mathbf{T}$ & .87 & 89 & 1.18 & 80 & 93 & .71 & .88 & 94 & 1.41 & 83 \\
\hline Mn & 26.0 & 33.5 & 31.5 & 262 & 332 & 30.4 & 26.6 & 35.2 & 192 & 24.7 \\
\hline Fo & 11.7 & 12.1 & 14.9 & 11.9 & 11.4 & 10.7 & 118 & 10.7 & 13.8 & 11.4 \\
\hline $\mathbf{A}$ & .45 & .70 & 85 & $A 5$ & so & .27 & .87 & 39 & 2.77 & $\cdot$ \\
\hline Co & .790 & 1.91 & 1.32 & .650 & 1.78 & .720 & 1.22 & 180 & 973 & .852 \\
\hline $\mathbf{N i}$ & .540 & .730 & .610 & .610 & .620 & .750 & 520 & 880 & A15 & .561 \\
\hline C. & 8.83 & 268 & 276 & 8.62 & 247 & 7.11 & 5.74 & 247 & 3.65 & 7.03 \\
\hline $\mathbf{M g}$ & 1.15 & 1.41 & 1.00 & 1.13 & 1.03 & 1.11 & 1.05 & 1.11 & 1.14 & 1.18 \\
\hline $\mathbf{K}$ & .41 & .58 & .49 & 42 & 51 & 51 & .47 & 55 & .87 & - \\
\hline $\mathbf{P}$ & 267 & 36 & .40 & 254 & 31 & 1.96 & 1.58 & .27 & .93 & 2.20 \\
\hline $\mathrm{H}_{2} \mathrm{O}$ & 11.9 & 15.7 & 15.1 & 15.9 & 15.0 & 15.1 & 9.0 & 18.0 & 8.5 & 7.3 \\
\hline $50^{\circ} \mathrm{C}$ & 8.6 & 8.5 & 9.8 & 8.3 & 10.9 & 8.7 & 9.1 & 9.0 & 9.3 & 9.3 \\
\hline $1000^{\circ} \mathrm{C}$ & 6.5 & 8.2 & 7.3 & 7.8 & 8.2 & 7.3 & 8.6 & 9.5 & 8.0 & 9.1 \\
\hline Sampled & Old & Top & Young & Old & Young & Old & Bulk & Bulk & Bulk & Bulk \\
\hline bervaril & • & - & • & - & • & • & - & - & $0.15 \mathrm{~mm}$ & $0-25 \mathrm{~mm}$ \\
\hline
\end{tabular}

\begin{tabular}{|c|c|c|c|c|c|c|c|c|c|c|}
\hline $\begin{array}{l}\text { Sequencel } \\
\text { Letitude }\end{array}$ & $\begin{array}{c}019003 \\
7.250\end{array}$ & $\begin{array}{l}020001 \\
8.747\end{array}$ & $\begin{array}{c}020002 \\
8.755\end{array}$ & $\begin{array}{l}052003 \\
18.699\end{array}$ & $\begin{array}{l}052003 \\
18.699\end{array}$ & $\begin{array}{l}052005 \\
18.643\end{array}$ & $\begin{array}{l}052005 \\
18.643\end{array}$ & $\begin{array}{l}052005 \\
18.643\end{array}$ & $\begin{array}{l}052005 \\
18.643\end{array}$ & $\begin{array}{l}052005 \\
18.643\end{array}$ \\
\hline Longitude & 171.008 & 169.738 & 169.792 & -158380 & -158.380 & -158.295 & -158.295 & -158.295 & -158.295 & -158.295 \\
\hline Depth & 2250 & 3550 & 2900 & 3261 & 3261 & 1924 & 1924 & 1924 & 1924 & 1924 \\
\hline Lab" & D-1 & $D-42$ & D-6-1 & CKB-2 & CKB-2OR/SVD & CKB-AAM & CKB-4G & CKB-5 & CKE-5GR/SVD & CKB-6 \\
\hline Si(wt.\%) & 2.60 & 299 & 1.93 & 6.58 & 6.64 & 6.69 & 6.88 & 5.23 & 5.24 & 5.57 \\
\hline $\mathbf{T i}$ & 97 & 1.13 & 1.24 & 1.78 & 1.77 & 1,42 & 1.29 & 1.38 & 1.39 & 1.44 \\
\hline Mn & 26.5 & 23.8 & 28.8 & 14.6 & 14.7 & 17.6 & 18.1 & 17.5 & 17.7 & 19.9 \\
\hline Po & 14.7 & 18.0 & 13.4 & 23.0 & 23.1 & 19.1 & 18.5 & 228 & $\mathbf{2 3 . 0}$ & 18.0 \\
\hline $\mathbf{A}$ & 57 & .70 & .93 & 272 & 2.66 & 254 & 2.59 & 1.73 & 1.59 & 208 \\
\hline$C_{0}$ & 1.27 & .837 & 1.33 & 360 & 350 & 550 & 487 & 480 & .470 & .480 \\
\hline $\mathbf{N i}$ & 550 & 304 & .655 & .070 & .069 & .240 & .270 & .120 & .120 & .285 \\
\hline C. & 247 & 223 & 2.28 & 2.11 & 2.10 & 224 & 2.22 & 2.05 & 2.05 & 2.17 \\
\hline $\mathbf{M g}_{\mathbf{g}}$ & 97 & 90 & 1.03 & 95 & 86 & .96 & .95 & 86 & 82 & .79 \\
\hline $\mathbf{K}$ & 49 & $\$ 2$ & 52 & 54 & 53 & .66 & .74 & .43 & 43 & .06 \\
\hline $\mathbf{P}$ & 37 & 36 & .29 & .44 & 43 & .40 & .41 & .43 & 43 & 34 \\
\hline $\mathrm{H}_{2} \mathrm{O}$ & 19.9 & 21.1 & 11.4 & 9.1 & 13.8 & 11.1 & 7.6 & 10.2 & 14.3 & 9.0 \\
\hline $50^{\circ} \mathrm{C}$ & 18.2 & 12.2 & 17.3 & 9.0 & 5.0 & 9.5 & 9.4 & 9.9 & 5.5 & 11.2 \\
\hline $1000^{\circ} \mathrm{C}$ & 6.4 & 9.1 & 6.2 & 5.5 & 9.2 & 8.6 & 7.6 & 6.7 & 10.0 & 6.7 \\
\hline Sampled & Bulk & Bulk & Bulk & Bulk & Bulk & Bulk & Bulk & Top & Top & Bottam \\
\hline indual & $0-10 \mathrm{~mm}$ & $0-3 \mathrm{~mm}$ & $0-20 \mathrm{~mm}$ & - & • & - & - & $\therefore$ & $\cdot$ & • \\
\hline
\end{tabular}


Table 2. Composition of ferromanganese crusts analyzed by USGS Woods Hole analytical laboratories (cont'd).

\begin{tabular}{|c|c|c|c|c|c|c|c|c|c|c|c|}
\hline Soquencell & 052006 & 052006 & 052006 & 052000 & 052007 & 052007 & 052008 & 052009 & 052010 & 052010 & 052011 \\
\hline Letitude & 18.643 & 18.643 & 18.643 & 18.643 & 18.643 & 18.643 & 18.643 & 18.637 & 18.637 & 18.637 & 18.637 \\
\hline Longitude & -158.295 & -158.295 & -158.295 & -158.295 & -158.295 & -158.295 & -158.295 & -158.313 & -158.313 & -158.313 & -158.313 \\
\hline Depth & 1924 & 1924 & 1924 & 1924 & 1924 & 1924 & 1924 & 2002 & 2002 & 2002 & 2002 \\
\hline Labu & CKB-7 & CKB-8 & CKB-9 & CKB-10 & CKB-10GR/SVD & CKB-11 & CKB-12 & CKB-13 & CKB-17 & CKB-18 & CKB-19 \\
\hline$S i(w t s)$ & 5.69 & 5.77 & 6.00 & 6.43 & 6.27 & 4.73 & 6.75 & 4.81 & 4,46 & 4.99 & 5.47 \\
\hline $\mathbf{T i}$ & 1.11 & 1.26 & .80 & 1.43 & 1.18 & 1.43 & 1.23 & 1.20 & 1.33 & 1.71 & 1.60 \\
\hline Mn & 19.6 & 19.1 & 19.9 & 18.8 & 18.8 & 19.8 & 20.4 & 19.2 & 203 & 20.7 & 17.9 \\
\hline Po & 18.4 & 19.6 & 18.1 & 18.7 & 18.7 & 21.7 & 17.1 & 21.5 & 20.4 & 19.5 & 21.6 \\
\hline Al & 2.00 & 1.89 & 2.14 & 2.55 & 2.10 & 1.66 & 2.45 & 1.44 & 1.73 & 2.20 & 2.10 \\
\hline $\mathbf{C o}$ & $\$ 70$ & 520 & 350 & .540 & .550 & 570 & .530 & .450 & 520 & .030 & 480 \\
\hline $\mathbf{N i}$ & .275 & 210 & 370 & .250 & .250 & .210 & 310 & .180 & .220 & .200 & .160 \\
\hline Ca & 2.23 & 222 & 2.29 & 2.13 & 2.09 & 225 & 2.49 & 2.11 & 220 & 2.21 & 2.13 \\
\hline $\mathbf{M}_{8}$ & 86 & 97 & .87 & 80 & 82 & .95 & 99 & .96 & .88 & .92 & .92 \\
\hline $\mathbf{K}$ & .66 & 56 & .88 & .89 & .88 & 46 & .67 & .42 & .49 & .51 & .50 \\
\hline $\mathbf{P}$ & 38 & .42 & .38 & 39 & 38 & .45 & 35 & .40 & .43 & 42 & .42 \\
\hline $\mathrm{H}_{2} \mathrm{O}$ & 9.1 & 11.3 & 6.4 & 9.7 & 12.9 & 10.2 & 9.7 & 7.6 & 6.6 & 6.8 & 5.7 \\
\hline $50^{\circ} 0^{\circ} \mathrm{C}$ & 9.6 & 10.2 & 9.6 & 9.6 & 5.7 & 10.8 & 9.9 & 10.1 & 11.6 & 10.6 & 11.3 \\
\hline $1000^{\circ} \mathrm{C}$ & 9.1 & 10.8 & 7.0 & 5.6 & 10.2 & 6.1 & 19 & 6.4 & 7.2 & 5.9 & 6.2 \\
\hline Sampled & Bulk & Top & Bottom & Bulk & Bulk & Bulk & Top & Bulk & Bulk & Bulk & Bulk \\
\hline Intorval & - & • & • & - & • & • & - & - & - & - & • \\
\hline
\end{tabular}

\begin{tabular}{|c|c|c|c|c|c|c|c|c|c|c|c|}
\hline Sequeno: & 052012 & 052015 & 052015 & 052015 & 052016 & 052016 & 052016 & 052017 & 052017 & 052017 & 052023 \\
\hline Latimde & 18.637 & 18.683 & 18.683 & 18.683 & 18.683 & 18.683 & 18.683 & 18.683 & 18.683 & 18.683 & 18.637 \\
\hline Longitude & -158.313 & -158.314 & -158.314 & -158.314 & -158.314 & -158.314 & -158.314 & -158.314 & -158.314 & -158.314 & -158.313 \\
\hline Depth & 2002 & 1047 & 1047 & 1047 & 1047 & 1047 & 1047 & 1047 & 1047 & 1047 & 2002 \\
\hline Leb" & CKB-20 & CKB-21 & CKB-22 & CKB.23 & CKB-24 & CKB-25 & CKB-26 & CKB-27 & CKB.28 & CKB-29 & CKB-16 \\
\hline Si(wt.s) & 4.39 & 294 & 330 & 2.65 & 4.16 & 3.48 & 4.39 & 3.16 & 3.22 & 2.31 & 5.71 \\
\hline $\mathrm{Ti}$ & 1.18 & 98 & 1.23 & .89 & 1.20 & 1.21 & .74 & 1.30 & 1.10 & .76 & 1.56 \\
\hline Mn & 20.6 & 23.5 & 25.5 & 22.1 & 24.6 & 258 & 16.6 & 220 & 25.4 & 19.9 & 18.6 \\
\hline Po & 19.9 & 14.5 & 16.0 & 12.6 & 15.4 & 15.7 & 11.4 & 14.0 & 15.5 & 11.1 & 19.7 \\
\hline Al & 1.37 & 1.13 & 1.39 & 1.01 & 1.65 & 1.42 & 1.68 & 1.60 & 1.29 & 81 & 2.33 \\
\hline Co & .530 & 560 & .750 & .440 & .740 & .730 & 310 & 520 & .640 & .370 & 500 \\
\hline $\mathrm{Ni}$ & .225 & 385 & 400 & .400 & .500 & .440 & 450 & .400 & 500 & .350 & .290 \\
\hline C. & 2.14 & 5.72 & 2.53 & 7.75 & 2.75 & 245 & 8.83 & 6.72 & 3.77 & 11.1 & 200 \\
\hline $\mathbf{M g}_{8}$ & 85 & 96 & .88 & 91 & .91 & .91 & 94 & 1.00 & 1.01 & 1.04 & .90 \\
\hline $\mathrm{K}$ & .46 & .48 & .54 & 51 & .63 & 52 & 82 & .48 & .54 & .44 & .62 \\
\hline $\mathbf{P}$ & 37 & 1.67 & .50 & 242 & 54 & 39 & 293 & 2.01 & .87 & 3.64 & .34 \\
\hline $\mathrm{H}_{2} \mathrm{O}$ & 6.8 & 6.5 & 6.8 & 6.1 & 7.4 & 6.1 & 5.1 & 8.5 & 11.6 & 8.1 & 5.9 \\
\hline $50^{\circ} \mathrm{C}$ & 10.0 & 11.3 & 12.1 & 10.8 & 9.9 & 11.4 & 7.8 & 10.7 & 10.1 & 8.7 & 115 \\
\hline $1000^{\circ} \mathrm{C}$ & 73 & 7.6 & 5.6 & 8.5 & 7.5 & 7.2 & 6.7 & 6.8 & 7.2 & - & 2.5 \\
\hline Sampled & Bulk & Bulk & Youns & Old & Bulk & Young & Old & Bulk & Young & Old & Bulk \\
\hline Intorval & • & • & - & - & - & - & - & - & - & - & - \\
\hline
\end{tabular}

\begin{tabular}{|c|c|c|c|c|c|c|c|c|c|c|c|}
\hline Sequencel: & 052023 & 053001 & 053002 & 053003 & 053004 & 053005 & 053006 & 053007 & 053008 & 053009 & 053018 \\
\hline Lutimds & 18.637 & 18.300 & 18.783 & 18.783 & 19.367 & 19.350 & 10.293 & 14.458 & 18.135 & 19.117 & 19.369 \\
\hline Langitudo & -158.313 & -161.767 & -162050 & .162 .050 & -162317 & -162.167 & -165995 & -168.978 & .169 .007 & -169.733 & -168.695 \\
\hline Depth & 2002 & 3111 & 4629 & 3147 & 4868 & 4209 & 1790 & 1750 & 2139 & 1805 & 2000 \\
\hline Labi\# & CKB-16GR/SVD & w-222532 & W-222533 & W-222534 & W-222535 & W-222536 & W-222567 & W-222568 & W-222569 & W-222570 & 21 \\
\hline Si(wt.\%) & 5.46 & 3.65 & 5.71 & 10.6 & 4.36 & 11.8 & 287 & 1.76 & 5.21 & 4.41 & 5.07 \\
\hline $\mathbf{T i}$ & 1.28 & 1.41 & 1.44 & 1.93 & 1.55 & 1.46 & 1.25 & 1.18 & 1.23 & 1.14 & 1.20 \\
\hline Mn & 19.8 & 27.1 & 20.2 & 13.2 & 23.5 & 13.0 & 30.1 & 32.9 & 25.7 & 23.8 & 21.4 \\
\hline $\mathrm{Fo}_{0}$ & 20.0 & 20.9 & 23.1 & 20.4 & 21.4 & 17.7 & 14.9 & 14.9 & 21.7 & 17.5 & 17.0 \\
\hline $\mathbf{N}$ & 1.92 & .77 & 1.45 & 4.02 & 1.21 & 4.34 & .81 & .40 & .96 & 1.20 & 1.86 \\
\hline$C_{0}$ & .485 & 1.02 & 558 & .249 & .674 & 357 & .679 & 1.27 & 910 & .784 & 1.05 \\
\hline $\mathrm{Ni}$ & 310 & 351 & .122 & .174 & .243 & .098 & .790 & .704 & 367 & .506 & 309 \\
\hline C. & 207 & 258 & 255 & 208 & 2.35 & 1.88 & 3.32 & 2.85 & 2.63 & 249 & 3.05 \\
\hline $\mathbf{M g}$ & 87 & 1.11 & 1.05 & 1.20 & 1.03 & 1.03 & 1.50 & 1.43 & 1.20 & 1.24 & 1.15 \\
\hline $\mathbf{K}$ & .64 & 49 & .36 & 1.04 & 40 & 1.43 & 53 & .54 & .52 & .46 & .61 \\
\hline $\mathbf{P}$ & 35 & .41 & .46 & .42 & A1 & $A 1$ & .47 & .40 & .53 & 53 & .42 \\
\hline $\mathrm{H}_{2} \mathrm{O}$ & 13.5 & 31.6 & 265 & 19.8 & 25.9 & 18.7 & 278 & 27.6 & 26,4 & 20.9 & 9.5 \\
\hline $50^{\circ} \mathrm{C}$ & 5.7 & - & - & - & - & . & . & - & 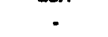 & - & 11.3 \\
\hline $1000^{\circ} \mathrm{C}$ & 10.0 & - & - & - & - & - & - & - & - & - & 8.5 \\
\hline Sampled & Bulk & Bulk & Bulk & Bulk & Bulk & Bulk & Bulk & Bulk & Bulk & Bulk & Bulk \\
\hline Interval &. & $0.12 \mathrm{~mm}$ & $0.10 \mathrm{~mm}$ & $0.7 \mathrm{~mm}$ & $0.5 \mathrm{~mm}$ & $0.12 \mathrm{~mm}$ & $0.7 \mathrm{~mm}$ & $0-9 \mathrm{~mm}$ & $0-14 \mathrm{~mm}$ & $0.10 \mathrm{~mm}$ & - \\
\hline
\end{tabular}


Table 2. Composition of ferromanganese crusts analyzed by USGS Woods Hole analytical laboratories (cont'd).

\begin{tabular}{|c|c|c|c|c|c|c|c|c|c|}
\hline Sequence: & 053020 & 053025 & 053026 & 053027 & 053029 & 053030 & 053032 & 053037 & 053037 \\
\hline Latitude & 19511 & 19.511 & 19.695 & 19.553 & 19.515 & 19.515 & 19515 & 13.115 & 13.115 \\
\hline Longitude & -168.835 & -168.835 & -168.995 & -168.832 & -168.855 & -168.855 & -168.855 & -165.552 & -165.552 \\
\hline Depth & 1800 & 1800 & 4500 & 2400 & 1790 & 1790 & 1790 & 4774 & 4774 \\
\hline Leby & 23 & 28 & 29 & 30 & 32 & 33 & 35 & MP1-CKB-28 & MP1-CKB-28* \\
\hline Si(wt.\%) & 5.42 & 3.43 & 8.15 & 4.99 & 3.08 & 2.17 & 1.85 & 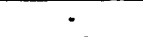 & $\cdot$ \\
\hline $\mathbf{T}$ & 1.14 & 1.17 & 155 & 1.20 & 92 & 1.10 & .84 & 2.15 & 1.37 \\
\hline Mn & 23.2 & 26.0 & 16.4 & 23.1 & 25.1 & 25.1 & 26.9 & 15.1 & - \\
\hline Po & 19.1 & 15.9 & 19.1 & 18.9 & 15.8 & 14.0 & 13.2 & 9.90 & - \\
\hline Al & 1.25 & 1.00 & 3.24 & 1.40 & 55 & .65 & 33 & • & $\cdot$ \\
\hline Co & .889 & 912 & .339 & 940 & .707 & .676 & .657 & .290 & .320 \\
\hline $\mathbf{N}$ & .324 & .431 & .397 & .348 & .362 & .408 & .445 & .140 & • \\
\hline ca & 2.32 & 287 & 1.74 & 240 & 4.67 & 5.62 & 5.58 & 1.41 & - \\
\hline Mg & 1.01 & 1.05 & 1.11 & 1.14 & 1.05 & 1.00 & 1.07 & - & - \\
\hline $\mathrm{K}$ & 54 & ss & .71 & .56 & .46 & .44 & 45 & - & - \\
\hline $\mathbf{P}$ & 44 & .46 & 28 & .43 & 1.23 & 1.44 & 1.43 & .28 & - \\
\hline $\mathrm{H}_{2} \mathrm{O}$ & 16.8 & 18.9 & 14.4 & 13.8 & 14.4 & 6.9 & 10.2 & 123 & 12.3 \\
\hline $50^{\circ} \mathrm{C}$ & 8.8 & 12.1 & 9.3 & 8.6 & 8.9 & 10.3 & 11.6 & • & - \\
\hline $1000^{\circ} \mathrm{C}$ & 33 & 7.9 & 6.2 & 6.8 & 7.8 & 10.7 & 9.2 & $\cdot$ & $\cdot$ \\
\hline Semplod & Top & Bulk & Top & Bulk & Bulk & Top & Bottom & Bulk & Bulk \\
\hline Interval & $\therefore$ & - & $\therefore$ & $\cdot$ & - & - & $\cdot$ & • & - \\
\hline
\end{tabular}

\begin{tabular}{|c|c|c|c|c|c|c|c|c|c|}
\hline Sequenceit & 053043 & 053043 & 053044 & 053044 & 053046 & 053046 & 053047 & 053047 & 053048 \\
\hline Latitude & 13.001 & 13.001 & 12.987 & 12.987 & 13.151 & 13.151 & 13.200 & 13.200 & 13.200 \\
\hline Longitude & -164.968 & -164.968 & -164.958 & -164958 & -165.485 & -165.485 & -165.462 & -165.462 & -165.462 \\
\hline Depth & 3040 & 3040 & 2055 & 2055 & $41 \$ 0$ & 4150 & 1508 & 1508 & 1508 \\
\hline
\end{tabular}

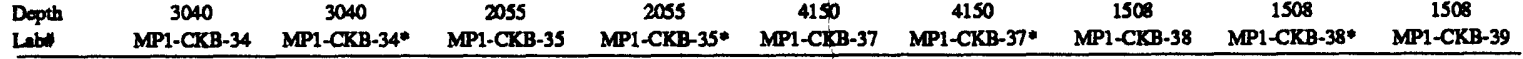

\begin{tabular}{|c|c|c|c|c|c|c|c|c|c|}
\hline Si(wt.s) & • & $\cdot$ & $\cdot$ & $\cdot$ & $\cdot$ & - & $\cdot$ & - & - \\
\hline $\mathrm{Ti}$ & 1.31 & 1.02 & 1.89 & .94 & 1.61 & .98 & 98 & .92 & 1.03 \\
\hline Mn & 25.9 & - & 27.3 & - & $24 A$ & $\cdot$ & 28.8 & - & 28.4 \\
\hline Fo & 16.3 & - & 17.1 & - & 15.6 & - & 18.0 & - & 17.8 \\
\hline $\mathbf{A} \mathbf{I}$ & - & $\cdot$ & $\cdot$ & $\cdot$ & -1 & - & - & - & • \\
\hline co & .590 & .680 & .850 & 820 & 570 & .680 & 1.29 & 1.39 & 1.31 \\
\hline$\sqrt{1}$ & .530 & - & .580 & $\cdot$ & .280 & - & .670 & - & .690 \\
\hline Ca & 2.64 & - & 2.54 & - & 237 & - & 2.75 & - & 2.62 \\
\hline $\mathbf{M}_{\mathbf{B}}$ & • & - & - & - & $\cdot$ & 。 & $\cdot$ & - & • \\
\hline $\mathrm{K}$ & - & - & - & - & $\cdot$ & - & - & $\cdot$ & - \\
\hline $\mathbf{P}$ & .44 & $\cdot$ & .41 & - & 49 & - & .49 & - & .36 \\
\hline $\mathrm{H}_{2} \mathrm{O}$ & 14.6 & 14.6 & 14.5 & 145 & 25.9 & 25.9 & 17.6 & 17.6 & 28.4 \\
\hline $500^{\circ} \mathrm{C}$ & $\cdot$ & . & • & . & $\cdot$ & - & • & • & - \\
\hline $1000^{\circ} \mathrm{C}$ & - & $\cdot$ & $\cdot$ & - & - & - & $\cdot$ & - & - \\
\hline Sarmpled & Bulk & Bulk & Bulk & Bulk & Bulk & Bulk & Bulk & Bulk & Bulk \\
\hline Interval & - & - & - & $\cdot$ & - & - & - & - & $\cdot$ \\
\hline
\end{tabular}

\begin{tabular}{|c|c|c|c|c|c|c|c|c|c|}
\hline Sequencoll & 053048 & 053048 & 053048 & 053049 & 053049 & 053049 & 053049 & 053103 & 053103 \\
\hline Lationds & 13.200 & 13.200 & 13.200 & 13.200 & 13.200 & 13.200 & 13.200 & 14.462 & 14.462 \\
\hline Longitude & -165.462 & -165.462 & -165.462 & -165.462 & -165.462 & -165.462 & -165.462 & -169.017 & -169.017 \\
\hline Depth & 1508 & 1508 & 1508 & 1508 & 1508 & 1508 & 1508 & 2110 & 2110 \\
\hline Lab" & MP1-CKB-39 & MP1-CKB-39* & MP1-CKB-39* & MP1-CKB-40 & MP1-CKB-40 & MP1-CKB-40* & MP1-CKB-40* & CKB-141 & CKB-142 \\
\hline si(wt.\%) & $\cdot$ & - & 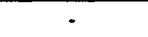 & $\cdot$ & - & $\cdot$ & $\cdot$ & 2.30 & 1.77 \\
\hline $\mathbf{T i}$ & 246 & - & 1.00 & .79 & .80 & $\cdot$ & 80 & 1.14 & 1.11 \\
\hline $\mathrm{Mn}$ & 28.8 & - & - & 22.7 & 18.8 & - & - & 28.0 & 25.8 \\
\hline Fo & 18.1 & - & - & 14.1 & 114 & $\cdot$ & - & 15.0 & 125 \\
\hline Al & • & - & $\cdot$ & $\cdot$ & - & $\cdot$ & $\cdot$ & .74 & .74 \\
\hline Co & 1.16 & 1.23 & 1.31 & 540 & 360 & 570 & .440 & 1.20 & .922 \\
\hline $\mathbf{N i}$ & .690 & $\cdot$ & - & .650 & 560 & - & - & .632 & .607 \\
\hline $\mathbf{c a}$ & 3.77 & - & - & 10.1 & 13.1 & - & - & 2.66 & 5.37 \\
\hline $\mathbf{M g}$ & - & - & - & - & - & - & - & 1.16 & 1.37 \\
\hline $\mathbf{K}$ & - & - & - & - & - & - & - & 51 & so \\
\hline $\mathbf{P}$ & .88 & - & • & 3.47 & 5.44 & - & $\cdot$ & .39 & 1.24 \\
\hline $\mathrm{H}_{2} \mathrm{O}$ & 30.5 & 28.4 & 30.5 & 24.5 & $\mathbf{2 3 . 1}$ & 245 & 23.1 & 9.7 & 7.8 \\
\hline $50^{\circ} \mathrm{C}$ & • & - & - & $\cdot$ & • & • & - & - & - \\
\hline $1000^{\circ} \mathrm{C}$ & - & - & $\cdot$ & - & $\cdot$ & $\cdot$ & $\cdot$ & $\cdot$ & - \\
\hline Sampled & Bulk & Bulk & Bulk & Bulk & Bulk & Bulk & Bulk & Young-Top & Old-Bottom \\
\hline leterval &. &. & - & . & • & - & - & $0-20 \mathrm{~mm}$ & $0-20 \mathrm{~mm}$ \\
\hline
\end{tabular}


Table 2. Composition of ferromanganese crusts analyzed by USGS Woods Hole analytical laboratories (cont'd).

\begin{tabular}{|c|c|c|c|c|c|c|c|c|c|c|c|}
\hline Sequencell & 053104 & 053104 & 053104 & 053104 & 053109 & 053109 & 053109 & 053110 & 053110 & 053113 & 053116 \\
\hline Lationde & 14,456 & 14.456 & 14.456 & 14.456 & 15.597 & 15.597 & 15.597 & 15.563 & 15.563 & 15.675 & 15.544 \\
\hline Longimde & -169.003 & -169.003 & -169.003 & -169.003 & -169.232 & -169.232 & -169.232 & -169.224 & -169.224 & -169.124 & -169.238 \\
\hline $\begin{array}{l}\text { Depth } \\
\text { Laby }\end{array}$ & $\begin{array}{c}1770 \\
\text { CKB-149 }\end{array}$ & $\begin{array}{c}1770 \\
\text { CKB-150 } \\
\end{array}$ & $\begin{array}{c}1770 \\
\text { CKB-151 } \\
\end{array}$ & $\begin{array}{c}1770 \\
\text { CKB-151 } \\
\end{array}$ & $\begin{array}{c}1630 \\
\text { CKB-206 }\end{array}$ & $\begin{array}{c}1630 \\
\text { CKB-207 }\end{array}$ & $\begin{array}{c}1630 \\
\text { CKB-208 } \\
\end{array}$ & $\begin{array}{c}1651 \\
\text { CKB-211 }\end{array}$ & $\begin{array}{c}1651 \\
\text { CKB-212 } \\
\end{array}$ & $\begin{array}{c}3443 \\
\text { CKB-213 } \\
\end{array}$ & $\begin{array}{c}1769 \\
\text { CKB-214 } \\
\end{array}$ \\
\hline Si(wt.s) & 3.62 & 2.62 & 1.92 & 2.14 & 205 & 86 & 1.38 & 254 & 3.53 & 3.73 & 243 \\
\hline $\mathrm{Ti}$ & .96 & 1.13 & 1.26 & 1.23 & 91 & 55 & .73 & 82 & .72 & 1.31 & 1.08 \\
\hline Mn & 24.6 & 26.8 & 29.0 & 28.2 & 273 & 24.6 & 26.7 & 28.9 & 12.2 & 227 & 27.1 \\
\hline Fo & 15.6 & 14.7 & 13.4 & 13.0 & 14.9 & 7.01 & 9.61 & 12.5 & 11.6 & 19.0 & 14.9 \\
\hline $\mathbf{A}$ & .80 & .79 & .66 & .84 & 47 &.$\infty$ & .75 & .79 & 1.19 & 1.03 & .62 \\
\hline Co & 1.35 & 1.16 & 1.07 & 121 & 1.34 & .507 & 829 & 1.21 & .400 & 947 & 1.26 \\
\hline $\mathrm{Ni}$ & 465 & .597 & .664 & .697 & 504 & .848 & 1.061 & .681 & 346 & .293 & 585 \\
\hline c & 2.52 & 251 & 279 & 272 & 258 & 129 & 7.76 & 3.22 & 12.2 & 2.53 & 275 \\
\hline $\mathbf{M g}$ & 1.11 & 1.16 & 1.17 & 1.20 & 1.11 & 2.14 & 1.97 & 1.33 & 1.91 & 1.09 & 1.10 \\
\hline $\mathbf{K}$ & .50 & 53 & .56 & 55 & 46 & .46 & .56 & .67 & .70 & A8 & 54 \\
\hline $\mathbf{P}$ & .43 & 38 & .35 & .37 & 45 & 3.84 & 2.17 & 54 & 3.79 & .42 & 46 \\
\hline $\mathrm{H}_{2} \mathrm{O}$ & 9.6 & 9.6 & 9.7 & 9.7 & 8.8 & 9.2 & 9.5 & 119 & 7.5 & 11.3 & 112 \\
\hline $50^{\circ} 0^{\circ} \mathrm{C}$ & - & - & • & - & - & • & • & • & - & • & - \\
\hline $1000^{\circ} \mathrm{C}$ & - & - & - & - & - & - & - & - & - & - & - \\
\hline $\begin{array}{l}\text { Sermpled } \\
\text { Enturval }\end{array}$ & $\begin{array}{l}\text { Young } \\
\text { O-10mm }\end{array}$ & $\begin{array}{l}\text { Middlo } \\
\text { O-10mm }\end{array}$ & $\begin{array}{l}\text { Old } \\
0-10 \mathrm{~mm}\end{array}$ & $\begin{array}{l}\text { Old } \\
0-10 \mathrm{~mm}\end{array}$ & $\begin{array}{c}\text { Young-Top } \\
\text { 0-20mm }\end{array}$ & $\begin{array}{l}\text { Old-Top } \\
20-30 \mathrm{~mm}\end{array}$ & $\begin{array}{c}\text { Bottam } \\
\text { Smin }\end{array}$ & $\begin{array}{l}\text { Young } \\
\text { O-Smm }\end{array}$ & $\underset{5-15 m m}{a d d}$ & $\begin{array}{c}\text { Bulk } \\
0-15 \text { inm }\end{array}$ & $\begin{array}{l}\text { Top } \\
\text { 15mm }\end{array}$ \\
\hline
\end{tabular}

\begin{tabular}{|c|c|c|c|c|c|c|c|c|c|c|c|}
\hline Sequencel & 053116 & 053116 & 053117 & 053117 & 053118 & 053118 & 053119 & 053119 & 053119 & $05312 \theta$ & 053130 \\
\hline Letitude & 15.544 & 15.544 & 15.615 & 15.615 & 15584 & 15.584 & 15.628 & 15.628 & 15.628 & $14 A 56$ & 15.597 \\
\hline $\begin{array}{l}\text { Longituda } \\
\text { Depth } \\
\text { Lab" }\end{array}$ & $\begin{array}{c}-169.238 \\
1769 \\
\text { CKB-214 }\end{array}$ & $\begin{array}{c}-169.238 \\
1769 \\
\text { CKB-215 }\end{array}$ & $\begin{array}{c}-169.227 \\
1498 \\
\text { CKB-224 }\end{array}$ & $\begin{array}{c}-169.227 \\
1498 \\
\text { CKB-225 }\end{array}$ & $\begin{array}{c}-169.205 \\
1605 \\
\text { CKB-228 }\end{array}$ & $\begin{array}{c}-169.205 \\
1605 \\
\text { CKB-220 }\end{array}$ & $\begin{array}{c}-169.239 \\
1552 \\
\text { CKB-230 }\end{array}$ & $\begin{array}{c}-169.239 \\
1552 \\
\text { CKB-231 }\end{array}$ & $\begin{array}{c}-169.239 \\
1552 \\
\text { CKB-231 }\end{array}$ & $\begin{array}{c}-169.003 \\
1770 \\
\text { CKB-152 }\end{array}$ & $\begin{array}{c}-169.232 \\
1630 \\
\text { CKB-209 }\end{array}$ \\
\hline Si(wt.\%) & 2.48 & 266 & 1.97 & 1.09 & 206 & 1.64 & 1.52 & .75 & .92 & 2.63 & 2.20 \\
\hline $\mathrm{Ti}$ & 1.08 & 1.17 & .97 & 85 & 91 & .76 & .88 & .72 & .73 & 1.00 & 1.07 \\
\hline Mn & 27.5 & 26.4 & 29.5 & 27.6 & 29.4 & 18.1 & 30.6 & 273 & 273 & 26.9 & 29.2 \\
\hline Fo & 15.0 & 12.1 & 13.6 & 8.83 & 14.9 & 11.0 & 14.2 & 8.11 & 8.04 & 12.5 & 14.0 \\
\hline Al & .62 & 1.00 & .59 & 32 & 37 & 55 & .27 & .11 & .15 & 1.11 & .61 \\
\hline Co & 1.33 & .761 & 1.28 & .832 & 134 & 336 & 1.48 & 829 & .739 & 1.10 & 1.26 \\
\hline $\mathrm{Ni}$ & .405 & .716 & .756 & 943 & .624 & .401 & .660 & .873 & 941 & .753 & 548 \\
\hline ca & 2.78 & 5.73 & 264 & 680 & 2.67 & 14.1 & 2.70 & 10.1 & 103 & 2.77 & 281 \\
\hline $\mathrm{Mg}$ & 1.13 & 1.49 & 1.25 & 1.56 & 1.17 & 2.16 & 1.22 & 1.84 & 1.97 & 1.27 & 1.15 \\
\hline $\mathbf{K}$ & .55 & .59 & .54 & 52 & .51 & 38 & .50 & .53 & .52 & .61 & 56 \\
\hline $\mathbf{P}$ & .46 & 1.36 & .39 & 1.72 & 43 & 4.56 & .42 & 2.74 & 289 & .46 & 45 \\
\hline $\mathrm{H}_{2} \mathrm{O}$ & 112 & 10.6 & 10.1 & 9.9 & 13.4 & 7.8 & 13.6 & 10.7 & 10.7 & 8.4 & 124 \\
\hline $50^{2} \mathrm{C}$ & - & $\cdot$ & - &. & . & - & - & - & . & - & . \\
\hline $1000^{\circ} \mathrm{C}$ & - & - & - & - & - & - & - & - & - & - & - \\
\hline $\begin{array}{l}\text { Sempled } \\
\text { Interval }\end{array}$ & $\begin{array}{c}\text { Top } \\
15 \mathrm{~mm}\end{array}$ & $\begin{array}{l}\text { Bottom } \\
15 \mathrm{smm}\end{array}$ & $\begin{array}{l}\text { Young } \\
10 \mathrm{~mm}\end{array}$ & $\begin{array}{l}\text { Old } \\
20 \mathrm{~mm}\end{array}$ & $\begin{array}{l}\text { Young } \\
25 \mathrm{~mm}\end{array}$ & $\begin{array}{l}\text { Old } \\
20 \mathrm{~mm}\end{array}$ & $\begin{array}{c}\text { Young-Top } \\
20 \mathrm{~mm}\end{array}$ & $\begin{array}{l}\text { Old-Bottom } \\
30 \mathrm{~mm}\end{array}$ & $\begin{array}{l}\text { Old-Bottom } \\
30 \mathrm{~mm}\end{array}$ & $\begin{array}{c}\text { Top } \\
10 \mathrm{~mm}\end{array}$ & $\begin{array}{l}\text { Young } \\
15 \mathrm{~mm}\end{array}$ \\
\hline
\end{tabular}

\begin{tabular}{|c|c|c|c|c|c|c|c|c|c|c|c|}
\hline Soquencoll & 053130 & 053130 & 053131 & 053131 & 053131 & 053131 & 053131 & 053132 & 053132 & 053132 & 053133 \\
\hline Latimds & 15.597 & 15.597 & 15544 & 15.544 & 15.544 & 15.544 & 15.544 & 15.544 & 15.544 & 15.544 & 15.615 \\
\hline Longitude & -169.232 & -169.232 & -169.238 & -169.238 & -169.238 & -169.238 & -169.238 & -169.238 & -169.238 & -169.238 & -169.227 \\
\hline Depth & 1630 & 1630 & 1769 & 1769 & 1769 & 1769 & 1769 & 1769 & 1769 & 1769 & 1498 \\
\hline Lab" & CKB-209 & CKB-210 & CKB-216 & CKB-217 & CKB-218 & CKB-219 & CKB-219 & CKB-221 & CKB-222 & CKB-223 & CKB-226 \\
\hline si(wt.s) & 2.32 & 1.92 & 2.50 & 2.12 & 99 & 1.38 & 1.49 & 2.26 & 1.77 & 1.15 & 1.42 \\
\hline $\mathrm{Ti}$ & 1.07 & .60 & .99 & 1.08 & 83 & 56 & .57 & .91 & 1.06 & .75 & 1.10 \\
\hline Mn & 29.0 & 18.6 & 27.8 & 292 & 27.0 & 16.5 & 16.7 & 27.3 & 28.9 & 23.8 & 32.3 \\
\hline $\mathrm{Fe}_{0}$ & 14.0 & 7.31 & 120 & 142 & 9.14 & 9.45 & 9.48 & 15.4 & 133 & 9.12 & 12.3 \\
\hline $\mathbf{A}$ & .69 & .60 & .58 & .60 & 31 & .33 & .40 & 49 & 45 & .29 & 46 \\
\hline Co & 1.44 & .433 & 1.30 & 1.17 & .661 & .298 & .266 & 1.40 & 1.19 & .574 & 1.52 \\
\hline $\mathrm{Ni}$ & .674 & .707 & .582 & .697 & .958 & .414 & .436 & 530 & .656 & .725 & .789 \\
\hline C. & 2.83 & 16.8 & 2.76 & 2.64 & 9.47 & 16.2 & 16.3 & 2.61 & 2.57 & 10.8 & 2.73 \\
\hline $\mathbf{M g}$ & 1.20 & 2.67 & 1.21 & 1.20 & 1.83 & 2.31 & 2.36 & 1.17 & 1.14 & 1.90 & 1.32 \\
\hline $\mathbf{K}$ & .54 & .48 & 51 & 54 & 50 & 34 & .34 & 47 & .50 & $A 5$ & 58 \\
\hline $\mathbf{P}$ & .46 & 5.55 & .49 & .38 & 258 & 5.25 & 5.33 & .45 & .36 & 3.13 & .35 \\
\hline $\mathrm{H}_{2} \mathrm{O}$ & 124 & 12.3 & 14.1 & 11.0 & 9.2 & 5.9 & 5.9 & 9.5 & 8.6 & 7.6 & 13.8 \\
\hline $50^{\circ} \mathrm{C}$ & - & - & - & - & - & - & - & - & - & - & - \\
\hline $1000^{\circ} \mathrm{C}$ & - & - & - & - & - & - & - & - & - & - & - \\
\hline $\begin{array}{l}\text { Sempled } \\
\text { Interval }\end{array}$ & $\begin{array}{l}\text { Young } \\
15 \text { mm }\end{array}$ & $\begin{array}{l}\text { Old } \\
15 \mathrm{~m} m \mathrm{~m}\end{array}$ & Young-Top & - & $\begin{array}{l}\text { Old-Top } \\
10 \mathrm{~mm}\end{array}$ & $\begin{array}{l}\text { Old-Bottom } \\
10 \mathrm{~mm}\end{array}$ & $\begin{array}{l}\text { Old-Bottom } \\
10 \mathrm{~mm}\end{array}$ & $\begin{array}{l}\text { Young } \\
15 \text { min }\end{array}$ & $\begin{array}{l}\text { Middle } \\
\text { ISmm }\end{array}$ & $\begin{array}{l}\text { Old } \\
40 \mathrm{~mm}\end{array}$ & $\begin{array}{c}\text { Young-Top } \\
20 \mathrm{~mm}\end{array}$ \\
\hline
\end{tabular}


Table 2. Composition of ferromanganese crusts analyzed by USGS Woods Hole analytical laboratories (cont'd).

\begin{tabular}{|c|c|c|c|c|c|c|c|c|c|}
\hline Sequencol & 053135 & 053135 & 053135 & 053135 & 053136 & 053136 & 053136 & 053137 & 053137 \\
\hline Latiende & 15.628 & 15.628 & 15.628 & 15.628 & 15.628 & 15.628 & 15.628 & 15.628 & 15.628 \\
\hline Longitude & -169.239 & -169.239 & -169.239 & -169.239 & -169.239 & -169.239 & -169.239 & -169.239 & -169.239 \\
\hline $\begin{array}{l}\text { Depth } \\
\text { Lebl }\end{array}$ & $\begin{array}{c}1552 \\
\text { CKB-232 }\end{array}$ & $\begin{array}{c}1552 \\
\text { CKB-233 }\end{array}$ & $\begin{array}{c}1552 \\
\text { CKB-234 }\end{array}$ & $\begin{array}{c}1552 \\
\text { CKB-235 }\end{array}$ & $\begin{array}{c}1552 \\
\text { CKB-236 }\end{array}$ & $\begin{array}{c}1552 \\
\text { CKB-236 }\end{array}$ & $\begin{array}{c}1552 \\
\text { CKB-237 }\end{array}$ & $\begin{array}{c}1552 \\
\text { CKB-239 }\end{array}$ & $\begin{array}{c}1552 \\
\text { CKB-240 }\end{array}$ \\
\hline$S i(w t . \%)$ & 1.85 & 95 & 1.20 & .91 & 1.40 & 1.58 & .90 & 1.69 & 1.36 \\
\hline$\pi$ & 1.03 & 57 & 1.04 & .54 & 93 & 92 & .71 & 94 & .98 \\
\hline Mn & 30.3 & 222 & 31.7 & 18.8 & 30.5 & 30.3 & 260 & 29.6 & 27.1 \\
\hline Po & 13.5 & 7.78 & 122 & 7.47 & 13.6 & 135 & 8.04 & 14.4 & 10.8 \\
\hline $\mathbf{A}$ & 54 & .14 & .62 & .16 & 36 & 37 & .20 & 34 & A5 \\
\hline Co & 1.21 & .439 & 1.29 & 399 & 1.41 & 1.55 & .712 & 1.44 & .723 \\
\hline $\mathrm{Ni}$ & .750 & .691 & .859 & .603 & .667 & .702 & 898 & .623 & .766 \\
\hline c & 289 & 14.3 & 2.81 & 15.7 & 2.71 & 2.69 & 10.7 & 2.67 & 6.69 \\
\hline $\mathrm{Mg}_{\mathbf{g}}$ & 1.26 & 2.24 & 1.24 & 234 & 1.15 & 1.17 & 2.04 & 1.24 & 1.52 \\
\hline $\mathbf{K}$ & 53 & .41 & 55 & .38 & 52 & 52 & .49 & 49 & 52 \\
\hline $\mathbf{P}$ & .13 & 4.37 & 37 & 4.91 & 38 & 39 & 3.10 & 12 & 1.64 \\
\hline $\mathrm{H}_{2} \mathrm{O}$ & 10.6 & 88 & 12.7 & 7.2 & 13.1 & 13.1 & 8.7 & 118 & 73 \\
\hline $50^{\circ} \mathrm{C}$ & - & 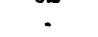 & 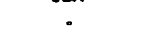 & 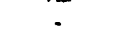 & - & 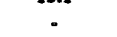 & - & - & . \\
\hline $1000^{\circ} \mathrm{C}$ & - & - & - & - & - & - & . & - & - \\
\hline $\begin{array}{l}\text { Samplod } \\
\text { Interval }\end{array}$ & $\begin{array}{l}\text { Youns-Top } \\
10 \mathrm{mmn}\end{array}$ & $\begin{array}{l}\text { Old-Top } \\
10 \mathrm{~mm}\end{array}$ & $\begin{array}{c}\text { Young-Bottom } \\
7 \mathrm{~mm}\end{array}$ & $\begin{array}{l}\text { Old-Bottam } \\
7 \mathrm{mmm}\end{array}$ & $\begin{array}{l}\text { Yount-Top } \\
15 \mathrm{~mm}\end{array}$ & $\begin{array}{l}\text { Young-Top } \\
15 \mathrm{mmm}\end{array}$ & $\begin{array}{l}\text { Old-Bottom } \\
25 \mathrm{~mm}\end{array}$ & $\begin{array}{l}\text { Young } \\
15 \operatorname{mm}\end{array}$ & $\begin{array}{l}\text { Old } \\
1 \text { smm }\end{array}$ \\
\hline
\end{tabular}

\begin{tabular}{lccccccccc}
\hline Sequencel & 053140 & 054001 & 054003 & 054003 & 054005 & 054005 & 054006 & 054006 \\
Latitude & 14.456 & 19.243 & 19.402 & 19.402 & 19.361 & 19.361 & 19.373 & 19.373 & 19.373 \\
Langitude & -169.003 & -179.668 & -171.173 & -171.173 & -171.064 & -171.064 & -170.991 & -170.991 & -170.991 \\
Depth & 1770 & 3224 & 2856 & 2856 & 2010 & 2010 & 1190 & 1190
\end{tabular}

\begin{tabular}{lccccccccc} 
Depth & 1770 & 3224 & 2856 & 2856 & 2010 & 2010 & 1190 & 1190 \\
Leb & $10 D K-S E M-63$ & W-222588 & MP1-CKB-42 & MP1-CKB-42* & MP1-CKB-45 & MP1-CKB-45* & MP1-CKB-46 & MP1-CKB-46* & MP1-CKB-47 \\
\hline
\end{tabular}

Si(wt.\%) $\quad 2.23 \quad 6.02$

\begin{tabular}{|c|c|c|c|c|c|c|c|c|c|}
\hline Sil(wt.\%) & 223 & 6.02 & - & - & - & $\bullet$ & - & - & - \\
\hline$\pi$ & 1.09 & 1.00 & 1.89 & 1.11 & 2.31 & 1.28 & .92 & - & .86 \\
\hline Mn & 26.2 & 17.1 & 21.0 & • & 25.0 & $\cdot$ & 27.1 & - & 24.5 \\
\hline$F_{0}$ & 13.4 & 15.9 & 13.8 & - & 15.8 & - & 17.1 & - & 15.3 \\
\hline $\mathbf{A}$ & 54 & 1.51 & - & - & - & - & - & - & - \\
\hline Co & 1.12 & .441 & .450 & 460 & .780 & .900 & 1.08 & 1.04 & .590 \\
\hline $\mathbf{N i}$ & .567 & .245 & .210 & $\cdot$ & .460 & - & .400 & $\cdot$ & .520 \\
\hline ca & 4.23 & 7.26 & 2.24 & - & 2.43 & - & 2.60 & = & 6.90 \\
\hline $\mathbf{M g}_{8}$ & 1.18 & 1.27 & $\cdot$ & - & - & - & $\cdot$ & - & • \\
\hline $\mathbf{K}$ & 54 & .67 & - & - & • & - & - & - & - \\
\hline $\mathbf{P}$ & .90 & 2.37 & .48 & - & .44 & - & .45 & - & 2.12 \\
\hline $\mathrm{H}_{2} \mathrm{O}$ & 18.9 & 18.4 & 93 & 9.5 & 13,3 & 13.3 & 28.8 & 28.8 & 26.3 \\
\hline $500^{\circ} \mathrm{C}$ & - & $\cdot$ & - & - & - & - & - & - & - \\
\hline $1000^{\circ} \mathrm{C}$ & - & - & - & - & - & - & - & - & • \\
\hline Semplod & Bulk & Bulk & Bulk & Bulk & Bulk & Bulk & Bulk & Bulk & Bulk \\
\hline brterval & $38 \mathrm{~mm}$ & $0-8 \mathrm{~mm}$ & • & - & • & • & • & • & - \\
\hline
\end{tabular}

\begin{tabular}{|c|c|c|c|c|c|c|c|c|c|}
\hline Sequencel & 054007 & 054008 & 054008 & 054012 & 054012 & 054013 & 054013 & 054014 & 054014 \\
\hline Latitude & 19373 & 19.373 & 19.373 & 19.408 & 19.408 & 19408 & 19.408 & 19.408 & 19.408 \\
\hline Langitude & -170.991 & -170.991 & -170.991 & -171.391 & -171.391 & -171.391 & -171.391 & -171.391 & -171.391 \\
\hline Depth & 1190 & 1190 & 1190 & 1925 & 1925 & 1925 & 1925 & 1925 & 1925 \\
\hline
\end{tabular}

$\begin{array}{lccccccccc}\text { Depth } & 1190 & 1190 & 1190 & 1925 & 1925 & 1925 & 1925 & 1925 \\ \text { Leb* } & \text { MP1-CKB-47* } & \text { MP1-CKB-48 } & \text { MP1-CKB-48* } & \text { MP1-CKB-52 } & \text { MP1-CKB-52* } & \text { MP1-CKB-53 } & \text { MP1-CKB-53* } & \text { MP1-CKB-54 } & \text { MP1-CKB-54* }\end{array}$ Si(sot $\%)$

\begin{tabular}{|c|c|c|c|c|c|c|c|c|c|}
\hline Si(wt.\%) & - & - & - & - & - & - & - & - & - \\
\hline $\mathbf{T i}$ & .76 & .97 & .94 & .63 & 59 & 1.04 & - & 98 & - \\
\hline Mn & - & 29.2 & - & 26.3 & - & 26.1 & - & 17.0 & - \\
\hline Fo & - & 18.3 & - & 165 & - & 15.4 & - & 10.4 & - \\
\hline Al & $\cdot$ & • & $\cdot$ & • & • & • & • & - & • \\
\hline Co & .660 & 1.70 & 156 & .470 & 520 & .540 & .610 & .430 & .420 \\
\hline $\mathrm{Ni}$ & $\cdot$ & .600 & - & .440 & - & .530 & - & .400 & $\cdot$ \\
\hline ca & - & 2.64 & - & 8.17 & - & 2.67 & - & 5.58 & - \\
\hline Mg & - & - & - & - & - & - & - & $\cdot$ & - \\
\hline $\mathbf{K}$ & - & - & - & - & - & - & - & - & - \\
\hline $\mathbf{P}$ & - & .39 & - & 2.64 & - & .40 & - & 188 & - \\
\hline $\mathrm{H}_{2} \mathrm{O}$ & 26.3 & 32.2 & 32.2 & 24.2 & 24.2 & 21.5 & 215 & 26.8 & 26.8 \\
\hline $50^{\circ} \mathrm{C}$ & - & - & - & $\cdot$ & • & • & - & - & - \\
\hline $1000^{\circ} \mathrm{C}$ & - & - & - & - & - & - & - & $\cdot$ & - \\
\hline Sampled & Bulk & Bulk & Bulk & Bulk & Bulk & Bulk & Bulk & Bulk & Bulk \\
\hline Intorval & - & . & - & • & - & - & - & - & - \\
\hline
\end{tabular}


Table 2. Composition of ferromanganese crusts analyzed by USGS Woods Hole analytical laboratories (cont'd).

\begin{tabular}{|c|c|c|c|c|c|c|c|c|c|c|}
\hline Sequencol" & 054019 & 054023 & 054023 & 054024 & 054024 & 054025 & 054025 & 054033 & 054033 & 054034 \\
\hline Latitude & 15.696 & 15.671 & 15.671 & 15.664 & 15.664 & 15.673 & 15.673 & 15.658 & 15.658 & 15.665 \\
\hline Langitods & -170.344 & -170.413 & -170.413 & -170.397 & -170.397 & -170.381 & -170.381 & -170.385 & -170.385 & .170 .386 \\
\hline $\begin{array}{l}\text { Depth } \\
\text { Leb" }\end{array}$ & $\begin{array}{c}2260 \\
\text { CKB-154 }\end{array}$ & $\begin{array}{c}1815 \\
\text { CKB-171 }\end{array}$ & $\begin{array}{c}1815 \\
\text { CKB-172 }\end{array}$ & $\begin{array}{c}1433 \\
\text { CKB-180 }\end{array}$ & $\begin{array}{c}1433 \\
\text { CKB-181 }\end{array}$ & $\begin{array}{c}1117 \\
\text { CKB-184 }\end{array}$ & $\begin{array}{c}1117 \\
\text { CKB-186 }\end{array}$ & $\begin{array}{c}1186 \\
\text { CXB-194 }\end{array}$ & $\begin{array}{c}1186 \\
\text { CKB-194 }\end{array}$ & $\begin{array}{c}1520 \\
\text { CKB-198 } \\
\end{array}$ \\
\hline Sil(wt.\%) & 4.50 & 3.11 & 2.94 & 2.14 & .98 & 4.05 & 1.10 & 92 & 1.08 & 1.25 \\
\hline $\mathbf{T i}$ & 1.07 & 96 & 1.22 & .86 & 50 & .77 & .71 & 86 & .85 & .75 \\
\hline Mn & 22.8 & 26.3 & 26.2 & 28.1 & 16.8 & 30.7 & 29.2 & 32.4 & 31.6 & 30.3 \\
\hline$C_{0}$ & .857 & 1.18 & .953 & 1.25 & .402 & 133 & 1.44 & 1.47 & 1.74 & 1.74 \\
\hline $\mathbf{N i}$ & 499 & .562 & .625 & .609 & .507 & .845 & .670 & .721 & .710 & 591 \\
\hline a & 2.60 & 262 & 3.45 & 4.20 & 17.6 & 3.59 & 292 & 277 & 2.70 & 2.65 \\
\hline Mg & 1.07 & 1.11 & 1.19 & 1.32 & 2.33 & 139 & 1.15 & 1.29 & 1.17 & 1.19 \\
\hline $\mathbf{K}$ & .61 & so & 57 & .50 & 35 & .69 & 51 & 53 & .50 & .47 \\
\hline $\mathbf{P}$ & .44 & .44 & .59 & .94 & 5.85 & .69 & .51 & 38 & .37 & .45 \\
\hline $\begin{array}{l}\text { Sarmpled } \\
\text { Interval }\end{array}$ & $\begin{array}{c}\text { Bulk } \\
10 \mathrm{~mm}\end{array}$ & $\begin{array}{c}\text { Top } \\
20 \mathrm{~mm}\end{array}$ & $\begin{array}{l}\text { Bottom } \\
20 \mathrm{~mm}\end{array}$ & $\underset{25 \mathrm{~mm}}{\text { Top }}$ & $\begin{array}{l}\text { Bottom } \\
25 \text { mm }\end{array}$ & $\begin{array}{l}\text { Young-Top } \\
15 \mathrm{~mm}\end{array}$ & $\begin{array}{c}\text { Young-Botarn } \\
8 \mathrm{~mm}\end{array}$ & $\begin{array}{c}\text { Top } \\
20 \mathrm{~mm}\end{array}$ & $\begin{array}{l}\text { Top } \\
20 \mathrm{~mm}\end{array}$ & Youns \\
\hline
\end{tabular}

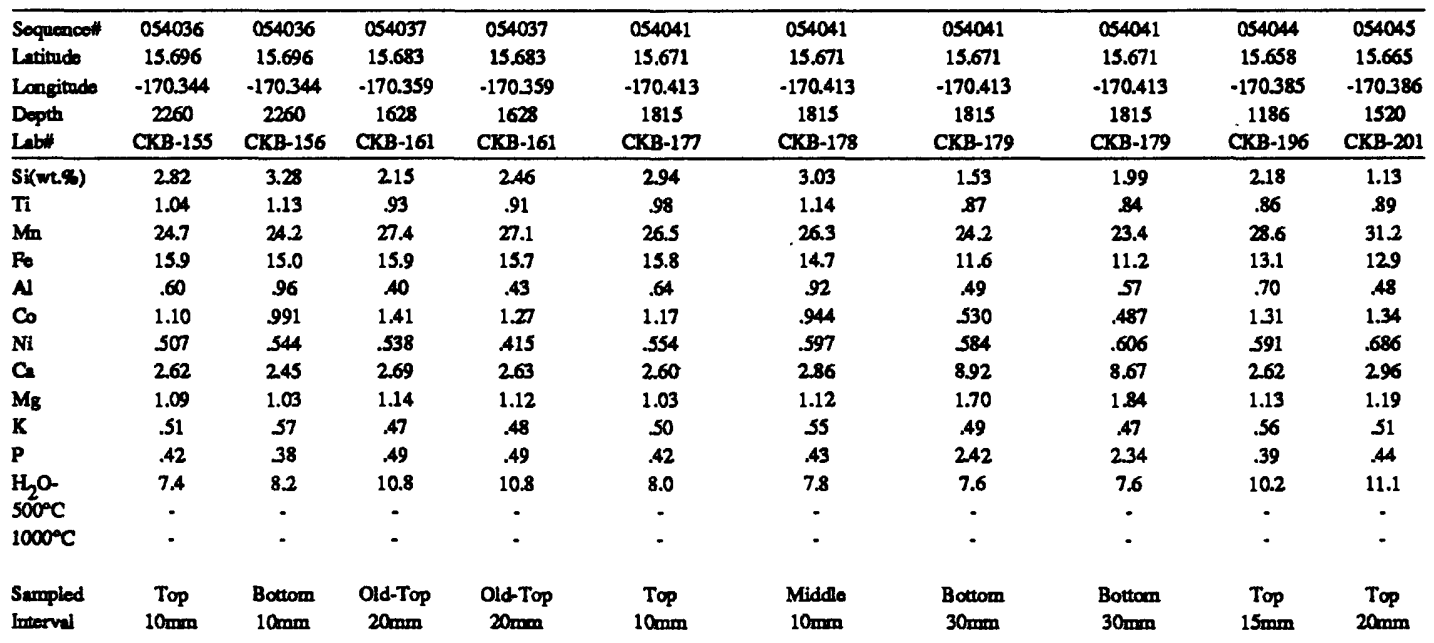

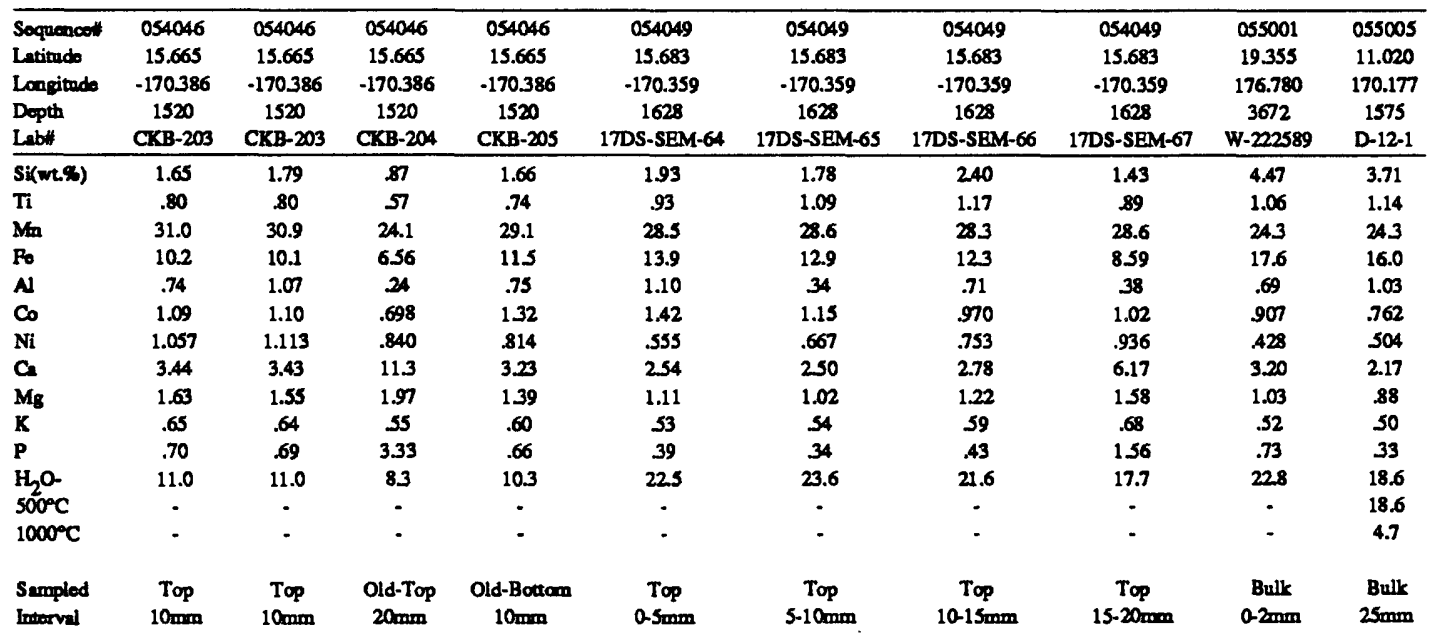


Table 2. Composition of ferromanganese crusts analyzed by USGS Woods Hole analytical laboratories (cont'd).

\begin{tabular}{|c|c|c|c|c|c|c|c|c|c|}
\hline Sequencoll & 055006 & 055000 & 056006 & 056008 & 056009 & 079001 & 080002 & 080003 & 080004 \\
\hline Latitude & 11.008 & 11.005 & 12.183 & 13.905 & 13.900 & 24.277 & 22.333 & 22.570 & 22.642 \\
\hline Langitudo & 170.203 & 170.230 & 168.995 & 167.627 & 167.653 & -68.107 & -72.085 & -72.375 & -72.850 \\
\hline Depth & 2000 & 1800 & 1300 & 1600 & 1600 & 5668 & 2250 & 3420 & 2390 \\
\hline Leb" & $D-13-4$ & D-14-3 & D-15 & D-17 & D-18 & W-222722 & W.222703 & W.2220704 & W-222005 \\
\hline Si(wt.\%) & 3.27 & 1.36 & 206 & 243 & 3.70 & 126 & 2.68 & 5.13 & 5.14 \\
\hline $\mathbf{T}$ & 1.14 & 1.20 & 93 & 1.03 & 1.01 & 92 & 1.07 & .87 & 56 \\
\hline Mn & 25.8 & 28.4 & 25.9 & 26.8 & 23.9 & 9.46 & 147 & 11.1 & 9.30 \\
\hline Fe & 16.0 & 13.2. & 15.8 & 14.6 & 17.2 & 16.5 & 27.2 & 255 & 21.3 \\
\hline $\mathbf{N}$ & 94 & .74 & 47 & .65 & 91 & 4.79 & 228 & 3.28 & 3.50 \\
\hline Co & 1.11 & 1.10 & .872 & .956 & .890 & .184 & .689 & 390 & .282 \\
\hline $\mathrm{Ni}$ & .489 & .619 & .461 & 546 & .410 & .034 & .119 & .110 & .106 \\
\hline C. & 219 & 3.21 & 240 & 246 & 231 & 92 & 2.12 & 4.24 & 7.93 \\
\hline $\mathbf{M g}$ & 91 & 1.00 & 85 & .98 & .89 & 1.45 & 156 & 1.76 & 1.91 \\
\hline $\mathrm{K}$ & 51 & .46 & .44 & .48 & so & 94 & .37 & .57 & 55 \\
\hline $\mathbf{P}$ & 33 & 55 & 39 & .35 & .41 & .03 & .62 & .69 & 53 \\
\hline $\mathrm{H}_{2} \mathrm{O}$ & 18.3 & 11.2 & 19.8 & 19.4 & 14.6 & 129 & 15.9 & 17.9 & 14.8 \\
\hline $50^{\circ} \mathrm{C}$ & 115 & 22.2 & 18.5 & 21.0 & 19.7 & - & $\cdot$ & 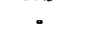 & - \\
\hline $1000^{\circ} \mathrm{C}$ & 11.0 & 3.8 & 74 & 49 & 3.5 & - & - & - & . \\
\hline $\begin{array}{l}\text { Sumplod } \\
\text { Intervel }\end{array}$ & $\begin{array}{l}\text { Bulk } \\
30 \mathrm{~mm}\end{array}$ & $\begin{array}{l}\text { Bulk } \\
10 \mathrm{~mm}\end{array}$ & $\begin{array}{l}\text { Bulk } \\
30 \mathrm{~mm}\end{array}$ & $\begin{array}{c}\text { Bulk } \\
20 \mathrm{mann}\end{array}$ & $\begin{array}{l}\text { Bulk } \\
30 \mathrm{~mm}\end{array}$ & $\begin{array}{c}\text { Bulk } \\
\text { a-12mm }\end{array}$ & $\underset{0.17 \mathrm{~mm}}{\text { Bulk }}$ & $\underset{\text { Bulkm }}{\text { Bulk }}$ & $\begin{array}{c}\text { Bulk } \\
0.28 \mathrm{mmm}\end{array}$ \\
\hline
\end{tabular}

\begin{tabular}{|c|c|c|c|c|c|c|c|c|c|}
\hline $\begin{array}{l}\text { Sequenoes } \\
\text { Latitude }\end{array}$ & $\begin{array}{l}080005 \\
23.157\end{array}$ & $\begin{array}{l}080006 \\
23.183\end{array}$ & $\begin{array}{l}080007 \\
23.487\end{array}$ & $\begin{array}{l}089001 \\
21.670\end{array}$ & $\begin{array}{l}089013 \\
22.208\end{array}$ & $\begin{array}{l}089014 \\
22256\end{array}$ & $\begin{array}{l}089016 \\
22.316\end{array}$ & $\begin{array}{l}089017 \\
21.718\end{array}$ & $\begin{array}{l}089020 \\
21.718\end{array}$ \\
\hline Langitude & .73 .752 & .73 .973 & .74 .200 & -167.953 & -166.882 & -166.854 & -166.899 & -167578 & -167.578 \\
\hline Depth & 3126 & 1315 & 2910 & 1690 & 2402 & 1970 & 2350 & 2400 & 2400 \\
\hline Lab" & W-222706 & W- 2222707 & W-222708 & W-222571 & 2 & 3 & 5 & 6 & 9 \\
\hline SH(wt.\%) & 3.73 & 2.30 & 1.67 & 7.87 & 7.12 & 5.93 & 4.17 & 5.53 & 3.93 \\
\hline $\mathrm{Ti}$ & 58 & 95 & 98 & 1.18 & 1.42 & 1.39 & 135 & 1.77 & 1.50 \\
\hline Mn & 644 & 16.8 & 18.2 & 23.2 & 17.1 & 19.9 & 224 & 21.2 & 23.8 \\
\hline Fo & 15.6 & 25.7 & 25.9 & 11.9 & 18.3 & 20.0 & 20.8 & 169 & 174 \\
\hline $\mathbf{N}$ & 1.76 & 2.61 & 2.23 & 2.94 & 286 & 2.01 & 138 & 2.20 & 1.74 \\
\hline Co & .198 & .722 & .775 & 317 & 497 & .659 & .734 & 562 & 1.22 \\
\hline $\mathbf{N i}$ & .011 & .253 & .305 & 915 & 307 & .266 & .287 & 387 & 392 \\
\hline C. & 15.3 & 259 & 2.14 & 1.43 & 232 & 242 & 243 & 233 & 299 \\
\hline $\mathbf{M}_{\mathbf{g}}$ & 2.19 & 1.82 & 1.78 & 1.73 & 1.05 & 1.04 & .99 & .91 & 1.23 \\
\hline $\mathrm{K}$ & .40 & .33 & .33 & .91 & .61 & .51 & .48 & .67 & 53 \\
\hline $\mathbf{P}$ & .41 & 59 & .58 & .20 & 38 & $A 0$ & .46 & .34 & .48 \\
\hline $\mathrm{H}_{2} \mathrm{O}$ & 9.1 & 21.0 & 21.3 & 14.8 & 5.5 & 135 & 12.9 & 19.9 & 5.6 \\
\hline $50^{\circ} \mathrm{C}$ & 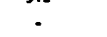 & 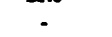 & - & 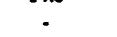 & 10.3 & 7.4 & 8.9 & 11.6 & 95 \\
\hline $1000^{\circ} \mathrm{C}$ & - & - & - & - & 9.6 & 8.6 & 3.6 & 8.9 & 10.1 \\
\hline Sempled & Bulk & Bulk & Bulk & Bulk & Bulk & Top & Bulk & Bottam & Top \\
\hline loterval & $0-5 \mathrm{~mm}$ & $0-30 \mathrm{cms}$ & $0-40 \mathrm{~mm}$ & $0-3 \mathrm{~mm}$ & - & $<\operatorname{mim}$ & - & • & $<\lim$ \\
\hline
\end{tabular}

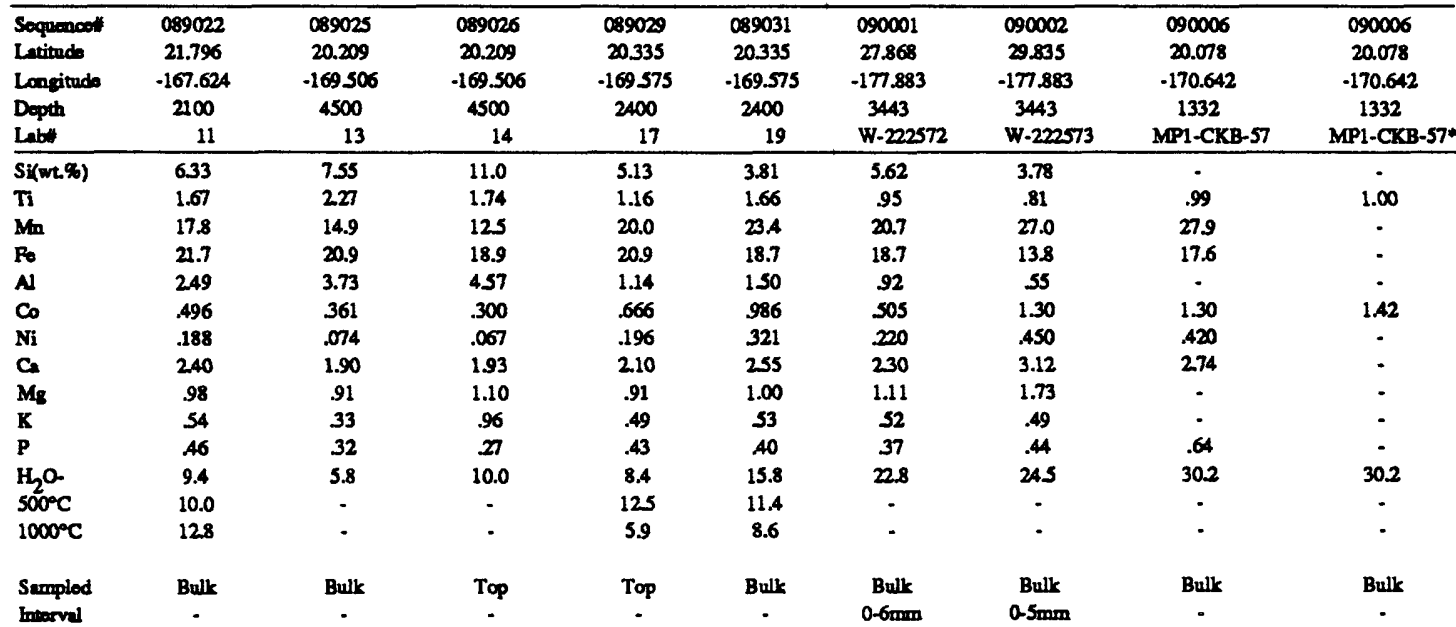


Table 2. Composition of ferromanganese crusts analyzed by USGS Woods Hole analytical laboratories (cont'd).

\begin{tabular}{|c|c|c|c|c|c|c|c|c|c|}
\hline $\begin{array}{l}\text { Soquencel } \\
\text { Letitude }\end{array}$ & $\begin{array}{l}090007 \\
20.078\end{array}$ & $\begin{array}{l}090007 \\
20.078\end{array}$ & $\begin{array}{l}090007 \\
20.078\end{array}$ & $\begin{array}{l}090007 \\
20.078\end{array}$ & $\begin{array}{c}090008 \\
20.078\end{array}$ & $\begin{array}{l}090008 \\
20.078\end{array}$ & $\begin{array}{l}090009 \\
20.220\end{array}$ & $\begin{array}{l}090009 \\
20.220\end{array}$ & $\begin{array}{l}090011 \\
20.133\end{array}$ \\
\hline $\begin{array}{l}\text { Longitade } \\
\text { Depth } \\
\text { Lab" }\end{array}$ & $\begin{array}{c}-170.642 \\
1332 \\
\text { MP1-CKB-58 }\end{array}$ & $\begin{array}{c}-170.642 \\
1332 \\
\text { MP1-CKB-58 }\end{array}$ & $\begin{array}{c}-170.642 \\
1332 \\
\text { MP1-CKB-58* }\end{array}$ & $\begin{array}{c}-170.642 \\
1332 \\
\text { MP1-CKB-58* }\end{array}$ & $\begin{array}{c}-170.642 \\
1332 \\
\text { MP1-CKB-59 }\end{array}$ & $\begin{array}{c}-170.642 \\
1332 \\
\text { MP1-CKB-59* }\end{array}$ & $\begin{array}{c}-170.568 \\
2573 \\
\text { MP1-CKB-60 }\end{array}$ & $\begin{array}{c}-170.568 \\
2573 \\
\text { MP1-CKB-60* }\end{array}$ & $\begin{array}{c}-170.681 \\
1476 \\
\text { CKB-124 }\end{array}$ \\
\hline Si(wt,\%) & - & - & • & • & • & • & - & - & 251 \\
\hline Ti & .74 & 86 & - & .72 & 1.20 & 1.03 & 1.70 & - & .96 \\
\hline Mn & 23.6 & 22.5 & - & . & 28.2 & • & 26.0 & - & 265 \\
\hline Po & 14.6 & 13.8 & - & - & 17.6 & - & 16.4 & - & 18.1 \\
\hline $\mathbf{N}$ & • & - & - & - & • & • & • & - & .65 \\
\hline$C_{0}$ & .680 & . 430 & 440 & .640 & .880 & .870 & 1.05 & 980 & 1.07 \\
\hline $\mathbf{N i}$ & .860 & .750 & $\cdot$ & $\cdot$ & .570 & • & 330 & • & .390 \\
\hline a & 11.1 & 9.76 & - & - & 289 & - & 290 & - & 2.40 \\
\hline Mg & • & • & - & - & - & - & • & - & .88 \\
\hline $\mathbf{K}$ & - & - & - & - & - & - & - & - & .43 \\
\hline $\mathbf{P}$ & 4.16 & 3.40 & - & - & .64 & - & .40 & - & .46 \\
\hline $\mathrm{H}_{2} \mathrm{O}$ & 26.6 & 26.7 & 26.7 & 26.6 & 13.9 & 13.9 & 37.9 & 37.9 & 135 \\
\hline $50^{\circ} 0^{\circ} \mathrm{C}$ & $\cdot$ & - & $\cdot$ & $\cdot$ & • & $\cdot$ & • & • & 11.2 \\
\hline $1000^{\circ} \mathrm{C}$ & . & - & - & - & - & - & - & - & 7.9 \\
\hline Semplod & Bulk & Bulk & Bulk & Bulk & Bulk & Bulk & Bulk & Bulk & Young \\
\hline Intervel & - & - & - & - & - & - & - & • & - \\
\hline
\end{tabular}

\begin{tabular}{|c|c|c|c|c|c|c|c|c|c|}
\hline Sequencelt & 090011 & 090011 & 090012 & 090012 & 090012 & 090013 & 090014 & 090015 & 090016 \\
\hline Latitude & 20.133 & 20.133 & 20.133 & 20.133 & 20.133 & 20.222 & 20.222 & 20.218 & 20.218 \\
\hline Longitude & -170.681 & $\cdot 170.681$ & -170.681 & -170.681 & -170.681 & -170.600 & -170.600 & -170.589 & -170.589 \\
\hline Depth & 1476 & 1476 & 1476 & 1476 & 1476 & • & - & 1480 & 1480 \\
\hline Lebi" & CKB-12S & CKB-126 & CKB-127 & CKB-128 & CKB-129 & CKB-130 & CKB-131 & CKB-132 & CKB-133 \\
\hline Sil(wt.\%) & 1.96 & 1.43 & 3.64 & 1.28 & 3.58 & 4.49 & 4.32 & 5.35 & 5.91 \\
\hline $\mathrm{Ti}$ & 1.20 & .64 & .98 & .75 & 1.24 & 1.16 & 1.24 & 1.15 & .05 \\
\hline Mn & 29.8 & 22.9 & 24.5 & 25.1 & 24.7 & 227 & 22.4 & 20.8 & 59.7 \\
\hline $\mathrm{Po}$ & 15.1 & 8.42 & 18.4 & 8.69 & 173 & 18.6 & 19.5 & 179 & 3.11 \\
\hline $\boldsymbol{N}$ & 89 & 37 & .81 & .46 & 1.28 & 1.29 & 1.25 & 1.43 & - \\
\hline$c_{0}$ & 1.01 & .370 & 1.05 & 530 & 940 & .710 & .700 & .710 & .002 \\
\hline $\mathrm{Ni}$ & .550 & .740 & 350 & .710 & $A 10$ & .420 & .310 & 310 & • \\
\hline Ca & 2.53 & 11.3 & 2.31 & 10.0 & 2.28 & 2.32 & 226 & 2.54 & - \\
\hline $\mathbf{M g}$ & .98 & 1.14 & .92 & 1.13 & .88 & 84 & 96 & .72 & . \\
\hline $\mathbf{K}$ & .49 & A4 & .45 & .50 & .52 & .48 & A7 & .52 & .75 \\
\hline $\mathbf{P}$ & 36 & 3.70 & A7 & 3.28 & .38 & A3 & .40 & .52 & .04 \\
\hline $\mathrm{H}_{2} \mathrm{O}$ & 123 & 9.8 & 13.5 & 11.2 & 124 & 14.0 & 128 & 12.2 & 15.5 \\
\hline $50^{2} \mathrm{C}$ & 10.6 & 9.6 & 6.0 & 5.8 & 10.4 & 64 & 6.2 & 95 & 7.3 \\
\hline $1000^{\circ} \mathrm{C}$ & 8.7 & 7.9 & 10.9 & 8.8 & 7.2 & 9.6 & 10.9 & 8.1 & 9.3 \\
\hline Samplod & Middle & Old & Young & Old & Middie & - & - & Bulk & Young \\
\hline Intorval & - & - & . & . & - & - & . & . & 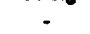 \\
\hline
\end{tabular}

\begin{tabular}{|c|c|c|c|c|c|c|c|c|c|}
\hline Sequencell & 090016 & 090016 & 091002 & 091004 & 092001 & 092002 & 093002 & 093003 & 093004 \\
\hline Letitude & 20.218 & 20.218 & 20.750 & 29.813 & 21.145 & 21.160 & 21.480 & 23.703 & 24.017 \\
\hline Longitude & -170.589 & -170.589 & 173.667 & 179.043 & 163.397 & 163.147 & 159.248 & 159.547 & 159,450 \\
\hline Depth & 1480 & 1480 & 3816 & 989 & 1777 & 1468 & 1602 & 2812 & 4079 \\
\hline Lab\# & CKB-134 & CKB-135 & W-222592 & W-222574 & W.222594 & W-222595 & W-222598 & W-222599 & W-222600 \\
\hline Si(wt.\%) & 3.02 & 1.16 & 250 & 1.66 & 9.90 & 241 & 5.17 & 8.96 & 7.83 \\
\hline $\mathbf{T i}$ & 1.39 & .68 & 90 & 86 & 1.02 & 94 & .86 & 1.03 & .85 \\
\hline $\mathbf{M n}$ & 26.8 & 25.5 & 28.0 & 31.8 & 16.9 & 28.3 & 22.2 & 17.6 & 20.3 \\
\hline $\mathbf{F o}_{0}$ & 16.4 & 7.72 & 14.9 & 123 & 15.8 & 16.5 & 18.3 & 18.2 & 16.9 \\
\hline $\mathbf{N}$ & 1.50 & .32 & .40 & .08 & 3.42 & .13 & .65 & 1.98 & 154 \\
\hline Co & .870 & .670 & .876 & 1.67 & .685 & 1.04 & .721 & .444 & .552 \\
\hline $\mathrm{Ni}$ & .520 & .690 & .631 & .653 & .232 & .466 & .298 & 246 & .270 \\
\hline Ca & 2.47 & 10.2 & 299 & 250 & 2.90 & 2.65 & 231 & 2.18 & 2.03 \\
\hline $\mathbf{M g}$ & 1.08 & 1.15 & 1.28 & 1.33 & 1.14 & 1.22 & .94 & .98 & 1.00 \\
\hline$K$ & 57 & .50 & 51 & .48 & .93 & .46 & 46 & .58 & .64 \\
\hline $\mathbf{P}$ & .40 & 3.31 & .61 & .45 & .52 & 52 & .54 & .45 & .39 \\
\hline $\mathrm{H}_{2} \mathrm{O}$ & 14.2 & 10.6 & 22.4 & 28.0 & 18.2 & 24.9 & 19.6 & 18.8 & 18.4 \\
\hline $50^{\circ} \mathrm{C}$ & 7.2 & 9.8 & - & - & - & . & - & - & - \\
\hline $1000^{\circ} \mathrm{C}$ & 11.5 & 7.5 & - & - & - & - & - & - & - \\
\hline Sampled & Middlo & Old & Bulk & Bulk & Bulk & Bulk & Bulk & Bulk & Bulk \\
\hline Interval & - & - & $0.5 \mathrm{~mm}$ & $0.7 \mathrm{~mm}$ & $0-4 \mathrm{~mm}$ & $0-9 \mathrm{mmn}$ & $0-12 \mathrm{~mm}$ & $0-10 \mathrm{~mm}$ & $0-6 \mathrm{cmm}$ \\
\hline
\end{tabular}


Table 2. Composition of ferromanganese crusts analyzed by USGS Woods Hole analytical laboratories (cont'd).

\begin{tabular}{|c|c|c|c|c|c|c|c|c|c|c|}
\hline Sequencel: & 093005 & 112001 & 115012 & 116003 & 116004 & 127013 & 127015 & 195002 & 195004 & 195006 \\
\hline Lationde & 29.490 & 30.213 & 39.517 & 30.883 & 30.983 & 31.793 & 30.978 & 55.107 & 55.127 & 55.127 \\
\hline Langitude & 153.348 & -39358 & -65350 & -78.783 & -78.217 & 174.325 & 175.878 & -140.462 & -140.423 & -140.423 \\
\hline $\begin{array}{l}\text { Depth } \\
\text { Labil }\end{array}$ & $\begin{array}{c}1991 \\
w-222601\end{array}$ & CHAIN-115D-9 & $\begin{array}{c}3382 \\
\text { A-296D-3-2 }\end{array}$ & $\begin{array}{c}836 \\
\text { A-266D40 }\end{array}$ & $\begin{array}{c}903 \\
\text { A-266D41 }\end{array}$ & $\begin{array}{c}2130 \\
\text { L8-82-D3 }\end{array}$ & $\begin{array}{c}1920 \\
\text { L8-82-D4 }\end{array}$ & $\begin{array}{c}2200 \\
56-79-1-4\end{array}$ & $\begin{array}{c}1400 \\
s 6-79-2-33\end{array}$ & $\begin{array}{c}1400 \\
56-79-2-34 B\end{array}$ \\
\hline$S^{\prime}(w t . \$)$ & 3.45 & 15.3 & 284 & 1.72 & 1.16 & 299 & 275 & 9.15 & 20.0 & 4.70 \\
\hline$\pi$ & 80 & .67 & .50 & 36 & .39 & 1.00 & .74 & .61 & 1.26 & .66 \\
\hline Mn & 243 & 698 & 16.6 & 154 & 16.9 & 25.8 & 25.0 & 19.2 & .10 & 23.1 \\
\hline$F_{0}$ & 17.4 & 16.2 & 25.7 & 145 & 14.1 & $15 . A$ & 173 & 17.7 & 8.94 & 18.8 \\
\hline $\mathbf{A} \mathbf{l}$ & .23 & 4.36 & 1.57 & 1.70 & 1.25 & 1.01 & .70 & 1.97 & 8.07 & 1.13 \\
\hline Co & $A 81$ & .079 & 314 & 318 & .401 & 867 & .806 & 311 & $\cdot$ & $A 76$ \\
\hline $\mathrm{Ni}$ & $A 05$ & .147 & .058 & 364 & 332 & 336 & .299 & .173 & $\cdot$ & .207 \\
\hline C. & 2.37 & .96 & 1.43 & 113 & 10.7 & 234 & 2.20 & 1.93 & 2.24 & 2.15 \\
\hline $\mathrm{Mg}$ & 99 & 1.38 & 80 & 3.56 & 3.14 & 96 & 95 & 97 & 1.91 & 85 \\
\hline $\mathbf{K}$ & .46 & 1.27 & .48 & .40 & .36 & .62 & 53 & .60 & 1.39 & 49 \\
\hline $\mathbf{P}$ & A7 & .27 & A2 & $A 3$ & .40 & 39 & 49 & 45 & 52 & $A 9$ \\
\hline $\mathrm{H}_{2} \mathrm{O}$ & 21.1 & 11.5 & 14.0 & 12.0 & 127 & 22.7 & 23.0 & 13.1 & 9.8 & 18.0 \\
\hline $50^{\circ} \mathrm{C}$ & - & - & - & - & • & - & • & • & - & • \\
\hline $1000^{\circ} \mathrm{C}$ & $\cdot$ & - & - & - & $\cdot$ & - & - & - & - & - \\
\hline Sempled & Bulk & Bulk & Bulk & Bulk & Bulk & Bulk & Bulk & Bulk & Bulk & Bulk \\
\hline Intorval & $0-8 \mathrm{~mm}$ & - & - & • & • & • & - & • & - & - \\
\hline
\end{tabular}

\begin{tabular}{|c|c|c|c|c|c|c|c|c|c|c|}
\hline Sequence" & 195008 & 195010 & 195011 & 195013 & 195015 & 196002 & 196004 & 196006 & 196008 & 196010 \\
\hline Letionde & 55.127 & 55.127 & 53.543 & 53543 & 53543 & 54.662 & 54662 & 54.662 & 54.662 & 54.662 \\
\hline Longitude & -140.423 & -140.423 & -144.373 & -144.373 & -144373 & -150.305 & -150.305 & -150305 & -150.305 & -150.305 \\
\hline Depth & 1400 & 1400 & 2100 & 2100 & 2100 & 1400 & 1400 & 1400 & 1400 & 1400 \\
\hline Lab" & $56-79-2-34 \mathrm{C}$ & S6.79-D2 & S-79-4-4 & $56-79-13$ & $56-79-4-15 A$ & $56-79-9-1$ & S6-79-9-9A & S6-79-9-9B & S6-79-9.11A & S6-79-9-11B \\
\hline Si(wt.\%) & 4.39 & 6.72 & 2.25 & 5.10 & 4.71 & 3.57 & 3.06 & 250 & 11.5 & 8.55 \\
\hline $\mathbf{T i}$ & .63 & .67 & 82 & 87 & .76 & 94 & 95 & 1.06 & 1.47 & .74 \\
\hline Mn & 239 & 14.3 & 30.0 & 24.7 & 23.7 & 25.8 & 288 & 27.6 & 10.5 & 18.8 \\
\hline Fo & 19.8 & 19.3 & 15.1 & 18.6 & 18.6 & 179 & 16.0 & 168 & 19.7 & $16 A$ \\
\hline $\mathbf{A}$ & 91 & 1.76 & .67 & 1.09 & .80 & 94 & 96 & .77 & 4.15 & 231 \\
\hline Co & 548 & .091 & .585 & 566 & .651 & .601 & 537 & $A 91$ & 355 & A44 \\
\hline $\mathrm{Ni}$ & .137 & .240 & .237 & 264 & .213 & 241 & .195 & 233 & .011 & .216 \\
\hline Ca & 235 & 229 & 2.74 & 251 & 232 & 238 & 256 & 247 & 1.47 & 1.88 \\
\hline $\mathbf{M g}$ & 92 & .95 & 1.18 & 1.02 & .95 & .90 & 96 & .77 & 1.23 & 1.12 \\
\hline $\mathbf{K}$ & $A 4$ & .42 & .42 & 44 & .53 & .46 & 54 & 44 & 1.01 & 85 \\
\hline $\mathbf{P}$ & .54 & 32 & 39 & .47 & .49 & 44 & $A 3$ & 12 & 35 & .41 \\
\hline $\mathrm{H}_{2} \mathrm{O}$ & 19.7 & 125 & 19.7 & 20.5 & 20.2 & 16.8 & 18.0 & 18.5 & 127 & 12.1 \\
\hline $500^{\circ} \mathrm{C}$ & - & • & - & - & - & - & - & - & - & - \\
\hline $1000^{\circ} \mathrm{C}$ & - & - & - & - & - & - & - & - & - & - \\
\hline Sempled & Bulk & Bulk & Bulk & Bulk & Bulk & Bulk & Bulk & Bulk & Bulk & Bulk \\
\hline hotorval &. & - &. & - & - &. &. & . & . &. \\
\hline
\end{tabular}

\begin{tabular}{|c|c|c|c|c|c|c|c|c|c|c|}
\hline Sequencell & 317010 & 317010 & 317010 & 317011 & 317014 & 353001 & 353002 & 380001 & 409001 & 409002 \\
\hline Letionde & -388 & -388 & -.388 & -.399 & -399 & -16.203 & -16.548 & -21.253 & -31.833 & -34.553 \\
\hline Langindo & -176.073 & -176.073 & -176.073 & -176.116 & -176.116 & -176.257 & -178.442 & $\$ 80.925$ & -13.578 & -15.147 \\
\hline Depth & 2107 & 2107 & 2107 & 1551 & 1551 & 1352 & 1499 & 2300 & 2984 & 2924 \\
\hline Lab" & CKB-136 & CKB-136 & CKB-138 & CKB-139 & 6DK-SEM-62 & W-222559 & $W-222560$ & W-222712 & AII-107.7-25 & AII-107-7-18 \\
\hline Si(wt.\%) & 3.02 & 3.18 & 222 & 242 & 224 & 754 & 734 & 5.51 & 4.00 & 4.43 \\
\hline $\mathrm{Ti}$ & 1.25 & 1.24 & 1.20 & 81 & $\pi$ & 80 & .74 & .78 & .56 & .69 \\
\hline Mn & 24.0 & 24.0 & 26.5 & 268 & 284 & 14.0 & 13.2 & 118 & 14.7 & 119 \\
\hline Fo & 16.6 & 16.5 & 15.5 & 14.7 & 14.9 & $23 A$ & 24.5 & 20.2 & 21.5 & 19.3 \\
\hline $\mathbf{A}$ & 1.10 & 1.09 & .78 & .66 & .28 & 296 & 2887 & 1.69 & 1.42 & 1.52 \\
\hline Co & 1.11 & 957 & 1.14 & 819 & 1.30 & 283 & .154 & 348 & 293 & 333 \\
\hline $\mathbf{N i}$ & 507 & 507 & .587 & .775 & .438 & .142 & .103 & .108 & .169 & - \\
\hline ca & 3.02 & 3.01 & 258 & 3.16 & 2.48 & 3.05 & 3.81 & 7.50 & 4.87 & 7.20 \\
\hline $\mathbf{M g}_{\mathbf{g}}$ & 1.17 & 1.18 & 1.19 & 1.31 & 1.20 & 1.29 & 139 & 1.38 & 1.52 & 200 \\
\hline $\mathbf{K}$ & 53 & 52 & .49 & so & .60 & 81 & 52 & .66 & $A 1$ & .40 \\
\hline$\vec{P}$ & 53 & 53 & 37 & .62 & .40 & 56 & 54 & 39 & .47 & AS \\
\hline $\mathrm{H}_{2} \mathrm{O}$ & 11.2 & 11.2 & 9.7 & 8.4 & 13.2 & 22.4 & 22.1 & 16.7 & 113 & 16.0 \\
\hline $50^{\circ} \mathrm{C}$ & 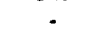 & - & - & $\cdot$ & - & . & 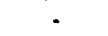 & - & - & - \\
\hline $1000^{\circ} \mathrm{C}$ & - & - & - & - & - & - & - & - & - & - \\
\hline Sampled & Bulk & Bulk & Top & Young & Top & Bulk & Bulk & Bulk & Bulk & Bulk \\
\hline Intorval & $30 \mathrm{~mm}$ & $30 \mathrm{~mm}$ & $15 \mathrm{~mm}$ & $20 \mathrm{~mm}$ & $0-5 \mathrm{~mm}$ & $0-1 \mathrm{mmn}$ & $0-10 \mathrm{~mm}$ & $0-20 \mathrm{~mm}$ & - & • \\
\hline
\end{tabular}


Table 2. Composition of ferromanganese crusts analyzed by USGS Woods Hole analytical laboratories (cont'd).

\begin{tabular}{|c|c|c|c|c|c|c|c|c|c|c|}
\hline Sequenco" & 409004 & 409007 & 409008 & 511001 & 515001 & 515002 & 515003 & 515005 & 515006 & 515007 \\
\hline Letitude & -35.278 & -36.558 & .32 .677 & -56.170 & -53.147 & -53.238 & $.53,418$ & .53 .428 & $-54,423$ & -54.442 \\
\hline Longitude & -15.735 & -17.587 & -14.018 & 41.800 & 9.192 & 9.193 & 9.198 & 9.152 & 1.570 & 1.622 \\
\hline Depth & 3638 & 2683 & 2634 & 3709 & 2438 & 3670 & 4418 & 5349 & 3240 & 4818 \\
\hline Lab* & AII-107-7-17 & All-107.7-15 & AII-107-7.23 & AII-107-6-1 & AII-107-6-66 & AII-107-6-65 & AII-107-6-61 & AII-107-6-60 & AII-107-6-40 & AII-107-6.39 \\
\hline$S i(w t . \%)$ & 3.83 & 5.72 & 4.08 & 16.7 & 8.30 & 7.36 & 7.12 & 13.7 & 13.6 & 13.8 \\
\hline $\mathbf{T i}$ & 84 & .61 & .79 & .59 & 1.75 & 1.73 & 1.53 & 1.06 & 1.00 & 93 \\
\hline Mn & 14.5 & 11.6 & 14.0 & 10.1 & 15.2 & 14.0 & 124 & 7.95 & 11.6 & 735 \\
\hline$F_{0}$ & 24.0 & 20.6 & 243 & 11.6 & 19.1 & 21.7 & 23.9 & 15.0 & 9.66 & 9.12 \\
\hline $\boldsymbol{N}$ & 1.40 & 1.98 & 1.99 & 3.44 & 218 & 203 & 212 & 3.00 & 259 & 194 \\
\hline$C_{0}$ & 415 & 211 & 364 & .207 & $2 n$ & .217 & 289 & .123 & .107 & .053 \\
\hline $\mathrm{Ni}$ & - & .004 & .073 & 316 & 386 & 220 & - & .006 & $A 83$ & .358 \\
\hline ca & 3.70 & 5.97 & 3.75 & 1.77 & 213 & 1.91 & 2.19 & 243 & 1.93 & 1.19 \\
\hline $\mathbf{M} \mathbf{8}$ & 1.17 & 1.77 & 1.30 & .95 & 98 & 91 & 93 & 3.50 & 4.86 & 9.98 \\
\hline $\mathbf{K}$ & .40 & .44 & .40 & 1.00 & .63 & 55 & 54 & .49 & 59 & 45 \\
\hline $\mathbf{P}$ & .46 & .44 & .51 & .17 & .27 & 31 & 37 & .16 & .11 &.$\infty$ \\
\hline $\mathrm{H}_{2} \mathrm{O}$ & 18.0 & 14.6 & 17.5 & 8.1 & 17.0 & 16.9 & 16.9 & 10.7 & 68 & 5.0 \\
\hline $50^{\circ} 0^{\circ} \mathrm{C}$ & - & - & - & - & $\cdot$ & - & - & - & $\cdot$ & $\cdot$ \\
\hline $1000^{\circ} \mathrm{C}$ & - & - & - & - & . & - & - & - & - & - \\
\hline Sempled & Bulk & Bulk & Bulk & Bulk & Bulk & Bulk & Bulk & Bulk & Bulk & Bulk \\
\hline Intarval & • & - & • & - & - & • & - & - & • & - \\
\hline
\end{tabular}


Table 3. Chemical composition of ferromanganese crusts analyzed by BOM Avondale analytical laboratories

\begin{tabular}{|c|c|c|c|c|c|c|c|c|c|c|c|c|c|}
\hline $\begin{array}{l}\text { Soquencoll } \\
\text { Latimde }\end{array}$ & $\begin{array}{c}017006 \\
8.414\end{array}$ & $\begin{array}{c}017007 \\
8.414\end{array}$ & $\begin{array}{c}017008 \\
8.414\end{array}$ & $\begin{array}{c}017009 \\
8.398\end{array}$ & $\begin{array}{c}017010 \\
8.398\end{array}$ & $\begin{array}{c}017011 \\
8.389\end{array}$ & $\begin{array}{c}017012 \\
8.353\end{array}$ & $\begin{array}{c}017013 \\
8.353\end{array}$ & $\begin{array}{c}017014 \\
8.308\end{array}$ & $\begin{array}{c}017015 \\
8.308\end{array}$ & $\begin{array}{c}017016 \\
8.308\end{array}$ & $\begin{array}{c}017019 \\
8.194\end{array}$ & $\begin{array}{c}017020 \\
8.194\end{array}$ \\
\hline Longitude & -164.289 & -164.239 & -164.289 & -164.359 & -164.359 & -164.294 & -164.317 & -164.317 & -164.346 & -164.346 & -164.346 & -164.200 & -164.200 \\
\hline Depth & 1850 & 1850 & 1850 & 2400 & 2400 & 1600 & 1150 & 1150 & 1400 & 1400 & 1400 & 1600 & 1600 \\
\hline Labi & 7504 & 7505 & 7506 & 7500 & 7508 & 7509 & 7510 & 7511 & 7512 & 7513 & 7514 & 7515 & 7516 \\
\hline$T(w / s)$ & 1.45 & 153 & 1.77 & 1.40 & 1.31 & 1.19 & 93 & 1.16 & 1.07 & 96 & .91 & 1.00 & 1.19 \\
\hline Man & 30.8 & 27.4 & 27.9 & 26.2 & 28.9 & 26.3 & 38.8 & 30.3 & 34.8 & 30.1 & 342 & 30.5 & 29.0 \\
\hline $\mathbf{F}_{0}$ & 18.4 & 18.6 & 19.4 & 18.2 & 18.7 & 15.7 & 9,97 & 148 & 15.0 & 14.9 & 13.8 & 15.1 & 16.8 \\
\hline $\mathbf{A}$ & 56 & .66 & .78 & 1.00 & .73 & 84 & $\$ 0$ & 30 & 30 & 28 & .32 & .38 & 32 \\
\hline Co & 1.14 & 840 & 1.01 & 1.02 & .960 & 1.37 & 3,02 & 1.41 & 1.41 & 1.07 & 1.97 & 1.17 & .860 \\
\hline $\mathrm{Ni}$ & .600 & .430 & .420 & $A 70$ & .480 & .680 & 980 & .430 & .790 & .660 & .780 & 510 & .520 \\
\hline$a$ & .100 & .100 & .100 & .090 & .100 & .090 & .000 & .050 & .050 & .040 & .060 & .060 & .080 \\
\hline Ca & 277 & 299 & 270 & 292 & 2.78 & 4.86 & 281 & 5.37 & 292 & 3.14 & 2.65 & 250 & 253 \\
\hline $\mathbf{M} 8$ & 1.28 & 1.07 & 1.15 & 1.33 & 1.26 & 1.40 & 220 & 1.26 & 1.34 & 1.12 & 1.37 & 1.12 & 1.01 \\
\hline $\mathrm{Na}$ & - & - & - & - & - & • & - & 1.61 & - & • & - & • & 1.61 \\
\hline $\mathbf{P}$ & 50 & .63 & .55 & .75 & 57 & 1.27 & .49 & $\pi 7$ & 49 & .62 & .36 & .41 & .43 \\
\hline Ba(ppen) & 2400 & 2700 & 3200 & 2700 & 2300 & 1900 & 2100 & 4300 & 2400 & 2600 & 1900 & 2900 & 3300 \\
\hline Mo & 700 & 800 & 700 & 700 & 800 & 600 & 900 & 1100 & 1100 & 1000 & 1000 & 1000 & 700 \\
\hline Po & 2600 & 1700 & 1600 & 1500 & 1500 & 2100 & 2500 & 2500 & 2600 & 1900 & 2200 & 2400 & 2300 \\
\hline St & 2000 & 2000 & 1900 & 1700 & 1800 & 1800 & 1500 & 2000 & 2100 & 2100 & 1900 & 2000 & 2000 \\
\hline $\mathbf{v}$ & 900 & 800 & 800 & 600 & 800 & 700 & 800 & 800 & 900 & 900 & 900 & 900 & 1000 \\
\hline $\mathbf{Z n}$ & 1100 & 1500 & 1400 & 1300 & 1200 & 1200 & 1500 & 1000 & 1200 & 1000 & 900 & 800 & 1000 \\
\hline Sarmplod & Top & Bulk & Bulk & Bulk & Bulk & Bulk & Top & Bulk & Bulk & Bottam & Top & Top & Bulk-minus \\
\hline Intorval & 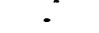 & • & • & • & - & $0-15$ mon & $\cdot$ & . & - & • & $\bullet$ & $\cdot$ & Top \\
\hline
\end{tabular}

\begin{tabular}{|c|c|c|c|c|c|c|c|c|c|c|c|c|c|}
\hline Sequencell & 017021 & 052019 & 052020 & 052021 & 052022 & 053017 & 053018 & 053019 & 053020 & 053021 & 053022 & 053023 & 053024 \\
\hline Lationde & 8.186 & 19.481 & 19.236 & 19.408 & 19.408 & 19.335 & 19.369 & 19.369 & 19.511 & 19.511 & 19.511 & 19.511 & 29.511 \\
\hline Longitudo & -164.202 & -157.054 & -157.172 & -157.080 & -157.080 & -168.665 & -168.695 & -168.695 & -168.835 & -168835 & -168835 & -168835 & -168.835 \\
\hline Depth & 1800 & 2750 & 3000 & 3050 & 3050 & 3791 & 2000 & 2000 & 1800 & 1800 & 1800 & 1800 & 1800 \\
\hline Lebi & 7517 & 1502 & 1830 & 1833 & 1835 & 7486 & 7487 & 7488 & 7489 & 7490 & 7491 & 7492 & 7493 \\
\hline $\mathrm{T}(w t .8)$ & 1.47 & 1.46 & 1.02 & 1.69 & 1.54 & 1.45 & 1.51 & 1.22 & 1.23 & 1.09 & 1.27 & 1.30 & 1.27 \\
\hline $\mathrm{Mn}$ & 26.1 & 19.5 & 16.2 & 20.0 & 16.1 & 21.8 & 22.7 & 23.4 & 20.5 & 32.3 & 27.4 & 24.1 & 30.9 \\
\hline $\mathrm{F}_{0}$ & 15.4 & 20.9 & 23.4 & 21.4 & 19.5 & 21.6 & 18.4 & 19.1 & 18.9 & 124 & 15.7 & 19.4 & 14.1 \\
\hline $\mathbf{N}$ & .95 & 1.10 & 1.71 & 122 & 2.13 & 1.05 & 1.44 & 1.04 & 97 & $A 0$ & .80 & 1.05 & 39 \\
\hline$C_{0}$ & 1.39 & .660 & .080 & .360 & .520 & .620 & 1.03 & 850 & 820 & .830 & .970 & 940 & .810 \\
\hline $\mathrm{Ni}$ & 560 & .270 & .240 & .340 & .190 & .400 & .440 & .470 & .420 & .640 & 580 & 480 & .500 \\
\hline Cre & .080 & .050 & .260 & .140 & .100 & .120 & .060 & .080 & .110 & 250 & .150 & .110 & .110 \\
\hline $\mathbf{c}$ & 288 & 209 & 158 & 2.18 & 2.62 & 243 & 297 & 3.30 & 213 & 4.89 & 3.75 & 2.25 & 4.33 \\
\hline $\mathbf{M}_{8}$ & 1.07 & 1.10 & 97 & 1.12 & 1.24 & 1.08 & 1.49 & 1.10 & 1.02 & 1.14 & 1.12 & 1.08 & 1.09 \\
\hline $\mathrm{Na}$ & • & • & - & $\cdot$ & - & $\cdot$ & $\bullet$ & - & - & 1.55 & 159 & - & $\cdot$ \\
\hline $\mathbf{P}$ & .46 & 30 & .30 & 30 & 50 & .42 & 59 & 52 & .55 & 1.55 & .86 & .43 & .93 \\
\hline$B d(p p m)$ & 1500 & 1700 & 3600 & 2800 & 1700 & 1800 & 1800 & 1800 & 2100 & 3800 & 2600 & 2200 & 3600 \\
\hline Mo & 500 & 300 & 600 & 400 & 300 & 600 & 400 & 500 & 500 & 900 & 700 & 500 & 800 \\
\hline Po & 2200 & 1900 & - & 400 & 900 & 2500 & 2000 & 2500 & 2600 & 3000 & 2700 & 2800 & 2400 \\
\hline St & 1600 & 1500 & 1500 & 1600 & 1300 & 1700 & 1700 & 1800 & 1800 & 2100 & 1900 & 1800 & 2100 \\
\hline $\mathbf{v}$ & 700 & 600 & 1300 & 1100 & 500 & 800 & 890 & 800 & 800 & 1000 & 800 & 800 & 900 \\
\hline $\mathrm{Zn}$ & 1000 & 300 & 500 & 400 & 300 & 800 & 900 & 900 & 1000 & 1200 & 900 & 1000 & 1100 \\
\hline Semplod & Bulk & Bulk & Bulk & Bulk & Bulk & Bulk & Bulk & Bulk & Top & Botan & Bulk & Top & Bottom \\
\hline Interval & $\cdot$ & $7-11 \mathrm{~mm}$ & $70-80 \mathrm{~mm}$ & $25-35 \mathrm{~mm}$ & $10-20 \mathrm{~mm}$ &. & 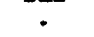 & - & $\bullet$ & - & $\cdot$ & $\bullet$ & - \\
\hline
\end{tabular}


Table 3. Chemical composition of ferromanganese crusts analyzed by BOM Avondale analytical laboratories (cont'd).

\begin{tabular}{|c|c|c|c|c|c|c|c|c|c|c|c|c|c|}
\hline $\begin{array}{l}\text { Sequencoll } \\
\text { Latirude }\end{array}$ & $\begin{array}{l}053025 \\
19.511\end{array}$ & $\begin{array}{l}053026 \\
19.695\end{array}$ & $\begin{array}{l}053027 \\
19.553\end{array}$ & $\begin{array}{l}053028 \\
19.553\end{array}$ & $\begin{array}{l}053029 \\
19.515\end{array}$ & $\begin{array}{l}053030 \\
19.515\end{array}$ & $\begin{array}{l}053031 \\
19.515\end{array}$ & $\begin{array}{l}053032 \\
19.515\end{array}$ & $\begin{array}{l}053033 \\
19.515\end{array}$ & $\begin{array}{l}053034 \\
19.515\end{array}$ & $\begin{array}{l}088011 \\
25.946\end{array}$ & $\begin{array}{l}088012 \\
25.944\end{array}$ & $\begin{array}{l}088013 \\
25951\end{array}$ \\
\hline Langinds & .168 .835 & -168995 & -168.832 & -168.832 & -168855 & -168.855 & -168.855 & -168.855 & -168.855 & -168.855 & -159.903 & -159.878 & -159.914 \\
\hline Depth & 1800 & 4500 & 2400 & 2400 & 1790 & 1790 & 1790 & 1790 & 1790 & 1790 & 3000 & 2600 & 3600 \\
\hline Lab" & 7494 & 7495 & 7496 & 7497 & 7498 & 7499 & 7500 & 7501 & 7502 & 7503 & 1426 & 1495 & 1499 \\
\hline Tr(wt.s) & 1.33 & 1.82 & 1.40 & 1.50 & 1.09 & 1.12 & 1.23 & 1.00 & 1.34 & 1.00 & 1.49 & 154 & 1.59 \\
\hline Mn & 27.7 & 16.8 & 24.5 & 23.6 & 279 & 20.2 & 24.7 & 304 & 23.8 & 30.7 & 15.1 & 20.7 & 20.1 \\
\hline $\mathbf{F e}$ & 16.0 & 18.4 & 19.2 & 20.6 & 17.1 & 16.3 & 19.2 & 149 & 205 & 15.9 & 185 & 227 & 19.6 \\
\hline $\mathbf{N}$ & 80 & 259 & 1.09 & 1.55 & .78 & .69 & 93 & 57 & 1.32 & .44 & 2.73 & 97 & 96 \\
\hline Co & 880 & 310 & .820 & .690 & .730 & .750 & .750 & .710 & .870 & s5o & 280 & .440 & .720 \\
\hline $\mathbf{N i}$ & 490 & $A 20$ & 390 & .420 & 410 & 140 & 360 & .480 & 340 & .430 & 240 & 310 & 310 \\
\hline $\mathrm{Co}$ & .100 & .200 & .130 & 220 & .140 & .160 & .130 & .170 & .110 & .140 & .120 & .100 & .050 \\
\hline $\mathbf{c}$ & 285 & 1.70 & 234 & 2.35 & 4.72 & 5.65 & 2.54 & 5.62 & 2.54 & 5.46 & 2.10 & 2.21 & 2.06 \\
\hline $\mathbf{M g}$ & 1.09 & 1.31 & 1.11 & 1.14 & 1.07 & 1.06 & 1.08 & 1.08 & 1.07 & 1.06 & 1.11 & 1.15 & 1.06 \\
\hline $\mathrm{Na}$ & - & 1.55 & • & • & 1.55 & • & • & • & • & • & • & • & • \\
\hline $\mathbf{P}$ & 52 & .60 & 59 & 51 & .79 & 1.19 & 56 & .84 & .44 & 1.11 & 30 & $A 0$ & 30 \\
\hline $\operatorname{Ba}(\mathrm{ppm})$ & 2800 & 2000 & 1700 & 1900 & 2600 & 3000 & 2000 & 3200 & 2000 & 3400 & 2100 & 2000 & 1800 \\
\hline Mo & 700 & 300 & 400 & 400 & 700 & 700 & 500 & 800 & 500 & 800 & 100 & 400 & 400 \\
\hline $\mathbf{P b}$ & 2100 & 1600 & 2200 & 2100 & 2700 & 2800 & 2700 & 3200 & 2800 & 2700 & 1200 & 2300 & 1900 \\
\hline Sr & 1800 & 1000 & 1800 & 1700 & 2100 & 2000 & 1900 & 2000 & 1700 & 2400 & 300 & 1800 & 1600 \\
\hline $\mathbf{v}$ & 800 & 500 & 700 & 700 & 900 & 900 & 700 & 900 & 700 & 1000 & 300 & 700 & 600 \\
\hline $\mathrm{Zn}$ & 1300 & 1100 & 1200 & 1100 & 1300 & 1500 & 1300 & 1600 & 1200 & 1200 & - & 400 & 400 \\
\hline Sampled & Bulk & Top & Bulk & Bulk & Bulk & Top & Top & Boxtom & Bulk & Bulk & Bulk & Bulk & Bulk \\
\hline Interval & - & $\cdot$ & • & • & • & $\cdot$ & $\cdot$ & - & - & • & $10-25 \mathrm{~mm}$ & 2-4mm & - \\
\hline
\end{tabular}

\begin{tabular}{|c|c|c|c|c|c|c|c|c|c|c|c|c|c|}
\hline $\begin{array}{l}\text { Sogprenoed } \\
\text { Latimade }\end{array}$ & $\begin{array}{l}088014 \\
25.944\end{array}$ & $\begin{array}{l}088015 \\
25.944\end{array}$ & $\begin{array}{l}088016 \\
25.944\end{array}$ & $\begin{array}{l}088017 \\
25.946\end{array}$ & $\begin{array}{l}088018 \\
25.951\end{array}$ & $\begin{array}{l}089011 \\
22.181\end{array}$ & $\begin{array}{l}089013 \\
22.208\end{array}$ & $\begin{array}{l}089014 \\
22.256\end{array}$ & $\begin{array}{l}089015 \\
22316\end{array}$ & $\begin{array}{l}089016 \\
22316\end{array}$ & $\begin{array}{l}039017 \\
21.718\end{array}$ & $\begin{array}{c}089018 \\
21.718\end{array}$ & $\begin{array}{l}089019 \\
21.718\end{array}$ \\
\hline Lanpituds & -159.878 & -159.878 & -159.878 & -159.903 & -159.914 & -166.810 & .166882 & -166.854 & -166.899 & -166.899 & -167.578 & -167.578 & -167.578 \\
\hline Depth & 2600 & 2600 & 2600 & 3000 & 3600 & 3950 & 2402 & 1970 & 2350 & 2350 & 2400 & 2400 & 2400 \\
\hline Lab" & 1800 & 1802 & 1812 & 1814 & 1817 & 7467 & 7468 & 7469 & 7470 & 7471 & 7472 & 7473 & 7474 \\
\hline$T i(w t .8)$ & 1.22 & 1.28 & 1.20 & 1.40 & 1.31 & 1.42 & 1.92 & 1.75 & 1.52 & 1.03 & 188 & 1.65 & 1.44 \\
\hline Mn & 24.1 & 24.5 & 16.8 & 23.4 & 179 & 20.7 & 20.4 & 225 & 23.7 & 244 & 25.1 & 22.7 & 21.5 \\
\hline Pe & 21.5 & 19.5 & 21.9 & 19.9 & 20.3 & 21.8 & 20.8 & 21.5 & 21.6 & 21.6 & 19.0 & 223 & 224 \\
\hline $\mathbf{A}$ & 85 & 88 & 1.36 & 85 & 1.40 & 1.89 & 245 & 1.79 & 1.77 & .98 & 1.85 & 1.61 & 193 \\
\hline$C_{0}$ & 830 & 910 & $A 10$ & 960 & 520 & .480 & 560 & .700 & .760 & .740 & .650 & .600 & .680 \\
\hline $\mathbf{N i}$ & 310 & 390 & .170 & 440 & 220 & .270 & .430 & 410 & 460 & $A 00$ & 500 & .370 & .330 \\
\hline $\mathrm{Ca}$ & .080 & .110 & .060 & .100 & .140 & .180 & 260 & .130 & .130 & .110 & .220 & .120 & .070 \\
\hline ca & 230 & 222 & 268 & 2.17 & 247 & 235 & 2.64 & 259 & 2.03 & 245 & 258 & 246 & 2.65 \\
\hline $\bar{M}_{8}$ & 1.21 & 1.22 & 1.04 & 1.20 & 1.06 & 1.52 & 1.17 & 1.12 & 1.52 & 1.14 & 1.11 & 1.09 & 1.06 \\
\hline $\mathrm{Na}$ & • & - & - & - & . & . & 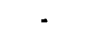 & - & . & 1.56 & - & - & - \\
\hline $\mathbf{P}$ & .40 & $A 0$ & .70 & 40 & .60 & .76 & .87 & .70 & .78 & .58 & $s 2$ & .56 & .73 \\
\hline$B a(p p e n)$ & 1800 & 2200 & 1800 & 1800 & 1900 & 1900 & 2500 & 2400 & 2100 & 2000 & 3400 & 2400 & 1700 \\
\hline Mo & 500 & 400 & 300 & 400 & 300 & 200 & 200 & 300 & 400 & 500 & 400 & 500 & 300 \\
\hline $\mathrm{Pb}$ & 1900 & 1300 & 1500 & 1500 & 1200 & 2200 & 2400 & 2700 & 2800 & 2300 & 1700 & 2300 & 2300 \\
\hline Sr & 1800 & 1700 & 1600 & 1600 & 1400 & 1500 & 1500 & 1700 & 1700 & 1800 & 1800 & 1700 & 1600 \\
\hline v & 700 & 600 & 1600 & 600 & 600 & 600 & 700 & 800 & 700 & 700 & 700 & 700 & 800 \\
\hline $\mathbf{z a}$ & 400 & 300 & 400 & 400 & 300 & 900 & 1200 & 1000 & 1100 & 1000 & 1100 & 1200 & 1000 \\
\hline Sempled & Bulk & Bulk & Bulk & Bulk & Bulk & Bulk & Bulk & Top & Bulk & Bulk & Bottom & Top & Top \\
\hline Interval & $46 \mathrm{~mm}$ & $6-8 \mathrm{~mm}$ & $5-8 \mathrm{~mm}$ & $7.10 \mathrm{~mm}$ & $7.25 \mathrm{~mm}$ &. & - & $<\operatorname{lmm}$ & - & . & - & - & $=$ \\
\hline
\end{tabular}


Table 3. Chemical composition of ferromanganese crusts analyzed by BOM Avondale analytical laboratories (cont'd).

\begin{tabular}{|c|c|c|c|c|c|c|c|c|c|c|c|c|c|}
\hline $\begin{array}{l}\text { Sequencell } \\
\text { Letimado }\end{array}$ & $\begin{array}{l}089020 \\
21.718\end{array}$ & $\begin{array}{l}089021 \\
21.796\end{array}$ & $\begin{array}{l}089022 \\
21.796\end{array}$ & $\begin{array}{l}089023 \\
21.796\end{array}$ & $\begin{array}{l}089025 \\
20.209\end{array}$ & $\begin{array}{l}089026 \\
20.209\end{array}$ & $\begin{array}{l}089027 \\
20.209\end{array}$ & $\begin{array}{l}089028 \\
20.209\end{array}$ & $\begin{array}{l}089029 \\
20.335\end{array}$ & $\begin{array}{l}0.89030 \\
20.335\end{array}$ & $\begin{array}{l}089031 \\
20.335\end{array}$ & $\begin{array}{l}089038 \\
24.322\end{array}$ & $\begin{array}{l}089039 \\
25.783\end{array}$ \\
\hline Langitude & -167.578 & -167.624 & -167.624 & -167.624 & -169.506 & -169.506 & -169506 & -169.506 & -169.575 & -169.575 & -169.575 & -168.083 & -167.733 \\
\hline Depth & 2400 & 2100 & 2100 & 2100 & 4500 & 4500 & 4500 & 4500 & 2400 & 2400 & 2400 & 1225 & 1600 \\
\hline Lab & 7475 & 7476 & 7477 & 7478 & 7479 & 7480 & 7481 & 7482 & 7483 & 7484 & 7485 & 1429 & 1460 \\
\hline$T(w h . s)$ & 1.79 & 2.14 & 200 & 1.25 & 2.53 & 2.10 & 1.75 & 2.63 & 1.50 & 1.54 & 1.99 & 1.62 & 52 \\
\hline Mm & 25.1 & 24.2 & 18.9 & 19.2 & 15.9 & 12.7 & 14.5 & 13.6 & 23.1 & 20.8 & 24.0 & 2.54 & 45.6 \\
\hline Po & 17.2 & 18.0 & 22.0 & 20.5 & 20.9 & 18.2 & 16.0 & 18.9 & 225 & 22.7 & 19.9 & 17.1 & 2.40 \\
\hline $\boldsymbol{N}$ & 3.28 & .79 & 1.69 & 1.48 & 251 & 3.79 & 3.52 & 3.00 & 1.01 & 1.12 & 80 & 5.26 & 1.33 \\
\hline Co & 1.14 & 1.04 & A5O & .540 & .330 & 270 & .330 & 280 & .650 & 550 & 930 & 200 & .070 \\
\hline $\mathbf{N}$ & 430 & .350 & .290 & .280 & .190 & .190 & 270 & .200 & 340 & 340 & 420 & .180 & 350 \\
\hline$c$ & .000 & .060 & .110 & .060 & .140 & .150 & .160 & .190 & .000 & .110 & .111 & .040 & .100 \\
\hline $\mathbf{c}$ & 2.82 & 2.25 & 237 & 2.10 & 202 & 1.97 & 1.93 & 1.76 & 217 & 2.08 & 2.47 & 4.92 & 1.07 \\
\hline $\mathbf{M g}$ & 1.16 & 1.12 & $1 . \infty$ & 1.03 & 1.09 & 1.39 & 1.55 & 1.11 & 1.07 & 1.02 & 1.07 & 2.63 & 2.38 \\
\hline $\mathrm{Na}$ & 1.87 & • & - & • & • & 1.55 & - & - & 1.54 & • & • & • & • \\
\hline $\mathbf{P}$ & .65 & 53 & 53 & .48 & A5 & .40 & .40 & 43 & 49 & 43 & 39 & .20 & .30 \\
\hline$B \times(p p m)$ & 1700 & 2000 & 2500 & 1600 & 2200 & 1800 & 1800 & 1900 & 2000 & 2900 & 2500 & 600 & 14000 \\
\hline Mo & 600 & 500 & 600 & 600 & 300 & 200 & 200 & 300 & 500 & 500 & 400 & 100 & 200 \\
\hline Pb & 2100 & 2300 & 2100 & 2400 & 2000 & 2000 & 1800 & 1700 & 2300 & 2400 & 2200 & 500 & • \\
\hline St & 1600 & 1600 & 1600 & 1500 & 1300 & 900 & 900 & 1000 & 1800 & 2000 & 1800 & 600 & 1400 \\
\hline V & 600 & 600 & 600 & 600 & 600 & 500 & 500 & 400 & 900 & 800 & 700 & 500 & 400 \\
\hline $\mathbf{Z n}$ & 1000 & 1200 & 900 & 700 & 800 & 700 & 800 & 700 & 800 & 800 & 900 & - & 1400 \\
\hline Sampled & Top & Bulk & Bulk & Top & Bulk & Top & Bottom & Bulk & Top & Bulk & Bulk & Bulk & Bulk \\
\hline Intorval & $<1 \mathrm{~mm}$ & . & . & - & - & - & . & . & - & . & - & - & $75-85 \mathrm{~mm}$ \\
\hline
\end{tabular}

\begin{tabular}{|c|c|c|c|c|c|c|c|c|c|c|c|c|c|}
\hline Sequencoll & 089040 & 089041 & 089042 & 089043 & 089044 & 089045 & 089046 & 089047 & 089048 & 089049 & 089050 & 089051 & 089052 \\
\hline Latinds & 25.929 & 23.672 & 25.685 & 25.135 & 25.276 & 25.728 & 25.260 & 25.783 & 25.454 & 25.489 & 25.783 & 25.783 & 24.322 \\
\hline Longitude & -167.755 & -164.891 & -160.180 & -160.027 & -162.068 & -160.201 & -162.046 & -167.733 & -168.683 & -168.672 & -167.733 & -167.733 & -168.083 \\
\hline Depth & 2500 & 1650 & 3000 & 2800 & 3000 & 2600 & 2300 & 1600 & 1115 & 1200 & 1600 & 1600 & 1225 \\
\hline Labll & 1470 & 1419 & 1420 & 1421 & 1422 & 1423 & 1424 & 1459 & 1474 & 1475 & 1476 & 1478 & 1481 \\
\hline Ti(wt.\%) & 1.17 & 1.10 & 1.45 & 1.18 & 1.26 & .89 & 1.13 & 57 & 1.02 & 1.09 & 53 & 34 & 1.52 \\
\hline Mn & 224 & 21.4 & 174 & 20.3 & 23.1 & 26.6 & 22.6 & 40.6 & 22.9 & 23.8 & 37.1 & 404 & 1.16 \\
\hline Fo & 20.0 & 19.8 & 18.0 & 19.1 & 18.0 & 18.4 & 19.9 & 4.94 & 14.2 & 143 & 4.19 & 3.12 & 179 \\
\hline $\boldsymbol{N}$ & .58 & 1.02 & 222 & 1.26 & 96 & .06 & 2.18 & 143 & 2.54 & 1.04 & 1.70 & 1.11 & 4.68 \\
\hline Co & .700 & .630 & 380 & .580 & .780 & 850 & .710 & .150 & 1.11 & 1.43 & .080 & .070 & .040 \\
\hline $\mathbf{N I}$ & 360 & .320 & 310 & 340 & 420 & 430 & 380 & 300 & 350 & 430 & 330 & 370 & .090 \\
\hline C. & .060 & .080 & .220 & .060 & .100 & .040 & .080 & .140 & .070 & .040 & .130 & .100 & .040 \\
\hline C. & 2.08 & 2.08 & 3.24 & 2.08 & 2.14 & 2.42 & 234 & 1.22 & 1.78 & 1.90 & 1.13 & 1.06 & 209 \\
\hline Mg & 1.10 & 1.18 & 1.17 & 1.09 & 1.22 & 1.30 & 1.21 & 2.19 & 1.32 & 1.22 & 211 & 210 & 2.32 \\
\hline $\mathrm{Ne}$ & • & • & $\cdot$ & - & - & • & - & • & • & • & - & • & - \\
\hline $\mathbf{P}$ & .40 & .40 & .60 & .30 & 30 & so & so & - & 30 & 30 & .20 & .20 & 30 \\
\hline Be(ppm) & 1700 & 1600 & 4200 & 1700 & 1800 & 1500 & 1800 & 14000 & 2200 & 1300 & 14000 & 15000 & 400 \\
\hline Mo & 500 & 500 & 300 & 400 & 400 & 700 & 400 & 200 & 800 & 500 & 200 & 200 & 900 \\
\hline $\mathbf{P b}$ & 1200 & 1900 & 1300 & 1600 & 1700 & 2200 & 2100 & 2400 & 1100 & 1000 & • & 1100 & 1800 \\
\hline Sr & 1700 & 1600 & 1600 & 1600 & 1600 & 1800 & 1600 & 1500 & 1300 & 1400 & 1400 & 1500 & 1500 \\
\hline $\mathbf{v}$ & 600 & 600 & 600 & 600 & 600 & 900 & 700 & 400 & 600 & 600 & 300 & 400 & 600 \\
\hline $\mathbf{Z n}$ & 100 & - & $\cdot$ & • & - & • & • & 1200 & 200 & 100 & 1000 & 800 & - \\
\hline Sampled & Bulk & Bulk & Bulk & Bulk & Bulk & Bulk & Bulk & Bulk & Bulk & Bulk & Bulk & Bulk & Bulk \\
\hline Intervel & $10-15 \mathrm{~mm}$ & $46 \mathrm{cmm}$ & $2-3 \mathrm{~mm}$ & $15-18 \mathrm{~mm}$ & $5-8 \mathrm{~mm}$ & - & $5.7 \mathrm{~mm}$ & $75-85 \mathrm{cmm}$ & $8-10 \mathrm{mon}$ & $2.22 \mathrm{~mm}$ & $75-85 \mathrm{~mm}$ & $75-85 \mathrm{~mm}$ & $0-1 \mathrm{~mm}$ \\
\hline
\end{tabular}


Table 3. Chemical composition of ferromanganese crusts analyzed by BOM Avondale analytical laboratories (cont'd).

\begin{tabular}{|c|c|c|c|c|c|c|c|c|c|c|c|c|c|}
\hline $\begin{array}{l}\text { Sequencol } \\
\text { Latitude }\end{array}$ & $\begin{array}{l}089053 \\
25.489\end{array}$ & $\begin{array}{l}089054 \\
25.489\end{array}$ & $\begin{array}{l}089055 \\
25.454\end{array}$ & $\begin{array}{l}089056 \\
24.322\end{array}$ & $\begin{array}{l}089057 \\
23.988\end{array}$ & $\begin{array}{l}089058 \\
25489\end{array}$ & $\begin{array}{l}089059 \\
25.489\end{array}$ & $\begin{array}{l}089060 \\
25.135\end{array}$ & $\begin{array}{r}089061 \\
23.915\end{array}$ & $\begin{array}{l}089062 \\
25.920\end{array}$ & $\begin{array}{l}089063 \\
25.926\end{array}$ & $\begin{array}{l}089064 \\
25.926\end{array}$ & $\begin{array}{l}089065 \\
25.783\end{array}$ \\
\hline Langitude & -168.672 & -168.672 & -168.683 & -168.083 & -164.485 & -168.672 & -168.672 & -162.027 & -164.408 & -167.755 & -167.710 & -167.710 & -167.733 \\
\hline Depth & 1200 & 1200 & 1115 & 1225 & 2100 & 1200 & 1200 & 2800 & 800 & 2500 & 3250 & 3250 & 1600 \\
\hline Lab: & 1483 & 1484 & 1485 & 1486 & 1425 & 1489 & 1490 & 1427 & 1428 & 1469 & 1471 & 1472 & 1473 \\
\hline$T i(m, \%)$ & 1.00 & 1.32 & 99 & 1.26 & 1.35 & 1.04 & 1.44 & 3.26 & 1.66 & 1.17 & 1.14 & 1.38 & 98 \\
\hline Mm & 22.0 & 3.53 & 23.2 & 19.7 & 18.9 & 22.9 & 16.0 & 4,42 & 19.6 & 21.6 & 13.0 & 3.53 & 19.9 \\
\hline Po & 18.2 & 10.5 & 17.5 & 19.3 & 16.9 & 17.8 & 17.8 & 18.0 & 19.5 & 19.5 & 15.5 & 4.06 & 16.9 \\
\hline A & .94 & 4.14 & 1.32 & 150 & 2.18 & 1.61 & 3.08 & 3.30 & 1.90 & .58 & 2.65 & 5.42 & 1.26 \\
\hline Co & 1.04 & .100 & .900 & .820 & .390 & .660 & .620 & .080 & 500 & .670 & 300 & .080 & .730 \\
\hline $\mathbf{N i}$ & 370 & .190 & .390 & 310 & .420 & .460 & .230 & .150 & .340 & .360 & .230 & .120 & 510 \\
\hline Cu & .060 & .050 & .070 & .060 & .160 & .080 & .060 & .060 & .100 & .050 & .150 & .050 & .080 \\
\hline $\mathbf{c}$ & 1.96 & 15.9 & 2.10 & 2.35 & 2.14 & 2.04 & 1.85 & 1.41 & 2.24 & 2.04 & 3.13 & 4.65 & 1.91 \\
\hline Ms & 1.22 & 1.20 & 1.10 & 1.36 & 1.19 & 1.44 & 1.80 & 239 & 1.17 & 1.18 & 2.14 & 4.01 & 1.28 \\
\hline $\mathrm{Na}$ & $\cdot$ & - & $\cdot$ & $\cdot$ & • & - & - & - & $\cdot$ & $\cdot$ & - & - & - \\
\hline $\mathbf{P}$ & .50 & 5.30 & 50 & $\mathbf{A O}$ & 30 & .50 & 40 & .70 & 30 & 30 & .30 & .80 & $\Delta 0$ \\
\hline Be(ppon) & 1500 & 600 & 1600 & 1600 & 2600 & 1700 & 1000 & 600 & 2200 & 1700 & 1300 & 500 & 1500 \\
\hline Mo & 600 & 100 & 600 & 400 & 300 & 500 & 400 & 100 & 300 & 600 & 300 & so & 500 \\
\hline Po & 3400 & 2000 & 3500 & 3700 & 1400 & 1400 & 1100 & - & 2000 & 1000 & 200 & $\cdot$ & 1100 \\
\hline St & 1600 & 900 & 1600 & 1500 & 1300 & 1600 & 1200 & 300 & 1600 & 1700 & 1100 & 400 & 1300 \\
\hline $\mathbf{v}$ & 600 & 300 & 700 & 600 & 500 & 700 & 600 & 200 & 500 & 700 & 600 & 300 & 700 \\
\hline $\mathbf{Z n}$ & 200 & - & 200 & 200 & • & 300 & 200 & - & - & 200 & 200 & - & 400 \\
\hline Samplod & Bulk & Bulk & Bulk & Bulk & Bulk & Bulk & Bulk & Bulk & Bulk & Bulk & Bulk & Bulk & Bulk \\
\hline Intorval & $3-18 \mathrm{~mm}$ & $1.2 \mathrm{~mm}$ & $10-15 \mathrm{~mm}$ & $1-10 \mathrm{~mm}$ & $10-15 \mathrm{~mm}$ & $15-20 \mathrm{~mm}$ & $1.5 \mathrm{~mm}$ & $2-6 \mathrm{~cm}$ & $3-12 \mathrm{~mm}$ & $10-25 \mathrm{~mm}$ & $4-8 \operatorname{mon}$ & $3.5 \mathrm{~mm}$ & $1.9 \mathrm{~mm}$ \\
\hline
\end{tabular}

\begin{tabular}{|c|c|c|c|c|c|c|c|c|c|c|c|c|c|}
\hline Sequencert & 089066 & 089067 & 089068 & 089069 & 089070 & 089071 & 089072 & 089073 & 089074 & 089075 & 089076 & 089077 & 089078 \\
\hline Letitude & 25.783 & 24.322 & 24.322 & 25489 & 25489 & 25.685 & 23.961 & 23.956 & 25.260 & 25.094 & 25.094 & 25.260 & 25.260 \\
\hline Longitude & -167.733 & -168.083 & -168.083 & -168.672 & -168.672 & -160.180 & -164.276 & -164414 & -162046 & -161.728 & -161.728 & -162.046 & .162 .046 \\
\hline Depth & 1600 & 1225 & 1225 & 1200 & 1200 & 3000 & 2650 & 1300 & 2300 & 2800 & 2800 & 2300 & 2300 \\
\hline Lab" & 1477 & 1479 & 1480 & 1482 & 1491 & 1494 & 1496 & 1497 & 1498 & 1500 & 1501 & 1801 & 1803 \\
\hline$T i(w t . \%)$ & .45 & 1.50 & 1.59 & 1.02 & 1.15 & .62 & 1.30 & 1.38 & 1.46 & 1.40 & 1.09 & 2.10 & 1.46 \\
\hline $\mathbf{M m}$ & 40.6 & 6.38 & 334 & 20.6 & 23.8 & 24.7 & 18.6 & 16.9 & 15.0 & 14.5 & 213 & 25.3 & 22.5 \\
\hline Po & 3.95 & 17.0 & 17.8 & 18.2 & 15.7 & 18.5 & 17.3 & 21.1 & 19.6 & 13.1 & 19.1 & 19.6 & 22.5 \\
\hline A & 1.30 & 4.20 & 5.11 & 1.21 & 1.32 & .56 & 1.84 & 1.66 & 181 & 3.24 & .69 & .70 & .74 \\
\hline Co & .090 & .200 & .110 & .940 & 1.30 & 1.29 & .550 & .330 & .500 & .260 & .600 & 1.02 & .530 \\
\hline $\mathrm{Ni}$ & 320 & .180 & .250 & .370 & .400 & .410 & .320 & 500 & .250 & .430 & .300 & .350 & .200 \\
\hline ca & .120 & .040 & .060 & .060 & .060 & .050 & .100 & .090 & .050 & .220 & .040 & .080 & .080 \\
\hline C. & 120 & 3.14 & 2.25 & 1.84 & 1.99 & 3.93 & 2.13 & 230 & 1.96 & 2.32 & 2.20 & 2.65 & 249 \\
\hline Ms & 2.23 & 256 & 258 & 1.25 & 1.30 & 1.47 & 1.28 & 1.10 & 1.20 & 1.24 & 1.08 & 1.30 & 1.12 \\
\hline $\mathrm{Na}$ & - & - & - & - & - & • & - & • & • & • & - & • & 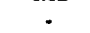 \\
\hline $\mathbf{P}$ & .20 & 30 & 30 & 50 & .40 & 1.10 & .40 & 40 & 50 & 50 & 30 & .30 & 40 \\
\hline Be(ppon) & 15000 & 800 & 600 & 1400 & 1300 & 1900 & 1600 & 2000 & 2000 & 2000 & 1500 & 2500 & 2400 \\
\hline Mo & 200 & 200 & 100 & 500 & 600 & 800 & 400 & 300 & 400 & 300 & 400 & 400 & 600 \\
\hline $\mathbf{P B}_{\mathbf{b}}$ & 900 & 1600 & 1100 & 3400 & 1500 & 2400 & 1800 & 1500 & 1600 & 1300 & 1800 & 1900 & 1400 \\
\hline St & 1500 & 600 & 300 & 1500 & 1400 & 1800 & 1400 & 1600 & 1200 & 1000 & 1600 & 1800 & 2000 \\
\hline $\mathbf{v}$ & 400 & 400 & 400 & 600 & 600 & 900 & 500 & 600 & 600 & 300 & 600 & 600 & 700 \\
\hline $\mathrm{Zn}$ & 1000 & - & - & 100 & 200 & 400 & 400 & 500 & 400 & 900 & 200 & 400 & 400 \\
\hline Samplod & Bulk & Bulk & Bulk & Bulk & Bulk & Bulk & Bulk & Bulk & Bulk & Bulk & Bulk & Bulk & Bulk \\
\hline Intarvil & $75-85 \mathrm{~mm}$ & $1-3 \mathrm{~mm}$ & $3-4 \mathrm{~mm}$ & $15.29 \mathrm{~mm}$ & 4-20mm & $1.2 \mathrm{mom}$ & $12-14 \mathrm{~mm}$ & S-9mon & $20-25 \mathrm{~mm}$ & $4-8 \mathrm{~mm}$ & $10-15 \mathrm{~mm}$ & $38-45 \mathrm{~mm}$ & $50-60 \mathrm{~mm}$ \\
\hline
\end{tabular}


Table 3. Chemical composition of ferromanganese crusts analyzed by BOM Avondale analytical laboratories (cont'd).

\begin{tabular}{|c|c|c|c|c|c|c|c|c|c|c|c|c|c|}
\hline $\begin{array}{l}\text { Sequencoll } \\
\text { Letituds }\end{array}$ & $\begin{array}{l}089079 \\
25.276\end{array}$ & $\begin{array}{l}089080 \\
25.980\end{array}$ & $\begin{array}{l}089081 \\
25.980\end{array}$ & $\begin{array}{l}089082 \\
25.980\end{array}$ & $\begin{array}{l}089083 \\
25.980\end{array}$ & $\begin{array}{l}089084 \\
25.980\end{array}$ & $\begin{array}{l}089085 \\
25.276\end{array}$ & $\begin{array}{l}089086 \\
23.672\end{array}$ & $\begin{array}{l}089087 \\
23.672\end{array}$ & $\begin{array}{l}089088 \\
25.260\end{array}$ & $\begin{array}{l}089089 \\
25.135\end{array}$ & $\begin{array}{l}089090 \\
25.276\end{array}$ & $\begin{array}{l}089091 \\
23.672\end{array}$ \\
\hline Longitnde & -162068 & -164.379 & -164.379 & .164 .379 & -164379 & -164379 & -162068 & -164.891 & -164.891 & -162.046 & -162.027 & -162.068 & -164.891 \\
\hline Depth & 3000 & 1800 & 1800 & 1800 & 1800 & 1800 & 3000 & 1650 & 1650 & $\mathbf{x} 00$ & 2800 & 3000 & 1650 \\
\hline Lab" & 1804 & 1805 & 1806 & 1807 & 1808 & 1809 & 1810 & 1811 & 1813 & 1815 & 1816 & 1818 & 1819 \\
\hline TI(wt.\%) & 184 & 1.26 & 1.20 & 1.32 & 1.10 & 1.25 & 1.40 & 1.51 & 1.38 & 1.48 & 1.31 & 151 & 1.44 \\
\hline Mn & 24.7 & 21.7 & 20.4 & 189 & 22.2 & 21.4 & 15.8 & 204 & 16.5 & 22.0 & 220 & 205 & 17.0 \\
\hline Fo & 199 & 23.3 & 228 & 23.0 & 21.7 & 20.4 & 203 & 214 & 224 & 22.5 & 204 & 21.6 & 21.6 \\
\hline $\mathbf{A}$ & .81 & 1.11 & 1.27 & 1.32 & .79 & 1.27 & 209 & 1.30 & 1.83 & .06 & 1.20 & 1.11 & 1.87 \\
\hline Co & .630 & .660 & 580 & 540 & .690 & .600 & 380 & 480 & $A 10$ & 580 & .700 & 500 & 570 \\
\hline $\mathrm{Ni}$ & 410 & .240 & .220 & .200 & 310 & .240 & .120 & .290 & .180 & 320 & 320 & 400 & 350 \\
\hline$\infty$ & .200 & .050 & .030 & .060 & .060 & .080 & .060 & .120 & .060 & .090 & .100 & .160 & .080 \\
\hline Ca & 2.32 & 1.72 & 2.28 & 2.17 & 2.20 & 2.26 & 257 & 2.27 & 2.50 & 214 & 2.28 & 216 & 270 \\
\hline $\mathbf{M g}_{\mathbf{8}}$ & 1.18 & 1.19 & 1.14 & 1.12 & 1.19 & 1.25 & 1.30 & 1.15 & 1.22 & 1.10 & 1.23 & 1.14 & 1.29 \\
\hline $\mathrm{Na}$ & • & - & • & $\cdot$ & • & - & • & • & • & • & - & • & - \\
\hline $\mathbf{P}$ & .40 & 40 & .40 & .40 & .40 & 40 & .40 & 40 & .40 & so & 40 & so & .40 \\
\hline Ba(ppm) & 3100 & 2100 & 2100 & 2000 & 2000 & 2000 & 2000 & 2200 & 2000 & 2200 & 1900 & 2200 & 1800 \\
\hline Mo & 500 & 500 & 500 & 500 & 600 & 500 & 300 & 400 & 400 & 500 & 400 & 400 & 300 \\
\hline $\mathrm{Pb}$ & 1600 & 1600 & 1800 & 1400 & 1900 & 1300 & 1300 & 2400 & 1300 & 1800 & 1300 & 1400 & 2700 \\
\hline Sr & 1900 & 1900 & 1900 & 1900 & 1900 & 1800 & 1600 & 1700 & 1600 & 1800 & 1700 & 1800 & 1600 \\
\hline $\mathbf{V}$ & 600 & 1100 & 1400 & 700 & 1400 & 700 & 600 & 600 & 700 & 700 & 700 & 600 & 600 \\
\hline $\mathbf{Z n}$ & 500 & 500 & 400 & 400 & 300 & 400 & 400 & 400 & 400 & 500 & 300 & 400 & 300 \\
\hline Sampled & $\begin{array}{c}\text { Balk } \\
25-60 \mathrm{~mm}\end{array}$ & $\begin{array}{c}\text { Bulk } \\
35-40 \mathrm{~mm}\end{array}$ & $\underset{30-35 \mathrm{mmm}}{\text { Bulk }}$ & $\begin{array}{c}\text { Bulk } \\
30-35 \mathrm{mmm}\end{array}$ & Balk & $\begin{array}{c}\text { Bulk } \\
14-18 \mathrm{~mm}\end{array}$ & $\begin{array}{c}\text { Bulk } \\
3-6 \mathrm{~mm}\end{array}$ & $\underset{\text { Bulk }}{\text { 6-8mm }}$ & $\begin{array}{c}\text { Bulk } \\
7-10 \mathrm{~mm}\end{array}$ & $\begin{array}{c}\text { Bulk } \\
25-30 \mathrm{~mm}\end{array}$ & $\underset{2-3 \mathrm{~mm}}{\text { Bulk }}$ & $\underset{7-13 \mathrm{~mm}}{\text { Bulk }}$ & $\underset{\text { 6-10 }}{\text { Bulk }}$ \\
\hline
\end{tabular}

\begin{tabular}{|c|c|c|c|c|c|c|c|c|c|c|c|c|c|}
\hline Sequencoll & 089092 & 089093 & 089094 & 089095 & 089096 & 089097 & 089098 & 089099 & 089100 & 090018 & 090019 & 090020 & 090021 \\
\hline Lationde & 25.276 & 25.260 & 25.303 & 25.276 & 25.303 & 23.979 & 23.979 & 25.135 & 25.094 & 28.895 & 28.895 & 29.106 & 29.167 \\
\hline Longitude & -162.068 & -162.046 & .162094 & -162068 &.-162.094 & -164.363 & -164.363 & -162.010 & -161.728 & -178.868 & -178.868 & -173.948 & -174.067 \\
\hline Depth & 3000 & 2300 & 3500 & 3000 & 3500 & 2200 & 2200 & 2175 & 2800 & 1280 & 1280 & 1850 & 1425 \\
\hline Lab" & 1820 & 1821 & 1822 & 1823 & 1824 & 1832 & 1834 & 1836 & 1839 & 1445 & 1446 & 1448 & 1449 \\
\hline $\operatorname{Ti}(w t . \%)$ & 1.30 & 1.61 & 1.38 & 1.48 & 120 & 1.46 & 1.46 & 1.60 & 1.12 & 84 & .96 & 1.08 & .90 \\
\hline Mn & 21.5 & 19.4 & 17.7 & 195 & 18.6 & 16.0 & 169 & 16.2 & 29.6 & 25.0 & 18.5 & 24.9 & 27.0 \\
\hline Po & 24.0 & 20.9 & 22.2 & 23.3 & 23.1 & 16.9 & 19.2 & 16.3 & 12.2 & 588 & 6.57 & 18.0 & 15.9 \\
\hline $\mathbf{A}$ & .82 & 1.66 & 1.77 & $1 A 2$ & 1.50 & 2.94 & 254 & 3.40 & 1.36 & 3.74 & 4.59 & 99 & s5 \\
\hline Co & .490 & .540 & 330 & 430 & 520 & .590 & 330 & 280 & 147 & .540 & .400 & .680 & .760 \\
\hline $\mathbf{N i}$ & 370 & .400 & 380 & 360 & 310 & .290 & .280 & .260 & .600 & .500 & .260 & 440 & ASO \\
\hline $\mathrm{Cu}$ & .090 & .120 & 200 & .130 & .060 & .060 & .090 & .150 & .060 & .060 & .040 & .080 & .040 \\
\hline ca & 224 & 208 & 222 & 2.36 & 206 & 2.02 & 255 & 3.20 & 251 & 262 & 1.50 & 232 & 232 \\
\hline $\mathbf{M g}_{\mathbf{g}}$ & 1.12 & 1.13 & 1.16 & 1.17 & 1.08 & 1.40 & 144 & 1.14 & 239 & 256 & 1.59 & 1.10 & 1.14 \\
\hline Ne & - & • & - & - & • & - & • & • & - & • & • & - & • \\
\hline $\mathbf{P}$ & .50 & 50 & 50 & .60 & .40 & 20 & 30 & so & 20 & 30 & 20 & so & .60 \\
\hline $\mathrm{Be}(\mathrm{ppm})$ & 2200 & 2700 & 3400 & 2300 & 1700 & 1400 & 1600 & 1800 & 1300 & 600 & 600 & 2000 & 1800 \\
\hline Mo & 500 & 400 & 300 & 400 & 400 & 300 & 300 & 200 & 800 & 300 & 300 & 700 & 800 \\
\hline Pb & 3000 & 3400 & 2300 & 3200 & 2600 & 800 & 500 & 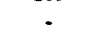 & 1600 & 2500 & 200 & 2300 & 2100 \\
\hline Sr & 1800 & 1800 & 1600 & 2000 & 1600 & 1000 & 1200 & 1200 & 1200 & 500 & 400 & 2000 & 1900 \\
\hline $\mathbf{V}$ & 800 & 700 & 600 & 700 & 600 & 200 & 500 & 500 & 500 & 500 & .400 & 800 & 800 \\
\hline $\mathbf{z n}$ & 400 & 300 & 400 & 300 & 200 & 300 & 400 & 200 & 400 & 2000 & 1400 & 1200 & 600 \\
\hline Surspled & Bulk & Bulk & Bulk & Bulk & Bulk & Bulk & Bulk & Nocule & Bulk & Bulk & Bulk & Bulk & Bulk \\
\hline Interval & $13-25 \mathrm{~mm}$ & $5-8 m$ & $2-4 \mathrm{~mm}$ & $10-15 \mathrm{mmm}$ & $5-8 \mathrm{~mm}$ & $2-5 m m$ & $3-6 \mathrm{mon}$ & . & $1.5 \mathrm{~mm}$ & $3-5 \mathrm{~mm}$ & $1.3 \mathrm{~mm}$ & $10-20 \mathrm{~mm}$ & $14-40 \mathrm{~mm}$ \\
\hline
\end{tabular}


Table 3. Chemical composition of ferromanganese crusts analyzed by BOM Avondale analytical laboratories (cont'd).

\begin{tabular}{|c|c|c|c|c|c|c|c|c|c|c|c|c|c|}
\hline $\begin{array}{l}\text { Sequencos" } \\
\text { Letirude }\end{array}$ & $\begin{array}{l}090022 \\
29.106\end{array}$ & $\begin{array}{l}090023 \\
29.167\end{array}$ & $\begin{array}{l}090024 \\
29.208\end{array}$ & $\begin{array}{l}090025 \\
28.895\end{array}$ & $\begin{array}{l}090027 \\
29.167\end{array}$ & $\begin{array}{l}090028 \\
29.167\end{array}$ & $\begin{array}{l}090029 \\
29.167\end{array}$ & $\begin{array}{l}090030 \\
29.185\end{array}$ & $\begin{array}{l}090031 \\
20.208\end{array}$ & $\begin{array}{l}090032 \\
29.167\end{array}$ & $\begin{array}{c}0900033 \\
29.062\end{array}$ & $\begin{array}{l}090034 \\
29.208\end{array}$ & $\begin{array}{l}090035 \\
28.895\end{array}$ \\
\hline Langitude & -173.948 & -174.067 & -174.112 & -178.868 & -174.067 & -174.067 & -174.067 & -174.010 & -174.112 & -174.067 & $-174,022$ & -174.112 & -178.868 \\
\hline Depth & 1850 & 1425 & 2200 & 1280 & 1425 & 1425 & 1425 & 985 & 2200 & 1425 & 1150 & 2200 & 1280 \\
\hline Labil & 1451 & 1452 & 1454 & 1434 & 1455 & 1457 & 1458 & 1462 & 1464 & 1465 & 1468 & 1467 & 1432 \\
\hline$T(w t . \%)$ & 91 & 1.35 & 1.22 & .70 & 1.26 & 82 & 94 & 1.16 & 1.33 & 1.22 & 1.08 & 1.17 & .67 \\
\hline Ma & 255 & 25.0 & 21.0 & 26.5 & 19.6 & 26.8 & 26.4 & 27.2 & 23.3 & 23.2 & 28.8 & 20.3 & 31.4 \\
\hline Po & 164 & 16.0 & 15.3 & 12.9 & 19.2 & 14.9 & 16.7 & 16.0 & 17.1 & 17.7 & 15.8 & 15.7 & 13.0 \\
\hline $\mathbf{A}$ & .54 & 87 & 1.10 & 93 & 1.12 & .64 & 58 & 1.39 & 1.34 & 1.30 & .64 & 1.95 & so \\
\hline$C_{0}$ & 860 & .630 & .690 & .930 & .720 & .820 & .930 & 1.10 & .640 & .770 & .670 & $\$ 40$ & 1.54 \\
\hline $\mathrm{Ni}$ & 450 & .450 & 380 & .550 & .310 & .530 & .460 & .440 & .420 & .480 & $A 00$ & .390 & .610 \\
\hline a & .060 & .070 & .120 & .040 & .080 & .060 & .040 & .050 & .120 & .060 & .040 & .120 & .050 \\
\hline Ca & 2.21 & 264 & 2.08 & 250 & 1.99 & 224 & 2.24 & 276 & 2.22 & 2.89 & 246 & 230 & 230 \\
\hline$\overline{\mathbf{M g}_{\mathbf{g}}}$ & 1.14 & 1.25 & 1.08 & 1.72 & 1.11 & 121 & 1.21 & 1.33 & 1.20 & 1.29 & 1.30 & 1.21 & 1.44 \\
\hline $\mathrm{Na}$ & • & - & - & • & - & - & - & • & • & • & • & - & - \\
\hline $\mathbf{P}$ & .50 & .70 & .60 & .20 & .70 & so & so & .80 & so & .80 & 40 & $A 0$ & 30 \\
\hline$B e(p p n)$ & 1600 & 2400 & 2800 & 1100 & 3500 & 1900 & 1600 & 2400 & 2700 & 1700 & 2400 & 3000 & 1400 \\
\hline Mo & 700 & 700 & 500 & 800 & 400 & 800 & 800 & 800 & 400 & 800 & 700 & 400 & 800 \\
\hline Po & 2000 & 2300 & 1700 & 2100 & 2700 & 1900 & 2300 & 3100 & 1800 & 2900 & 1400 & 1200 & 2500 \\
\hline St & 1700 & 1800 & 1500 & 1400 & 2000 & 1700 & 1700 & 1900 & 1600 & 1800 & 1900 & 1400 & 1600 \\
\hline $\mathbf{v}$ & 600 & 700 & 600 & 700 & 600 & 800 & 700 & 800 & 600 & 800 & 800 & 500 & 800 \\
\hline $\mathrm{Zn}$ & 600 & 800 & 600 & $\cdot$ & 600 & 600 & 200 & 400 & 700 & 600 & 400 & 400 & • \\
\hline Samplod & Bulk & Bulk & Bulk & Bulk & Bulk & Bulk & Bulk & Bulk & Bulk & Bulk & Bulk & Bulk & Bulk \\
\hline Inorval & $25-33 \mathrm{~mm}$ & $15-20 \mathrm{~mm}$ & $15-20 \mathrm{~mm}$ & $1.2 \mathrm{~mm}$ & $10.25 \mathrm{~mm}$ & $25-35 \mathrm{~mm}$ & $15-20 \mathrm{~mm}$ & • & $15.25 \mathrm{~mm}$ & $412 \mathrm{~mm}$ & $90-110 \mathrm{~mm}$ & $20-25 \mathrm{~mm}$ & $10-12 \mathrm{~mm}$ \\
\hline
\end{tabular}

\begin{tabular}{|c|c|c|c|c|c|c|c|c|c|c|c|c|c|}
\hline $\begin{array}{l}\text { Sequenos: } \\
\text { Letitude }\end{array}$ & $\begin{array}{l}090036 \\
28.881\end{array}$ & $\begin{array}{l}090026 \\
28.895\end{array}$ & $\begin{array}{l}090038 \\
28.895\end{array}$ & $\begin{array}{l}090039 \\
28.739\end{array}$ & $\begin{array}{l}090040 \\
29.106\end{array}$ & $\begin{array}{l}090041 \\
29.106\end{array}$ & $\begin{array}{l}090042 \\
29.167\end{array}$ & $\begin{array}{l}090043 \\
29.167\end{array}$ & $\begin{array}{l}090044 \\
29.106\end{array}$ & $\begin{array}{l}090045 \\
29.106\end{array}$ & $\begin{array}{l}090046 \\
29.106\end{array}$ & $\begin{array}{l}090047 \\
29.106\end{array}$ & $\begin{array}{l}090048 \\
28.881\end{array}$ \\
\hline Langitude & -179.745 & -178.868 & -178.868 & -178.783 & -173.948 & -174.020 & -174.067 & -174.067 & -173.948 & -173.948 & -173.948 & -173.948 & -179.745 \\
\hline Depth & 2000 & 1280 & 1280 & 3000 & 1850 & 1475 & 1425 & 1425 & 1850 & 1850 & 1850 & 1850 & 2000 \\
\hline Lab" & 1440 & 1441 & 1447 & 1450 & 1453 & 1461 & 1463 & 1466 & 1826 & 1827 & 1828 & 1820 & 1837 \\
\hline $\operatorname{Ti}(w t . \%)$ & .68 & .74 & .88 & 94 & 1.22 & .88 & 1.05 & 1.05 & 1.08 & 1.16 & .99 & 93 & 1.20 \\
\hline Mn & 26.9 & 26.9 & 30.5 & 19.7 & 14.1 & 28.5 & 25.9 & 23.5 & 24.4 & 25.6 & 272 & 29.1 & 26.0 \\
\hline Fo & 23.1 & 13.9 & 11.9 & 19.6 & 20.6 & 125 & 16.6 & 15.0 & 17.3 & 17.9 & 17.6 & 127 & 17.6 \\
\hline $\mathbf{N}$ & 1.17 & .74 & .92 & .73 & 1.62 & 1.24 & 1.55 & 2.23 & 1.11 & .72 & .61 & 152 & 1.02 \\
\hline Co & .620 & 1.32 & 1.75 & .470 & .790 & 1.42 & .680 & .500 & .700 & .840 & .870 & 580 & .760 \\
\hline $\mathrm{Ni}$ & 940 & 510 & .740 & .280 & .210 & .620 & .460 & .460 & .480 & .510 & $A 00$ & 1.150 & .420 \\
\hline ar & 200 & .070 & .060 & .040 & .060 & .050 & .060 & .080 & .080 & .070 & .060 & .130 & .060 \\
\hline Ca & 1.98 & 2.94 & 2.16 & 1.98 & 1.40 & 2.32 & 2.20 & 2.34 & 276 & 236 & 276 & 260 & 250 \\
\hline Mg & 1.60 & 1.42 & 1.68 & 1.02 & 1.17 & 1.35 & 1.28 & 1.23 & 1.27 & 1.30 & 127 & 1.81 & 1.26 \\
\hline $\mathrm{Na}$ & - & - & • & - & - & - & - & - & - & - & - & - & - \\
\hline $\mathbf{P}$ & .30 & 30 & .40 & 50 & .60 & .60 & .60 & .60 & .70 & so & so & $A 0$ & .30 \\
\hline Be(ppen) & 1400 & 1600 & 1400 & 1400 & 2000 & 1400 & 2200 & 3800 & 3000 & 2000 & 2600 & 3000 & 1500 \\
\hline Mo & 600 & 800 & 800 & 400 & 200 & 700 & 600 & 600 & 600 & 600 & 800 & 600 & 500 \\
\hline Pb & 1500 & 2100 & 2700 & 1400 & 2500 & 3100 & 2400 & 2300 & 2700 & 3000 & 3600 & 2000 & 600 \\
\hline Sr & 1500 & 1800 & 1300 & 1600 & 1700 & 1400 & 1700 & 1600 & 1700 & 1800 & 1900 & 1300 & 1600 \\
\hline $\mathbf{v}$ & 600 & 800 & 700 & 600 & 500 & 700 & 800 & 600 & 600 & 700 & 800 & 600 & 600 \\
\hline $2 n$ & 1000 & 600 & 1300 & 600 & 500 & 3800 & 600 & 700 & 300 & 200 & 200 & 800 & 300 \\
\hline Sumplod & Bulk & Bulk & Bulk & Bulk & Bulk & Bulk & Bulk & Bulk & Bulk & Bulk & Bulk & Bulk & Bulk \\
\hline Interval & $1.3 \mathrm{~mm}$ & - & $1-9 \mathrm{~mm}$ & $8-10 \mathrm{~mm}$ & $4-10 \mathrm{~mm}$ & - & $20-25 \mathrm{~mm}$ & $15.30 \mathrm{~mm}$ & $13-25 \mathrm{~mm}$ & $13-20 \mathrm{~mm}$ & $3-5 \mathrm{~mm}$ & $48 \mathrm{~mm}$ & 6-8mm \\
\hline
\end{tabular}


Table 3. Chemical composition of ferromanganese crusts analyzed by BOM Avondale analytical laboratories (cont'd).

\begin{tabular}{|c|c|c|c|c|c|c|c|c|c|c|c|c|c|}
\hline $\begin{array}{l}\text { Soquencoll } \\
\text { Letitude }\end{array}$ & $\begin{array}{l}090049 \\
28.895\end{array}$ & $\begin{array}{l}091005 \\
29.857\end{array}$ & $\begin{array}{l}091006 \\
29.643\end{array}$ & $\begin{array}{l}091007 \\
29.857\end{array}$ & $\begin{array}{l}091008 \\
29.839\end{array}$ & $\begin{array}{l}091009 \\
29.839\end{array}$ & $\begin{array}{l}091010 \\
20.634\end{array}$ & $\begin{array}{l}091011 \\
29.641\end{array}$ & $\begin{array}{c}091012 \\
29869\end{array}$ & $\begin{array}{r}091013 \\
29.820\end{array}$ & $\begin{array}{l}091014 \\
29.641\end{array}$ & $\begin{array}{l}091015 \\
29.634\end{array}$ & $\begin{array}{l}091016 \\
29.839\end{array}$ \\
\hline Longitude & -178.869 & 179.083 & 179.282 & 179.083 & 179.065 & 179.065 & 179.288 & 179.282 & 179.098 & 179.103 & 179.282 & 179.288 & 179.065 \\
\hline Depth & 1280 & 2250 & 1300 & 2250 & 2100 & 2100 & 1865 & 1300 & 2900 & 1700 & 1300 & 1865 & 2100 \\
\hline Lab: & 1838 & 1435 & 1442 & 1487 & 1430 & 1431 & 1433 & 1456 & 1492 & 1438 & 1436 & 1437 & 1439 \\
\hline$T(w t s)$ & 1.06 & 123 & .69 & 1.12 & 1.24 & 98 & .82 & 1.02 & 156 & 1.28 & .84 & .72 & 99 \\
\hline $\mathrm{Mn}$ & 279 & 23.0 & 20.0 & 20.1 & 144 & 23.8 & 25.7 & 219 & 22.7 & 21.4 & 27.3 & 23.7 & 14.2 \\
\hline $\mathbf{F}_{0}$ & 165 & 16.6 & 14.8 & 20.1 & 165 & 16.0 & 18.6 & 19.6 & 18.2 & 17.2 & 17.6 & 18.0 & 22.6 \\
\hline A & 88 & 1.00 & 51 & 1.25 & 278 & 1.20 & 54 & .62 & 1.04 & 156 & .69 & 34 & 190 \\
\hline$c_{0}$ & .740 & 570 & 1.28 & .500 & 460 & .800 & .640 & 1.23 & .730 & .620 & 840 & .600 & 520 \\
\hline $\mathrm{Ni}$ & 510 & .400 & 510 & 380 & 360 & .380 & 380 & 320 & 380 & .460 & .400 & 390 & 320 \\
\hline cre & .070 & .090 & .070 & .080 & .120 & .050 & .080 & .040 & .080 & .060 & .040 & .060 & .060 \\
\hline Ca & 239 & 2.26 & 224 & 1.93 & 284 & 2.06 & 252 & 204 & 230 & 2.69 & 2.46 & 209 & 1.72 \\
\hline $\mathbf{M g}_{\mathbf{g}}$ & 2.02 & 1.17 & 1.30 & 1.24 & 1.98 & 1.22 & 1.22 & 1.22 & 1.16 & 1.48 & 1.27 & 1.07 & 1.24 \\
\hline $\mathrm{Na}$ & - & • & - & - & - & - & • & - & - & • & - & - & - \\
\hline $\mathbf{P}$ & .40 & .30 & 30 & .40 & 30 & 40 & .40 & .60 & .40 & .30 & .30 & 30 & .40 \\
\hline Besp(pon) & 2000 & 3000 & 1400 & 2000 & 2700 & 1300 & 1400 & 2400 & 2400 & 1300 & 1500 & 1400 & 3200 \\
\hline Mo & 700 & 600 & 900 & 500 & 200 & 600 & 700 & 600 & 400 & 600 & 800 & 700 & 200 \\
\hline $\mathrm{Pb}_{\mathrm{b}}$ & 1600 & 2000 & 2100 & 3300 & 2100 & 2000 & 2400 & 2600 & 1600 & 1900 & 2500 & 2500 & 3100 \\
\hline Sr & 1600 & 1600 & 1800 & 1600 & 1200 & 2300 & 1800 & 2000 & 1500 & 1600 & 1700 & 1800 & 1600 \\
\hline $\mathbf{v}$ & 700 & 700 & 700 & 600 & 500 & 700 & 800 & 600 & 500 & 700 & 800 & 800 & 700 \\
\hline $\mathbf{z a}$ & 500 & 600 & 500 & 400 & - & • & - & 400 & 300 & 500 & 400 & 500 & 700 \\
\hline Strmplod & Bulk & Bulk & Bulk & Bulk & Bulk & Balk & Bulk & Bulk & Bulk & Bulk & Bulk & Bulk & Bulk \\
\hline Intorval & $3-5 \mathrm{~mm}$ & - & $2.12 \mathrm{~mm}$ & $1-6 \mathrm{~mm}$ & $2-6 \mathrm{~mm}$ & $5-15 \mathrm{~mm}$ & $7-8 \mathrm{~mm}$ & . & - & $5-6 \mathrm{~mm}$ & - & $8-12 \mathrm{~mm}$ & $1-3 \mathrm{~mm}$ \\
\hline
\end{tabular}

\begin{tabular}{|c|c|c|c|c|c|}
\hline Sequence & 091017 & 091018 & 091019 & 091020 & 091021 \\
\hline Latitude & 29.634 & 29.634 & 29.866 & 29.869 & 29.820 \\
\hline Longitude & 179.288 & 179.288 & 179.083 & 179.098 & 179.104 \\
\hline Depth & 1865 & 1865 & 2250 & 2900 & 1700 \\
\hline Lab" & 1443 & 1444 & 1488 & 1493 & 1825 \\
\hline$T(w t, \%)$ & .81 & .70 & 1.01 & 1.04 & 237 \\
\hline Mn & 24.6 & 20.8 & 22.0 & 18.5 & 9.04 \\
\hline $\mathrm{Po}_{0}$ & 18.1 & 173 & 18.2 & 17.3 & 14.7 \\
\hline $\mathbf{N}$ & .78 & 1.19 & .88 & 1.60 & 3.89 \\
\hline Co & .640 & 480 & 580 & 390 & .250 \\
\hline $\mathrm{Ni}$ & .400 & $A 00$ & 340 & .400 & .130 \\
\hline $\mathrm{Cu}$ & .050 & .060 & .060 & .120 & .040 \\
\hline C. & 214 & 1.84 & 2.18 & 2.00 & 3.82 \\
\hline $\mathbf{M g}$ & 1.01 & 1.18 & 1.12 & 1.30 & 250 \\
\hline $\mathrm{Na}$ & • & • & - & - & - \\
\hline $\mathbf{P}$ & 30 & .30 & .40 & .40 & .40 \\
\hline $\mathrm{Ba}(\mathrm{ppm})$ & 1700 & 1400 & 1600 & 2000 & 700 \\
\hline Mo & 700 & 600 & 500 & 400 & 300 \\
\hline Po & 2400 & 1500 & 1500 & 700 & 1300 \\
\hline $\mathbf{S r}$ & 1900 & 1700 & 1700 & 1300 & 700 \\
\hline $\mathbf{v}$ & 800 & 800 & 600 & 500 & 400 \\
\hline $\mathbf{z n}$ & 500 & 600 & 300 & 300 & 200 \\
\hline Sampled & Bulk & Bulk & Bulk & Bulk & Bulk \\
\hline Interval & $8-15 \mathrm{~mm}$ & $7-10 \mathrm{~mm}$ & $1-3 \mathrm{nmm}$ & $8-10 \mathrm{~mm}$ & • \\
\hline
\end{tabular}


Table 4. Composition of ferromanganese crusts analyzed by TUCLZ (Halbach et al.) and German Geological Survey (Marchig et al.)

\begin{tabular}{|c|c|c|c|c|c|c|c|c|c|c|c|}
\hline $\begin{array}{l}\text { Sequancoll } \\
\text { Latitude }\end{array}$ & $\begin{array}{c}017006 \\
8.414\end{array}$ & $\begin{array}{c}017007 \\
8.414\end{array}$ & $\begin{array}{c}017008 \\
8.414\end{array}$ & $\begin{array}{c}017009 \\
8398\end{array}$ & $\begin{array}{c}017010 \\
8.398\end{array}$ & $\begin{array}{c}017011 \\
8389\end{array}$ & $\begin{array}{c}017012 \\
8.353\end{array}$ & $\begin{array}{c}017013 \\
8.353\end{array}$ & $\begin{array}{c}017014 \\
8.308\end{array}$ & $\begin{array}{c}017015 \\
8.308\end{array}$ & $\begin{array}{c}017015 \\
8.308\end{array}$ \\
\hline Longitude & -164.289 & -164.289 & -164.289 & -164.359 & -164.359 & .164 .294 & .164317 & -164.317 & -164.346 & -164.346 & -164.346 \\
\hline Depth & 1850 & 1850 & 1850 & 2400 & 2400 & 1600 & 1150 & 1150 & 1400 & 1400 & 1400 \\
\hline Labil & 38 & 39 & 40 & 41 & 42 & 43 & 44 & 45 & 46 & 47 & 47 \\
\hline si(mts) & 208 & 278 & 266 & 3.49 & 4.32 & 236 & 1.17 & 1.33 & .72 & .67 & 1.30 \\
\hline $\mathrm{TI}$ & 1.14 & 1.12 & 1.46 & 1.00 & 1.13 & 1.05 &.$\pi$ & 93 & 92 & 90 & 88 \\
\hline Mn & 27.3 & 24.9 & 239 & 25.8 & 23.0 & 23.7 & 33.9 & 27.6 & 318 & 32.4 & 29.1 \\
\hline Re & 16.0 & 18.1 & 18.8 & 16.9 & 184 & 15.3 & 8.47 & 13.4 & 13.8 & 14.1 & 13.8 \\
\hline $\mathbf{N}$ & $s 1$ & .63 & .68 & .66 & 94 & 92 & 51 & 27 & 37 & 34 & 31 \\
\hline Co & 1.01 & 808 & 871 & 294 & 903 & 1.20 & 256 & 1.24 & 1.14 & 1.06 & 1.08 \\
\hline $\mathrm{Ni}$ & 566 & 511 & .446 & 536 & 478 & .633 & 948 & 410 & .676 & .643 & .654 \\
\hline a & .053 & .068 & .085 & .091 & .085 & .076 & .073 & .040 & .035 & .041 & .040 \\
\hline C. & 2.65 & 3.21 & 260 & 263 & 285 & 5.08 & 2.49 & 5.38 & 287 & 3.44 & 6.15 \\
\hline Ms & 1.13 & 1.08 & 1.07 & 1.11 & 1.17 & 1.39 & 1.77 & 1.17 & 1.21 & 1.20 & 1.18 \\
\hline $\mathbf{P}$ & 36 & 57 & 39 & 45 & 54 & 1.45 & .40 & 1.51 & 37 & .57 & .60 \\
\hline $\mathrm{H}_{2} \mathrm{O}$ & 17.0 & 15.8 & 10.4 & 49 & 5.9 & 68 & 4.0 & 49 & 9.7 & 9.8 & 9.8 \\
\hline$A^{2}(p p o n)$ & $\bullet$ & • & • & • & • & - & - & - & • & - & - \\
\hline$a$ & 81 & 53 & 61 & 49 & 58 & 85 & 43 & 37 & 37 & 43 & 41 \\
\hline $\mathbf{P b}_{0}$ & 1400 & 1600 & 1600 & 1400 & 1200 & 1700 & 1800 & 1200 & 1100 & 1500 & 1500 \\
\hline St & 1300 & 2200 & 2100 & 1900 & 1800 & 1800 & 1500 & 2200 & 2100 & 2000 & 2200 \\
\hline $2 n$ & 1000 & 1000 & 950 & 810 & 800 & 900 & 1100 & 700 & 870 & 950 & 1000 \\
\hline Sampled & Top & Bulk & Bulk & Bulk & Bulk & Balk & Top & Bulk & Bulk & Bottom & Bottom \\
\hline Interval & - & - & - & - &. & $0-15 \mathrm{~cm}$ & - &. & - & 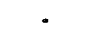 & - \\
\hline
\end{tabular}

\begin{tabular}{|c|c|c|c|c|c|c|c|c|c|c|c|}
\hline $\begin{array}{l}\text { Sequencoitl } \\
\text { Letitude }\end{array}$ & $\begin{array}{c}017016 \\
8.308\end{array}$ & $\begin{array}{c}017016 \\
8.308\end{array}$ & $\begin{array}{c}017019 \\
8.194\end{array}$ & $\begin{array}{c}017020 \\
8.194\end{array}$ & $\begin{array}{c}017021 \\
8.186\end{array}$ & $\begin{array}{c}017030 \\
9.081\end{array}$ & $\begin{array}{c}017035 \\
9.148\end{array}$ & $\begin{array}{c}017036 \\
9.148\end{array}$ & $\begin{array}{c}017037 \\
9.148\end{array}$ & $\begin{array}{c}017038 \\
9.148\end{array}$ & $\begin{array}{c}017041 \\
9.142\end{array}$ \\
\hline Longitudo & -164.346 & -164346 & -164.200 & -164.200 & -164.202 & -164.727 & -164.808 & -164808 & .164 .808 & -164.808 & -164.784 \\
\hline Depth & 1400 & 1400 & 1600 & 1600 & 1800 & 4301 & 2600 & 2600 & 2600 & 2600 & 2465 \\
\hline Lebill & 48 & 48 & 50 & 51 & 52 & MP1-CKB11 & MP1-CKB18 & MP1-CKB19 & MP1-CKB20 & MP1-CKB21 & MP1-CKB24 \\
\hline Si(wtW) & .98 & 1.08 & 1.66 & 1.26 & 3.60 & 4.93 & 256 & 1.59 & 1.01 & 3.58 & - \\
\hline $\mathbf{T}$ & .79 & .76 & 88 & 1.02 & 1.27 & 98 & .77 & .65 & .56 & 1.07 & - \\
\hline Mm & 328 & 314 & 30.6 & 20.7 & 245 & 23.5 & 25.8 & 28.1 & 20.1 & 23.7 & 33.2 \\
\hline Fo & 12.4 & 12.1 & 14.7 & 16.5 & 15.0 & 15.0 & 143 & 13.2 & 9.60 & 145 & 10.7 \\
\hline $\mathbf{N}$ & 36 & .23 & 42 & 33 & 1.22 & 1.21 & .32 & 25 & .12 & .66 & - \\
\hline Co & 1.87 & 1.82 & 1.45 & .872 & 1.26 & .380 & .920 & 1.13 & 530 & .780 & 1.62 \\
\hline $\mathbf{N i}$ & .750 & .728 & 498 & A95 & .535 & .500 & $A 60$ & 560 & 360 & 380 & .770 \\
\hline ca & .021 & .022 & .047 & .063 & .038 & .390 & .050 & .060 & .070 & .050 & .040 \\
\hline ca & 283 & 268 & 281 & 2.81 & 299 & 290 & 3.40 & 2.62 & 9.50 & 3.46 & . \\
\hline Ms & 1.36 & 1.35 & 1.23 & 1.11 & 1.25 & 90 & .90 & .76 & .68 & 86 & - \\
\hline $\mathbf{P}$ & 41 & 37 & .44 & 42 & 52 & .14 & .03 & .14 & 2.05 & 31 & - \\
\hline $\mathrm{H}_{2} \mathrm{O}$ & 9.3 & 9.3 & 9.7 & 7.1 & 10.3 & $\cdot$ & - & • & - & - & - \\
\hline Ax(ppon) & - & - & - & - & - & - & - & - & - & - & - \\
\hline a & 31 & 58 & 37 & 45 & 79 & 29 & 40 & 18 & 20 & 48 & - \\
\hline$P_{0}$ & 1400 & 1800 & 1200 & 1100 & 1500 & 740 & 900 & 1000 & 1100 & 1300 & - \\
\hline Sr & 1800 & 1800 & 2200 & 2300 & 1700 & • & • & • & - & - & - \\
\hline $\mathbf{Z n}$ & 820 & 760 & 740 & 1000 & 760 & 700 & - & 700 & 800 & - & - \\
\hline Sampled & Top & Top & Top & Bulk-minus & Bulk & Bulk & Bulk & Bulk & Old & Young & Bulk \\
\hline Interval & $=$ & - & - & Top & • & • & - & - & - & - & - \\
\hline
\end{tabular}


Table 4. Composition of ferromanganese crusts analyzed by TUCLZ (Halbach et al.) and German Geological Survey (Marchig et al.) (cont'd).

\begin{tabular}{|c|c|c|c|c|c|c|c|c|c|c|c|c|}
\hline $\begin{array}{l}\text { Sequencell } \\
\text { Letitude }\end{array}$ & $\begin{array}{c}017042 \\
9.142\end{array}$ & $\begin{array}{c}017043 \\
9.142\end{array}$ & $\begin{array}{c}017105 \\
9.081\end{array}$ & $\begin{array}{c}017106 \\
9.081\end{array}$ & $\begin{array}{c}017107 \\
9.081\end{array}$ & $\begin{array}{c}017108 \\
9.081\end{array}$ & $\begin{array}{c}017109 \\
9.081\end{array}$ & $\begin{array}{c}017110 \\
9.081\end{array}$ & $\begin{array}{c}017111 \\
9.081\end{array}$ & $\begin{array}{c}017112 \\
9.148\end{array}$ & $\begin{array}{c}017113 \\
9.148\end{array}$ & $\begin{array}{c}017114 \\
9.148\end{array}$ \\
\hline $\operatorname{si}(w t . \%)$ & .71 & .23 & 248 & 269 & 15.4 & 2.59 & 3.02 & 231 & 276 & 1.24 & 213 & 1.54 \\
\hline $\mathrm{Ti}$ & $A 3$ & .12 & • & $\cdot$ & - & - & • & • & - & • & • & • \\
\hline $\mathbf{N}$ & .18 & .11 & .48 & .62 & 5.29 & 52 & .64 & $s 1$ & .57 & .22 & .45 & 27 \\
\hline Co & 1.18 & .790 & .540 & .580 & .120 & .550 & .580 & .610 & .610 & 1.25 & 1.12 & 1.26 \\
\hline $\mathbf{N i}$ & .710 & .780 & .380 & .410 & .370 & .390 & .380 & .410 & 380 & .750 & .670 & .650 \\
\hline Cu & .050 & .040 & .091 & .110 & .360 & .094 & .087 & .089 & .086 & .044 & .056 & .072 \\
\hline Ca & 3.89 & 9.40 & 1.64 & 259 & 2.94 & 1.91 & 1.85 & 259 & 284 & 1.76 & 3.67 & 203 \\
\hline $\mathbf{M} \mathbf{g}$ & .92 & 1.06 & .87 & 99 & 1.63 & 89 & 93 & 1.00 & 1.02 & 1.04 & 1.04 & 1.06 \\
\hline $\mathbf{P b}$ & 1500 & 1700 & 960 & 1100 & 300 & 960 & 980 & 1100 & 1100 & 1300 & 860 & 1200 \\
\hline St & • & • & 1500 & 1400 & 200 & 1400 & 1400 & 1400 & 1400 & 980 & 1500 & 1500 \\
\hline $\mathbf{2 n}$ & 700 & 1200 & 520 & 740 & 610 & 560 & 540 & 610 & 640 & 910 & 1700 & 810 \\
\hline Sarmpled & Young & Old & Bulk & Bulk & Bulk & Bulk & Bulk & Bulk & Bulk & Bulk & Bulk & Bulk \\
\hline Interval & - & • & 13.0 & 13.0 & - & 15.0 & 120 & 12.0 & 17.0 & 25.0 & 25.0 & 60.0 \\
\hline
\end{tabular}

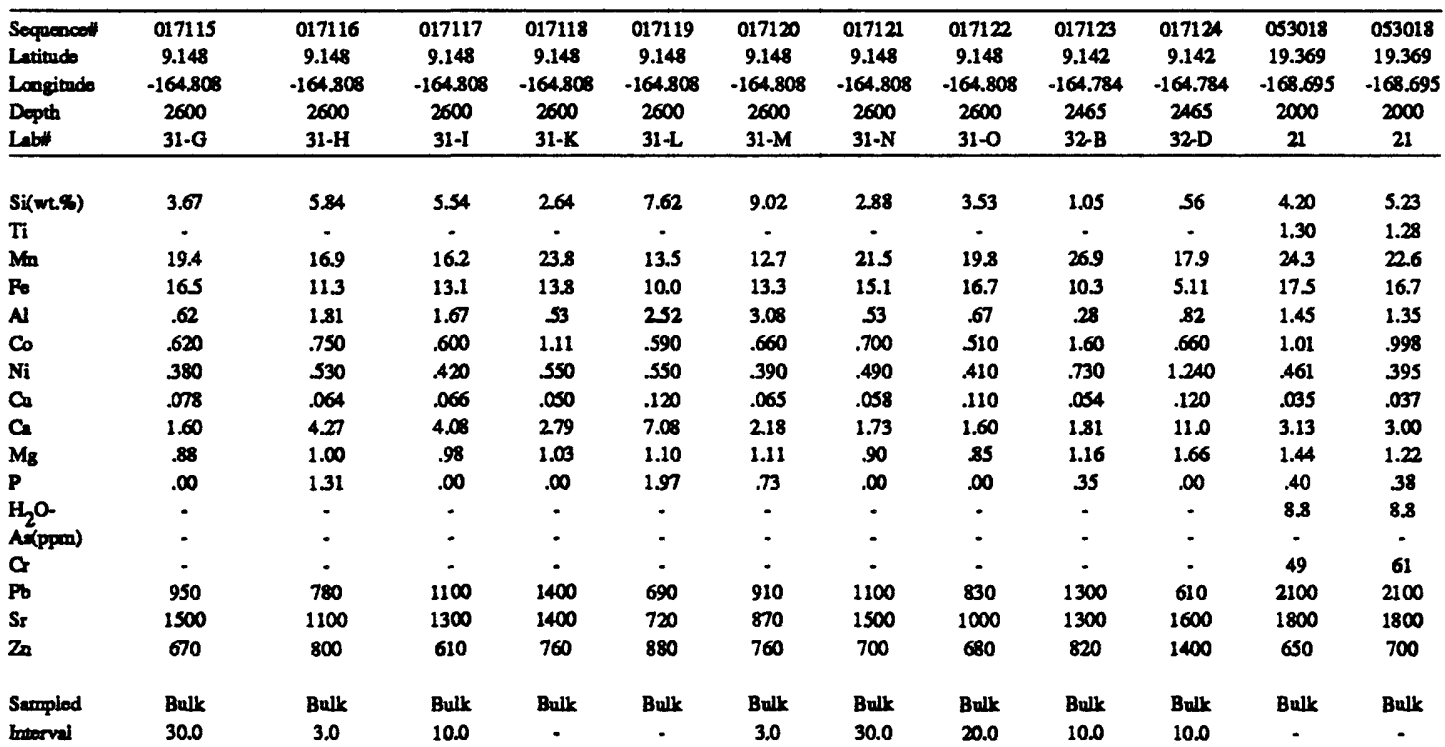


Table 4. Composition of ferromanganese crusts analyzed by TUCLZ (Halbach et al.) and German Geological Survey (Marchig et al.) (cont'd).

\begin{tabular}{|c|c|c|c|c|c|c|c|c|c|c|c|}
\hline Sequenooll & 053019 & 053020 & 053021 & 053023 & 053024 & 053026 & 053027 & 053027 & 053028 & 053020 & 053030 \\
\hline Leatitude & 19.369 & 19511 & 19511 & 19511 & 19.511 & 19.695 & 19.553 & 19553 & 19553 & 19.515 & 19515 \\
\hline Longitude & -168.695 & -168.835 & -168.835 & -168.835 & -168.835 & -168.995 & -168.832 & -168.832 & -168.832 & -168.855 & -168.855 \\
\hline Depth & 2000 & 1800 & 1800 & 1800 & 1800 & 4500 & 2400 & 2400 & 2400 & 1790 & 1790 \\
\hline Leb* & 2 & 23 & 24 & 26 & 27 & 29 & 30 & 30 & 31 & 32 & 33 \\
\hline Sx(wtS) & 4.32 & 4.70 & 1.36 & 4.66 & 1.46 & 8.61 & 3.99 & 452 & 5.10 & 2.26 & 2.71 \\
\hline $\mathbf{T}$ & 1.05 & 1.11 & 1.01 & 1.16 & 1.09 & 1.63 & 1.15 & 1.15 & 1.23 & .92 & 1.00 \\
\hline $\mathbf{M n}$ & 21.2 & 23.1 & 323 & 225 & 29.5 & 15.8 & 24.2 & 228 & 22.3 & 25.7 & 25.2 \\
\hline$P_{0}$ & 17.4 & 18.6 & 11.1 & 17.6 & 12.7 & 19.2 & 18.1 & 19.0 & 18.5 & 15.3 & 148 \\
\hline Al & 1.09 & 1.12 & .27 & .11 & 27 & 270 & 87 & 1.17 & 1.34 & 57 & .53 \\
\hline Co & .744 & 849 & .798 & 847 & .762 & 331 & .820 & .798 & .703 & .690 & .657 \\
\hline $\mathbf{M i}$ & .383 & 407 & .641 & .423 & 549 & .437 & .472 & .416 & .434 & .437 & 449 \\
\hline Cu & .050 & .061 & .150 & .044 & .068 & .230 & .094 & .090 & .130 & .094 & .110 \\
\hline Ca & 3.60 & 234 & 5.07 & 2.34 & 4.62 & 1.81 & 238 & 269 & -2.44 & 5.15 & 5.51 \\
\hline $\mathbf{M} 8$ & 1.08 & 1.00 & 1.14 & 1.07 & 1.06 & 1.31 & 1.12 & 1.15 & 1.16 & 1.03 & 1.03 \\
\hline $\mathbf{P}$ & $A 4$ & $A 1$ & 1.03 & 34 & 86 & 26 & .40 & .41 & $A 0$ & 123 & 1.33 \\
\hline $\mathrm{H}_{2} \mathrm{O}$ & 11.3 & 16.4 & 17.3 & 19.7 & 19.9 & 15.3 & 11.0 & 11.0 & 14.7 & 13.0 & 8.7 \\
\hline As(ppos) & - & - & - & - & . & - & . & - & - & - & $\cdot$ \\
\hline a & 53 & so & 24 & 56 & 25 & 122 & 47 & 47 & 52 & 43 & 43 \\
\hline Pb & 2000 & 1800 & 1700 & 2000 & 2000 & 1700 & 2100 & 1500 & 1700 & 1700 & 1700 \\
\hline Sx & 1700 & 1900 & 2200 & 1800 & 2200 & 1200 & 1900 & 1800 & 1800 & 2100 & 2000 \\
\hline $2 n$ & 590 & 710 & 960 & 670 & 860 & 860 & 750 & 740 & 820 & 840 & 920 \\
\hline Samplod & Bulk & Top & Bottom & Top & Bottom & Top & Bulk & Bulk & Bulk & Bulk & Top \\
\hline berval & - & $\because$ & - & 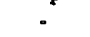 & - & 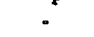 &. &. & - & - & 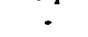 \\
\hline
\end{tabular}

\begin{tabular}{|c|c|c|c|c|c|c|c|c|c|c|c|}
\hline Sequencoll & 053031 & 053032 & 053033 & 053033 & 053034 & 053034 & 053037 & 053043 & 053044 & 053046 & 053047 \\
\hline Letitude & 19.515 & 19515 & 19315 & 19.515 & 19.515 & 19.515 & 13.115 & 13.001 & 12987 & 13.151 & 13.200 \\
\hline Langitude & -168.855 & -168.855 & -168.855 & -168.855 & -168.855 & -168.855 & -165.552 & -164.968 & -164.958 & -165485 & -165.462 \\
\hline Depth & 1790 & 1790 & 1790 & 1790 & 1790 & 1790 & 4774 & 3040 & 2055 & 4150 & 1508 \\
\hline Leb" & 34 & 35 & 36 & 36 & 37 & 37 & MP1-CKB28 & MP1-CKB34 & MP1-CKB35 & MP1-CKB37 & MP1-CKB38 \\
\hline$S i(w t . \%)$ & 4.07 & 151 & 4.03 & 4.20 & 1.20 & 1.47 & 11.1 & 3.67 & 289 & 280 & 1.58 \\
\hline $\mathbf{T I}$ & 1.04 & .89 & 1.03 & 1.15 & 87 & 86 & 1.38 & 98 & 35 & .31 & 99 \\
\hline Mn & 22.9 & 27.5 & 22.0 & 21.1 & 28.3 & 285 & 13.0 & 24.1 & 27.4 & 18.9 & 30.7 \\
\hline$F_{0}$ & 18.1 & 13.5 & 17.5 & 18.3 & 14.1 & 13.7 & 123 & 14.8 & 14.6 & 14.3 & 122 \\
\hline $\mathbf{A}$ & 85 & 39 & 86 & 1.12 & .43 & .43 & 298 & .76 & 56 & .38 & .28 \\
\hline Co & .717 & .669 & .836 & 836 & 547 & 547 & 310 & .030 & .840 & .620 & 1.21 \\
\hline $\mathrm{Ni}$ & .435 & $A 91$ & .424 & .412 & .419 & .488 & 200 & $A 80$ & 530 & .270 & .620 \\
\hline$C$ & .064 & .130 & .047 & .047 & .070 & .068 & .110 & .150 & .060 & .100 & .050 \\
\hline Ca & 2.44 & 5.98 & 227 & 2.31 & 5.64 & 5.72 & 2.22 & 3.65 & 3.25 & 2.84 & 386 \\
\hline M8 & 1.07 & 1.06 & 1.02 & 1.05 & .99 & 99 & 91 & 91 & 91 & .71 & 1.00 \\
\hline $\mathbf{P}$ & .48 & 1.45 & .40 & 38 & 1.31 & 1.35 & .19 & .23 & 25 & .22 & .27 \\
\hline $\mathrm{H}_{2} \mathrm{O}$ & 14.9 & 10.3 & 19.9 & 19.9 & 14.0 & 14.0 & - & - & . & $\cdot$ & - \\
\hline$A^{2}(p p m)$ & 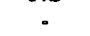 & 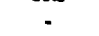 & 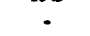 & . & 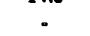 & - & - & - & - & - & - \\
\hline$a$ & 44 & 31 & 59 & 56 & 43 & 52 & 129 & 29 & 22 & 26 & 22 \\
\hline $\mathbf{P b}$ & 1800 & 1300 & 1900 & 1900 & 1500 & 1900 & 760 & 710 & 510 & 1000 & 1600 \\
\hline sr & 1900 & 2100 & 1800 & 1600 & 2300 & 2400 & - & 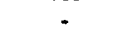 & . & $\cdot$ & - \\
\hline $\mathbf{z n}$ & 790 & 890 & 660 & 670 & 830 & 830 & 500 & 800 & 700 & - & 700 \\
\hline Sampled & Top & Bottom & Bulk & Bulk & Bulk & Bulk & Bulk & Young & Youns & Bulk & Bulk \\
\hline Intorval & $\bullet$ & • & • & - & - & - & - & - & . & . & . \\
\hline
\end{tabular}


Table 4. Composition of ferromanganese crusts analyzed by TUCLZ (Halbach et al.) and German Geological Survey (Marchig et al.) (cont'd).

\begin{tabular}{|c|c|c|c|c|c|c|c|c|c|c|c|c|}
\hline Sequenos" & 053048 & 053049 & 053141 & 053142 & 053143 & 053144 & 053145 & 053146 & 053147 & 053148 & 053149 & 053150 \\
\hline Latimde & 13.200 & 13.200 & 13.001 & 13.001 & 13.001 & 13.001 & 13.001 & 13.001 & 13.001 & 12987 & 12987 & 12987 \\
\hline Longitude & $-165,462$ & -165.462 & -164.968 & -164.968 & -164.968 & -164.968 & -164.968 & -164.968 & -164.968 & -164.958 & -164958 & -164.958 \\
\hline Depth & 1508 & 1508 & 3040 & 3040 & 3040 & 3040 & 3040 & 3040 & 3040 & 2055 & 2055 & 2055 \\
\hline Lab" & MP1-CKB39 & MP1-CKB40 & 43-A & 43-B1 & 43-B2 & 43-C & 43-D & 43-B & 43-P & 44-A & 44B & 44-B \\
\hline $\operatorname{si}(w, \%)$ & 99 & 210 & 3.72 & 3.67 & 1.17 & 4.84 & 8.09 & 4.16 & 3.23 & 7.92 & 6.22 & 5.19 \\
\hline $\mathrm{Ti}$ & .58 & .74 & - & - & - & • & - & $\cdot$ & - & - & • & • \\
\hline Mn & 30.0 & 221 & 18.4 & 19.1 & 20.8 & 18.3 & 126 & $17 A$ & 20.3 & 3.56 & 17.6 & 3.19 \\
\hline $\mathbf{P o}_{0}$ & 11.5 & 8.00 & 15.6 & $15 A$ & 15.1 & 13.4 & 14.9 & $15 A$ & 15.3 & 5.50 & 120 & 33.7 \\
\hline A & .15 & .44 & .72 & 85 & .69 & 1.39 & 256 & 1.03 & 49 & .72 & 206 & .62 \\
\hline$C_{0}$ & 1.14 & .710 & .600 & .590 & .740 & .620 & .480 & .620 & .780 & .070 & 560 & .110 \\
\hline $\mathbf{N i}$ & 570 & 560 & 350 & .460 & .510 & .570 & .250 & 350 & $A 10$ & .160 & .630 & .090 \\
\hline $\mathrm{Ca}$ & .050 & .050 & .056 &.$\infty 1$ & .105 & .190 & .046 & .060 & .063 & .030 & .130 & .056 \\
\hline$a$ & 4.19 & 10.00 & 240 & 1.64 & 1.62 & 262 & 298 & 260 & 1.64 & 23.3 & 4.73 & 7.46 \\
\hline $\mathbf{M g}$ & .82 & .88 & .86 & 86 & 90 & .91 & 96 & .64 & 88 & .64 & 1.18 & .64 \\
\hline $\mathbf{P}$ & .38 & 2.64 & .40 &.$\infty$ & 28 & $A 3$ & .89 &.$\infty$ & 33 & 7.02 & 1.44 &.$\infty$ \\
\hline $\mathrm{H}_{2} \mathrm{O}$ & • & • & • & $\cdot$ & - & • & • & - & • & - & • & - \\
\hline$A^{2}(\mathrm{ppm})$ & - & - & - & 83 & 74 & - & - & 137 & - & 40 & - & 159 \\
\hline$a$ & 15 & 55 & • & - & $\cdot$ & • & • & - & • & - & • & - \\
\hline Po & 1200 & 1000 & 1100 & 700 & 910 & 430 & 780 & 920 & 980 & 570 & 850 & 280 \\
\hline Sr & . & - & 1500 & 950 & 1400 & 920 & 730 & 1400 & 1500 & 1300 & 920 & 550 \\
\hline $\mathbf{z n}$ & 600 & 800 & 760 & 640 & 620 & 820 & 510 & 660 & 630 & 390 & 1100 & 810 \\
\hline Sampled & Youns & Old & Bulk & Bulk & Bulk & Bulk & Bulk & Bulk & Bulk & Bulk & Bulk & Bulk \\
\hline heovial & - & - & 20.0 & 50.0 & 50.0 & - & 5.0 & 20.0 & - & - & 30.0 & • \\
\hline
\end{tabular}

\begin{tabular}{|c|c|c|c|c|c|c|c|c|c|c|c|c|}
\hline Seqpencoll & 053151 & 053152 & 053153 & 053154 & 053155 & 053156 & 053157 & 053158 & 053159 & 053160 & 053161 & 053162 \\
\hline Letionds & 12987 & 13.151 & 13.151 & 13.151 & 13.200 & 13.200 & 13200 & 13.200 & 13.200 & 13200 & 13.200 & 13.200 \\
\hline Longitude & -164.958 & -165.485 & -165.485 & $-165 A 85$ & -165.462 & -165.462 & -165.462 & -165.462 & -165.462 & -165.462 & -165462 & -165.462 \\
\hline Depth & 2055 & 4150 & 4150 & 4150 & 1508 & 1508 & 1508 & 1508 & 1508 & 1508 & 1508 & 1508 \\
\hline Lebil & 441 & 57-A & 57-C & 57-D & 58-A1 & 58-A2 & 58-B & 58-C & 58-D & 58-E1 & 58-P & 58-G \\
\hline Sil(wt.\%) & 6.73 & 3.55 & 9.68 & 8.02 & 1.57 & 1.82 & 1.80 & 3.34 & 1.31 & 4.96 & 1.87 & 3.23 \\
\hline $\mathrm{Ti}$ & - & - & - & - & - & - & - & - & - & - & - & - \\
\hline $\mathbf{M n}$ & 14.6 & 19.6 & 10.4 & 144 & 24.3 & 23.9 & 27.6 & 22.5 & 24.6 & 16.9 & 25.3 & 22.4 \\
\hline$P_{0}$ & 15.2 & 17.6 & 14.1 & 15.7 & 126 & 129 & 13.3 & 10.7 & 12.3 & 11.1 & 12.8 & 12.4 \\
\hline $\mathbf{A}$ & 199 & 55 & 3.77 & 271 & 26 & 31 & 33 & .10 & 27 & 1.50 & 55 & .87 \\
\hline Co & .750 & 580 & 340 & .400 & 1.34 & 1.40 & 1.42 & 1.30 & 1.44 & 970 & 1.30 & 1.26 \\
\hline $\mathrm{Ni}$ & 310 & 300 & 200 & .250 & .530 & 530 & .590 & .530 & 540 & .400 & 500 & 550 \\
\hline$c_{0}$ & .032 & 210 & .054 & .170 & .027 & .026 & .036 & .040 & .038 & .049 & .036 & .037 \\
\hline C. & 200 & 2.65 & 2.26 & 1.93 & 1.73 & 1.85 & 3.04 & 3.78 & 1.94 & 6.06 & 243 & 1.93 \\
\hline $\mathbf{M g}$ & .97 & .93 & 85 & .84 & 91 & 98 & 1.15 & 1.12 & 1.02 & 99 & 1.03 & 1.09 \\
\hline $\mathbf{P}$ & .68 & .43 & .50 & $A 8$ & $A 2$ & .0 & 46 &.$\infty$ & .44 &.$\infty$ & 51 & 54 \\
\hline $\mathrm{H}_{2} \mathrm{O}$ & $\cdot$ & - & - & - & $\cdot$ & $\cdot$ & - & $\cdot$ & $\cdot$ & - & $\cdot$ & $\cdot$ \\
\hline$A^{2}(\mathrm{ppm})$ & - & 135 & 80 & 97 & - & - & - & - & 179 & - & 102 & 118 \\
\hline$a$ & $\cdot$ & - & • & - & - & - & - & - & - & • & - & - \\
\hline Po & 400 & 1200 & 870 & 810 & 1300 & 660 & 1400 & 1300 & 1500 & 1300 & 1300 & 1300 \\
\hline Sz & 930 & 1400 & 990 & 1100 & 1600 & 1400 & 1600 & 1300 & 1000 & 850 & 1600 & 1300 \\
\hline $\mathbf{Z n}$ & 610 & 650 & 610 & 700 & 560 & 620 & 700 & 950 & 630 & 540 & 610 & 630 \\
\hline Sampled & Bulk & Bulk & Bulk & Bulk & Bulk & Bulk & Bulk & Bulk & Bulk & Bulk & Bulk & Bulk \\
\hline lotocival & 1.0 & 2.0 & 3.0 & 5.0 & 30.0 & 30.0 & 50.0 & 3.0 & 8.0 & 20 & 7.0 & 5.0 \\
\hline
\end{tabular}


Table 4. Composition of ferromanganese crusts analyzed by TUCLZ (Halbach et al.) and German Geological Survey (Marchig et al.) (cont'd).

\begin{tabular}{|c|c|c|c|c|c|c|c|c|c|c|c|}
\hline Sequence: & 053163 & 054003 & 054005 & 054006 & 054007 & 054008 & 054012 & 054013 & 054014 & 054050 & 054051 \\
\hline Letimds & 13.200 & $19 A 02$ & 19.361 & 19.373 & 19.373 & 19.373 & 19.408 & $19 A 08$ & 19.408 & 19.402 & $19 A 02$ \\
\hline Langituds & -165.462 & -171.173 & -171.064 & -170.991 & -170.991 & -170.991 & -171.391 & -171.391 & -171.391 & -171.173 & -171.173 \\
\hline Depth & 1508 & 2856 & 2010 & 1190 & 1190 & 1190 & 1925 & 1925 & 1925 & 2856 & 2856 \\
\hline Labil & $58-X$ & MP1-CKB42 & MP1-CKBAS & MP1-CKB46 & MP1-CKBA7 & MP1-CKB48 & MP1-CKBS2 & MP1-CKB53 & MP1-CKBS4 & 73-A & 73-B \\
\hline Si(wt.\%) & 1 AS & 6.40 & 3.72 & 2.19 & 1.55 & 1.64 & 1.13 & - & - & 11.1 & 9.58 \\
\hline TI & $\cdot$ & 1.16 & 1.06 & 89 & 1.02 & .71 & $A 7$ & • & • & • & - \\
\hline Mn & 26.0 & 19.0 & 23.4 & 20.0 & 21.6 & 20.0 & 24.2 & 21.8 & 18.4 & 11.7 & 128 \\
\hline Po & 11.1 & 16.6 & 14.6 & 15.5 & 7.90 & 15.6 & 11.1 & 13.7 & 10.9 & 13.2 & 14.9 \\
\hline $\mathbf{N}$ & 27 & 1.10 & .66 & 33 & 31 & 25 & 22 & - & - & 295 & 2.35 \\
\hline Co & 1.33 & .560 & 830 & 1.02 & 500 & 1.38 & .450 & 550 & sso & 380 & .400 \\
\hline $\mathbf{N i}$ & .700 & .130 & 380 & $\$ 40$ & .620 & 510 & .390 & 510 & 380 & 250 & .250 \\
\hline a & .048 & .120 & .090 & .040 & .110 & .030 & .060 & .150 & .110 & .000 & .130 \\
\hline $\mathrm{Ce}$ & 1.79 & 3.26 & 3.65 & 3.77 & 9.51 & 439 & 9.20 & - & - & 1.19 & 1.22 \\
\hline Mg & 1.09 & 92 & .96 & 97 & 1.02 & 1.07 & .76 & - & - & 1.45 & 1.37 \\
\hline$P$ & 32 & 31 & .26 & 34 & 208 & 35 & 1.29 & - & - & .36 & $A 0$ \\
\hline $\mathrm{H}_{2} \mathrm{O}$ & - & - & - & - & - & • & • & - & - & - & - \\
\hline$A$ Axppon) & $\cdot$ & - & - & $\cdot$ & - & $\cdot$ & - & - & $\cdot$ & - & - \\
\hline$a$ & $\cdot$ & 41 & 38 & 22 & 25 & 27 & 16 & - & - & - & • \\
\hline Po & 1300 & 1500 & 1600 & 1900 & 1800 & 2000 & 2300 & - & - & 1200 & 1000 \\
\hline Sr & 1500 & - & • & • & - & • & - & - & $\cdot$ & 600 & 680 \\
\hline $\mathrm{Zn}$ & 720 & - & - & 500 & 900 & 300 & 700 & $\cdot$ & - & 550 & 580 \\
\hline Semplod & Bulk & Bulk & Youns & Bulk & Old & Young & Old & Bulk & Bulk & Bulk & Bulk \\
\hline Interval & • & - & - & • & - & - & $\cdot$ & • & - & 3.0 & 3.0 \\
\hline
\end{tabular}

\begin{tabular}{|c|c|c|c|c|c|c|c|c|c|c|c|}
\hline Sequence: & 054052 & 054053 & 054054 & 054055 & 054056 & 054057 & 054058 & 054059 & 054060 & 054061 & 054062 \\
\hline Lationds & 19.402 & 19.402 & 19.402 & 19.402 & 19.402 & 19.361 & 19.361 & 19.361 & 19.361 & 19.361 & 19.361 \\
\hline Longitude & -171.173 & -171.173 & -171.173 & -171.173 & -171.173 & -171.064 & -171.064 & -171.064 & -171.064 & -171.064 & -171.064 \\
\hline Depth & 2856 & 2856 & 2856 & 2856 & 2856 & 2010 & 2010 & 2010 & 2010 & 2010 & 2010 \\
\hline Lab* & 73-C & 73-B & 73-P & $73-G$ & 73-H & 75-A & 75-B & $75-C$ & 75-D & 75-B & $75-G$ \\
\hline$S i(w t . \%)$ & 5.03 & 788 & 9.72 & 7.62 & 8.46 & 358 & 4.75 & 4.58 & 3.97 & 3.A1 & 5.12 \\
\hline $\mathbf{T}$ & • & - & - & - & - & - & • & - & $\dot{ }$ & • & - \\
\hline Mn & 19.6 & 127 & 120 & 15.6 & 14.2 & 21.1 & 16.7 & 18.1 & 18.9 & $21 . A$ & 18.1 \\
\hline Fo & 16.7 & 15.8 & 15.6 & 16.1 & 15.6 & 163 & 16.0 & 175 & 164 & 16.1 & 17.1 \\
\hline $\boldsymbol{N}$ & .10 & 2.45 & 2.40 & 20 & 237 & .64 & 1.03 & .48 & .69 & .59 & 1.27 \\
\hline Co & .700 & .490 & 380 & 480 & 490 & 850 & .580 & .690 & .710 & .840 & .540 \\
\hline $\mathbf{N I}$ & 350 & .300 & .260 & $A 30$ & 280 & 400 & .300 & 350 & .350 & .400 & .330 \\
\hline C & .083 & .140 & .094 & .104 & .120 & .054 & .047 & .057 & .059 & .048 & .093 \\
\hline Ce & 2.62 & 1.98 & 1.04 & 220 & 1.81 & 269 & 240 & 1.57 & 1.83 & 266 & 2.56 \\
\hline $\mathrm{Mg}$ & 1.17 & 1.21 & 1.46 & 1.10 & 1.37 & 1.02 & 85 & .90 & .89 & $1 . \infty$ & 91 \\
\hline $\mathbf{P}$ & .00 & 47 & .39 & so & .40 & .49 &.$\infty$ & 52 & .47 & .38 & .46 \\
\hline $\mathrm{H}_{2} \mathrm{O}$ & $\cdot$ & - & $\cdot$ & • & - & $\cdot$ & - & $\cdot$ & $\cdot$ & $\cdot$ & $\cdot$ \\
\hline A (ppon) & 103 & $\cdot$ & - & - & - & - & - & - & - & - & 73 \\
\hline$c$ & • & - & - & • & - & - & - & • & • & - & - \\
\hline $\mathbf{P b}$ & 1500 & 1300 & 1300 & 1300 & 1200 & 3400 & 1400 & 1500 & 1400 & 1300 & 1500 \\
\hline Sr & 1300 & 1200 & 650 & 1100 & 1200 & 1500 & 1500 & 1400 & 1500 & 1500 & 1500 \\
\hline $2 n$ & 650 & 650 & 630 & 860 & 660 & 710 & 570 & 700 & 590 & 650 & 720 \\
\hline Sampled & Bulk & Bulk & Bulk & Bulk & Bulk & Bulk & Bulk & Bulk & Bulk & Bulk & Bulk \\
\hline inorvi: & 5.0 & 5.0 & 20 & 5.0 & 20 & $\mathbf{2 0 . 0}$ & 1.0 & 30.0 & 2.0 & 2.0 & 5.0 \\
\hline
\end{tabular}


Table 4. Composition of ferromanganese crusts analyzed by TUCLZ (Halbach et al.) and German Geological Survey (Marchig et al.) (cont'd).

\begin{tabular}{|c|c|c|c|c|c|c|c|c|c|c|c|c|c|}
\hline Sequencell & 054063 & 054064 & 054065 & 054066 & 054057 & 054068 & 054069 & 054070 & 054071 & 054072 & 054073 & 054074 & 054075 \\
\hline Latitude & 19.373 & 19.373 & 19.373 & 19.373 & 19.373 & 19.373 & 19.373 & 19.408 & 19.408 & 19.408 & 19.408 & 19.408 & 19.408 \\
\hline Longitude & -170.991 & -170.991 & -170.991 & -170.991 & -170.991 & -170.991 & -170.991 & -171391 & -171391 & -171391 & -171391 & -171.391 & -171.391 \\
\hline Depth & 1190 & 1190 & 1190 & 1190 & 1190 & 1190 & 1190 & 1925 & 1925 & 1925 & 1925 & 1925 & 1925 \\
\hline Lab: & 76-A & 76-B & 76-D & 76-B & 76-P & $76-0$ & $76-\mathrm{H}$ & 78-A & 78-B & $78-\mathrm{C}$ & 78-D & 78-B & 78- $\mathrm{F}$ \\
\hline Six (wt.s) & 208 & 1.68 & 215 & 250 & 2.48 & 1.96 & 222 & $25 s$ & 3.06 & 2.52 & 3.83 & 5.14 & 5.26 \\
\hline Ti & 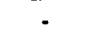 & - & - & 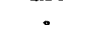 & . & . & . & - & . & 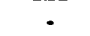 & - & - & - \\
\hline Mn & 24.3 & 23.6 & 225 & 213 & 213 & 22.3 & 219 & 22.1 & 209 & 303 & 2.05 & 173 & 15.9 \\
\hline Fe & 15.6 & 12.6 & 15.6 & 14.9 & 15.1 & 14.6 & 15.6 & 126 & 13.7 & 8.75 & 1.83 & 16.8 & 14.7 \\
\hline $\mathbf{A}$ & .04 & 35 & 37 & A5 & 43 & 31 & 38 & .72 & .86 & .70 & 151 & 1.45 & 1.31 \\
\hline Co & 1.10 & 133 & 1.02 & 970 & 820 & 950 & 930 & .680 & .740 & $\$ 60$ & .040 & .590 & .620 \\
\hline $\mathbf{N i}$ & .40 & 540 & 400 & 380 & 390 & $A 10$ & $A 10$ & 520 & 500 & 340 & .080 & .360 & .370 \\
\hline a & .031 & .039 & .031 & .036 & .040 & .037 & .036 & .180 & .094 & .150 & .040 & .075 & .074 \\
\hline Ca & 289 & 1.68 & 1.86 & 1.66 & 1.71 & 1.75 & 1.75 & 260 & 250 & 1.86 & 32.0 & 437 & 4.01 \\
\hline $\mathbf{M g}$ & 1.08 & 1.03 & .97 & 91 & 91 & .95 & 96 & .93 & .97 & 1.08 & .63 & 94 & 1.05 \\
\hline $\mathbf{P}$ & .44 & 51 &.$\infty$ & .00 & 57 & .00 & .00 & .30 &.$\infty$ &.$\infty$ & .00 & .72 & 1.37 \\
\hline $\mathrm{H}_{2} \mathrm{O}$ & - & - & - & - & • & - & - & • & - & • & - & - & - \\
\hline$A^{2}$ (ppon) & - & - & - & . & - & - & . & . & . & - & - & 81 & - \\
\hline$a$ & • & - & - & • & • & • & • & - & • & • & - & - & - \\
\hline $\mathbf{P b}$ & 3800 & 1600 & 1600 & 1500 & 1500 & 1500 & 1400 & 1300 & 1400 & 310 & 208 & 1200 & 1500 \\
\hline Sr & 1600 & 1500 & 1600 & 1600 & 1600 & 1700 & 1600 & 1600 & 1500 & 2300 & 1800 & 1500 & 840 \\
\hline $\mathbf{Z n}$ & 570 & 590 & 690 & 490 & 530 & 520 & 520 & 770 & 690 & 800 & 300 & 730 & 620 \\
\hline Sampled & Bulk & Bulk & Bulk & Bulk & Bulk & Bulk & Bulk & Bulk & Bulk & Bulk & Bulk & Bulk & Bulk \\
\hline letarval & 30.0 & 40.0 & 20.0 & 20.0 & 25.0 & 15.0 & 5.0 & 40.0 & - & 4.0 & 3.0 & 4.0 & 3.0 \\
\hline
\end{tabular}

\begin{tabular}{|c|c|c|c|c|c|c|c|c|c|c|c|c|c|}
\hline Sequence" & 054076 & 054077 & 054078 & 054079 & 054080 & 054081 & 089011 & 089013 & 089017 & 089018 & 089019 & 089020 & 089021 \\
\hline Lationde & 19.408 & 19.408 & 19.408 & 19.408 & 19.408 & 19.408 & 22.181 & 22208 & 21.718 & 21.718 & 21.718 & 21.718 & 21.796 \\
\hline Longitnde & -171.391 & -171.391 & -171.391 & -171.391 & -171.391 & -171.391 & -166.810 & -166.882 & -167.578 & -167.578 & -167.578 & -167.578 & -167.624 \\
\hline Depth & 1925 & 1925 & 1925 & 1925 & 1925 & 1925 & 3950 & 2402 & 2400 & 2400 & 2400 & 2400 & 2100 \\
\hline Leb: & $78-G$ & 78-H & $78-1$ & 78-L & $78-M$ & $78 \mathrm{~N}$ & 1 & 2 & 6 & 7 & 8 & 9 & 10 \\
\hline Si(wt.\%) & 278 & 6.19 & 4.56 & 288 & 3.06 & 128 & 6.88 & 6.87 & 5.50 & 5.38 & 7.10 & 3.91 & 3.74 \\
\hline $\mathbf{T i}$ & • & - & • & • & • & - & 1.20 & 1.49 & 1.42 & 1.27 & 1.16 & 1.64 & 1.87 \\
\hline Mn & 228 & 12.4 & 18.6 & 219 & 203 & 240 & 19.3 & 17.1 & 22.0 & 20.5 & 18.3 & 25.0 & 23.1 \\
\hline $\mathbf{F o}_{0}$ & 14.7 & 11.7 & 15.4 & 14.2 & 15.6 & 4.56 & 21.4 & 18.7 & 16.5 & 20.7 & 19.7 & 18.1 & 18.7 \\
\hline A & 39 & 1.61 & .12 & 38 & 50 & 6.00 & 1.88 & 2.36 & 1.53 & 1.41 & 1.69 & 97 & 85 \\
\hline Co & .710 & $A 20$ & .630 & .830 & .650 & .060 & 458 & .465 & 570 & 587 & .559 & 1.21 & .768 \\
\hline $\mathbf{N i}$ & .420 & 340 & 350 & 470 & 340 & 200 & 256 & .465 & .461 & 329 & .263 & .487 & .374 \\
\hline C. & .059 & .085 & .063 & .070 & .042 & .065 & .160 & .220 & .160 & .077 & .042 & .072 & .052 \\
\hline $\mathrm{Ca}$ & 2.74 & 6.94 & 245 & 1.97 & 1.85 & 17.7 & 245 & 2.49 & 2.53 & 2.45 & 245 & 3.02 & 2.49 \\
\hline $\mathrm{Mg}$ & .97 & .83 & 1.00 & .99 & 1.02 & .76 & 1.23 & 1.16 & 1.01 & 1.04 & 98 & 1.22 & 1.18 \\
\hline $\mathbf{P}$ &.$\infty$ & 3.03 & .00 &.$\infty$ &.$\infty$ & 5.49 & 34 & 34 & .28 & .39 & 49 & 37 & 31 \\
\hline $\mathrm{H}_{2} \mathrm{O}$ & • & • & • & - & - & - & 6.2 & 7.5 & 17.6 & 14.8 & 124 & 5.6 & 63 \\
\hline$A^{2}(p p m)$ & - & 50 & - & - & 133 & - & - & - & - & . & . & 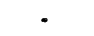 & - \\
\hline$a$ & • & . & - & - & . & - & 65 & 79 & 53 & 53 & 78 & 57 & 67 \\
\hline $\mathrm{Pb}$ & 1400 & 830 & 1800 & 1300 & 1300 & 300 & 1700 & 1800 & 1300 & 1200 & 1800 & 2300 & 2100 \\
\hline Sr & 1400 & 900 & 1300 & 1600 & 1600 & 1000 & 1600 & 1600 & 1800 & 1800 & 1600 & 1800 & 1800 \\
\hline $\mathrm{Zn}$ & 740 & 590 & 700 & 660 & 580 & 540 & 750 & 940 & 840 & 800 & 960 & 780 & 690 \\
\hline Semplod & Bulk & Bulk & Bulk & Bulk & Bulk & Bulk & Bulk & Bulk & Bottom & Top & Top & Top & Bulk \\
\hline Inourval & - & 3.0 & 3.0 & 3.0 & 30.0 & - & • & - & • & $\cdot$ & $\cdot$ & $<\mathrm{lmm}$ & • \\
\hline
\end{tabular}


Table 4. Composition of ferromanganese crusts analyzed by TUCLZ (Halbach et al.) and German Geological Survey (Marchig et al.) (cont'd).

\begin{tabular}{|c|c|c|c|c|c|c|c|c|c|c|c|c|}
\hline Sequencel" & 089022 & 089023 & 089025 & 089026 & 089027 & 089030 & 089031 & 090006 & 090007 & 090008 & 090009 & 090070 \\
\hline Letitude & 21.796 & 21.796 & 20.209 & 20.209 & 20.209 & 20.335 & 20.335 & 20.078 & 20.078 & 20.078 & 20.220 & 20.078 \\
\hline Longitude & -167.624 & -167.624 & .169 .506 & -169.506 & -169.506 & -169.575 & -169.575 & $\cdot 170.642$ & -170.642 & -170.642 & -170.568 & -170.642 \\
\hline Depth & 2100 & 2100 & 4500 & 4500 & 4500 & 2400 & 2400 & 1332 & 1332 & 1332 & 2573 & 1332 \\
\hline Labil & 11 & 12 & 13 & 14 & 15 & 18 & 19 & MP1-CKBS7 & MP1-CKBS8 & MP1-CKB59 & MP1-CKB60 & 111-B \\
\hline si(wt.s) & 640 & 6.36 & 7.68 & 10.9 & 9.30 & 5.42 & 3.62 & 1.77 & .75 & 250 & - & 3.06 \\
\hline $\mathbf{T}$ & 1.67 & 1.08 & 219 & 1.78 & 1.53 & 1.40 & 1.71 & .81 & .80 & .64 & - & - \\
\hline Mn & 19.1 & 17.9 & 15.0 & 11.7 & 14.7 & 21.8 & 22.6 & 268 & 24.6 & 27.0 & 24.0 & 20.3 \\
\hline Fe & 21.6 & 20.3 & 228 & 18.9 & 19.3 & 21.6 & 17.6 & 15.7 & 7.30 & 13.8 & 14.6 & 128 \\
\hline $\mathbf{N}$ & 158 & 1.52 & 256 & 3.93 & 3.81 & 1.05 & .91 & .25 & .25 & .18 & - & 92 \\
\hline Co & 506 & .563 & 330 & 252 & .358 & 552 & .926 & 1.10 & .750 & $\$ 40$ & 1.10 & 1.02 \\
\hline $\mathrm{Ni}$ & 334 & 258 & .192 & .168 & 246 & .293 & .293 & .440 & 820 & 460 & 350 & .560 \\
\hline$\infty$ & .087 & .045 & .120 & .150 & .160 & .073 & .079 & .020 & .180 & .040 & .070 & .057 \\
\hline ca & 253 & 220 & 2.11 & 2.11 & 2.16 & 207 & 2.70 & 3.10 & 9.80 & 4.30 & • & 4.20 \\
\hline Ms & 1.00 & 98 & 1.15 & 1.37 & 1.41 & 1.00 & 1.11 & .76 & 96 & 1.02 & - & 1.21 \\
\hline $\mathbf{P}$ & .48 & $A 2$ & .31 & .26 & .26 & 37 & 38 & 31 & 1.98 & $A 2$ & - & 1.44 \\
\hline $\mathrm{H}_{2} \mathrm{O}$ & 7.2 & 14.7 & 6.2 & 4.9 & 10.5 & 11.2 & 14.7 & - & - & - & - & - \\
\hline$A^{2}(p p m)$ & - & - & - & - & • & - & • & - & - & - & - & - \\
\hline$c$ & 56 & 65 & 90 & 112 & 151 & 53 & 52 & 15 & 17 & 27 & - & • \\
\hline Po & 1500 & 1700 & 1900 & 1400 & 1300 & 2000 & 1700 & 1700 & 1400 & 2000 & - & 1600 \\
\hline Sr & 1800 & 1700 & 1500 & 1100 & 1100 & 1900 & 1800 & - & - & - & - & 1500 \\
\hline $2 n$ & 820 & 690 & 710 & 570 & 700 & 790 & 750 & 700 & 1400 & - & - & 700 \\
\hline Sempled & Bulk & Top & Bulk & Top & Bottoon & Bulk & Bulk & Balk & Old & Young & Bulk & Bulk \\
\hline Intorval & - & $\cdot$ & - & - & - & - & - & • & - & - & - & 5.0 \\
\hline
\end{tabular}

\begin{tabular}{|c|c|c|c|c|c|c|c|c|c|c|c|}
\hline Soquencoll & 090071 & 090072 & 090073 & 090074 & 090075 & 090076 & 09007 & 090078 & 090079 & 090080 & 090081 \\
\hline Letitude & 20.078 & 20.078 & 20.078 & 20.078 & 20.078 & 20.220 & 20.220 & 20.220 & 20.220 & 20.220 & 20.220 \\
\hline Longitude & -170.642 & -170.642 & -170.642 & -170.642 & -170.642 & -170.568 & -170568 & $-170 \leq 68$ & -170.568 & -170.568 & -170.568 \\
\hline Depth & 1332 & 1332 & 1332 & 1332 & 1332 & 2573 & 2573 & 2573 & 2573 & 2573 & 2573 \\
\hline Lab: & $111-\mathrm{P}$ & $111-G$ & $111 \cdot \mathrm{HI}$ & $111 \cdot 12$ & $111-I$ & 113-A & 113-B & $113-\mathrm{C}$ & 113-D & 113-E & 113-F \\
\hline si(wt.S) & 299 & 2.24 & 245 & 96 & 2.17 & 220 & 7.69 & 4.49 & 5.40 & 5.49 & 17.1 \\
\hline $\mathrm{Ti}$ & - & - & - & - & - & - & - & - & - & - & - \\
\hline Mn & 20.4 & 223 & 21.7 & 23.6 & 17.1 & 20.6 & 13.3 & 165 & 15.5 & 17.4 & 3.27 \\
\hline $\mathbf{P B}_{\mathbf{B}}$ & 13.7 & 14.4 & 14.0 & 5.69 & 145 & 13.6 & 175 & 16.2 & 18.8 & 15.4 & 11.8 \\
\hline $\mathbf{A l}$ & .68 & A2 & .48 & .13 & .66 & 47 & 1.81 & 1.12 & 1.08 & .15 & 5.60 \\
\hline Co & 1.000 & 1.19 & 1.16 & .480 & 1.02 & 1.09 & 390 & 520 & 420 & 580 & .100 \\
\hline $\mathbf{N i}$ & .430 & 420 & .440 & 940 & 480 & 410 & .230 & .310 & .230 & .330 & .180 \\
\hline$\infty$ & .041 & .025 & .038 & .074 & .074 & .039 & .088 & .077 & .074 & .210 & .096 \\
\hline ca & 3.13 & 1.76 & 289 & 6.94 & 1.85 & 2.78 & 1.75 & 2.31 & 151 & 284 & 1.46 \\
\hline M8 & 1.14 & 97 & 1.02 & 1.01 & 1.02 & .90 & 1.08 & 92 & .85 & 1.02 & 1.62 \\
\hline $\mathbf{P}$ & 1.34 &.$\infty$ &.$\infty$ & .00 &.$\infty$ & .36 & .63 & .00 & .41 &.$\infty$ & .45 \\
\hline $\mathrm{H}_{2} \mathrm{O}$ & • & $\cdot$ & - & $\cdot$ & $\cdot$ & - & - & - & - & - & - \\
\hline$A^{2}(p p m)$ & - & 121 & - & - & 185 & - & - & - & 66 & - & - \\
\hline$a$ & - & - & - & - & - & - & - & • & - & • & • \\
\hline$P_{b}$ & 1600 & 1700 & 1600 & 1100 & 1500 & 1500 & 1200 & 1500 & 1400 & 1200 & 320 \\
\hline Sr & 1600 & 1600 & 1500 & 1600 & 1100 & 1500 & 760 & 1500 & 880 & 1200 & 310 \\
\hline $\mathbf{Z n}$ & 530 & 700 & 550 & 1100 & 630 & 600 & 580 & 600 & 600 & 740 & 430 \\
\hline Samplod & Bulk & Bulk & Bulk & Bulk & Bulk & Bulk & Bulk & Bulk & Bulk & Bulk & Bulk \\
\hline Inervel & 20.0 & 5.0 & 20 & 2.0 & - & 7.0 & 5.0 & .5 & 10.0 & 5.0 & 2 \\
\hline
\end{tabular}


Table 5. Composition of ferromanganese crusts analyzed onboard SP Lee L5-83HW and L5-84CP by Portaspec XRF

\begin{tabular}{|c|c|c|c|c|c|c|c|c|c|c|c|c|c|}
\hline $\begin{array}{l}\text { Sequencoll } \\
\text { Latitude }\end{array}$ & $\begin{array}{c}017006 \\
8.414\end{array}$ & $\begin{array}{c}017007 \\
8.414\end{array}$ & $\begin{array}{c}017008 \\
8.414\end{array}$ & $\begin{array}{c}017009 \\
8.398\end{array}$ & $\begin{array}{c}017010 \\
8.398\end{array}$ & $\begin{array}{c}017011 \\
8.389\end{array}$ & $\begin{array}{c}017012 \\
8.353\end{array}$ & $\begin{array}{c}017013 \\
8.353\end{array}$ & $\begin{array}{c}017014 \\
8.308\end{array}$ & $\begin{array}{c}017015 \\
8.308\end{array}$ & $\begin{array}{c}017016 \\
8.308\end{array}$ & $\begin{array}{c}017017 \\
8.297\end{array}$ & $\begin{array}{c}017019 \\
8.194\end{array}$ \\
\hline Longimde & -164.289 & -164.289 & -164.289 & -164.359 & -164.359 & -164.204 & -164.317 & -164.317 & -164346 & -164346 & -164346 & -164.369 & -164.200 \\
\hline Depth & 1850 & 1850 & 1850 & 2400 & 2400 & 1600 & 1150 & 1150 & 1400 & 1400 & 1400 & 1700 & 1600 \\
\hline Labill & 38 & 39 & 40 & 41 & 42 & 43 & 4 & 45 & 46 & - & 48 & 49 & so \\
\hline $\operatorname{Mn}(w . s)$ & 25.0 & 222 & 23.4 & 24.6 & 22.1 & 228 & 33.6 & 25.2 & 28.9 & 28.4 & 30.8 & 203 & 28.0 \\
\hline Fo & 15.8 & 17.5 & 18.8 & 17.4 & 20.0 & 14.6 & $8: 30$ & 11.7 & 12.6 & 13.7 & 120 & 10.4 & 14.1 \\
\hline Co & 1.12 & .800 & .980 & .930 & 1.01 & 1.37 & 248 & 1.26 & 1.10 & 1.10 & 1.000 & 1.24 & 1.49 \\
\hline $\mathbf{N i}$ & $\mathbf{A 3 0}$ & .200 & .200 & .370 & 310 & .470 & $\$ 10$ & 210 & 480 & .460 & 590 & 390 & .330 \\
\hline C. & .020 & .040 & .050 & .040 & .040 & - & .030 & $<.030$ & $<.030$ & $<.030$ & $<.030$ & 390 & $<.030$ \\
\hline$P b(p p m)$ & 1000 & 1000 & 1100 & 940 & 960 & 1000 & 850 & 1100 & 1000 & 970 & 1000 & 1000 & 1100 \\
\hline $2 n$ & 710 & 750 & 750 & 600 & 610 & 750 & 670 & 410 & 570 & 650 & - & $\cdot$ & 400 \\
\hline Samplod & Top & Bulk & Bulk & Bulk & Bulk & Bulk & Top & Bulk & Bulk & Botrom & Top & Bulk & Top \\
\hline Intervil & • & • & • & - & - & $0-15 x m$ & • & - & - & • & - & - & • \\
\hline
\end{tabular}

\begin{tabular}{|c|c|c|c|c|c|c|c|c|c|c|c|c|c|}
\hline Sequencoit: & 017020 & 017021 & 019003 & 020001 & 020002 & 053017 & 053018 & 053019 & 053020 & 053021 & 053022 & 053023 & 053024 \\
\hline Latitude & 8.194 & 8.186 & 7.250 & 8.747 & 8.755 & 19.335 & 19.369 & 19.369 & 19.511 & 19.511 & 19.511 & 19.511 & 19.511 \\
\hline Longitude & -164.200 & -164.202 & 171.008 & 169.738 & 169.792 & -168.665 & $-16 t .695$ & -168.695 & -168.835 & -168.835 & -168.835 & -168.835 & -168.835 \\
\hline Depth & 1600 & 1800 & 2250 & 3550 & 2900 & 3791 & 2000 & 2000 & 1800 & 1800 & 1800 & 1800 & 1800 \\
\hline Lab" & 51 & 52 & D-1 & $D-42$ & D-6-1 & 20 & 21 & 22 & 23 & 24 & 25 & 26 & 27 \\
\hline $\operatorname{Mn}(w t . \%)$ & 27.0 & 24.0 & 24.4 & 21.6 & 26.5 & 20.2 & 20.5 & 20.6 & 21.2 & 28.7 & 23.0 & 21.5 & 26.0 \\
\hline Re & 16.2 & 14.4 & 14.5 & 17.0 & 12.7 & 20.3 & 17.0 & 18.3 & 17.4 & 9.60 & 13.6 & 16.2 & 10.3 \\
\hline$C_{0}$ & 890 & 1.45 & 1.22 & .810 & 1.25 & .064 & 1.18 & .900 & .910 & .770 & 970 & 1.01 & .880 \\
\hline $\mathbf{N i}$ & 340 & .410 & .490 & .490 & .030 & .070 & .170 & .180 & 200 & .420 & .310 & .250 & .280 \\
\hline C. & $<000$ & $<\infty 0$ & .090 & .080 & .110 & . & .010 & .030 & .040 & .140 & .180 & .030 & .040 \\
\hline$P b(p p m)$ & 1000 & 970 & - & - &. & 1200 & 1000 & 1100 & 1100 & 1000 & 1100 & 1200 & 1100 \\
\hline $2 n$ & 630 & 350 & - & - & - & 800 & 800 & 800 & 450 & 600 & 560 & 460 & 560 \\
\hline Samplod & Bulk-minus & Bulk & Bulk & Bulk & Bulk & Bulk & Bulk & Bulk & Top & Bottom & Bulk & Top & Bottam \\
\hline Intorval & Top & - & $0-10$ mm & $0-3 \mathrm{~mm}$ & $0-20 \mathrm{~mm}$ & - & - & - & $\cdot$ & . & - & $\cdot$ & - \\
\hline
\end{tabular}

\begin{tabular}{|c|c|c|c|c|c|c|c|c|c|c|c|c|c|}
\hline Sequencoll & 053025 & 053026 & 053027 & 053028 & 053029 & 053030 & 053031 & 053032 & 053053 & 053034 & 053035 & 055005 & 055006 \\
\hline Latituds & 19.511 & 19.695 & 19553 & 19.553 & 19.515 & 19.515 & 19.515 & 19.515 & 19.515 & 19.515 & 16.678 & 11.020 & 11.008 \\
\hline Longitude & -168.835 & -168.995 & -168.832 & -168.832 & -168.855 & -168.855 & -168.855 & -168.855 & -168.855 & -168.855 & .169 .359 & 170.177 & 170.203 \\
\hline Depth & 1800 & 4500 & 2400 & 2400 & 1790 & 1790 & 1790 & 1790 & 1790 & 1790 & 1400 & 1575 & 2000 \\
\hline Labi" & 28 & 29 & 30 & 31 & 32 & 33 & 34 & 35 & 36 & 37 & 53 & D-12-1 & D-13-4 \\
\hline $\operatorname{Mn}(w . \%)$ & 23.5 & 12.0 & 20.7 & 18.0 & 24.0 & 24.0 & 22.2 & 25.5 & 20.1 & 263 & $<.50$ & 225 & 235 \\
\hline$F_{0}$ & 12.9 & 18.7 & 17.8 & 18.6 & 14.4 & 13.8 & 18.4 & 12.3 & 18.8 & 13.0 & 103 & 15.8 & 15.5 \\
\hline Co & .870 & .320 & .920 & .690 & .680 & .710 & 800 & .064 & .930 & .490 & $<100$ & .660 & 1.07 \\
\hline $\mathbf{N i}$ & .270 & .200 & .180 & .200 & .270 & 290 & .240 & 320 & .210 & .280 & $<100$ & .470 & .480 \\
\hline c. & .040 & .230 & .060 & .200 & .090 & .100 & .060 & .120 & .050 & .070 & - & .100 & .090 \\
\hline$P(p p m)$ & 1200 & 960 & 1100 & 1100 & 1400 & 1200 & 1400 & 1100 & 1200 & 1500 & 590 & - & - \\
\hline $\mathrm{Zn}$ & 580 & 680 & 450 & 620 & 580 & 590 & 570 & 550 & 540 & 560 & 370 & - & $\cdot$ \\
\hline Samplod & Bulk & Top & Bulk & Bulk & Bulk & Top & Top & Bottom & Bulk & Bulk & Bulk & Bulk & Bulk \\
\hline Interval & • & - & • & • & - & $\cdot$ & - & - & • & • & - & $25 \mathrm{~mm}$ & $30 \mathrm{~mm}$ \\
\hline
\end{tabular}


Table 5. Composition of ferromanganese crusts analyzed onboard SP Lee L5-83HW and L5-84CP by Portaspec XRF(cont'd).

\begin{tabular}{|c|c|c|c|c|c|c|c|c|c|c|c|c|c|}
\hline $\begin{array}{l}\text { Sequencel } \\
\text { Lationdo }\end{array}$ & $\begin{array}{l}055007 \\
11.005\end{array}$ & $\begin{array}{l}056006 \\
12.183\end{array}$ & $\begin{array}{l}056008 \\
13.905\end{array}$ & $\begin{array}{l}056009 \\
13.900\end{array}$ & $\begin{array}{l}089011 \\
22181\end{array}$ & $\begin{array}{l}089013 \\
22208\end{array}$ & $\begin{array}{l}089014 \\
22256\end{array}$ & $\begin{array}{r}089015 \\
22316\end{array}$ & $\begin{array}{r}089016 \\
22316\end{array}$ & $\begin{array}{l}089017 \\
21.718\end{array}$ & $\begin{array}{l}089018 \\
21.718\end{array}$ & $\begin{array}{l}089019 \\
21.718\end{array}$ & $\begin{array}{l}089020 \\
21.718\end{array}$ \\
\hline Langitude & 170.230 & 168.995 & 167.627 & 167.653 & -166.810 & -166.882 & -166.854 & -166.899 & -166.899 & -167.578 & -167.578 & -167.578 & -167.578 \\
\hline Depth & 1800 & 1300 & 1600 & 1600 & 3950 & 2402 & 1970 & 2350 & 2350 & 2400 & 2400 & 2400 & 2400 \\
\hline Leb" & D-14-3 & D-15 & D-17 & D-18 & 1 & 2 & 3 & 4 & 5 & 6 & 7 & 8 & 9 \\
\hline $\operatorname{Mn}(w . \$)$ & 25.5 & 24.0 & 220 & 21.2 & 17.3 & 15.6 & 17.0 & 182 & 20.0 & 20.0 & 19.5 & 17.0 & 23.0 \\
\hline Po $_{0}$ & 125 & 135 & 155 & 17.0 & 20.0 & 18.0 & 18.5 & 19.7 & 20.0 & 15.2 & 20.3 & 19.4 & 15.8 \\
\hline Co & 990 & .800 & .740 & .680 & 440 & 530 & .670 & .740 & .680 & 520 & .550 & .600 & 1.000 \\
\hline $\mathbf{N i}$ & 590 & 590 & $\mathbf{A 4 0}$ & 390 & .150 & .250 & .230 & 265 & 210 & .270 & .180 & .150 & .260 \\
\hline$\infty$ & .120 & .180 & .110 & .110 & .140 & .200 & .070 & .072 & .050 & .170 & .070 & .040 & .050 \\
\hline$P Q$ (ppm) & - & - & - & $\cdot$ & - & 1000 & 1200 & 1200 & 1200 & 1000 & 1000 & 1200 & 1200 \\
\hline $\mathbf{Z n}$ & - & - & - & - & - & - & - & - & - & 680 & 500 & 440 & 440 \\
\hline Sumpled & Bulk & Bulk & $\begin{array}{c}\text { Bulk } \\
20 \mathrm{~mm}\end{array}$ & Bulk & Bulk & Bulk & Top & Bulk & Bulk & $\begin{array}{c}\text { Bottam } \\
.\end{array}$ & Top & Top & Top \\
\hline
\end{tabular}

\begin{tabular}{|c|c|c|c|c|c|c|c|c|c|c|c|}
\hline Sequencels & 089021 & 089022 & 089023 & 089024 & 089025 & 089026 & 089027 & 089028 & 089029 & 089030 & 089031 \\
\hline Latitude & 21.796 & 21.796 & 21.796 & 21.841 & 20.209 & 20.209 & 20.209 & 20.209 & 20.335 & 20.335 & 20.335 \\
\hline Longitudo & -167.624 & -167.624 & -167.624 & -167.666 & -169.506 & -169506 & -169506 & .169 .506 & -169.575 & .169 .575 & -169.575 \\
\hline Depth & 2100 & 2100 & 2100 & 4123 & 4500 & 4500 & 4500 & 4500 & 2400 & 2400 & 2400 \\
\hline Lab: & 10 & 11 & 12 & PC4 & 13 & 14 & 15 & 16 & 17 & 18 & 19 \\
\hline $\operatorname{Mrn}(w . s)$ & 23.5 & 16.5 & 16.8 & 10.3 & 14.0 & 11.0 & 13.8 & 123 & 18.5 & 18.0 & 20.5 \\
\hline Po $_{0}$ & 16.0 & 23.1 & 192 & 24.0 & 20.4 & 18.6 & 16.1 & 192 & 20.7 & 19.7 & 16.8 \\
\hline Co & 1.10 & 540 & .064 & $A 70$ & 380 & 340 & $A 20$ & 360 & .710 & .630 & 1.02 \\
\hline $\mathrm{Ni}$ & .210 & .160 & .140 & .370 & .100 & .100 & .160 & .120 & .070 & .060 & .160 \\
\hline Co & .050 & .060 & .040 & 340 & .100 & .130 & .160 & .160 & .020 & .050 & .070 \\
\hline$P(p p m)$ & 1200 & 1200 & 1200 & . & 900 & 800 & 800 & 900 & 1100 & 1100 & 1000 \\
\hline $\mathbf{Z n}$ & 450 & 610 & 480 & 870 & 550 & 500 & 550 & 650 & 400 & 450 & 800 \\
\hline Samplod & Bulk & Bulk & Top & Bulk & Bulk & Top & Bottom & Bulk & Top & Bulk & Bulk \\
\hline Intorval & • & - & - & - & - & • & - & - & - & - & - \\
\hline
\end{tabular}


Table 5a. Composition of ferromanganese crusts analyzed onboard R/V Sonne Midpac2a and Midpac2b by XRF

\begin{tabular}{|c|c|c|c|c|c|c|c|c|c|c|c|c|}
\hline $\begin{array}{l}\text { Sequencell } \\
\text { Letitude }\end{array}$ & $\begin{array}{c}017047 \\
9.694\end{array}$ & $\begin{array}{c}017048 \\
9.694\end{array}$ & $\begin{array}{c}017049 \\
9.694\end{array}$ & $\begin{array}{c}017050 \\
9.694\end{array}$ & $\begin{array}{c}017051 \\
9.696\end{array}$ & $\begin{array}{c}017051 \\
9.696\end{array}$ & $\begin{array}{c}017052 \\
9.696\end{array}$ & $\begin{array}{c}017052 \\
9.696\end{array}$ & $\begin{array}{c}017053 \\
9.696\end{array}$ & $\begin{array}{c}017053 \\
9.696\end{array}$ & $\begin{array}{c}017059 \\
9.716\end{array}$ & $\begin{array}{c}017059 \\
9.716\end{array}$ \\
\hline Langinde & -162.178 & -162.178 & -162.178 & -162178 & -162.181 & -162.181 & -162.181 & -162.181 & -162.181 & -162181 & -162167 & -162167 \\
\hline $\begin{array}{l}\text { Dopth } \\
\text { Laby }\end{array}$ & $\begin{array}{c}1993 \\
\text { CKB-30 }\end{array}$ & $\begin{array}{c}1993 \\
\text { CKB-31 } \\
\end{array}$ & $\begin{array}{c}1993 \\
\text { CKB-32 } \\
\end{array}$ & $\begin{array}{c}1993 \\
\text { CKB-33 } \\
\end{array}$ & $\begin{array}{c}1927 \\
\text { CKB-34 } \\
\end{array}$ & $\begin{array}{c}1927 \\
\text { CKB-35 }\end{array}$ & $\begin{array}{c}1927 \\
\text { CKB-36 } \\
\end{array}$ & $\begin{array}{c}1927 \\
\text { CKB-37 }\end{array}$ & $\begin{array}{c}1927 \\
\text { CKB-38 } \\
\end{array}$ & $\begin{array}{c}1927 \\
\text { CKB-39 }\end{array}$ & $\begin{array}{c}1602 \\
\text { CKB-40 } \\
\end{array}$ & $\begin{array}{c}1602 \\
\text { CKB-41 }\end{array}$ \\
\hline Si(wt.s) & 2,00 & 2.00 & 4.10 & 3.00 & 1.50 & 1.70 & 2.20 & 2.00 & 240 & 2.10 & 1.20 & 1.10 \\
\hline $\mathbf{T i}$ & 1.09 & 1.33 & 1.43 & 1.70 & 81 & 95 & 1.38 & 1.05 & 1.32 & 1.08 & 93 & 84 \\
\hline $\mathrm{Mm}$ & 27.7 & 30.9 & 28.4 & 30.1 & 21.7 & 31.0 & 30.9 & 17.3 & 28.8 & 30.5 & 32.9 & 229 \\
\hline $\mathrm{Pe}$ & 168 & 14.2 & 17.6 & 17.6 & 123 & 145 & 15.5 & 120 & 16.1 & 13.2 & $13 A$ & 113 \\
\hline $\mathbf{A}$ & .40 & 36 & .81 & 53 & 26 & .27 & 38 & .34 & $s 1$ & .70 & 33 & 30 \\
\hline Co & 870 & 1.41 & 1.02 & 1.40 & 380 & 1.23 & 1.17 & 330 & 980 & 1.19 & 1.45 & .470 \\
\hline $\mathbf{N i}$ & .440 & .660 & .470 & .590 & 200 & .660 & .650 & .460 & 590 & .860 & .720 & .480 \\
\hline $\mathrm{Cr}$ & .048 & .058 & .039 & .048 & .048 & .039 & .048 & .058 & .058 & .07 & .048 & .048 \\
\hline Ca & 3.64 & 3.34 & 393 & 5.62 & 19.4 & 3.39 & 4.22 & 25.0 & 4.10 & 4.40 & 4.10 & 20.1 \\
\hline $\mathbf{P}$ & .45 & $2 n$ & 42 & • & 3.36 & .45 & 50 & 5.38 & .63 & .71 & 53 & 3.70 \\
\hline$P b(p p m)$ & 1600 & 1700 & 1700 & 1800 & 2000 & 2100 & 2000 & 1700 & 2100 & 2000 & 2300 & 2000 \\
\hline St & $\therefore$ & - & 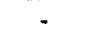 & - & $\cdot$ & . & 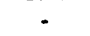 & - & . & . & • & 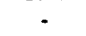 \\
\hline $\mathrm{Zn}$ & 700 & 960 & 770 & 970 & 800 & 920 & 980 & 970 & 1000 & 1200 & 910 & 850 \\
\hline Sempled & Bulk & Bulk & Bulk & Paroes & ad & Youns & Young & Old & Bulk & Top & Young & ald \\
\hline Imporval & • & - & - & - & . & . & - & • & - & $\cdot$ & - & - \\
\hline
\end{tabular}

\begin{tabular}{|c|c|c|c|c|c|c|c|c|c|c|c|c|}
\hline Sequencell & 017059 & 017059 & 017060 & 017062 & 017063 & 017064 & 017064 & 017070 & 017071 & $0170 m$ & 017078 & 017079 \\
\hline Latitudo & 9.716 & 9.716 & 9.716 & 9.682 & 9.705 & 9.705 & 9.705 & 6.516 & 6.503 & 6.271 & 6.274 & 6.281 \\
\hline Langitude & -162.167 & -162.167 & -162167 & -162184 & -162.184 & -162.184 & -162.184 & -162408 & -162370 & -162876 & -162886 & -162.878 \\
\hline Dopth & 1602 & 1602 & 1602 & 2706 & 1940 & 1940 & 1940 & 2349 & 2572 & 2957 & 2217 & 1376 \\
\hline Lab: & CKB- 42 & CKB-43 & CKB-44 & CKB-45 & CKB-46 & CKB-47 & CKB-48 & CKB-SOAM & CKB-S1 & CKB-52AM & CKB-53AM & CKB-54AM \\
\hline si(wt.\%) & 1.40 & 90 & 1.20 & 7.30 & 180 & 1.50 & 1.70 & 250 & 4.10 & 6.20 & 4.40 & 5.70 \\
\hline $\mathrm{Ti}$ & .93 & .65 & .89 & 1.04 & 97 & 57 & 81 & 96 & 1.04 & .94 & 99 & 1.14 \\
\hline Mn & 25.7 & 27.8 & 31.2 & 17.8 & 2.9 & 12.7 & 28.1 & 25.3 & 23.7 & 19.0 & 20.1 & 26.2 \\
\hline Fo & 11.7 & 7.60 & 11.6 & 12.1 & 125 & 7.50 & 109 & 14.3 & 15.7 & 13.7 & 12.6 & 9.80 \\
\hline Al & .39 & .66 & 34 & 2.47 & .44 & .78 & .47 & 37 & .83 & 1.39 & 1.26 & 2.09 \\
\hline Co & .850 & 1.05 & 1.52 & 990 & A30 & .480 & 1.44 & .930 & .730 & .570 & 1.08 & 1.93 \\
\hline $\mathbf{N i}$ & 560 & 1.350 & .780 & .380 & .400 & .640 & .670 & .620 & .470 & .400 & .450 & .600 \\
\hline $\mathrm{Cu}$ & .048 & .116 & .048 & .029 & .087 & .068 & .048 & .058 & $.0 m$ & .048 & .019 & .019 \\
\hline$c_{2}$ & 13.2 & 11.2 & 3.50 & 13.7 & 13.3 & 27.7 & 4.10 & 2.60 & 3.50 & 6.10 & 5.00 & 4.30 \\
\hline $\mathbf{P}$ & 247 & 2.36 & .51 & 2.66 & 256 & 7.24 & .74 & AS & .68 & 1.36 & .45 & .50 \\
\hline $\mathrm{Pb}(\mathrm{ppm})$ & 2100 & 1800 & 2300 & 1500 & 2200 & 1600 & 2200 & 1500 & 1500 & 1200 & 1400 & 1700 \\
\hline Sr & - & • & - & • & • & • & - & - & • & - & - & - \\
\hline $\mathbf{Z n}$ & 900 & 1600 & 1000 & 650 & 950 & 1000 & 810 & 800 & 780 & 760 & 710 & 870 \\
\hline Samplod & Bulk & Bulk & Top & Top & Bulk & ad & Young & - & - & Bulk & Bulk & Bulk \\
\hline Interval & - & - & - & - & - & - & - & - & - & - & - & - \\
\hline
\end{tabular}


Table 5a. Composition of ferromanganese crusts analyzed onboard RN Sonne Midpac2a and Midpac2b by XRF(cont'd).

\begin{tabular}{|c|c|c|c|c|c|c|c|c|c|c|c|c|}
\hline $\begin{array}{l}\text { Sequencell } \\
\text { Latitude }\end{array}$ & $\begin{array}{c}017080 \\
6.281\end{array}$ & $\begin{array}{c}017082 \\
6.263\end{array}$ & $\begin{array}{c}017082 \\
6.263\end{array}$ & $\begin{array}{c}017083 \\
6.263\end{array}$ & $\begin{array}{c}017084 \\
6.263\end{array}$ & $\begin{array}{c}017085 \\
6.290\end{array}$ & $\begin{array}{c}017085 \\
6.290\end{array}$ & $\begin{array}{c}017085 \\
6.290\end{array}$ & $\begin{array}{c}017085 \\
6.290\end{array}$ & $\begin{array}{c}017085 \\
6.290\end{array}$ & $\begin{array}{c}017086 \\
9.164\end{array}$ & $\begin{array}{c}017087 \\
9.172\end{array}$ \\
\hline $\begin{array}{l}\text { Longinds } \\
\text { Dopth } \\
\text { Laby }\end{array}$ & $\begin{array}{c}-162.878 \\
1376 \\
\text { CKB-55AM } \\
\end{array}$ & $\begin{array}{c}-162.963 \\
2887 \\
\text { CKB-57AM }\end{array}$ & $\begin{array}{c}-162.963 \\
2887 \\
\text { CKB-58AM }\end{array}$ & $\begin{array}{c}-162.963 \\
2887 \\
\text { CKB-59 }\end{array}$ & $\begin{array}{c}-162.963 \\
2887 \\
\text { CKB-60 }\end{array}$ & $\begin{array}{c}-162970 \\
2486 \\
\text { CKB-61 }\end{array}$ & $\begin{array}{c}-162.970 \\
2486 \\
\text { CKB-61AM }\end{array}$ & $\begin{array}{c}-162970 \\
2486 \\
\text { CKB-62 }\end{array}$ & $\begin{array}{c}-162.970 \\
2486 \\
\text { CKB-63 }\end{array}$ & $\begin{array}{c}-162970 \\
2486 \\
\text { C.B-64 }\end{array}$ & $\begin{array}{c}-164.842 \\
2291 \\
\text { CKB-65 }\end{array}$ & $\begin{array}{c}-164.826 \\
1853 \\
\text { CKB-66 }\end{array}$ \\
\hline si(wt.\%) & 3.70 & 5.40 & 4.10 & 4.90 & 5.80 & 270 & 240 & 3.40 & 3.20 & 3.10 & 390 & 240 \\
\hline $\mathbf{T}$ & 1.06 & .98 & 1.02 & 1.25 & 1.33 & 1.48 & 1.14 & 1.37 & 1.50 & 1.46 & 1.03 & 1.32 \\
\hline Mn & 25.7 & 23.9 & 232 & 25.6 & 23.6 & 28.8 & 25.2 & 29.6 & 27.0 & 27.4 & 243 & 28.0 \\
\hline $\mathbf{P e}$ & 9.60 & 18.9 & 16.0 & 18.4 & 18.8 & 21.1 & 17.4 & 203 & 213 & 21.8 & 182 & 13.4 \\
\hline $\mathbf{N}$ & 1.16 & 89 & 81 & .88 & 1.20 & 51 & 44 & 55 & .70 & 55 & .61 & .75 \\
\hline Co & 1.97 & 540 & 870 & 800 & .720 & .710 & .620 & .640 & 560 & .610 & .720 & 1.41 \\
\hline $\mathrm{Ni}$ & 590 & 370 & $A 50$ & 480 & .440 & 490 & .430 & .540 & 440 & .440 & 120 & .610 \\
\hline $\mathrm{Cr}$ & .019 & .058 & .039 & .058 & .077 & .068 & .048 & .048 & .106 & .087 & .048 & .068 \\
\hline Ca & 4.70 & 4.10 & 290 & 4.30 & 5.60 & 6.00 & 3.60 & 5.90 & 5.60 & 5.70 & 3.80 , & 5.00 \\
\hline $\mathbf{P}$ & 46 & .72 & A5 & .59 & .87 & .64 & 51 & .78 & .76 & .79 & .56 & .61 \\
\hline$P b(p p m)$ & 1700 & 1300 & 1200 & 1500 & 1500 & 1600 & 1600 & 1800 & 1500 & 1600 & 1500 & 1900 \\
\hline St & 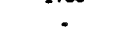 & . & 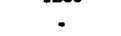 & - & 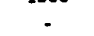 & . & - & . & - & - & 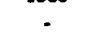 & . \\
\hline $\mathbf{Z n}$ & 870 & no & 730 & 850 & 830 & 1100 & 870 & 1000 & 1200 & 1300 & 770 & 890 \\
\hline Semplod & Bulk & - & - & Bulk & Bulk & Bulk & Bulk & Top & Botrom & Middle & Bulk & Bulk \\
\hline Intorval & - & - & - & . & - & - & - & - & . & - & - & - \\
\hline
\end{tabular}

\begin{tabular}{|c|c|c|c|c|c|c|c|c|c|c|c|c|}
\hline Sequencest & 017087 & 017088 & 017088 & 017088 & 017088 & 017088 & 017088 & 017089 & 017090 & 017093 & 017093 & 017093 \\
\hline Lationde & 9.172 & 9.179 & 9.179 & 9.179 & 9.179 & 9.179 & 9.179 & 9.166 & 9.140 & 9.225 & 9.225 & 9.225 \\
\hline Longitude & -164.826 & -164830 & -164.830 & -164.830 & -164.830 & -164.830 & -164830 & -164.798 & -164.789 & -164.780 & -164.780 & -164.780 \\
\hline Dopth & 1853 & 1263 & 1203 & 1263 & 1263 & 1263 & 1263 & 1427 & 2638 & 1456 & 1456 & 1456 \\
\hline Laby & CKB-67 & CKB-68 & CKB-69 & CKB-70AM & CKB-71 & CKB-72 & CKB-72AM & CKB-73 & CKB-74 & CKB-75 & CKB-76 & CKB-76AM \\
\hline Si(wt.\%) & 16.7 & 1.17 & 214 & .91 & 1.44 & 1.13 & 1.23 & 1.45 & 82 & 2.15 & 1.71 & .85 \\
\hline $\mathrm{Ti}$ & 1.13 & 1.06 & 1.33 & 1.04 & 1.25 & .94 & 86 & 1.00 & 86 & 1.28 & 1.16 & .87 \\
\hline Mn & - & 303 & 32.6 & 325 & 333 & 29.4 & 28.5 & 29.5 & 33.6 & 30.8 & 27.1 & 26.0 \\
\hline $\mathrm{Pe}$ & 6.70 & 11.9 & 15.3 & 9.50 & 13.9 & 115 & 11.1 & 11.1 & 113 & 14.4 & 127 & 113 \\
\hline $\mathbf{N}$ & 7.78 & 31 & 41 & 32 & .36 & 32 & 37 & 49 & .26 & AS & .41 & .28 \\
\hline Co & .020 & 1.26 & 1.38 & 1.76 & 134 & .930 & .920 & 1.30 & 1.71 & 1.35 & .950 & 960 \\
\hline $\mathbf{N i}$ & .030 & .720 & .610 & 840 & .740 & .700 & .710 & 650 & 800 & .650 & 580 & .610 \\
\hline Ca & .002 & .039 & .039 & .058 & .039 & .039 & .039 & .058 & .058 & .039 & .058 & .039 \\
\hline $\mathrm{Ca}$ & 12.2 & 5.30 & 5.00 & 290 & 5.60 & 113 & 12.7 & 12.3 & 3.80 & 4.90 & 9.50 & 10.3 \\
\hline $\mathbf{P}$ & 249 & .68 & .68 & .34 & .60 & 1.78 & 1.89 & 1.87 & 40 & .71 & 1.65 & 130 \\
\hline$P b$ (ppm) & 290 & 2100 & 2200 & 2300 & 2300 & 2200 & 2300 & 2100 & 2200 & 2200 & 1900 & 1700 \\
\hline Sr & - & - & • & - & • & - & - & - & . & - & - & - \\
\hline $\mathbf{Z n}$ & 227 & 980 & 870 & 1000 & 970 & 1000 & 1000 & 900 & 920 & 900 & 930 & 910 \\
\hline Semplod & Bulk & Young & Old & Young & Bulk & Youms & Young & - & - & Young & Old & Old \\
\hline Interval & - & - & - & - & . & - & - & - & - & - & - & 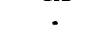 \\
\hline
\end{tabular}


Table 5a. Composition of ferromanganese crusts analyzed onboard R/V Sonne Midpac2a and Midpac2b by XRF(cont'd).

\begin{tabular}{|c|c|c|c|c|c|c|c|c|c|c|c|c|}
\hline $\begin{array}{l}\text { Sequencell } \\
\text { Latitude }\end{array}$ & $\begin{array}{c}017093 \\
9.225\end{array}$ & $\begin{array}{c}017095 \\
9.247\end{array}$ & $\begin{array}{c}017095 \\
9.247\end{array}$ & $\begin{array}{c}017096 \\
9.247\end{array}$ & $\begin{array}{c}017096 \\
9.247\end{array}$ & $\begin{array}{c}017097 \\
9.247\end{array}$ & $\begin{array}{c}017098 \\
9.247\end{array}$ & $\begin{array}{c}017098 \\
9.247\end{array}$ & $\begin{array}{c}017099 \\
9.200\end{array}$ & $\begin{array}{c}017099 \\
9.200\end{array}$ & $\begin{array}{c}017099 \\
9.200\end{array}$ & $\begin{array}{c}017099 \\
9.200\end{array}$ \\
\hline Longitude & -164.780 & -164.846 & -164.846 & -164.846 & -164.846 & -164.846 & -164.846 & -164.846 & -164.929 & -164.929 & -164.920 & -164929 \\
\hline $\begin{array}{l}\text { Depth } \\
\text { Labs }\end{array}$ & $\begin{array}{c}1456 \\
\text { CKB-77AM }\end{array}$ & CKB-79 & CKB-80 & CKB-81 & CKB-82 & CKB-83 & CKB-84 & CKB-85 & CKB-86AM & CKB-87 & CKB-88 & CKB-89AM \\
\hline sí(wt.\$) & 1.18 & 150 & 1.70 & 150 & 1.20 & 260 & 2.00 & 1.20 & 3.00 & 1.20 & .90 & 1.20 \\
\hline $\mathrm{Ti}$ & 1.08 & 1.24 & 1.23 & 1.25 & 1.04 & 1.11 & 1.55 & 93 & .47 & 1.14 & .73 & .89 \\
\hline Mn & 29.8 & 31.8 & 27.0 & 32.1 & 26.4 & 33.1 & 320 & 26.3 & 11.7 & 34.1 & 27.6 & 27.9 \\
\hline $\mathbf{F}_{0}$ & 11.3 & 14.3 & 13.1 & 148 & 11.7 . & 13.1 & 15.6 & 11.9 & 5.40 & 11.7 & 9.90 & 129 \\
\hline $\mathbf{A l}$ & 30 & 40 & AS & .33 & 28 & 81 & .44 & 35 & 1.28 & .28 & .26 & .29 \\
\hline Co & 1.75 & 1.38 & .870 & 140 & .820 & 1.80 & 1.32 & .070 & .460 & 1.71 & .700 & 1.43 \\
\hline $\mathrm{Ni}$ & .620 & .720 & .600 & .660 & 570 & .770 & .680 & .630 & .630 & .780 & .770 & .550 \\
\hline $\mathrm{Cu}$ & .020 & .043 & .048 & .039 & .039 & .048 & .048 & .048 & .097 & .048 & .039 & .019 \\
\hline Ca & 80 & 6.20 & 19.0 & 650 & 209 & 6.40 & 6.50 & 20.1 & 29.0 & 4.20 & 13.4 & 280 \\
\hline $\bar{P}$ & 49 & .71 & 3.60 & .64 & 3.92 & 59 & .70 & 3.77 & 7.44 & 47 & 2.61 & 50 \\
\hline Pu(ppm) & 2100 & 2200 & 1900 & 2200 & 1800 & 2200 & 2300 & 1700 & 1000 & 2200 & 2000 & 2200 \\
\hline $\mathrm{St}$ & - & • & • & - & - & • & - & • & - & • & • & • \\
\hline $\mathrm{Zn}$ & 800 & 940 & 1000 & 880 & 950 & 910 & 1000 & 1000 & 920 & 940 & 1100 & 700 \\
\hline Semplod & Top & Youns & Old & Youns & ad & Top & Young & Old & - & Young & Old & Top \\
\hline Interval & 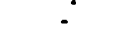 & - & - & - & - & - & . & - & - & . & - & $\because$ \\
\hline
\end{tabular}

\begin{tabular}{|c|c|c|c|c|c|c|c|c|c|c|c|c|}
\hline Soquencell & 017101 & 017102 & 052002 & 052003 & 052004 & 052005 & 052005 & 052005 & 052005 & 052006 & 052006 & 052006 \\
\hline Latitude & 9.716 & 9.223 & 18.699 & 18.699 & 18.699 & 18.643 & 18.643 & 18.643 & 18.643 & 18.643 & 18.643 & 18.643 \\
\hline Longitude & .162 .167 & -164.858 & .158 .380 & -158.380 & -158.380 & -158.295 & -158.295 & -158.295 & -158.295 & -158.295 & -158.295 & -158.295 \\
\hline Depth & 1602 & 1420 & 3261 & 3261 & 3261 & 1924 & 1924 & 1924 & 1924 & 1924 & 1924 & 1924 \\
\hline Lab" & CKB-49 & CKB-78 & CKB-1 & CKB-2 & CKB-3 & CKB-4AM & $\mathbf{C K B}+40$ & CKB-5 & CKB-6 & CKB-7 & CKB-8 & CKB-8AM \\
\hline
\end{tabular}

\begin{tabular}{|c|c|c|c|c|c|c|c|c|c|c|c|c|}
\hline $\operatorname{si}(w t . s)$ & 1.90 & 90 & 6.90 & 6.70 & 10.6 & 6.90 & 7.50 & 5.30 & 6.00 & 5.80 & 6.60 & 6.60 \\
\hline $\mathrm{Ti}$ & 1.06 & 1.04 & 1.67 & 186 & 2.51 & 1.57 & 152 & 1.36 & 1.12 & 1.07 & 1.44 & .14 \\
\hline Min & 26.3 & 31.4 & 15.7 & 15.9 & 4.60 & 19.2 & 19.7 & 17.5 & 19.7 & 19.4 & 20.9 & 19.6 \\
\hline Fe & 12.3 & 9.80 & 20.1 & 208 & 20.5 & 173 & 20.2 & 20.1 & 16.5 & 16.2 & 19.7 & 20.0 \\
\hline $\mathbf{A}$ & 57 & 28 & 1.63 & 1.60 & 238 & 1.88 & 1.86 & 89 & 1.41 & 1.52 & 1.53 & 1.48 \\
\hline Co & 1.24 & 1.46 & .380 & 360 & .110 & .660 & .540 & .470 & .490 & .480 & 560 & .550 \\
\hline $\mathrm{Ni}$ & .630 & .810 & .180 & .160 & .050 & 350 & .200 & .180 & .200 & .290 & 300 & .270 \\
\hline $\mathrm{Cu}$ & .058 & .048 & .087 & .087 & .058 & .107 & .116 & .048 & .145 & .136 & .126 & .107 \\
\hline C. & 11.9 & 3.70 & 1.45 & 1.90 & .40 & 270 & 3.30 & 1.70 & 2.00 & 210 & 3.00 & 270 \\
\hline $\mathbf{P}$ & 208 & .36 & 34 & 39 & 28 & .48 & 54 & .44 & 33 & 38 & .53 & 54 \\
\hline
\end{tabular}

$\begin{array}{lcccccccccccc}\text { Pb(ppm) } & 2100 & 2100 & 1600 & 1500 & 1100 & 1400 & 1500 & 1800 & 1200 & 1100 & 1400 & 1500 \\ \text { Sr } & - & - & - & - & - & - & - & - & - & - & - \\ \text { Zn } & 960 & 990 & 790 & 770 & 780 & 710 & 770 & 730 & 660 & 670 & 750 & 730 \\ \text { Sampiod } & \text { Bulk } & - & \text { Bulk } & \text { Bulk } & \text { Bulk } & \text { Bulk } & \text { Bulk } & \text { Top } & \text { Bottom } & \text { Bulk } & \text { Top } & \text { Top } \\ \text { Intorval } & - & - & - & - & - & - & - & - & - & - & - & -\end{array}$


Table 5a. Composition of ferromanganese crusts analyzed onboard RN Sonne Midpac2a and Midpac2b by XRF(cont'd).

\begin{tabular}{|c|c|c|c|c|c|c|c|c|c|c|c|c|}
\hline Sequence" & 052006 & 052007 & 052007 & 052007 & 052008 & 052000 & 052010 & 052010 & 052011 & 052012 & 052015 & 052015 \\
\hline Letitudo & 18.643 & 18.643 & 18.643 & 18.643 & 18.643 & 18.637 & 18.637 & 18.637 & 18.637 & 18.637 & 18.683 & 18.683 \\
\hline Langinide & -158.295 & -158.295 & -158.295 & -158.25 & -158.295 & -158.313 & -158.313 & -158.313 & -158313 & -158313 & -158.314 & -158314 \\
\hline Deppth & 1924 & 1924 & 1924 & 1924 & 1924 & 2002 & 2002 & 2002 & 2002 & 2002 & 1047 & 1047 \\
\hline Laby & CKB-9 & CXB-10 & CKB-10 can & CKB-11 & CKB-12 & CKB-13 & CKB-17 & CKB-18 & CKB-19 & CKB-20 & CKB-21 & CKB-22 \\
\hline Si(wt.\%) & 5.70 & 10.6 & 10.6 & 4.90 & 6.20 & 4.70 & 4.60 & 4.70 & 5.40 & 4.30 & 2.70 & 4.00 \\
\hline $\mathrm{Ti}$ & .78 & 90 & .79 & 1.36 & 1.26 & 1.28 & 1.33 & 135 & 1.42 & 1.26 & 1.04 & 1.49 \\
\hline Mn & 193 & 13.0 & 10.1 & 21.1 & 21.4 & 21.1 & 21.6 & 21.6 & 192 & 21.8 & 22.8 & 269 \\
\hline Fo & 163 & 13.8 & 12.0 & 20.8 & 16.6 & 21.4 & 20.1 & 20.1 & 20.6 & 195 & 14.4 & 16.6 \\
\hline Al & 1.73 & 3.54 & 351 & 85 & 1.61 & 85 & .90 & 1.01 & 1.03 & 80 & .69 & .96 \\
\hline$C_{0}$ & 360 & 300 & 230 & .560 & .520 & .470 & 530 & .660 & 500 & 540 & .530 & .790 \\
\hline $\mathbf{N i}$ & 400 & .260 & .200 & .270 & .350 & .260 & 270 & 360 & 240 & 280 & .440 & .480 \\
\hline Cos & 252 & .155 & .136 & .058 & .165 & .078 & .097 & .087 & .058 & .106 & .116 & .107 \\
\hline $\mathrm{Ca}$ & 2.20 & 1.00 & 4.30 & 2.20 & 260 & 200 & 2.20 & 250 & 1.90 & 200 & 8.00 & 3.90 \\
\hline$P$ & .40 & 36 & 1.05 & A5 & 33 & $A 1$ & 37 & 53 & .42 & 37 & 1.39 & .71 \\
\hline $\mathrm{Pb}(\mathrm{ppm})$ & 850 & 1100 & 750 & 1600 & 1100 & 1500 & 1500 & 1800 & 1500 & 1400 & 1500 & 1700 \\
\hline St & - & - & $\cdot$ & - & - & - & - & . & - & . & - & - \\
\hline $\mathrm{Zn}$ & 750 & 670 & 610 & 800 & 780 & 850 & 780 & 790 & 810 & 750 & 810 & 870 \\
\hline Sampled & Bottan & Bulk & Bulk & Bulk & Top & Bulk & Bulk & Bulk & Bulk & Bulk & Bulk & Young \\
\hline Inerval & - & . &. &. & 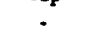 &. & . & - & - &. & . &. \\
\hline
\end{tabular}

\begin{tabular}{|c|c|c|c|c|c|c|c|c|c|c|c|c|}
\hline Sequencell & 052015 & 052016 & 052016 & 052016 & 052017 & 052017 & 052017 & 052023 & 053103 & 053103 & 053104 & 053104 \\
\hline Latitude & 18.683 & 18.683 & 18.683 & 18.683 & 18.683 & 18.683 & 18.683 & 18.637 & 14.462 & 14.462 & 14.456 & 14.456 \\
\hline Langituds & .158 .314 & .158 .314 & -158314 & -158.314 & -158.314 & -158.314 & -158.314 & -158.313 & -169.017 & -169.017 & -169.003 & -169.003 \\
\hline Dopth & 1047 & 1047 & 1047 & 1047 & 1047 & 1047 & 1047 & 2002 & 2110 & 2110 & 1770 & 1770 \\
\hline Lab: & CKB-23 & CKB-24 & CKB-25 & CKB-26 & CKB-27 & CKB-2B & CKB-29 & CKB-16 & CKB-141 & CKB-142 & CKB-149 & CKB-150 \\
\hline Si(wt.\%) & 2.30 & 3.70 & 3.60 & 3.60 & 280 & 3.00 & 1.50 & 9.20 & 1.94 & 1.53 & 331 & 2.42 \\
\hline $\mathrm{Ti}$ & .89 & 130 & 1.20 & .67 & .98 & 1.11 & .62 & 207 & 1.14 & 1.05 & 88 & 1.12 \\
\hline Mn & 215 & 24.7 & 25.4 & 16.9 & 224 & 24.9 & 19.4 & 25.9 & 26.8 & 23.7 & 22.2 & 23.8 \\
\hline $\mathrm{Fo}$ & 12.0 & 15.2 & 19.2 & 10.7 & 13.9 & 15.0 & 10.8 & 24.1 & 14.1 & 11.1 & 14.0 & 125 \\
\hline Al & .65 & .98 & 82 & 1.12 & .73 & .74 & 32 & 2.06 & .70 & so & .90 & .80 \\
\hline Co & .450 & .720 & .730 & .320 & .500 & .620 & 340 & 580 & 1.09 & 840 & 1.09 & 1.05 \\
\hline $\mathrm{Ni}$ & $\$ 70$ & .540 & .490 & .440 & .410 & .470 & .270 & 340 & 570 & .600 & .460 & 550 \\
\hline $\mathrm{Cu}$ & .184 & .116 & .116 & .184 & .126 & .126 & .107 & .155 & .057 & .098 & .026 & .050 \\
\hline Ca & 11.6 & 3.12 & 289 & 11.6 & 9.82 & 4.79 & 143 & 530 & 257 & - & 2.25 & 2.61 \\
\hline $\mathbf{P}$ & 227 & 54 & so & 2.38 & 1.76 & 24 & 2.66 & 54 & .37 & .87 & 30 & 30 \\
\hline$P b(p p m)$ & 1200 & 1600 & 1700 & 1300 & 1600 & 1500 & 1700 & 1700 & 1700 & 1300 & 1700 & 1600 \\
\hline Sr & • & - & - & - & - & - & - & - & 1300 & 1400 & 1200 & 1200 \\
\hline $\mathbf{Z n}$ & 870 & 920 & 830 & 810 & 750 & 790 & 650 & 920 & 210 & 820 & 620 & 720 \\
\hline Samplod & Old & Bulk & Young & Old & Bulk & Youns & add & Bulk & Young-Top & Old-Bottom & Young & Middle \\
\hline Inorval & $\cdot$ & - & - & $\cdot$ & - & - & - &. & $0-20 \mathrm{~mm}$ & $0-20 \mathrm{mon}$ & $0-10 \mathrm{~mm}$ & $0-10 \mathrm{~mm}$ \\
\hline
\end{tabular}


Table 5a. Composition of ferromanganese crusts analyzed onboard R/N Sonne Midpac2a and Midpac2b by XRF(cont'd).

\begin{tabular}{|c|c|c|c|c|c|c|c|c|c|c|c|c|}
\hline Sequencell & 053104 & 053109 & 053109 & 053109 & 053110 & 053110 & 053113 & 053116 & 053116 & 053116 & 053117 & 053117 \\
\hline Latitudo & 14.456 & 15.597 & 15.597 & 15.597 & 15563 & 15.563 & 15.675 & 15.544 & 15.544 & 15.544 & 15.615 & 15.615 \\
\hline Longitude & -169.003 & -169.232 & -169.232 & -169.232 & -169.224 & -169.224 & -169.124 & -169.238 & -169.238 & -169.238 & -169.227 & -169.227 \\
\hline $\begin{array}{l}\text { Dopth } \\
\text { Lab }\end{array}$ & $\begin{array}{c}1770 \\
\text { CXB-151 }\end{array}$ & $\begin{array}{c}1630 \\
\text { CKB-206 }\end{array}$ & $\begin{array}{c}1630 \\
\text { CKB-207 }\end{array}$ & $\begin{array}{c}1630 \\
\text { CKB-208 }\end{array}$ & $\begin{array}{c}1651 \\
\text { CX8-211 } \\
\end{array}$ & $\begin{array}{c}1651 \\
\text { CXB-212 } \\
\end{array}$ & $\begin{array}{c}3443 \\
\text { CKB-213 }\end{array}$ & $\begin{array}{c}1769 \\
\text { CXB-214 }\end{array}$ & $\begin{array}{c}1769 \\
\text { CXB-214 } \\
\end{array}$ & $\begin{array}{c}1769 \\
\text { CXB-215 } \\
\end{array}$ & $\begin{array}{c}1498 \\
\text { CKB-224 }\end{array}$ & $\begin{array}{c}1498 \\
\text { CKB-225 }\end{array}$ \\
\hline Si(wt.\$) & 1.88 & 1.95 & 30 & .74 & 209 & 265 & 412 & 3.30 & 3.44 & 3.24 & 3.17 & 96 \\
\hline $\mathbf{T i}$ & 1.16 & 1.01 & .42 & .74 & 87 & .64 & 1.44 & 1.25 & 1.17 & 1.18 & 1.03 & 94 \\
\hline $\mathrm{Mm}$ & 253 & 27.4 & 20.1 & 24.6 & 2.3 & 15.1 & 21.1 & 20.9 & 20.9 & 232 & 38.3 & 32.6 \\
\hline $\mathrm{Pe}$ & 113 & 15.1 & 5.56 & 8.75 & 124 & 9.96 & 18.9 & 17.4 & 175 & 14.4 & 21.0 & 13.2 \\
\hline $\mathbf{A}$ & .80 & .60 & 30 & .10 & .73 & 35 & 99 & 90 & .97 & .65 & 1.17 & 35 \\
\hline Co & 940 & 1.28 & .480 & 890 & 1.25 & 360 & 1.10 & 1.22 & 120 & .770 & 1.49 & 980 \\
\hline $\mathbf{N i}$ & .670 & 530 & .820 & 960 & .680 & .410 & 380 & 570 & .600 & .740 & .840 & 990 \\
\hline $\mathrm{Cu}$ & .082 & .024 & .045 & .119 & .162 & .145 & .058 & .056 & .056 & .126 & .090 & .138 \\
\hline Ca & $2 \pi$ & 2.82 & 128 & 7.48 & 3.20 & 10.4 & 247 & 3.75 & 3.44 & 5.97 & 3.18 & 8.62 \\
\hline $\mathbf{P}$ & .30 & .40 & 3.93 & 1.74 & .41 & 282 & 35 & so & .57 & 1.60 & 42 & 213 \\
\hline Pb(ppon) & 1400 & 1900 & 1300 & 1500 & 1800 & 1400 & 1500 & 1700 & 1700 & 1300 & 1900 & 1400 \\
\hline Sx & 1300 & 1300 & 1400 & 1200 & 1200 & 1500 & 1200 & 1200 & 1200 & 1300 & 1300 & 1400 \\
\hline $\mathrm{Zn}$ & 820 & 640 & 1000 & 990 & 800 & 800 & 660 & 670 & 640 & 790 & 810 & 1000 \\
\hline $\begin{array}{l}\text { Semplod } \\
\text { Interval }\end{array}$ & $\begin{array}{l}\text { Old } \\
\text { O-10mm }\end{array}$ & $\begin{array}{c}\text { Youne-Top } \\
\text { O-20mman }\end{array}$ & $\begin{array}{l}\text { Old-Top } \\
20-30 \mathrm{mmm}\end{array}$ & $\begin{array}{c}\text { Bottom } \\
\text { Smm }\end{array}$ & $\begin{array}{l}\text { Youns } \\
0-5 \text { mm }\end{array}$ & $\begin{array}{c}\text { Old } \\
\text { 5-15mm }\end{array}$ & $\begin{array}{c}\text { Bulk } \\
\text { 0-15imm }\end{array}$ & $\underset{15 \mathrm{~mm}}{T o p}$ & $\begin{array}{c}\text { Top } \\
15 \mathrm{cmm}\end{array}$ & $\begin{array}{l}\text { Bottom } \\
15 \mathrm{~mm}\end{array}$ & $\begin{array}{l}\text { Young } \\
\text { 10mm }\end{array}$ & $\begin{array}{l}\text { Old } \\
20 \mathrm{~mm}\end{array}$ \\
\hline
\end{tabular}

\begin{tabular}{|c|c|c|c|c|c|c|c|c|c|c|c|c|}
\hline Sequencell & 053118 & 053118 & 053119 & 053119 & 053128 & 053128 & 053128 & 053128 & 053128 & 053128 & 053129 & 053129 \\
\hline Letitude & 15.584 & 15.584 & 15.628 & 15.628 & 14,462 & 14.462 & 14.462 & 14462 & 14.462 & 14.462 & 14,456 & 14.456 \\
\hline Longitude & -169.205 & -169.205 & -169.239 & -169.239 & -169.017 & -169.017 & -169.017 & -169.017 & .169 .017 & -169.017 & -169.003 & -169.003 \\
\hline Dopth & 1605 & 1605 & 1552 & 1552 & 2110 & 2110 & 2110 & 2110 & 2110 & 2110 & 1770 & 1770 \\
\hline Lab & CKB-228 & CXB-229 & CKB-230 & CKB-231 & CKB-143 & CKB-144 & CKB-145 & CKB-146 & CKB-147 & CKB-148 & CKB-152 & CKB-153 \\
\hline Si(wt.\%) & 3.01 & 1.34 & 2.15 & .28 & 3.30 & 3.35 & 277 & 200 & 1.74 & .98 & 2.42 & 2.15 \\
\hline $\mathbf{T i}$ & 1.01 & .65 & 97 & .63 & 93 & 1.05 & 1.14 & 1.02 & 1.10 & 54 & 94 & .67 \\
\hline Mn & 36.0 & 21.8 & 37.0 & 30.5 & 23.8 & 23.4 & 23.4 & 24.3 & 25.0 & 11.0 & 25.1 & 19.3 \\
\hline Fe & 20.5 & 13.7 & 19.8 & 10.5 & 15.1 & 145 & 13.7 & 121 & 11.6 & 6.76 & 11.4 & 7.81 \\
\hline $\mathbf{A}$ & 1.03 & 30 & .97 & 30 & 90 & 1.10 & 1.00 & .80 & .80 & so & 1.10 & .70 \\
\hline Co & 1.47 & 450 & 156 & 810 & 1.03 & 890 & .700 & 1.04 & .880 & 320 & 1.03 & 510 \\
\hline $\mathrm{Ni}$ & .690 & 530 & .740 & 1.040 & 480 & 560 & 560 & 560 & .630 & .380 & .750 & 1.060 \\
\hline $\mathrm{Cu}$ & .042 & .099 & .042 & .110 & .046 & .078 & .133 & .055 & .089 & .100 & .110 & .248 \\
\hline c & 3.25 & 143 & 3.29 & 11.4 & 232 & 257 & 284 & 274 & 2.75 & 11.3 & 2.67 & 5.79 \\
\hline $\mathbf{P}$ & .47 & 4.74 & .46 & 3.72 & 36 & .12 & .13 & 31 & .34 & 4.00 & 40 & 1.73 \\
\hline$P b(p p m)$ & 2000 & 1600 & 1900 & 1300 & 1600 & 1500 & • & 1600 & 1500 & 1200 & 1700 & 1400 \\
\hline Sx & 1300 & 1700 & 1300 & 1400 & 1200 & 1300 & 1400 & 1200 & 1300 & 1600 & 1200 & 1100 \\
\hline $\mathbf{Z n}$ & 670 & 810 & 700 & 980 & 670 & 740 & 800 & 700 & 790 & 680 & 850 & 1800 \\
\hline $\begin{array}{l}\text { Semplod } \\
\text { Intarval }\end{array}$ & $\begin{array}{l}\text { Youns } \\
25 \text { mon }\end{array}$ & $\begin{array}{l}\text { Old } \\
20 \mathrm{~mm}\end{array}$ & $\begin{array}{l}\text { Young-Top } \\
20 \mathrm{~mm}\end{array}$ & $\begin{array}{l}\text { Old-Bottom } \\
30 \mathrm{~mm}\end{array}$ & $\begin{array}{c}\text { Top } \\
10 \mathrm{~mm}\end{array}$ & $\begin{array}{c}\text { Young-Top } \\
\text { 15mon }\end{array}$ & $\begin{array}{c}\text { Young-Bottom } \\
15 \text { mom }\end{array}$ & $\underset{20 \mathrm{cmm}}{\text { ald }}$ & $\begin{array}{l}\text { Young } \\
\text { 10ming }\end{array}$ & $\begin{array}{l}\text { Old } \\
10 \mathrm{~mm}\end{array}$ & $\begin{array}{c}\text { Old } \\
10 \mathrm{~mm}\end{array}$ & $\begin{array}{l}\text { Bottom } \\
10 \text { omm }\end{array}$ \\
\hline
\end{tabular}


Table 5a. Composition of ferromanganese crusts analyzed onboard R/V Sonne Midpac2a and Midpac2b by XRF(cont'd).

\begin{tabular}{|c|c|c|c|c|c|c|c|c|c|c|c|c|}
\hline Sequencell & 053130 & 053130 & 053131 & 053131 & 053131 & 053131 & 053131 & 053132 & 053132 & 053132 & 053133 & 053133 \\
\hline Latimade & 15.597 & 15.597 & 15.544 & 15.544 & 15.544 & 15.544 & 15.544 & 15.544 & 15.544 & 15.544 & 15.615 & 15.615 \\
\hline Longitude & -169.232 & -169.232 & -169.238 & -169.238 & -169.238 & -169.238 & -169.238 & -169.238 & -169.238 & -169.238 & -169.227 & -169.227 \\
\hline $\begin{array}{l}\text { Dopth } \\
\text { Leby }\end{array}$ & $\begin{array}{c}1630 \\
\text { CKR-209 }\end{array}$ & $\begin{array}{c}1630 \\
\text { CKB-210 }\end{array}$ & $\begin{array}{c}1769 \\
\text { CKB-216 }\end{array}$ & $\begin{array}{c}1769 \\
\text { CKB-217 }\end{array}$ & $\begin{array}{c}1769 \\
\text { CKB-218 }\end{array}$ & $\begin{array}{c}1769 \\
\text { CKB-219 }\end{array}$ & $\begin{array}{c}1769 \\
\text { CKB-220 }\end{array}$ & $\begin{array}{c}1769 \\
\text { CKB-221 }\end{array}$ & $\begin{array}{c}1769 \\
\text { CKB-222 }\end{array}$ & $\begin{array}{c}1769 \\
\text { CKRB-223 }\end{array}$ & $\begin{array}{c}1498 \\
\text { CKB-226 }\end{array}$ & $\begin{array}{c}1498 \\
\text { CKB-227 }\end{array}$ \\
\hline Si(wt.\%) & 250 & .58 & 3.03 & 3.30 & .98 & 1.25 & 3.06 & 3.82 & 2.79 & 1.13 & 2.36 & 30 \\
\hline Ti & 1.17 & .56 & 1.04 & 1.21 & .76 & 46 & 99 & 1.03 & 1.20 & .65 & 1.18 & 16 \\
\hline Mn & 282 & 174 & 30.6 & 345 & 31.7 & 21.1 & 33.7 & 363 & 38.2 & 20.8 & 37,4 & 26.1 \\
\hline Fo & 13.6 & 6.61 & 18.4 & 20.7 & 13.9 & 120 & 17.0 & 223 & 20.9 & 14.1 & 17.0 & 8.77 \\
\hline $\mathbf{A}$ & .75 & .30 & 85 & 1.08 & .30 & 20 & 1.23 & 1.09 & 1.09 & 30 & 1.12 & .20 \\
\hline Co & 1.28 & $\$ 90$ & 1.20 & 1.36 & .790 & 330 & 1.07 & 1.64 & 1.41 & .720 & 1.59 & .700 \\
\hline $\mathbf{N i}$ & .640 & .740 & 590 & .770 & 1.020 & .600 & 1350 & .600 & .800 & 910 & 910 & 1.140 \\
\hline $\mathrm{Cu}$ & .055 & .103 & .042 & .097 & .136 & .100 & 313 & .031 & .069 & .107 & .076 & .120 \\
\hline c. & 2.91 & 125 & 3.32 & 3.84 & 10.7 & 16.3 & 6.03 & 3.63 & 3.57 & 11.9 & 3.20 & 14.6 \\
\hline $\mathbf{P}$ & 36 & 3.84 & 58 & .41 & 328 & 6.05 & 1.66 & 31 & $\mathbf{A 8}$ & 3.95 & .34 & 5.16 \\
\hline$P(p p e n)$ & 1800 & 1200 & 1800 & 1700 & 1300 & 1300 & 1500 & 2000 & 1900 & 1400 & 1900 & 1100 \\
\hline St & 1300 & 1500 & 1200 & 1300 & 1400 & 1600 & 1100 & 1300 & 1300 & 1500 & 1200 & 1400 \\
\hline $\begin{array}{l}\text { Samplod } \\
\text { Intarval }\end{array}$ & $\begin{array}{l}\text { Young } \\
\text { 15mm }\end{array}$ & $\begin{array}{c}\text { Old } \\
15 \text { man }\end{array}$ & Young-Top & Top-Midde & $\begin{array}{l}\text { Old-Top } \\
10 \mathrm{~mm}\end{array}$ & $\begin{array}{l}\text { Old-Botrom } \\
10 \mathrm{~mm}\end{array}$ & $\begin{array}{c}\text { Youns-Bottam } \\
10 \mathrm{~mm}\end{array}$ & $\begin{array}{l}\text { Youns } \\
\text { 15romm }\end{array}$ & $\begin{array}{l}\text { Middlo } \\
15 \mathrm{~mm}\end{array}$ & $\begin{array}{l}\text { Old } \\
40 \mathrm{~mm}\end{array}$ & $\begin{array}{l}\text { Youns-Top } \\
20 \mathrm{~mm}\end{array}$ & $\begin{array}{l}\text { Old-Top } \\
\text { 30mm }\end{array}$ \\
\hline
\end{tabular}

\begin{tabular}{|c|c|c|c|c|c|c|c|c|c|c|c|c|}
\hline Sequencell & 053135 & 053135 & 053135 & 053135 & 053136 & 053136 & 053136 & 053137 & 053137 & 054019 & 054021 & 054021 \\
\hline Latitudo & 15.628 & 15.628 & 15.628 & 15.628 & 15.628 & 15.628 & 15.628 & 15.628 & 15.628 & 15.696 & 15.683 & 15.683 \\
\hline Langituds & -169.239 & -169.239 & -169.239 & -169.239 & -169.239 & -169.239 & -169.239 & -169.239 & -169.239 & -170.344 & -170.359 & -170.359 \\
\hline Dopth & 1552 & 1552 & 1552 & 1552 & 1552 & 1552 & 1552 & 1552 & 1552 & 2260 & 1628 & 1628 \\
\hline Lub* & CKB-232 & CKB-233 & CKB-234 & CKB-235 & CKB-236 & CKB-236 & CKB-237 & CKB-239 & CKB-240 & CKB-154 & CKB-157 & CKB-158 \\
\hline Si(wt.8) & 288 & .21 & 1.99 & 45 & 2.31 & 2.33 & .43 & 2.67 & 1.71 & 4.39 & 2.33 & 214 \\
\hline $\mathbf{T i}$ & 1.13 & .48 & 1.16 & .44 & 1.01 & 1.03 & .67 & 1.05 & .98 & 1.11 & .97 & 1.10 \\
\hline Mn & 37.2 & 24.0 & 37.4 & 22.1 & 36.2 & 36.7 & 20.2 & 36.7 & 32.9 & 21.8 & 255 & 27.6 \\
\hline $\mathbf{P e}$ & 19.4 & 10.1 & 17.2 & 10.3 & 19.6 & 19.2 & 10.9 & 19.6 & 15.4 & 14.7 & 12.2 & 14.7 \\
\hline $\mathbf{N}$ & 1.10 & .35 & 1.19 & 30 & 1.20 & 1.09 & 35 & 1.00 & .15 & 1.02 & .56 & .96 \\
\hline$C_{0}$ & 1.35 & .460 & 1.31 & 420 & 153 & 1.53 & .760 & 1.44 & .840 & .870 & 800 & 1.07 \\
\hline $\mathrm{Ni}$ & .830 & .800 & .930 & .760 & 800 & .800 & 1.000 & .700 & .900 & 500 & .690 & .590 \\
\hline Cr & .078 & .087 & .144 & .107 & .064 & .065 & .115 & .045 & .117 & .067 & .088 & .051 \\
\hline ca & 3.51 & 15.4 & 3.42 & 15.0 & 3.43 & 3.31 & 125 & 3.28 & 7.64 & 243 & 4.93 & 2.72 \\
\hline $\mathbf{P}$ & 47 & 5.05 & 38 & 5.53 & .48 & .43 & 3.70 & .42 & 2.10 & 35 & 1.17 & 37 \\
\hline Pb(ppon) & 1900 & 1400 & 1800 & 1200 & 1900 & 1900 & 1200 & 1900 & 1300 & 1600 & 1600 & 1700 \\
\hline Sx & 1300 & 1500 & 1300 & 1500 & 1300 & 1300 & 1400 & 1300 & 1400 & 1200 & 1300 & 1300 \\
\hline $\mathbf{z a}$ & 820 & 940 & 920 & 930 & 750 & 750 & 1000 & 710 & 900 & 680 & 820 & 730 \\
\hline $\begin{array}{l}\text { Semplod } \\
\text { Imerval }\end{array}$ & $\begin{array}{l}\text { Youns Top } \\
10 \mathrm{~mm}\end{array}$ & $\begin{array}{l}\text { Old-Top } \\
10 \mathrm{~mm}\end{array}$ & $\begin{array}{c}\text { Young-Bottom } \\
7 \operatorname{man}\end{array}$ & $\begin{array}{l}\text { Old-Bottom } \\
7 \mathrm{~mm}\end{array}$ & $\begin{array}{l}\text { Young-Top } \\
\text { 15romm }\end{array}$ & $\begin{array}{l}\text { Young-Top } \\
\text { 15men }\end{array}$ & $\begin{array}{l}\text { Old-Bottom } \\
25 \mathrm{~mm}\end{array}$ & $\begin{array}{l}\text { Young } \\
15 \text { mm }\end{array}$ & $\begin{array}{l}\text { Old } \\
15 \mathrm{~mm}\end{array}$ & $\begin{array}{c}\text { Bulk } \\
10 \mathrm{~mm}\end{array}$ & $\begin{array}{l}\text { Top } \\
10 \mathrm{~mm}\end{array}$ & $\begin{array}{l}\text { Middle } \\
10 \mathrm{~mm}\end{array}$ \\
\hline
\end{tabular}


Table 5a. Composition of ferromanganese crusts analyzed onboard R/V Sonne Midpac2a and Midpac2b by XRF(cont'd).

\begin{tabular}{|c|c|c|c|c|c|c|c|c|c|c|c|c|}
\hline $\begin{array}{l}\text { Sequencent } \\
\text { Latitade }\end{array}$ & $\begin{array}{l}054021 \\
15.683\end{array}$ & $\begin{array}{l}054022 \\
15.649\end{array}$ & $\begin{array}{l}054022 \\
15.649\end{array}$ & $\begin{array}{l}054022 \\
15.649\end{array}$ & $\begin{array}{l}054023 \\
15.671\end{array}$ & $\begin{array}{l}054023 \\
15.671\end{array}$ & $\begin{array}{l}054023 \\
15.671\end{array}$ & $\begin{array}{l}054024 \\
15.664\end{array}$ & $\begin{array}{l}054024 \\
15.664\end{array}$ & $\begin{array}{l}054025 \\
15.673\end{array}$ & $\begin{array}{l}054025 \\
15.673\end{array}$ & $\begin{array}{l}054025 \\
15.673\end{array}$ \\
\hline Longitude & -170359 & -170.413 & -170.413 & -170.413 & -170.413 & -170.413 & -170.413 & -170.397 & -170.397 & -170.381 & -170.381 & -170381 \\
\hline $\begin{array}{l}\text { Depth } \\
\text { Lebis }\end{array}$ & $\begin{array}{c}1628 \\
\text { CKB-159 }\end{array}$ & $\begin{array}{c}2180 \\
\text { CKB-163 }\end{array}$ & $\begin{array}{c}2180 \\
\text { CKB-164 }\end{array}$ & $\begin{array}{c}2180 \\
\text { CKB-165 }\end{array}$ & $\begin{array}{c}1815 \\
\text { CKB-171 }\end{array}$ & $\begin{array}{c}1815 \\
\text { CKB-171 }\end{array}$ & $\begin{array}{c}1815 \\
C \times 8-172\end{array}$ & $\begin{array}{c}1433 \\
\text { CKB-180 }\end{array}$ & $\begin{array}{c}1433 \\
\text { CKB-181 }\end{array}$ & $\begin{array}{c}1117 \\
\text { CKB-184 }\end{array}$ & $\begin{array}{c}1117 \\
\text { CKB-185 }\end{array}$ & $\begin{array}{c}1117 \\
\text { CKB-185 }\end{array}$ \\
\hline $\operatorname{si}(w t \%)$ & .50 & 4.60 & 5.72 & 1.36 & 3.49 & 350 & 3.28 & 2.20 & $<.50$ & 1.15 & so & .60 \\
\hline $\mathbf{T i}$ & .47 & $1 . \infty 9$ & 1.22 & .67 & 95 & .98 & 1.24 & .87 & .33 & .78 & .43 & .41 \\
\hline Mn & 163 & 23.8 & 23.0 & 19.7 & 26.5 & 27.3 & 27.0 & 28.8 & 15.5 & 29.9 & 16.8 & 175 \\
\hline Fo & 752 & 17.4 & 169 & 10.2 & 15.7 & 16.1 & 14.2 & 14.0 & 5.67 & 10.3 & 6.51 & 6.50 \\
\hline $\mathbf{A} \mathbf{l}$ & $<50$ & 1.07 & 1.60 & 50 & 1.03 & 1.02 & 1.12 & .66 & $<.30$ & .70 & $<30$ & $<30$ \\
\hline Co & 330 & 1.07 & 970 & .600 & 1.07 & 1.12 & 810 & 1.22 & $A 40$ & 1.15 & 360 & 370 \\
\hline $\mathrm{Ni}$ & 560 & .420 & 530 & .650 & 540 & .560 & .680 & .650 & .600 & .760 & 540 & 550 \\
\hline $\mathrm{Cu}$ & .040 & .047 & .098 & .137 & .034 & .034 & .104 & .040 & .050 & .066 & .022 & .023 \\
\hline Ca & 127 & 2.88 & 2.46 & 9.28 & 260 & 271 & 3.62 & 4.23 & 16.0 & 4.10 & 13.9 & 14.2 \\
\hline $\mathbf{P}$ & 4.28 & .38 & 32 & 2.97 & 37 & 40 & .63 & 92 & 6.01 & .61 & 5.13 & 4.84 \\
\hline $\mathrm{Pb}$ (ppm) & 1300 & 1600 & 1500 & 1000 & 1700 & 1800 & 1500 & 1800 & 1300 & 1700 & 1700 & 1700 \\
\hline Sx & 1500 & 1300 & 1200 & 1400 & 1300 & 1300 & 1300 & 1300 & 1700 & 1200 & 1500 & 1600 \\
\hline $2 n$ & 830 & 650 & 760 & 780 & 660 & 680 & 860 & 700 & 810 & 770 & 690 & 720 \\
\hline Sampled & Bottom & Top & Middle & Bottom & Top & Top & Bottom & Top & Bottan & Young-Top & Old-Top & Old-Top \\
\hline Interval & $10 \mathrm{~mm}$ & $10 \mathrm{~mm}$ & $10 \mathrm{~mm}$ & $10 \mathrm{~mm}$ & $20 \mathrm{nom}$ & $20 \mathrm{~mm}$ & $20 \mathrm{mem}$ & $25 \mathrm{~mm}$ & $25 \operatorname{men}$ & $15 \mathrm{~mm}$ & 15 mem & $15 \operatorname{mm}$ \\
\hline
\end{tabular}

\begin{tabular}{|c|c|c|c|c|c|c|c|c|c|c|c|c|}
\hline Sequencent & 054025 & 054031 & 054032 & 054032 & 054032 & 054033 & 054033 & 054034 & 054034 & 054034 & 054036 & 054036 \\
\hline Lationde & 15.673 & 15.631 & 15.637 & 15.637 & 15.637 & 15.658 & 15.658 & 15.665 & 15.665 & 15.665 & 15.696 & 15.696 \\
\hline Langituds & -170381 & -170.402 & -170.406 & -170.406 & -170.406 & -170.385 & -170.385 & -170.386 & -170.386 & -170.386 & -170.344 & -170.344 \\
\hline Depth & 1117 & 2200 & 1770 & 1770 & 1770 & 1186 & 1186 & 1520 & 1520 & 1520 & 2260 & 2260 \\
\hline Labu & CKB-186 & CKB-187 & CKB-188 & CKB-189 & CKB-190 & CKB-194 & CKB-195 & CXB-198 & CKB-199 & CKB-200 & CKB-155 & CKB-156 \\
\hline Si(wts) & 1.04 & 3.19 & 4.77 & 4.00 & 222 & .78 & .30 & 95 & .31 & $\boldsymbol{2 0}$ & 3.23 & 3.89 \\
\hline $\mathrm{Ti}$ & .78 & 1.24 & 1.02 & 1.24 & 1.25 & .89 & .49 & .79 & .67 & .11 & 1.11 & 1.16 \\
\hline Mn & 30.9 & 283 & 26.1 & 28.4 & 30.0 & 329 & 23.4 & 33.3 & 25.9 & 4.64 & 24.7 & 24.9 \\
\hline Fo & 13.4 & 16.3 & 18.0 & 17.5 & 143 & 129 & 7.73 & 16.2 & 10.7 & 55 & 15.6 & 15.4 \\
\hline Al & 1.06 & .95 & 1.06 & 1.03 & 91 & .85 & .30 & .68 & .30 & $<30$ & .83 & 1.12 \\
\hline Co & 1.44 & 1.000 & 1.14 & .970 & 910 & 1.45 & 520 & 1.66 & .880 & .060 & 1.14 & 1.03 \\
\hline $\mathrm{Ni}$ & .700 & 580 & .450 & .620 & .720 & .760 & .770 & .620 & .710 & .410 & .480 & 580 \\
\hline $\mathrm{Cu}$ & .039 & .078 & .028 & .091 & .158 & .034 & .020 & .022 & .052 & .045 & .042 & .087 \\
\hline ca & 3.18 & 3.13 & 279 & 280 & 3.83 & 289 & 10.7 & 3.09 & 8.98 & 20.6 & 299 & 2.66 \\
\hline $\mathbf{P}$ & .56 & .34 & .40 & 39 & .63 & 36 & 3.50 & .47 & 2.71 & 8.54 & .40 & .34 \\
\hline Pb(ppon) & 1900 & 1500 & 1700 & 1500 & 1300 & 1900 & 1900 & 1900 & 1700 & 5100 & 1600 & 1500 \\
\hline St & 1300 & 1300 & 1300 & 1300 & 1400 & 1200 & 1400 & 1300 & 1300 & 2100 & 1300 & 1200 \\
\hline $2 n$ & 680 & 750 & 610 & 690 & 830 & 680 & 840 & 570 & 740 & 790 & 650 & 720 \\
\hline $\begin{array}{l}\text { Sampied } \\
\text { Interval }\end{array}$ & $\begin{array}{c}\text { Young-Bottom } \\
8 \mathrm{~mm}\end{array}$ & $\begin{array}{c}\text { Bull } \\
\text { 25mm }\end{array}$ & $\begin{array}{c}\text { Top } \\
10 \mathrm{~mm}\end{array}$ & $\begin{array}{l}\text { Middlo } \\
10 \mathrm{~mm}\end{array}$ & $\begin{array}{c}\text { Botom } \\
-\end{array}$ & $\begin{array}{c}\text { Top } \\
20 \mathrm{~mm}\end{array}$ & $\begin{array}{l}\text { Bottom } \\
20 \mathrm{~mm}\end{array}$ & $\begin{array}{c}\text { Young } \\
-\end{array}$ & Old & Old & $\begin{array}{c}\text { Youmg-Top } \\
10 \mathrm{~mm}\end{array}$ & $\begin{array}{l}\text { Bottom } \\
10 \mathrm{~mm}\end{array}$ \\
\hline
\end{tabular}


Table 5a. Composition of ferromanganese crusts analyzed onboard $R / V$ Sonne Midpac2a and Midpac2b by XRF(cont'd).

\begin{tabular}{|c|c|c|c|c|c|c|c|c|c|c|c|c|}
\hline Sequence" & 054037 & 054037 & 054037 & 054038 & 054038 & 054038 & 054038 & 054038 & 054039 & OS4039 & 054040 & 054040 \\
\hline Letimde & 15.683 & 15.683 & 15.683 & 15.649 & 15.649 & 15.649 & 15.649 & 15.649 & 15.671 & 15.671 & 15.671 & 15.671 \\
\hline Longimds & -170.359 & -170.359 & .170 .359 & -170.413 & -170.413 & -170.413 & -170.413 & -170.413 & -170413 & -170.413 & -170.413 & -170.413 \\
\hline $\begin{array}{l}\text { Depth } \\
\text { Labu }\end{array}$ & $\begin{array}{c}1628 \\
\text { CKB-160 }\end{array}$ & $\begin{array}{c}1628 \\
\text { CKB-161 }\end{array}$ & $\begin{array}{c}1628 \\
\text { CKB-162 }\end{array}$ & $\begin{array}{c}2180 \\
\text { CKB-166 }\end{array}$ & $\begin{array}{c}2180 \\
\text { CKB-167 }\end{array}$ & $\begin{array}{c}2180 \\
\text { CKB-168 }\end{array}$ & $\begin{array}{c}2180 \\
\text { CKB-169 }\end{array}$ & $\begin{array}{c}2180 \\
\text { CKB-170 }\end{array}$ & $\begin{array}{c}1815 \\
\text { CKB-173 }\end{array}$ & $\begin{array}{c}1815 \\
\text { CKB-174 }\end{array}$ & $\begin{array}{c}1815 \\
\text { CKB-175 }\end{array}$ & $\begin{array}{c}1815 \\
\text { CKB-176 } \\
\end{array}$ \\
\hline Si(wts) & 3.62 & 2.36 & 1.65 & 3.68 & 3.36 & 2.13 & 1.72 & .60 & 289 & 284 & 3.54 & 3.03 \\
\hline $\mathbf{T i}$ & 1.03 & .92 & 43 & 1.08 & 1.25 & 1.09 & .59 & so & 1.07 & 95 & 94 & 1.13 \\
\hline Mn & 26.0 & 27.5 & 13.2 & 24.6 & 25.2 & 25.3 & 14.4 & 16.3 & 27.0 & 22.3 & 27.6 & 27.7 \\
\hline Fo & 14.7 & 16.0 & 7.77 & 16.9 & 15.7 & 12.5 & 10.7 & 8.19 & 14.7 & 11.4 & 16.0 & 14.7 \\
\hline A & 1.15 & .96 & so & $n$ & 99 & .48 & $<50$ & $<30$ & 1.09 & 34 & .82 & 95 \\
\hline Co & 1.15 & 1.29 & 500 & 1.11 & 970 & .710 & .330 & .430 & 990 & .670 & 1.10 & .830 \\
\hline $\mathrm{Ni}$ & .640 & 540 & .670 & .420 & 520 & .640 & 380 & .340 & .650 & .700 & .570 & .740 \\
\hline $\mathrm{Cr}$ & .075 & .028 & .117 & .031 & .007 & .114 & .099 & .142 & .080 & .170 & .041 & .109 \\
\hline c. & 254 & 288 & 11.4 & 246 & 282 & 6.06 & 11.8 & 13.0 & 2.75 & 6.71 & 260 & 3.13 \\
\hline $\mathbf{P}$ & 37 & 54 & 4.33 & .41 & .30 & 1.33 & 4.23 & 4.40 & .37 & 1.62 & 31 & $A 7$ \\
\hline $\mathrm{Pb}(\mathrm{ppm})$ & 1800 & 1900 & 1200 & 1800 & 1500 & 1200 & 1100 & 1500 & 1600 & 1300 & 1700 & 1400 \\
\hline st & 1200 & 1300 & 1500 & 1400 & 1300 & 1400 & 1600 & 1600 & 1200 & 1300 & 1200 & 1200 \\
\hline $\mathbf{Z n}$ & 760 & 650 & 860 & 640 & 720 & 840 & 700 & 690 & 760 & 930 & 700 & 850 \\
\hline Samplad & Young-Top & Old-Top & Botom & - & - & $\cdot$ & $\cdot$ & - & $T_{o p}$ & Botrom & $T c p$ & Botom \\
\hline Interval & $20 \mathrm{mom}$ & $20 \mathrm{~mm}$ & $10 \mathrm{mmn}$ & - & - & - & - & - & $20 \mathrm{mon}$ & $20 \mathrm{~mm}$ & $20 \mathrm{~mm}$ & $10 \mathrm{~mm}$ \\
\hline
\end{tabular}

\begin{tabular}{|c|c|c|c|c|c|c|c|c|c|c|c|c|}
\hline Sequence" & 054041 & 054041 & 054041 & 054042 & 054042 & 054043 & 054043 & 054043 & 054044 & 054044 & 054045 & 054045 \\
\hline Latioude & 15.671 & 15.671 & 15.671 & 15.664 & 15.664 & 15.637 & 15.637 & 15.637 & 15.658 & 15.658 & 15.665 & 15.665 \\
\hline
\end{tabular}

\begin{tabular}{|c|c|c|c|c|c|c|c|c|c|c|c|c|}
\hline si(wt.\%) & 3.49 & 3.24 & 1.25 & .20 & 50 & 3.68 & $n$ & 1.15 & 1.97 & 3.12 & .74 & .20 \\
\hline $\mathbf{T i}$ & 1.00 & 1.06 & .79 & .35 & $A 4$ & 1.14 & .65 & 51 & .94 & 1.19 & 95 & .65 \\
\hline $\mathrm{Mn}$ & 275 & 27.7 & 23.4 & 9.76 & 19.2 & 28.7 & 21.8 & 15.8 & 30.9 & 29.6 & 29.0 & 255 \\
\hline Fo & 16.4 & 16.1 & 115 & 5.08 & 7.61 & 16.2 & 9.03 & 9.16 & 15.0 & 15.0 & 120 & 6.16 \\
\hline A & 1.13 & .87 & .30 & $<30$ & $<30$ & 1.04 & .30 & 30 & .75 & 89 & 58 & 35 \\
\hline Co & 1.15 & 940 & 550 & .220 & $A 10$ & 1.10 & 540 & .200 & 1.29 & 1.09 & 1.31 & .880 \\
\hline $\mathrm{Ni}$ & 550 & .600 & .640 & .440 & .600 & .640 & 810 & $A 50$ & .620 & .630 & .640 & 970 \\
\hline $\mathrm{Cr}$ & .034 & .065 & .101 & .050 & .034 & .060 & .103 & .073 & .086 & .078 & .043 & .066 \\
\hline c & 293 & 2.87 & 8.58 & 17.2 & 13.1 & 275 & 10.1 & 12.6 & 3.27 & 3.24 & 3.38 & 8.64 \\
\hline $\mathbf{P}$ & .49 & .38 & 2.25 & 5.57 & 455 & 32 & 3.02 & 3.42 & 30 & .36 & 33 & 227 \\
\hline$P(p p m)$ & 1800 & 1600 & 1300 & 1200 & 1500 & 1700 & 1100 & 1100 & 1800 & 1800 & 1800 & 1400 \\
\hline St & 1300 & 1300 & 1500 & 1800 & 1500 & 1200 & 1400 & 1600 & 1300 & 1300 & 1300 & 1300 \\
\hline $\mathbf{Z n}$ & 700 & 730 & 830 & 790 & 850 & 740 & 920 & 700 & 7000 & 7000 & 700 & 1000 \\
\hline Samplod & Top & Middle & Botrom & - & Old & Top & Middle & Bottom & Top & Bottom & Top & Bottom \\
\hline Interval & $10 \mathrm{~mm}$ & $10 \mathrm{~mm}$ & $30 \mathrm{~mm}$ & . & - & $15 \mathrm{~mm}$ & $10 \mathrm{~mm}$ & $20 \mathrm{~mm}$ & $15 \mathrm{~mm}$ & $15 \mathrm{~mm}$ & $20 \mathrm{~mm}$ & $\checkmark$ \\
\hline
\end{tabular}


Table 5a. Composition of ferromanganese crusts analyzed onboard R/V Sonne Midpac2a and Midpac2b by XRF(cont'd).

\begin{tabular}{|c|c|c|c|c|c|c|c|c|c|c|c|c|}
\hline Sequencel & 054046 & 054046 & 054046 & 090011 & 090011 & 090011 & 090012 & 090012 & 090012 & 090013 & 090014 & 090015 \\
\hline Lationde & 15.665 & 15.665 & 15.665 & 20.133 & 20.133 & 20.133 & 20.133 & 20.133 & 20.133 & 20.222 & 20.222 & 20.218 \\
\hline $\begin{array}{l}\text { Longitude } \\
\text { Dopth } \\
\text { Lab: }\end{array}$ & $\begin{array}{c}-170.386 \\
1520 \\
\text { CXB-203 }\end{array}$ & $\begin{array}{c}-170.386 \\
1520 \\
\text { CKB-204 }\end{array}$ & $\begin{array}{c}-170.386 \\
1520 \\
\text { CKB-205 } \\
\end{array}$ & $\begin{array}{c}-170.681 \\
1476 \\
C K B-124 \\
\end{array}$ & $\begin{array}{c}-170.681 \\
1476 \\
\text { CKB-125 } \\
\end{array}$ & $\begin{array}{c}-170.681 \\
1476 \\
\text { CK8-126 } \\
\end{array}$ & $\begin{array}{c}-170.681 \\
1476 \\
\text { CKB-12 }\end{array}$ & $\begin{array}{c}-170.681 \\
1476 \\
\text { CKB-128 } \\
\end{array}$ & $\begin{array}{c}-170.681 \\
1476 \\
\text { CKB-120 } \\
\end{array}$ & $\begin{array}{c}-170.600 \\
- \\
\text { CKB-130 } \\
\end{array}$ & $\begin{array}{c}-170.600 \\
- \\
\text { CKB-131 } \\
\end{array}$ & $\begin{array}{c}-170589 \\
1480 \\
\text { CKB-132 } \\
\end{array}$ \\
\hline Si(wts) & 1.50 & .30 & 157 & 3.10 & 2.20 & 140 & 4.20 & 1.50 & 5.10 & 5.90 & 5.60 & 8.10 \\
\hline $\mathrm{Ti}$ & .84 & .49 & 80 & 1.08 & 1.31 & .71 & 1.14 & 94 & 1.62 & 1.46 & 1.58 & .99 \\
\hline Mn & 20.8 & 21.5 & 28.4 & 26.7 & 28.9 & 228 & 24.8 & 26.6 & 27.0 & 24.3 & 24.4 & 23.1 \\
\hline Fo & 10.2 & 5.59 & 11.3 & 18.3 & 14.8 & 8.00 & 18.4 & 9.30 & 18.8 & 19.5 & 20.7 & 19.5 \\
\hline $\mathbf{A}$ & 83 & .30 & .76 & 51 & .42 & .39 & .76 & $A 2$ & 1.86 & 1.28 & 1.05 & 1.86 \\
\hline$C_{0}$ & 1.10 & 590 & 1.22 & 1.07 & .980 & 410 & 1.01 & 580 & .970 & .750 & .760 & .760 \\
\hline $\mathbf{N i}$ & 1.010 & 880 & 850 & .440 & .640 & .790 & 390 & .740 & .470 & .470 & 370 & 360 \\
\hline $\mathrm{Cu}$ & .124 & .062 & .102 & .029 & .058 & .087 & .020 & .136 & .058 & .194 & .058 & .048 \\
\hline C. & 3.39 & 11.1 & 2.77 & 340 & 3.40 & 20.3 & 3.20 & 21.4 & 3.90 & 4.00 & 3.60 & 4.40 \\
\hline $\mathbf{P}$ & 58 & 3.23 & 38 & .68 & .49 & 4.58 & .64 & 4.47 & .63 & .35 & .60 & .81 \\
\hline$P b$ (ppm) & 1600 & 1500 & 1600 & 2500 & 2000 & 1500 & 2400 & 1800 & 2100 & 2100 & 2100 & 2100 \\
\hline Sr & 1100 & 1400 & 1100 & - & - & • & $\cdot$ & - & - & - & - & • \\
\hline $\mathbf{Z n}$ & 1000 & 990 & 780 & 670 & 880 & 1000 & 620 & 1200 & 760 & 910 & 780 & 680 \\
\hline Seropiled & Young-Top & Old-Top & Old-Bottom & Young & Middle & otd & Young & Old & Middle & - & - & Bulk \\
\hline Imtarval & $10 \mathrm{~mm}$ & $20 \mathrm{~mm}$ & $10 \mathrm{~mm}$ & - & • & • & $\cdot$ & - & - & - & - & • \\
\hline
\end{tabular}

\begin{tabular}{|c|c|c|c|c|c|c|}
\hline Sequencell & 090016 & 317010 & 317010 & 317010 & 317011 & 317011 \\
\hline Letitudo & 20.218 & -.388 & -388 & -.388 & -.399 & -.399 \\
\hline Longitude & -170.589 & -176.073 & -176.073 & -176.073 & -176.116 & -176.116 \\
\hline Depth & 1480 & 2107 & 2107 & 2107 & 1551 & 1551 \\
\hline Lab & CKB-133 & CKB-136 & CKB-137 & CKB-138 & CKB-139 & CKB-140 \\
\hline Si(wt\%) & 5.10 & 3.51 & 4.10 & 2.73 & 293 & .69 \\
\hline $\mathbf{T i}$ & 1.62 & 1.43 & 1.67 & 1.32 & .89 & .65 \\
\hline Mn & 26.6 & 28.2 & 26.0 & 31.7 & 32.1 & 24.7 \\
\hline Fe & 19.7 & 20.0 & 19.7 & 19.8 & 19.4 & 10.2 \\
\hline $\mathbf{A}$ & 89 & 1.00 & 1.30 & 80 & $1 . \infty$ & - \\
\hline Co & 1.06 & 1.06 & 1.000 & 1.28 & .780 & .730 \\
\hline $\mathrm{Ni}$ & .410 & 540 & .470 & .650 & .880 & .700 \\
\hline Cu & .038 & .088 & .111 & .078 & .074 & .062 \\
\hline a & 4.00 & 332 & 4.32 & 3.22 & 387 & 11.7 \\
\hline $\mathbf{P}$ & .76 & .58 & .63 & $A 1$ & .83 & 4.03 \\
\hline Pb(ppon) & 2400 & 1500 & 1300 & 1600 & 1500 & 1300 \\
\hline Sr & • & 1400 & 1400 & 1300 & 1400 & 1600 \\
\hline $\mathrm{Zn}$ & 640 & 830 & 900 & 840 & 870 & 930 \\
\hline $\begin{array}{l}\text { Semplod } \\
\text { Itterval }\end{array}$ & Yourng & $\underset{30 \mathrm{mon}}{\text { Bulk }}$ & $\begin{array}{l}\text { Bottom } \\
10 \mathrm{mmn}\end{array}$ & $\underset{15 \mathrm{~mm}}{\text { Top }}$ & $\begin{array}{l}\text { Young } \\
20 \mathrm{~mm}\end{array}$ & $\underset{20 \mathrm{~mm}}{O d d}$ \\
\hline
\end{tabular}


Table 6. Composition of ferromanganese crusts analyzed by Japanese (unpublished data).

\begin{tabular}{|c|c|c|c|c|c|c|c|c|c|c|c|c|}
\hline Sequencell & 094007 & 094008 & 094008 & 094009 & 094009 & 094009 & 094010 & 094011 & 094012 & 094013 & 094013 & 094013 \\
\hline Lationde & 26.430 & 25.987 & 25.987 & 26.166 & 26.166 & 26.166 & 26.164 & 26.081 & 25.934 & 25314 & 25.314 & 25.314 \\
\hline Langinede & 144.280 & 143.499 & 143.499 & 144.252 & 144.252 & 144.252 & 144.182 & 144352 & 144.275 & 143.913 & 143.913 & 143.913 \\
\hline $\begin{array}{l}\text { Dopth } \\
\text { Lab* }\end{array}$ & $\begin{array}{c}2780 \\
\text { D845-6 }\end{array}$ & $\begin{array}{c}2390 \\
\text { D849-10C }\end{array}$ & $\begin{array}{c}2390 \\
\text { D849-10N }\end{array}$ & $\begin{array}{c}905 \\
\text { D851.14 }\end{array}$ & $\begin{array}{c}905 \\
\text { D851-15 }\end{array}$ & $\begin{array}{c}905 \\
\text { D851-18 }\end{array}$ & $\begin{array}{c}790 \\
\text { D853-12 }\end{array}$ & $\begin{array}{c}955 \\
\text { D854-14 }\end{array}$ & $\begin{array}{c}1870 \\
D 856-4\end{array}$ & $\begin{array}{c}1515 \\
\text { D858-1A }\end{array}$ & $\begin{array}{c}1515 \\
\text { D858-1B }\end{array}$ & $\begin{array}{c}1515 \\
\text { D858-1C }\end{array}$ \\
\hline Si(wt.\%) & 5.03 & 5.72 & 257 & 121 & .61 & 2.46 & 1.71 & 3.99 & 8.17 & 2.80 & 272 & 95 \\
\hline Mn & 18.1 & 17.4 & 21.4 & 11.5 & 32.1 & 24.7 & 129 & 23.0 & 14.4 & 21.7 & 24.9 & 242 \\
\hline Po & 18.6 & 17.3 & 11.4 & 7,42 & 730 & 16.4 & 5.48 & 10.2 & 19.4 & 18.9 & 145 & 7.88 \\
\hline $\mathbf{A}$ & 1.27 & 1.50 & 1.05 & .65 & 36 & .75 & $1 \mathrm{A4}$ & 1.90 & 1.86 & 98 & 85 & 35 \\
\hline Co & 530 & 570 & 560 & .740 & sso & 1.11 & .300 & .600 & .340 & .810 & 580 & 460 \\
\hline $\mathbf{N i}$ & 260 & 310 & .570 & .270 & 1.050 & .370 & .480 & .600 & .170 & .310 & .480 & 800 \\
\hline $\mathrm{Cn}$ & .065 & .041 & .100 & .097 & 264 & .060 & .059 & .081 & .088 & .041 & .057 & .097 \\
\hline $\mathrm{H} 2 \mathrm{O}+$ & 8.7 & 8.8 & 8.1 & 5.2 & 9.1 & 10.5 & 6.1 & 9.3 & 8.8 & 10.5 & 9.9 & 7.6 \\
\hline$P b(p p m)$ & 1500 & 1600 & 2800 & 2200 & 3000 & 2600 & 200 & 1700 & 1800 & 2000 & 2200 & 1500 \\
\hline $\mathrm{Zn}$ & 610 & 520 & 870 & 580 & 1200 & 490 & 1300 & 840 & 520 & 560 & 600 & 780 \\
\hline Samplod & Bulk & Bulk & Noculo & Young-Nodule & Young-Nodulo & Bulk & Bulk & Bulk & Bulk & Top & Midalo & Bottam \\
\hline Interval & 8.0 & 6.0 & 6.0 & 35.0 & 17.0 & 10.0 & 20.0 & 20.0 & 6.0 & 15.0 & 30.0 & 30.0 \\
\hline
\end{tabular}

\begin{tabular}{|c|c|c|c|c|c|c|c|c|c|c|c|c|}
\hline Sequencel & 094014 & 094014 & 094015 & 094016 & 094016 & 094017 & 094017 & 094018 & 094019 & 094020 & 094021 & 094022 \\
\hline Latinude & 25.223 & 25.223 & 25.272 & 26.172 & 26.172 & 26.250 & 26.250 & 26.902 & 25.846 & 25.751 & 25.797 & 25.793 \\
\hline Longinde & 143.928 & 143.928 & 144.082 & 145.168 & 145.168 & 145.154 & 145.154 & 145.507 & 147.798 & 147992 & 146.747 & 146.783 \\
\hline Depth & 1560 & 1560 & 2500 & 1250 & 1250 & 1100 & 1100 & 1250 & 1410 & 1375 & 1470 & 1395 \\
\hline Labi & D859-11A & D859-11B & D862-1 & D866-1A & D866-1B & D867-1 & D867.3 & D869-2 & D879-9 & D881-1 & D883-1 & D884-1 \\
\hline Si(wt,\%) & 254 & 4.32 & 5.66 & 11.4 & 582 & 4.16 & .70 & 4.20 & 7.55 & 4.09 & 1.29 & 5.13 \\
\hline Mn & 20.6 & 229 & 159 & 12.4 & 19.1 & 21.4 & 27.6 & 23.3 & 15.1 & 22.5 & 24.8 & 21.5 \\
\hline Fo & 10.9 & 13.8 & 19.5 & 15.9 & 16.4 & 18.2 & 5.98 & 14.0 & 17.6 & 17.1 & 4.77 & 15.2 \\
\hline Al & .67 & 1.52 & 1.53 & 4.79 & 216 & 1.05 & 31 & 1.45 & 2.38 & 85 & .68 & 1.75 \\
\hline Co & 380 & .720 & .330 & .210 & .400 & .560 & .630 & 530 & .730 & .760 & .500 & .670 \\
\hline $\mathrm{Ni}$ & .500 & .550 & 260 & 370 & 390 & .280 & .840 & .770 & .280 & .370 & 1.060 & 330 \\
\hline $\mathrm{Cr}$ & .130 & .081 & .073 & .092 & .074 & .046 & .082 & .070 & .086 & .048 & .151 & .078 \\
\hline $\mathrm{H} 2 \mathrm{O}+$ & 7.5 & 98 & 10.1 & 6.7 & 11.1 & 11.9 & 9.2 & 113 & 9.4 & 11.6 & 9.0 & 11.1 \\
\hline PL(ppon) & 1400 & 1800 & 1500 & 300 & 1800 & 2200 & 1700 & 1600 & 2000 & 2300 & 1600 & 1800 \\
\hline $\mathrm{Zn}_{\mathbf{n}}$ & 630 & 630 & 620 & 700 & 600 & 480 & 780 & 810 & 560 & 470 & 1300 & 600 \\
\hline Sampiod & Bulk & Bulk & Bulk & Top & Bottom & Bulk & Bulk & Nodule & Bulk & Bulk & Bulk & Bulk \\
\hline Irterval & 50.0 & 20.0 & 20.0 & 20.0 & 30.0 & 20.0 & 40.0 & 15.0 & 30.0 & 20.0 & 12.0 & 8.0 \\
\hline
\end{tabular}


Table 7. Composition of ferromanganese crusts analyzed by Analytical Services Company

\begin{tabular}{|c|c|c|c|c|c|c|c|c|c|c|c|c|}
\hline Sequonce" & 052019 & 052020 & 052021 & 052022 & 088011 & 088012 & 088013 & 088014 & 088015 & 088016 & 088017 & 088018 \\
\hline Latimde & 19.481 & 19.236 & 19.408 & 19.408 & 25.946 & 25.944 & 25.951 & 25.944 & 25.944 & 25.944 & 25.946 & 25.951 \\
\hline Dopth & 2750 & 3000 & 3050 & 3050 & 3000 & 2600 & 3600 & 2600 & 2600 & 2600 & 3000 & 3600 \\
\hline $\operatorname{Mn}(w h . s)$ & 19.6 & 16.0 & 19.6 & 15.1 & 15.1 & 20.0 & 16.7 & 22.2 & 229 & 15.9 & 23.3 & 18.1 \\
\hline Po & 20.2 & 22.6 & 20.0 & 15.2 & 18.0 & 21.4 & 20.5 & 18.4 & $17 A$ & 20.0 & 18.0 & 19.5 \\
\hline $\mathrm{Ni}$ & 255 & 309 & .336 & .284 & 247 & .292 & .195 & 369 & $A 03$ & .192 & $A 00$ & .247 \\
\hline $\mathrm{Cu}$ & .056 & .245 & .131 & .158 & .148 & .093 & .092 & .058 & .093 & .064 & .081 & .129 \\
\hline $\begin{array}{l}\text { Semplod } \\
\text { Imorval }\end{array}$ & $\begin{array}{c}\text { Bulk } \\
7-11 \mathrm{~mm}\end{array}$ & $\begin{array}{c}\text { Bulk } \\
70-80 \mathrm{~mm}\end{array}$ & $\begin{array}{c}\text { Bulk } \\
25-35 \mathrm{~mm}\end{array}$ & $\begin{array}{c}\text { Bulk } \\
10-20 \mathrm{mmm}\end{array}$ & $\begin{array}{c}\text { Bulk } \\
10-25 \mathrm{~mm}\end{array}$ & $\begin{array}{c}\text { Bulk } \\
2-4 \mathrm{~mm}\end{array}$ & $\begin{array}{c}\text { Bulk } \\
\text { - }\end{array}$ & $\begin{array}{c}\text { Bulk } \\
46 \operatorname{mmn}\end{array}$ & $\underset{\text { 6-8mmm }}{\text { Bulk }}$ & $\begin{array}{c}\text { Bulk } \\
\text { 5-8mmn }\end{array}$ & $\underset{7-10 \text { malk }}{\text { Bulk }}$ & $\begin{array}{c}\text { Bulk } \\
7-25 \mathrm{~mm}\end{array}$ \\
\hline
\end{tabular}

\begin{tabular}{|c|c|c|c|c|c|c|c|c|c|c|c|c|}
\hline Sequencel & 089038 & 089039 & 089040 & 089041 & 089042 & 089043 & 089044 & 089045 & 089046 & 089047 & 089048 & 089049 \\
\hline Latitude & 24.322 & 25.783 & 25.929 & 23.672 & 25.685 & 25.135 & 25.276 & 25.728 & 25.260 & 25.783 & $25 A 54$ & 25.489 \\
\hline Depeh & 1225 & 1600 & 2500 & 1650 & 3000 & 2800 & 3000 & 2600 & 2300 & 1600 & 1115 & 1200 \\
\hline $\operatorname{Mn}(w t . s)$ & 2.57 & 42.2 & 22.2 & 20.8 & 15.6 & 20.1 & 228 & 25.6 & 22.6 & 38.3 & 23.7 & 25.7 \\
\hline Pe & 17.2 & 1.82 & 19.3 & 19.6 & 27.6 & 19.7 & 17.9 & 172 & 18.5 & 4.48 & 14.5 & 15.0 \\
\hline $\mathrm{Ni}$ & .133 & 367 & 311 & 314 & .295 & . 292 & $A 17$ & 425 & 381 & 342 & 344 & .426 \\
\hline $\mathrm{Cu}$ & .026 & .114 & .046 & .069 & 214 & .054 & .096 & .021 & .080 & .144 & .061 & .036 \\
\hline $\begin{array}{l}\text { Semplod } \\
\text { Intarval }\end{array}$ & Bulk & $\begin{array}{c}\text { Bulk } \\
75-85 \mathrm{~mm}\end{array}$ & $\begin{array}{c}\text { Bulk } \\
\text { 10-15mon }\end{array}$ & $\begin{array}{c}\text { Bulk } \\
\text { 4-6mm }\end{array}$ & $\begin{array}{c}\text { Bulk } \\
2-3 \mathrm{~mm}\end{array}$ & $\begin{array}{c}\text { Bulk } \\
15-18 \mathrm{~mm}\end{array}$ & $\begin{array}{c}\text { Bulk } \\
5-8 \mathrm{mmm}\end{array}$ & Bulk & $\begin{array}{c}\text { Bulk } \\
\text { 5-7mm }\end{array}$ & $\begin{array}{c}\text { Bulk } \\
75-85 \text { mmon }\end{array}$ & $\begin{array}{l}\text { Bulk } \\
\text { 8-10mim }\end{array}$ & $\underset{2-22 \mathrm{~mm}}{\text { Bulk }}$ \\
\hline
\end{tabular}

\begin{tabular}{|c|c|c|c|c|c|c|c|c|c|c|c|c|}
\hline Sequence: & 089050 & 089051 & 089052 & 089053 & 089054 & 089055 & 089056 & 089057 & 089058 & 089059 & 089060 & 089061 \\
\hline Latinuds & 25.783 & 25.783 & 24.322 & 25.489 & 25.489 & 25.454 & 24.322 & 23.988 & 25,489 & 25489 & 25.135 & 23.915 \\
\hline Depth & 1600 & 1600 & 1225 & 1200 & 1200 & 1115 & 1225 & 2100 & 1200 & 1200 & 2800 & 800 \\
\hline $\operatorname{Mn}(w L . \$)$ & 37.6 & 43.0 & 2.65 & 23.1 & 3.53 & 23.6 & 194 & 18.8 & 22.2 & 15.3 & 4.50 & 19.0 \\
\hline $\mathrm{Pe}$ & 3.37 & 244 & 20.0 & 17.7 & 9.76 & 17.1 & 18.2 & 165 & 16.9 & $17 \mathrm{~A}$ & 16.9 & 18.8 \\
\hline $\mathrm{Ni}$ & 286 & 327 & .081 & 314 & .153 & .346 & .263 & .431 & 517 & .244 & .140 & .307 \\
\hline $\mathrm{Cr}$ & .142 & .099 & .008 & .032 & .033 & .046 & .040 & .155 & .062 & .037 & .043 & .106 \\
\hline $\begin{array}{l}\text { Sarnpled } \\
\text { Interval }\end{array}$ & $\begin{array}{c}\text { Bulk } \\
75-85 \mathrm{~mm}\end{array}$ & $\begin{array}{c}\text { Bulk } \\
75-85 \mathrm{mmon}\end{array}$ & $\underset{0-1 \mathrm{~mm}}{\text { Bulk }}$ & $\begin{array}{c}\text { Bulk } \\
\text { 3-18mm }\end{array}$ & $\begin{array}{c}\text { Bulk } \\
\text { 1-2mm }\end{array}$ & $\begin{array}{c}\text { Bulk } \\
\text { 10-15 mmon }\end{array}$ & $\begin{array}{c}\text { Bulk } \\
1.10 \mathrm{~mm}\end{array}$ & $\underset{10-15 \mathrm{~mm}}{\text { Bulk }}$ & $\begin{array}{c}\text { Bulk } \\
15-20 \text { man }\end{array}$ & $\begin{array}{c}\text { Bulk } \\
1.5 \mathrm{~mm}\end{array}$ & $\begin{array}{c}\text { Bulk } \\
26 \mathrm{~mm}\end{array}$ & $\underset{3-12 \mathrm{mmm}}{\text { Bulk }}$ \\
\hline
\end{tabular}

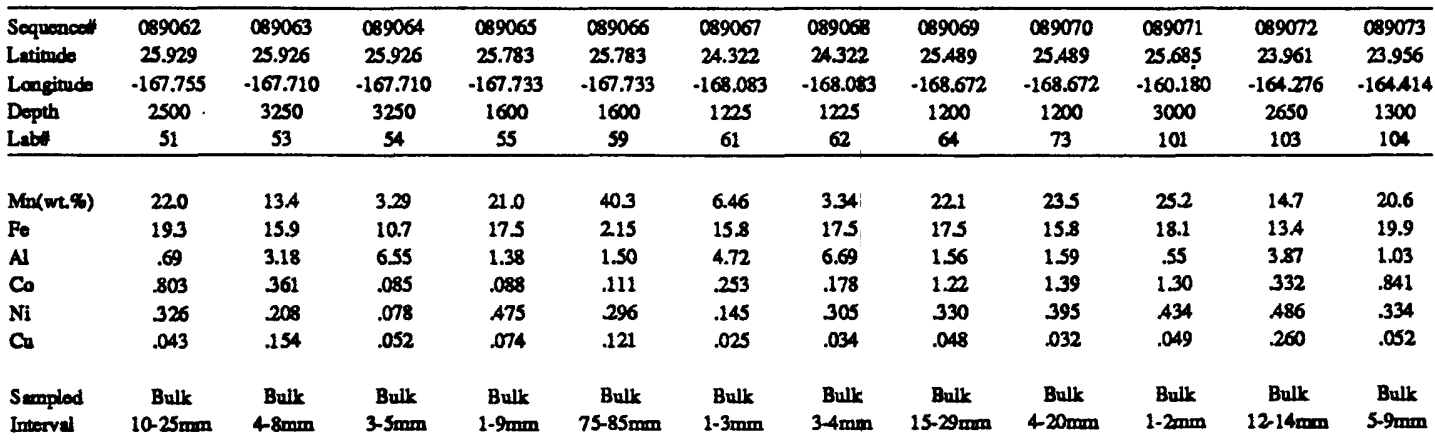


Table 7. Composition of ferromanganese crusts analyzed by Analytical Services Company (cont'd).

\begin{tabular}{|c|c|c|c|c|c|c|c|c|c|c|c|c|}
\hline $\begin{array}{l}\text { Sequenoell } \\
\text { Lutinude }\end{array}$ & $\begin{array}{l}089074 \\
25.260\end{array}$ & $\begin{array}{l}089075 \\
25.094\end{array}$ & $\begin{array}{l}089076 \\
25.094\end{array}$ & $\begin{array}{l}08907 \\
25.260\end{array}$ & $\begin{array}{l}089078 \\
25.260\end{array}$ & $\begin{array}{l}089079 \\
25.276\end{array}$ & $\begin{array}{l}089080 \\
25.980\end{array}$ & $\begin{array}{l}089081 \\
25.980\end{array}$ & $\begin{array}{l}089082 \\
25.980\end{array}$ & $\begin{array}{l}089083 \\
25.980\end{array}$ & $\begin{array}{l}089084 \\
25.980\end{array}$ & $\begin{array}{l}089085 \\
25.276\end{array}$ \\
\hline Langinds & -162046 & -161.728 & -161.728 & -162046 & -162.046 & -162068 & -164.379 & -164.379 & -164.379 & -164.379 & -164.379 & -162.068 \\
\hline $\begin{array}{l}\text { Depth } \\
\text { Laby }\end{array}$ & $\begin{array}{l}2300 \\
105\end{array}$ & $\begin{array}{l}2800 \\
107\end{array}$ & $\begin{array}{l}2800 \\
108\end{array}$ & $\begin{array}{l}2300 \\
202\end{array}$ & $\begin{array}{l}2300 \\
204\end{array}$ & $\begin{array}{c}3000 \\
205\end{array}$ & $\begin{array}{c}1800 \\
206\end{array}$ & $\begin{array}{l}1800 \\
207\end{array}$ & $\begin{array}{c}1800 \\
208\end{array}$ & $\begin{array}{l}1800 \\
209\end{array}$ & $\begin{array}{l}1800 \\
210\end{array}$ & $\begin{array}{l}3000 \\
211\end{array}$ \\
\hline $\operatorname{Mn}(w t \%)$ & 14.6 & 18.5 & 224 & 23.8 & 20.5 & 222 & 19.6 & 19.5 & 18.0 & 22.0 & 20.9 & 19.4 \\
\hline Pa & 195 & 17.1 & 19.8 & 17.7 & 20.6 & 18.1 & 20.5 & 21.3 & 20.9 & 20.0 & 19.4 & 19.9 \\
\hline $\mathbf{A} \mathbf{l}$ & 3.32 & 232 & .72 & .71 & 83 & .88 & 1.25 & 1.43 & 1.55 & 84 & 1.41 & 1.48 \\
\hline Co & 599 & .738 & .732 & 1.03 & 540 & .726 & .717 & 594 & .575 & .723 & .651 & 564 \\
\hline $\mathbf{M}$ & .248 & 310 & 329 & $A 0 B$ & 290 & .442 & .278 & 237 & .223 & 312 & .434 & 308 \\
\hline $\mathrm{Cu}$ & .043 & .087 & .041 & .061 & .072 & .176 & .029 & .040 & .037 & 320 & .054 & .103 \\
\hline $\begin{array}{l}\text { Samplod } \\
\text { Intarval }\end{array}$ & $\begin{array}{c}\text { Bulk } \\
20-25 \mathrm{~mm}\end{array}$ & $\begin{array}{l}\text { Bulk } \\
48 \mathrm{~mm}\end{array}$ & $\begin{array}{c}\text { Bulk } \\
\text { 10-15rmm }\end{array}$ & $\begin{array}{c}\text { Bulk } \\
38-45 \mathrm{~mm}\end{array}$ & $\begin{array}{c}\text { Bulk } \\
\text { so-60.mm }\end{array}$ & $\begin{array}{c}\text { Bulk } \\
25-60 \mathrm{~mm}\end{array}$ & $\begin{array}{c}\text { Bulk } \\
35-40 \mathrm{~mm}\end{array}$ & $\begin{array}{c}\text { Bulk } \\
\text { 30-35imm }\end{array}$ & $\begin{array}{c}\text { Bulk } \\
\text { 30-35rmm }\end{array}$ & $\begin{array}{c}\text { Bulk } \\
35-40 \mathrm{~mm}\end{array}$ & $\begin{array}{c}\text { Bulk } \\
\text { 14-18mm }\end{array}$ & $\begin{array}{c}\text { Bulk } \\
3-6 \mathrm{~mm}\end{array}$ \\
\hline
\end{tabular}

\begin{tabular}{|c|c|c|c|c|c|c|c|c|c|c|c|c|}
\hline $\begin{array}{l}\text { Sequencell } \\
\text { Latitude }\end{array}$ & $\begin{array}{l}089086 \\
23.672\end{array}$ & $\begin{array}{l}089087 \\
23.672\end{array}$ & $\begin{array}{l}089088 \\
25.260\end{array}$ & $\begin{array}{l}089089 \\
25.135\end{array}$ & $\begin{array}{l}089090 \\
25.276\end{array}$ & $\begin{array}{l}089091 \\
23.672\end{array}$ & $\begin{array}{l}089092 \\
25.276\end{array}$ & $\begin{array}{l}089093 \\
25.260\end{array}$ & $\begin{array}{l}089094 \\
25303\end{array}$ & $\begin{array}{l}089095 \\
25.276\end{array}$ & $\begin{array}{l}089096 \\
25.303\end{array}$ & $\begin{array}{l}089097 \\
23.979\end{array}$ \\
\hline Langinds & -164.891 & -164.891 & -162.046 & -162027 & -162.068 & -164891 & -162.068 & -162.046 & -162094 & -162.068 & -162094 & -164.363 \\
\hline Depth & 1650 & 1650 & 2300 & 2800 & 3000 & 1650 & 3000 & 2300 & 3500 & 3000 & 3500 & 2000 \\
\hline Laby & 212 & 214 & 216 & 217 & 219 & 220 & 221 & 222 & 223 & 224 & 225 & 308 \\
\hline$M n(w t . \%)$ & 14.9 & 158 & 21.9 & 21.6 & 20.7 & 17.2 & 21.4 & 19.9 & 17.8 & 17.4 & 17.6 & 15.0 \\
\hline Po & 20.1 & 21.0 & 21.3 & 19.1 & 20.4 & 20.1 & 21.6 & 18.9 & 19.4 & 18.5 & 18.5 & 15.7 \\
\hline $\mathbf{A}$ & 1.95 & 219 & .69 & 1.31 & 1.20 & 230 & .93 & 1.89 & 1.77 & 1.40 & 1.92 & 269 \\
\hline Co & 448 & A24 & .647 & 827 & .554 & 593 & 514 & .621 & .302 & .452 & .563 & .667 \\
\hline $\mathrm{Ni}$ & .191 & .238 & 304 & 328 & 333 & .246 & 277 & 344 & .311 & .254 & .225 & 327 \\
\hline $\mathrm{Cu}$ & .038 & .044 & .058 & .079 & .124 & .043 & .058 & .094 & .139 & .089 & .053 & .065 \\
\hline $\begin{array}{l}\text { Samplod } \\
\text { Imterval }\end{array}$ & $\begin{array}{c}\text { Bulk } \\
\text { 6-8mm }\end{array}$ & $\begin{array}{c}\text { Bulk } \\
7-10 \mathrm{mms}\end{array}$ & $\begin{array}{c}\text { Bulk } \\
\text { 25-30mon }\end{array}$ & $\begin{array}{c}\text { Bulk } \\
2.3 \mathrm{~mm}\end{array}$ & $\underset{7-13 \mathrm{~mm}}{\text { Bulk }}$ & $\begin{array}{c}\text { Bulk } \\
\text { 6-10mm }\end{array}$ & $\begin{array}{c}\text { Bulk } \\
13-25 \mathrm{~mm}\end{array}$ & $\underset{\text { 5-8min }}{\text { Bulk }}$ & $\begin{array}{c}\text { Bulk } \\
2-4 \text { am }\end{array}$ & $\begin{array}{c}\text { Bulk } \\
10-15 \text { sum }\end{array}$ & $\underset{\text { S-8ming }}{\text { Bulk }}$ & $\begin{array}{c}\text { Bulk } \\
2.5 \operatorname{monn}\end{array}$ \\
\hline
\end{tabular}

\begin{tabular}{|c|c|c|c|c|c|c|c|c|c|c|c|c|}
\hline Sequencell & 089098 & 089099 & 089100 & 090018 & 090019 & 090020 & 090021 & 090022 & 090023 & 090024 & 090025 & 090027 \\
\hline Letitude & 23.979 & 25.135 & 25.094 & 28.895 & 28.895 & 20.106 & 20.167 & 29.106 & 29.167 & 20.208 & 28.895 & 20.167 \\
\hline Longitude & -164.363 & -162010 & -161.728 & -178.868 & -178.868 & -173.948 & $-174,067$ & -173.948 & -174.007 & -174.112 & -178.868 & -174.067 \\
\hline Depth & 2200 & 2175 & 2800 & 1280 & 1280 & 1850 & 1425 & 1850 & 1425 & 2200 & 1280 & 1425 \\
\hline Laby & 310 & 312 & 315 & $2 n$ & 28 & 30 & 31 & 33 & 34 & 36 & 16 & 37 \\
\hline $\operatorname{Mn}(w t .8)$ & 15.7 & 24.5 & 168 & 23.8 & 17.5 & 23.0 & 278 & 24.5 & 24.2 & 215 & 27.1 & 19.0 \\
\hline Pe & 19.1 & 164 & 18.0 & 5.36 & 6.08 & 15.4 & 16.0 & 15.9 & 14.4 & 15.9 & 128 & 18.9 \\
\hline $\mathbf{A}$ & 234 & 1.06 & 200 & 3.84 & 4.45 & .61 & 52 & 54 & 80 & 1.24 & 99 & 1.11 \\
\hline Co & 389 & 838 & 538 & 598 & $A 36$ & .711 & 889 & 925 & .677 & .851 & 1.16 & .787 \\
\hline $\mathrm{Ni}$ & 324 & 470 & .245 & .420 & .225 & 398 & A51 & A17 & .558 & .419 & 540 & .296 \\
\hline $\mathrm{Cu}$ & .090 & .060 & .098 & .043 & .027 & .058 & .038 & .044 & .064 & .127 & .035 & .070 \\
\hline $\begin{array}{l}\text { Semplod } \\
\text { Interval }\end{array}$ & $\begin{array}{l}\text { Bulk } \\
3-6 \mathrm{~mm}\end{array}$ & $\begin{array}{c}\text { Noctulo } \\
.\end{array}$ & $\begin{array}{c}\text { Bulk } \\
\text { 1-Simm }\end{array}$ & $\begin{array}{l}\text { Bulk } \\
\text { 3-5mm }\end{array}$ & $\begin{array}{c}\text { Bulk } \\
1-3 \mathrm{~mm}\end{array}$ & $\begin{array}{c}\text { Bulk } \\
10-20 \text { mmm }\end{array}$ & $\begin{array}{c}\text { Bulk } \\
14-40 \text { mm }\end{array}$ & $\begin{array}{c}\text { Bulk } \\
25-33 \mathrm{~mm}\end{array}$ & $\begin{array}{c}\text { Bulk } \\
15-20 \mathrm{~mm}\end{array}$ & $\begin{array}{c}\text { Buik } \\
15-20 \text {.mm }\end{array}$ & $\begin{array}{c}\text { Bulk } \\
1.2 \mathrm{~mm}\end{array}$ & $\begin{array}{c}\text { Bulk } \\
10-25 \mathrm{~mm}\end{array}$ \\
\hline
\end{tabular}

\begin{tabular}{|c|c|c|c|c|c|c|c|c|c|c|c|c|}
\hline Sequencel" & 090028 & 090029 & 090030 & 090031 & 090032 & 090033 & 090034 & 090035 & 090036 & 090026 & 090038 & 090039 \\
\hline Latitudo & 29.167 & 29.167 & 29.185 & 29.208 & 29.167 & 29.062 & 29.208 & 28.895 & 28.881 & 28.895 & 28.895 & 28.739 \\
\hline Longitude & -174.067 & -174.067 & -174.010 & -174.112 & -174.067 & -174.022 & -174.112 & -178.868 & -179.745 & -178.868 & -178.868 & -178.783 \\
\hline Depth & 1425 & 1425 & 985 & 2200 & 1425 & 1150 & 2200 & 1280 & 2000 & 1280 & 1280 & 3000 \\
\hline Labil & 39 & 40 & 44 & 46 & 47 & 50 & 49 & 14 & 2 & 23 & 29 & 32 \\
\hline $\operatorname{Mn}(w t . \%)$ & 27.5 & 25.9 & 29.8 & 22.3 & 25.8 & 27.2 & 18.8 & 31.1 & 27.5 & 28.4 & 29.0 & 19.0 \\
\hline Fe & 14.9 & 15.3 & 13.4 & 17.3 & 16.0 & 15.6 & 15.7 & 12.6 & 126 & 16.0 & 10.5 & 19.1 \\
\hline $\mathbf{N}$ & .63 & 48 & .63 & 1.18 & 35 & .64 & 2.14 & .26 & 1.33 & .73 & 57 & .70 \\
\hline co & .902 & .986 & 1.66 & .756 & .904 & .779 & 563 & 1.43 & .778 & 1.32 & 1.61 & 576 \\
\hline $\mathrm{Ni}$ & .556 & 493 & .685 & 379 & .424 & 379 & 381 & .642 & .959 & .546 & .690 & 271 \\
\hline Cu & .053 & .035 & .052 & .121 & .037 & .043 & .134 & .037 & .197 & .047 & .041 & .042 \\
\hline $\begin{array}{l}\text { Sempiod } \\
\text { Interval }\end{array}$ & $\begin{array}{c}\text { Bulk } \\
25-35 \mathrm{~mm}\end{array}$ & $\underset{15-20 \mathrm{mmn}}{\text { Bulk }}$ & Bulk & $\underset{15-25 \mathrm{~mm}}{\text { Bulk }}$ & $\begin{array}{c}\text { Bulk } \\
4-12 \mathrm{~mm}\end{array}$ & $\begin{array}{c}\text { Bulk } \\
90-110 \mathrm{~mm}\end{array}$ & $\frac{\text { Bulk }}{20-25 \mathrm{~mm}}$ & $\underset{10-12 \mathrm{mmn}}{\text { Bulk }}$ & $\begin{array}{c}\text { Bulk } \\
1-3 \mathrm{~mm}\end{array}$ & Bulk & $\begin{array}{l}\text { Bulk } \\
1.9 \mathrm{mmm}\end{array}$ & $\begin{array}{c}\text { Bulk } \\
\text { 8-10mm }\end{array}$ \\
\hline
\end{tabular}


Table 7. Composition of ferromanganese crusts analyzed by Analytical Services Company (cont'd).

\begin{tabular}{|c|c|c|c|c|c|c|c|c|c|c|c|c|}
\hline $\begin{array}{l}\text { Sequencel } \\
\text { Latitude }\end{array}$ & $\begin{array}{r}090040 \\
29.106\end{array}$ & $\begin{array}{l}090041 \\
29.106\end{array}$ & $\begin{array}{l}090042 \\
29.167\end{array}$ & $\begin{array}{l}090043 \\
29.167\end{array}$ & $\begin{array}{l}090044 \\
29.106\end{array}$ & $\begin{array}{l}090045 \\
29.106\end{array}$ & $\begin{array}{l}090046 \\
29.106\end{array}$ & $\begin{array}{l}090047 \\
29.106\end{array}$ & $\begin{array}{l}090048 \\
28.881\end{array}$ & $\begin{array}{l}090049 \\
28.895\end{array}$ & $\begin{array}{l}091005 \\
29.857\end{array}$ & $\begin{array}{l}091006 \\
29.641\end{array}$ \\
\hline Langituds & -173.948 & -174.020 & -174.067 & -174.067 & -173.948 & .173 .948 & -173.948 & -173.948 & $\cdot 179.745$ & -178.869 & 179.083 & 179.282 \\
\hline Dopth & 1850 & 1475 & 1425 & 1425 & 1850 & 1850 & 1850 & 1850 & 2000 & 1280 & 2250 & 1300 \\
\hline Lab: & 35 & 43 & 45 & 48 & 302 & 303 & 304 & 305 & 313 & 314 & 17 & 24 \\
\hline $\operatorname{Mn}(w t . \%)$ & 14.2 & 29.3 & 243 & 23.4 & 23.6 & 25.1 & 26.4 & 28.0 & 264 & 27.3 & 222 & 30.4 \\
\hline Fe & 20.5 & 127 & 16.0 & 15.6 & 16.0 & 15.2 & 15.8 & 10.8 & 15.2 & 11.2 & 16.1 & 13.8 \\
\hline $\boldsymbol{N}$ & 1.89 & 35 & 1.00 & 1.34 & 1.22 & .81 & .60 & 1.78 & 91 & 1.88 & .98 & 26 \\
\hline Co & 866 & 1.63 & .752 & .665 & .746 & 918 & 1.01 & .681 & 814 & 1.61 & .720 & 1.26 \\
\hline $\mathbf{N i}$ & .179 & .648 & $\$ 70$ & .463 & .443 & .489 & A45 & 1.160 & 553 & .640 & $A 0 B$ & .603 \\
\hline $\mathrm{Cu}$ & .028 & .036 & .047 & .070 & .059 & .054 & .040 & .131 & .067 & .046 & .105 & .043 \\
\hline Semplod & Bulk & Bulk & Bulk & Bulk & Bulk & Bulk & Bulk & Bulk & Bulk & Bulk & Bulk & Bulk \\
\hline Interval & 4-10 $\mathrm{cmm}$ & - & $20-25 \mathrm{~mm}$ & $15.30 \mathrm{cmm}$ & 13-25man & $13.20 \mathrm{~mm}$ & $3-500 m$ & $48 \mathrm{~mm}$ & 6-8 & 3-5rom & - & $2-12 \mathrm{~mm}$ \\
\hline
\end{tabular}

\begin{tabular}{lcccccccccccc}
\hline Sequence & 091007 & 091008 & 091009 & 091010 & 091011 & 091012 & 091013 & 091014 & 091015 & 091016 & 091017 & 091018 \\
Letitude & 29.857 & 29.839 & 29.839 & 29.634 & 29.641 & 29.869 & 29.820 & 29.641 & 29.634 & 29.839 & 29.634 & 29.634 \\
Longitide & 179.083 & 179.065 & 179.065 & 179.288 & 179.282 & 179.098 & 179.103 & 179.282 & 179.288 & 179.065 & 179.288 & 179.288 \\
Dopth & 250 & 2100 & 2100 & 1865 & 1300 & 2900 & 1700 & 1300 & 1865 & 2100 & 1865 & 1865 \\
Lebl & 69 & 12 & 13 & 15 & 38 & 74 & 20 & 18 & 19 & 21 & 25 & 26 \\
\hline
\end{tabular}

\begin{tabular}{|c|c|c|c|c|c|c|c|c|c|c|c|c|}
\hline $\operatorname{Mn}(w t . x)$ & 19.0 & 13.6 & 23.8 & 24.7 & 21.7 & 21.8 & 21.4 & 26.6 & 25.7 & 15.3 & 25.1 & 19.9 \\
\hline Fo & 18.7 & 16.8 & 16.2 & 17.6 & 18.5 & 17.4 & 16.4 & 16.7 & 17.9 & 22.7 & 17.6 & 16.1 \\
\hline $\mathbf{A} \mathbf{l}$ & 1.46 & 270 & 1.22 & .42 & .59 & 1.12 & 1.70 & A4 & .48 & 1.64 & .57 & 1.15 \\
\hline Co & .615 & .578 & 963 & .766 & 1.24 & 848 & .755 & 1.08 & .792 & .683 & .788 & 559 \\
\hline $\mathrm{Ni}$ & 249 & .389 & 397 & 348 & .293 & 394 & 396 & $A 10$ & 388 & 307 & 365 & 367 \\
\hline $\mathrm{Cn}$ & .056 & .070 & .044 & .027 & .028 & .067 & .030 & .028 & .024 & .057 & .026 & .033 \\
\hline $\begin{array}{l}\text { Sempiod } \\
\text { Interval }\end{array}$ & $\underset{1-6 \mathrm{~mm}}{\text { Bulk }}$ & $\begin{array}{c}\text { Bulk } \\
2-6 \mathrm{~mm}\end{array}$ & $\underset{5-15 \mathrm{~mm}}{\text { Bulk }}$ & $\underset{7-8 \operatorname{mm}}{\text { Bulk }}$ & $\begin{array}{c}\text { Bulk } \\
\text { - }\end{array}$ & $\begin{array}{c}\text { Bulk } \\
-\end{array}$ & $\begin{array}{l}\text { Bulk } \\
\text { 5- } 6 \mathrm{~mm}\end{array}$ & Bulk & $\begin{array}{c}\text { Bulk } \\
\text { 8-12mm }\end{array}$ & $\underset{1-3 \mathrm{mmm}}{\text { Bulk }}$ & $\begin{array}{c}\text { Bulk } \\
\text { 8-15mmm }\end{array}$ & $\begin{array}{c}\text { Bulk } \\
7-10 \mathrm{~mm}\end{array}$ \\
\hline
\end{tabular}

\begin{tabular}{lccc}
\hline Soquencell & 091019 & 091020 & 091021 \\
Latitude & 29.857 & 29.869 & 29.820 \\
Langitude & 179.083 & 179.098 & 179.104 \\
Depth & 2250 & 2900 & 1700 \\
Lebt & 70 & 75 & 301 \\
\hline & & & \\
Min(wr.5) & 21.9 & 17.7 & 8.39 \\
Po & 17.7 & 16.4 & 12.7 \\
A & .94 & 1.77 & 4.67 \\
Co & .722 & .447 & .296 \\
Ni & 376 & .439 & .186 \\
Cu & .049 & .114 & .012 \\
& & & \\
Sumplod & Bulk & Bulk & Bulk \\
Imterval & $1-3 \mathrm{~mm}$ & $8-10 \mathrm{~mm}$ &.
\end{tabular}


Table 8. Composition of crusts analyzed by Aplin (PhD. thesis)

\begin{tabular}{|c|c|c|c|c|c|c|c|c|c|c|c|c|}
\hline Sequences: & 016008 & 016009 & 016010 & 053051 & 053052 & 053053 & 053054 & 053055 & 053056 & 053057 & 053058 & 053059 \\
\hline Lationde & -2083 & 3.633 & 4.367 & 10.817 & 10.817 & 10.817 & 12517 & 13.467 & 13,467 & 13.407 & 13,467 & 14.983 \\
\hline Langimds & -157.350 & -159.517 & -159.083 & -165550 & -165550 & -165.550 & -167.050 & -165.417 & .165 .417 & -165417 & -165.417 & -166.100 \\
\hline Dopth & 1350 & 2800 & 2150 & 1150 & 1150 & 1150 & 3000 & 3850 & 3850 & 3850 & 3850 & 2800 \\
\hline Lab" & $041-1 / 1$ & $054-1 / 1$ & $055-3 / 3$ & $058-1 / 2$ & $058-2 / 2 A$ & $058-2 / 2 \mathrm{~B}$ & $059-7 \pi$ & $060-2 / 4 A$ & $060-2 / 4 \mathrm{~B}$ & $060-3 / 4$ & $060-4 / 4$ & $061-2 / 5 \mathrm{~A}$ \\
\hline Ti(wts) & 1.06 & 97 & 51 & .39 & 1.01 & .77 & 1.48 & 1.38 & 1.26 & 1.32 & 134 & 1.20 \\
\hline $\mathbf{M n}$ & 29.3 & 23.7 & 15.4 & 21.6 & 29.2 & 279 & 20.3 & 19.7 & 20.3 & 19.2 & 23.1 & 219 \\
\hline Fo & $14 A$ & 15.3 & 16.1 & 21.0 & 13.0 & 123 & 19.5 & 18.2 & 182 & 18.6 & 20.7 & 19.0 \\
\hline $\mathbf{A}$ & 52 & .70 & 87 & .62 & 38 & 52 & 87 & 1.41 & .88 & .95 & .74 & 1.13 \\
\hline Co & 1.16 & .800 & .232 & .660 & 1.57 & 1.12 & .547 & .483 & .496 & .520 & 560 & 540 \\
\hline $\mathrm{Ni}$ & 570 & .530 & 370 & .310 & .690 & .700 & .280 & 260 & 298 & .260 & 330 & 370 \\
\hline $\mathrm{Cu}$ & .031 & .047 & .059 & .080 & 959.05 & .061 & .121 & .095 & .099 & .096 & .136 & .201 \\
\hline C. & 2.64 & 265 & 4.53 & 2.29 & 256 & 5.10 & 2.15 & 230 & 2.15 & 202 & 243 & 249 \\
\hline $\mathbf{M}_{8}$ & 1.18 & 1.20 & 1.32 & .96 & 1.26 & 1.22 & 99 & 1.08 & 1.02 & 1.03 & 99 & .98 \\
\hline $\mathrm{Ne}$ & 1.69 & 2.29 & 3.72 & 1.46 & 1.60 & 1.44 & 154 & 1.53 & 1.65 & 1.72 & 1.55 & 1.62 \\
\hline $\mathbf{K}$ & .33 & 34 & .39 & .38 & 40 & 10 & 35 & $A 5$ & 34 & 36 & 36 & .43 \\
\hline $\mathbf{P}$ & 35 & .31 & 1.18 & .32 & .33 & 1.10 & 31 & 32 & 32 & 33 & 30 & 30 \\
\hline Be(ppon) & 1100 & 960 & 1800 & 1400 & 840 & 620 & 1300 & 1200 & 1400 & 1400 & 1400 & 1600 \\
\hline$C d$ & 6.3 & 55 & 3.1 & 4.3 & 8.1 & 7.0 & 39 & 3.3 & 4.2 & 4.0 & 4.0 & 3.6 \\
\hline C & 52 & 49 & 29 & 30 & 32 & 36 & 28 & 2 & 31 & 33 & 32 & 33 \\
\hline Mo & 660 & 450 & 280 & 480 & 780 & 740 & 340 & 370 & 450 & 370 & 500 & 450 \\
\hline $\mathrm{Pb}$ & 1600 & 770 & 310 & 1400 & 1700 & 1600 & 1200 & 1100 & 1100 & 1200 & 1100 & 930 \\
\hline $\mathbf{S r}$ & 1300 & 1200 & 1500 & 1300 & 1300 & 1400 & 1200 & 1100 & 1300 & 1200 & 1300 & 1200 \\
\hline $\mathbf{v}$ & 630 & 550 & 450 & 590 & 620 & 580 & 580 & 530 & 580 & 550 & 600 & 540 \\
\hline $\mathbf{Z n}$ & 680 & 590 & 510 & 590 & 700 & 720 & 540 & 520 & 550 & 540 & 570 & 560 \\
\hline Thiclmead (mm) & 5.0 & 6.0 & 20 & 10.0 & 30.0 & 30.0 & 5.0 & 20.0 & 10.0 & 8.0 & 13.0 & 20.0 \\
\hline
\end{tabular}

\begin{tabular}{|c|c|c|c|c|c|c|c|c|c|c|c|c|}
\hline Sequencell & 053060 & 053061 & 053062 & 053063 & 053064 & 053065 & 053066 & 053067 & 053068 & 053069 & 053070 & 053071 \\
\hline Latimde & 14.983 & 14.983 & 14.983 & 14983 & 14.983 & 15.150 & 15.150 & 15.150 & 15.150 & 15.150 & 15.150 & 15.150 \\
\hline Langituds & -166.100 & -166.100 & -166.100 & -166.100 & -166.100 & -168.100 & -168.100 & -168.100 & .168 .100 & -168.100 & -168.100 & .168 .100 \\
\hline Dopth & 2800 & 2800 & 2800 & 2300 & 0 & 2000 & 2700 & 2700 & 2700 & 2700 & 2700 & 2700 \\
\hline Labu & $061-2 / 5 B$ & $061-4 / 5 A$ & $061-4 / 5 B 1$ & $061-4 / 5 B 2$ & $061-4 / 5 B 3$ & $062-1 / 3 A$ & 062-1/3B & $062-1 / 3 C$ & $062-1 / 3 D$ & 062-1/3E & $062-1 / 3 P$ & $062-3 / 3 A$ \\
\hline Ti(wt.\%) & 1.37 & 1.40 & 1.23 & 1.42 & 1.12 & 1.38 & 97 & 1.20 & 1.43 & 1.10 & 1.74 & 1.10 \\
\hline Mn & 21.8 & 23.1 & 26.3 & 21.0 & 24.2 & 20.8 & 19.9 & 20.9 & 18.5 & 19.3 & 18.0 & 17.7 \\
\hline Fe & 19.3 & 19.9 & 18.3 & 14.3 & 18.0 & 185 & 16.9 & 195 & 20.6 & 17.8 & 19.6 & 16.0 \\
\hline $\mathbf{A}$ & 1.20 & 96 & .36 & .19 & .80 & 94 & 1.95 & 1.00 & 1.24 & 1.27 & 1.43 & 2.97 \\
\hline Co & 354 & .590 & 940 & A95 & 513 & .464 & .354 & .291 & .238 & .235 & .244 & .431 \\
\hline $\mathrm{Ni}$ & 358 & .370 & .440 & 490 & 380 & 330 & 358 & .325 & 335 & .348 & 237 & 360 \\
\hline $\mathrm{Cu}$ & 273 & .137 & .094 & .169 & .175 & .177 & .273 & 325 & 347 & .316 & 255 & 365 \\
\hline$c_{2}$ & 2.54 & 2.41 & 2.46 & 3.29 & .29 & 2.16 & 2.10 & 2.13 & 1.88 & 1.97 & 208 & 286 \\
\hline $\mathbf{M}_{8}$ & .98 & 97 & 1.01 & 1.15 & 1.01 & .95 & 92 & .95 & .97 & .91 & 87 & .94 \\
\hline $\mathrm{Na}$ & 1.56 & 152 & 1.44 & 1.54 & 1.35 & 1.38 & 1.37 & 1.20 & 1.10 & 1.18 & 1.11 & 1.75 \\
\hline $\mathbf{K}$ & $A 5$ & 41 & 34 & .78 & .43 & 40 & .67 & 48 & 54 & .73 & .71 & 1.01 \\
\hline $\mathbf{P}$ & .30 & .33 & 59 & .39 & .27 & .23 & .24 & .22 & .20 & 30 & 35 & .33 \\
\hline Bappon) & 2400 & 1500 & 1400 & 1500 & 1900 & 1700 & 2400 & 3000 & 1200 & 1600 & 1900 & 2000 \\
\hline $\mathrm{Cd}$ & 3.7 & 4.0 & 5.2 & 4.8 & 3.7 & 3.5 & 25 & 20 & 2.9 & 26 & 3.1 & 3.1 \\
\hline$c$ & 34 & 20 & $\boldsymbol{x}$ & 49 & 43 & 30 & 37 & 58 & 33 & 30 & 34 & 33 \\
\hline Mo & 400 & 310 & 600 & 400 & 470 & 440 & 400 & 710 & 620 & 650 & 490 & 250 \\
\hline Pb & 580 & 1000 & 1300 & 710 & 680 & 1000 & 580 & 1000 & 1400 & 1800 & 2400 & 710 \\
\hline St & 1300 & 1300 & 1300 & 1100 & 1400 & 1400 & 1300 & 1500 & 1400 & 1400 & 1600 & 1100 \\
\hline $\mathbf{v}$ & 560 & 580 & 610 & 490 & 620 & 570 & 560 & 840 & 840 & 750 & 750 & 390 \\
\hline $\mathrm{Zn}$ & 640 & 570 & 570 & 550 & 620 & 590 & 640 & 780 & 900 & 770 & 830 & 550 \\
\hline Thiclonost(mm) & 15.0 & 25.0 & 35.0 & 35.0 & 35.0 & 70.0 & 70.0 & 70.0 & 70.0 & 70.0 & 70.0 & 62.0 \\
\hline
\end{tabular}


Table 8. Composition of crusts analyzed by Aplin (PhD. thesis) cont'd.

\begin{tabular}{|c|c|c|c|c|c|c|c|c|c|c|c|c|}
\hline Sequencell & 053072 & 053073 & 053074 & 053075 & 053076 & 053077 & 053078 & 053079 & 053080 & 053081 & 053082 & 053083 \\
\hline Letitude & 15.150 & 15.150 & 16.433 & 16.433 & 16.433 & 16.433 & 16.433 & 16.433 & 16.433 & 16.433 & 16.433 & 16.433 \\
\hline Longitendo & -168.100 & -168.100 & -168.150 & -168.150 & -168.150 & -168.150 & -168.150 & -168.150 & .168 .150 & -168.150 & -168.150 & -168.150 \\
\hline Despth & 2700 & 2700 & 2325 & 2325 & 2325 & 2325 & 2325 & 2325 & 2325 & 2325 & 2325 & 2400 \\
\hline Labi & $062-3 / 3 \mathrm{~B}$ & $062-3 / 3 \mathrm{~F}$ & $063-1 / 9$ & $003-2 / 9 \mathrm{~A}$ & 063-2/9B & $063-29 \mathrm{C}$ & $003-5 / 9 A$ & $003-5 / 9 B$ & $063-6 / 9 A$ & $063-6,9 \mathrm{~B}$ & $063-8 / 9$ & $0642 \pi$ \\
\hline$T i(w t \%)$ & 1.27 & 1.32 & 1.39 & 1.37 & 1.40 & 1.53 & 1.52 & 1.33 & 1.46 & 1.31 & 1.33 & 1.53 \\
\hline $\mathbf{M n}$ & 19.6 & 195 & 21.3 & 24.7 & 22.0 & 23.2 & 21.0 & 15.6 & 22.8 & 13.2 & 20.4 & 21.8 \\
\hline Pe & 21.6 & 15.8 & 17.4 & 163 & 15.5 & 16.1 & 21.9 & 17.6 & 18.5 & 16.0 & 18.5 & 208 \\
\hline $\mathbf{A}$ & 93 & 2.74 & 1.00 & .62 & 1.21 & 94 & .86 & 1.51 & 57 & 231 & 82 & 1.05 \\
\hline Co & .494 & .530 & .770 & .710 & 570 & .750 & .600 & .444 & 840 & 320 & .630 & 540 \\
\hline $\mathbf{N I}$ & .250 & .420 & 410 & $A 10$ & .430 & .440 & .290 & .290 & .330 & .300 & .267 & .350 \\
\hline $\mathrm{Cu}$ & .113 & .247 & .115 & .112 & .160 & .134 & .075 & .075 & .055 & .124 & .058 & .128 \\
\hline Ca & 2.13 & 2.40 & 223 & 226 & 2.85 & 236 & 225 & 2.16 & 2.20 & 5.03 & 2.14 & 245 \\
\hline $\mathbf{M}_{\mathbf{8}}$ & .98 & 97 & 1.12 & .99 & 1.00 & 1.03 & 99 & 1.43 & 1.02 & 1.02 & 1.03 & 99 \\
\hline $\mathrm{Na}$ & 1.35 & 1.83 & 1.54 & 1.44 & 1.53 & 1.59 & 1.83 & 3.96 & 1.50 & 1.67 & 1.67 & 1.39 \\
\hline $\mathbf{K}$ & 35 & 1.03 & $A 4$ & .37 & 56 & $\mathbf{A 5}$ & 39 & .73 & 38 & .59 & 39 & 39 \\
\hline $\mathbf{P}$ & 32 & 33 & 30 & .46 & .29 & 35 & 33 & 35 & 1.12 & .39 & 34 & .75 \\
\hline Ba(ppm) & 1400 & 1700 & 1400 & 1300 & 1400 & 1500 & 1300 & 320 & 1300 & 1600 & 1300 & 1500 \\
\hline Cd & 3.2 & 3.6 & 43 & 38.0 & 43 & 4.6 & 3.6 & 33 & 3.7 & 3.5 & 3.4 & 3.5 \\
\hline$c$ & 20 & 35 & 41 & 28 & 40 & 35 & 37 & 38 & 20 & 42 & 12 & 35 \\
\hline Mo & 410 & 310 & 460 & 510 & 420 & 470 & 440 & 231 & 480 & 155 & 440 & 450 \\
\hline $\mathrm{Pb}$ & 1300 & 730 & 1300 & 1500 & 1100 & 1400 & 1400 & 1200 & 1500 & 850 & 1400 & 1300 \\
\hline Sz & 1300 & 1100 & 1300 & 1300 & 1300 & 1300 & 1300 & 1000 & 1400 & 990 & 1300 & 1300 \\
\hline $\mathbf{v}$ & 580 & 400 & 560 & 580 & 530 & 540 & 560 & 420 & 580 & 360 & 580 & 560 \\
\hline $\mathbf{Z n}$ & 560 & 550 & 580 & 570 & 630 & 590 & 540 & 570 & 530 & 560 & 540 & 560 \\
\hline Thichnosu(mon) & 62.0 & 62.0 & 10.0 & 25.0 & 25.0 & 25.0 & 2.0 & 1.0 & 20.0 & 5.0 & 20.0 & 30.0 \\
\hline
\end{tabular}

\begin{tabular}{|c|c|c|c|c|c|c|c|c|c|c|c|c|}
\hline Sequencell & 053084 & 053085 & 053086 & 053087 & 053088 & 053089 & 315003 & 315004 & 315005 & 350007 & 350008 & 350009 \\
\hline Letitude & 16.433 & 16.433 & 16.433 & 16.433 & 16.433 & 16.433 & .7583 & -9.067 & -9.007 & -15.017 & -13.033 & -13.033 \\
\hline Longitede & -168.150 & -168.150 & -168.150 & -168.150 & -168.150 & -168.150 & $\cdot-151.550$ & -150.700 & -150.700 & $\cdot 149.033$ & -147.933 & -147.933 \\
\hline Dopth & 2400 & 2400 & 2400 & 2400 & 2400 & 2400 & 1880 & 1300 & 1300 & 3400 & 4700 & 4700 \\
\hline Leb" & $064-4 \pi A$ & $064-4 / 7 \mathrm{~B}$ & $0645 / 7 A$ & $0645 / 7 \mathrm{~B}$ & $0647 / 7 \mathrm{~A}$ & $0647 / 7 \mathrm{~B}$ & $044-2 / 2$ & $045-6 / 13 A$ & $045-6 / 138$ & $052-47$ & $053-1 / 1 A$ & $053-1 / 1 \mathrm{~B}$ \\
\hline
\end{tabular}

\begin{tabular}{|c|c|c|c|c|c|c|c|c|c|c|c|c|}
\hline$T(w . s)$ & 1.55 & 1.66 & 1.42 & 1.37 & 1.42 & 1.49 & 1.18 & 1.38 & 1.35 & 1.06 & 87 & 51 \\
\hline Mn & 16.4 & 20.3 & 20.4 & 20.6 & 22.2 & 23.1 & 26.1 & 27.6 & 28.5 & 17.2 & 11.7 & 532 \\
\hline $\mathbf{P e}$ & 15.8 & 18.0 & 22.7 & 20.8 & 18.3 & 20.0 & 18.3 & 13.7 & 123 & 17.0 & 11.5 & 7.65 \\
\hline Al & 298 & 1.98 & 87 & .94 & 137 & 1.19 & 33 & .46 & 32 & 1.19 & 5.02 & 5.02 \\
\hline Co & .270 & .456 & .700 & .770 & .540 & .483 & 930 & 1.05 & 1.15 & A85 & .252 & .089 \\
\hline $\mathrm{Ni}$ & .390 & .400 & .290 & 300 & .420 & .390 & 540 & .690 & .760 & .256 & 440 & 200 \\
\hline Cu & .250 & .186 & .067 & .078 & .187 & .190 & .043 & .069 & .063 & .058 & .225 & .107 \\
\hline $\mathrm{Ca}$ & 3.50 & 270 & 226 & 2.20 & 2.32 & 2.39 & 2.64 & 2.44 & 266 & 2.18 & 204 & 244 \\
\hline Mg & 1.21 & 1.13 & .98 & .99 & 1.05 & 1.09 & 1.05 & 1.12 & 1.17 & 1.42 & 1.26 & 1.02 \\
\hline $\mathrm{Na}$ & 1.65 & 1.74 & 1.43 & 1.42 & 1.69 & 1.61 & 1.60 & 1.55 & 1.59 & 1.88 & 243 & 283 \\
\hline $\mathbf{x}$ & .71 & 52 & 38 & .38 & 54 & AS & 31 & $A 0$ & 40 & 32 & 210 & 291 \\
\hline $\mathbf{P}$ & AS & 33 & 33 & .25 & .26 & 36 & 34 & 29 & .28 & .34 & $A 8$ & .70 \\
\hline Bespen) & 2800 & 1600 & 1400 & 1400 & 1600 & 1600 & 1100 & 860 & 890 & 520 & 960 & 690 \\
\hline Cd & 3.8 & 3.4 & 3.5 & 3.8 & 3.9 & 3.8 & 5.4 & 6.9 & 7.4 & 3.2 & 3.6 & 1.4 \\
\hline$a$ & 50 & 42 & 32 & 36 & 47 & 32 & 28 & 31 & 31 & 35 & 45 & 49 \\
\hline Mo & 193 & 330 & 410 & 390 & 470 & 470 & 560 & 550 & 600 & 270 & 170 & 70 \\
\hline $\mathrm{Pb}$ & 780 & 970 & 1400 & 1400 & 940 & 880 & 1400 & 1300 & 1600 & 760 & 390 & 160 \\
\hline Sz & 1000 & $11 \infty$ & 1300 & 1200 & 1300 & 1400 & 1400 & 1300 & 1300 & 1100 & 500 & 330 \\
\hline $\mathbf{v}$ & 340 & 500 & 570 & 540 & 550 & 560 & 610 & 570 & 560 & 500 & 320 & 170 \\
\hline Zn & 570 & 950 & 570 & 570 & 630 & 600 & 620 & 760 & 790 & 500 & 500 & 260 \\
\hline Thicknosid(mm) & 15.0 & 15.0 & 120 & 15.0 & 15.0 & 3.0 & 5.0 & 220 & 220 & 10 & 6.0 & 6.0 \\
\hline
\end{tabular}


Table 8. Composition of crusts analyzed by Aplin (PhD. thesis) cont'd.

\begin{tabular}{|c|c|c|c|c|c|c|c|c|c|c|c|}
\hline Sequencell & 350010 & 350011 & 351007 & 351008 & 351009 & 351010 & 351011 & 351012 & 351013 & 351014 & 351015 \\
\hline Letitude & -13.033 & -13.033 & -11.050 & -11.050 & -12.883 & -12.883 & -12.883 & -12.883 & -12883 & -12883 & -12.883 \\
\hline Langitude & -147.933 & -147.933 & -150.100 & -150.100 & -150.767 & -150.767 & -150.767 & -150.767 & -150.767 & -150.767 & -150.767 \\
\hline Dopth & 4700 & 4700 & 4250 & 4250 & 3000 & 3000 & 3000 & 3000 & 3000 & 3000 & 3000 \\
\hline Lab: & $053-1 / 1 C$ & 053-1/1D & $050-4 / 6 \mathrm{~A}$ & $050-4 / 6 \mathrm{~B}$ & $051-2 \pi$ & $051-3 / 7 A$ & OS1-2/B & $051-577 \mathrm{~A}$ & $051.5 / 7 \mathrm{~B}$ & $051.5 / 7 \mathrm{C}$ & $051-6 / 7 A$ \\
\hline$T i(w t . \%)$ & AS & 46 & 1.28 & .91 & 93 & .78 & .87 & .91 & 94 & 98 & .80 \\
\hline Mn & 5.75 & 839 & 25.0 & 20.6 & 19.9 & 17.4 & 18.0 & 20.5 & 20.2 & 20.5 & 17.6 \\
\hline Fo & 5.68 & 6.24 & 19.8 & 18.5 & 17.9 & -16.1 & 17.1 & 21.0 & 22.0 & 22.3 & 15.8 \\
\hline $\mathbf{A}$ & 6.12 & 6.70 & .76 & 1.92 & .78 & 288 & 262 & 181 & 1.40 & 1.43 & 3.00 \\
\hline Co & .091 & .107 & .600 & $A 60$ & .380 & 316 & 316 & 377 & 406 & 384 & 309 \\
\hline Ni & 250 & .450 & 370 & .390 & 320 & $A \infty O$ & 430 & $A 10$ & 380 & 370 & 420 \\
\hline $\mathrm{Cr}$ & .120 & .246 & .101 & .124 & .124 & 309 & 224 & .191 & .194 & .196 & 331 \\
\hline $\mathrm{Ca}_{\mathrm{a}}$ & 1.96 & 2,00 & 2.62 & 2.64 & 2.27 & 2.04 & 2.19 & 297 & 256 & 262 & 2.03 \\
\hline $\mathbf{M g}$ & .92 & 1.11 & 1.03 & 1.01 & 1.24 & .99 & 1.09 & 1.04 & 1.03 & 1.00 & 1.08 \\
\hline $\mathrm{Na}$ & 3.01 & 2.77 & 1.59 & 1.58 & 241 & 2.05 & 1.83 & 1.49 & $1 \mathrm{A1}$ & $1 . A 1$ & 1.97 \\
\hline $\mathbf{K}$ & 3.42 & 296 & 32 & .48 & .33 & 1.00 & .76 & AS & 39 & 40 & .96 \\
\hline $\overrightarrow{\mathbf{P}}$ & 35 & .20 & .29 & .42 & .35 & .30 & .32 & 12 & .43 & .38 & .28 \\
\hline$B a(p p m)$ & 610 & 630 & 1300 & 1300 & 1000 & 1200 & 1300 & 1300 & 1300 & 1300 & 1300 \\
\hline Cd & 1.7 & 3.7 & 4.0 & 3.0 & 3.5 & 3.0 & 3.7 & 3.3 & 5.1 & 3.1 & 3.4 \\
\hline$c$ & 41 & ss & 30 & 33 & 32 & 48 & 48 & 49 & 66 & 47 & 41 \\
\hline $\mathbf{M o}$ & 100 & 150 & 600 & 440 & 410 & 320 & 300 & 410 & 450 & 420 & 300 \\
\hline $\mathbf{P b}_{\mathbf{b}}$ & 130 & 160 & 860 & 700 & 690 & 460 & 520 & 480 & 540 & 560 & 460 \\
\hline Sx & 270 & 310 & 1300 & 1100 & 1200 & 970 & 1000 & 1200 & 1200 & 1200 & 950 \\
\hline $\mathbf{V}$ & 160 & 200 & 610 & 510 & 590 & 470 & 480 & 520 & 560 & 550 & 440 \\
\hline $\mathrm{Zn}_{n}$ & 250 & 380 & 460 & 480 & 540 & 570 & 650 & 520 & 580 & 570 & 610 \\
\hline Thichopes(mm) & 6.0 & 6.0 & 30.0 & 30.0 & 20 & 12.0 & 120 & 30.0 & 30.0 & 30.0 & 15.0 \\
\hline
\end{tabular}

Table 8a. Rare earth elements of crusts analyzed by Aplin (PhD. thesis) cont'd.

\begin{tabular}{|c|c|c|c|c|c|c|c|c|c|c|c|}
\hline $\begin{array}{l}\text { Sequencell } \\
\text { Latitude }\end{array}$ & $\begin{array}{c}016008 \\
2.083\end{array}$ & $\begin{array}{c}016010 \\
4.367\end{array}$ & $\begin{array}{l}053051 \\
10.817\end{array}$ & $\begin{array}{l}053053 \\
10.817\end{array}$ & $\begin{array}{l}053054 \\
12517\end{array}$ & $\begin{array}{l}053056 \\
13.467\end{array}$ & $\begin{array}{l}053059 \\
14983\end{array}$ & $\begin{array}{l}053065 \\
15.150\end{array}$ & $\begin{array}{l}053068 \\
15.150\end{array}$ & $\begin{array}{l}053070 \\
15.150\end{array}$ & $\begin{array}{l}053075 \\
16.433\end{array}$ \\
\hline Langitude & -157.350 & -159.083 & -165.550 & -165.550 & $-167.050^{\circ}$ & -165.417 & -166.100 & -168.100 & -168.100 & -168.100 & -168.150 \\
\hline Depth(m) & 1350 & 2150 & 1150 & 1150 & 3000 & 3850 & 2800 & 2700 & 2700 & 2700 & 2325 \\
\hline Laby & $041-1 / 1$ & 055-3/3 & 058-1/2 & $058-2 / 28$ & $059-7 \pi$ & $060-2 / 4 \mathrm{~B}$ & $\infty 61-2 / 5$ & $062-1 / 3 A$ & C62-1/3D & $0.52-1 / 3 P$ & $063-2 / 9 A$ \\
\hline
\end{tabular}

\begin{tabular}{|c|c|c|c|c|c|c|c|c|c|c|c|}
\hline La(ppm) & 175 & 172 & 330 & 223 & 340 & 330 & 310 & 300 & 320 & 460 & 310 \\
\hline Ce & 610 & 300 & 940 & 1100 & 1300 & 1000 & 1000 & 700 & 1500 & 2900 & 890 \\
\hline $\mathbf{P r}$ & 26 & 28 & 64 & 30 & 78 & 70 & 59 & 58 & 57 & 58 & 54 \\
\hline Hd & 131 & 139 & 300 & 158 & 360 & 330 & 280 & 270 & 260 & 280 & 260 \\
\hline Sm & 26 & 27 & 63 & 30 & 76 & 68 & 57 & 54 & 47 & 56 & 50 \\
\hline $\mathrm{Eu}$ & 7 & 7 & 16 & 8 & 19 & 17 & 14 & 13 & 11 & 14 & 12 \\
\hline Gd & 31 & 31 & 66 & 36 & 73 & 68 & 59 & 54 & 42 & 60 & 54 \\
\hline Dy & 34 & 32 & 60 & 40 & 68 & 66 & 56 & 52 & 38 & 48 & 55 \\
\hline $\mathrm{Er}$ & 26 & 21 & 33 & 31 & 36 & 37 & 32 & 31 & 21 & 30 & 34 \\
\hline$\overline{Y_{b}}$ & 24 & 19 & 29 & 27 & 30 & 31 & 20 & 27 & 18 & 27 & 30 \\
\hline La & 4 & 3 & 4 & 4 & 4 & 5 & 4 & 4 & 3 & 4 & 4 \\
\hline Thichnead (mon) & 5.0 & 20 & 10.0 & 30.0 & 5.0 & 10.0 & 20.0 & 70.0 & 70.0 & 70.0 & 25.0 \\
\hline
\end{tabular}

\begin{tabular}{lcccccccc}
\hline Sequencell & 053078 & 053087 & 315003 & 315005 & 350011 & 351007 & 351008 & 351015 \\
Latitude & 16.433 & 16.433 & -7.583 & -9.067 & -13.033 & -11.050 & -11.050 & -12.883 \\
Longitudi & -168.150 & -168.150 & -151.550 & -150.700 & -147.933 & -150.100 & -150.100 & -150.767 \\
Depth(m) & 2325 & 2400 & 1880 & 1300 & 4700 & 4250 & 4250 & 3000 \\
LebH & $063-5 / 9 \mathrm{~A}$ & $064-5 / 7 \mathrm{~B}$ & $044-2 / 2$ & $045-6 / 13 \mathrm{~B}$ & $053-1 / 1 \mathrm{D}$ & $050-4 / 6 \mathrm{~A}$ & $050-4 / 6 \mathrm{~B}$ & $051-6 / 7 \mathrm{~A}$ \\
\hline
\end{tabular}

\begin{tabular}{|c|c|c|c|c|c|c|c|c|}
\hline Le(ppon) & 350 & 350 & 218 & 165 & 228 & 390 & 280 & 139 \\
\hline co & 990 & 1100 & 500 & 710 & 219 & 1400 & 900 & 320 \\
\hline Pr & 65 & 70 & 32 & 22 & 43 & 65 & 55 & 24 \\
\hline Hd & 310 & 330 & 160 & 109 & 201 & 290 & 260 & 113 \\
\hline $\mathrm{Sm}$ & 63 & 77 & 30 & 19 & 48 & 67 & 53 & 21 \\
\hline $\mathrm{Eu}$ & 16 & 17 & 8 & 5 & 12 & 17 & 13 & 5 \\
\hline Gd & 67 & 68 & 35 & 24 & 56 & 70 & 53 & 23 \\
\hline Dy & 63 & 66 & 40 & 28 & 50 & 60 & 52 & 24 \\
\hline $\mathrm{Br}$ & 36 & 38 & 28 & 22 & 31 & 35 & 32 & 16 \\
\hline $\mathbf{Y b}$ & 31 & 32 & 26 & 21 & 29 & 34 & 28 & 14 \\
\hline La & 5 & 5 & 4 & 3 & 5 & 6 & 4 & 2 \\
\hline 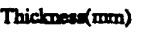 & 20 & 15.0 & 5.0 & 22.0 & 6.0 & 30.0 & 30.0 & 15.0 \\
\hline
\end{tabular}


Table 9. Composition of crusts from the Scripps Nodule Databank

\begin{tabular}{|c|c|c|c|c|c|c|c|c|c|c|c|}
\hline Sequence & $0800024-01$ & $0870004-00$ & $0890009-00$ & $0910002-00$ & $0910005-01$ & $0910005-02$ & $1120022-00$ & $1130048-00$ & $1130061-\infty$ & $1130062-01$ & 1130063-01 \\
\hline Latitude & -62850 & -64.517 & -64.183 & -63.067 & -63.100 & -63.100 & -56.617 & -55.933 & -53.042 & -59.142 & -53.058 \\
\hline Langitude & .74 .758 & -145.967 & -165.933 & 178.483 & 177.617 & 177.617 & -34.800 & -45.202 & -48.883 & -48.883 & -48.950 \\
\hline Dopth & 4005 & 3775 & 2932 & 3583 & 3383 & 3383 & 3696 & 3740 & 3118 & 3850 & 3255 \\
\hline Si(wt.\%) & $\cdot$ & - & 11.3 & 155 & - & $\cdot$ &. & - & - & $\cdot$ & - \\
\hline $\mathrm{TI}$ & - & .91 & .03 & .46 & .78 & 1.16 & • & • & 53 & - & - \\
\hline $\mathbf{M n}$ & 10.3 & 18.1 & 14.8 & 880 & 135 & 121 & 200 & 26.6 & 7.40 & 12.3 & 27.7 \\
\hline$F_{0}$ & 25.7 & 17.3 & 11.0 & 124 & 224 & 23.0 & 19.2 & 13.0 & 19.9 & 23.6 & 14.5 \\
\hline $\mathbf{A}$ & • & - & 240 & 3.90 & - & - & - & - & . & - & - \\
\hline Co & .140 & 570 & .170 & .140 & .330 & .350 & 480 & .120 & 340 & .150 & 210 \\
\hline $\mathrm{Ni}$ & .100 & 200 & .700 & .270 & 200 & .130 & 260 & 990 & .060 & 2200 & .770 \\
\hline $\mathrm{Cu}$ & .100 & .090 & .200 & .130 & .080 & .080 & .050 & .410 & .090 & .100 & .160 \\
\hline ca & 228 & - . & 1.52 & 1.79 & - & $\cdot$ & 224 & 2.00 & • & 214 & 1.65 \\
\hline $\mathbf{M} 8$ & 299 & - & - & - & - & - & 96 & 1.90 & $\cdot$ & 1.82 & 1.63 \\
\hline $\mathrm{Na}$ & 216 & - & - & - & - & - & 1.57 & 3.57 & - & 297 & 278 \\
\hline $\mathbf{K}$ & 98 & - & - & - & - & - & 34 & 1.07 & - & 99 & 1.07 \\
\hline $\mathbf{P}$ & - & - & • & - & • & - & - & . & - & $\cdot$ & . \\
\hline $\mathrm{H}_{2} \mathrm{O}$ & - & 17.1 & 17.4 & 12.2 & 19.1 & 20.4 & - & $\cdot$ & 15.0 & • & $\cdot$ \\
\hline A(ppon) & - & - & - & - & - & • & - & - & • & - & - \\
\hline $\mathrm{Ba}$ & - & 1200 & 3900 & 2500 & 1700 & 2800 & - & - & 1100 & - & - \\
\hline Cd & $\cdot$ & - & - & • & • & • & - & - & • & - & - \\
\hline Co & $\cdot$ & - & - & - & - & - & - & - & - & - & - \\
\hline$a$ & $\cdot$ & - & - & - & - & - & - & - & - & - & - \\
\hline Mo & - & 1300 & 110 & 50 & 1100 & 620 & - & - & 510 & - & - \\
\hline $\mathrm{Pb}$ & 2000 & - & 1500 & 1400 & - & $\because$ & 3100 & 900 & $\cdot$ & 2600 & 800 \\
\hline St & - & 1300 & 1000 & 1100 & 1300 & 1300 & - & - & 860 & - & • \\
\hline v & - & 560 & - & - & 1200 & 920 & - & - & 490 & - & - \\
\hline $\mathbf{Y}$ & $\cdot$ & - & • & - & • & $\cdot$ & - & - & • & - & - \\
\hline $\mathrm{Zn}$ & 640 & 400 & 1000 & 580 & 600 & 500 & 1800 & 2700 & 300 & 870 & 1600 \\
\hline Semple & Mn-coutrod & Noctule & Crax & Crust & Crust & Crast & Crust & Mn-costad & Soveral & Crast & Crust \\
\hline & rock & • & • & • & • & • & • & rock & crusts & • & - \\
\hline Portion & nnknown & without & croses & croses & outser & artion & unknown & unknown & miknown & unknown & nolonowe \\
\hline memplod & - & nuclous & nection & section & cont & cruat & - & - & - & - & - \\
\hline Nuclous & • & Clay & • & - & - & - & - & - & • & • & • \\
\hline Referenos & A368 & A227 & A197 & A197 & $A 2 x$ & A2x & A368 & A368 & $A 221$ & A368 & A368 \\
\hline Sequencell & $1130065-\infty 0$ & $1140011-01$ & $1140029-01$ & $1140040-05$ & $1140051-\infty$ & $1140081-01$ & $1140081-02$ & $1140088-01$ & $1140119-\infty$ & $1140127-00$ & $1140127-01$ \\
\hline Latitude & -59.033 & -53.000 & .57 .538 & -55.100 & .55 .733 & -56.207 & -56.267 & -56.233 & .55 .642 & .52833 & .52 .833 \\
\hline Langitude & 48.978 & -52.900 & -55.138 & -55.833 & -56.050 & -58.275 & -58.275 & -58.700 & -55.912 & -50.500 & -50.500 \\
\hline Dopth & 3862 & 3101 & 4075 & 2871 & 3914 & 4087 & 4087 & 4133 & 4085 & 2842 & 2842 \\
\hline Si(wt.\%) & - & 13.8 & - & $\cdot$ & - & - & - & $\cdot$ & $\cdot$ & • & - \\
\hline $\mathrm{Ti}$ & - & .25 & - & - & .37 & .40 & 47 & - & - & - & - \\
\hline Mn & 18.4 & 10.8 & 11.3 & 14.0 & 3.90 & 1.00 & 1.00 & 1.56 & 6.20 & 9.50 & 9.20 \\
\hline Fe & 21.1 & 15.8 & 17.9 & 28.2 & 19.2 & 14.5 & 16.8 & 40.3 & 20.8 & 14.7 & 18.9 \\
\hline $\mathbf{A}$ & - & 270 & - & $\cdot$ & - & - & - & • & $\cdot$ & 3.57 & 3.00 \\
\hline Co & .160 & .150 & .160 & 280 & .120 & .130 & .150 & .020 & .100 & .100 & .140 \\
\hline $\mathrm{Ni}$ & .500 & 390 & .240 & .230 & .120 & .290 & .130 & .030 & .110 & .240 & .070 \\
\hline Cu & 250 & .080 & .080 & .070 & .090 & .160 & .160 & .060 & .040 & .100 & .090 \\
\hline c. & 1.65 & 1.19 & 1.66 & 1.35 & - & - & - & 1.51 & $\cdot$ & - & $\cdot$ \\
\hline $\mathbf{M} 8$ & 1.70 & - & 252 & 1.54 & . & - & - & 1.75 & - & - & . \\
\hline $\mathrm{Na}$ & 3.08 & - & 4.07 & 209 & - & - & - & 219 & - & - & - \\
\hline $\mathbf{x}$ & 96 & - & 259 & .77 & - & - & - & .91 & - & - & - \\
\hline $\mathbf{P}$ & $\cdot$ & - & - & - & - & - & - & • & - & - & - \\
\hline $\mathrm{H}_{2} \mathrm{O}$ & - & 13.0 & - & - & 7.8 & 10.1 & 7.7 & $\cdot$ & - & - & - \\
\hline Ax(ppon) & - & - & - & - & • & - & - & - & - & - & - \\
\hline $\mathrm{Ba}$ & - & 3000 & - & - & 580 & 400 & 510 & - & - & - & - \\
\hline Cd & - & . & - & - & $\cdot$ & • & 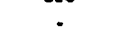 & - & - & - & - \\
\hline C. & - & - & - & - & - & - & - & - & - & $\cdot$ & $\cdot$ \\
\hline $\mathrm{Cr}$ & - & - & - & - & - & - & - & - & - & 52 & 14 \\
\hline Mo & • & 140 & • & - & 510 & 260 & 290 & • & - & 160 & 230 \\
\hline $\mathbf{P b}$ & 2100 & 1200 & 1100 & 3200 & - & • & $\cdot$ & 2400 & - & 600 & 1100 \\
\hline Sr & - & 800 & - & - & 760 & 480 & 520 & - & - & - & - \\
\hline $\mathbf{v}$ & - & $\cdot$ & - & - & 710 & 230 & 660 & - & - & 350 & 570 \\
\hline $\mathbf{Y}$ & • & - & - & - & - & - & $\cdot$ & - & - & - & - \\
\hline $\mathrm{zn}$ & 1100 & 670 & 520 & 900 & 300 & 460 & 450 & 660 & - & 720 & 1700 \\
\hline Semplo & Crust & Crust & Crust & Crust & Crat & Crust & Crust & Mn-coeted & Crust & Crust & Crust \\
\hline type & - & - & • & - & • & - & $\cdot$ & rock & $\cdot$ & - & - \\
\hline Portion & unknown & cross & mnkown & unkmown & outer & unknown & nnknown & anknown & unknown & anknown & unknown \\
\hline sampled & • & soction & • & - & crust & • & $\cdot$ & - & - & - & - \\
\hline Naclous & - & - & - & - & - & - & - & • & • & - & • \\
\hline Roferenos & A368 & A197 & A368 & A368 & A227 & A227 & A227 & A368 & A333 & A333 & A333 \\
\hline
\end{tabular}


Table 9. Composition of crusts from the Scripps Nodule Databank (cont'd).

\begin{tabular}{|c|c|c|c|c|c|c|c|c|c|c|c|}
\hline Sequencell & $1140128-\infty 0$ & $1150001-\infty$ & $1150191-\infty$ & $1160020-02$ & $1160025-02$ & $1180019-\infty$ & $1230002-00$ & $1230002-01$ & $1230006-00$ & $1230007-00$ & $1230011-\infty$ \\
\hline Latimade & .51 .517 & .57 .050 & .58 .467 & .57 .983 & -57.083 & -52017 & .52 .167 & -52.167 & -59.850 & -58.083 & -59.133 \\
\hline Langituds & .52 .433 & -60.017 & -60.625 & .70 .883 & -70.983 & -99.950 & -142.133 & -142.133 & .144 .867 & -144950 & -145.133 \\
\hline Dopth & 2052 & 3438 & 3039 & 4007 & 3767 & 4418 & 2295 & 2205 & 3219 & 3025 & 3155 \\
\hline Sir(wt\%) & - & - & - & - & - & $\cdot$ & $\cdot$ & . & . & - & . \\
\hline $\mathrm{Ti}$ & - & .60 & • & - & - & .67 & 1.08 & 1.43 & 1.14 & 1.03 & .71 \\
\hline Mn & 20 & 7.60 & 11.7 & 10.4 & 14.7 & 21.2 & 21.2 & 20.5 & 10.6 & 11.7 & 125 \\
\hline Po & 15.3 & 233 & 26.8 & 159 & 255 & 14.6 & 20.7 & 17.1 & 224 & 21.3 & 18.4 \\
\hline $\mathbf{A}$ & 260 & 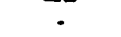 & . & . & . & 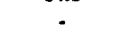 & 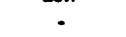 & 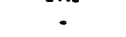 & 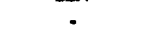 & $\because$ & 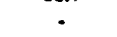 \\
\hline Co & .010 & .220 & .210 & 570 & .160 & .180 & .740 & 139 & 440 & .420 & 350 \\
\hline $\mathbf{N I}$ & .040 & .130 & .080 & .210 & 310 & .840 & 100 & 390 & .170 & .180 & 520 \\
\hline $\mathrm{Cn}$ & .010 & .130 & .070 & .070 & .140 & 320 & .110 & .060 & .110 & .080 & 280 \\
\hline c. & • & - & 241 & • & 2.11 & • & • & - & - & • & - \\
\hline $\mathbf{M g}$ & - & - & 152 & - & 200 & - & - & - & - & - & - \\
\hline $\mathrm{Na}$ & - & - & 2.49 & - & 2.71 & - & - & - & - & - & - \\
\hline $\mathbf{K}$ & - & - & 58 & - & 1.12 & - & - & - & - & - & - \\
\hline $\mathbf{P}$ & - & • & - & - & - & • & • & - & • & • & • \\
\hline $\mathrm{H}_{2} \mathrm{O}$ & - & 13.1 & - & 17.0 & - & 19.5 & 10.1 & 8.1 & 15.7 & 19.2 & 159 \\
\hline$A x(p p m)$ & - & - & - & - & - & - & - & • & • & - & - \\
\hline Ba & - & 2600 & - & - & - & 900 & 1000 & 1000 & 650 & 830 & 2300 \\
\hline Cd & - & - & - & - & - & - & - & • & - & - & - \\
\hline Co & • & - & - & - & - & - & - & - & - & - & - \\
\hline$c$ & 128 & • & - & - & - & • & • & • & • & - & • \\
\hline Mo & 50 & 820 & - & - & 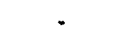 & 550 & 630 & 640 & 1100 & 410 & 350 \\
\hline $\mathbf{P b}$ & • & • & 3300 & - & 2100 & - & - & - & . & - & . \\
\hline Sr & • & 910 & - & - & • & 1000 & 1400 & 1200 & 910 & 1100 & 810 \\
\hline $\mathbf{v}$ & 200 & 890 & - & - & - & 540 & 1000 & 800 & 850 & 760 & 540 \\
\hline $\mathbf{Y}$ & - & - & - & - & - & - & - & - & $\cdot$ & - & - \\
\hline $\mathrm{Zn}$ & 330 & 500 & 720 & - & 740 & 700 & 550 & 410 & 600 & 500 & 700 \\
\hline Semplo & Crust & Crust & Merconted & Nodule & Crust & Crust & Unlonown & Noctulo & Cruxt & Crust & Crux \\
\hline type & • & • & rock & - & - & • & • & • & - & • & • \\
\hline Partion & fremonts & outor & mnknown & whole & unterown & outror & unkenown & whole & outsor & whole & outer \\
\hline anuplod & - & crust & - & - & • & cruat & $\cdot$ & - & crust & - & coust \\
\hline Neclow & - & Cry & - & - & • & Sediment & • & - & Undoterminod & non-spperent & Palagcaits \\
\hline References & $\mathbf{A 3 3 3}$ & A227 & A368 & A227 & $A 368$ & A227 & $A 227$ & A22 & A227 & A227 & A227 \\
\hline Sequencer: & $1250012-00$ & $1270003-00$ & $1280029-00$ & $1200010-00$ & $1290032-00$ & $1290032-01$ & $1200032-02$ & $1290032-03$ & $1290062-00$ & $1300013-00$ & $1300013-01$ \\
\hline Latitude & -54.500 & -50.317 & -50.783 & .56 .367 & .55 .158 & -55.158 & -55.158 & -35.158 & -56.683 & .56 .417 & $-56,417$ \\
\hline Longitude & -163.317 & 176.383 & 169.958 & 158.467 & 150.050 & 150.050 & 150.050 & 150.050 & 158.767 & 140.017 & 140.017 \\
\hline Depth & 4650 & 2377 & 896 & 1595 & 3866 & 3866 & 3866 & 3866 & 1280 & 3750 & 3750 \\
\hline Si(wt.s) & 9.80 & 1.50 & 150 & $\cdot$ & . & - & $\cdot$ & - & 4.60 & 31.3 & 11.2 \\
\hline Ti & .61 & 1.00 & 1.00 & 1.15 & • & • & • & • & • & 55 & 1.01 \\
\hline Mn & 14,4 & 11.1 & 5.10 & 215 & 124 & 10.9 & 13.5 & 11.5 & 15.2 & .20 & 12.4 \\
\hline Fe & 149 & 12.2 & 24.4 & 20.4 & 16.2 & 18.0 & 15.4 & 145 & 20.8 & 5.25 & 129 \\
\hline $\mathbf{N}$ & 200 & 2.00 & 2.00 & - & . & - & - & - & - & 7.30 & 1.90 \\
\hline$c_{0}$ & .300 & 300 & .200 & .730 & $A 50$ & .290 & 320 & .130 & 300 & .010 & .100 \\
\hline $\mathbf{N i}$ & 370 & .150 & .200 & 380 & 260 & .190 & .840 & 360 & .250 & - & - \\
\hline Cu & .150 & - & - & .040 & .060 & .040 & .040 & .060 & .110 & .010 & .220 \\
\hline c. & 1.32 & 2.00 & 2.00 & - & • & - & - & $\cdot$ & 1.83 & .41 & 2.14 \\
\hline $\mathbf{M g}_{\mathbf{g}}$ & - & 1.00 & 1.50 & - & - & . & - & - & . & 1.50 & 1.20 \\
\hline $\mathrm{Na}$ & - & 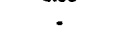 & . & - & - & - & - & - & - & 1.28 & 1.59 \\
\hline $\mathbf{K}$ & - & • & • & - & - & - & - & - & • & 277 & .70 \\
\hline$P$ & - & 1.00 & 3.00 & - & - & . & - & - & $.2 n$ & .03 & .15 \\
\hline $\mathrm{H}_{2} \mathrm{O}$ & 165 & 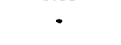 & - & 27.0 & - & - & - & - & $\cdot$ & 1.0 & 18.2 \\
\hline$A($ ppon) & - & - & - & - & - & - & - & - & 270 & - & - \\
\hline Ba & 4200 & - & - & 1500 & - & - & - & - & $\cdot$ & 100 & 800 \\
\hline $\mathrm{Cd}$ & - & - & - & - & - & - & - & - & - & - & - \\
\hline co & . & - & - & - & - & - & - & - & - & - & - \\
\hline$c$ & • & 40 & 100 & - & - & - & - & - & • & 70 & 70 \\
\hline Mo & 170 & 100 & 100 & 720 & - & - & - & - & 440 & - & • \\
\hline $\mathbf{P b}$ & 1700 & 1500 & 1500 & - & - & - & - & - & 1600 & 300 & 1100 \\
\hline Sr & 1000 & • & • & 1600 & - & - & - & - & 1400 & 200 & 900 \\
\hline $\mathbf{v}$ & • & 500 & 800 & 1100 & - & - & - & - & - & 200 & 300 \\
\hline $\mathbf{Y}$ & - & • & - & - & • & • & • & - & 175 & • & - \\
\hline $\mathbf{Z n}_{n}$ & 570 & • & - & 620 & 190 & 160 & 190 & 160 & 910 & 200 & 600 \\
\hline Semplo & Coust & Crust & Mercostod & Noctule & Mn-conted & Min-contod & Mn-costad & Mn-conted & Mo-costred & Mn-costod & Mo-costed \\
\hline typo & • & - & rock & - & rock & rock & rock & rock & rock & rock & rock \\
\hline Partice & croses & unicrown & mbown & whole & outor & onter & outar & outor & outer & melous & outor \\
\hline sempled & soction & . & . & - & $\cos x$ & const & crust & crose & crust & - & crost \\
\hline Nucleus & - & • & - & • & - & - & - & • & volcenic & Rock & Rock \\
\hline Referencos & A197 & A260 & A260 & $A 227$ & A250 & A250 & A250 & A250 & A388 & A247 & A247 \\
\hline
\end{tabular}


Table 9. Composition of crusts from the Scripps Nodule Databank (cont'd).

\begin{tabular}{|c|c|c|c|c|c|c|c|c|c|c|c|}
\hline Sequencell & $1300013-02$ & $1300013-03$ & $1300013-04$ & 1300013.05 & $1300013-06$ & $1300013-07$ & $1300013-08$ & $1300013-09$ & $1300013-10$ & $1300013-11$ & $1300013-13$ \\
\hline Latimde & -56.417 & $-56,417$ & -56.417 & .56 .417 & $.56,417$ & -56.417 & -56.417 & -56.417 & -56.417 & -56.417 & $-56,417$ \\
\hline Longitude & 140.017 & 140.017 & 140.017 & 140.017 & 140.017 & 140.017 & 140.017 & 140.017 & 140.017 & 140.017 & 140.017 \\
\hline Dopth & 3750 & 3750 & 3750 & 3750 & 3750 & 3750 & 3750 & 3750 & 3750 & 3750 & 3750 \\
\hline Si(wt\%) & 20.0 & 20.0 & 23.0 & 15.0 & 19.6 & 31.0 & 20.1 & 16.0 & 10.7 & 30.0 & 6.00 \\
\hline $\mathbf{T i}$ & 35 & 54 & .47 & 51 & 1.00 & 20 & 58 & .67 & .62 & 24 & .22 \\
\hline Mn & 3.70 & 2.40 & .49 & 11.0 & 8.80 & 238 & 4.94 & 7.01 & 18.5 & 20 & 28.9 \\
\hline $\mathrm{F}_{0}$ & 4.13 & 17.1 & 6.01 & 8.18 & 9.93 & $3 A 5$ & 14.3 & 128 & 13.4 & 270 & 6.01 \\
\hline $\mathbf{A}$ & 630 & 2.20 & 8.60 & 440 & 430 & 6.67 & 2.90 & 2.80 & 1.40 & 8.10 & 1.10 \\
\hline$C_{0}$ & .020 & .020 & .010 & .060 & .030 & .010 & .020 & .040 & .230 & .010 & .120 \\
\hline $\mathbf{N i}$ & .020 & .060 & .020 & 510 & 280 & .010 & .170 & .200 & .610 & .010 & 1.130 \\
\hline $\mathrm{Cr}$ & .020 & .050 & .020 & 220 & .130 & .010 & .090 & .150 & 260 & .010 & .410 \\
\hline ca & 2.93 & 1.04 & 3.34 & 248 & 2.17 & 1.09 & 94 & 1.37 & 1.77 & 1.52 & 1.98 \\
\hline Ms & 1.35 & 1.72 & 3.11 & 227 & 1.16 & .74 & 1.19 & 139 & 1.25 & $A 6$ & 1.69 \\
\hline $\mathrm{Na}$ & • & 1.66 & 2.14 & 205 & . & - & 1.94 & 1.95 & 1.90 & 3.15 & 2.15 \\
\hline $\mathbf{K}$ & - & 1.24 & 1.24 & 86 & • & - & 1.74 & 1.01 & .71 & 2.18 & .78 \\
\hline $\mathbf{P}$ & .05 & .06 & .05 & .07 & .36 & - & . & .10 & .14 & .03 & .11 \\
\hline $\mathrm{H}_{2} \mathrm{O}$ & 29 & 11.1 & 6.4 & 10.6 & 6.4 & 26 & 9.2 & 15.2 & 10.9 & 4.1 & 13.9 \\
\hline
\end{tabular}

\begin{tabular}{|c|c|c|c|c|c|c|c|c|c|c|c|}
\hline$A(p p m)$ & $\cdot$ & $\cdot$ & $\cdot$ & $\cdot$ & • & - 1 & • & • & - & - & - \\
\hline $\mathrm{Ba}$ & 400 & 200 & 200 & 500 & 400 & 500 & 400 & 500 & 900 & 400 & 1300 \\
\hline Cd & - & - & - & - & • & - & - & - & - & - & - \\
\hline co & - & $\cdot$ & - & - & - &. & - & - & - & - & - \\
\hline$C$ & 70 & 140 & 70 & 70 & 70 & 70 & 70 & 70 & 70 & 70 & 70 \\
\hline Mo & 200 & 500 & 100 & 400 & 500 & 200 & 100 & 200 & 300 & 100 & 600 \\
\hline $\mathrm{Pb}$ & - & 1200 & 200 & 600 & - & - & 900 & 900 & 900 & 900 & 700 \\
\hline Sz & - & 300 & 300 & 600 & - & - & 500 & 700 & 800 & 200 & 800 \\
\hline V & 100 & 200 & 300 & 400 & 500 & 200 & 200 & 300 & 300 & 100 & 500 \\
\hline $\mathbf{Y}$ & - & • & - & • & - & $\cdot$ & - & - & - & - & - \\
\hline $\mathrm{Zn}_{\mathbf{n}}$ & • & 300 & 300 & 1000 & - & • & 600 & 600 & 1000 & 80 & 3400 \\
\hline $\begin{array}{l}\text { Semple } \\
\text { type }\end{array}$ & $\begin{array}{l}\text { Mn-coutod } \\
\text { rock }\end{array}$ & $\begin{array}{l}\text { Mn-coutod } \\
\text { rock }\end{array}$ & $\begin{array}{l}\text { Mn-contod } \\
\text { rock }\end{array}$ & $\begin{array}{l}\text { Mn-controd } \\
\text { rock }\end{array}$ & $\begin{array}{l}\text { Mn-coited } \\
\text { rock }\end{array}$ & $\begin{array}{l}\text { Mor-costed } \\
\text { rock }\end{array}$ & $\begin{array}{l}\text { Mn-costed } \\
\text { rock }\end{array}$ & $\begin{array}{l}\text { Mn-coutod } \\
\text { rock }\end{array}$ & $\begin{array}{l}\text { Mor-conted } \\
\text { rock }\end{array}$ & $\begin{array}{l}\text { Min-coutod } \\
\text { rock }\end{array}$ & $\begin{array}{l}\text { Min-costed } \\
\text { rock }\end{array}$ \\
\hline Partion & whole & whole & rndeus & outer & whole & whole & whole & whole & whole & whole & whole \\
\hline earoplod & - & • & - & cout & • & - & - & • & • & • & - \\
\hline Naclous & Rock & Clay & Rock & Rock & Rock & Rock & Rock & Clay & Rock & Rock & . \\
\hline
\end{tabular}

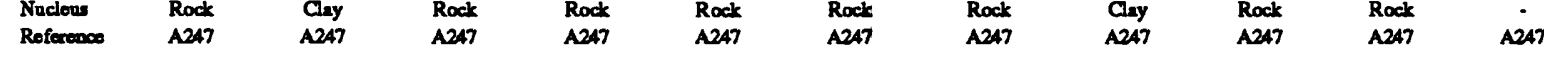

\begin{tabular}{|c|c|c|c|c|c|c|c|c|c|c|c|}
\hline Sequencell & $1300013-14$ & $1300013-15$ & $1300013-16$ & $1340035-00$ & $1350032-00$ & $1360009-00$ & $1360023-01$ & $1430013-00$ & $1440015-00$ & $1490051-00$ & $1490055-00$ \\
\hline Latiouds & -56.417 & $-56,417$ & .56 .417 & -56.555 & -54833 & .52083 & -55.880 & .52 .167 & -50.367 & 49.633 & 49.650 \\
\hline Longitude & 140.017 & 140.017 & 140.017 & 100.068 & 90.140 & 84.547 & 81.118 & 10,483 & 8.750 & 42.467 & -44.117 \\
\hline Dopth & 3750 & 3750 & 3750 & 3409 & 4662 & 4232 & 4086 & 2561 & 4686 & 2202 & 2952 \\
\hline Si(wt.\%) & 20.0 & 33.6 & 24.3 & - & $\cdot$ & - & . & $\cdot$ & $\cdot$ & $\cdot$ & $\cdot$ \\
\hline $\mathrm{Ti}$ & 36 & .48 & .60 & - & - & - & - & - & - & - & - \\
\hline Mn & 3.40 & .20 & .19 & 11.0 & 1.00 & 16.3 & 8.90 & 24.0 & 21.0 & 14.8 & 15.2 \\
\hline Po & 6.40 & 3.87 & 11.2 & 14.1 & 15.2 & 16.6 & 10.6 & 19.5 & 9.80 & 14.8 & 17.7 \\
\hline $\mathbf{A}$ & 7.80 & 4.80 & 3.20 & $\cdot$ & • & - & - & $\cdot$ & - & 1.88 & 1.40 \\
\hline Co & .010 & .010 & .010 & .050 & • & .110 & • & .600 & .080 & 240 & .170 \\
\hline $\mathrm{Ni}$ & .170 & .010 & .050 & .180 & 310 & .410 & .200 & 380 & .600 & .450 & .270 \\
\hline $\mathrm{Cu}$ & .080 & .010 & .100 & $\$ 70$ & 370 & .260 & .210 & .040 & .200 & .200 & .120 \\
\hline C & 1.43 & 1.32 & .71 & 1.30 & 1.00 & 1.50 & 1.50 & - & 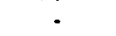 & - & - \\
\hline Ms & 2.23 & 1.51 & 1.71 & - & - & . & . & - & - & - & - \\
\hline $\mathrm{Na}$ & .09 & .58 & 2.33 & - & - & - & - & . & - & - & . \\
\hline $\mathbf{K}$ & 3.75 & 1.91 & 1.57 & - & - & - & - & - & - & . & - \\
\hline $\mathbf{P}$ & .07 & .03 & .05 & - & . & - & - & - & - & - & - \\
\hline $\mathrm{H}_{2} \mathrm{O}$ & 10.7 & 27 & 10.6 & - & - & - & $\cdot$ & - & • & - & - \\
\hline$A-(p p m)$ & - & - & - & - & . & - & - & - & - & - & - \\
\hline $\mathrm{Ba}$ & 800 & 400 & 300 & - & - & - & - & - & - & - & - \\
\hline Cd & - & $\cdot$ & - & - & - & - & - & - & - & - & - \\
\hline co & - & - & - & - & . & - & - & - & - & - & . \\
\hline Cr & 70 & 70 & 70 & - & - & - & - & - & . & • & - \\
\hline Mo & 300 & 100 & 100 & • & • & - & • & - & - & 180 & 260 \\
\hline $\mathrm{Pb}$ & 500 & 300 & 900 & 1300 & 1100 & 1400 & 1300 & - & - & 800 & 1300 \\
\hline Sr & 400 & 80 & 200 & $\therefore$ & 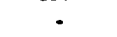 & . & - & - & - & - & $\cdot$ \\
\hline v & 200 & 100 & 200 & - & - & - & - & - & - & - & - \\
\hline $\mathbf{Y}$ & . & $\cdot$ & $\cdot$ & $\cdot$ & $\cdot$ & - & - & - & - & - & - \\
\hline $\mathbf{Z n}$ & 200 & 200 & 300 & 900 & 700 & 700 & 700 & - & - & 960 & 820 \\
\hline Serople & Mn-coatrod & Mn-costred & Mn-couted & Mn-coutrod & Crust & Crust & Mn-encrusted & Mn-coatrod & Mn-costod & Crust & Coust \\
\hline typo & rock & rock & rock & rock & • & • & sediment & rock & rock & $\cdot$ & $\cdot$ \\
\hline Partion & whole & whole & whole & withoust & fragments & fragment: & belf & anknown & unknown & unknown & unknown \\
\hline semplod & - & • & - & macleus & 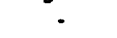 & . & $\vec{\cdot}$ & - & . & . & - \\
\hline Nuclews & Chout & Rock & Rock & Rocik & - & - & - & - & • & . & - \\
\hline Reforence & A247 & $A 247$ & $A 247$ & A422 & $\mathbf{A 4 2 2}$ & $A 422$ & A422 & A333 & A333 & A333 & A333 \\
\hline
\end{tabular}


Table 9. Composition of crusts from the Scripps Nodule Databank (cont'd).

\begin{tabular}{|c|c|c|c|c|c|c|c|c|c|c|c|}
\hline Sequenced & $1490056-00$ & $1500059-00$ & $1500059-01$ & $1500129-00$ & $1530001-\infty 0$ & $1530003-\infty 0$ & $15500005-07$ & $1580001-01$ & $1590005-02$ & $1630063-00$ & $1630064-00$ \\
\hline Letionde & -49.617 & -47.933 & -47.933 & -47.762 & -42.067 & -42083 & -41.983 & -42.017 & 45.100 & -49.667 & -49.400 \\
\hline Lancitudo & 44.100 & -57.100 & .57 .100 & -57.642 & -81.983 & -86.050 & .102 .017 & -130.033 & -145.183 & 179.883 & 177.983 \\
\hline Depth & 2932 & 2585 & 2585 & 3652 & 3200 & 3012 & 4200 & 4839 & 4954 & 2286 & 978 \\
\hline$\overline{S i(w t . \%)}$ & - & . & $\cdot$ & $\cdot$ &. & $\cdot$ & . & $\cdot$ & $\cdot$ & 150 & 150 \\
\hline $\mathbf{T i}$ & • & - & - & - & 53 & 1.15 & • & 1.15 & • & 150 & 1.00 \\
\hline Mn & 5.80 & 23.0 & 18.6 & .09 & 123 & 10.7 & 245 & 10.7 & 18.7 & 14.7 & 18.8 \\
\hline $\overrightarrow{P_{0}}$ & 11.0 & 18.0 & 22.2 & 450 & 27.6 & 28.2 & 8.20 & 28.2 & 9.80 & 17.1 & 7.07 \\
\hline $\mathbf{A}$ & 3.68 & - & - & • & - & • & - & - & - & 200 & .60 \\
\hline Co & 080 & 1.20 & .320 & • & 260 & .240 & .100 & 240 & .190 & .600 & .600 \\
\hline $\mathbf{N i}$ & .080 & 1.100 & .210 & .010 & .130 & .130 & 920 & .130 & .770 & .300 & 1.000 \\
\hline $\mathrm{Cu}$ & .070 & .080 & .060 & .010 & 000 & .090 & 180 & 090 & .460 & - & - \\
\hline c & 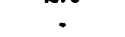 & - & 184 & 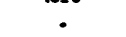 & . & - & 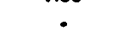 & - & 1.60 & 200 & 1.00 \\
\hline Ms & - & - & 93 & - & - & - & - & - & - & 1.00 & .70 \\
\hline Na & - & - & 1.43 & - & - & - & - & - & - & - & 1.00 \\
\hline $\mathbf{K}$ & - & - & 31 & . & - & - & - & - & - & • & - \\
\hline $\mathbf{P}$ & - & . & - & - & - & • & - & - & - & 150 & - \\
\hline $\mathrm{H}_{2} \mathrm{O}$ & - & - & - & - & 21.9 & 25.2 & - & 25.2 & - & - & - \\
\hline As(ppon) & - & - & - & - & - & • & • & • & - & - & • \\
\hline Be & - & - & - & - & 1200 & 1100 & 1600 & 1100 & - & - & 1000 \\
\hline Cd & - & - & - & - & . & . & - & 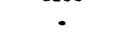 & - & - & . \\
\hline$c_{0}$ & - & . & - & - & - & - & - & - & - & - & - \\
\hline$c$ & - & - & - & - & - & - & - & - & - & 40 & 20 \\
\hline Mo & 100 & - & • & - & 510 & 620 & - & 620 & • & 200 & 150 \\
\hline $\mathbf{P b}_{\mathbf{b}}$ & 600 & - & 2600 & . & - & • & - & - & 900 & 2000 & 1000 \\
\hline St & - & - & . & - & 1300 & 1000 & - & 1000 & $\cdot$ & • & 1000 \\
\hline $\mathbf{v}$ & - & - & - & - & 1300 & 810 & . & 810 & - & 500 & 700 \\
\hline $\mathbf{Y}$ & - & . & • & - & 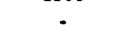 & - & - & - & • & - & 100 \\
\hline $\mathbf{Z n}$ & 370 & - & 700 & - & 500 & 500 & 1800 & 500 & 800 & • & • \\
\hline Semplo & Cout & Crast & Crust & Mn-conted & Crust & Crast & Crust & Crust & Min-conted & Noctalo & Nodule \\
\hline type & - & - & - & rock & • & • & - & - & rock & • & • \\
\hline Portion & unlonown & unknown & monown & uxtonown & ontex & outur & unknown & outor & half & unknown & unknown \\
\hline axpolod & - & - & - & - & costex & cruat & . & cont & - & - & - \\
\hline Neclows & • & - & - & - & Basalt & • & • & Pelaganito & Pumice & Undbtermined & • \\
\hline Roferenos & A333 & A179 & $A 368$ & 1419 & A227 & A221 & A323 & A227 & A459 & A260 & A260 \\
\hline Soquencell & 163006401 & $1630065-01$ & $1630066-00$ & $1630066-01$ & 181002400 & $1810025-\infty$ & $1840038-00$ & $1840039-00$ & $1890007-00$ & $1890007-01$ & $1890013-00$ \\
\hline Latitude & -49.400 & -49.983 & -49.367 & -49.367 & .33 .800 & .33800 & -30.033 & -30.500 & -33.050 & -33.050 & -33.042 \\
\hline Lengitude & 177.983 & 177533 & 176.267 & 176.267 & -1.233 & -1.233 & -38.950 & .35 .933 & -82050 & -82.050 & -83.942 \\
\hline Depth & 978 & 1335 & 1280 & 1280 & 1489 & 1553 & 939 & 1000 & 3881 & 3881 & 3650 \\
\hline$S i(w t . \%)$ & 332 & 1.96 & 150 & 3.88 & $\cdot$ & . & $\cdot$ & - & $\cdot$ & . & . \\
\hline $\mathrm{Ti}$ & $\cdot$ & - & 1.20 & - & - & - & - & - & 91 & 1.18 & .71 \\
\hline $\mathrm{Mn}$ & 10.4 & 122 & 22.2 & 194 & 25.0 & 23.4 & 26.0 & 24.8 & 17.5 & - & 19.1 \\
\hline Fo & 6.78 & 7.19 & 15.2 & 758 & 19.3 & 19.6 & 17.2 & 19.4 & 18.7 & 19.9 & 18.9 \\
\hline $\boldsymbol{N}$ & • & - & 200 & - & - & - & - & - & - & • & - \\
\hline$C_{0}$ & A20 & .180 & 1.000 & $A 60$ & .730 & .590 & 1.01 & 900 & .170 & .060 & .190 \\
\hline $\mathbf{N i}$ & .610 & 530 & 1.000 & 920 & 360 & .410 & .530 & 360 & .490 & .160 & .460 \\
\hline Cu & .080 & .050 & - & .100 & .060 & .090 & .060 & .040 & .210 & .220 & .190 \\
\hline ca & 37 & 8.69 & 200 & 3.79 & - & - & 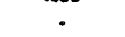 & . & . & . & 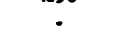 \\
\hline Ms & - & - & 150 & - & - & . & - & - & - & - & - \\
\hline $\mathrm{Na}$ & - & - & - & - & - & - & - & - & - & - & - \\
\hline $\mathbf{K}$ & • & • & - & - & - & - & . & - & . & - & - \\
\hline $\mathbf{P}$ & 33 & 244 & - & 97 & - & - & . & - & - & - & • \\
\hline $\mathrm{H}_{2} \mathrm{O}$ & - & - & - & - & - & - & - & - & 18.1 & 8.3 & 22.7 \\
\hline As(ppon) & 91 & 115 & - & 113 & - & - & - & - & - & - & - \\
\hline$B_{\mathbf{n}}$ & 3100 & 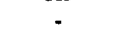 & - & 4300 & - & - & . & - & 1200 & 100 & 1200 \\
\hline Cd & - & - & - & • & . & - & - & - & - & $\cdot$ & - \\
\hline C. & - & - & - & - & - & - & - & - & - & . & - \\
\hline$c$ & - & - & - & - & - & - & - & - & - & - & - \\
\hline Mo & 74 & 430 & 200 & 420 & - & - & - & - & 820 & 230 & 200 \\
\hline $\mathrm{Pb}$ & 400 & 700 & 1500 & 700 & . & - & - & - & . & . & 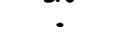 \\
\hline Sr & 1300 & 1400 & - & 1100 & - & - & - & - & 1100 & 230 & 810 \\
\hline $\mathbf{v}$ & - & - & 500 & - & - & - & - & - & 730 & 610 & 360 \\
\hline $\mathbf{Y}$ & 122 & 112 & - & 104 & - & - & - & - & - & $\cdot$ & - \\
\hline $\mathrm{Zn}_{\mathrm{n}}$ & 740 & 1100 & - & 1400 & - & • & • & - & $6 \infty$ & 800 & 500 \\
\hline Semplo & Mn-coutod & Mn-conted & Noctulo & Mn-conted & Nodulo & Noctula & Nodule & Unknown & Crust & Crust & Cust \\
\hline type & rock & rock & - & rock & - & - & - & - & - & - & • \\
\hline Portion & outor & outor & minnown & couturer & nnknown & monown & unknown & uniknown & coter & medous & contar \\
\hline ampiod & coust & crost & - & crust & . & - & $\cdot$ & - & crust & - & crut \\
\hline Nuclous & - & - & - & - & - & - & - & • & any & $a_{x y}$ & Clay \\
\hline References & A388 & A388 & A260 & A388 & A333 & A333 & A333 & A333 & A220 & A227 & 1227 \\
\hline
\end{tabular}


Table 9. Composition of crusts from the Scripps Nodule Databank (cont'd).

\begin{tabular}{|c|c|c|c|c|c|c|c|c|c|c|c|}
\hline Sequences & $1890013-01$ & $1890016-00$ & $1890016-01$ & $1890022-01$ & $1900007-00$ & $1900014-00$ & $1900014-01$ & $1930004-\infty$ & $1940005-00$ & $1940005-01$ & $1940005-02$ \\
\hline Letitude & -33.042 & -32967 & -32.967 & -33.075 & -39.850 & -39.892 & -39.892 & -37.067 & -35.967 & -35.967 & -35.967 \\
\hline Langitude & -83.942 & -88.000 & $\$ 88.000$ & -89.550 & -96.867 & -96908 & -96.908 & -129.967 & -134.300 & -134.300 & -134.300 \\
\hline Dopth & 3650 & 3619 & 3619 & 3795 & 3639 & 3557 & 3557 & 3548 & 4696 & 4696 & 4696 \\
\hline si(wt.\%) & - & $\cdot$ & - & - & $\cdot$ & - & $\cdot$ & $\cdot$ & $\cdot$ & $\cdot$ & $\cdot$ \\
\hline $\mathrm{Ti}$ & 1.19 & .37 & 1.17 & - & .23 & - & - & 1.20 & .78 & 94 & 96 \\
\hline Mn & - & 22.5 & • & 17.8 & 28.6 & 27.2 & 21.7 & 21.9 & 226 & 21.7 & 183 \\
\hline Po & 19.6 & 15.8 & 20.6 & 8.60 & 10.3 & 15.1 & 13.0 & 168 & 13.6 & 14.4 & 16.5 \\
\hline $\mathbf{A}$ & • & • & • & - & - & - & - & • & • & • & • \\
\hline Co & .020 & .190 & .040 & .040 & .120 & .050 & .050 & 1.02 & .340 & 390 & $\mathbf{4 0 0}$ \\
\hline $\mathbf{N i}$ & .110 & 1.060 & .010 & 1.200 & 1.730 & 1.180 & .600 & .370 & .750 & .740 & 390 \\
\hline $\mathrm{Ca}$ & .160 & 370 & .090 & 490 & 440 & .90 & .500 & .060 & .200 & .180 & .110 \\
\hline c. & • & • & - & 1.60 & - & 250 & 1.90 & • & • & - & • \\
\hline Mg & - & - & - & - & - & - & - & - & - & - & - \\
\hline $\mathrm{Na}$ & - & - & - & - & - & - & - & - & . & . & - \\
\hline $\mathbf{x}$ & - & - & - & - & . & - & - & - & - & - & - \\
\hline $\mathbf{P}$ & - & • & - & - & - & - & . & - & • & - & - \\
\hline $\mathrm{H}_{2} \mathrm{O}$ & 8.0 & 20.5 & 8.8 & - & 19.2 & - & - & 23.6 & 21.1 & 21.7 & 22.6 \\
\hline As(ppon) & - & - & - & - & - & - & - & - & - & - & - \\
\hline $\mathrm{Bs}$ & 100 & 1200 & 100 & - & 1500 & • & - & 1200 & 1000 & 930 & 800 \\
\hline Cd & $\cdot$ & • & • & - & - & - & - & • & - & $\cdot$ & • \\
\hline Co & - & - & - & - & - & - & . & - & - & - & - \\
\hline$c$ & - & - & - & - & - & - & - & - & $\cdot$ & - & - \\
\hline Mo & 680 & 610 & 110 & - & 590 & - & - & 430 & 560 & 490 & 250 \\
\hline $\mathrm{Pb}$ & - & - & • & 500 & - & 300 & 300 & • & • & - & - \\
\hline Sr & 250 & 1000 & 160 & - & 840 & - & - & 1500 & 1300 & 1200 & 1300 \\
\hline $\mathbf{v}$ & 410 & 740 & 460 & - & 740 & - & - & 770 & 540 & 600 & 550 \\
\hline $\mathbf{Y}$ & - & - & • & - & - & • & - & - & $\cdot$ & $\cdot$ & - \\
\hline $\mathrm{Zn}$ & 800 & 800 & 200 & 1100 & 1200 & 1800 & 1600 & 300 & 400 & 400 & 300 \\
\hline Samplo & Crust & Crust & Crust & Mn-eacrusted & Coust & Crust & Crust & Crust & Cruat & Cruat & Crust \\
\hline type & - & - & • & sodimeat & - & - & • & • & • & • & - \\
\hline Partion & melons & outer & melous & half & ouver & milnown & unicnown & outer & outor & outser & outor \\
\hline numplod & - & crust & • & $\cdot$ & crust & - & - & crest & crust & cruat & crust \\
\hline Nuclous & Clay & Cay & Oay & Sediment & Palagonito & - & - & • & $\cdot$ & - & Palagonits \\
\hline Referenco & 1227 & $A 227$ & A220 & A459 & 1227 & A459 & A459 & A227 & A227 & A227 & $A 227$ \\
\hline Sequence & $1940009-00$ & $1970019-00$ & $1970033-00$ & $1980025-01$ & $1980025-02$ & $1980049-00$ & $2010001-\infty 0$ & $2010001-01$ & $2010001-02$ & $2010001-03$ & $2040011-\infty 0$ \\
\hline Letionde & -35.183 & -32.675 & -38.643 & -35.850 & -35.850 & -33.000 & -39.750 & -39.750 & -39.750 & -39.750 & -37.465 \\
\hline Langitude & -135.533 & -160.013 & -167.918 & -178.517 & -178.517 & -171.652 & 159.650 & 159.650 & 159.650 & 159.650 & 120.255 \\
\hline Dopth & 4700 & 5300 & 1420 & 6715 & 6715 & 3225 & $\cdot$ & - & $\cdot$ & $\cdot$ & 4316 \\
\hline Si(wt.\$) & - & 738 & . & . & 6.03 & $\cdot$ & . & . & 9.40 & 7.80 & . \\
\hline $\mathrm{Ti}$ & 1.34 & - & - & - & - & 1.01 & - & • & - & - & - \\
\hline $\mathbf{M n}$ & 19.6 & 14.6 & 23.4 & 205 & 17.6 & 16.9 & 26.2 & 27.3 & 22.0 & 23.4 & 2.30 \\
\hline Fo & 14.2 & 23.3 & 16.1 & 20.4 & 19.5 & 21.5 & 8.19 & 7.33 & 7.89 & 7.20 & 5.90 \\
\hline $\mathbf{A}$ & • & - & - & - & $\cdot$ & $\cdot$ & - & - & $\cdot$ & - & 4.90 \\
\hline Co & .730 & - & 1.000 & .250 & .180 & .460 & .190 & .180 & .150 & .130 & .080 \\
\hline $\mathrm{Ni}$ & .330 & .200 & .540 & .200 & .210 & .370 & 1.250 & 1.300 & 1.100 & 1.170 & .070 \\
\hline c. & .050 & .420 & .100 & .070 & .080 & .90 & .600 & .600 & .560 & 550 & .060 \\
\hline c. & • & 1.64 & 3.50 & 1.90 & 1.68 & $\cdot$ & 2.36 & 159 & 2.20 & 1.47 & - \\
\hline $\mathbf{M g}$ & - & - & - & • & - & - & - & - & • & - & - \\
\hline Na & - & - & - & - & - & - & - & - & - & - & - \\
\hline $\mathbf{K}$ & - & - & - & - & - & - & - & - & - & - & - \\
\hline $\mathbf{P}$ & - & - & - & - & .19 & - & - & - & 40 & .09 & - \\
\hline $\mathrm{H}_{2} \mathrm{O}$ & - & 47.1 & - & - & - & - & - & - & - & • & - \\
\hline$A=$ (ppm) & - & - & - & - & 260 & - & - & - & 94 & 122 & - \\
\hline $\mathrm{Ba}$ & 90 & - & - & - & - & - & - & - & - & - & - \\
\hline Cd & - & - & - & - & - & - & 14.0 & 14.0 & - & - & - \\
\hline co & - & - & - & - & - & - & - & - & - & - & - \\
\hline c. & 31 & - & - & - & - & - & - & - & - & . & 53 \\
\hline Mo & 330 & - & - & - & 700 & - & - & - & 410 & 440 & 100 \\
\hline $\mathbf{P b}$ & 600 & - & 2100 & 1800 & 1300 & - & 800 & 700 & 600 & 400 & 400 \\
\hline st & - & - & - & - & 1000 & - & - & - & 570 & 480 & • \\
\hline $\mathbf{v}$ & 440 & - & - & - & - & * & - & - & - & - & - \\
\hline $\mathbf{Y}$ & - & - & • & - & 174 & - & - & - & 151 & 68 & • \\
\hline $\mathrm{Zn}_{n}$ & - & 800 & 800 & 320 & 400 & * & 1100 & 1100 & 1100 & 1100 & 210 \\
\hline Samplop & Nodule & Mn-conted & Crust & Mn-encrusted & Mn-encrussod & Crust & Mn-controd & Mn-conted & Mo-costrod & Mn-coated & Mn-encrustod \\
\hline type & • & rock & - & sodimeat & sodimest & 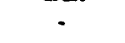 & ergonicnet. & rock & organicmat. & rock & sodiment \\
\hline Portica & half & uniknown & fragmonts & cuter & outer & unknown & unicnown & unknown & outur & outer & whole \\
\hline sempiod & . & 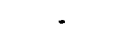 & 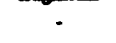 & crost & crust & $=$ & $\cdot$ & - & crust & crunt & - \\
\hline Nuclous & - & - & • & $a_{x y}$ & any & - & Bone & Pumice & Bane & Pumice & Sediment \\
\hline Roferencos & $A 240$ & A293 & A405 & $A 200$ & A388 & A343 & $A 200$ & A200 & A388 & A388 & A407 \\
\hline
\end{tabular}


Table 9. Composition of crusts from the Scripps Nodule Databank (cont'd).

\begin{tabular}{|c|c|c|c|c|c|c|c|c|c|c|c|}
\hline Sequencell & $2040014-\infty$ & $2040016-00$ & $2050057-\infty 0$ & $2060016-00$ & $2070001-00$ & $2070001-01$ & $2070019-00$ & $2070085-00$ & $2100024-01$ & $2100027-00$ & $2100027-01$ \\
\hline Latioude & -37.500 & -37.162 & -38.185 & -32800 & -31.500 & -31.500 & -31.283 & -31.710 & -30.443 & -32667 & -32.667 \\
\hline Longinde & 120.167 & 121.223 & 118.675 & 103.872 & 99.283 & 99.283 & 94.207 & 91.365 & 60.805 & 66383 & 66.383 \\
\hline Dopth & 3736 & 4243 & 4023 & 5300 & 2412 & 2412 & 2074 & 4298 & 5731 & 4910 & 4910 \\
\hline Si(wt.\%) & - & - & . & $\cdot$ & $\cdot$ & $\cdot$ & - & - & $\cdot$ & $\cdot$ & 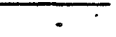 \\
\hline TI & - & - & - & 49 & - & - & • & - & - & - & • \\
\hline Mn & 13.9 & 15.3 & 18.7 & 14.5 & 220 & 23.4 & 1.87 & 19.1 & 12.3 & 13.7 & 129 \\
\hline Fo & 19.8 & 14.4 & 21.3 & 129 & 7.89 & 7.20 & 163 & 19.7 & 17.9 & 15.1 & 17.5 \\
\hline A & - & - & - & 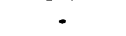 & - & - & 5.66 & 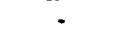 & 255 & 3.71 & 3.26 \\
\hline co & .170 & .230 & .280 & 240 & .150 & .130 & 080 & 320 & .280 & 20 & 210 \\
\hline $\mathbf{N i}$ & .150 & 470 & .250 & .490 & 1.100 & 1.170 & .100 & 300 & .140 & 300 & 260 \\
\hline $\mathrm{Cu}$ & .140 & 320 & .110 & 300 & 560 & 550 & .070 & 120 & .090 & .190 & .180 \\
\hline Ca & 290 & 1.50 & 2.90 & - & - & - & 230 & 3.70 & 2.14 & 1.91 & 1.34 \\
\hline $\mathbf{M}_{\mathbf{g}}$ & - & - & - & - & - & . & - & - & - & • & - \\
\hline $\mathrm{Na}$ & - & - & - & - & - & - & - & - & - & - & - \\
\hline $\mathbf{K}$ & - & - & - & - & - & - & - & - & - & - & - \\
\hline P & - & - & - & . & - & - & . & - & - & - & - \\
\hline $\mathrm{H}_{2} \mathrm{O}$ & - & - & - & - & - & - & - & - & - & - & - \\
\hline Ax(ppm) & - & - & . & - & - & . & - & . & - & - & . \\
\hline $\mathrm{Ba}$ & - & - & - & 690 & - & - & - & - & - & - & - \\
\hline cd & - & - & - & - & - & - & - & - & - & - & - \\
\hline co & - & - & - & - & - & - & - & - & - & - & . \\
\hline$a$ & - & - & - & 6 & - & - & - & - & - & . & - \\
\hline Mo & - & - & • & 380 & 410 & 440 & - & - & - & - & - \\
\hline Pb & 800 & 900 & 1200 & 200 & - & • & 100 & 1200 & 1400 & 1100 & 1000 \\
\hline St & $\cdot$ & - & 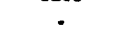 & $\cdot$ & - & - & 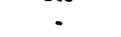 & 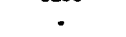 & 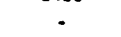 & - & $\cdot$ \\
\hline $\mathbf{v}$ & - & - & - & 470 & - & - & - & - & - & - & - \\
\hline $\mathbf{Y}$ & - & - & • & - & - & - & - & - & - & - & . \\
\hline $\mathbf{Z a}$ & 800 & 600 & 500 & • & 1100 & 1100 & 540 & 500 & 440 & - & - \\
\hline Semplo & Cruat & Crus & Const & Crust & Mn-conted & Mo-conted & Min-controd & Crust & Crust & Crust & Crust \\
\hline type & - & - &. & • & argeniemat. & rock & rook & - & - & • & - \\
\hline Partion & fragmonts & fngmonts & fragments & outar & ourer & $\infty$ & anter & fremouts & unknown & unknown & uninown \\
\hline acuplod & - & - & . & cout & crust & croust & crut & . & - & - & - \\
\hline Nuclous & • & • & • & • & - & • & - & • & • & • & • \\
\hline Referenos & A405 & A405 & 1405 & $A 240$ & A388 & A388 & $\mathbf{A 4 6 7}$ & A40s & A467 & A467 & A467 \\
\hline Sequencelly & $2120046-00$ & $2130069-00$ & $2130059-04$ & $2130070-00$ & 213007001 & $2140009-\infty 0$ & $2140010-00$ & $2140022-01$ & $2140058-00$ & $2140071-00$ & $2140073-00$ \\
\hline Latitude & -36.750 & -32.917 & -32917 & -31.900 & -31.900 & $.37,433$ & -38.633 & -39.533 & -39350 & -35.333 & -35.783 \\
\hline Longinude & 44.767 & 35.317 & 35.317 & 34350 & 34.350 & 26.467 & 26.133 & 24.833 & 25.050 & 22.642 & 22.383 \\
\hline Depth & 2265 & 1250 & 1250 & 2700 & 2700 & 2867 & 2960 & 2961 & 2875 & 320 & 1070 \\
\hline$\overline{S i(w t \%)}$ & . & 3.30 & 1.60 & 4.40 & - & 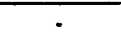 & 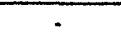 & . & - & 3.20 & 4.90 \\
\hline $\mathrm{Ti}$ & - & 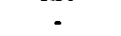 & - & - & - & - & • & - & - & - & - \\
\hline Mn & 19.5 & 15.2 & 19.1 & 16.2 & 16.0 & 15.0 & 11.8 & 17.2 & 23.6 & 10.9 & 15.8 \\
\hline Pe & 17.8 & 9.60 & 15.5 & 15.2 & 19.0 & 20.2 & 19.7 & 2.18 & 11.0 & 9.00 & 18.4 \\
\hline Al & 1.31 & - & - & . & 1.47 & . & . & . & - & - & - \\
\hline Co & .690 & 530 & .770 & .620 & .610 & .540 & 580 & 300 & .240 & .180 & 370 \\
\hline $\mathrm{Ni}$ & 370 & .650 & .320 & 350 & 340 & .360 & 580 & .120 & 1.140 & 420 & 580 \\
\hline $\mathrm{Cu}$ & 080 & .130 & .050 & .100 & .090 & .140 & .170 & .070 & .190 & .030 & .040 \\
\hline $\mathrm{Ca}$ & 201 & 5.46 & 1.89 & 137 & 1.23 & 1.50 & 1.15 & 2.74 & . & 6.50 & 5.30 \\
\hline$M_{8}$ &. & 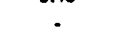 & - & . & . & 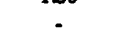 & 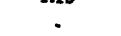 & 1.23 & . & - & - \\
\hline $\mathrm{Na}$ & . & . & - & . & . & . & . & 2.02 & - & - & . \\
\hline $\mathbf{K}$ & - & . & - & - & . & . & . & .49 & . & . & - \\
\hline$\vec{P}$ & - & 1.65 & .20 & .23 & - & - & - & 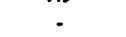 & - & 4.10 & 1.80 \\
\hline $\mathrm{H}_{2} \mathrm{O}$ & - & $\cdot$ & 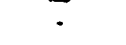 & $\cdot$ & - & - & . & - & - & 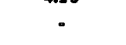 & - \\
\hline$A x$ (ppon) & - & 310 & 360 & 243 & - & - & . & - & - & 209 & 380 \\
\hline $\mathrm{Ba}$ & - & 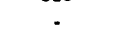 & 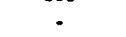 & 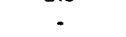 & - & - & . & - & - & . & 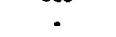 \\
\hline Cd & - & - & - & . & . & . & . & - & - & . & - \\
\hline co & - & - & . & . & . & - & . & . & . & . & . \\
\hline$c$ & . & • & - & - & - & - & . & . & . & - & - \\
\hline Mo & • & 430 & 720 & 490 & • & - & - & - & - & 182 & 430 \\
\hline $\mathrm{Pb}$ & 2200 & 1500 & 2400 & 1800 & 2200 & 1700 & 1200 & 2500 & . & 500 & 700 \\
\hline Sr & - & 1300 & 1700 & 1200 & - & 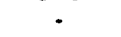 & $\cdot$ & - & - & 1600 & 1200 \\
\hline v & - & - & $\cdot$ & - & - & - & - & - & - & • & - \\
\hline $\mathbf{Y}$ & • & 510 & 180 & 112 & $\cdot$ & - & - & - & - & 60 & 141 \\
\hline $\mathbf{Z n}_{n}$ & 700 & 690 & 570 & 670 & 680 & 700 & 590 & 690 & • & 840 & 1000 \\
\hline Semple & Crust & Noctule & Nodule & Nodale & Unkonown & Nodulo & Nodule & Cruat & Crust & Mn-conted & Crust \\
\hline type & - & - & - & - & - & . & - & - & - & rock & - \\
\hline Partion & unionown & half & outer & half & unionown & fratomonts & unimown & unkoown & unknown & cutor & unknown \\
\hline sampled & - & • & $\cos x$ & • & . & . & - & . & - & cout & . \\
\hline Nucieus & • & - & - & - & - & - & - & - & - & Rock & - \\
\hline Referenco & A467 & A388 & A388 & A388 & A467 & $A 422$ & A200 & A368 & A333 & A466 & A466 \\
\hline
\end{tabular}


Table 9. Composition of crusts from the Scripps Nodule Databank (cont'd).

\begin{tabular}{|c|c|c|c|c|c|c|c|c|c|c|c|}
\hline Sequencell & $2140074-00$ & $2140074-01$ & $2140075-\infty$ & $2140076-00$ & $2150025-01$ & $2150026-01$ & $2150026-02$ & 215005404 & $2150058-02$ & $2150086-00$ & $2160038-00$ \\
\hline Latitude & -35.433 & -35.433 & -35.617 & -35.650 & -37550 & -37.550 & -37.550 & -34.600 & -34.700 & .32 .367 & -32.325 \\
\hline Longinude & 22367 & 22367 & 22.367 & 22217 & 18.100 & 18.100 & 18.100 & 17.000 & 16.900 & 15.017 & 2.158 \\
\hline Dopth & 1700 & 1700 & 230 & 202 & 3147 & 1796 & 1796 & 2740 & 3200 & 2941 & 2047 \\
\hline si(wt.s) & 90 & 1.10 & 1.70 & 90 & . & $\cdot$ & - & 7.67 & 5.24 & - & . \\
\hline $\mathrm{Ti}$ & - & . & 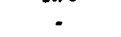 & - & - & 13 & 40 & - & - & - & • \\
\hline Mn & .18 & 13.6 & 11.2 & 22 & 18.2 & 19.0 & 21.0 & 168 & 23.9 & 17.9 & 17.0 \\
\hline Fe & 37.6 & 7.60 & 4.90 & 42.0 & 218 & 17.5 & 21.0 & 16.6 & 13.8 & 19.3 & 16.0 \\
\hline $\mathbf{A}$ & - & - & 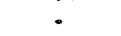 & - & - & 24 & .65 & - & - & - & $\cdot$ \\
\hline$C_{0}$ & - & .160 & .140 & - & 370 & .064 & .700 & 290 & .230 & .150 & 840 \\
\hline $\mathbf{N i}$ & .010 & .660 & .570 & .020 & 200 & .360 & 370 & 540 & .830 & 350 & 560 \\
\hline $\mathrm{Cr}$ & $\cdot$ & .020 & .080 & - & .040 & .050 & .040 & .070 & .110 & .160 & .100 \\
\hline ca & 4.29 & 6.70 & 9.20 & 239 & 2.19 & 7.12 & 4.96 & 1.63 & 1.70 & 1.91 & - \\
\hline $\mathbf{M g}$ & - & - & - & - & 1.12 & 1.60 & 1.00 & - & 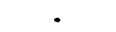 & 1.54 & - \\
\hline $\mathrm{Na}$ & - & - & - & - & 181 & 1.19 & .21 & - & - & 211 & - \\
\hline $\mathbf{K}$ & • & - & - & - & .19 & 36 & 33 & - & - & .72 & - \\
\hline $\mathbf{P}$ & 1.50 & 4.10 & 480 & 1.00 & - & 1.96 & 1.43 & .21 & 21 & $\cdot$ & - \\
\hline $\mathrm{H}_{2} \mathrm{O}$ & • & - & • & - & - & • & - & • & • & - & - \\
\hline As(ppm) & 630 & 2003 & 144 & 1100 & - & - & - & - & 215 & - & - \\
\hline $\mathrm{Ba}$ & 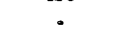 & - & 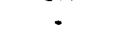 & 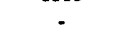 & - & - & - & - & 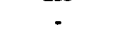 & - & - \\
\hline Cd & - & - & - & - & - & - & - & - & - & - & - \\
\hline Co & - & - & - & - & - & - & - & - & - & - & - \\
\hline$c$ & - & - & - & - & - & - & - & - & - & - & - \\
\hline Mo & 16 & 260 & 237 & 23 & • & 180 & 160 & - & 350 & - & - \\
\hline $\mathrm{Pb}$ & 100 & 700 & 300 & 100 & 2700 & 1600 & 2000 & - & 300 & 1700 & . \\
\hline Sr & 380 & 2800 & 2000 & 205 & 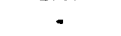 & . & - & - & 860 & - & - \\
\hline $\mathrm{v}$ & . & - & - & . & . & - & . & . & . & - & - \\
\hline $\mathbf{Y}$ & 17 & 51 & 50 & 14 & - & - & . & - & 68 & - & . \\
\hline $\mathrm{Zn}_{n}$ & 260 & 740 & 750 & 320 & 600 & . & - & 50 & 1200 & 1900 & - \\
\hline Semplo & Crust & Crust & Crust & Crust & Crant & Nodule & Nodulo & Mn-costed & Mo-cottod & Crust & Unkenown \\
\hline type & - & - & - & - & - & - & - & material & material & - & - \\
\hline Partion & uniknown & unlonown & unknown & unknown & unlonown & unknown & unionown & outur & outre & unionown & unknown \\
\hline samplad & - & • & - & - & - & - & . & crust & crust & - & • \\
\hline Nucleus & - & - & - & - & - & - & . & arganic & arganic & - & - \\
\hline Roferencos & A466 & A466 & A466 & A466 & A368 & A419 & A419 & A388 & A388 & A368 & A179 \\
\hline Seqpence & $2160038-01$ & $2160039-00$ & $2160041-\infty$ & $2160041-01$ & 2160041.02 & $2160050-01$ & $2160052-01$ & 2160058-00 & $2190040-01$ & $2190042-01$ & $2190045-01$ \\
\hline Lationde & -32.325 & -32.367 & .32350 & -32350 & -32.350 & -31.483 & -31.450 & -33.517 & -28.500 & -28.533 & -28.533 \\
\hline Longinds & 2.158 & 2.150 & 2.117 & 2117 & 2.117 & 1.033 & 950 & 2467 & -28.967 & -29.000 & -29.000 \\
\hline Depth & 2047 & 1786 & 1675 & 1675 & 1675 & 3043 & 4136 & 4477 & 3781 & 3566 & 4361 \\
\hline Si(wt.\%) & . & - & - & . & . & $\cdot$ & . & $\cdot$ &. & - & . \\
\hline $\mathrm{Ti}$ & - & - & - & - & - & - & - & - & - & - & - \\
\hline Mn & 20.4 & 26.8 & 17.0 & 220 & 19.0 & 18.0 & 173 & 7.10 & 18.6 & 134 & 18.6 \\
\hline Fe & 19.5 & 15.8 & 18.0 & 19.0 & 18.0 & 22.3 & 17.7 & 4.30 & 19.6 & 143 & 19.6 \\
\hline A & - & - & $\cdot$ &. & - & 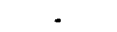 & - & - & - & - & - \\
\hline Co & .670 & .700 & .840 & .070 & .920 & .630 & .200 & .050 & .480 & .370 & 490 \\
\hline $\mathrm{Ni}$ & .250 & 840 & .360 & 260 & 360 & .230 & 300 & 360 & 310 & 280 & .280 \\
\hline $\mathrm{Cu}$ & .030 & .180 & .030 & .030 & .040 & .060 & .130 & 200 & .130 & .130 & .080 \\
\hline Ca & 248 & 1.79 & - & 292 & - & 2.12 & 3.67 & - & 240 & 5.14 & 230 \\
\hline $\mathbf{M g}$ & 1.04 & 198 & - & 1.03 & - & 1.18 & 1.42 & - & 1.36 & 1.02 & 1.57 \\
\hline $\mathrm{Ne}$ & 1.48 & 1.50 & - & 1.42 & - & 1.46 & 2.32 & - & 1.78 & 3.36 & 1.87 \\
\hline $\mathbf{K}$ & 31 & .48 & . & 33 & - & 35 & .95 & - & .64 & 2.71 & 35 \\
\hline $\mathbf{P}$ & $\therefore$ & - & - & - & - & 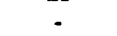 & - & - & - & - & 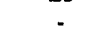 \\
\hline $\mathrm{H}_{2} \mathrm{O}$ & - & - & - & - & - & - & - & - & - & - & - \\
\hline As(ppon) & - & - & - & - & - & - & - & - & - & - & - \\
\hline Ba & - & - & - & - & - & - & - & - & - & - & - \\
\hline cd & - & - & - & - & - & - & - & - & - & - & - \\
\hline Ce & - & - & - & . & - & - & . & - & - & - & - \\
\hline$c$ & - & - & - & - & - & . & - & - & - & . & - \\
\hline Mo & - & - & - & - & - & - & - & - & - & - & - \\
\hline $\mathrm{Pb}$ & 2900 & 22000 & - & 3000 & - & 1900 & 1700 & - & 1300 & 1000 & 1900 \\
\hline Sx & $\cdot$ & - & - & - & - & - & - & - & $\cdot$ & 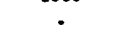 & - \\
\hline $\mathrm{v}$ & - & - & - & - & - & - & - & - & - & - & - \\
\hline $\mathbf{Y}$ & - & - & - & - & - & - & - & - & - & - & - \\
\hline $\mathrm{Zn}$ & 580 & 1100 & - & 1900 & - & 580 & 600 & - & 810 & 4600 & 580 \\
\hline Semplo & Nodule & Nodule & Unknown & Nodule & Noctale & Nadule & Crust & Mn-costed & Crust & Crust & Crast \\
\hline type & - & - & • & - & - & • & • & rock & - & - & • \\
\hline Partion & fragments & unknown & unknown & fragments & unionown & unknown & unknown & outer & monown & unknown & unknown \\
\hline samplod & - & • & • & - & • & - & $\cdot$ & crust & $\cdot$ & - & - \\
\hline Nucleus & - & • & - & - & • & - & • & Sediment & - & • & - \\
\hline Reforence & A368 & A368 & A179 & A368 & A419 & $A 368$ & $A 368$ & A333 & A368 & A368 & A368 \\
\hline
\end{tabular}


Table 9. Composition of crusts from the Scripps Nodule Databank (cont'd).

\begin{tabular}{|c|c|c|c|c|c|c|c|c|c|c|c|}
\hline Sequencell & 2190045-02 & $2210032-01$ & $2250007-01$ & $2250007-05$ & $2310033-00$ & $2310055-01$ & $2320002-00$ & $2320002-01$ & $2320015-\infty$ & $2320015-01$ & $2320019-00$ \\
\hline Letimado & -28.533 & -28.367 & -25.517 & -25.517 & -20.610 & -20.390 & .22583 & -22.583 & -24.683 & -24.683 & -24.812 \\
\hline Langituds & -29.000 & -41.217 & -85.233 & .85 .233 & -147588 & -148.020 & -150.800 & -150.800 & -155.250 & -155.250 & -157.488 \\
\hline Depth & 4361 & 3280 & 920 & 920 & 4570 & 4630 & $80 \pi$ & 807 & 4542 & 4542 & 4901 \\
\hline$S i(w t \%)$ & . & . & 260 & $\cdot$ & $\cdot$ & . & $\cdot$ & . & 4.40 & 5.10 & $\cdot$ \\
\hline $\mathbf{T i}$ & - & - & 59 & - & - & 1.00 & 1.29 & - & 88 & .97 & - \\
\hline Mn & 17.2 & 12.2 & 37.3 & 21.5 & 20.3 & 16.2 & 16.1 & 47.5 & 15.7 & 14.0 & 19.5 \\
\hline Fo & 20.8 & 18.7 & 11.2 & 9.40 & 15.4 & 17.9 & 118 & 138 & 17.0 & 189 & 16.7 \\
\hline $\mathbf{N}$ & 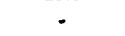 & - & .70 & - & 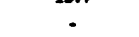 & 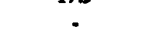 & . & - & 220 & 220 &. \\
\hline co & 540 & 330 & .700 & 1.34 & 510 & 520 & 257 & 1.89 & 570 & 580 & .580 \\
\hline $\mathrm{Ni}$ & .270 & 230 & 560 & 340 & .600 & 420 & 410 & 830 & $A 10$ & .270 & .520 \\
\hline Cu & .140 & .070 & .160 & .060 & 300 & .130 & .050 & .090 & .260 & 310 & .310 \\
\hline ca & 3.65 & - & 2.10 & - & 250 & • & • & - & 1.73 & 1.69 & 270 \\
\hline $\mathbf{M g}$ & 1.23 & - & 210 & - & 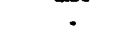 & - & - & . & 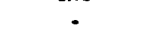 & 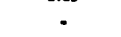 & 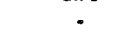 \\
\hline $\mathrm{Na}$ & 1.49 & - & 240 & - & - & - & - & - & - & - & - \\
\hline $\mathbf{K}$ & 4 & - & so & - & . & . & - & - & - & - & - \\
\hline $\mathbf{P}$ & - & - & - & - & - & - & - & - & - & - & - \\
\hline $\mathrm{H}_{2} \mathrm{O}$ & - & - & - & - & - & - & - & - & 18.7 & 17.4 & - \\
\hline$A \times(\mathrm{ppm})$ & - & - & - & - & - & - & - & - & - & - & - \\
\hline $\mathrm{Ba}$ & - & - & 1800 & - & - & - & 5900 & - & 6500 & 6200 & - \\
\hline Cd & - & - & - & - & . & - & - & - & • & - & - \\
\hline$c_{0}$ & - & - & - & - & - & - & - & - & - & - & - \\
\hline$c$ & - & - & - & . & - & . & - & - & - & - & - \\
\hline Mo & • & . & $\infty \infty$ & - & - & - & 400 & - & 250 & 210 & - \\
\hline Pb & 1600 & - & 1900 & - & 900 & 800 & 5100 & 4100 & 1500 & 1600 & 1200 \\
\hline Sr & - & - & 740 & - & - & - & - & • & 1000 & 1100 & - \\
\hline $\mathbf{v}$ & - & - & 600 & - & - & 210 & 690 & . & - & - & - \\
\hline $\mathbf{Y}$ & - & - & 260 & - & . & 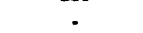 & - & - & - & - & - \\
\hline$z_{n}$ & 670 & - & 400 & 500 & 700 & 550 & • & - & 630 & 590 & 700 \\
\hline Semple & Nodule & Soverel & Nodule & Crust & Nodule & Noctule & Nochlob & Nodulo & Nocule & Nodulo & Notule \\
\hline typo & $\cdot$ & conests & - & - & - & • & $\cdot$ & $\cdot$ & - & • & $\cdot$ \\
\hline Partion & unknown & unknown & coter & nnionown & helf & croses & half & $\operatorname{crose}$ & croses & outer & bulf \\
\hline sempiod & - & • & coust & - & - & soction & - & soction & soction & const & - \\
\hline Nuclous & - & - & - & - & Undoterminod & Undeturmined & - & • & - & • & Undetermined \\
\hline Referencos & $A 368$ & A333 & A261 & A323 & A437 & A248. & A240 & A166 & A197 & A197 & A437 \\
\hline Sequencel & $2320022-00$ & $2320026-01$ & $2320028-01$ & $2320028-02$ & $2320028-04$ & $2330001-00$ & $2330001-01$ & $2330023-03$ & $2330024-01$ & $2330024-02$ & $2330027-\infty$ \\
\hline Latioude & -26.033 & -29.583 & .27 .943 & -27.943 & .27 .943 & -27.523 & .27 .523 & -23.535 & -22.937 & -22.937 & -21.897 \\
\hline Longitude & -157.967 & -159.100 & -159.785 & -159.785 & -159.785 & -160.652 & -160.652 & -162.902 & -162.080 & -162.080 & -160.913 \\
\hline Depth & 5038 & 5252 & 4558 & 4558 & 4558 & 4990 & 4990 & 4993 & 4848 & 4848 & 4817 \\
\hline$\overline{S i(w t \%)}$ & 5.90 & 6.30 & . & . & - & . & . & 6.76 & 5.85 & 5.84 & 638 \\
\hline $\mathbf{T i}$ & 98 & .89 & • & • & - & • & - & - & - & - & 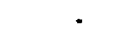 \\
\hline Mn & 14.1 & 13.4 & 16.0 & 14.6 & 15.1 & 17.3 & 17.5 & 15.9 & 178 & 21.8 & $19.6^{\circ}$ \\
\hline Fo & 18.2 & 18.4 & 18.4 & 16.4 & 16.6 & 175 & 17.8 & 26.9 & 23.5 & 23.5 & 20.0 \\
\hline $\mathbf{N}$ & 250 & 3.10 & 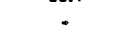 & - & . & . & - & 275 & 286 & 286 & 3.18 \\
\hline co & .730 & 540 & .500 & 590 & 500 & 520 & 510 & 540 & 580 & 580 & 520 \\
\hline $\mathbf{N i}$ & 320 & 330 & .420 & .250 & 340 & 430 & 410 & 230 & 440 & .440 & .470 \\
\hline $\mathrm{Cr}$ & .140 & 230 & 250 & .150 & 210 & 240 & .210 & .120 & 250 & .250 & .230 \\
\hline $\mathrm{Ca}_{2}$ & 1.37 & 1.19 & 1.50 & 1.70 & 1.60 & 1.70 & 1.60 & 1.88 & 1.68 & 1.72 & 2.14 \\
\hline $\mathrm{Mg}$ & . & . & - & . & 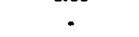 & 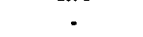 & 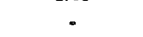 & - & . & - & - \\
\hline $\mathrm{Na}$ & - & - & - & - & . & - & - & . & - & . & - \\
\hline $\mathbf{K}$ & - & - & - & - & - & - & - & - & - & - & - \\
\hline $\mathbf{P}$ & - & • & - & - & . & - & - & • & - & - & - \\
\hline $\mathrm{H}_{2} \mathrm{O}$ & 16.4 & 17.1 & - & - & - & - & - & 41.9 & 32.9 & 32.9 & 29.4 \\
\hline$A d(p p m)$ & - & - & - & - & - & - & - & - & - & - & - \\
\hline $\mathrm{Ba}$ & 7200 & 6800 & - & - & - & - & - & . & - & - & - \\
\hline Cd & . & - & - & - & - & - & . & - & . & . & - \\
\hline Co & - & - & - & - & - & . & - & - & - & . & - \\
\hline$c$ & - & - & - & . & - & - & - & • & • & - & • \\
\hline Mo & 200 & 210 & - & • & • & • & - & 200 & 300 & 300 & 400 \\
\hline Pb & 1800 & 1700 & 1100 & 1100 & 1000 & 1100 & 1100 & 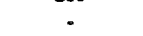 & - & . & . \\
\hline St & 930 & 890 & - & - & - & - & - & - & - & . & . \\
\hline $\mathbf{v}$ & - & - & - & - & - & . & - & - & - & . & . \\
\hline $\mathbf{Y}$ & - & - & - & - & - & • & • & - & - & - & - \\
\hline $\mathrm{Zn}$ & 520 & 560 & $6 \infty$ & 300 & 500 & 600 & 600 & 700 & 800 & 800 & 700 \\
\hline Semplo & Nodule & Nodule & Nodule & Nodule & Nodulo & Noduis & Noctule & Nodule & Nodule & Nodule & Nodule \\
\hline typo & - & $\cdot$ & • & • & • & • & • & - & - & . & • \\
\hline Partion & croess & croses & half & quertor & croses & whole & whale & outor & 2-5noctalos: & outeor & 2-Sinodules: \\
\hline samplod & moction & extion & - & 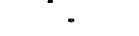 & rection & • & • & coust & componito & croust & comporite \\
\hline Nuclous & - & - & noor-4peront & Undetermined & Rock & Undetermined & Undetermined & Undeterminod & Undetermined & volenic & Underemined \\
\hline References & A197 & A197 & A405 & A40S & A405 & A40S & A405 & $A 293$ & A293 & A293 & A293 \\
\hline
\end{tabular}


Table 9. Composition of crusts from the Scripps Nodule Databank (cont'd).

\begin{tabular}{|c|c|c|c|c|c|c|c|c|c|c|c|}
\hline $\begin{array}{l}\text { Soquencoll } \\
\text { Letitude } \\
\text { Longitude } \\
\text { Depth } \\
\end{array}$ & $\begin{array}{c}2330035-01 \\
-27.652 \\
-160.015 \\
4982 \\
\end{array}$ & $\begin{array}{c}2330053-00 \\
-22.598 \\
-160.708 \\
4465 \\
\end{array}$ & $\begin{array}{c}2330056-04 \\
-22.780 \\
-160.727 \\
4850 \\
\end{array}$ & $\begin{array}{c}2330063-\infty 0 \\
-22.758 \\
-160.780 \\
4850 \\
\end{array}$ & $\begin{array}{c}2330065-01 \\
-22.662 \\
-160.788 \\
4830 \\
\end{array}$ & $\begin{array}{c}2330069-03 \\
-22.667 \\
-160822 \\
4850 \\
\end{array}$ & $\begin{array}{c}2330074-\infty 0 \\
-22808 \\
-160863 \\
2950 \\
\end{array}$ & $\begin{array}{c}2330077-\infty \\
-22802 \\
-160.872 \\
3055 \\
\end{array}$ & $\begin{array}{c}2330077-01 \\
-22.802 \\
-160.872 \\
3055 \\
\end{array}$ & $\begin{array}{c}2330082-02 \\
-22.693 \\
-160.920 \\
4535 \\
\end{array}$ & $\begin{array}{c}2330088-00 \\
-23.213 \\
-160.973 \\
4630 \\
\end{array}$ \\
\hline Si(wt\%) & 6.85 & $\cdot$ & $\cdot$ & $\cdot$ & 5.78 & 5.13 & $\cdot$ & $\cdot$ & $\cdot$ & $\cdot$ & $\cdot$ \\
\hline $\mathrm{Ti}$ & - & 1.29 & 1.10 & .06 & 1.27 & 1.35 & 1.08 & 1.27 & 1.27 & 1.19 & 1.00 \\
\hline Mn & 16.0 & 14.8 & 15.6 & 39.3 & 15.3 & 25.2 & 15.0 & 18.6 & 19.1 & 16.3 & 15.1 \\
\hline Po & 26.4 & 18.4 & 147 & 1.74 & 18.7 & 19.6 & 18.8 & 228 & 19.9 & 14.1 & 19.8 \\
\hline $\mathbf{A}$ & 2.43 & • & • & • & 3.09 & 292 & - & • & • & • & • \\
\hline Co & .570 & $\$ 40$ & .650 & .060 & 510 & 530 & .530 & .710 & 520 & .760 & 300 \\
\hline $\mathbf{N i}$ & .260 & 330 & .320 & .150 & 200 & 210 & .280 & $A 60$ & $A 10$ & .310 & .340 \\
\hline $\mathrm{Cu}$ & .110 & .180 & .110 & .100 & • & • & .090 & .100 & .070 & .090 & .120 \\
\hline ca & 1.79 & - & • & • & 159 & 1.59 & • & • & • & • & • \\
\hline $\mathrm{Mg}_{\mathrm{g}}$ & $\cdot$ & - & - & - & 1.39 & 1.39 & . & . & - & . & . \\
\hline $\mathrm{N}_{2}$ & - & - & - & - & 1.48 & 1.36 & - & - & - & - & - \\
\hline $\mathbf{K}$ & - & - & - & - & 54 & 52 & - & - & - & - & - \\
\hline $\mathbf{P}$ & . & - & - & - & .27 & $2 n$ & . & . & - & . & - \\
\hline $\mathrm{H}_{2} \mathrm{O}$ & 38.8 & - & - & - & 8.3 & 8.1 & - & $\cdot$ & - & $\cdot$ & - \\
\hline Ax(ppm) & . & . & . & . & - & - & - & - & - & . & . \\
\hline $\mathrm{Ba}$ & - & - & . & - & - & - & . & - & - & - & - \\
\hline Cd & - & - & - & - & - & - & - & - & - & - & - \\
\hline$c_{0}$ & - & . & - & - & - & . & . & - & - & . & - \\
\hline$c r$ & - & - & . & - & - & . & - & - & - & - & - \\
\hline Mo & 200 & - & - & - & - & - & . & - & - & . & . \\
\hline $\mathrm{Pb}$ & . & - & 1800 & 400 & - & . & - & 2700 & 1400 & - & - \\
\hline St & . & - & - & - & - & - & - & - & • & - & - \\
\hline v & - & - & - & - & - & . & - & - & - & . & - \\
\hline $\mathbf{Y}$ & • & - & - & - & - & - & - & - & - & - & - \\
\hline $\mathbf{Z n}$ & 700 & • & 520 & 900 & • & • & - & 600 & 700 & • & • \\
\hline Semplo & Nodule & Court & Court & Coust & Nocule & Nodule & Coust & Court & Coust & Nodulo & Court \\
\hline type & • & - & • & - & • & • & • & - & - & $\cdot$ & $\cdot$ \\
\hline Partion & outer & anknown & unkrown & maknown & 2.5nochlos & outer & mbnown & topeide & underride & underside & unbrown \\
\hline sumplod & cours & - & • & - & compositio & $\cos x$ & - & $\because$ & - & • & • \\
\hline Nucleus & Undoterminod & - & - & • & - & • & - & • & • & • & - \\
\hline Refecronos & A293 & A343 & A343 & A343 & A390 & A390 & A343 & A343 & A343 & A343 & A343 \\
\hline $\begin{array}{l}\text { Sequencest } \\
\text { Laciude }\end{array}$ & $\begin{array}{c}2330090-00 \\
-24.920\end{array}$ & $\begin{array}{c}2330091-00 \\
-23.218\end{array}$ & $\begin{array}{c}2330092-00 \\
-27.233\end{array}$ & $\begin{array}{c}2330092-01 \\
-27.233\end{array}$ & $\begin{array}{c}2360113-00 \\
-23.550\end{array}$ & $\begin{array}{c}236011400 \\
-22090\end{array}$ & $\begin{array}{c}2360115-\infty 0 \\
-22.550\end{array}$ & $\begin{array}{c}2420023-00 \\
-21.200\end{array}$ & $\begin{array}{c}2420023-01 \\
-21.200\end{array}$ & $\begin{array}{c}2430025-01 \\
-22.363\end{array}$ & $\begin{array}{c}2450014-00 \\
-22.683\end{array}$ \\
\hline Longitude & .161 .337 & -161.417 & .162 .247 & .162247 & 168.808 & 168.500 & 167.963 & 102800 & 102800 & 91.633 & 76.017 \\
\hline Depth & 4960 & 4875 & 4260 & 4260 & 1345 & 800 & 1875 & 4962 & 4962 & 5280 & 4940 \\
\hline Si(wt\%) & - & $\cdot$ & $\cdot$ & - & 3.21 & 3.25 & 6.46 & $\cdot$ & $\cdot$ & 5.88 & - \\
\hline $\mathrm{Ti}$ & .43 & 1.17 & 1.18 & 1.22 & • & - & - & - & - & .64 & - \\
\hline Mn & 9.13 & 16.1 & 155 & 15.4 & 19.9 & 148 & 10.6 & 1.40 & .70 & 19.1 & 1.80 \\
\hline Fo & 9.93 & 17.9 & 20.4 & 21.4 & 25.1 & 240 & 32.2 & 4.20 & 4.60 & 13.1 & 6.36 \\
\hline $\mathbf{N}$ & - & • & • & - & - & - & - & • & • & 2.20 & 6.94 \\
\hline Co & .280 & 520 & 540 & .500 & .650 & .370 & .480 & .010 & .010 & .230 & .040 \\
\hline $\mathrm{Ni}$ & .500 & .470 & .230 & .250 & 510 & 360 & .420 & .100 & .040 & .450 & .050 \\
\hline cu & .240 & .110 & .120 & .110 & .110 & .120 & .090 & .050 & .030 & .180 & .040 \\
\hline a & - & - & - & • & • & $\cdot$ & - & • & - & 2.03 & 1.03 \\
\hline$M_{\mathbf{B}}$ & - & - & - & - & - & - & - & . & . & 1.01 & $\cdot$ \\
\hline $\mathrm{Na}$ & - & . & - & - & - & - & - & - & . & • & - \\
\hline $\mathbf{k}$ & - & . & . & . & . & . & . & . & . & . & . \\
\hline P & - & - & - & . & - & - & - & - & - & .27 & . \\
\hline $\mathrm{H}_{2} \mathrm{O}-$ & - & • & $\cdot$ & - & $\cdot$ & $\cdot$ & $\cdot$ & - & - & $\cdot$ & $\cdot$ \\
\hline Ax(ppon) & - & . & - & - & - & . & - & - & - & - & - \\
\hline $\mathrm{Ba}$ & - & - & - & - & - & - & - & - & - & 1300 & - \\
\hline Cd & - & - & - & - & - & - & - & - & . & • & . \\
\hline$c_{0}$ & - & - & - & . & - & . & . & - & - & - & - \\
\hline Cr & - & - & - & - & 28 & 36 & 30 & - & - & 3 & - \\
\hline Mo & - & - & - & - & - & - & - & - & - & 380 & - \\
\hline $\mathrm{Pb}$ & - & 1700 & - & . & - & . & - & - & - & - & 200 \\
\hline Sr & - & $\cdot$ & - & - & - & . & - & - & - & $\cdot$ & - \\
\hline v & - & - & - & - & . & - & - & - & - & 450 & - \\
\hline $\mathbf{Y}$ & - & - & - & . & - & . & . & - & - & . & - \\
\hline $\mathrm{zn}_{\mathbf{n}}$ & - & 1000 & • & - & - & - & • & • & - & • & 280 \\
\hline Samplo & Coust & Noctule & Nocule & Nodule & Mn-cound & Mn-coursod & Mn-coned & Court & Crust & Crum & Court \\
\hline type & $\cdot$ & • & • & - & rock & rock & rock & - & • & • & • \\
\hline Partion & unknown & sverasos & outer & modeus & average & avenge & outer & unknown & unknown & octor & unknown \\
\hline ampled & - & analyais & cores & - & analyais & analyais & coust & - & • & croust & - \\
\hline Nucleus & - & - & - & . & Basedt & Benalt & Bendt & . & - & - & - \\
\hline Roforenose & A343 & $A 343$ & A343 & A343 & A349 & A349 & A349 & A333 & A333 & A220 & A467 \\
\hline
\end{tabular}


Table 9. Composition of crusts from the Scripps Nodule Databank (cont'd).

\begin{tabular}{|c|c|c|c|c|c|c|c|c|c|c|c|}
\hline $\begin{array}{l}\text { Sequenoull } \\
\text { Latitude } \\
\text { Longitude } \\
\text { Depth }\end{array}$ & $\begin{array}{c}2450016-01 \\
-23.267 \\
74.983 \\
4520\end{array}$ & $\begin{array}{c}2450016-03 \\
-23.267 \\
74.983 \\
4520\end{array}$ & $\begin{array}{c}2450028-01 \\
-24.117 \\
72.433 \\
4050\end{array}$ & $\begin{array}{c}2460021-06 \\
-25.833 \\
65.517 \\
4450\end{array}$ & $\begin{array}{c}2460037-00 \\
-27.250 \\
61.033 \\
5588\end{array}$ & $\begin{array}{c}2460040-00 \\
-29.300 \\
60.650 \\
5415\end{array}$ & $\begin{array}{c}2460041-01 \\
-29.298 \\
60.602 \\
4564\end{array}$ & $\begin{array}{c}2460041-05 \\
-29.298 \\
60.602 \\
4564\end{array}$ & $\begin{array}{c}2460041-06 \\
-29.298 \\
60.602 \\
4564\end{array}$ & $\begin{array}{c}2460049-00 \\
-22.600 \\
64.983 \\
4449\end{array}$ & $\begin{array}{c}2470085-00 \\
-25.220 \\
57.390 \\
3977\end{array}$ \\
\hline Si(wt.\%) & - & $\cdot$ & $\cdot$ & - & 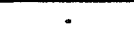 & • & - & . & . & - & - \\
\hline $\mathrm{Ti}$ & .57 & - & - & $\cdot$ & $\cdot$ & • & $\cdot$ & • & $\cdot$ & • & • \\
\hline $\mathbf{M n}$ & 13.2 & 19.0 & 10.8 & 13.8 & 10.4 & 8.50 & 11.8 & 13.7 & 8.76 & 4.60 & 15.2 \\
\hline Pe & 16.0 & 11.9 & 24.0 & 19.2 & 15.0 & 129 & 18.7 & 19.1 & 15.8 & 11.9 & 20.0 \\
\hline Al & • & • & 231 & 2.73 & • & 3.04 & • & 1.85 & 206 & 641 & - \\
\hline Co & .210 & .170 & .160 & .300 & 240 & .160 & .270 & 340 & .230 & .040 & .500 \\
\hline $\mathbf{M}$ & .410 & .740 & .230 & 300 & .140 & .170 & .160 & 210 & .150 & 200 & 220 \\
\hline $\mathrm{Cu}$ & .190 & $\$ 40$ & .170 & .140 & .150 & .110 & .060 & .090 & .060 & .150 & .100 \\
\hline Ca & • & - & 1.82 & 1.89 & 1.30 & 1.84 & 1.80 & 1.84 & 1.66 & 1.41 & - \\
\hline Ms & - & - & • & • & • & • & • & • & • & • & - \\
\hline $\mathrm{Na}$ & - & - & $\cdot$ & - & - & - & - & - & $\cdot$ & - & - \\
\hline $\mathbf{K}$ & - & - & - & - & - & - & - & - & - & - & - \\
\hline $\bar{P}$ & - & - & - & - & - & - & - & - & - & - & - \\
\hline $\mathrm{H}_{2} \mathrm{O}$ & - & - & $\cdot$ & $\cdot$ & $\cdot$ & $\cdot$ & $\cdot$ & - & $\cdot$ & $\cdot$ & - \\
\hline$A x$ (ppm) & - & - & - & - & - & - & - & - & - & - & - \\
\hline $\mathrm{Ba}$ & 1900 & 1100 & - & - & - & - & $\cdot$ & - & $\cdot$ & $\cdot$ & - \\
\hline Cd & • & - & - & - & - & - & - & - & - & - & - \\
\hline Ce & - & - & - & - & - & - &. & - & - & - & - \\
\hline$c$ & 14 & 25 & - & - & - & - & . & - & - & - & - \\
\hline Mo & 220 & $\because$ & - & - & • & • & - & - & - & - & - \\
\hline $\mathbf{P b}$ & 300 & - & 900 & 1100 & 1200 & 800 & 1200 & 1400 & 900 & 200 & - \\
\hline Sr & - & - & - & $\cdot$ & $\cdot$ & - & - & - & $\cdot$ & $\cdot$ & - \\
\hline $\mathbf{v}$ & 390 & - & - & - & - & - & - & - & - & - & - \\
\hline $\mathbf{Y}$ & $\cdot$ & • & - & - & • & - & - & - & - & - & - \\
\hline$Z_{n}$ & • & 1000 & 570 & 540 & 500 & 340 & 500 & 510 & 380 & • & • \\
\hline Semplo & Crust & Crust & Crust & Crust & Mn-encruited & Conut & Mn-enerustod & Crust & Crus & Croxt & Nodule \\
\hline type & - & - & - & - & sodiment & - & odiment & • & - & • & • \\
\hline Partion & fragmonts & minnown & minown & monown & monkown & minown & without & unknown & monown & unknown & everage \\
\hline amplod & - & - & - & • & - & - & nuclons & - & - & - & analyzis \\
\hline Nucleus & - & - & - & $\cdot$ & - & - & Sodiment & - & - & - & • \\
\hline Refarenos & A240 & A323 & A467 & A467 & 1422 & A467 & A40s & A467 & A467 & A467 & 1426 \\
\hline Sequencell & 249003401 & $2510100-00$ & 252008400 & $2630033-00$ & $2640048-00$ & $2650020-00$ & $2660032-01$ & $2670023-00$ & $2670071-\infty 0$ & $2670098-00$ & $2670098-01$ \\
\hline Latitude & -29.100 & -29.617 & -28.200 & -10.633 & -12732 & -18.600 & -15.383 & -17.600 & -11.983 & -16.483 & -16.483 \\
\hline Langitede & 36.733 & 10.133 & 2750 & -109.600 & -113.608 & -126.500 & -136.300 & -141.917 & .144 .367 & -145.550 & -145.550 \\
\hline Dopth & 3880 & 4838 & 3129 & 2134 & 3455 & 3477 & 4480 & 2132 & 4875 & 1270 & 1270 \\
\hline Si(wt.\%) & 5.50 & - & $\cdot$ & 5.80 & - & 6.96 & - & 4.50 & - & 1.30 & 200 \\
\hline $\mathbf{T i}$ & • & • & • & • & - & - & 21 & 1.40 & - & 1.18 & 1.40 \\
\hline Mn & 12.2 & 13.2 & 18.8 & 19.7 & 11.6 & 19.2 & 21.5 & 228 & 17.2 & 22.4 & 29.7 \\
\hline Po & 15.4 & 6.00 & 18.8 & 17.8 & 34.0 & 19.1 & 8.14 & 23.1 & 13.1 & 13.8 & 17.5 \\
\hline Al & - & 6.52 & - & 1.64 & - & 2.43 & $\cdot$ & 1.60 & - & .70 & 1.30 \\
\hline Co & .220 & .070 & .590 & .680 & .020 & 390 & .070 & .700 & .250 & 1.10 & 1.40 \\
\hline $\mathbf{N i}$ & .140 & 800 & .430 & .320 & .100 & 880 & 1.290 & 480 & .380 & 580 & .770 \\
\hline $\mathrm{Cr}$ & .060 & .330 & .160 & • & 200 & .120 & .740 & .130 & .380 & .170 & .140 \\
\hline C. & 1.15 & - & - & 3.72 & 1.80 & - & • & 280 & 2.10 & 2.92 & 260 \\
\hline $\mathbf{M}_{\mathbf{g}}$ & - & - & - & 24 & - & - & - & 1.20 & - & - & 210 \\
\hline Na & - & - & - & $\because$ & - & - & - & 220 & - & - & 2.20 \\
\hline $\mathrm{K}$ & - & - & - & 3.15 & - & - & - & so & - & .32 & - \\
\hline P & .18 & - & - & - & - & - & - & - & - & .17 & - \\
\hline $\mathrm{H}_{2} \mathrm{O}$ & $\cdot$ & : & - & $\cdot$ & $\cdot$ & - & $\cdot$ & - & - & 27.5 & - \\
\hline A.ppon) & 300 & - & - & - & - & - & - & - & - & - & - \\
\hline Ba & 900 & - & - & - & - & - & 1300 & 1300 & - & 5100 & 1200 \\
\hline Cd & 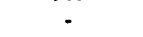 & - & - & - & - & - & - & - & - & - & - \\
\hline Ce & - & - & - & - & - & - & - & - & - & - & - \\
\hline$c$ & - & - & - & - & - & - & 5 & 6 & - & • & - \\
\hline Mo & 370 & 190 & - & - & - & - & 400 & 500 & $\cdot$ & 560 & 420 \\
\hline $\mathbf{P b}$ & 1500 & 300 & - & - & 600 & - & 200 & 800 & 600 & 2500 & 3100 \\
\hline Sr & 1000 & $\cdot$ & - & - & - & - & - & 1100 & - & 1600 & 1500 \\
\hline $\mathbf{v}$ & - & $\cdot$ & - & - & - & - & 270 & 800 & - & - & 700 \\
\hline $\mathbf{Y}$ & 126 & - & - & - & - & - & $\cdot$ & 410 & - & - & 340 \\
\hline $\mathrm{Zn}_{n}$ & 400 & 80 & • & - & 700 & • & - & 500 & 600 & 670 & 400 \\
\hline Semplo & Mn-encrusted & Crus & Nodule & Mn-contiod & Crust & Mn-contod & Crust & Noctulo & Coust & Nodule & Nodulo \\
\hline type & sodiment & • & - & rock & - & rock & • & - & • & • & • \\
\hline Partica & outer & outer & without & outer & fragments & outer & fingments & 2-5roctules & outer & whole & 2-5nodules \\
\hline anplod & $\cos x$ & coust & nuclens & crust & - & crust & - & componito & croust & - & cocaposito \\
\hline Nucleus & - & - & Undotormined & - & - & - & - & - & - & - & - \\
\hline References & A388 & A333 & A333 & $\mathbf{A 2 3 0}$ & A405 & A230 & A240 & A261 & A437 & A197 & A261 \\
\hline
\end{tabular}


Table 9. Composition of crusts from the Scripps Nodule Databank (cont'd).

\begin{tabular}{|c|c|c|c|c|c|c|c|c|c|c|c|}
\hline Sequencell & $2670098-02$ & $2570098-03$ & 2670098-05 & $2670098-06$ & $2670098-07$ & $2670098-08$ & $2670098-10$ & $2670098-11$ & $2670098-12$ & $2670098-13$ & $2670098-14$ \\
\hline Letitude & -16.483 & -16.483 & -16.483 & -16.483 & -16.483 & $-16,483$ & -16.483 & -16.483 & -16.483 & -16.483 & .16 .483 \\
\hline Longinude & -145.550 & -145.550 & -145.550 & -145.550 & -145550 & -145.550 & -145.550 & -145550 & -145.550 & -145.550 & -145550 \\
\hline Dopth & 1270 & 1270 & 1270 & 1270 & 1290 & 1270 & 1270 & 1270 & 1270 & 1270 & 1270 \\
\hline si(wt.\%) & 1.50 & 1.70 & - & . & 240 & 2.00 & 260 & 1.26 & • & - & 1.68 \\
\hline $\mathbf{T i}$ & 1.50 & 1.30 & • & 1.14 & 1.10 & 1.10 & . & 1.23 & 94 & 1.10 & - \\
\hline Mn & 30.3 & 322 & 41.1 & 16.9 & - & 24.5 & 20.5 & 223 & 25.4 & 24.5 & 29.4 \\
\hline Fo & 18.9 & 17.5 & 13.8 & 11.3 & 150 & 11.5 & 11.6 & 13.0 & 14.3 & 115 & 14.8 \\
\hline $\mathbf{A}$ & .70 & 1.10 & • & - & - & 1.50 & $\bullet$ & .69 & - & - & - \\
\hline Co & 1.60 & 1.40 & 1.16 & 1.80 & 130 & 1.15 & .770 & 1.06 & 1.09 & 1.15 & 1.64 \\
\hline $\mathbf{N i}$ & .064 & .720 & 960 & .620 & .750 & .700 & 580 & . & .680 & .700 & 860 \\
\hline ar & .120 & .120 & .120 & .090 & .140 & .250 & .090 & • & .130 & 250 & .140 \\
\hline ca & 3.10 & 3.00 & . & . & - & 2.10 & 94 & 207 & 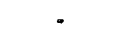 & 2.10 & 2.84 \\
\hline $\mathrm{Mg}_{8}$ & 1.60 & 1.40 & - & . & 230 & 1.00 & .58 & 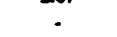 & - & 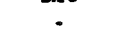 & 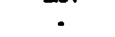 \\
\hline $\mathrm{Na}$ & 220 & 180 & - & - & - & - & . & - & - & - & . \\
\hline $\mathbf{K}$ & .40 & - & - & . & - & .40 & • & - & - & .40 & - \\
\hline P & - & - & - & - & . & 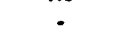 & 1.32 & .08 & - & - & 33 \\
\hline $\mathrm{H}_{2} \mathrm{O}$ & - & - & - & - & - & - & - & - & - & - & - \\
\hline As(ppm) & • & - & - & - & - & - & - & - & - & - & 270 \\
\hline $\mathrm{Ba}$ & 1600 & 1900 & - & 5800 & - & - & . & . & - & - & - \\
\hline Cd & - & - & - & - & - & - & - & - & - & - & - \\
\hline co & - & - & - & - & - & - & . & - & - & - & - \\
\hline Cr & • & • & - & - & . & - & - & - & - & - & - \\
\hline Mo & 700 & 570 & - & 590 & 720 & - & - & - & 330 & - & 570 \\
\hline Pb & 2000 & 1900 & 3300 & 4700 & - & . & - & - & 1100 & - & 1900 \\
\hline s8 & 1400 & 1700 & . & 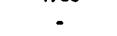 & • & - & - & - & 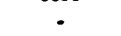 & - & 1400 \\
\hline $\mathbf{v}$ & 1000 & 800 & - & 650 & 750 & - & - & - & 2300 & . & - \\
\hline $\mathbf{Y}$ & 260 & 240 & - & . & 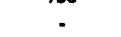 & - & . & . & 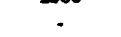 & . & 186 \\
\hline $\mathbf{Z n}_{n}$ & - & 500 & • & - & • & 1000 & - & - & 1300 & 1000 & 820 \\
\hline Semple & Crust & Unknown & Nodule & Nodule & Unlanown & Nodule & Unknown & Unknown & Unbnown & Noctule & Nodule \\
\hline typo & - & $\cdot$ & • & - & • & - & - & - & - & - & - \\
\hline Partion & cross & anknown & cross & haif & unknown & croses & unknown & unknown & unknown & unknown & whole \\
\hline ancoled & soction & - & rection & • & - & soction & - & - & - & - & - \\
\hline Nuclous & - & - & - & - & • & - & - & - & - & - & - \\
\hline References & A261 & A261 & A166 & 1240 & A201 & A232 & A261 & A261 & A261 & A381 & A388 \\
\hline Sequence" & $2070098-15$ & $2570117-01$ & $2670119-\infty$ & 2670119.01 & $2670119-02$ & $2670119-03$ & $2670119-04$ & $2670130-01$ & $2670130-03$ & $2670130-06$ & $2670130-07$ \\
\hline Letiondo & .16 .483 & -16.167 & -17.000 & -17.000 & -17.000 & -17.000 & -17.000 & -18.917 & -18.917 & -18.917 & -18.917 \\
\hline Langitude & -145550 & -146.000 & $-146,000$ & -146.000 & -146.000 & -146.000 & $-146,000$ & -146.383 & -146.383 & -146.383 & -146.383 \\
\hline Dopth & 1270 & 1190 & 1700 & 1700 & 1700 & 1700 & $17 \infty$ & 4465 & 4465 & 4465 & 4465 \\
\hline Si(wt\%) & - & .70 & .70 & 1.30 & .52 &. & . & 6.30 & $\cdot$ & . & $\cdot$ \\
\hline $\mathrm{Ti}$ & - & - & 1.11 & 1.50 & 1.04 & 1.16 & - & 1.20 & - & - & - \\
\hline Mn & . & 9.50 & 23.2 & 27.8 & 23.9 & 21.2 & 41.9 & 15.0 & 25.2 & 21.2 & 19.1 \\
\hline Fo & - & 7.00 & 12.6 & 18.2 & 115 & 10.8 & 13.7 & 16.1 & 18.3 & 17.8 & 18.6 \\
\hline Al & - & 1.30 & .40 & 1.20 & .56 & 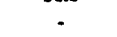 & 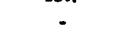 & 3.40 & - & 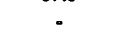 &. \\
\hline Co & 1.20 & 460 & 1.53 & 230 & 152 & 253 & 1.52 & 500 & .580 & .650 & .600 \\
\hline $\mathrm{Ni}$ & 800 & 300 & .580 & 800 & 310 & .350 & 910 & .230 & .460 & 340 & 300 \\
\hline $\mathrm{Cu}$ & .200 & .080 & .950 & .100 & .060 & .070 & .090 & .170 & .180 & .140 & .150 \\
\hline Ca & - & 4.00 & 3.14 & 3.60 & 2.87 & • & . & 208 & - & 242 & 2.26 \\
\hline $\mathbf{M}_{\mathbf{8}}$ & - & 2.10 & - & 240 & 1.65 & - & - & • & - & - & - \\
\hline Na & - & 1.30 & - & 2.70 & - & - & - & - & - & - & - \\
\hline $\mathbf{K}$ & - & . & 34 & 30 & - & - & - & .50 & - & - & - \\
\hline $\mathrm{P}$ & - . & - & .12 & 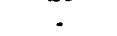 & .21 & - & - & .21 & - & - & - \\
\hline $\mathrm{H}_{2} \mathrm{O}$ & - & - & 24.5 & - & • & - & - & 17.7 & . & - & - \\
\hline$A v(p p m)$ & - & - & - & - & - & - & . & - & - & - & - \\
\hline Ba & - & - & 6600 & 1200 & - & 16000 & - & 5400 & - & - & - \\
\hline Cd & - & . & - & - & - & - & - & - & . & - & 3.0 \\
\hline co & - & - & - & - & - & - & - & - & - & - & - \\
\hline$c$ & - & - & • & 8 & - & $\cdot$ & - & - & - & - & - \\
\hline Mo & . & 110 & 500 & 640 & . & 580 & - & 310 & - & - & - \\
\hline $\mathrm{Pb}$ & - & 1300 & 2800 & 4000 & - & 3900 & 3600 & 1200 & 2300 & 1200 & 1200 \\
\hline Sz & . & - & 1500 & 1400 & - & 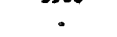 & . & 1200 & - & 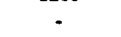 & - \\
\hline v & - & - & - & 1000 & - & 650 & - & 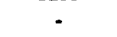 & - & - & - \\
\hline $\mathbf{Y}$ & - & - & - & 450 & - & - & - & • & - & - & - \\
\hline $\mathrm{Zn}_{n}$ & - & • & 620 & 600 & - & - & - & 570 & • & 450 & 460 \\
\hline Semple & Nodule & Crust & Nodule & Crust & Crust & Crust & Noctule & Nodule & Nodule & Nodule & Nocule \\
\hline typo & - & - & - & - & • & • & - & - & - & • & • \\
\hline Partion & unknown & ankenown & $\operatorname{cros}$ & cross & nolonown & balf & cross & cross & croses & without & without \\
\hline unoplod & . & - & section & section & - & . & soction & soction & soction & melous & nuclous \\
\hline Nuclous & - & • & - & • & - & • & • & • & - & Cany & Clay \\
\hline Reference & A381 & A261 & A197 & A261 & A178 & 1240 & A166 & A197 & Al66 & $A 200$ & $A 200$ \\
\hline
\end{tabular}


Table 9. Composition of crusts from the Scripps Nodule Databank (cont'd).

\begin{tabular}{|c|c|c|c|c|c|c|c|c|c|c|c|}
\hline $\begin{array}{l}\text { Sequencels } \\
\text { Latitude } \\
\text { Longitude } \\
\text { Depth }\end{array}$ & $\begin{array}{c}2070130-10 \\
-18.917 \\
-146.383 \\
4465\end{array}$ & $\begin{array}{c}2670202-00 \\
-11.433 \\
-149.283 \\
5106\end{array}$ & $\begin{array}{c}2670224-01 \\
-13.567 \\
-149.300 \\
4520\end{array}$ & $\begin{array}{c}2670243-00 \\
-16.583 \\
-146,400 \\
1900\end{array}$ & $\begin{array}{c}267025200 \\
-13.250 \\
-148.500 \\
4650\end{array}$ & $\begin{array}{c}2670252-01 \\
-13.250 \\
-148.500 \\
4650\end{array}$ & $\begin{array}{c}2670252-02 \\
-13.250 \\
-148.500 \\
4650\end{array}$ & $\begin{array}{c}2680002-\infty 0 \\
-14483 \\
-150.017 \\
1789\end{array}$ & $\begin{array}{c}2680008-02 \\
-13.883 \\
-150.583 \\
3623\end{array}$ & $\begin{array}{c}2680008-03 \\
-13.883 \\
-150.583 \\
3623 \\
\end{array}$ & $\begin{array}{c}2680009-00 \\
-13.883 \\
-150.583 \\
3695 \\
\end{array}$ \\
\hline Si(wt.\%) & 5.10 & 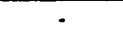 & $\cdot$ & $\cdot$ & 24.9 & 6.10 & 9.30 & $\cdot$ & $\cdot$ & - & - \\
\hline $\mathbf{T i}$ & • & 1.34 & - & .40 & .71 & 134 & 1.50 & • & .68 & .46 & • \\
\hline Mn & 18.0 & 18.1 & 163 & 21.1 & .55 & 20.4 & 15.6 & 12.5 & 14.4 & 17.4 & 19.0 \\
\hline Fo & 16.9 & 15.5 & 15.6 & 14.4 & 9.20 & 17.4 & 14.6 & 16.7 & 15.8 & 17.4 & 17.0 \\
\hline $\mathbf{N}$ & - & - & $\cdot$ & - & 6.50 & 30 & 50 & - & • & • & • \\
\hline co & 560 & .650 & 530 & .500 & - & - & - & 460 & .500 & 510 & 500 \\
\hline $\mathrm{Ni}$ & 320 & 800 & ASO & .400 & .020 & - & . & 400 & .350 & 330 & .400 \\
\hline Ca & .160 & 320 & .190 & 300 & .050 & • & • & .180 & .220 & 300 & .200 \\
\hline ca & 207 & • & • & 1.90 & 1.10 & 230 & 1.90 & • & • & - & • \\
\hline $\mathbf{M g}$ & • & - & - & . & 2.60 & 1.35 & 1.28 & - & - & - & - \\
\hline $\mathrm{Na}$ & - & - & - & - & 2.60 & 1.36 & 1.98 & - & - & - & - \\
\hline $\mathbf{K}$ & - & - & - & .20 & 2.95 & .83 & 1.41 & - & - & - & - \\
\hline $\mathbf{P}$ & 22 & - & - & - & - & - & • & - & - & - & - \\
\hline $\mathrm{H}_{2} \mathrm{O}$ & $\cdot$ & - & - & $\cdot$ & - & - & - & - & - & - & - \\
\hline As(ppm) & 165 & - & - & - & - & - & - & - & - & - & - \\
\hline $\mathrm{Ba}$ & - & 2900 & - & - & 133 & - & - & - & 1100 & 780 & - \\
\hline Cd & - & - & - & - & - & - & - & - & - & - & - \\
\hline$c_{0}$ & - & - & - & - & 46 & - & - & - & . & - & - \\
\hline Cr & - & 8 & - & - & . & - & - & - & 7 & 5 & - \\
\hline Mo & 320 & 580 & - & - & - & - & . & - & 250 & 290 & - \\
\hline $\mathbf{P b}$ & 800 & 400 & . & - & - & - & - & - & 300 & 200 & - \\
\hline Sz & 1000 & - & - & - & 75 & . & - & . & $\cdot$ & - & - \\
\hline $\mathbf{v}$ & . & $\$ 40$ & . & . & - & . & - & - & 430 & 370 & - \\
\hline $\mathbf{Y}$ & 164 & - & - & - & - & - & - & - & • & - & - \\
\hline $\mathrm{zn}$ & 530 & . & . & 1000 & 270 & 20 & 30 & - & . & . & - \\
\hline Sumplo & Nodule & Noctulo & Nodule & Noctule & Mn-ancrestad & Mo-opcrustod & Mn-encrustod & Crost & Noctule & Noctule & Noctule \\
\hline typo & • & • & $\cdot$ & $\cdot$ & sodimeat & ndiment & sodiment & - & • & • & • \\
\hline Portion & without & whole & $n$ & unknown & melous & outor & layar-edjacent & minown & balf & half & composite \\
\hline eamplod & maclons & - & - & • & • & crost & to-suclous & • & • & • & • \\
\hline Nuclous & Clay & • & - & - & Sediment & Sodiment & Sodiment & - & - & . & • \\
\hline Roferences & A388 & A240 & A389 & A381 & A463 & 1463 & A463 & A179 & A240 & A240 & A379 \\
\hline Sequenoedt & $2680009-01$ & 268000902 & $2680009-07$ & $2680009-09$ & $2680026-00$ & $2680027-00$ & $2080028-00$ & $2680035-00$ & $2680039-\infty 0$ & $2680041-\infty 0$ & $2680060-00$ \\
\hline Letionde & $\cdot \mathbf{- 1 3 . 8 8 3}$ & -13.883 & -13.883 & -13.883 & -17.817 & -17.808 & -17.792 & -17.867 & -17.89 & -18.997 & -11.583 \\
\hline $\begin{array}{l}\text { Loopitude } \\
\text { Deph }\end{array}$ & $\begin{array}{c}-150.583 \\
3695 \\
\end{array}$ & $\begin{array}{c}-150.583 \\
3695\end{array}$ & $\begin{array}{c}-150.583 \\
3695\end{array}$ & $\begin{array}{c}-150.583 \\
3695\end{array}$ & $\begin{array}{c}-154.017 \\
1260\end{array}$ & $\begin{array}{c}-154.033 \\
983\end{array}$ & $\begin{array}{c}-154.058 \\
1586\end{array}$ & $\begin{array}{c}-154.133 \\
1836 \\
\end{array}$ & $\begin{array}{c}-154.883 \\
4790\end{array}$ & $\begin{array}{c}-155.005 \\
4605\end{array}$ & $\begin{array}{c}-158.517 \\
5338 \\
\end{array}$ \\
\hline Si(wL\%) & $\cdot$ & - &. & $\cdot$ & $\cdot$ & . & $\cdot$ & $\cdot$ & $\cdot$ & $\cdot$ & $\cdot$ \\
\hline $\mathrm{Ti}$ & 200 & 1.50 & - & - & - & - & - & - & • & - & 1.24 \\
\hline Mn & 19.3 & 18.8 & 19.6 & . & 8.10 & 20.7 & 23.0 & 15.6 & 14.0 & 16.0 & 14.8 \\
\hline $\mathbf{F o}$ & 17.2 & 168 & 18.8 & - & 13.6 & 16.5 & 18.0 & 129 & 15.5 & 19.1 & 14.4 \\
\hline $\mathbf{A}$ & - & • & $\cdot$ & . & • & • & 80 & - & • & • & - \\
\hline Co & .600 & 500 & .520 & 500 & 540 & 1.48 & 1.20 & .064 & .580 & 540 & .730 \\
\hline $\mathrm{Ni}$ & 350 & 400 & 320 & .400 & 200 & .500 & .460 & .420 & .240 & 270 & 260 \\
\hline Cu & 200 & .150 & .180 & 200 & .090 & .150 & .080 & .080 & .080 & .180 & .180 \\
\hline ce & 1.30 & 1.10 & 250 & - & - & - & 3.12 & 3.80 & - & 230 & • \\
\hline $\mathbf{M g}$ & - & . & - & . & - & - & 1.32 & - & - & • & . \\
\hline $\mathrm{Na}$ & - & . & . & . & . & . & 1.10 & . & . & . & . \\
\hline $\mathbf{K}$ & .25 & .30 & - & - & - & - & 10 & - & - & - & - \\
\hline P & $\cdot$ & . & - & . & - & - & .42 & - & . & - & - \\
\hline $\mathrm{H}_{2} \mathrm{O}$ & $\cdot$ & $\cdot$ & - & - & - & - & $\cdot$ & $\cdot$ & $\cdot$ & $\cdot$ & - \\
\hline As(ppm) & - & - & - & - & - & - & - & . & . & . & . \\
\hline $\mathrm{Ba}$ & - & - & - & - & - & - & - & - & . & . & 2300 \\
\hline Cd & - & . & . & - & - & . & . & . & . & - & . \\
\hline$c_{0}$ & - & - & - & . & . & - & - & . & . & . & - \\
\hline Cr & . & . & . & - & - & . & . & . & . & . & 8 \\
\hline$M_{0}$ & . & . & . & . & . & - & 280 & . & . & . & 380 \\
\hline $\mathrm{Pb}$ & . & . & 1000 & - & . & . & . & . & . & 1200 & 300 \\
\hline St & . & . & - & . & - & . & . & . & . & - & . \\
\hline $\mathbf{v}$ & . & . & . & . & - & - & . & - & - & - & 610 \\
\hline $\mathbf{Y}$ & . & . & . & - & . & . & . & - & . & . & - \\
\hline zn & 1500 & 500 & 500 & - & - & - & - & • & • & 600 & - \\
\hline Sumplo & Nodule & Nodulo & Nodule & Nocdule & Coust & Crust & Nocule & Nodule & Nodule & Nodule & Noctuie \\
\hline typo & - & • & • & - & - & - & • & - & • & • & $\cdot$ \\
\hline Pation & unbrown & unknown & half & anknown & unknown & mblnown & unkown & molnown & unlown & helf & balf \\
\hline remplod & $\cdot$ & • & $\cdot$ & • & • & - & - & $\cdot$ & - & $\cdot$ & - \\
\hline Nucleus & . & - & nor-eppervent & . & - & - & - & . & - & Sediment & - \\
\hline Reforencese & A381 & A381 & A422 & A381 & A179 & A179 & A419 & A179 & Al79 & A437 & $A 240$ \\
\hline
\end{tabular}


Table 9. Composition of crusts from the Scripps Nodule Databank (cont'd).

\begin{tabular}{|c|c|c|c|c|c|c|c|c|c|c|c|}
\hline Sequencell & $2680069-00$ & $2680070-00$ & 2680076-01 & $2680076-02$ & $2680077-01$ & 2680077.02 & $2680078-01$ & $2680095-00$ & $2690013-00$ & $2690013-01$ & $2690019-00$ \\
\hline Letitude & -11.667 & -12450 & .13 .917 & -13.917 & .14 .050 & -14.050 & -13.833 & -11.062 & .12 .700 & -12.700 & -12700 \\
\hline Longinude & -159.267 & -159.383 & $-150,550$ & -150.550 & -150.003 & -150.633 & -150.550 & -156.165 & -160.617 & -160.617 & -160.750 \\
\hline Dopth & 5146 & 4990 & 3755 & 3755 & 3760 & 3760 & $\cdot$ & 4820 & 4753 & 4753 & 3953 \\
\hline Si(wt\%) & - &. & $\cdot$ & $\cdot$ &. &. & - & $\cdot$ & 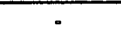 & $\cdot$ & - \\
\hline $\mathrm{Ti}$ & 1.12 & • & - & - & • & - & - & 81 & - & - & - \\
\hline Mn & $15 A$ & 17.7 & 20.0 & 18.1 & 18.1 & 16.9 & 14.8 & 19.2 & 18.7 & - & 18.6 \\
\hline Po & 15.4 & 174 & 125 & 11.3 & 14.8 & 13.8 & 123 & 15.9 & 19.4 & - & 20.5 \\
\hline $\mathbf{A}$ & • & - & $\cdot$ & - & $\cdot$ & $\cdot$ & - & - & 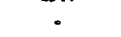 & - & . \\
\hline Co & 540 & 500 & .030 & .570 & 540 & 500 & .540 & 360 & .680 & .560 & .610 \\
\hline $\mathrm{NI}$ & .980 & $A 10$ & $A 60$ & .420 & 580 & 540 & .420 & 430 & 330 & . & 220 \\
\hline $\mathrm{Cr}$ & $A 30$ & 310 & 220 & .200 & 250 & 230 & .270 & .250 & .210 & - & .100 \\
\hline C. & • & 1.80 & • & - & - & - & - & - & 3.10 & $\cdot$ & 280 \\
\hline $\mathbf{M}$ & - & - & - & - & - & $\dot{1}$ & - & - & - & - & - \\
\hline $\mathrm{Na}$ & - & - & - & - & - & - & - & - & - & - & - \\
\hline $\mathrm{x}$ & - & - & - & - & - & - & - & - & - & - & - \\
\hline $\mathbf{P}$ & - & - & - & - & - & - & - & - & - & - & - \\
\hline $\mathrm{H}_{2} \mathrm{O}$ & • & - & • & - & - & - & - & - & - & - & . \\
\hline At(ppm) & • & - & . & - & - & . & • & - & - & - & - \\
\hline $\mathrm{Ba}$ & 1200 & - & - & - & - & 9 & - & - & - & $\cdot$ & - \\
\hline Cd & • & - & - & - & - & \& & - & - & - & 5.0 & - \\
\hline co & - & - & - & - & - & . & - & - & - & $\cdot$ & - \\
\hline$c$ & 15 & - & - & - & - & - & - & - & - & • & - \\
\hline Mo & 400 & - & - & - & - & . & - & - & - & 340 & - \\
\hline $\mathrm{Pb}$ & 100 & 900 & - & - & - & - & - & - & 1300 & 1300 & 1200 \\
\hline Sr & $\cdot$ & $\cdot$ & - & - & - & - & - & - & - & $\cdot$ & 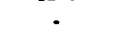 \\
\hline $\mathbf{V}$ & - & - & - & - & - & . & - & - & - & - & - \\
\hline $\mathbf{Y}$ & - & - & - & - & - & - & - & - & - & • & • \\
\hline $2 n$ & - & • & - & - & • & $\bullet$ & - & • & 2400 & 1800 & 1200 \\
\hline Semplo & Notule & Nodulo & Nodulo & Nodule & Nodule & Noduio & Nodule & Crust & Nodule & Noctule & Nodule \\
\hline type & - & - & • & - & - & • & - & - & - & • & • \\
\hline Portion & whole & whole & $n$ & without & $n$ & without & without & unknown & uniknown & unknown & fragments \\
\hline ampiod & - & - & - & madous & $\cdot$ & malows & ruclous & $\cdot$ & - & - & - \\
\hline Nuclows & - & Undetermined & - & • & - & + & - & - & - & - & • \\
\hline Roferencos & $A 240$ & A422 & A389 & A389 & A389 & A359 & A389 & A343 & 1422 & $A 303$ & A422 \\
\hline Sequence & $2690019-03$ & $2690022-01$ & $2690022-02$ & $2690023-00$ & $2690023-01$ & $2690003-02$ & $2690030-00$ & $2690033-00$ & $2690006-00$ & $2690065-00$ & $2690132-01$ \\
\hline Letitude & -12.700 & -12700 & -12.700 & -11.850 & -11.850 & $-11 B 50$ & -12750 & -12733 & .12275 & -18.597 & -12910 \\
\hline Longitudo & -160.750 & -160.817 & -160.817 & -160.850 & -160.850 & -160.850 & -160.925 & .160 .933 & -161.058 & -161.527 & -160.747 \\
\hline Dopth & 3953 & 3587 & 3587 & 3803 & 3803 & 3803 & 3500 & 3610 & 2720 & 4998 & 4300 \\
\hline si(wt.\%) & - & $\cdot$ & $\cdot$ & $\cdot$ & $\cdot$ & , & . & - & . & 5.84 & - \\
\hline $\mathrm{Ti}$ & - & - & - & .79 & 81 & 96 & - & - & - & 1.11 & 1.20 \\
\hline Mn & - & 17.8 & - & 16.9 & 15.7 & 16.6 & 15.8 & 15.9 & 19.1 & 14.4 & 13.8 \\
\hline Fo & - & 19.9 & - & 18.0 & 18.1 & 18.5 & 13.9 & 133 & 18.3 & 18.4 & 17.0 \\
\hline A & - & 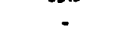 & - & 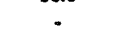 &. & 4 & - & . & . & 3.96 & 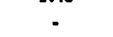 \\
\hline Co & .500 & - & 410 & 510 & .660 & 590 & .540 & • & - & .560 & .250 \\
\hline $\mathrm{Ni}$ & - & .240 & - & .300 & 240 & .230 & .350 & .350 & 410 & .110 & .360 \\
\hline Cu & - & .150 & - & .170 & .160 & 200 & .290 & 260 & .120 & .120 & 200 \\
\hline C. & - & - & - & • & - & + & 270 & . & - & 2.29 & - \\
\hline $\mathbf{M}_{8}$ & - & - & - & - & - & - & • & - & - & .78 & - \\
\hline $\mathrm{Na}$ & - & - & - & - & - & - & - & - & - & 150 & - \\
\hline $\mathbf{K}$ & - & - & - & - & - & - & - & - & - & .67 & - \\
\hline $\mathbf{P}$ & - & - & - & - & - & - & - & - & - & $\cdot$ & - \\
\hline $\mathrm{H}_{2} \mathrm{O}$ & - & - & - & $\cdot$ & - & - & - & - & - & 20.2 & - \\
\hline As(ppm) & - & - & - & - & • & . & . & - & - & . & - \\
\hline $\mathrm{Ba}$ & - & - & - & 1100 & 440 & 1300 & - & - & - & - & - \\
\hline Cd & 4.0 & - & 1.0 & • & • & • & . & - & - & - & . \\
\hline co & $\cdot$ & - & - & - & - & - & - & - & - & - & - \\
\hline$c$ & - & - & - & 5 & 4 & 7 & - & - & - & - & - \\
\hline Mo & 340 & - & 270 & 270 & 240 & 210 & $\cdot$ & - & - & • & - \\
\hline Pb & 1200 & - & 800 & 300 & 300 & 2200 & 700 & - & - & 1900 & - \\
\hline Sr & - & - & $\cdot$ & - & - & 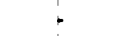 & • & - & - & - & - \\
\hline v & - & - & . & 420 & 380 & 420 & - & - & - & - & - \\
\hline $\mathbf{Y}$ & • & - & - & • & - & $\cdot$ & • & - & - & - & - \\
\hline $\mathbf{Z n}$ & 1000 & • & 2000 & - & • & - & 1800 & • & - & • & - \\
\hline Semplo & Nodulo & Crust & Crust & Nodule & Nodule & Nodule & Crust & Crust & Crust & Nodule & Crust \\
\hline typo & - & - & • & • & - & 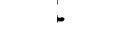 & • & $\cdot$ & - & - & - \\
\hline Portion & fragments & frugments & unknown & half & whole & ottres & unknown & fragments & unknown & without & fragments \\
\hline sumpled & . & . & $\cdot$ & $\cdot$ & - & const & $\cdot$ & • & • & nucleus & - \\
\hline Nucleos & • & • & - & • & - & • & • & - & • & • & • \\
\hline References & A303 & A321 & $A 303$ & A240 & A240 & A343 & A405 & A321 & A321 & A343 & A343 \\
\hline
\end{tabular}


Table 9. Composition of crusts from the Scripps Nodule Databank (cont'd).

\begin{tabular}{|c|c|c|c|c|c|c|c|c|c|c|c|}
\hline Sequencout & $2690132-04$ & $2690132-12$ & $2690137-00$ & $2690138-00$ & $2690139-\infty 0$ & $2690142-00$ & $2690144-\infty 0$ & $2690146-\infty$ & $2690188-00$ & 2690197.00 & $2710120-\infty 0$ \\
\hline Leritude & -12.910 & -12.910 & -19.767 & -19.710 & -19.680 & -19.775 & -19.752 & -15573 & -15.140 & -11.917 & -11.317 \\
\hline Longitude & -160.747 & -160.747 & .161 .783 & -161.833 & -161.887 & -161.908 & .161 .942 & -164.590 & .166 .900 & -169.533 & 178.200 \\
\hline Dopth & $\$ 300$ & 4300 & 4070 & 4825 & 4330 & 4985 & 5060 & 5540 & 5372 & - & 770 \\
\hline$S i(w t . \%)$ & • & - & - & - & $\cdot$ & - & - & $\cdot$ & - & - & - \\
\hline $\mathrm{Ti}$ & 132 & 1.27 & 1.57 & 1.41 & 1.53 & 1.27 & 1.01 & 1.32 & - & - & - \\
\hline Mn & 125 & 15.3 & 11.8 & 13.0 & 13.2 & 13.8 & 168 & 18.6 & 3,40 & 19.9 & 34.2 \\
\hline $\mathrm{Fe}$ & 17.8 & 16.2 & 19.5 & 19.6 & 21.3 & 15.7 & 155 & 14.7 & 21.0 & 16.1 & 17.4 \\
\hline $\mathbf{N}$ & - & - & - & - & - & - & - & • & - & • & - \\
\hline Co & 330 & 570 & .540 & 310 & 510 & 320 & 330 & .550 & .290 & .690 & .850 \\
\hline $\mathbf{N i}$ & 390 & .290 & .280 & .190 & .350 & 500 & .064 & 390 & .030 & .200 & .540 \\
\hline Co & .250 & .140 & .170 & .110 & .100 & .220 & 330 & .210 & .090 & .010 & .050 \\
\hline Ca & • & • & • & • & • & • & - & • & • & 260 & • \\
\hline $\mathrm{M}_{\mathrm{s}}$ & - & - & - & - & - & - & - & - & - & • & - \\
\hline $\mathrm{Na}$ & - & - & - & - & - & - & - & - & - & - & - \\
\hline $\mathbf{x}$ & - & - & . & - & - & - & - & - & - & - & - \\
\hline $\mathbf{P}$ & - & - & . & - & - & - & - & - & - & - & - \\
\hline $\mathrm{H}_{2} \mathrm{O}$. & $\cdot$ & - & - & - & - & - & - & • & - & - & - \\
\hline$A_{s}(p p m)$ & . & - & - & - & - & - & . & - & - & - & - \\
\hline $\mathrm{Ba}$ & - & - & - & - & - & - & . & - & - & - & - \\
\hline Cd & - & - & - & - & - & - & - & - & - & - & - \\
\hline co & - & - & - & - & - & - & - & - & - & - & - \\
\hline$c$ & - & - & - & - & - & - & - & - & - & - & - \\
\hline Mo & - & - & - & - & - & - & - & • & - & • & • \\
\hline $\mathbf{P b}$ & - & - & - & . & - & - & - & 1300 & . & 1700 & 3500 \\
\hline Sz & - & - & - & - & - & - & - & - & - & - & - \\
\hline $\mathbf{v}$ & . & - & . & - & . & - & . & - & - & - & - \\
\hline $\mathbf{Y}$ & . & - & - & . & . & - & . & - & - & . & . \\
\hline $\mathbf{z n}$ & • & • & • & • & - & • & • & 550 & - & 300 & • \\
\hline Semplo & Crust & Nodubo & Noduse & Cras & Nodulo & Me-coutod & Mo-conted & Nodulo & Crost & Crose & Nodulo \\
\hline type & • & • & - & - & • & rock & $\operatorname{sock}$ & • & - & • & • \\
\hline Partion & frigmonts & layoredjecent & without & unknown & without & outer & oulore & ontor & whole & valnown & croos \\
\hline semplod & . & tonandous & nuclous & • & nedous & coust & const & crust & - & • & section \\
\hline Nuclous & - & - & - & - & - & - & - & - & - & - & - \\
\hline Reforencos & A343 & A343 & A343 & A343 & A343 & A343 & A343 & A343 & A414 & A437 & A166 \\
\hline Sequencoll & $2710120-01$ & $2720050-\infty 0$ & $2070039-\infty 0$ & $2770039-01$ & $2780082-\infty 0$ & $2780095-00$ & $2780098-\infty$ & $2790066-00$ & $2790094-00$ & $2790095-\infty 0$ & 2790095-01 \\
\hline Letitude & -11.317 & $-17,450$ & -10.850 & -10.850 & -15.883 & -10.833 & -11.817 & -13.250 & -11.833 & -17.883 & -17.883 \\
\hline Lociginde & 178.200 & 163.667 & 113.017 & 113.017 & 102.950 & 104.633 & 102.900 & 93.200 & 96.967 & 99.100 & 99.100 \\
\hline Dopth & 770 & 1310 & 5150 & 5150 & 5579 & 3850 & 5078 & .5325 & 3080 & 5471 & 5471 \\
\hline$S i(w t . \%)$ & - & 2.56 & - & 120 & - & - & $\cdot$ & . & - & - & - \\
\hline $\mathrm{Ti}$ & - & - & • & • & - & - & - & .63 & • & - & - \\
\hline Mn & 11.0 & 23.0 & 18.4 & 16.0 & 20.8 & 10.6 & 15.8 & 15.4 & 1.60 & 10.6 & 15.2 \\
\hline $\mathrm{Pe}$ & 10.8 & 18.6 & 12.6 & 12.2 & 15.4 & 14.3 & 12.8 & 10.5 & 18.1 & 10.6 & 124 \\
\hline N & - & 1.20 & • & - & - & $\cdot$ & - & • & - & • & - \\
\hline Co & .440 & .600 & .090 & .050 & .320 & 270 & .110 & .120 & .040 & .170 & .200 \\
\hline $\mathrm{Ni}$ & .260 & 330 & .680 & .610 & 530 & .180 & 510 & 570 & .030 & .190 & .540 \\
\hline Cr & .070 & .070 & .190 & .190 & .310 & .120 & .270 & .830 & .050 & .130 & .240 \\
\hline$C_{2}$ & - & 3.18 & 202 & 1.84 & 1.80 & - & • & • & • & - & • \\
\hline Ms & - & 1.32 & - & - & - & - & - & . & - & - & - \\
\hline $\mathrm{Na}$ & - & 1.64 & - & - & - & - & . & - & - & - & - \\
\hline $\mathbf{K}$ & - & .36 & - & - & . & - & . & - & . & - & - \\
\hline $\mathbf{P}$ & - & - & - & .07 & - & - & . & - & . & - & . \\
\hline $\mathrm{H}_{2} \mathrm{O}$ & - & - & - & $\cdot$ & - & $\cdot$ & $\cdot$ & • & - & - & - \\
\hline As(ppom) & - & - & - & - & - & - & . & • & . & - & . \\
\hline $\mathrm{Ba}_{\mathbf{z}}$ & 890 & - & - & - & . & - & . & 1700 & . & - & . \\
\hline Cd & - & - & 4.0 & - & . & - & - & - & . & - & - \\
\hline$c_{0}$ & - & - & - & - & . & - & . & - & - & . & . \\
\hline$c$ & 24 & 16 & - & - & . & . & . & 12 & . & . & . \\
\hline Mo & - & - & - & - & • & - & . & 280 & - & - & - \\
\hline $\mathbf{P b}$ & . & - & 1000 & - & 1300 & . & . & 500 & - & - & . \\
\hline Sx & - & - & - & - & - & - & .. & - & - & - & . \\
\hline $\mathbf{v}$ & - & - & - & - & . & - & - & 430 & - & - & - \\
\hline $\mathbf{Y}$ & • & - & - & - & - & . & . & - & - & - & - \\
\hline $2 n$ & 5000 & • & 850 & 1100 & 600 & • & • & - & • & - & • \\
\hline Semplo & Crast & Crust & Mro-encrustod & Ma-encrustod & Mn-cotiod & Crust & Mn-contod & Crant & Mn-coatod & Crust & Crast \\
\hline typo & - & - & rodiment & sodimant & rock & • & rock & - & rock & • & • \\
\hline Partion & anknown & crows & outer & ouver & outor & unbrown & outer & frequents & unicnown & whole & outor \\
\hline amoplod & - & soction & coust & crust & const & $\cdot$ & coust & - & - & $\cdot$ & croust \\
\hline Nuclous & • & - & Cay & Clay & - & • & • & - & - & • & • \\
\hline Reforencos & A323 & A349 & $A 200$ & A388 & A422 & A451 & A451 & A240 & A333 & A333 & A333 \\
\hline
\end{tabular}


Table 9. Composition of crusts from the Scripps Nodule Databank (cont'd).

\begin{tabular}{|c|c|c|c|c|c|c|c|c|c|c|c|}
\hline Sequencell & $2790105-00$ & $2790106-00$ & $2790113-\infty 0$ & $2790115-00$ & $2790119-00$ & 2790123-01 & $2790126-00$ & $2790127-00$ & $2800039-01$ & $2800081-00$ & $2800082-00$ \\
\hline Latitude & -13.017 & -13.017 & -13.017 & -12583 & -15.767 & -15.767 & -15.767 & -15.767 & -17.333 & -12767 & -12.767 \\
\hline Longitude & 91.250 & 91.250 & 91.250 & 95.400 & 99.900 & 99.900 & 99900 & 99.900 & 84383 & 88.900 & 88.900 \\
\hline Depth & 4400 & 4260 & 4760 & 1500 & 5000 & 5600 & 3760 & 3420 & 5010 & 5180 & 4480 \\
\hline $\operatorname{Si}(w t . \$)$ & - & $\cdot$ & $\cdot$ & $\cdot$ & $\cdot$ & $\cdot$ & $\cdot$ & $\cdot$ & $\cdot$ & $\cdot$ & $\cdot$ \\
\hline $\mathrm{Ti}$ & - & - & $\cdot$ & $\cdot$ & - & - & • & • & - & - & $\cdot$ \\
\hline $\mathbf{M n}$ & 13.8 & 19.1 & 18.8 & 21.8 & 18.6 & 9.89 & 16.7 & 17.1 & 352 & 5.60 & 143 \\
\hline $\mathbf{F}_{0}$ & 125 & 126 & 15.2 & 10.9 & 125 & 14.7 & 135 & 14.9 & 5.89 & 6.20 & 10.6 \\
\hline $\mathbf{A}$ & • & - & • & - & - & - & • & • & 7.60 & • & - \\
\hline $\mathrm{Co}$ & 240 & 240 & .270 & .560 & 260 & .100 & .680 & 530 & .070 & .050 & .170 \\
\hline $\mathbf{M}$ & .340 & 470 & .500 & .560 & 360 & .530 & .650 & 280 & .080 & .180 & 350 \\
\hline$\infty$ & .180 & .170 & .090 & .100 & .130 & .200 & .130 & .060 & .060 & .120 & .190 \\
\hline c. & • & - & - & • & - & • & - & • & .58 & - & - \\
\hline $\mathbf{M g}$ & - & - & - & - & - & - & - & $\cdot$ & $\bullet$ & - & $\cdot$ \\
\hline $\mathrm{Na}$ & - & - & - & - & - & - & - & - & - & - & - \\
\hline $\mathbf{K}$ & - & - & - & - & - & - & - & - & - & - & - \\
\hline $\mathbf{P}$ & - & - & - & - & - & - & - & - & - & - & - \\
\hline $\mathrm{H}_{2} \mathrm{O}$ & $\cdot$ & $\cdot$ & - & $\cdot$ & - & $\cdot$ & $\cdot$ & $\cdot$ & $\cdot$ & $\cdot$ & • \\
\hline$A \propto(p p m)$ & $\cdot$ & - & $\cdot$ & - & - & - & - & - & - & - & - \\
\hline $\mathrm{Ba}$ & - & - & - & - & - & - & - & - & - & - & - \\
\hline Cd & - & - & - & - & - & - & - & - & - & - & - \\
\hline co & - & - & - & - & - & - & - & . & - & - & - \\
\hline$C_{x}$ & - & - & - & - & - & - & - & - & $\cdot$ & - & - \\
\hline Mo & - & - & - & - & - & - & - & - & $\cdot$ & - & - \\
\hline $\mathbf{P b}$ & - & - & - & - & - & - & - & - & 400 & - & - \\
\hline Sr & - & $\cdot$ & - & - & - & - & - & - & - & - & $\cdot$ \\
\hline $\mathbf{v}$ & - & - & - & - & - & - & - & $\cdot$ & - & - & - \\
\hline $\mathbf{Y}$ & - & - & - & - & - & - & - & - & - & - & - \\
\hline $\mathbf{Z n}$ & - & - & • & - & • & - & • & - & 210 & - & - \\
\hline Samplo & Crust & Crust & Crust & Crust & Crust & Soverdil & Nodule & Nodule & Crast & Crust & Crust \\
\hline typo & - & - & - & - & • & crusts & - & - & • & - & - \\
\hline Portion & nnkmown & unknown & monknown & anknown & unknown & minnown & unknown & anknown & unknown & outer & unknown \\
\hline samplod & • & - & - & • & - & • & • & • & • & crust & - \\
\hline Nuclews & $\cdot$ & • & - & - & • & - & - & • & - & - & - \\
\hline References & A451 & A451 & A451 & A451 & A451 & A451 & A451 & A451 & A467 & A451 & A451 \\
\hline Sequencest: & $28000033-00$ & 280008400 & $2800086-00$ & $2810005-00$ & $2820002-00$ & $28200039-00$ & $2820060-00$ & $2820062-00$ & $2820130-00$ & $2840074-00$ & $2890019-00$ \\
\hline Latitude & -12767 & -12767 & -10.017 & -18.800 & -10.092 & -17.588 & -18.342 & -10.417 & -19.232 & -14.167 & -9.960 \\
\hline Longitudo & 88.900 & 88.900 & 88.017 & 78.150 & 66.418 & 65.653 & 63.638 & 63.250 & 61.022 & 41.567 & -5.387 \\
\hline Dopth & 4750 & 4830 & 4620 & 4934 & 2422 & 2502 & 3451 & 3115 & 3260 & 1485 & 1394 \\
\hline Si(wt.\%) & - & $\cdot$ & $\cdot$ & - & $\cdot$ & - & $\cdot$ & $\cdot$ & . & $\cdot$ & $\cdot$ \\
\hline $\mathbf{T i}$ & - & • & - & - & • & • & - & • & - & - & • \\
\hline $\mathbf{M n}$ & 15.2 & 19.3 & 15.4 & 128 & 1.50 & 14.8 & 6.93 & 11.9 & 11.8 & 14.7 & 24.0 \\
\hline Fo & 10.6 & 8.55 & 13.7 & 11.2 & 9.61 & 22.7 & 15.7 & 220 & 23.8 & 17.3 & 18.0 \\
\hline $\mathbf{A}$ & - & - & - & - & 7,46 & 1.09 & 4.45 & 1.63 & $\cdot$ & - & - \\
\hline Co & 200 & .140 & .130 & .160 & .030 & .180 & .100 & .170 & .220 & .280 & 1.44 \\
\hline $\mathrm{Ni}$ & 580 & 1.010 & .360 & $A 60$ & .060 & .210 & .120 & .150 & .170 & .180 & 430 \\
\hline $\mathrm{Cu}$ & 230 & 480 & .280 & .270 & .070 & .090 & .110 & .000 & .130 & - & .050 \\
\hline Ca & - & - & - & 1.50 & 1.17 & 2.95 & 222 & 205 & 2.31 & - & - \\
\hline $\mathbf{M}$ & - & - & - & - & • & - & - & - & - & - & - \\
\hline $\mathrm{Na}$ & - & - & - & - & - & - & - & $\cdot$ & - & - & $\cdot$ \\
\hline $\mathbf{K}$ & - & - & - & - & - & - & - & $\cdot$ & $\cdot$ & - & . \\
\hline $\mathbf{P}$ & - & - & - & - & - & - & - & - & - & - & - \\
\hline $\mathrm{H}_{2} \mathrm{O}$ & - & - & - & - & - & - & $\cdot$ & - & - & - & $\cdot$ \\
\hline$A \&$ (ppm) & - & - & - & - & - & - & - & - & - & - & - \\
\hline $\mathrm{Ba}$ & . - & - & - & - & - & - & - & - & - & - & - \\
\hline Cd & - & - & - & - & - & - & $\cdot$ & $\cdot$ & - & - & $\cdot$ \\
\hline Co & - & - & - & - & - & - & - & - & - & - & $\cdot$ \\
\hline$C_{r}$ & - & - & - & - & - & - & - & - & - & - & - \\
\hline Mo & - & - & - & - & $\cdot$ & $\cdot$ & $\cdot$ & $\cdot$ & $\cdot$ & - & - \\
\hline $\mathbf{P b}$ & - & $\cdot$ & - & - & 200 & 600 & 400 & 700 & 800 & - & • \\
\hline Sx & - & - & - & - & $\cdot$ & $\cdot$ & - & $\cdot$ & $\cdot$ & $\cdot$ & $\cdot$ \\
\hline $\mathbf{v}$ & - & - & - & - & - & - & $\cdot$ & - & - & - & - \\
\hline $\mathbf{Y}$ & - & - & - & $\cdot$ & $\cdot$ & $\cdot$ & $\cdot$ & $\cdot$ & $\cdot$ & - & $\cdot$ \\
\hline $\mathbf{Z n}_{n}$ & • & - & - & - & 350 & 540 & 370 & 590 & 580 & • & • \\
\hline Samplo & Crust & Crust & Const & Crust & Crust & Coux & Crust & Crust & Crent & Crust & Crust \\
\hline typo & - & • & - & - & - & - & - & - & $\bullet$ & • & $\cdot$ \\
\hline Portion & unknown & anlonown & monlmown & unlonown & unknown & unbown & unknown & unknown & unknown & unlenown & unknown \\
\hline semplod & - & - & • & • & • & - & - & - & - & - & • \\
\hline Nuclews & • & • & • & • & • & - & - & • & $\cdot$ & $\cdot$ & • \\
\hline Roference & A451 & A451 & AAS1 & A179 & A467 & A467 & A467 & A467 & A467 & A416 & A179 \\
\hline
\end{tabular}


Table 9. Composition of crusts from the Scripps Nodule Databank (cont'd).

\begin{tabular}{|c|c|c|c|c|c|c|c|c|c|c|c|}
\hline Sequence: & $2900014-00$ & 290001401 & $2900024-00$ & $2900024-01$ & $2070068-00$ & $2990009-00$ & $2990020-00$ & 299008000 & $3040029-00$ & $3040103-\infty 0$ & $3040105-\infty$ \\
\hline Latioude & -5.783 & -5.783 & -8.350 & -8.350 & -1.762 & -8.125 & -7.833 & -3.713 & .5 .817 & -4.978 & -4.982 \\
\hline Langitade & -11.417 & -11.417 & -13.200 & -13.200 & -85.903 & -104.175 & -108.133 & -102870 & -154.650 & -154.993 & -154.983 \\
\hline Dopth & 2380 & 2380 & 2265 & 2265 & 2611 & 3814 & 1567 & 3649 & 4768 & 4800 & 4990 \\
\hline si(wt\%) & $\cdot$ & - & $\cdot$ & - & . & 4.70 & 12.1 & $\cdot$ & $\cdot$ & $\cdot$ & - \\
\hline $\mathrm{Ti}$ & • & - & • & $\cdot$ & • & .14 & .88 & - & - & - & - \\
\hline Mn & 25.2 & 11.3 & 124 & 11.5 & 239 & 23.0 & 5.59 & 23.2 & 4.20 & • & 14.1 \\
\hline Po & 229 & 21.5 & 23.1 & 21.7 & 15.2 & 14.3 & 13.0 & 243 & 9.50 & 9.70 & 8.90 \\
\hline $\mathbf{N}$ & $\cdot$ & $\cdot$ & • & • & • & 1.30 & 3.44 & • & • & - & • \\
\hline$C_{0}$ & .620 & 240 & .480 & $A 40$ & .070 & .550 & • & 230 & • & .140 & .190 \\
\hline $\mathrm{Ni}$ & 340 & .140 & .150 & .150 & .880 & 1.000 & - & 320 & .270 & .140 & .890 \\
\hline ca & .050 & .070 & .040 & .030 & .100 & .700 & • & .010 & .250 & .130 & .580 \\
\hline C. & $\cdot$ & 250 & 530 & 480 & • & 1.39 & 4.54 & .60 & • & 1.70 & 1.00 \\
\hline $\mathbf{M}$ & - & 1.61 & • & - & $\cdot$ & • & .02 & • & - & - & • \\
\hline $\mathrm{Na}$ & - & 3.18 & - & - & - & - & .00 & - & - & - & - \\
\hline $\mathbf{K}$ & - & .34 & - & - & - & 49 & 54 & - & - & - & $\cdot$ \\
\hline $\mathbf{P}$ & - & • & - & - & - & .11 & - & - & - & - & - \\
\hline $\mathrm{H}_{2} \mathrm{O}$ & $\cdot$ & $\cdot$ & - & $\cdot$ & • & 17.8 & - & $\cdot$ & $\cdot$ & - & $\cdot$ \\
\hline As(ppon) & $\cdot$ & $\cdot$ & - & - & $\cdot$ & - & - & - & - & - & $\cdot$ \\
\hline Ba & - & - & - & - & - & 5000 & - & - & - & - & - \\
\hline Cd & - & - & - & - & - & - & - & - & - & - & - \\
\hline C. & - & $\cdot$ & $\cdot$ & - & - & - & - & - & - & $\cdot$ & - \\
\hline$C$ & - & - & $\cdot$ & - & - & - & $\cdot$ & $\cdot$ & - & - & • \\
\hline Mo & - & - & $\cdot$ & - & $\cdot$ & 320 & - & $\cdot$ & - & - & - \\
\hline $\mathbf{P b}$ & 3500 & 1200 & 1100 & 1100 & 400 & 500 & - & 800 & - & 300 & 300 \\
\hline $\mathbf{S z}$ & - & - & - & - & $\cdot$ & 820 & - & - & - & - & $\cdot$ \\
\hline $\mathbf{v}$ & - & - & - & - & - & - & - & - & - & - & - \\
\hline $\mathbf{Y}$ & - & - & - & - & - & - & - & - & - & - & • \\
\hline $\mathbf{Z n}$ & - & 600 & 500 & 500 & 910 & 1100 & • & 190 & • & 500 & 1200 \\
\hline Semplo & Noctule & Crent & Mr-coatiod & Mn-contiod & Crast & Nodule & Min-contod & Crust & Crust & Crust & Mo-coated \\
\hline typo & $\cdot$ & - & rock & roct & $\cdot$ & $\cdot$ & rock & $\cdot$ & - & - & rock \\
\hline Portion & croes & unknown & without & without & $n$ & whole & ankoown & untroown & unknown & fragmonts & half \\
\hline amplod & esction & - & maclous & miclous & - & - & $\cdot$ & - & • & • & $\cdot$ \\
\hline Neclow & • & • & - & $\cdot$ & • & - & - & - & Undoterminod & non-apperent & • \\
\hline Reference & Al66 & $A 368$ & A405 & A405 & A291 & Al97 & $\mathbf{A 2 3 0}$ & A376 & A321 & A376 & A376 \\
\hline Sequencelly & $3040105-01$ & $3040115-00$ & $3050010-00$ & $3050029-00$ & $3050029-03$ & $3050039-00$ & $3050039-02$ & $3050009-03$ & $3050072-01$ & $3050076-01$ & $3050090-\infty 0$ \\
\hline Latioude & -4.982 & -2018 & -8.080 & -2.292 & -2202 & -9.805 & -9.805 & -9.805 & -4.233 & -4.233 & -8.275 \\
\hline Longitude & -154.983 & -158.523 & -160.550 & -163.958 & -163.958 & -164.743 & -164.743 & -164.743 & -168.283 & -168.283 & -168.508 \\
\hline Depth & 4990 & 5080 & 4243 & 5243 & 5243 & 4199 & 4199 & 4199 & 5204 & 5230 & 4565 \\
\hline Si(wt\%) & - & - & $\cdot$ & - & $\cdot$ & - & - & $\cdot$ & $\cdot$ & - & $\cdot$ \\
\hline $\mathrm{Ti}$ & - & • & - & - & - & - & - & - & • & - & - \\
\hline Mn & - & 128 & 14.9 & - & 18.6 & 20.3 & 19.5 & - & 199 & $\cdot$ & 23.1 \\
\hline $\mathrm{Pe}$ & - & 8.00 & 21.1 & - & 11.5 & - & 319 & - & 18.3 & - & - \\
\hline $\mathbf{A}$ & - & - & - & - & • & $\cdot$ & - & - & - & - & 236 \\
\hline Co & - & .150 & - & 280 & 330 & .460 & - & - & 580 & 530 & .500 \\
\hline $\mathbf{N i}$ & - & .770 & .100 & - & .650 & .200 & .280 & - & 300 & • & .300 \\
\hline a & - & 540 & .110 & - & 410 & .130 & .100 & - & .160 & - & .150 \\
\hline C. & - & 2.20 & • & - & 1.90 & - & $\cdot$ & - & 260 & - & - \\
\hline $\mathrm{M}_{8}$ & - & - & - & - & 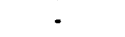 & - & - & - & - & - & - \\
\hline $\mathrm{Na}$ & - & - & - & - & - & - & - & - & - & - & - \\
\hline $\mathbf{K}$ & - & - & - & - & $\cdot$ & - & - & - & - & - & - \\
\hline $\mathbf{P}$ & - & $\cdot$ & - & - & - & - & - & - & • & - & - \\
\hline $\mathrm{H}_{2} \mathrm{O}$ & $\cdot$ & $\cdot$ & - & - & - & - & 33.0 & - & 27.8 & - & - \\
\hline Ax(ppon) & 235 & - & - & - & - & - & - & 330 & - & - & - \\
\hline $\mathrm{Bn}$ & 980 & - & - & $\cdot$ & - & - & $\cdot$ & 1800 & - & $\cdot$ & 3 \\
\hline Cd & 12.0 & - & - & 4.0 & - & 4.0 & - & 4.0 & - & 4.0 & 5.0 \\
\hline Co & • & - & - & $\cdot$ & - & - & - & - & - & $\cdot$ & - \\
\hline$c$ & 25 & - & - & - & - & 17 & - & 13 & - & - & - \\
\hline Mo & $\cdot$ & $\cdot$ & - & 260 & • & 450 & $\cdot$ & - & - & 310 & 270 \\
\hline $\mathbf{P b}$ & - & 500 & - & 700 & 700 & - & - & - & 1000 & 1200 & 1100 \\
\hline St & - & $\cdot$ & - & $\cdot$ & • & - & - & - & - & $\cdot$ & $\cdot$ \\
\hline v & - & - & - & - & - & 690 & - & - & - & - & 630 \\
\hline $\mathbf{Y}$ & - & - & - & - & - & - & - & - & $\cdot$ & - & $\cdot$ \\
\hline $\mathbf{Z n}$ & • & 800 & - & 520 & 800 & 690 & • & • & 600 & 570 & 490 \\
\hline Semplo & Mn-coutiod & Mn-coetod & Min-contad & Crust & Crust & Crust & Crust & Crust & Noctule & Nodule & Nocule \\
\hline typo & rock & rock & rock & - & • & • & - & - & $\cdot$ & • & • \\
\hline Portion & half & half & outer & fragments & fragments & frigments & unknown & unknown & $\operatorname{cross}$ & naknown & half \\
\hline andeplod & • & - & const & 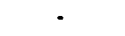 & - & 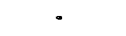 & • & • & rection & • & $\cdot$ \\
\hline Nuclous & - & - & axy & • & - & - & • & • & Clay & • & noo-yperent \\
\hline Roferences & A475 & A376 & A321 & A303 & A422 & A.301 & A321 & A475 & A422 & $A 303$ & A303 \\
\hline
\end{tabular}


Table 9. Composition of crusts from the Scripps Nodule Databank (cont'd).

\begin{tabular}{|c|c|c|c|c|c|c|c|c|c|c|c|}
\hline $\begin{array}{l}\text { Sequenooll } \\
\text { Latimide } \\
\text { Lenginde } \\
\text { Depth }\end{array}$ & $\begin{array}{c}3050091-00 \\
-8.283 \\
-168.520 \\
4751 \\
\end{array}$ & $\begin{array}{c}3050092-00 \\
-8.287 \\
-168.528 \\
4758 \\
\end{array}$ & $\begin{array}{c}3050095-03 \\
-8.817 \\
-168.550 \\
4550\end{array}$ & $\begin{array}{c}3050095-04 \\
-8.817 \\
-168550 \\
4550\end{array}$ & $\begin{array}{c}3050095-05 \\
-8 \$ 17 \\
-168.550 \\
4550\end{array}$ & $\begin{array}{c}3050095-06 \\
-8.817 \\
-168.550 \\
4550\end{array}$ & $\begin{array}{c}3050095-07 \\
-8.817 \\
-168.550 \\
4550 \\
\end{array}$ & $\begin{array}{c}3050095-08 \\
-8.817 \\
-168.550 \\
4550 \\
\end{array}$ & $\begin{array}{c}3050100-\infty \\
-7.517 \\
-168.817 \\
5288 \\
\end{array}$ & $\begin{array}{c}3050100-01 \\
-7.517 \\
-168.817 \\
5288 \\
\end{array}$ & $\begin{array}{c}3050104-00 \\
-7.675 \\
-161.613 \\
4540 \\
\end{array}$ \\
\hline Si(wt\%) & - & - & - & - & - & 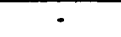 & $\cdot$ & $\cdot$ & - & . & - \\
\hline $\mathrm{Ti}$ & - & - & - & • & - & - & - & - & - & = & • \\
\hline Min & 220 & 21.8 & - & 18.3 & 19.6 & - & - & - & - & 15.4 & 21.1 \\
\hline Fo & - & • & - & 16.6 & 178 & - & - & - & - & 20.2 & 16.8 \\
\hline $\mathbf{N}$ & - & 2.16 & - & • & • & $=$ & - & - & • & • & • \\
\hline Co & 530 & .660 & 350 & • & - & - & - & .490 & 310 & 430 & 390 \\
\hline $\mathrm{Ni}$ & .260 & .330 & - & 350 & .380 & - & - & - & • & .140 & 390 \\
\hline $\mathrm{Cu}$ & .150 & .110 & - & .220 & .200 & - & - & - & - & .130 & .130 \\
\hline c. & - & • & - & - & • & - & - & - & - & 220 & 29 \\
\hline $\mathbf{M g}$ & - & - & - & - & - & - & - & - & - & • & - \\
\hline $\mathrm{Na}$ & - & - & - & - & - & - & - & - & - & - & - \\
\hline $\mathbf{K}$ & - & - & - & - & - & - & - & - & - & - & - \\
\hline $\mathbf{P}$ & - & - & - & - & - & - & - & - & - & - & - \\
\hline $\mathrm{H}_{2} \mathrm{O}$ & - & - & - & 27.9 & 31.2 & - & - & - & - & - & - \\
\hline$A x$ (ppm) & - & - & - & - & - & - & - & - & - & - & - \\
\hline Be & - & 3 & - & - & - & - & - & - & - & - & - \\
\hline Cd & 4.0 & 5.0 & 6.0 & - & - & - & - & 4.0 & 6.0 & - & - \\
\hline Co & $\cdot$ & $\cdot$ & - & - & - & - & - & - & - & - & - \\
\hline$C$ & 13 & - & - & - & - &. & - & - & - & - & - \\
\hline$\dot{\dot{M}_{0}}$ & 370 & 350 & 188 & - & - & - & - & 270 & 178 & - & - \\
\hline $\mathrm{Pb}$ & - & 1100 & 900 & - & - & - & - & 800 & 1000 & 1000 & - \\
\hline Sr & - & - & - & - & - & - & - & - & - & - & - \\
\hline $\mathbf{v}$ & 660 & 660 & - & - & - & - & - & $\cdot$ & - & - & - \\
\hline $\mathbf{Y}$ & • & - & - & - & - & - & - & - & • & - & - \\
\hline $2 n$ & 600 & 470 & 500 & - & - & - & - & 390 & 460 & 600 & 570 \\
\hline Samplo & Notale & Nodale & Crust & Crut & Crast & Crust & Crust & Crust & Mn-encrusted & Mn-encrusted & Crust \\
\hline typo & $\cdot$ & • & $\cdot$ & - & • & - & - & • & sedimeat & sedimont & • \\
\hline Partion & unimown & half & whole & whole & monknown & unknown & unknown & unknown & conter & outter & unionown \\
\hline senoplod & - & • & - & - & - & - & - & - & crust & crust & - \\
\hline Nuclens & • & Cry & - & - & • & - & - & - & Sediment & Sodiment & - \\
\hline Roferences & A301 & 1303 & $A 303$ & A321 & A321 & $A 30 B$ & $A 30 B$ & A303 & $A 303$ & A422 & A324 \\
\hline Sequencell & $3050104-01$ & $3050115-02$ & $3050117-00$ & $3050117-01$ & $3050127-\infty$ & $3050129-02$ & $3050130-01$ & $3060045-00$ & $3140168-01$ & $3150023-00$ & $3150065-00$ \\
\hline Latioude & -7.675 & -1.265 & -1.297 & -1.207 & -8.317 & -7.740 & -7.397 & -6.233 & -9.133 & -5.383 & -6.733 \\
\hline Langinds & -161.613 & -168.243 & -168.390 & -168.390 & -168.550 & $-168 . \$ 53$ & -168.548 & -176.717 & 104.667 & 97.483 & 90.207 \\
\hline Dopth & 4540 & 6965 & 6730 & 6730 & 4482 & 4700 & 5471 & 5853 & 5330 & 3558 & 3906 \\
\hline Si(wt.\%) & $\cdot$ & . & - & - & 4.90 & - & $\cdot$ & - & - & $\cdot$ & - \\
\hline $\mathrm{Ti}$ & • & - & - & • & 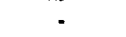 & - & • & - & - & .29 & • \\
\hline Mn & 18.3 & 19.9 & 19.6 & 20.9 & 20.2 & - & 18.5 & 15.9 & 15.2 & 14.6 & 4.60 \\
\hline Pe & 14.6 & 15.9 & 20.7 & 20.3 & 18.1 & - & 17.8 & 175 & 11.8 & 15.9 & 3.80 \\
\hline Al & - & - & - & - & 1.75 & • & - & - & • & • & • \\
\hline Co & $A 80$ & .810 & 320 & 380 & .660 & 550 & 520 & .250 & .090 & .070 & .090 \\
\hline $\mathrm{Ni}$ & .440 & .360 & .250 & 230 & .300 &. & .440 & .310 & 580 & 360 & 210 \\
\hline $\mathrm{Cu}$ & .060 & .090 & .170 & .140 & .030 & - & 320 & .200 & .260 & 300 & .180 \\
\hline C. & .60 & 2.40 & 1.90 & 2.20 & 226 & - & 2.50 & 90 & . & $\because$ & $\cdot$ \\
\hline Mg & • & - & - & - & 1.21 & - & - & - & - & - & - \\
\hline $\mathrm{Na}$ & - & - & - & - & - & - & - & - & - & - & - \\
\hline $\mathbf{K}$ & - & - & - & - & - & - & - & - & - & - & - \\
\hline $\mathbf{P}$ & - & - & - & - & - & - & - & - & - & - & - \\
\hline $\mathrm{H}_{2} \mathrm{O}$ & - & - & - & $\cdot$ & - & - & - & - & - & . & $\cdot$ \\
\hline$A s$ (ppm) & - & - & - & - & - & - & - & - & - & - & - \\
\hline $\mathrm{Ba}$ & - & - & - & - & - & - & - & - & - & 1300 & - \\
\hline Cd & - & - & - & - & - & 9.0 & - & - & - & - & - \\
\hline co & - & - & - & - & - & - & - & - & - & - & - \\
\hline$c$ & - & - & - & - & - & - & - & - & - & 3 & - \\
\hline Mo & - & • & - & • & - & 212 & • & - & - & 180 & - \\
\hline $\mathbf{P b}$ & - & 1000 & 900 & 1000 & 1200 & 900 & 900 & - & - & 400 & - \\
\hline Sr & - & . & - & - & - & - & - & - & - & - & . \\
\hline $\mathrm{v}$ & - & - & - & - & - & - & - & - & . & 350 & - \\
\hline $\mathbf{Y}$ & - & - & - & $\cdot$ & - & - & - & • & - & - & - \\
\hline $\mathbf{Z n}_{n}$ & 630 & 400 & 400 & 400 & 540 & 480 & 600 & 580 & • & • & - \\
\hline Semple & Cout & Nodule & Crust & Crust & Nodule & Nodule & Noculo & Crust & Mn-costed & Crunt & Mn-contod \\
\hline type & - & - & - & - & - & - & • & - & rock & • & material \\
\hline Portion & unionown & quertuer & frigmont & frigments & minnown & ours & balf & unknown & onter & frigments & outer \\
\hline sampled & - & - & . & . & - & crust & $\cdot$ & - & coust & $\cdot$ & crust \\
\hline Nuclous & - & noo-sppareat & - & • & - & Rock & Tooth & $=$ & Bemilt & • & argenic \\
\hline Roference & A324 & A405 & A405 & A405 & A284 & A303 & A422 & A 324 & A451 & A240 & A333 \\
\hline
\end{tabular}


Table 9. Composition of crusts from the Scripps Nodule Databank (cont'd).

\begin{tabular}{|c|c|c|c|c|c|c|c|c|c|c|c|}
\hline $\begin{array}{l}\text { Soquonoul } \\
\text { Letitude } \\
\text { Longinude } \\
\text { Depth }\end{array}$ & $\begin{array}{c}3150077-00 \\
-9.600 \\
90.483 \\
4850\end{array}$ & $\begin{array}{c}3190018-00 \\
-7.332 \\
57.937 \\
2421\end{array}$ & $\begin{array}{c}3190028-00 \\
-2.133 \\
57.400 \\
4133\end{array}$ & $\begin{array}{c}3190029-00 \\
-2.100 \\
57.383 \\
4041\end{array}$ & $\begin{array}{c}3190029-01 \\
-2100 \\
57.383 \\
4041\end{array}$ & $\begin{array}{c}3190044-00 \\
-9.650 \\
56.417 \\
2800\end{array}$ & $\begin{array}{c}3190044-01 \\
-9.650 \\
56417 \\
2800\end{array}$ & $\begin{array}{c}3190078-00 \\
-6.667 \\
51.900 \\
2000\end{array}$ & $\begin{array}{c}3190078-01 \\
-6.607 \\
51.900 \\
2000\end{array}$ & $\begin{array}{c}3190078-02 \\
-6.607 \\
51.900 \\
2900 \\
\end{array}$ & $\begin{array}{c}3190080-00 \\
-6.600 \\
51.567 \\
2990 \\
\end{array}$ \\
\hline Sí(wt.\%) & $\cdot$ & $\cdot$ & $\cdot$ & $\cdot$ & $\cdot$ & $\cdot$ & $\cdot$ & $\cdot$ & $\cdot$ & $\cdot$ & $\cdot$ \\
\hline $\mathrm{Ti}$ & - & - & 1.76 & .91 & 1.20 & 140 & - & 1.14 & - & - & - \\
\hline M & 15.0 & 4.50 & 18.4 & 15.4 & 145 & 15.2 & 19.6 & 135 & 32.1 & 22.6 & 23.8 \\
\hline Fo & 15.1 & 15.7 & 16.4 & 17.9 & 19.1 & 13.6 & 19.7 & 25.6 & 16.7 & 17.2 & 18.8 \\
\hline $\mathbf{N}$ & • & 4.20 & • & - & • & • & - & - & - & • & • \\
\hline Co & .160 & .280 & $\$ 10$ & 590 & 350 & 1.000 & 880 & $\mathbf{8 8 0}$ & .600 & 1.22 & .680 \\
\hline $\mathbf{N i}$ & .360 & .090 & .470 & 340 & 320 & 390 & $A 80$ & 340 & .580 & 530 & $A 80$ \\
\hline$c u$ & .220 & .090 & .050 & .080 & .060 & .070 & .130 & .050 & .080 & .080 & 300 \\
\hline$C_{2}$ & • & 250 & • & - & - & • & - & • & • & - & • \\
\hline $\mathbf{M}_{8}$ & - & • & - & - & - & - & - & - & - & - & - \\
\hline $\mathrm{Na}$ & - & - & - & - & - & - & - & - & - & - & - \\
\hline $\mathbf{K}$ & - & - & - & - & - & - & - & - & - & - & - \\
\hline $\mathbf{P}$ & - & - & - & - & - & - & - & - & - & - & . \\
\hline $\mathrm{H}_{2} \mathrm{O}-$ & - & $\cdot$ & $\cdot$ & $\cdot$ & - & - & 29.3 & $\cdot$ & - & 32.3 & 29.6 \\
\hline$A \simeq(p p m)$ & - & - & - & - & - & - & - & - & - & - & - \\
\hline Ba & - & - & 1400 & 1400 & 1800 & 4000 & - & 2200 & - & - & - \\
\hline Cd & - & - & - & • & - & - & - & • & - & - & - \\
\hline Co & - & - & - & - & - & - & - & - & - & - & - \\
\hline c & - & - & 14 & 20 & 21 & 5 & - & 13 & - & - & . \\
\hline Mo & - & • & 280 & 350 & 200 & 470 & - & 470 & - & - & - \\
\hline Pb & - & 1600 & 800 & 400 & 1400 & 2600 & - & 2500 & 2400 & - & - \\
\hline Sr & - & - & 810 & - & - & • & - & • & • & - & - \\
\hline $\mathbf{v}$ & - & - & 860 & 590 & 840 & 740 & - & 650 & - & - & - \\
\hline $\mathbf{Y}$ & - & - & 140 & • & - & - & - & • & - & - & - \\
\hline $\mathbf{Z n}$ & - & 440 & 470 & - & • & - & • & - & • & - & • \\
\hline Samplo & Coust & Crust & Crast & Court & Crose & Crust & Uninown & Count & Nodule & Unbown & Unlonown \\
\hline type & • & • & • & • & • & • & • & • & • & • & • \\
\hline Portion & unkown & antrown & unbown & fragmonts & fragments & fingerentar & unboown & fragnom & crom & mentrown & unionown \\
\hline exuplod & - & • & • & • & • & • & • & • & soction & • & • \\
\hline Nuclens & - & - & - & - & - & - & - & . & . & . & - \\
\hline Reforencose & A451 & A467 & A203 & A240 & A240 & A240 & A409 & A240 & A166 & A409 & A409 \\
\hline Sequenoodil & $3190080-01$ & $3190093-00$ & $3190093-01$ & $3190093-02$ & $3190093-03$ & $3190093-04$ & 3190093-05 & $3190093-06$ & $3190093-07$ & $3190093-08$ & $3190093-09$ \\
\hline Latitude & -6.600 & -2555 & -2.555 & -2555 & -2555 & -2.555 & -2.535 & .2535 & -2.555 & -2535 & -2555 \\
\hline Longinade & 51.56 & 57.278 & 57.278 & 57.278 & 57.278 & 57.278 & 57.278 & 57.278 & 57.278 & 57.278 & 57.278 \\
\hline Depth & 2990 & 3398 & 3398 & 3398 & 3398 & 3398 & 3398 & 3398 & 3398 & 3398 & 3398 \\
\hline Si(wts) & - & $\cdot$ & $\cdot$ & $\cdot$ & $\cdot$ & $\cdot$ & $\cdot$ & - & $\cdot$ & $\cdot$ & $\cdot$ \\
\hline $\mathbf{T i}$ & • & • & • & - & .70 & .70 & .75 & .70 & .74 & .71 & .74 \\
\hline Mn & 38.3 & 18.8 & 16.2 & 15.5 & 16.1 & 14.4 & 15.0 & 12.7 & 16.0 & 14.3 & 14.1 \\
\hline Fo & 17.1 & 20.1 & 20.6 & 16.2 & 19.9 & 21.0 & 17.8 & 16.0 & 21.3 & 19.7 & 20.2 \\
\hline A & • & $\cdot$ & 1.63 & 2.55 & 1.62 & 1.06 & 202 & 250 & .88 & 2.18 & 1.20 \\
\hline Co & .720 & 390 & 360 & .180 & 530 & .300 & .280 & 260 & .270 & 240 & .280 \\
\hline $\mathrm{Ni}$ & .710 & 360 & .270 & .440 & 260 & .170 & .240 & .230 & .180 & .220 & .190 \\
\hline cu & .080 & .070 & .080 & 260 & .090 & .050 & .110 & .110 & .050 & .080 & .050 \\
\hline$C_{2}$ & • & 3.00 & 203 & 1.71 & 1.93 & 1.72 & 1.71 & 1.69 & 236 & 159 & 1.76 \\
\hline$M_{6}$ & - & • & • & • & 1.08 & 95 & 1.02 & 1.09 & 1.04 & 1.04 & 1.03 \\
\hline $\mathrm{Na}$ & - & - & • & - & $\cdot$ & . & $\cdot$ & • & $\cdot$ & $\cdot$ & $\cdot$ \\
\hline $\mathbf{x}$ & - & - & - & . & - & - & . & - & . & . & . \\
\hline $\mathbf{P}$ & - & - & - & . & . & . & . & - & . & . & . \\
\hline $\mathrm{H}_{2} \mathrm{O}$ & $\cdot$ & • & $\cdot$ & - & - & $\cdot$ & $\cdot$ & $\cdot$ & - & $\cdot$ & $\cdot$ \\
\hline As(ppon) & • & - & - & - & - & - & - & . & . & . & - \\
\hline $\mathrm{Ba}$ & - & - & - & - & - & . & . & - & . & . & . \\
\hline Cd & - & - & - & - & 7.0 & 5.0 & 6.0 & 6.0 & 6.0 & 5.0 & - \\
\hline$c_{0}$ & - & - & - & - & - & - & - & - & - & - & - \\
\hline Cr & - & - & - & - & . & - & - & - & - & - & . \\
\hline Mo & - & • & - & • & • & • & . & - & $\cdot$ & - & - \\
\hline $\mathrm{Pb}$ & 2900 & 1300 & 1400 & 1100 & 1200 & 1200 & 1000 & 800 & 1100 & 1000 & 1100 \\
\hline Sx & $\cdot$ & - & • & - & . & • & • & - & - & - & - \\
\hline v & - & - & - & - & - & - & - & - & - & - & - \\
\hline $\mathbf{Y}$ & - & . & . & • & . & • & - & - & . & - & . \\
\hline $\mathbf{Z n}$ & • & 490 & 510 & 750 & 520 & 510 & 450 & 420 & 580 & 350 & 500 \\
\hline Samplo & Noctule & Croust & Crust & Coust & Cresx & Crox & Coust & Crust & Crust & Coust & Crust \\
\hline type & • & $\cdot$ & • & • & - & • & • & • & • & • & • \\
\hline Portion & croess & fragneats & unlnown & unlonown & unkoown & unlnown & unkown & unknown & unlnown & unlonown & untrown \\
\hline amplod & moction & 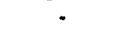 & $\cdot$ & $\cdot$ & $\cdot$ & • & • & • & • & • & • \\
\hline Nucloos & • & • & - & - & • & - & . & • & . & • & $\cdot$ \\
\hline Refierencos & A166 & A376 & A433 & A433 & A433 & A433 & A433 & A433 & A433 & A433 & A433 \\
\hline
\end{tabular}


Table 9. Composition of crusts from the Scripps Nodule Databank (cont'd).

\begin{tabular}{|c|c|c|c|c|c|c|c|c|c|c|c|}
\hline Sequencell & $3190093-10$ & $3190093-11$ & $3190093-12$ & $3190093-13$ & $3190093-14$ & $3190093-15$ & $3190093-16$ & $3190093-17$ & $3190093-18$ & $3190101-\infty 0$ & $3190101-01$ \\
\hline Latimude & -2555 & -2.555 & -2555 & .2555 & -2355 & .2555 & -2555 & -2.555 & -2555 & -1.225 & -1.225 \\
\hline Longinde & 57.278 & 57.278 & 57.278 & 57.278 & 57.278 & 57.278 & 57.278 & 57.278 & 57.278 & 56.025 & 56.025 \\
\hline Depth & 3398 & 3398 & 3398 & 3398 & 3398 & 3398 & 3398 & 3398 & 3398 & 4480 & 4480 \\
\hline Si(wt\%) & . & $\cdot$ & - & - & $\cdot$ & $\cdot$ & - & - & - & - & $\cdot$ \\
\hline $\mathbf{T i}$ & 38 & .64 & 58 & .80 & .81 & .82 & .75 & .83 & .69 & - & 1.13 \\
\hline Mn & 145 & 13.4 & 128 & 165 & 15.8 & 14.9 & 19.6 & 13.4 & 15.4 & 18.3 & 19.1 \\
\hline Pe & 20.5 & 19.6 & 18.2 & 20.7 & 18.3 & 17.7 & 20.4 & 215 & 21.1 & 19.4 & 18.0 \\
\hline $\mathbf{A}$ & 87 & 2.15 & 248 & 98 & 1.63 & 1.87 & 59 & 1.68 & 1.01 & 1.43 & - \\
\hline Co & 230 & .200 & .170 & .370 & 340 & .320 & .410 & .200 & .270 & 540 & .630 \\
\hline $\mathrm{Ni}$ & .160 & .190 & .170 & .210 & .280 & .250 & 240 & 240 & .170 & 200 & .280 \\
\hline $\mathrm{Cr}$ & .050 & .060 & .060 & .040 & .090 & .100 & .000 & .130 & .080 & .070 & .050 \\
\hline ca & 2.18 & 1.77 & 1.80 & 2.12 & 2.80 & 1.70 & 238 & 2.01 & 1.84 & 1.85 & 1.80 \\
\hline $\mathbf{M s}$ & 97 & 1.01 & 1.07 & 1.02 & 1.10 & 1.16 & 98 & 1.10 & 96 & • & 1.02 \\
\hline Na & - & - & • & - & • & • & - & • & - & - & - \\
\hline $\mathbf{K}$ & - & - & - & . & - & - & - & - & . & . & - \\
\hline $\mathbf{P}$ & - & - & - & - & - & - & . & - & - & - & - \\
\hline $\mathrm{H}_{2} \mathrm{O}$ & - & - & . & - & - & - & - & - & - & $=$ & - \\
\hline$A x(p p m)$ & - & - & - & - & - & - & - & - & - & - & - \\
\hline $\mathrm{B} \mathbf{2}$. & $\bullet$ & - & • & • & • & • & • & $\cdot$ & • & - & $\cdot$ \\
\hline Cd & 7.0 & 8.0 & 7.0 & 6.0 & 6.0 & 7.0 & 6.0 & 6.0 & 7.0 & - & 6.0 \\
\hline C. & - & - & • & - & - & - & • & - & - & - & - \\
\hline$c$ & - & - & - & - & 。 & . & . & - & - & - & - \\
\hline Mo & • & • & $\cdot$ & - & - & • & • & • & • & - & - \\
\hline $\mathrm{Pb}$ & 1000 & 900 & 700 & 1200 & 1100 & 1100 & 1200 & 1000 & 1200 & 1600 & 1300 \\
\hline Sr & • & • & $\cdot$ & • & • & • & • & • & • & • & • \\
\hline $\mathbf{v}$ & - & - & - & - & - & - & - & - & - & - & - \\
\hline $\mathbf{Y}$ & - & - & . & - & - & - & - & - & - & - & - \\
\hline $\mathbf{Z n}$ & 590 & 530 & 540 & 530 & 490 & 480 & 590 & 550 & 520 & 500 & 490 \\
\hline Semplop & Crust & Crust & Crust & Crust & Crust & Cont & Crust & Crust & Crust & Crust & Crust \\
\hline type & - & - & • & - & • & • & • & - & • & • & - \\
\hline Partion & unionown & unkonown & unitoown & unlonown & unlonown & unknown & unlonown & unlonown & monlonown & unlonown & unionown \\
\hline emorpled & - & • & • & - & • & • & • & - & • & - & - \\
\hline Nucless & - & - & • & • & - & • & - & - & . & - & - \\
\hline Roference & A433 & A433 & A433 & A433 & A433 & A433 & A433 & A433 & A433 & A433 & A433 \\
\hline Sequencell & $3190101-02$ & $3190101-03$ & $3260221-\infty$ & $3260221-01$ & $3260221-02$ & $3280016-01$ & $3280016-02$ & $3290001-\infty 0$ & $3350037-\infty$ & $3350037-01$ & $3350075-\infty 0$ \\
\hline Latiouds & -1.225 & -1.225 & .350 & .350 & 350 & 6.050 & 6.050 & 9.650 & 9.950 & 9.950 & 9.138 \\
\hline Langinde & 56.025 & 56.025 & -17517 & -17.517 & -17517 & -32367 & -32367 & $-40,450$ & -108.800 & -108.800 & -105.450 \\
\hline Dopth & 4480 & 4480 & 1712 & 1712 & 1712 & 3020 & 3020 & 2388 & 1228 & 1228 & 2651 \\
\hline si(wt.\%) & . & . & . & . & . & - & - & . & 4.40 & $\cdot$ & . \\
\hline $\mathrm{Ti}$ & 1.22 & 1.14 & - & - & • & - & - & - & - & .70 & - \\
\hline $\mathbf{M n}$ & 18.0 & 16.9 & 17.3 & 23.1 & 17.7 & 9.01 & 125 & 15.2 & 31.5 & 44.4 & 19.4 \\
\hline Fo & 17.6 & 19.0 & 14.8 & 133 & 20.4 & 19.7 & 245 & 22.2 & 10.3 & 8.07 & 20.2 \\
\hline $\mathbf{A}$ & 1.23 & 1.77 & - & • & - & - & - & - & - & - & - \\
\hline Co & .530 & .450 & 320 & .720 & 500 & .430 & .280 & 510 & .500 & .760 & .130 \\
\hline $\mathrm{Ni}$ & .290 & .280 & .280 & .430 & 200 & .140 & .160 & .170 & .740 & 940 & .660 \\
\hline $\mathrm{Cr}$ & .090 & .120 & .060 & .030 & .070 & .090 & .070 & .020 & .030 & .040 & .290 \\
\hline C. & 1.83 & 1.66 & $\cdot$ & 5.10 & - & - & 266 & 2.42 & - & - & 200 \\
\hline Ms & 1.05 & 1.09 & - & 1.25 & - & - & 1.43 & 1.40 & - & - & - \\
\hline $\mathrm{Na}$ & - & - & - & 3.23 & - & - & 1.43 & 1.40 & - & - & - \\
\hline $\mathbf{K}$ & - & - & - & 40 & - & - & 31 & .20 & - & - & - \\
\hline $\mathbf{P}$ & - & - & - & - & - & - & - & - & - & - & - \\
\hline $\mathrm{H}_{2} \mathrm{O}$ & - & - & - & • & - & • & • & - & - & . & - \\
\hline Ax(ppon) & - & - & - & - & . & - & - & - & - & - & - \\
\hline Ba & - & - & - & - & - & - & - & - & - & - & - \\
\hline Cd & 5.0 & 6.0 & - & - & - & - & - & - & - & - & - \\
\hline Co & • & - & - & - & - & - & - & - & - & - & - \\
\hline$C$ & - & - & - & - & - & - & - & - & - & - & - \\
\hline Mo & • & • & - & • & - & $\cdot$ & $\cdot$ & - & - & 700 & - \\
\hline $\mathrm{Pb}$ & 13000 & 14000 & - & 1600 & - & 200 & 900 & 1400 & 2200 & 1800 & 400 \\
\hline St & - & - & - & - & - & $\cdot$ & - & 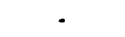 & - & - & $\cdot$ \\
\hline v & . & - & - & - & - & - & - & - & - & - & - \\
\hline $\mathbf{Y}$ & - & - & . & - & - & - & - & - & - & - & • \\
\hline $\mathrm{Zn}$ & 490 & 470 & - & 630 & • & - & 570 & 580 & • & • & 1000 \\
\hline Semplo & Cust & Crust & Crust & Notule & Nodule & Crust & Crust & Crust & Nodulo & Noctule & Mn-conted \\
\hline typo & - & • & - & • & • & - & - & • & • & • & rock \\
\hline Partion & uncoown & un'mown & unknown & unknown & unknown & fragments & unlonown & unlopown & nuclous & cross & topside \\
\hline samplod & • & • & - & - & - & • & • & • & • & rection & • \\
\hline Naclous & - & - & • & • & - & • & - & - & • & • & - \\
\hline Reference & A433 & A433 & A179 & A368 & A419 & A240 & A368 & A368 & A240 & Al66 & A422 \\
\hline
\end{tabular}


Table 9. Composition of crusts from the Scripps Nodule Databank (cont'd).

\begin{tabular}{|c|c|c|c|c|c|c|c|c|c|c|c|}
\hline $\begin{array}{l}\text { Sequenod } \\
\text { Leatitude } \\
\text { Longitude } \\
\text { Depph }\end{array}$ & $\begin{array}{c}3350075-01 \\
9.138 \\
-105.450 \\
2651 \\
\end{array}$ & $\begin{array}{c}3350075-02 \\
9.138 \\
-105,450 \\
2651 \\
\end{array}$ & $\begin{array}{c}3350075-03 \\
9.138 \\
-105.450 \\
2651 \\
\end{array}$ & $\begin{array}{c}3350075-04 \\
9.138 \\
-105.450 \\
2651 \\
\end{array}$ & $\begin{array}{c}3350090-08 \\
8.157 \\
-104.457 \\
3325 \\
\end{array}$ & $\begin{array}{c}3350090-09 \\
8.157 \\
-104.457 \\
3325 \\
\end{array}$ & $\begin{array}{c}3350109-\infty 0 \\
8.803 \\
-103.892 \\
1822 \\
\end{array}$ & $\begin{array}{c}3350124-\infty 0 \\
8.825 \\
-103.917 \\
2095 \\
\end{array}$ & $\begin{array}{c}3350124-03 \\
8.825 \\
-103.917 \\
2095 \\
\end{array}$ & $\begin{array}{c}3350124-\infty \\
8.825 \\
-103.917 \\
2095 \\
\end{array}$ & $\begin{array}{c}3380111-02 \\
9.100 \\
-139.750 \\
5040 \\
\end{array}$ \\
\hline Si(wt.\%) & - & - & - & - & $\cdot$ & - & - & - & - & - & $\cdot$ \\
\hline $\mathrm{Ti}$ & • & . & . & - & 34 & • & - & • & • & .01 & .33 \\
\hline Mn & 8.06 & 13.6 & - & - & 145 & 13.2 & 22.1 & 12.8 & 42.6 & - & 17.6 \\
\hline Po & 19.6 & 24.7 & - & . & 22.4 & 23.9 & 168 & 20.6 & • & - & 6.70 \\
\hline Al & • & • & • & . & • & • & • & • & - & .09 & • \\
\hline$c_{0}$ & . & • & .070 & .060 & .130 & • & .070 & .070 & - & • & .064 \\
\hline $\mathbf{N i}$ & .110 & .250 & • & • & .270 & .140 & .150 & .170 & - & - & 1.090 \\
\hline Cu & .120 & .100 & - & - & 210 & .120 & .020 & .040 & • & - & 1.220 \\
\hline c. & • & • & - & - & 1.90 & • & 3.00 & 3.10 & 9.00 & - & 1.00 \\
\hline Me & - & - & - & - & - & - & • & • & • & .92 & • \\
\hline $\mathrm{Na}$ & - & - & - & - & - & - & - & - & - & 3.14 & - \\
\hline $\mathbf{K}$ & - & - & - & - & - & - & - & - & - & • & - \\
\hline $\mathbf{P}$ & . & . & - & . & . & . & . & . & - & . & - \\
\hline $\mathrm{H}_{2} \mathrm{O}-$ & $\cdot$ & - & - & - & - & - & - & - & - & - & - \\
\hline$A x(p p m)$ & - & - & - & - & - & - & - & - & - & . & - \\
\hline $\mathrm{Bu}_{\mathrm{a}}$ & - & . & - & . & - & - & . & - & - & 920 & . \\
\hline Cd & - & - & 10.0 & 4.0 & - & - & - & - & - & - & - \\
\hline co & - & - & . & • & - & - & - & - & - & - & - \\
\hline Cr & - & - & • & . & - & - & - & - & - & - & - \\
\hline Mo & . & . & 310 & 175 & - & - & • & - & . & - & - \\
\hline Pb & . & - & 200 & 200 & 400 & - & 1200 & 400 & - & . & 500 \\
\hline Sz & - & - & . & • & • & - & • & • & - & - & . \\
\hline $\mathbf{v}$ & - & - & . & - & - & - & - & - & - & - & - \\
\hline $\mathbf{Y}$ & - & - & • & • & • & - & - & • & - & - & • \\
\hline $\mathbf{Z n}$ & • & • & 1100 & 720 & 600 & • & $6 \infty$ & $6 \infty$ & • & - & 1000 \\
\hline Sminple & Mo-costod & Mn-conted & Mn-contod & Mo-costiod & Crust & Crust & Mran-ontod & Crust & Crust & Crast & Nodule \\
\hline type & sock & rock & rock & rock & $\cdot$ & $\cdot$ & rock & - & $\cdot$ & - & • \\
\hline $\begin{array}{l}\text { Partion } \\
\text { semplod }\end{array}$ & $\begin{array}{c}\text { leyer-adjecont } \\
\text { to-medous }\end{array}$ & $\begin{array}{c}\text { underside } \\
\text {. }\end{array}$ & $\begin{array}{c}\text { underside } \\
-\end{array}$ & topside & fremonts & fragneats & $\begin{array}{l}\text { outer } \\
\text { const }\end{array}$ & frements & fraponeats & fraponents & without \\
\hline Nuclens & - & - & . & . & - & . & . & valconic & . & . & . \\
\hline Referencose & A342 & A342 & A303 & A303 & A342 & A342 & A405 & A437 & A437 & A465 & A405 \\
\hline Seqrencent & $3390080-01$ & $3390102-01$ & $3390348-\infty 0$ & $3400111-04$ & $3400135-05$ & $3400233-00$ & $3400236-\infty$ & $3400241-02$ & $3400241-03$ & $3400265-07$ & $3410013-00$ \\
\hline Letiondo & 8.033 & 8.89 & 9.293 & 7.750 & 7.433 & 8.718 & 8.695 & 9.333 & 9.333 & 7.970 & 5.872 \\
\hline Longitucto & -149.900 & -146.492 & -146.050 & $\cdot 150.683$ & .151 .783 & -150.235 & -150.312 & $\cdot-150.807$ & -150.807 & -152.287 & -160.848 \\
\hline Dopth & 5073 & 5225 & 5000 & $\cdot$ & - & 4978 & 5009 & 4839 & 4839 & 5189 & 1604 \\
\hline Si(wt.\%) & - & - & - & 13.7 & 1.54 & - & . & • & - & - & - \\
\hline $\mathrm{Ti}$ & .78 & - & • & • & • & • & • & • & • & - & • \\
\hline Mn & 14.0 & 27.6 & 24.0 & 17.9 & 4.50 & 23.4 & 17.6 & 21.6 & 16.7 & 27.4 & 23.0 \\
\hline Po & 21.3 & 6.60 & 6.50 & 7.00 & 1.10 & 11.9 & 11.8 & 10.8 & 146 & 5.26 & 16.6 \\
\hline A $\mathbf{I}$ & - & - & - & - & - & - & . & . & - & - & - \\
\hline$C_{0}$ & 510 & .220 & • & .200 & .050 & .680 & .530 & 540 & 560 & 570 & .550 \\
\hline $\mathrm{Ni}$ & .064 & 1.550 & 1.100 & 1.000 & .250 & 940 & .630 & .790 & .670 & 1.530 & 400 \\
\hline Cu & .950 & 1.130 & 800 & .860 & .220 & 320 & .670 & .630 & .700 & 1.580 & .020 \\
\hline Ca & • & 1.40 & - & • & • & 220 & 1.70 & - & • & 1.70 & 7.20 \\
\hline Mg & - & - & - & - & - & • & • & - & . & - & • \\
\hline $\mathbf{N a}$ & - & - & - & - & - & - & - & - & - & - & - \\
\hline $\mathbf{K}$ & - & - & - & - & - & - & - & - & - & - & - \\
\hline $\mathbf{P}$ & . & - & - & - & - & - & - & - & - & - & - \\
\hline $\mathrm{H}_{2} \mathrm{O}$ & $\cdot$ & - & $\cdot$ & - & - & - & - & $\cdot$ & - & - & 28.6 \\
\hline As(ppm) & - & . & - & . & - & . & . & - & . & - & - \\
\hline Ba & 7500 & - & . & . & - & . & . & - & . & - & - \\
\hline Cd & - & . & . & - & . & - & - & . & . & . & - \\
\hline$c_{0}$ & - & - & . & . & . & - & - & - & - & . & - \\
\hline cr & 10 & . & . & . & . & . & . & . & . & . & . \\
\hline Mo & 180 & - & . & . & - & - & . & - & . & . & - \\
\hline Pb & 1600 & 500 & . & 500 & 200 & 900 & 800 & . & . & 400 & 1500 \\
\hline St & - & - & . & - & - & . & - & . & - & - & - \\
\hline $\mathbf{v}$ & 420 & . & . & . & . & . & . & . & - & - & . \\
\hline $\mathbf{Y}$ & - & • & - & - & - & - & - & - & . & • & . \\
\hline $\mathbf{Z n}$ & • & 1300 & - & 1100 & $6 \infty$ & 800 & 800 & 600 & 700 & 1400 & 900 \\
\hline Sumplo & Unknown & Crust & Crust & Min-costod & Mre-cound & Nodulo & Nodule & Nodule & Nodule & Nodule & Crust \\
\hline type & • & • & - & rock & material & • & • & • & • & • & • \\
\hline Portion & unlonown & unknown & ankonown & unkoown & unknown & Gragments & whole & balf & half & helf & outorer \\
\hline semplod & $\cdot$ & • & $\cdot$ & • & $\cdot$ & • & $\cdot$ & • & $\cdot$ & - & const \\
\hline Nuclows & - & • & - & volcenic & orgmic & • & - & • & - & Mn-axide & • \\
\hline Reforences & A189 & A405 & A446 & $\mathbf{A 3 2 6}$ & A326 & A376 & A376 & A448 & 1448 & A376 & A422 \\
\hline
\end{tabular}


Table 9. Composition of crusts from the Scripps Nodule Databank (cont'd).

\begin{tabular}{|c|c|c|c|c|c|c|c|c|c|c|c|}
\hline $\begin{array}{l}\text { Sequencell } \\
\text { Latimde } \\
\text { Langitude } \\
\text { Depth }\end{array}$ & $\begin{array}{c}3410013-01 \\
5.872 \\
-160.848 \\
1604\end{array}$ & $\begin{array}{c}3410021-00 \\
5.232 \\
-161.497 \\
1307\end{array}$ & $\begin{array}{c}3410021-01 \\
5.232 \\
-161.497 \\
1307\end{array}$ & $\begin{array}{c}3410021-02 \\
5.232 \\
-161.497 \\
1307\end{array}$ & $\begin{array}{c}3410021-03 \\
5.232 \\
-161.497 \\
1307\end{array}$ & $\begin{array}{c}3410170-00 \\
8.330 \\
-164.362 \\
1519\end{array}$ & $\begin{array}{c}3410170-01 \\
8.330 \\
-164.362 \\
1519\end{array}$ & $\begin{array}{c}3410170-02 \\
8.330 \\
-164.362 \\
1519\end{array}$ & $\begin{array}{c}3410170-03 \\
8330 \\
-164.362 \\
1519\end{array}$ & $\begin{array}{c}3410170-04 \\
8.330 \\
-164.362 \\
1519\end{array}$ & $\begin{array}{c}3410186-00 \\
8340 \\
-164390 \\
1640\end{array}$ \\
\hline Si(wt.\%) & $\cdot$ & $\cdot$ & $\cdot$ & . & $\cdot$ & - & $\cdot$ & $\cdot$ & - &. & .75 \\
\hline $\mathbf{T i}$ & - & • & - & - & - & • & • & - & - & • & .89 \\
\hline Mn & - & 18.9 & 26.0 & - & - & 28.5 & 27.7 & - & - & 34.9 & 263 \\
\hline $\mathbf{P}_{0}$ & - & 11.8 & 14.5 & - & • & 11.8 & 228 & - & - & 13.4 & 9.88 \\
\hline $\mathbf{A}$ & • & - & - & - & - & $\cdot$ & • & • & - & - & .47 \\
\hline Co & 1.33 & - & - & 1.17 & .410 & - & . & 1.24 & .850 & 1.61 & 1.02 \\
\hline $\mathrm{Ni}$ & - & .620 & .440 & - & - & .680 & .70 & . & - & 830 & .790 \\
\hline $\mathrm{Cr}$ & - & .020 & .020 & - & - & .030 & .050 & - & - & .050 & .080 \\
\hline $\mathrm{C}$ & - & - & • & - & - & - & • & - & - & • & 206 \\
\hline M8 & - & - & - & - & - & - & - & - & - & - & 1.28 \\
\hline $\mathrm{Na}$ & - & - & - & - & - & - & . & - & . & - & - \\
\hline $\mathbf{K}$ & - & - & - & . & - & . & - & - & - & . & - \\
\hline $\mathbf{P}$ & • & • & • & . & - & • & • & - & . & • & 27 \\
\hline $\mathrm{H}_{2} \mathrm{O}$ & - & 35.1 & 34.4 & - & - & 35.9 & 33.7 & - & - & - & - \\
\hline
\end{tabular}

\begin{tabular}{|c|c|c|c|c|c|c|c|c|c|c|c|}
\hline As(ppm) & - & - & - & - & - & - & - & - & $\cdot$ & - & - \\
\hline Ba & • & - & $\cdot$ & - & - & - & - & - & $\cdot$ & - & - \\
\hline Cd & 4.0 & - & - & 3.0 & 5.0 & - & - & 4.0 & 6.0 & - & - \\
\hline Co & • & - & - & • & • & - & - & $\cdot$ & • & - & - \\
\hline C & - & $\cdot$ & $\cdot$ & $\cdot$ & - & $\cdot$ & $\cdot$ & $\cdot$ & $\cdot$ & - & - \\
\hline Mo & 1000 & - & - & 950 & 1100 & - & - & 860 & 810 & - & - \\
\hline $\mathbf{P b}$ & 1500 & - & - & 2000 & 1900 & - & - & 1900 & 2000 & - & - \\
\hline Sr & - & - & - & - & - & - & $\cdot$ & • & • & - & - \\
\hline $\mathbf{V}$ & - & - & - & - & - & - & - & - & - & - & - \\
\hline $\mathbf{Y}$ & - & - & - & $\cdot$ & • & - & - & $\cdot$ & - & - & $\cdot$ \\
\hline $2 n$ & 650 & • & • & 490 & 560 & • & • & 690 & 740 & • & 800 \\
\hline Semplo & Crust & Crust & Crust & Crust & Crest & Crust & Crust & Crust & Crust & Nodule & Crust \\
\hline type & - & • & • & • & • & • & • & • & - & - & • \\
\hline Partica & unknown & unknown & monown & unknown & nn'mown & unknown & unlonown & unknown & unknown & unknowa & average \\
\hline sempled & • & - & • & • & • & • & $\cdot$ & - & • & - & anelyzis \\
\hline Nuclens & • & - & • & - & - & - & $\cdot$ & • & - & • & $\cdot$ \\
\hline References & A303 & A321 & A321 & A303 & $A 303$ & A321 & A321 & $A 303$ & $A 303$ & A418 & A363 \\
\hline Sequencel & $3420007-01$ & $3420015-00$ & $3420039-00$ & $3420039-01$ & $3420100-00$ & $3420113-\infty$ & $3420113-01$ & $3430021-\infty 0$ & $3470359-00$ & $3470359-01$ & $3470359-02$ \\
\hline Latimde & 9.817 & 7.067 & 7317 & 7.317 & 900 & 5.002 & 5.002 & 8.317 & 7.455 & 7.455 & 7.455 \\
\hline Longituds & -170.983 & -171.700 & -175.467 & $-175 A 67$ & -179.333 & -173.012 & -173.012 & 176.417 & 136.407 & 136.407 & 136.407 \\
\hline Depth & 4875 & 5386 & 5190 & 5190 & 3091 & 5280 & 5280 & 5097 & 3555 & 3555 & 3555 \\
\hline Si(wts) & - & - & 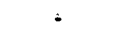 & - & - & - & $\cdot$ & . & • & - & - \\
\hline $\mathrm{Ti}$ & .69 & .93 & 98 & 53 & - & - & - & - & - & - & - \\
\hline $\mathbf{M n}$ & 18.2 & 18.6 & 19.0 & 18.0 & 226 & 19.3 & 20.7 & 17.2 & 21.6 & 18.1 & - \\
\hline $\mathbf{P}_{0}$ & 13.3 & 16.7 & 129 & 17.1 & 20.6 & 12.0 & 13.1 & 10.8 & 17.2 & 21.1 & - \\
\hline $\mathbf{A}$ & - & - & - & - & - & - & - & - & - & - & - \\
\hline Co & 500 & 510 & .560 & .690 & 380 & .540 & 580 & 370 & - & - & .480 \\
\hline $\mathbf{N i}$ & 860 & .470 & .830 & .710 & 340 & .630 & .680 & 1.040 & .380 & .200 & - \\
\hline $\mathrm{Cu}$ & .690 & .210 & .800 & .790 & .110 & .540 & 580 & .600 & .050 & .050 & - \\
\hline Ca & - & - & - & - & - & - & - & - & - & - & - \\
\hline $\mathbf{M g}$ & - & - & - & - & - & - & $\cdot$ & - & - & - & - \\
\hline $\mathrm{Na}$ & - & - & - & - & - & - & - & - & - & - & $\cdot$ \\
\hline $\mathbf{K}$ & - & - & - & - & - & - & $\cdot$ & - & - & - & - \\
\hline $\mathbf{P}$ & - & - & - & - & - & - & • & - & - & - & - \\
\hline $\mathrm{H}_{2} \mathrm{O}$ & - & - & - & - & - & 24.3 & 25.9 & - & - & - & - \\
\hline$A \approx(p p m)$ & • & - & - & - & - & - & - & - & - & - & - \\
\hline $\mathrm{Ba}$ & 1600 & 1900 & 2000 & 600 & - & - & - & - & - & - & - \\
\hline Cd & - & - & - & - & - & - & - & - & - & - & 5.0 \\
\hline Co & - & - & - & - & - & - & - & - & - & - & • \\
\hline$c$ & 6 & 4 & 2 & 2 & - & - & - & - & - & - & $\cdot$ \\
\hline Mo & 390 & 450 & 320 & 280 & - & - & - & - & - & - & 540 \\
\hline Pb & 500 & 200 & 100 & 700 & - & 600 & 600 & - & - & - & 100 \\
\hline Sr & • & • & • & • & - & $\cdot$ & - & - & - & - & $\cdot$ \\
\hline $\mathbf{V}$ & 390 & 600 & 460 & 370 & - & $\cdot$ & - & - & - & - & - \\
\hline $\mathbf{Y}$ & - & • & - & $\cdot$ & - & - & - & - & - & - & $\cdot$ \\
\hline $\mathbf{Z n}$ & - & - & - & - & • & 680 & 720 & - & • & • & 680 \\
\hline Sempopos & Nodule & Unknown & Nodule & Nodulo & Crust & Nodule & Nodule & Crust & Crust & Crust & Crust \\
\hline type & - & $\cdot$ & • & • & • & - & - & - & • & • & - \\
\hline Portica & quartar & fragments & balf & half & unknown & whole & whole & uniknown & fragments & fragments & uniknown \\
\hline sampled & 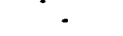 & 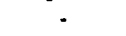 & - & - & - & • & • & - & • & • & - \\
\hline Nocleus & • & - & - & - & - & $\cdot$ & • & - & - & $\cdot$ & - \\
\hline Refarence & A240 & A240 & A240 & $A 240$ & A333 & A361 & A361 & A179 & A342 & $A 342$ & $A 303$ \\
\hline
\end{tabular}


Table 9. Composition of crusts from the Scripps Nodule Databank (cont'd).

\begin{tabular}{|c|c|c|c|c|c|c|c|c|c|c|c|}
\hline $\begin{array}{l}\text { Sequencell } \\
\text { Latitude } \\
\text { Langiterde } \\
\text { Depth }\end{array}$ & $\begin{array}{c}3470359-03 \\
7.455 \\
136.407 \\
3555 \\
\end{array}$ & $\begin{array}{c}3470363-00 \\
7.433 \\
133.715 \\
3869 \\
\end{array}$ & $\begin{array}{c}3470363-01 \\
7.433 \\
133.715 \\
3869 \\
\end{array}$ & $\begin{array}{c}3470366-00 \\
5.028 \\
130.400 \\
1600 \\
\end{array}$ & $\begin{array}{c}3530147-00 \\
.000 \\
73.033 \\
1728 \\
\end{array}$ & $\begin{array}{c}3540040-00 \\
5.600 \\
61.883 \\
2176 \\
\end{array}$ & $\begin{array}{c}3540041-00 \\
5567 \\
61.867 \\
2331 \\
\end{array}$ & $\begin{array}{c}3540066-00 \\
2.942 \\
60.057 \\
4042\end{array}$ & $\begin{array}{c}3540066-01 \\
2.942 \\
60.057 \\
4042\end{array}$ & $\begin{array}{c}3540066-02 \\
2.942 \\
60.057 \\
4042\end{array}$ & $\begin{array}{c}3540066-03 \\
2942 \\
60.057 \\
4042\end{array}$ \\
\hline$S \dot{s}(w t . \%)$ & - & - & - & - & - & $\cdot$ & - & 8.70 & 19.5 & 7.10 & 7.90 \\
\hline $\mathbf{T i}$ & - & - & - & - & - & .69 & so & .69 & 33 & 59 & .62 \\
\hline Mn & - & .07 & - & 6.00 & 8.61 & 13.8 & 16.6 & 16.5 & 730 & 175 & 16.7 \\
\hline Fo & - & 297 & - & 125 & 15.6 & 19.8 & 225 & 18.0 & 12.6 & 19.3 & 16.7 \\
\hline $\boldsymbol{N}$ & - & - & - & - & 1.91 & - & • & • & • & • & - \\
\hline$C_{0}$ & 350 & - & .030 & 260 & 260 & $A 10$ & 260 & 240 & .100 & 230 & .230 \\
\hline $\mathbf{N i}$ &. & .030 & - & .170 & .190 & .280 & 250 & .210 & .190 & 230 & 250 \\
\hline $\mathrm{Cu}$ & - & $A 20$ & - & .020 & .030 & .030 & .050 & .000 & .150 & .060 & .060 \\
\hline$a$ & - & - & - & - & 8.14 & • & - & • & - & - & $\therefore$ \\
\hline Mg & - & - & - & - & - & - & - & - & - & - & - \\
\hline $\mathrm{Na}$ & - & - & - & - & - & - & - & - & - & - & - \\
\hline $\mathbf{k}$ & - & - & - & - & - & - & - & - & - & - & - \\
\hline$\vec{P}$ & - & - & - & - & - & - & - & - & - & - & - \\
\hline $\mathrm{H}_{2} \mathrm{O}$ & - & - & - & - & - & - & - & - & - & - & - \\
\hline A (ppon) & - & - & - & - & - & - & - & - & - & - & - \\
\hline $\mathrm{Be}$ & - & - & - & - & - & 1800 & 1700 & - & - & - & - \\
\hline Cd & 4.0 & - & 8.0 & - & - & - & - & - & - & - & - \\
\hline co & • & - & . & - & . & - & . & - & - & - & - \\
\hline$a$ & $\cdot$ & - & • & - & - & 18 & 16 & 20 & 59 & 14 & 16 \\
\hline Mo & 450 & - & 23 & - & - & 420 & 430 & 650 & 560 & 750 & 810 \\
\hline $\mathbf{P b}$ & 100 & - & 100 & 900 & 1100 & $\infty \infty$ & 200 & 1200 & 200 & 700 & 700 \\
\hline Sr & • & - & • & $\cdot$ & • & - & - & - & - & • & • \\
\hline $\mathbf{v}$ & - & - & - & - & - & 690 & 670 & 820 & 760 & 940 & 910 \\
\hline $\mathbf{Y}$ & - & - & - & - & - & - & - & • & - & - & - \\
\hline $\mathbf{Z n}$ & 660 & - & 200 & 420 & 470 & - & - & 370 & 190 & 240 & 550 \\
\hline Stomplo & Crust & Crust & Coust & Crust & Crust & Crut & Coust & Crust & Crut & Crust & Crut \\
\hline type & - & - & - & • & • & - & - & - & - & • & - \\
\hline Partica & nnknown & fragments & unlonown & nnkonown & nalenown & fragmons & framents & topseide & underside & unlonown & topside \\
\hline somplod & $\cdot$ & . & - & $\cdot$ & - & - & 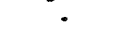 & 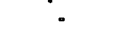 & - & • & $\because$ \\
\hline Nucleus & - & • & - & • & - & - & - & - & - & - & - \\
\hline Roferences & $A 303$ & $A 342$ & $A 303$ & A343 & A467 & $A 240$ & A240 & A217 & A217 & A217 & A217 \\
\hline $\begin{array}{l}\text { Sequenosill } \\
\text { Letitude }\end{array}$ & $\begin{array}{c}3540066-04 \\
2942\end{array}$ & $\begin{array}{c}3540066-27 \\
2.942\end{array}$ & $\begin{array}{c}3540066-28 \\
2.942\end{array}$ & $\begin{array}{c}3540066-29 \\
2942\end{array}$ & $\begin{array}{c}3540066-30 \\
2.942\end{array}$ & $\begin{array}{c}3540066-31 \\
2.942\end{array}$ & $\begin{array}{c}3540066-32 \\
2.942\end{array}$ & $\begin{array}{c}3540070-00 \\
2.967\end{array}$ & $\begin{array}{c}3540070-01 \\
2967\end{array}$ & $\begin{array}{c}3540070-02 \\
2.967\end{array}$ & $\begin{array}{c}3540070-03 \\
2967\end{array}$ \\
\hline Langitude & 60.057 & 60.057 & 60.057 & 60.057 & 60.057 & 60.057 & 60.057 & 60.047 & 60.047 & 60.047 & 60.047 \\
\hline Depth & 4042 & 4042 & 4042 & 4042 & 4042 & 4042 & 4042 & 4093 & 4093 & 4093 & 4093 \\
\hline$s i(w L \%)$ & 14.8 & - & . & - &. & . & . & 8.10 & 10.1 & 6.40 & 9.50 \\
\hline $\mathrm{Ti}$ & 28 & .59 & .71 & .44 & .61 & 54 & .56 & .43 & 55 & $A 5$ & .78 \\
\hline Mn & 113 & 173 & 15.5 & 21.4 & 17.1 & 17.7 & 16.7 & 20.9 & 15.1 & 16.0 & 18.7 \\
\hline Fo & 13.9 & 168 & 18.1 & 12.6 & 15.8 & 14.9 & 16.9 & 13.1 & 15.4 & 17.0 & 14.7 \\
\hline $\mathbf{N}$ & - & 1.14 & 1.69 & 1.55 & 156 & 1.44 & 1.90 & . & - & - & . \\
\hline Co & .150 & .170 & .180 & .100 & .140 & .110 & .160 & .160 & .260 & .220 & 360 \\
\hline $\mathrm{Ni}$ & .210 & 350 & .270 & 830 & $A 20$ & .480 & 340 & 960 & .350 & 280 & 350 \\
\hline $\mathrm{Cu}$ & .160 & .140 & .120 & 310 & 2200 & .170 & 200 & .220 & .100 & .050 & .060 \\
\hline C & - & 1.90 & 1.86 & 1.70 & 1.61 & 1.87 & 1.55 & - & - & - & • \\
\hline Ms & - & 1.05 & 1.11 & 1.30 & 1.17 & 1.15 & 1.13 & - & - & - & - \\
\hline $\mathrm{Na}$ & - & • & - & - & - & - & - & - & - & - & - \\
\hline $\mathbf{x}$ & - & - & - & - & - & - & - & - & - & - & - \\
\hline $\mathbf{P}$ & - & - & - & - & . & - & - & - & - & - & - \\
\hline $\mathrm{H}_{2} \mathrm{O}$ & - & - & - & - & - & - & - & - & : & - & - \\
\hline Ax(ppon) & - & - & - & - & . & - & - & . & - & - & - \\
\hline Ba & - & - & - & - & - & • & • & . & - & - & - \\
\hline Cd & - & 5.0 & 4.0 & 11.0 & 6.0 & 6.0 & 6.0 & - & - & - & - \\
\hline Co & - & - & - & - & - & - & - & - & - & - & - \\
\hline $\mathrm{C}$ & 27 & - & - & - & - & - & - & 25 & 18 & 9 & 17 \\
\hline Mo & 530 & • & - & - & - & • & • & 500 & 470 & 550 & 360 \\
\hline $\mathbf{P b}$ & 500 & 1000 & 1100 & 800 & 1100 & 900 & 1200 & 700 & 700 & 700 & 700 \\
\hline $\mathbf{S r}$ & - & - & - & - & - & - & - & - & - & - & - \\
\hline $\mathbf{v}$ & 650 & - & - & - & - & - & - & 820 & 840 & 890 & 690 \\
\hline $\mathbf{Y}$ & - & - & - & - & - & • & - & $\cdot$ & - & - & - \\
\hline $\mathbf{Z n}$ & 250 & 660 & 580 & 940 & 700 & 680 & 660 & 660 & 490 & 410 & 330 \\
\hline Semplo & Crust & Cruat & Crust & Crust & Crunt & Crust & Crust & Crust & Crust & Crust & Crust \\
\hline typo & - & - & - & • & - & • & - & • & - & • & - \\
\hline Partion & underside & unkmows & unknown & unlonown & unionown & unknown & nolonown & topside & underzides & unknown & unionown \\
\hline rempled & $\cdot$ & - & - & - & $\cdot$ & • & - & 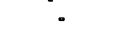 & - & - & - \\
\hline Nuclous & - & • & - & - & - & - & • & - & - & - & - \\
\hline Refarencos & A217 & A433 & A433 & A433 & A433 & A433 & A433 & A217 & A217 & A217 & A217 \\
\hline
\end{tabular}


Table 9. Composition of crusts from the Scripps Nodule Databank (cont'd).

\begin{tabular}{|c|c|c|c|c|c|c|c|c|c|c|c|}
\hline $\begin{array}{l}\text { Sequencell } \\
\text { Latitude } \\
\text { Langitude } \\
\text { Depth } \\
\end{array}$ & $\begin{array}{c}3540072-01 \\
2.790 \\
60.042 \\
3816 \\
\end{array}$ & $\begin{array}{c}3540072-10 \\
2.790 \\
60.042 \\
3816 \\
\end{array}$ & $\begin{array}{c}3540072-11 \\
2.790 \\
60.042 \\
3816 \\
\end{array}$ & $\begin{array}{c}3540072-12 \\
2.790 \\
60.042 \\
3816 \\
\end{array}$ & $\begin{array}{c}3540072-13 \\
2790 \\
60.042 \\
3816 \\
\end{array}$ & $\begin{array}{c}3540072-14 \\
2.790 \\
60.042 \\
3816\end{array}$ & $\begin{array}{c}3540072-19 \\
2.790 \\
60.042 \\
3816\end{array}$ & $\begin{array}{c}3540072-20 \\
2790 \\
60.042 \\
3816 \\
\end{array}$ & $\begin{array}{c}3540072-21 \\
2.790 \\
60.042 \\
3816 \\
\end{array}$ & $\begin{array}{c}3540072-22 \\
2.790 \\
60.042 \\
3816 \\
\end{array}$ & $\begin{array}{c}3540072-23 \\
2.790 \\
60.042 \\
3816 \\
\end{array}$ \\
\hline Si(wt.\%) & 13.4 & 780 & 5.90 & 7.10 & 8.90 & 6.70 & - & $\cdot$ & • & • & $\cdot$ \\
\hline $\mathbf{T i}$ & .49 & 1.00 & 90 & 1.02 & .88 & 35 & .62 & .71 & 90 & 96 & .82 \\
\hline Mn & 11.4 & 16.0 & 18.5 & 17.2 & 15.6 & 16.9 & 16.2 & 15.4 & 15.9 & 15.8 & 17.7 \\
\hline $\mathrm{Fo}_{0}$ & 15.4 & 153 & 15.2 & 16.0 & 15.2 & 16.1 & 18.8 & 18.8 & 16.6 & 14.7 & 148 \\
\hline $\mathbf{A l}$ & • & • & • & • & • & • & 1.58 & 1.70 & 2.11 & 1.88 & 2.27 \\
\hline Co & $\mathbf{2 3 0}$ & 530 & .064 & .080 & $A S O$ & .190 & .140 & .180 & 310 & .270 & .220 \\
\hline $\mathbf{N i}$ & 280 & 430 & 520 & 100 & 390 & .400 & 200 & .210 & .270 & .350 & 550 \\
\hline Cr & .120 & .080 & .100 & .040 & .050 & .140 & .150 & .110 & .100 & .130 & .210 \\
\hline $\mathrm{Ca}$ & • & • & • & • & • & - & 1.58 & 1.72 & 1.89 & 2.62 & 1.54 \\
\hline Ms & - & - & - & - & - & - & 1.11 & 1.04 & 1.15 & 1.23 & 1.52 \\
\hline $\mathrm{Ne}$ & $\cdot$ & - & - & - & - & - & • & • & - & • & • \\
\hline $\mathbf{K}$ & - & - & - & - & - & - & - & - & - & - & - \\
\hline $\mathbf{P}$ & - & - & - & - & - & - & - & - & - & - & - \\
\hline $\mathrm{H}_{2} \mathrm{O}$ & $\cdot$ & $\cdot$ & $\cdot$ & $\cdot$ & • & - & - & • & - & • & - \\
\hline$A s$ (ppm) & $\cdot$ & - & - & $\cdot$ & $\cdot$ & - & $\cdot$ & - & - & • & - \\
\hline $\mathrm{Be}$ & - & - & - & - & - & - & - & - & - & $\cdot$ & $\cdot$ \\
\hline $\mathrm{Cd}$ & - & - & - & - & - & - & 10.0 & 5.0 & 5.0 & 6.0 & 6.0 \\
\hline co & $\cdot$ & - & - & - & - & - & • & $\cdot$ & $\cdot$ & $\cdot$ & - \\
\hline$c$ & 31 & 25 & 13 & 11 & 18 & 15 & - & - & - & - & - \\
\hline Mo & 73 & 550 & 570 & 400 & 470 & 480 & $\cdot$ & - & - & $\cdot$ & • \\
\hline $\mathbf{P b}$ & 1100 & 1500 & 1100 & 1000 & 1000 & 700 & 900 & 1100 & 1100 & 1200 & 1000 \\
\hline St & - & • & • & • & • & • & • & - & • & • & • \\
\hline $\mathbf{v}$ & 620 & 1000 & 860 & 810 & 860 & 1000 & - & - & - & - & - \\
\hline $\mathbf{Y}$ & - & - & • & - & • & • & - & - & $\cdot$ & • & $\cdot$ \\
\hline $\mathbf{Z n}$ & 330 & 400 & 410 & 440 & 390 & 510 & 540 & 540 & 530 & 570 & 680 \\
\hline Semplo & Cruat & Crest & Crust & Cust & Crust & Crust & Crust & Crust & Crust & Crust & Crest \\
\hline typo & - & • & - & - & $\bullet$ & - & • & - & - & • & - \\
\hline Partion & unlonown & unknown & unknown & unknown & unknown & monown & unknown & unknown & unitoown & unknown & unkrown \\
\hline sumplod & - & - & • & - & • & - & - & - & - & • & - \\
\hline Nuclous & Clay & $\cdot$ & - & - & - & - & - & • & • & • & - \\
\hline References & A217 & A217 & A217 & A217 & A217 & A217 & A433 & A433 & A433 & A433 & A433 \\
\hline Sequencell & $3540072-24$ & $3540088-01$ & $3540088-02$ & $3540088-03$ & $3540088-04$ & $3540088-05$ & $3540088-06$ & $3540088-07$ & $3540088-08$ & $3540088-09$ & $3540088-10$ \\
\hline Latitude & 2.790 & 6.872 & 6.872 & 6.872 & 6.872 & 6.872 & 6.872 & 6.872 & 6.872 & 6.872 & 6.872 \\
\hline Langinde & 60.042 & 62.893 & 62.893 & 62893 & 62893 & 62.893 & 62893 & 62.893 & 62.893 & 62893 & 62.893 \\
\hline Dopth & 3816 & 3640 & 3640 & 3640 & 3640 & 3640 & 3640 & 3640 & 3640 & 3640 & 3640 \\
\hline Si(wt.\%) & - & - & - & - & - & $\cdot$ & - & - & - & $\cdot$ & - \\
\hline $\mathrm{Ti}$ & 85 & - & • & .44 & .40 & 52 & 51 & .28 & .41 & 58 & 53 \\
\hline $\mathbf{M n}$ & 14.4 & 17.2 & 18.1 & 163 & 15.7 & 17.1 & 13.4 & 243 & 18.9 & • & 11.0 \\
\hline Po & 153 & 21.7 & 18.3 & 20.2 & 19.8 & 20.0 & 19.4 & 9.40 & 17.0 & 23.0 & 23.2 \\
\hline $\mathbf{A}$ & 1.91 & 1.14 & 1.55 & 1.17 & 1.01 & 1.05 & 1.44 & 1.88 & 1.51 & 1.71 & 1.73 \\
\hline$C_{0}$ & 200 & 370 & .210 & .180 & .170 & .170 & .170 & .180 & .210 & .180 & .210 \\
\hline $\mathbf{N i}$ & 270 & 250 & .270 & .210 & .170 & .200 & .170 & .380 & .440 & .200 & .210 \\
\hline $\mathrm{Cu}$ & .110 & .090 & .100 & .050 & .040 & .050 & .060 & 220 & .190 & .100 & .110 \\
\hline $\mathrm{C}_{2}$ & 1.75 & 1.76 & 1.68 & 1.79 & 1.82 & 1.83 & 1.68 & 1.16 & 1.10 & 1.46 & 1.28 \\
\hline $\mathrm{Mg}$ & 1.20 & - & - & 1.00 & 1.01 & 1.00 & 95 & 204 & 1.72 & 1.32 & 1.15 \\
\hline $\mathrm{Na}$ & $\cdot$ & - & - & • & • & - & • & - & • & • & • \\
\hline $\mathbf{K}$ & - & - & - & - & - & - & - & - & - & - & - \\
\hline $\mathbf{P}$ & - & - & - & - & - & - & - & $\because$ & - & - & - \\
\hline $\mathrm{H}_{2} \mathrm{O}$ - & - & - & - & - & $\cdot$ & $\cdot$ & - & $\cdot$ & - & $\cdot$ & - \\
\hline As(ppm) & - & - & - & - & - & - & - & - & - & - & - \\
\hline Ba & - & - & - & - & - & - & $\cdot$ & • & • & - & $\cdot$ \\
\hline Cd & 4.0 & - & $\cdot$ & 5.0 & 6.0 & 6.0 & 6.0 & 5.0 & 6.0 & 5.0 & 5.0 \\
\hline Co & - & - & - & - & - & - & • & • & - & - & - \\
\hline$c$ & - & - & - & - & - & - & - & - & - & - & - \\
\hline Mo & • & • & - & • & • & • & • & - & - & • & • \\
\hline Pb & 1000 & 1300 & 1000 & 1000 & 1000 & 900 & 1100 & 500 & 800 & 1300 & 1200 \\
\hline Sz & - & - & - & - & - & $\cdot$ & - & $\cdot$ & - & - & - \\
\hline $\mathrm{v}$ & - & - & - & - & - & - & - & - & - & - & - \\
\hline $\mathbf{Y}$ & $\cdot$ & • & - & $\cdot$ & $\cdot$ & $\cdot$ & $\therefore$ & - & $\dot{0}$ & - & - \\
\hline $\mathbf{Z n}$ & 490 & 490 & 550 & 510 & 500 & 500 & 530 & 550 & 720 & 690 & 690 \\
\hline Samplo & Crust & Crust & Crust & Crust & Crust & Coust & Crust & Crat & Crust & Cout & Crust \\
\hline type & $\cdot$ & $\cdot$ & $\cdot$ & $\cdot$ & $\cdot$ & $\cdot$ & $\cdot$ & - & - & $\cdot$ & - \\
\hline Partion & unknown & unkonown & uninown & unionown & unknowa & uninown & unknown & unknown & unknown & unknown & unknown \\
\hline sampled & - & - & - & - & - & - & - & - & - & - & - \\
\hline Nuclous & - & - & • & • & - & - & - & - & - & - & - \\
\hline Referenco & A433 & A433 & A433 & A433 & A433 & A433 & A433 & A433 & A433 & A433 & A433 \\
\hline
\end{tabular}


Table 9. Composition of crusts from the Scripps Nodule Databank (cont'd).

\begin{tabular}{|c|c|c|c|c|c|c|c|c|c|c|c|}
\hline 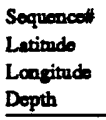 & $\begin{array}{c}3540088-11 \\
6.872 \\
62893 \\
3640 \\
\end{array}$ & $\begin{array}{c}3540088-12 \\
6.872 \\
62.893 \\
3640 \\
\end{array}$ & $\begin{array}{c}3540088-13 \\
6.872 \\
62893 \\
3640 \\
\end{array}$ & $\begin{array}{c}3540088-14 \\
6.872 \\
62893 \\
3640 \\
\end{array}$ & $\begin{array}{c}3540088-15 \\
6.872 \\
62.893 \\
3640 \\
\end{array}$ & $\begin{array}{c}3540088-16 \\
6.872 \\
62893 \\
3640\end{array}$ & $\begin{array}{c}3540088-17 \\
6.872 \\
62893 \\
3640\end{array}$ & $\begin{array}{c}3540088-18 \\
6.872 \\
62893 \\
3640 \\
\end{array}$ & $\begin{array}{c}3540088-19 \\
6.872 \\
62.893 \\
3640 \\
\end{array}$ & $\begin{array}{c}3540088-20 \\
6.872 \\
62893 \\
3640 \\
\end{array}$ & $\begin{array}{c}3540088-21 \\
6.872 \\
62893 \\
3640 \\
\end{array}$ \\
\hline Sitwt.\%) & $\cdot$ & . & $\cdot$ & $\cdot$ & - & $\cdot$ & $\cdot$ & $\cdot$ & $\cdot$ & $\cdot$ & $\cdot$ \\
\hline $\mathbf{T i}$ & AO & .63 & .60 & 59 & .42 & .63 & 56 & .70 & .65 & .61 & AB \\
\hline Mn & 21.2 & 164 & 9.80 & 10.8 & 21.0 & 11.8 & 11.6 & 15.8 & 13.7 & 15.7 & 16.6 \\
\hline $\mathrm{Fe}$ & 13.1 & 19.2 & 23.4 & 225 & 173 & 24.1 & 23.3 & 195 & 21.0 & 20.4 & 20.2 \\
\hline Al & 1.85 & 1.81 & 1.96 & 2.19 & 1.70 & 1.43 & 1.57 & 1.10 & 1.48 & 235 & 94 \\
\hline Co & 210 & 320 & .200 & .240 & 210 & .200 & .180 & 200 & .230 & .220 & .200 \\
\hline $\mathbf{N i}$ & $A 20$ & 540 & .170 & .200 & $A 30$ & .220 & .200 & .230 & .170 & 230 & .200 \\
\hline cu & .220 & .210 & .100 & .110 & .160 & .090 & .110 & .040 & .070 & .100 & .040 \\
\hline ce & 1.02 & 1.10 & 1.06 & 1.23 & 131 & 1.26 & 1.07 & 1.70 & 1.71 & 1.29 & 1.67 \\
\hline Mg & 1.88 & 1.61 & 1.09 & 1.25 & 1.46 & 1.02 & 1.01 & 1.13 & 1.02 & 1.20 & 1.07 \\
\hline $\mathrm{Na}$ & - & $\cdot$ & • & • & - & • & • & $\cdot$ & • & • & • \\
\hline $\mathbf{K}$ & - & $\cdot$ & - & - & - & - & - & . & . & - & - \\
\hline $\mathbf{P}$ & - & - & - & - & - & - & - & - & - & - & - \\
\hline $\mathrm{H}_{2} \mathrm{O}$ & $\cdot$ & $\cdot$ & • & - & $\cdot$ & • & $\cdot$ & $\cdot$ & • & - & - \\
\hline Ax(ppm) & - & - & - & - & - & - & - & - & - & - & - \\
\hline Ba & - & - & • & • & - & - & • & $\cdot$ & • & • & - \\
\hline Cd & 6.0 & 8.0 & 6.0 & 7.0 & 9.0 & 6.0 & 7.0 & 9.0 & 7.0 & 6.0 & 8.0 \\
\hline$c_{0}$ & . & - & - & - & - & - & $\cdot$ & - & - & - & - \\
\hline Cr & - & - & - & - & - & - & . & - & - & - & - \\
\hline Mo & - & . & - & . & . & - & - & . & - & . & - \\
\hline Pb & 700 & 1100 & 1100 & 1200 & 900 & 1200 & 1200 & 1000 & 700 & 1000 & 1100 \\
\hline St & - & $\cdot$ & • & • & • & $\cdot$ & • & $\cdot$ & $\cdot$ & • & - \\
\hline v & - & - & - & . & - & - & . & . & - & - & - \\
\hline $\mathbf{Y}$ & - & • & - & • & - & • & - & - & . & - & . \\
\hline $\mathbf{z n}$ & 580 & 820 & 620 & 590 & 650 & 710 & 670 & 500 & 490 & 500 & 520 \\
\hline Samplo & Crost & Crust & Crest & Court & Crust & Crust & Court & Crust & Crest & Court & Court \\
\hline type & • & • & • & -. & - & • & • & • & • & • & - \\
\hline Portion & untorown & unlown & mown & unknown & unknown & minown & unkown & untrown & mbmown & unknown & untown \\
\hline semplod & • & • & • & - & - & - & • & - & - & • & - \\
\hline Nucleus & - & - & - & - & . & - & - & . & - & - & • \\
\hline Refecrenos & A433 & A433 & $\mathbf{A 4 3 3}$ & A433 & A433 & A433 & A433 & A433 & A433 & A433 & A433 \\
\hline Sequencoll & $3540088-22$ & $3540088-23$ & $3540088-24$ & $3540088-25$ & $3540088-26$ & $3540088-27$ & $3540088-28$ & $3540088-29$ & $3540088-30$ & $3540088-31$ & $3540088-32$ \\
\hline Letitude & 6.872 & 6.872 & 6.872 & 6.872 & 6.872 & 6.872 & 6.872 & 6.872 & 6.872 & 6.872 & 6.872 \\
\hline Longitude & 62893 & 62.893 & 62.893 & 62.893 & 62.893 & 62.893 & 62.893 & 62.893 & 62.893 & 62.893 & 62893 \\
\hline Depth & 3640 & 3640 & 3640 & 3640 & 3640 & 3640 & 3640 & 3640 & 3640 & 3640 & 3640 \\
\hline Si(wt.\%) & - & - & - & - & - & - & $\cdot$ & $\cdot$ & $\cdot$ & $\cdot$ & $\cdot$ \\
\hline $\mathrm{Ti}$ & $A 8$ & .49 & .60 & 52 & .47 & 47 & .46 & .83 & 20 & .43 & 57 \\
\hline Mn & 15.2 & 17.0 & 15.3 & 14.9 & 123 & 122 & 13.2 & 14.7 & 224 & 20.2 & 13.0 \\
\hline Po & 20.3 & 193 & 20.4 & 21.6 & 19.7 & 18.6 & 203 & 22.1 & 8.60 & 15.4 & 22.1 \\
\hline A & 1.14 & 1.01 & 133 & 1.45 & 1.54 & 299 & . & .98 & 1.77 & 1.73 & 1.41 \\
\hline$c_{0}$ & .170 & .150 & .190 & .180 & .170 & .170 & .190 & 270 & .140 & 210 & .190 \\
\hline $\mathbf{N i}$ & .160 & .190 & .180 & .180 & .130 & .210 & .160 & .170 & .360 & 500 & .240 \\
\hline ca & .040 & .060 & .070 & .080 & .070 & .110 & .100 & .070 & .230 & .230 & .110 \\
\hline$c_{2}$ & 254 & 1.76 & 154 & 221 & 1.80 & 3.82 & 1.80 & 1.70 & 1.04 & 1.35 & 1.18 \\
\hline Ms & 1.05 & 1.04 & 1.02 & • & 1.01 & 1.22 & 1.07 & 1.04 & 1.90 & 1.61 & 1.13 \\
\hline Na & - & - & - & - & - & • & - & - & - & - & - \\
\hline $\mathbf{K}$ & . & - & . & . & . & . & - & - & . & . & . \\
\hline P & - & - & . & - & - & . & - & . & - & . & - \\
\hline $\mathrm{H}_{2} \mathrm{O}-$ & - & . & $\cdot$ & - & - & - & - & - & - & - & - \\
\hline Ax(ppm) & $\because$ & - & - & . & . & - & - & - & . & - & . \\
\hline $\mathrm{Ba}$ & $\because$ & $\cdot$ & - & $\cdot$ & - & . & . & - & . & - & - \\
\hline Cd & 6.0 & 5.0 & 5.0 & 5.0 & 6.0 & 5.0 & 5.0 & 5.0 & 5.0 & 5.0 & 5.0 \\
\hline co & - & $\cdot$ & - & - & • & $\cdot$ & - & - & - & . & - \\
\hline Cr & - & - & - & . & . & . & . & - & . & . & . \\
\hline Mo & • & - & - & • & • & . & . & - & . & . & . \\
\hline $\mathbf{P b}$ & 1000 & 900 & 1200 & 1100 & 1100 & 800 & 1000 & 1100 & 300 & 800 & 1100 \\
\hline Sx & • & - & • & - & • & - & - & - & - & • & - \\
\hline v & . & . & . & . & - & . & . & . & - & . & . \\
\hline $\mathbf{Y}$ & . & . & - & - & . & - & - & . & - & . & . \\
\hline Zn & 510 & 440 & 460 & 320 & 500 & 480 & 470 & 500 & 300 & 690 & 720 \\
\hline Samplos & Coust & Crust & Coust & Court & Crust & Crust & Court & Crust & Crust & Crust & Crust \\
\hline type & • & - & • & • & • & • & • & • & • & • & • \\
\hline Partion & untorown & unknown & mbown & unkrown & unknown & monown & unknown & unknown & monown & unkmown & untrown \\
\hline sumpled & - & • & • & • & • & - & - & $\cdot$ & $\cdot$ & - & • \\
\hline Nuclous & . & - & . & - & - & - & - & . & - & . & - \\
\hline Referenoses & A433 & A433 & $\mathbf{A 4 3 3}$ & A433 & A433 & A433 & A433 & A433 & A433 & A433 & A433 \\
\hline
\end{tabular}


Table 9. Composition of crusts from the Scripps Nodule Databank (cont'd).

\begin{tabular}{|c|c|c|c|c|c|c|c|c|c|c|c|}
\hline $\begin{array}{l}\text { Sequencell } \\
\text { Lativudo } \\
\text { Longitude } \\
\text { Depth }\end{array}$ & $\begin{array}{c}3540088-33 \\
6.872 \\
62.893 \\
3640 \\
\end{array}$ & $\begin{array}{c}3540088-34 \\
6.872 \\
62.893 \\
3640 \\
\end{array}$ & $\begin{array}{c}3540088-35 \\
6.872 \\
62.893 \\
3640 \\
\end{array}$ & $\begin{array}{c}3540088-36 \\
6.872 \\
62893 \\
3640 \\
\end{array}$ & $\begin{array}{c}3540088-37 \\
6.872 \\
62.893 \\
3640 \\
\end{array}$ & $\begin{array}{c}3540088-38 \\
6.872 \\
62.893 \\
3640 \\
\end{array}$ & $\begin{array}{c}3540088-39 \\
6.872 \\
62893 \\
3640 \\
\end{array}$ & $\begin{array}{c}3540088-40 \\
6.872 \\
62.893 \\
3640 \\
\end{array}$ & $\begin{array}{c}3540088-41 \\
6.872 \\
62.893 \\
3640 \\
\end{array}$ & $\begin{array}{c}3540088-42 \\
6.872 \\
62.893 \\
3640 \\
\end{array}$ & $\begin{array}{c}3540088-43 \\
6872 \\
62.893 \\
3640 \\
\end{array}$ \\
\hline Si(wt.\%) & - & - & $\cdot$ & - & - & 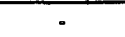 & - & $\cdot$ & - & . & . \\
\hline $\mathbf{T i}$ & 55 & .46 & 34 & $A 1$ & .58 & 49 & $A 7$ & .47 & 52 & .45 & so \\
\hline Mn & 11.0 & 9.50 & 20.5 & 21.9 & 10.7 & 1.00 & 14.6 & 15.4 & 125 & 12.8 & 9.10 \\
\hline Po & 228 & 20.3 & 10.6 & 13.5 & 209 & 23.0 & 18.6 & 18.7 & 222 & 18.9 & 17.1 \\
\hline $\mathbf{A}$ & 1.71 & 2.13 & 1.81 & 1.70 & 1.77 & 1.79 & 1.06 & 1.12 & 1.61 & 2.22 & 3.79 \\
\hline Co & .180 & .160 & .190 & .200 & .180 & .160 & .160 & .160 & .160 & .170 & .080 \\
\hline $\mathbf{N i}$ & .120 & .160 & .500 & 490 & .220 & .200 & .170 & .220 & .140 & .200 & .170 \\
\hline Cr & .190 & .110 & .260 & 240 & .120 & .130 & .040 & .070 & .070 & .100 & .110 \\
\hline C. & 1.40 & 142 & 99 & 1.13 & 1.27 & 1.27 & 201 & • & 1.60 & 1.49 & 1.39 \\
\hline $\mathbf{M}_{8}$ & 1.04 & 1.03 & 1.97 & 1.68 & 1.09 & 1.11 & 1.02 & 1.06 & 1.03 & 1.20 & 1.23 \\
\hline $\mathrm{Na}$ & - & - & - & - & - & - & - & - & - & - & - \\
\hline $\mathbf{K}$ & - & - & - & - & - & - & - & - & - & - & - \\
\hline $\mathbf{P}$ & - & - & - & - & - & - & - & - & - & - & - \\
\hline $\mathrm{H}_{2} \mathrm{O}$ & - & - & - & $\cdot$ & - & - & - & $\cdot$ & $\cdot$ & - & - \\
\hline$A r(p p m)$ & - & - & - & - & - & - & - & - & - & - & - \\
\hline $\mathrm{Ba}$ & • & - & - & - & - & - & - & - & - & - & - \\
\hline Cd & 4.0 & 3.0 & 6.0 & 5.0 & 4.0 & 4.0 & 6.0 & 3.0 & 3.0 & 4.0 & 4.0 \\
\hline Ce & - & - & - & - & - & - & - & - & $\cdot$ & • & - \\
\hline $\mathrm{Cr}$ & - & . & - & - & - & - & - & - & - & - & - \\
\hline Mo & • & • & $\cdot$ & $\cdot$ & • & • & - & • & $\bullet$ & • & - \\
\hline $\mathbf{P b}$ & 1200 & 1200 & 600 & 700 & 1100 & 1200 & 900 & 900 & 1100 & 900 & 500 \\
\hline Sr & - & - & $\cdot$ & - & - & - & - & - & - & - & - \\
\hline $\mathbf{v}$ & - & - & - & - & - & - & - & - & - & - & - \\
\hline $\mathbf{Y}$ & - & - & - & - & • & - & - & - & • & - & - \\
\hline $2 n$ & 1000 & 620 & 600 & 630 & 660 & 700 & 470 & 510 & 490 & 470 & 430 \\
\hline Semple & Crust & Crest & Crest & Crust & Crast & Crust & Crust & Crust & Const & Crust & Crust \\
\hline type & - & - & - & • & - & $\cdot$ & - & • & • & • & - \\
\hline Partion & unlonown & unkenown & monknown & unknown & unkrown & untnown & unknown & unknown & unknown & unknown & unknown \\
\hline semplod & • & - & - & - & 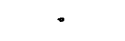 & - & - & - & - & - & - \\
\hline Nuclous & - & - & - & • & - & - & - & - & - & - & - \\
\hline References & A433 & A433 & A433 & A433 & A433 & A433 & A433 & A433 & A433 & A433 & A433 \\
\hline Sequencell & $3540090-01$ & $3540090-02$ & $3550004-\infty$ & $3550004-01$ & 355000402 & 355000403 & $3550004-04$ & $3550004-05$ & $3550004-06$ & $3550004-07$ & 355000408 \\
\hline Letitudo & 5.010 & 5.010 & 2.755 & 2.755 & 2755 & 2.755 & 2.755 & 2755 & 2755 & 2755 & 2755 \\
\hline Langitude & 61548 & 61548 & 59.903 & 59.903 & 59.903 & 59.903 & 59.903 & 59.903 & 59.903 & 59.903 & 59.903 \\
\hline Depth & 3000 & 3000 & 3975 & 3975 & 3975 & 3975 & 3975 & 3975 & 3975 & 3975 & 3975 \\
\hline Si(wt.\%) & - & $\cdot$ & 7.80 & 4.30 & 9.90 & 11.4 & 5.90 & 6.60 & 8.50 & 15.7 & 8.30 \\
\hline $\mathrm{Ti}$ & - & - & .80 & 99 & .71 & .61 & 1.02 & 1.16 & 52 & 37 & 1.10 \\
\hline Mn & 18.1 & 13.6 & 14.1 & 16.5 & 13.4 & 13.1 & 17.0 & 15.6 & 14.1 & 9.10 & 15.6 \\
\hline Po & 22.3 & 20.4 & 14.1 & 15.2 & 13.1 & 13.7 & 14.2 & 15.2 & 14.7 & 10.2 & 14.2 \\
\hline $\mathbf{A}$ & $A 4$ & 1.60 & - & - & - & - & 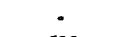 & - & - & - & - \\
\hline Co & .180 & .160 & .400 & 490 & .270 & .250 & 590 & 560 & .180 & .120 & 520 \\
\hline $\mathbf{N i}$ & 230 & .190 & .310 & 280 & 230 & .280 & 350 & .280 & .170 & .400 & 310 \\
\hline $\mathrm{Cr}$ & .030 & .050 & .060 & .050 & .060 & .070 & .060 & .030 & .040 & .110 & .040 \\
\hline c. & 2.19 & 2.26 & - & - & • & • & - & - & - & - & - \\
\hline $\mathbf{M}_{8}$ & - & - & - & - & - & - & - & . & - & - & - \\
\hline $\mathrm{Na}$ & - & - & - & - & - & - & - & - & - & - & - \\
\hline $\mathbf{K}$ & - & - & - & - & - & - & - & . & - & - & - \\
\hline P & - & - & - & - & - & - & - & - & - & - & - \\
\hline $\mathrm{H}_{2} \mathrm{O}$ & - & - & - & - & - & - & - & $\cdot$ & - & - & - \\
\hline As(ppm) & . & - & - & - & . & - & - & - & - & . & - \\
\hline $\mathrm{Ba}$ & - & - & - & - & - & - & - & - & - & - & - \\
\hline Cd & - & - & - & - & - & - & - & - & - & - & - \\
\hline C. & - & - & - & - & - & - & - & - & - & - & - \\
\hline $\mathrm{Cr}$ & - & - & 19 & 12 & 16 & 23 & 12 & 10 & 14 & 46 & 12 \\
\hline Mo & • & - & 350 & 380 & 330 & 280 & 430 & 320 & 420 & 204 & 410 \\
\hline Pb & 1200 & 1000 & 700 & 800 & 700 & 600 & 1000 & 800 & 700 & 300 & 700 \\
\hline Sr & - & - & - & - & - & - & - & - & • & • & - \\
\hline $\mathbf{v}$ & - & - & 670 & 820 & 610 & 580 & 820 & 650 & 700 & 400 & 690 \\
\hline $\mathbf{Y}$ & - & - & - & • & - & - & - & - & $\cdot$ & - & $\cdot$ \\
\hline $\mathbf{Z n}$ & 510 & 560 & 130 & 150 & 250 & 200 & 300 & 230 & 250 & 230 & 200 \\
\hline Semplo & Cust & Crost & Crust & Crust & Crust & Crut & Cust & Crust & Crust & Const & Crust \\
\hline typo & - & - & • & • & • & - & - & • & • & - & • \\
\hline Partion & unknown & unknown & unknown & unknown & unknown & unknown & unknown & unimown & unimown & enknown & unknown \\
\hline sempled & • & - & - & $\cdot$ & • & • & $\cdot$ & $\cdot$ & • & • & • \\
\hline Nuclous & - & - & - & - & • & - & - & - & - & • & • \\
\hline Roference & A433 & A433 & A217 & A217 & A217 & A217 & A217 & A217 & A217 & A217 & $A 217$ \\
\hline
\end{tabular}


Table 9. Composition of crusts from the Scripps Nodule Databank (cont'd).

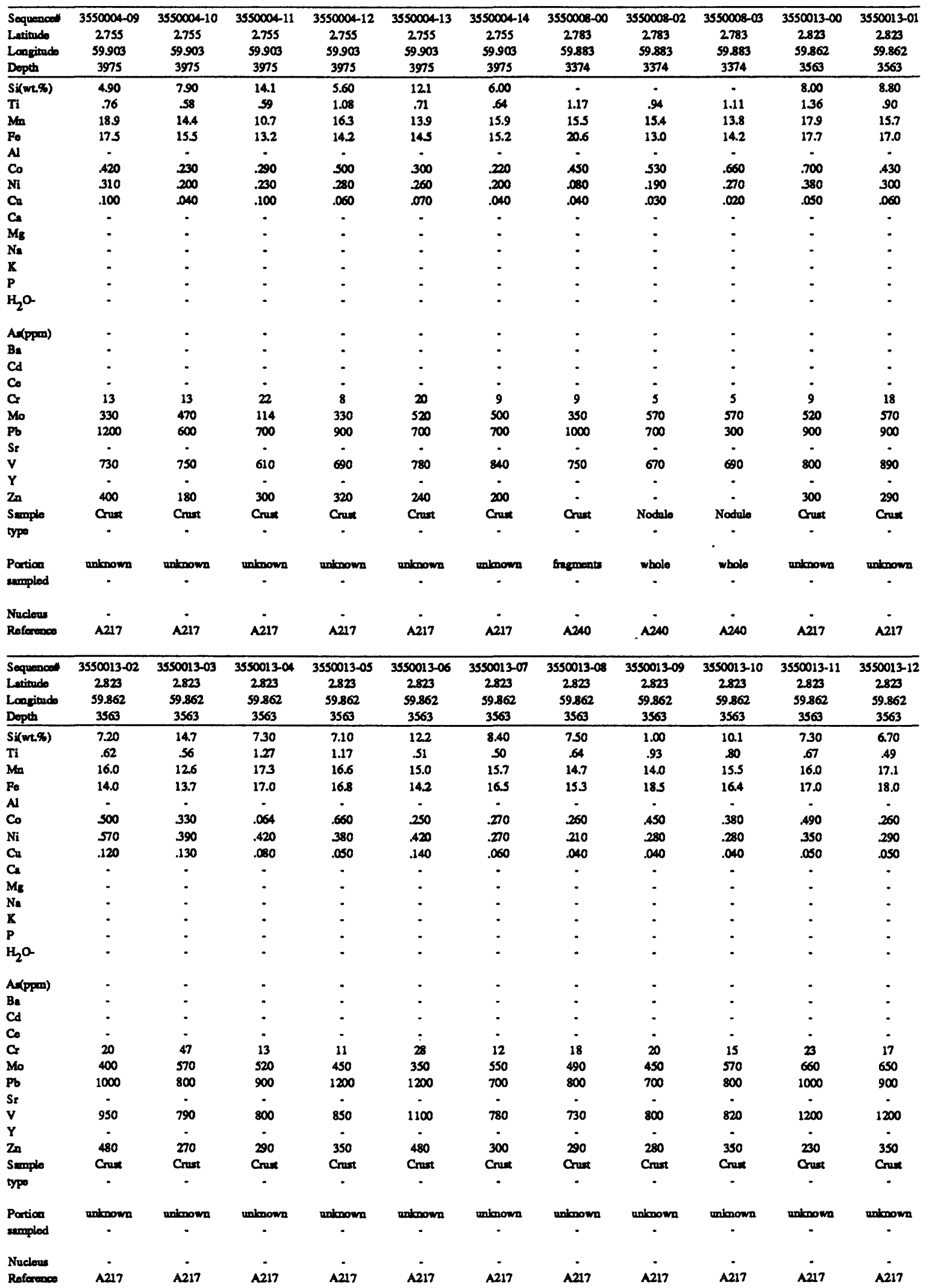


Table 9. Composition of crusts from the Scripps Nodule Databank (cont'd).

\begin{tabular}{|c|c|c|c|c|c|c|c|c|c|c|c|}
\hline $\begin{array}{l}\text { Sequencell } \\
\text { Latitude } \\
\text { Longinudo } \\
\text { Depth }\end{array}$ & $\begin{array}{c}3550013-13 \\
2.823 \\
59.862 \\
3563\end{array}$ & $\begin{array}{c}3550013-14 \\
2.823 \\
59.862 \\
3563\end{array}$ & $\begin{array}{c}3550013-15 \\
2.823 \\
59.862 \\
3563\end{array}$ & $\begin{array}{c}3550014-\infty 0 \\
2.798 \\
59.862 \\
3776\end{array}$ & $\begin{array}{c}3550014-01 \\
2798 \\
59.862 \\
3776\end{array}$ & $\begin{array}{c}355001402 \\
2.798 \\
59.862 \\
3776\end{array}$ & $\begin{array}{c}3550015-00 \\
2.863 \\
59.855 \\
3617\end{array}$ & $\begin{array}{c}3550015-01 \\
2.863 \\
59.855 \\
3617\end{array}$ & $\begin{array}{c}3550015-02 \\
2.863 \\
59.855 \\
3617\end{array}$ & $\begin{array}{c}3550015-03 \\
2.863 \\
59.855 \\
3617\end{array}$ & $\begin{array}{c}3550015-04 \\
2863 \\
59.855 \\
3617\end{array}$ \\
\hline $\mathrm{Si}\left(\mathrm{wt} . \mathrm{w}_{)}\right)$ & 9.50 & 8.70 & 9.60 & 4.80 & 5.30 & 8.50 & 13.9 & 7.20 & 6.10 & 6.20 & 143 \\
\hline $\mathrm{Ti}$ & 59 & .68 & 50 & .89 & .71 & 53 & 42 & .72 & 90 & .81 & 42 \\
\hline $\mathbf{M n}$ & 15.7 & 15.8 & 15.5 & 17.2 & 18.4 & 15.8 & 114 & 153 & 16.3 & 163 & 10.9 \\
\hline Fo & 17.1 & 18.5 & 16.6 & 13.9 & 13.3 & 14.7 & 132 & 15.2 & 16.2 & 16.7 & 13.0 \\
\hline A & - & - & • & - & - & - & - & • & - & - & - \\
\hline Co & 310 & 300 & .280 & .460 & 420 & .240 & 270 & 470 & .570 & 560 & .240 \\
\hline $\mathbf{N i}$ & 310 & 270 & .360 & 300 & 390 & 260 & 400 & 330 & .340 & $A 10$ & 430 \\
\hline $\mathrm{Cu}$ & .040 & .040 & .070 & .040 & .070 & .050 & .160 & .060 & .040 & .050 & .120 \\
\hline c. & . & - & $\cdot$ & - & - & - & - & - & - & - & - \\
\hline $\mathrm{M}_{8}$ & . & . & 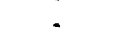 & . & . & . & - & - & . & - & . \\
\hline $\mathrm{Na}$ & - & - & - & - & - & . & - & - & - & - & - \\
\hline $\mathbf{K}$ & - & - & - & - & - & - & - & - & - & - & - \\
\hline $\mathbf{P}$ & - & - & - & - & - & - & - & - & . & . & . \\
\hline $\mathrm{H}_{2} \mathrm{O}$ & - & $\cdot$ & - & - & - & - & - & - & - & - & $\cdot$ \\
\hline$A x(p p m)$ & - & - & - & - & - & - & - & . & - & - & - \\
\hline $\mathrm{Be}$ & - & - & - & - & - & - & - & - & - & - & - \\
\hline$c d$ & - & - & - & - & - & - & - & - & - & - & - \\
\hline$c_{0}$ & - & - & - & - & - & - & - & - & - & - & - \\
\hline cr & 17 & 13 & 21 & 26 & 8 & 10 & 34 & 22 & 22 & 16 & 48 \\
\hline Mo & 600 & 500 & 640 & 410 & 350 & 630 & 370 & 520 & 350 & 450 & 340 \\
\hline $\mathbf{P b}_{b}$ & 1100 & 800 & 900 & 700 & 700 & 600 & 800 & 1200 & 1300 & 1200 & 700 \\
\hline Sr & - & $\cdot$ & - & - & $\cdot$ & $\cdot$ & $\cdot$ & $\cdot$ & - & - & - \\
\hline $\mathbf{v}$ & 1100 & 850 & 1000 & 710 & 670 & 880 & 880 & 980 & 880 & 1000 & 740 \\
\hline $\mathbf{Y}$ & 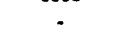 &. &. & - & - & $\cdot$ & 0 & 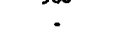 & 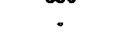 & 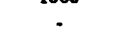 & - \\
\hline $\mathbf{Z n}_{\mathbf{n}}$ & 560 & 480 & 430 & 110 & 230 & 250 & 460 & 330 & 430 & 320 & 270 \\
\hline Sampio & Crust & Crust & Crux & Crust & Crent & Crus & Crux & Crut & Crust & Crust & Crust \\
\hline typo & $\cdot$ & - & - & • & - & - & - & • & 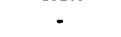 & . & . \\
\hline Portion & unknown & unknown & unknown & unlorown & unknown & unknown & unknown & unknown & unknown & topsido & underside \\
\hline anmpled & - & $\cdot$ & • & - & - & - & - & • & • & $\because$ & • \\
\hline Nucleus & - & . & - & - & . & - & - & . & - & - & - \\
\hline Referenco & A217 & A217 & A217 & A217 & A217 & A217 & A217 & A217 & $A 217$ & A217 & $A 217$ \\
\hline Sequencell & $3550015-05$ & $3550015-06$ & $3550015-07$ & $3550015-08$ & $3550015-09$ & $3550015-10$ & $3550015-11$ & $3550015-12$ & $3550015 \cdot 13$ & $3550015-15$ & $3550018-00$ \\
\hline Latitude & 2.863 & 2.863 & 2.863 & 2.863 & 2.863 & 2863 & 2.863 & 2.863 & 2863 & 2.863 & 2.803 \\
\hline Longitude & 59.855 & 59.855 & 59.855 & 59.855 & 59.855 & 59.855 & 59.855 & 59.855 & 59.855 & 59.855 & 59.768 \\
\hline Depth & 3617 & 3617 & 3617 & 3617 & 3617 & 3617 & 3617 & 3617 & 3617 & 3617 & 3432 \\
\hline$\overline{S i(w t .9)}$ & 6.10 & 125 & 6.90 & 113 & 8.70 & 8.40 & 9.30 & 8.70 & 10.7 & 11.4 & 9.00 \\
\hline $\mathrm{Ti}$ & 1.04 & .46 & 1.24 & .93 & .67 & .65 & .67 & .55 & .60 & .63 & .60 \\
\hline $\mathrm{Mn}$ & 14.2 & 11.1 & 17.3 & 14.5 & 14.5 & 14.8 & 15,6 & 15.6 & 13.0 & 14.0 & 15.2 \\
\hline Fo & 16.4 & 13.2 & 16.1 & 17.0 & 17.0 & 15.7 & 8.20 & 17.1 & 15.7 & 14.7 & 15.3 \\
\hline A & 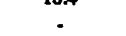 &. & $=$ & - & . & . & - & $\cdot$ & - & 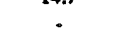 & - \\
\hline Co & .490 & .210 & .660 & 300 & 360 & .220 & .250 & 300 & .370 & .290 & .270 \\
\hline $\mathrm{Ni}$ & 280 & 360 & .420 & .230 & .240 & .280 & 310 & .250 & .290 & 390 & .250 \\
\hline $\mathrm{Cu}$ & .050 & .150 & .030 & .040 & .040 & .080 & .070 & .040 & .050 & .100 & .040 \\
\hline ca & • & - & - & - & - & $\cdot$ & - & - & - & • & - \\
\hline$M_{8}$ & - & . & - & - & - & - & - & - & - & - & - \\
\hline $\mathrm{Ne}$ & - & - & - & - & - & . & - & - & - & - & - \\
\hline $\mathbf{K}$ & - & . & - & - & . & . & . & - & - & - & . \\
\hline P & - & . & - & - & . & . & . & . & . & . & . \\
\hline $\mathrm{H}_{2} \mathrm{O}$ & - & . & - & - & - & - & - & - & - & - & . \\
\hline$A \Omega(p p m)$ & - & - & - & - & - & - &. & . & - & - & - \\
\hline Be & . & . & . & . & . & - & - & - & - & - & - \\
\hline Cd & - & - & - & - & - & - & . & - & - & - & - \\
\hline Co & - & - & - & - & - & - & - & - & - & - & - \\
\hline $\mathrm{Cr}$ & 19 & 38 & 11 & 19 & 20 & 79 & 18 & 15 & 26 & 20 & 16 \\
\hline Mo & 390 & 330 & 540 & 810 & 500 & 700 & 610 & 320 & 480 & 330 & 460 \\
\hline $\mathrm{Pb}$ & 1300 & 1000 & 1200 & 900 & 1100 & 700 & 800 & 800 & 1000 & 1100 & 600 \\
\hline Sr & $\cdot$ & $\cdot$ & - & - & 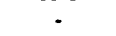 & - & - & $\cdot$ & - & $\cdot$ & $\cdot$ \\
\hline $\mathrm{v}$ & 860 & 620 & 930 & 1000 & 950 & 1100 & 1200 & 700 & 950 & 850 & 850 \\
\hline$Y$ & - &. & 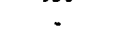 & . & . & 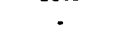 & . & - & $\cdot$ & - & $\cdot$ \\
\hline $\mathbf{Z n}$ & 250 & 370 & 370 & 300 & 360 & 450 & 480 & 400 & 300 & 490 & 190 \\
\hline Samplo & Crust & Cruat & Crust & Crust & Crust & Crust & Crust & Crust & Crat & Mn-coatred & Crust \\
\hline typo & - & 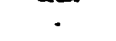 & . & 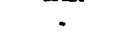 & . & - & + & - & - & rock & - \\
\hline Partica & topside & undersido & topside & underside & unknown & unknown & unknown & unknown & unknown & cuter & unknown \\
\hline umplod & . & . & - & - & - & - & - & 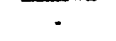 & - & coust & - \\
\hline Nucleus & - & • & - & - & - & . & • & • & • & • & - \\
\hline Reforences & A217 & A217 & A217 & A217 & A217 & $A 217$ & A217 & A217 & A217 & A217 & A217 \\
\hline
\end{tabular}


Table 9. Composition of crusts from the Scripps Nodule Databank (cont'd).

\begin{tabular}{|c|c|c|c|c|c|c|c|c|c|c|c|}
\hline Sequencell & $3550018-01$ & $3550018-02$ & $3550018-03$ & $3550018-04$ & $3550018-05$ & $3550018-06$ & $3550066-00$ & $3550069-\infty$ & $3550069-01$ & $3550069-02$ & $3550069-03$ \\
\hline Latitude & 2.803 & 2.803 & 2803 & 2.803 & 2.803 & 2.803 & 1.210 & 1.365 & 1.365 & 1365 & 1.365 \\
\hline Langitude & 59.768 & 59.768 & 59.768 & 59.768 & 59.768 & 59.768 & 56.615 & 56.612 & 56.612 & 56.612 & 56.612 \\
\hline Dopth & 3432 & 3432 & 3432 & 3432 & 3432 & 3432 & 930 & 2940 & 2940 & 2940 & 2940 \\
\hline$\overline{S i(w t \%)}$ & 7.50 & 7.50 & 7.50 & 7.20 & 7.90 & 9.80 & $\cdot$ & - & - & - & - \\
\hline $\mathrm{Ti}$ & .65 & .61 & 58 & .75 & 51 & .63 & • & • & • & .91 & 1.07 \\
\hline $\mathbf{M m}$ & 14.4 & 16.9 & 17.8 & 179 & 16.9 & 15.5 & 3.72 & 6.20 & 13.0 & 8.10 & 6.50 \\
\hline$P_{0}$ & 15.2 & 18.1 & 18.5 & 17.5 & 17.6 & 18.2 & 794 & 29.0 & 15.8 & 25.9 & 26.7 \\
\hline Al & . & 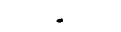 & 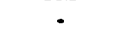 & 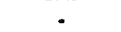 & - & - & 54 & 1.66 & 3.18 & 1.49 & 1.85 \\
\hline Co & 310 & 320 & 310 & .660 & 300 & .360 & 230 & 220 & .180 & 300 & .220 \\
\hline $\mathbf{N i}$ & .230 & 230 & .240 & 300 & 280 & .300 & 200 & .120 & .180 & .130 & .130 \\
\hline Co & .040 & .040 & .040 & .040 & .060 & .050 & .030 & .040 & .120 & .040 & .050 \\
\hline c. & - & . & 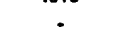 & . & . & 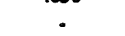 & 680 & 1.39 & 237 & 1.62 & 1.52 \\
\hline $\mathrm{M}_{\mathbf{8}}$ & - & - & - & - & - & - & - & - & - & 1.25 & 1.22 \\
\hline $\mathrm{Na}$ & - & - & - & - & - & - & - & - & - & • & • \\
\hline $\mathbf{K}$ & - & - & - & - & - & . & - & - & - & - & - \\
\hline $\mathbf{P}$ & . & - & - & - & . & . & . & - & - & - & - \\
\hline $\mathrm{H}_{2} \mathrm{O}$ & - & - & - & - & - & - & - & - & . & - & - \\
\hline$A=$ (ppon) & - & - & - & - & - & - & - & - & - & - & - \\
\hline Ba & - & - & - & - & - & - & - & - & - & - & - \\
\hline Cd & - & - & - & - & - & . & - & - & - & 5.0 & 6.0 \\
\hline co & - & - & - & - & - & - & . & - & - & - & - \\
\hline Cr & -11 & 13 & 13 & 15 & 19 & 18 & - & . & - & - & - \\
\hline Mo & 410 & 710 & 770 & 630 & 710 & 570 & - & - & - & - & - \\
\hline $\mathbf{P b}$ & 700 & 800 & 700 & 900 & 1000 & 700 & 1200 & $17 \infty 0$ & 700 & 1400 & 1400 \\
\hline sr & - & - & • & . & - & - & - & 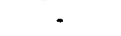 & $\cdot$ & - & - \\
\hline $\mathrm{v}$ & 720 & 890 & 920 & 850 & 1000 & 880 & - & - & - & - & - \\
\hline $\mathbf{Y}$ & 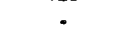 & - & 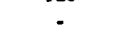 & . & $\cdot$ & . & • & • & • & • & - \\
\hline $\mathbf{z n}$ & 310 & 270 & 230 & 290 & 330 & 300 & 380 & 550 & 400 & 590 & 540 \\
\hline Sample & Crust & Crust & Crus & Crust & Crut & Crus & Orust & Crust & Crus & Crust & Crusx \\
\hline type & - & - & • & - & - & • & - & • & • & - & - \\
\hline Partion & unknown & unknown & monown & unknown & unknown & unlonown & unknown & unknown & unknown & unkrown & unknown \\
\hline semplod & • & - & • & - & - & - & • & - & • & - & - \\
\hline Nuclous & - & - & • & • & • & - & • & • & • & • & - \\
\hline References & A217 & $\mathbf{A 2 1 7}$ & A217 & A217 & A217 & A217 & A467 & A433 & A433 & $\mathbf{A 4 3 3}$ & A433 \\
\hline Sequencell & $3550071-\infty$ & $3550071-01$ & $3550071-02$ & $3550071-03$ & $3550071-04$ & $3550071-05$ & $3550071-06$ & $3550011-07$ & $3550071-08$ & $3550071-09$ & $3550071-10$ \\
\hline Latimde & 1.320 & 1320 & 1320 & 1320 & 1320 & 1.320 & 1320 & 1320 & 1320 & 1320 & 1320 \\
\hline Langitude & 56.593 & 56.593 & 56.593 & 56.593 & 56.593 & 56.593 & 56.593 & 56.593 & 56.593 & 56.593 & 56.593 \\
\hline Dopth & 2180 & 2180 & 2180 & 2180 & 2180 & 2180 & 2180 & 2180 & 2180 & 2180 & 2180 \\
\hline $\mathrm{si}(\mathrm{wt} \%)$ & - & - & $\cdot$ & . & $\cdot$ & $\cdot$ & . & $\cdot$ & $\cdot$ & . &. \\
\hline $\mathrm{Ti}$ & • & .72 & 80 & .89 & .69 & .63 & .69 & .72 & .67 & .71 & .74 \\
\hline Mn & 16.0 & 16.8 & 12.6 & 10.8 & 20.8 & 17.3 & 20.7 & 19.7 & 16.2 & 17.0 & 18.4 \\
\hline Fo & 21.7 & 19.6 & 22.2 & 20.5 & 143 & 19.0 & $16 A$ & 19.1 & 17.8 & 19.7 & 17.4 \\
\hline Al & 1.18 & .71 & 1.27 & 1.93 & 1.85 & 55 & 1.27 & .94 & 156 & .63 & 1.22 \\
\hline Co & 410 & 540 & .350 & 440 & .40 & .420 & 370 & 390 & .390 & 510 & 390 \\
\hline $\mathrm{Ni}$ & .260 & .260 & .230 & .240 & 1.030 & .280 & .660 & 380 & .420 & .250 & $A 10$ \\
\hline $\mathrm{Cr}$ & .050 & .030 & .050 & .060 & .160 & .040 & .100 & .060 & .060 & .030 & .080 \\
\hline ca & 1.95 & 2.48 & 150 & 1.40 & 1.60 & 2.15 & 1.93 & - & 1.87 & 2.12 & 1.84 \\
\hline $\mathbf{M g}$ & - & 1.12 & 1.22 & 1.21 & 2.03 & .98 & 1.39 & 1.07 & 132 & - & 1.10 \\
\hline Na & - & - & . & . & - & - & - & • & - & . & - \\
\hline $\mathbf{K}$ & - & - & - & - & - & - & - & - & - & - & . \\
\hline P & - & . & - & - & - & . & - & - & . & - & - \\
\hline $\mathrm{H}_{2} \mathrm{O}$ & - & - & - & - & - & - & - & - & - & - & - \\
\hline As(ppm) & . & . & - & - & - & . & - & - & . & - & - \\
\hline $\mathrm{Ba}_{2}$ & . & - & . & . & - & . & . & - & . & - & . \\
\hline Cd & - & 7.0 & 4.0 & 6.0 & 10.0 & 6.0 & 9.0 & 7.0 & 11.0 & 7.0 & 8.0 \\
\hline Co & . & - & • & • & - & • & . & . & • & - & - \\
\hline $\mathrm{Cr}$ & . & - & . & - & - & . & - & - & . & . & - \\
\hline Mo & . & - & - & - & - & - & - & - & - & - & - \\
\hline $\mathrm{Pb}$ & 2100 & 1800 & 1600 & 1600 & 1300 & 1700 & 1700 & 1600 & 1300 & 1700 & 1500 \\
\hline Sr & - & - & • & - & - & • & - & • & - & • & • \\
\hline $\mathbf{v}$ & . & - & . & . & . & . & - & . & - & - & - \\
\hline $\mathbf{Y}$ & - & - & - & - & - & - & - & - & - & - & - \\
\hline $2 n$ & 580 & 630 & 610 & 560 & 1000 & 600 & 720 & 620 & 640 & 550 & 570 \\
\hline Sampie & Coust & Crust & Cruse & Cust & Crut & Cras & Crust & Crust & Cunt & Curt & Crus \\
\hline typo & - & • & • & - & - & • & • & • & - & - & • \\
\hline Portion & unimown & unknown & unknown & unknown & unknown & unknown & unknown & unknown & unknown & unknown & unknown \\
\hline amplod & . & - & - & - & - & • & • & • & • & - & • \\
\hline Nuclous & - & - & - & - & • & - & • & - & • & • & - \\
\hline References & A433 & A433 & A433 & A433 & A433 & A433 & A433 & A433 & A433 & $\mathbf{A 4 3 3}$ & A433 \\
\hline
\end{tabular}


Table 9. Composition of crusts from the Scripps Nodule Databank (cont'd).

\begin{tabular}{|c|c|c|c|c|c|c|c|c|c|c|c|}
\hline $\begin{array}{l}\text { Sequencell } \\
\text { Latitude } \\
\text { Longitude } \\
\text { Dopth } \\
\end{array}$ & $\begin{array}{c}3550073-\infty 0 \\
1.283 \\
56.575 \\
1865 \\
\end{array}$ & $\begin{array}{c}3550075-00 \\
.17 \\
55.605 \\
1358\end{array}$ & $\begin{array}{c}350075-01 \\
.177 \\
55.605 \\
1358 \\
\end{array}$ & $\begin{array}{c}3550075-02 \\
.177 \\
55.605 \\
1358 \\
\end{array}$ & $\begin{array}{c}3550076-00 \\
.187 \\
55588 \\
1600 \\
\end{array}$ & $\begin{array}{c}3550076-01 \\
.187 \\
55.588 \\
1600 \\
\end{array}$ & $\begin{array}{c}3550076-02 \\
.187 \\
55.588 \\
1600 \\
\end{array}$ & $\begin{array}{c}3550076-03 \\
.187 \\
55588 \\
1600 \\
\end{array}$ & $\begin{array}{c}3550076-04 \\
.187 \\
55.588 \\
1600 \\
\end{array}$ & $\begin{array}{c}3550076-05 \\
.187 \\
55.588 \\
1600 \\
\end{array}$ & $\begin{array}{c}3550076-06 \\
.187 \\
55.588 \\
1600 \\
\end{array}$ \\
\hline$\overline{S i(w t \%)}$ & $\cdot$ & - & $\cdot$ & $\cdot$ & - & $\cdot$ & $\cdot$ & $\cdot$ & $\cdot$ & $\cdot$ & $\cdot$ \\
\hline $\mathrm{Ti}$ & - & - & .75 & .79 & - & .62 & .70 & .65 & .00 & .70 & so \\
\hline Mn & 16.9 & 17.6 & 17.6 & 19.3 & 18.9 & 18.7 & 9.80 & 18.7 & 18.8 & 7.80 & 13.5 \\
\hline Pe & 30.7 & 16.7 & 15.5 & 16.5 & 19.3 & 19.9 & 18.5 & 19.5 & 19.4 & 18.9 & 228 \\
\hline Al & 34 & .36 & 55 & 39 & .83 & 53 & 287 & .77 & .46 & 3.08 & 1.70 \\
\hline$c_{0}$ & 390 & 890 & .980 & 930 & 300 & .350 & 320 & 360 & .340 & 300 & .40 \\
\hline $\mathrm{Ni}$ & 250 & 320 & 300 & 310 & 340 & .270 & .150 & .270 & .230 & .120 & .130 \\
\hline $\mathrm{Cu}$ & .050 & .020 & .020 & .020 & .040 & .040 & .070 & .050 & .020 & .070 & .070 \\
\hline$C_{2}$ & 1.79 & 5.98 & 4.74 & 3.06 & 2.04 & - & 1.37 & 1.87 & 2.31 & 1.47 & 2.07 \\
\hline $\mathbf{M}_{\mathbf{8}}$ & • & • & 1.10 & 1.14 & • & - & 99 & .98 & 94 & • & 1.04 \\
\hline $\mathbf{N a}$ & - & - & - & • & - & - & - & - & - & - & - \\
\hline $\mathbf{K}$ & - & - & - & - & - & - & - & - & - & - & - \\
\hline $\mathbf{P}$ & - & - & - & - & - & - & - & - & - & - & - \\
\hline $\mathrm{H}_{2} \mathrm{O}-$ & $\cdot$ & $\cdot$ & - & $\cdot$ & - & - & - & - & - & $\cdot$ & - \\
\hline As(ppm) & - & - & - & - & - & - & - & - & . & . & . \\
\hline $\mathrm{Ba}$ & - & - & - & - & - & - & . & . & - & . & - \\
\hline $\mathrm{Cd}$ & - & - & 8.0 & 6.0 & - & 6.0 & 5.0 & 5.0 & 5.0 & 6.0 & 5.0 \\
\hline co & - & - & - & - & - & - & - & - & - & - & - \\
\hline Cr & - & - & - & - & - & - & - & - & - & - & - \\
\hline Mo & • & - & - & - & • & - & • & • & • & - & - \\
\hline $\mathbf{P b}$ & 1900 & 2300 & 1900 & 2200 & 1800 & 1600 & 1400 & 1600 & 1700 & 1500 & 2100 \\
\hline$S_{\mathbf{r}}$ & - & - & - & - & • & • & - & • & - & - & - \\
\hline $\mathbf{v}$ & . & - & - & - & - & . & - & - & - & . & - \\
\hline $\mathbf{Y}$ & - & . & - & . & - & . & . & . & . & . & . \\
\hline $\mathbf{z n}$ & 450 & 470 & 500 & 500 & 610 & 600 & 500 & 600 & 580 & 520 & 760 \\
\hline Sumplo & Court & Cruat & Crust & Court & Cruat & Crust & Cust & Cruat & Crust & Crust & Crust \\
\hline type & • & - & • & • & • & - & - & • & - & • & - \\
\hline Portion & unkrown & unknown & unlonown & unknown & unkonown & unknown & unknown & unknown & malnown & underown & uniknown \\
\hline sampled & • & • & $\cdot$ & • & $\cdot$ & $\cdot$ & • & $\cdot$ & • & $\cdot$ & $\cdot$ \\
\hline Nuclous & • & - & • & - & - & • & • & - & - & • & • \\
\hline Referencos & A467 & A433 & A433 & A433 & A433 & A433 & A433 & A433 & A433 & A433 & A433 \\
\hline Sequenoell & $3550076-07$ & $3550076-08$ & $3550076-09$ & $3550076-10$ & $3550079-\infty$ & 3550081.00 & $3550081-01$ & $3550081-02$ & $3550081-03$ & $3630202-\infty 0$ & $3640015-00$ \\
\hline Letitude & .187 & .187 & .187 & .187 & .178 & .145 & .145 & .145 & .145 & 15.350 & 17.900 \\
\hline Longinide & 55.588 & 55.588 & 55.588 & 55.588 & 55.518 & 55.165 & 55.165 & 55.165 & 55.165 & -21.867 & .31 .950 \\
\hline Depth & 1600 & 1600 & 1600 & 1600 & 1200 & 1800 & 1800 & 1800 & 1800 & . & 4868 \\
\hline Si(wt.\%) & $\cdot$ & $\cdot$ & $\cdot$ & $\cdot$ & - & $\cdot$ & • & • & $\cdot$ & - & - \\
\hline $\mathrm{Ti}$ & 55 & .57 & .77 & - & . & - & • & .69 & 1.11 & . & - \\
\hline Mn & 18.3 & 19.4 & 7.70 & 2.23 & 24.7 & 20.1 & 165 & 18.5 & 5.50 & 18.9 & 15.2 \\
\hline Po & • & 15.8 & • & 15.2 & 155 & 23.4 & 18.3 & 19.3 & 15.3 & 23.2 & 23.8 \\
\hline Al & .37 & 1.53 & 2.76 & 51 & 58 & • & 1.73 & .83 & 4.12 & • & - \\
\hline Co & 300 & 340 & .230 & 1.45 & 1.04 & .610 & .530 & 510 & .230 & 1.23 & 300 \\
\hline $\mathrm{Ni}$ & .280 & 920 & .140 & - & . & .320 & 360 & .250 & .180 & .190 & .170 \\
\hline $\mathrm{Cu}$ & .030 & .160 & .060 & .420 & .480 & .060 & .040 & .040 & .030 & .010 & .120 \\
\hline ca & 2.15 & 1.48 & 1.42 & 220 & 3.07 & • & 2.29 & 1.67 & 6.86 & 3.00 & 1.50 \\
\hline $\mathbf{M g}_{\mathbf{g}}$ & .96 & 1.73 & 1.02 & - & - & - & - & 1.02 & 1.03 & • & - \\
\hline $\mathbf{N a}$ & $\cdot$ & • & $\cdot$ & - & - & - & - & $\cdot$ & $\cdot$ & - & - \\
\hline $\mathbf{K}$ & - & - & - & - & - & - & - & - & - & - & - \\
\hline $\mathbf{P}$ & - & - & - & - & - & - & - & - & - & - & - \\
\hline $\mathrm{H}_{2} \mathrm{O}$ & - & $\cdot$ & - & $\cdot$ & $\cdot$ & $\cdot$ & - & - & - & - & - \\
\hline$A=(p p m)$ & - & - & . & - & - & . & - & - & - & . & - \\
\hline $\mathrm{Ba}_{2}$ & - & - & - & - & . & . & - & - & - & . & - \\
\hline Cd & 4.0 & 8.0 & 20 & . & - & - & - & 11.0 & 3.0 & - & - \\
\hline$c_{0}$ & - & - & - & - & - & - & - & • & - & - & - \\
\hline Cr & - & - & - & - & - & - & . & - & - & - & - \\
\hline Mo & - & • & $\cdot$ & $\cdot$ & - & - & $\cdot$ & - & $\cdot$ & - & - \\
\hline $\mathbf{P b}$ & 1600 & 1200 & 1800 & 2400 & 2500 & - & 1900 & 1800 & 600 & 2500 & - \\
\hline St & $\cdot$ & $\cdot$ & $\cdot$ & . & - & . & 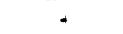 & - & - & - & - \\
\hline $\mathbf{v}$ & . & . & - & . & - & - & • & - & - & - & - \\
\hline $\mathbf{Y}$ & . & - & - & - & - & - & $\cdot$ & - & - & . & - \\
\hline $\mathrm{Zn}$ & 610 & 950 & 570 & 170 & 430 & - & 580 & 600 & 470 & 100 & • \\
\hline Sample & Crust & Cruent & Crust & Crosst & Crust & Nodule & Crust & Crust & Crusx & Crunt & Crust \\
\hline typo & • & $\cdot$ & • & & 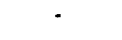 & - & • & $\cdot$ & - & - & • \\
\hline Partion & unknown & unknown & unknown & unknown & unkrown & unkrown & unknown & unknown & unknown & quarter & unknown \\
\hline resupled & • & $\cdot$ & - & • & - & & • & - & $\cdot$ & $\cdot$ & $\cdot$ \\
\hline Nuclous & - & . & . & - & - & - & . & - & $\cdot$ & • & • \\
\hline Roferencos & A433 & A433 & A433 & A467 & A467 & A 418 & A433 & A433 & A433 & A459 & A179 \\
\hline
\end{tabular}


Table 9. Composition of crusts from the Scripps Nodule Databank (cont'd).

\begin{tabular}{|c|c|c|c|c|c|c|c|c|c|c|c|}
\hline Sequencell & $3710037-00$ & $3710047-00$ & $3710047-01$ & $3710131-\infty$ & $3710149-00$ & $3710149-02$ & $3710153-00$ & $3710153-01$ & $3710154-01$ & $3710159-03$ & $3710167-\infty 0$ \\
\hline Lentitude & 12.833 & 12.833 & 12.833 & 10.402 & 13.767 & 13.767 & 14.725 & 14.725 & 12.525 & 11.500 & 11.497 \\
\hline Longituda & -100.533 & -107.150 & -107.150 & -108.793 & -101.872 & -101.872 & -102.575 & -102575 & -103.200 & -103.292 & -103.957 \\
\hline Dopth & 3455 & 3517 & 3517 & 1130 & 1693 & 1693 & 1843 & 1843 & 2007 & 2703 & 2035 \\
\hline Si(wt.\%) & $\cdot$ & $\cdot$ & - & $=$ & - & - & $\cdot$ & - & $\cdot$ & $\cdot$ & - \\
\hline $\mathrm{Ti}$ & - & - & • & - & $\cdot$ & - & - & - & • & $\cdot$ & $\cdot$ \\
\hline Mn & 7.20 & 18.0 & 16.0 & 30.7 & 220 & 18.8 & 7.90 & 19.8 & 17.1 & 13.3 & 11.8 \\
\hline Pe & 4.00 & 4.20 & 8.40 & 16.5 & 18.3 & 20.9 & 13.3 & 19.6 & 21.4 & 23.7 & 20.9 \\
\hline Al & - & • & - & .40 & - & - & • & - & - & - & • \\
\hline Co & .020 & .050 & .070 & 1.10 & 570 & .810 & 280 & 520 & .250 & .010 & - \\
\hline $\mathrm{Ni}$ & .240 & .790 & .550 & 560 & .430 & .280 & .130 & 310 & .280 & .170 & .130 \\
\hline $\mathrm{Cu}$ & 1.220 & 820 & .460 & .040 & .110 & .070 & .010 & .080 & .090 & .160 & .110 \\
\hline $\mathrm{Ca}$ & - & • & • & - & 200 & 240 & 4.20 & 250 & 2.40 & 200 & 270 \\
\hline $\mathbf{M}_{\mathbf{8}}$ & - & - & - & - & - & - & • & $\cdot$ & • & - & - \\
\hline $\mathrm{Na}$ & - & - & - & - & - & - & - & - & - & - & - \\
\hline $\mathbf{K}$ & - & - & - & - & - & - & - & - & - & - & - \\
\hline $\mathbf{P}$ & - & - & - & - & - & - & - & - & - & - & - \\
\hline $\mathrm{H}_{2} \mathrm{O}$ & - & - & - & - & • & - & - & - & - & - & $\cdot$ \\
\hline$A(p p \infty)$ & - & - & - & - & - & - & - & - & - & - & - \\
\hline $\mathrm{Ba}$ & - & - & - & - & - & - & - & - & - & - & - \\
\hline Cd & - & - & - & - & - & - & - & - & - & - & - \\
\hline co & - & - & - & - & - & - & - & - & - & - & - \\
\hline$c$ & - & - & - & 42 & - & - & - & - & - & - & - \\
\hline Mo & - & - & - & 1300 & - & - & - & - & - & - & • \\
\hline $\mathrm{Pb}$ & - & - & - & 1700 & 1200 & 1400 & 800 & 1200 & 800 & 400 & 400 \\
\hline Sr & - & - & - & - & - & - & - & - & - & - & - \\
\hline v & - & - & - & 125 & - & - & - & - & - & - & - \\
\hline $\mathbf{Y}$ & - & - & - & - & • & • & • & • & • & - & - \\
\hline $\mathrm{Zn}$ & • & - & • & 710 & 600 & 400 & 400 & 500 & 500 & 600 & 500 \\
\hline Semple & Crust & Crust & Crust & Nocule & Crux & Unlenown & Coust & Crust & Crust & Crust & Mn-coated \\
\hline typo & • & $\cdot$ & - & • & • & • & • & • & $\cdot$ & - & rock \\
\hline Portion & unimown & fragonersts & fregments & whole & fragments & fragments & fragments & fragments & fragments & fragments & without \\
\hline encoplod & - & - & - & - & - & • & 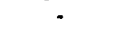 & - & 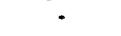 & $\cdot$ & suclous \\
\hline Nucleus & - & - & - & - & - & - & - & - & volcanic & - & Besalt \\
\hline Reforence & A333 & A333 & A333 & A334 & A437 & A437 & A437 & A437 & A437 & A437 & A437 \\
\hline Sequencest & $3720005-00$ & $3720078-01$ & $3720079-00$ & $3730005-00$ & $3730026-01$ & $3740019-00$ & $3740063-00$ & $3750069-01$ & $3750090-00$ & $3750090-06$ & $3750099-00$ \\
\hline Latitude & 14.607 & 16.083 & 13.450 & 12.275 & 13.052 & 14.370 & 11.017 & 13.167 & 10.008 & 10.008 & 11.050 \\
\hline Longitude & -117.293 & -112.633 & -118.417 & -120.158 & -125.480 & -133.115 & -139.967 & -147.750 & -143.320 & -143.320 & -140.000 \\
\hline Dopth & 4035 & 3069 & 4208 & 4471 & 4400 & 4684 & 4877 & 5610 & 5075 & 5075 & 4780 \\
\hline Si(wt.\%) & $\cdot$ & $\cdot$ & - & $\cdot$ & 11.2 & - & - & $\cdot$ & - & $\cdot$ & - \\
\hline $\mathrm{Ti}$ & - & - & - & • & .20 & • & - & 1.67 & • & - & - \\
\hline Mm & 8.80 & 40.5 & 31.5 & 315 & 27.8 & 28.4 & 31.5 & 20.6 & 28.4 & 26.6 & 8.00 \\
\hline$F_{0}$ & 7.00 & .70 & 4.20 & 5.08 & 240 & - & 5.60 & 14.2 & 5.30 & 8.60 & 7.30 \\
\hline $\mathbf{A}$ & $\cdot$ & $\cdot$ & - & • & 4.60 & • & • & 2.70 & - & • & - \\
\hline Co & .050 & - & .550 & .160 & .100 & .560 & .700 & 240 & .700 & .680 & .170 \\
\hline $\mathrm{Ni}$ & .580 & 320 & .990 & 1.680 & .900 & 1.300 & 840 & .330 & 1.430 & 1.290 & 530 \\
\hline $\mathrm{Cu}$ & .570 & .120 & 1.100 & 1.400 & 1.000 & .970 & .920 & 360 & .980 & .770 & 570 \\
\hline C. & .70 & • & - & 1.30 & .90 & $\cdot$ & - & 200 & 1.80 & 1.90 & .70 \\
\hline Ms & $\cdot$ & - & - & - & 2.00 & . & - & 1.28 & - & - & - \\
\hline $\mathrm{Na}$ & - & - & - & - & 260 & - & - & 1.06 & - & - & $\cdot$ \\
\hline $\mathrm{K}$ & - & - & - & - & 1.40 & . & . & 80 & - & - & - \\
\hline $\mathbf{P}$ & - & - & . & - & - & - & - & .20 & - & - & - \\
\hline $\mathrm{H}_{2} \mathrm{O}$ & - & - & - & - & - & - & - & - & - & $\cdot$ & - \\
\hline$A M(p p m)$ & - & - & - & - & - & - & - & - & - & - & - \\
\hline Ba & - & - & - & - & 23000 & - & - & - & - & - & . \\
\hline Cd & - & - & - & - & - & - & - & - & - & - & - \\
\hline co & - & - & - & - & $\cdot$ & - & - & - & - & - & - \\
\hline$c$ & - & - & - & - & 12 & - & - & - & - & - & - \\
\hline Mo & $\cdot$ & - & - & - & 380 & - & - & 280 & - & • & - \\
\hline $\mathbf{P b}$ & 300 & - & - & - & 300 & - & - & 800 & 400 & 500 & 200 \\
\hline Sr & - & - & - & - & 990 & - & - & $\cdot$ & $\cdot$ & • & $\cdot$ \\
\hline $\mathbf{v}$ & - & - & - & - & 600 & - & - & - & - & - & . \\
\hline $\mathbf{Y}$ & • & - & - & - & 60 & - & - & - & - & - & $\cdot$ \\
\hline $\mathrm{Zn}$ & 700 & - & - & - & 400 & - & - & - & 1700 & 1200 & 800 \\
\hline Semple & Mnencrustod & Mn-conted & Nodule & Cust & Crust & Unimown & Nodulo & Min-ancrusted & Nodulo & Nocule & Cruat \\
\hline typo & modiment & rock & - & • & - & • & • & sodimoent & • & • & - \\
\hline Portion & whole & unknown & unknown & uniknown & wbole & unknown & unknown & unknown & without & topside & uninnown \\
\hline sumpled & - & - & • & • & - & - & - & - & melous & - & • \\
\hline Nucleus & nor-spperent & - & - & - & - & - & • & • & - & • & - \\
\hline Referenco & A437 & A333 & A333 & A179 & A261 & A395 & A419 & A419 & A376 & A405 & A405 \\
\hline
\end{tabular}


Table 9. Composition of crusts from the Scripps Nodule Databank (cont'd).

\begin{tabular}{|c|c|c|c|c|c|c|c|c|c|c|c|}
\hline Sequencell & $3750101-\infty$ & $3750101-01$ & $3750103-02$ & 3750103-03 & $3750125-00$ & $3750128-03$ & $3750131-01$ & $3750131-02$ & $3750131-03$ & $3750132-02$ & $3750156-23$ \\
\hline Latitude & 11.100 & 11.100 & 11.100 & 11.100 & 11.035 & 11.043 & 10.950 & 10.950 & 10.950 & 10.965 & $11.1 \infty$ \\
\hline Longitude & -140.017 & -140.017 & -140.017 & -140.017 & -140.088 & -140.070 & -140.158 & -140.158 & -140.158 & -140.148 & -147.500 \\
\hline Depth & 4800 & 4800 & 4800 & 4800 & 4909 & 4895 & 4223 & 4223 & 4223 & 4619 & $\cdot$ \\
\hline Si(wt.\%) & $\cdot$ & - & $\cdot$ & $\cdot$ & $\cdot$ & $\cdot$ & - & $\cdot$ & $\cdot$ & $\cdot$ & 7.60 \\
\hline $\mathbf{T i}$ & • & - & • & - & $\cdot$ & - & - & - & 1.18 & - & 1.20 \\
\hline $\mathbf{M n}$ & 16.0 & 17.8 & 18.0 & - & 20.2 & 24.5 & 25.0 & 21.7 & • & 29.4 & 18.6 \\
\hline $\mathbf{P e}$ & 6.90 & 7.30 & 8.60 & - & 2.60 & 8.50 & 10.2 & 10.7 & 11.4 & 5.20 & 11.8 \\
\hline Al & • & - & - & - & • & • & • & • & • & - & 4.60 \\
\hline Co & .240 & 340 & .320 & - & .130 & 510 & 410 & 590 & - & 510 & .290 \\
\hline $\mathrm{Ni}$ & 1.090 & 1.200 & 1.110 & - & .890 & 1.100 & 1.080 & .980 & - & 1.580 & .600 \\
\hline cu & .810 & 840 & .770 & - & .810 & 900 & 590 & .660 & - & 1.200 & .280 \\
\hline C. & 1.00 & 1.20 & 1.30 & - & 9.60 & 1.50 & 1.90 & 2.10 & - & 1.90 & .98 \\
\hline $\mathbf{M g}$ & • & $\cdot$ & • & - & - & • & - & • & • & • & .51 \\
\hline $\mathrm{Na}$ & - & - & - & - & - & - & - & - & 1.64 & - & 5.80 \\
\hline $\mathbf{K}$ & - & - & - & - & - & - & - & - & - & - & 1.20 \\
\hline $\mathbf{P}$ & - & - & - & $\cdot$ & $\cdot$ & $\cdot$ & - & $\cdot$ & $\cdot$ & - & - \\
\hline $\mathrm{H}_{2} \mathrm{O}$ & $\cdot$ & - & $\cdot$ & $\cdot$ & $\cdot$ & - & - & - & - & - & - \\
\hline$A=(p p m)$ & - & - & - & 208 & - & $\cdot$ & - & $\cdot$ & $\cdot$ & - & - \\
\hline $\mathrm{Ba}$ & - & - & - & 3900 & - & - & - & - & - & - & 4800 \\
\hline Cd & - & - & - & 8.0 & - & - & $\cdot$ & - & - & - & 13.0 \\
\hline co & - & - & - & - & - & - & . & - & - & - & 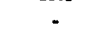 \\
\hline$c r$ & - & - & - & 17 & - & - & - & - & - & - & $\cdot$ \\
\hline Mo & - & - & - & 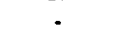 & - & • & - & - & - & - & 590 \\
\hline $\mathbf{P b}$ & 400 & 500 & 500 & $\cdot$ & 200 & 1100 & 700 & 900 & $\cdot$ & 400 & 600 \\
\hline $\mathbf{S r}$ & $\cdot$ & $\cdot$ & $\cdot$ & $\cdot$ & - & • & • & $\cdot$ & - & $\cdot$ & 68 \\
\hline $\mathbf{v}$ & - & - & - & - & - & - & - & - & - & - & 400 \\
\hline $\mathbf{Y}$ & • & • & • & - & • & • & - & - & . & - & • \\
\hline $\mathrm{Zn}$ & 1100 & 1200 & 1000 & - & 1800 & 1500 & 1100 & 900 & $\cdot$ & 1800 & 520 \\
\hline Sample & Crust & Soveral & Crust & Crut & Mn-conted & Nodule & Mn-coutrod & Mn-conted & Croust & Noctulo & Crust \\
\hline typo & $\cdot$ & crusts & • & $\cdot$ & rock & - & rock & rock & $\cdot$ & • & - \\
\hline Partion & unknown & croses & frigments & Engments & half & whole & outor & outer & fregments & whole & unknown \\
\hline enmpled & • & rection & $\cdot$ & $\cdot$ & $\cdot$ & $\cdot$ & crust & crust & $\cdot$ & - & - \\
\hline Nucleus & • & • & - & - & Rock & non-apperent & • & - & - & non-appariont & - \\
\hline References & A405 & A422 & A422 & A475 & A438 & A438 & A438 & A438 & A465 & A376 & A474 \\
\hline Sequencell & $3750156-24$ & $3750156-25$ & $3750156-26$ & $3750163-02$ & $3750183-01$ & $3750358-03$ & $3750890-00$ & $3750410-01$ & $3750438-\infty$ & $3760027-00$ & $3760034-\infty 0$ \\
\hline Latimde & 11.100 & 11.100 & 11.100 & 11.028 & 11.018 & 11.513 & 13,567 & 13.160 & 12583 & 14.317 & 11.850 \\
\hline Longitude & -147.500 & -147500 & -147.500 & -140.113 & -140.147 & -148.578 & -140.633 & -141.008 & -142.150 & -152617 & -152.950 \\
\hline Depth & - & $\cdot$ & $\cdot$ & 4868 & $\cdot$ & 5125 & 4721 & 6010 & $\cdot$ & 5490 & 5221 \\
\hline Si(wt.\%) & 1.00 & 10.3 & 14.6 & $\cdot$ & . & . & 9.19 & $\cdot$ & $\cdot$ & 13.2 & - \\
\hline $\mathrm{Ti}$ & .88 & .88 & 84 & • & • & • & - & • & - & $\cdot$ & 1.22 \\
\hline Mn & 153 & 8.40 & 10.7 & 13.2 & 227 & 11.5 & 22.0 & 24.5 & 31.3 & 18.4 & 11.0 \\
\hline Fe & 8.50 & 5.30 & 5.70 & 9.90 & 17.1 & 11.1 & 9.24 & - & 4.91 & 16.2 & 6.92 \\
\hline $\mathbf{A}$ & 3.90 & 5.20 & 6.30 & - & - & $\cdot$ & 3,04 & - & • & 1.67 & - \\
\hline Co & .270 & .130 & .180 & 330 & 390 & .090 & $\$ 20$ & .500 & .210 & 500 & 800 \\
\hline $\mathrm{Ni}$ & 1.020 & .720 & .760 & .750 & .440 & 350 & $\$ 90$ & .530 & 1.250 & 1.000 & 540 \\
\hline Cu & 360 & 310 & .320 & .500 & .130 & 320 & .480 & 390 & 1.210 & 1.500 & 570 \\
\hline ca & .88 & .14 & 98 & 1.30 & 2.70 & 1.20 & .14 & - & • & 1.54 & $\cdot$ \\
\hline $\mathbf{M}_{\mathbf{B}}$ & 56 & 1.14 & .89 & - & - & - & 1.88 & - & - & 1.18 & - \\
\hline $\mathrm{Ne}$ & 5.16 & 5.58 & 6.08 & - & - & - & 1.32 & - & - & - & - \\
\hline $\mathbf{K}$ & 1.78 & 2.12 & 2.20 & - & - & - & 50 & - & - & - & - \\
\hline $\mathbf{P}$ & - & - & - & - & - & - & 25 & $\cdot$ & - & - & - \\
\hline $\mathrm{H}_{2} \mathrm{O}$ & - & - & • & $\cdot$ & - & - & $\cdot$ & - & - & 8.9 & $\cdot$ \\
\hline$A s(p p m)$ & • & - & • & - & - & - & - & - & - & - & - \\
\hline Be & 5300 & 4200 & 4200 & $\cdot$ & • & $\cdot$ & - & - & $\cdot$ & - & 2500 \\
\hline Cd & 16.0 & 16.0 & 14.0 & - & - & - & - & - & - & - & • \\
\hline Co & - & - & • & - & - & - & - & - & - & - & - \\
\hline $\mathrm{Cr}$ & 10 & 9 & 7 & - & - & - & - & - & $\cdot$ & - & 6 \\
\hline Mo & 400 & 390 & 300 & $\cdot$ & • & • & - & - & 620 & - & 200 \\
\hline $\mathbf{P b}$ & 500 & 500 & 300 & 600 & 1200 & 1000 & - & - & $\cdot$ & - & 400 \\
\hline Sr & 40 & 248 & 160 & $\cdot$ & - & $\cdot$ & - & - & $\cdot$ & - & - \\
\hline $\mathbf{v}$ & 340 & 240 & 200 & $\cdot$ & $\cdot$ & $\cdot$ & $\cdot$ & - & $\cdot$ & - & 410 \\
\hline $\mathbf{Y}$ & $\cdot$ & - & $\cdot$ & - & $\cdot$ & $\cdot$ & - & $\cdot$ & - & - & $\cdot$ \\
\hline $\mathbf{Z n}$ & 640 & 500 & 600 & 700 & 570 & 1000 & • & - & $\cdot$ & • & - \\
\hline Semple & Crust & Crust & Crust & Crust & Mn-conted & Mn-encrusted & Nocule & Noctule & Crust & Nodule & Nodule \\
\hline type & - & $\cdot$ & - & • & rock & sodiment & - & - & - & - & - \\
\hline Partion & unkown & unknown & unknown & cross & outer & whole & unknown & composite & unbnown & whole & wholo \\
\hline sampled & . & - & • & soction & crost & • & • & 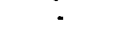 & - & • & • \\
\hline Nucleus & - & • & • & - & Basalt & Sodimem & - & - & - & - & - \\
\hline References & A474 & A474 & A474 & A405 & $A 376$ & A437 & A392 & A393 & A392 & A012 & A240 \\
\hline
\end{tabular}


Table 9. Composition of crusts from the Scripps Nodule Databank (cont'd).

\begin{tabular}{|c|c|c|c|c|c|c|c|c|c|c|c|}
\hline $\begin{array}{l}\text { Sequencent } \\
\text { Letitude } \\
\text { Longitude } \\
\text { Depth }\end{array}$ & $\begin{array}{c}3760118-00 \\
15.817 \\
-157.067 \\
5118 \\
\end{array}$ & $\begin{array}{c}3760145-01 \\
19.100 \\
-158.233 \\
3131 \\
\end{array}$ & $\begin{array}{c}3760145-02 \\
19.100 \\
-158.233 \\
3131 \\
\end{array}$ & $\begin{array}{c}3760147-02 \\
18.350 \\
-158.450 \\
1317 \\
\end{array}$ & $\begin{array}{c}3760147-03 \\
18.350 \\
-158.450 \\
1317 \\
\end{array}$ & $\begin{array}{c}3760147-04 \\
18.350 \\
-158.450 \\
1317 \\
\end{array}$ & $\begin{array}{c}3760208-00 \\
10.938 \\
-153.303 \\
4800 \\
\end{array}$ & $\begin{array}{c}3760223-00 \\
18.717 \\
-158.283 \\
475 \\
\end{array}$ & $\begin{array}{c}3760223-01 \\
18.717 \\
-158.283 \\
475 \\
\end{array}$ & $\begin{array}{c}3770020-00 \\
16.100 \\
-161.750 \\
2400\end{array}$ & $\begin{array}{c}3770020-01 \\
16.100 \\
-161.750 \\
2400 \\
\end{array}$ \\
\hline Si(wt.\%) & . & $\cdot$ & $\cdot$ & . & $\cdot$ & - & • & - & - & 6.00 & 4.20 \\
\hline $\mathbf{T i}$ & • & - & - & - & - & - & .87 & - & - & 84 & .88 \\
\hline Mn & 20.3 & • & - & 19.9 & • & - & 235 & 6.30 & - & 16.0 & 15.0 \\
\hline Fe & 15.3 & 16.0 & - & 16.9 & 129 & - & 126 & 8.40 & - & 17.2 & 7.60 \\
\hline $\mathbf{A}$ & $\cdot$ & • & • & - & - & - & . & - & - & 1.69 & 1.76 \\
\hline Co & .300 & 300 & 200 & 870 & 520 & 280 & 320 & .790 & .360 & .730 & .600 \\
\hline $\mathrm{Ni}$ & .540 & .150 & • & .420 & .170 & • & .530 & .700 & - & .250 & .250 \\
\hline $\mathrm{Cu}$ & .380 & .040 & . & .040 & .020 & - & .420 & .090 & - & .040 & .050 \\
\hline Ca & 2.80 & 250 & - & 290 & 1.70 & - & . & 730 & - & 201 & 1.70 \\
\hline $\mathbf{M g}$ & $\cdot$ & $\cdot$ & - & - & • & . & - & • & - & - & 1.00 \\
\hline $\mathrm{Ne}$ & - & $\cdot$ & - & - & - & - & . & - & - & - & .89 \\
\hline $\mathbf{K}$ & - & - & . & - & - & - & - & - & - & - & .68 \\
\hline $\mathbf{P}$ & . & - & - & - & - & . & - & - & - & - & .24 \\
\hline $\mathrm{H}_{2} \mathrm{O}$ & . & - & - & - & - & - & $\cdot$ & - & - & 20.6 & - \\
\hline A《(ppm) & - & - & - & - & - & - & - & - & - & - & $\cdot$ \\
\hline $\mathrm{Be}$ & - & - & - & . & - & $\cdot$ & . & - & $\cdot$ & 4500 & 200 \\
\hline Cd & - & - & 5.0 . & - & - & 4.0 & - & - & 9.0 & - & - \\
\hline ce & - & - & - & . & - & $\cdot$ & - & - & $\cdot$ & - & - \\
\hline$C$ & - & - & - & - & - & - & - & - & $\cdot$ & $\cdot$ & $\cdot$ \\
\hline Mo & - & • & 125 & • & $\cdot$ & 132 & - & • & 710 & 250 & 100 \\
\hline $\mathbf{P b}$ & 800 & 1000 & 800 & 1900 & 1000 & 700 & - & 1400 & 1600 & 1900 & $\cdot$ \\
\hline Sr & $\cdot$ & $\cdot$ & $\cdot$ & $\cdot$ & $\cdot$ & $\cdot$ & - & $\cdot$ & - & 1200 & - \\
\hline $\mathbf{v}$ & - & - & - & - & - & - & - & - & - & • & - \\
\hline $\mathbf{Y}$ & - & • & - & - & - & • & - & - & • & • & $\cdot \cdot$ \\
\hline $\mathbf{Z n}$ & 800 & 600 & 490 & 560 & 400 & 340 & - & 860 & 810 & 530 & - \\
\hline Semplo & Crust & Mn-encrustod & Mn-socrustod & Nodule & Mo-encrustod & Morecruted & Crust & Crust & Crust & Nodule & Noctulo \\
\hline type & $\cdot$ & modimene & sodiment & $\cdot$ & rediment & modiment & • & $\cdot$ & - & - & - \\
\hline Portican & half & fragments & fragments & oustar & fragments & unionown & unknown & fraponents & fragments & crows & unlonown \\
\hline samplod & $\cdot$ & - & 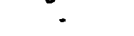 & cous & - & - & $\cdot$ & 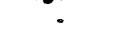 & - & soction & - \\
\hline Nucious & Undetermined & • & - & - & - & - & - & - & - & - & - \\
\hline Referencos & A437 & A376 & A303 & A376 & A376 & A303 & A343 & A376 & A303 & A197 & A290 \\
\hline Sequencell & $3770022-02$ & $3770025-01$ & $3770025-02$ & $3770026-01$ & $3770026-02$ & $3770026-03$ & $3770055-00$ & $3770055-01$ & $3770056-\infty$ & $3770061-\infty 0$ & $3770061-01$ \\
\hline Latimda & 18.783 & 19.383 & 19.383 & 19.367 & 19.367 & 19.367 & 10.500 & 10.500 & 12.070 & 15.600 & 15.600 \\
\hline Longitude & -162050 & -162167 & -162.167 & -162317 & -162.317 & -162.317 & -165.550 & -165.550 & -165.838 & -166.667 & -166.667 \\
\hline Dopth & 3148 & 4160 & 4160 & 4868 & 4868 & 4868 & 4341 & 4341 & 1488 & 5440 & 5440 \\
\hline Si(wt.\%) & - & - &. & $\cdot$ &. & - &. & $\cdot$ &. &. & 200 \\
\hline $\mathrm{Ti}$ & • & - & • & • & - & - & 1.38 & 1.12 & • & 1.22 & 1.20 \\
\hline Mn & 14.8 & - & 15.0 & 15.2 & - & - & 14.2 & 148 & 26.0 & 16.7 & 19.5 \\
\hline Po & 18.4 & . & 24.0 & 13.9 & 13.1 & - & 16.4 & 14.2 & 5.60 & 17.0 & 14.6 \\
\hline $\mathbf{A}$ & - & - & - & - & - & - & - & - & - & - & 1.10 \\
\hline$C_{0}$ & .570 & .260 & .410 & .710 & .490 & 370 & 880 & .680 & .400 & 840 & 550 \\
\hline $\mathrm{Ni}$ & 310 & $\cdot$ & .100 & .300 & .210 & - & .660 & 540 & 1.060 & 340 & 300 \\
\hline $\mathrm{Cu}$ & .160 & - & .050 & .140 & .090 & - & 320 & .420 & .140 & .160 & .090 \\
\hline Ca & 210 & . & 200 & 3.80 & 4.40 & - & - & 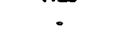 & 240 & - & 1.10 \\
\hline $\mathbf{M g}_{\mathbf{g}}$ & - & - & 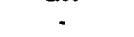 & - & - & - & - & - & 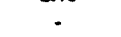 & - & 1.10 \\
\hline $\mathrm{Ne}$ & - & - & - & - & . & - & - & - & - & - & 1.80 \\
\hline $\mathbf{K}$ & . & - & - & - & - & - & - & - & - & - & .50 \\
\hline $\bar{P}$ & - & - & - & - & - & - & - & - & - & - & .18 \\
\hline $\mathrm{H}_{2} \mathrm{O}$ & . & - & . & - & - & . & - & - & - & - & $\cdot$ \\
\hline Ax(ppm) & . & . & - & - & - & - & - & - & - & - & - \\
\hline Ba & - & - & - & - & - & - & 3500 & 3400 & - & 1500 & 820 \\
\hline Cd & - & 6.0 & . & - & - & 5.0 & - & - & - & - & $\cdot$ \\
\hline Ce & - & - & - & - & - & - & - & - & - & - & - \\
\hline Cr & - & • & . & . & - & - & 4 & 3 & - & 9 & - \\
\hline Mo & - & 280 & - & - & - & 235 & 360 & 350 & - & 430 & 300 \\
\hline $\mathrm{Pb}$ & 1100 & 1500 & 1600 & 800 & 1200 & 900 & 400 & 100 & 1000 & 700 & . \\
\hline Sx & - & - & - & - & 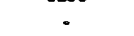 & - &. & 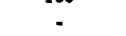 & - & 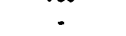 & . \\
\hline $\mathbf{v}$ & - & - & - & - & - & . & 540 & 470 & - & 630 & . \\
\hline $\mathbf{Y}$ & - & - & - & . & - & • & - & 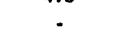 & • & 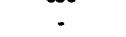 & - \\
\hline $\mathbf{Z n}$ & 720 & 690 & 390 & 480 & 490 & 440 & - & - & 1400 & • & . \\
\hline Semplo & Nodule & Crus & Crust & Nodule & Crux & Crust & Nodule & Noctulo & Mo-coatiod & Unknown & Nocule \\
\hline type & - & $\cdot$ & - & - & - & - & - & - & rock & - & - \\
\hline Partica & whole & mbonown & fragments & fragments & fragments & fragments & half & half & without & fragments & unlonown \\
\hline sampled & . & . & 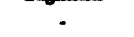 & - & 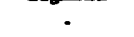 & 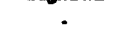 & $\cdot$ & 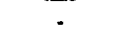 & nucleus & 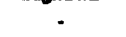 & $\cdot$ \\
\hline Nucleus & - & • & - & - & • & - & - & • & Rock & - & - \\
\hline References & A376 & A303 & $A 376$ & $A 376$ & A376 & $A 303$ & $A 240$ & $A 240$ & A405 & $\mathbf{A 2 4 0}$ & $A 290$ \\
\hline
\end{tabular}


Table 9. Composition of crusts from the Scripps Nodule Databank (cont'd).

\begin{tabular}{|c|c|c|c|c|c|c|c|c|c|c|c|}
\hline Sequencell & $3770073-00$ & $3770101-\infty$ & $3770101-01$ & $3770103-\infty 0$ & $3770103-01$ & $3770103-03$ & $3770121-\infty$ & $3770123-\infty$ & $3770138-\infty 0$ & $3770138-01$ & $3770138-02$ \\
\hline Latimido & 14.933 & 19.117 & 19.117 & 19.117 & 19.117 & 19.117 & 10.063 & 10.362 & 18.300 & 18.300 & 18.300 \\
\hline Longitude & -167.567 & -169.733 & -169.733 & -169.733 & -169.733 & -169.733 & .165 .330 & -166.983 & -161.767 & $\cdot 161.767$ & .161 .767 \\
\hline Depth & 4315 & 1740 & 1740 & 1740 & 1740 & 1740 & 1330 & 2865 & 3060 & 3060 & 3060 \\
\hline Si(wt.\%) & $\cdot$ & 3.70 & $\cdot$ & $\cdot$ & 3.30 & 3.00 & 1.39 & 1.98 & . & - & $\cdot$ \\
\hline $\mathrm{Ti}$ & - & 1.40 & 1.19 & • & 1.13 & 1.50 & 58 & 1.04 & 1.20 & - & - \\
\hline Mn & 21.3 & 24.6 & 13.4 & 40.1 & 20.5 & • & 18.8 & 223 & 39.8 & 18.8 & - \\
\hline Pe & 16.1 & 20.3 & 12.7 & 18.3 & 14.5 & - & 8.69 & 13.1 & 20.4 & 18.0 & - \\
\hline $\mathbf{A} \mathbf{l}$ & - & 1.30 & • & • & 1.00 & .70 & .76 & .51 & • & - & • \\
\hline Co & 590 & 1.40 & 1.28 & .710 & .950 & 1.50 & .800 & .880 & .520 & .064 & 520 \\
\hline $\mathrm{Ni}$ & .400 & .630 & .40 & .064 & .420 & .700 & .610 & 530 & .310 & 340 & .060 \\
\hline $\mathrm{Cu}$ & .270 & .090 & .070 & .100 & .100 & .150 & .060 & .090 & .060 & .100 & .110 \\
\hline Ca & 2.30 & 3.60 & - & - & 2.07 & 7.00 & 10.5 & 204 & - & 2.70 & - \\
\hline Ms & • & 1.40 & - & - & - & 1.50 & 1.24 & .99 & - & • & - \\
\hline $\mathrm{Ne}$ & - & 240 & - & - & - & 3.00 & • & • & - & - & - \\
\hline $\mathbf{K}$ & - & 30 & - & - & .40 & .70 & - & - & - & - & - \\
\hline $\mathbf{P}$ & - & - & . & - & .03 & - & 3.67 & .28 & - & - & - \\
\hline $\mathrm{H}_{2} \mathrm{O}$ & - & - & - & - & 28.2 & - & - & - & 24.0 & - & . \\
\hline$A<(\mathrm{ppm})$ & - & - & - & - & - & - & - & - & . & - & - \\
\hline Ba & - & 1300 & 3300 & - & 5000 & 1500 & - & . & . & - & - \\
\hline Cd & - & 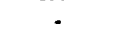 & - & - & - & . & - & - & - & - & - \\
\hline Co & - & - & - & - & - & 1500 & - & $=$ & - & - & - \\
\hline$C r$ & - & 25 & 6 & - & $\cdot$ & 7 & - & - & • & - & - \\
\hline Mo & - & 700 & 560 & - & 600 & 700 & - & - & 1100 & - & - \\
\hline $\mathbf{P b}$ & 1000 & 1900 & 2600 & 3700 & 2400 & 3000 & - & - & - & 1200 & - \\
\hline Sr & • & 1300 & - & - & 1500 & 3000 & - & - & - & • & - \\
\hline $\mathbf{V}$ & - & 700 & 570 & - & - & 700 & - & - & - & - & - \\
\hline $\mathbf{Y}$ & . & 350 & - & - & - & 300 & - & - & - & - & - \\
\hline $\mathrm{Zn}$ & 500 & • & - & - & 620 & • & 800 & 600 & • & 600 & - \\
\hline Semplo & Noctule & Unbonown & Crut & Nocule & Nodule & Nocule & Crust & Crust & Nodule & Nodulo & Noctule \\
\hline type & • & • & - & - & - & • & • & - & • & • & • \\
\hline Portion & quarter & fragments & fragments & cross & croess & unknown & average & average & without & cross & unknown \\
\hline sampled & 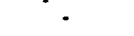 & - & 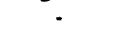 & section & soction & - & enalynis & malyais & nuclous & soction & - \\
\hline Nucleus & non-spparour & • & • & • & - & • & • & - & • & - & Undetermined \\
\hline Roferencos & A437 & A261 & A240 & A166 & A197 & $A 262$ & A363 & A366 & A346 & A376 & A395 \\
\hline Sequencell & $3770138-04$ & $3770138-05$ & $3770145-\infty 0$ & $3770153-\infty$ & $3770153-01$ & $3770153-02$ & $3770153-03$ & $3770153-04$ & $3770153-05$ & $3780004-00$ & $3780004-02$ \\
\hline Latitude & 18.300 & 18.300 & 19.978 & 19.512 & 19.512 & 19.512 & 19.512 & 19.512 & 19.512 & 10.383 & 10.383 \\
\hline Langitude & -161.767 & -161.767 & -166.083 & -168.875 & .168 .875 & -168.875 & -168.875 & -168.875 & -168.875 & -170.950 & -170.950 \\
\hline Depth & 3060 & 3060 & 4938 & 1805 & 1805 & 1805 & 1805 & 1805 & 1805 & 4469 & 4469 \\
\hline Si(wt.\%) & - & - & - & . & $\cdot$ & - & $\cdot$ & $\cdot$ & - & - & - \\
\hline $\mathrm{Ti}$ & - & • & - & • & • & - & - & - & - & 1.11 & .99 \\
\hline Mn & 16.7 & 20.8 & 6.59 & 21.5 & 23.2 & 19.2 & 21.6 & 23.4 & 224 & 17.0 & 20.0 \\
\hline Pe & 19.3 & 19.4 & 11.0 & 13.1 & 17.2 & 15.1 & 16.2 & 15.8 & 16.7 & 14.5 & 16.2 \\
\hline Al & - & - & - & - & • & - & - & - & - & - & - \\
\hline$c_{0}$ & 500 & .660 & .100 & .690 & .850 & .750 & .890 & 900 & .910 & .690 & .880 \\
\hline $\mathbf{N i}$ & 200 & .200 & 360 & .510 & .470 & .370 & .470 & 540 & .480 & .770 & 940 \\
\hline $\mathrm{Cu}$ & .040 & .070 & .220 & .110 & .040 & .040 & .080 & .120 & .060 & .630 & .690 \\
\hline $\mathrm{Ca}$ & 2.20 & 2.90 & - & 5.70 & 290 & 5.20 & 2.70 & - & 2.70 & - & - \\
\hline $\mathbf{M}_{\mathbf{8}}$ & - & . & - & - & - & • & . & - & - & - & - \\
\hline $\mathrm{Ne}$ & . & - & - & - & . & - & - & - & - & - & - \\
\hline $\mathbf{K}$ & . & - & - & - & - & - & - & - & . & - & - \\
\hline $\mathbf{P}$ & - & - & - & - & - & - & - & - & - & - & - \\
\hline $\mathrm{H}_{2} \mathrm{O}$ & - & - & - & • & - & - & - & - & - & - & - \\
\hline Ax(ppon) & - & - & - & - & - & - & - & - & - & • & • \\
\hline $\mathrm{Be}$ & - & - & - & - & - & - & - & - & - & 2800 & 850 \\
\hline Cd & - & - & - & - & - & - & - & - & - & - & - \\
\hline$c_{0}$ & - & - & - & - & - & - & - & - & - & - & - \\
\hline $\mathrm{Cr}$ & - & - & - & - & - & - & - & - & - & - & 2 \\
\hline Mo & • & - & - & • & • & • & - & - & - & 390 & 350 \\
\hline $\mathrm{Pb}$ & 1600 & 1500 & - & 1700 & 1700 & 1500 & 1600 & 1500 & 1500 & 100 & 500 \\
\hline Sr & - & - & - & - & - & - & - & - & - & - & - \\
\hline$\vec{v}$ & - & - & - & - & - & - & - & - & - & 490 & 530 \\
\hline $\mathbf{Y}$ & - & - & - & - & $\cdot$ & - & $\cdot$ & - & - & $\cdot$ & - \\
\hline $\mathbf{z n}_{\mathbf{n}}$ & 500 & 540 & 460 & 700 & 600 & 500 & 600 & 600 & 600 & • & • \\
\hline Sample & Cruat & Nodulo & Crust & Nocule & Nodule & Nocule & Nodule & Nocule & Nodule & Noctule & Nochule \\
\hline typo & - & - & - & • & - & - & • & - & - & - & - \\
\hline Portion & fragments & without & unknown & whole & whole & half & without & helf & cross & half & half \\
\hline sempled & - & medeus & - & • & - & $\cdot$ & muclous & - & rection & • & • \\
\hline Nucleus & • & • & - & non-epparent & Noctule-fragment & Rock & Bano & Nochula-fragment & Noctulo-fregmont & - & - \\
\hline Reference & A376 & A376 & A383 & A422 & A422 & A422 & A422 & A422 & A422 & A240 & A240 \\
\hline
\end{tabular}


Table 9. Composition of crusts from the Scripps Nodule Databank (cont'd).

\begin{tabular}{|c|c|c|c|c|c|c|c|c|c|c|c|}
\hline Sequencell & $3780005-00$ & $3780005-01$ & $3780005-02$ & $3780005-05$ & $3780020-00$ & $3780022-\infty 0$ & $3780028-00$ & $3780032-00$ & $3780032-01$ & $3780032-02$ & $3780035-\infty$ \\
\hline Latirude & 19.417 & 19.417 & 19.417 & 19.417 & 12.267 & 12.517 & 18.333 & 15.713 & 15.713 & 15.713 & 17.900 \\
\hline Longitude & -171.000 & -171.000 & -171.000 & -171.000 & -172.800 & -172850 & -173.383 & -174.050 & .174 .050 & -174.050 & -174.267 \\
\hline Dopth & 1409 & 1409 & 1409 & 1409 & 2708 & 5464 & 3861 & 5670 & 5670 & 5670 & 1749 \\
\hline$s i(w . \%)$ & 270 & $\cdot$ & . & - & $\cdot$ & - & 7.10 & 4.66 & 3.53 & 6.00 & 8.40 \\
\hline $\mathrm{Ti}$ & 1.13 & 1.54 & • & • & 1.26 & 1.38 & 1.10 & 1.00 & .80 & 1.00 & 1.10 \\
\hline Mn & 22.7 & 123 & 27.4 & 22.2 & 13.0 & 15.9 & 13.1 & 165 & 18.0 & 15.7 & 17.1 \\
\hline Fo & 13.3 & 11.7 & 16.2 & 13.2 & 16.0 & 129 & 14.6 & 14.9 & 13.9 & 13.6 & 21.7 \\
\hline Al & 1.00 & - & - & - & - & - & 230 & 155 & 1.00 & 241 & 2.30 \\
\hline Co & .950 & 1.17 & .610 & 800 & .900 & .720 & .420 & 360 & .390 & 340 & .560 \\
\hline $\mathrm{Ni}$ & .600 & .470 & .900 & 520 & 310 & 530 & 300 & 200 & .240 & .210 & .240 \\
\hline $\mathrm{Cu}$ & .150 & .060 & .140 & - & - & .430 & .170 & .100 & .110 & .140 & .030 \\
\hline C. & 224 & - & • & - & - & • & $1.5 t$ & 1.87 & 1.98 & 1.93 & 3.20 \\
\hline $\mathbf{M}$ & - & - & - & - & - & - & - & 1.07 & 98 & .91 & 1.20 \\
\hline Ne & - & - & - & - & - & - & - & - & - & - & 1.90 \\
\hline $\mathbf{K}$ & 38 & - & - & . & . & - & 52 & - & - & - & 40 \\
\hline $\mathbf{P}$ & .07 & - & - & - & - & - & • & .17 & 24 & 21 & - \\
\hline $\mathrm{H}_{2} \mathrm{O}$ & 23.9 & - & - & - & - & - & 25.2 & $\cdot$ & - & - & - \\
\hline$A x$ (ppm) & • & - & - & - & • & - & • & - & - & - & - \\
\hline Be & 5800 & 2300 & - & - & 2900 & 4300 & 6100 & - & - & - & 1100 \\
\hline Cd & - & - & - & - & • & • & - & - & - & - & • \\
\hline Co & - & - & - & - & - & - & - & - & - & - & - \\
\hline $\mathrm{Cr}$ & - & 6 & - & . & 4 & 4 & - & - & - & - & 18 \\
\hline Mo & 710 & 480 & - & - & 390 & 360 & 270 & - & - & - & 470 \\
\hline $\mathbf{P b}$ & 2300 & 700 & 2700 & - & 800 & 100 & 2200 & 1100 & - & - & 1400 \\
\hline st & 1400 & - & 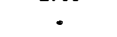 & - & $\cdot$ & - & 1200 & - & - & . & 1100 \\
\hline $\mathbf{v}$ & - & 710 & - & - & 750 & 540 & - & - & - & - & 700 \\
\hline $\mathbf{Y}$ & - & $\cdot$ & - & - & - & $\cdot$ & $\cdot$ & - & - & . & 300 \\
\hline $\mathrm{Zn}$ & 620 & - & - & - & - & - & 430 & 530 & - & - & - \\
\hline Semple & Noctulo & Noctule & Noctule & Nodule & Unknown & Nodule & Crest & Crust & Crust & Crust & Unknown \\
\hline typo & • & • & • & • & • & - & $\cdot$ & • & • & $\cdot$ & • \\
\hline Portion & $\operatorname{croses}$ & whole & 0000 & wholo & fraements & whole & outer & outer & middlo & layer-adjacent & frapments \\
\hline sampled & rection & - & ection & • & . & • & crust & crust & layex & to-mucleus & - \\
\hline Nucleus & - & • & - & - & - & - & - & • & - & - & - \\
\hline Referenos & A197 & A240 & A166 & A255 & 1240 & $A 240$ & A197 & A178 & Al78 & A178 & A261 \\
\hline Sequencell & $3780046-\infty 0$ & $3780046-01$ & $3780046-02$ & $3780046-03$ & $3780068-00$ & $3780068-01$ & $3780070-00$ & $3780070-01$ & $3780084-\infty 0$ & $3780089-01$ & $3780090-00$ \\
\hline Letitude & 17.800 & 17.800 & 17.800 & 17.800 & 17.167 & 17.167 & 17.067 & 17.067 & 19.243 & 12,000 & 17.282 \\
\hline Longitude & -174.367 & -174.367 & -174.367 & -174367 & -177.167 & -177.167 & -177.250 & -177.250 & -179.668 & -175.717 & -176.303 \\
\hline Dopth & 2288 & 2238 & 2288 & 2288 & 2050 & 2050 & 2013 & 2013 & 2870 & 5340 & 4400 \\
\hline Si(wt\%) & . & 4.90 & . & 3.00 & . & . & 4.10 & - & . & $\cdot$ & . \\
\hline $\mathrm{Ti}$ & - & .89 & .82 & 1.50 & - & .87 & 1.00 & .81 & - & - & - \\
\hline $\mathrm{Mn}$ & 35.9 & 14.4 & 11.1 & . & 35.4 & 15.0 & 13.0 & 21.0 & 18.0 & 17.7 & 17.2 \\
\hline Fo & 15.9 & 14.0 & 14.5 & - & 15.9 & 13.3 & 10.7 & 13.4 & 16.6 & 12.1 & 12.2 \\
\hline Al & - & 1.80 & $\cdot$ & .70 & $\cdot$ & - & 220 & .39 & - & 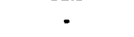 & - \\
\hline Co & .700 & .700 & .890 & 1.50 & .740 & .880 & .450 & 540 & .490 & 500 & .200 \\
\hline $\mathrm{Ni}$ & 560 & .290 & .230 & 300 & .660 & .340 & .470 & 580 & .320 & .430 & .280 \\
\hline $\mathrm{Cr}$ & .050 & .070 & .080 & .150 & .090 & .090 & .190 & .470 & .130 & .230 & .160 \\
\hline Ca & • & 6.85 & - & 7.00 & - & $\cdot$ & 8.48 & . & - & . & - \\
\hline $\mathbf{M}_{8}$ & - & - & - & 1.50 & - & - & - & - & - & - & - \\
\hline $\mathrm{Ne}$ & - & - & - & 1.50 & - & - & - & - & - & - & - \\
\hline $\mathbf{K}$ & - & .39 & - & .70 & - & . & .43 & • & - & - & - \\
\hline $\mathbf{P}$ & - & .08 & - & - & - & . & .14 & .54 & - & . & - \\
\hline $\mathrm{H}_{2} \mathrm{O}-$ & - & 19.4 & - & - & - & - & 22.5 & . & - & - & - \\
\hline$A s(p p m)$ & - & - & - & • & - & • & • & - & - & - & - \\
\hline $\mathrm{Ba}$ & - & 4300 & 1500 & 1500 & . & 2600 & 5600 & - & . & . & - \\
\hline Cd & - & - & - & - & . & - & - & - & - & - & - \\
\hline Ce & - & - & - & 1500 & - & - & - & - & - & - & - \\
\hline $\mathrm{Cr}$ & - & - & 11 & 15 & . & 36 & - & . & - & - & . \\
\hline Mo & . & 430 & 280 & 300 & - & 370 & 490 & . & 420 & - & - \\
\hline $\mathbf{P b}$ & 3000 & 2000 & 1900 & 3000 & 2700 & 1800 & 1900 & . &. & - & - \\
\hline Sr & • & 1400 & $\cdot \cdot$ & 3000 & - & - & 1700 & - & - & - & - \\
\hline $\mathbf{v}$ & - & • & 440 & 700 & - & 560 & - & - & - & - & - \\
\hline $\mathbf{Y}$ & - & - & $\cdot$ & 300 & . & - & - & - & - & - & - \\
\hline $\mathrm{Zn}$ & • & 400 & - & • & • & • & 570 & - & - & • & - \\
\hline Semple & Nodnio & Crust & Crux & Nodulo & Noctule & Crux & Crust & Unknown & Mn-conted & Nodule & Mn-contod \\
\hline typo & • & • & • & • & - & • & • & • & rock & - & rock \\
\hline Portion & cosess & crows & frugments & unknown & crows & fragmonts & cross & unknown & outser & $n$ & $n$ \\
\hline amplod & noction & rection & . & - & soction & - & roction & - & crux & $\cdot$ & - \\
\hline Nuclous & - & - & • & - & - & - & • & - & - & - & Undetrorninod \\
\hline Referenco & A166 & A197 & $A 240$ & A262 & A166 & A240 & A197 & Al65 & A352 & A389 & A249 \\
\hline
\end{tabular}


Table 9. Composition of crusts from the Scripps Nodule Databank (cont'd).

\begin{tabular}{|c|c|c|c|c|c|c|c|c|c|c|c|}
\hline Sequencell & $3780090-01$ & $3780090-04$ & $3780090-05$ & $3780090-06$ & $3780090-09$ & $3780091-00$ & $3780095-00$ & $3780096-00$ & $3780096-01$ & $3780096-02$ & $3780096-03$ \\
\hline Letitude & 17.282 & 17.282 & 17.282 & 17.282 & 17.282 & 13.115 & 19.980 & 18.517 & 18.517 & 18.517 & 18.517 \\
\hline Longitude & -176.303 & -176.303 & -176.303 & -176.303 & -176.303 & -173.988 & -170.667 & -175.100 & -175.100 & -175.100 & -175.100 \\
\hline Depth & 4400 & 4400 & 4400 & 4400 & 4400 & 5610 & 2150 & 1052 & 1052 & 1052 & 1052 \\
\hline Si(wLg) & $\cdot$ & $\cdot$ & $\cdot$ & - & $\cdot$ & 6.11 & $\cdot$ & $\cdot$ & $\cdot$ & - & $\cdot$ \\
\hline $\mathrm{Ti}$ & • & . & $\cdot$ & - & - & 84 & 1.25 & 84 & .69 & .53 & .72 \\
\hline Mn & 13.2 & 15.7 & 14.6 & 12.1 & 132 & 19.3 & 17.5 & 22.1 & 18.8 & 10.7 & 20.6 \\
\hline Fe & 20.5 & 12.0 & 10.4 & 18.8 & 10.1 & 10.6 & 15.4 & 11.0 & 12.1 & 6.55 & 10.1 \\
\hline A & $\cdot$ & 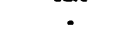 & . & 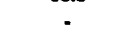 & . & 250 &. & 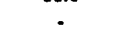 & - & - & $\because$ \\
\hline co & .370 & .420 & 250 & .340 & 350 & 300 & 580 & .830 & .650 & .180 & .940 \\
\hline $\mathrm{Ni}$ & .210 & .590 & .240 & .190 & .500 & .750 & 140 & .900 & .620 & .420 & .610 \\
\hline $\mathrm{Cu}$ & .130 & .300 & .140 & .120 & 250 & 300 & 200 & .090 & .100 & .090 & .070 \\
\hline Ca & $\cdot$ & - & $\cdot$ & $\cdot$ & - & 1.67 & - & - & $\cdot$ & $\cdot$ & $\cdot$ \\
\hline $\mathbf{M}$ & - & - & - & - & - & 1.36 & - & - & - & - & $\cdot$ \\
\hline $\mathrm{Na}$ & - & - & - & - & - & 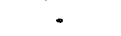 & - & - & - & - & . \\
\hline $\mathbf{K}$ & - & - & - & - & - & - & - & - & - & - & - \\
\hline $\mathbf{P}$ & - & - & - & - & - & .23 & - & - & - & - & - \\
\hline $\mathrm{H}_{2} \mathrm{O}$ & - & - & - & - & - & - & - & - & $\cdot$ & $\cdot$ & $\cdot$ \\
\hline$A x(p p m)$ & - & - & - & - & - & - & - & - & . & . & . \\
\hline $\mathrm{Ba}$ & - & - & - & - & - & - & - & - & - & - & - \\
\hline Cd & - & - & - & - & - & - & - & - & - & . & - \\
\hline co & - & - & - & . & - & - & - & - & - & - & - \\
\hline $\mathrm{Cr}$ & - & - & - & - & - & - & - & - & - & - & - \\
\hline Mo & - & - & - & - & - & - & - & - & - & - & - \\
\hline $\mathrm{Pb}$ & - & - & - & - & - & - & - & - & - & - & - \\
\hline Sr & - & - & - & - & - & - & - & - & - & - & - \\
\hline $\mathbf{v}$ & - & - & - & - & - & - & - & - & - & - & - \\
\hline $\mathbf{Y}$ & . & - & - & - & - & $\cdot$ & - & - & - & - & - \\
\hline $\mathrm{Zn}$ & • & • & • & • & • & 600 & - & - & • & • & • \\
\hline Semple & Mn-conted & Mo-conted & Mn-conted & Mn-conted & Mo-conted & Crust & Nodule & Crust & Crust & Crux & Crust \\
\hline typo & rock & rock & rock & rock & rock & • & • & - & $\cdot$ & • & $\cdot$ \\
\hline Portion & $n$ & $n$ & outor & outor & outor & average & avernge & average & outer & middle & underside \\
\hline sampled & $\cdot$ & $\cdot$ & crust & cruat & crust & malyzis & anelyzir & malyois & court & layer & $\cdot$ \\
\hline Nucleus & Undetermined & Undetermined & Undetermined & Undetermined & Undetermined & • & - & - & - & • & • \\
\hline References & A249 & A249 & A249 & A249 & A249 & A363 & A343 & A343 & A343 & A343 & A343 \\
\hline Sequenock & $3780097-00$ & $3790020-00$ & $3790020-01$ & $3790044-00$ & $3790045-00$ & $3790046-00$ & $3790046-01$ & $3800024-01$ & $3800024-02$ & $3800025-00$ & $3800025-02$ \\
\hline Latimade & 18.320 & 19.313 & 19.313 & 18.527 & 18.617 & 19.047 & 19.047 & 12.050 & 12050 & 11.950 & 11.950 \\
\hline Longitade & -178.347 & 176.735 & 176.735 & 177.187 & 171.977 & 171.148 & 171.148 & 165.000 & 165.000 & 164.983 & 164.983 \\
\hline Dopth & 1630 & 1753 & 1753 & 2000 & 2440 & 1300 & 1300 & 2090 & 2090 & 2105 & 2105 \\
\hline Si(wt.d) & . & $\cdot$ & $\cdot$ & $\cdot$ & $\cdot$ & $\cdot$ & $\cdot$ & .90 & - & 1.70 & $\cdot$ \\
\hline $\mathrm{Ti}$ & .96 & • & - & 1.20 & 1.02 & .77 & .43 & .70 & 1.18 & 1.10 & • \\
\hline Mn & 21.8 & 19.0 & - & 20.8 & 18.7 & 21.8 & 16.0 & 24.0 & 23.6 & 19.5 & 43.3 \\
\hline Fo & 14.6 & 16.2 & - & 15.1 & 16.7 & 15.8 & 5.56 & 10.5 & 15.6 & 11.5 & 13.1 \\
\hline $\mathbf{A}$ & - & • & - & - & • & • & • & 50 & .29 & .50 & • \\
\hline $\mathrm{Co}_{0}$ & .790 & .680 & - & .530 & .660 & .710 & .330 & .340 & .610 & 1.05 & 1.12 \\
\hline $\mathrm{Ni}$ & .600 & .440 & - & .410 & .350 & .490 & .670 & .750 & .610 & .420 & 1.020 \\
\hline $\mathrm{Cu}$ & .120 & .130 & - & .120 & .180 & .090 & .140 & .090 & .470 & .110 & .140 \\
\hline ca & - & 250 & - & $\cdot$ & $\cdot$ & $\cdot$ & $\cdot$ & 2.20 & $\cdot$ & 1.60 & $\cdot$ \\
\hline $\mathbf{M g}_{8}$ & - & . & - & - & - & - & . & 1.60 & . & . & - \\
\hline $\mathrm{Na}$ & - & . & - & - & - & . & . & 2.30 & - & - & - \\
\hline $\mathrm{K}$ & . & . & - & . & - & - & - & . & - & .20 & - \\
\hline $\mathbf{P}$ & - & - & - & - & - & - & - & - & .41 & - & - \\
\hline $\mathrm{H}_{2} \mathrm{O}$ & $\cdot$ & - & - & - & $\cdot$ & $\cdot$ & $\cdot$ & $\cdot$ & $\cdot$ & - & $\cdot$ \\
\hline As(ppon) & - & - & 203 & . & - & - & - & • & - & • & - \\
\hline $\mathrm{Ba}$ & - & - & 2000 & - & - & - & - & 2100 & - & 1500 & - \\
\hline Cd & - & - & 24.0 & - & - & - & - & • & - & - & - \\
\hline$c_{0}$ & - & - & $\cdot$ & - & - & - & - & - & $\cdot$ & - & - \\
\hline $\mathrm{Cr}$ & - & - & 13 & - & - & - & - & - & - & $\cdot$ & - \\
\hline Mo & - & - & . & - & - & - & - & 700 & - & 390 & - \\
\hline $\mathrm{Pb}$ & - & 1500 & - & - & - & - & . & 1800 & - & 1500 & 2600 \\
\hline Sr & - & $\cdot$ & - & - & - & - & - & 1900 & - & 1100 & $\cdot$ \\
\hline $\mathrm{v}$ & - & - & - & - & - & - & - & 700 & - & $\cdot$ & - \\
\hline $\mathbf{Y}$ & - & - & - & - & - & - & . & 570 & - & - & - \\
\hline $\mathrm{Zn}$ & • & 600 & • & - & - & - & - & 600 & - & • & - \\
\hline Semplo & Crust & Crust & Crus & Mn-conted & Nodule & Mn-conted & Mn-costed & Crust & Nodule & Unknown & Nodule \\
\hline typo & - & - & 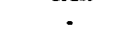 & sock & - & rock & rock & 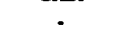 & - & - & - \\
\hline Portion & average & fragments & fragments & outer & average & topside & underside & outer & unicoown & unibown & croses \\
\hline ampled & analyois & - & . & crost & malysis & . & - & $\cos x$ & - & - & soction \\
\hline Nuclous & • & • & - & - & - & • & - & - & • & - & 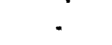 \\
\hline Reforence & $A 343$ & A405 & A475 & $A 343$ & A343 & A343 & A343 & A261 & A165 & A197 & Al66 \\
\hline
\end{tabular}


Table 9. Composition of crusts from the Scripps Nodule Databank (cont'd).

\begin{tabular}{|c|c|c|c|c|c|c|c|c|c|c|c|}
\hline Sequence: & $3800025-06$ & $3800027-00$ & $3800027-01$ & $3800031-00$ & $3800031-01$ & $3800084-01$ & $3800085-00$ & $3810070-00$ & $3810070-01$ & $3810070-02$ & $3820417-00$ \\
\hline Latinude & 11.950 & 12.050 & 12.050 & 12.150 & 12.150 & 11.800 & 11.950 & 16.892 & 16.892 & 16.892 & 17.445 \\
\hline Longitude & 164.983 & 164.950 & 164.950 & 164.683 & 164.683 & 165.000 & 164.950 & 155.133 & 155.133 & 155.133 & 144565 \\
\hline Depth & 2105 & 2105 & 2105 & 1830 & 1830 & 1620 & 1330 & 5775 & 5775 & 5775 & 3857 \\
\hline Si(wt\%) & 260 & $\cdot$ & 4.10 & 280 & - & . & $\cdot$ & $\cdot$ & $\cdot$ & - & $\cdot$ \\
\hline $\mathrm{Ti}$ & 1.70 & • & 1.20 & 1.70 & 1.35 & • & • & - & $=$ & • & - \\
\hline Mn & 29.7 & 38.4 & 25.9 & 29.7 & 194 & 45.5 & 50.3 & 16.7 & 18.0 & 18.0 & 5.00 \\
\hline Po & 17.5 & 16.0 & 21.0 & 18.9 & 13.5 & 13.0 & 15.8 & 15.7 & 16.0 & 16.0 & 11.0 \\
\hline N & .70 & - & 1.10 & .70 & . & 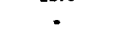 & 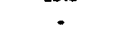 & - & 208 & . & 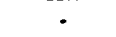 \\
\hline Co & 1.60 & 840 & 1.10 & 1.30 & 1.60 & .790 & 1.50 & .540 & 500 & .500 & .010 \\
\hline $\mathrm{NI}$ & .064 & .720 & .600 & .630 & .520 & 1.000 & .880 & .220 & .220 & .220 & .030 \\
\hline $\mathrm{Cr}$ & .160 & .110 & .170 & .180 & .040 & .120 & .090 & .230 & .140 & .140 & .050 \\
\hline C. & 240 & - & 2.00 & 2.40 & • & - & - & 1.90 & 1.96 & - & 2.40 \\
\hline $\mathbf{M}$ & 1.50 & - & 1.50 & 1.30 & - & - & - & $\cdot$ & 1.14 & - & - \\
\hline $\mathrm{Ne}$ & 230 & - & 200 & 1.90 & - & - & - & - & 1.70 & - & - \\
\hline $\mathbf{K}$ & 30 & - & 30 & 30 & - & - & - & - & .60 & - & - \\
\hline $\mathbf{P}$ & - & - & - & - & - & - & - & - & .23 & - & . \\
\hline $\mathrm{H}_{2} \mathrm{O}$ & - & $\cdot$ & $\cdot$ & $\cdot$ & $\cdot$ & $\cdot$ & - & $\cdot$ & • & - & $\cdot$ \\
\hline$A s$ (ppm) & - & . & - & - & - & - & - & - & - & - & - \\
\hline Ba & 2200 & - & 1400 & 1700 & 5100 & - & - & - & - & - & - \\
\hline Cd & - & . & - & - & - & - & - & - & - & - & - \\
\hline$c_{0}$ & - & - & - & - & - & . & . & - & - & - & - \\
\hline c. & . & . & . & - & - & - & - & . & - & . & . \\
\hline Mo & 590 & - & 420 & 590 & 470 & - & • & - & 320 & - & - \\
\hline $\mathbf{P b}$ & 2300 & 2900 & 3200 & 1600 & 2500 & 2500 & 3100 & 900 & 1000 & - & 200 \\
\hline Sr & 1700 & • & 1600 & 1300 & - & - & - & - & • & - & • \\
\hline $\mathbf{v}$ & 700 & - & 800 & 700 & 440 & - & - & - & - & - & - \\
\hline $\mathbf{Y}$ & 250 & - & 200 & 230 & $\cdot$ & - & - & - & - & - & - \\
\hline $\mathrm{Zn}$ & - & • & 500 & 500 & - & • & • & 7300 & • & • & 400 \\
\hline Sample & Cout & Noctule & Crust & Cout & Crust & Nodule & Nodule & Noctule & Noctule & Natulo & Coust \\
\hline typo & • & $\cdot$ & $\cdot$ & $\cdot$ & $\cdot$ & $\cdot$ & • & $\bullet$ & - & $\cdot$ & $\cdot$ \\
\hline Portion & fragments & crosen & fragments & fragments & fragments & $\cos 0$ & arous & quartor & unknown & unlonown & whole \\
\hline semplod & - & section & $\cdot$ & 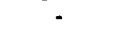 & $\cdot$ & soction & section & $\cdot$ & • & - & • \\
\hline Nucleus & - & • & - & $\cdot$ & - & - & - & Updeterminod & - & - & - \\
\hline References & $A 261$ & A166 & A261 & A261 & $A 240$ & A166 & A166 & A40S & A419 & A419 & A405 \\
\hline Sequencell & $3820533-00$ & $3830052-00$ & $3830063-00$ & $3830210-00$ & $3830211-\infty 0$ & $3830273-00$ & $3830273-01$ & $3840299-00$ & 3920032.00 & $3980907-00$ & $3990010-02$ \\
\hline Latinude & 12.283 & 15.533 & 18.953 & 19.667 & 19.667 & 13.313 & 13.313 & 18.613 & 12.728 & 26.517 & 25.750 \\
\hline Longitude & 144.533 & 136.400 & 135.793 & 135.950 & 135.950 & 134.560 & 134.560 & 128.130 & 47.373 & -11.775 & .20 .233 \\
\hline Depth & 6125 & 4184 & 3195 & 1717 & 2678 & 2010 & 2010 & 4550 & 1170 & 1600 & 2791 \\
\hline Si(wt.q) & $\cdot$ & $\cdot$ & - & . & $\cdot$ & $\cdot$ & $\cdot$ & . & 4.40 & $\cdot$ & 2.80 \\
\hline $\mathrm{Ti}$ & • & .80 & - & - & - & • & • & - & .43 & .74 & 1.30 \\
\hline $\mathbf{M n}$ & 11.8 & 15.7 & 19.5 & 17.2 & 15.6 & 12.3 & 13.9 & 129 & 17.7 & 12.3 & 21.5 \\
\hline Fo & 19.6 & 24.6 & 13.8 & 20.4 & 19.8 & 15.7 & 173 & 17.6 & 16.6 & 18.8 & 25.9 \\
\hline $\mathbf{N}$ & $\cdot$ & 234 & - & - & - & - & $\cdot$ & - & - & 1.48 & 1.40 \\
\hline$C_{0}$ & .280 & 310 & 540 & 500 & 380 & .200 & .240 & .180 & .460 & .550 & .680 \\
\hline $\mathrm{Ni}$ & 260 & .190 & .520 & 280 & .280 & .260 & .270 & 280 & 370 & .240 & 310 \\
\hline $\mathrm{Cn}$ & .230 & .140 & .100 & .050 & .140 & .110 & .120 & .140 & .020 & .040 & .050 \\
\hline c. & - & 1.12 & - & - & • & $\cdot$ & - & - & . & 1.70 & 3.40 \\
\hline $\mathbf{M}_{\mathbf{8}}$ & - & .88 & - & - & - & - & - & - & - & - & 1.40 \\
\hline $\mathrm{Ne}$ & - & 282 & - & - & - & - & - & - & - & - & 1.60 \\
\hline $\mathbf{K}$ & - & .60 & . & - & - & - & - & - & - & - & - \\
\hline $\mathbf{P}$ & - & .27 & - & - & - & - & - & - & - & - & - \\
\hline $\mathrm{H}_{2} \mathrm{O}$ & - & - & - & - & - & - & - & - & - & - & - \\
\hline$A x$ (ppm) & - & - & - & - & - & - & - & - & - & - & - \\
\hline Be & - & - & 690 & . & - & - & - & - & - & - & 1200 \\
\hline Cd & - & - & $\cdot$ & . & - & . & - & - & - & 10.0 & $\cdot$ \\
\hline $\mathrm{Co}_{0}$ & - & - & - & - & - & - & - & - & . & • & - \\
\hline$C r$ & - & $\cdot$ & - & - & - & - & - & - & 27 & 150 & 11 \\
\hline Mo & - & 360 & - & - & - & • & - & - & 660 & • & 560 \\
\hline $\mathbf{P b}$ & $\cdot$ & 1500 & - & . & - & 1000 & 1200 & - & 1300 & 2600 & 800 \\
\hline Sr & . & • & - & - & - & • & - & - & $\cdot$ & - & $\cdot$ \\
\hline $\mathbf{v}$ & - & - & - & - & . & - & - & - & 900 & - & 1100 \\
\hline $\mathbf{Y}$ & . & - & - & . & - & - & - & - & $\cdot$ & $\cdot$ & 1400 \\
\hline$Z_{n}$ & - & - & 700 & - & - & 450 & 550 & $\cdot$ & 460 & 580 & - \\
\hline Sample & Mn-contiod & Mn-eacrustod & Nodule & Mn-coated & Crus & Crust & Crust & Crust & Crux & Unknown & Unknown \\
\hline type & rock & sediment & $\cdot$ & orgunic & $\cdot$ & - & - & - & • & - & . \\
\hline Portion & unknown & unknown & unknown & unknown & fragments & unknown & unionown & unknown & unknown & unknown & unibrows \\
\hline sampled & - & - & • & - & - & - & • & • & $\cdot$ & - & - \\
\hline Nuclous & • & • & - & • & • & • & • & • & • & $\cdot$ & - \\
\hline Roforences & A333 & A419 & A323 & A333 & A333 & A343 & A343 & A343 & A195 & A467 & A261 \\
\hline
\end{tabular}


Table 9. Composition of crusts from the Scripps Nodule Databank (cont'd).

\begin{tabular}{|c|c|c|c|c|c|c|c|c|c|c|c|}
\hline Sequencell & $3990101-\infty 0$ & $3990102-00$ & $3990103-\infty 0$ & $4010035-03$ & $4010046-00$ & $4010064-00$ & $4010081-\infty$ & $4010081-01$ & $4010090-\infty$ & $4010103-00$ & $4020004-00$ \\
\hline Latitude & 26.067 & 26.008 & 28.875 & 23.367 & 26.333 & 24.517 & 23.768 & 23.768 & 23.950 & 24.390 & 24.778 \\
\hline Longitude & .22 .367 & .22367 & -25.442 & -43.650 & -44.850 & -45.517 & -46.617 & -46.617 & -47.483 & 48.990 & .50 .435 \\
\hline Depth & 2300 & 2286 & 4648 & 4565 & 2500 & 3400 & 3155 & 3155 & 4124 & 5396 & 5726 \\
\hline si(wt.\%) & $\cdot$ & - & $\cdot$ & $\cdot$ & $\cdot$ & $\cdot$ & . & $\cdot$ & $\cdot$ & $\cdot$ & - \\
\hline $\mathbf{T i}$ & - & • & - & - & $\cdot$ & - & + & - & - & - & - \\
\hline Mn & 18.1 & 16.3 & 17.6 & 11.8 & 10.5 & 8.95 & 10.4 & 112 & 7.88 & 13.0 & 14.0 \\
\hline $\mathbf{P e}$ & 25.7 & 243 & 21.8 & 13.5 & 19.1 & 26.2 & 19.0 & 24.8 & 29.3 & 23.9 & 18.8 \\
\hline $\boldsymbol{N}$ & • & - & - & • & - & • & - & - & - & - & - \\
\hline Co & .600 & .470 & .410 & .880 & .340 & .250 & 380 & $\mathbf{4 1 0}$ & .210 & .260 & 360 \\
\hline $\mathrm{Ni}$ & .310 & 250 & .260 & .330 & .110 & .120 & .180 & .150 & .140 & .230 & .300 \\
\hline $\mathrm{Cr}$ & .090 & .070 & .130 & .070 & .050 & .090 & .120 & .080 & .130 & .090 & .150 \\
\hline C. & • & 2.18 & 1.72 & • & $A$ & 3.86 & • & 6.25 & 1.51 & 1.38 & - \\
\hline Mg & - & 1.35 & 1.25 & - & 1.21 & 1.56 & - & 1.35 & 2.17 & 1.68 & - \\
\hline Na & - & 1.53 & 1.56 & - & 2.37 & 1.56 & - & .82 & 2.02 & 1.89 & - \\
\hline $\mathbf{K}$ & - & .24 & 37 & - & 26 & 30 & - & .19 & 1.54 & .61 & - \\
\hline $\mathbf{P}$ & $\cdot$ & - & - & - & - & • & - & • & - & - & - \\
\hline $\mathrm{H}_{2} \mathrm{O}$ & - & $\cdot$ & - & - & $\cdot$ & - & $\cdot$ & $\cdot$ & - & $\cdot$ & - \\
\hline$A=(p p m)$ & - & $\cdot$ & - & - & - & - & - & - & - & - & - \\
\hline Ba & - & - & - & - & - & - & - & - & - & - & - \\
\hline Cd & - & - & - & - & - & - & • & - & - & - & - \\
\hline Co & - & - & - & - & - & - & - & - & - & - & - \\
\hline $\mathrm{Cr}$ & ' & - & - & - & - & - & :- & - & - & - & - \\
\hline Mo & $\cdot$ & • & - & - & • & - & - & $\cdot$ & $\cdot$ & - & - \\
\hline $\mathbf{P b}$ & - & 2200 & 1500 & - & 1100 & 700 & - & 900 & 700 & 1000 & - \\
\hline Sr & - & • & - & - & - & • & - & • & - & - & - \\
\hline $\mathbf{v}$ & - & - & - & - & - & - & - & - & - & - & - \\
\hline$Y$ & - & • & - & - & • & $\cdot$ & - & - & $\cdot$ & $\cdot$ & - \\
\hline $\mathbf{Z n}$ & • & 620 & 590 & • & 410 & 530 & • & 510 & 550 & 490 & - \\
\hline Semplo & Nodule & Crat & Cruat & Micronodules & Crust & Crust & Crust & Crant & Crust & Cruart & Crant \\
\hline typo & $\cdot$ & $\cdot$ & - & $\cdot$ & $\cdot$ & • & - & $\cdot$ & - & - & - \\
\hline Portion & unkorown & unkoown & unkerown & unkrowa & unknown & unknown & unkhown & untrnown & untenown & unkoown & unknown \\
\hline sumpled & • & $\cdot$ & - & - & $\cdot$ & $\cdot$ & - & - & - & • & $\cdot$ \\
\hline Nucleus & • & • & • & - & • & - & $\cdot$ & • & • & • & - \\
\hline Reference & A354 & $A 368$ & $A 368$ & A419 & A368 & $A 368$ & A179 & $A 368$ & A368 & A368 & A179 \\
\hline Sequenced & $4020004-01$ & $4020045-01$ & $4020058-00$ & $4020070-00$ & $4020092-00$ & $4020094-00$ & $4030605-\infty 0$ & $4030014-\infty$ & $4030109-01$ & $4040109-02$ & $4040232-00$ \\
\hline Latinude & 24.778 & 21.850 & 29.283 & 26.430 & 27.417 & 26.583 & 25.967 & 27.433 & 20.817 & 22453 & 24.742 \\
\hline Longinsde & -50.435 & -56.150 & -57.383 & -58.630 & -59.717 & -59.867 & -60.317 & -61.300 & -66.917 & -71.602 & .73 .777 \\
\hline Depth & 5726 & 4049 & 5840 & 5141 & 5842 & 5297 & 5868 & 5762 & 4878 & 5192 & 5312 \\
\hline Si(wt.q) & - & $\cdot$ & 6.30 & - & $\cdot$ & - & - & • & - & - & $\cdot$ \\
\hline $\mathrm{Ti}$ & - & • & .65 & - & - & - & - & - & $\cdot$ & - & - \\
\hline Mn & 13.9 & 15.0 & 16.2 & 20.0 & 15.4 & 14.0 & 125 & 16.0 & 13.0 & 42.5 & 16.4 \\
\hline $\mathbf{F e}$ & 25.1 & 18.9 & 15.9 & 944 & 16.6 & 18.2 & 20.8 & 17.0 & 29.3 & 15.0 & 21.7 \\
\hline $\mathbf{A} \mathbf{l}$ & - & • & 4.30 & - & 1.14 & 95 & - & - & • & • & - \\
\hline Co & .330 & .270 & .540 & .240 & .390 & 280 & 560 & 530 & .240 & 3.00 & .070 \\
\hline $\mathrm{Ni}$ & .270 & .310 & .490 & 1.560 & .340 & .180 & $\$ 40$ & .410 & .160 & .900 & .220 \\
\hline $\mathrm{Cr}$ & .130 & .120 & .280 & .410 & .090 & .090 & .600 & .180 & .140 & .800 & .200 \\
\hline C. & 1.42 & 238 & $A 1$ & $\cdot$ & • & - & • & $\cdot$ & 1.59 & - & 2.08 \\
\hline $\mathbf{M}_{8}$ & 1.51 & 2.03 & - & - & - & - & - & $\cdot$ & 1.36 & - & 2.78 \\
\hline $\mathrm{Na}$ & 1.86 & 3.96 & - & - & - & - & - & $\cdot$ & 1.59 & - & 2.85 \\
\hline $\mathbf{K}$ & .44 & .47 & 51 & - & . & - & - & - & 35 & - & .74 \\
\hline $\mathbf{P}$ & • & - & .10 & - & - & - & - & - & - & - & - \\
\hline $\mathrm{H}_{2} \mathrm{O}$ & $\cdot$ & $\cdot$ & 18.1 & - & $\cdot$ & $\cdot$ & $\cdot$ & $\cdot$ & $\cdot$ & $\cdot$ & $\cdot$ \\
\hline$A s(p p m)$ & $\cdot$ & - & - & - & - & - & - & - & - & - & - \\
\hline Be & $\cdot$ & $\cdot$ & 6900 & - & - & - & $\cdot$ & - & - & - & - \\
\hline Cd & $\cdot$ & - & - & - & - & - & - & - & - & - & - \\
\hline co & - & $\cdot$ & - & - & - & - & - & - & - & - & - \\
\hline $\mathrm{Cr}$ & - & - & $\cdot$ & - & - & - & - & - & $\cdot$ & - & - \\
\hline Mo & - & - & 310 & - & - & - & - & - & • & - & $\cdot$ \\
\hline $\mathbf{P b}$ & 1000 & 900 & 1300 & - & - & - & $\cdot$ & - & 1100 & $\cdot$ & 600 \\
\hline Sr & - & - & 770 & - & - & - & - & $\cdot$ & • & - & $\cdot$ \\
\hline $\mathbf{v}$ & - & - & - & - & - & - & - & - & - & - & - \\
\hline $\mathbf{Y}$ & - & - & - & - & • & - & - & - & $\cdot$ & - & • \\
\hline $\mathbf{Z n}$ & 500 & 490 & 670 & - & 400 & 460 & $\cdot$ & $\cdot$ & 720 & - & 670 \\
\hline Sample & Cruat & Cruat & Nodulo & Crust & Const & Cruat & Nodulo & Micronodulos & Crust & Notule & Const \\
\hline typo & • & $\cdot$ & - & $\cdot$ & - & - & - & $\cdot$ & $\cdot$ & $\cdot$ & $\cdot$ \\
\hline Partion & unknowa & unknown & outer & uninown & unknown & outer & uninown & unlnown & unknown & unknown & unknown \\
\hline sarmplod & $\cdot$ & - & const & - & - & crust & $\cdot$ & - & - & - & • \\
\hline Nucleus & - & • & • & $\cdot$ & $\cdot$ & • & • & - & - & - & $\cdot$ \\
\hline References & A368 & A368 & A197 & A179 & A333 & A333 & A179 & A419 & $A 368$ & A419 & $A 368$ \\
\hline
\end{tabular}


Table 9. Composition of crusts from the Scripps Nodule Databank (cont'd).

\begin{tabular}{|c|c|c|c|c|c|c|c|c|c|c|c|}
\hline Sequencell & $4040837-00$ & $4040839-00$ & $4040840-00$ & $4070161-01$ & $4070166-00$ & $4070166-01$ & $4070195-02$ & $4070216-\infty 0$ & $4070216-01$ & $4070217-\infty 0$ & $4070218-00$ \\
\hline Latitude & 27.233 & 26.875 & 26.883 & 22.833 & 22.817 & 22.817 & 22838 & 21.450 & 21.450 & 23.050 & 20.150 \\
\hline Longituds & -76.433 & .76 .633 & -76.637 & -109.278 & -109.242 & -109.242 & -109.753 & -108300 & -108.300 & -108.750 & -109.000 \\
\hline Dopth & 4710 & 1535 & 1545 & 1125 & 846 & 846 & 1326 & 1540 & 1540 & $\cdot$ & 1900 \\
\hline Si(wt.\%) & $\cdot$ & $\cdot$ & . & $\cdot$ & - & - & - & - & $\cdot$ & - & $\cdot$ \\
\hline $\mathrm{Ti}$ & - & - & - & • & - & - & - & . & . & - & - \\
\hline Mn & 9.30 & 13.9 & 15.3 & 14.7 & 13.0 & 16.3 & 13.7 & 15.1 & 12.3 & 29.2 & 23.8 \\
\hline Fo & 213 & 24.0 & 24.0 & 13.0 & 13.3 & 15.3 & 16.0 & 22.2 & 13.3 & 10.8 & 13.0 \\
\hline $\mathbf{A}$ & - & - & - & - & - & - & - & - & - & - & - \\
\hline Co & 1.03 & 540 & .460 & .180 & .170 & .180 & .220 & 350 & .190 & .140 & .160 \\
\hline $\mathbf{N i}$ & .160 & .220 & .260 & .180 & .200 & .270 & .180 & .150 & .180 & 500 & 390 \\
\hline Cu & .180 & .060 & .080 & .010 & .020 & .020 & .010 & .060 & .080 & .090 & .090 \\
\hline c. & . & 3.65 & 2.33 & 1.40 & 1.40 & 1.70 & 1.70 & 1.60 & 130 & 1.40 & 1.30 \\
\hline $\mathbf{M g}_{\mathbf{g}}$ & - & 1.63 & 1.67 & - & - & - & . & . & - & - & - \\
\hline Na & - & 1.05 & 1.19 & - & - & - & - & - & - & - & - \\
\hline $\mathbf{K}$ & . & .21 & 24 & - & - & - & - & - & - & - & - \\
\hline $\mathbf{P}$ & . & - & - & - & - & - & - & - & - & - & - \\
\hline $\mathrm{H}_{2} \mathrm{O}$ & - & - & - & - & - & - & - & - & - & - & - \\
\hline$A \varepsilon$ (ppm) & - & - & - & - & - & - & - & - & - & - & . \\
\hline Ba & - & - & - & - & - & - & - & - & - & - & - \\
\hline Cd & - & - & - & - & - & . & - & - & - & - & - \\
\hline co & - & - & - & - & - & - & - & - & - & - & - \\
\hline $\mathrm{Cr}$ & . & - & - & - & - & - & - & - & - & - & - \\
\hline Mo & . & - & - & - & - & - & • & - & - & - & - \\
\hline Pb & 600 & 1300 & 1400 & 1000 & 1200 & 1300 & 1500 & 1400 & 900 & 300 & 400 \\
\hline St & • & - & - & - & - & - & - & - & . & - & • \\
\hline $\mathbf{v}$ & - & - & - & - & - & - & . & - & - & - & - \\
\hline $\mathbf{Y}$ & • & - & - & - & - & - & - & - & • & - & - \\
\hline $\mathbf{Z n}_{0}$ & 500 & 530 & 600 & 400 & 400 & 500 & 300 & 500 & 300 & 1100 & 1000 \\
\hline Sample & Nodulo & Crust & Crust & Coust & Crust & Crust & Crust & Soveral & Severd & Soveral & Soveral \\
\hline typo & • & • & - & - & - & $\cdot$ & - & crostos & crostes & crust & cousts \\
\hline Partion & unknown & unknown & monown & croses & fragments & frapments & frapments & fragments & frapments & frapments & fragments \\
\hline samplod & $\cdot$ & . & - & section & 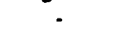 & 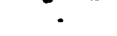 & 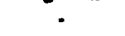 & 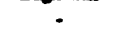 & 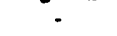 & - & - \\
\hline Nuclew & • & - & • & • & - & - & - & - & - & - & - \\
\hline References & A272 & A368 & A368 & A422 & $A 422$ & $\mathbf{A 4 2}$ & 1422 & A437 & A437 & A437 & A437 \\
\hline Sequencell & $4070225-00$ & $4080106-00$ & $4080106-01$ & $4080121-05$ & $4080121-06$ & $4080253-\infty 0$ & $4080253-01$ & $4080253-02$ & $4080306-\infty 0$ & $4080310-01$ & $4080310-02$ \\
\hline Latitude & 20.850 & 20.750 & 20.750 & 21.800 & 21.800 & 20517 & 29.517 & 29517 & 27.717 & 25.250 & 25.250 \\
\hline Langitude & -109.217 & -112783 & -112.783 & -113.050 & -113.050 & -117.283 & -117.283 & -117.283 & -119.283 & -119.667 & -119.667 \\
\hline Depth & 1825 & 1711 & 1711 & 3450 & 3450 & 820 & 820 & 820 & 1310 & 64 & 64 \\
\hline Si(wt.\%) & . & $\cdot$ & . & . &. &. & . & 1.10 & 7.90 & . & 1.70 \\
\hline Ti & • & - & - & - & - & - & 84 & $\cdot$ & .70 & • & .60 \\
\hline Mn & 10.2 & 18.8 & 19.0 & 30.0 & 24.5 & 42.2 & 17.3 & 21.1 & 17.7 & 424 & 29.7 \\
\hline $\mathrm{Pe}$ & 23.7 & 22.1 & 21.2 & 5.90 & 123 & 14.4 & 12.8 & 15.6 & 23.1 & 13.5 & 19.6 \\
\hline Al & . & . & $\cdot$ & - & . & 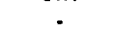 & . & 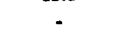 & 2.10 & . & 1.00 \\
\hline Co & 240 & • & - & .090 & .180 & .650 & .920 & .730 & .430 & .680 & 900 \\
\hline $\mathbf{N i}$ & .080 & .250 & .180 & .700 & .660 & .660 & 200 & .220 & .200 & .680 & 520 \\
\hline Cu & .070 & .080 & .010 & .500 & 480 & .090 & .030 & .040 & .010 & .040 & .070 \\
\hline c. & 1.40 & - & - & - & - & . & - & 214 & 1.60 & - & 3.10 \\
\hline Mg & - & - & - & - & - & - & - & - & 1.40 & - & 2.10 \\
\hline $\mathrm{Na}$ & . & . & - & - & - & - & - & - & 3.50 & - & 250 \\
\hline $\mathbf{K}$ & - & - & - & - & . & - & - & .20 & 30 & - & .40 \\
\hline $\mathbf{P}$ & - & - & - & . & - & - & - & 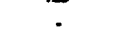 & - & - & 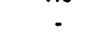 \\
\hline $\mathrm{H}_{2} \mathrm{O}$ & - & . & - & - & - & - & - & - & - & - & - \\
\hline Axppm) & - & - & . & - & . & . & - & - & - & - & - \\
\hline $\mathrm{Ba}$ & - & - & - & 3700 & 4400 & - & $\$ 200$ & 8800 & 1100 & . & 1100 \\
\hline cd & . & . & - & 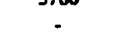 & - & . & - & 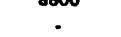 & . &. & . \\
\hline co & . & . & - & . & . & . & - & . & - & . & . \\
\hline $\mathrm{Cr}$ & - & - & - & 15 & 33 & . & 15 & . & 14 & . & - \\
\hline Mo & • & - & - & . & - & • & 750 & - & 470 & - & 1500 \\
\hline Pb & $11 \infty$ & - & - & . & - & 5000 & 3100 & - & 1000 & 3400 & 1500 \\
\hline St & - & - & - & - & . & . & . & 1800 & 1300 & 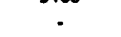 & 1100 \\
\hline v & - & - & . & - & - & - & 670 & . & 700 & . & 1100 \\
\hline $\mathbf{Y}$ & . & - & - & . & . & - & - & - & 200 & - & 260 \\
\hline $\mathbf{Z n}_{\mathbf{n}}$ & 500 & - & - & 2000 & 1600 & - & - & - & . & • & - \\
\hline Sempiplo & Several & Crust & Crust & Cun & Crust & Nodulo & Soveral & Crust & Crust & Nodule & Nodule \\
\hline typo & crusts & - & - & - & • & - & crusts & - & • & - & • \\
\hline Partion & fragments & underside & topside & unkpown & unknown & cross & cormposito & unknown & croses & croses & ouver \\
\hline ampled & - & • & - & - & • & soction & $>12$ crusts & - & soction & soction & crust \\
\hline Nuclous & • & - & - & - & • & - & - & • & - & • & - \\
\hline Roferences & A437 & A 342 & A342 & A323 & A323 & A166 & A189 & A197 & A261 & A166 & A261 \\
\hline
\end{tabular}


Table 9. Composition of crusts from the Scripps Nodule Databank (cont'd).

\begin{tabular}{|c|c|c|c|c|c|c|c|c|c|c|c|}
\hline Sequencell & $4080336-00$ & 4080336-01 & $4080342-00$ & $4080386-00$ & $4080399-00$ & $4080464-00$ & $4090002-04$ & $4090002-05$ & $4090021-00$ & $4100004-00$ & $4100063-01$ \\
\hline Latinude & 20.883 & 20.883 & 21.100 & 20.500 & 20.517 & 22.000 & 21.450 & 21.450 & 23.717 & 20.067 & 23.283 \\
\hline Longitude & -114.083 & -114.083 & -114.150 & -114.350 & -114.533 & -114.000 & -126.717 & -126.717 & -124.100 & -130.067 & -138.250 \\
\hline Depth & 2560 & 2560 & 3560 & 3790 & 3100 & 3400 & 4300 & 4300 & 3787 & 4959 & 4890 \\
\hline Si(wt\%) & $\cdot$ & $\cdot$ & . & - & . & . & 7.00 & 215 & $\cdot$ & . & 6.50 \\
\hline $\mathbf{T i}$ & - & - & - & - & - & .30 & 1.10 & .40 & - & - & 1.61 \\
\hline Mn & 18.4 & $20 A$ & 25.9 & 17.6 & 13.1 & 26.5 & 24.6 & 7.00 & 6.10 & 25.0 & 15.9 \\
\hline Pe & 20.4 & . & 10.9 & 16.8 & 19.6 & 9.50 & 18.2 & 7.90 & 11.7 & 6.10 & 14.1 \\
\hline A & . & .79 & - & - & . & . & 230 & 6.30 & 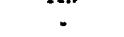 & - & 250 \\
\hline Co & - & 350 & 590 & - & - & 1.000 & 500 & .070 & .230 & 360 & 520 \\
\hline $\mathrm{Ni}$ & .310 & 330 & 1.020 & 370 & 260 & 1.250 & 460 & 390 & $A 50$ & 1.040 & 350 \\
\hline Ca & .030 & .040 & .670 & .120 & .160 & .250 & 360 & .280 & 210 & 1.000 & 200 \\
\hline ce & • & • & 1.40 & • & - & 1.40 & 1.80 & .60 & .90 & - & 1.35 \\
\hline $\mathrm{Mg}_{\mathrm{g}}$ & - & - & 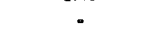 & - & - & $\cdot$ & 1.30 & 1.60 & • & - & . \\
\hline $\mathrm{Na}$ & - & - & - & - & - & - & 200 & 3.50 & - & - & - \\
\hline $\mathbf{K}$ & - & - & - & - & - & .30 & .70 & 280 & . & - & .62 \\
\hline P & - & - & . & . & - & . & . & . & - & - & - \\
\hline $\mathrm{H}_{2} \mathrm{O}$ & - & - & - & - & - & - & - & - & - & - & 21.9 \\
\hline As(ppm) & - & - & - & - & . & . & - & - & - & - & - \\
\hline $\mathrm{Ba}$ & - & - & - & - & - & . & 1600 & 700 & - & 3000 & 7900 \\
\hline Cd & - & 3.0 & - & . & - & . & - & - & - & - & - \\
\hline co & . & . & - & . & . & . & . & - & . & . & - \\
\hline $\mathrm{Cr}$ & - & - & . & . & . & . & - & 15 & . & 23 & - \\
\hline Mo & - & 590 & • & . & - & - & 610 & 100 & $\cdot$ & . & 280 \\
\hline $\mathbf{P b}$ & - & 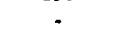 & 500 & . & - & - & 1500 & 500 & 900 & . & 1700 \\
\hline Sr & - & - & - & - & - & - & 900 & 420 & - & . & 1100 \\
\hline $\mathbf{v}$ & - & 930 & - & - & - & - & 600 & 190 & - & - & 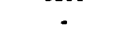 \\
\hline $\mathbf{Y}$ & - & - & - & - & - & - & 170 & 63 & - & - & - \\
\hline $\mathrm{Zn}$ & • & 530 & 1600 & - & • & 1500 & - & ? & 650 & 1100 & 640 \\
\hline Samplo & Crust & Crux & Noctule & Cour & Crust & Nodule & Noctule & Crust & Crust & Crost & Nodule \\
\hline type & - & - & - & - & $\cdot$ & - & • & - & - & - & - \\
\hline Partion & unknown & unknown & querter & fragments & quartor & unkmown & outer & cross & fragments & unknown & cross \\
\hline anoplod & - & - & - & - & . & . & crust & section & - & . & ection \\
\hline Nucleus & - & - & Noctulo-fragment & - & - & - & - & - & • & - & - \\
\hline References & A298 & $A 303$ & A422 & A298 & A298 & A381 & A261 & A261 & A376 & A323 & A197 \\
\hline Sequencel & $4120320-01$ & $4120323-00$ & $4120379-00$ & $4120379-01$ & $4120380-00$ & $4120382-00$ & $4120382-01$ & $4120383-00$ & $4120383-01$ & $4120383-02$ & $4120389-00$ \\
\hline Latinde & 28.400 & 23.017 & 21.617 & 21.617 & 21.892 & 21.925 & 21.925 & 21.833 & 21.833 & 21.833 & 21.718 \\
\hline Lengitude & -159.183 & -159.350 & -158.688 & -158.688 & -158.767 & -158.550 & -158.550 & -158.617 & -158.617 & -158.617 & -158.700 \\
\hline Depth & 5676 & 4856 & 1680 & 1680 & 1495 & 1330 & 1330 & 1255 & 1255 & 1255 & 700 \\
\hline si(wt.\%) & - & $\cdot$ & 4.41 & . & . & 4.57 & 4.02 & 6.31 & 1.75 & 246 & 4.00 \\
\hline Ti & - & - & 266 & - & - & 1.46 & 2.19 & 201 & .80 & 1.02 & 290 \\
\hline Mn & 5,43 & .40 & 14.9 & 23.5 & 4.37 & 15.2 & 17.1 & 21.0 & 17.5 & 19.1 & 8.96 \\
\hline $\mathrm{Pe}$ & 25.5 & 3.00 & 22.4 & 8.17 & 10.5 & 229 & 18.4 & 15.7 & 13.9 & 16.8 & 24.8 \\
\hline $\mathbf{A}$ & - & - & 1.28 & - & - & $1 A 1$ & 2.08 & 3.42 & .46 & 99 & 1.77 \\
\hline Co & .080 & .010 & - & 310 & .180 & - & - & - & - & - & - \\
\hline $\mathrm{Ni}$ & .150 & .010 & 260 & 490 & 240 & .140 & 210 & .890 & .230 & .490 & .090 \\
\hline $\mathrm{Cu}$ & .250 & .020 & . & .070 & .030 & - & - & - & 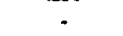 & - & 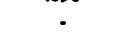 \\
\hline ca & $\because$ & - & 1.74 & 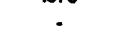 & 1.88 & 1.80 & 1.92 & 1.22 & 1.78 & 1.80 & 1.26 \\
\hline $\mathrm{Mg}_{\mathbf{g}}$ & - & . & 1.16 & . & . & 1.01 & 1.03 & 3.39 & .98 & 1.30 & 1.28 \\
\hline Na & - & - & .98 & - & - & 1.28 & 85 & 1.51 & 1.48 & 1.85 & 2.52 \\
\hline $\mathrm{K}$ & - & - & .29 & - & - & .32 & 36 & .38 & .18 & .25 & 33 \\
\hline $\mathbf{P}$ & . & . & . & . & . & - & . & . & . & . & . \\
\hline $\mathrm{H}_{2} \mathrm{O}$ & - & - & $20 A$ & - & - & 29.5 & 31.8 & 17.5 & 47.0 & 36.5 & 31.9 \\
\hline$A=(p p m)$ & . & . & - & . & - & - & . & . & . & - & - \\
\hline $\mathrm{Be}$ & . & - & . & . & . & . & - & - & - & - & . \\
\hline Cd & - & - & - & . & - & - & - & - & - & - & - \\
\hline co & . & - & • & - & - & - & - & - & • & - & - \\
\hline $\mathrm{Cr}$ & . & - & 2300 & - & - & 340 & - & 640 & 2000 & 4300 & . \\
\hline Mo & - & - &. & . & . & $\cdot$ & . & - & - & - & -. \\
\hline $\mathrm{Pb}$ & - & - & - & - & - & - & . & - & - & - & - \\
\hline Sr & - & - & - & - & - & - & - & - & . & - & - \\
\hline $\mathbf{v}$ & - & - & - & - & . & - & - & - & . & . & - \\
\hline $\mathbf{Y}$ & - & - & . & • & - & - & - & - & - & - & - \\
\hline $\mathrm{Zn}$ & - & - & - & 1700 & 410 & - & - & • & - & - & - \\
\hline Semple & Mn-encrustod & Crost & Mn-couted & Crost & Crust & Mn-conted & Mn-conted & Mn-costed & Mn-coated & Mn-costed & Mn-costed \\
\hline typo & sodimernt & - & rock & - & - & rock & rock & rock & rock & rock & rock \\
\hline Portion & uninown & unkmown & middle & unionown & unknown & outer & middle & middle & outer & middle & outer \\
\hline sampled & - & - & layor & - & - & crust & layer & layor & crust & layor & crust \\
\hline Nucleus & - & - & • & - & - & - & - & - & - & - & - \\
\hline Roforanos & A419 & A179 & A254 & A324 & A324 & A254 & A254 & A254 & A254 & A254 & A254 \\
\hline
\end{tabular}


Table 9. Composition of crusts from the Scripps Nodule Databank (cont'd).

\begin{tabular}{|c|c|c|c|c|c|c|c|c|c|c|c|}
\hline Sequencell & $4120390-00$ & $4120453-00$ & $4120453-01$ & $4120457-00$ & $4120458-00$ & $4120459-\infty$ & $4120461-\infty 0$ & $4120462-00$ & $4120463-\infty 0$ & $4120463-01$ & $4120464-\infty 0$ \\
\hline Latinude & 21.820 & 20.137 & 20.137 & 21.727 & 21.675 & 21.692 & 21.850 & 21.905 & 21.965 & 21.965 & 21.782 \\
\hline Longitude & -158.803 & -156.265 & -156.265 & -158.292 & -158.457 & -158.495 & -158.687 & -158.635 & .158 .610 & -158.610 & -158.743 \\
\hline Dopth & 1300 & 1220 & 1220 & 795 & 920 & 985 & 1340 & 1840 & 1985 & 1985 & 1120 \\
\hline Si(wt.x) & 279 & $\cdot$ & $\cdot$ & $\cdot$ & $\cdot$ & $\cdot$ & $\cdot$ & $\cdot$ & $\cdot$ & $\cdot$ & - \\
\hline $\mathrm{Ti}$ & 1.24 & 1.23 & 1.51 & 1.21 & 1.13 & 1.12 & 1.17 & 1.27 & • & 1.83 & - \\
\hline Mm & 2.1 & 14.9 & 8.20 & 24.3 & 24.3 & 27.4 & 22.4 & 19.5 & 24.0 & 22.1 & 25.8 \\
\hline Pe & 179 & 10.8 & 10.8 & 20.3 & 224 & 19.5 & 17.5 & 21.8 & 19.3 & 20.8 & 17.2 \\
\hline $\mathbf{A}$ & 94 & 3.40 & - & . & - & - & - & - & - & - & - \\
\hline Co & - & .060 & .020 & 1.10 & 920 & 1.17 & 870 & 590 & .800 & .740 & .760 \\
\hline $\mathbf{N i}$ & .250 & .120 & .030 & 340 & .260 & .440 & 400 & .280 & .370 & 360 & .690 \\
\hline $\mathrm{Ca}$ & . & .010 & .010 & .030 & .020 & .030 & .030 & .030 & .030 & .040 & .070 \\
\hline $\mathrm{Ca}$ & 1.71 & • & • & • & • & - & - & • & • & $\cdot$ & $\cdot$ \\
\hline $\mathbf{M}_{8}$ & 1.08 & - & - & - & - & - & - & - & - & - & - \\
\hline $\mathrm{Na}$ & 1.52 & - & - & - & - & - & - & - & - & - & - \\
\hline $\mathbf{K}$ & 31 & - & - & - & - & - & - & - & - & - & - \\
\hline $\mathbf{P}$ & • & - & - & - & - & - & - & - & - & - & - \\
\hline $\mathrm{H}_{2} \mathrm{O}$ & 31.6 & - & - & - & - & - & $\cdot$ & - & - & - & - \\
\hline$A \subseteq$ (ppm) & - & - & - & - & - & - & - & - & - & - & - \\
\hline $\mathrm{Ba}$ & - & - & - & - & - & - & - & - & - & - & - \\
\hline cd & - & - & - & - & - & - & - & - & - & - & - \\
\hline$c_{0}$ & - & - & - & - & . & . & - & - & - & - & - \\
\hline$C_{r}$ & . & - & . & . & - & - & - & - & - & - & - \\
\hline Mo & - & - & - & - & - & - & - & - & - & - & - \\
\hline $\mathbf{P b}$ & - & - & - & - & - & - & - & - & - & - & - \\
\hline Sr & - & - & - & - & - & - & - & - & - & - & - \\
\hline $\mathbf{v}$ & - & - & - & - & - & - & - & - & - & - & - \\
\hline $\mathbf{Y}$ & - & - & - & - & - & - & - & - & - & - & . \\
\hline $\mathbf{Z n}_{\mathbf{n}}$ & - & - & - & - & - & - & - & - & - & - & - \\
\hline Sample & Mn-couted & Min-coented & Mn-costod & Count & Cruat & Crust & Cust & Crunt & Crust & Crust & Crust \\
\hline typo & rock & rock & rock & $\cdot$ & $\cdot$ & - & $\cdot$ & • & - & - & - \\
\hline Portion & outor & unitenown & unknown & unknown & unkenown & unknown & unicnown & uniknown & unknown & unknown & unknown \\
\hline sempled & crust & - & • & • & • & - & • & • & - & - & $\cdot$ \\
\hline Nuclew & - & - & - & • & • & • & - & - & • & - & • \\
\hline Refarence & A254 & A380 & A380 & A380 & A380 & A380 & A380 & A380 & A380 & A380 & A380 \\
\hline Sequencell & $4120466-00$ & $4120466-01$ & $4120466-02$ & $4120466-03$ & $4120466-04$ & $4120466-05$ & $4120466-06$ & $4120467-00$ & $4120468-\infty 0$ & $4120468-01$ & $4120468-02$ \\
\hline Latitude & 21.833 & 21.833 & 21.833 & 21.833 & 21.833 & 21.833 & 21.833 & 21.923 & 21.872 & 21.872 & 21.872 \\
\hline Langitude & -158.667 & -158.667 & -158.667 & -158.667 & -158.667 & -158.667 & -158.667 & -158.795 & -158.823 & -158.823 & -158.823 \\
\hline Dopth & 1265 & 1265 & 1265 & 1265 & 1265 & 1265 & 1265 & 1910 & 1115 & 1115 & 1115 \\
\hline $\operatorname{Si}(w t . \%)$ & - & - & - & - & - & $\cdot$ & $\cdot$ & - & $\cdot$ & - & - \\
\hline $\mathrm{Ti}$ & 1.49 & 1.04 & 1.25 & 1.20 & 1.31 & 1.36 & 1.08 & .25 & 1.35 & 1.04 & 1.00 \\
\hline Mn & 4.70 & 24.6 & 21.6 & 21.6 & 18.0 & 13.0 & 225 & 7.60 & 8.90 & 17.2 & 27.2 \\
\hline Pe & 11.6 & 19.3 & 18.3 & 20.5 & 17.1 & 15.8 & 18.6 & 22.6 & 13.6 & 16.0 & 163 \\
\hline $\mathbf{A}$ & 5.40 & • & • & - & • & • & • & - & - & • & - \\
\hline Co & .180 & 800 & .780 & .670 & 550 & .390 & .760 & .060 & .380 & 540 & 860 \\
\hline $\mathbf{N i}$ & .140 & 340 & .320 & .260 & 280 & .280 & 340 & 380 & .150 & 300 & .710 \\
\hline $\mathrm{Cu}$ & .020 & .020 & .040 & .030 & .030 & .040 & .030 & .050 & .030 & .020 & .060 \\
\hline $\mathrm{C}$ & • & • & • & • & - & - & • & - & - & • & • \\
\hline $\mathbf{M}_{\mathbf{6}}$ & - & - & - & - & - & - & - & - & - & - & - \\
\hline $\mathrm{Na}$ & - & - & - & - & - & - & - & - & - & - & - \\
\hline $\mathbf{K}$ & - & - & - & - & - & - & - & - & - & - & - \\
\hline $\mathbf{P}$ & - & - & - & - & - & - & - & . & - & - & - \\
\hline $\mathrm{H}_{2} \mathrm{O}$ & - & - & - & - & - & - & - & - & - & - & - \\
\hline$A$ (ppm) & - & - & - & . & - & . & - & - & - & - & - \\
\hline Ba & - & - & - & - & - & - & - & - & - & - & - \\
\hline Cd & - & - & - & - & - & - & . & - & - & - & - \\
\hline Co & - & - & - & - & - & - & - & - & - & - & - \\
\hline $\mathrm{Cr}$ & - & - & - & - & - & - & - & - & - & - & - \\
\hline Mo & - & - & - & - & - & - & - & - & - & - & - \\
\hline $\mathbf{P b}$ & - & - & - & - & - & - & - & - & - & - & - \\
\hline Sx & - & - & - & - & - & - & - & - & - & - & - \\
\hline $\mathbf{v}$ & - & - & - & - & - & - & - & - & - & - & - \\
\hline $\mathbf{Y}$ & - & - & - & - & . & - & - & - & - & - & - \\
\hline $\mathbf{Z n}$ & - & - & • & • & - & • & • & • & • & - & • \\
\hline Semple & Mn-couted & Crust & Mn-costrod & Crust & Mn-costod & Mn-contod & Mn-contod & Mn-costed & Mn-conted & Mn-couted & Crust \\
\hline typo & rock & $\cdot$ & rock & $\cdot$ & rock & rock & rock & rock & rock & rock & • \\
\hline Partion & unknown & unknown & unknown & unknown & unicnown & unknown & unknown & unknown & unknown & unknown & unkpown \\
\hline semplod & • & - & - & $\cdot$ & • & - & - & - & - & - & $\cdot$ \\
\hline Nucleus & - & - & - & - & - & • & - & - & - & - & • \\
\hline Reference & A380 & A380 & A380 & A380 & A380 & A380 & A380 & A380 & A380 & A380 & A380 \\
\hline
\end{tabular}


Table 9. Composition of crusts from the Scripps Nodule Databank (cont'd).

\begin{tabular}{|c|c|c|c|c|c|c|c|c|c|c|c|}
\hline Sequencall & $4120475-00$ & $4130055-00$ & $4130055-01$ & $4130165-\infty 0$ & $4130165-01$ & $4130165-02$ & $4130216-00$ & $4130216-01$ & $4130216-02$ & $4130216-03$ & $4130217-00$ \\
\hline Latioude & 21.847 & 28.138 & 28.138 & 21.535 & 21.535 & 21.535 & 25.675 & 25.675 & 25.675 & 25.675 & 25.488 \\
\hline Longitude & -159.372 & -162.278 & -162278 & -167.930 & -167.930 & -167.930 & -168.697 & -168.697 & -168.697 & -168.697 & .168 .657 \\
\hline Depth & 900 & 2561 & 2561 & 1690 & 1690 & 1690 & $\cdot$ & • & 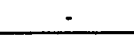 & $\bullet$ & $\cdot$ \\
\hline Si(wt.\%) & $\cdot$ & $\cdot$ & $\cdot$ & $\cdot$ & $\cdot$ & . & - & $\cdot$ & - & - & $\cdot$ \\
\hline $\mathrm{Ti}$ & • & • & • & - & $\cdot$ & • & .93 & .97 & 1.12 & .89 & .73 \\
\hline Mn & 19.6 & 13.4 & 19.7 & 16.9 & 17.7 & 18.8 & 23.5 & 21.6 & 198 & 22.0 & 23.7 \\
\hline Fo & 20.2 & 121 & 14.2 & 17.1 & 17.0 & 15.4 & 16.9 & 15.9 & 15.7 & 17.5 & 15.4 \\
\hline $\mathbf{A}$ & • & • & - & • & - & - & 80 & 1.40 & 1.70 & 1.00 & 1.20 \\
\hline Co & .650 & .600 & .740 & .730 & .770 & .760 & .800 & .740 & .670 & .770 & .780 \\
\hline $\mathrm{Ni}$ & .140 & .370 & .460 & 390 & .370 & .420 & .410 & 590 & $\mathbf{A 4 0}$ & .350 & 530 \\
\hline $\mathrm{Cu}$ & .070 & .090 & .170 & .010 & .060 & .080 & .070 & .120 & .080 & .070 & .070 \\
\hline C. & - & 3.30 & 2.60 & 270 & 2.40 & 4.10 & - & - & - & - & - \\
\hline Mg & - & - & - & - & • & - & - & - & - & - & - \\
\hline $\mathrm{Na}$ & - & - & - & - & - & - & - & - & . & - & - \\
\hline $\mathbf{K}$ & $\cdot$ & - & $\cdot$ & - & - & - & - & - & - & $\cdot$ & $\cdot$ \\
\hline $\mathbf{P}$ & $\cdot$ & - & - & $\cdot$ & - & - & - & - & $\cdot$ & - & $\cdot$ \\
\hline $\mathrm{H}_{2} \mathrm{O}$ & - & - & $\cdot$ & $\cdot$ & $\cdot$ & - & - & - & $\cdot$ & - & - \\
\hline As(ppon) & - & - & $\cdot$ & - & - & - & - & - & - & - & - \\
\hline Be & - & - & - & - & - & - & - & - & - & - & - \\
\hline Cd & - & - & - & - & - & - & - & - & . & - & - \\
\hline $\mathrm{Co}_{0}$ & - & - & - & . & - & - & - & - & - & - & - \\
\hline $\mathrm{Cr}$ & • & $\therefore$ & $\cdot$ & - & - & - & - & - & $\cdot$ & - & - \\
\hline Mo & 400 & - & • & - & - & • & - & - & - & - & - \\
\hline $\mathrm{Pb}$ & $\cdot$ & 1000 & 1200 & 1400 & 1600 & 1700 & - & - & - & - & - \\
\hline Sr & - & - & - & - & - & - & - & - & - & - & - \\
\hline $\mathbf{v}$ & - & - & - & - & - & - & - & - & $\cdot$ & - & - \\
\hline $\mathbf{Y}$ & - & - & $\cdot$ & - & - & - & - & - & - & - & - \\
\hline $\mathbf{Z n}$ & • & 500 & 600 & 700 & 600 & 500 & • & $\cdot$ & • & - & - \\
\hline Semplo & Nodulo & Mn-costod & Nodule & Nodulo & Noctule & Noctule & Crut & Crust & Mn-controd & Crus & Crust \\
\hline typo & - & rock & • & $\cdot$ & • & • & $\bullet$ & $\cdot$ & rock & $\cdot$ & 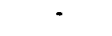 \\
\hline Portion & cormposits & $\operatorname{crcos}$ & without & quarter & querter & half & untom wh & unknown & monowns & unknown & unknown \\
\hline remplod & 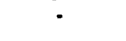 & wection & madeus & 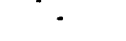 & 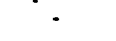 & - & - & - & $\cdot$ & - & - \\
\hline Nocleus & • & Pumico & volcanic & - & Sodiment & Rock & - & • & Undetermined & - & - \\
\hline Reference & A386 & $A 405$ & A405 & A405 & A405 & A22 & A380 & A380 & A380 & A380 & A380 \\
\hline Sequencell & $4130217-01$ & $4130227-00$ & $4130230-00$ & $4130232-00$ & $4130251-00$ & $4140067-01$ & $4140067-02$ & $4140067-03$ & $4140067-04$ & $4140057-05$ & $4140221-00$ \\
\hline Latioude & 25.488 & 24.202 & 23817 & 23.367 & 28.965 & 23.917 & 23.917 & 23.917 & 23.917 & 23.917 & 28.817 \\
\hline Longitude & -168.657 & -166.730 & -164.420 & -163.567 & .162 .022 & -173.655 & -173.655 & -173.655 & -173.655 & -173.655 & -178.950 \\
\hline Depth & - & $\cdot$ & $\cdot$ & $\cdot$ & 3050 & 4000 & 4000 & 4000 & 4000 & 4000 & - \\
\hline Si(wL\%) & - & $\cdot$ & $\dot{ }$ & $\cdot$ & $\cdot$ & 4.41 & 4.56 & 5.06 & 4.96 & 11.7 & $\cdot$ \\
\hline $\mathrm{Ti}$ & 83 & .89 & .76 & 83 & - & $\cdot$ & - & $\cdot$ & $\cdot$ & - & .67 \\
\hline Mn & 244 & 23.5 & 23.5 & 26.2 & 20.9 & 18.5 & 18.7 & 19.0 & 18.2 & 19.1 & 31.8 \\
\hline $\mathbf{F o}$ & 16.2 & 16.7 & 16.7 & 14.4 & 18.9 & 127 & 14.5 & 13.5 & 15.4 & 13.4 & 11.7 \\
\hline Al & 1.20 & .80 & .90 & 50 & • & - & - & • & • & • & 30 \\
\hline Co & 1.000 & 1.04 & 1.000 & 1.36 & .760 & - & - & $\cdot$ & $\cdot$ & - & 1.64 \\
\hline $\mathrm{Ni}$ & .490 & .300 & .300 & 370 & .270 & $\cdot$ & - & 370 & 340 & .380 & .620 \\
\hline $\mathrm{Cu}$ & .070 & .030 & .040 & .040 & .050 & - & - & - & - & - & .050 \\
\hline C. & - & - & $\cdot$ & - & 2.90 & 1.81 & 1.93 & 1.73 & 1.80 & 1.60 & - \\
\hline Mg & - & - & , & - & - & - & - & • & - & • & - \\
\hline $\mathrm{Na}$ & - & - & . & - & - & - & - & - & $\cdot$ & - & - \\
\hline $\mathbf{K}$ & - & - & - & - & - & - & - & - & - & - & - \\
\hline $\mathbf{P}$ & - & - & - & . & - & - & - & .30 & .40 & .42 & - \\
\hline $\mathrm{H}_{2} \mathrm{O}$ & - & $\cdot$ & - & $\cdot$ & - & - & $\cdot$ & $\cdot$ & - & $\cdot$ & - \\
\hline$A=(p p m)$ & - & - & - & - & - & - & - & - & - & - & - \\
\hline $\mathrm{Ba}$ & - & - & - & - & - & - & . & - & - & - & . \\
\hline Cd & - & - & - & - & - & - & - & - & - & - & - \\
\hline Ce & - & - & - & - & - & $\cdot$ & - & - & - & - & $\cdot$ \\
\hline$C_{r}$ & - & - & - & - & - & - & - & - & - & $\cdot$ & - \\
\hline Mo & - & $\cdot$ & - & $\cdot$ & $\cdot$ & - & - & - & $\cdot$ & $\cdot$ & - \\
\hline Pb & - & - & - & $\cdot$ & 1800 & - & - & - & - & $\cdot$ & - \\
\hline Sr & - & - & - & - & - & - & - & - & - & - & - \\
\hline $\mathbf{v}$ & - & - & . & $\cdot$ & - & - & - & - & - & - & - \\
\hline $\mathbf{Y}$ & - & - & - & $\cdot$ & - & - & - & - & - & - & - \\
\hline $\mathbf{Z n}$ & - & - & • & - & 300 & - & - & • & • & - & - \\
\hline Semplo & Crust & Mn-costod & Crust & Crust & Crust & Crust & Crent & Crax & Crux & Crun & Crun \\
\hline typo & • & rock & • & - & - & • & • & - & • & • & $\cdot$ \\
\hline Partion & unknown & unknown & unknown & unknown & outer & middle & middlo & middle & middlo & whole & unknown \\
\hline sempled & - & - & • & $\cdot$ & croust & Inyer & layer & layex & layex & • & - \\
\hline Nuclous & - & - & - & • & - & - & 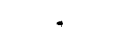 & • & - & - & • \\
\hline Reforence & A380 & A380 & A380 & A380 & A459 & A276 & A276 & A276 & A276 & A276 & A380 \\
\hline
\end{tabular}


Table 9. Composition of crusts from the Scripps Nodule Databank (cont'd).

\begin{tabular}{|c|c|c|c|c|c|c|c|c|c|c|c|}
\hline $\begin{array}{l}\text { Sequencell } \\
\text { Letitude } \\
\text { Longinude } \\
\text { Depth }\end{array}$ & $\begin{array}{c}4140223-00 \\
27.702 \\
-175.683 \\
-\end{array}$ & $\begin{array}{c}4140230-00 \\
20.695 \\
-170.548 \\
1930\end{array}$ & $\begin{array}{c}4140230-01 \\
20.695 \\
-170.548 \\
1930\end{array}$ & $\begin{array}{c}4140230-03 \\
20.695 \\
-170.548 \\
1930\end{array}$ & $\begin{array}{c}4140230-04 \\
20.695 \\
-170.548 \\
1930 \\
\end{array}$ & $\begin{array}{c}4140232-00 \\
20.517 \\
-170.830 \\
2980 \\
\end{array}$ & $\begin{array}{c}4150100-00 \\
20.750 \\
173.667 \\
3385\end{array}$ & $\begin{array}{c}4150104-\infty \\
20.752 \\
173.440 \\
1623\end{array}$ & $\begin{array}{c}4150104-01 \\
20.752 \\
173.440 \\
1623 \\
\end{array}$ & $\begin{array}{c}4150106-\infty 0 \\
20.788 \\
173.340 \\
1278 \\
\end{array}$ & $\begin{array}{c}4150106-01 \\
20.788 \\
173.340 \\
1278 \\
\end{array}$ \\
\hline$s i(n t \%)$ & . & - & . & - & - & - & - & - & . & . & . \\
\hline $\mathbf{T i}$ & .63 & .91 & 95 & 1.12 & 84 & 1.09 & • & - & - & • & • \\
\hline Mn & 28.6 & 20.2 & 17.3 & 17.1 & 19.8 & 15.4 & 18.4 & 22.1 & - & 23.8 & 24.1 \\
\hline Po & 7.10 & 14.4 & 15.3 & 15.9 & 15.1 & 17.4 & 175 & 16.3 & - & 17.4 & 16.3 \\
\hline $\boldsymbol{N}$ & 1.80 & • & - & • & • & • & • & • & - & • & • \\
\hline Co & .600 & 570 & .520 & .590 & $\mathbf{5 9 0}$ & .380 & 390 & .920 & - & 1.01 & 1.06 \\
\hline $\mathbf{N i}$ & 190 & A70 & .420 & .600 & 530 & .330 & .290 & .460 & - & $\mathbf{A S O}$ & 500 \\
\hline $\mathrm{Cu}$ & .070 & .130 & .130 & .140 & .150 & .280 & .160 & .050 & - & .040 & .030 \\
\hline $\mathbf{C a}$ & - & • & • & • & • & - & - & 3.00 & - & 290 & 2.80 \\
\hline$M 8$ & - & - & - & - & - & - & - & - & - & . & • \\
\hline $\mathrm{Na}$ & - & - & - & - & - & - & - & - & - & - & $\cdot$ \\
\hline $\mathbf{K}$ & - & - & - & - & - & - & - & - & - & - & - \\
\hline $\mathbf{P}$ & - & - & - & - & - & - & - & - & - & - & - \\
\hline $\mathrm{H}_{2} \mathrm{O}$ & - & - & - & - & - & $\cdot$ & - & $\cdot$ & $\cdot$ & - & - \\
\hline$A \times$ (ppon) & - & - & - & - & - & - & - & - & 350 & - & - \\
\hline $\mathrm{Ba}$ & - & - & - & - & - & - & - & - & 1900 & - & - \\
\hline Cd & - & - & - & - & - & - & - & - & 5.0 & - & - \\
\hline co & - & - & - & - & - & - & - & - & $\cdot$ & - & - \\
\hline $\mathrm{Cr}$ & - & - & - & - & - & - & - & - & 10 & - & - \\
\hline Mo & - & - & - & - & - & - & 420 & - & $\cdot$ & • & • \\
\hline $\mathrm{Pb}$ & - & - & - & - & - & - & - & 1700 & - & 1800 & 1900 \\
\hline St & - & - & - & - & - & - & - & • & - & • & • \\
\hline $\mathbf{v}$ & - & - & - & - & - & - & - & . & - & - & - \\
\hline $\mathbf{Y}$ & - & - & - & - & - & - & - & $\cdot$ & - & - & $\cdot$ \\
\hline $\mathrm{Zn}$ & - & - & - & - & • & - & - & 600 & • & 500 & 400 \\
\hline Semplo & Mn-controd & Crunt & Nodule & Nocule & Crust & Crust & Mn-controd & Crust & Crust & Cruit & Crunt \\
\hline typo & rock & • & • & • & • & • & rock & • & $\cdot$ & $\cdot$ & • \\
\hline Partion & unitonown & unlonown & without & without & uniconown & unknown & outer & frugments & fragmeats & fragments & frapmonts \\
\hline semplod & - & - & macions & nuclous & . & - & crunt & 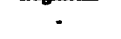 & 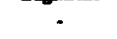 & 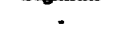 & 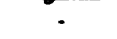 \\
\hline Nucleus & - & - & - & - & • & - & - & - & • & - & - \\
\hline Referenoses & A380 & A343 & A343 & - $A 343$ & A343 & A343 & A352 & A40s & A475 & A405 & A405 \\
\hline Sequencell & $4150176-\infty$ & $4150177-00$ & $4160105-\infty$ & $4160105-01$ & $4160105-02$ & $4160136-00$ & $4160136-01$ & $4160136-02$ & $4160196-\infty 0$ & $4160196-01$ & $4160224-01$ \\
\hline Lationde & $26.9 n$ & 22.133 & 21.145 & 21.145 & 21.145 & 21.160 & 21.160 & 21.160 & 21.688 & 21.688 & 24.065 \\
\hline Longinuda & 177.302 & 175.370 & 163.397 & 163.397 & 163.397 & 163.147 & 163.147 & 163.147 & 161.875 & 161.875 & 160.765 \\
\hline Depth & 2750 & 1500 & 1575 & 1575 & 1575 & 1437 & 1437 & 1437 & 1479 & 1479 & 3951 \\
\hline $\mathrm{Si}(w t . \%)$ & . & - & . & . & $\cdot$ & . & $\cdot$ & $\cdot$ & $\therefore$ & . & 5.44 \\
\hline $\mathrm{Ti}$ & .82 & .64 & - & - & - & - & - & - & $\because$ & - & 1.08 \\
\hline $\mathbf{M n}$ & 16.0 & 20.2 & 21.2 & 14.5 & - & 22.3 & 23.9 & - & 18.3 & 20.8 & 228 \\
\hline$P_{0}$ & 16.0 & 13.9 & 17.5 & 14.3 & - & 16.8 & 9.50 & - & 14.8 & 16.7 & 13.2 \\
\hline Al & - & - & - & - & - & - & - & - & - & - & 1.70 \\
\hline Co & .500 & $A 70$ & .730 & .540 & - & .780 & 590 & - & .630 & .700 & .510 \\
\hline $\mathbf{N i}$ & 200 & 550 & .410 & .320 & - & .430 & .710 & - & .500 & 420 & A90 \\
\hline Cu & .080 & .150 & .070 & .040 & - & .150 & .120 & - & .140 & .020 & .150 \\
\hline Ca & • & - & - & 3.60 & - & 2.60 & 7.50 & - & - & 280 & 2.21 \\
\hline $\mathbf{M}$ & - & - & - & • & - & • & - & - & - & • & .72 \\
\hline $\mathrm{Ne}$ & - & - & - & - & - & - & - & - & - & - & 1.68 \\
\hline $\mathbf{K}$ & - & - & - & - & - & - & - & - & - & - & - \\
\hline $\mathbf{P}$ & - & - & - & - & - & - & - & - & - & - & - \\
\hline $\mathrm{H}_{2} \mathrm{O}$ & $\cdot$ & - & - & - & - & - & - & - & • & $\cdot$ & 20.6 \\
\hline$A x(p p m)$ & - & - & - & - & 310 & . & - & 270 & - & - & - \\
\hline $\mathrm{Ba}$ & - & - & - & - & 1600 & - & - & 1800 & . & - & - \\
\hline Cd & - & - & - & - & 3.0 & . & - & 5.0 & . & - & - \\
\hline ce & . & - & - & - & . & - & - & . & . & . & - \\
\hline$C$ & - & - & - & - & 34 & - & - & 14 & $\cdot$ & - & - \\
\hline Mo & - & • & 640 & - & - & - & - & - & 500 & • & - \\
\hline $\mathbf{P b}$ & 1900 & 2300 & . & 1400 & . & 1700 & 1400 & - & . & 1800 & . \\
\hline Sr & - & - & - & - & - & 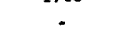 & . & - & . & 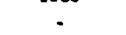 & - \\
\hline $\mathbf{v}$ & - & - & - & - & - & - & - & - & . & - & - \\
\hline $\mathbf{Y}$ & - & - & - & - & - & - & - & - & . & - & - \\
\hline$Z_{n}$ & 530 & 990 & • & 500 & - & 500 & 1000 & • & • & 500 & - \\
\hline Semplo & Nodule & Cruat & Mn-costod & Mn-encrustod & Mo-encrusted & Crust & Cunt & Crus & Me-conted & Mn-coutod & Noculo \\
\hline type & • & • & rock & sediment & rediment & • & • & • & rock & rock & • \\
\hline Portion & outor & unknown & outer & croms & cross & fragment & fragmonts & frugments & outor & outer & ouver \\
\hline sempled & cout & - & cruat & extion & ection & . & - & - & crost & crust & $\cos 2 x$ \\
\hline Nuclens & - & • & - & - & - & . & . & . & - & Rock & - \\
\hline Referencos & A343 & A343 & A352 & A405 & A475 & A405 & A422 & A475 & A352 & A405 & A343 \\
\hline
\end{tabular}


Table 9. Composition of crusts from the Scripps Nodule Databank (cont'd).

\begin{tabular}{|c|c|c|c|c|c|c|c|c|c|c|c|}
\hline $\begin{array}{l}\text { Sequencoll } \\
\text { Latimude } \\
\text { Loaginude } \\
\text { Depth }\end{array}$ & $\begin{array}{c}4160231-00 \\
25.550 \\
168.020 \\
2870 \\
\end{array}$ & $\begin{array}{c}4160237-00 \\
21.167 \\
163.220 \\
3047 \\
\end{array}$ & $\begin{array}{c}4160237.01 \\
21.167 \\
163.220 \\
3047 \\
\end{array}$ & $\begin{array}{c}4160238-02 \\
27.853 \\
162.928 \\
3090 \\
\end{array}$ & $\begin{array}{c}4160238-03 \\
27.853 \\
162.928 \\
3090 \\
\end{array}$ & $\begin{array}{c}4160239-00 \\
22.333 \\
161.527 \\
1315 \\
\end{array}$ & $\begin{array}{c}4160240-00 \\
22.658 \\
160.870 \\
1280 \\
\end{array}$ & $\begin{array}{c}4170002-00 \\
21.483 \\
159.538 \\
1265 \\
\end{array}$ & $\begin{array}{c}4170004-00 \\
24.027 \\
159.450 \\
3332 \\
\end{array}$ & $\begin{array}{c}4170005-01 \\
23.815 \\
159.437 \\
1711 \\
\end{array}$ & $\begin{array}{c}4170035.00 \\
29.495 \\
153.362 \\
1719 \\
\end{array}$ \\
\hline Si(wtS) & • & - & - & - & $\cdot$ & 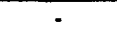 & - & $\cdot$ & $\cdot$ & - & $\cdot$ \\
\hline $\mathrm{Ti}$ & 85 & .79 & 98 & .91 & 1.27 & .7 & .77 & - & - & - & - \\
\hline Mn & 20.3 & 18.4 & 19.8 & 16.1 & 23.0 & 23.0 & 19.6 & 21.6 & 226 & 19.4 & 19.6 \\
\hline Po & 13.4 & 16.7 & 16.0 & 16.3 & 122 & 14.6 & 15.4 & 16.3 & 14.8 & 16.9 & 17.2 \\
\hline $\mathbf{A}$ & • & - & - & • & • & • & - & $\cdot$ & • & $\cdot$ & $\cdot$ \\
\hline Co & 570 & 530 & .580 & .130 & .830 & .790 & .780 & .780 & .620 & 580 & 560 \\
\hline $\mathbf{N i}$ & .420 & 500 & .370 & 200 & 550 & 530 & 370 & 460 & .450 & 360 & 360 \\
\hline $\mathrm{Cr}$ & .150 & .140 & .130 & .060 & .130 & • & • & .090 & .150 & .070 & .040 \\
\hline Ca & • & • & • & • & • & - & - & • & - & 250 & • \\
\hline $\mathbf{M}_{\mathbf{8}}$ & - & - & - & • & - & - & - & - & - & • & - \\
\hline Na & - & - & - & - & - & - & - & - & - & - & - \\
\hline $\mathbf{K}$ & - & - & - & - & - & - & - & $\cdot$ & - & - & $\cdot$ \\
\hline $\mathbf{P}$ & - & - & - & - & - & - & - & - & - & $\cdot$ & - \\
\hline $\mathrm{H}_{2} \mathrm{O}$ & $\cdot$ & - & - & - & - & $\cdot$ & - & - & - & $\cdot$ & - \\
\hline Ax(ppen) & - & - & - & - & - & - & - & - & - & • & . \\
\hline Ba & - & - & - & - & - & $\cdot$ & - & $\cdot$ & $\cdot$ & $\cdot$ & - \\
\hline $\mathrm{Cd}$ & - & - & - & - & - & - & • & - & - & - & - \\
\hline co & - & - & - & - & - & - & - & - & - & $\cdot$ & - \\
\hline$c$ & - & - & - & - & - & - & - & • & - & - & - \\
\hline Mo & $\cdot$ & - & - & - & - & - & - & 500 & 540 & - & 560 \\
\hline $\mathrm{Pb}$ & - & - & - & - & - & - & - & - & $\cdot$ & 1700 & - \\
\hline Sr & $\cdot$ & - & - & $\cdot$ & - & $\cdot$ & $\cdot$ & - & - & - & - \\
\hline $\mathbf{v}$ & $\cdot$ & - & - & $\cdot$ & $\cdot$ & - & - & - & - & - & $\cdot$ \\
\hline $\mathbf{Y}$ & $\cdot$ & - & - & - & - & - & - & - & - & $\cdot$ & $\cdot$ \\
\hline$Z_{n}$ & • & - & • & • & - & • & • & - & - & 500 & - \\
\hline Semple & Nodule & Nocule & Nodule & Crust & Crust & Nodule & Nodule & Mn-couted & Noctule & Unknown & Mn-conted \\
\hline typo & • & $\cdot$ & - & $\cdot$ & $\cdot$ & • & • & rock & • & - & rock \\
\hline Portion & without & without & without & middle & underside & without & without & outer & whole & fragments & outer \\
\hline semopled & meleus & nuclous & macleus & leyer & • & nucleus & mactous & crust & - & • & court \\
\hline Nucleus & • & $\cdot$ & - & $\cdot$ & $\cdot$ & - & $\cdot$ & • & - & - & - \\
\hline Reference & A343 & A343 & A343 & A343 & A343 & A343 & A343 & A352 & A352 & $A 405$ & A352 \\
\hline Sequencell & $4170035-01$ & $4170035-02$ & $4170066-00$ & $4170066-01$ & $4170067-02$ & $4180009-00$ & $4180011-00$ & $4180011-01$ & $4180272-00$ & $4180403-01$ & $4340358-00$ \\
\hline Latitude & 29.495 & 29.495 & 23.533 & 23.533 & 26.553 & 28.383 & 27.933 & 27.933 & 20.912 & 27.818 & 34.867 \\
\hline Longituce & 153.362 & 153.362 & 157.393 & 157.393 & 152.217 & 148.250 & 147.650 & 147.650 & 142532 & 145.700 & -16.517 \\
\hline Depth & 1719 & 1719 & 1425 & 1425 & 3200 & 2423 & 2728 & 2728 & 2423 & 1116 & 1480 \\
\hline Si(wt.s) & $\cdot$ & - & - & $\cdot$ & - & - & - & - & - & - & - \\
\hline $\mathbf{T i}$ & - & - & .77 & .88 & 1.49 & - & - & - & - & - & $\cdot$ \\
\hline $\mathrm{Mn}$ & 23.6 & 27.7 & 224 & 20.8 & 18.2 & 21.0 & 25.7 & 8.70 & 9.50 & 28.6 & 15.9 \\
\hline Fe & 13.8 & 10.5 & 13.1 & 14.2 & 15.4 & 19.8 & 125 & 5.40 & 13.4 & 10.3 & 17.5 \\
\hline $\mathbf{A} \mathbf{N}$ & - & - & - & • & - & • & - & - & • & - & - \\
\hline Co & .660 & .750 & .650 & .630 & .700 & .580 & .050 & .170 & .070 & 520 & .630 \\
\hline $\mathbf{N i}$ & .600 & 850 & .840 & .620 & .350 & .330 & .210 & .220 & .110 & 1.290 & .270 \\
\hline $\mathrm{Cu}$ & .060 & .110 & .230 & .120 & .120 & .020 & .060 & .080 & .060 & .070 & .030 \\
\hline C. & 290 & 290 & - & - & - & 2.60 & 3.50 & 4.10 & • & 210 & 4.42 \\
\hline Mg & - & • & - & - & - & - & • & • & - & • & 211 \\
\hline Ne & $\cdot$ & - & - & - & - & - & - & - & $\cdot$ & - & 3.04 \\
\hline K & - & - & - & - & - & - & - & - & - & - & .27 \\
\hline $\mathbf{P}$ & • & - & - & - & - & - & - & - & • & • & - \\
\hline $\mathrm{H}_{2} \mathrm{O}$ & $\cdot$ & - & - & $\cdot$ & $\cdot$ & - & - & - & - & - & - \\
\hline As(ppon) & $\cdot$ & - & - & $\cdot$ & $\cdot$ & - & - & - & $\cdot$ & - & - \\
\hline $\mathrm{Be}$ & - & - & - & - & - & - & - & - & 1100 & - & - \\
\hline Cd & - & - & - & $\cdot$ & - & - & - & - & • & - & - \\
\hline co & - & - & - & - & - & - & - & - & $\cdot$ & - & - \\
\hline $\mathrm{Cr}$ & - & - & - & - & - & - & - & - & 23 & - & - \\
\hline Mo & - & - & - & - & - & $\cdot$ & - & - & $\cdot$ & • & - \\
\hline $\mathbf{P b}$ & 1700 & 1600 & - & - & - & 2000 & 300 & 600 & - & 1200 & 3400 \\
\hline Sr & • & - & - & - & - & • & $\cdot$ & $\cdot$ & - & - & - \\
\hline $\mathbf{v}$ & - & - & $\cdot$ & $\cdot$ & - & - & - & - & - & - & - \\
\hline $\mathbf{Y}$ & - & • & - & - & - & - & • & $\cdot$ & $\cdot$ & - & $\cdot$ \\
\hline $\mathrm{Zn}$ & 600 & 800 & - & - & - & 500 & 600 & 400 & 500 & 1100 & 550 \\
\hline Sermplo & Crust & Nocule & Nodule & Noctule & Nocule & Cust & Crust & Crust & Crust & Nodulo & Coust \\
\hline typo & - & - & - & - & - & • & • & - & - & • & - \\
\hline Portion & fragments & fragments & without & without & undenside & fragments & fragments & fragments & unknown & $\operatorname{cros}$ & unknown \\
\hline samoplod & $\cdot$ & • & mudeus & maclens & - & • & • & • & - & section & - \\
\hline Nucleus & - & • & • & • & • & - & , & $\cdot$ & • & Undetorminod & - \\
\hline Reference & A405 & A405 & A343 & A343 & A343 & A405 & A405 & A40S & $A 323$ & A422 & A368 \\
\hline
\end{tabular}


Table 9. Composition of crusts from the Scripps Nodule Databank (cont'd).

\begin{tabular}{|c|c|c|c|c|c|c|c|c|c|c|c|}
\hline $\begin{array}{l}\text { Sequencoul } \\
\text { Letitude } \\
\text { Longitude } \\
\text { Depeth }\end{array}$ & $\begin{array}{c}4340396-00 \\
36.308 \\
-12.575 \\
3800 \\
\end{array}$ & $\begin{array}{c}4340397-00 \\
36.292 \\
-12583 \\
3700 \\
\end{array}$ & $\begin{array}{c}4340397-01 \\
36.292 \\
-12583 \\
3700 \\
\end{array}$ & $\begin{array}{c}4340397-02 \\
36.292 \\
-12583 \\
3700 \\
\end{array}$ & $\begin{array}{c}4340397-03 \\
36.292 \\
-12583 \\
3700 \\
\end{array}$ & $\begin{array}{c}4340397-04 \\
36.292 \\
-12583 \\
3700 \\
\end{array}$ & $\begin{array}{c}4340397-05 \\
36.292 \\
-12583 \\
3700 \\
\end{array}$ & $\begin{array}{c}4340398-00 \\
36.033 \\
-12.850 \\
4600 \\
\end{array}$ & $\begin{array}{c}4340399-\infty 0 \\
35.133 \\
-12.933 \\
1450 \\
\end{array}$ & $\begin{array}{c}4340399-01 \\
35.133 \\
-12933 \\
1450 \\
\end{array}$ & $\begin{array}{c}4360183-00 \\
36.943 \\
-33.065 \\
2670 \\
\end{array}$ \\
\hline Si(wt.\%) & $\cdot$ & $\cdot$ & . & . & - & $\cdot$ & 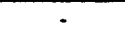 & $\cdot$ & . & $\cdot$ & 7.80 \\
\hline $\mathbf{T i}$ & 58 & .42 & .46 & 50 & .48 & 54 & .56 & .45 & .81 & 91 & .05 \\
\hline Mn & 260 & 11.8 & 6.96 & 114 & 7.00 & 7.82 & 8.10 & 9.63 & 13.9 & 13.8 & 26.8 \\
\hline $\mathrm{F}_{0}$ & 279 & 15.5 & 26.4 & 134 & 143 & 12.7 & 17.2 & 20.5 & 19.5 & 18.7 & 114 \\
\hline $\mathbf{A l}$ & 3.23 & 3.18 & 284 & 3.26 & 3.76 & 5.09 & 3.82 & 297 & 1.70 & 1.66 & 90 \\
\hline Co & .030 & .140 & .110 & .130 & .180 & .220 & .190 & .170 & .790 & .740 & .010 \\
\hline $\mathbf{N i}$ & .140 & .190 & .200 & 200 & .110 & .160 & .130 & .220 & .250 & 300 & .060 \\
\hline Cur & 200 & .150 & .200 & .140 & .120 & .120 & .130 & .180 & .040 & .050 & .030 \\
\hline Ca & 31 & 1.80 & .49 & 1.50 & .82 & 1.60 & 97 & .69 & 3.60 & 6.70 & 1.60 \\
\hline $\mathbf{M g}$ & • & • & • & - & - & - & - & • & • & • & 2.23 \\
\hline Ne & - & - & - & - & - & - & - & - & - & - & 1.83 \\
\hline $\mathbf{K}$ & - & - & - & - & - & - & - & - & - & - & .81 \\
\hline $\mathbf{P}$ & - & - & - & - & - & - & - & - & - & - & $\cdot$ \\
\hline $\mathrm{H}_{2} \mathrm{O}$ & - & $\cdot$ & - & - & - & - & - & $\cdot$ & - & - & - \\
\hline$A \Phi$ (ppon) & - & - & - & . & - & - & - & - & - & - & • \\
\hline $\mathrm{Ba}$ & - & • & $\cdot$ & • & - & - & - & • & • & • & 590 \\
\hline Cd & 6.0 & 8.0 & 7.0 & 14.0 & 10.0 & 13.0 & 120 & 7.0 & 10.0 & 10.0 & • \\
\hline co & • & • & $=$ & - & • & • & - & $\cdot$ & - & - & - \\
\hline $\mathrm{Cr}$ & 65 & 38 & 68 & 50 & 55 & 80 & 45 & 45 & 80 & 50 & 13 \\
\hline Mo & - & - & - & - & - & - & • & • & • & - & - \\
\hline $\mathrm{Pb}$ & 1100 & 1100 & 1300 & 1100 & 900 & 900 & 1300 & 1500 & 2600 & 2700 & - \\
\hline Sr & - & • & - & - & - & - & - & - & - & - & 350 \\
\hline $\mathbf{v}$ & - & - & - & - & - & - & - & - & - & - & 320 \\
\hline $\mathbf{Y}$ & - & - & - & - & - & • & - & - & - & - & - \\
\hline $\mathrm{Zn}$ & 540 & 550 & 660 & 490 & 420 & 420 & 550 & 560 & 570 & 520 & 130 \\
\hline Sesmple & Crust & Crust & Crust & Crust & Coust & Crust & Crust & Crust & Unlmown & Crust & Crust \\
\hline type & • & $\cdot$ & • & $\cdot$ & • & $\cdot$ & • & $\cdot$ & - & • & - \\
\hline Portion & unionown & unknown & unknown & unknown & unknown & unknown & unknown & unkonown & unkonown & unknown & unkoown \\
\hline sompled & • & • & • & • & - & • & • & • & • & • & • \\
\hline Nuclous & - & - & - & - & - & - & - & - & - & - & - \\
\hline References & A467 & A467 & A467 & A467 & A467 & A467 & A467 & A467 & A467 & A467 & A464 \\
\hline Sequencell & $4360184-\infty$ & $4360185-00$ & $4360186-\infty 0$ & $4360187-00$ & $4360188-\infty 0$ & $4360189-\infty 0$ & $4360190-00$ & $4370030-01$ & $4370045-00$ & $4380089-00$ & $4380090-00$ \\
\hline Letitude & 36.968 & 36.950 & 36.945 & 36.953 & 36.950 & 36.833 & 36.937 & 31.817 & 30.817 & 32428 & 32.428 \\
\hline Langitude & -33.063 & -33.058 & -33.067 & -33.078 & -33.070 & -33.178 & -33.167 & -43.417 & -44.550 & .58 .968 & .58 .968 \\
\hline Dopth & 2100 & 2680 & 2690 & 2560 & 2700 & - & $\ldots$ & 3700 & 3540 & 3453 & 3453 \\
\hline Si(wt.\%) & 3.50 & 3.50 & 4.10 & 3.70 & 4.10 & 6.10 & 4.00 & 4.21 & 2.60 &. & . \\
\hline $\mathrm{Ti}$ & 1.07 & .76 & .02 & 80 & .86 & .98 & .66 & . & 87 & - & - \\
\hline Mn & 12.4 & 9.20 & 29.0 & 10.2 & 9.60 & 7.90 & 7.40 & 14.5 & 11.5 & 11.9 & 14.4 \\
\hline Po & 25.7 & 20.1 & 9.60 & 24.1 & 26.6 & 23.2 & 212 & 26.6 & 25.9 & 20.2 & 14.0 \\
\hline $\mathbf{N}$ & 240 & 1.90 & .40 & 240 & 280 & 4.10 & 240 & $\cdot$ & 2.60 & - & - \\
\hline Co & 300 & 230 & .010 & 250 & 230 & .190 & .180 & .620 & .790 & 340 & .420 \\
\hline $\mathbf{N i}$ & .110 & .070 & .020 & .080 & .100 & .070 & .070 & .230 & .160 & .240 & 320 \\
\hline $\mathrm{Cu}$ & .040 & .030 & .010 & .040 & .050 & .050 & .040 & .120 & .070 & .140 & .140 \\
\hline$c_{2}$ & 5.20 & 5.20 & 5.20 & 8.00 & 5.90 & 6.50 & 5.30 & 245 & 203 & - & • \\
\hline $\mathbf{M g}$ & 2.21 & 1.88 & 1.62 & 1.79 & 1.80 & 213 & 1.39 & - & - & - & - \\
\hline $\mathrm{Ne}$ & 95 & .67 & 1.09 & 59 & .99 & .95 & .65 & - & - & . & . \\
\hline $\mathbf{K}$ & .17 & .12 & .66 & .09 & .18 & .19 & .17 & . & - & - & - \\
\hline $\mathbf{P}$ & - & $\cdot$ & - & - & - & - & . & .46 & - & - & - \\
\hline $\mathrm{H}_{2} \mathrm{O}$ & - & - & - & - & - & - & - & - & 17.1 & - & - \\
\hline$A \leq(p p m)$ & - & - & - & • & - & - & • & - & - & - & - \\
\hline $\mathrm{Ba}$ & 920 & 740 & 700 & 970 & 850 & 670 & 750 & - & 6700 & - & - \\
\hline $\mathrm{Cd}$ & - & - & - & - & • & - & - & - & - & - & - \\
\hline$C_{0}$ & • & - & - & • & - & • & - & - & - & - & - \\
\hline $\mathrm{Cr}$ & 75 & 86 & 9 & 110 & 82 & 190 & 71 & - & • & - & - \\
\hline Mo & - & - & - & - & - & - & . & - & 170 & - & - \\
\hline $\mathrm{Pb}$ & - & - & - & - & • & - & - & - & 2000 & - & - \\
\hline Sr & 3200 & 2300 & 620 & 2800 & 2500 & 1700 & 2500 & - & 900 & - & - \\
\hline v & 1500 & 1300 & 108 & 1600 & 1500 & 1300 & 1400 & - & - & - & - \\
\hline $\mathbf{Y}$ & - & - & - & • & - & - & - & • & - & - & - \\
\hline $\mathbf{Z n}$ & 380 & 280 & 40 & 420 & 380 & 300 & 320 & 630 & 510 & • & - \\
\hline Samplo & Mn-conted & Mn-conted & Crust & Mn-conted & Mn-costed & Mn-costed & Mn-conted & Unknown & Nodule & Crust & Crunt \\
\hline typo & rock & rock & - & rock & rock & rock & rock & $\cdot$ & $\cdot$ & • & - \\
\hline Partion & outer & outer & unknown & outor & outer & outer & ouser & unkonown & wholo & unknown & unknown \\
\hline samplod & crust & crust & - & crust & crust & crust & crust & - & • & • & - \\
\hline Nucleus & volcenic & volcanic & - & volcanic & volcanic & volcanic & volcmic & - & - & - & - \\
\hline References & A464 & A464 & A464 & A464 & A464 & A464 & A464 & A388 & A197 & Al79 & A179 \\
\hline
\end{tabular}


Table 9. Composition of crusts from the Scripps Nodule Databank (cont'd).

\begin{tabular}{|c|c|c|c|c|c|c|c|c|c|c|c|}
\hline $\begin{array}{l}\text { Sequerceall } \\
\text { Letitudo } \\
\text { Longitude } \\
\text { Dopth }\end{array}$ & $\begin{array}{c}4380090-01 \\
32.428 \\
-58.968 \\
3453\end{array}$ & $\begin{array}{c}4380112-00 \\
32.033 \\
-59.000 \\
2150\end{array}$ & $\begin{array}{c}4380113-00 \\
32.133 \\
.59 .025 \\
3475\end{array}$ & $\begin{array}{c}4390002-01 \\
38.067 \\
-60.217 \\
3007\end{array}$ & $\begin{array}{c}4390042-00 \\
34.867 \\
.62500 \\
1460\end{array}$ & $\begin{array}{c}4390213-00 \\
31.015 \\
-67.113 \\
4764\end{array}$ & $\begin{array}{c}4390353-00 \\
39.000 \\
-61.000 \\
.\end{array}$ & $\begin{array}{c}4390358-00 \\
39.000 \\
-60.950 \\
2000\end{array}$ & $\begin{array}{c}4390358-02 \\
39.000 \\
-60.950 \\
2000\end{array}$ & $\begin{array}{c}4390358-03 \\
39.000 \\
-60.950 \\
2000\end{array}$ & $\begin{array}{c}4390358-04 \\
39.000 \\
-60.950 \\
2000\end{array}$ \\
\hline Si(wtg) & $\cdot$ & $\cdot$ & - & - & .90 & $\cdot$ & .88 & $\cdot$ & 1.10 & 2.30 & 3.20 \\
\hline $\mathrm{Ti}$ & • & • & - & - & .57 & - & 52 & - & 55 & 50 & ss \\
\hline Mn & 15.2 & 19.8 & 21.1 & 14.0 & 169 & 13.3 & 16.1 & 20.8 & 16.4 & 16.1 & 135 \\
\hline $\mathrm{Fe}$ & 23.0 & 19.8 & 17.9 & 25.2 & 18.9 & 20.6 & 13.0 & 24.6 & 19.3 & 18.6 & 18.5 \\
\hline $\mathbf{A}$ & - & - & • & - & 1.40 & - & 31 & - & .83 & 1.20 & .98 \\
\hline$c_{0}$ & 510 & .600 & .600 & 360 & 910 & .180 & .200 & .630 & .200 & .220 & .290 \\
\hline $\mathrm{Ni}$ & 250 & .270 & .740 & 200 & 270 & .200 & .130 & 330 & .110 & 250 & .100 \\
\hline $\mathrm{Cr}$ & .110 & .090 & .210 & .100 & .040 & .120 & .070 & .110 & .050 & .060 & .070 \\
\hline$c_{2}$ & 1.89 & 280 & 1.64 & 1.98 & 223 & - & 7.60 & - & 2.00 & 1.90 & 200 \\
\hline$M_{8}$ & 1.55 & 1.29 & 2.62 & 156 & • & - & - & - & - & - & - \\
\hline $\mathrm{Na}$ & 1.83 & 132 & 1.25 & 150 & - & - & - & - & - & - & - \\
\hline $\mathrm{x}$ & .80 & .28 & 31 & 23 & - & - & 32 & - & .47 & .74 & .74 \\
\hline $\mathbf{P}$ & - & - & - & - & - & - & - & - & - & - & - \\
\hline $\mathrm{H}_{2} \mathrm{O}$ & - & - & - & - & 21.5 & - & - & - & - & - & - \\
\hline$A x(p p m)$ & - & - & - & - & - & - & 190 & - & 190 & 250 & 190 \\
\hline $\mathrm{Ba}_{\mathrm{a}}$ & . & - & - & - & 7100 & - & 1900 & - & 1600 & 1700 & 1200 \\
\hline Cd & . & - & . & - & - & . & - & . & • & . & . \\
\hline$c_{0}$ & - & - & - & - & - & - & - & - & - & - & - \\
\hline cr & - & - & - & - & - & - & - & - & - & - & - \\
\hline Mo & • & - & • & • & 390 & - & 360 & - & 370 & 320 & 280 \\
\hline $\mathrm{Pb}$ & 1300 & 1500 & 1100 & 1400 & 2300 & - & 800 & - & 600 & 600 & 600 \\
\hline Sr & • & - & - & • & 1300 & - & 2400 & - & 1700 & 1600 & 1500 \\
\hline $\mathbf{v}$ & . & . & . & - & - & - & 1200 & - & 1500 & 1300 & 1600 \\
\hline $\mathbf{Y}$ & - & - & - & . & . & - & 410 & - & 250 & 250 & 290 \\
\hline Zn & 570 & 590 & 980 & 610 & 530 & • & 900 & • & 850 & 900 & 750 \\
\hline Sample & Court & Court & Crust & Curst & Crust & Crust & Crust & Nodule & Crust & Crust & Crust \\
\hline typo & • & • & • & • & - & - & - & • & $\cdot$ & • & • \\
\hline Portion & unknown & unkoown & unknown & unknown & croses & unknown & unknown & unkrown & topeide & middle & underside \\
\hline sempled & - & - & . & $\cdot$ & exction & - & $\cdot$ & - & $\cdot$ & leyer & - \\
\hline Nuclous & - & • & - & - & - & - & . & - & . & • & . \\
\hline Referencos & A368 & A368 & A368 & A368 & Alg & Al79 & A283 & A354 & A283 & A283 & A283 \\
\hline Sequencedt & 4390358-05 & $4400456-00$ & $4400536-01$ & $400536-03$ & $4400539-\infty 0$ & $4400539-01$ & $4400548-\infty 0$ & $4400564-02$ & $4400565-\infty$ & $4400569-01$ & $440097-00$ \\
\hline Lesinedo & 39.000 & 30.000 & 30.850 & 30.850 & 31.213 & 31.213 & 31.383 & 30.883 & 31.680 & 31.253 & 31.283 \\
\hline Longinde & -60.950 & -77.000 & -78.450 & .78 .450 & -78.483 & -78.483 & -78.667 & -78.783 & -78.800 & -78.983 & -117.583 \\
\hline Depth & 2000 & 2640 & 732 & 732 & 648 & 648 & 512 & 815 & 543 & 546 & 2100 \\
\hline$S i(w t \%)$ & $\cdot$ & $\cdot$ & 2.00 & 1.31 & .51 & .61 & 1.26 & $\cdot$ & .61 & 28 & 125 \\
\hline $\mathrm{Ti}$ & - & - & .49 & - & .26 & .35 & .09 & . & .06 & .26 & .25 \\
\hline Mn & 17.7 & 40.9 & 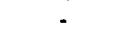 & 195 & 123 & 12.1 & 6.95 & 17.9 & 13.1 & 11.1 & 134 \\
\hline $\mathrm{Fo}$ & 22.4 & 14.6 & 18.7 & 16.7 & 6.78 & 12.5 & 1.60 & 13.4 & 5.10 & 6.64 & 11.4 \\
\hline A & 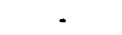 & - & - & . & .58 & 1.85 & .74 & - & .95 & 37 & 3.90 \\
\hline co & .390 & .660 & .550 & .730 & . & 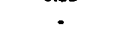 & $\because$ & .270 & . & 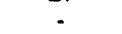 & .080 \\
\hline $\mathbf{N i}$ & .170 & 840 & 520 & .600 & 310 & .310 & .220 & .430 & .440 & 300 & 340 \\
\hline $\mathrm{Cx}$ & .030 & .060 & .070 & .080 & .100 & .110 & .100 & .050 & .180 & .120 & .060 \\
\hline$c_{2}$ & 1.89 & - & . & 7.72 & 7.08 & 9.93 & 8.73 & .16 & 2.01 & 1.08 & .94 \\
\hline Mg & 1.10 & . & 3.70 & - & 133 & 1.93 & 151 & 2.13 & 205 & 1.09 & - \\
\hline $\mathrm{Na}$ & 1.27 & . & - & - & . & - & - & 1.04 & - & - & - \\
\hline $\mathbf{K}$ & .25 & - & - & . & .23 & .17 & .24 & .26 & 36 & .24 & .92 \\
\hline $\mathbf{P}$ & - & - & - & 32 & 2.40 & .10 & .43 & $\cdot$ & 5.63 & 3.84 & - \\
\hline $\mathrm{H}_{2} \mathrm{O}-$ & - & - & - & $\cdot$ & • & $\cdot$ & $\cdot$ & $\cdot$ & $\cdot$ & • & 15.4 \\
\hline Axppmo) & - & . & . & 390 & - & . & . & . & . & . & . \\
\hline Ba & - & . & . & . & . & - & - & . & . & . & 4100 \\
\hline Cd & - & . & - & . & - & - & - & . & - & - & • \\
\hline$c_{0}$ & - & - & - & - & . & - & - & - & . & - & - \\
\hline Cr & - & . & . & - & - & - & - & - & - & - & - \\
\hline Mo & - & - & 680 & 600 & . & - & - & - & - & - & 360 \\
\hline $\mathrm{Pb}$ & 1800 & 2500 & - & 1100 & - & . & - & 1500 & - & • & 800 \\
\hline Sr & • & - & - & 1400 & 2500 & 840 & 1700 & • & 22000 & 3400 & 740 \\
\hline v & - & - & 840 & $\cdot$ & • & • & • & - & - & $\cdot$ & $\cdot$ \\
\hline $\mathbf{Y}$ & - & . & . & 219 & . & . & . & . & - & - & - \\
\hline Zn & 620 & - & - & 550 & . & • & • & 540 & . & • & 670 \\
\hline Sumple & Crust & Nodule & Nodule & Nodule & Crost & Crust & Count & Crant & Crust & Crust & Crust \\
\hline type & & • & $\cdot$ & • & • & $\cdot$ & $\cdot$ & - & • & & • \\
\hline Partion & unknown & cooss & unknown & whole & unkrown & unkrown & unkrown & unkrown & unkrown & unknown & cross \\
\hline sampled & 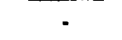 & soction & 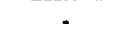 & - & - & & • & $\cdot$ & • & - & section \\
\hline Nuclous & - & - & . & - & . & . & • & • & - & - & - \\
\hline Referencos & A368 & A166 & A201 & A388 & A374 & A374 & A374 & A368 & A374 & A374 & A197 \\
\hline
\end{tabular}


Table 9. Composition of crusts from the Scripps Nodule Databank (cont'd).

\begin{tabular}{|c|c|c|c|c|c|c|c|c|c|c|c|}
\hline $\begin{array}{l}\text { Sequencoll } \\
\text { Latitude } \\
\text { Longitude } \\
\text { Depth }\end{array}$ & $\begin{array}{c}4440102-\infty \\
30.200 \\
-117.633 \\
1300\end{array}$ & $\begin{array}{c}4440102-01 \\
30.200 \\
-117.633 \\
1300\end{array}$ & $\begin{array}{c}4440102-02 \\
30.200 \\
-117.633 \\
1300\end{array}$ & $\begin{array}{c}4440106-01 \\
30.300 \\
-117.667 \\
1060\end{array}$ & $\begin{array}{c}4440106-02 \\
30.300 \\
-117.667 \\
1060\end{array}$ & $\begin{array}{c}4440137-\infty 0 \\
31.383 \\
-118.050 \\
1040\end{array}$ & $\begin{array}{c}4440137-01 \\
31.383 \\
-118.050 \\
1040\end{array}$ & $\begin{array}{c}440318-01 \\
31.083 \\
-118.617 \\
1650 \\
\end{array}$ & $\begin{array}{c}4460084-00 \\
32.933 \\
-132500 \\
702\end{array}$ & $\begin{array}{c}4460085-00 \\
38.538 \\
-133.650 \\
4427 \\
\end{array}$ & $\begin{array}{c}4460085-01 \\
38.538 \\
-133.650 \\
4427 \\
\end{array}$ \\
\hline si(wt.\%) & 10.9 & . & . & 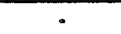 & . & 9.80 & . & . & . & - & - \\
\hline $\mathrm{TI}$ & 53 & 58 & . & - & .46 & .63 & 53 & .24 & - & - & - \\
\hline Mn & 10.7 & 14.2 & 16.4 & 33.4 & 144 & 13.7 & 19.4 & 14.6 & 25.6 & 12.6 & 3.40 \\
\hline Fo & 14.7 & 15.1 & 20.8 & 183 & 11.9 & 145 & 129 & 9.22 & 11.4 & 5.90 & 5.60 \\
\hline Al & 3.50 & - & - & - & - & 270 & . & - & - & - & • \\
\hline Co & .400 & 560 & - & .550 & 590 & 530 & .580 & 200 & 900 & .100 & .020 \\
\hline $\mathbf{N i}$ & .180 & 200 & .180 & 350 & 590 & .230 & .450 & 480 & 400 & 810 & .250 \\
\hline $\mathrm{Cu}$ & .040 & .030 & .020 & .040 & .040 & .050 & .060 & .130 & - & 460 & .170 \\
\hline $\mathrm{ca}$ & 1.48 & - & - & - & - & 1.65 & . & . & - & - & - \\
\hline $\mathbf{M}_{8}$ & - & - & . & - & - & - & - & - & - & - & - \\
\hline $\mathrm{Ne}$ & - & - & - & - & - & - & - & - & - & - & - \\
\hline $\mathbf{K}$ & .5s & - & . & - & - & .42 & - & - & . & - & - \\
\hline P & - & . & . & . & - & - & - & - & - & - & - \\
\hline $\mathrm{H}_{2} \mathrm{O}$ & 19.0 & - & - & - & - & 20.6 & - & - & - & - & $\cdot$ \\
\hline$A x$ (ppm) & - & - & . & - & - & • & - & - & - & . & - \\
\hline Be & 4600 & 3900 & - & . & 4400 & 6100 & 6400 & 5700 & - & - & - \\
\hline Cd & . & 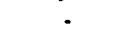 & . & - & - & . & - & - & . & . & . \\
\hline co & - & - & - & - & - & - & - & - & - & - & - \\
\hline $\mathrm{Cr}$ & - & 49 & - & - & 86 & - & 46 & 12 & - & . & - \\
\hline Mo & 4100 & 290 & - & • & 340 & 480 & 420 & 320 & - & . & . \\
\hline $\mathrm{Pb}$ & 2400 & 1100 & - & 3400 & 300 & 2100 & 1200 & 400 & - & - & . \\
\hline Sr & 1300 & - & - & - & $\cdot$ & 1500 & . & - & - & - & - \\
\hline $\mathbf{v}$ & - & 540 & - & - & 670 & - & 930 & 520 & - & - & - \\
\hline $\mathbf{Y}$ & - & - & - & - & . & - & . & . & . & . & . \\
\hline $\mathbf{Z n}_{n}$ & 360 & . & - & - & . & 400 & - & - & - & - & . \\
\hline Semple & Crust & Crux & Mn-couted & Noctuio & Crus & Crant & Crust & Cruar & Crust & Cruxt & Crust \\
\hline typo & - & - & rock & - & - & - & - & - & - & - & - \\
\hline Partion & crows & fragments & without & unknown & fruemenss & crows & fragments & fregmons & unknown & unknown & untenown \\
\hline samolod & soction & 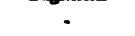 & medons & . & . & soction &. & . & - & . & 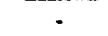 \\
\hline Nuclous & - & - & - & - & - & - & - & - & - & - & - \\
\hline References & A197 & A240 & A321 & Al66 & A240 & A197 & A240 & $A 240$ & A255 & A333 & A333 \\
\hline Sequencoll & $4460089-03$ & $4470008-00$ & $4480024-01$ & $4480030-08$ & $4490019-00$ & $4490039-04$ & $4490043-00$ & $4490047-00$ & 4490047.01 & $4490048-00$ & $4490048-01$ \\
\hline Letinude & 32927 & 35.700 & 30.717 & 36.000 & 30.350 & 31.730 & 32.143 & 33.928 & 33.928 & 31.935 & 31.935 \\
\hline Longituds & -133.883 & -140.850 & -159.567 & -157.000 & -163.852 & -165.717 & -160.272 & -162095 & -162095 & -162.240 & -162240 \\
\hline Depth & 5385 & 5207 & 5770 & - & 2476 & 5597 & 4000 & 2430 & 2430 & 2800 & 2800 \\
\hline$\overline{\text { Si(wt.\%) }}$ &. & . & . & . &. &. & . & . & . & - & - \\
\hline $\mathrm{Ti}$ & • & 1.76 & • & . & - & • & - & • & - & • & • \\
\hline Mn & 280 & 3.00 & 18.4 & 213 & 24.0 & 6.70 & 16.9 & 21.9 & 22.1 & 21.0 & 16.8 \\
\hline$F_{0}$ & 7.70 & 31.0 & 17.8 & 13.6 & 18.2 & 7.90 & 16.3 & 14.7 & 16.9 & 18.8 & 14.3 \\
\hline Al & - & 2.17 & 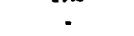 & . & - & 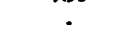 & . & - & 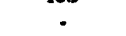 & - &. \\
\hline Co & .050 & .140 & .280 & 520 & .760 & .010 & $\$ 40$ & .770 & .690 & .710 & .510 \\
\hline $\mathbf{N i}$ & .080 & .010 & 200 & .600 & 370 & .120 & $A 00$ & .660 & 570 & 300 & 350 \\
\hline Cu & .080 & .100 & 170 & .260 & .050 & .160 & .290 & .170 & .130 & .100 & .180 \\
\hline $\mathrm{Ca}$ & $=$ & 36 & . &. & 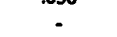 & 1.40 & 1.70 & 280 & 250 & 250 & 280 \\
\hline $\mathbf{M g}_{\mathbf{g}}$ & . & .70 & - & - & . & 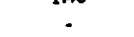 & . &. & . & . & . \\
\hline $\mathrm{Ne}$ & - & 35 & - & . & . & . & . & . & - & . & . \\
\hline $\mathbf{K}$ & - & 50 & - & - & - & - & - & . & - & . & - \\
\hline $\mathbf{P}$ & . & .28 & - & - & - & - & . & . & - & . & . \\
\hline $\mathrm{H}_{2} \mathrm{O}$ & - & 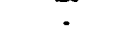 & . & - & - & - & - & - & - & - & - \\
\hline AN(ppm) & - & - & - & - & - & - & - & - & - & - & - \\
\hline Be & - & - & - & - & - & - & - & - & - & - & - \\
\hline Cd & . & - & . & . & . & - & - & - & - & - & - \\
\hline Co & - & - & - & - & - & . & . & - & - & . & - \\
\hline $\mathrm{Cr}$ & - & • & - & - & - & - & - & - & - & . & - \\
\hline Mo & - & 1000 & - & - & 740 & • & . & - & - & - & - \\
\hline $\mathrm{Pb}$ & - & 1600 & - & - & $\cdot$ & 500 & 1200 & 1200 & 1400 & 1400 & 900 \\
\hline St & . & . & - & - & - & $\cdot$ & 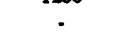 & . & 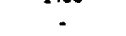 & . & . \\
\hline v & - & - & - & - & - & - & . & - & - & - & - \\
\hline $\mathbf{Y}$ & - & - & - & - & - & - & - & - & - & - & - \\
\hline $\mathbf{Z n}_{\mathbf{n}}$ & - & . & - & - & - & 400 & 400 & 500 & 500 & 300 & 500 \\
\hline Semple & Cour & Mn-cocrusted & Mn-encrustod & Noctulo & Mn-conted & Mn-oncrunted & Crust & Nodule & Mn-conted & Mn-costed & Mn-coated \\
\hline typo & • & sediment & odiment & • & rock & sediment & • & . & rock & rock & rock \\
\hline Partion & unknown & unknown & unknown & whole & outer & whole & outer & outer & without & outer & ouver \\
\hline ampled & - & . & $\cdot$ & $\cdot$ & crust & - & crust & crust & meclous & crust & crust \\
\hline Nucleus & - & - & - & - & • & Pumico & - & Baralt & Rock & Rock & • \\
\hline Reference & A333 & $A 419$ & $A 419$ & A277 & A352 & 1437 & A459 & 1459 & A459 & A459 & A459 \\
\hline
\end{tabular}


Table 9. Composition of crusts from the Scripps Nodule Databank (cont'd).

\begin{tabular}{|c|c|c|c|c|c|c|c|c|c|c|c|}
\hline $\begin{array}{l}\text { Sequencell } \\
\text { Letitude } \\
\text { Longitude } \\
\text { Depth }\end{array}$ & $\begin{array}{c}4490049-00 \\
33.217 \\
-162.338 \\
2900\end{array}$ & $\begin{array}{c}4490050-00 \\
31.118 \\
-162.365 \\
2400\end{array}$ & $\begin{array}{c}4490051-00 \\
31.802 \\
-162843 \\
2500\end{array}$ & $\begin{array}{c}4490051-01 \\
31.802 \\
-162843 \\
2500\end{array}$ & $\begin{array}{c}4490051-02 \\
31.802 \\
-162.843 \\
2500\end{array}$ & $\begin{array}{c}449005200 \\
31.645 \\
-162.872 \\
2600\end{array}$ & $\begin{array}{c}4490052-01 \\
31.645 \\
-162872 \\
2600\end{array}$ & $\begin{array}{c}4490054-\infty 0 \\
31.660 \\
-163.017 \\
2450\end{array}$ & $\begin{array}{c}4490054-01 \\
31.660 \\
-163.017 \\
2450 \\
\end{array}$ & $\begin{array}{c}4490055-00 \\
33.627 \\
-165.098 \\
2770\end{array}$ & $\begin{array}{c}4490055-01 \\
33.627 \\
-165.098 \\
2770 \\
\end{array}$ \\
\hline Si(wt\%) & - & - & $\cdot$ & - & $\cdot$ & $\cdot$ & 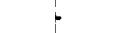 & - & - & - & - \\
\hline $\mathrm{Ti}$ & - & - & - & • & - & • & - & • & - & • & - \\
\hline Mn & 20.6 & 23.1 & 18.5 & 194 & 20.4 & 20.5 & 17.2 & 19.9 & 173 & 21.0 & 20.9 \\
\hline Po & 17.4 & 15.1 & 17.5 & 194 & 19.7 & 18.6 & 14.5 & 18.6 & 18.8 & 16.6 & 16.2 \\
\hline $\mathbf{A}$ & - & - & • & - & - & • & $\cdot$ & - & - & • & - \\
\hline Co & 1.000 & .870 & 580 & .780 & .800 & .860 & .810 & .750 & .790 & .770 & .790 \\
\hline $\mathbf{N i}$ & 360 & .610 & 320 & .270 & .250 & 300 & .500 & .250 & .270 & 410 & $A 80$ \\
\hline $\mathrm{Cu}$ & .060 & .160 & .130 & .080 & .080 & .060 & .170 & .100 & .090 & .160 & .140 \\
\hline C. & 230 & 2.60 & 2.60 & 230 & 250 & 250 & 240 & 240 & 2.20 & 260 & 230 \\
\hline Mg & - & $\cdot$ & - & - & $\cdot$ & • & - & • & • & - & • \\
\hline $\mathrm{Ne}$ & $\cdot$ & $\cdot$ & - & $\cdot$ & $\cdot$ & $\cdot$ & $\cdot$ & $\cdot$ & $\cdot$ & $\cdot$ & $\cdot$ \\
\hline $\mathbf{K}$ & - & - & - & $\cdot$ & - & $\cdot$ & • & - & $\cdot$ & - & $\cdot$ \\
\hline $\mathbf{P}$ & - & - & - & - & - & - & - & - & - & - & - \\
\hline $\mathrm{H}_{2} \mathrm{O}$ & $\cdot$ & $\cdot$ & $\cdot$ & $\cdot$ & $\cdot$ & $\cdot$ & $\cdot$ & $\cdot$ & - & - & $\cdot$ \\
\hline As(ppm) & - & - & $\cdot$ & - & - & - & t & - & $\cdot$ & - & - \\
\hline $\mathrm{Be}$ & - & - & - & - & - & - & 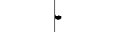 & $\cdot$ & - & - & - \\
\hline $\mathrm{Cd}$ & $\cdot$ & - & - & - & $\cdot$ & $\cdot$ & t & - & $\cdot$ & - & $\cdot$ \\
\hline Co & $\cdot$ & $\cdot$ & - & $\cdot$ & $\cdot$ & - & $b$ & - & - & - & - \\
\hline$C r$ & $\cdot$ & - & - & - & $\cdot$ & - & - & - & - & - & $\cdot$ \\
\hline Mo & - & - & • & - & - & - & - & - & - & - & $\cdot$ \\
\hline $\mathrm{Pb}$ & 1600 & 1400 & 1200 & 1400 & 1400 & 1600 & 1300 & 1500 & 1600 & 1200 & 1400 \\
\hline Sr & • & $\cdot$ & $\cdot$ & - & $\cdot$ & $\cdot$ & $\cdot$ & • & $\cdot$ & $\cdot$ & $\cdot$ \\
\hline $\mathbf{y}$ & $\cdot$ & - & - & - & - & - & - & - & $\cdot$ & $\cdot$ & $\cdot$ \\
\hline $\mathbf{Y}$ & - & - & - & - & $\cdot$ & - & - & - & - & - & - \\
\hline $\mathrm{Zn}$ & 300 & 500 & 400 & 300 & 300 & 300 & 500 & 300 & 400 & 400 & 400 \\
\hline Sumple & Mn-controd & Nocule & Crut & Crant & Crus & Crust & Crust & Cront & Mn-coutad & Nodule & Mar-coutred \\
\hline typo & rock & - & - & • & - & - & - & $\cdot$ & rock & • & rock \\
\hline $\begin{array}{l}\text { Portion } \\
\text { sempled }\end{array}$ & $\begin{array}{l}\text { without } \\
\text { muclous }\end{array}$ & $\begin{array}{l}\text { without } \\
\text { nucleus }\end{array}$ & $\begin{array}{l}\text { layeredjacent } \\
\text { tome cleus }\end{array}$ & $\begin{array}{l}\text { middio } \\
\text { layer }\end{array}$ & $\begin{array}{l}\text { outer } \\
\text { corsin }\end{array}$ & $\begin{array}{l}\text { outer } \\
\text { corust }\end{array}$ & $\begin{array}{l}\text { outer } \\
\text { crust }\end{array}$ & $\begin{array}{l}\text { outer } \\
\text { crust }\end{array}$ & $\begin{array}{l}\text { outer } \\
\text { crust }\end{array}$ & $\begin{array}{l}\text { outer } \\
\text { crust }\end{array}$ & $\begin{array}{l}\text { outor } \\
\text { crust }\end{array}$ \\
\hline Nucleus & Rock & Rock & Basalt & Besalt & Basedt & • & - & - & Rock & Nodulefingment & Rock \\
\hline Referencos & A459 & A459 & A459 & A459 & A459 & A459 & A459 & A459 & A459 & A459 & A459 \\
\hline Sequencell & $4490055-02$ & $4490056-00$ & $4490056-01$ & 4490057.00 & $4490058-00$ & 4490058-01 & $4500034-\infty$ & 4500037.00 & $4500042-02$ & 4510017.00 & $4510023-\infty$ \\
\hline Letitude & 33.627 & 32.468 & 32.468 & 34.077 & 33.863 & 33.863 & 37.725 & 38.677 & 38.940 & 32.137 & 35.582 \\
\hline Longitude & -165.098 & -165.800 & -165.800 & -166.502 & -166.515 & -166.515 & -174.485 & -177.390 & -178.622 & 172.262 & 170.953 \\
\hline Depth & 2770 & 3000 & 3000 & 4500 & 3450 & 3450 & 5500 & 6401 & 5396 & 1309 & 1607 \\
\hline$S i(w t . \%)$ & $\cdot$ & $\cdot$ & $\cdot$ & $\cdot$ & $\cdot$ & $\cdot$ & $\cdot$ & $\cdot$ & $\cdot$ & $\cdot$ & - \\
\hline $\mathrm{Ti}$ & $\cdot$ & - & $\cdot$ & - & - & - & - & $\cdot$ & - & - & - \\
\hline Mn & 20.6 & 175 & 20.5 & 16.5 & 18.1 & 21.8 & 22.4 & 21.7 & 10.9 & 12.8 & 220 \\
\hline Pe & 15.5 & 14.3 & 18.9 & 10.6 & 139 & 16.2 & 8.20 & 7.80 & 9.60 & 9.70 & 1.00 \\
\hline $\mathbf{N}$ & - & - & - & - & • & - & - & $\cdot$ & • & - & - \\
\hline Co & .710 & 530 & .830 & 300 & 510 & .690 & .090 & .120 & .020 & .450 & .040 \\
\hline $\mathbf{N i}$ & 500 & .440 & .300 & 1.060 & .430 & .470 & .330 & 1.150 & 350 & 360 & .090 \\
\hline Cur & .150 & .190 & .080 & .610 & .250 & .190 & .360 & .700 & 310 & .040 & .020 \\
\hline Ca & 250 & 2.70 & 2.40 & 2.30 & 240 & 2.40 & • & - & 1.20 & 5.20 & 4.20 \\
\hline $\mathbf{M g}_{8}$ & - & - & - & - & . & - & - & - & • & - & • \\
\hline $\mathrm{Ne}$ & - & - & - & - & - & - & - & - & - & - & - \\
\hline $\mathbf{K}$ & - & - & - & . & - & - & - & - & - & - & - \\
\hline $\mathbf{P}$ & - & $\cdot$ & - & $\cdot$ & - & $\cdot$ & - & $\cdot$ & - & $\cdot$ & $\cdot$ \\
\hline $\mathrm{H}_{2} \mathrm{O}$ & * & - & • & - & $\cdot$ & $\cdot$ & $\cdot$ & • & - & - & $\cdot$ \\
\hline$A s(p p m)$ & - & $\cdot$ & - & - & - & - & - & - & - & - & - \\
\hline B. & - & $\cdot$ & - & - & - & $\cdot$ & - & - & $\cdot$ & - & - \\
\hline Cd & - & - & - & - & - & - & - & - & - & - & $\cdot \cdot$ \\
\hline Co & - & - & - & - & - & - & - & - & - & - & $\cdot$ \\
\hline $\mathrm{Cr}$ & $\cdot$ & $\cdot$ & $\cdot$ & - & $\cdot$ & $\cdot$ & - & $\cdot$ & $\cdot$ & $\cdot$ & - \\
\hline Mo & - & • & - & $\cdot$ & $\cdot$ & • & 300 & 300 & $\cdot$ & - & $\cdot$ \\
\hline $\mathbf{P b}$ & 1200 & 1100 & 1500 & 700 & 900 & 1200 & . & - & 800 & 1100 & 100 \\
\hline Sr & $\cdot$ & - & $\cdot$ & $\bullet$ & $\cdot$ & $\cdot$ & - & $\cdot$ & $\cdot$ & $\cdot$ & $\cdot$ \\
\hline $\mathbf{v}$ & - & $\cdot$ & - & - & - & - & - & - & - & - & - \\
\hline $\mathbf{Y}$ & - & $\cdot$ & $\cdot$ & • & $\cdot$ & $\cdot$ & - & - & $\cdot$ & • & - \\
\hline $\mathrm{Zn}$ & 400 & 500 & 600 & 1100 & 600 & 500 & - & - & 500 & 500 & 500 \\
\hline Sample & Mon-contod & Mn-conted & Mn-couted & Crust & Nodule & Nocule & Crust & Cruxt & Mn-enerustod & Crust & Crust \\
\hline typo & rock & rock & rock & • & - & • & $\cdot$ & $\cdot$ & sediment & $\cdot$ & - \\
\hline Partion & middle & outer & outer & fragments & outer & without & composite & composite & without & fragments & fragments \\
\hline sumpled & layer & crust & crust & . & crest & nucleus & 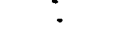 & - & nucleus & . & - \\
\hline Nucieus & Rock & Banalt & Basalt & - & non-apparent & Beselt & $\cdot$ & • & Sediment & - & - \\
\hline Referencos & A459 & A459 & A459 & A459 & A459 & A459 & A386 & A386 & A437 & A422 & A422 \\
\hline
\end{tabular}


Table 9. Composition of crusts from the Scripps Nodule Databank (cont'd).

\begin{tabular}{|c|c|c|c|c|c|c|c|c|c|c|c|}
\hline Sequencell & $4520015-01$ & $4520015-02$ & $4540006-00$ & $4540006-01$ & $4540010-00$ & $4540010-02$ & $4540030-00$ & $4540033-00$ & $4540200-00$ & $4540200-01$ & $4670471-00$ \\
\hline Latimde & 37.267 & 37.267 & 32.018 & 32018 & 32383 & 32383 & 38.000 & 34.010 & 36.028 & 36.028 & 38.563 \\
\hline Longitude & 162400 & 162.400 & 149.273 & 149.273 & 148,425 & 148.425 & 146.000 & 145.945 & 143.412 & 143412 & 14.180 \\
\hline Dopth & 1295 & 1205 & 1605 & 1605 & 5751 & 5751 & 3500 & 2515 & $\cdot$ & - & 740 \\
\hline si(wt.\%) & - & $\cdot$ & $\cdot$ & - & - & $\cdot$ & 3.30 & . & . & . & 12.2 \\
\hline $\mathbf{T i}$ & - & - & - & - & 30 & - & so & - & - & - & .14 \\
\hline $\mathrm{Mn}$ & 25.7 & 22.0 & 18.4 & 19.7 & 8.80 & 13.0 & 19.8 & 153 & 7.10 & 3.76 & 8.30 \\
\hline Fo & 125 & 14.1 & 17.0 & 15.3 & 16.0 & 17.0 & 13.9 & 20.0 & 6.81 & 3.61 & 17.6 \\
\hline Al & . & - & - & - & - & - & .62 & . & - & - & 5.60 \\
\hline Co & .610 & 500 & .550 & 490 & .080 & .110 & .290 & 410 & .130 & .070 & $.080^{\circ}$ \\
\hline$\overline{\mathrm{Ni}}$ & .670 & 370 & .810 & 850 & .060 & .110 & 380 & .190 & .070 & .040 & .180 \\
\hline $\mathrm{Cu}$ & .120 & .070 & .130 & .150 & .060 & .090 & .100 & .020 & .080 & .040 & .070 \\
\hline ca & 3.68 & 230 & 150 & 150 & 204 & 1.20 & 212 & 230 & . & 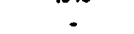 & 1.20 \\
\hline $\mathrm{Mg}$ & 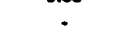 &. & 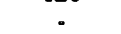 &. & 32 & .38 & 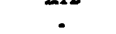 & . & . & - & 1.60 \\
\hline $\mathrm{Na}$ & - & - & - & - & 1.36 & 1.40 & - & . & . & . & . \\
\hline K & - & . & - & . & 46 & .46 & . & . & . & . & . \\
\hline $\mathbf{P}$ & . & - & - & . & .15 & .19 & . & . & . & . & . \\
\hline $\mathrm{H}_{2} \mathrm{O}$ & - & - & - & - & $\cdot$ & $\cdot$ & 21.7 & - & - & . & - \\
\hline$A x(p p m)$ & - & - & - & - & - & - & - & . & - & . & - \\
\hline Be & - & - & - & - & - & - & 7200 & - & - & - & - \\
\hline $\mathrm{Cd}$ & - & - & - & - & - & - & - & - & . & - & . \\
\hline $\mathrm{Co}_{0}$ & - & - & - & - & - & - & - & 。 & - & - & 160 \\
\hline c & - & - & - & - & - & - & - & . & - & - & . \\
\hline Mo & - & • & - & - & 260 & 180 & 480 & - & - & . & - \\
\hline $\mathrm{Pb}$ & 1800 & 2200 & 1600 & 1500 & 500 & 1100 & 1500 & 1600 & . & - & - \\
\hline St & . & . & . & - & 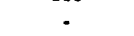 & . & 1300 & . & - & - & • \\
\hline v & . & . & . & . & . & . & . & . & . & . & 360 \\
\hline $\mathbf{Y}$ & . & - & . & . & . & . & . & . & . & . & . \\
\hline $\mathbf{Z n}$ & 900 & 400 & 800 & 900 & - & . & 570 & 500 & . & . & . \\
\hline Semple & Nodulo & Crust & Mn-contod & Mn-conted & Mn-encrusted & Mn-encrustod & Crunt & Mn-encrutted & Mn-contod & Mn-conted & Mn-conted \\
\hline type & - & 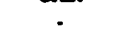 & rock & rock & sedimeat & sediment & - & rodimont & rock & rock & rock \\
\hline Partion & outor & fragment: & quarter & without & unknown & unknown & croses & outer & $n$ & outor & outer \\
\hline sompled & crust & 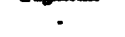 & 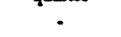 & nucleus & - & . & roction & crust & - & crust & cruse \\
\hline Nuclens & • & - & Rock & Rock & • & . & - & - & • & Undetermined & volcanic \\
\hline Refarences & A405 & A405 & A405 & A405 & A419 & A419 & A197 & A405 & A249 & A249 & $A 477$ \\
\hline Sequences & $4670471-01$ & $4670471-02$ & $4670471-03$ & $4670471-04$ & $4670472-00$ & $4670472-01$ & $4670472-02$ & $4700278-00$ & $4700278-01$ & $4700280-00$ & $4700280-01$ \\
\hline Latimde & 38.563 & 38.563 & 38.563 & 38.563 & 38.640 & 38.640 & 38.640 & 41.350 & 41.350 & 42858 & 42858 \\
\hline Longitudo & 14.180 & 14.180 & 14.180 & 14.180 & 14.017 & 14.017 & 14.017 & -14475 & -14.475 & -19.933 & -19.933 \\
\hline Depth & 740 & 740 & 740 & 740 & 690 & 690 & 690 & 5005 & 5005 & 2692 & 2692 \\
\hline si(wt\%) & 11.6 & 13.9 & 20.4 & 200 & 135 & 17.7 & 9.20 &. &. &. & - \\
\hline Ti & .71 & .04 & .19 & .02 & .10 & .07 & .02 & - & - & - & - \\
\hline Mn & 11.3 & 1.22 & 4.60 & .29 & .73 & 480 & 200 & 15.4 & 20.3 & 19.1 & 215 \\
\hline $\mathrm{Po}_{0}$ & 11.0 & 28.0 & 8.60 & 7.20 & 25.8 & 19.6 & 355 & 23.1 & 20.0 & 20.7 & 16.3 \\
\hline $\mathbf{A}$ & 7.70 & 3.40 & 6.20 & .08 & 3.20 & 280 & 80 & - & - & - & - \\
\hline Co & .080 & .030 & .040 & - & .030 & .030 & .020 & 320 & 300 & .420 & .480 \\
\hline$\overline{\mathrm{Ni}}$ & 200 & • & .130 & .010 & - & .020 & .010 & .230 & 370 & 260 & 530 \\
\hline $\mathrm{Cu}$ & .080 & .010 & .050 & 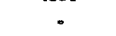 & - & .030 & - & .100 & .170 & .070 & .180 \\
\hline Ca & 1.80 & .43 & 150 & 19.7 & .68 & 51 & .47 & 1.77 & 1.87 & 220 & 208 \\
\hline $\mathrm{Mg}_{\mathbf{g}}$ & 1.80 & .48 & 1.10 & 240 & .60 & 1.00 & .36 & 1.59 & 1.56 & 1.23 & 1.91 \\
\hline $\mathrm{Na}$ & - & 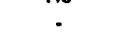 & - &. & 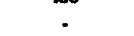 & - & . & 1.54 & 1.75 & 1.28 & 1.42 \\
\hline $\mathbf{K}$ & - & - & - & - & - & - & - & .25 & .44 & .28 & 52 \\
\hline $\mathbf{P}$ & - & - & - & . & - & - & - & . & - & - & - \\
\hline $\mathrm{H}_{2} \mathrm{O}$ & - & . & - & - & - & - & - & - & - & - & - \\
\hline$A \wedge(p p m)$ & - & . & - & - & - & . & - & . & . & - & - \\
\hline Ba & - & - & . & - & - & - & - & - & . & - & - \\
\hline Cd & - & . & - & - & . & . & . & - & . & . & . \\
\hline co & 160 & 42 & 138 & 15 & 62 & 30 & 56 & . & . & - & - \\
\hline C & - & - & 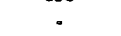 & . & - & . & - & . & . & . & - \\
\hline Mo & . & - & . & - & . & . & . & - & - & • & - \\
\hline $\mathrm{Pb}$ & . & - & - & . & - & . & . & 2400 & 2700 & 1700 & 1200 \\
\hline Sr & • & - & . & - & - & . & . & . & . & . &. \\
\hline v & 370 & 95 & 240 & 300 & 105 & 59 & 165 & - & - & - & - \\
\hline $\mathbf{Y}$ & - & . &. & - & - & 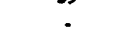 & - & . & - & - & - \\
\hline $\mathbf{Z n}$ & - & - & - & - & • & - & - & 510 & 650 & 630 & 850 \\
\hline Semple & Mn-conted & Mn-conted & Mn-conted & Mn-conted & Mn-contod & Mn-conted & Mn-couted & Crust & Crust & Crust & Crut \\
\hline type & rock & rock & rock & rock & rock & rock & rock & 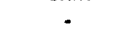 & - & $\cdot$ & - \\
\hline Partion & outor & ouver & outer & outer & outer & outer & outer & unkonown & unknown & unknown & unkonown \\
\hline amplod & crust & coust & crust & crust & crust & crust & crust & - & - & . & . \\
\hline Nucleus & volcenic & volcanic & volcenic & volcanic & volcenic & volcenic & volemic & - & - & - & - . \\
\hline Reference & $\mathbf{A 4 7 7}$ & A477 & $A 477$ & A477 & $\mathrm{A} 477$ & $A 477$ & $\mathrm{~A} 477$ & A368 & A368 & A368 & A368 \\
\hline
\end{tabular}


Table 9. Composition of crusts from the Scripps Nodule Databank (cont'd).

\begin{tabular}{|c|c|c|c|c|c|c|c|c|c|c|c|}
\hline $\begin{array}{l}\text { Sequencoll } \\
\text { Latimude } \\
\text { Longitude } \\
\text { Dopth }\end{array}$ & $\begin{array}{c}4710180-00 \\
42.900 \\
-20.142 \\
3288 \\
\end{array}$ & $\begin{array}{c}4710180-01 \\
42.900 \\
-20.142 \\
3288 \\
\end{array}$ & $\begin{array}{c}4710182-00 \\
42.910 \\
-20.187 \\
3222 \\
\end{array}$ & $\begin{array}{c}4710183-00 \\
42.903 \\
-20.213 \\
3194 \\
\end{array}$ & $\begin{array}{c}4710184-00 \\
42.907 \\
-20.223 \\
3294 \\
\end{array}$ & $\begin{array}{c}4710185-00 \\
42.900 \\
-20.250 \\
3345 \\
\end{array}$ & $\begin{array}{c}4710185-01 \\
42.900 \\
-20.250 \\
3345 \\
\end{array}$ & $\begin{array}{c}4710187-\infty 0 \\
44.088 \\
-21.927 \\
1675 \\
\end{array}$ & $\begin{array}{c}4710188-00 \\
44.038 \\
-21.948 \\
2275 \\
\end{array}$ & $\begin{array}{c}4710189-00 \\
44.135 \\
-22295 \\
2885 \\
\end{array}$ & $\begin{array}{c}4710190-00 \\
45.583 \\
-27.050 \\
2262 \\
\end{array}$ \\
\hline $\begin{array}{l}\text { Si(wr.q) } \\
\mathrm{Ti}\end{array}$ & . & : & : & . & : & . & - & $\dot{.}$ & . & $\dot{.}$ & $\dot{-}$ \\
\hline Mn & 19.5 & 183 & 18.2 & 27.4 & 17.0 & 18.1 & 179 & 22.5 & 19.5 & 17.5 & 13.7 \\
\hline Po & 16.5 & 21.1 & 20.9 & 751 & 203 & 20.2 & 199 & 17.1 & 19.4 & 20.6 & 22.8 \\
\hline $\mathbf{A}$ & • & • & $\cdot$ & • & • & $\cdot$ & • & • & • & • & • \\
\hline$C_{0}$ & .290 & 530 & .550 & 200 & 560 & .440 & .520 & 1.01 & .540 & 520 & 120 \\
\hline $\mathbf{N i}$ & 530 & 270 & .270 & .680 & .260 & .260 & 310 & 320 & .350 & 300 & .180 \\
\hline $\mathrm{Cu}$ & .240 & .100 & .100 & 340 & .080 & .080 & .120 & .040 & .060 & .090 & .040 \\
\hline$c_{2}$ & 199 & 1.87 & 1.95 & 233 & 2.99 & 2.75 & 209 & 334 & 2.46 & 244 & 2.71 \\
\hline $\mathbf{M}_{8}$ & 220 & 1.39 & 1.41 & 2.66 & 1.37 & 1.26 & 1.61 & 1.41 & 1.62 & 1.64 & 205 \\
\hline $\mathrm{Na}$ & 1.85 & 1.33 & 1.39 & 239 & 137 & 1.54 & 1.78 & 1.46 & 1.35 & 1.60 & 1.89 \\
\hline K & .96 & .32 & .28 & 1.70 & .20 & 30 & 26 & .30 & 30 & .27 & .25 \\
\hline $\mathbf{P}$ & . & - & - & • & • & - & • & - & - & - & - \\
\hline $\mathrm{H}_{2} \mathrm{O}$. & $\cdot$ & $\cdot$ & - & $\cdot$ & - & - & . & . & $\cdot$ & - & - \\
\hline As(ppon) & - & - & - & - & - & - & - & - & - & - & - \\
\hline $\mathrm{Ba}_{2}$ & - & - & - & - & - & - & $\cdot$ & - & - & - & - \\
\hline Cd & - & - & - & - & - & - & - & - & - & - & - \\
\hline co & - & - & - & - & - & - & - & - & . & . & - \\
\hline Cr & . & - & - & . & - & - & - & - & . & - & • \\
\hline Mo & • & - & - & • & • & • & • & - & - & • & - \\
\hline $\mathrm{Pb}$ & 1100 & 1500 & 1500 & 600 & 1500 & 1400 & 1600 & 3000 & 1900 & 2000 & 1000 \\
\hline Sr & $\cdot$ & • & $\cdot$ & • & - & • & - & $\cdot$ & • & • & • \\
\hline v & - & - & - & - & - & - & • & - & - & - & - \\
\hline $\mathbf{Y}$ & • & - & • & - & • & - & - & - & - & . & . \\
\hline $\mathbf{z n}$ & 850 & 610 & 600 & 1400 & 610 & 640 & 670 & 490 & 630 & 720 & 590 \\
\hline Sample & Crust & Crust & Crust & Crust & Coust & Crust & Crunt & Crust & Crust & Court & Crux \\
\hline typo & $\cdot$ & • & • & • & • & • & • & • & $\cdot$ & • & $\cdot$ \\
\hline Portion & unknown & unkroown & unknown & unkrown & unkrown & unknown & unkrown & unkrown & unknown & unkrown & unkrown \\
\hline samplod & • & • & - & - & • & $\cdot$ & • & • & - & • & $\cdot$ \\
\hline Nuclous & • & • & • & • & . & $\cdot$ & - & $\cdot$ & • & • & - \\
\hline Referencos & A368 & A368 & A368 & A368 & A368 & A368 & A368 & A368 & A368 & A368 & A368 \\
\hline $\begin{array}{l}\text { Sequenod } \\
\text { Letitude }\end{array}$ & $\begin{array}{c}4710190-01 \\
45583\end{array}$ & $\begin{array}{c}4710192-00 \\
45.350\end{array}$ & $\begin{array}{c}4710193-00 \\
45.300\end{array}$ & $\begin{array}{c}4710194-00 \\
45.617\end{array}$ & $\begin{array}{c}4710195-00 \\
45.767\end{array}$ & $\begin{array}{c}4710196-00 \\
45.833\end{array}$ & $\begin{array}{c}4710197-\infty \\
45.750\end{array}$ & $\begin{array}{c}4710198-00 \\
45.700\end{array}$ & $\begin{array}{c}4710199-00 \\
45.767\end{array}$ & $\begin{array}{c}4710200-00 \\
45.800\end{array}$ & $\begin{array}{c}4710201-\infty 0 \\
45.750\end{array}$ \\
\hline Longitude & -27.050 & .27 .167 & -27383 & .27 .717 & -27.967 & -28.300 & -28.947 & .28 .933 & .28 .983 & -29.117 & -29.233 \\
\hline Depth & 2262 & 2097 & 2575 & 2231 & 1938 & 2421 & 2189 & 2865 & 2558 & 2295 & 2096 \\
\hline$S i\left(w r . x_{0}\right)$ & . & - & • & - & . & - & - & • & $\cdot$ & $\cdot$ & - \\
\hline $\mathrm{Ti}$ & - & • & • & - & - & • & - & - & . & - & - \\
\hline Mn & 12.2 & 127 & 13.3 & 12.5 & 5.59 & 6.88 & 11.9 & 14.0 & 14.4 & 16.6 & 15.0 \\
\hline Po & 23.8 & 21.9 & 24.3 & 23.9 & 229 & 15.5 & 20.9 & 220 & 21.6 & 21.7 & 20.3 \\
\hline Al & • & - & $\cdot$ & • & - & . & - & • & - & - & • \\
\hline Co & 280 & .290 & .320 & .190 & .150 & .250 & .120 & .410 & .430 & $A 60$ & 460 \\
\hline $\mathrm{Ni}$ & .180 & .140 & .130 & .120 & .100 & .160 & .150 & .210 & .190 & .190 & .270 \\
\hline $\mathrm{Cu}$ & .040 & .030 & .040 & .050 & .060 & .030 & .030 & .060 & .050 & .030 & .070 \\
\hline$C_{2}$ & 267 & 2.46 & 2.35 & 4.40 & 3.64 & 5.97 & 4.26 & 2.58 & 2.77 & 2.39 & 1.57 \\
\hline $\mathbf{M g}_{\mathbf{g}}$ & 232 & 1.91 & 1.46 & 1.50 & 5.39 & .14 & 201 & 1.81 & 1.53 & 1.39 & 1.88 \\
\hline $\mathrm{Na}$ & 1.91 & 2.03 & 1.39 & 1.28 & 1.66 & .70 & 1.71 & 1.27 & 1.38 & 1.20 & 1.51 \\
\hline $\mathbf{K}$ & .28 & .29 & .21 & .25 & .59 & .11 & 30 & .28 & .21 & .22 & 31 \\
\hline $\mathbf{P}$ & - & . & - & - & - & - & - & - & - & - & - \\
\hline $\mathrm{H}_{2} \mathrm{O}$. & $\cdot$ & $\cdot$ & - & $\cdot$ & $\cdot$ & $\cdot$ & • & $\cdot$ & - & - & $\cdot$ \\
\hline Ax(ppon) & - & - & - & - & - & - & - & - & - & - & - \\
\hline $\mathrm{Ba}$ & - & . & - & - & . & - & - & - & . & - & - \\
\hline Cd & - & - & - & . & - & - & - & - & - & - & - \\
\hline$c_{0}$ & - & - & - & . & . & - & - & - & - & - & - \\
\hline C. & - & - & - & - & - & - & - & - & - & - & - \\
\hline Mo & • & $\cdot$ & • & $\cdot$ & . & • & • & $\cdot$ & • & • & $\cdot$ \\
\hline $\mathrm{Pb}$ & 1200 & 1100 & 1200 & 600 & 500 & 500 & 1200 & 1100 & 1100 & 1100 & 1000 \\
\hline Sr & • & $\cdot$ & $\cdot$ & • & • & - & . & - & 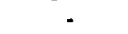 & - & • \\
\hline v & - & . & . & . & . & - & - & . & - & . & - \\
\hline $\mathbf{Y}$ & . & - & . & - & - & . & . & - & - & . & - \\
\hline $\mathrm{zn}$ & 650 & 590 & 620 & 530 & 480 & 340 & 550 & 600 & 590 & 520 & 670 \\
\hline Semple & Crust & Crust & Crust & Crust & Crust & Cous & Crust & Cruar & Crust & Crust & Crust \\
\hline type & $\cdot$ & $\cdot$ & $\cdot$ & $\cdot$ & $\cdot$ & $\cdot$ & • & • & $\cdot$ & $\cdot$ & • \\
\hline Portion & unkonown & unknown & unknown & unknown & unknown & unknown & uniknown & unknown & unlonown & unkrown & unknown \\
\hline semplod & - & - & $\cdot$ & - & . & $\cdot$ & $\cdot$ & $\cdot$ & $\cdot$ & $\cdot$ & $\cdot$ \\
\hline Naclous & - & . & . & - & - & - & . & . & • & - & $\cdot$ \\
\hline References & A368 & A368 & A368 & A368 & A368 & A368 & A368 & A368 & A368 & A368 & A368 \\
\hline
\end{tabular}


Table 9. Composition of crusts from the Scripps Nodule Databank (cont'd).

\begin{tabular}{|c|c|c|c|c|c|c|c|c|c|c|c|}
\hline $\begin{array}{l}\text { Sequenced } \\
\text { Letitude } \\
\text { Longitude } \\
\text { Depth }\end{array}$ & $\begin{array}{c}4710202-00 \\
45.267 \\
-29.433 \\
2660 \\
\end{array}$ & $\begin{array}{c}4710203-\infty 0 \\
45.517 \\
-20.567 \\
2752 \\
\end{array}$ & $\begin{array}{c}4710204-00 \\
45.233 \\
-20.800 \\
2468 \\
\end{array}$ & $\begin{array}{c}4710204-01 \\
45.233 \\
-29.800 \\
2468 \\
\end{array}$ & $\begin{array}{c}4710206-\infty 0 \\
45.233 \\
-20.833 \\
2468 \\
\end{array}$ & $\begin{array}{c}4710207-00 \\
45.217 \\
-29.867 \\
2395 \\
\end{array}$ & $\begin{array}{c}4810487-01 \\
40.383 \\
-127.983 \\
1260 \\
\end{array}$ & $\begin{array}{c}4810487.03 \\
40.383 \\
-127.983 \\
1260 \\
\end{array}$ & $\begin{array}{c}4810492-00 \\
42.750 \\
-128.050 \\
2520 \\
\end{array}$ & $\begin{array}{c}4840006-04 \\
40.235 \\
-155.917 \\
5033 \\
\end{array}$ & $\begin{array}{c}4840006-05 \\
40.235 \\
-155.917 \\
5033 \\
\end{array}$ \\
\hline $\operatorname{si}(w c \%)$ & $\cdot$ & $\cdot$ & - & - & $\cdot$ & - & - & - & 13.5 & $\cdot$ & - \\
\hline $\mathrm{Ti}$ & - & - & - & - & - & - & - & - & $A 1$ & - & - \\
\hline Mn & 15.7 & 15.9 & 15.4 & 16.3 & 15.4 & 16.2 & 21.0 & 1.00 & 15.2 & 15.0 & - \\
\hline Fo & 22.3 & 22.2 & 229 & 225 & 20.1 & 21.6 & 14.1 & 15.5 & 21.7 & 7.60 & 9.00 \\
\hline $\mathbf{A}$ & - & • & $\cdot$ & • & - & - & • & - & 1.80 & - & $\cdot$ \\
\hline Co & 360 & 420 & $A 30$ & .450 & 410 & .570 & 500 & 280 & .170 & .210 & .290 \\
\hline $\mathrm{Ni}$ & 200 & .190 & .180 & .200 & .360 & .280 & 420 & .140 & .160 & 530 & .200 \\
\hline $\mathrm{Cu}$ & .030 & .030 & .040 & .040 & .140 & .080 & .020 & .040 & .060 & .440 & .210 \\
\hline c. & 223 & 2.62 & 2.72 & 231 & 2.03 & 2.60 & 238 & - & 1.80 & - & - \\
\hline $\mathbf{M}_{\mathbf{B}}$ & 1.53 & 1.33 & $1.4 S$ & 1.48 & 4.18 & 1.99 & • & - & 1.00 & - & - \\
\hline Na & 1.00 & 1.05 & 1.19 & 1.09 & 1.23 & 1.34 & $\cdot$ & - & 2.10 & - & - \\
\hline $\mathbf{K}$ & 20 & .21 & .22 & 21 & .26 & .24 & - & - & so & - & $\cdot$ \\
\hline P & - & - & $\cdot$ & - & $\cdot$ & - & - & - & - & - & - \\
\hline $\mathrm{H}_{2} \mathrm{O}$ & - & • & $\cdot$ & - & - & - & - & - & $\cdot$ & - & - \\
\hline$A n(p p m)$ & - & - & - & - & - & - & - & - & • & - & - \\
\hline $\mathrm{Ba}$ & - & $\cdot$ & $\cdot$ & - & $\cdot$ & - & • & - & 1200 & 3100 & 1800 \\
\hline $\mathrm{Cd}$ & - & - & - & - & - & - & 3.0 & - & - & - & - \\
\hline co & - & - & - & - & - & - & - & - & - & - & - \\
\hline cr & - & - & - & - & - & - & - & 50 & 14 & - & 26 \\
\hline Mo & - & - & • & - & $\cdot$ & • & - & - & 500 & - & $\cdot$ \\
\hline $\mathbf{P b}$ & 1100 & 1100 & 1300 & 1000 & 900 & 1000 & 1400 & - & 1000 & - & - \\
\hline Sr & • & - & - & • & - & - & • & - & 1300 & - & - \\
\hline $\mathbf{v}$ & - & - & - & - & - & - & - & - & 460 & - & - \\
\hline $\mathbf{Y}$ & - & - & - & - & - & - & - & - & 170 & - & - \\
\hline $\mathbf{Z n}$ & 550 & 510 & 550 & 540 & 700 & 610 & 390 & 400 & 400 & 800 & 400 \\
\hline Semple & Crust & Crust & Cruat & Crat & Crust & Crust & Nocule & Crast & Crust & Crut & Crust \\
\hline type & • & • & • & - & - & - & - & - & - & • & - \\
\hline Partion & unkonown & unkenows & unknown & uniknown & unknown & unimown & fragment & unknown & croese & outer & middle \\
\hline samplod & • & • & $\cdot$ & + & $\cdot$ & - & 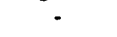 & - & ecction & cant & layer \\
\hline Nucleus & - & - & - & • & - & • & • & - & • & • & - \\
\hline References & A368 & A368 & A368 & A368 & A368 & A368 & 1200 & $A 323$ & A261 & A.323 & $A 323$ \\
\hline Sequencell & $4870015-00$ & $4870015-01$ & $4870025-04$ & $5190054-01$ & $5200002-02$ & $52000003-00$ & & & & & \\
\hline Letitudes & 40.267 & 40.267 & 40.340 & 56.167 & 52.783 & 54.537 & & & & & \\
\hline Longituds & 170.333 & 170.333 & 175.630 & -145.250 & -150.083 & -150.368 & & & & & \\
\hline Depth & 2037 & 2037 & 5304 & - & $\cdot$ & 972 & & & & & \\
\hline Si(wt.\%) & $\cdot$ & - & - & - & - & . & & & & & \\
\hline $\mathbf{T i}$ & .04 & .65 & - & • & • & • & & & & & \\
\hline Mn & 20.6 & 26.5 & 10.4 & 20.9 & 23.2 & 18.0 & & & & & \\
\hline Fo & 13.0 & $17 A$ & 8.30 & 134 & 9.60 & 26.0 & & & & & \\
\hline Al & 1.10 & • & - & - & - & $\cdot$ & & & & & \\
\hline Co & 510 & 550 & .020 & .400 & .500 & .860 & & & & & \\
\hline $\mathrm{Ni}$ & .610 & .480 & .380 & 370 & .630 & .240 & & & & & \\
\hline Cu & .050 & .050 & 300 & 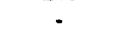 & $\cdot$ & .040 & & & & & \\
\hline c. & 1.19 & - & 1.60 & - & - & - & & & & & \\
\hline$M_{8}$ & 1.08 & - & • & - & - & . & & & & & \\
\hline $\mathrm{Na}$ & 200 & - & - & - & - & - & & & & & \\
\hline $\mathbf{K}$ & 50 & - & - & - & - & - & & & & & \\
\hline $\mathbf{P}$ & .40 & - & - & - & - & - & & & & & \\
\hline $\mathrm{H}_{2} \mathrm{O}$ & 16.3 & - & - & - & - & - & & & & & \\
\hline As(ppm) & - & - & - & - & - & - & & & & & \\
\hline $\mathrm{Be}$ & - & - & $\cdot$ & - & $\cdot$ & $\cdot$ & & • & & & \\
\hline Cd & - & - & $\cdot$ & - & - & - & & & & & \\
\hline ce & - & - & - & - & - & . & & & & & \\
\hline$C r$ & - & - & - & - & - & - & & & & & \\
\hline Mo & 760 & 2800 & $\cdot$ & - & - & - & & & & & \\
\hline $\mathrm{Pb}$ & 2300 & - & 500 & - & - & - & & & & & \\
\hline St & $\cdot$ & . & $\cdot$ & . & - & . & & & & & \\
\hline v & - & - & - & - & - & - & & & & & \\
\hline $\mathbf{Y}$ & - & - & $\cdot$ & - & - & - & & & & & \\
\hline $\mathbf{Z n}$ & - & - & 600 & - & - & - & & & & & \\
\hline Semple & Unbnown & Noctule & Mn-encruted & Cruat & Crust & Nodulo & & & & & \\
\hline typo & • & $\cdot$ & rdiment & - & $\cdot$ & • & & & & & \\
\hline Partion & unknown & without & without & unknown & unknown & unknown & & & & & \\
\hline sampled & • & nucleus & muclous & • & $\cdot$ & • & & & & & \\
\hline Nucleus & - & - & Sediment & - & - & • & & & & & \\
\hline Referencos & $A 200$ & A346 & A437 & A255 & A255 & A419 & & & & & \\
\hline
\end{tabular}


Table 10. Trace Analyses of crusts from Scripps Nodule Databank

\begin{tabular}{|c|c|c|c|c|c|c|c|c|c|c|c|}
\hline Sequencell & $0870004-00$ & $0910005-01$ & $0910005-02$ & $1130061-00$ & $1140051-00$ & $1140081-01$ & $1140081-02$ & $1150001-\infty 0$ & $1180019-\infty$ & $1230002-00$ & $1230002-01$ \\
\hline Latitude & -64.517 & -63.100 & -63.100 & -53.042 & -55.733 & -56.267 & -56.267 & -57.050 & .52017 & -52.167 & -52.167 \\
\hline Langitude & -145.967 & 177.617 & 177.617 & -48.883 & -56.050 & -58.275 & -58.275 & -60.017 & -99.950 & -142.133 & -142.133 \\
\hline Depth(m) & 3775 & 3383 & 3383 & 3118 & 3914 & 4087 & 4087 & 3438 & 4418 & 2295 & 2295 \\
\hline$S n$ (ppon) & $80^{\circ}$ & $80^{\circ}$ & $130^{*}$ & $80^{\circ}$ & $150^{*}$ & $50^{\circ}$ & $60^{\circ}$ & $130^{*}$ & $200^{\circ}$ & $400^{\circ}$ & . \\
\hline $\mathbf{z}$ & 760 & 90 & 550 & 760 & 480 & 330 & 790 & 420 & • & 400 & 400 \\
\hline Reference & A227 & A227 & 1227 & A227 & A220 & A227 & $A 227$ & A227 & A227 & A227 & A227 \\
\hline
\end{tabular}

\begin{tabular}{|c|c|c|c|c|c|c|c|c|c|c|c|}
\hline Sequencel & $1230006-00$ & $1230007-\infty 0$ & $1230011-\infty$ & $1270003-00$ & $1280029-00$ & $1290010-00$ & $1290062-00$ & $1300013-00$ & $1300013-01$ & $1300013-02$ & $1300013-0$ \\
\hline Latimde & .59 .850 & .58 .083 & -59.133 & -50.317 & -50.783 & -56.367 & -56.683 & -56.417 & $-56,417$ & -56.417 & -56.417 \\
\hline Longitude & -144.867 & -144.950 & -145.133 & 176.383 & 169.958 & 158.467 & 158.767 & 140.017 & 140.017 & 140.017 & 140.017 \\
\hline Depth(m) & 3219 & 3025 & 3155 & 2377 & 896 & 1595 & 1280 & 3750 & 3750 & 3750 & 3750 \\
\hline B (ppona) & - & - & - & $700^{\circ}$ & $900^{\circ}$ & - & - & - & - & $\cdot$ & - \\
\hline Bo & - & - & - & $10^{*}$ & $10^{\circ}$ & - & - & - & - & $100^{\circ}$ & $100^{*}$ \\
\hline C & - & - & - & - & - & - & - & 300 & 1600 & 4300 & 3300 \\
\hline C] & - & - & - & - & - & - & - & 400 & 3900 & • & • \\
\hline G. & - & - & - & - & - & - & 17 & - & - & - & - \\
\hline $\mathbf{s}$ & • & • & - & - & - & • & . & 500 & 1100 & 800 & 1000 \\
\hline Sn & $260^{\circ}$ & $2200^{\circ}$ & $130^{*}$ & - & - & $10^{*}$ & - & - & - & - & - \\
\hline $\mathrm{TI}$ & - & - & - & - & - & - & - & 100 & 200 & - & 100 \\
\hline Z & - & 680 & 1200 & - & • & 2100 & 950 & 200 & 500 & 200 & 500 \\
\hline Reference & A227 & A227 & A227 & A260 & A260 & A227 & A388 & A247 & A247 & A247 & A247 \\
\hline
\end{tabular}

\begin{tabular}{|c|c|c|c|c|c|c|c|c|c|c|c|}
\hline Sequencel & $1300013-04$ & $1300013-05$ & $1300013-06$ & $1300013-07$ & $1300013-08$ & $1300013-09$ & $1530001-\infty$ & $1530003-00$ & $1550005-07$ & $1580001-01$ & $1630063-00$ \\
\hline Latitude & .56 .417 & .56 .417 & -56.417 & -56.417 & -56.417 & -56417 & -42.067 & -42.083 & -41.983 & -42.017 & -49.667 \\
\hline Longitede & 140.017 & 140.017 & 140.017 & 140.017 & 140.017 & 140.017 & -81.983 & -86.050 & -102.017 & -130.033 & 179.883 \\
\hline Depth(m) & 3750 & 3750 & 3750 & 3750 & 3750 & 3750 & 3200 & 3012 & 4200 & 4839 & 2286 \\
\hline B (ppm) & - & - & - & $\because$ & $\ddots$ & - & - & - & - & - & $800^{\circ}$ \\
\hline Bo & $\cdot$ & • & - & $100^{\circ}$ & $100^{\circ}$ & • & - & - & - & - & $10^{\circ}$ \\
\hline C & 2000 & 2500 & 4000 & 5200 & 2900 & 2300 & - & - & - & - & • \\
\hline a & - & • & - & • & 4300 & 4600 & - & - & - & - & - \\
\hline $\mathbf{s}$ & 500 & 800 & 1400 & 1600 & 600 & 1000 & - & - & $\cdot$ & - & - \\
\hline Sc & $\cdot$ & $\cdot$ & • & • & • & • & - & $=$ & 7 & $\cdot$ & - \\
\hline Sn & - & - & - & - & - & - & $80^{\circ}$ & $80^{\circ}$ & - & $80^{\circ}$ & - \\
\hline Th & • & - & - & - & - & $\cdot$ & - & - & 8 & $\cdot$ & - \\
\hline $\mathbf{T l}$ & 100 & 100 & $\cdot$ & $\cdot$ & 200 & 100 & - & $\cdot$ & - & $\cdot$ & - \\
\hline$Z_{x}$ & 200 & 200 & 200 & 200 & 300 & 400 & 420 & 760 & - & 760 & - \\
\hline References & A247 & A247 & A247 & A247 & A247 & A247 & A2m & A227 & A323 & A227 & A260 \\
\hline
\end{tabular}

\begin{tabular}{|c|c|c|c|c|c|c|c|c|c|c|c|}
\hline Sequence" & $1630064-00$ & $1630064-01$ & $1630065-01$ & $1630066-00$ & $1630066-01$ & $1890007-\infty 0$ & $1890007-01$ & $1890013-00$ & $1890013-01$ & $1890016-00$ & 189001601 \\
\hline Lationde & -49.400 & -49.400 & -49.983 & -49.367 & -49.367 & -33.050 & .33 .050 & -33.042 & -33.042 & .32 .967 & .32 .967 \\
\hline Langituds & 177.983 & 177.983 & 177.533 & 176.267 & 176.267 & -82050 & -82.450 & .83 .942 & -83.942 & -88.000 & -88.000 \\
\hline Depth(m) & 978 & 978 & 1335 & 1280 & 1280 & 3881 & 3881 & 3650 & 3650 & 3619 & 3619 \\
\hline B (ppen) & $400^{\circ}$ & - & - & $600^{\circ}$ & - & - & - & - & - & - & - \\
\hline Bo & $10^{*}$ & . & . & $10^{*}$ & - & - & - & . & - & - & - \\
\hline G. & $\bullet$ & 10 & 13 & - & 18 & $\ddots$ & - & $\bullet$ & $\bullet$ & $\bullet$ & $\ddots$ \\
\hline Sn & $100^{\circ}$ & . & - & . & . & $180^{\circ}$ & $360^{\circ}$ & $200^{*}$ & $260^{\circ}$ & $180^{\circ}$ & $260^{\circ}$ \\
\hline $\mathbf{Y b}$ & $100^{\circ}$ & • & - & - & - & - & - & • & - & - & - \\
\hline Z & 300 & 230 & 380 & - & 270 & 440 & 260 & 660 & 430 & 410 & 100 \\
\hline Reference & A260 & A388 & A388 & A260 & A388 & 1227 & A227 & A227 & A227 & A227 & A227 \\
\hline
\end{tabular}

\begin{tabular}{|c|c|c|c|c|c|c|c|c|c|c|c|}
\hline Sequence" & $1900007-00$ & $1930004-00$ & $1940005-\infty 0$ & $1940005-01$ & $1940005-02$ & $1980025-01$ & $1980025-02$ & $2010001-\infty 0$ & 2010001-01 & $2010001-02$ & 2010001.00 \\
\hline Latitude & -39.850 & -37.067 & -35.967 & -35.967 & -35.967 & -35.850 & -35.850 & -39.750 & -39.750 & -39.750 & -39.750 \\
\hline Langitude & -96.867 & -129.967 & -134.300 & .134 .300 & -134.300 & .178 .517 & -178.517 & 159.650 & 159.650 & 159.650 & 159.650 \\
\hline Dopth(m) & 3639 & 3548 & 4696 & 4696 & 4696 & 6715 & 6715 & - & - & $\cdot$ & - \\
\hline Bi (ppon) & • & - & - & - & - & 10 & $\cdot$ & 3 & 1 & - & - \\
\hline G. & - & - & - & - & - & - & 14 & . & - & 17 & 14 \\
\hline$s$ & • & 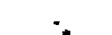 & - & • & $\cdot$ & - & $22000^{\circ}$ & - & - & $18000^{\circ}$ & $18000^{\circ}$ \\
\hline Sn & $180^{\circ}$ & $40^{\circ}$ & $60^{\circ}$ & $130^{\circ}$ & $60^{\circ}$ & - & . & - & - & - & - \\
\hline $\mathrm{TI}$ & - & - & - & - & - & 100 & 100 & 118 & 175 & 118 & 175 \\
\hline $\mathbf{z}$ & 210 & - & 510 & 440 & 440 & . & 570 & • & - & 243 & 220 \\
\hline Reforencos & A227 & A227 & A227 & A227 & A227 & $A 200$ & A388 & $A 200$ & $A 200$ & A388 & A388 \\
\hline
\end{tabular}


Table 10. Trace Analyses of crusts from Scripps Nodule Databank

\begin{tabular}{|c|c|c|c|c|c|c|c|c|c|c|c|}
\hline Sequencest & $2130069-00$ & $2130069-04$ & $2130070-\infty 0$ & $2140009-00$ & $2140010-00$ & $2140071-\infty 0$ & $2140073-00$ & 214007400 & $2140074-01$ & $2140075-00$ & $2140076-00$ \\
\hline Letipuda & -32.917 & .32 .917 & -31.900 & -37.433 & -38.633 & -35.333 & -35.783 & -35.433 & -35.433 & .35 .617 & .35 .650 \\
\hline Longitude & 35.317 & 35.317 & 34.350 & 26.467 & 26.133 & 2.642 & 22.383 & 22.367 & 22.367 & 22.367 & 22.217 \\
\hline Depth(m) & 1250 & 1250 & 2700 & 2867 & 2960 & 320 & 1070 & 1700 & 1700 & 230 & 202 \\
\hline$F$ (ppon) & - & - & - & $30000^{\circ}$ & - & - & - & - & - & - & - \\
\hline Ga & 17 & 20 & 18 & - & - & - & - & - & - & - & - \\
\hline So & - & - & - & - & 2 & - & - & - & - & - & - \\
\hline $\mathrm{TI}$ & - & - & - & - & 260 & . & - & - & - & - & - \\
\hline $\mathbf{z x}$ & 320 & 450 & 500 & - & - & $167^{\circ}$ & $236^{*}$ & $31^{\circ}$ & $137^{\circ}$ & $101^{*}$ & $266^{*}$ \\
\hline Referencoso & A388 & A388 & A388 & A422 & A200 & A466 & A466 & A466 & 1466 & A466 & A466 \\
\hline
\end{tabular}

\begin{tabular}{|c|c|c|c|c|c|c|c|c|c|c|c|}
\hline Sequenoull & $2150054-04$ & $2150058-02$ & $2250007-01$ & $2250007-05$ & $2310055-01$ & 2330065-01 & $2330069-03$ & $2430025-01$ & $2450016-03$ & $2490034-01$ & $2670023-\infty$ \\
\hline Latimde & -34.600 & -34.700 & -25.517 & -25.517 & .20390 & -22662 & -22.667 & -22363 & -23.267 & -29.100 & -17.600 \\
\hline Langitude & 17.000 & 16.900 & .85 .233 & -85.233 & -148.020 & -160.788 & -160.822 & 91.633 & 74.983 & 36.733 & -141.917 \\
\hline Depth(m) & 2740 & 3200 & 920 & 920 & 4630 & 4830 & 4850 & 5280 & 4520 & 3880 & 2132 \\
\hline
\end{tabular}

\begin{tabular}{|c|c|c|c|c|c|c|c|c|c|c|c|}
\hline$B$ (ppm) & • & - & 230 & - & $\cdot$ & - & - & - & - & - & 400 \\
\hline $\mathbf{B i}$ & - & - & . & - & $50^{*}$ & • & • & - & - & - & - \\
\hline C & - & - & - & - & - & 330 & 1000 & 2000 & - & - & - \\
\hline CI & - & - & - & - & - & 8700 & 9000 & • & - & - & - \\
\hline Ga & $\cdot$ & 9 & - & - & - & - & - & - & - & 15 & - \\
\hline s & $11000^{*}$ & $17000^{*}$ & - & - & - & - & - & 1900 & - & - & - \\
\hline Se & - & - & 10 & 2 & - & - & - & - & 8 & - & 14 \\
\hline Th & - & - & - & 86 & - & - & - & - & $\mathbf{2 3}$ & - & - \\
\hline $\mathbf{T I}$ & 56 & 350 & - & - & - & . & - & . & - & - & - \\
\hline Yb & - & • & 57 & - & - & . & - & - & - & - & 53 \\
\hline $\mathbf{Z x}$ & - & 211 & 260 & - & - & - & - & 520 & - & 500 & 1000 \\
\hline References & A388 & A388 & A261 & A323 & $A 248$ & A390 & A390 & A2200 & $A 323$ & A388 & A261 \\
\hline
\end{tabular}

\begin{tabular}{|c|c|c|c|c|c|c|c|c|c|c|c|}
\hline Sequences. & $2070098-01$ & $2670098-02$ & 2670098-03 & $2670098-07$ & $2670117-01$ & $2670119-01$ & $2570130-06$ & $2670130-07$ & $2670252-00$ & $2690013-01$ & $2690019-03$ \\
\hline Latitude & -16.483 & -16.483 & -16.483 & -16.483 & -16.167 & -17.000 & -18.917 & -18.917 & -13.250 & -12700 & .12700 \\
\hline Longitude & -145.550 & -145.550 & -145.550 & -145.550 & -146.000 & -146.000 & -146.383 & -146.383 & -148.500 & -160.617 & -160.750 \\
\hline Depth(m) & 1270 & 1270 & 1270 & 1270 & 1190 & 1700 & 4465 & 4465 & 4650 & 4753 & 3953 \\
\hline
\end{tabular}

\begin{tabular}{|c|c|c|c|c|c|c|c|c|c|c|c|}
\hline$B$ (ppm) & 400 & 300 & 300 & - & 500 & 600 & - & - & 124 & - & - \\
\hline $\mathbf{B i}$ & 27 & • & 36 & - & • & • & 1 & 8 & • & - & - \\
\hline $\mathrm{Bu}$ & - & - & - & - & - & - & - & - & 2 & - & - \\
\hline G. & - & - & - & - & - & - & - & - & 7 & - & - \\
\hline Nd & . & - & - & - & - & - & - & - & 22 & - & - \\
\hline Se & - & - & - & - & 13 & 10 & - & - & - & - & - \\
\hline Sm & - & - & - & - & - & - & - & - & $\mathbf{s}$ & - & - \\
\hline Sn & - & - & - & - & . & - & - & 4 & - & - & - \\
\hline Tb & - & - & - & - & - & - & - & - & 1 & - & - \\
\hline Th & - & - & . & • & - & - & - & - & . & 64 & 20 \\
\hline $\mathrm{n}$ & - & - & - & 180 & - & - & 340 & 340 & - & 175 & 164 \\
\hline $\mathbf{U}$ & - & - & - & • & - & - & • & • & : & 10 & 15 \\
\hline $\mathbf{Y b}$ & 48 & 53 & 31 & - & 53 & 57 & - & - & 2 & - & - \\
\hline $\mathbf{Z}$ & 800 & 720 & 620 & - & - & 650 & • & • & - & • & - \\
\hline Referenos & A261 & A261 & A261 & A201 & A261 & A261 & $A 200$ & $A 200$ & $A 463$ & A303 & A303 \\
\hline
\end{tabular}

\begin{tabular}{|c|c|c|c|c|c|c|c|c|c|c|c|}
\hline Sequence. & $2690022-02$ & $2690065-00$ & $2710120-01$ & $2770039-\infty 0$ & $2770039-01$ & $3040105-01$ & $3050029-00$ & $3050039-00$ & $3050039-03$ & $3050076-01$ & $3050090-\infty$ \\
\hline Letitude & -12.700 & -18.597 & -11.317 & -10.850 & -10.850 & -4.982 & -2.292 & -9.805 & -9.805 & -4.233 & -8.275 \\
\hline Longitude & -160.817 & -161.527 & 178.200 & 113.017 & 113.017 & -154.983 & -163.958 & -164.743 & -164.743 & -168.283 & -168.508 \\
\hline Dopth(m) & 3587 & 4998 & 770 & 5150 & 5150 & 4990 & 5243 & 4199 & 4199 & 5230 & 4565 \\
\hline Ag(ppon) & - & - & - & - & - & 11 & - & - & - & - & - \\
\hline Bo & - & - & - & - & . & . & - & 3 & - & - & - \\
\hline$B i$ & - & - & - & 13 & - & - & - & - & - & - & - \\
\hline C & - & 800 & - & - & - & - & - & - & - & . & - \\
\hline$H_{B}$ (ppb) & - & $\cdot$ & - & - & - & 93 & - & - & 2800 & - & $\cdot$ \\
\hline $\mathrm{s}$ & - & - & - & - & $28000^{\circ}$ & - & - & - & - & - & - \\
\hline Se & - & - & 5 & - & • & - & - & - & - & - & - \\
\hline So & - & - & - & - & - & 57 & $\cdot$ & • & 50 & - & - \\
\hline Te & - & - & - & - & - & - & - & 223 & - & - & - \\
\hline Th & 28 & - & - & - & - & - & - & • & - & 80 & - \\
\hline $\mathrm{TI}$ & 121 & - & - & 68 & 68 & - & 158 & - & - & 171 & 164 \\
\hline $\mathbf{U}$ & 10 & - & • & - & • & - & • & 14 & • & 13 & $\cdot$ \\
\hline Referenos & $A 303$ & A343 & $A 323$ & A200 & A388 & A475 & A303 & A301 & A475 & A303 & A303 \\
\hline
\end{tabular}


Table 10. Trace Analyses of crusts from Scripps Nodule Databank

\begin{tabular}{|c|c|c|c|c|c|c|c|c|c|c|c|}
\hline Sequencill & $3050091-\infty$ & $3050092-00$ & $3050095-03$ & $3050095-06$ & $3050095-07$ & $3050095-08$ & $3050100-\infty 0$ & $3050129-02$ & $3190028-00$ & $3350075-03$ & 3350075.04 \\
\hline Latitude & -8.283 & -8.287 & -8.817 & -8.817 & -8.817 & -8.817 & .7517 & -7.740 & -2.133 & 9.138 & 9.138 \\
\hline Langitude & -168.520 & -168.528 & -168.550 & -168.550 & -168.550 & -168.550 & -168.817 & -168.553 & 57.400 & -105.450 & -105.450 \\
\hline Depth(m) & 4751 & 4758 & 4550 & 4550 & 4550 & 4550 & 5288 & 4700 & 4133 & 2651 & 2651 \\
\hline Bo (ppm) & 3 & - & - & - & - & - & - & - & - & - & - \\
\hline Te & 210 & - & - & - & - & - & - & - & . & - & - \\
\hline Th & - & - & - & 23 & 31 & - & 51 & - & - & 20 & 10 \\
\hline $\mathrm{TI}$ & - & 184 & 186 & . & . & 173 & 85 & 168 & - & 137 & 97 \\
\hline $\mathbf{U}$ & 14 & . & - & 10 & 7 & - & 11 & . & - & 4 & 4 \\
\hline $\mathbf{z}$ & - & - & - & . & - & - & 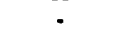 & - & 520 & - & - \\
\hline Referenos & A301 & A303 & 4303 & A303 & A3OB & A303 & A303 & A303 & A203 & A303 & A303 \\
\hline
\end{tabular}

\begin{tabular}{|c|c|c|c|c|c|c|c|c|c|c|c|}
\hline Sequencelt & $3410013-01$ & $3410021-02$ & $3410021-03$ & $3410170-02$ & $3410170-03$ & $3470359-02$ & $3470359-03$ & $3470363-01$ & $3540066-00$ & $3540066-01$ & $3540066-02$ \\
\hline Latitude & 5.872 & 5.232 & 5.232 & 8.330 & 8.330 & 7.455 & 7.455 & 7.433 & 2.942 & 2.942 & 2942 \\
\hline Langitude & -160.848 & -161.497 & -161.497 & $\cdot 164.362$ & -164.362 & 136.407 & 136.407 & 133.715 & 60.057 & 60.057 & 60.057 \\
\hline Doptt(m) & 1604 & 1307 & 1307 & 1519 & 1519 & 3555 & 3555 & 3869 & 4042 & 4042 & 4042 \\
\hline
\end{tabular}

\begin{tabular}{|c|c|c|c|c|c|c|c|c|c|c|c|}
\hline $\mathbf{T h}$ & 7 & 11 & 6 & 7 & 10 & 39 & 31 & 32 & - & - & - \\
\hline TI & 193 & 250 & 280 & 340 & 300 & 148 & 86 & 74 & - & - & - \\
\hline $\mathbf{U}$ & 17 & 17 & 15 & 15 & 15 & 8 & 7 & 48 & • & $\cdot$ & $\cdot$ \\
\hline $\mathbf{Z x}$ & $\cdot$ & - & - & - & - & - & - & - & 620 & 280 & 540 \\
\hline References & A303 & A303 & A303 & A303 & A303 & A303 & A303 & A303 & A217 & A217 & A217 \\
\hline
\end{tabular}

\begin{tabular}{|c|c|c|c|c|c|c|c|c|c|c|c|}
\hline Sequencel & $3540066-03$ & $3540066-04$ & $3540070-00$ & $3540070-01$ & $3540070-02$ & $3540070-03$ & $3540072-01$ & $3550004-00$ & $3550004-01$ & $3550004-02$ & 355000403 \\
\hline Latimde & 2.942 & 2.942 & 2.967 & 2.967 & 2967 & 2.967 & 2790 & 2.755 & 2755 & 2755 & 2755 \\
\hline Langitude & 60.057 & 60.057 & 60.047 & 60.047 & 60.047 & 60.047 & 60.042 & 59.903 & 59.903 & 59.903 & 59.903 \\
\hline Depth(m) & 4042 & 4042 & 4093 & 4093 & 4093 & 4093 & 3816 & 3975 & 3975 & 3975 & 3975 \\
\hline$Z x$ (ppan) & 620 & 209 & 250 & 260 & 350 & 520 & 410 & 380 & 740 & 300 & 310 \\
\hline Reference & A217 & A217 & $A 217$ & A217 & A217 & A217 & A217 & A217 & A217 & A217 & A217 \\
\hline
\end{tabular}

\begin{tabular}{|c|c|c|c|c|c|c|c|c|c|c|c|}
\hline $\begin{array}{l}\text { Sequenceill } \\
\text { Latumide }\end{array}$ & $\begin{array}{c}3550004-04 \\
2755\end{array}$ & $\begin{array}{c}3550004-05 \\
2.755\end{array}$ & $\begin{array}{c}355000406 \\
2.755\end{array}$ & $\begin{array}{c}3550004-07 \\
2.755\end{array}$ & $\begin{array}{c}3550004-08 \\
2.755\end{array}$ & $\begin{array}{c}3550004-09 \\
2755\end{array}$ & $\begin{array}{c}3550013-\infty 0 \\
2823\end{array}$ & $\begin{array}{c}3550013-01 \\
2823\end{array}$ & $\begin{array}{c}3550013-02 \\
2.823\end{array}$ & $\begin{array}{c}3550013-03 \\
2.823\end{array}$ & $\begin{array}{c}3550013-04 \\
2,823\end{array}$ \\
\hline Longitude & 59.903 & 59.903 & 59.903 & 59.903 & 59.903 & 59.903 & 59.862 & 59.862 & 59.862 & 59.862 & 59.862 \\
\hline Depth(m) & 3975 & 3975 & 3975 & 3975 & 3975 & 3975 & 3563 & 3563 & 3563 & 3563 & 3563 \\
\hline$Z x$ (ppor) & 720 & 550 & 580 & 99 & 800 & 580 & 1300 & 1000 & 480 & 260 & 1200 \\
\hline Reference & A217 & A217 & A217 & A217 & A217 & A217 & A217 & A217 & A217 & A217 & A217 \\
\hline
\end{tabular}

\begin{tabular}{|c|c|c|c|c|c|c|c|c|c|c|c|}
\hline Sequencel & $3550013-05$ & $3550013-06$ & $3550013-07$ & $3550013-08$ & $3550013-09$ & $3550014-\infty$ & $3550014-01$ & $3550014-02$ & $3550015-\infty$ & $3550015-01$ & $3550015-02$ \\
\hline Letionde & 2.823 & 2823 & 2.823 & 2.823 & 2823 & 2798 & 2.798 & 2.798 & 2863 & 2863 & 2863 \\
\hline Langituds & 59.862 & 59.862 & 59.862 & 59.862 & 59.862 & 59.862 & 59.862 & 59.862 & 59.855 & 59.855 & 59.855 \\
\hline Dopth(m) & 3563 & 3563 & 3563 & 3563 & 3563 & 3776 & 3776 & 3776 & 3617 & 3617 & 3617 \\
\hline$Z x$ (ppon) & 1400 & 360 & 380 & 630 & 880 & 580 & 450 & .460 & 164 & 540 & 790 \\
\hline
\end{tabular}

\begin{tabular}{|c|c|c|c|c|c|c|c|c|c|c|c|}
\hline Sequencel & $3550015-03$ & $3550015-04$ & $3550015-05$ & $3550015-06$ & $3550015-07$ & $3550015-08$ & $3550015-09$ & $3550018-00$ & $3550018-01$ & $3550018-02$ & $3550018-03$ \\
\hline Latibude & 2.863 & 2863 & 2.863 & 2.863 & 2.863 & 2.863 & 2863 & 2803 & 2803 & 2803 & 2803 \\
\hline Longituds & 59.855 & 59.855 & 59.855 & 59.855 & 59.855 & 59.855 & 59.855 & 59.768 & 59.768 & 59.768 & 59.768 \\
\hline Depth(m) & 3617 & 3617 & 3617 & 3617 & 3617 & 3617 & 3617 & 3432 & 3432 & 3432 & 3432 \\
\hline$Z=$ (ppon) & 830 & 151 & 980 & 249 & 1500 & 1000 & 670 & 640 & 480 & 620 & 510 \\
\hline Reforence & A217 & A217 & A217 & A217 & A217 & A217 & A217 & A217 & A217 & A217 & A217 \\
\hline
\end{tabular}


Table 10. Trace Analyses of crusts from Scripps Nodule Databank

\begin{tabular}{|c|c|c|c|c|c|c|c|c|c|c|c|}
\hline Sequencell & $3550018-04$ & $3550018-05$ & $3550018-06$ & $3730026-01$ & 3750103-03 & $3750390-00$ & $3760145-02$ & $3760147-04$ & $3760223-01$ & $3770025-01$ & $3770026-03$ \\
\hline Latitude & 2.803 & 2.803 & 2.803 & 13.052 & 11.100 & 13.567 & 19.100 & 18.350 & 18.717 & 19.383 & 19.367 \\
\hline Langituds & 59.768 & 59.768 & 59.768 & -125.480 & -140.017 & -140.633 & -158.233 & -158.450 & -158.283 & -162.167 & -162.317 \\
\hline Depth(m) & 3432 & 3432 & 3432 & 4400 & 4800 & 4721 & 3131 & 1317 & 475 & 4160 & 4868 \\
\hline B (ppon) & - & - & • & 120 & - & • & - & - & $\cdot$ & - & - \\
\hline $\mathrm{Oe}$ & - & - & - & 30 & - & - & - & - & - & - & - \\
\hline $\mathrm{Hg}_{\mathrm{g}}(\mathrm{ppb})$ & - & - & - & - & 204 & • & - & - & - & - & - \\
\hline $\mathrm{s}$ & - & - & - & - & • & 3500 & - & - & - & - & - \\
\hline Se & - & - & - & - & 41 & • & - & - & • & - & - \\
\hline$\pi$ & - & - & - & - & - & - & 86 & 88 & 200 & 100 & 139 \\
\hline $\mathbf{Y b}$ & - & - & - & 18 & - & - & - & - & • & • & • \\
\hline $\mathbf{z}$ & 470 & 420 & 570 & 240 & - & • & - & • & • & • & • \\
\hline References & A217 & A217 & A217 & A261 & A475 & A392 & A303 & 1303 & A303 & A303 & $A 303$ \\
\hline
\end{tabular}

\begin{tabular}{|c|c|c|c|c|c|c|c|c|c|c|c|}
\hline Sequencell & $3770101-\infty$ & $3770103-03$ & $3770138-\infty 0$ & $3780035-00$ & $3780046-03$ & $3780070-01$ & $3790020-01$ & $3800024-01$ & $3800024-02$ & $3800025-06$ & $3800027-01$ \\
\hline Lationde & 19.117 & 19.117 & 18.300 & 17.900 & 17.800 & 17.067 & 19.313 & 12.050 & 12.050 & 11.950 & 12.050 \\
\hline Longitude & -169.733 & -169.733 & -161.767 & -174.267 & -174.367 & -177.250 & 176.735 & 165.000 & 165.000 & 164.983 & 164.950 \\
\hline Depth(m) & 1740 & 1740 & 3060 & 1749 & 2288 & 2013 & 1753 & 2090 & 2090 & 2105 & 2105 \\
\hline B (ppm) & 300 & 150 & - & 300 & 150 & - & - & 190 & - & 500 & 400 \\
\hline Bo & - & 3 & - & - & 3 & - & - & $\cdot$ & - & - & - \\
\hline Bi & . & 30 & - & - & 30 & - & - & - & - & 27 & - \\
\hline$H_{g}$ (ppb) & - & $\because$ & - & - & $\cdot$ & - & 82 & - & - & - & - \\
\hline Nd & . & $300^{\circ}$ & . & - & $300^{\circ}$ & - & . & - & - & . & - \\
\hline Sc & 11 & - & - & 11 & . & - & - & 20 & - & 10 & - \\
\hline So & - & - & - & - & - & - & 61 & . & - & - & - \\
\hline Th & - & - & 35 & - & • & - & - & - & - & - & - \\
\hline$\pi$ & - & 300 & - & - & 150 & . & . & - & . & - & - \\
\hline $\mathbf{U}$ & - & - & 9 & - & - & - & - & - & - & - & - \\
\hline Yb & 66 & 30 & - & 53 & 30 & - & . & 66 & $\cdot$ & 53 & 40 \\
\hline $\mathrm{Zx}$ & 800 & 700 & - & 900 & 700 & $56^{*}$ & - & 470 & $82^{*}$ & 800 & 670 \\
\hline Reference & A261 & A262 & A346 & A261 & A262 & A165 & A475 & A261 & A165 & A261 & A261 \\
\hline
\end{tabular}

\begin{tabular}{|c|c|c|c|c|c|c|c|c|c|c|c|}
\hline Sequencell & $3800031-00$ & $3830063-00$ & $3920032-00$ & $3990010-02$ & $3990101-\infty 0$ & $4080121-05$ & $4080121-06$ & $4080306-00$ & $4080310-02$ & $4080336-01$ & $4090002-0$ \\
\hline Latitude & 12.150 & 18.953 & 12.728 & 25.750 & 26.067 & 21.800 & 21.800 & 27.717 & 25.250 & 20.883 & 21.450 \\
\hline Longitude & 164.683 & 135.793 & 47.373 & -20.233 & -22.367 & -113.050 & -113.050 & -119.283 & -119.667 & -114.083 & -126.717 \\
\hline Depth(m) & 1830 & 3195 & 1170 & 2791 & 2300 & 3450 & 3450 & 1310 & 64 & 2560 & 4300 \\
\hline Ag(ppm) & - & - & - & $\cdot$ & - & - & - & $\cdot$ & $\cdot$ & - & 2 \\
\hline B & 300 & - & - & 400 & - & - & - & 500 & 200 & - & 400 \\
\hline Ge & - & - & - & • & - & - & - & • & - & - & 7 \\
\hline Sc & 9 & 8 & $\cdot$ & 21 & - & 5 & 1 & $\cdot$ & 10 & - & 16 \\
\hline Sn & $\cdot$ & - & - & $\cdot$ & 6 & - & - & - & $\cdot$ & $\cdot$ & $\cdot$ \\
\hline Th & $\cdot$ & 31 & - & - & $\cdot$ & 10 & 20 & - & - & 35 & - \\
\hline $\mathbf{n}$ & - & $\cdot$ & - & - & - & $\cdot$ & - & - & - & 113 & - \\
\hline $\mathbf{U}$ & - & - & - & - & - & - & - & - & - & 15 & - \\
\hline $\mathbf{Y b}$ & 44 & - & $\cdot$ & 66 & - & - & - & 48 & 57 & $\cdot$ & 44 \\
\hline $\mathbf{z}$ & 1000 & $\cdot$ & 480 & 640 & $\cdot$ & $\cdot$ & $\cdot$ & 530 & 480 & $\cdot$ & 1000 \\
\hline Reforences & A261 & A323 & A195 & A261 & A354 & $A 323$ & A323 & A261 & A261 & $A 303$ & A261 \\
\hline
\end{tabular}

\begin{tabular}{|c|c|c|c|c|c|c|c|c|c|c|c|}
\hline Sequencell & $4090002-05$ & $4100004-00$ & $4150104-01$ & $4160105-02$ & $4160136-02$ & $4180272-00$ & $4360183-\infty 0$ & $4360184-00$ & $4360185-\infty 0$ & $4360186-00$ & $4360187-00$ \\
\hline Latioude & 21.450 & 20.067 & 20.752 & 21.145 & 21.160 & 20.912 & 36.943 & 36.968 & 36.950 & 36.945 & 36.953 \\
\hline Longituds & -126.717 & -130.067 & 173,440 & 163.397 & 163.147 & 142532 & -33.065 & -33.063 & -33.058 & -33.067 & .33 .078 \\
\hline Depth(m) & 4300 & 4959 & 1623 & 1575 & 1437 & 2423 & 2670 & 2100 & 2680 & 2690 & 2560 \\
\hline B (ppm) & 200 & - & - & - & - & - & $204^{*}$ & $400^{\circ}$ & $280^{\circ}$ & $170^{*}$ & $390^{\circ}$ \\
\hline $\mathrm{Ga}$ & 7 & - & • & - & - & - & 5 & 20 & 20 & 9 & 27 \\
\hline $\mathrm{H}_{\mathrm{g}}$ (ppb) & - & - & 114 & 81 & 137 & - & - & - & - & - & - \\
\hline Sc & 14 & 7 & - & - & - & 10 & - & - & - & - & - \\
\hline So & - & - & 46 & 44 & 47 & - & - & - & - & - & . \\
\hline Th & - & 39 & - & - & - & 1 & - & - & - & - & - \\
\hline Yb & 9 & . & - & - & - & - & . & - & - & - & - \\
\hline $\mathbf{z}$ & 380 & - & - & . & . & - & - & . & - & . & - \\
\hline Reference & A261 & A323 & A475 & A475 & A475 & $\mathbf{A 3 2 3}$ & A464 & A464 & A464 & A464 & A464 \\
\hline
\end{tabular}


Table 10. Trace Analyses of crusts from Scripps Nodule Databank

\begin{tabular}{|c|c|c|c|c|c|c|c|c|c|c|c|}
\hline Sequencel & $4360188-00$ & $4360189-00$ & $4360190-\infty 0$ & $4370030-01$ & $4390353-00$ & $4390358-\infty 0$ & $4390358-02$ & $4390358-03$ & $4390358-04$ & $4400536-01$ & $4400536-03$ \\
\hline Latinude & 36.950 & 36.833 & 36.937 & 31.817 & 39.000 & 39.000 & 39.000 & 39.000 & 39.000 & 30.850 & 30.850 \\
\hline Langituds & -33.070 & -33.178 & -33.167 & 43.417 & .61 .000 & -60.950 & -60.950 & -60.950 & -60.950 & -78.450 & -78.450 \\
\hline Depth(m) & 2700 & - & - & 3700 & - & 2000 & 2000 & 2000 & 2000 & 732 & 732 \\
\hline B (ppon) & $400^{\circ}$ & $350^{\circ}$ & $330^{\circ}$ & - & 120 & - & 170 & 190 & 170 & - & . \\
\hline G. & 16 & 2 & 27 & - & • & - & - & $\cdot$ & - & . & 10 \\
\hline s & $\cdot$ & - & - & $25000^{\circ}$ & $\cdot$ & - & - & $\cdot$ & $\cdot$ & - & $9000^{\circ}$ \\
\hline Sb & - & - & - & - & 45 & - & 60 & 45 & 25 & - & - \\
\hline Sc & - & - & - & . & $28^{*}$ & - & $28^{*}$ & $42^{*}$ & $39^{\circ}$ & - & - \\
\hline $\mathrm{Sn}$ & - & - & - & $\cdot$ & - & 4 & • & $\cdot$ & • & - & $\cdot$ \\
\hline$\pi$ & - & - & - & 244 & - & - & - & - & - & 55 & 206 \\
\hline $\mathrm{Yb}$ & - & - & - & $\cdot$ & 38 & - & 33 & 32 & 39 & - & • \\
\hline $2 x$ & . & - & - & - & 690 & - & 790 & 1100 & 890 & . & 450 \\
\hline Reference & A464 & A464 & A464 & A388 & A283 & A 354 & A283 & A283 & A283 & $A 201$ & A388 \\
\hline
\end{tabular}

\begin{tabular}{|c|c|c|c|c|c|c|c|c|c|c|c|}
\hline Sequencell & $4670471-\infty$ & $4670471-01$ & $4670471-02$ & $4670471-03$ & $4670471-04$ & $4670472-00$ & $4670472-01$ & $4670472-02$ & $4810487-01$ & $4810487-03$ & $4810492-00$ \\
\hline Latinude & 38.563 & 38.563 & 38.563 & 38.563 & 38.563 & 38.640 & 38.640 & 38.640 & 40.383 & 40.383 & 42.750 \\
\hline Longitudo & 14.180 & 14.180 & 14.180 & 14.180 & 14.180 & 14.017 & 14.017 & 14.017 & -127.983 & -127.983 & -128.050 \\
\hline Depth(m) & 740 & 740 & 740 & 740 & 740 & 690 & 690 & 690 & 1260 & 1260 & 2520 \\
\hline
\end{tabular}

\begin{tabular}{|c|c|c|c|c|c|c|c|c|c|c|c|}
\hline Depth(m) & 740 & 740 & 740 & 740 & 740 & 690 & 690 & 690 & 1260 & 1260 & 2520 \\
\hline B (ppon) & - & - & - & - & - & - & - & - & - & - & 600 \\
\hline $\mathrm{Bi}$ & - & - & - & - & - & - & - & - & 6 & - & $\cdot$ \\
\hline $\mathbf{E u}$ & 4 & 4 & 1 & 3 & 1 & 2 & - & 1 & - & - & - \\
\hline Lu & 1 & 1 & $\cdot$ & 1 & - & 1 & - & - & - & - & - \\
\hline Nd & 73 & 74 & 18 & 49 & 9 & 33 & 10 & 23 & - & - & - \\
\hline Se & - & - & - & - & - & $\cdot$ & - & $\cdot$ & - & 16 & 13 \\
\hline Sm & 15 & 14 & 3 & 11 & 2 & 7 & 1 & 4 & - & - & - \\
\hline Sn & - & - & - & - & - & - & - & - & 3 & - & - \\
\hline Tb & 2 & 2 & - & 2 & - & .1 & - & - & - & - & - \\
\hline Th & . & . & - & - & - & $\because$ & - & - & $\cdot$ & 34 & - \\
\hline $\mathbf{T i}$ & $\because$ & $=$ & $\because$ & : & $\because$ & 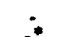 & : & : & 102 & $\cdot$ & - \\
\hline Yo & $8^{*}$ & $7^{*}$ & $1^{*}$ & $5^{*}$ & $2^{*}$ & $3^{*}$ & $1^{*}$ & 1 & $\cdot$ & - & 35 \\
\hline $\mathbf{z}$ & - & • & • & • & • & - & • & • & • & • & 560 \\
\hline Reference & A477 & A47 & $A 47$ & A477 & $A 47$ & A47 & A477 & A47 & $A 200$ & A323 & A261 \\
\hline
\end{tabular}

\begin{tabular}{lcccc}
\hline Sequenced & $4840006-04$ & $4840006-05$ & $4870015-01$ & $5200002-02$ \\
Latinude & 40.235 & 40.235 & 40.267 & 52783 \\
Longitude & -155.917 & -155.917 & 170.333 & -150.083 \\
Depth(m) & 5033 & 5033 & 2037 & - \\
\hline & & & & \\
He (ppb) & - & - & - & 470 \\
Sc & 14 & 11 & 9 & - \\
Th & 138 & 131 & - & - \\
Reference & A323 & A323 & A346 & A255
\end{tabular}


Table 11. Composition of Mn oxide majors as reported by NGDC

\begin{tabular}{|c|c|c|c|c|c|c|c|c|c|}
\hline $\begin{array}{l}\text { MMBIB } \\
\text { Sampleit }\end{array}$ & $\begin{array}{l}\text { APLLAC8501 } \\
45\end{array}$ & $\begin{array}{l}\text { BURNWC7705 } \\
1052\end{array}$ & $\begin{array}{l}\text { CANNJR7701 } \\
6243-1\end{array}$ & $\begin{array}{l}\text { CANNJR7701 } \\
6243-2\end{array}$ & $\begin{array}{l}\text { CANNJR7701 } \\
6243-3\end{array}$ & $\begin{array}{c}\text { CANNJR7701 } \\
6243-4\end{array}$ & $\begin{array}{l}\text { CANNJR7701 } \\
\text { 6243-5 }\end{array}$ & $\begin{array}{l}\text { CANNIR7701 } \\
6243-6\end{array}$ & $\begin{array}{c}\text { CORLJB7801 } \\
\text { A-1 }\end{array}$ \\
\hline Analysiell & 1 & 1 & 1 & 1 & 1 & 1 & 1 & 1 & 1 \\
\hline Sumpletypo & 1 & 8 & 8 & 8 & 8 & 8 & 8 & 8 & 19 \\
\hline Letitudo & -8.550 & 2.286 & 12550 & 12.550 & 12.550 & 12550 & 12.550 & 12550 & .600 \\
\hline Longitude & -167.000 & -101.018 & 47.651 & 47.651 & 47.651 & 47.651 & 47.651 & 47.651 & -86.117 \\
\hline Depth & 3940 & 3150 & 2550 & 2550 & 2550 & 2550 & 2550 & 2550 & $\cdot$ \\
\hline$A(w t . \%)$ & 1.80 & $\cdot$ & 1.11 & 1.11 & 84 & .06 & .69 & 34 & .09 \\
\hline c. & 230 & $\cdot$ & 1.41 & 1.74 & 1.49 & 150 & 1.55 & 1.52 & 1.07 \\
\hline Co & 390 & .051 & .004 & $.00 n$ & .003 & .01 & $\cdot$ & .002 & $\cdot$ \\
\hline Ca & .140 & .073 & .008 & .011 & .020 & .001 & .001 & .008 & .004 \\
\hline$F_{0}$ & 17.8 & 21.8 & 6.39 & 1.31 & 1.53 & 4.52 & .76 & 1.51 & 27.3 \\
\hline $\mathbf{K}$ & $\cdot$ & - & 1.13 & 1.54 & 1.57 & 1.28 & 1.21 & 1.62 & $\cdot$ \\
\hline Ms & - & $\cdot$ & 1.09 & 2.21 & 2.01 & 1.60 & 1.50 & 2.17 & $\cdot$ \\
\hline Mn & 19.2 & 16.7 & 34.2 & 34.9 & 37.3 & 38.8 & 39.8 & 425 & 7.29 \\
\hline Na & $\cdot$ & $\cdot$ & 3.53 & 2.57 & 2.75 & 3.04 & 290 & 3.07 & • \\
\hline $\mathbf{N i}$ & .320 & .195 & .039 & .090 & .067 & .001 & .002 & .038 & .007 \\
\hline $\mathbf{P}$ & - & $\cdot$ & • & • & • & $\cdot$ & - & • & - \\
\hline $\mathbf{S i}$ & - & - & 4.79 & 5.38 & 4.04 & 232 & 3.38 & 1.97 & 15.10 \\
\hline $\mathrm{Ti}$ & 1.15 & - & .20 & .16 & .11 & .02 & .07 & .08 & • \\
\hline $\mathrm{H} 2 \mathrm{O}$ & $\cdot$ & $\cdot$ & $\cdot$ & $\cdot$ & $\cdot$ & • & • & $\cdot$ & $\cdot$ \\
\hline Axppm) & - & - & • & • & • & • & • & • & 60 \\
\hline Be & - & - & 1700 & 2000 & 1100 & 1000 & 530 & 660 & 320 \\
\hline$C d$ & - & - & $\cdot$ & • & • & • & • & • & $\cdot$ \\
\hline ce & - & - & - & - & - & - & - & - & - \\
\hline$a$ & - & - & - & - & - & . & - & - & 10 \\
\hline Mo & - & - & - & - & - & - & - & - & - \\
\hline $\mathrm{Pb}$ & 790 & - & - & • & - & - & • & • & - \\
\hline Pt & - & . & 380 & 350 & 320 & 340 & 220 & 340 & - \\
\hline $\mathbf{v}$ & - & - & • & • & - & - & - & - & - \\
\hline $\mathbf{Y}$ & • & • & 10 & 10 & 10 & • & . & 10 & - \\
\hline $\mathbf{Z n}$ & 510 & 540 & 220 & 450 & 780 & 20 & 20 & 380 & 150 \\
\hline
\end{tabular}

\begin{tabular}{|c|c|c|c|c|c|c|c|c|c|}
\hline $\begin{array}{l}\text { MMBIB } \\
\text { Samploty }\end{array}$ & $\begin{array}{c}\text { CORLBB7801 } \\
\text { A-2 }\end{array}$ & $\begin{array}{c}\text { CORLJB7801 } \\
\text { DM-1 }\end{array}$ & $\begin{array}{c}\text { CRLJB7801 } \\
\text { DM-2 }\end{array}$ & $\begin{array}{c}\text { CORLJB7801 } \\
\text { N-1 }\end{array}$ & $\begin{array}{c}\text { CORLJB7801 } \\
\text { N-2 }\end{array}$ & $\begin{array}{c}\text { CORLJB7801 } \\
\text { N.3 }\end{array}$ & $\underset{N-4}{\text { CORLJB7801 }}$ & $\begin{array}{c}\text { CORLJB7801 } \\
\text { N.S }\end{array}$ & $\begin{array}{c}\text { CORLJB7801 } \\
\text { TB-1 }\end{array}$ \\
\hline Analyain & 1 & 1 & 1 & 1 & 1 & 1 & 1 & 1 & 1 \\
\hline Semplotypo & 19 & 19 & 19 & 19 & 19 & 19 & 19 & 19 & 19 \\
\hline Latitude & 583 & .417 & .417 & .600 & .600 & .583 & 583 & .600 & .600 \\
\hline Longitude & -86.100 & -86.100 & -86.100 & 86.117 & -86.117 & -86.100 & .86 .100 & -86.117 & -86.117 \\
\hline Depeh & $\cdot$ & $\cdot$ & - & $\cdot$ & $\cdot$ & - & $\cdot$ & - & $\cdot$ \\
\hline$A(w t S)$ & .55 & 1.33 & 1.32 & .01 & .02 & .26 & 1.12 & .83 & .20 \\
\hline C. & 1.70 & 2.46 & 2.10 & .49 & .08 & 1.02 & 6.70 & 17.2 & 1.19 \\
\hline Co & - & .050 & .025 & - & - & - & .001 &.$\infty 1$ & .001 \\
\hline $\mathrm{Cu}$ & .006 & .040 & .038 & - & .001 & - & .015 & .009 & .010 \\
\hline Fo & 27.6 & 17.3 & 10.2 & 25.7 & 23.2 & 27.4 & 11.8 & 8.33 & .31 \\
\hline $\mathbf{K}$ & - & - & 46 & 1.74 & 1.86 & .83 & .64 & .50 & .55 \\
\hline Ms & - & - & 88 & 1.44 & 1.71 & 1.27 & 1.01 & 1.01 & 1.97 \\
\hline Mn & 8.41 & 22.6 & 15.1 & .20 & .18 & 35 & 34 & 1.14 & 51.1 \\
\hline $\mathrm{Ne}$ & - & • & 1.11 & 1.19 & 1.03 & 1.21 & .09 & .20 & 2.20 \\
\hline $\mathbf{N i}$ & .005 &.$m$ & .454 & $\cdot$ & .001 & .003 & .010 & .006 & .050 \\
\hline $\mathbf{P}$ & - & - & - & • & • & - & • & • & - \\
\hline Si & 11.40 & 5.17 & 17.50 & 22.40 & 24.80 & 18.80 & 20.80 & 13.40 & .65 \\
\hline $\mathrm{Ti}$ & - & • & - & . & - & - & . & . & . \\
\hline 420 & - & - & - & - & . & - & - & . & - \\
\hline$A \times(p p m)$ & 20 & 180 & 120 & 10 & . & 10 & • & - & - \\
\hline Be & 1100 & 1400 & 760 & 70 & 70 & 600 & 1900 & 2100 & 2500 \\
\hline Cd & • & . & - & - & - & - & . & - & - \\
\hline$\infty$ & - & 210 & 110 & - & - & . & . & - & - \\
\hline$a$ & 10 & 40 & 20 & - & . & 10 & 20 & 10 & 10 \\
\hline Mo & - & - & - & - & - & . & - & . & - \\
\hline $\mathbf{P b}$ & - & . & - & - & - & - & - & . & . \\
\hline Pt & - & - & - & - & - & - & - & . & . \\
\hline $\mathrm{v}$ & - & . & . & . & - & - & - & . & - \\
\hline$Y$ & . & - & - & - & . & - & - & - & - \\
\hline $\mathrm{Zn}_{\mathbf{n}}$ & 150 & 770 & 520 & 30 & 30 & so & 380 & 240 & 300 \\
\hline
\end{tabular}


Table 11. Composition of Mn oxide majors as reported by NGDC (cont'd).

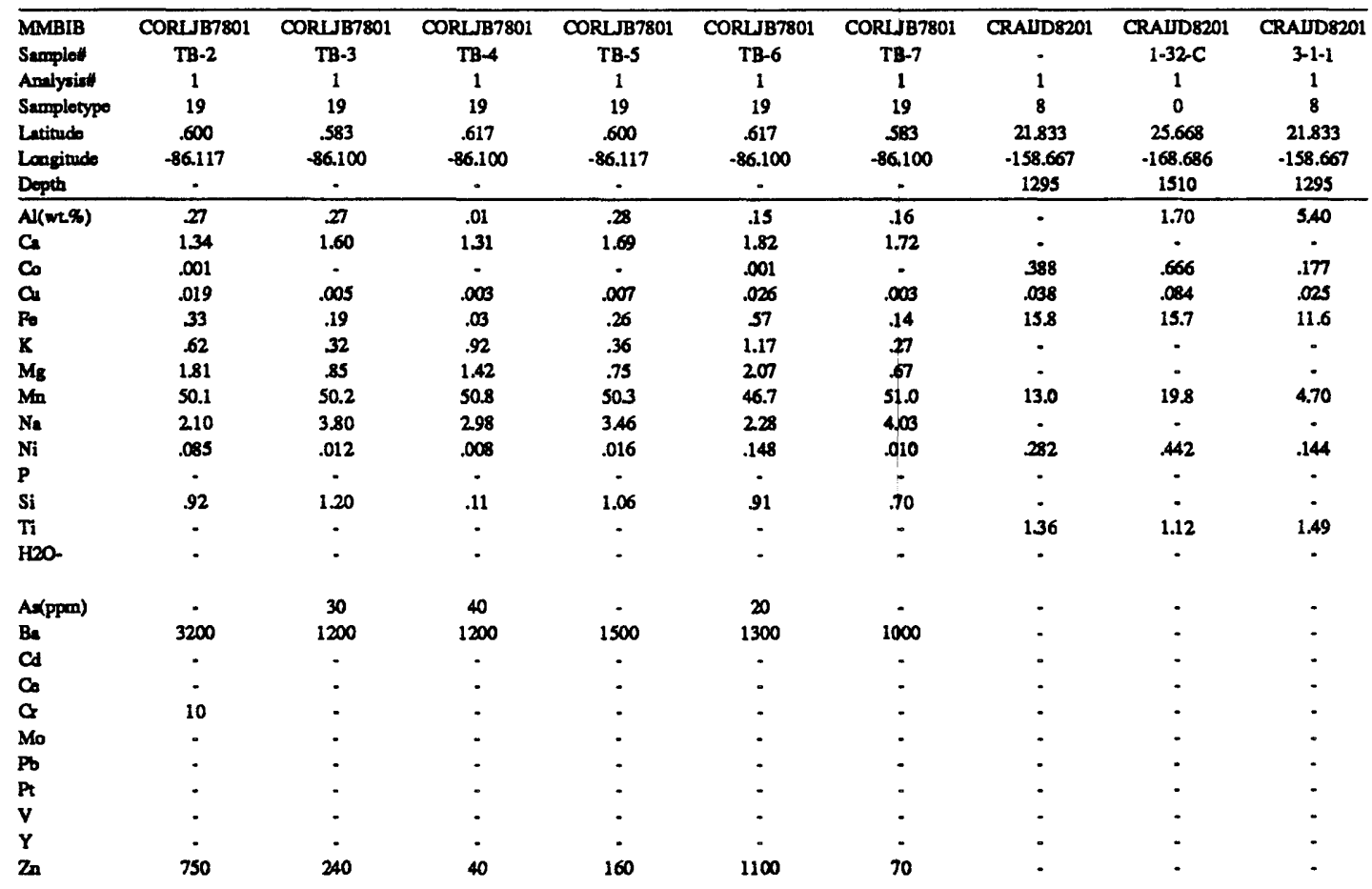

\begin{tabular}{|c|c|c|c|c|c|c|c|c|c|}
\hline MMBIB & $\begin{array}{l}\text { CRALD8201 } \\
\text { 3-1-2A }\end{array}$ & $\begin{array}{l}\text { CRAUD8201 } \\
3-2-4 A\end{array}$ & $\begin{array}{c}\text { CRALDS201 } \\
3-2.5 A\end{array}$ & $\begin{array}{c}\text { CRALDD201 } \\
\text { 3-2-6 }\end{array}$ & $\begin{array}{c}\text { CRAUD8201 } \\
3-28 \mathrm{~A}\end{array}$ & $\begin{array}{c}\text { CRAID8201 } \\
5+10\end{array}$ & $\begin{array}{c}\text { CRAIJD8201 } \\
\text { 5-11-A }\end{array}$ & $\begin{array}{c}\text { CRAUD8201 } \\
5-12-A\end{array}$ & $\begin{array}{c}\text { CRAID8201 } \\
\text { 5-5-A }\end{array}$ \\
\hline Anniyni= & 1 & 9 & 1 & 1 & 1 & 1 & 1 & 1 & 1 \\
\hline Sampletype & 8 & 8 & 8 & 8 & 8 & 8 & 8 & 8 & 8 \\
\hline Latinde & 21.833 & 21.833 & 21.833 & 21.833 & 21.833 & 38.617 & 40.218 & 37.752 & 28.785 \\
\hline Langiande & -158.667 & -158.667 & -158.667 & .158 .667 & -158.667 & 171.034 & 170.367 & 170.285 & -178.801 \\
\hline Depth & 1295 & 1295 & 1295 & 1295 & 1295 & 1104 & 2030 & 1800 & 880 \\
\hline$A \mid(w t s)$ & - & . & - & - & . & 80 & .40 & .70 & 30 \\
\hline $\mathrm{Ca}$ & - & - & • & - & . & - & - & - & - \\
\hline Co & .798 & .782 & .668 & 554 & - & 1,12 & .411 & .463 & 1.41 \\
\hline$C_{u}$ & .024 & .036 & .027 & .032 & - & .055 & .052 & .047 & .065 \\
\hline Pe & 19.3 & 18.3 & 20.5 & 17.1 & 18.6 & 19.3 & 13.1 & 15.3 & 8.90 \\
\hline $\mathbf{K}$ & - & - & - & - & - & 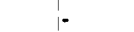 & - & - & - \\
\hline $\mathbf{M g}$ & - & - & - & - & - & - & - & • & - \\
\hline $\mathrm{Mn}$ & 24.6 & 21.6 & 21.6 & 18.0 & 225 & 25.0 & 27.2 & 19.5 & 30.9 \\
\hline $\mathrm{Ne}$ & . & - & - & - & . & . & - & - & - \\
\hline $\mathrm{Ni}$ & 341 & .317 & 264 & .282 & - & .246 & .680 & 364 & .982 \\
\hline$P$ & 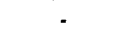 & • & - & • & - & . & • & • & $\cdot$ \\
\hline Si & - & - & - & - & - & - & . & . & . \\
\hline $\mathrm{Ti}$ & 1.04 & 1.25 & 1.20 & 1.31 & - & - & - & - & - \\
\hline $\mathrm{H} 2 \mathrm{O}$ & - & - & - & - & - & - & - & - & - \\
\hline$A \leq(p p e n)$ & - & . & - & . & . & - & - & - & - \\
\hline Be & - & - & - & - & . & - & . & . & - \\
\hline Cd & . & . & - & - & - & . & . & . & - \\
\hline Co & - & - & - & - & - & - & - & - & . \\
\hline$a$ & . & . & - & - & - & - & - & - & - \\
\hline Mo & - & - & - & . & - & - & - & - & - \\
\hline $\mathrm{Pb}$ & - & - & - & - & . & - & - & . & - \\
\hline Pt & . & . & - & - & . & . & - & - & - \\
\hline v & - & - & - & - & . & - & - & - & - \\
\hline$Y$ & - & - & - & - & - & - & - & . & - \\
\hline $\mathrm{Zn}_{\mathbf{n}}$ & - & - & . & - & . & - & - & - & - \\
\hline
\end{tabular}


Table 11. Composition of Mn oxide majors as reported by NGDC (cont'd).

\begin{tabular}{|c|c|c|c|c|c|c|c|c|c|}
\hline $\begin{array}{l}\text { MMBIB } \\
\text { Samplot }\end{array}$ & $\begin{array}{c}\text { CRAUD8201 } \\
\text { 5-5-B }\end{array}$ & $\begin{array}{c}\text { CRAUD8201 } \\
\text { 5-7-C }\end{array}$ & $\begin{array}{c}\text { CRALDD8201 } \\
\text { 5-7-B }\end{array}$ & $\begin{array}{c}\text { CRAUD8201 } 1 \\
\text { S-8-B }\end{array}$ & $\begin{array}{c}\text { CRAUD8201 } \\
1-20-A\end{array}$ & $\begin{array}{c}\text { CRAUD8201 } \\
1-24\end{array}$ & $\begin{array}{c}\text { CRAUDS201 } \\
1.32 \mathrm{~A}\end{array}$ & $\begin{array}{c}\text { CRAUD8201 } \\
1.32 \mathrm{~B}\end{array}$ & $\begin{array}{c}\text { CRAUDS201 } \\
\text { 1-32-D }\end{array}$ \\
\hline Antyai= & 1 & 1 & 1 & 1 & 1 & 1 & 1 & 1 & 1 \\
\hline Sempletype & 8 & 8 & 8 & 8 & 8 & 8 & 8 & 8 & 8 \\
\hline Latitude & 28.785 & 27.751 & 27.751 & 26451 & 28.817 & 27.700 & 25.668 & 25.668 & 25.668 \\
\hline Longitude & -178.801 & -175.636 & -175.636 & -177.852 & -178.950 & -175.683 & -168.686 & -168.686 & -168.686 \\
\hline Depth & - & 850 & 850 & 930 & 980 & 1090 & 1510 & 1510 & 1510 \\
\hline$\overline{A l(w t . \%)}$ & 30 & .40 & .40 & .60 & 30 & 1.80 & 80 & 1.40 & 1.00 \\
\hline c & - & • & - & $\cdot$ & - & $\cdot$ & - & - & • \\
\hline Co & 1.16 & 969 & .939 & .656 & 1.64 & .597 & .800 & .741 & .771 \\
\hline ar & .057 & .059 & .050 & .120 & .053 & .066 & .070 & .125 & .068 \\
\hline Fo & 7.80 & 11.6 & 13.6 & 6.20 & 11.7 & 7.10 & 169 & 15.9 & 17.5 \\
\hline $\mathbf{K}$ & - & - & . & . & 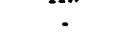 & 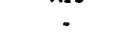 & $\cdot$ & . & 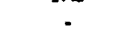 \\
\hline Ms & - & - & - & . & - & - & - & - & - \\
\hline Min & 33.0 & 25.8 & 27.4 & 19.6 & 31.8 & 28.6 & 23.5 & 21.6 & 22.0 \\
\hline $\mathrm{Na}$ & - & - & - & - & - & - & - & - & $\cdot$ \\
\hline $\mathbf{N i}$ & 1.107 & 689 & 570 & .609 & .624 & A88 & $A 11$ & 592 & 349 \\
\hline $\mathbf{P}$ & - & $\cdot$ & . & . & . & - & - & - & $\because$ \\
\hline Si & . & - & . & . & . & . & . & . & . \\
\hline$\pi$ & - & - & - & - & .67 & .63 & 93 & 97 & 89 \\
\hline H2O & - & - & . & - & . & 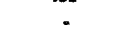 & $?$ & 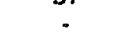 & 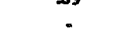 \\
\hline$A \times$ (ppm) & - & - & - & - & - & - & - & - & - \\
\hline $\mathrm{Ba}$ & - & - & . & - & - & - & - & - & - \\
\hline Cd & - & - & - & . & - & . & - & . & - \\
\hline$\infty$ & - & - & . & - & . & . & . & - & - \\
\hline$a$ & - & . & . & - & - & - & . & - & - \\
\hline Mo & - & - & . & . & - & . & . & - & - \\
\hline $\mathrm{Pb}$ & . & . & . & . & . & . & . & . & . \\
\hline $\mathrm{Pt}$ & - & - & . & - & - & - & - & - & - \\
\hline v & . & . & - & - & - & - & . & . & . \\
\hline $\mathbf{Y}$ & . & . & . & - & - & - & - & - & - \\
\hline $\mathrm{Zn}$ & . & . & - & - & - & . & . & - & - \\
\hline
\end{tabular}

\begin{tabular}{|c|c|c|c|c|c|c|c|c|c|}
\hline MMBIB & CRAVD8201 & CRALD8201 & CRAUD8201 & CRALID201 & CRAUD8201 & CRAUD8201 & CRAULD201 & CRAUD8201 & CRAUDD201 \\
\hline Surmplot & 1-33-A & 1-33-B & $1-43$ & 1-47-A & $1-49$ & $21011 A$ & $2-101 A$ & $2-1214 A$ & 2-8-8A \\
\hline Amiyris: & 1 & 1 & 1 & 1 & 1 & 1 & 3 & 1 & 1 \\
\hline Sampletypo & 8 & 8 & 8 & 8 & 8 & 8 & 8 & 8 & 8 \\
\hline Latiude & 25.484 & 25.484 & 24.285 & 23.817 & 23.367 & 21.953 & 21.953 & 21.769 & 21.850 \\
\hline Longitude & -168.651 & -168.651 & -166.719 & $-164,417$ & -163.567 & -158.602 & -158.602 & -158.735 & -158.684 \\
\hline Depth & 1075 & 1075 & 1115 & 870 & 870 & 1985 & 1985 & 1120 & 1340 \\
\hline$\overline{A l\left(w t x_{0}\right)}$ & 120 & 1.20 & 80 & .90 & 50 & $\cdot$ & . & . & $\cdot$ \\
\hline a & $\cdot$ & $\cdot$ & - & - & - & - & . & - & - \\
\hline$c_{0}$ & .775 & 1.00 & 1.04 & 1.00 & 1.36 & .740 & - & .761 & .867 \\
\hline C. & .069 & .068 & .029 & .035 & .037 & .038 & .027 & .069 & .028 \\
\hline Po & 15.4 & 16.2 & 16.7 & 16.7 & 14.4 & 20.8 & 19.3 & 17.2 & 17.5 \\
\hline $\mathbf{K}$ & - & . & $\cdot$ & 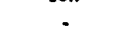 & 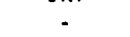 & - & - & - & 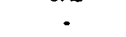 \\
\hline $\mathbf{M}_{\mathbf{s}}$ & - & - & - & - & - & - & - & - & . \\
\hline $\mathrm{Mn}$ & 23.7 & 24.4 & 23.5 & 23.5 & 26.2 & 22.1 & 24.0 & 25.8 & 22.4 \\
\hline $\mathrm{Na}$ & - & $\cdot$ & - & - & - & - & - & $\cdot$ & - \\
\hline $\mathrm{Ni}$ & .525 & 492 & 299 & 299 & 370 & 362 & 371 & .692 & .401 \\
\hline $\mathbf{P}$ & - & $\cdot$ & 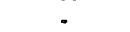 & - & - & . & 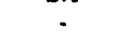 & 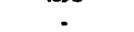 & 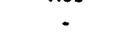 \\
\hline Si & - & - & - & - & - & - & . & - & • \\
\hline$\pi$ & .73 & .83 & 89 & .76 & 83 & 1.83 & . & . & 8.17 \\
\hline H2O & - & - & $\cdot$ & • & - & - & - & - & - \\
\hline As(ppen) & - & - & - & , & - & . & - & . & . \\
\hline $\mathrm{Be}$ & - & - & - & - & - & - & - & - & - \\
\hline Cd & - & - & - & - & . & . & . & - & . \\
\hline$\infty$ & - & - & - & . & - & - & . & - & . \\
\hline$a$ & - & - & - & - & - & - & - & . & - \\
\hline Mo & - & - & . & - & - & - & - & - & - \\
\hline $\mathrm{Pb}$ & - & - & - & - & - & - & - & - & - \\
\hline $\mathrm{Pt}$ & - & - & - & - & - & - & . & - & - \\
\hline $\mathbf{v}$ & - & - & . & - & . & - & - & . & - \\
\hline $\mathbf{Y}$ & - & - & - & . & - & - & - & - & - \\
\hline $\mathbf{z n}$ & - & - & - & - & - & - & - & - & - \\
\hline
\end{tabular}


Table 11. Composition of Mn oxide majors as reported by NGDC (cont'd).

\begin{tabular}{|c|c|c|c|c|c|c|c|c|c|}
\hline $\begin{array}{l}\text { MMBIB } \\
\text { Samplot }\end{array}$ & $\begin{array}{l}\text { CRAUD8201 } \\
\text { 2-9-9A }\end{array}$ & $\begin{array}{c}\text { CRALD8201 } \\
\text { 3-3-11 }\end{array}$ & $\begin{array}{c}\text { CRALD8201 } \\
\text { 3-4-14 }\end{array}$ & $\begin{array}{c}\text { CRALDD201 } \\
\text { 3-4-15A }\end{array}$ & $\begin{array}{c}\text { CRAUD8201 } \\
3-4-17\end{array}$ & $\begin{array}{c}\text { CRAUDA201 } \\
\text { 3-5-22 }\end{array}$ & $\begin{array}{l}\text { CRALID8201 } \\
\text { 3-8-21A }\end{array}$ & $\begin{array}{c}\text { CRALID8201 } \\
4-10\end{array}$ & $\begin{array}{c}\text { CRALD8201 } \\
411\end{array}$ \\
\hline Analysix" & 1 & 1 & 1 & 1 & 1 & 1 & 1 & 1 & 1 \\
\hline Sampletype & 8 & 8 & 8 & 8 & 8 & 8 & 8 & 8 & 8 \\
\hline Letínde & 21.901 & 21.918 & 21.868 & 21.868 & 21.868 & 20.134 & 20.134 & 23.168 & 23.117 \\
\hline Longitudo & -158.634 & -158.785 & -158.818 & -158.818 & -158.818 & -156.253 & -156.253 & -162433 & -162467 \\
\hline Dopth & 1840 & 1945 & 1150 & 1150 & 1150 & 1440 & 1440 & 2065 & 1865 \\
\hline$A(w t . \%)$ & $\cdot$ & $\cdot$ & - & $\cdot$ & $\cdot$ & • & 3.40 & .60 & 50 \\
\hline C & • & $\cdot$ & $\cdot$ & - & - & • & • & • & $\cdot$ \\
\hline Co & 591 & .062 & 378 & .538 & .856 & .021 & .055 & 1.23 & .722 \\
\hline Cu & .026 & .053 & .025 & .020 & .059 & .012 & .012 & .036 & .047 \\
\hline Fo & 21.8 & 22.6 & 13.6 & 16.0 & 16.3 & 10.8 & 10.8 & 19.0 & 15.7 \\
\hline $\mathbf{K}$ & - & • & • & - & • & - & • & - & - \\
\hline Mg & $\cdot$ & $\cdot$ & • & • & • & • & - & • & - \\
\hline Mn & 19.5 & 7.60 & 8.90 & 17.2 & 27.2 & 8.20 & 14.9 & 26.1 & 21.4 \\
\hline $\mathrm{Na}$ & • & - & • & • & • & • & - & • & • \\
\hline $\mathrm{Ni}$ & .281 & 375 & .149 & 304 & .711 & .034 & .119 & A76 & 387 \\
\hline $\mathbf{P}$ & - & - & - & - & - & - & - & $\cdot$ & 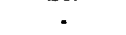 \\
\hline Si & - & - & - & - & - & - & - & - & . \\
\hline $\mathrm{Ti}$ & 1.27 & .25 & 1.35 & 1.04 & 1.00 & 1.51 & 1.23 & - & - \\
\hline $\mathrm{H} 2 \mathrm{O}$ & • & - & • & - & - & $\cdot$ & • & - & - \\
\hline$A x$ (ppon) & - & - & - & - & - & - & - & - & - \\
\hline $\mathrm{Ba}$ & - & - & - & - & - & - & - & - & - \\
\hline Cd & - & - & - & - & - & - & - & - & - \\
\hline$c_{0}$ & - & . & . & - & - & - & - & - & - \\
\hline$a$ & - & - & . & - & - & - & - & - & - \\
\hline Mo & - & - & • & - & - & - & - & - & - \\
\hline $\mathbf{P b}$ & - & - & - & - & - & - & - & - & - \\
\hline $\mathbf{P t}$ & - & - & - & - & - & - & - & - & - \\
\hline $\mathbf{v}$ & - & - & - & - & - & - & - & - & - \\
\hline $\mathbf{Y}$ & - & - & - & . & - & - & - & - & - \\
\hline $\mathbf{Z n}$ & . & . & - & . & - & - & - & - & - \\
\hline
\end{tabular}

\begin{tabular}{|c|c|c|c|c|c|c|c|c|c|}
\hline $\begin{array}{l}\text { MMBIB } \\
\text { Sampleit }\end{array}$ & $\begin{array}{c}\text { CRALD8201 } \\
4-3\end{array}$ & $\begin{array}{c}\text { CRALD8201 } \\
45\end{array}$ & $\begin{array}{c}\text { CRALDD8201 } \\
47\end{array}$ & $\begin{array}{c}\text { CRALD8201 } \\
4-8\end{array}$ & $\begin{array}{c}\text { CRAIDS201 } \\
4-9\end{array}$ & $\begin{array}{c}\text { CRAUDD2201 } \\
24-2\end{array}$ & $\begin{array}{l}\text { CRAUDD201 } \\
\text { 2-5.3A }\end{array}$ & $\begin{array}{c}\text { CRAUD8201 } \\
2-6-6\end{array}$ & $\begin{array}{l}\text { CRONDS8201 } \\
\text { U23-1 }\end{array}$ \\
\hline Analyaist & 1 & 1 & 1 & 1 & 1 & 1 & 1 & 1 & 1 \\
\hline Sermpletype & 8 & 8 & 8 & 8 & 8 & 8 & 8 & 8 & 8 \\
\hline Letínde & 25.650 & 25.500 & 25.683 & 24.235 & 23.702 & 21.718 & 21.718 & 21.685 & -26.933 \\
\hline Longitude & -168.785 & .168 .235 & -167.686 & -166.068 & -165.600 & -158.285 & -158.451 & -158.485 & -177.617 \\
\hline Depth & 1860 & 1440 & 1340 & 1840 & 1330 & 795 & 920 & 985 & 1700 \\
\hline $\mathrm{Al}(\mathrm{wt} \%)$ & 1.60 & .60 & 90 & .80 & 1.20 & - & . & - & .25 \\
\hline c. & - & - & - & $\cdot$ & - & - & $\cdot$ & - & 210 \\
\hline Co & .774 & 1.09 & .122 & 1.11 & .776 & 1.10 & .923 & 1.17 & • \\
\hline Cu & .061 & .036 & .024 & .075 & .040 & .026 & .015 & .025 & .010 \\
\hline Fo & 16.3 & 17.8 & 13.5 & 16.3 & 13.8 & 20.3 & 22.4 & 19.5 & .24 \\
\hline $\mathbf{K}$ & • & - & - & - & - & - & • & - & • \\
\hline $\mathbf{M}_{\mathbf{B}}$ & - & - & $\cdot$ & • & - & - & • & - & - \\
\hline $\mathrm{Mn}$ & 18.0 & 22.2 & 21.2 & 22.2 & 17.0 & 24.3 & 24.3 & $27 A$ & 52.0 \\
\hline $\mathrm{Na}$ & . & - & . & . & . & . & . & . & . \\
\hline $\mathrm{Ni}$ & .358 & .343 & .406 & 368 & 393 & .337 & .262 & .443 & .032 \\
\hline $\mathbf{P}$ & - & - & - & • & - & - & - & - & • \\
\hline $\mathbf{S i}$ & - & - & - & - & - & - & - & - & - \\
\hline $\mathrm{Ti}$ & - & - & - & - & - & 1.21 & 1.13 & 1.12 & - \\
\hline $\mathrm{H} 2 \mathrm{O}$ & $\cdot$ & - & - & - & - & • & • & • & - \\
\hline$A x(p p m)$ & - & - & - & . & - & - & - & - & - \\
\hline Ba & - & - & - & - & - & - & - & - & - \\
\hline Cd & . & - & . & - & . & - & . & - & - \\
\hline Co & - & - & - & . & - & - & - & - & - \\
\hline$c$ & . & - & - & - & - & - & - & . & - \\
\hline Mo & - & . & - & - & . & - & - & - & - \\
\hline Pb & - & - & - & - & - & - & - & • & - \\
\hline $\mathrm{Pt}$ & - & . & - & - & - & . & - & - & - \\
\hline v & - & - & - & . & . & - & - & . & - \\
\hline $\mathbf{Y}$ & . & . & . & . & . & . & . & - & - \\
\hline $\mathrm{Zn}$ & - & . & . & . & - & - & . & . & 490 \\
\hline
\end{tabular}


Table 11. Composition of Mn oxide majors as reported by NGDC (cont'd).

\begin{tabular}{|c|c|c|c|c|c|c|c|c|c|}
\hline MMBIB & CRONDS8201 & CRONDS8201 & CRONDS8201 & CRONDS8201 & CRONDS8201 & CRONDS8201 & CRONDS8201 & CRONDS8201 & DHLJG8201 \\
\hline Sample: & U23-1 & U23-1 & U23-1 & U23-1 & U23-1 & U23-1 & U23-2 & U23-2 & ID \\
\hline Analysiet & 2 & 3 & 4 & 5 & 6 & 7 & 1 & 2 & 1 \\
\hline Sampletype & 8 & 8 & 8 & 8 & 8 & 8 & 8 & 8 & 1 \\
\hline Latitude & -26.933 & -26.933 & -26.933 & -26.933 & -26.933 & -26.933 & .26 .933 & -26.933 & -38.600 \\
\hline Longitude & -177.617 & -177.617 & -177.617 & -177.617 & -177.617 & -177.617 & -177.617 & -177.617 & -167900 \\
\hline Depth & 1700 & 1700 & 1700 & 1700 & 1700 & 1700 & 1700 & 1700 & 1420 \\
\hline$A(w t . \%)$ & .03 & .07 & .02 & .03 & .08 & .28 & 1.10 & 15.6 & $\cdot$ \\
\hline C. & 220 & 3.60 & 4.90 & 5.80 & 6.80 & 7.90 & 1.10 & 260 & - \\
\hline Co & .001 & - & $\cdot$ & - & - & .001 & .005 & $\cdot$ & - \\
\hline $\mathrm{Cu}$ & .002 & .003 & .003 & .003 & .003 & .017 & .020 & .003 & - \\
\hline Re & .05 & .06 & .01 & .02 & .08 & .32 & 1.50 & 270 & - \\
\hline $\mathbf{K}$ & $\cdot$ & - & - & - & - & - & - & $\because$ & - \\
\hline Mg & - & - & - & • & - & - & - & - & - \\
\hline $\mathbf{M n}$ & - & 50.0 & 49.0 & 49.0 & 520 & 46.0 & 38.0 & 49.0 & - \\
\hline $\mathrm{Na}$ & $\cdot$ & $\cdot$ & - & - & . & - & - & - & - \\
\hline $\mathbf{N i}$ & .009 & .015 & .004 & .004 & .010 & .048 & .073 & .002 & - \\
\hline $\mathbf{P}$ & - & • & - & - & - & • & • & • & - \\
\hline Si & - & - & - & - & - & - & - & - & - \\
\hline $\mathrm{Ti}$ & - & - & - & - & - & - & - & - & - \\
\hline $\mathrm{H} 20 \mathrm{O}$ & $\cdot$ & - & - & - & $\cdot$ & • & $\cdot$ & $\cdot$ & $\cdot$ \\
\hline$A x(p p m)$ & - & - & - & - & - & - & - & - & $\cdot$ \\
\hline Ba & - & - & - & - & - & - & - & - & 30 \\
\hline Cd & - & - & - & - & - & $\cdot$ & - & - & - \\
\hline$\infty$ & - & - & - & - & - & - & - & - & - \\
\hline$a$ & - & - & - & - & - & - & - & - & - \\
\hline Mo & - & - & - & - & - & - & - & - & - \\
\hline $\mathrm{Pb}$ & - & - & - & - & - & - & - & - & - \\
\hline $\mathbf{P t}$ & - & - & - & - & - & - & - & - & - \\
\hline $\mathbf{v}$ & - & - & - & - & - & - & - & - & - \\
\hline $\mathbf{Y}$ & - & - & - & - & - & - & . & . & - \\
\hline $\mathbf{Z n}$ & 110 & 170 & 30 & so & 150 & 710 & 760 & 90 & - \\
\hline
\end{tabular}

\begin{tabular}{|c|c|c|c|c|c|c|c|c|c|}
\hline MMBIB & DHLJG8201 & DULJG8201 & EHRLAM6803 & EHRLAM6803 & EHRLAM6803 & EHRLAM6803 & BHRLAM6803 & EHRLAM6803 & BHRLAM6803 \\
\hline Sample: & 143D-C & 143D-E & $113-10$ & $2-9$ & 11.1 & $2390-12$ & $2390-12$ & $2390-12$ & 2390-12 \\
\hline Analyzi= & 1 & 1 & 1 & 1 & 1 & 1 & 2 & 3 & 4 \\
\hline Sumpletype & 1 & 1 & 7 & 1 & 1 & 8 & 8 & 8 & 8 \\
\hline Letitude & 19.500 & 19.500 & 9.019 & 35.583 & -1.617 & 31.202 & 31.202 & 31.202 & 31.202 \\
\hline Longitude & -168.900 & -168.900 & -42.669 & -67.917 & 58.617 & -78.483 & -78.483 & -78.483 & -78.483 \\
\hline Depth & 1805 & 1805 & 2300 & 3000 & 3940 & 648 & 648 & 648 & 648 \\
\hline $\boldsymbol{A}(w \cdot s)$ & - & - & - & - & . & - & . & - & - \\
\hline c. & - & - & - & - & - & - & - & - & - \\
\hline Co & - & - & - & - & - & - & - & - & - \\
\hline $\mathrm{Cu}$ & - & - & - & - & - & - & - & - & - \\
\hline Fo & - & - & - & - & - & - & - & - & - \\
\hline $\mathbf{K}$ & - & - & - & - & - & - & - & - & - \\
\hline $\mathbf{M g}$ & - & - & - & - & - & - & - & - & - \\
\hline Mn & - & - & - & . & . & - & - & - & - \\
\hline Na & - & - & - & - & - & - & - & - & - \\
\hline $\mathbf{N i}$ & - & - & . & - & . & - & - & - & - \\
\hline $\mathbf{P}$ & - & - & - & - & - & . & . & - & - \\
\hline Si & - & - & - & - & - & - & - & . & - \\
\hline $\mathbf{T i}$ & - & - & - & . & - & - & - & - & - \\
\hline H2O & - & - & - & - & - & - & - & - & - \\
\hline$A x(p p \infty)$ & - & . & - & . & - & - & - & - & . \\
\hline Ba & 40 & 20 & - & - & - & - & - & . & . \\
\hline Cd & - & - & - & • & - & • & - & • & - \\
\hline$\infty$ & - & - & so & 910 & 2800 & 630 & 470 & 530 & 780 \\
\hline$\alpha$ & - & . & . & - & - & - & - & - & • \\
\hline Mo & - & - & - & - & . & - & . & . & . \\
\hline $\mathbf{P b}$ & - & - & - & - & - & - & - & - & . \\
\hline $\mathbf{P t}$ & - & - & - & - & - & . & - & - & - \\
\hline $\mathbf{v}$ & - & - & - & . & - & - & . & . & . \\
\hline $\mathbf{Y}$ & - & - & 20 & 80 & 170 & 80 & 70 & 60 & 160 \\
\hline $\mathbf{Z n}$ & - & . & . & . & - & - & - & . & . \\
\hline
\end{tabular}


Table 11. Composition of Mn oxide majors as reported by NGDC (cont'd).

\begin{tabular}{|c|c|c|c|c|c|c|c|c|c|}
\hline $\begin{array}{l}\text { MMBIB } \\
\text { Serroplont }\end{array}$ & $\begin{array}{l}\text { EHRLAM6803 } \\
\text { 5-12A }\end{array}$ & $\begin{array}{c}\text { BHRLAM6803 } \\
4\end{array}$ & $\begin{array}{l}\text { FRIBGHBDO1 } \\
\text { FMC-1 }\end{array}$ & $\begin{array}{l}\text { FRIBGH8001 } \\
\text { FMC-1 }\end{array}$ & $\begin{array}{l}\text { FRIBGHBOO1 } \\
\text { FMC-1 }\end{array}$ & $\begin{array}{l}\text { FRIBGHBCO1 } \\
\text { PMC-1 }\end{array}$ & $\begin{array}{l}\text { FRIEGH8001 } \\
\text { FMC-1 }\end{array}$ & $\begin{array}{l}\text { FRIBGH8001 } \\
\text { FMC-1 }\end{array}$ & $\begin{array}{l}\text { FRIBGH } 8001 \\
\text { FMC-1 }\end{array}$ \\
\hline Analysia* & 1 & 1 & 1 & 10 & 11 & 12 & 13 & 14 & 15 \\
\hline Sampletype & 1 & 1 & 8 & 8 & 8 & 8 & 8 & 8 & 8 \\
\hline Lestitude & 31.934 & -25.517 & 9.300 & 9.300 & 9.300 & 9,300 & 9.300 & 9.300 & 9.300 \\
\hline Longitude & -77.250 & -85.233 & -146.050 & -146.050 & -146.050 & -146.050 & -146.050 & -146.050 & -146.050 \\
\hline Dopth & 787 & 920 & 4830 & 4830 & 4830 & 4830 & 4830 & 4830 & 4830 \\
\hline$A \mathcal{N}(w t . s)$ & - & $\cdot$ & 1.20 & .98 & 1.09 & 1.11 & 1.00 & 1.55 & 1.62 \\
\hline Ca & $\cdot$ & - & - & • & $\cdot$ & - & - & - & $\cdot$ \\
\hline Co & - & - & $A 20$ & .130 & .130 & 110 & .110 & .100 & .090 \\
\hline Cu & - & - & .180 & .210 & 200 & 200 & .230 & .270 & .290 \\
\hline Fo & - & - & 17.6 & 28.8 & 27.8 & 28.0 & 26.7 & 28.0 & 29.0 \\
\hline $\mathbf{K}$ & - & - & - & • & $\cdot$ & - & • & - & $\cdot$ \\
\hline Mg & - & $\cdot$ & - & - & • & $\cdot$ & $\cdot$ & - & $\cdot$ \\
\hline Mn & $\cdot$ & . & 26.2 & 18.3 & 19.0 & 19.0 & 20.2 & 17.8 & 17.2 \\
\hline $\mathrm{Na}$ & - & - & - & - & - & - & - & - & - \\
\hline $\mathbf{N i}$ & $\cdot$ & $\cdot$ & 430 & .210 & .230 & 240 & .300 & 220 & 240 \\
\hline $\mathbf{P}$ & $\cdot$ & - & $\cdot$ & $\cdot$ & - & $\cdot$ & $\cdot$ & $\cdot$ & - \\
\hline $\mathbf{S i}$ & - & - & 6.00 & 7.00 & 680 & 6.80 & 2.99 & 6.40 & 6.80 \\
\hline $\mathbf{T i}$ & - & - & - & - & $\cdot$ & • & • & - & - \\
\hline $\mathrm{H}_{2} \mathrm{O}$ & - & - & - & - & - & - & - & - & $\cdot$ \\
\hline$A \times(p p m)$ & - & - & - & - & • & • & - & • & • \\
\hline $\mathrm{Ba}$ & - & - & 1400 & 2300 & 2600 & 2600 & 1400 & 3000 & 3000 \\
\hline Cd & • & - & - & - & • & - & - & - & - \\
\hline$\infty$ & 970 & 60 & 1000 & 390 & 470 & 470 & 230 & 580 & 590 \\
\hline$a$ & • & $\cdot$ & • & $\cdot$ & $\cdot$ & • & - & $\cdot$ & • \\
\hline Mo & - & - & - & - & - & - & - & - & - \\
\hline $\mathrm{Pb}$ & - & . & - & - & - & - & . & - & . \\
\hline Pt & - & - & - & - & - & - & - & - & - \\
\hline $\mathbf{V}$ & - & . & - & - & - & - & - & - & - \\
\hline $\mathbf{Y}$ & 180 & 10 & - & - & - & - & - & - & $\cdot$ \\
\hline $\mathbf{Z n}$ & - & . & 560 & 950 & 930 & 900 & 900 & 890 & 920 \\
\hline
\end{tabular}

\begin{tabular}{|c|c|c|c|c|c|c|c|c|c|}
\hline MMBIB & FRIRGH8001 & FRIBGH8001 & FRIBGHEO01 & FRIEGH8001 & FRIROH8CO1 & FRIBGHBOO1 & FRIEGHBOO1 & FRIEGH8001 & FRIBGH8CO1 \\
\hline Sermplo: & FMC-1 & FMC-1 & FMC-1 & FMC-1 & FMC-1 & FMC-1 & PMC-1 & PMC-1 & FMC-1 \\
\hline Sampletype & 8 & 8 & 8 & 8 & 8 & 8 & 8 & 8 & 8 \\
\hline Longitude & -146.050 & -146.050 & -146.050 & -146.050 & -146.050 & -146.050 & -146.050 & -146.050 & -146.050 \\
\hline Dopth & 4830 & 4830 & 4830 & 4830 & 4830 & 4830 & 4830 & 4830 & 4830 \\
\hline$A(w t \%)$ & 1.88 & 2.58 & 3.47 & 4.25 & .85 & .86 & .86 & .43 & .90 \\
\hline ca & .290 & .270 & .270 & .240 & .230 & 220 & .200 & .180 & .180 \\
\hline Fo & 26.7 & 28.6 & 28.3 & 27.1 & 16.0 & 17.1 & 20.5 & 26.0 & 26.5 \\
\hline $\mathbf{K}$ & - & - & - & - & - & - & $\cdot$ & - & $\cdot$ \\
\hline $\mathbf{M}_{\mathbf{B}}$ & - & - & - & - & $\cdot$ & - & - & - & - \\
\hline $\mathrm{Mn}$ & 18.4 & 15.7 & 14.5 & 14.2 & 28.0 & 27.5 & 24.8 & 20.5 & 20.3 \\
\hline $\mathrm{Ne}$ & - & - & - & - & - & - & - & - & - \\
\hline As(ppon) & - & - & - & - & - & - & - & - & - \\
\hline $\mathrm{Ba}$ & 3100 & 3000 & 3200 & 3300 & 1100 & 800 & 1400 & 2100 & 2500 \\
\hline Cd & - & - & - & - & $\cdot$ & $\cdot$ & 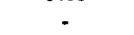 & 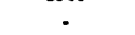 & - \\
\hline C. & 630 & 730 & 770 & 680 & 640 & 450 & 500 & 450 & 600 \\
\hline a & $\cdot$ & $\cdot$ & • & $\cdot$ & $\cdot$ & - & - & - & $\cdot$ \\
\hline Mo & $\cdot$ & - & - & $\cdot$ & - & - & - & - & - \\
\hline $\mathrm{Pb}$ & - & - & - & $\cdot$ & - & - & $\cdot$ & - & $\cdot$ \\
\hline $\mathbf{P t}$ & - & - & - & - & - & - & $\cdot$ & - & • \\
\hline $\mathbf{v}$ & - & - & - & - & - & - & . & - & - \\
\hline $\mathbf{Y}$ & $\cdot$ & - & $\cdot$ & $\cdot$ & - & - & - & $\cdot$ & - \\
\hline $\mathbf{Z n}$ & 860 & 820 & 800 & 830 & 580 & 570 & 580 & 780 & 760 \\
\hline
\end{tabular}


Table 11. Composition of Mn oxide majors as reported by NGDC (cont'd).

\begin{tabular}{|c|c|c|c|c|c|c|c|c|c|}
\hline MMBIB & FRIEGH8001 & FRIEGH8001 & FRIEGH8001 & GLASGP7202 & GLASGP7202 & GLASGP7202 & GLASGP7202 & GLASGP7402 & GLASGP7402 \\
\hline Sampleal & FMC-1 & FMC-1 & FMC-1 & 2139A & 2139B & $2139 \mathrm{C}$ & 2139P & 6249 & 6252 \\
\hline Analynis: & 7 & 8 & 9 & 1 & 1 & 1 & 1 & 1 & 1 \\
\hline Serpoletype & 8 & 8 & 8 & 8 & 8 & 8 & 8 & 8 & 8 \\
\hline Lationde & 9.300 & 9.300 & 9.300 & .55 .151 & -55.151 & .55 .151 & -55.151 & 2718 & 2.752 \\
\hline Langinide & -146.050 & -146.050 & -146.050 & 150.050 & 150.050 & 150.050 & 150.050 & 59.902 & 59.852 \\
\hline Depth & 4830 & 4830 & 4830 & 3865 & 3865 & 3865 & 3865 & 3998 & 3833 \\
\hline$A l(w t . \%)$ & .92 & 92 & 98 & - & - & - & $\cdot$ & - & $\cdot$ \\
\hline Ca & - & $\cdot$ & - & - & $\cdot$ & - & $\cdot$ & $\cdot$ & $\cdot$ \\
\hline$C_{0}$ & .150 & .150 & .130 & 449 & .289 & 317 & .129 & 333 & 343 \\
\hline a & .200 & .200 & .210 & .058 & .038 & .042 & .060 & .057 & .062 \\
\hline $\mathbf{F}_{0}$ & 26.6 & 26.7 & 28.2 & 124 & 10.9 & 135 & 11.5 & 14.3 & 13.6 \\
\hline $\mathbf{K}$ & • & $\cdot$ & $\cdot$ & • & $\cdot$ & - & • & $\cdot$ & • \\
\hline $\mathbf{M} \mathbf{8}$ & - & • & - & $\bullet$ & • & - & • & - & • \\
\hline $\mathbf{M m}$ & 20.1 & 20.0 & 18.9 & 132 & 18.6 & 12.6 & 11.9 & 14.8 & 162 \\
\hline $\mathrm{Na}$ & - & - & - & - & - & • & - & - & - \\
\hline $\mathbf{N i}$ & .240 & .240 & .200 & .258 & .194 & .840 & .356 & .281 & 315 \\
\hline $\mathbf{P}$ & • & • & - & • & - & . & - & - & - \\
\hline Si & 6.40 & 6.40 & 7.00 & - & - & - & - & 8.88 & 7.06 \\
\hline $\mathrm{Ti}$ & 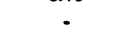 & . & 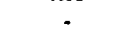 & - & . & - & - & .74 & .64 \\
\hline $\mathrm{H} 20$ & - & $\cdot$ & - & - & - & - & - & • & - \\
\hline$A x(p p \infty)$ & • & • & - & - & . & - & - & - & - \\
\hline Ba & 2000 & 2500 & 2700 & - & . & - & - & - & - \\
\hline Cd & - & - & - & - & . & - & - & - & . \\
\hline$\infty$ & 480 & 570 & 460 & . & . & - & - & - & . \\
\hline$\alpha$ & • & • & • & - & . & - & - & 20 & 20 \\
\hline Mo & . & . & - & - & - & - & - & 350 & 410 \\
\hline $\mathbf{P b}$ & - & - & - & - & - & - & - & 730 & 610 \\
\hline $\mathbf{P}$ & - & - & - & - & - & - & - & • & • \\
\hline $\mathbf{V}$ & - & - & - & - & - & - & - & 680 & 720 \\
\hline $\mathbf{Y}$ & - & • & - & - & • & - & - & - & • \\
\hline $2 n$ & 860 & 860 & 920 & 200 & 190 & 190 & 90 & 260 & 200 \\
\hline
\end{tabular}

\begin{tabular}{|c|c|c|c|c|c|c|c|c|c|}
\hline $\begin{array}{l}\text { MMBIB } \\
\text { Semploto }\end{array}$ & $\begin{array}{c}\text { GLASGP7402 } \\
6253\end{array}$ & $\begin{array}{c}\text { OLASGP7402 } \\
6256\end{array}$ & $\begin{array}{c}\text { GLASGP7402 } \\
6257\end{array}$ & $\begin{array}{c}\text { GLASGP7402 } \\
6273\end{array}$ & $\begin{array}{l}\text { GLASGP7504 } \\
\text { 37DT-20 }\end{array}$ & $\begin{array}{l}\text { GLASGP7504 } \\
\text { 38DT-22 }\end{array}$ & $\begin{array}{l}\text { OLASGP7504 } \\
\text { 52DT-31 }\end{array}$ & $\begin{array}{l}\text { GLASGPB202 } \\
\text { 137DK }\end{array}$ & $\begin{array}{c}\text { OLASGP8202 } \\
\text { 137DK }\end{array}$ \\
\hline Andyais: & 1 & 1 & 1 & 1 & 1 & 1 & 1 & 1 & 2 \\
\hline Sampletype & 8 & 8 & 8 & 8 & 15 & 7 & 8 & 8 & 8 \\
\hline Letiondo & 2.817 & 2.818 & 2.768 & 2.902 & -21.684 & -22.535 & -32.668 & -10.000 & -10.000 \\
\hline Longitude & 59.834 & 59.851 & 59.768 & 60.036 & -159.950 & -160.117 & -160.002 & -134.000 & -134.000 \\
\hline Depth & 3579 & 3472 & 3516 & 4189 & 3970 & 4817 & 5300 & 5000 & 5000 \\
\hline$A l(w t . \%)$ & $\cdot$ & $\cdot$ & - & $\cdot$ & 7.18 & 7.43 & $\cdot$ & - & - \\
\hline C. & - & $\cdot$ & $\cdot$ & - & 6.68 & 1.77 & 3.12 & - & - \\
\hline Co & 371 & 372 & .372 & .167 & 581 & .270 & .794 & .460 & .420 \\
\hline $\mathrm{Ca}$ & .078 & .072 & .046 & 233 & .116 & 364 & - & 500 & .150 \\
\hline$F_{0}$ & 16.0 & 15.0 & 17.2 & 13.8 & 39.1 & 19.0 & 44.0 & 23.2 & 24.7 \\
\hline $\mathbf{K}$ & - & - & - & - & - & - & - & - & - \\
\hline $\mathbf{M}_{\mathbf{g}}$ & - & - & - & - & - & - & - & - & - \\
\hline $\mathbf{M n}$ & 15.8 & 14.4 & 16.4 & 18.1 & 17.2 & 17.4 & 27.6 & 24.5 & 19.5 \\
\hline $\mathrm{Na}$ & $\cdot$ & • & - & - & - & - & - & $\cdot$ & - \\
\hline $\mathrm{Ni}$ & .384 & 372 & .261 & .711 & .252 & .864 & 378 & .120 & .290 \\
\hline $\mathbf{P}$ & • & • & • & - & - & - & • & - & - \\
\hline $\mathrm{Si}$ & 9.19 & 9.32 & 8.04 & 9.94 & 22.67 & 17.17 & 13.96 & - & - \\
\hline $\mathbf{T i}$ & .70 & .72 & .63 & .45 & . & - & . & - & - \\
\hline $\mathrm{H} 20$ & - & - & - & - & 48.4 & 25.9 & 47.1 & - & - \\
\hline$A \simeq$ (ppon) & - & - & - & - & - & - & - & - & - \\
\hline Ba & - & - & . & - & - & . & . & - & - \\
\hline $\mathrm{cd}$ & - & . & - & - & . & . & . & . & - \\
\hline$c_{0}$ & . & . & - & - & . & - & . & . & . \\
\hline$a$ & 20 & 30 & 20 & 1800 & - & - & . & - & . \\
\hline Mo & 510 & 480 & 610 & 420 & 190 & 400 & . & - & - \\
\hline $\mathrm{Pb}$ & 920 & 1000 & 760 & 670 & . & - & - & - & - \\
\hline $\mathbf{P}_{\mathbf{t}}$ & . & - & . & . & . & - & - & - & - \\
\hline v & 910 & 910 & 830 & 730 & . & . & - & . & . \\
\hline $\mathbf{Y}$ & - & - & - & - & - & - & - & - & - \\
\hline $\mathbf{Z n}$ & 380 & 360 & 270 & 400 & 970 & 1100 & 1500 & 800 & 600 \\
\hline
\end{tabular}


Table 11. Composition of Mn oxide majors as reported by NGDC (cont'd).

\begin{tabular}{|c|c|c|c|c|c|c|c|c|c|}
\hline $\begin{array}{l}\text { MMBIB } \\
\text { Sumpleat }\end{array}$ & $\begin{array}{l}\text { GLASGP8202 } \\
\text { 137DK }\end{array}$ & $\begin{array}{l}\text { GLASGP8202 } \\
\text { 137DK }\end{array}$ & $\begin{array}{l}\text { GLASGP8202 } \\
151 \text {-GB }\end{array}$ & $\begin{array}{l}\text { GLASGP8202 } \\
\text { 211-GB }\end{array}$ & $\begin{array}{l}\text { GLASGP8202 } \\
\text { 218-GB }\end{array}$ & $\begin{array}{l}\text { GLASGP8202 } \\
\text { \&-GB }\end{array}$ & $\begin{array}{l}\text { GLASGP8302 } \\
\text { 153-GB }\end{array}$ & $\begin{array}{c}\text { GLASGP8302 } \\
\text { 153-GB }\end{array}$ & $\begin{array}{c}\text { GLASGP8302 } \\
154-G B\end{array}$ \\
\hline Amplyzist" & 3 & 4 & 2 & 1 & 1 & 1 & 1 & 2 & 6 \\
\hline Sampletypo & 8 & 8 & 1 & 8 & 8 & 8 & 1 & 1 & 1 \\
\hline Latitude & -10.000 & -10.000 & -17.717 & -22000 & -22.017 & -7.000 & -17.417 & -17.417 & -17.400 \\
\hline Longitude & -134.000 & -134.000 & -159.967 & -161.900 & .161 .967 & -132.000 & -159.600 & -159.600 & -159.617 \\
\hline Depth & 5000 & 5000 & 5186 & 4433 & 4473 & 3000 & 5027 & 5027 & 5112 \\
\hline$A(w t . \%)$ & - & - & - & - & - & - & 4.92 & 450 & 3.76 \\
\hline a & . & - & . & - & - & - & • & • & • \\
\hline$c_{0}$ & .180 & .210 & 510 & .120 & 270 & .130 & 510 & 530 & .500 \\
\hline a & 510 & 310 & .170 & .080 & .070 & .730 & .110 & .090 & .130 \\
\hline$F_{0}$ & 22.3 & 25.5 & 26.1 & 25.6 & 31.6 & 22.6 & 114 & 14.9 & 19.2 \\
\hline $\mathbf{K}$ & • & - & $\cdot$ & • & • & - & • & • & - \\
\hline $\mathbf{M}_{\mathbf{8}}$ & - & - & - & - & - & - & - & - & - \\
\hline Mn & 23.9 & 20.0 & 18.9 & 6.11 & 7.94 & 15.9 & 16.8 & 16.0 & 16.2 \\
\hline $\mathrm{Na}$ & - & - & - & - & - & - & • & - & - \\
\hline $\mathrm{Ni}$ & .730 & .450 & 300 & .150 & .120 & .750 & .260 & .230 & 300 \\
\hline $\mathbf{P}$ & • & - & - & - & • & - & $\cdot$ & - & - \\
\hline $\mathbf{s i}$ & - & . & - & . & - & - & 9.77 & 9.02 & 7.76 \\
\hline $\mathbf{T i}$ & - & - & - & - & . & - & - & - & • \\
\hline H2O. & - & - & - & - & - & - & - & - & - \\
\hline Aspppon) & - & - & - & - & - & - & • & - & - \\
\hline Be & - & - & - & - & - & - & 1300 & 1400 & 1800 \\
\hline Cd & - & - & - & - & - & - & • & • & - \\
\hline Co & - & - & - & - & - & - & - & - & - \\
\hline G & • & - & . & - & . & - & - & - & - \\
\hline Mo & - & - & - & - & . & • & - & - & • \\
\hline $\mathrm{Pb}$ & - & - & - & . & . & - & - & - & - \\
\hline $\mathrm{Pt}$ & . & . & - & - & - & - & - & • & . \\
\hline$v$ & • & - & - & . & - & . & - & - & - \\
\hline $\mathbf{Y}$ & . & . & . & • & . & • & • & • & . \\
\hline Zn & 1100 & 1100 & 700 & 800 & 700 & 1200 & 600 & 600 & 700 \\
\hline
\end{tabular}

\begin{tabular}{|c|c|c|c|c|c|c|c|c|c|}
\hline MMBIB & GLASGP8302 & GLASGP8302 & GLASGP8302 & GLASGP8302 & GLASGP8302 & GLASGP8302 & GLASGP8302 & GLASGP8302 & GLASGP8302 \\
\hline Sumplett & $157 \mathrm{~GB}$ & $165 \mathrm{~GB}$ & $165 G B$ & $165 G B$ & 165GB & 165GB & $165 \mathrm{~GB}$ & 165GB & $165 \mathrm{~GB}$ \\
\hline Anelynist" & 7 & 1 & 2 & 3 & 4 & 5 & 6 & 7 & 8 \\
\hline Sumpletypo & 1 & 1 & 1 & 1 & 1 & 1 & 1 & 1 & 1 \\
\hline Letioude & -17.383 & -19.017 & -19.017 & -19.017 & -19.017 & -19.017 & -19.017 & -19.017 & -19.017 \\
\hline $\begin{array}{l}\text { Loagitude } \\
\text { Depth }\end{array}$ & $\begin{array}{c}-159.617 \\
5148\end{array}$ & $\begin{array}{c}-161.667 \\
4990\end{array}$ & $\begin{array}{c}-161.667 \\
4990\end{array}$ & $\begin{array}{c}-161.667 \\
4990\end{array}$ & $\begin{array}{c}-161.667 \\
4990\end{array}$ & $\begin{array}{c}-161.667 \\
4990\end{array}$ & $\begin{array}{c}-161.667 \\
4990\end{array}$ & $\begin{array}{c}-161.667 \\
4990\end{array}$ & $\begin{array}{c}-161.667 \\
4990\end{array}$ \\
\hline$A(w t . \%)$ & 3.39 & 4.45 & 4.07 & 4.07 & 3.86 & 3.86 & 6.56 & 4.23 & 4.29 \\
\hline c & - & - & - & • & - & - & - & - & - \\
\hline$c_{0}$ & .590 & 550 & 550 & 520 & 550 & 550 & 550 & 540 & 500 \\
\hline$\infty$ & .170 & .150 & .120 & .100 & .100 & .100 & .090 & .110 & .100 \\
\hline$F_{0}$ & 20.1 & 17.8 & 19.1 & 19.5 & 19.7 & 19.3 & 19.1 & 18.1 & 18.1 \\
\hline $\mathbf{K}$ & • & • & • & • & • & • & • & • & - \\
\hline $\mathbf{M}_{8}$ & - & - & - & - & - & • & - & • & • \\
\hline Mn & 18.1 & 17.9 & 165 & 15.0 & 16.0 & 15.2 & 15.9 & 14.4 & 14.1 \\
\hline $\mathbf{N} \mathbf{a}$ & • & - & - & • & • & - & - & - & - \\
\hline $\mathbf{N i}$ & A10 & 340 & .270 & 230 & 230 & 230 & 230 & .220 & .210 \\
\hline $\mathbf{P}$ & - & - & • & - & - & - & - & • & • \\
\hline $\mathbf{S i}$ & 6.73 & 7.76 & 7.99 & 8.42 & 7.90 & 790 & 13.46 & 9.07 & 9.32 \\
\hline Ti & • & • & - & • & • & - & - & - & - \\
\hline H2O. & - & - & - & - & - & - & - & - & - \\
\hline Axppm) & - & - & - & - & $\cdot$ & - & - & - & - \\
\hline Be & 1500 & 1300 & 1400 & 1500 & 1500 & 1500 & 1600 & 1500 & 1400 \\
\hline Cd & - & - & - & - & • & - & • & - & - \\
\hline$\infty$ & - & - & - & . & - & - & • & • & • \\
\hline$\alpha$ & $\cdot$ & . & . & . & . & . & . & . & . \\
\hline Mo & - & - & - & - & - & - & - & • & . \\
\hline $\mathrm{Pb}$. & - & - & - & - & - & - & • & - & - \\
\hline $\mathrm{Pt}^{\circ}$ & . & . & . & - & . & . & . & - & - \\
\hline v & . & - & . & - & . & . & - & - & - \\
\hline$Y$ & - & - & - & . & - & . & . & $\cdot$ & . \\
\hline $\mathbf{z n}$ & 700 & 600 & 600 & 600 & 600 & 600 & 600 & 600 & 600 \\
\hline
\end{tabular}


Table 11. Composition of $\mathrm{Mn}$ oxide majors as reported by NGDC (cont'd).

\begin{tabular}{|c|c|c|c|c|c|c|c|c|c|}
\hline MMBIB & GLASGP8302 & GLASGP8302 & GLASGP8302 & GLASGP8302 & GLASGP8302 & GLASGP8302 & GLASGP8302 & GLASGP8302 & GLASGP8302 \\
\hline Samplott & $165 G B$ & 167GB & 167GB & 167GB & 167GB & 169B & $167 \mathrm{~GB}$ & $167 \mathrm{~GB}$ & 167GB \\
\hline Analynist" & 9 & 1 & 2 & 3 & 4 & 5 & 6 & 7 & 8 \\
\hline Sumpletypo & 1 & 1 & 1 & 1 & 1 & 1 & 1 & 1 & 1 \\
\hline Letitude & -19.017 & -19.000 & -19.000 & -19.000 & -19.000 & -19.000 & -19.000 & -19.000 & -19.000 \\
\hline $\begin{array}{l}\text { Longitude } \\
\text { Deppth }\end{array}$ & $\begin{array}{c}-161.667 \\
4990\end{array}$ & $\begin{array}{c}-161.650 \\
4925\end{array}$ & $\begin{array}{c}-161.650 \\
4925\end{array}$ & $\begin{array}{c}-161.650 \\
4925\end{array}$ & $\begin{array}{c}-161.650 \\
4925\end{array}$ & $\begin{array}{c}-161.650 \\
4925\end{array}$ & $\begin{array}{c}-161.650 \\
4925\end{array}$ & $\begin{array}{c}-161.650 \\
4925\end{array}$ & $\begin{array}{c}-161.650 \\
4925\end{array}$ \\
\hline$A \mid(w L \%)$ & 4.45 & 4.60 & 4.07 & 3.70 & 3.55 & 3.65 & 3.65 & 4.71 & 4.13 \\
\hline a & • & - & • & - & - & • & - & - & - \\
\hline$c_{0}$ & 540 & 530 & 570 & .570 & .590 & 580 & 570 & 510 & 510 \\
\hline $\mathrm{Cu}$ & .110 & .150 & .140 & .130 & .110 & .110 & .110 & .110 & .120 \\
\hline Fo & 18.8 & 18.8 & 19.0 & 20.0 & 20.4 & 19.7 & 20.2 & 17.4 & 18.9 \\
\hline $\mathbf{K}$ & - & • & - & - & - & - & - & - & - \\
\hline$M_{8}$ & • & - & • & - & - & - & - & • & • \\
\hline Mn & 15.6 & 16.3 & 16.9 & 17.2 & 16.9 & 16.2 & 16.4 & 143 & 15.1 \\
\hline $\mathrm{Na}$ & - & - & - & • & • & • & • & - & - \\
\hline $\mathrm{Ni}$ & .250 & 300 & 300 & .290 & .270 & .250 & .250 & .230 & .240 \\
\hline $\mathbf{P}$ & - & - & - & $\cdot$ & - & - & - & - & - \\
\hline $\mathbf{S i}$ & 8.93 & 8.51 & 734 & 7.48 & 7.25 & 7.34 & 7,48 & 9.68 & 8.51 \\
\hline $\mathbf{T i}$ & $\cdot$ & - & - & - & - & - & - & - & - \\
\hline H2O- & - & - & - & - & - & - & - & - & - \\
\hline$A x(p p m)$ & - & - & - & - & - & - & - & - & - \\
\hline Ba & 1500 & 1400 & 1500 & 1500 & 1600 & 1600 & 1600 & 1600 & 1500 \\
\hline Cd & - & - & - & - & - & - & - & - & - \\
\hline$c_{0}$ & - & - & - & - & - & - & - & - & - \\
\hline$a$ & - & - & - & . & - & . & . & - & - \\
\hline Mo & • & - & . & - & - & . & - & . & - \\
\hline $\mathrm{Pb}$ & - & - & - & - & - & - & . & - & - \\
\hline $\mathrm{Pt}$ & • & - & - & • & . & - & - & - & . \\
\hline$v$ & - & - & . & - & . & . & . & - & - \\
\hline $\mathbf{Y}$ & $\cdot$ & - & . & - & - & . & . & . & - \\
\hline $\mathbf{Z n}$ & 600 & 600 & 600 & 600 & 600 & 600 & 600 & 500 & 600 \\
\hline
\end{tabular}

\begin{tabular}{|c|c|c|c|c|c|c|c|c|c|}
\hline MMBIB & GLASGP8302 & GLASGP8302 & GLASGP8302 & GLASGP8302 & GLASGP8302 & GLASGP8302 & GLASGP8302 & GLASGP8302 & GLASGP8302 \\
\hline Samplet & $167 \mathrm{~GB}$ & $168 \mathrm{~GB}$ & $168 C B$ & $168 \mathrm{~GB}$ & $168 \mathrm{~GB}$ & $168 G B$ & $168 \mathrm{~GB}$ & $170 \mathrm{~GB}$ & $170 \mathrm{~GB}$ \\
\hline Analynist" & 9 & 10 & 2 & 3 & 4 & 5 & 9 & 1 & 2 \\
\hline Sampletype & 1 & 1 & 1 & 1 & 1 & 1 & 1 & 1 & 1 \\
\hline Letitude & -19.000 & -18.983 & -18.983 & -18.983 & -18.983 & -18.983 & -18.983 & -18.983 & -18.983 \\
\hline $\begin{array}{l}\text { Longitude } \\
\text { Depth }\end{array}$ & $\begin{array}{c}-161.650 \\
4925\end{array}$ & $\begin{array}{c}-161.650 \\
4936\end{array}$ & $\begin{array}{c}-161.650 \\
4936\end{array}$ & $\begin{array}{c}-161.650 \\
4936\end{array}$ & $\begin{array}{c}-161.650 \\
4936\end{array}$ & $\begin{array}{c}-161.650 \\
4936\end{array}$ & $\begin{array}{c}-161.650 \\
4936\end{array}$ & $\begin{array}{c}-161.633 \\
4897\end{array}$ & $\begin{array}{c}-161.633 \\
4897\end{array}$ \\
\hline$\overline{A l(w t . \%)}$ & 4.02 & 3.23 & 4.39 & 4.02 & 4.02 & 3.86 & 4.02 & 4.29 & 3.92 \\
\hline ce & - & - & - & - & - & - & - & - & - \\
\hline$C_{0}$ & 550 & .600 & 520 & 500 & 510 & 520 & 530 & 530 & sso \\
\hline $\mathrm{cu}$ & .150 & .130 & .190 & .160 & .130 & .120 & .140 & .130 & .110 \\
\hline Fo & 19.4 & 21.7 & 19.6 & 20.2 & 20.2 & 19.2 & 19.3 & 17.9 & 18.8 \\
\hline $\mathbf{K}$ & - & - & . & . & - & - & - & - & - \\
\hline $\mathrm{Mg}_{8}$ & - & - & . & . & - & . & - & - & . \\
\hline Mn & 16.2 & 15.2 & 16.4 & 15.6 & 14.7 & 145 & 16.1 & 17.1 & 16.6 \\
\hline $\mathrm{Ne}$ & - & • & . & • & - & - & - & . & - \\
\hline $\mathrm{Ni}$ & 270 & 300 & 370 & 320 & 300 & .290 & 300 & 300 & .260 \\
\hline $\mathbf{P}$ & - & - & • & • & • & - & • & • & - \\
\hline $\mathbf{S i}$ & 7.95 & 7.20 & 7.85 & 7.48 & 7.99 & 7.90 & 7.76 & 8.04 & 7.76 \\
\hline $\mathrm{Ti}$ & • & - & • & • & - & • & • & - & • \\
\hline $\mathrm{H} 20$ - & - & - & . & - & - & . & - & - & - \\
\hline Ax(ppon) & - & - & - & - & - & . & - & . & · \\
\hline $\mathrm{Ba}$ & 1500 & 1600 & 1400 & 1500 & 1500 & 1700 & 1900 & 1400 & 1500 \\
\hline $\mathrm{Cd}$ & $\cdot$ & - & - & - & - & . & . & . & 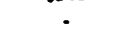 \\
\hline$c_{0}$ & - & . & . & . & . & - & , & . & . \\
\hline$\alpha$ & . & . & . & . & . & - & . & . & - \\
\hline Mo & . & - & . & . & . & - & . & . & . \\
\hline $\mathrm{Pb}$ & . & . & . & . & . & . & . & . & . \\
\hline $\mathrm{Pt}_{\mathrm{t}}$ & . & . & . & . & . & . & . & . & - \\
\hline $\mathbf{v}$ & - & . & - & - & - & - & . & . & - \\
\hline $\mathbf{Y}$ & . & - & . & . & . & - & . & - & . \\
\hline $2 n$ & 600 & 700 & 700 & 700 & 600 & 600 & 600 & 600 & 600 \\
\hline
\end{tabular}


Table 11. Composition of Mn oxide majors as reported by NGDC (cont'd).

\begin{tabular}{|c|c|c|c|c|c|c|c|c|c|}
\hline MMBIB & GLASGP8302 & GLASGP8302 & GLASGP8302 & GLASGP8302 & GLASGP8302 & GLASGP8302 & GLASGP8302 & GLASGP8302 & GLASGP8302 \\
\hline Semple\#" & $170 G \mathrm{~B}$ & $170 \mathrm{~GB}$ & $170 \mathrm{~GB}$ & $170 \mathrm{~GB}$ & $170 \mathrm{~GB}$ & $171 G B$ & 171GB & $172 \mathrm{KG}$ & $172 \mathrm{KG}$ \\
\hline Analysist & 3 & 4 & 5 & 6 & 8 & 3 & 6 & 1 & 2 \\
\hline Sumpletype & 1 & 1 & 1 & 1 & 1 & 1 & 1 & 1 & 1 \\
\hline Letitudo" & -18.983 & -18.983 & -18.983 & -18.983 & -18.983 & -18.967 & -18.967 & -18.983 & -18.983 \\
\hline $\begin{array}{l}\text { Longitude } \\
\text { Depth }\end{array}$ & $\begin{array}{c}-161.633 \\
4897\end{array}$ & $\begin{array}{c}-161.633 \\
4897\end{array}$ & $\begin{array}{c}-161.633 \\
4897\end{array}$ & $\begin{array}{c}-161.633 \\
4897\end{array}$ & $\begin{array}{c}-161.633 \\
4897\end{array}$ & $\begin{array}{c}-161.633 \\
4955\end{array}$ & $\begin{array}{c}-161.633 \\
4955\end{array}$ & $\begin{array}{c}-161.683 \\
4944\end{array}$ & $\begin{array}{c}-161.683 \\
4944\end{array}$ \\
\hline$\overline{A l(w t . \%)}$ & 4.02 & 4.02 & 3.86 & 4.18 & 4.07 & 4.18 & 4.18 & 4.07 & 4.02 \\
\hline Ca & - & • & - & - & • & • & • & • & • \\
\hline$C_{0}$ & .550 & .550 & .580 & 520 & 540 & .500 & 510 & .560 & 550 \\
\hline ca & .100 & .110 & .110 & .130 & .110 & .140 & .110 & .130 & .110 \\
\hline Po & 19.0 & 19.4 & 19.1 & 18.4 & 18.7 & 18.7 & 18.7 & 19.7 & 19.6 \\
\hline $\mathbf{K}$ & • & - & - & - & - & - & - & - & - \\
\hline $\mathbf{M}_{\mathbf{8}}$ & - & - & - & - & • & • & • & - & - \\
\hline Mn & 15.8 & 15.1 & 16.7 & 15.4 & 15.9 & 15.4 & 14.5 & 172 & 16.1 \\
\hline Na & • & - & • & - & - & - & - & • & • \\
\hline $\mathbf{N i}$ & .240 & .230 & .250 & 250 & .250 & .330 & .230 & 290 & .270 \\
\hline $\mathbf{P}$ & - & - & - & - & • & - & • & - & • \\
\hline Si & 8.04 & 8.32 & 7.62 & 8.70 & 8.18 & 8.04 & 8.79 & 7.62 & 8.04 \\
\hline $\mathrm{Ti}$ & - & - & • & • & - & • & • & • & • \\
\hline H2O & - & - & - & - & - & - & - & - & - \\
\hline Axppen) & - & - & - & - & - & - & - & - & - \\
\hline Be & 1500 & 1600 & 1500 & 1700 & 1500 & 1600 & 1500 & 1400 & 1600 \\
\hline cd & • & • & • & - & • & - & $\cdot$ & $\cdot$ & - \\
\hline$C_{0}$ & • & - & - & - & - & - & - & - & - \\
\hline$\sigma$ & - & - & - & - & - & - & - & - & - \\
\hline Mo & - & - & . & - & - & - & - & - & - \\
\hline $\mathbf{P b}$ & - & - & - & - & - & - & - & - & - \\
\hline $\mathrm{Pt}$ & - & - & - & - & - & - & - & - & - \\
\hline$v$ & - & . & - & - & - & - & - & - & - \\
\hline $\mathbf{Y}$ & . & . & - & - & - & . & . & . & . \\
\hline$z_{n}$ & 600 & 600 & 600 & 600 & 600 & 700 & 600 & 600 & 600 \\
\hline
\end{tabular}

\begin{tabular}{|c|c|c|c|c|c|c|c|c|c|}
\hline MMBIB & GLASGP8302 & GLASGP8302 & GLASGP8302 & GLASGP8302 & GLASGP8302 & GLASGP8302 & GLASGP8302 & GLASGP8302 & GLASGP8302 \\
\hline Sermplot & $172 \mathrm{KG}$ & $172 \mathrm{KG}$ & $174 \mathrm{KG}$ & $174 \mathrm{KG}$ & $174 \mathrm{KG}$ & $174 \mathrm{KG}$ & $174 \mathrm{KG}$ & $174 \mathrm{KO}$ & $179 \mathrm{KG}$ \\
\hline Anelysist & 4 & 6 & 1 & 2 & 3 & 4 & 6 & 8 & 1 \\
\hline Sempletype & 1 & 6 & 1 & 1 & 1 & 1 & 1 & 1 & 1 \\
\hline Latitudo & -18.983 & -18.983 & -19.033 & -19.033 & -19.033 & -19.033 & -19.033 & -19.033 & -19.350 \\
\hline $\begin{array}{l}\text { Longitude } \\
\text { Depth }\end{array}$ & $\begin{array}{c}-161.683 \\
4944\end{array}$ & $\begin{array}{c}-161.683 \\
4944\end{array}$ & $\begin{array}{c}-161.667 \\
5088\end{array}$ & $\begin{array}{c}-161.667 \\
5088\end{array}$ & $\begin{array}{c}-161.667 \\
5088\end{array}$ & $\begin{array}{c}-161.667 \\
5088\end{array}$ & $\begin{array}{c}-161.667 \\
5088\end{array}$ & $\begin{array}{c}-161.667 \\
5088\end{array}$ & $\begin{array}{c}-162.267 \\
4616\end{array}$ \\
\hline$\overline{A l(w L . \%)}$ & 4.45 & 3.86 & 4.18 & 4.66 & 7.90 & 4.66 & 4.55 & 3.23 & 4.23 \\
\hline a & - & · & - & - & - & - & - & 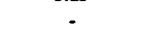 & - \\
\hline$c_{0}$ & 520 & 580 & 540 & .520 & .470 & .540 & .510 & .630 & .610 \\
\hline $\mathrm{Cu}$ & .130 & .100 & .130 & .150 & .170 & .110 & .140 & .120 & .090 \\
\hline Fo & 18.8 & 19.0 & 19.6 & 18.8 & 193 & 18.3 & 18.5 & 20.2 & 18.1 \\
\hline $\mathbf{K}$ & - & - & - & - & - & - & - & - & - \\
\hline $\mathbf{M g}_{\mathbf{g}}$ & - & - & - & . & - & - & - & - & . \\
\hline Mn & 15.4 & 17.6 & 15.8 & 16.9 & 16.3 & 15.0 & 15.5 & 17.7 & 17.6 \\
\hline $\mathbf{N a}$ & - & - & - & - & - & - & - & • & - \\
\hline $\mathbf{N i}$ & 290 & .270 & 320 & 320 & 390 & .290 & 320 & 310 & .250 \\
\hline $\mathbf{P}$ & • & - & - & - & - & - & - & • & - \\
\hline $\mathbf{S i}$ & 8.98 & 8.37 & 8.42 & 8.18 & 8.65 & 9.63 & 9.07 & 6.92 & 9.07 \\
\hline $\mathrm{Ti}$ & . & - & - & - & • & - & - & • & • \\
\hline $\mathrm{H} 2 \mathrm{O}$ & - & - & - & - & - & - & - & - & - \\
\hline Axppona) & - & - & - & - & - & - & - & - & - \\
\hline Be & 1400 & 1400 & 1500 & 1400 & 1400 & 1300 & 1400 & 1500 & 1100 \\
\hline ca & - & • & - & . & - & - & . & . & . \\
\hline$c_{0}$ & - & . & - & - & - & - & - & - & . \\
\hline$\alpha$ & - & - & . & . & - & - & - & - & - \\
\hline Mo & - & - & - & - & - & - & - & - & - \\
\hline $\mathbf{P b}$ & - & - & - & - & - & - & - & - & • \\
\hline Pt & - & - & - & - & - & - & - & - & - \\
\hline$v$ & - & - & - & - & - & - & - & - & - \\
\hline $\mathbf{Y}$ & - & - & - & . & - & $\cdot$ & - & $\cdot$ & - \\
\hline $\mathrm{Zn}_{n}$ & 600 & 600 & 600 & 600 & 700 & 600 & 600 & 600 & 600 \\
\hline
\end{tabular}


Table 11. Composition of Mn oxide majors as reported by NGDC (cont'd).

\begin{tabular}{|c|c|c|c|c|c|c|c|c|c|}
\hline $\begin{array}{l}\text { MMBIB } \\
\text { Sumplett }\end{array}$ & $\begin{array}{l}\text { GLASGP8302 } \\
\text { 179KO }\end{array}$ & $\begin{array}{l}\text { GLASGP8302 } \\
\text { 179KG }\end{array}$ & $\begin{array}{l}\text { GLASGP8302 } \\
\text { 179KG }\end{array}$ & $\begin{array}{l}\text { GLASGP8302 } \\
\text { 179KG }\end{array}$ & $\begin{array}{l}\text { GLASGP8302 } \\
191 G B\end{array}$ & $\begin{array}{l}\text { GLASGPB302 } \\
191 G B\end{array}$ & $\begin{array}{l}\text { GLASGP8302 } \\
\text { 1920B }\end{array}$ & $\begin{array}{l}\text { GLASGP8302 } \\
1926 B\end{array}$ & $\begin{array}{c}\text { GLASGP8302 } \\
\text { 192GB }\end{array}$ \\
\hline Analyzist" & 2 & 3 & 4 & 6 & 3 & 5 & 2 & 3 & 4 \\
\hline Sampletypo & 1 & 1 & 1 & 1 & 1 & 1 & 1 & 1 & 1 \\
\hline Letitude" & -19.350 & .19 .350 & -19.350 & -19.350 & -19.933 & -19.933 & -19.933 & -19.933 & -19.933 \\
\hline Longitude & -162.267 & -162.267 & -162.267 & -162.267 & -162.000 & -162.000 & -162.000 & -162.000 & -162.000 \\
\hline Depth & 4616 & 4616 & 4616 & 4616 & 4958 & 4958 & 4900 & 4900 & 4900 \\
\hline$A(w L . \%)$ & 4.29 & 3.92 & 4.13 & 3.49 & 3.70 & 3.33 & 4.02 & 3.97 & 3.92 \\
\hline ca & - & - & . & - & - & - & - & - & - \\
\hline$C_{0}$ & 550 & 530 & 560 & 520 & 550 & 560 & 510 & 510 & 510 \\
\hline$a$ & .080 & .080 & .080 & .130 & .180 & .200 & 230 & .180 & .150 \\
\hline$F_{0}$ & 18.2 & 18.9 & 18.4 & 19.8 & 18.2 & 18.6 & 17.8 & 18.9 & 18.7 \\
\hline $\mathbf{K}$ & - & - & - & - & - & • & - & - & - \\
\hline Mg & . & - & . & . & . & . & . & - & - \\
\hline Mn & 16.3 & 16.8 & 16.9 & 16.4 & 17.2 & 18.6 & 18.6 & 17.0 & 15.9 \\
\hline Na & - & • & - & - & - & - & - & - & - \\
\hline $\mathrm{Ni}$ & .250 & .240 & .250 & .230 & .360 & $A 10$ & .480 & 380 & .330 \\
\hline $\mathbf{P}$ & . & - & - & 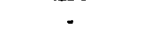 & - & - & - & - & - \\
\hline $\mathbf{S i}$ & 9.35 & 8.65 & 9.02 & 7.62 & 7.71 & 6.83 & 6.78 & 7.43 & 7.76 \\
\hline $\mathrm{Ti}$ & - & - & - & - & - & - & - & - & - \\
\hline $\mathrm{H} 2 \mathrm{O}$ & - & - & - & - & - & - & - & - & - \\
\hline$A \times p p \infty n)$ & • & - & - & - & - & - & - & . & - \\
\hline Ba & 1200 & 1500 & 1300 & 1700 & 1700 & 1800 & 1500 & 2500 & 1600 \\
\hline Cd & . & - & - & - & - & - & . & - & . \\
\hline$c$ & . & - & . & - & . & . & - & . & . \\
\hline$\sigma$ & . & - & . & . & . & - & . & . & . \\
\hline Mo & . & . & . & . & - & - & - & - & - \\
\hline $\mathrm{Pb}$ & . & - & . & . & . & - & . & . & . \\
\hline $\mathrm{Pt}$ & . & . & - & - & - & . & - & . & . \\
\hline$v$ & . & . & - & . & . & - & - & - & • \\
\hline $\mathbf{Y}$ & $\cdot$ & - & . & . & . & - & . & . & - \\
\hline $\mathrm{Zn}$ & 600 & 600 & 600 & 600 & 700 & 700 & 700 & 700 & 700 \\
\hline
\end{tabular}

\begin{tabular}{|c|c|c|c|c|c|c|c|c|c|}
\hline $\begin{array}{l}\text { MMBIB } \\
\text { Semplet }\end{array}$ & $\begin{array}{l}\text { GLASGP8302 } \\
1920 B\end{array}$ & $\begin{array}{l}\text { GLASGP8302 } \\
193 G B\end{array}$ & $\begin{array}{l}\text { GLASGP8302 } \\
\text { 194GB }\end{array}$ & $\begin{array}{l}\text { GLASGPB302 } \\
\text { 194GB }\end{array}$ & $\begin{array}{l}\text { GLASGP8302 } \\
196 \mathrm{~GB}\end{array}$ & $\begin{array}{l}\text { GLASGP8302 } \\
196 G B\end{array}$ & $\begin{array}{c}\text { GLASGP8302 } \\
\text { 196GB }\end{array}$ & $\begin{array}{l}\text { GLASGP8302 } \\
\text { 196GB }\end{array}$ & $\begin{array}{c}\text { GLASGP8302 } \\
\text { 197GB }\end{array}$ \\
\hline $\begin{array}{l}\text { Sampleft } \\
\text { Analyzist }\end{array}$ & 6 & 4 & 3 & 4 & 2 & 3 & 4 & $\begin{array}{l}190018 \\
5\end{array}$ & $\begin{array}{c}\text { 197GB } \\
2\end{array}$ \\
\hline Sumpletypo & 1 & 1 & 1 & 1 & 1 & 1 & 1 & 1 & 1 \\
\hline Latitudo" & -19.933 & -19.917 & -19.917 & -19.917 & -20.483 & -20.483 & -20.483 & .20 .483 & .20 .467 \\
\hline Longitade & -162.000 & -162.000 & .162 .000 & -162.000 & .161 .233 & -161.233 & -161.233 & -161.233 & -161.233 \\
\hline Depth & 4900 & 4857 & 4938 & 4938 & 4907 & 4907 & 4907 & 4907 & 4913 \\
\hline $\mathrm{Al}(w \mathrm{w}, \%)$ & 3.76 & 3.92 & 4.29 & 4.29 & 4.13 & 3.76 & 4.07 & 4.02 & 4.23 \\
\hline c & - & - & - & - & $\cdot$ & - & $\cdot$ & - & - \\
\hline$C_{0}$ & .500 & 520 & .500 & .520 & 510 & .470 & 540 & 500 & 510 \\
\hline $\mathrm{Cu}$ & .120 & .220 & .130 & .100 & .240 & .180 & .160 & .220 & .220 \\
\hline Po & 19.2 & 19.2 & 19.0 & 18.2 & 16.8 & 17.6 & 17.2 & 16.9 & 16.8 \\
\hline $\mathbf{K}$ & $\cdot$ & $\cdot$ & $\cdot$ & - & • & . & - & - & - \\
\hline Mg & - & - & - & - & - & . & - & - & - \\
\hline Mn & 14.2 & 25.5 & 14.3 & 14.4 & 19.6 & 18.0 & 17.4 & 19.0 & 18.4 \\
\hline $\mathbf{N a}$ & • & • & • & • & - & • & - & - & - \\
\hline $\mathrm{Ni}$ & 300 & 300 & .290 & .270 & 530 & .410 & .400 & 500 & 480 \\
\hline $\mathbf{P}$ & - & - & - & • & • & • & - & $\cdot$ & - \\
\hline Si & 7.90 & 7.90 & 8.51 & 8.74 & 7.15 & 7.43 & 8.37 & 7.43 & 729 \\
\hline Ti & • & • & - & • & • & • & - & • & - \\
\hline $\mathrm{H} 2 \mathrm{O}$ & - & - & - & - & . & - & - & - & - \\
\hline As(ppn) & - & - & . & - & . & - & . & - & - \\
\hline $\mathrm{Ba}$ & 1600 & 1500 & 1400 & 1300 & 1500 & 1500 & 1500 & 1500 & 1600 \\
\hline Cd & - & - & • & - & - & - & - & • & $\cdot$ \\
\hline$c$ & - & - & - & - & - & - & . & - & - \\
\hline$a$ & - & - & - & - & - & - & - & • & - \\
\hline Mo & . & . & . & - & . & - & . & - & . \\
\hline $\mathrm{Pb}$ & . & - & . & . & . & . & • & $\cdot$ & • \\
\hline $\mathrm{Pt}$ & $\cdot$ & - & - & . & - & . & - & - & - \\
\hline $\mathbf{v}$ & . & - & - & • & - & - & - & - & - \\
\hline Y & - & - & . & . & - & . & - & . & . \\
\hline & 700 & 600 & & 600 & 700 & 600 & 600 & 700 & 700 \\
\hline
\end{tabular}


Table 11. Composition of Mn oxide majors as reported by NGDC (cont'd).

\begin{tabular}{|c|c|c|c|c|c|c|c|c|c|}
\hline MMBIB & GLASGP8302 & GLASGP8302 & GLASGP8302 & GLASGP8302 & GLASOP8302 & GLASGP8302 & GLASGP8302 & GLASGP8302 & GLASGP8302 \\
\hline Sumplot: & 197GB & $197 \mathrm{~GB}$ & 197GB & 197GB & $197 \mathrm{~GB}$ & $198 \mathrm{KO}$ & $211 \mathrm{~GB}$ & 218KAL & $220 \mathrm{CB}$ \\
\hline Analygist" & 3 & 4 & 5 & 6 & 7 & 1 & 1 & 1 & 1 \\
\hline Sumpletype & 1 & 1 & 1 & 1 & 1 & 1 & 6 & 1 & 1 \\
\hline Letitude" & -20.467 & -20.467 & -20.467 & -20.467 & -20.467 & -20.483 & -21.600 & -21.617 & -21.500 \\
\hline Longitude & -161.233 & -161.233 & -161.233 & -161.233 & -161.233 & -161.217 & -161.533 & -161.583 & -162417 \\
\hline Depth & 4913 & 4913 & 4913 & 4913 & 4913 & 4995 & 4433 & 4473 & 4913 \\
\hline $\mathbf{A}(w t . \%)$ & 4.23 & 3.86 & 4.18 & 4.82 & 3.81 & 4.07 & 5,40 & 4.50 & 3.60 \\
\hline a & - & - & - & - & - & . & - & - & - \\
\hline co & 520 & 550 & 510 & 520 & .550 & 530 & .070 & .180 & 540 \\
\hline$\infty$ & .180 & .160 & .220 & .160 & .190 & .170 & .090 & .080 & .300 \\
\hline Fo & 17.4 & 17.4 & 16.9 & 15.5 & 18.8 & 18.0 & 12.3 & 18.2 & 16.0 \\
\hline $\mathbf{K}$ & • & - & • & • & - & - & - & - & - \\
\hline Mt & - & - & - & - & - & - & • & - & - \\
\hline Mn & 17.8 & 173 & 18.5 & 18.3 & 16.5 & 18.0 & 3,40 & 630 & 23.3 \\
\hline Na & - & - & - & - & - & - & - & - & - \\
\hline $\mathbf{N i}$ & .420 & 380 & .480 & 360 & .420 & $A 20$ & .240 & .210 & .680 \\
\hline $\mathbf{P}$ & • & • & • & - & - & - & • & - & • \\
\hline Si & 7.62 & 7.76 & 7.39 & 8.84 & 7.20 & 7.76 & 15.10 & 1215 & 5.70 \\
\hline $\mathrm{Ti}$ & - & - & • & - & - & - & • & • & • \\
\hline $\mathrm{H} 2 \mathrm{O}$ & $\cdot$ & - & - & - & - & - & - & - & $\cdot$ \\
\hline Ax(ppm) & - & - & • & • & - & - & • & - & - \\
\hline Be & 1600 & 1600 & 1600 & 1500 & 1600 & 1500 & 1000 & 1400 & 1700 \\
\hline Cd & - & - & - & - & - & - & - & . & . \\
\hline$c_{0}$ & . & . & - & . & . & . & - & . & - \\
\hline$a$ & . & . & - & - & • & . & . & - & . \\
\hline Mo & . & . & . & . & - & . & . & - & . \\
\hline $\mathrm{Pb}$ & . & - & . & - & - & - & - & - & $\cdot$ \\
\hline $\mathrm{Pt}$ & - & - & - & - & - & - & - & - & - \\
\hline v & . & - & - & . & • & - & - & - & . \\
\hline $\mathbf{Y}$ & - & - & - & $\cdot$ & . & - & • & - & - \\
\hline Zn & 700 & 600 & 700 & 500 & 700 & 600 & 300 & 600 & 800 \\
\hline
\end{tabular}

\begin{tabular}{|c|c|c|c|c|c|c|c|c|c|}
\hline $\begin{array}{l}\text { MMBIB } \\
\text { Sumple:4 }\end{array}$ & $\begin{array}{c}\text { GLASGP8302 } \\
225 G B\end{array}$ & $\begin{array}{l}\text { GLASGP } 8302 \\
225 G B\end{array}$ & $\begin{array}{c}\text { GLASGP8302 } \\
225 G B\end{array}$ & $\begin{array}{l}\text { GLASGP8302 } \\
226 G B\end{array}$ & $\begin{array}{l}\text { GLASGP8302 } \\
226 \mathrm{~GB}\end{array}$ & $\begin{array}{c}\text { GLASGP8302 } \\
226 G B\end{array}$ & $\begin{array}{l}\text { GLASGP8302 } \\
2260 B\end{array}$ & $\begin{array}{l}\text { GLASGP8302 } \\
227 \mathrm{~GB}\end{array}$ & $\begin{array}{c}\text { GLASGP8302 } \\
227 G B\end{array}$ \\
\hline Analygist" & 2 & 3 & 4 & 2 & 3 & 4 & 6 & 1 & 2 \\
\hline Sumpietype & 1 & 1 & 1 & 1 & 1 & 1 & 1 & 1 & 1 \\
\hline Letitude & .21 .350 & -21.350 & -21.350 & -21.350 & -21.350 & -21.350 & .21 .350 & -21.367 & -21.367 \\
\hline Longitude & -162.967 & -162.967 & -162967 & -162.967 & -162.967 & -162.967 & -162.967 & -162.933 & -162.933 \\
\hline Depth & 4964 & 4964 & 4964 & 4944 & 4944 & 4944 & 4944 & 4861 & 4861 \\
\hline$\overline{A(w t . \%)}$ & 4.13 & 4.34 & 4.18 & 4.02 & 4.07 & 4.23 & 4.34 & 4.13 & 4.87 \\
\hline c & - & - & - & - & - & - & - & • & - \\
\hline$c_{0}$ & 520 & 500 & .500 & 510 & 530 & 510 & 500 & 510 & .640 \\
\hline$\infty$ & .220 & .210 & .240 & .260 & .220 & .220 & 250 & .160 & .240 \\
\hline Fo & 16.8 & 16.7 & 163 & 16.5 & 17.1 & 17.1 & 163 & 17.9 & 17.2 \\
\hline $\mathrm{x}$ & - & - & - & • & - & • & - & - & - \\
\hline Mg & - & - & - & - & - & • & - & - & - \\
\hline Mn & 20.4 & 19.1 & 20.6 & 20.5 & 19.7 & 17.2 & 19.1 & 19.7 & 18.3 \\
\hline Na & • & . & - & - & • & - & - & - & - \\
\hline $\mathbf{N i}$ & 530 & .450 & 550 & 590 & .180 & .430 & .460 & 510 & 510 \\
\hline $\mathbf{P}$ & - & - & • & - & - & - & - & • & - \\
\hline $\mathbf{S i}$ & 7.34 & 7.76 & 7.25 & 6.83 & 7.29 & 8.23 & 7.76 & 7.29 & 9.72 \\
\hline $\mathrm{Ti}$ & - & - & - & - & - & - & - & - & - \\
\hline H2O- & - & - & - & - & - & - & - & . & - \\
\hline As(ppm) & . & - & - & . & - & . & - & - & - \\
\hline Ba & 1600 & 1600 & 1600 & 1600 & 1700 & 1600 & 1600 & 1600 & 1500 \\
\hline$\overline{c d}$ & . & - & - & . & . & . & . & - & - \\
\hline$c_{0}$ & . & - & - & . & - & . & . & . & . \\
\hline a & . & . & - & - & . & . & . & • & . \\
\hline Mo & . & - & . & . & . & - & • & . & . \\
\hline $\mathrm{Pb}$ & . & . & . & - & . & . & • & - & - \\
\hline $\mathrm{Pt}$ & - & . & . & . & - & - & • & - & • \\
\hline v & . & . & . & - & - & - & • & - & . \\
\hline $\mathbf{Y}$ & - & . & . & - & - & - & $\cdot$ & $\cdot$ & . \\
\hline $\mathbf{z n}$ & 700 & 700 & 700 & 800 & 700 & 700 & 700 & 700 & 900 \\
\hline
\end{tabular}


Table 11. Composition of Mn oxide majors as reported by NGDC (cont'd).

\begin{tabular}{|c|c|c|c|c|c|c|c|c|c|}
\hline $\begin{array}{l}\text { MMBIB } \\
\text { Semplet }\end{array}$ & $\begin{array}{l}\text { GLASGP8302 } \\
227 G B\end{array}$ & $\begin{array}{c}\text { GLASGPB302 } \\
229 \mathrm{~GB}\end{array}$ & $\begin{array}{c}\text { GLASGP8302 } \\
2290 B\end{array}$ & $\begin{array}{l}\text { GLASGP8302 } \\
229 \mathrm{~GB}\end{array}$ & $\begin{array}{c}\text { GLASGP8302 } \\
2290 \mathrm{~B}\end{array}$ & $\begin{array}{l}\text { GLASGP8302 } \\
240 G B\end{array}$ & $\begin{array}{l}\text { GLASGP8302 } \\
241 \mathrm{X}\end{array}$ & $\begin{array}{c}\text { GLASGP8302 } \\
\text { LOC-26 }\end{array}$ & $\begin{array}{l}\text { HALBPAB301 } \\
\text { 111DK2 }\end{array}$ \\
\hline Anelyoist & 3 & 1 & 2 & 3 & 5 & 9 & 3 & 1 & 1 \\
\hline Sempletype & 1 & 1 & 1 & 1 & 1 & 8 & 1 & $i$ & 8 \\
\hline Leatude & .21 .367 & -21.367 & -21.367 & -21.367 & .21 .367 & -22.317 & .22 .033 & -19.350 & 20.069 \\
\hline Longitude & -162.933 & -162.917 & -162.917 & -162.917 & -162.917 & -164.250 & -164.283 & -162.267 & -170.635 \\
\hline Depth & 4861 & 4861 & 4861 & 4861 & 4861 & 5216 & 5211 & 4616 & 1240 \\
\hline$A l(w L \%)$ & 4.50 & 4.02 & 4.13 & 3.86 & 4.23 & 6.03 & 3.76 & 4.13 & . \\
\hline$c_{2}$ & $\cdot$ & - & $\cdot$ & - & - & - & - & - & - \\
\hline$c_{0}$ & .580 & .520 & 540 & .560 & .520 & 270 & .580 & .560 & 1.30 \\
\hline$c_{2}$ & 200 & .280 & .230 & .200 & .230 & .190 & .110 & .080 & .020 \\
\hline Fo & 17.6 & 17.0 & 17.0 & 17.7 & 17.0 & 16.3 & 18.7 & 18.4 & 14.6 \\
\hline $\mathbf{K}$ & • & - & - & - & - & - & - & - & - \\
\hline M\& & - & - & - & - & - & . & • & . & - \\
\hline Mn & 19.0 & 21.8 & 20.7 & 19.5 & 19.7 & 11.5 & 16.4 & 16.9 & 26.7 \\
\hline Na & - & • & - & - & - & - & - & - & - \\
\hline $\mathrm{Ni}$ & 510 & .630 & 560 & .450 & 510 & 370 & .270 & 250 & .480 \\
\hline $\mathbf{P}$ & $\cdot$ & - & - & - & - & - & - & - & - \\
\hline $\mathbf{S i}$ & 8.51 & 6.36 & 6.50 & 7.06 & 7.29 & 12.15 & 8.56 & 9.02 & - \\
\hline $\mathrm{Ti}$ & - & $\cdot$ & - & - & · & - & - & - & . \\
\hline $\mathrm{H} 20 \mathrm{O}$ & - & - & - & - & - & - & - & - & - \\
\hline Ax(ppon) & - & - & - & - & - & - & $\cdot$ & - & - \\
\hline Be & 1600 & 2700 & 1600 & 1700 & 1700 & 1100 & 1300 & 1300 & - \\
\hline cd & - & - & - & - & - & - & . & . & . \\
\hline$\infty$ & - & - & - & - & . & • & - & . & . \\
\hline$\alpha$ & - & - & • & - & . & . & . & . & . \\
\hline Mo & . & - & • & - & . & - & . & . & . \\
\hline $\mathrm{Pb}$ & . & • & • & - & - & - & - & . & - \\
\hline $\mathrm{Pt}$ & - & - & - & - & . & - & . & . & . \\
\hline $\mathbf{v}$ & . & - & . & . & - & . & . & . & . \\
\hline Y & . & . & . & . & . & . & . & . & . \\
\hline $\mathbf{z}$ & 800 & 800 & 800 & 700 & 700 & 500 & 500 & 600 & - \\
\hline
\end{tabular}

\begin{tabular}{|c|c|c|c|c|c|c|c|c|c|}
\hline МMBIB & HALBPA8301 & HALBPA8301 & HALBPA8301 & HALBPA8301 & HALBPA8301 & HANAKN8001 & HANAKN8001 & ISKOJM8201 & ISKOIM8201 \\
\hline Samplo: & 31DKS & $320 \times 2$ & $570 K 2$ & 73DK7 & 76DK & 2P.50 & DWHD-16 & MDH-20 & RC12-D4 \\
\hline Analysis: & 1 & 1 & 1 & 1 & 1 & 1 & 1 & 1 & 1 \\
\hline Sampletype & 8 & 8 & 8 & 8 & 8 & 1 & $i$ & $i$ & 1 \\
\hline Lentitude & 9.136 & 9.400 & 13.150 & 19.400 & 19.350 & -13.883 & -16.483 & 28.817 & -37.050 \\
\hline Longitude & -164.801 & -164.783 & -165.484 & -171.168 & -170.983 & .150 .583 & -145.550 & -178.950 & -7.183 \\
\hline Depth & 2100 & 1120 & 3280 & 2860 & 1190 & 3620 & 1270 & 1090 & 3100 \\
\hline$\overline{\mathrm{A}(w \mathrm{wt} \%)}$ & $\cdot$ & - & - & . & $\cdot$ & . & $\cdot$ & . & $\cdot$ \\
\hline C. & - & - & - & - & - & - & - & - & - \\
\hline$C_{0}$ & .780 & 200 & .620 & .570 & 910 & .600 & 1.40 & .690 & .420 \\
\hline co & .050 & .050 & .100 & .120 & .040 & .200 & 300 & .040 & .190 \\
\hline Fo $_{0}$ & 14.5 & 9.50 & 19.0 & 17.5 & 16.7 & 20.1 & 13.6 & 15.8 & 20.1 \\
\hline $\mathbf{K}$ & 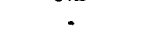 & - & 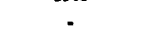 & - & - & - & . & - & . \\
\hline $\mathrm{M}_{8}$ & • & - & - & - & - & . & . & • & . \\
\hline Mn & 23.7 & 31.4 & 21.4 & 29.9 & 25.7 & 225 & 29.5 & 127 & 18.6 \\
\hline $\mathrm{Na}$ & - & - & . & - & . & . & . & . & - \\
\hline $\mathrm{Ni}$ & 380 & .620 & .270 & .250 & .470 & 500 & 800 & .280 & .540 \\
\hline $\mathbf{P}$ & - & - & - & - & - & . &. & . & - \\
\hline $\mathbf{S i}$ & - & . & - & . & . & . & . & . & . \\
\hline $\mathrm{Ti}$ & - & - & - & . & - & . & . & - & . \\
\hline H2O & - & . & . & . & - & - & . & . & - \\
\hline$A \times(p p m)$ & - & . & - & - & - & - & - & - & . \\
\hline Ba & - & . & . & . & - & . & . & - & . \\
\hline Cd & - & . & - & - & - & - & . & . & . \\
\hline$\infty$ & . & . & . & . & . & . & . & . &. \\
\hline$a$ & - & - & . & . & . & . & . & . & . \\
\hline Mo & - & - & - & . & . & . & . & - & - \\
\hline $\mathrm{Pb}$ & . & . & - & . & . & . & . & - & - \\
\hline $\mathrm{Pt}$ & . & . & - & - & . & . & . & . & . \\
\hline $\mathbf{v}$ & - & - & - & - & - & . & . & . & - \\
\hline $\mathbf{Y}$ & . & . & . & . & . & . & . & - & - \\
\hline $\mathbf{Z n}$ & - & . & . & - & - & - & - & . & - \\
\hline
\end{tabular}


Table 11. Composition of Mn oxide majors as reported by NGDC (cont'd).

\begin{tabular}{|c|c|c|c|c|c|c|c|c|c|}
\hline $\begin{array}{l}\text { MMBIB } \\
\text { Serrolef }\end{array}$ & $\begin{array}{l}\text { ISKOMM8201 } \\
\text { RCI4-D3 }\end{array}$ & $\begin{array}{l}\text { ISKOJM8201 } \\
\text { RC15-DS }\end{array}$ & $\begin{array}{l}\text { ISKOJM8201 } \\
\text { V16SBT3 }\end{array}$ & $\begin{array}{l}\text { ISKOJM8201 } \\
\text { V18-D32 }\end{array}$ & $\begin{array}{l}\text { ISKOJM8201 } \\
\text { V25-D14 }\end{array}$ & $\begin{array}{l}\text { ISKOJM8201 } \\
\text { V27-256 }\end{array}$ & $\begin{array}{l}\text { JONBHA8001 } \\
\text { PC34-13 }\end{array}$ & $\begin{array}{l}\text { JONEHA8001 } \\
\text { PC34-21 }\end{array}$ & $\begin{array}{c}\text { JONEHA8001 } \\
\text { PC36-15 }\end{array}$ \\
\hline Analynis" & 1 & 1 & 1 & 1 & 1 & 1 & 1 & 1 & 1 \\
\hline Sampletype & 1 & 1 & 1 & 1 & 1 & 1 & 1 & 1 & 1 \\
\hline Latitude & -36.750 & -48.467 & -13.067 & -14.300 & 24.950 & 25.417 & -45.200 & .52 .083 & -56.583 \\
\hline Langitude & 44.767 & -55.233 & -24.683 & -149.533 & -51.750 & -29.983 & 145.067 & 135.100 & 150.283 \\
\hline Depth & 2220 & 2650 & 440 & 2000 & 5960 & 5080 & 3968 & 3367 & 3516 \\
\hline$\overline{A l(w t . \%)}$ & $\cdot$ & $\cdot$ & - & . & - & - & . & - & $\cdot$ \\
\hline C. & - & • & • & - & $\cdot$ & $\cdot$ & $\cdot$ & - & $\cdot$ \\
\hline$C_{0}$ & .380 & .310 & .430 & .690 & 580 & .600 & .070 & .050 & .120 \\
\hline $\mathrm{Cu}$ & .150 & .090 & 270 & .190 & 180 & .180 & .140 & .180 & .120 \\
\hline Fo & 16.2 & 26.3 & 19.8 & 23.3 & 19.6 & 21.3 & 19.0 & 20.0 & 14.0 \\
\hline $\mathbf{K}$ & - & - & - & - & - & - & - & - & - \\
\hline $\mathbf{M B}_{\mathrm{B}}$ & - & - & - & - & $\cdot$ & - & • & - & - \\
\hline $\mathbf{M n}$ & 17.6 & 19.8 & 20,4 & 25.3 & 20.2 & 18.8 & 7.00 & 8.50 & 1.00 \\
\hline $\mathrm{Ne}$ & • & - & - & • & • & - & • & - & . \\
\hline $\mathrm{Ni}$ & .560 & .290 & .660 & 570 & 540 & 310 & .120 & .190 & .230 \\
\hline $\mathbf{P}$ & - & - & • & - & - & - & - & - & - \\
\hline $\mathbf{S i}$ & - & - & - & - & - & - & - & - & . \\
\hline $\mathbf{T}$ & - & - & - & - & - & - & - & - & . \\
\hline $\mathrm{H} 2 \mathrm{O}$ - & - & - & - & - & - & - & - & - & - \\
\hline As(ppm) & - & - & - & - & - & - & - & - & - \\
\hline Ba & . & - & - & - & - & - & - & - & - \\
\hline Cd & - & - & - & - & . & - & - & - & - \\
\hline$\infty$ & - & - & - & - & - & - & . & - & - \\
\hline$a$ & - & - & - & - & - & - & - & - & - \\
\hline Mo & - & - & - & - & - & - & - & - & - \\
\hline $\mathrm{Pb}$ & - & - & - & - & - & - & - & - & - \\
\hline $\mathrm{Pt}$ & - & - & - & - & - & . & . & - & - \\
\hline v & - & - & - & - & - & - & - & - & - \\
\hline $\mathbf{Y}$ & - & - & - & - & - & - & - & - & . \\
\hline $\mathbf{Z n}$ & - & - & - & - & - & - & - & - & - \\
\hline
\end{tabular}

\begin{tabular}{|c|c|c|c|c|c|c|c|c|c|}
\hline $\begin{array}{l}\text { MMBIB } \\
\text { Sample\#" }\end{array}$ & $\begin{array}{l}\text { JONEHA8001 } \\
\text { PC36-18 }\end{array}$ & $\begin{array}{l}\text { JONEHA8001 } \\
\text { PC36-37 }\end{array}$ & $\begin{array}{l}\text { JONEHA8001 } \\
\text { PC38-10 }\end{array}$ & $\begin{array}{l}\text { JONEHAB } 001 \\
\text { PC4409 }\end{array}$ & $\begin{array}{l}\text { JONEHA8CO1 } \\
\text { PC44-28 }\end{array}$ & $\begin{array}{l}\text { JONEHA8001 } \\
\text { RD38-02 }\end{array}$ & $\begin{array}{l}\text { JONBHA8C01 } \\
\text { RD38-03 }\end{array}$ & $\begin{array}{c}\text { JONEHA8001 } \\
\text { KD13 }\end{array}$ & $\begin{array}{c}\text { JONEHA8001 } \\
\text { KD3 }\end{array}$ \\
\hline Analyaiv" & 1 & 1 & 1 & 1 & 1 & 1 & 1 & 1 & 1 \\
\hline Sampletype & 1 & 1 & 1 & 1 & 1 & 1 & 1 & 1 & 1 \\
\hline Latitude & -53.017 & -58.667 & .57 .467 & -53.500 & -51.983 & -61.883 & -57.000 & -12900 & .13 .100 \\
\hline Longitude & 150.000 & 159.517 & 150.467 & 163.517 & 119.633 & 149.283 & 150.000 & 119.867 & 120.083 \\
\hline Depth & 3878 & 3876 & 3255 & 2689 & 3905 & 3657 & 3291 & 2100 & 2300 \\
\hline$A(w t . \%)$ & - & - & $\cdot$ & $\cdot$ & - & $\cdot$ & - & - & $\cdot$ \\
\hline C. & - & - & - & - & - & - & - & • & $\cdot$ \\
\hline $\mathbf{C o}_{0}$ & .160 & .010 & .290 & .160 & .110 & .210 & .150 & .350 & .260 \\
\hline $\mathrm{Ca}$ & .070 & .040 & .180 & .060 & .070 & 350 & .130 & .060 & .040 \\
\hline Ro & 17.0 & 7.50 & 15.0 & 17.9 & 20.0 & 9.40 & 120 & 18.3 & 19.4 \\
\hline K & - & - & - & - & - & - & - & - & - \\
\hline $\mathrm{MB}_{\mathbf{B}}$ & • & • & - & $\cdot$ & - & - & • & - & - \\
\hline $\mathrm{Mn}$ & 9.50 & 1.50 & 14.0 & 105 & 10.7 & 16.0 & 135 & 17.5 & 17.5 \\
\hline $\mathrm{Na}$ & - & - & • & . & . & - & . & . & . \\
\hline $\mathrm{Ni}$ & .130 & .040 & 390 & .090 & .080 & .790 & .430 & .380 & 300 \\
\hline $\mathbf{P}$ & • & • & • & - & - & • & - & - & • \\
\hline Si & - & - & - & - & - & - & - & - & - \\
\hline$\pi$ & - & - & - & - & - & - & - & - & - \\
\hline $\mathrm{H} 20$ & - & - & - & - & - & - & - & - & - \\
\hline As(ppn) & - & - & - & - & - & - & - & - & - \\
\hline Be & - & - & - & - & - & - & - & - & - \\
\hline Cd & - & - & - & - & - & - & - & - & - \\
\hline$C_{0}$ & - & - & - & - & . & . & - & - & - \\
\hline$a$ & - & - & - & . & - & - & - & - & - \\
\hline Mo & - & - & - & - & - & - & - & - & - \\
\hline $\mathrm{Pb}$ & - & - & - & - & - & - & - & - & - \\
\hline Pt & - & - & - & - & - & - & - & - & - \\
\hline $\mathbf{v}$ & - & - & . & - & - & - & - & - & - \\
\hline $\mathbf{Y}$ & - & - & - & - & - & - & - & - & - \\
\hline $\mathbf{Z n}$ & - & - & - & - & - & - & - & - & - \\
\hline
\end{tabular}


Table 11. Composition of Mn oxide majors as reported by NGDC (cont'd).

\begin{tabular}{|c|c|c|c|c|c|c|c|c|c|}
\hline $\begin{array}{l}\text { MMBIB } \\
\text { Semplot }\end{array}$ & $\begin{array}{c}\text { JONEHA8CO1 } \\
\text { KD4 }\end{array}$ & $\begin{array}{c}\text { XOBAKA8401 } \\
2-124\end{array}$ & $\begin{array}{c}\text { KOBAKA } 8401 \\
\text { 2-306 }\end{array}$ & $\begin{array}{c}\text { KOBAKA } 4401 \\
27-017\end{array}$ & $\begin{array}{c}\text { КОВAKA8401 } \\
27-017\end{array}$ & $\begin{array}{c}\text { XOBAKA } 8401 \\
\text { 3-606 }\end{array}$ & $\begin{array}{c}\text { KOBAKA } 8401 \\
3-606\end{array}$ & $\begin{array}{c}\text { KOBAKAS401 } \\
3.607\end{array}$ & $\begin{array}{c}\text { KOBAKA8401 } \\
5.33\end{array}$ \\
\hline Analyais" & 1 & 1 & 1 & 1 & 3 & 1 & 2 & 1 & 1 \\
\hline Sampletype & 1 & 1 & 1 & 1 & 1 & 1 & 1 & 1 & 1 \\
\hline Latitude & -13.150 & 26.267 & 26.267 & 26.200 & 26.200 & 26.117 & 26.117 & 26.083 & 26.217 \\
\hline Longitude & 120.083 & 142.967 & 142.967 & 144.183 & 144.183 & 144.317 & 144.317 & 144.317 & 144.083 \\
\hline Depth & 2100 & 1430 & 1430 & 890 & 890 & 1050 & 1050 & 1050 & 2430 \\
\hline$A l(w t . \%)$ & $\cdot$ & - & - & 33 & $\cdot$ & 1.06 & $\cdot$ & - & - \\
\hline $\mathrm{Ca}$ & • & 1.42 & 1.45 & 1.01 & 2.27 & 2.59 & 2.59 & 1.31 & 1.75 \\
\hline Co & .320 & .180 & 280 & 576 & 580 & .473 & .480 & .430 & 220 \\
\hline $\mathrm{Cu}$ & .030 & .078 & .071 & .046 & .046 & .091 & .091 & .080 & .043 \\
\hline Fo & 18.1 & 16.3 & 16.9 & 17.9 & 17.9 & 7.83 & 7.83 & 934 & 18.1 \\
\hline $\mathbf{K}$ & - & .31 & .29 & 23 & .20 & .84 & 84 & 37 & .31 \\
\hline Ms & $\cdot$ & .90 & 88 & .10 & 1.01 & 1.01 & 1.01 & .90 & .85 \\
\hline Mn & 21.2 & 14.3 & 14.4 & 23.7 & 23.7 & 25.0 & 25.0 & 20.1 & 13.4 \\
\hline $\mathrm{Na}$ & - & .85 & .81 & 29 & 95 & 1.37 & 1.37 & 1.06 & 1.03 \\
\hline $\mathrm{Ni}$ & .470 & .200 & .200 & .360 & 360 & .634 & .630 & .310 & .170 \\
\hline $\mathbf{P}$ & • & • & • & - & - & • & - & • & • \\
\hline Si & - & • & - & 1.22 & - & .68 & - & . & • \\
\hline $\mathrm{Ti}$ & - & .63 & .79 & .95 & .71 & .60 & .60 & .68 & .65 \\
\hline $\mathrm{H} 2 \mathrm{O}$ & - & - & $\cdot$ & - & - & • & - & - & - \\
\hline As(ppon) & - & 180 & 150 & • & 380 & - & 150 & 180 & 200 \\
\hline Ba & - & 2200 & 1800 & 130 & 3100 & 2000 & 2500 & 3600 & 2300 \\
\hline Cd & - & • & - & • & - & . & - & - & . \\
\hline C. & - & 870 & 940 & . & 1800 & - & 1800 & 2100 & 920 \\
\hline$a$ & - & • & • & - & • & - & - & = & • \\
\hline Mo & - & 290 & 270 & - & 750 & - & 820 & 770 & 180 \\
\hline $\mathbf{P b}_{\mathbf{b}}$ & - & • & • & • & • & • & • & • & • \\
\hline $\mathrm{Pt}$ & - & 1100 & 1200 & 1800 & 1800 & 1200 & 1200 & 1900 & 1200 \\
\hline v & - & 510 & 560 & 810 & 810 & 650 & 650 & 700 & 450 \\
\hline $\mathbf{Y}$ & - & 130 & 140 & 190 & 190 & 80 & 80 & 270 & 170 \\
\hline $\mathbf{Z n}$ & - & 530 & 470 & 510 & 510 & 80 & 820 & 540 & 490 \\
\hline
\end{tabular}

\begin{tabular}{|c|c|c|c|c|c|c|c|c|c|}
\hline MMBIB & KOBAKA8401 & KRAETA7401 & KRABTA7401 & KRAETA7401 & KRAETA7401 & KRAETA7401 & KRABTA7401 & KRAETA7401 & KRAETA7401 \\
\hline Semple\# & $8-51$ & E15-13 & E15-13 & E15.13 & B15-13 & B21 4 & E21 4 & B21 4 & B21-4 \\
\hline Analyais: & 1 & 1 & 2 & 3 & 4 & 1 & 2 & 3 & 4 \\
\hline Semplotypo & 1 & 8 & 8 & 8 & 8 & 8 & 8 & 8 & 8 \\
\hline Latinde & 25.500 & -55.383 & -55.383 & -55.383 & -55.383 & -33.050 & -33.050 & -33.050 & -33.050 \\
\hline Longitnde & 142533 & -145.033 & -145.033 & -145.033 & -145.033 & -82.050 & -82050 & -82050 & -82.050 \\
\hline Depth' & 2010 & . & - & - & $\cdot$ & $\cdot$ & - & - & $\cdot$ \\
\hline $\mathrm{Al}(\mathrm{wt} . \%)$ & $\cdot$ & - & - & . & $\cdot$ & - & - & - & - \\
\hline Ca & 3.07 & - & - & $\cdot$ & - & - & • & - & - \\
\hline$C_{0}$ & .500 & .090 & .090 & .084 & .084 & .128 & .126 & .140 & .130 \\
\hline a & .017 & .022 & .022 & .022 & .020 & .078 & .048 & .054 & .062 \\
\hline Pe & 14.9 & 15.2 & 14.7 & 15.2 & 13.5 & 15.6 & 15.5 & 16.4 & 17.4 \\
\hline $\mathbf{K}$ & .20 & • & . & . & - & . & - & . & . \\
\hline $\mathrm{M}_{\mathbf{B}}$ & .90 & • & - & - & . & - & . & - & . \\
\hline Mn & 18.4 & 7.50 & 7.80 & 7.40 & 6.60 & 13.2 & 13.1 & 13.2 & 13.7 \\
\hline Na & 1.18 & - & - & - & - & . & 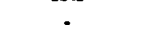 & 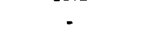 & 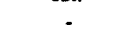 \\
\hline $\mathrm{Ni}$ & .310 & .028 & .040 & .038 & .056 & .264 & .262 & .272 & 248 \\
\hline $\mathrm{P}$ & . & - & . & . & - &. &. & . & . \\
\hline Si & - & . & - & - & . & . & . & . &. \\
\hline $\mathrm{Ti}$ & 56 & - & - & - & - & - & - & . & - \\
\hline $\mathrm{H} 2 \mathrm{O}$ & . & . & - & - & . & . & - & - & - \\
\hline Ax(ppm) & 330 & - & - & - & . & - & - & - & - \\
\hline $\mathrm{Ba}$ & 3200 & - & - & - & - & - & . &. & . \\
\hline Cd & • & - & - & - & . & - & - & - & - \\
\hline Co & 1200 & - & . & . & . & . & - & - & - \\
\hline$c$ & - & - & . & . & - & . & . & - & . \\
\hline Mo & 470 & • & - & - & - & . & . & • & . \\
\hline $\mathrm{Pb}$ & - & 720 & 720 & 700 & 620 & 820 & 760 & 740 & 760 \\
\hline $\mathrm{Pt}$ & 1400 & - & . & - & . & . & . & - &. \\
\hline $\mathbf{v}$ & 650 & - & - & - & - & . & . & . & - \\
\hline $\mathbf{Y}$ & 190 & . & - & - & - & . & - & . & - \\
\hline $\mathbf{Z n}_{n}$ & 490 & 300 & 360 & 380 & 360 & 600 & 580 & 600 & 620 \\
\hline
\end{tabular}


Table 11. Composition of Mn oxide majors as reported by NGDC (cont'd).

\begin{tabular}{|c|c|c|c|c|c|c|c|c|c|}
\hline $\begin{array}{l}\text { MMBIB } \\
\text { Samplett }\end{array}$ & $\begin{array}{l}\text { KRAETA7401 } \\
\text { E21-4 }\end{array}$ & $\begin{array}{l}\text { KRABTA7401 } \\
\text { E21 } 4\end{array}$ & $\begin{array}{c}\text { KRAETA7401 } \\
\text { E24-15C }\end{array}$ & $\begin{array}{l}\text { KRABTA7401 } \\
\text { E24-15C }\end{array}$ & $\begin{array}{l}\text { KRABTA7401 } \\
\text { E24-15C }\end{array}$ & $\begin{array}{l}\text { KRISSA7201 } \\
\text { 2P-50 }\end{array}$ & $\begin{array}{c}\text { KRISSA7201 } \\
\text { TF-2 }\end{array}$ & $\begin{array}{l}\text { KRISSA7201 } \\
\text { TRPD-2D }\end{array}$ & $\begin{array}{c}\text { KRISSA7201 } \\
\text { ZBTES3D }\end{array}$ \\
\hline Analyain: & 5 & 6 & 1 & 2 & 3 & 1 & 1 & 1 & 1 \\
\hline Surupletype & 8 & 8 & 8 & 8 & 8 & 1 & 1 & 1 & 1 \\
\hline Letitude & -33.050 & -33.050 & -35.967 & -35.967 & -35.967 & -13.883 & -13.867 & 20.750 & 40.267 \\
\hline Longitude & -82.050 & -82.050 & -134.833 & -134.833 & -134.833 & -150.583 & -150.583 & -112.783 & 170.333 \\
\hline Depth & - & $\cdot$ & - & $\cdot$ & $\cdot$ & 3695 & 3623 & 3000 & 3000 \\
\hline $\operatorname{Al}(w t \%)$ & - & - & - & - & - & - & - & - & - \\
\hline c & - & - & - & - & - & - & - & - & - \\
\hline Co & .136 & .102 & .356 & .310 & .270 & .390 & .420 & 367 & 545 \\
\hline Cu & .048 & .256 & .036 & .042 & .048 & .145 & .194 & .021 & .045 \\
\hline $\mathbf{F}_{\mathbf{0}}$ & 16.5 & 10.0 & 128 & 12.2 & 12.4 & 20.5 & 20.0 & 8.60 & $17 A$ \\
\hline $\mathbf{K}$ & - & - & - & - & - & - & - & - & - \\
\hline $\mathbf{M}_{\mathbf{8}}$ & • & • & - & $\cdot$ & • & - & - & • & • \\
\hline Mn & 13.5 & 13.3 & 13.7 & 14.0 & 13.8 & 18.0 & 18.6 & 18.9 & 26.5 \\
\hline $\mathrm{Na}$ & - & - & $\cdot$ & • & $\cdot$ & $\cdot$ & $\cdot$ & $\cdot$ & - \\
\hline $\mathrm{Ni}$ & .234 & .662 & .332 & .312 & .288 & 314 & .302 & .253 & .480 \\
\hline $\mathbf{P}$ & - & - & - & - & - & - & - & - & - \\
\hline $\mathbf{S i}$ & - & - & - & - & - & $\cdot$ & - & - & - \\
\hline $\mathrm{Ti}$ & $\cdot$ & - & - & $\cdot$ & $\cdot$ & 1.34 & 1.14 & .65 & .65 \\
\hline $\mathrm{H} 2 \mathrm{O}$ & $\cdot$ & $\cdot$ & $\cdot$ & $\cdot$ & $\cdot$ & $\cdot$ & $\cdot$ & - & $\cdot$ \\
\hline$A x(p p m)$ & $\cdot$ & $\cdot$ & - & - & - & $\cdot$ & - & $\cdot$ & $\cdot$ \\
\hline Be & $\cdot$ & - & - & $\cdot$ & - & $\cdot$ & - & - & $\cdot$ \\
\hline Cd & - & - & - & - & - & - & - & - & - \\
\hline Co & - & - & - & - & - & - & - & - & - \\
\hline$a$ & $\cdot$ & $\cdot$ & - & $\cdot$ & - & $\cdot$ & 30 & $\cdot$ & 20 \\
\hline Mo & - & $\cdot$ & $\cdot$ & $\cdot$ & - & 760 & $\cdot$ & 590 & 2800 \\
\hline $\mathrm{Pb}$ & 760 & 440 & 940 & 940 & 1000 & $\cdot$ & - & - & - \\
\hline $\mathbf{P t}$ & $\cdot$ & $\cdot$ & $\cdot$ & $\cdot$ & $\cdot$ & - & $\cdot$ & $\cdot$ & $\cdot$ \\
\hline $\mathbf{v}$ & $\cdot$ & - & $\cdot$ & - & $\cdot$ & - & - & - & $\cdot$ \\
\hline $\mathbf{Y}$ & - & $\cdot$ & - & - & - & - & - & - & - \\
\hline $\mathbf{Z n}_{\mathbf{n}}$ & 540 & 450 & 450 & 420 & 400 & - & - & $\cdot$ & $\cdot$ \\
\hline
\end{tabular}

\begin{tabular}{|c|c|c|c|c|c|c|c|c|c|}
\hline $\begin{array}{l}\text { MMBIB } \\
\text { Sample: }\end{array}$ & $\begin{array}{c}\text { KRISSA7201 } \\
\text { ZETES3D }\end{array}$ & $\begin{array}{l}\text { LAVRVM7301 } \\
\text { STN363 }\end{array}$ & $\begin{array}{c}\text { LAVRVM7301 } \\
\text { STN363 }\end{array}$ & $\begin{array}{l}\text { LONSPP8007 } \\
\text { B-CRUST }\end{array}$ & $\begin{array}{l}\text { LONSPF8007 } \\
\text { DPSD3-C }\end{array}$ & $\begin{array}{l}\text { LONSPF8007 } \\
\text { DPSD3-N }\end{array}$ & $\begin{array}{l}\text { LONSPP8007 } \\
\text { DPSD4N }\end{array}$ & $\begin{array}{l}\text { LONSPF8007 } \\
\text { DPSDS-C }\end{array}$ & $\begin{array}{c}\text { LONSPF8CO7 } \\
\text { NOD-B }\end{array}$ \\
\hline Analyais" & 2 & 1 & 2 & 1 & 1 & 1 & 1 & 1 & 2 \\
\hline Saxplotypo & 1 & 1 & 1 & 7 & 7 & 1 & 1 & 7 & 1 \\
\hline Latitudo & 40.267 & 33.500 & 33.500 & 8.701 & 8.701 & 8.701 & 8.701 & 8.701 & 8.701 \\
\hline Longitude & 170.333 & -29.933 & -29.933 & -103.886 & -103.886 & -103.886 & -103.886 & -103.886 & -103.886 \\
\hline Depth & 3000 & 2370 & 2370 & 1875 & 1875 & 1875 & 1875 & 1875 & 1875 \\
\hline$A l(w t \%)$ & - & 5.94 & 5.00 & - & - & - & $\cdot$ & - & - \\
\hline Ca & • & 1.69 & 2.14 & • & - & • & • & - & - \\
\hline$C_{0}$ & .490 & - & $\cdot$ & .710 & .120 & .100 & .120 & .130 & .410 \\
\hline$C_{n}$ & .041 & $\cdot$ & - & .010 & .440 & .140 & .270 & .290 & .090 \\
\hline $\mathbf{F}_{\mathbf{0}}$ & 18.0 & 17.1 & 18.6 & 17.0 & 14.0 & 20.3 & 21.2 & 20.2 & 16.7 \\
\hline $\mathbf{K}$ & - & .58 & .30 & • & • & - & - & - & - \\
\hline $\mathbf{M} 8$ & - & 1.74 & 1.92 & - & - & $\cdot$ & - & - & - \\
\hline Mn & 26.6 & 11.8 & 13.3 & 21.3 & 21.4 & 11.4 & 19.6 & 19.4 & 25.2 \\
\hline Na & • & 1.19 & .89 & - & - & - & - & - & $\cdot$ \\
\hline $\mathbf{N i}$ & .464 & $\cdot$ & $\cdot$ & .400 & .630 & .220 & .630 & .660 & .570 \\
\hline $\mathbf{P}$ & • & .22 & .22 & • & • & • & - & $\cdot$ & - \\
\hline $\mathbf{S i}$ & • & 4.90 & 2.63 & - & - & - & - & . & - \\
\hline $\mathrm{Ti}$ & .70 & 1.60 & 1.53 & - & - & - & - & - & - \\
\hline H2O. & - & - & - & - & - & - & - & - & $\cdot$ \\
\hline$A=(p p m)$ & - & - & - & - & - & $\cdot$ & - & - & - \\
\hline Be & - & - & - & - & - & - & - & - & - \\
\hline Cd & - & - & - & - & - & . & - & - & - \\
\hline Ce & - & - & - & - & - & - & - & - & - \\
\hline$\alpha$ & - & - & - & - & . & - & - & - & - \\
\hline Mo & - & - & - & - & - & - & - & - & - \\
\hline Pb & - & - & - & 1200 & 200 & 300 & 400 & 400 & 1000 \\
\hline $\mathbf{P t}$ & - & - & . & . & - & . & $\cdot$ & $\cdot$ & - \\
\hline $\mathbf{V}$ & - & - & - & - & - & - & - & - & - \\
\hline $\mathbf{Y}$ & . & - & - & • & - & - & - & - & • \\
\hline$Z_{n}$ & - & - & - & 500 & 900 & 800 & 900 & 1000 & 1000 \\
\hline
\end{tabular}


Table 11. Composition of Mn oxide majors as reported by NGDC (cont'd).

\begin{tabular}{|c|c|c|c|c|c|c|c|c|c|}
\hline MMBIB & LONSPFBOOT & MCGOPJ7901 & MCGOPJ7901 & MCGOPJ7901 & MCOOPJ7901 & MCGOPJ7901 & MCGOPJ7901 & MEYLMA7801 & MEYLMA7801 \\
\hline Surmplo" & SIQD4-C & 2A3-30 & $2 A 3-31$ & 2A3-32 & $2 A 4-30$ & $2 \mathrm{B2}$ & $2 B 8$ & G-1001 & G-1002 \\
\hline Analyois: & 1 & 1 & 1 & 1 & 1 & 1 & 1 & 1 & 2 \\
\hline Semplerypo & $i$ & 8 & 8 & 8 & 8 & 8 & 8 & 18 & 7 \\
\hline Letinuds & 8.701 & 26.152 & 26.152 & 26.152 & 26.152 & 26.117 & 26.117 & -21.684 & -22.535 \\
\hline Longitude & -103.886 & -44.784 & -44.784 & -44.784 & -44.784 & -25.350 & .25 .350 & -159.950 & -160.117 \\
\hline Depth & 1875 & 3148 & 3148 & 3148 & 3148 & 2600 & 2600 & 3970 & 4817 \\
\hline$A l(w t \%)$ &. & .07 & .07 & 25 & .27 & 3.11 & 2.17 & 3.70 & 5.50 \\
\hline C. & - & - & $\cdot$ & - & - & - & 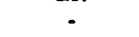 & 3.45 & 1.32 \\
\hline $\mathrm{Co}_{0}$ & 1.15 & - & .001 & - & .002 & .005 & .245 & 300 & 200 \\
\hline$w$ & .240 & - & - & - & . & - & 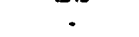 & .060 & .270 \\
\hline Fo & 224 & .04 & .10 & .48 & 34 & 205 & 20.1 & 20.2 & 14.1 \\
\hline $\mathbf{K}$ & . & .87 & .87 & 87 & .77 & .42 & .48 & 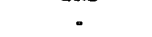 & . \\
\hline $\mathbf{M}_{8}$ & - & 1.35 & 132 & 135 & 1.18 & 1.02 & 98 & - & • \\
\hline $\mathbf{M n}$ & 16.6 & $34 A$ & 383 & 34.0 & 36.4 & 12.0 & 11.7 & 8.90 & 12.9 \\
\hline $\mathrm{Na}$ & • & - & - & - & - & - & - & - & - \\
\hline $\mathrm{Ni}$ & .380 & - & - & - & - & - & - & .130 & .640 \\
\hline $\mathbf{P}$ & . & - & - & - & - & - & . & - & - \\
\hline Si & - & - & - & . & - & - & - & 11.70 & 12.72 \\
\hline$\pi$ & - & - & . & - & - & - & - & 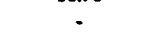 & 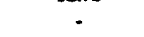 \\
\hline $\mathrm{H} 2 \mathrm{O}$ & - & - & - & - & - & - & - & - & - \\
\hline$A \in(p p n)$ & - & - & - & - & - & - & - & - & - \\
\hline Ba & - & . & - & - & - & - & - & - & - \\
\hline$\overline{c d}$ & - & - & - & - & - & - & - & . & - \\
\hline$\infty$ & - & - & - & - & - & - & - & . & . \\
\hline$a$ & - & so & - & 30 & 30 & so & so & . & . \\
\hline Mo & - & . & - & . & - & - & - & - & - \\
\hline Po & 400 & . & - & - & - & - & - & - & - \\
\hline $\mathrm{Pt}$ & - & - & - & - & - & - & - & - & - \\
\hline v & - & . & - & - & . & - & - & - & . \\
\hline $\mathbf{Y}$ & - & . & - & - & - & - & - & - & - \\
\hline $\mathbf{Z n}$ & 700 & - & . & - & . & - & - & - & - \\
\hline
\end{tabular}

\begin{tabular}{|c|c|c|c|c|c|c|c|c|c|}
\hline MMBIB & MBYLMA7801 & MBYLMA7801 & MEYLMA7801 & MEYLMA7801 & MEYLMA7801 & MBYLMA7801 & MEZUAA7501 & MEDAA7501 & MEZUAA8101 \\
\hline Sampleat & G-1007 & G-993B & G-993B & G-994 & G-996B & I-166 & 111 & 113 & D315 \\
\hline Amlynis: & 2 & 4 & 5 & 2 & 1 & 3 & 1 & 1 & 1 \\
\hline Sumpletype & 1 & 16 & 1 & 16 & 16 & 6 & 8 & 8 & 1 \\
\hline Latitude & -27.650 & -23.534 & -23534 & .22934 & -21.886 & -15.134 & 10.052 & 8.335 & 10.555 \\
\hline Longitude & -160.003 & -162900 & -162.900 & -162069 & -160.902 & -166.900 & -165.319 & -164.385 & -168.003 \\
\hline Depth & 4982 & 4993 & 4993 & 4848 & 4817 & 5348 & 1340 & 1660 & 1609 \\
\hline$\overline{A l(w t . \%)}$ & $\cdot$ & 2.80 & 3.80 & 2.90 & 3.20 & $\cdot$ & .76 & .47 & $\cdot$ \\
\hline a & 1.79 & 1.88 & 2.33 & 1.68 & 214 & - & 10.5 & 2.06 & • \\
\hline Co & .570 & 540 & - & .580 & 520 & .166 & .800 & 1.02 & 1.87 \\
\hline Co & .110 & .120 & - & .250 & .230 & .053 & .060 & .080 & .040 \\
\hline Fo & 26.4 & 26.9 & 24.6 & 235 & 20.0 & 22.7 & 8.69 & 9.88 & 13.2 \\
\hline $\mathbf{K}$ & - & - & .76 & - & • & - & - & - & - \\
\hline $\mathbf{M g}_{8}$ & - & - & 1.44 & - & - & • & 1.24 & 1.28 & - \\
\hline $\mathbf{M n}$ & 16.0 & 15.9 & 16.1 & 17.8 & 19.6 & 7.02 & 15.3 & 215 & 36.2 \\
\hline $\mathrm{Ne}$ & • & - & 1.45 & - & . & - & - & 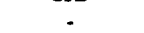 & . \\
\hline $\mathrm{Ni}$ & .260 & .230 & $\cdot$ & .440 & $A 70$ & .035 & .610 & .790 & .920 \\
\hline $\mathbf{P}$ & • & $\cdot$ & .12 & - & - & - & 3.67 & .27 & - \\
\hline $\mathbf{S i}$ & 6.86 & 6.76 & 9.52 & 5.85 & 6.39 & . & 1.39 & .75 & - \\
\hline$\pi$ & - & - & 1.93 & - & $\bullet$ & - & 58 & 89 & - \\
\hline $\mathrm{H} 2 \mathrm{O}$ & - & - & • & - & $\cdot$ & $\cdot$ & - & - & 25.7 \\
\hline Ax(ppon) & - & - & - & - & - & - & - & - & - \\
\hline $\mathrm{Ba}$ & - & - & . & - & . & - & - & - & - \\
\hline Cd & - & - & - & - & - & . & - & - & - \\
\hline$\infty$ & - & . & - & - & - & - & - & - & - \\
\hline$a$ & - & - & - & - & - & - & - & - & - \\
\hline Mo & - & - & - & - & - & - & - & - & - \\
\hline $\mathrm{Pb}$ & - & - & - & - & - & - & - & - & 2000 \\
\hline Pt & - & - & - & - & - & - & - & - & 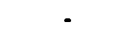 \\
\hline $\mathbf{v}$ & - & - & - & - & . & . & - & - & . \\
\hline $\mathbf{Y}$ & - & - & - & - & - & - & . & - & . \\
\hline $\mathrm{Zn}$ & - & - & - & - & - & - & 800 & 800 & 890 \\
\hline
\end{tabular}


Table 11. Composition of $\mathrm{Mn}$ oxide majors as reported by NGDC (cont'd).

\begin{tabular}{|c|c|c|c|c|c|c|c|c|c|}
\hline $\begin{array}{l}\text { MMBIB } \\
\text { Sumplot }\end{array}$ & $\begin{array}{l}\text { MIZUAA8201 } \\
\text { B15 }\end{array}$ & $\begin{array}{l}\text { MIZUAA8201 } \\
\text { B15 }\end{array}$ & $\begin{array}{l}\text { MIZUAA18201 } \\
\text { FG219-2 }\end{array}$ & $\begin{array}{l}\text { MIZUAA8201 } \\
\text { FG251-2 }\end{array}$ & $\begin{array}{l}\text { MIZUAA8201 } \\
\text { P171 }\end{array}$ & MOORWS7601 & $\begin{array}{l}\text { MOORWS7601 } \\
\text { D.9 }\end{array}$ & $\begin{array}{l}\text { MOORWS7601 } \\
\text { D.9 }\end{array}$ & $\begin{array}{c}\text { MOORWS7601 } \\
\text { D-9 }\end{array}$ \\
\hline $\begin{array}{l}\text { Semplet } \\
\text { Anelygisal }\end{array}$ & 4 & 5 & 2 & 1 & 9 & 1 & $\begin{array}{c}D-9 \\
2\end{array}$ & 3 & $\begin{array}{c}D-9 \\
4\end{array}$ \\
\hline Sumpletype & 1 & 1 & 1 & 1 & 11 & 8 & 8 & 8 & 8 \\
\hline Latiucule & -13.794 & -13.794 & -13.788 & 16.169 & -1.513 & 2.953 & 2.953 & 2953 & 2.953 \\
\hline Longitude & -159.476 & $-159,476$ & $-159,474$ & -179.339 & -165881 & .95 .167 & -95.167 & .95 .167 & -95.167 \\
\hline Depth & 5162 & 5162 & 5147 & 5291 & 5537 & . & $\cdot$ & 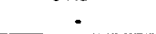 & 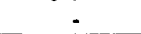 \\
\hline$A l(w t . \%)$ & $\cdot$ & $\cdot$ & - & - & $\cdot$ & - & - & - & - \\
\hline c & - & - & - & - & - & - & - & - & - \\
\hline$c_{0}$ & 563 & 568 & 592 & .579 & 895 & .157 & .091 & .005 & .017 \\
\hline $\mathrm{Cu}$ & .203 & .170 & 201 & .284 & .028 & .050 & .094 & .007 & .174 \\
\hline Fo & 19.2 & 24.5 & 19.8 & 18.4 & 4.96 & • & - & - & • \\
\hline $\mathbf{K}$ & - & • & . & • & - & . & . & - & . \\
\hline $\mathbf{M g}_{\mathbf{g}}$ & - & • & • & • & - & • & - & • & • \\
\hline $\operatorname{Mn}$ & 21.0 & 17.7 & 220 & 227 & .70 & 27.2 & 25.5 & 36.4 & 44.8 \\
\hline $\mathbf{N}_{\mathbf{2}}$ & • & • & • & • & . & • & • & • & - \\
\hline $\mathrm{Ni}$ & .371 & $22 \pi$ & .402 & 500 & .013 & .490 & .630 & .054 & 2.300 \\
\hline $\mathbf{P}$ & - & - & - & - & - & • & - & • & - \\
\hline si & - & - & . & . & - & - & - & - & - \\
\hline $\mathrm{Ti}$ & - & . & - & - & . & - & - & . & - \\
\hline H2O & 11.2 & 120 & 15.5 & 120 & 14.0 & . & - & - & - \\
\hline Axpppon) & - & - & - & - & - & - & - & - & - \\
\hline $\mathrm{Ba}$ & - & - & - & - & - & - & - & . & - \\
\hline Cd & . & . & - & - & - & . & - & . & - \\
\hline$\infty$ & - & . & . & - & - & - & - & - & - \\
\hline$\alpha$ & • & - & . & . & - & . & . & - & . \\
\hline Mo & . & - & . & . & - & . & . & - & - \\
\hline$P_{0}$ & 1500 & 2100 & 1300 & 1500 & 40 & - & - & - & - \\
\hline $\mathrm{Pt}$ & - & - & - & • & . & - & - & - & • \\
\hline $\mathbf{v}$ & - & - & - & - & . & - & - & - & • \\
\hline $\mathbf{Y}$ & $\cdot$ & - & . & $\cdot$ & . & $\cdot$ & - & - & . \\
\hline $\mathbf{Z n}$ & 620 & 570 & 630 & 650 & 140 & 1000 & 1800 & 580 & 4500 \\
\hline
\end{tabular}

\begin{tabular}{|c|c|c|c|c|c|c|c|c|c|}
\hline MMBIB & MOORWS7601 & MOORWS7601 & MOORWS7601 & MOORWS7601 & MOORWS7601 & MOORWS7601 & MOORWS7601 & MOORWS8101 & MOORWS8101 \\
\hline Samplei" & D6 & D6 & D6 & D6 & D6 & D6 & D6 & $20-2$ & $20-2$ \\
\hline Anelynist & 1 & 2 & 3 & 4 & 5 & 6 & 7 & 11 & 12 \\
\hline Sampletype & 8 & 8 & 8 & 8 & 8 & 8 & 8 & 1 & 1 \\
\hline Letitude & 2685 & 2685 & 2.685 & 2.685 & 2685 & 2685 & 2685 & 11.117 & 11.117 \\
\hline Longitude & -95.235 & -95.235 & -95.235 & -95.235 & -95.235 & -95.235 & -95.235 & -140.083 & -140.083 \\
\hline Depth & $\cdot$ & - & $\cdot$ & $\cdot$ & . & $\cdot$ & $\cdot$ & 4722 & 4722 \\
\hline $\mathbf{A}\left(\mathbf{w}, x_{0}\right)$ & . & . & . & . & - & $\cdot$ & - & 1.17 & 1.08 \\
\hline c & - & - & - & - & - & - & - & 1.64 & 1.63 \\
\hline$C_{0}$ & - & .002 & .002 & .002 & .004 & .004 & .002 & .485 & $521^{\circ}$ \\
\hline$\infty$ & .005 & .004 & .001 & .001 & .002 & .002 & .020 & .799 & 911 \\
\hline Fo & • & - & - & - & - & - & - & 9.46 & 13.2 \\
\hline $\mathbf{K}$ & - & - & - & . & - & . & - & .51 & .35 \\
\hline $\mathbf{M g}_{\mathbf{g}}$ & - & • & . & • & - & - & - & .85 & 1.28 \\
\hline Mn & 49.1 & 52.7 & 65.2 & 49.6 & 60.7 & 58.2 & 46.8 & 26.1 & 27.5 \\
\hline $\mathbf{N a}$ & • & - & - & - & - & • & - & 1.31 & .90 \\
\hline $\mathrm{Ni}$ & - & .010 & .006 & .007 & - & - & .050 & 1.037 & 2.430 \\
\hline $\mathbf{P}$ & - & • & - & - & - & - & - & • & - \\
\hline $\mathbf{S i}$ & - & - & - & - & - & - & - & 3.01 & 2.94 \\
\hline $\mathbf{T i}$ & - & - & - & - & - & - & - & .74 & .75 \\
\hline $\mathrm{H} 2 \mathrm{O}$ & - & - & - & - & - & - & - & - & - \\
\hline Ax(ppm) & - & - & - & - & - & - & - & - & - \\
\hline Ba & . & - & - & . & - & . & . & 4300 & 3900 \\
\hline$c d$ & . & - & . & - & - & - & - & • & • \\
\hline$c_{e}$ & . & . & . & - & - & . & . & - & - \\
\hline $\bar{c}$ & . & - & - & . & - & . & . & . & . \\
\hline Mo & . & - & - & . & - & . & - & . & - \\
\hline Po & - & . & - & - & - & - & - & - & - \\
\hline $\mathrm{Pt}$ & - & . & - & - & - & - & - & - & - \\
\hline v & - & . & - & . & - & - & . & - & . \\
\hline $\mathbf{Y}$ & . & . & . & . & . & . & . & - & - \\
\hline $\mathbf{z n}$ & 280 & 100 & 90 & 140 & 4600 & 4100 & 4900 & - & - \\
\hline
\end{tabular}


Table 11. Composition of $\mathrm{Mn}$ oxide majors as reported by NGDC (cont'd).

\begin{tabular}{|c|c|c|c|c|c|c|c|c|c|}
\hline $\begin{array}{l}\text { MMBIB } \\
\text { Sumple: }\end{array}$ & $\begin{array}{c}\text { MOORWS8102 } \\
20.2\end{array}$ & $\begin{array}{c}\text { MOORWS810: } \\
20.2\end{array}$ & $\begin{array}{c}\text { MOORWS8101 } \\
20.2\end{array}$ & $\begin{array}{c}\text { MOORWS8101 } \\
20-2\end{array}$ & $\begin{array}{c}\text { MOORWS8101 } \\
20-2\end{array}$ & $\begin{array}{c}\text { MOORWS8101 } \\
20-2\end{array}$ & $\begin{array}{c}\text { MOORWS8101 } \\
20-2\end{array}$ & $\begin{array}{c}\text { MOORWS8101 } \\
20-2\end{array}$ & $\begin{array}{c}\text { MOORWS8101 } \\
20-2\end{array}$ \\
\hline Analyaial & 13 & 14 & 15 & 16 & 18 & 22 & 24 & 25 & 26 \\
\hline Surpletype & 1 & 1 & 1 & 1 & 1 & 1 & 1 & 1 & 1 \\
\hline Latitude & 11.117 & 11.117 & 11.117 & 11.127 & 11.117 & 11.117 & 11.117 & 11.117 & 11.117 \\
\hline Longitude & -140.083 & -140.083 & -140.083 & -140.083 & -140.083 & -140.083 & -140.083 & -140.083 & -140.083 \\
\hline Depth & 4722 & 4722 & 4722 & 4722 & 4722 & 4722 & 4722 & 4722 & 4722 \\
\hline$A l(w t s)$ & 1.18 & 1.49 & 1.16 & 1.76 & 1.86 & 1.65 & 283 & .76 & 1.14 \\
\hline C. & 2.13 & 1.91 & 1.89 & 1.66 & 1.18 & 2.04 & 1.29 & 2.64 & 1.54 \\
\hline Co & .470 & .712 & .433 & 521 & 520 & 595 & 397 & 367 & 800 \\
\hline Cu & 351 & .615 & - & 895 & .655 & 320 & 1.486 & .120 & 375 \\
\hline $\mathbf{F}_{\mathbf{0}}$ & 18.5 & 17.8 & 13.9 & 13.0 & 7.40 & 10.7 & 5.36 & 8.21 & 12.1 \\
\hline $\mathbf{K}$ & .26 & .34 & .32 & .30 & .68 & .47 & .58 & .52 & .47 \\
\hline $\mathbf{M g}_{\mathbf{g}}$ & .65 & 1.02 & .75 & 2.15 & 2.63 & 1.80 & 3,40 & .84 & 1.37 \\
\hline Mn & 13.1 & 345 & 19.2 & 21.9 & 31.2 & 25.7 & 30.9 & 148 & 20,4 \\
\hline Na & .90 & 1.08 & .79 & 1.08 & 1.97 & 1.72 & 1.28 & 1.21 & 1.42 \\
\hline $\mathbf{N i}$ & 330 & .605 & 550 & 1.147 & 1.878 & 1.045 & 2774 & 338 & .754 \\
\hline $\mathbf{P}$ & • & • & - & - & • & • & • & • & • \\
\hline Si & 5.41 & 5.45 & 3.26 & 4.90 & 3.38 & 3.47 & 284 & 2.30 & 3.21 \\
\hline $\mathrm{Ti}$ & 1.82 & 1.49 & 1.02 & 1.09 & .71 & .95 & .45 & .59 & .83 \\
\hline $\mathrm{H} 2 \mathrm{O}$ & - & • & - & • & - & $\cdot$ & - & - & - \\
\hline$A x(p p n)$ & • & • & • & • & • & • & • & • & - \\
\hline $\mathrm{Be}$ & 4300 & 5600 & 3600 & 3100 & 3100 & 2800 & 1800 & 2500 & 4400 \\
\hline Cd & - & • & - & - & - & - & • & • & • \\
\hline$\infty$ & - & - & - & . & . & - & . & - & - \\
\hline$\alpha$ & - & - & - & - & - & - & - & - & - \\
\hline Mo & - & - & - & - & - & - & - & - & . \\
\hline $\mathrm{Pb}$ & - & - & - & - & - & - & - & - & - \\
\hline Pt & - & - & - & - & . & - & - & . & . \\
\hline v & - & - & - & . & - & - & - & . & - \\
\hline $\mathbf{Y}$ & - & - & - & - & - & - & - & - & - \\
\hline $\mathrm{Zn}$ & - & - & - & - & - & - & - & - & - \\
\hline
\end{tabular}

\begin{tabular}{|c|c|c|c|c|c|c|c|c|c|}
\hline MMBIB & MOORWS8101 & MOORWS8101 & MOORWS8101 & MOORWS8101 & MOORWS8101 & MOORWS8101 & MOORWS8101 & MOORWS8101 & MOORWS8101 \\
\hline Sampleit & $20-2$ & 20.2 & 20.2 & $20-2$ & 20.2 & $20-2$ & 20.2 & $20-2$ & 20.2 \\
\hline Analyoist & 27 & 28 & 29 & 30 & 31 & 32 & 5 & 6 & 7 \\
\hline Sarpletype & 1 & 1 & 1 & 1 & 1 & 1 & 1 & 1 & 1 \\
\hline Latitude & 11.117 & 11.117 & 11.117 & 11.117 & 11.117 & 11.117 & 11.117 & 11.117 & 11.117 \\
\hline Longitnde & -140.083 & -140.083 & -140.083 & -140.083 & -140.083 & -140.083 & -140.083 & -140.083 & -140.083 \\
\hline Depth & 4722 & 4722 & 4722 & 4722 & 4722 & 4722 & 4722 & 4722 & 4722 \\
\hline $\mathrm{Al}(w \mathrm{t} . \%)$ & 1.05 & .81 & .72 & 2.61 & 2.72 & 2.64 & 1.01 & 1.34 & 1.54 \\
\hline Ca & 2.16 & 1.87 & 2.11 & 1.13 & 1.29 & 1.30 & 1.84 & 1.77 & 1.37 \\
\hline Co & .698 & .485 & .470 & .463 & .411 & 433 & .617 & 866 & 529 \\
\hline $\mathrm{Cu}$ & .471 & 599 & 311 & 1.070 & .256 & 1.789 & .304 & 343 & 503 \\
\hline $\mathrm{Fe}_{\mathrm{e}}$ & 14.2 & 12.8 & 9.02 & 4.08 & 8.40 & 2.31 & 14.7 & 16.0 & 7.13 \\
\hline $\mathbf{K}$ & .47 & .38 & .43 & .73 & .35 & .71 & .55 & .37 & .71 \\
\hline $\mathbf{M}_{\mathbf{g}}$ & 1.19 & 1.70 & 152 & 2.76 & .80 & 3.82 & 1.10 & 1.12 & 1.87 \\
\hline $\mathrm{Mn}$ & 25.9 & 23.1 & 26.2 & 24.3 & 13.4 & 349 & 28.7 & 27.3 & 27.9 \\
\hline $\mathrm{Ne}$ & 2.11 & 2.13 & 2.03 & 1.52 & 2.93 & 2.19 & 1.10 & 1.64 & .96 \\
\hline $\mathrm{Ni}$ & .762 & 1.352 & 1.147 & 1.902 & 346 & 3.151 & .652 & 542 & 1.163 \\
\hline $\mathbf{P}$ & . & - & • & - & $\cdot$ & - & - & 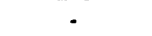 & - \\
\hline $\mathbf{S i}$ & 2.96 & 2.31 & 2.72 & 8.03 & 7.78 & 2.27 & 3.21 & 3.46 & 3.20 \\
\hline Ti & 1.29 & .67 & .58 & .41 & .78 & .26 & 1.09 & 1.19 & .33 \\
\hline $\mathrm{H} 20$ & • & - & - & - & - & . & - & $\cdot$ & - \\
\hline Ax(ppon) & • & - & . & - & - & . & • & - & - \\
\hline $\mathrm{Ba}$ & 5700 & 4100 & 4700 & 2900 & 3100 & 3800 & 4900 & 4700 & 1500 \\
\hline Cd & - & $\cdot$ & 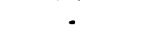 & 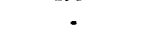 & - & - & - & • & - \\
\hline$\infty$ & . & . & . & - & . & - & . & . & . \\
\hline$a$ & . & - & . & . & - & - & . & - & . \\
\hline Mo & . & - & . & . & - & . & . & - & . \\
\hline $\mathrm{Pb}$ & . & - & . & . & . & . & - & . & - \\
\hline Pt & . & - & - & - & . & - & - & - & . \\
\hline $\mathbf{v}$ & . & . & - & . & . & . & - & - & - \\
\hline $\mathbf{Y}$ & . & - & . & . & - & . & - & - & - \\
\hline $\mathrm{Zn}$ & . & - & . & . & - & - & - & - & - \\
\hline
\end{tabular}


Table 11. Composition of Mn oxide majors as reported by NGDC (cont'd).

\begin{tabular}{|c|c|c|c|c|c|c|c|c|c|}
\hline $\begin{array}{l}\text { MMBIB } \\
\text { Semplo* }\end{array}$ & $\begin{array}{c}\text { MOORWS8101 } \\
20-2\end{array}$ & $\begin{array}{c}\text { MOORWS8401 } \\
91\end{array}$ & $\begin{array}{l}\text { MORITA8105 } \\
\text { FG91-2 }\end{array}$ & $\begin{array}{l}\text { MORITA8105 } \\
\text { G622 }\end{array}$ & $\begin{array}{l}\text { MORITA8105 } \\
\text { G622 }\end{array}$ & $\begin{array}{l}\text { NOAKLC7501 } \\
\text { ELTANOS }\end{array}$ & $\begin{array}{c}\text { NOAKLC7501 } \\
\text { ELTAN13 }\end{array}$ & $\begin{array}{l}\text { NOAKLC7501 } \\
\text { ELTAN14 }\end{array}$ & $\begin{array}{l}\text { NOAKLC7501 } \\
\text { ELTAN15 }\end{array}$ \\
\hline Analyaia: & 8 & 3 & 1 & 3 & 3 & 1 & 1 & 1 & 1 \\
\hline Sempletype & 1 & 8 & 1 & 1 & 1 & 1 & 1 & 1 & 1 \\
\hline Letitude & 11.117 & 11.000 & 12986 & 12.985 & 12.985 & -45.184 & -51.969 & .52069 & .53 .017 \\
\hline Longitude & -140.083 & -140.000 & 179.986 & 179.983 & 179.983 & 145.052 & 119.619 & 135.100 & 150.000 \\
\hline Depth & 4722 & - & 4762 & 5053 & 5053 & 3969 & 3905 & 3367 & 3878 \\
\hline$\overline{A l(w t . \%)}$ & .94 & - & $\cdot$ & - & - & $\cdot$ & - & - & - \\
\hline a & 1.81 & - & - & - & - & - & - & - & - \\
\hline$C_{0}$ & 587 & - & .691 & .652 & .699 & .070 & .110 & .050 & .160 \\
\hline Ca & .288 & .653 & .243 & .268 & .246 & .140 & .070 & .180 & .070 \\
\hline$F_{0}$ & 11.7 & 6.26 & 19.2 & 19.4 & 20.6 & 19.0 & 20.0 & 20.0 & $17.0^{\circ}$ \\
\hline $\mathbf{K}$ & .34 & - & - & 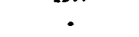 & - & 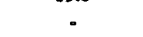 & - & - & - \\
\hline $\mathrm{Mg}_{8}$ & 1.43 & - & - & • & - & - & - & • & - \\
\hline Mn & 27.0 & 229 & 24.7 & 25.0 & 25.5 & 7.00 & 10.7 & 8.50 & 9.50 \\
\hline $\mathbf{N a}$ & 1.51 & • & • & - & - & - & • & - & - \\
\hline $\mathrm{Ni}$ & .620 & .655 & .576 & 550 & .500 & .120 & .080 & .190 & .130 \\
\hline P & . & - & . & - & . & - & . & . & - \\
\hline Si & 2.53 & . & - & - & - & - & . & - & - \\
\hline $\mathrm{Ti}$ & .99 & - & - & • & - & . & - & - & - \\
\hline H2O & • & - & 21.8 & 21.8 & 227 & - & . & - & - \\
\hline$A x(p p m)$ & • & - & . & - & - & - & - & - & - \\
\hline Ba & 3700 & 2700 & . & . & . & . & - & . & . \\
\hline cd & - & - & - & - & - & - & - & . & - \\
\hline$c$ & - & - & - & - & . & - & - & - & - \\
\hline$a$ & - & . & - & . & - & - & . & - & - \\
\hline Mo & - & . & • & . & - & - & - & - & . \\
\hline $\mathrm{Pb}$ & - & . & 1300 & 1300 & 1300 & . & - & - & - \\
\hline$P_{t}$ & - & - & - & - & - & - & - & - & - \\
\hline $\mathbf{v}$ & - & . & . & - & - & . & - & - & . \\
\hline $\mathbf{Y}$ & - & - & • & - & - & . & - & - & . \\
\hline $\mathbf{z}$ & . & 750 & 690 & 670 & 670 & - & - & - & . \\
\hline
\end{tabular}

\begin{tabular}{|c|c|c|c|c|c|c|c|c|c|}
\hline MMBIB & NOAKLC7501 & NOHAMA7601 & NOHAMA7601 & NOHAMA7601 & NOHAMA7601 & NOHAMA7601 & NOHAMA7601 & NOHAMA7601 & NOHAMA7601 \\
\hline Samplet & ELTAN16 & 03 & 04 & 05 & 06 & 07 & $\infty$ & 10 & 11 \\
\hline Analysiat & 1 & 1 & 1 & 1 & 1 & 1 & 1 & 1 & 1 \\
\hline Sampletypo & 1 & 8 & 8 & 8 & 8 & 8 & 8 & 8 & 8 \\
\hline Letitude & -53.500 & 44.617 & 44.617 & 44.550 & 44.500 & 37.117 & -38.000 & 38.983 & 36.067 \\
\hline Longitude & 163.517 & 170.333 & 170.300 & 170.283 & 170.417 & 162.650 & -169.967 & 146.000 & 143.533 \\
\hline Depth & 2689 & 1385 & 1365 & 1232 & 1290 & 2920 & 5070 & 3450 & 3350 \\
\hline$A l(w t . \%)$ & $\cdot$ & .11 & .13 & .15 & .11 & .93 & 1.25 & .64 & 1.38 \\
\hline c & $\cdot$ & $\cdot$ & $\cdot$ & - & - & $\cdot$ & $\cdot$ & $\cdot$ & - \\
\hline$c_{0}$ & .160 & 538 & 559 & 527 & .619 & .186 & .213 & .195 & .243 \\
\hline $\mathrm{Cu}$ & .060 & .047 & .064 & .050 & .059 & .066 & .088 & .110 & .237 \\
\hline Po & 17.0 & 17.2 & 15.2 & 16.3 & 17.5 & 17.2 & 20.3 & 18.5 & 17.3 \\
\hline $\mathbf{K}$ & - & • & - & - & - & - & - & • & - \\
\hline $\mathbf{M g}$ & $\cdot$ & - & • & - & - & $\cdot$ & - & $\cdot$ & - \\
\hline $\mathrm{Mn}$ & 10.5 & 27.5 & 25.9 & 26.0 & 28.3 & 10.2 & 17.3 & 16.5 & 12.3 \\
\hline $\mathrm{Na}$ & • & • & • & • & - & - & - & - & • \\
\hline $\mathbf{N i}$ & .090 & .609 & 585 & .578 & .624 & 222 & .262 & .411 & 341 \\
\hline $\mathbf{P}$ & - & - & - & - & - & - & - & - & • \\
\hline Si & - & - & $\cdot$ & - & $\cdot$ & $\cdot$ & - & - & - \\
\hline $\mathrm{Ti}$ & - & .71 & .68 & .69 & .85 & 1.24 & .93 & 94 & 1.43 \\
\hline $\mathrm{H} 20$ - & $\cdot$ & $\cdot$ & - & - & $\cdot$ & $\cdot$ & - & • & • \\
\hline$A \times(p p m)$ & - & - & - & - & - & - & - & - & - \\
\hline $\mathrm{Ba}$ & - & - & - & - & - & - & - & - & - \\
\hline Cd & - & - & - & - & - & - & - & - & - \\
\hline co & - & - & - & - & - & - & - & - & - \\
\hline$a$ & - & - & - & - & - & - & - & - & - \\
\hline Mo & - & - & • & - & - & - & $\cdot$ & $\cdot$ & - \\
\hline $\mathrm{Pb}$ & - & 1900 & 1800 & 2000 & 2200 & 1600 & 1100 & 1200 & 1400 \\
\hline Pt & - & $\cdot$ & • & - & $\cdot$ & • & - & • & $\cdot$ \\
\hline $\mathbf{v}$ & - & - & - & - & - & - & - & - & - \\
\hline $\mathbf{Y}$ & - & • & - & - & - & • & $\cdot$ & • & - \\
\hline $\mathbf{Z n}$ & - & 710 & 640 & 650 & 650 & 500 & 550 & 690 & 700 \\
\hline
\end{tabular}


Table 11. Composition of $\mathrm{Mn}$ oxide majors as reported by NGDC (cont'd).

\begin{tabular}{|c|c|c|c|c|c|c|c|c|c|}
\hline MMBIB & NOHAMA7601 & NOHAMA7601 & NOHAMA7601 & NOHAMA7601 & RONAPAB403 & RONAPA 8403 & $\begin{array}{l}\text { RONAPAB403 } \\
1247-1\end{array}$ & $\begin{array}{l}\text { RONAPA8403 } \\
1247-2\end{array}$ & ROONGS8001 \\
\hline Analynis:" & 1 & 1 & 1 & 1 & 1 & 1 & 1 & 1 & 23 \\
\hline Sempletypo & 8 & 8 & 1 & 1 & 19 & 19 & 19 & 19 & 8 \\
\hline Letisude & 37.117 & 23.817 & .20 .383 & 28.083 & 28.134 & 26.134 & 26.134 & 26.134 & 11.017 \\
\hline $\begin{array}{l}\text { Longitude } \\
\text { Depth }\end{array}$ & $\begin{array}{c}145.300 \\
2630\end{array}$ & $\begin{array}{c}148.767 \\
1250\end{array}$ & $\begin{array}{c}-148.017 \\
4618\end{array}$ & $\begin{array}{c}131.633 \\
1260\end{array}$ & $\begin{array}{c}-44.769 \\
2944\end{array}$ & $\begin{array}{c}-26.768 \\
2896\end{array}$ & $\begin{array}{c}44.769 \\
2058\end{array}$ & $\begin{array}{c}\mathbf{4 4 . 7 6 9} \\
2051\end{array}$ & $\begin{array}{c}-147.635 \\
5308\end{array}$ \\
\hline $\mathrm{Al}(\mathrm{wt} . \mathbf{x})$ & 82 & 59 & 1.07 & 1.64 & .05 & .03 & .09 & .05 & 4.60 \\
\hline ca & - & - & - & - & .17 & 1.56 & 1.39 & .92 & .98 \\
\hline$c_{0}$ & .198 & .791 & 532 & .230 & .010 & .020 & .010 & .010 & 290 \\
\hline$a_{0}$ & .056 & .062 & .169 & .089 & .010 & .010 & .010 & .010 & 280 \\
\hline Po & 20.7 & 12.8 & 19.7 & 20.7 & 324 & .10 & 9.57 & 41.5 & 11.8 \\
\hline $\mathbf{k}$ & - & - & - & - & 1.34 & .43 & 51 & .90 & 1.20 \\
\hline $\mathbf{M g}_{\mathbf{g}}$ & - & - & • & . & 1.86 & .92 & 1.05 & .62 & 51 \\
\hline Mn & 17.2 & 23.8 & 19.8 & 16.1 & .67 & 523 & 415 & 243 & 18.6 \\
\hline $\mathrm{N}_{\mathbf{2}}$ & $\cdot$ & - & - & - & - & - & - & - & 5.80 \\
\hline $\mathbf{N i}$ & .293 & 564 & .415 & 529 & .010 & .010 & .030 & .010 & .600 \\
\hline $\mathbf{P}$ & . & - & - & - & - & - & - & - & - \\
\hline si & - & . & - & . & 1215 & .06 & 1.24 & 5.88 & 7.60 \\
\hline $\mathrm{Ti}$ & .75 & .76 & 2.16 & .60 & 94 & .31 & 37 & .65 & 1.20 \\
\hline H2O- & - & .. & - & - & 6.9 & 175 & 19.2 & 13.6 & - \\
\hline Axppon) & - & - & - & - & - & - & - & - & - \\
\hline Ba & - & - & . & . & - & - & - & - & 4800 \\
\hline Cd & - & - & - & - & - & - & - & - & 10.0 \\
\hline$c_{0}$ & . & . & . & . & - & - & - & - & - \\
\hline$a$ & . & . & . & . & . & . & . & . & . \\
\hline Mo & . & • & . & - & . & - & - & . & 590 \\
\hline $\mathrm{Pb}$ & 1500 & 2800 & 870 & 2100 & - & - & - & - & 580 \\
\hline $\mathbf{P t}$ & . & . & 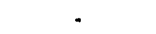 & - & - & . & - & - & 70 \\
\hline v & - & - & . & - & - & - & - & • & 400 \\
\hline$Y$ & • & - & - & - & - & - & - & - & - \\
\hline $\mathrm{za}$ & 620 & 710 & 550 & 780 & 100 & 100 & 100 & 100 & 520 \\
\hline
\end{tabular}

\begin{tabular}{|c|c|c|c|c|c|c|c|c|c|}
\hline MMBIB & ROONGS8DO1 & ROONOS8COI & ROONOS8001 & SCOTMR7401 & SCOTMR7401 & SCOTMR7401 & SCOTMR7401 & SCOTMR7401 & SCOTMR7401 \\
\hline Samplo: & 109 & 109 & 109 & $148-2 B$ & $160-100$ & 253-13 & 253-13 & 253-13 & $253-13$ \\
\hline Analyois:" & 24 & 25 & 26 & 1 & 1 & 1 & 2 & 3 & 4 \\
\hline Sumpletype & 8 & 8 & 8 & 6 & 6 & 6 & 6 & 6 & 6 \\
\hline Letitudo & 11.017 & 11.017 & 11.017 & 26.117 & 30.133 & 26.133 & 26.133 & 26.133 & 26.133 \\
\hline Longinde & -147.635 & -147.635 & -147.635 & -25.350 & -42.483 & -44.750 & -4.750 & 44.750 & 44.750 \\
\hline Depth & 5308 & 5308 & 5308 & - & - & $\cdot$ & - & $\cdot$ & $\cdot$ \\
\hline$A l(w t . \%)$ & 3.90 & 5.20 & 6.30 & - & $\cdot$ & - & . & - & - \\
\hline Ca & .88 & .14 & .98 & - & - & - & • & - & - \\
\hline Co & .270 & .130 & .180 & .720 & .272 & .002 & .002 & .002 & .002 \\
\hline Con & 360 & 310 & 320 & .075 & .088 & .001 & .012 & .009 & - \\
\hline Fo & 8.50 & 5.30 & 5.70 & 16.1 & 18.1 & .01 & .08 & .11 & .07 \\
\hline $\mathbf{K}$ & 1.78 & 212 & 2.20 & - & $\cdot$ & - & $\cdot$ & - & - \\
\hline $\mathbf{M}_{\mathbf{B}}$ & 56 & 1.14 & .89 & • & - & - & • & - & - \\
\hline $\mathrm{Mn}$ & 15.3 & 8.40 & 10.7 & 14.1 & 980 & 39.2 & 38.5 & 38.6 & 39.4 \\
\hline $\mathrm{Na}$ & . & 5.58 & 6.08 & 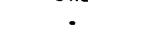 & 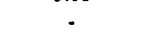 & 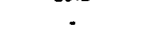 & - & . & 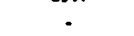 \\
\hline $\mathrm{Ni}$ & 1.020 & .720 & .760 & .220 & .128 & .010 & .079 & .066 & .020 \\
\hline $\mathbf{P}$ & • & - & • & - & - & - & - & - & - \\
\hline Si & 10.00 & 10.30 & 14.60 & . & . & - & . & - & - \\
\hline $\mathrm{Ti}$ & 88 & 88 & .84 & - & - & - & - & - & - \\
\hline H2O & - & . & - & - & . & - & - & - & - \\
\hline$A x(p p m)$ & - & - & . & - & - & - & - & - & - \\
\hline $\mathrm{Ba}$ & 5300 & 4200 & 4200 & . & . & - & . & - & . \\
\hline Cd & 20.0 & 20.0 & 10.0 & - & - & - & - & - & - \\
\hline$C_{0}$ & - & - & . & . & . & . & . & . & - \\
\hline$a$ & 10 & 10 & 10 & . & - & - & . & . & - \\
\hline Mo & 400 & 390 & 300 & - & . & - & - & - & - \\
\hline $\mathrm{Pb}$ & 480 & 480 & 340 & - & - & - & - & - & . \\
\hline Pt & 40 & 250 & 160 & - & • & . & - & . & - \\
\hline $\mathbf{v}$ & 340 & 240 & 200 & - & - & . & - & . & . \\
\hline $\mathbf{Y}$ & - & - & - & - & - & . & - & - & - \\
\hline $\mathbf{z n}$ & 640 & 500 & 600 & - & - & - & - & - & - \\
\hline
\end{tabular}


Table 11. Composition of Mn oxide majors as reported by NGDC (cont'd).

\begin{tabular}{|c|c|c|c|c|c|c|c|c|c|}
\hline MMBIB & SCOTMR7401 & SCOTMR7401 & SCOTMR7401 & SCOTMR7401 & SCOTMR7401 & SIEGMD8101 & SKORNS7501 & SKORNS7501 & $\begin{array}{c}\text { SKORNS7501 } \\
6298-9\end{array}$ \\
\hline $\begin{array}{l}\text { Samplet } \\
\text { Analynist" }\end{array}$ & $\underset{5}{253-13}$ & $\begin{array}{c}253-13 \\
6\end{array}$ & $\begin{array}{c}253-13 \\
7\end{array}$ & $\begin{array}{c}254-15 \\
1\end{array}$ & $\begin{array}{c}255-19 \\
1\end{array}$ & $\underset{5}{019-1}$ & $\begin{array}{c}6288-13 \\
1\end{array}$ & $\begin{array}{c}6298-53 \\
1\end{array}$ & $\begin{array}{c}6298-9 \\
1\end{array}$ \\
\hline Sempletypo & 6 & 6 & 6 & 6 & 6 & 1 & $i$ & 1 & 1 \\
\hline Letitude & 26.133 & 26.133 & 26.133 & 26.553 & 26.269 & 10.950 & -22.733 & -22.733 & .22733 \\
\hline Longitude & 44.750 & 44.750 & 44.750 & 44.500 & -45.101 & -140.163 & -160.817 & -160.817 & -160.817 \\
\hline Depth & - & - & - & - & • & 4223 & 4530 & 4530 & 3180 \\
\hline$A(w t . \%)$ & - & - & - & - & . & 1.49 & - & ? & - \\
\hline c & - & - & . & . & . & 1.26 & . & - & . \\
\hline$c_{0}$ & .002 & .002 &.$\infty 1$ & 948 & .495 & 551 & 920 & 570 & .565 \\
\hline$\infty$ & .002 & .002 &.$\infty 1$ & .030 & .041 & .415 & .080 & .091 & .104 \\
\hline $\mathrm{P}_{0}$ & .07 & .04 & .04 & 16.4 & 18.6 & 10.7 & 16.7 & 19.0 & 17.9 \\
\hline $\mathbf{K}$ & . & - & - & - & - & .42 & - & - & - \\
\hline $\mathbf{M g}_{\mathbf{g}}$ & . & - & - & . & . & .71 & - & - & . \\
\hline Mn & 39.2 & 39.1 & 39.3 & 11.2 & 11.0 & 224 & 226 & 15.4 & 16.6 \\
\hline Na & - & - & $\cdot$ & . & • & .43 & - & . & • \\
\hline $\mathbf{N i}$ & .040 & .027 & .005 & .090 & .111 & 597 & .420 & .180 & .450 \\
\hline $\mathbf{P}$ & • & - & - & - & - & - & - & • & • \\
\hline $\mathbf{s i}$ & . & . & . & . & . & 4.60 & - & - & . \\
\hline $\mathrm{Ti}$ & . & - & - & - & . & .57 & - & - & . \\
\hline $\mathrm{H} 2 \mathrm{O}$ & - & . & . & . & . & - & . & . & - \\
\hline As(ppon) & - & - & - & - & - & - & - & - & . \\
\hline Ba & - & . & - & . & . & 1800 & - & - & - \\
\hline Cd & - & - & - & - & . & - & - & - & - \\
\hline$c_{0}$ & - & . & - & - & . & - & - & . & . \\
\hline$a$ & - & - & - & - & . & - & - & . & - \\
\hline Mo & . & . & - & . & . & . & . & - & - \\
\hline $\mathrm{Pb}$ & . & . & - & . & . & . & - & . & . \\
\hline $\mathrm{Pt}_{\mathrm{t}}$ & . & . & . & . & . &. & - & . & . \\
\hline v & - & . & . & . & . & . & . & . & . \\
\hline Y & . & . & - & . & . & . & . & . & . \\
\hline$Z_{n}$ & . & . & . & . & . & 2600 & 700 & 440 & 660 \\
\hline
\end{tabular}

\begin{tabular}{|c|c|c|c|c|c|c|c|c|c|}
\hline MMBIB & STOFPA8101 & SUESEA7701 & SUESEA7701 & SUESEA7701 & SUESBA7701 & SUESEA7701 & SUESEA7701 & SUESEA7701 & SUESEA7701 \\
\hline Samplet & 215KO & 1 & 1 & 1 & 1 & 1 & 1 & 1 & 1 \\
\hline Analynas: & 1 & 1 & 10 & 11 & 12 & 13 & 14 & 2 & 3 \\
\hline Sampletype & 3 & 1 & 1 & 1 & 1 & 1 & 1 & 1 & 1 \\
\hline Letitude & -21.983 & 54.767 & 54.77 & 54.767 & 54.767 & 54.767 & 54.767 & 54.767 & 54.77 \\
\hline Longitude & -161.900 & 10.083 & 10.083 & 10.083 & 10.083 & 10.083 & 10.083 & 10.083 & 10.083 \\
\hline Depth & 447 & 25 & 25 & 25 & 25 & 25 & 25 & 25 & 25 \\
\hline$A(w t \%)$ & - & - & - & - & - & - & - & - & - \\
\hline ce & - & - & - & - & - & - & - & - & - \\
\hline$C_{0}$ & .032 & - & - & - & - & & . & - & . \\
\hline ca & .050 & .019 & .004 & .003 & .004 & .004 & .003 & .027 & .026 \\
\hline Po & 16.3 & 6.61 & 11.8 & 9.61 & 7.73 & 6.71 & 4.30 & 17.9 & 14.4 \\
\hline K & - & • & • & • & - & - & • & - & - \\
\hline $\mathbf{M g}_{\mathbf{g}}$ & • & • & $\cdot$ & • & - & - & - & - & - \\
\hline Mn & 3.81 & 34.6 & 30.6 & 30.8 & 32.6 & 34.4 & 38.7 & 11.0 & 16.6 \\
\hline $\mathrm{Na}$ & - & - & - & • & • & • & • & - & • \\
\hline $\mathrm{Ni}$ & .090 & - & . & - & - & - & - & - & - \\
\hline $\mathbf{P}$ & $\cdot$ & - & - & - & - & - & - & - & - \\
\hline $\mathbf{S i}$ & - & - & . & - & . & - & . & . & . \\
\hline Ti & - & - & . & . & - & - & - & - & - \\
\hline H2O- & - & - & - & - & - & - & - & - & - \\
\hline$A x(p p m)$ & - & - & - & - & - & - & - & - & - \\
\hline $\mathrm{Be}$ & 700 & - & - & - & . & - & - & - & - \\
\hline $\mathrm{Cd}$ & • & 10.0 & . & . & . & . & - & 10.0 & 10.0 \\
\hline co & - & • & - & . & - & - & - & $\cdot$ & $\cdot$ \\
\hline$a$ & - & - & - & . & - & . & - & - & - \\
\hline Mo & - & - & - & - & . & - & - & . & - \\
\hline $\mathbf{P b}$ & - & 160 & 60 & 40 & 30 & 30 & 20 & 120 & 140 \\
\hline $\mathrm{Pt}$ & - & - & . & . & - & - & - & - & - \\
\hline v & . & . & . & . & - & . & . & . & . \\
\hline $\mathbf{Y}$ & . & • & . & . & - & . & . & • & . \\
\hline $\mathbf{Z n}$ & 740 & 1500 & 240 & 170 & 190 & 200 & 120 & 1500 & 1700 \\
\hline
\end{tabular}


Table 11. Composition of Mn oxide majors as reported by NGDC (cont'd).

\begin{tabular}{|c|c|c|c|c|c|c|c|c|c|}
\hline MMBIB & SUESEA7701 & SUESEA 701 & SUESEA7701 & SUESEA7701 & SUESEAT7OI & SUESEA770I & TAKBNA790I & TAKENA7901 & TAKENA7901 \\
\hline Sumple" & 1 & 1 & 1 & 1 & 1 & 1 & Ol-KI & o6-N4 & 12 \\
\hline Andyrish & 4 & 5 & 6 & 7 & 8 & 9 & 1 & 1 & 1 \\
\hline Sampletype & 1 & 1 & 1 & 1 & 1 & 1 & 1 & 1 & 1 \\
\hline Letinide & $\$ 4.767$ & 54.76 & 54.767 & $\$ 4.767$ & $\$ 4.767$ & 54.767 & 44.500 & 17.269 & 50.101 \\
\hline Longitude & 10.083 & 10.083 & 10.083 & 10.083 & 10.083 & 10.083 & 170.417 & -176301 & -123.785 \\
\hline Depth & 25 & 25 & 25 & 25 & 25 & 25 & 1280 & 4400 & 350 \\
\hline $\boldsymbol{N}(\mathbf{w t . \% )}$ & - & - & - & - & - & • & - & - & - \\
\hline c & . & - & - & . & . & - & - & - & - \\
\hline$c_{0}$ & • & • & - & - & • & • & 371 & sor & .022 \\
\hline c. & .009 & .010 & .007 & .008 & .007 & .007 & .047 & .180 & .008 \\
\hline Fo & 25.1 & 22.3 & 9.92 & 9.84 & 16.1 & 14.4 & 1.10 & 124 & 8.89 \\
\hline $\mathbf{K}$ & - & - & - & - & - & - & - & • & • \\
\hline Mg & - & - & - & • & • & . & - & . & - \\
\hline $\operatorname{Mn}$ & 11.5 & 10.5 & 28.4 & 24.3 & 22.8 & 22.7 & 22.7 & 15.2 & 224 \\
\hline Na & • & • & . & - & - & - & - & - & - \\
\hline $\mathrm{Ni}$ & - & - & - & - & - & - & 375 & 349 & .025 \\
\hline $\mathbf{P}$ & - & - & - & - & - & . & • & - & - \\
\hline $\mathbf{S i}$ & - & - & - & - & - & . & - & - & - \\
\hline $\mathbf{T i}$ & - & - & . & . & - & . & . & - & . \\
\hline H2O- & - & - & - & - & - & - & - & - & - \\
\hline Ax(ppon) & - & - & - & . & - & - & - & - & . \\
\hline $\mathrm{Ba}$ & - & - & - & . & . & - & - & - & - \\
\hline Cd & - & - & - & . & - & - & - & - & - \\
\hline$\infty$ & - & - & - & - & - & - & - & . & - \\
\hline$\alpha$ & - & - & - & - & - & - & - & - & - \\
\hline Mo & - & . & . & - & . & - & - & - & - \\
\hline $\mathrm{Pb}$ & 120 & 140 & 60 & 90 & 50 & so & - & - & - \\
\hline $\mathrm{Pt}$ & • & - & . & . & - & - & - & - & - \\
\hline v & - & - & - & - & - & - & - & - & - \\
\hline $\mathbf{Y}$ & $\cdot$ & - & $\cdot$ & • & • & - & • & • & • \\
\hline $\mathbf{z}$ & 1000 & 980 & 1000 & 1500 & & & 480 & 550 & 190 \\
\hline
\end{tabular}

\begin{tabular}{|c|c|c|c|c|c|c|c|c|c|}
\hline $\begin{array}{l}\text { MMBIB } \\
\text { Samplett }\end{array}$ & $\begin{array}{c}\text { TAKBNA7901 } \\
13\end{array}$ & $\begin{array}{l}\text { THUAA8101 } \\
\text { 137-DK }\end{array}$ & $\begin{array}{l}\text { THUAA8101 } \\
\text { 226-GB }\end{array}$ & $\begin{array}{c}\text { UCHITA7601 } \\
9-7\end{array}$ & $\begin{array}{c}\text { UCHITA7601 } \\
9-7\end{array}$ & $\begin{array}{c}\text { UCHITA760I } \\
9-7\end{array}$ & $\begin{array}{c}\text { UCHIrA7601 } \\
9.7\end{array}$ & $\begin{array}{c}\text { UCHITA7601 } \\
9-7\end{array}$ & $\begin{array}{c}\text { UCHITA7601 } \\
9-7\end{array}$ \\
\hline Amelysist" & 1 & 1 & 1 & 10 & 11 & 12 & 13 & 14 & 15 \\
\hline Sampletype & 1 & 8 & 1 & 1 & 1 & 1 & 1 & 1 & 1 \\
\hline Letitude & 50.101 & -10.149 & -21.300 & 44.617 & 44.617 & 44.617 & 44.617 & 44.617 & 44.617 \\
\hline Longitude & -123.785 & -134.000 & -162.951 & 170.300 & 170.300 & 170.300 & 170.300 & 170.300 & 170.300 \\
\hline Depth & 350 & 4453 & 4944 & 1365 & 1365 & 1365 & 1365 & 1365 & 1365 \\
\hline$A(w L \%)$ & - & - & 8.00 & .75 & 32 & .22 & .20 & .22 & .24 \\
\hline $\mathrm{C}_{\mathbf{a}}$ & - & . & - & 1.38 & 1.72 & 1.77 & 1.64 & 1.80 & 1.72 \\
\hline co & .020 & .050 & 510 & .350 & 800 & .620 & .670 & .710 & .640 \\
\hline cu & .009 & .270 & .220 & .114 & .056 & .060 & .046 & .046 & .040 \\
\hline Po & 9.32 & 14.2 & 17.1 & 10.0 & 13.5 & 12.8 & 13.4 & 13.8 & 14.5 \\
\hline K & - & $\cdot$ & - & - & - & $\cdot$ & - & - & - \\
\hline M8 & $\cdot$ & - & - & 1.36 & 95 & .95 & 91 & 92 & 87 \\
\hline Mn & 223 & 6.45 & 17.2 & 29.8 & 25.4 & 27.1 & 26.1 & 25.6 & 23.7 \\
\hline $\mathbf{N}_{\mathbf{2}}$ & - & - & - & - & • & - & - & $\cdot$ & - \\
\hline $\mathrm{Ni}$ & .038 & .440 & .430 & 1.000 & .440 & .560 & .460 & 440 & 360 \\
\hline $\mathbf{P}$ & - & 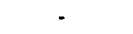 & - & - & - & - & - & • & - \\
\hline si & - & - & 17.60 & 2.47 & 1.81 & 1.61 & 1.71 & 1.50 & 205 \\
\hline$\pi$ & - & - & . & - & - & $\cdot$ & - & $\cdot$ & • \\
\hline $\mathrm{H} 2 \mathrm{O}$ & - & - & - & - & - & - & - & • & $\cdot$ \\
\hline$A x(p p m)$ & - & - & - & - & - & - & - & - & - \\
\hline $\mathrm{Ba}_{\mathrm{a}}$ & - & . & 1600 & - & - & - & - & . & - \\
\hline Cd & - & - & - & - & - & . & - & - & - \\
\hline ce & • & . & . & . & . & . & - & . & . \\
\hline$\alpha$ & - & - & - & • & . & . & . & . & - \\
\hline Mo & - & - & • & . & - & $\cdot$ & • & • & . \\
\hline Po & $\cdot$ & - & - & 840 & 1100 & 840 & 1100 & 1100 & 840 \\
\hline $\mathrm{Pt}$ & - & - & - & $\cdot$ & $\cdot$ & $\cdot$ & - & $\cdot$ & $\cdot$ \\
\hline $\mathbf{v}$ & - & . & - & . & . & . & . & . & . \\
\hline $\mathbf{Y}$ & . & $\cdot$ & . & . & . & . & . & - & - \\
\hline $\mathbf{Z n}$ & 230 & 700 & 700 & - & - & . & . & - & - \\
\hline
\end{tabular}


Table 11. Composition of Mn oxide majors as reported by NGDC (cont'd).

\begin{tabular}{|c|c|c|c|c|c|c|c|c|c|}
\hline MMBIB & UCHITA7601 & UCHITA7601 & UCHITA7601 & UCHTTA7601 & UCHITA7601 & UCHITA7601 & UCHITA7601 & UCHITA7601 & VANDCH7602 \\
\hline Sumplek & $9-7$ & 9.7 & $9-7$ & 9.7 & $9-7$ & 9.7 & 9.7 & $9-7$ & 01 \\
\hline Analygist & 17 & 3 & 4 & 5 & 6 & 7 & 8 & 9 & 1 \\
\hline Sumpletype & 1 & 1 & 1 & 1 & 1 & 1 & 1 & 1 & 1 \\
\hline Letitude & 44.617 & 44.617 & 44.617 & 44.617 & 44.617 & 44.617 & 44.617 & 44.617 & -16.483 \\
\hline Longitude & 170.300 & 170.300 & 170.300 & 170.300 & 170.300 & 170.300 & 170.300 & 170.300 & -145.550 \\
\hline Depth & 1365 & 1365 & 1365 & 1365 & 1365 & 1365 & 1365 & 1365 & 1270 \\
\hline$N(w t . \%)$ & 1.50 & $\cdot$ & 1.24 & 1.50 & 1.37 & $\cdot$ & 59 & 26 & $\cdot$ \\
\hline a & 1.29 & 11.8 & 2.37 & 1.17 & 1.41 & 2.02 & 1.69 & 1.75 & - \\
\hline$c_{0}$ & .190 & .250 & .190 & .440 & .360 & .430 & 330 & .700 & 1.03 \\
\hline$a$ & .168 & .120 & .098 & .160 & .136 & .098 & .092 & .052 & .120 \\
\hline Pe & 5.11 & 3.94 & 6.42 & 8.59 & 9.89 & 10.9 & 11.1 & 13.5 & 13.0 \\
\hline K & • & • & • & • & • & • & • & • & • \\
\hline$M_{8}$ & 1.96 & 5.32 & 1.09 & 1.90 & 1.47 & 1.42 & 1.04 & 1.03 & • \\
\hline $\operatorname{Mn}$ & 33.6 & 19.2 & 28.0 & 28.8 & 28.6 & 29.8 & 27.1 & 27.4 & 21.5 \\
\hline $\mathrm{Ne}$ & - & • & • & • & $\cdot$ & - & - & • & - \\
\hline $\mathbf{N i}$ & 1.440 & 880 & 1.400 & 1.320 & .960 & .960 & .480 & 520 & .560 \\
\hline $\mathbf{P}$ & • & - & • & • & • & • & - & $\cdot$ & • \\
\hline si & 3.94 & .62 & 2.59 & 3.98 & 3.89 & 1.96 & 274 & 1.74 & - \\
\hline $\mathrm{Ti}$ & • & - & • & - & • & • & • & $\cdot$ & - \\
\hline $\mathrm{H} 2 \mathrm{O}$ & - & - & - & - & . & - & - & - & - \\
\hline Axppon) & - & - & - & - & - & - & - & - & - \\
\hline Be & - & - & . & - & - & - & $\therefore$ & . & . \\
\hline $\mathrm{Cd}$ & - & - & • & - & - & - & - & - & - \\
\hline Co & - & - & - & - & - & - & - & - & - \\
\hline$\alpha$ & - & - & - & - & - & - & - & - & . \\
\hline Mo & - & - & • & • & • & • & - & - & . \\
\hline Pb & 340 & - & 840 & 1000 & 1100 & 1100 & 840 & 790 & 1700 \\
\hline $\mathrm{Pt}$ & • & . & • & - & $\cdot$ & $\cdot$ & $\cdot$ & - & $\cdot$ \\
\hline $\mathbf{v}$ & - & - & - & - & . & - & - & - & - \\
\hline $\mathbf{Y}$ & - & - & - & . & . & . & - & . & . \\
\hline $\mathrm{Zn}$ & - & - & . & - & - & - & - & - & 800 \\
\hline
\end{tabular}

\begin{tabular}{|c|c|c|c|c|c|c|c|c|c|}
\hline $\begin{array}{l}\text { MMBIB } \\
\text { Samplet }\end{array}$ & $\begin{array}{c}\text { VANDCH7602 } \\
04\end{array}$ & $\begin{array}{c}\text { VANDCH7602 } \\
04\end{array}$ & $\begin{array}{l}\text { VONSUA7801 } \\
\text { KD13-1 }\end{array}$ & $\begin{array}{l}\text { VONSUA7801 } \\
\text { KD13-1 }\end{array}$ & $\begin{array}{c}\text { VONSUA7801 } \\
\text { KD13-1 }\end{array}$ & $\begin{array}{l}\text { VONSUA7801 } \\
\text { KD13-1 }\end{array}$ & $\begin{array}{l}\text { VONSUA8401 } \\
\text { 237KD }\end{array}$ & $\begin{array}{l}\text { VONSUA3401 } \\
\text { 237KD }\end{array}$ & $\begin{array}{l}\text { VONSUA8401 } \\
237 \mathrm{KD}\end{array}$ \\
\hline Analygis:" & 1 & 2 & 1 & 2 & 3 & 4 & 0 & 1 & 2 \\
\hline Sempletypo & 1 & 1 & 8 & 8 & 8 & 8 & 8 & 8 & 8 \\
\hline Letitude & 38.067 & 38.057 & -12.910 & -12.910 & -12910 & -12.910 & 9.300 & 9.300 & 9.300 \\
\hline Longitude & -60.217 & -60.217 & 119.882 & 119.882 & 119.882 & 119.882 & -146.050 & -146.050 & -146.050 \\
\hline Depth & 3200 & 3200 & 2100 & 2100 & 2100 & 2100 & 4830 & 4830 & 4830 \\
\hline$\overline{N(w t . \%)}$ & - & $\cdot$ & .07 & .08 & .08 & .07 & - & .79 & 53 \\
\hline a & - & - & 3.19 & 243 & 249 & 25.3 & 1.25 & 1.18 & 2.50 \\
\hline$c_{0}$ & .360 & 360 & .240 & 310 & 330 & .083 & .470 & .330 & .390 \\
\hline $\mathrm{Cu}$ & .140 & .160 & .052 & .042 & .067 & .047 & .150 & .170 & .250 \\
\hline Po & 18.3 & 195 & 16.6 & 18.8 & 18.4 & 10.8 & 12.6 & 15.9 & 16.6 \\
\hline $\mathbf{K}$ & - & - & $\cdot$ & - & . & - & - & - & - \\
\hline $\mathbf{M}_{8}$ & • & - & 98 & 90 & .99 & .49 & 1.11 & .93 & 86 \\
\hline Mn & 11.3 & 120 & 18.6 & 17.0 & 17.3 & 3.66 & 20.1 & 18.8 & 19.8 \\
\hline $\mathrm{Na}$ & - & - & - & . & . & - & 1.11 & 213 & .60 \\
\hline $\mathrm{Ni}$ & .230 & .230 & .420 & 310 & 320 & .120 & .490 & 350 & .500 \\
\hline $\mathbf{P}$ & . & - & - & - & - & - & - & . & $\cdot$ \\
\hline $\mathbf{S i}$ & . & . & 4.27 & 3.96 & 4.82 & 251 & . & . & . \\
\hline $\mathrm{Ti}$ & . & . & - & - & - & - & . & - & . \\
\hline $\mathrm{H} 20$ & . & . & . & - & . & . & . & . & . \\
\hline$A x(p p m)$ & . & - & . & . & . & . & 280 & 230 & 90 \\
\hline Be & . & . & . & - & . & . & - & - & . \\
\hline cd & - & - & . & - & - & . & - & • & . \\
\hline$C_{0}$ & • & . & . & . & . & . & 800 & 1100 & 540 \\
\hline$\sigma$ & - & - & 60 & 70 & 70 & 90 & - & . & . \\
\hline Mo & • & . & • & - & . & • & - & . & . \\
\hline $\mathbf{P b}$ & 1000 & 1000 & 1800 & 2400 & 1600 & 720 & 850 & 720 & 690 \\
\hline $\mathrm{Pt}$ & . & . & - & - & - & - & . & - & - \\
\hline $\mathrm{v}$ & . & . & - & . & . & . & . & . & . \\
\hline $\mathrm{Y}$ & . & . & - & . & . & . & . & . & . \\
\hline $\mathrm{Zn}_{\mathbf{n}}$ & 800 & 800 & 760 & 720 & 810 & 460 & 670 & 980 & 620 \\
\hline
\end{tabular}


Table 11. Composition of Mn oxide majors as reported by NGDC (cont'd).

\begin{tabular}{|c|c|c|c|c|c|c|c|c|c|}
\hline $\begin{array}{l}\text { MMBIB } \\
\text { Samplo: }\end{array}$ & $\begin{array}{c}\text { VONSUAS401 } \\
\text { 237KD }\end{array}$ & $\begin{array}{c}\text { VONSUA8401 } \\
237 \mathrm{KD}\end{array}$ & $\begin{array}{c}\text { VONSUA8401 } \\
237 \mathrm{KD}\end{array}$ & $\begin{array}{c}\text { XAVIAA8201 } \\
\text { D41 }\end{array}$ & $\begin{array}{l}\text { XAVIAA8201 } \\
\text { D42-1 }\end{array}$ & $\begin{array}{l}\text { XAVIAAB201 } \\
\text { D42-1-1 }\end{array}$ & $\begin{array}{l}\text { XAVIAA8201 } \\
\text { D42F }\end{array}$ & $\begin{array}{c}\text { XAVIAAB201 } \\
\text { D43B }\end{array}$ & $\begin{array}{l}\text { XAVLAA8201 } \\
\text { D46-1 }\end{array}$ \\
\hline Analyois: & 21 & 22 & 3 & 1 & 1 & 1 & 1 & 1 & 1 \\
\hline Sempletype & 8 & 8 & 8 & 1 & 1 & 1 & 1 & 1 & 1 \\
\hline Latipude & 9.300 & 9.300 & 9.300 & -8.000 & -8.000 & .8 .000 & -8.000 & 8.000 & -8.000 \\
\hline Langitude & -146.050 & -146.050 & -146.050 & -34.000 & -34.000 & -34.000 & -34.000 & -34.000 & -34.000 \\
\hline Depth & 4830 & 4830 & 4830 & . & $\cdot$ & $\cdot$ & 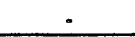 & - & - \\
\hline$\overline{\mathrm{A}(w t . q)}$ & .70 & .53 & $\cdot$ & 1.13 & 1.22 & 1.12 & 1.93 & 4.38 & 1.90 \\
\hline C. & 241 & 2.50 & 1.38 & 2.83 & 6.73 & 1.25 & 4.99 & 148 & 1.42 \\
\hline$c_{0}$ & 320 & 390 & 320 & .120 & .430 & .460 & .190 & .190 & 230 \\
\hline cu & .160 & .250 & .250 & .013 & .038 & .021 & .025 & .034 & .037 \\
\hline Po & 15.0 & 126 & 14.0 & 30.6 & 19.6 & 26.4 & 17.1 & 13.4 & 23.5 \\
\hline $\mathbf{K}$ & - & 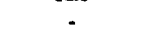 & • & .67 & .66 & .68 & 204 & 249 & 240 \\
\hline $\mathbf{M g}_{\mathbf{g}}$ & .78 & .86 & .94 & - & - & $\cdot$ & • & $\cdot$ & - \\
\hline $\mathrm{Mn}$ & 18.3 & 21.0 & 20.9 & 10.9 & 16.8 & 12.5 & 9.93 & 6.81 & 11.7 \\
\hline $\mathrm{Ne}$ & - & . & 1.67 & • & . &. & . & . & . \\
\hline $\mathbf{N i}$ & .400 & .500 & .400 & .260 & 1.020 & 270 & 370 & .670 & .360 \\
\hline $\mathbf{P}$ & • & - & • & .59 & 52 & .51 & 2.98 & 53 & 32 \\
\hline Si & - & - & - & 2.26 & 1.61 & 3.13 & 5.12 & 11.57 & 4.73 \\
\hline $\mathrm{Ti}$ & - & - & . & .10 & .28 & 32 & .21 & 1.17 & 27 \\
\hline $\mathrm{H} 20$ & - & - & - & - & . & - & . & 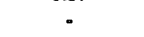 & . \\
\hline$A x(p p \infty)$ & - & - & 190 & - & - & - & - & - & • \\
\hline Ba & - & - & 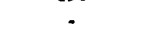 & 1500 & 3500 & 2400 & - & - & 4000 \\
\hline Cd & - & . & - & $\cdot$ & - & - & - & - & - \\
\hline$C_{e}$ & - & - & 1300 & - & - & . & . & - & - \\
\hline$a$ & - & - & . & - & . & . & . & . & . \\
\hline Mo & - & - & - & - & . & . & - & . & . \\
\hline $\mathrm{Pb}$ & 790 & 690 & 490 & - & . & . & - & - & - \\
\hline$P_{t}$ & $\cdot$ & - & - & • & . & . & - & • & - \\
\hline$v$ & - & - & - & 1100 & 800 & 1100 & 280 & 280 & 900 \\
\hline $\mathbf{Y}$ & - & • & - & - & - & . & . & . & . \\
\hline $\mathbf{Z n}$ & 560 & 620 & 540 & 900 & 1400 & 1000 & 730 & 530 & 1000 \\
\hline
\end{tabular}

\begin{tabular}{|c|c|c|c|c|c|c|c|c|c|}
\hline $\begin{array}{l}\text { MMBIB } \\
\text { Samplell }\end{array}$ & $\begin{array}{l}\text { XAVIAA8201 } \\
\text { D46-1-1 }\end{array}$ & $\begin{array}{l}\text { XAVLAA8201 } \\
\text { D48-1-1 }\end{array}$ & $\begin{array}{c}\text { XAVLAA8201 } \\
\text { D48A }\end{array}$ & $\begin{array}{c}\text { XAVIAA8201 } \\
\text { D49A }\end{array}$ & $\begin{array}{l}\text { XAVLAA8201 } \\
\text { DAB1 }\end{array}$ & $\begin{array}{l}\text { XAVIAA8201 } \\
\text { DS3 }\end{array}$ & $\begin{array}{c}\text { XAVIAA8201 } \\
\text { D53-1 }\end{array}$ & $\begin{array}{l}\text { XAVIAA8201 } \\
\text { DS3-1-1 }\end{array}$ & $\begin{array}{c}\text { XAVIAA8201 } \\
\text { DS3B }\end{array}$ \\
\hline Analynis" & 1 & 1 & 1 & 1 & 1 & 1 & 1 & 1 & 1 \\
\hline Sampletype & 1 & 1 & 1 & 1 & 1 & 1 & 1 & 1 & 1 \\
\hline Latitude & -8.000 & -8.000 & -8.000 & -8.000 & -8.000 & -8.000 & -8.000 & 8.000 & -8.000 \\
\hline Longitude & -34.000 & -34.000 & -34.000 & -34.000 & -34.000 & -34.000 & -34.000 & -34.000 & .34 .000 \\
\hline Dopth & $\cdot$ & $\cdot$ & $\cdot$ & $\cdot$ & $\cdot$ & • & • & $\cdot$ & • \\
\hline$A(w t . \%)$ & 1.43 & 1.68 & 1.55 & 1.37 & 2.92 & .77 & .68 & 1.13 & .90 \\
\hline a & 5.91 & .99 & 1.23 & .94 & 1.52 & 1.60 & 1.51 & 1.91 & 1.23 \\
\hline$C_{0}$ & 340 & .270 & .260 & 310 & .160 & 340 & 320 & .600 & 290 \\
\hline$C$ & .068 & .050 & .019 & .033 & .022 & .015 & .022 & .022 & .013 \\
\hline$F_{0}$ & 21.2 & 18.9 & 18.5 & 23.3 & 13.0 & 24.7 & 23.2 & 29.2 & 21.3 \\
\hline $\mathbf{K}$ & .65 & .70 & .62 & 1.90 & 1.51 & 87 & .64 & .64 & 1.08 \\
\hline $\mathbf{M g}$ & - & - & - & - & - & $\cdot$ & - & - & - \\
\hline Mn & 15.2 & 11.0 & 10.9 & 10.5 & 5.47 & 208 & 178 & 13.5 & 16.3 \\
\hline Ne & • & $\cdot$ & - & - & - & - & - & • & - \\
\hline $\mathbf{N i}$ & 1.160 & .190 & .160 & .150 & .120 & .280 & .230 & .150 & .220 \\
\hline $\mathbf{P}$ & 2.20 & .27 & .43 & .47 & 39 & .40 & .40 & .64 & 36 \\
\hline Si & 2.14 & 4.98 & 5.52 & 4.72 & 7.42 & 1.68 & 1.31 & 2.84 & 203 \\
\hline $\mathbf{T i}$ & .24 & .46 & .40 & .46 & .70 & .30 & .20 & .50 & .19 \\
\hline H2O- & • & • & - & - & - & - & - & • & - \\
\hline$A x(p p m)$ & - & - & - & - & - & • & • & - & - \\
\hline $\mathrm{Ba}$ & 2400 & 1900 & - & 900 & - & 4700 & 5900 & 1600 & 3700 \\
\hline Cd & - & - & - & - & - & - & . & - & - \\
\hline$c$ & - & - & - & - & - & - & . & - & - \\
\hline$a$ & - & - & - & - & - & - & . & - & - \\
\hline Mo & - & . & - & . & . & - & . & . & . \\
\hline $\mathrm{Pb}$ & . & - & . & . & . & . & . & - & . \\
\hline $\mathbf{P t}$ & - & • & - & - & • & - & • & • & • \\
\hline V & 900 & 600 & 170 & 880 & 350 & 1200 & 1200 & 1300 & 1600 \\
\hline $\mathbf{Y}$ & • & - & • & - & • & - & . & - & - \\
\hline $\mathbf{Z n}$ & 1300 & 700 & 360 & 480 & 330 & 960 & 800 & 800 & 840 \\
\hline
\end{tabular}


Table 11. Composition of Mn oxide majors as reported by NGDC (cont'd).

\begin{tabular}{|c|c|c|c|c|c|c|c|c|c|}
\hline $\begin{array}{l}\text { MMBIB } \\
\text { Samploy }\end{array}$ & $\begin{array}{c}\text { XAVIAA8201 } \\
\text { D7-1 }\end{array}$ & $\begin{array}{c}\text { XAVLAA8201 } \\
\text { D7-1-1 }\end{array}$ & $\begin{array}{c}\text { XAVIAA8201 } \\
\text { D78-1-1 }\end{array}$ & $\begin{array}{c}\text { XAVIAA8201 } \\
\text { D7B1 }\end{array}$ & $\begin{array}{l}\text { XAVIAA8201 } \\
\text { D7C }\end{array}$ & $\begin{array}{l}\text { XAVIAA8201 } \\
\text { D7CPL-2 }\end{array}$ & $\begin{array}{l}\text { XAVIAA8201 } \\
\text { D7CPL1 }\end{array}$ & $\begin{array}{l}\text { XAVIAA8201 } \\
\text { DTD }\end{array}$ & $\begin{array}{c}\text { XAVLAA8201 } \\
\text { P81 }\end{array}$ \\
\hline Analyais: & 1 & 1 & 1 & 1 & 1 & 1 & 1 & 1 & 1 \\
\hline Sarmpletype & 1 & 1 & 1 & 1 & 1 & 1 & 1 & 1 & 1 \\
\hline Letitude & -8.000 & -8.000 & -8.000 & 8.000 & .8 .000 & -8.000 & -8.000 & -8.000 & -8.000 \\
\hline Longitude & -34.000 & -34.000 & .34 .000 & $-34,000$ & .34 .000 & -34.000 & -34.000 & .34 .000 & -37.000 \\
\hline Depth & - & $\cdot$ & - & - & $\cdot$ & - & - & 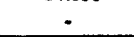 & 260 \\
\hline$\overline{\mathrm{Al}(\mathrm{w} t \mathrm{~L})}$ & .76 & 1.25 & $\cdot$ & 1.65 & 239 & 1.63 & 1.36 & 1.62 & 51 \\
\hline a & 156 & 1.84 & 7.11 & 6.33 & 6.54 & .79 & 1.36 & 1.17 & 2.04 \\
\hline$c_{0}$ & .770 & .670 & .170 & .120 & .160 & 350 & 1.02 & 540 & .170 \\
\hline $\overrightarrow{C a}$ & .021 & .020 & .065 & .063 & .074 & .025 & .018 & .018 & .018 \\
\hline Po & 25.1 & 278 & 10.4 & 8.05 & 10.1 & 18.4 & 21.8 & 24.7 & 26.8 \\
\hline $\mathbf{K}$ & 59 & .65 & 1.40 & 96 & 1.36 & 1.06 & .76 & 1.22 & 51 \\
\hline $\mathrm{Mg}$ & - & . & 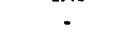 & 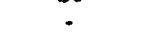 & - & - & 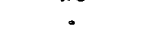 & 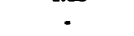 & $\because$ \\
\hline $\mathrm{Mn}$ & 175 & 14.9 & 13.3 & 9.19 & 10.5 & 8.77 & 16.3 & 125 & 14.3 \\
\hline $\mathrm{Ne}$ & . & . & . & 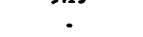 &. & . & . & 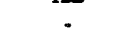 &. \\
\hline $\mathrm{Ni}$ & 240 & .180 & 1.390 & 940 & 1.280 & ,180 & .340 & .220 & .570 \\
\hline $\mathbf{P}$ & .51 & 54 & 2.76 & 1.44 & 288 & .40 & 51 & 50 & .69 \\
\hline $\mathbf{S i}$ & 1.10 & 1.79 & 4.28 & 3.30 & 4.28 & 2.86 & 1.83 & 2.85 & .86 \\
\hline $\mathrm{Ti}$ & .45 & 51 & .58 & 51 & .60 & .33 & .64 & 42 &.$\infty 9$ \\
\hline $\mathrm{H} 20 \mathrm{O}$ & - & - & - & - & - & - & - & - & - \\
\hline$A x(p p m)$ & - & . & - & . & . & - & . & . & • \\
\hline Ba & $\mathbf{2 8 0 0}$ & 2600 & - & 1400 & 1200 & 1000 & - & - & 2400 \\
\hline Cd & . & . & - & . & . & . & . & . & - \\
\hline Ce & - & . & - & - & - & . & - & - & - \\
\hline$\alpha$ & - & . & - & - & - & - & - & - & . \\
\hline Mo & - & . & - & - & - & - & - & - & - \\
\hline $\mathrm{Pb}$ & - & - & . & - & - & - & - & . & - \\
\hline $\mathrm{Pt}_{\mathrm{t}}$ & • & - & - & - & - & - & • & • & - \\
\hline v & 1100 & 1200 & 330 & 340 & 470 & 430 & 590 & 960 & 1400 \\
\hline $\mathbf{Y}$ & .. & 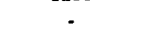 & . & 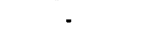 & - & - & . & . & - \\
\hline $\mathrm{Zn}$ & 950 & 750 & 1200 & 710 & 810 & 560 & 430 & 570 & 800 \\
\hline
\end{tabular}

\begin{tabular}{|c|c|}
\hline MMBIB & XAVIAA8201 \\
\hline Samplef & P9OA \\
\hline Analysis" & 1 \\
\hline Sampletype & 1 \\
\hline Letitude & -8.000 \\
\hline Longitude & .37 .000 \\
\hline Dopth & 260 \\
\hline$A l(w t . \%)$ & 1.25 \\
\hline Ca & 1.23 \\
\hline Co & .270 \\
\hline Cu & .022 \\
\hline$P_{0}$ & 19.6 \\
\hline $\mathbf{K}$ & .68 \\
\hline $\mathbf{M g}$ & • \\
\hline $\mathrm{Mn}$ & 8.94 \\
\hline $\mathrm{Na}$ & • \\
\hline $\mathbf{N i}$ & .150 \\
\hline $\mathbf{P}$ & .36 \\
\hline $\mathbf{S i}$ & 4.02 \\
\hline $\mathbf{T i}$ & .44 \\
\hline $\mathrm{H} 20$ & - \\
\hline$A x$ (ppren) & - \\
\hline Ba & - \\
\hline Cd & - \\
\hline ce & - \\
\hline$a$ & - \\
\hline Mo & - \\
\hline $\mathrm{Pb}$ & - \\
\hline $\mathrm{Pt}$ & • \\
\hline $\mathbf{v}$ & 720 \\
\hline $\mathbf{Y}$ & • \\
\hline $\mathbf{Z n}$ & 560 \\
\hline
\end{tabular}


Table 12.Chemical composition of ferromanganese crusts as reported in

Glasby (written communication), Dillard and Crowther (1984) and

Exon (1982).

\begin{tabular}{|c|c|c|c|c|c|c|c|c|c|}
\hline Sequence: & 316004 & 351016 & 352001 & 352002 & 352000 & 352004 & 352005 & 352006 & 387001 \\
\hline Letitude & -9.997 & -19.920 & -12752 & -10.033 & -10.097 & -11.138 & -11.555 & -19.225 & -21.303 \\
\hline Langituda & -161.033 & -157.655 & -160.713 & -161.150 & -161.090 & -165.567 & -165.445 & -169.913 & -159.738 \\
\hline Depth & 1843 & 1744 & 4469 & 1863 & 2600 & 1913 & 1207 & 2520 & 1272 \\
\hline Lab* & U-314 & U-367 & U-299B & U-315 & $\mathrm{U}-316 \mathrm{~B}$ & U-333B & U-336B & U-358 & U.375 \\
\hline$F o(w t . \%)$ & 19.9 & 20.5 & 21.6 & 19.5 & 14.8 & 20.0 & 23.0 & 23.5 & 4.50 \\
\hline $\mathrm{Mn}$ & 23.5 & 20.1 & 14.8 & 27.1 & 16.1 & 27.0 & 215 & 17.6 & 41.3 \\
\hline $\mathrm{Cu}$ & .050 & .070 & .160 & .080 & .120 & .080 & .050 & .050 & .250 \\
\hline $\mathrm{Ni}$ & .430 & .310 & 300 & 530 & .510 & .480 & .320 & .180 & 380 \\
\hline Co & 960 & .640 & .350 & .850 & .480 & .930 & .650 & 340 & .230 \\
\hline $\mathbf{S i}$ & 10.2 & 9.89 & 19.6 & 7.02 & 23.3 & 6.90 & 9.25 & 12.3 & 4.21 \\
\hline Al & 2.36 & 2.47 & 6.18 & 1.48 & 6.08 & 1.43 & 232 & 282 & 3.37 \\
\hline $\mathbf{T i}$ & 1.85 & 1.81 & 1.90 & 1.56 & 2.65 & 1.78 & 1.62 & 1.29 & 1.01 \\
\hline ca & 5.44 & 8.30 & 4.04 & 5.00 & 5.54 & 4.45 & 5.92 & 8.05 & 6.97 \\
\hline $\mathbf{K}$ & 35 & .39 & 1.45 & 36 & 1.80 & .35 & 51 & 31 & .16 \\
\hline $\mathbf{P}$ & 1.03 & 1.17 & 1.72 & 1.14 & 1.41 & 1.06 & 1.37 & 1.14 & .44 \\
\hline As(ppm) & - & - & - & - & - & - & - & - & - \\
\hline $\mathrm{Ba}$ & - & $:$ & - & - & - & - & - & - & - \\
\hline Mo & $\cdot$ & - & • & - & $\cdot$ & $\cdot$ & - & - & - \\
\hline $\mathbf{P b}$ & 61 & 440 & 118 & 410 & 164 & 440 & 380 & 176 & 215 \\
\hline Sr & - & • & - & - & - & • & • & • & - \\
\hline $\mathbf{Y}$ & • & • & - & - & - & - & - & - & - \\
\hline Zn & 740 & 690 & 740 & 740 & 810 & 740 & 710 & 640 & 3300 \\
\hline $\mathbf{z}$ & - & - & - & - & - & • & • & • & • \\
\hline
\end{tabular}

\begin{tabular}{|c|c|c|c|c|c|c|c|c|c|}
\hline Sequencoll & c5s3164 & 350006 & 351017 & 387002 & 353007 & 389002 & 389003 & 389004 & 389005 \\
\hline Letitude & 12.050 & -16.483 & -13.883 & -22.583 & -14.137 & -22766 & -22.768 & -23.025 & -21.850 \\
\hline Longitude & 165.000 & -145.550 & -150.583 & -150.800 & -171.120 & -175.804 & -175.628 & -175.957 & -175.077 \\
\hline Depth & 2090 & 1270 & 3695 & 807 & 3100 & 800 & 700 & 650 & 750 \\
\hline Lebi & MP43 & DWHDI6 & M2P50 & AMPH9D & WS-79(1)-10 & $3-9$ & $5-10$ & 8.5 & 1.2 \\
\hline$F o(w t . \%)$ & 11.1 & 135 & 15.8 & 124 & 15.9 & 220 & 25.1 & 21.6 & 25.1 \\
\hline Mn & 22.6 & 226 & 17.0 & 24.2 & 10.3 & 18.8 & 18.0 & 16.1 & 18.0 \\
\hline Cu & .197 & .186 & .214 & .160 & .200 & .053 & .042 & .042 & .039 \\
\hline $\mathrm{Ni}$ & .700 & .359 & .294 & .414 & .130 & .310 & .270 & .250 & .280 \\
\hline Co & .805 & 1.18 & .483 & 1.99 & .160 & .570 & .560 & .440 & 510 \\
\hline $\mathbf{S i}$ & .81 & .93 & 4.91 & $<40$ & - & - & - & - & - \\
\hline Al & .48 & .44 & 1.32 & .10 & - & - & - & - & - \\
\hline $\mathbf{T i}$ & 80 & 1.02 & 1.08 & 1.17 & - & - & - & - & - \\
\hline Ca & 3.14 & 2.28 & 1.77 & .29 & - & - & - & - & - \\
\hline $\mathbf{K}$ & .26 & .36 & 55 & 237 & - & - & - & $\cdot$ & - \\
\hline $\mathbf{P}$ & .75 & .40 & .37 & .37 & $\cdot$ & - & - & - & - \\
\hline As(ppm) & 190 & 230 & 180 & 310 & - & - & - & - & - \\
\hline Ba & 2500 & 2100 & 1800 & 2200 & - & - & - & - & - \\
\hline Mo & 810 & 520 & 0 & 560 & - & $\cdot$ & - & - & - \\
\hline $\mathrm{Pb}$ & 1900 & 2400 & 1100 & 2900 & $\cdot$ & - & $\cdot$ & $\cdot$ & - \\
\hline Sr & 1600 & 1600 & 1400 & 1700 & - & - & - & - & - \\
\hline $\mathbf{Y}$ & 140 & 155 & 200 & 120 & - & - & $\cdot$ & - & - \\
\hline $\mathbf{Z n}$ & 1100 & 910 & 700 & 650 & - & - & - & - & - \\
\hline $\mathbf{Z}$ & 570 & 525 & 620 & 410 & - & - & - & - & - \\
\hline
\end{tabular}


Table 13.Chemical composition of ferromanganese crusts as reported in Volkov (1976) and Dymond and others (1984)

\begin{tabular}{|c|c|c|c|c|c|c|c|c|}
\hline Sequencoll & 055012 & Sequencolit & 010012 & 010013 & 010014 & Sequencoil & 010013 & 010014 \\
\hline Latitudo & 17.000 & Latitude & 6.658 & 6.658 & 6.658 & Latitude & 6.658 & 6.658 \\
\hline Langitude & 170.500 & Longitude & -92.842 & -92842 & -92842 & Longitude & -92.842 & -92.842 \\
\hline Depth & 4090 & Depth & 3450 & 3450 & 3450 & Depth & 3450 & 3450 \\
\hline Lab" & 611 & Lab" & MP9842 & MP9843 & MP9844 & Leb" & MP9843 & MP9844 \\
\hline $\mathrm{Fo}(w t, \%)$ & 16.7 & $\mathrm{Na}$ (wt.\%) & 1.91 & 1.78 & 1.83 & $\mathrm{~F} \alpha(w \mathrm{t} \%)$ & 14.0 & 145 \\
\hline Mn & 14.7 & $\mathbf{M g}$ & 1.18 & 1.16 & 1.12 & $\mathrm{Ba}$ & 1800 & 1300 \\
\hline $\mathbf{T i}$ & 1.28 & Al & 1.12 & 1.17 & 1.21 & Sc(ppm) & 7.6 & 8.1 \\
\hline $\mathbf{P}$ & AO & $\mathbf{S i}$ & 4.91 & 4.78 & 5.10 & $a$ & 19.2 & 11.6 \\
\hline $\mathrm{Ba}(\mathrm{ppm})$ & 900 & $\mathbf{K}$ & .51 & .51 & .51 & Co & 999.9 & 1155. \\
\hline a & .090 & C. & 2.63 & 2.46 & 242 & Sb & 16 & 16 \\
\hline $\mathrm{Ni}$ & .279 & $\mathbf{T i}$ & .45 & .49 & .52 & Le & 136 & 131 \\
\hline Co & .319 & Mn & 22.3 & 21.7 & 21.2 & Hf & 11. & 11. \\
\hline$V(p p m)$ & 470 & Fo & 15.8 & 18.1 & 18.4 & Th & 12 & 13. \\
\hline$Z x(p p m)$ & 600 & Co & .114 & .117 & .132 & $\mathbf{U}$ & 7.5 & 8.3 \\
\hline$W($ ppen) & 88 & $\mathbf{N i}$ & .583 & .521 & .527 & & & \\
\hline Mo(ppm) & 176 & $\mathrm{Cu}$ & .070 & .065 & .061 & & & \\
\hline \multirow[t]{2}{*}{$A x(p p m)$} & 115 & $Z n$ (ppon) & 970 & 750 & 770 & & & \\
\hline & & $B a(p p m)$ & 1700 & 1400 & 1400 & & & \\
\hline
\end{tabular}


Table 14. Chemical composition of ferromanganese crusts as reported in Goddard and others (1987).

\begin{tabular}{|c|c|c|c|c|c|c|c|c|c|c|}
\hline Sequencel" & 002003 & 002004 & 003002 & 003003 & 003004 & 003005 & 003006 & 003007 & 003008 & 003009 \\
\hline Latitude & 8.917 & 8.917 & 9.017 & 9.017 & 9.017 & 9.017 & 4.283 & 4.217 & 7.917 & 7.917 \\
\hline Longitudo & -19.767 & -19.767 & -21.033 & -21.033 & -21.033 & -21.033 & -20.867 & -21.017 & -20.983 & -20.983 \\
\hline $\begin{array}{l}\text { Depth } \\
\text { Sample:f }\end{array}$ & $\begin{array}{c}1611 \\
s-1224-2\end{array}$ & $\begin{array}{c}1611 \\
S-1224-5\end{array}$ & $\begin{array}{c}834 \\
\text { s-1225-4 }\end{array}$ & $\begin{array}{c}834 \\
\text { S-1225-6 }\end{array}$ & $\begin{array}{c}834 \\
\text { s-1225-7 }\end{array}$ & $\begin{array}{c}834 \\
\text { S. } 1225-10\end{array}$ & $\begin{array}{c}2934 \\
S 1233-1\end{array}$ & $\begin{array}{c}3350 \\
s 1234-1\end{array}$ & $\begin{array}{c}1621 \\
S 1240-1\end{array}$ & $\begin{array}{c}1621 \\
S 1240-2\end{array}$ \\
\hline Fo & 25.2 & 23.5 & 17.9 & 18.3 & 17.1 & 17.7 & 21.8 & 20.4 & 21.7 & 19.6 \\
\hline Mn & 15.7 & 16.1 & 21.3 & 10.8 & 22.2 & 21.5 & 17.9 & 17.2 & 19.1 & 19.6 \\
\hline $\mathrm{Ne}$ & .79 & .75 & 1.26 & 3.17 & 1.19 & 2.04 & .83 & 1.00 & .70 & .67 \\
\hline $\mathbf{K}$ & .15 & .14 & .20 & .15 & .22 & 23 & .19 & .21 & .16 & .11 \\
\hline Ca & 1.97 & 2.11 & 3.41 & 4.78 & 2.33 & 230 & 1.92 & 1.53 & 1.89 & 1.47 \\
\hline $\mathbf{M g}$ & 1.20 & 1.32 & 1.38 & 2.29 & 1.52 & 1.65 & 1.29 & 1.50 & 1.55 & 1.86 \\
\hline Ca & .029 & .037 & .032 & .029 & .027 & .030 & .066 & .067 & .770 & .044 \\
\hline $\mathbf{Z n}$ & .069 & .068 & .049 & .048 & .046 & .059 & .071 & .063 & .089 & .064 \\
\hline $\mathbf{N i}$ & .177 & .237 & .243 & .215 & 271 & .262 & .247 & .252 & 373 & .379 \\
\hline Co & .435 & 552 & .921 & .693 & 1.000 & 1.01 & 355 & .692 & .477 & .412 \\
\hline $\mathrm{Ti}$ & $\cdot$ & - & $\cdot$ & $\cdot$ & - & $\cdot$ & $\cdot$ & $\cdot$ & .880 & 1.16 \\
\hline Thicknear(mm.) & 39 & 39 & 5 & 2 & 5 & 2 & 15 & 13 & 20 & 20 \\
\hline Substrate & Basalt & Basealt & Phospharito & Phoopharite & Phoopharite & Phosphorite & & & & Sediment \\
\hline
\end{tabular}

\begin{tabular}{|c|c|c|c|c|c|c|c|c|c|c|}
\hline Sequencei" & 115016 & 115017 & 114007 & 114008 & 114009 & 114010 & 115018 & 003010 & $\infty 03011$ & 003012 \\
\hline Latitude & 39.867 & 38.833 & 36.850 & 36.850 & 36.867 & 36.517 & 38.300 & 1.483 & 1.483 & 1.483 \\
\hline Langitude & -67.367 & -64.067 & -58.817 & -58.817 & -58.800 & -59.383 & -62.933 & -24.833 & -24.833 & .24 .833 \\
\hline Depth & 3423 & 3793 & 3660 & 3660 & 2550 & 3175 & 3660 & 2210 & 2210 & 2210 \\
\hline Sempio\# & All85-2-2 & AII85-11-24 & Al185-16-10 & AII85-16-17 & An85-17-11 & All85-18-1 & KNR61-28-15 & S-1197B-1 & S-1197B-2 & S-11978-3 \\
\hline Fo & 16.7 & 11.0 & 15.0 & 13.9 & 21.6 & 16.8 & 10.1 & 19.5 & 21.6 & 155 \\
\hline Mn & 15.4 & 13.8 & 16.8 & 16.4 & 18.5 & 17.3 & 13.7 & 19.4 & 16.9 & 11.1 \\
\hline $\mathrm{Na}$ & 54 & .56 & .45 & .48 & .76 & .61 & 53 & .93 & .93 & 1.26 \\
\hline $\mathbf{K}$ & .20 & .23 & .15 & .14 & .12 & .14 & .18 & .24 & .22 & .85 \\
\hline a & .61 & 1.03 & 1.09 & 1.39 & 2.63 & 1.39 & 2.00 & 2.03 & 1.54 & 5.20 \\
\hline $\mathbf{M} \mathbf{8}$ & 1.87 & 1.44 & 1.54 & 1.01 & 1.03 & 1.08 & 1.16 & 132 & 1.26 & 1.82 \\
\hline $\mathrm{Cu}$ & .032 & .083 & .050 & .038 & .035 & .031 & .060 & .089 & .078 & .098 \\
\hline $\mathbf{Z n}$ & .043 & .038 & .039 & .038 & .059 & .037 & .047 & .074 & .061 & .076 \\
\hline $\mathbf{N i}$ & 337 & .240 & .117 & .143 & .150 & .112 & .225 & .348 & .188 & .478 \\
\hline Co & .090 & .048 & .062 & .066 & .135 & .112 & .063 & .533 & .649 & .151 \\
\hline$\pi$ & .447 & .245 & .745 & .718 & 1.39 & 1.14 & 348 & $\cdot$ & 1.07 & $\cdot$ \\
\hline Thickenesx (mom.) & 70 & 25 & 12 & 8 & 10 & 4 & 3 & 72 & 22 & 26 \\
\hline Substrate & Basalt & Limestane & Baralt & $\begin{array}{c}\text { Montmorillonite } \\
\text { day }\end{array}$ & $\begin{array}{l}\text { Phillipaito } \\
\text { clay }\end{array}$ & Besalt & $\begin{array}{l}\text { Glecial } \\
\text { ematic }\end{array}$ & Basalt & $\begin{array}{l}\text { Basalt } \\
\text { broccia }\end{array}$ & Banalt \\
\hline
\end{tabular}


Table 14. Chemical composition of ferromanganese crusts as reported in Goddard and others (1987).

\begin{tabular}{|c|c|c|c|c|c|c|c|c|c|c|}
\hline Sequencell & 003013 & 003014 & 301002 & 301003 & 303001 & 004001 & 004002 & 304001 & 002005 & 002006 \\
\hline Letitude & 1.483 & 1.483 & -4.117 & -3.800 & -.900 & .600 & .933 & -.167 & .400 & .233 \\
\hline Langitudo & -24.833 & -24.833 & -12133 & -11.083 & -30.133 & -32933 & -32.933 & 43.367 & -18.483 & -16.933 \\
\hline Depth & 2210 & 2210 & 3371 & 3440 & 3941 & 3652 & 2643 & 4240 & 6470 & 3108 \\
\hline Surmpleat & S-1197B-4 & S-1197B-6 & All42-1-8 & All42-2-6 & CH35-4-1 & CHBS-2-1B & AII20-34-13 & AII20-9-2 & Al120-11-16 & AII20-14-2 \\
\hline Fo & 18.6 & 19.5 & 19.1 & 8.23 & 15.6 & 27.7 & 19.6 & 9.10 & 13.8 & 24.5 \\
\hline $\mathrm{Mn}$ & 17.3 & 18.7 & 14.4 & 9.15 & 13.6 & 12.4 & 10.4 & 15.5 & 7.50 & 18.9 \\
\hline $\mathrm{Na}$ & 96 & .96 & 89 & .67 & 1.00 & 1.14 & 90 & .80 & 91 & .66 \\
\hline $\mathbf{K}$ & 30 & .20 & .18 & .14 & .21 & .16 & .15 & .21 & 52 & .14 \\
\hline C. & 2.76 & 2.12 & 1.89 & 6.75 & 230 & 186 & 3.08 &.$\pi$ & 1.13 & 1.58 \\
\hline $\mathbf{M g}$ & 1.39 & 1.58 & 1.38 & 3.62 & .96 & 1.12 & 83 & 281 & 1.87 & 1.16 \\
\hline c. & .083 & .088 & .123 & .020 & .042 & .069 & .041 & .196 & .069 & .037 \\
\hline $\mathbf{z n}$ & .072 & .065 & .065 & .020 & .037 & .048 & .041 & .071 & .037 & .057 \\
\hline $\mathrm{Ni}$ & .249 & .331 & .361 & .226 & .239 & .128 & .128 & .450 & .113 & .120 \\
\hline $\mathrm{Co}_{0}$ & .584 & .641 & .119 & .271 & .420 & 202 & .225 & .030 & .065 & .125 \\
\hline $\mathbf{T i}$ & • & - & .479 & .615 & 1.57 & 1.01 & .987 & .275 & .544 & 1.05 \\
\hline Thickness(mm.) & 38 & 38 & 13 & 1 & 1 & 2 & 2 & 4 & 1 & 40 \\
\hline Substrato & Bessalt & Besalt & Metabasalt & Metagabbro & Besalt & Besalt & Besalt & Serpentinite & Besalt & \\
\hline
\end{tabular}

\begin{tabular}{|c|c|c|c|c|c|c|}
\hline Sequencol & 002007 & 003015 & 002008 & 301004 & 301005 & 002009 \\
\hline Letitude & .733 & .100 & .067 &. .133 & -.133 & .317 \\
\hline Longitude & -17.167 & -20.433 & -18.300 & -18.317 & -18.383 & -17.317 \\
\hline Depth & 4523 & 3320 & 1425 & 2135 & 3200 & 2950 \\
\hline Samplet & AII20-16-93 & AII20-23-61 & AII60-14-2 & All60-15-7 & All60-17-14 & All60-19-2 \\
\hline Fe & 7.32 & 16.2 & 26.4 & 17.5 & 14.4 & 15.0 \\
\hline Mn & 4.47 & 13.9 & 8.78 & 125 & 10.4 & 13.7 \\
\hline Na & .31 & .75 & .74 & .68 & 56 & 98 \\
\hline $\mathbf{K}$ & .18 & .19 & .11 & .12 & .15 & .21 \\
\hline C. & 1.02 & 1.58 & 1.37 & 1.87 & 1.36 & 1.23 \\
\hline $\mathbf{M g}$ & 217 & .89 & 1.17 & .91 & 2.01 & 1.13 \\
\hline $\mathrm{Cu}$ & .048 & .076 & .147 & .055 & .061 & .107 \\
\hline $\mathbf{z n}$ & .026 & .051 & .062 & .041 & .027 & .038 \\
\hline $\mathrm{Ni}$ & .064 & .247 & .142 & .100 & .088 & .182 \\
\hline Co & .029 & .179 & .234 & .118 & .074 & .069 \\
\hline $\mathrm{Ti}$ & .364 & .940 & 1.25 & .755 & 547 & 532 \\
\hline Thickness(mm.) & 1 & 5 & 8 & 6 & 1 & 14 \\
\hline \multirow[t]{2}{*}{ Substrato } & Metagabbro & Gabbro & Metagabbro & Metagabbro & Amphibolits & Metagabbro \\
\hline &. & • & Besalt & . & 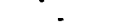 & 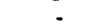 \\
\hline
\end{tabular}


Table 15. Chemical composition of ferromanganese crust layers as reported in Goddard and others (1987).

\begin{tabular}{|c|c|c|c|c|c|c|c|c|c|c|c|}
\hline Soquancoll & 003010 & 003010 & 003010 & 003011 & 003011 & 003012 & 003012 & 003012 & 003013 & 003013 & 003013 \\
\hline Latitude & 1.483 & 1.483 & 1.483 & 1.483 & 1.483 & 1.483 & 1.483 & 1.483 & 1.483 & 1.483 & 1.483 \\
\hline Langitude & -24.833 & -24.833 & -24.833 & -24.833 & -24.833 & -24.833 & -24.833 & -24.833 & -24.833 & -24.833 & .24 .833 \\
\hline Depth & 2210 & 2210 & 2210 & 2210 & 2210 & 2210 & 2210 & 2210 & 2210 & 2210 & 2210 \\
\hline Sub-senoplot" & S-1197B-1a & S-1197B-1b & S-1197B-lc & S-1197B-2a & S-1197B-2b & S-1197B-3a & S-1197B-3b & S-1197B-3c & S-1197B-4a & S-1197B-4b & S-1197B-4c \\
\hline \multicolumn{12}{|l|}{ Position from } \\
\hline Surfaco(mon) & $0-3$ & $3-27$ & $27-49$ & $0-6$ & $6-22$ & $0-7$ & 7.14 & 1426 & $0-6$ & $6-23$ & $23-38$ \\
\hline$P_{0}$ & 18.7 & 19.4 & 19.9 & 20.4 & 21.8 & 20.4 & 10.1 & 16.5 & 18.1 & 18.5 & 19.2 \\
\hline Mn & 17.7 & 20.8 & 19.9 & 123 & 16.8 & 153 & 5.7 & 13.0 & 13.2 & 19.6 & 19.2 \\
\hline $\mathrm{Na}$ & 1.04 & 1.03 & .71 & 84 & 1.10 & 1.06 & 1.35 & 131 & .79 & 1.11 & .99 \\
\hline $\mathbf{K}$ & .26 & .27 & .19 & .24 & .22 & .43 & 1.24 & .78 & .33 & .29 & 28 \\
\hline c. & 286 & 1.54 & 1.69 & 5.75 & 1.51 & 1.59 & 9.09 & 4.11 & 5.33 & 1.43 & 1.47 \\
\hline $\mathbf{M g}_{\mathbf{8}}$ & 1.48 & 1.38 & 1.11 & 158 & 1.31 & 207 & 1.79 & 159 & 1.33 & 1.43 & 1.44 \\
\hline $\mathrm{Cu}$ & .089 & .118 & .064 & .087 & .084 & .108 & .072 & .117 & .072 & .093 & .085 \\
\hline $\mathbf{N i}$ & .369 & .377 & .298 & .245 & .196 & .499 & .299 & .684 & .221 & .258 & .270 \\
\hline$C_{0}$ & .454 & .649 & .495 & .405 & .749 & .175 & .080 & .201 & .352 & .755 & .651 \\
\hline $\mathrm{Zn}$ & .081 & .067 & .075 & .063 & .062 & .106 & .049 & .077 & .068 & .067 & .082 \\
\hline
\end{tabular}

\begin{tabular}{|c|c|c|c|c|c|c|c|c|c|c|c|}
\hline Sequencel & 003014 & 003014 & 003014 & 002003 & 002003 & 002003 & 002004 & 002004 & 002004 & 003006 & 003006 \\
\hline Latitude & 1.483 & 1.483 & 1.483 & 8.917 & 8.917 & 8.917 & 8.917 & 8.917 & 8.917 & 4.283 & 4.283 \\
\hline Longitude & -24.833 & -24.833 & .24 .833 & -19.767 & -19.767 & -19.767 & -19.767 & -19.767 & -19.767 & -20.867 & -20.867 \\
\hline Depth & 2210 & 2210 & 2210 & 1611 & 1611 & 1611 & 1611 & 1611 & 1611 & 2934 & 2934 \\
\hline Sub-semplot & S-1197B-6a & S-1197B-6b & S-1197B-6c & $s-1224-2 x$ & $S-1224-2 b$ & $S-1224-2 c$ & S-1224-5a & $s-1224-5 b$ & $S-1224-5 c$ & S1233-1 & S1233-1b \\
\hline
\end{tabular}

Powition from

\begin{tabular}{|c|c|c|c|c|c|c|c|c|c|c|c|}
\hline Surfaco(tom) & $0-5$ & $5-26$ & $26-38$ & 0.7 & $7-24$ & $24-39$ & $0-7$ & $7-22$ & 22-39 & 0.7 & $7-15$ \\
\hline $\mathrm{Pe}_{0}$ & 17.9 & 20.5 & 20.2 & 25.6 & 25.6 & 24.6 & 220 & 24.8 & 23.5 & 22.1 & 21.6 \\
\hline Mn & 19.3 & 17.2 & 19.5 & 16.2 & 15.4 & 15.6 & 15.6 & 17.2 & 16.7 & 175 & 18.2 \\
\hline $\mathbf{N a}$ & .79 & 1.02 & 1.09 & .83 & .83 & .71 & .88 & .61 & .91 & .85 & .82 \\
\hline $\mathbf{K}$ & .37 & .24 & .27 & .17 & .16 & .14 & .16 & .14 & .18 & .17 & 21 \\
\hline C. & 3.56 & 1.43 & 1.61 & 2.26 & 11.9 & 1.75 & 2.66 & 1.87 & 1.82 & 1.96 & 1.88 \\
\hline $\mathbf{M g}$ & 206 & 1.34 & 1.34 & 1.23 & 1.20 & 1.20 & 1.17 & 151 & 1.28 & 1.21 & 1.37 \\
\hline Cn & .085 & .090 & .092 & .027 & .034 & .027 & .017 & .053 & .044 & .061 & .072 \\
\hline $\mathbf{N i}$ & .533 & .202 & .254 & .170 & .194 & .170 & .279 & .232 & .203 & .219 & .377 \\
\hline Co & 533 & .732 & .659 & 398 & .465 & .443 & .478 & .599 & .583 & .276 & 333 \\
\hline $\mathrm{Zn}$ & .059 & .070 & .066 & .069 & .063 & .076 & .062 & .065 & .077 & .070 & .071 \\
\hline
\end{tabular}


Table 16.Chemical composition of ferromanganese crusts as reported in DeCarlo and ohers (1987).

\begin{tabular}{|c|c|c|c|c|c|c|c|c|c|c|}
\hline Sequence" & 089101 & 089102 & 089103 & 089104 & 089105 & 089106 & 090050 & 090051 & 090052 & 090053 \\
\hline Latinude & 25.926 & 25.920 & 25.929 & 25.783 & 25.489 & 25.489 & 28.904 & 28.904 & 28.895 & 28.895 \\
\hline Langinde & -167.710 & -167.755 & -167.755 & -167.733 & -168.672 & -168.672 & -178.957 & -178.957 & -178.868 & -178.868 \\
\hline Depth & 3250 & 2500 & 2500 & 1600 & 1200 & 1200 & 1500 & 1500 & 1280 & 1280 \\
\hline Lab" & $67-39-2$ & $67-40-1$ & $67-40-2$ & $69-10-3$ & $71-11-2$ & $71-11-7$ & $59-29-1$ & $59-29-2$ & $59-30-7$ & $59-30-8$ \\
\hline$A l(w t \%)$ & 3.20 & .62 & .70 & 1.40 & .66 & 1.10 & .65 & .51 & .53 & .25 \\
\hline C. & 2.64 & 2.45 & 240 & 1.17 & 1.57 & 236 & 2.48 & 250 & 2.62 & 2.72 \\
\hline Co & .540 & .750 & .960 & .090 & 1.39 & 1.31 & 1.28 & 1.10 & 1.35 & 1.32 \\
\hline co & .195 & .056 & .047 & .107 & .044 & .056 & .037 & .041 & .043 & .034 \\
\hline Fo & 17.0 & 20.3 & 19.4 & 1.80 & 18.2 & 17.1 & 17.4 & 16.1 & 13.8 & 125 \\
\hline $\mathbf{K}$ & .65 & AS & 38 & 51 & 32 & .41 & .56 & .37 & .60 & .53 \\
\hline $\mathbf{M B}$ & 1.50 & 1.11 & 1.03 & 2.10 & 1.20 & 2.35 & 1.06 & 1.03 & 1.32 & 1.17 \\
\hline Mn & 17.0 & 21.2 & 23.2 & 41.8 & 25.3 & 23.7 & 22.7 & 26.3 & 29.1 & 30.3 \\
\hline Na & 1.40 & 1.70 & 1.20 & 1.04 & 1.04 & 1.20 & 1.78 & .89 & 1.95 & 1.96 \\
\hline $\mathbf{N i}$ & .330 & .380 & 410 & 370 & .400 & 410 & .300 & .460 & .590 & .630 \\
\hline $\mathbf{S i}$ & 7.80 & 3.55 & 4.07 & 2.30 & 2.60 & 4.25 & 3.50 & 2.20 & 210 & 1.30 \\
\hline $\mathrm{Ti}$ & 1.14 & 1.20 & 1.26 & .55 & 84 & .66 & 1.00 & .84 & .70 & .60 \\
\hline A\&(ppm) & 2.4 & .3 & 3.6 & 3.7 & .6 & 5 & 2.5 & 5 & 3.6 & 1.0 \\
\hline Ba & 1200 & 1400 & 1400 & 9800 & 1000 & 1100 & 2200 & 1100 & 1200 & 1100 \\
\hline Cd & 6.4 & 7.2 & 5.9 & 58.0 & 8.4 & 7.5 & 6.2 & 7.5 & 11.1 & 10.6 \\
\hline $\mathbf{P b}$ & 1900 & 2300 & 2000 & 200 & 2800 & 2400 & 3000 & 2400 & 2700 & 2800 \\
\hline v & 570 & 690 & 620 & 246 & 820 & 790 & 810 & 680 & 760 & 850 \\
\hline $\mathbf{Z n}$ & 670 & 660 & 650 & 2000 & 540 & 580 & 580 & 550 & 560 & 640 \\
\hline LOI(wt\%) & 129 & 15.2 & 15.6 & 16.4 & 16.2 & 15.4 & 15.8 & 16.2 & 18.0 & 17.8 \\
\hline Sequence" & 090054 & 090055 & 090056 & 090057 & 090058 & 090059 & 090060 & 090061 & 090062 & 090063 \\
\hline Latitude & 28.895 & 28.895 & 28.739 & 29.106 & 29.106 & 29.167 & 29.167 & 29.167 & 29.167 & 29.167 \\
\hline Longiunde & -178.868 & -178.868 & -178.783 & -173.948 & -173.948 & -174.067 & -174.067 & -174.067 & -174.067 & -174.067 \\
\hline Depth & 1280 & 1280 & 3000 & 1850 & 1850 & 1425 & 1425 & 1425 & 1425 & 1425 \\
\hline Labb" & $59-30-12$ & $59-30-15$ & $60-33-2$ & $61-34-5$ & $61-346$ & $62-6-5$ & $62-6-6$ & $62-6-7$ & $62-6-9$ & $62-6-10$ \\
\hline $\mathbf{A l}(w t 5)$ & 58 & 5.00 & .90 & .70 & .75 & .55 & 1.03 & .80 & 1.54 & 1.38 \\
\hline Ca & 2.64 & 1.60 & 230 & 2.55 & 2.57 & 2.82 & 2.64 & 2.77 & 2.00 & 2.38 \\
\hline Co & 1.47 & .570 & .600 & $.840^{\circ}$ & .800 & .870 & .780 & .840 & .710 & .720 \\
\hline co & .045 & .031 & .044 & .054 & .067 & .046 & .042 & .062 & .064 & .055 \\
\hline Fo & 13.1 & 6.20 & 19.1 & 17.0 & 17.0 & 16.4 & 163 & 15.6 & 18.4 & 16.2 \\
\hline $\mathbf{K}$ & .54 & 1.46 & .60 & 56 & 55 & .53 & .60 & .62 & .61 & .63 \\
\hline $\mathbf{M g}$ & .96 & 2.22 & .95 & .96 & 1.00 & 1.03 & 1.17 & 1.15 & 1.10 & 1.22 \\
\hline $\mathbf{M n}$ & 30.5 & 18.7 & 19.6 & 25.1 & 24.6 & 25.3 & 24.4 & 26.0 & 19.1 & 23.0 \\
\hline Na & 1.70 & 2.93 & 1.65 & 1.73 & 1.72 & 1.73 & 1.77 & 1.95 & 1.80 & 1.80 \\
\hline $\mathbf{N i}$ & .650 & .270 & 310 & .440 & .460 & .490 & .450 & .640 & .330 & .500 \\
\hline $\mathbf{S i}$ & 1.70 & 125 & 5.75 & 3.94 & 3.53 & 2.55 & 4.40 & 3.30 & 7.60 & 4.90 \\
\hline $\mathrm{T}$ & .78 & .88 & 1.00 & .97 & 1.10 & 1.14 & .90 & 1.05 & 1.18 & 1.00 \\
\hline Ag(ppen) & .8 & .2 & 3.3 & 3.6 & 3.2 & .5 & 3 & .9 & .4 & .4 \\
\hline Ba & 1100 & 600 & 1400 & 1700 & 1700 & 1600 & 1500 & 1400 & 2600 & 1400 \\
\hline Cd & 13.4 & 31.2 & 4.6 & 6.5 & 6.6 & 7.0 & 13.6 & 10.8 & 6.0 & 7.7 \\
\hline $\mathrm{Pb}$ & 2100 & 140 & 2200 & 2500 & 2600 & 2400 & 2400 & 2400 & 2200 & 2100 \\
\hline $\mathbf{v}$ & 760 & 260 & 670 & 780 & 780 & 810 & 750 & 720 & 660 & 760 \\
\hline $\mathbf{Z n}$ & 590 & 1100 & 670 & 680 & 760 & 720 & 630 & 880 & 610 & 650 \\
\hline LOI(wt\%) & 17.2 & 13.3 & 14.8 & 16.2 & 15.6 & 16.2 & 15.1 & 17.5 & 13.5 & 15.0 \\
\hline
\end{tabular}


Table 16. Chemical composition of ferromanganese crusts as reported in DeCarlo and others (1987).

\begin{tabular}{|c|c|c|c|c|c|c|c|c|c|c|}
\hline Sequencoll & 090064 & 090065 & 090066 & 090067 & 090068 & 090069 & 091024 & 091025 & 091026 & 091027 \\
\hline Latitude & 29.167 & 29.167 & 29.208 & 29.208 & 29.185 & 29.185 & 29.866 & 29.839 & 29.820 & 29.643 \\
\hline Longitude & -174.067 & -174.067 & -174.112 & -174.112 & -174.010 & -174.010 & 179.083 & 179.065 & 179.103 & 179.282 \\
\hline Depth & 1425 & 1425 & 2200 & 2200 & 985 & 985 & 2250 & 2100 & 1700 & 1300 \\
\hline Lab\# & $62-6-11$ & $62-6-12$ & $62-35-10$ & $62-35-11$ & $63-7-1$ & $63-7-3$ & $55-23-4$ & $55-24-4$ & $56-25-7$ & $57-28.5$ \\
\hline$A(w t \%)$ & 1.04 & 1.30 & .77 & 1.80 & 55 & .65 & 1.70 & 1.80 & 1.70 & .42 \\
\hline ce & 2.63 & 244 & 200 & 2.30 & 2.64 & 2.80 & 2.57 & 1.72 & 257 & 2.72 \\
\hline Co & .930 & .670 & .730 & 500 & 1.23 & 1.18 & .720 & .630 & .750 & 1.28 \\
\hline $\mathrm{Cu}$ & .042 & .069 & .101 & .113 & .054 & .057 & .050 & .078 & .041 & .040 \\
\hline $\mathrm{Pe}$ & 14.8 & 15.4 & 15.7 & 15.7 & 16.5 & 16.1 & 17.3 & 18.5 & 15.4 & 13.2 \\
\hline K & .68 & .63 & .62 & .83 & .54 & .56 & .88 & .70 & .83 & .57 \\
\hline $\mathbf{M}_{\mathbf{B}}$ & 1.08 & 1.02 & .78 & .90 & 1.17 & 1.15 & 1.10 & 1.33 & 1.15 & 1.16 \\
\hline Mn & 24.5 & 22.9 & 21.6 & 18.8 & 26.9 & 25.8 & 20.0 & 19.9 & 222 & 29.6 \\
\hline $\mathrm{Na}$ & 1.79 & 1.88 & .96 & 1.41 & 1.92 & 1.74 & 1.56 & 1.48 & 1.56 & 191 \\
\hline $\mathbf{N i}$ & 500 & 530 & .410 & .340 & .460 & .470 & 360 & .410 & .450 & 590 \\
\hline $\mathbf{S i}$ & 4.20 & 5.50 & 4.60 & 730 & 1.13 & 1.50 & 6.40 & 6.02 & 6.08 & 1.53 \\
\hline $\mathrm{Ti}$ & .90 & .93 & 1.20 & 1.14 & 1.06 & 1.07 & 1.00 & 1.02 & .84 & .66 \\
\hline$A_{g}$ (ppon) & 3.0 & .7 & 3 & 2 & .8 & 1.4 & .3 & 3 & 1.1 & 3.1 \\
\hline $\mathrm{Ba}$ & 2000 & 2700 & 1700 & 2400 & 1800 & 1600 & 1000 & 2800 & 1000 & 1200 \\
\hline Cd & 7.8 & 7.1 & 6.4 & 6.9 & 9.2 & 8.0 & 7.2 & 8.3 & 8.8 & 10.8 \\
\hline $\mathrm{Pb}$ & 2400 & 2200 & 2100 & 1600 & 3000 & 2900 & 2200 & 2200 & 2300 & 2800 \\
\hline $\mathbf{v}$ & 740 & 680 & 620 & 560 & 870 & 920 & 610 & 590 & 660 & 860 \\
\hline $\mathbf{z n}$ & 690 & 660 & 660 & 610 & 760 & 800 & 580 & 760 & 580 & 640 \\
\hline LOI(wt\%) & 16.9 & 15.9 & 15.2 & 14.2 & 12.2 & 15.9 & 145 & 14.7 & 15.3 & 16.2 \\
\hline
\end{tabular}


Table 17.Chemical composition of ferromanganese crusts as reported in DeCarlo and others (1987).

\begin{tabular}{|c|c|c|c|c|c|c|c|c|c|c|}
\hline Sequencell & 317015 & 317016 & 317017 & 317018 & 317019 & 317020 & 317021 & 317022 & 317023 & 317024 \\
\hline Letitude & -3.837 & .3 .837 & -3.837 & -3.837 & -3.837 & -3.837 & -3.837 & -3.837 & -3.804 & .3 .804 \\
\hline Longirude & -173.329 & -173.329 & -173.329 & -173.329 & -173.329 & -173.329 & -173.329 & -173.329 & -173.490 & -173.490 \\
\hline Depth & 2265 & 2265 & 2265 & 2265 & 2265 & 2265 & 2265 & 2265 & 4800 & 4800 \\
\hline Lab\# & 6RD-008-01 & 6RD-008-02 & 6RD-008-03 & 6RD-C08-04 & GRD-COB-05 & 6RD-CO8- 06 & GRD-COS-CB & GRD-C08-S8 & 6RD-009-01A & GRD- $009-1 \mathrm{AF}$ \\
\hline$A l(w+\%)$ & .95 & .86 & .66 & .69 & 55 & .44 & 51 & .46 & 1.25 & 85 \\
\hline Ca & 2.63 & 3.21 & 258 & 275 & 288 & 3.40 & 5.77 & 5.34 & 2.29 & 2.24 \\
\hline Co & .742 & .820 & .855 & .871 & .783 & .818 & .770 & .723 & .495 & .504 \\
\hline cu & .098 & .092 & .120 & .113 & .097 & .096 & .122 & .103 & .128 & .086 \\
\hline Pe & 18.0 & 17.0 & 15.9 & 16.7 & 15.6 & 15.3 & 14.1 & 14.0 & 16.6 & 20.9 \\
\hline $\mathbf{M g}$ & 1.04 & 1.20 & 1.21 & 1.19 & 1.09 & 1.13 & 1.23 & 1.10 & 1.05 & 1.05 \\
\hline Mn & 23.5 & 23.1 & 26.3 & 25.5 & 24.8 & 26.7 & 25.4 & 245 & 23.5 & 21.9 \\
\hline $\mathrm{Ni}$ & .586 & .596 & .672 & .648 & .586 & .646 & .651 & .620 & .382 & .251 \\
\hline $\mathbf{P}$ & .577 & .730 & .508 & 563 & .622 & .778 & 1.59 & 1.51 & .404 & .524 \\
\hline $\mathbf{S i}$ & 4.07 & 3.65 & 274 & 297 & 236 & 2.29 & 2.14 & 2.25 & 4.96 & 3,48 \\
\hline $\mathrm{n}$ & 1.11 & 1.12 & 1.16 & 1.20 & 1.22 & 1.15 & 1.27 & 1.16 & 1.09 & 1.25 \\
\hline v & .074 & .068 & .077 & .076 & .075 & .000 & .073 & .076 & .074 & .091 \\
\hline Thicknesax (mm.) & 20.0 & 40.0 & 60.0 & 50.0 & 45.0 & 60.0 & 60.0 & 60.0 & 85.0 & 85.0 \\
\hline
\end{tabular}

\begin{tabular}{|c|c|c|c|c|c|c|c|c|c|c|}
\hline Sequencell & 317025 & 317026 & 317027 & 317028 & 317029 & 317030 & 317031 & 317032 & 317033 & 317034 \\
\hline Letinude & -3.804 & -3.804 & -3.804 & -3.804 & -3.804 & -2.992 & -2.992 & -2992 & -2992 & -2992 \\
\hline Longitude & -173.490 & -173.490 & $-173,490$ & $-173,490$ & -173.490 & -171.380 & -171.380 & -171.380 & -171.380 & -171.380 \\
\hline Depth & 4800 & 4800 & 4800 & 4800 & 4800 & 1465 & 1465 & 1465 & 1465 & 1465 \\
\hline Lab" & 6RD-009-02 & 6RD-009-03 & GRD-009-04 & 6RD-009-05 & 6RD-009-06 & 7RD-011-X & 7RD-011-01 & 7RD-011-02 & $7 R D-011-03$ & 7RD-011-04 \\
\hline$A \mathrm{Al}(w+\%)$ & 1.06 & 1.40 & 94 & .60 & .91 & .22 & .60 & .20 & .69 & .29 \\
\hline Ca & 2.20 & 215 & 2.25 & 215 & 1.86 & 277 & 295 & 2.88 & 19.1 & 4.82 \\
\hline$C_{0}$ & .514 & .556 & 599 & .728 & .711 & 1.37 & 1.70 & 1.63 & .427 & 1.18 \\
\hline $\mathrm{Cu}$ & .153 & .116 & .094 & .085 & .115 & .057 & .112 & .042 & .127 & .098 \\
\hline Re & 19.9 & 19.2 & 18.7 & 17.2 & $18.2^{\circ}$ & 12.9 & 127 & 14.6 & 3.92 & 8.95 \\
\hline Mg & 1.04 & 1.02 & 1.08 & 1.26 & 1.08 & 1.30 & 1.62 & 1.32 & 1.72 & 1.44 \\
\hline $\mathrm{Mn}$ & 23.5 & 22.0 & 23.1 & 23.8 & 22.8 & 31.7 & 30.9 & 29.5 & 15.7 & 29.3 \\
\hline $\mathrm{Ni}$ & .350 & .298 & 321 & 377 & 338 & .855 & .682 & .660 & 1.127 & 1.035 \\
\hline $\mathbf{P}$ & .530 & .425 & 512 & .408 & .365 & .553 & .753 & .629 & 6.57 & 1.32 \\
\hline $\mathbf{S i}$ & 3.85 & 4.44 & 3.04 & 202 & 2.99 & 1.13 & 1.56 & 1.11 & .68 & .68 \\
\hline $\mathrm{n}$ & 1.26 & 1.25 & 1.34 & 1.33 & 1.42 & .90 & 1.02 & .79 & .22 & .68 \\
\hline $\mathbf{v}$ & .089 & .079 & .084 & .078 & .077 & .087 & .093 & .083 & .032 & .064 \\
\hline Thickneses(mm.) & 60.0 & 70.0 & 30.0 & 70.0 & 60.0 & 20.0 & 35.0 & 8.0 & 20.0 & 10.0 \\
\hline
\end{tabular}

\begin{tabular}{|c|c|c|c|c|c|c|c|c|c|c|}
\hline Sequencoll & 317035 & 317036 & 317037 & 317038 & 317039 & 317040 & 317041 & 317042 & 317043 & 317044 \\
\hline Latinude & -2.992 & -2.992 & -2.992 & -2992 & -2992 & -2992 & -2992 & -2.992 & -2.992 & -2992 \\
\hline Longitude & -171.380 & .171 .380 & -171.380 & -171.380 & -171.380 & -171.380 & -171.380 & -171.380 & -171.380 & -171.380 \\
\hline Depth & 1465 & 1465 & 1465 & 1465 & 1465 & 1465 & 1465 & 1465 & 1465 & 1465 \\
\hline Lab" & 7RD-011.05 & 7RD-011-06 & 7RD-011-07 & 7RD-011-08 & 7RD-011-10 & 7RD-011-11 & TRD-011-12 & 7RD-011-13 & TRD-011-14 & 7RD-011-15 \\
\hline$A l(w t \%)$ & .27 & .23 & .20 & 36 & .46 & .22 & .47 & .29 & 36 & 36 \\
\hline Ca & 2.60 & 275 & 3.51 & 5.49 & 3.41 & 2.38 & 3.10 & 8.73 & 6.92 & 3.65 \\
\hline Co & 1.26 & 1.48 & 1.30 & 1.29 & 1.24 & 1.48 & 1.62 & .987 & 1.22 & 1.32 \\
\hline $\mathrm{Cu}$ & .053 & .035 & .057 & .082 & .089 & .058 & .116 & .083 & .049 & .067 \\
\hline Fe & 12.5 & 14.4 & 127 & 9.98 & 10.1 & 10.6 & 8.14 & 10.1 & 10.0 & 124 \\
\hline $\mathbf{M g}_{8}$ & 1.37 & 1.26 & 1.33 & 1.56 & 1.41 & 1.43 & 1.84 & 1.15 & 1.24 & 1.31 \\
\hline Mn & 28.1 & 28.7 & 31.4 & 29.3 & 30.7 & 30.7 & 33.8 & 25.9 & 27.3 & 27.0 \\
\hline $\mathrm{Ni}$ & .790 & .694 & .828 & 1.134 & .890 & .915 & 1.263 & .688 & .733 & .784 \\
\hline $\mathbf{P}$ & .523 & .612 & .750 & 1.64 & .752 & .440 & .689 & 2.82 & 2.05 & .892 \\
\hline $\mathbf{S i}$ & 1.07 & 1.27 & 1.09 & .78 & .92 & .83 & .64 & .91 & 1.26 & 1.20 \\
\hline $\mathbf{T i}$ & .83 & .90 & .87 & .68 & 85 & .84 & .53 & .85 & 90 & 87 \\
\hline $\mathbf{v}$ & .081 & .092 & .084 & .065 & .100 & .072 & .094 & .085 & .084 & .073 \\
\hline Thickness (mm.) & 10.0 & 7.0 & 25.0 & 7.0 & 7.0 & 10.0 & 7.0 & 18.0 & 13.0 & 10.0 \\
\hline
\end{tabular}


Table 17. Chemical composition of ferromanganese crusts as reported in DeCarlo and others (1987).

\begin{tabular}{|c|c|c|c|c|c|c|c|c|c|c|}
\hline Sequencolt & 317045 & 317046 & 318001 & 318002 & 318003 & 318004 & 318005 & 318006 & $31800 n$ & 318008 \\
\hline Letitude & -3.016 & -3.016 & -7.451 & -7.451 & -7.408 & -7.408 & -7.408 & -7.408 & -7.408 & -7.408 \\
\hline Longitude & -171.446 & .171 .446 & 179.024 & 179.024 & 179.046 & 179.046 & 179.046 & 179.046 & 179.046 & 179.046 \\
\hline Depth & 3130 & 3130 & 1230 & 1230 & 1690 & 1690 & 1690 & 1690 & 1690 & 1690 \\
\hline Leb" & 7RD-012-1A & $7 R D-012-1 B$ & 4MD-D1-SO1 & 4MD-01-SO2 & 4RD-007-01 & 4RD-COT-02 & 4RD-007-03 & 4RD-007-04 & 4RD-007-05 & 4RD-007-07 \\
\hline$A \perp(w t \%)$ & .43 & 58 & .26 & .22 & .94 & 1.08 & 56 & .52 & 1.28 & .99 \\
\hline ca & 251 & 245 & 254 & 334 & 253 & 3.14 & 5.22 & 3.47 & 275 & 3.60 \\
\hline Co & .664 & .691 & 1.92 & 1.52 & .709 & .683 & 856 & 857 & .624 & .780 \\
\hline Cu & .125 & .146 & .067 & .063 & .069 & .100 & .126 & .100 & .109 & .130 \\
\hline Fo & 17.4 & 17.7 & 11.3 & 11.6 & 17.4 & 17.4 & 155 & 16.1 & 16.8 & 16.0 \\
\hline Mg & 1.11 & 1.03 & 1.56 & 1.38 & 1.12 & 1.17 & 1.12 & 1.18 & 1.22 & 1.26 \\
\hline $\mathrm{Mn}$ & 26.2 & 26.6 & 320 & 33.0 & 22.7 & 22.0 & 24.6 & 25.6 & 21.6 & 24.2 \\
\hline $\mathbf{N i}$ & 515 & 558 & .885 & .892 & .517 & .525 & 543 & .558 & .523 & .675 \\
\hline $\mathbf{P}$ & .474 & .448 & .411 & .565 & .528 & .764 & 1.35 & .766 & .637 & .965 \\
\hline Si & 246 & 286 & .98 & 88 & 3.21 & 3.77 & 208 & 2.20 & 3.88 & 3.10 \\
\hline $\mathrm{Ti}$ & 1.21 & 1.20 & 1.14 & .96 & 1.11 & 1.20 & 1.35 & 1.27 & 1.41 & 1.16 \\
\hline $\mathbf{v}$ & .090 & .088 & .072 & .081 & .074 & .on & .082 & .088 & .076 & .085 \\
\hline Thicknesx(mm.) & 50.0 & 54.0 & 10.0 & 15.0 & 10.0 & 23.0 & 40.0 & 40.0 & 50.0 & 50.0 \\
\hline
\end{tabular}

\begin{tabular}{|c|c|c|c|c|c|c|c|c|c|c|}
\hline Sequencel & 318009 & 318010 & 318011 & 318012 & 318013 & 318014 & 318015 & 318016 & 318017 & 318018 \\
\hline Letinde & -7.408 & -7.408 & $-7,408$ & -7.408 & -7.408 & -7.408 & -7.408 & -7.408 & .7 .408 & -7.408 \\
\hline Langitude & 179.046 & 179.046 & 179.046 & 179.046 & 179.046 & 179.046 & 179.046 & 179.046 & 179.046 & 179.046 \\
\hline Depth & 1690 & 1690 & 1690 & 1690 & 1690 & 1690 & 1690 & 1690 & 1690 & 1690 \\
\hline Lab" & 4RD-007-09 & 4RD-007-10 & 4RD-007-11 & 4RD-007-12 & 4RD-007.13 & 4RD-007-14 & 4RD-007-15 & 4RD-007-16 & 4RD-007-17 & 4RD-007-18 \\
\hline$A \mathbf{N}(w t \%)$ & .58 & .77 & 1.12 & .67 & .82 & 1.21 & .74 & .41 & .70 & .69 \\
\hline C. & 3.16 & 3.38 & 3.77 & 2.71 & 2.44 & 248 & 3.42 & 6.58 & 2.36 & 2.29 \\
\hline Co & .854 & .812 & .579 & .822 & .751 & .691 & .934 & .868 & .804 & 875 \\
\hline$\infty$ & .107 & .121 & .115 & .096 & .068 & .093 & .087 & .106 & .087 & .075 \\
\hline Fe & 15.1 & 16.7 & 16.0 & 16.6 & 16.9 & 17.8 & 15.8 & 11.4 & 16.0 & 15.6 \\
\hline $\mathbf{M g}$ & 1.13 & 1.16 & 1.23 & 1.22 & 1.10 & 1.23 & 1.17 & 1.08 & 1.11 & 1.17 \\
\hline $\operatorname{Mn}$ & 23.9 & 24.8 & 21.6 & 25.5 & 22.9 & 21.9 & 23.6 & 23.7 & 23.6 & 24.5 \\
\hline $\mathrm{Ni}$ & .571 & 547 & .632 & .606 & 553 & 562. & 586 & .659 & .588 & .601 \\
\hline$p$ & .748 & .784 & 1.08 & .579 & 507 & .618 & .852 & 1.98 & .537 & .475 \\
\hline $\mathbf{S i}$ & 2.05 & 272 & 3.50 & 2.43 & 294 & 3.81 & 240 & 1.20 & 256 & 237 \\
\hline $\mathrm{Ti}$ & 1.26 & 1.30 & 1.04 & 1.26 & 1.10 & 1.25 & 1.27 & 1.28 & 1.09 & 1.12 \\
\hline $\mathbf{v}$ & .074 & .081 & .070 & .085 & .074 & .074 & .069 & .068 & .073 & .077 \\
\hline Thicknesx (mm.) & 40.0 & 40.0 & 40.0 & 35.0 & 40.0 & 40.0 & 45.0 & 40.0 & 40.0 & 60.0 \\
\hline
\end{tabular}


Table 18. Chemical composition of ferromanganese crusts as reported in OF87-281 (Hein and others, 1987).

\begin{tabular}{|c|c|c|c|c|c|c|c|c|c|c|c|}
\hline Sequence:" & 058008 & 058009 & 058010 & 058010 & 058010 & 058011 & 058012 & 058013 & 094023 & 094024 & 094025 \\
\hline Letitudio & 17.936 & 19.149 & 19.149 & 19.149 & 19.149 & 19.149 & 19.455 & 17.148 & 20.025 & 20.112 & 20.396 \\
\hline Longitude & 145.503 & 145.498 & 145.498 & 145.498 & 145.498 & 145.498 & 145.491 & 145.657 & 145.223 & 145.046 & 144.977 \\
\hline Depth & 1940 & 2880 & 2880 & 2880 & 2880 & 2880 & 1380 & 2975 & 172 & 1650 & 1930 \\
\hline Lab\# & D6-1 & D8-5A & D8-12A & D8-12B & $D 8-12 C$ & D8-20 & D11-1A & D3-3 & D12-2 & D13-1-A & D15-1-A \\
\hline$S i$ (wt.\%) & 17.6 & 10.4 & 14.0 & 19.6 & 15.2 & 17.7 & 200 & 57 & 10.1 & 18.4 & 15.3 \\
\hline $\mathbf{A}$ & .40 & 3.60 & 4.10 & 7.90 & 5.30 & 6.50 & .83 & 20 & 4.00 & 6.90 & 4.90 \\
\hline Ro & 8.10 & 16.8 & 12.3 & 5.70 & 14.8 & 4.70 & 1.56 & 23 & 2.75 & 4.70 & 15.9 \\
\hline $\mathbf{M}_{\mathbf{g}}$ & 6.60 & 1.61 & 2.00 & 1.80 & 1.48 & 268 & 1.76 & .68 & 2.25 & 2.22 & 1.79 \\
\hline $\mathrm{Ce}$ & 6.20 & 3.30 & 2.70 & 8.50 & 3.40 & 6.60 & 1.07 & 2.13 & 3.60 & 6.30 & 3.20 \\
\hline $\mathrm{Na}$ & 1.28 & 1.65 & 1.62 & 211 & 1.63 & 2.17 & 1.54 & 3.50 & 2.21 & 245 & 1.46 \\
\hline $\mathbf{K}$ & .41 & .63 & 1.14 & 56 & 1.01 & .85 & 1.55 & .40 & .78 & 36 & .93 \\
\hline $\mathbf{T i}$ & $A 1$ & .81 & 29 & .44 & .88 & 32 & .10 & .02 & .19 & 35 & .27 \\
\hline $\mathbf{P}$ & .04 & 30 & .14 & .06 & .27 & .07 & .06 & .03 & .04 & .03 & .16 \\
\hline Mn & 9.80 & 12.3 & 13.6 & 4.40 & 7.10 & 11.9 & 44.3 & 48.0 & 28.9 & 12.2 & 9.10 \\
\hline $\mathrm{H}_{2} \mathrm{O}^{\circ}$ & 1.5 & 4.7 & 26 & 23 & 4.0 & 1.2 & 3.9 & 4.6 & 3.0 & 1.2 & 3.6 \\
\hline $\mathrm{H}_{2}^{2} \mathrm{O}^{+}$ & 1.8 & 6.3 & 5.4 & 1.9 & 6.1 & 22 & 7.4 & 6.9 & 5.3 & 24 & 5.3 \\
\hline $\mathrm{C}_{2}$ & .01 & .18 & .05 & 3.14 & .19 & .83 & .06 & .04 & .06 & 43 & .08 \\
\hline
\end{tabular}

\begin{tabular}{|c|c|c|c|c|c|c|c|c|c|c|c|}
\hline Ax(ppon) & 10 & 180 & 90 & 13 & 120 & 12 & 110 & 140 & 37 & $<1$ & 180 \\
\hline Ba & 250 & 1100 & 2800 & 260 & 880 & 290 & 5000 & 500 & 1500 & 230 & 850 \\
\hline Cd & 8.40 & 5.80 & 6.20 & 1.70 & 1.90 & .65 & 16.00 & 1.40 & 18.00 & .35 & 1.85 \\
\hline $\mathrm{Co}_{0}$ & 50 & 930 & 740 & 18 & 530 & 140 & 85 & 25 & 12 & 27 & 180 \\
\hline$\alpha$ & 140 & 20 & 35 & 21 & 24 & 71 & 6 & 4 & 15 & 39 & 26 \\
\hline $\overrightarrow{a_{2}}$ & 210 & 580 & 4000 & 120 & 400 & 900 & 400 & 28 & 130 & 220 & 130 \\
\hline Mo & 73 & 140 & 230 & 3 & 19 & 56 & 840 & 1300 & 240 & 38 & 190 \\
\hline $\mathbf{N i}$ & 320 & 1200 & 1400 & 130 & 730 & 120 & 840 & 82 & 150 & 38 & 260 \\
\hline $\mathrm{Pb}$ & $<49$ & 650 & 300 & 25 & 330 & 25 & 100 & 10 & 25 & $<49$ & $<49$ \\
\hline Sr & 210 & 1000 & 540 & 410 & 800 & 400 & 1100 & 400 & 530 & 300 & 360 \\
\hline v & 460 & 580 & 310 & 260 & 340 & 220 & 410 & $<4$ & 620 & 250 & 230 \\
\hline $\mathbf{Z n}$ & 310 & 560 & 480 & 140 & 370 & 83 & 840 & 110 & 160 & 85 & 220 \\
\hline Thicknesd (mma) & - & - & - & - & 8.0 & - & - & $\cdot$ & 20 & - & 8.0 \\
\hline
\end{tabular}

\begin{tabular}{|c|c|c|c|c|c|c|c|c|c|c|c|}
\hline$Y$ (ppon) & 17 & 130 & 39 & 38 & 110 & 96 & 38 & 3 & 12 & 17 & 20 \\
\hline La & 5 & $\cdot$ & - & - & - & 121 & - & 2 & - & $\cdot$ & - \\
\hline Co & 9 & 310 & 53 & $<10$ & 210 & 220 & 22 & 2 & $<10$ & $<10$ & 17 \\
\hline $\operatorname{Pr}$ & 1 & - & - & . & - & 27 & - & $<1$ & • & $\cdot$ & • \\
\hline Nd & 7 & - & - & - & - & 118 & - & $<$ & - & - & - \\
\hline Sm & 2 & - & - & - & - & 26 & - & $<1$ & - & - & - \\
\hline $\mathrm{Eu}$ & .66 & - & - & - & - & 6.8 & - & .13 & - & - & - \\
\hline Gd & 3 & - & - & - & - & 29 & - & 1 & - & - & - \\
\hline Tb & $<1$ & - & - & - & - & 5 & - & $<1$ & - & - & - \\
\hline Dy & 3 & - & - & - & . & - & - & $<1$ & . & - & - \\
\hline Ho & .64 & - & - & . & - & 5.2 & - & .12 & - & - & - \\
\hline Er & 2.2 & $\cdot$ & - & - & - & 14 & - & $<5$ & - & - & - \\
\hline Tm & .3 & - & - & - & - & 22 & - & $<2$ & - & - & - \\
\hline $\mathrm{Yb}$ & 19 & - & - & - & - & 14 & - & $<5$ & - & - & - \\
\hline $\mathbf{L u}$ & 30 & - & - & $\cdot$ & - & 2.1 & - & $<05$ & • & - & - \\
\hline $\mathrm{Pt}$ & .005 & .084 & .026 & .00 & .039 & .006 & .054 & $<005$ & .010 & $<005$ & .017 \\
\hline Pd & .002 & .0022 & .0024 & .0026 & .0022 & .004 & .015 & $<010$ & .002 & .0056 & .0026 \\
\hline $\mathbf{R h}$ & $<001$ & $<.001$ & $<001$ & $<.001$ & $<001$ & $<.001$ & $<001$ & $<\infty D_{1}$ & $<001$ & $<\infty 1$ & $<001$ \\
\hline
\end{tabular}


Table 18. Chemical composition of ferromanganese crusts as reported in OF87-281 (Hein and others, 1987).

\begin{tabular}{|c|c|c|c|c|c|c|c|c|c|c|c|}
\hline Sequiencott & 094026 & 094027 & 094028 & 094029 & 094030 & 094031 & 094032 & 094033 & 094033 & 094034 & 094035 \\
\hline Latitude & 20.612 & 21.052 & 21.141 & 21.141 & 21.141 & 21.141 & 21.765 & 22.082 & 22.082 & 22.082 & 22293 \\
\hline Longitude & 144.653 & 143.832 & 143.904 & 143.904 & 143.904 & 143.904 & 143.720 & 143.400 & 143.400 & 143.400 & 143.226 \\
\hline $\begin{array}{l}\text { Depth } \\
\text { Lab* }\end{array}$ & $\begin{array}{c}1640 \\
D 20-1-A \\
\end{array}$ & $\begin{array}{c}2290 \\
\text { D27-1-A }\end{array}$ & $\begin{array}{c}1715 \\
\text { D28-3-A }\end{array}$ & $\begin{array}{c}1715 \\
D 28-4-A\end{array}$ & $\begin{array}{c}1715 \\
\text { D28-6-A }\end{array}$ & $\begin{array}{c}1715 \\
\text { D28-7-A }\end{array}$ & $\begin{array}{c}1170 \\
\text { D33-2-3-A }\end{array}$ & $\begin{array}{c}1950 \\
\text { D36-2A }\end{array}$ & $\begin{array}{c}1950 \\
\text { D36-2-B }\end{array}$ & $\begin{array}{l}1950 \\
\text { D36-4 }\end{array}$ & $\begin{array}{c}1572 \\
\text { D37-1 }\end{array}$ \\
\hline Si(wt.\%) & 1.60 & 252 & 20.8 & 15.7 & 15.4 & 830 & . & 13.0 & 12.4 & 123 & 7.60 \\
\hline Al & 57 & 84 & 7.60 & 5.80 & 5.70 & 270 & 3.10 & 4.70 & 4.30 & 4.30 & 2.63 \\
\hline$F_{0}$ & $\mathbf{s i}$ & 5.30 & 6.60 & 4.80 & 5.60 & 17.4 & 8.80 & 14.6 & 14.7 & 15.2 & 18.0 \\
\hline Ms & .82 & 1.59 & 3.40 & 2.49 & 241 & 1.08 & 1.29 & 1.36 & 1.28 & 1.38 & .99 \\
\hline $\mathrm{ca}$ & 2.36 & 1.45 & 6.20 & 5.00 & 5.20 & 259 & 2.00 & 290 & 2.37 & 269 & 230 \\
\hline $\mathrm{N}_{\mathbf{2}}$ & 3.26 & 1.70 & 2.00 & 2.12 & 2.11 & 1.69 & - & 1.82 & 1.49 & 1.43 & 1.55 \\
\hline $\mathbf{K}$ & 51 & 1.33 & .48 & $\pi$ & .72 & 58 & .84 & 1.03 & 1.08 & 1.00 & .61 \\
\hline $\mathbf{T i}$ & .04 & .19 & 38 & 34 & .41 & .67 & .14 & .87 & .80 & .86 & .70 \\
\hline $\mathbf{P}$ & $\angle 03$ & .18 & .06 & .04 & .05 & 38 & .10 & .23 & 23 & .25 & .43 \\
\hline Mn & 46.3 & 36.8 & 5.60 & 15.9 & 15.6 & 13.1 & 14.5 & 4.90 & 5.90 & 6.10 & 12.1 \\
\hline $\mathrm{H}_{2} \mathrm{O}^{-}$ & 3.0 & 6.2 & 15 & 2.6 & 2.0 & 10.3 & • & 14.8 & 16.7 & 15.8 & 14.4 \\
\hline $\mathrm{H}_{2} \mathrm{O}^{+}$ & 7.6 & 7.4 & 1.6 & 3.7 & 3.8 & 7.0 & . & 4.4 & 4.0 & 5.2 & 5.5 \\
\hline $\mathrm{CO}_{2}$ & .04 & .14 & .02 & .75 & .93 & 31 & - & .14 & .15 & .18 & .28 \\
\hline
\end{tabular}

\begin{tabular}{|c|c|c|c|c|c|c|c|c|c|c|c|}
\hline Ax(ppm) & 110 & 160 & 12 & 14 & 19 & 230 & $\cdot$ & 110 & 110 & 120 & 270 \\
\hline Ba & 450 & 9800 & 310 & 620 & 890 & 1100 & 510 & 760 & 720 & 760 & 910 \\
\hline Cd & 2.75 & 27.00 & .45 & 7.40 & 16.00 & 2.30 & 30 & 1.10 & 1.40 & 1.25 & 1.55 \\
\hline Co & 9 & 1000 & 42 & 33 & 51 & 1400 & $<4$ & 470 & 560 & 580 & 1700 \\
\hline$\alpha$ & $<1$ & 6 & 85 & 8 & 9 & 12 & 7 & 32 & 35 & 35 & 15 \\
\hline $\mathrm{Cr}$ & 39 & 1700 & 140 & 96 & 190 & 420 & 45 & 330 & 440 & 380 & 340 \\
\hline Mo & 980 & 1000 & $<4$ & 67 & 70 & 170 & 220 & $<4$ & $<4$ & 18 & 250 \\
\hline $\mathrm{Ni}$ & 120 & 2400 & 54 & 57 & 300 & 1700 & 69 & 380 & 580 & 530 & 1500 \\
\hline $\mathrm{Pb}$ & 70 & 460 & $<49$ & $<49$ & $<49$ & 1100 & $<49$ & 400 & 460 & 440 & 1200 \\
\hline Sr & 360 & 1100 & 290 & 360 & 420 & 1200 & 410 & 670 & 680 & 730 & 1100 \\
\hline $\mathbf{V}$ & 30 & 530 & 260 & 320 & 460 & 640 & 320 & 270 & 280 & 290 & 520 \\
\hline Zn & 720 & 940 & 110 & 110 & 390 & 470 & 320 & 360 & 360 & 390 & 430 \\
\hline Thickness (mom.) & - & 12.0 & 12.0 & - & - & 30.0 & - & 13.0 & 7.0 & 25.0 & 20.0 \\
\hline
\end{tabular}

\begin{tabular}{|c|c|c|c|c|c|c|c|c|c|c|c|}
\hline$Y$ (ppon) & .5 & 125 & 17 & 45 & 34 & 150 & 35 & 86 & 97 & 99 & 191 \\
\hline Le & • & 157 & • & 32 & • & - & - & • & - & . & 253 \\
\hline Co & $<10$ & 303 & $<10$ & 51 & $<10$ & 430 & 17 & 250 & 250 & 260 & 550 \\
\hline $\operatorname{Pr}$ & - & 34 & - & 6 & - & - & - &. & 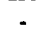 & - & 53 \\
\hline Nd & - & 148 & . & 29 & . & . & - & - & . & - & 230 \\
\hline Sm & - & 33 & . & 6 & . & - & . & . & - & . & so \\
\hline $\mathrm{Eu}$ & - & 8.4 & . & 1.6 & - & - & - & - & - & . & 12.6 \\
\hline Gd & - & 37 & . & 7 & - & - & - & - & - & - & 56 \\
\hline Tb & - & 6 & - & 2 & - & - & - & - & . & . & 8 \\
\hline Dy & - & 27 & - & . & - & - & - & . & . & - & 40 \\
\hline Ho & - & 65 & . & 1.6 & - & - & . & . & - & . & 9.9 \\
\hline $\mathbf{E} x$ & - & 18 & . & 5 & - & - & - & - & . & - & 28 \\
\hline $\operatorname{Tm}$ & - & 27 & - & .7 & - & - & - & - & . & . & 4 \\
\hline $\mathrm{Yb}$ & . & 18 & . & 4.3 & - & - & . & . & - & - & 26 \\
\hline La & - & 28 & - & .73 & - & - & - & - & - & . & 4 \\
\hline $\mathrm{Pt}$ & $<.005$ & .064 & .007 & .011 & .008 & .069 & - & - & - & - & .090 \\
\hline Pd & .001 & .0012 & .0046 & .0046 & .0046 & .0066 & - & . & - & - &.$\infty 2$ \\
\hline $\mathbf{R h}$ & $<.001$ & $<.001$ & $<001$ & $<.001$ & $<001$ & .0023 & - & - & . & . & .0046 \\
\hline
\end{tabular}


Table 18. Chemical composition of ferromanganese crusts as reported in OF87-281 (Hein and others, 1987).

\begin{tabular}{|c|c|c|c|c|c|c|c|c|c|c|c|}
\hline Sequencel & 094036 & 094037 & 094038 & 094039 & 094040 & 094041 & 094042 & 094042 & 094042 & 094043 & 094044 \\
\hline Latitude & 22.293 & 22.293 & 22.462 & 22.829 & 22.922 & 23.587 & 23.618 & 23.618 & 23.618 & 23.618 & 23.618 \\
\hline Longitude & 143.226 & 143.226 & 142.999 & 142.585 & 142.526 & 141.825 & 141.819 & 141.819 & 141.819 & 141.819 & 141.819 \\
\hline Depth & 1572 & 1572 & 1230 & 2450 & 2000 & 1400 & 1105 & 1105 & 1105 & 1105 & 1105 \\
\hline Lab* & D37-4 & D37-5 & D41-2B-A & D42-1 & D441 & DS1-1 & DS2-4-A & D52-4-B & Ds2-4-C & D52-5 & DS2-9 \\
\hline Si(wt.\%) & 9.60 & 9.30 & 2.05 & 4.20 & 7.10 & 5.90 & 8.00 & .18 & 9.90 & 530 & 6.40 \\
\hline Al & 3.30 & 3.20 & 57 & 1.10 & .82 & 2.70 & 254 & .11 & 4.20 & .10 & 2.28 \\
\hline Fe & 18.7 & 19.5 & 1.09 & 18.5 & 5.40 & 1.90 & 6.50 & .17 & 3.40 & 6.30 & 3.60 \\
\hline $\mathbf{M g}$ & 1.03 & 1.07 & 2.16 & .98 & 1.14 & 2.32 & 202 & 1.98 & 2.33 & 1.44 & 2.20 \\
\hline ca & 250 & 2.62 & 1.38 & 2.19 & 1.26 & 2.76 & 1.98 & 1.48 & 3.10 & 1.54 & 1.96 \\
\hline Na & 1.80 & 1.67 & 1.69 & 1.74 & 3.10 & 2.70 & 1.78 & 2.40 & 2.30 & 2.01 & 1.90 \\
\hline $\mathbf{K}$ & .67 & .65 & 1.55 & .42 & .91 & 1.30 & 1.61 & 1.04 & 1.89 & 1.40 & 1.63 \\
\hline $\mathbf{T i}$ & .86 & .78 & .04 & .83 & .04 & .14 & .14 & .01 & .23 & .07 & .13 \\
\hline $\mathbf{P}$ & .42 & .44 & .03 & .47 & $<.03$ & .06 & .11 & $<03$ & .09 & .16 & .08 \\
\hline $\mathrm{Mn}$ & 10.1 & 11.5 & 44.3 & 15.8 & 34.7 & 33.4 & 29.6 & 47.2 & 27.1 & 35.0 & 33.7 \\
\hline $\mathrm{H}_{2} \mathrm{O}^{-}$ & 9.7 & 7.3 & 6.2 & 16.2 & 6.2 & 5.1 & 4.4 & 4.2 & 27 & 6.5 & 4.7 \\
\hline $\mathrm{H}_{2} \mathrm{O}^{+}$ & 73 & 7.5 & 73 & 7.0 & 5.7 & 5.5 & 6.5 & 8.6 & 5.9 & 6.5 & 6.9 \\
\hline$\infty_{2}$ & .25 & .28 & .03 & .46 & .01 & 1.50 & .04 & .02 & .03 & .03 & .02 \\
\hline
\end{tabular}

\begin{tabular}{|c|c|c|c|c|c|c|c|c|c|c|c|}
\hline Ax(ppon) & 250 & 280 & 78 & 280 & 50 & 34 & 57 & 28 & 21 & 98 & So \\
\hline Be & 980 & 980 & 2100 & 920 & 1700 & 870 & 2000 & 530 & 580 & 1300 & 1300 \\
\hline $\mathrm{Cd}$ & 1.65 & 1.60 & 23.00 & 2.15 & $<20$ & 10.00 & 9.80 & .50 & 5.90 & $<.20$ & 6.90 \\
\hline Co & 1400 & 1600 & 31 & 2600 & 170 & 44 & 87 & 190 & 35 & 230 & 100 \\
\hline$\alpha$ & 21 & 17 & 3 & 5 & 2 & 2 & 3 & 0 & 12 & 7 & 5 \\
\hline Cr & 360 & 310 & 120 & 200 & 300 & 60 & 67 & 18 & 100 & 62 & 62 \\
\hline Mo & 160 & 210 & 1200 & 180 & 670 & 250 & 230 & 610 & 490 & 530 & 420 \\
\hline $\mathrm{Ni}$ & 1200 & 1400 & 360 & 2200 & 130 & 150 & 210 & 30 & 190 & 280 & 270 \\
\hline $\mathbf{P b}$ & 1100 & 1300 & 30 & 1400 & 15 & 18 & 28 & $\theta$ & 43 & 38 & 43 \\
\hline Sr & 1000 & 1200 & 680 & 1300 & 280 & 580 & 700 & 450 & 500 & 520 & 550 \\
\hline v & 500 & 530 & 130 & 540 & $<4$ & 160 & 130 & $<4$ & 58 & $<4$ & 59 \\
\hline $\mathbf{Z n}$ & 460 & 460 & 440 & 530 & 35 & 190 & 180 & 18 & 210 & 140 & 200 \\
\hline Thicknesu(mm.) & 25.0 & 20.0 & - & 2.0 & - & - & - & - & $\cdot$ & $\cdot$ & $\cdot$ \\
\hline
\end{tabular}

\begin{tabular}{|c|c|c|c|c|c|c|c|c|c|c|c|}
\hline$Y($ ppon) & 150 & 170 & 60 & 201 & 8 & 9 & 13 & .5 & 16 & 10 & 12 \\
\hline La & - & • & 13 & 313 & 5 & 7 & - & • & • & 12 & - \\
\hline Co & 520 & 540 & 18 & 790 & 14 & 9 & 18 & $<10$ & 18 & 21 & 27 \\
\hline $\mathrm{Pr}$ & - & - & 2 & 70 & 1 & 1 & - & $\cdot$ & . & 2 & . \\
\hline Nd & - & - & 11 & 290 & 5 & 5 & - & . & . & 9 & - \\
\hline Sm & - & - & 2 & 64 & 1 & 1 & - & - & - & 2 & - \\
\hline Eu & - & - & .68 & 16.2 & 39 & .26 & . & - & - & .51 & - \\
\hline Gd & - & - & 3 & 68 & 1 & 1 & - & - & - & 2 & - \\
\hline Tb & - & - & 1 & 10 & $<1$ & $<1$ & - & - & - & $<1$ & - \\
\hline Dy & - & - & 3 & 51 & 1 & $<1$ & - & - & - & $<1$ & . \\
\hline Ho & - & - & .96 & 11.3 & .4 & .24 & - & - & - & .37 & - \\
\hline Ex & - & - & 3.1 & 31 & 1.4 & .8 & - & - & - & 1.2 & - \\
\hline $\operatorname{Tm}$ & - & - & 3 & 4.6 & $<2$ & $<2$ & . & - & - & $<2$ & - \\
\hline Yb & - & - & 22 & 29 & 1.5 & 8 & . & . & - & 1 & - \\
\hline Lu & - & - & .4 & 4.5 & .21 & .14 & - & - & - & .17 & - \\
\hline $\mathbf{P t}$ & - & - & .008 & .130 & .013 & $<005$ & - & - & - & $<005$ & - \\
\hline Pd & . & - & .001 & .0028 & .0028 & .0012 & - & - & - & .0012 & - \\
\hline $\mathbf{R h}$ & - & - & $<.001$ & $<.001$ & $<\infty 01$ & $<. \infty 01$ & - & - & - & $<\infty 1$ & . \\
\hline
\end{tabular}


Table 18. Chemical composition of ferromanganese crusts as reported in OF87-281 (Hein and others, 1987).

\begin{tabular}{|c|c|c|c|c|c|c|c|c|c|c|c|}
\hline Sequenco" & 094045 & 094046 & 094047 & 094048 & 094049 & 094050 & 094051 & 094052 & 094053 & 094054 & 094055 \\
\hline Latitude & 23.817 & 24.028 & 24.028 & 24.075 & 24.075 & 24.043 & 24.043 & 23.193 & 23.193 & 23.193 & 23.193 \\
\hline Longitudo & 141.727 & 141.588 & 141.588 & 141.553 & 141.553 & 141.670 & 141.670 & 141.395 & 141.395 & 141.395 & 141.395 \\
\hline Depth & 1640 & 1780 & 1780 & 1500 & 1500 & 1730 & 1730 & 1080 & 1080 & 1080 & 1080 \\
\hline Lab* & DS4-2 & DS5-3 & DS5-4 & D56-1-B & D56-2 & DS7.2 & DS7-4 & D58-3 & D58-4 & DS8-7 & DS8.11 \\
\hline$S i(w t \%)$ & 14.8 & 16.5 & .19 & 14.7 & 6.90 & 12.0 & 17.4 & 3.80 & 2.60 & 4.50 & 5.60 \\
\hline A I & 6.10 & 5.90 & .14 & 6.10 & 265 & 3.40 & 7.30 & 1.41 & 91 & 1.23 & 1.54 \\
\hline Pe & 6.50 & 4.40 & .11 & 5.30 & 1.61 & 9.10 & 5.60 & 20.4 & 20.0 & 20.0 & 19.9 \\
\hline Ms & 3.20 & 1.99 & 2.41 & 2.43 & 1.89 & 2.11 & 3.50 & 1.02 & 1.03 & 99 & 1.00 \\
\hline Ca & 5.10 & 3.70 & 1.69 & 4.60 & 7.70 & 2.37 & 6.60 & 2.22 & 2.19 & 2.14 & 2.11 \\
\hline Ne & 1.96 & 2.40 & 2.07 & 2.20 & 2.40 & 1.94 & 1.68 & 1.44 & 1.39 & 1.32 & 1.37 \\
\hline $\mathbf{K}$ & 1.49 & 2.03 & 1.65 & 1.65 & 1.09 & 1.60 & 1.02 & A2 & 34 & 38 & 12 \\
\hline $\mathbf{T i}$ & .43 & 30 & .01 & .35 & .13 & .16 & 30 & 56 & 53 & 56 & .57 \\
\hline $\mathbf{P}$ & .10 & .09 & .03 & .12 & .05 & .13 & .07 & .62 & .64 & .61 & 58 \\
\hline Mn & 14.5 & 15.5 & 44.2 & 15.7 & 27.4 & 19.9 & 10.5 & 16.9 & 17.7 & 16.8 & 15.3 \\
\hline $\mathrm{H}_{2} \mathrm{O}^{\circ}$ & 29 & 28 & 5.9 & 27 & 3.4 & 4.8 & 1.7 & 11.1 & 13.7 & 14.9 & 15.8 \\
\hline $\mathrm{H}_{2}^{2} \mathrm{O}^{+}$ & 3.9 & 3.5 & 6.7 & 6.0 & 5.4 & 5.5 & 2.8 & 8.1 & 8.3 & 7.8 & 7.4 \\
\hline $\mathrm{CO}_{2}$ & .03 & .02 & .04 & 59 & 5.90 & .02 & .01 & 31 & 34 & 36 & .33 \\
\hline
\end{tabular}

\begin{tabular}{|c|c|c|c|c|c|c|c|c|c|c|c|}
\hline Axppon) & 14 & 18 & 48 & 24 & 20 & 62 & 22 & 410 & 430 & 410 & 380 \\
\hline Ba & 1000 & $11 \infty$ & 900 & 660 & 910 & 1000 & 790 & 970 & 930 & 950 & 900 \\
\hline $\mathrm{Cd}$ & 9.60 & 2200 & 13.00 & 14.00 & 4.20 & 12.00 & 2.10 & 2.45 & 2.65 & 2.42 & 2.35 \\
\hline $\mathrm{Co}_{0}$ & 33 & 20 & 40 & 61 & 40 & 220 & 220 & 3000 & 3300 & 3000 & 2600 \\
\hline$a$ & 14 & 17 & 0 & 11 & 4 & 17 & 77 & 13 & 12 & 13 & 17 \\
\hline a & 200 & 130 & 120 & 210 & 44 & 77 & 310 & 200 & 200 & 220 & 210 \\
\hline Mo & 83 & 55 & 520 & 64 & 100 & 240 & 140 & 530 & 570 & 510 & 450 \\
\hline $\mathrm{Ni}$ & 170 & 240 & 340 & 340 & 73 & 300 & 110 & 2000 & 2100 & 2000 & 1800 \\
\hline $\mathrm{Pb}$ & 23 & 28 & 10 & ss & 20 & 20 & 5 & 2100 & 240 & 2100 & 2000 \\
\hline Sr & 800 & 630 & 590 & 680 & 620 & 480 & 620 & 1500 & 1500 & 1500 & 1300 \\
\hline $\mathbf{v}$ & 310 & 190 & 150 & 240 & 81 & 170 & 210 & 730 & 800 & 730 & 680 \\
\hline $\mathbf{Z n}$ & 220 & 350 & 260 & 400 & 85 & 210 & 110 & 490 & 550 & 510 & 480 \\
\hline Thickress(mm.) & - & $\cdot$ & 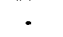 & - & - & $\cdot$ & - & 18.0 & 8.0 & 15.0 & 30.0 \\
\hline
\end{tabular}

\begin{tabular}{|c|c|c|c|c|c|c|c|c|c|c|c|}
\hline$Y(p p m)$ & 19 & 22 & 6.9 & 25 & 13 & 14 & 13 & 240 & 240 & 257 & 247 \\
\hline La & - & 13 & - & - & - & - & - & - & - & 354 & 320 \\
\hline co & 39 & 63 & $<10$ & 73 & 14 & 31 & 15 & 580 & 570 & 600 & 590 \\
\hline $\mathbf{P z}_{\mathbf{z}}$ & - & 7 & • & - & - & - & $\cdot$ & - & • & 73 & 67 \\
\hline Nd & - & 27 & - & - & - & - & $\cdot$ & - & $\cdot$ & 310 & 290 \\
\hline Sm & - & 5 & - & - & - & - & - & - & - & 67 & 62 \\
\hline Eu & - & 1.31 & - & - & - & - & - & - & - & 16.9 & 15.7 \\
\hline Gd & - & 5 & - & - & - & $\cdot$ & - & - & • & 75 & 70 \\
\hline $\mathrm{Tb}$ & - & $<1$ & - & - & - & - & - & - & - & 11 & 10 \\
\hline Dy & - & $<1$ & - & - & - & - & - & - & - & 58 & 54 \\
\hline Ho & - & .76 & - & - & - & $\cdot$ & - & - & - & 129 & 12.3 \\
\hline $\mathrm{Er}$ & - & 2.2 & - & - & - & - & - & - & - & 36 & 34 \\
\hline Tm & • & 3 & - & - & - & $\cdot$ & - & - & - & 5.2 & 5 \\
\hline $\mathbf{Y b}$ & - & 23 & - & - & - & - & - & - & - & 33 & 32 \\
\hline Lu & - & 37 & • & $\cdot$ & • & $\cdot$ & - & $\cdot$ & - & 5.1 & 5 \\
\hline $\mathrm{Pt}$ & - & .005 & - & - & $\cdot$ & - & - & - & - & .140 & .190 \\
\hline Pd & - & .0022 & - & - & - & - & - & - & - & .0014 & .0014 \\
\hline $\mathbf{R h}$ & - & $<. \infty 1$ & - & - & . & - & - & - & - & .002 & .0018 \\
\hline
\end{tabular}


Table 18. Chemical composition of ferromanganese crusts as reported in OF87-281 (Hein and others, 1987).

\begin{tabular}{|c|c|c|c|c|c|c|c|c|}
\hline Sequencelt & 094056 & 094057 & 094058 & 094059 & 094060 & 094061 & 094062 & 094063 \\
\hline Letinde & 23.095 & 22.447 & 22.447 & 22.447 & 25.122 & 25.122 & 20.648 & 25.122 \\
\hline Longitude & 141.388 & 141.928 & 141.928 & 141.928 & 141.273 & 141.273 & 144.449 & 141.273 \\
\hline Depth & 1470 & 1660 & 1660 & 1660 & 1620 & 1620 & 2200 & 1620 \\
\hline Lab" & D59-4 & D71-1 & D71-2 & D71-4 & D73-1A & D73-1P & D21-1.A & D73-2 \\
\hline Si(wt. $\left.\sigma_{0}\right)$ & 5.70 & 10.7 & 11.3 & 10.3 & 16.9 & 55 & 128 & 5.40 \\
\hline Al & 1.91 & 3.80 & 296 & 3.80 & 5.70 & .51 & 4.60 & 1.64 \\
\hline Fo & 21.1 & 17.5 & 18.0 & 17.0 & 6.40 & 34 & 14.7 & 19.5 \\
\hline Mg & .92 & 1.02 & 1.01 & 1.15 & 225 & 2.05 & 1.44 & 1.02 \\
\hline C. & 2.07 & 2.42 & 2.28 & 2.63 & 4.00 & 1.61 & 3.30 & 2.21 \\
\hline $\mathbf{N a}$ & 1.47 & 1.74 & 1.58 & 1.68 & 2.40 & 2.05 & 1.65 & 1.60 \\
\hline $\mathbf{K}$ & .46 & 85 & .69 & 83 & .95 & 1.05 & .86 & 49 \\
\hline $\mathbf{T i}$ & .69 & .76 & .66 & .72 & .42 & .02 & .75 & 80 \\
\hline P & .58 & 37 & .40 & 38 & .08 & .01 & .32 & 56 \\
\hline Mn & 13.6 & 9.80 & 11.8 & 10.7 & 12.7 & 45.2 & 9.50 & 15.2 \\
\hline $\mathrm{H}_{2} \mathrm{O}^{\circ}$ & 10.1 & 8.6 & 13.6 & 8.1 & 2.6 & 6.0 & 6.9 & 11.0 \\
\hline $\mathrm{H}_{2}^{2} \mathrm{O}^{+}$ & 6.2 & 6.7 & 6.1 & 6.6 & 3.5 & 7.7 & 4.7 & 8.4 \\
\hline $\mathrm{C}_{2}$ & .28 & .14 & .25 & .21 & .14 & 30 & .24 & .25 \\
\hline As(ppon) & 380 & 220 & 250 & 220 & 20 & 52 & 150 & 320 \\
\hline Ba & 1100 & 910 & 910 & 910 & 460 & 5000 & 970 & 940 \\
\hline Cd & 1.88 & 1.40 & 1.70 & 1.80 & 8.00 & 285 & 2.20 & 2.35 \\
\hline Co & 2100 & 900 & 1100 & 970 & 31 & 6 & 790 & 2700 \\
\hline$a$ & 15 & 15 & 16 & 16 & 6 & 3 & 330 & 15 \\
\hline $\mathrm{Cu}$ & 220 & 470 & 430 & 440 & 160 & 43 & 510 & 180 \\
\hline Mo & 350 & 160 & 250 & 210 & 85 & 200 & 100 & 260 \\
\hline $\mathrm{Ni}$ & 1500 & 1100 & 1400 & 1300 & 170 & 54 & 1500 & 1900 \\
\hline $\mathrm{Pb}$ & 1800 & 860 & 1100 & 870 & 20 & 5 & 890 & 1600 \\
\hline St & 1400 & 1000 & 1100 & 1100 & 430 & 570 & 930 & 1300 \\
\hline $\mathbf{v}$ & 700 & 440 & 490 & 470 & 310 & no & 390 & 640 \\
\hline $\mathbf{Z n}$ & 520 & 440 & 430 & 420 & 290 & 50 & 400 & 500 \\
\hline Thiclonese(mm.) & 25.0 & 40.0 & 35.0 & 320 & - & - & 12.0 & 5.0 \\
\hline$Y(p p m)$ & 220 & 150 & 195 & 150 & 25 & 22 & 104 & 200 \\
\hline Le & $\cdot$ & - & 246 & $\cdot$ & - & 11 & 119 & - \\
\hline Ce & 610 & 380 & 440 & 360 & $<10$ & 4 & 215 & 830 \\
\hline $\operatorname{Pr}$ & - & - & so & - & - & 2 & 27 & • \\
\hline Nd & • & - & 220 & . & - & 11 & 118 & - \\
\hline Sm & - & - & 47 & - & - & 2 & 26 & - \\
\hline Eu & - & - & 12.2 & - & - & .83 & 6.9 & - \\
\hline Gd & - & - & 55 & - & - & 3 & 30 & - \\
\hline Tb & - & - & 9 & - & - & 1 & 5 & - \\
\hline Dy & . & - & 39 & - & - & 3 & . & . \\
\hline Ho & - & - & 9.8 & - & - & .73 & 55 & - \\
\hline Ex & - & - & 28 & - & - & 23 & 15 & - \\
\hline $\mathrm{Tm}$ & . & - & 4.1 & - & - & 3 & 2.3 & . \\
\hline$Y_{b}$ & - & - & 26 & - & - & 2.1 & 15 & - \\
\hline Lu & - & - & 4.1 & - & - & 37 & 23 & - \\
\hline $\mathrm{Pt}$ & - & - & .110 & - & - & .015 & .075 & - \\
\hline $\mathrm{Pd}$ & - & - & .002 & . & . & .0066 & .0032 & . \\
\hline $\mathbf{R h}$ & - & - & .0018 & - & - & $<.001$ & $<.001$ & - \\
\hline
\end{tabular}

